\title{
Summary of Significant Floods in the United States and Puerto Rico, 1994 Through 1998 Water Years
}

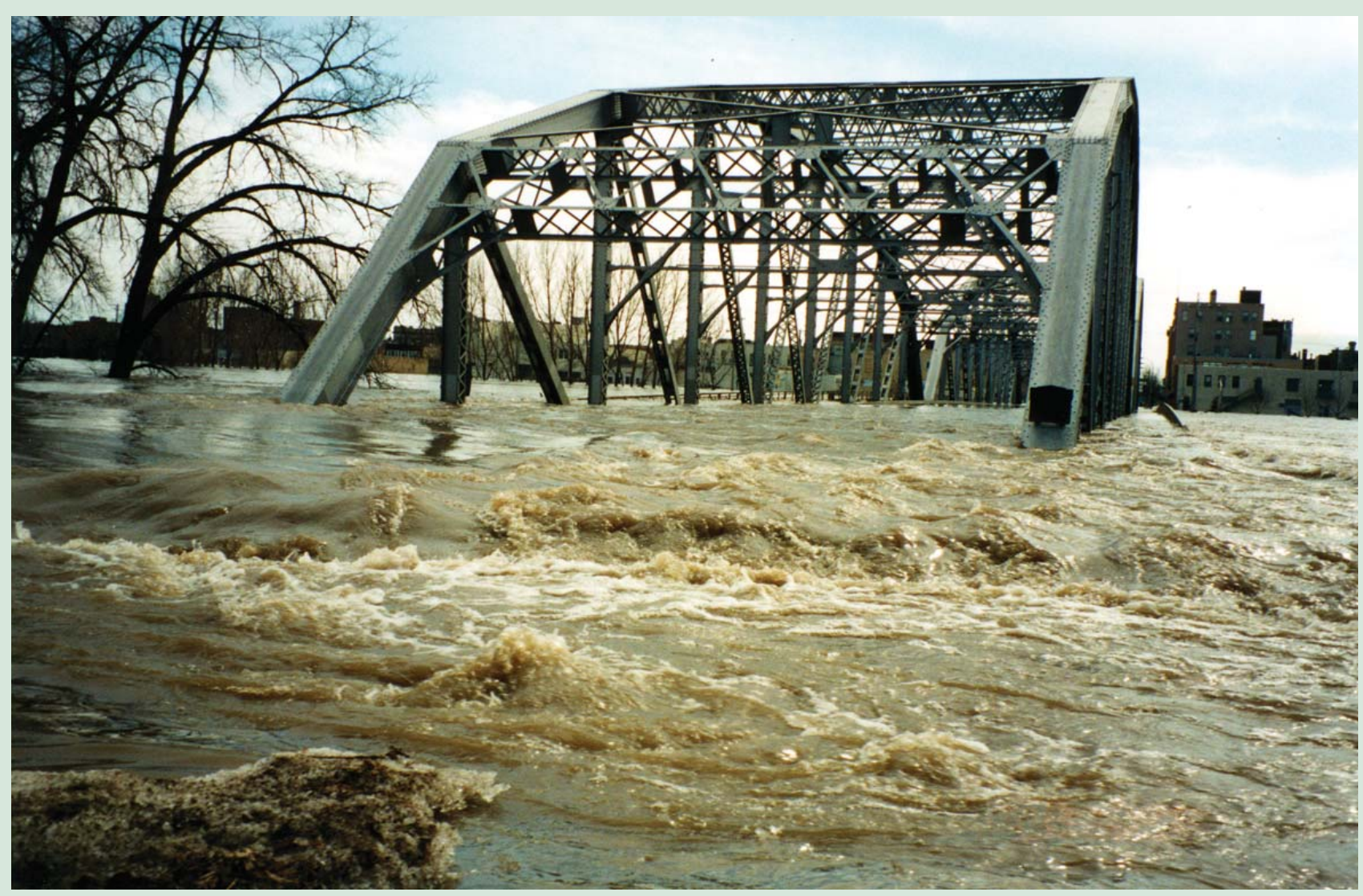

Scientific Investigations Report 2005-5194 


\title{
Summary of Significant Floods in the United States and Puerto Rico, 1994 Through 1998 Water Years
}

\author{
By C.A. Perry
}

Scientific Investigations Report 2005-5194 


\title{
U.S. Department of the Interior Gale A. Norton, Secretary
}

\author{
U.S. Geological Survey \\ P. Patrick Leahy, Acting Director
}

\section{U.S. Geological Survey, Reston, Virginia: 2005}

For sale by U.S. Geological Survey, Information Services

Box 25286, Denver Federal Center

Denver, CO 80225

For more information about the USGS and its products:

Telephone: 1-888-ASK-USGS

World Wide Web: http://www.usgs.gov/

Any use of trade, product, or firm names in this publication is for descriptive purposes only and does not imply endorsement by the U.S. Government.

Although this report is in the public domain, permission must be secured from the individual copyright owners to reproduce any copyrighted materials contained within this report.

Suggested citation:

Perry, C.A., 2005, Summary of significant floods in the United States and Puerto Rico, 1994 through 1998 water years: U.S. Geological Survey Scientific Investigations Report 2005-5194, 327 p.

Front cover: Spring 1997 flood on the Red River of the North in Grand Forks, North Dakota (photograph taken by Steven Norbeck, U.S. Geological Survey, Grand Forks, North Dakota).

Back cover: Aftermath of fire during spring 1997 flood in Grand Forks, North Dakota (photograph taken by U.S. Geological Survey personnel, Grand Forks, North Dakota).

Prepared by the U.S. Geological Survey in Lawrence, Kansas (http://ks.water.usgs.gov) 


\section{Contents}

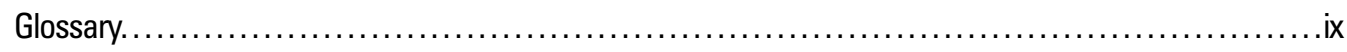

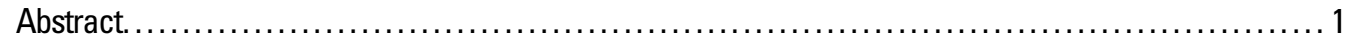

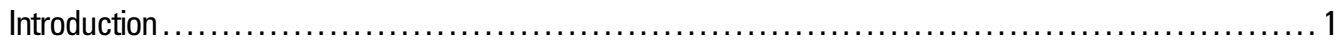

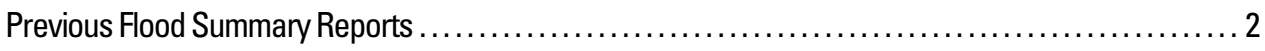

Determination of Flood Stages and Discharges.................................... 2

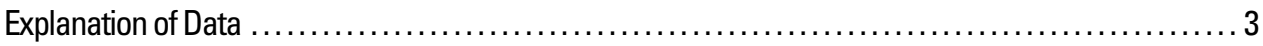

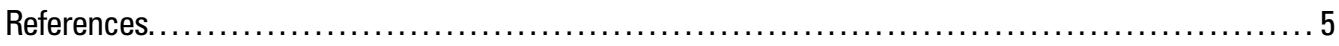

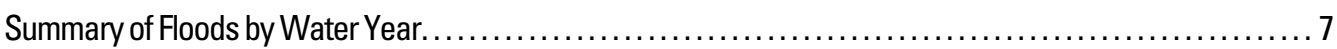

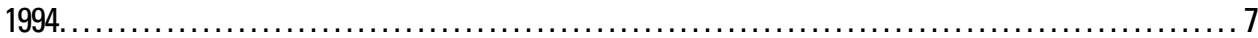

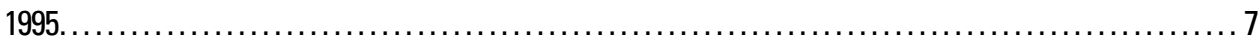

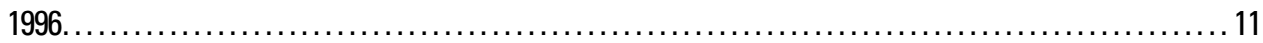

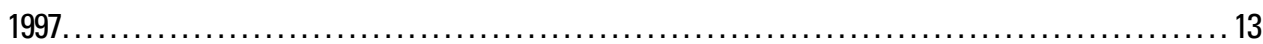

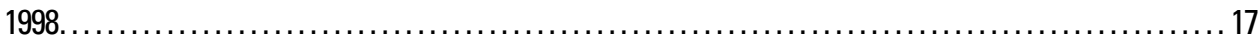

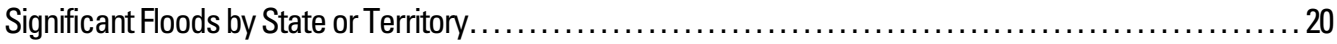

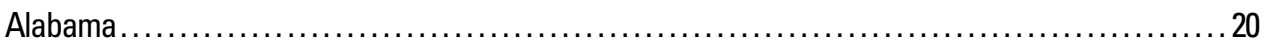

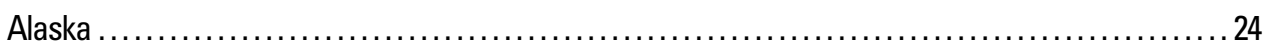

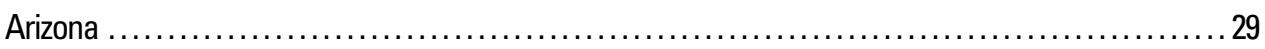

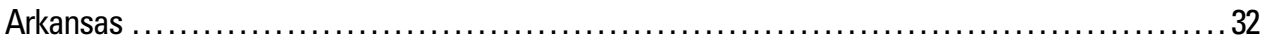

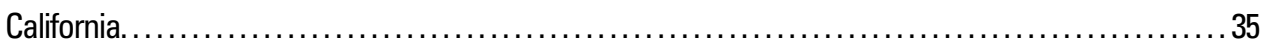

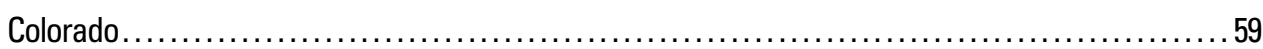

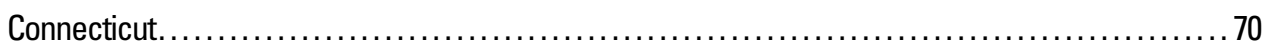

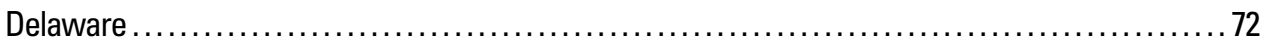

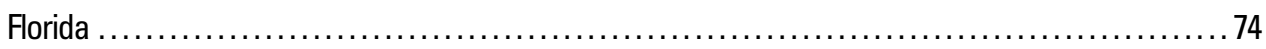

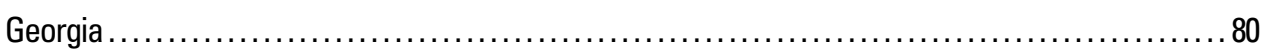

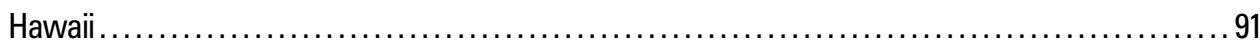

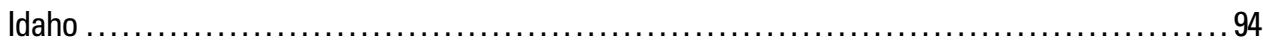

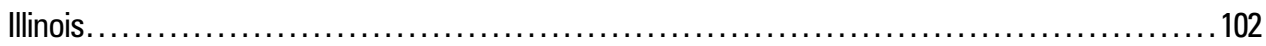

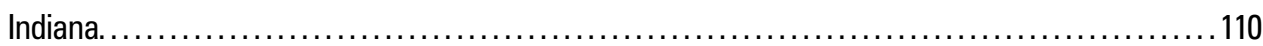

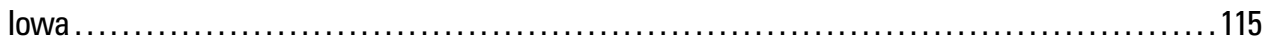

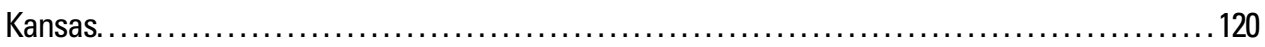

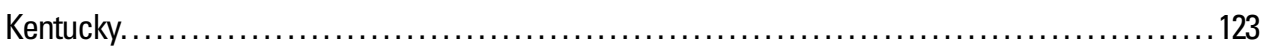

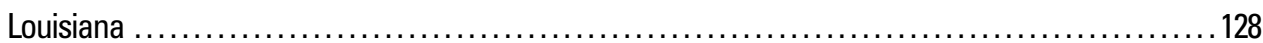

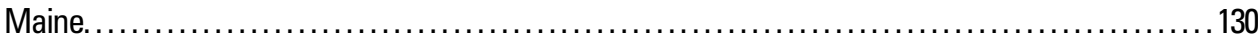

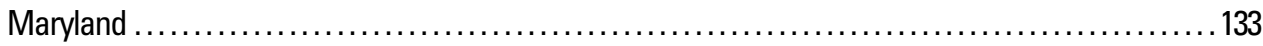

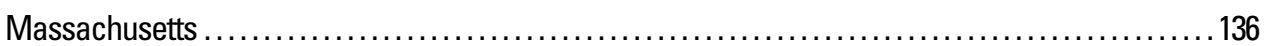

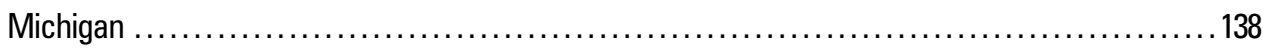

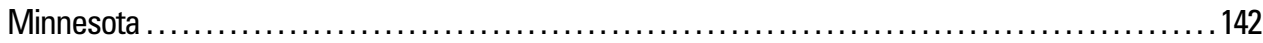

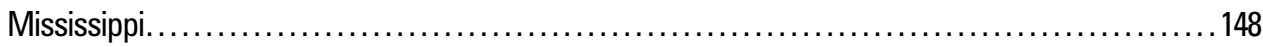

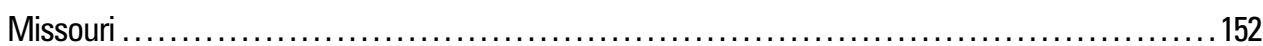

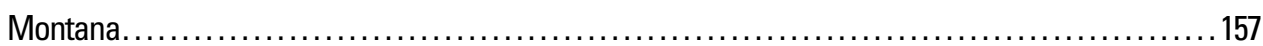

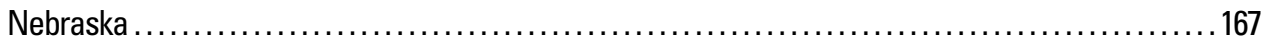

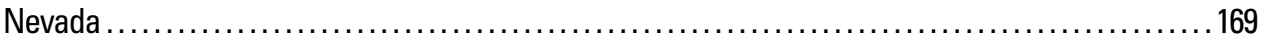




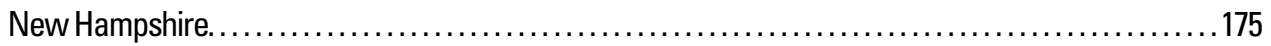

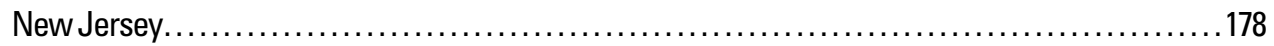

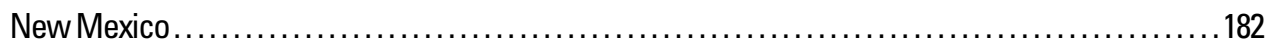

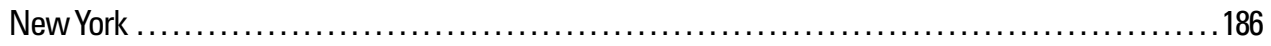

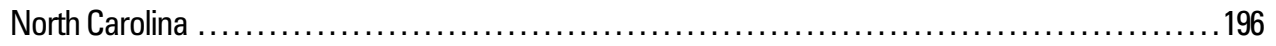

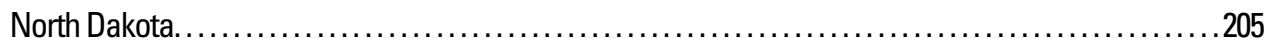

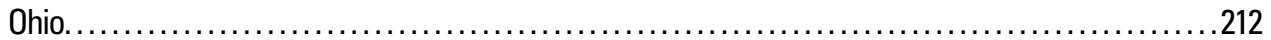

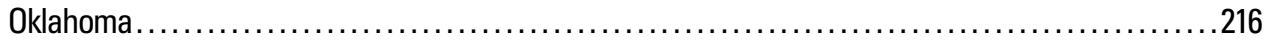

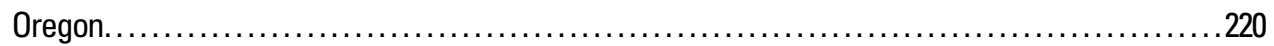

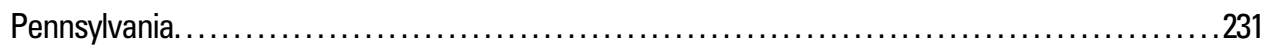

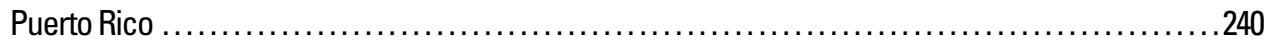

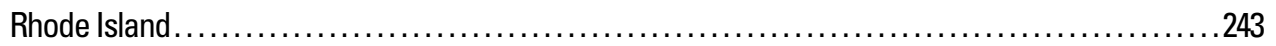

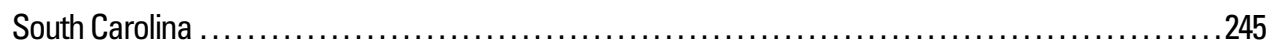

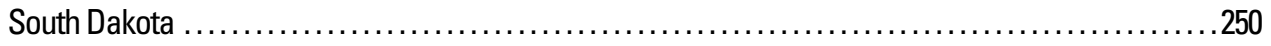

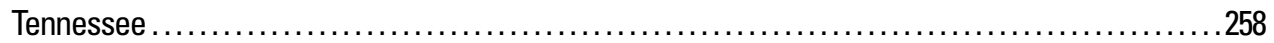

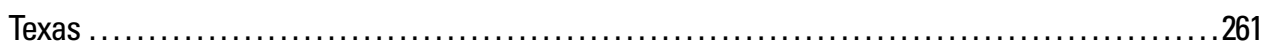

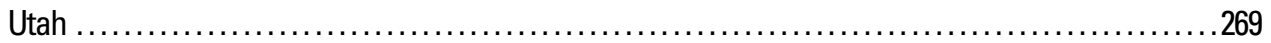

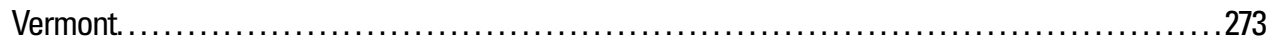

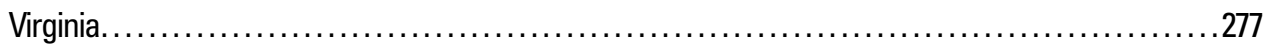

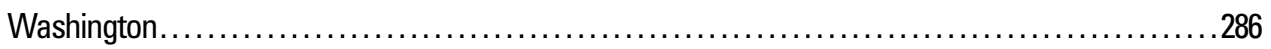

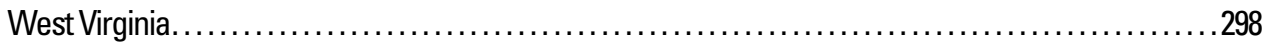

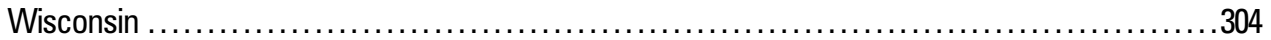

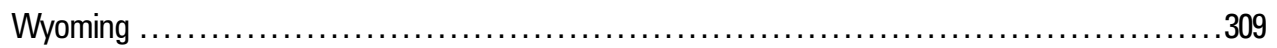

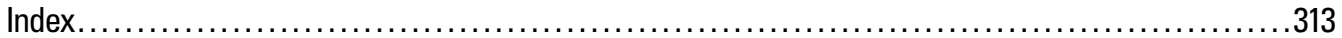

\section{Figures}

1. Graph showing example of a stage-discharge relation and upward extension of relation to observed maximum stage.

2. Graph showing maximum discharge in relation to drainage area and envelope curve for a region. ....

3-12. Map showing:

3. Percentage of streamgages in each State or territory recording greater than approximate 20-year recurrence-interval flooding during 1994 water year. ...

4. Standardized 1994 water year precipitation deviations from long-term mean precipitation in conterminous United States

5. Percentage of streamgages in each State or territory recording greater than approximate 20-year recurrence-interval flooding during 1995 water year. .

6. Standardized 1995 water year precipitation deviations from long-term mean precipitation in conterminous United States

7. Percentage of streamgages in each State or territory recording greater than approximate 20-year recurrence-interval flooding during 1996 water year.

8. Standardized 1996 water year precipitation deviations from long-term mean precipitation in conterminous United States. ...

9. Percentage of streamgages in each State or territory recording greater than approximate 20-year recurrence-interval flooding during 1997 water year. 
10. Standardized 1997 water year precipitation deviations from long-term mean precipitation in

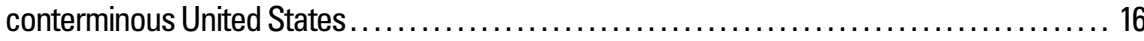

11. Percentage of streamgages in each State or territory recording greater than approximate 20 -year recurrence-interval flooding during 1998 water year.

12. Standardized 1998 water year precipitation deviations from long-term mean precipitation in conterminous United States

13-63. Maps showing location of streamgages with significant floods during 1994-98 water years for:

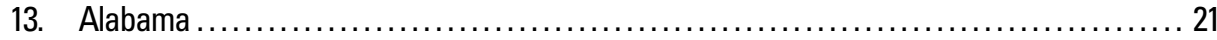

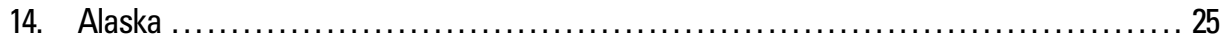

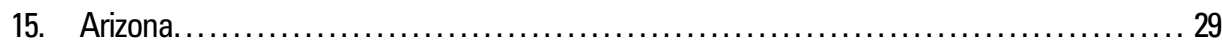

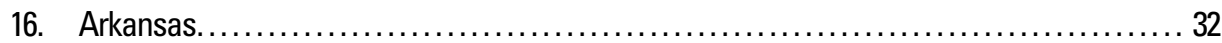

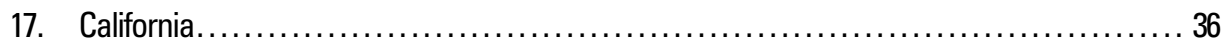

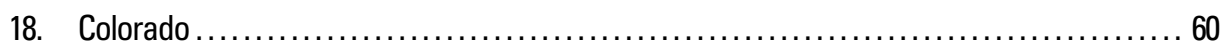

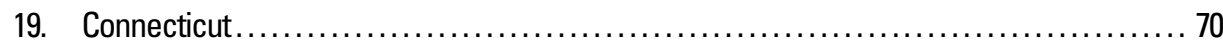

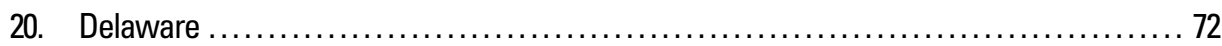

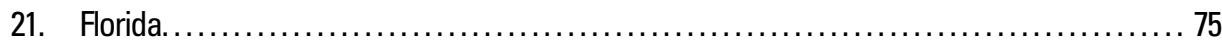

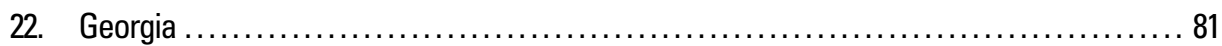

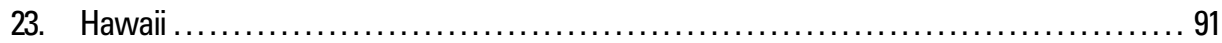

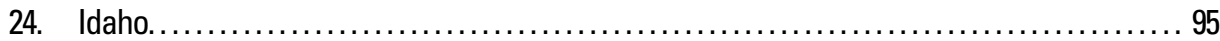

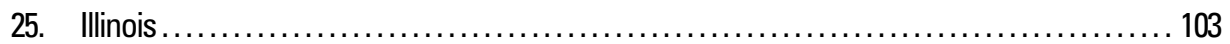

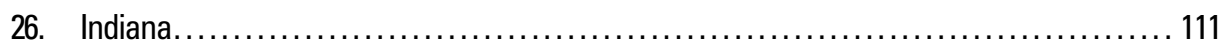

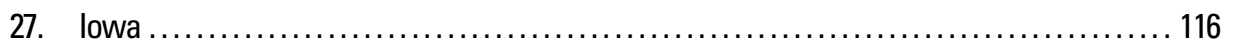

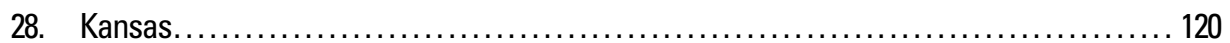

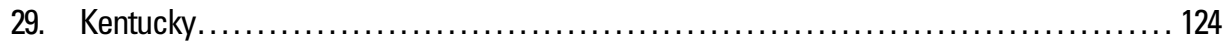

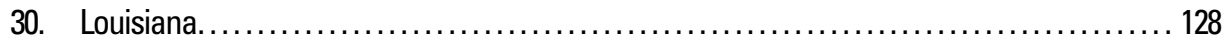

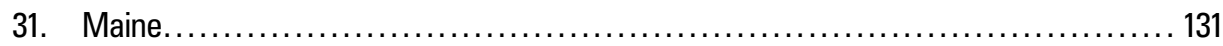

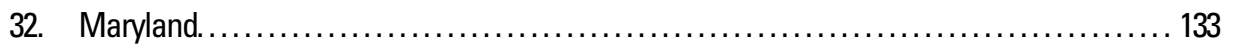

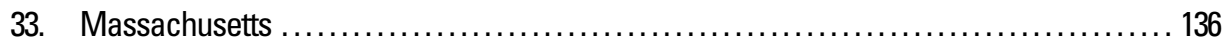

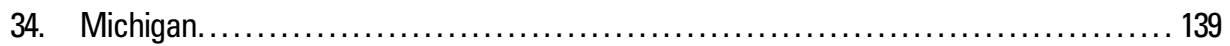

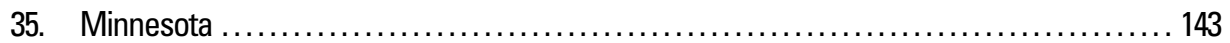

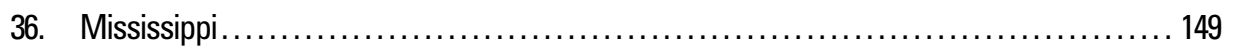

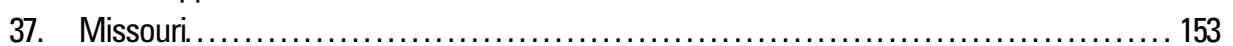

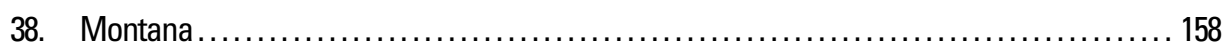

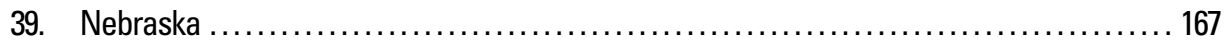

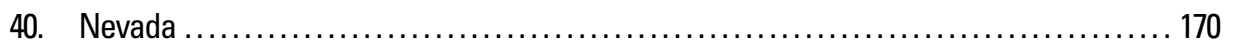

41. New Hampshire................................................. 175

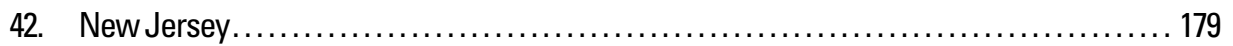

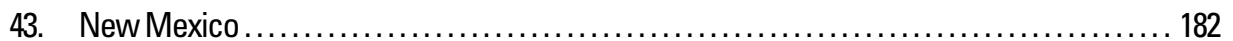

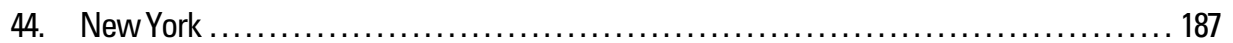

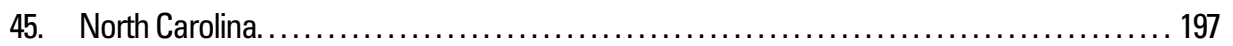

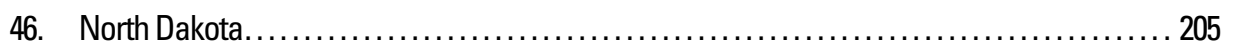

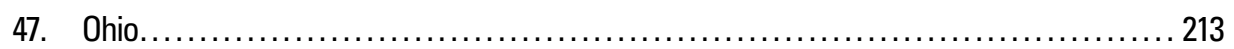

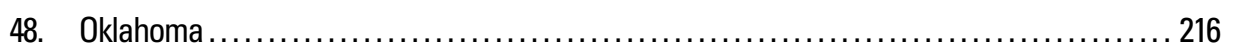

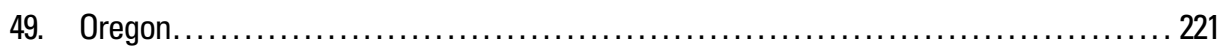

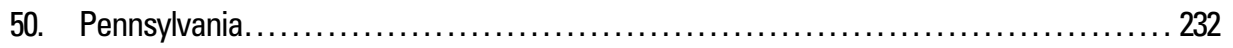

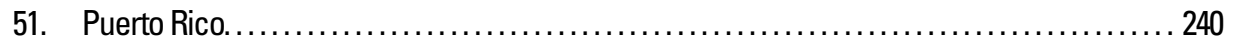

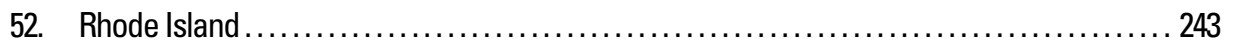

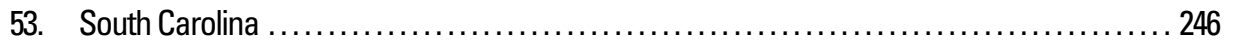

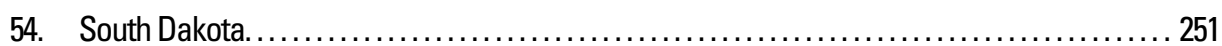




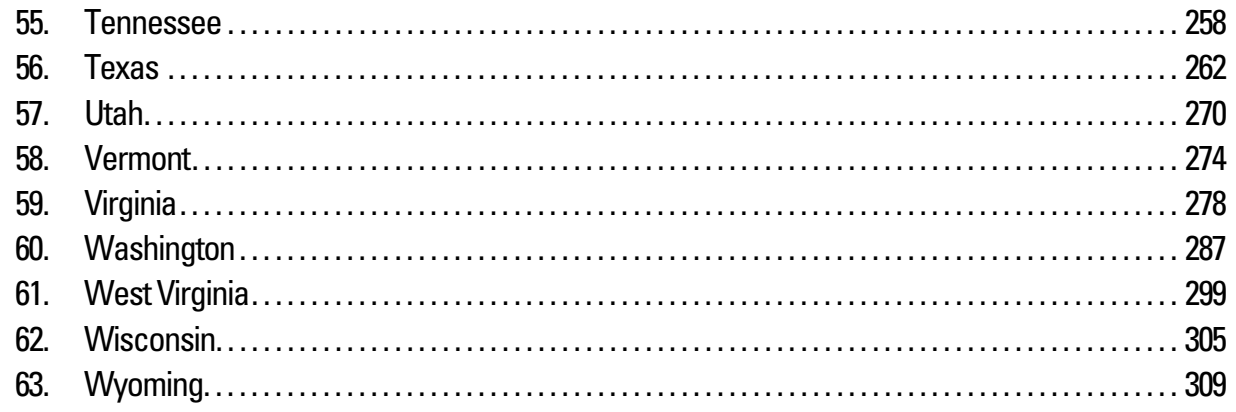

\section{Tables}

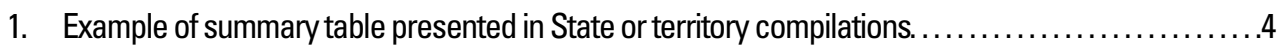

2-52. Maximum stage and discharge for period of record for streamgages having significant floods during 1994-98 water years in:

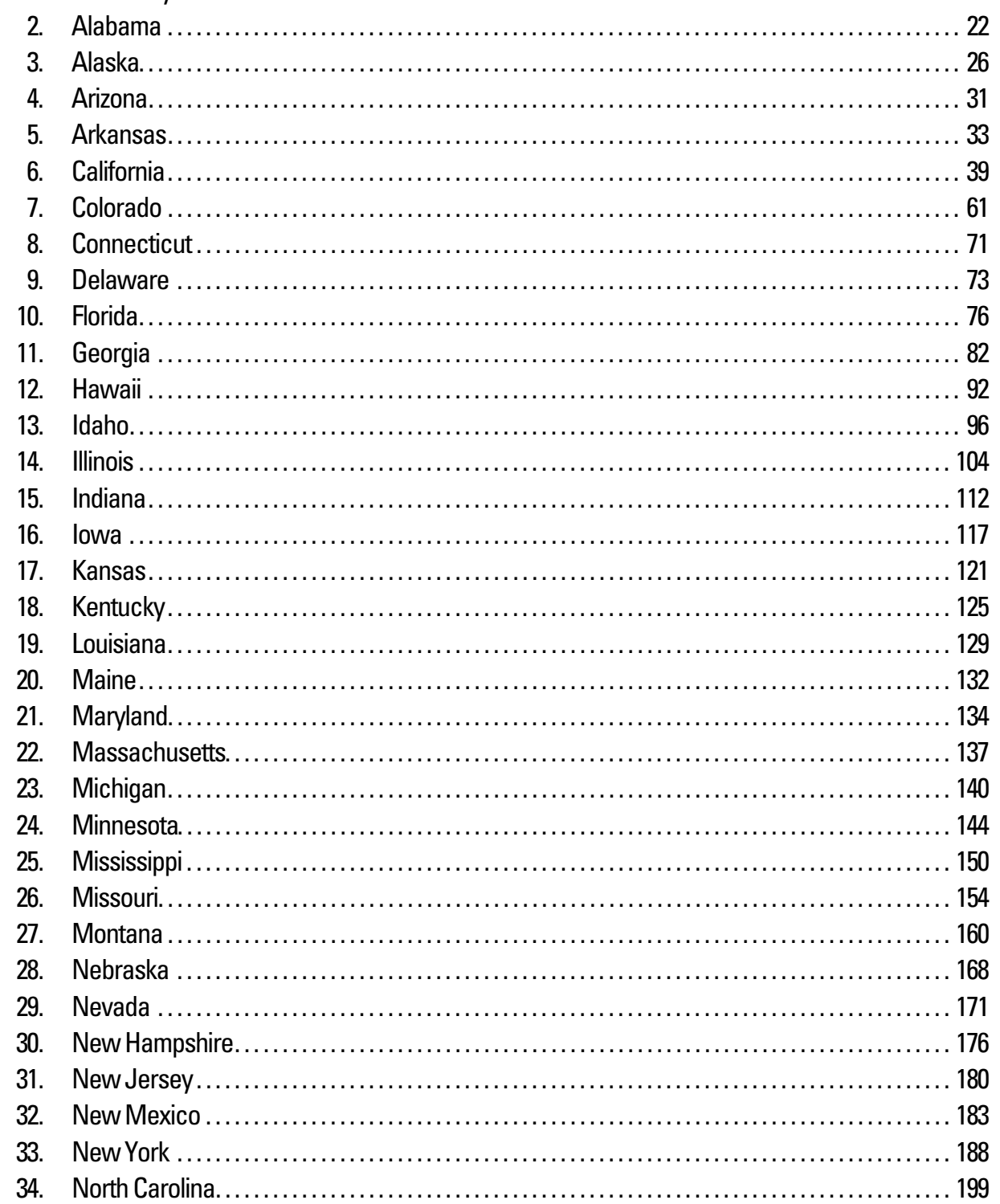




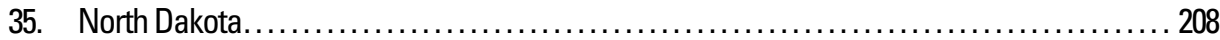

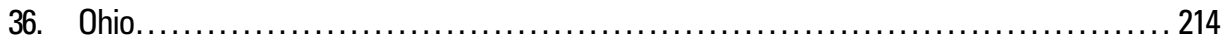

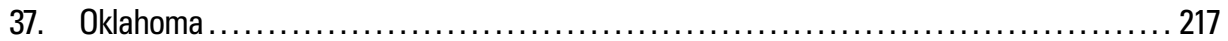

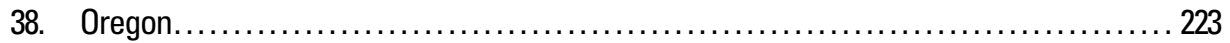

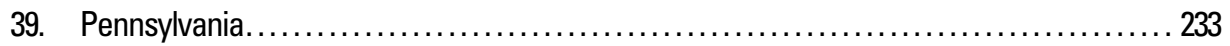

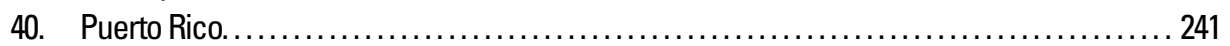

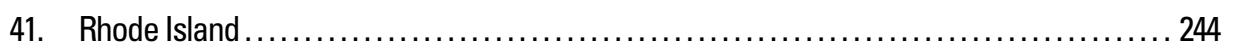

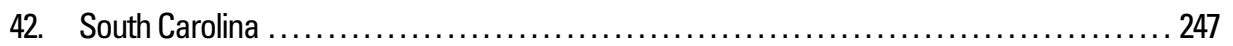

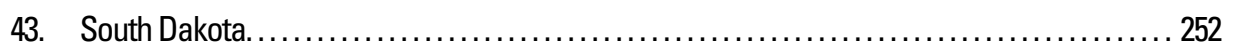

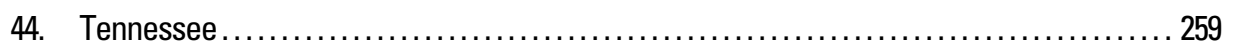

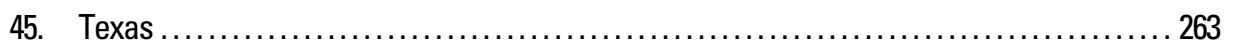

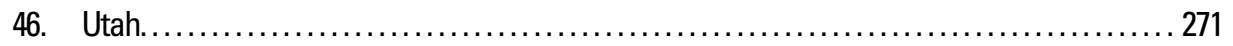

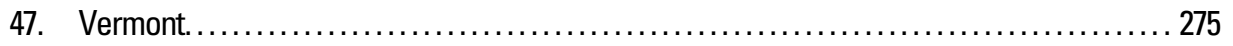

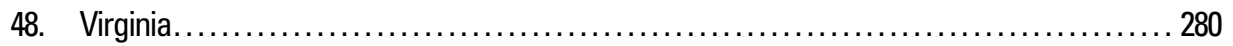

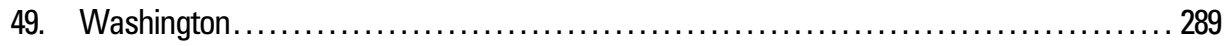

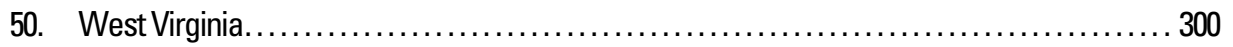

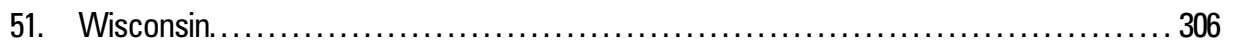

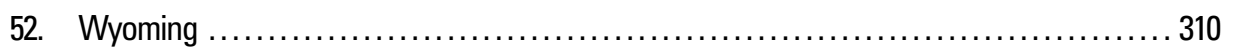


Conversion Factors and Datums

\begin{tabular}{lcl}
\hline Multiply & By & To obtain \\
\hline acre & 4,047 & square meter \\
cubic foot per second & 0.02832 & cubic meter per second \\
cubic foot per second per square mile & 0.01093 & cubic meter per second per kilometer \\
foot & 0.3048 & meter \\
foot per second & 0.3048 & meter per second \\
inch & 25.4 & millimeter \\
inch per hour & 25.4 & millimeter per hour \\
mile & 1.609 & kilometer \\
mile per hour & 1.609 & kilometer per hour \\
square foot & 0.09290 & square meter \\
square mile & 2.590 & square kilometer \\
yard & 0.9144 & meter \\
\hline
\end{tabular}

Temperature can be converted to degrees Celsius $\left({ }^{\circ} \mathrm{C}\right)$ or degrees Fahrenheit $\left({ }^{\circ} \mathrm{F}\right)$ by the equations:

${ }^{0} \mathrm{C}=5 / 9\left({ }^{\circ} \mathrm{F}-32\right)$

${ }^{0} \mathrm{~F}=9 / 5\left({ }^{\circ} \mathrm{C}\right)+32$.

Horizontal coordinate information is referenced to the North American Datum of 1983 (NAD 83).

Vertical coordinate information is referenced to the North American Vertical Datum of 1988 (NAVD 88). 


\section{Glossary}

Although much of the terminology used in this report is widely understood, some terms have specialized meanings in hydrology or are unfamiliar outside of hydrologic usage. Most of the definitions given here are from Langbein and Iseri (1960), with slight modifications, and explain the terms as they are generally used by hydrologists in the U.S. Geological Survey.

Absorption The entrance of water into the soil or rocks by all natural processes. It includes the infiltration of precipitation or snowmelt.

Bank The margins of a channel. Banks are called right or left as viewed facing the direction of the flow.

Convective precipitation Precipitation that falls from storms with localized updrafts (thunderstorms).

Cubic feet per second A unit expressing rates of discharge. One cubic foot per second is equal to the discharge of a stream of rectangular cross section, 1 foot wide and 1 foot deep, flowing water at an average velocity of 1 foot per second.

Current meter An instrument for measuring the velocity of flowing water. The U.S. Geological Survey typically uses a rotating cup meter.

Discharge In its simplest concept, discharge means outflow; therefore, the use of this term is not restricted as to course or location, and it can be applied to describe the flow of water from a pipe or from a drainage basin. If the discharge occurs in some course or channel, it is correct to speak of the discharge of a canal or of a river.

Drainage area The drainage area of a stream at a specified location is that area, measured in a horizontal plane, that is enclosed by a drainage divide.

Drainage basin A part of the surface of the Earth that is occupied by a drainage system, which consists of a surface stream or a body of impounded surface water together with all tributary surface streams and bodies of impounded surface water.
El Niño A warming of the eastern tropical Pacific Ocean that affects weather patterns worldwide.

Flood An overflow or inundation that comes from a river or other body of water (Barrows, 1948, p. 4) and causes or threatens damage. Any relatively high streamflow overtopping the natural or artificial banks in any reach of a stream (Leopold and Maddock, 1954, p. 249_ 251).

Flood plain The lowland that borders a river, usually dry but subject to flooding (Hoyt and Langbein, 1955, p. 12).

Flood stage The stage at which overflow of the natural banks of a stream begins to cause damage in the reach in which the elevation is measured.

Isohyetal map A map or chart showing lines that connect points that received the same amount of precipitation.

Overland flow The flow of rainwater or snowmelt over the land surface toward stream channels.

Peak flow The greatest rate of flow during a flood.

Regulation The artificial manipulation of the flow of a stream.

Reservoir A pond, lake, or basin, either natural or artificial, for the storage, regulation, and control of water.

Runoff That part of the precipitation that appears in surface streams.

Stage The height of a water surface above an established datum plane (also gage height).

Stage-discharge curve A graph showing the relation between the gage height, usually plotted as ordinate, and the amount of water flowing (discharge) in a channel, expressed as volume per unit of time, usually plotted as abscissa.

Stage-discharge relation The relation expressed by the stage-discharge curve.

Standardized Precipitation Index An index that is based on the probability of precipitation for any time scale. 
Streamflow The discharge that occurs in a natural channel. Although the term discharge can be applied to the flow of a canal, the word "streamflow" uniquely describes the discharge in a surface stream course. The term "streamflow" is more general than runoff as streamflow may be applied to discharge whether or not it is affected by diversion or regulation.

Streamgage A gaging station where a record of discharge of a stream is obtained.

Surface runoff That part of the runoff that travels over the soil surface to the nearest stream channel. It also is defined as that part of the runoff of a drainage basin that has not passed beneath the surface following precipitation.

Surface water Water on the surface of the Earth.

Water equivalent of snow The amount of water that would be obtained if the snow should be completely melted. Water content may be merely the amount of liquid water in the snow at the time of observation (Wilson, 1942, p. 153-154).

Water year In U.S. Geological Survey reports, water year is the 12-month period, October 1 through September 30. The water year is designated by the year in which it ends. Thus, the year ending September 30, 1994, is called the "1994 water year." 


\title{
Summary of Significant Floods in the United States and Puerto Rico, 1994 Through 1998 Water Years
}

\author{
By C.A. Perry
}

\section{Abstract}

This volume is a compilation of significant floods that occurred at streamgages throughout the United States and Puerto Rico from October 1, 1993, through September 30, 1998. A significant flood in this report refers to a peak-flow discharge (instantaneous or time averaged) that is in the top 5 percent of all the annual peak flows recorded at streamgages during their total period of record. Most of these floods are approximately equal to or greater than the 20 -year recurrence interval flood ( 0.05 probability of occurrence in any 1 year) for that streamgage.

A summary of the most devastating floods according to number of lives lost and amount of damage is provided for each water year from 1994 through 1998. Significant interstate floods also are described. For each year, national maps are provided showing percentage of streamgages in each State recording the significant floods and standardized deviations from long-term (1950-95) mean precipitation.

Compilations arranged by State for each of the 50 United States and Puerto Rico also are presented. Each State compilation includes: (1) State maps to locate the streamgages recording significant floods and (2) tables of data that allow the reader to compare each significant flood during water years 1994 through 1998 with the maximum flood for the entire period of record at each streamgage.

\section{Introduction}

Maximum floodflows for selected locations are compiled annually by State and Puerto Rico offices of the U.S. Geological Survey (USGS). Each office publishes these data along with other data including daily flow, water-quality, and groundwater information in the USGS Water-Data Report series. The peak discharges for each streamgage also are placed in the Peak Flow File of the USGS National Water Information System (NWIS) and is available on the World Wide Web (http://water.data.usgs.gov/nwis). A publication was needed that compiled significant floods nationwide and provided a relative measure of the severity in a single publication. These publications have become the National Flood Summary series of which this volume is a part. This publication, in addition to providing a list of floods, provides a description of major or noteworthy floods and provides some information as to cause, loss of life, damage, and cost. During the period October 1, 1993, through September 30, 1998 (1994-98 water years) approximately 5,000 county declarations of disaster were tabulated for the United States; 54 percent of these declarations were for flooding and another 10 percent were for hurricanes during which flooding is inherent.

Innumerable combinations of variable meteorologic and physiographic factors produce floods of all degrees and severity. Some meteorologic factors that affect floods are the form, amount, duration, and intensity of precipitation; the amount of previous precipitation, which would affect the moisture absorption of the soil; the air temperature, which may cause frozen soil or may determine the rate of snowmelt; and the direction of storm movement. The principal physiographic features of a drainage basin that determine floodflows are drainage area, elevation, character of soil, shape, slope, direction of slope, and vegetative or other land cover. With the exception of vegetative cover and soil preconditions, the physiographic features are fixed for any given drainage basin. The combination of the magnitude and intensity of meteorologic phenomena, the antecedent moisture conditions, and the effect of inherent physiographic features on runoff determines what the magnitude of a flood will be.

Human factors also affect the magnitude of flooding. Levees, designed to protect parts of the flood plain from inundation, can raise flood elevations. Flood-control reservoirs store floodwaters and can lower flood elevations and peak discharges. Urban areas have increased impervious areas that can increase flood elevations and discharges significantly.

Flood damages frequently are difficult to assess. Dollar amounts given in this report should be used as a general indication of flood losses rather than as definite values. Even if detailed surveys and estimates have been made, there is little consistency among methods used and types of losses included. Some estimates may exclude certain locations (such as mountainous areas) or types of loss (either insured or uninsured) or type of property (either private or public). Some estimates include traffic interruptions and flood-mitigation costs; others include strictly physical damage. Estimates may be based on replacement costs or on depreciated values. For floods not described in detailed published reports, the only damage estimates available usually are the preliminary figures contained in 


\section{Summary of Significant Floods in the United States and Puerto Rico, 1994 Through 1998 Water Years}

newspapers, National Oceanic and Atmospheric Administration (NOAA) climatological data, or other sources published shortly after the flood. A statement that a disaster declaration was issued indicates that the damage was severe and that financial aid to victims was authorized by the governmental entity making the declaration.

Some of the flood descriptions in this volume give the amount of rainfall and duration of the storm associated with the flooding. Recurrence intervals for these storms may be determined from a rainfall-frequency atlas of the United States (U.S. Weather Bureau, 1961) or from a simplified set of equalrainfall maps and charts contained in a report by Rostvedt (1965).

Continuing investigation of surface-water resources within the United States is performed by the USGS in cooperation with more than 800 agencies and organizations, including State agencies, the U.S. Army Corps of Engineers, the Bureau of Reclamation, and other Federal, tribal, or local agencies. The National Weather Service, in addition to collecting and compiling data on meteorological phenomena, also collect data on stream stages in some areas.

\section{Previous Flood Summary Reports}

During the 1950s and 1960s the USGS summarized floods of each year in an annual series of Water-Supply Papers entitled, "Summary of Floods In the United States." A summary was published for each calendar year from 1950 through 1969. Water-Supply Paper 1137-I, the first in the series (U.S. Geological Survey, 1954), states the purpose of the series as being:

"To assemble in a single volume information relating to all known severe floods in the United States whether local or of wide areal extent. For floods that are described in... other publications of the Geological Survey or in reports by other Federal and State agencies, only very brief mention including references to the reports containing detailed descriptions, will be given here. Local floods for which no individual reports have been prepared are described briefly."

In the first volume of that Water-Supply Paper series, each flood was described in a maximum of three or four paragraphs. Later volumes contained longer articles including maps. The series was discontinued after the 1969 volume; however, in 1987 a program was begun to prepare and publish summaries for 1970 and succeeding years. Two National Flood Summary publications (one for the calendar years 1990 and for 1991 and one for 1992 through September 1993) were published with the longer article format. The period from January 1, 1970, to December 31, 1989, was published with an abbreviated format for the 50 United States, Puerto Rico, and the Virgin Islands. Water-Supply Papers 2474 and 2499 cover the periods 1990 to 1991 (Jordan and Combs, 1996) and 1992 to 1993 (Perry and Combs, 1998), respectively. Water-Supply Paper 2502 covers the calendar year period from 1970 to 1989 (Perry, Aldridge, and Ross, 2001). This volume is published in the abbreviated format for the 1994-98 water years. Much of the explanation contained in the next two sections of this volume is paraphrased from previous reports (Rostvedt, 1972; Jordan and Combs, 1996; Perry and Combs, 1998; Perry, Aldridge, and Ross, 2001).

\section{Determination of Flood Stages and Discharges}

The usual method of determining stream discharges at a streamgage is the application of a stage-discharge relation to a known stage. This relation usually is defined by current-meter measurements made through as wide a range of stage as possible (fig. 1). If the maximum discharge exceeds the range of the current-meter measurements, short extensions may be made to a graph of the stage-discharge relation by logarithmic extrapolation, by velocity-area studies, or by the use of other measurable hydraulic factors (Kennedy, 1983).

Maximum discharges that are substantially greater than the range of the defined stage-discharge relation at streamgage and maximum discharges at miscellaneous streamgages that have no developed stage-discharge relation generally are determined by various types of indirect measurements. In addition, adverse conditions often make it impossible to obtain current-meter or acoustic doppler measurements at some streamgages during major floods. Maximum discharges at these streamgages are determined, after the floods have subsided, by indirect methods, which involve determination of water-surface elevations from high-water marks, surveying cross sections, and computing

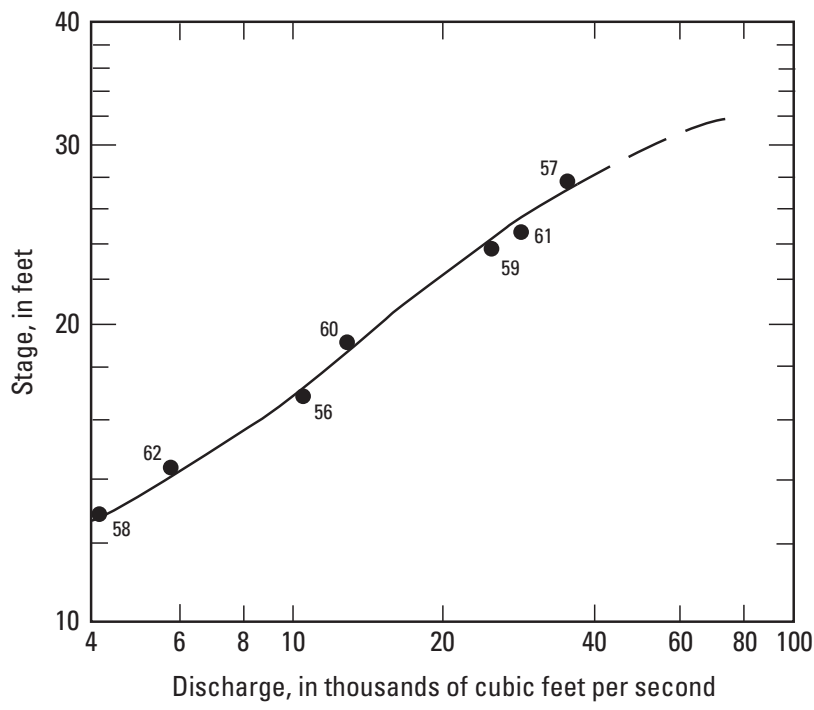

\section{EXPLANATION}

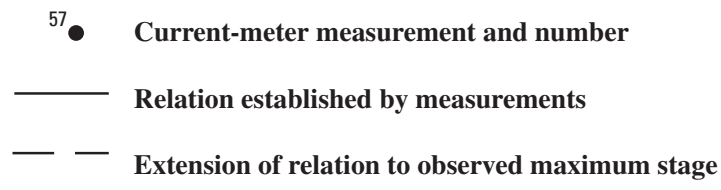

Figure 1. Example of a stage-discharge relation and upward extension of relation to observed maximum stage. 
discharge from hydraulic equations rather than from direct measurement of stream velocity by use of a current meter or acoustic doppler. Indirect methods are described by Dalrymple and Benson (1967), Hulsing (1967), Matthai (1967), Bodhaine (1968), and Benson and Dalrymple (1987).

The accuracy of indirect measurements depends on streamgage conditions and the experience of personnel who select streamgage locations and make the surveys, and generally is poorer than for current-meter or acoustic-doppler measurements. The indirect measurements used in determining maximum discharges for floods are not identified as such in this volume. Information as to the source and quality of discharge data in this volume can be obtained from the USGS office in the State in which the reported streamgage is located.

\section{Explanation of Data}

The floods in this volume are described by State and in downstream order. The data for each State include: (1) a short narrative of selected floods that occurred during the 1994-98 water years and references for published reports on precipitation data, floods, and flood damage for the State, (2) a summary table of flood data for those streamgages with significant floods of approximately 20-year recurrence interval or greater during the 1994 through 1998 water years, and (3) a State map showing location of all streamgages where a significant flood was recorded.

In the example of a summary table (table 1), the first two columns identify the streamgage, which may be a continuousrecord streamgage, a partial-record streamgage, or another streamgage at which data have been obtained. The first column gives the USGS permanent streamgage number (downstreamorder number). The second column gives the name of the streamgage.

Total drainage in the summary table is the total area, as measured on a flat projection map, that constitutes the stream basin (divide to divide). The actual drainage area contributing to runoff may be smaller than the total drainage area if the total area includes areas of extremely rapid infiltration rates that do not produce surface runoff or closed subbasins within the larger basin that do not have surface outlets.

The column headed "Period of record" shows the water years for which the stage or discharge shown in the sixth and seventh columns is known to be a maximum. For most streamgages, this period corresponds to the period of systematic collection of streamflow data. For other streamgages, written or oral history may indicate that a flood stage was the highest since people have observed the stream or was the highest since some known date. For some streamgages, two or more periods are given. The use of two periods separated by a comma indicates a break in the period of record. Maximum stages or discharges during the intervening period are unknown.
The fifth column shows the water year in which the maximum stage and discharge for the indicated period occurred. The sixth and seventh columns show the stage and discharge of that maximum. Separate listings are made when maximum stage and maximum discharge did not occur concurrently.

The last five columns present data for the significant floods during the period October 1, 1993, through September 30,1998 . The data include the date on which the maximum occurred, maximum stage, and maximum discharge, whether the stream was regulated during the flood and, where available, the recurrence interval of the discharge.

The probability of a given discharge being equaled or exceeded in any given year frequently is used as an indication of a flood's relative magnitude and for comparison with floods at other streamgages. The relative magnitude also can be expressed in terms of recurrence interval, which is the reciprocal of the flood probability. A third way of expressing the relative flood magnitude is the percent chance of occurrence, which is 100 times the flood probability. A discharge that will be equaled or exceeded on an average (over a long period of time) of once in 10 years has a recurrence interval of 10 years; is termed a "10-year flood"; has a probability of 0.10 ; and has a 10 -percent chance of occurring in any given year. A 100-year flood has a recurrence interval of 100 years; a probability of 0.01 ; and a 1-percent chance of occurring in any given year. Because recurrence interval is most commonly used by Federal agencies (for example, in the context of flood insurance), it is used in this volume even though percent chance avoids the unintended connotations of regularity of occurrence that accompany the term "recurrence interval."

Equivalence of flood probability and percent-chance values to selected recurrence-interval values are as follows:

\begin{tabular}{ccc}
\hline $\begin{array}{c}\text { Probability } \\
\text { (dimensionless) }\end{array}$ & Percent chance & $\begin{array}{c}\text { Recurrence } \\
\text { interval } \\
\text { (years) }\end{array}$ \\
\hline 0.50 & 50 & 2 \\
.20 & 20 & 5 \\
.10 & 10 & 10 \\
.04 & 4 & 25 \\
.02 & 2 & 50 \\
.01 & 1 & 100 \\
\hline
\end{tabular}

In addition to probability or percent chance of a given magnitude of discharge occurring in any 1 year, the probability or percent chance of occurrence during a given period of consecutive years also can be calculated. Results of such calculations for selected combinations of recurrence interval and length of period are as follows (*means greater than 99.9 but less than a 100-percent chance): 
Table 1. Example of summary table presented in State or territory compilations.

$\left[\mathrm{mi}^{2}\right.$, square miles; ft, feet above an arbitrary datum; $\mathrm{ft}^{3} / \mathrm{s}$, cubic feet per second; --, not determined or not applicable; ST, state name; >, greater than; <, less than. Source: Recurrence intervals calculated from U.S. Geological Survey data. Other data from U.S. Geological Survey reports or databases]

\begin{tabular}{|c|c|c|c|c|c|c|c|c|c|c|c|}
\hline \multirow[b]{2}{*}{$\begin{array}{c}\text { Streamgage } \\
\text { number }\end{array}$} & \multirow[b]{2}{*}{ Streamgage name } & \multirow[b]{2}{*}{$\begin{array}{c}\text { Total } \\
\text { drainage } \\
\left(\mathrm{mi}^{2}\right)\end{array}$} & \multicolumn{4}{|c|}{$\begin{array}{l}\text { Maximum stage and discharge for period } \\
\text { of record through } 1998 \text { water year }\end{array}$} & \multicolumn{5}{|c|}{ Significant floods $1994-98$ water years } \\
\hline & & & $\begin{array}{l}\text { Period of } \\
\text { record } \\
\text { (water } \\
\text { years) }\end{array}$ & $\begin{array}{l}\text { Water } \\
\text { year }\end{array}$ & $\begin{array}{l}\text { Stage } \\
(\mathrm{ft})\end{array}$ & $\begin{array}{l}\text { Dis- } \\
\text { charge } \\
\left(\mathrm{ft}^{3} / \mathrm{s}\right)\end{array}$ & $\begin{array}{c}\text { Date } \\
\text { (month/day/ } \\
\text { year) }\end{array}$ & $\begin{array}{c}\text { Stage } \\
(\mathrm{ft})\end{array}$ & $\begin{array}{c}\text { Discharge } \\
\left(\mathrm{ft}^{3} / \mathrm{s}\right)\end{array}$ & $\begin{array}{l}\text { Regu- } \\
\text { lated } \\
\text { during } \\
\text { flood }^{1}\end{array}$ & $\begin{array}{l}\text { Recur- } \\
\text { rence } \\
\text { interval } \\
\text { (years) }\end{array}$ \\
\hline 05551212 & Hypothetical Creek near Town, ST & 21.0 & $1961-97$ & 1961 & 13.1 & -- & $\begin{array}{l}02 / 02 / 94 \\
11 / 30 / 97\end{array}$ & $\begin{array}{l}12.22 \\
12.67\end{array}$ & $\begin{array}{l}4,200 \\
5,500\end{array}$ & $\mathrm{~N}$ & $\begin{array}{c}25 \\
25-50\end{array}$ \\
\hline 05555000 & Hypothetical River at City, ST & 1,212 & $\begin{array}{l}1939 \\
1955-98\end{array}$ & 1995 & 21.21 & 82,800 & $09 / 12 / 95$ & 21.21 & 82,800 & $\mathrm{~N}$ & $>100$ \\
\hline 06930030 & Hypo River near Metropolis, ST & 3,333 & 1919-98 & 1943 & 33.33 & 99,900 & $12 / 23 / 96$ & 25.55 & 33,000 & $\mathrm{Y}$ & $10-25$ \\
\hline
\end{tabular}

${ }^{1}$ Regulated during flood: N, no; Y, yes. 


\begin{tabular}{rrrrrc}
\hline $\begin{array}{c}\text { Recur- } \\
\text { rence } \\
\begin{array}{c}\text { interval } \\
\text { (years) }\end{array}\end{array}$ & \multicolumn{5}{c}{ Percent chance for indicated time period, in years } \\
\cline { 2 - 6 } & 5 & 10 & 50 & 100 & 500 \\
\hline 2 & 97 & 99.9 & $*$ & $*$ & $*$ \\
10 & 41 & 65 & 99.5 & $*$ & $*$ \\
50 & 10 & 18 & 64 & 87 & $*$ \\
100 & 5 & 10 & 39 & 63 & 99.3 \\
\hline
\end{tabular}

Recurrence intervals during any given flood may differ from streamgage to streamgage because of nonuniform distribution of runoff and uncertainty in the computed recurrence values. Operational patterns for reservoirs generally are not defined adequately to permit recurrence intervals to be computed for maximum discharges on regulated streams.

Another method of indicating a flood's relative magnitude is by comparison of its maximum discharge and the stream's drainage area with values on a regional "envelope curve." A flood-envelope curve is one drawn on a graph in which maximum known discharges are plotted in relation to the drainage area of each streamgage (fig. 2). The envelope curve is a smooth curve drawn to equal or exceed all the plotted discharges in relation to the drainage areas. Envelope curves are given for 17 regions of the conterminous United States in Crippen and Bue (1977). This method is better than the formerly used calculation of "unit discharge" (division of the discharge by the drainage area) because unit discharges for greatly different sizes of drainage area are not comparable. If the unit discharges for a very small and a very large drainage area are the same, the unit discharge is much more unusual for the large drainage area.

The Standardized Precipitation Index (SPI) is an index that looks at the probability for precipitation for any time scale (McKee and others, 1993). Calculation of the SPI value is based on long-term precipitation records for a specific time period and

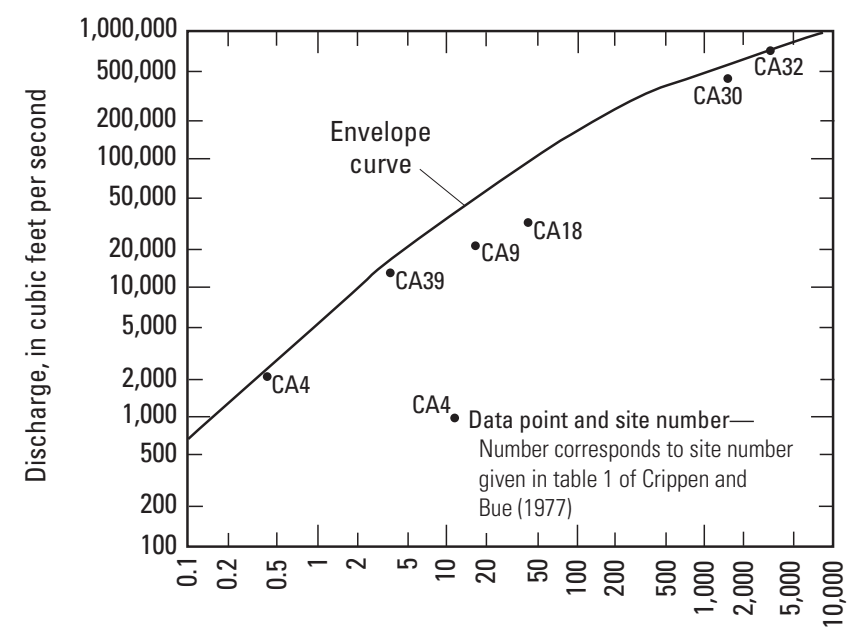

Drainage area, in square miles

Figure 2. Maximum discharge in relation to drainage area and envelope curve for a region (modified from Crippen and Bue, 1977, p. 15). can be done for any location desired. The record is put into probability and normal distributions so that the mean SPI value for that location is zero. Positive values are an indication of greater than median precipitation amount, whereas a negative value indicates a less than median precipitation amount. Because the SPI is standardized, both drought and wet conditions can be represented for all climate types.

\section{References}

Barrows, H.K., 1948, Floods, their hydrology and control: New York, McGraw-Hill Book Co., 432 p.

Benson, M.A., and Dalrymple, Tate, 1987, General field and office procedures for indirect measurements: U.S. Geological Survey Techniques of Water-Resources Investigations, book 3, chap. A1, 29 p.

Bodhaine, G.L., 1968, Measurement of peak discharge at culverts by indirect methods: U.S. Geological Survey Techniques of Water-Resources Investigations, book 3, chap. A3, $60 \mathrm{p}$.

Crippen, J.R., and Bue, C.D., 1977, Maximum floodflows in the conterminous United States: U.S. Geological Survey WaterSupply Paper 1887, $52 \mathrm{p}$.

Dalrymple, Tate, and Benson, M.A., 1967, Measurement of peak discharge by slope-area method: U.S. Geological Survey Techniques of Water-Resources Investigations, book 3, chap. A2, 12 p.

Hoyt, W.G., and Langbein, W.B., 1955, Floods: Princeton, New Jersey, Princeton University Press, 469 p.

Hulsing, Harry, 1967, Measurement of peak discharge at dams by indirect methods: U.S. Geological Survey Techniques of Water-Resources Investigations, book 3, chap. A5, 29 p.

Jordan, P.R., and Combs, L.J., eds., 1996, Summary of floods in the United States during 1990 and 1991: U.S. Geological Survey Water-Supply Paper 2474, 257 p.

Kennedy, E.J., 1983, Computation of continuous records of streamflow: U.S. Geological Survey Techniques of WaterResources Investigations, book 3, chap. A13, 53 p.

Langbein, W.B., and Iseri, K.T., 1960, General introduction and hydrologic definitions, in Manual of hydrology, part 1, General surface-water techniques: U.S. Geological Survey Water-Supply Paper 1541-A, 29 p.

Leopold, L.B., and Maddock, Thomas, Jr., 1954, The flood control controversy: New York, Ronald Press Co., 278 p.

Matthai, H.F., 1967, Measurement of peak discharge at width contractions by indirect methods: U.S. Geological Survey Techniques of Water-Resources Investigations, book 3, chap. A4, 44 p.

McKee, T.B., Doesken, N.J., and Kleist, J., 1993, The relationship of drought frequency and duration to time scales: Preprints, 8th Conference on Applied Climatology, January 1722, 1992, Anaheim, California, p. 179-184. 


\section{Summary of Significant Floods in the United States and Puerto Rico, 1994 Through 1998 Water Years}

Perry, C.A., and Combs, L.J., eds., 1998, Summary of floods in the United States during 1992 and 1993: U.S. Geological Survey Water-Supply Paper 2499, 286 p.

Perry, C.A., Aldridge, B.N., and Ross, H.C., 2001, Summary of significant floods in the United States, Puerto Rico, and the Virgin Islands, 1970 through 1989: U.S. Geological Survey Water-Supply Paper 2502, 597 p.

Rostvedt, J.O., 1965, Summary of floods in the United States during 1960: U.S. Geological Survey Water-Supply Paper 1790-B, $147 \mathrm{p}$.

Rostvedt, J.O.,1972, Summary of floods in the United States during 1968: U.S. Geological Survey Water-Supply Paper 1970-B, 73 p.
U.S. Geological Survey, 1954, Summary of floods in the United States during 1950: U.S. Geological Survey Water-Supply Paper 1137-I, p. 957-991.

U.S. Weather Bureau, 1961, Rainfall frequency atlas of the United States: U.S. Weather Bureau Technical Paper 40, $115 \mathrm{p}$.

Wilson, W.T., 1942, Melting characteristics of snow and its contribution to runoff, in June 30-July 2, 1941, Hydrology Conference Proceedings: University Park, Pennsylvania State College, School of Engineering Technical Bulletin 27, p. 153-165. 


\section{Summary of Floods By Water Year}

\section{Water Year}

The 1994 water year began quietly after the great floods in the Mississippi River Basin subsided during the late summer and fall of 1993. Figure 3 shows the percentage of streamgages in each State recording significant floods (top 5 percent of the peak-flow record) during 1994 water year. Georgia had 25 percent of its streamgages with significant flooding (top 5 percent) and Missouri had 21 percent. Alabama, Alaska, Delaware, Illinois, Indiana, North Carolina, Tennessee, and West Virginia had more than 5 percent of their total number of streamgages with significant flooding. Standardized precipitation deviations for the 1994 water year (fig. 4) show a similar trend. On the basis of 1950-95 long-term mean precipitation, much of the Southeast and the Northeast had above-normal annual precipitation. Most of the western two-thirds of the United States experienced below-normal precipitation.

Excessive rains during November 1993 in Missouri caused flooding on the Big, Bourbeuse, Current, Gasconade, James, Meramec, and Osage Rivers (see fig. 37 in the State compilations). Floods came again to this same region during April 1994. These floods extended into Illinois, with the Embarras, Illinois, Sangamon, and Vermilion Rivers flooding, and also into Indiana, with the Eel, Maumee, St. Mary, Tippecanoe, Vermilion, Wabash, and White Rivers flooding (see figs. 25 and 26 in the State compilations). These floods were part of a wet spring with floods in West Virginia during February 1994, and floods in Tennessee and North Carolina during March 1994.

Spring floods also occurred in North Dakota during April and May 1994 with melting of an excessive accumulation of winter snowfall. Spring floods also occurred in Alaska during May and June 1994. The Alaska flooding was exacerbated during August 1994 by excessive rainfall in the Koyukuk River Basin (see fig. 14 in the State compilations).

The 1994 tropical storm season started early with the first named storm, Tropical Storm Alberto (June 30 through July 7 , 1994), affecting the southeastern States of Alabama, Florida, Georgia, and Mississippi. The remnants of Alberto also caused flooding in North Carolina, South Carolina, and Tennessee. Thirty-one deaths and more than $\$ 500$ million in damage resulted from this slow-moving storm (Stamey, 1997). Tropical Storm Beryl lingered in the central Gulf Coast region during August 14-19, 1994, and caused flooding in Alabama, Florida, and Georgia.

\section{USGS Published Reports on Flooding During 1994 Water Year}

Hale, T.W., and Stamey, T.C, 1995a, Flooding in southeastern United States from Tropical Storm Alberto, July 1994, in Espey, W.H., Jr., and Combs, P.G, eds., Proceedings of the 1995 First International Conference on Water Resources
Engineering: American Society of Civil Engineers, p. 765769.

Hale, T.W., and Stamey, T.C, 1995b, The Independence Day flood of 1994: U.S. Geological Survey Yearbook, fiscal year 1994, p. 17-20.

Liscum, Fred, and East, J.W., 1995, Floods in southeast Texas, October 1994: U.S. Geological Survey Fact Sheet 94-0073, $2 \mathrm{p}$.

Meyer, D.F., 1995, Flooding in the middle Koyukuk River basin, Alaska, August 1994: U.S. Geological Survey WaterResources Investigations Report 95-4118, 8 p., 2 sheets.

Stamey, T.C., 1997, Flooding in southeastern United States from Tropical Storm Alberto, July 1994, in Leavesley, G.H., Lins, H.F., Nobilis, Franz, Parker, R.S., Schneider, V.R., and van de Ven, F.H.M., eds., Destructive water-water-caused natural disasters, their abatement and control: International Association of Hydrological Sciences-Association Internationale des Sciences Hydrologiques Publication, v. 239, p. 33-38.

\section{Water Year}

The 1995 water year recorded flooding in many States. Figure 5 shows the percentage of streamgages in each State recording significant floods (top 5 percent of the peak-flow record) during the 1995 water year. Wyoming had the highest percentage of streamgages with significant floods at 26 percent, and Colorado and South Dakota had 19 and 17 percent, respectively. Alaska, California, Florida, Georgia, Kansas, Louisiana, Nebraska, North Carolina, Oklahoma, South Carolina, Texas, Utah, Vermont, Virginia, and West Virginia all had from 6 to 15 percent of their streamgages recording significant floods. Standardized precipitation deviations for the 1995 water year (fig. 6) showed above-normal conditions in much of the country. The only areas that had a rainfall deficit were parts of the Northeast, the Southern Appalachian Mountains, western Tennessee, eastern Arkansas, and extreme southern Texas, and some of the areas around the Great Lakes.

The 1995 water year began with Tropical Storm Gordon (November 8-21, 1994) crossing the southern part of the Florida peninsula, moving northeastward into the Atlantic Ocean, then doubling back and striking Florida again near Melbourne (see fig. 21 in State compilations). Damage was estimated at $\$ 400$ million (National Oceanic and Atmospheric Administration, 1994).

Pacific winter storms lashed southern California in January 1995 causing many streams to flood. Southern Utah experienced excessive snowfall in March 1995, which melted rapidly and caused flooding. Snowmelt also was a problem in South Dakota in the James River Basin (see fig. 54 in State compilations) in April 1995. During April and May 1995, intermittent intense thunderstorms from Louisiana and Texas, through Oklahoma and Kansas, and north to South Dakota caused floods and flash floods. New Orleans, Louisiana (fig. 30), experienced more than $\$ 3$ billion in damage from the intense rains (National 

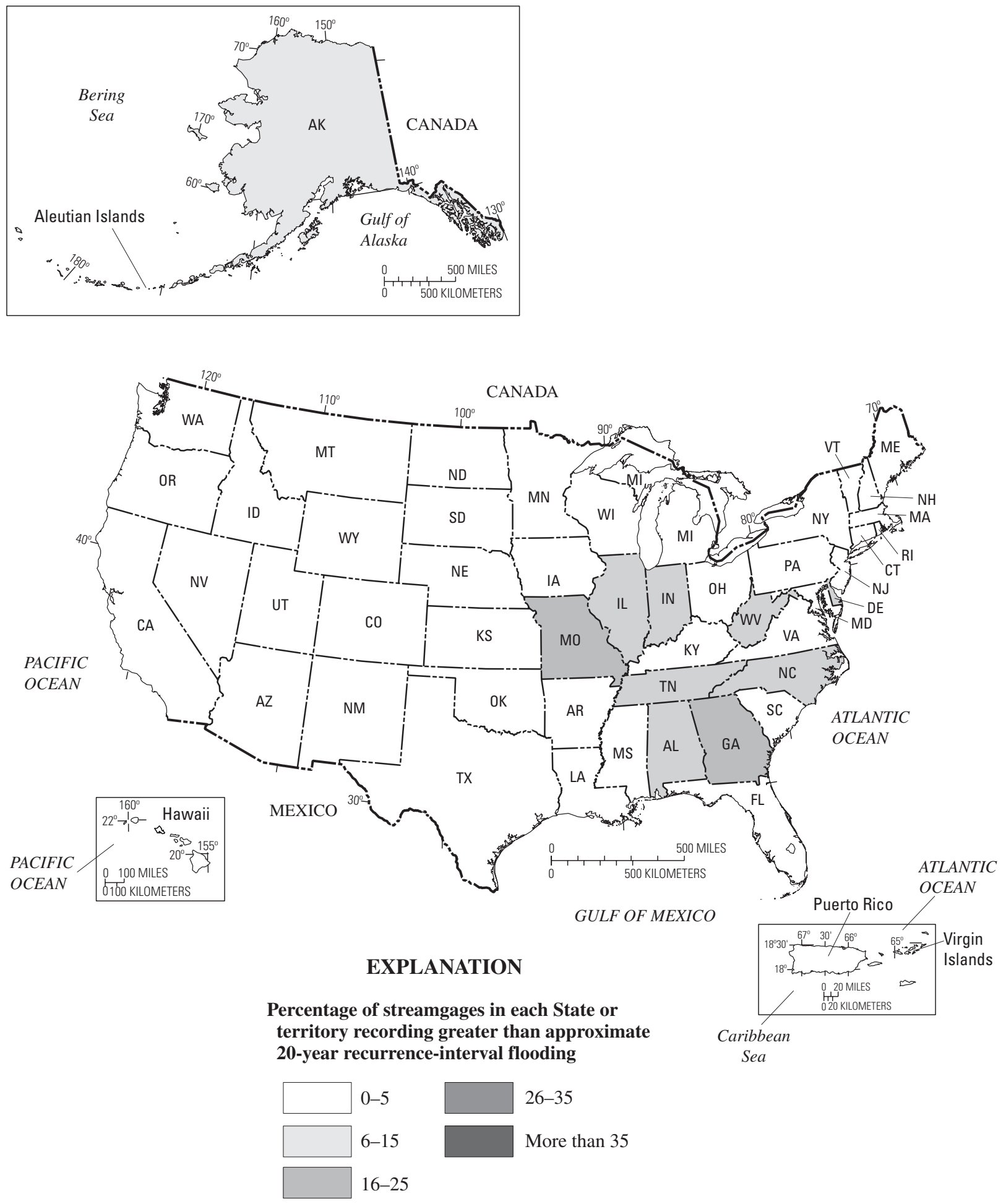

Figure 3. Percentage of streamgages in each State or territory recording greater than approximate 20-year recurrenceinterval flooding during 1994 water year. 


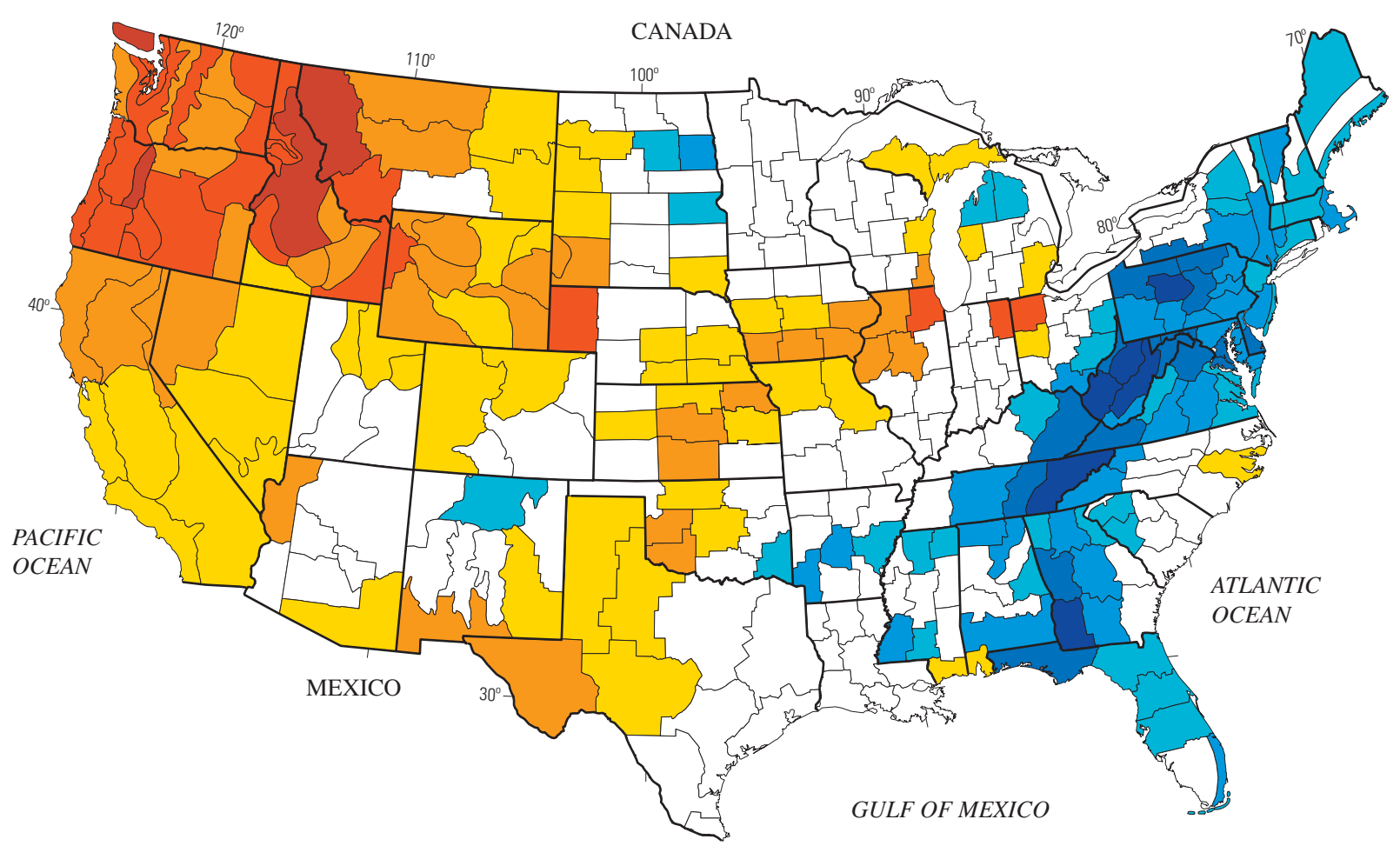

EXPLANATION

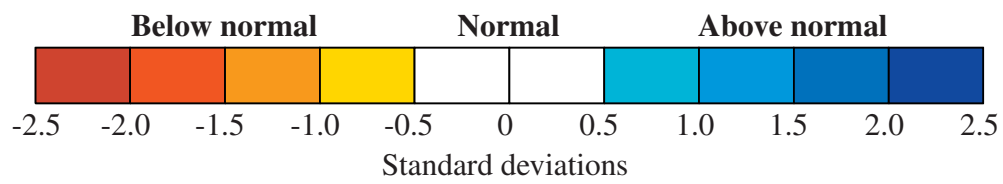

Boundary of National Weather Service meteorological divisions

Figure 4. Standardized 1994 water year precipitation deviations from long-term mean precipitation (1950-95) in conterminous United States (source of data: National Oceanic and Atmospheric Administration, Climate Diagnostics Center, at URL http://www.cdc.noaa.gov/USclimate/USclimdivs.html).

Oceanic and Atmospheric Administration, 1995). In Dallas, Texas (see fig. 56 in State compilations), excessive rainfall and flash flooding caused 17 deaths (National Oceanic and Atmospheric Administration, 1995).

The mountains of Colorado and Wyoming had deep snowpacks from the 1994-95 winter that persisted into late spring 1995 because of cooler than normal temperatures. However, the snowpack melted rapidly in late May and June 1995 causing flooding on many streams originating in the Rocky Mountains. Excessive precipitation in the Midwest caused the Mississippi and Ohio Rivers to flood in May and June 1995 in Illinois, Kentucky, and Missouri (see figs. 25, 29, and 37 in State compilations).

The 1995 hurricane season in the Atlantic Ocean and Gulf of Mexico was quite active with 15 named storms through October 1, 1995. Hurricane Allison (June 3-6, 1995) struck the Panhandle of Florida, southern Georgia, and the eastern edge of North Carolina and South Carolina. Tropical Storm Dean (July 28-August 2, 1995) made landfall south of HouSston and moved northwest of Dallas (see fig. 56 in State compilations) and dissipated. Hurricane Erin (July 31-August 6, 1995) crossed the Florida peninsula from east to west, curved up to Mobile, Alabama (see fig. 13 in State compilations), crossed Mississippi, and then traveled up the Ohio River until it dissipated over West Virginia. Tropical Storm Jerry (August 22-28, 1995) traveled the length of the Peninsula of Florida, through central Georgia, and dissipated over eastern South Carolina. The center of Hurricane Marilyn (September 12-22, 1995) passed just east of Puerto Rico and caused flooding on the eastern part of the island.

\section{USGS Published Reports on Flooding During 1995 Water Year}

Allen, D.V., 1996, Floods, runoff, and snow pack in Utah, 1995: U.S. Geological Survey Fact Sheet 106-96, 2 p.

Morgan, B.A., Wieczorek, G.F., and Campbell, R.H., 1999a, Historical and potential debris-flow and flood hazard map of the area affected by the June 27, 1995, storm in Madison County, Virginia: U.S. Geological Survey Geologic Investigations I-2623-B, 1 sheet. 

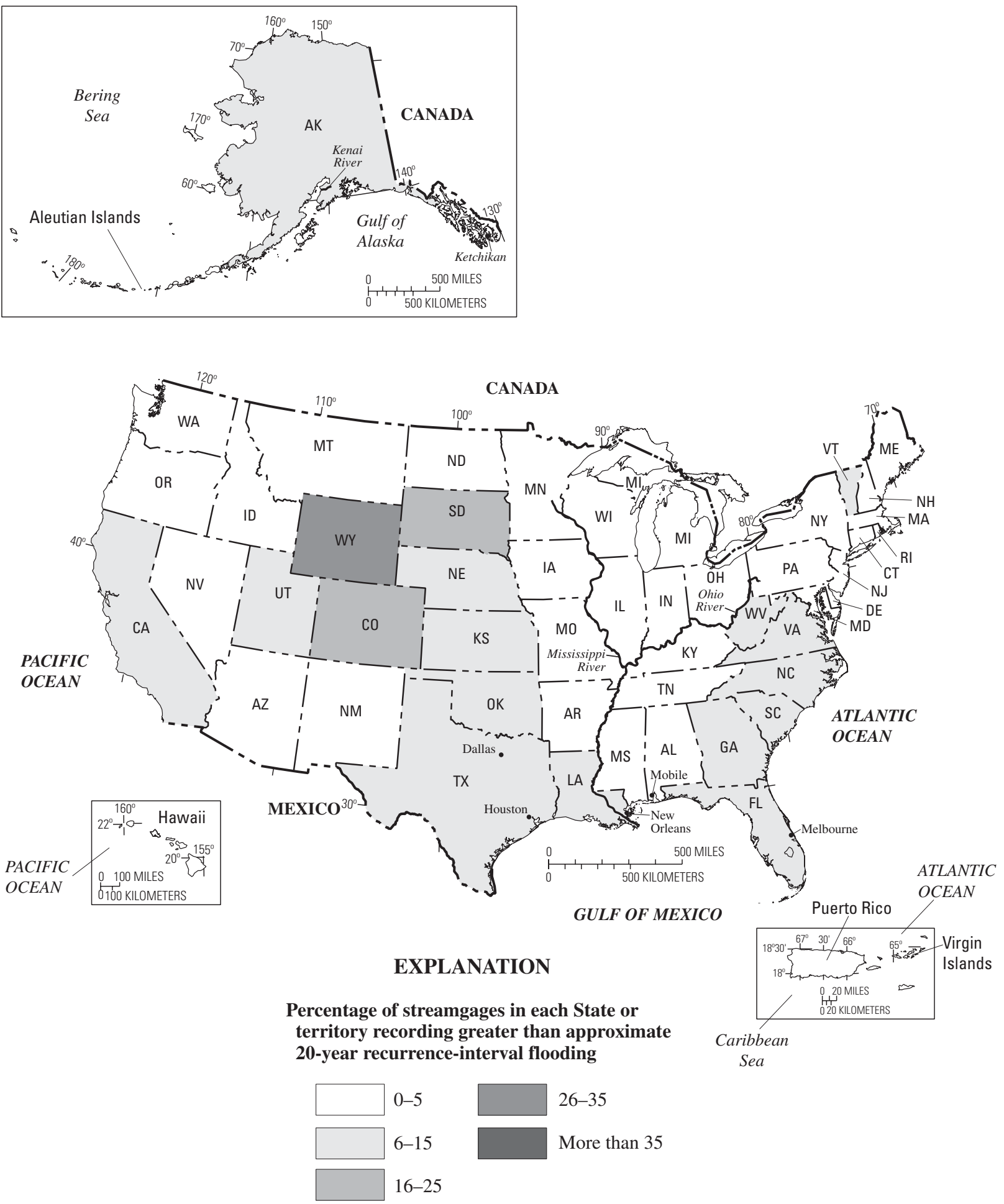

Figure 5. Percentage of streamgages in each State or territory recording greater than approximate 20-year recurrenceinterval flooding during 1995 water year. 


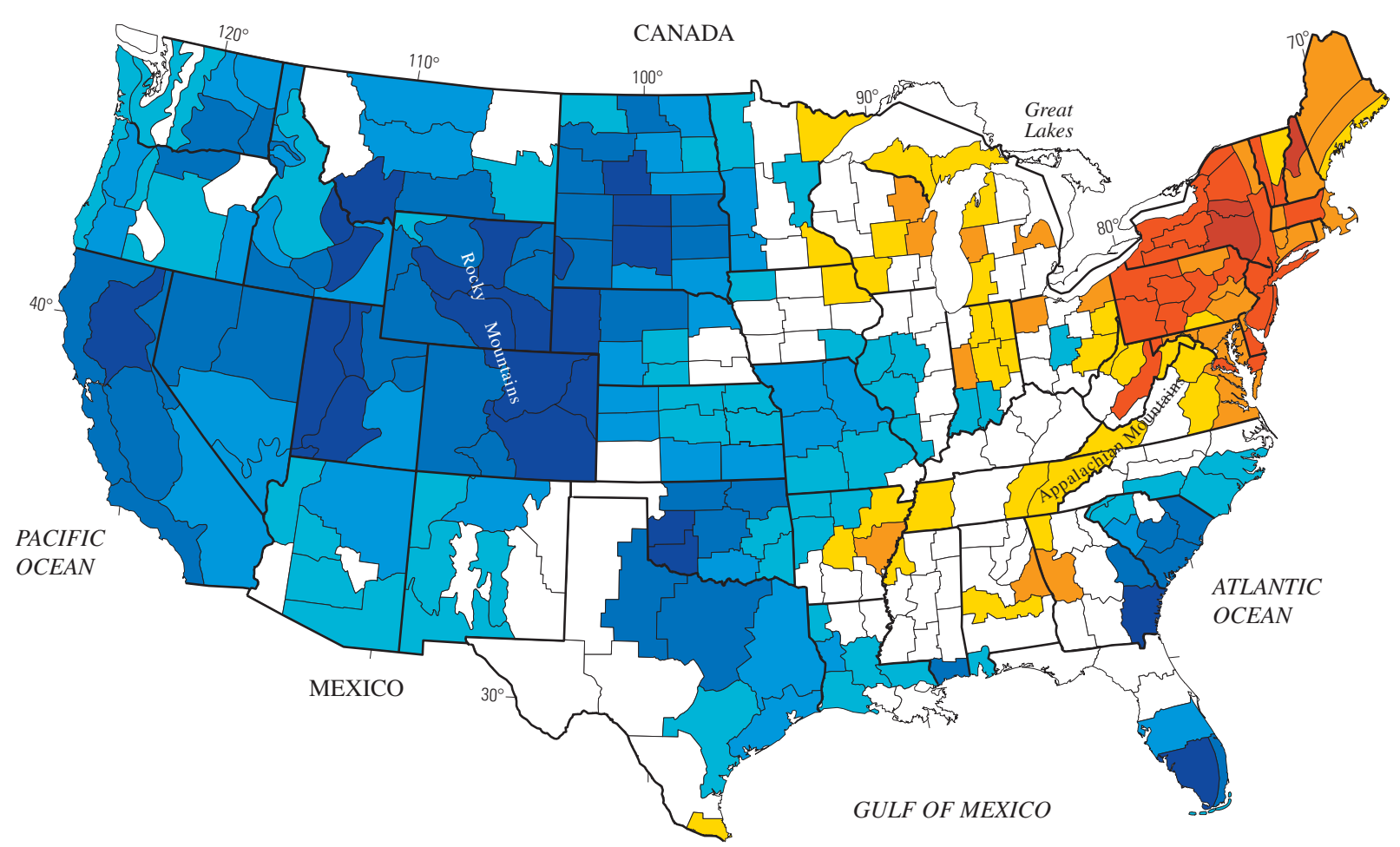

EXPLANATION

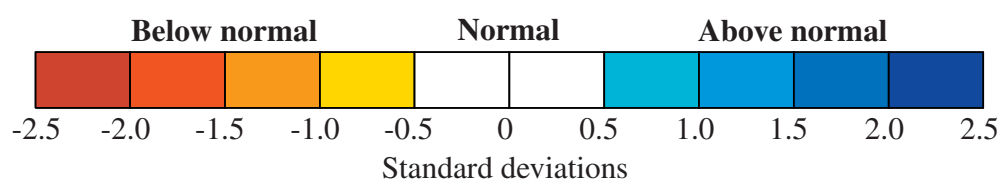

Boundary of National Weather Service meteorological divisions

Figure 6. Standardized 1995 water year precipitation deviations from long-term mean precipitation (1950-95) in conterminous United States (source of data: National Oceanic and Atmospheric Administration, Climate Diagnostics Center, at URL http://www.cdc.noaa.gov/USclimate/USclimdivs.html).

Morgan, B.A., Wieczorek, G.F., and Campbell, R.H., 1999b, Map of rainfall, debris flows, and flood effects of the June 27, 1995, storm in Madison County, Virginia: U.S. Geological Survey Geologic Investigations I-2623-A, 1 sheet.

National Oceanic and Atmospheric Administration (NOAA), 1994, Storm data (by State): Asheville, North Carolina, National Climatic Data Center, various months.

National Oceanic and Atmospheric Administration (NOAA), 1995, Storm data (by State): Asheville, North Carolina, National Climatic Data Center, various months.

Robinson, J.B., Hazell, W.F., and Young, W.S., 1998, Effects of August 1995 and July 1997 storms in the city of Charlotte and Mecklenburg County, North Carolina: U.S. Geological Survey Fact Sheet 036-98, 2 p.

Teller, R.W., Burr, M.J., and Rahder, D.L., 1995, Floods in South Dakota, spring 1995: U.S. Geological Survey Fact Sheet 164-95, 4 p.

Torres-Sierra, Heriberto, 1998, Storm-tide elevations caused by Hurricane Marilyn on the U.S. Virgin Islands, September 15-
16, 1995: U.S. Geological Survey Open-File Report 960440, 31 p., 16 sheets.

U.S. Geological Survey, 1995, Northern California storms and floods of January 1995: U.S. Geological Survey Fact Sheet 062-95, 2 p.

\section{Water Year}

The 1996 water year had flooding in many of the States in the northern one-half of the country. Figure 7 shows the percentage of streamgages in each State recording significant floods (top 5 percent of the peak-flow record) during the 1996 water year. Hardest hit were the Eastern and Northeastern States from North Carolina to New York. West Virginia had 41 percent of its streamgages record significant floods during 1996. Streamgages in Idaho, Oregon, and Washington also had a high percentage of floods. This tendency for flooding extended eastward from Montana and Wyoming to Indiana. Standardized 1996 water year precipitation deviations showed an abundance 

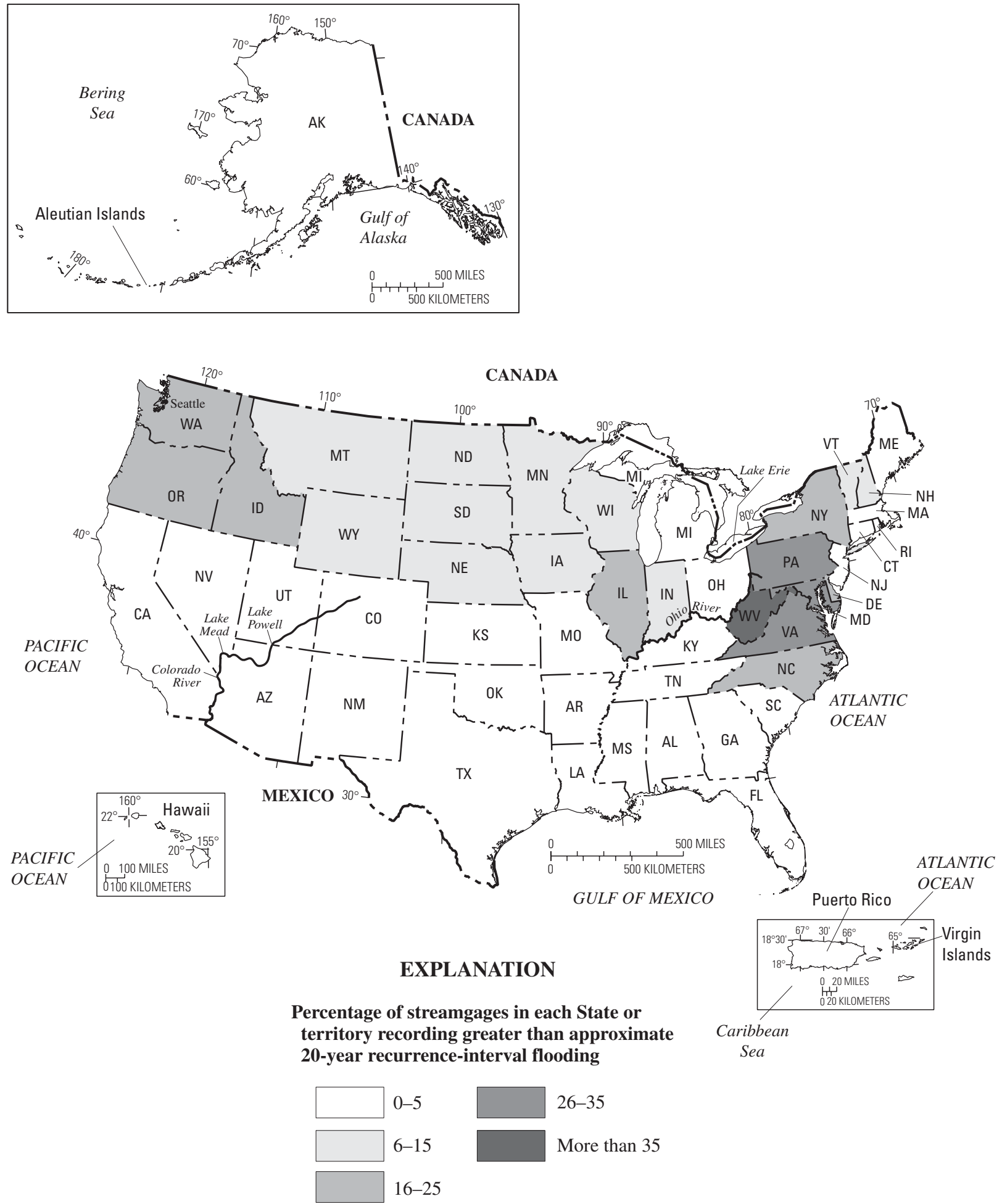

Figure 7. Percentage of streamgages in each State or territory recording greater than approximate 20-year recurrenceinterval flooding during 1996 water year. 
of precipitation in the Eastern States and in the Pacific Northwest (fig. 8).

The 1996 water year began with flooding from Hurricane Opal in eastern Alabama and western Georgia on October 4-5, 1995. The water year ended with a strong Pacific storm moving onshore over Oregon and Washington, and moving inland to Idaho and Wyoming. The worst flooding from this storm occurred in the Seattle area (fig. 7) and also in the southwestern part of Oregon. This storm and the many more to follow in January and February 1996 resulted in widespread flooding in the Pacific Northwest States. Many peak floods of record occurred from northern California to Washington and Idaho.

January 1996 was also the month for flooding in the Northeastern States of Virginia, Maryland, Delaware, New Jersey, Pennsylvania, New York, and Vermont. This major regional flood was a result of rain falling on a deep snowpack. There were 35 deaths from this flood. A major rain and snowmelt flood traveled down the Ohio River Valley from Pennsylvania to Kentucky in January 1996.

In March and April an artificial flood was released down the Colorado River below Lake Powell to Lake Mead to benefit river-bar formation (fig. 7). Many rivers in Illinois were above flood levels from May through July 1996 as a result of persistent summer thunderstorms. Hurricane Bertha (July 5-14, 1996) struck Puerto Rico, the Virgin Islands, and North and South Carolina causing flooding and 12 deaths. Hurricane Fran (August 23-September 6, 1996) caused the greatest flooding of any of the tropical storms for 1996. Hurricane Fran made landfall in southern North Carolina on September 5, travelled through the center of North Carolina, Virginia, and West Virginia, western Pennsylvania, and crossed Lake Erie into Canada. Many streamgages in these States had their highest flow of record during this time. Hardest hit were North Carolina, Virginia, Maryland, and Pennsylvania. Hurricane Hortense (September 3-16, 1996) caused flooding and 21 deaths in Puerto Rico.

\section{USGS Published Reports on Flooding During 1996 Water Year}

Beckwith, M.A., 1996, Water-quality data collected during floods in the Coeur D'Alene River, northern Idaho, February 1996: U.S. Geological Survey Fact Sheet 219-96, 4 p.

Beckwith, M.A., Berenbrock, C.E., and Backsen, R.L., 1996, Magnitude of floods in northern Idaho, February 1996: U.S. Geological Survey Fact Sheet 222-96, 2 p.

Berris, S.N., Hess, G.W., Taylor, R.L., and Bohman, L.R., 1997, Flood-control effects of Truckee River basin reservoirs, December 31, 1996, through January 4, 1997, California and Nevada: U.S. Geological Survey Fact Sheet 037-97, p. 4.

Carpenter, M.C., 1996, Monitoring erosion and deposition during the spring 1996 controlled-flood experiment on the Colorado River in the Grand Canyon using an array of loadcell scour sensors, in Wanted-water for rural Arizona:
Proceedings of the Arizona Hydrological Society Annual Symposium, v. 9, p. 29-31.

Fischer, E.E., and Eash, D.A., 1998, Statistical summaries of selected Iowa streamflow data through September 1996: U.S. Geological Survey Open-File Report 98-170, 681 p.

Graf, J.B., 1996, Velocity and longitudinal dispersion of the Colorado River in Grand Canyon during the controlled flood of March 1996 [abs.]: EOS, Transactions of American Geophysical Union, v. 77, no. 46, supplement, p. 271.

Hodgkins, G.A., and Stewart, G.J., 1997, Flood of October 1996 in southern Maine: U.S. Geological Survey WaterResources Investigations Report 97-4189, 28 p.

Holmes, R.R., Jr., and Kapadia, Amit, 1997, Floods in northern Illinois, July 1996: U.S. Geological Survey Fact Sheet 09797, 4 p.

Holmes, R.R., Jr., and Kupka, Amanda, 1997, Floods of July 18-20, 1996, in northern Illinois: U.S. Geological Survey Open-File Report 97-425, 29 p, 1 sheet.

Konieczki, A.D., Graf, J.B., and Carpenter, M.C., 1997, Streamflow and sediment data collected to determine the effects of a controlled flood in March and April 1996 on the Colorado River between Lees Ferry and Diamond Creek, Arizona: U.S. Geological Survey Open-File Report 97-224, $55 \mathrm{p}$.

Lumia, Richard, 1998, Flood of January 19-20, 1996, in New York State: U.S. Geological Survey Water-Resources Investigations Report 97-4252, 61 p.

Melis, T.S., Griffiths, P.G., and Webb, R.H., 1996, Reworking of recently-aggraded debris fans by the 1996 controlled flood on the Colorado River in Grand Canyon, Arizona [abs.]: EOS, Transactions of American Geophysical Union, v. 77, no. 46, supplement, p. 271.

O'Day, C.M., and Graf, J.B., 1996, Effects of the 1996 controlled flood on channel-sand storage in the Colorado River, Grand Canyon, Arizona [abs.]: EOS, Transactions of American Geophysical Union, v. 77 no. 46, supplement, p. 271.

Thompson, R.E., Jr., 1996, Statewide floods in Pennsylvania, January 1996: U.S. Geological Survey Fact Sheet 103-96, $2 \mathrm{p}$.

Thompson, R.E., Jr., and Durlin, R.R., 1997, 1996—a historical year for statewide and regional floods in the Commonwealth of Pennsylvania [abs.], in Geological Society of America, Northeastern Section, 32nd Annual Meeting, Abstracts with Programs: Geological Society of America, v. 29, no. 1, p. 85.

Torres-Sierra, Heriberto, 1998, Flood of September 10, 1996, Bayamon, Puerto Rico: U.S. Geological Survey WaterResources Investigations Report 98-4120, 2 sheets.

Zynjuk, L.D., and Majedi, B.F.,1996, January 1996 floods deliver large loads of nutrients and sediment to the Chesapeake Bay: U.S. Geological Survey Fact Sheet 140-96, 2 p.

\section{Water Year}

The 1997 water year saw flooding in many of the States in the northern and western parts of the country. Figure 9 shows 


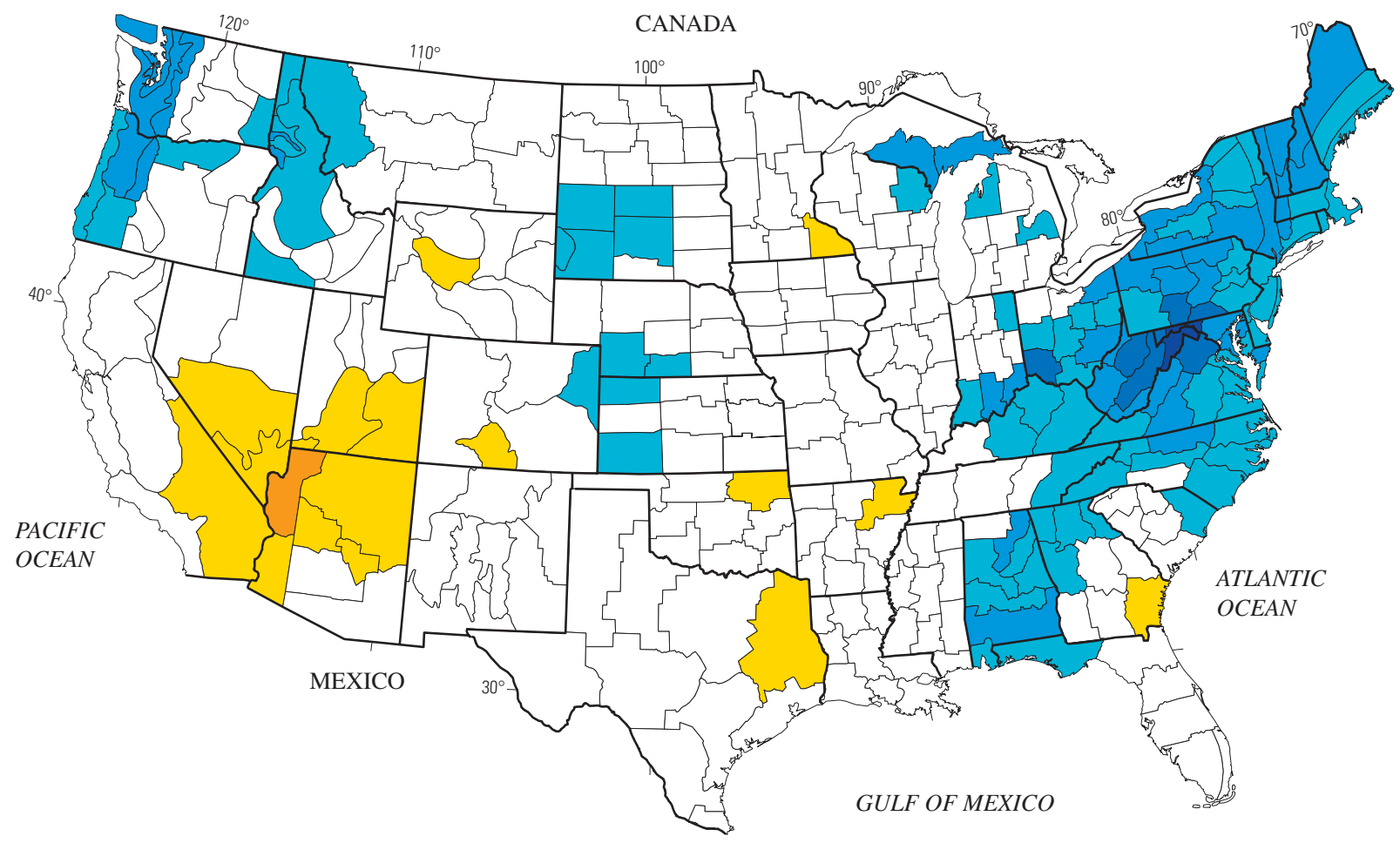

EXPLANATION

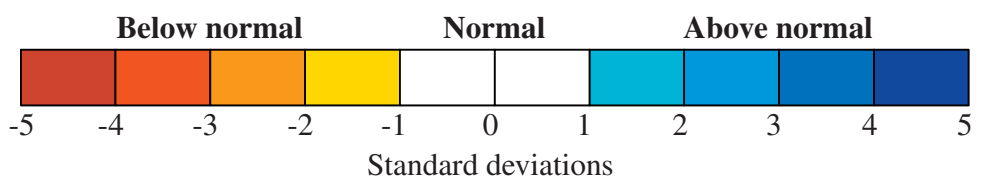

Boundary of National Weather Service meteorological divisions

Figure 8. Standardized 1996 water year precipitation deviations from long-term mean precipitation (1950-95) in conterminous United States (source of data: National Oceanic and Atmospheric Administration, Climate Diagnostics Center, at URL http://www.cdc.noaa.gov/USclimate/USclimdivs.html).

the percentage of streamgages in each State recording significant floods (top 5 percent of the peak-flow record) during the 1997 water year. Extreme rain and snowmelt flooding occurred from Idaho to Minnesota. North Dakota had 40 percent of its streamgages experience a significant flood in 1997. Idaho and California had 36 and 28 percent of its streamgages, respectively, experience flooding. Standardized precipitation deviations for the 1997 water year showed much of the Western United States with above-normal precipitation. There were very few below-normal areas nationwide (fig. 10).

Excessive snowfall in November and December 1996 throughout the Pacific Northwest augmented flooding that began during a January 1997 warming in California, Idaho, Nevada, Oregon, and Washington. Excessive winter snowfall extended eastward into Montana, North Dakota, South Dakota, and Minnesota. The deep snowpack and warming temperatures in March 1997 started flooding on the Red River of the North (fig. 9). An intense blizzard in early April 1997 brought a severe drop in temperatures and 70-mile-per-hour winds that compounded the flooding problems. Additional runoff from the snow, sleet, and rain forced the Red River of the North to record levels. Severe flooding continued for more than a month in North Dakota and Minnesota.

Intense early spring thunderstorms during the first week of March 1997 from Arkansas to Pennsylvania caused extensive flooding in those States and in Indiana, Kentucky, Missouri, Ohio, Tennessee, and West Virginia. The Ohio River was above flood stage from Pittsburgh, Pennsylvania, to Cairo, Illinois (fig. 9). Severe flooding occurred on the Licking River in Kentucky.

The only tropical storm during the 1997 water year that affected the United States was Hurricane Danny (July 16-26, 1997). It made landfall on July 17 on the Louisiana Delta, skirting southern Mississippi, travelling the length of Alabama before turning east and cutting through northern Georgia, northern South Carolina, and going out to sea near the North Carolina/Virginia border. Flooding occurred in Alabama, Florida, Georgia, Mississippi, North Carolina, and South Carolina. 

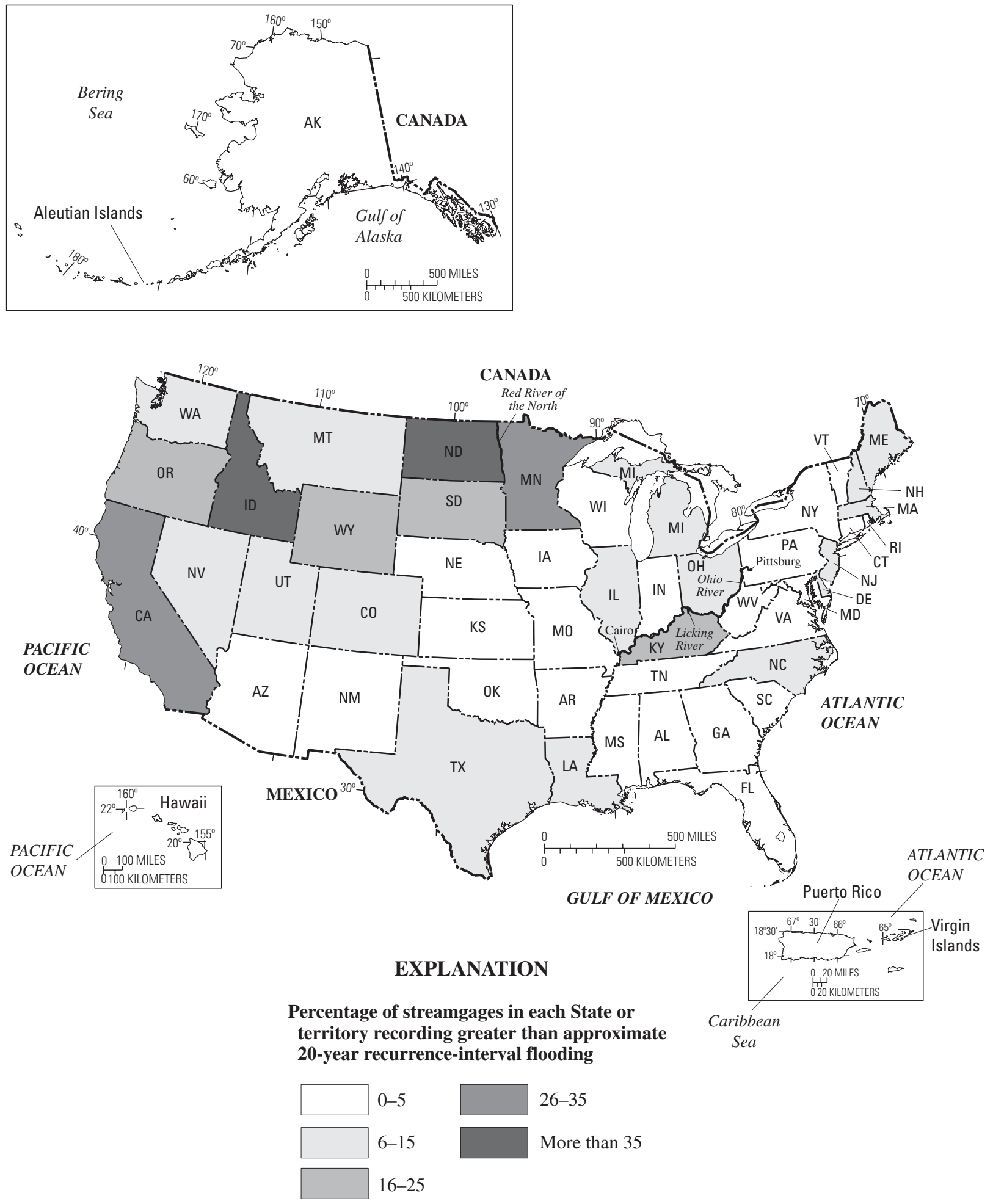

Figure 9. Percentage of streamgages in each State or territory recording greater than approximate 20-year recurrenceinterval flooding during 1997 water year. 


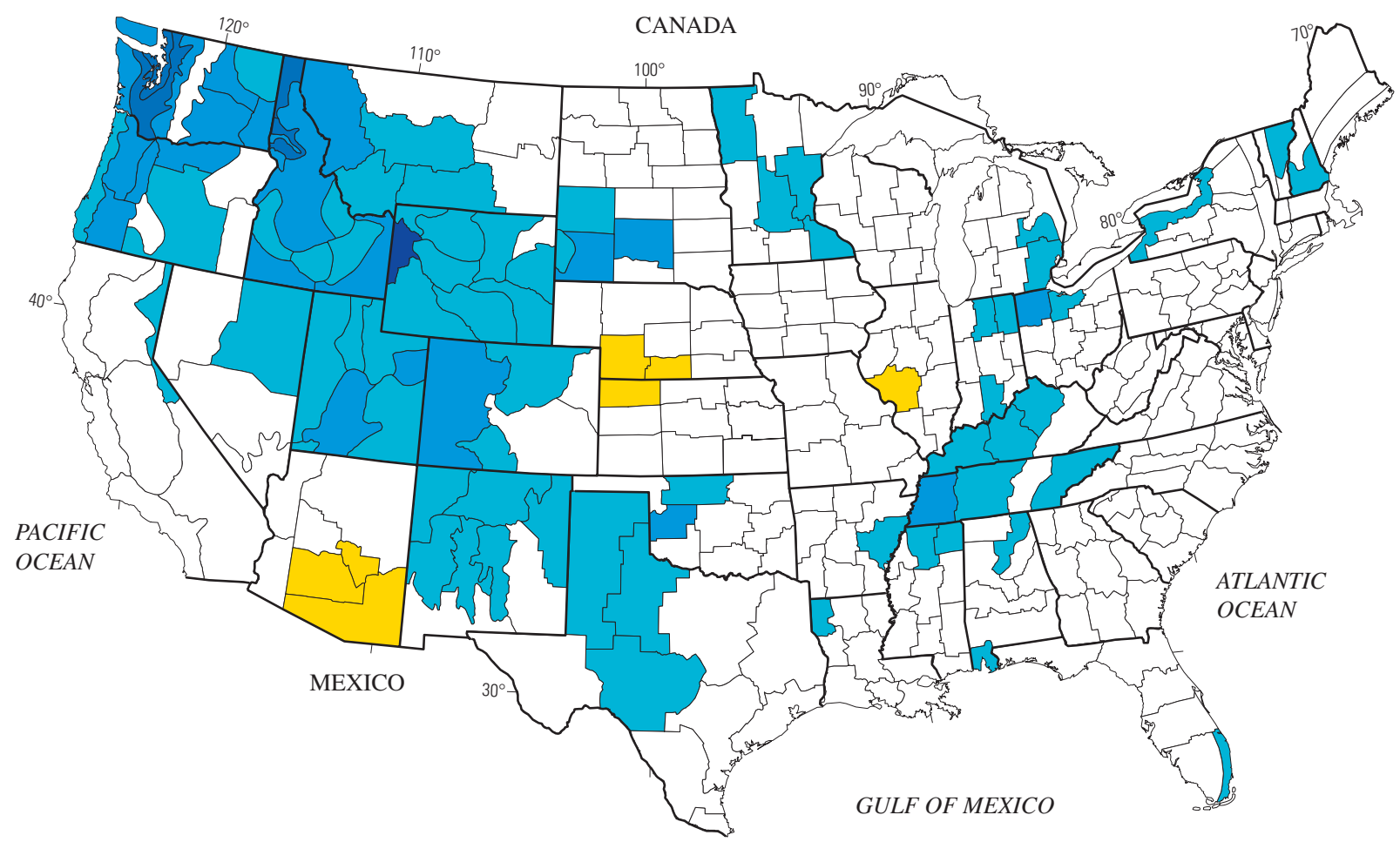

EXPLANATION

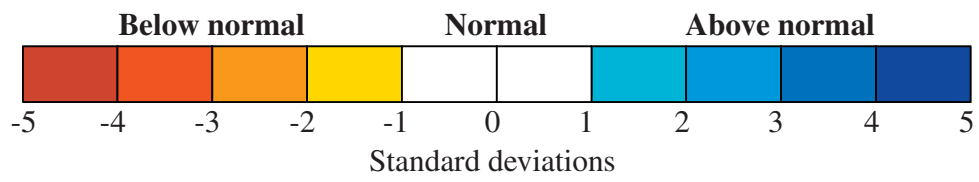

Boundary of National Weather Service meteorological divisions

Figure 10. Standardized 1997 water year precipitation deviations from long-term mean precipitation (1950-95) in conterminous United States (source of data: National Oceanic and Atmospheric Administration, Climate Diagnostics Center, at URL http://www.cdc.noaa.gov/USclimate/USclimdivs.html).

On July 28, 1997, excessive rainfall in Colorado created flash floods in which five persons lost their lives. Flash floods in Arizona claimed 11 lives on August 12.

\section{USGS Published Reports on Flooding During 1997 Water Year}

Berris, S.N., Hess, G.W., Taylor, R.L., and Bohman, L.R., 1997, Flood-control effects of Truckee River basin reservoirs, December 31, 1996, through January 4, 1997, California and Nevada: U.S. Geological Survey Fact Sheet 037-97, $4 \mathrm{p}$.

Brigham, M.E., and Lorenz, D.L., 1997, Water quality in the Red River of the North during the spring flood of 1997, in Hartman, J.H. ed., Symposium on the Red River flood of 1997-involving science in future watershed management decisions: Proceedings of the North Dakota Academy of Science, v. 51, supplement 1, p. 30.
Hess, G.W., and Williams, R.P., 1997, Flood of January 1997 in the Truckee River basin, western Nevada: U.S. Geological Survey Fact Sheet 123-97, 2 p.

Hunrichs, R.A., Pratt, D.A., and Meyer, R.W., 1998, Magnitude and frequency of the floods of January 1997 in northern and central California - preliminary determinations: U.S. Geological Survey Open-File Report 98-626, 120 p.

Jackson, K.S., Vivian, S.A., Diam, F.J., and Creceluis, C.J., 1997, Flood of March 1997 in southern Ohio: U.S. Geological Survey Water-Resources Investigations Report 97-4149, $21 \mathrm{p}$.

Macek-Rowland, K.M., 1997, Floods in the Red River of the North and Missouri River basins in North Dakota and western Minnesota: U.S. Geological Survey Open-File Report 97-0575, 9 p.

Rigby, J.G, Crompton, E.J., Berry, K.A., Yildirim, Unal, Hickman, S.F., and Davis, D.A., 1998, The 1997 New Year's floods in western Nevada: Nevada Bureau of Mines and Geology, Special Publication 23, 111 p. 
Robinson, J.B., Hazell, W.F., and Young, W.S., 1998, Effects of August 1995 and July 1997 storms in the city of Charlotte and Mecklenburg County, North Carolina: U.S. Geological Survey Fact Sheet 036-98, 6 p.

Teller, R.W., and Burr, M.J., 1998, Floods in north-central and eastern South Dakota, spring 1997: U.S. Geological Survey Fact Sheet 021-98, 4 p.

Thomas, K.A., and Hess, G.W., 1997, Flood of January 1997 in the Walker River basin, California and Nevada: U.S. Geological Survey Fact Sheet 182-97, 2 p.

Thomas, K.A., and Williams, R.P., 1997, Flood of January 1997 in the Carson River basin, California and Nevada: U.S. Geological Survey Fact Sheet 183-97, 2 p.

Wiche, G.J., Martin, C.R., Albright, L.L., and Wald, G.B., 1997a, Flood tracking chart for the Red River of the North basin: U.S. Geological Survey Open-File Report 97-193, 1 sheet.

Wiche, G.J., Martin, C.R., Albright, L.L., and Wald, G.B., 1997b, Flood tracking chart for the Sheyenne River basin: U.S. Geological Survey Open-File Report 97-177, 1 sheet.

\section{Water Year}

The 1998 water year experienced flooding in the southeastern and northeastern parts of the United States. California also had some significant floods. Figure 11 shows the percentage of streamgages in each State recording significant floods (top 5 percent of the peak-flow record) during the 1998 water year. Much of the country had above-normal standardized precipitation deviations for the 1998 water year (fig. 12). The same areas just mentioned had above-normal precipitation of more than two standard deviations for the 12-month period from October 1997 to September 1998.

A major storm that struck the eastern United States during January 4-9, 1998, caused flooding from Texas and Oklahoma across the southeast, on up the eastern seaboard, and finally out to sea east of Maine. This storm was followed a month later in February 1998 by an intense Atlantic storm that caused coastal flooding from North Carolina to New Jersey. Strong February storms also caused record peak flows in California from the San Francisco Bay area on south to the Los Angeles area (fig. 11).

Although there were several episodes of severe weather in the spring and summer 1998, there were no significant floods.
However, the 1998 hurricane season brought flooding to the Southeastern States by August. Hurricane Bonnie (August 1930, 1998) came onshore in eastern North Carolina on August 27 and caused widespread wind damage and coastal flooding.

Tropical Storm Charlie (August 21-24, 1998) brought torrential rain and flooding to southern Texas. Hurricane Earl

(August 31-September 3, 1998) raced from the western Gulf of Mexico across Florida, Georgia, and South Carolina and caused minimal flooding. Tropical Storm Frances (September 8-13, 1998) wandered into southern Texas and caused flooding there. Hurricane Georges (September 15-October 1, 1998) first struck Puerto Rico on September 21 with high winds and torrential rainfall. Its track carried it up the center of the islands of Hispaniola and Cuba where it lost some of its intensity before making landfall in southern Mississippi. There, Georges turned abruptly east and tracked along the Gulf Coast until crossing Florida and dissipating. The storm surge and excessive rain caused flooding along the Gulf Coast.

\section{USGS Published Reports on Flooding During 1998 Water Year}

Bowers, J.C., 2001, Floods in Cuyama Valley, California, February 1998: U.S. Geological Survey Fact Sheet 162-00, 6 p.

Fischer, E.E., 1999, Flood of June 15-17, 1998, Nishnabotna and East Nishnabotna Rivers, southwest Iowa: U.S. Geological Survey Open-File Report 99-70, 15 p.

Koltun, G.F., 1999, Floods of June 28-29, 1998, in Ohio: U.S. Geological Survey Water-Resources Investigations Report 99-4192, $26 \mathrm{p}$.

Parker, G.W., Ries, K.G., III, and Socolow, R.S., 1998, The flood of June 1998 in Massachusetts and Rhode Island: U.S. Geological Survey Fact Sheet 110-98, 4 p.

Slade, R.M., Jr., and Persky, Kristie, 1999, Floods in the Guadalupe and San Antonio River basins in Texas, October 1998: U.S. Geological Survey Fact Sheet 147-99, 4 p.

Turnipseed, D.P., Giese, G.L., Pearman, J.L., Farris, G.S., Krohn, M.D., and Sallenger, A.H., Jr., 1998, Hurricane Georges-headwater flooding, storm surge, beach erosion, and habitat destruction on the central Gulf Coast: U.S. Geological Survey Water-Resources Investigations Report 984231, $6 \mathrm{p}$. 

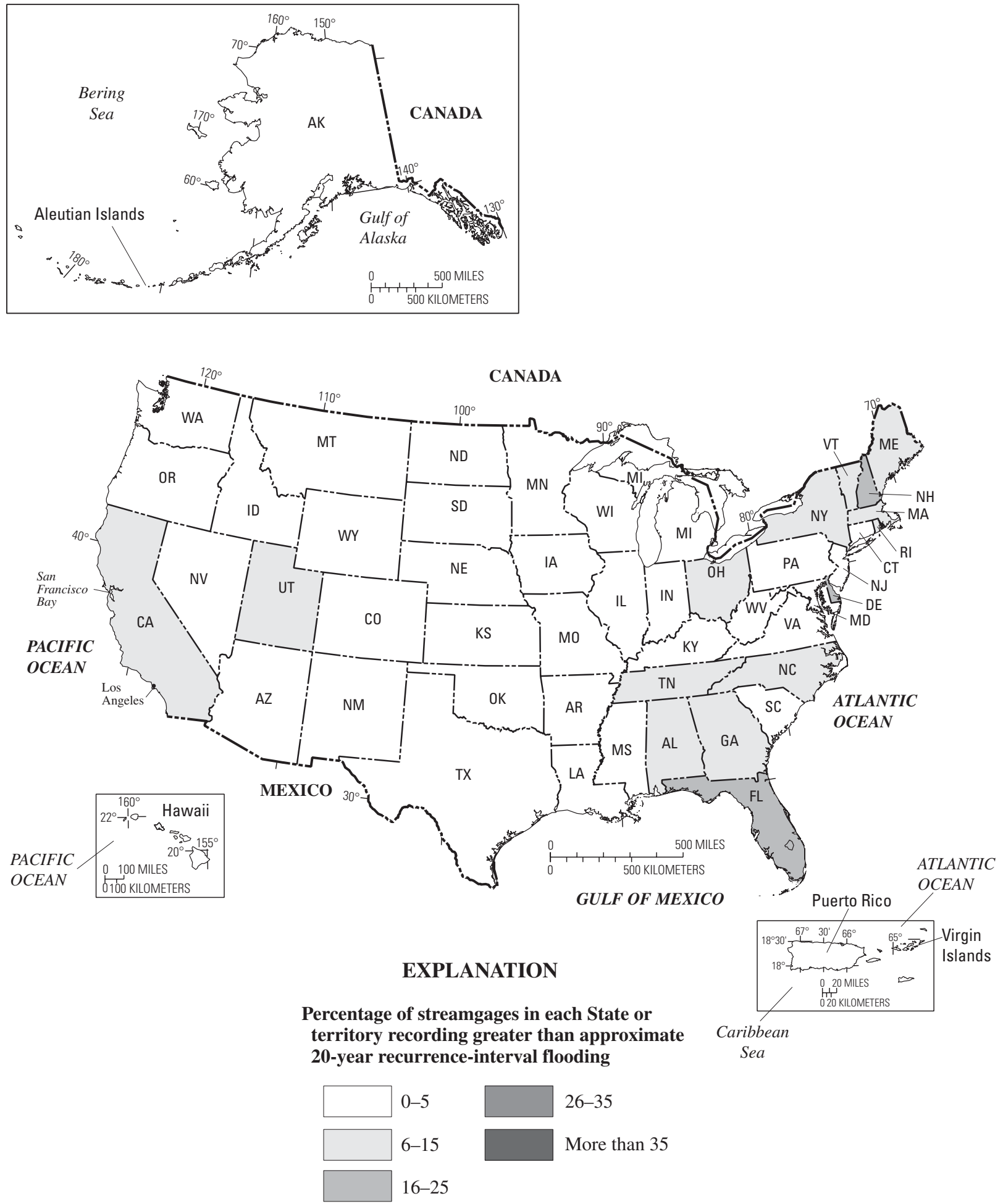

Figure 11. Percentage of streamgages in each State or territory recording greater than approximate 20-year recurrenceinterval flooding during 1998 water year. 


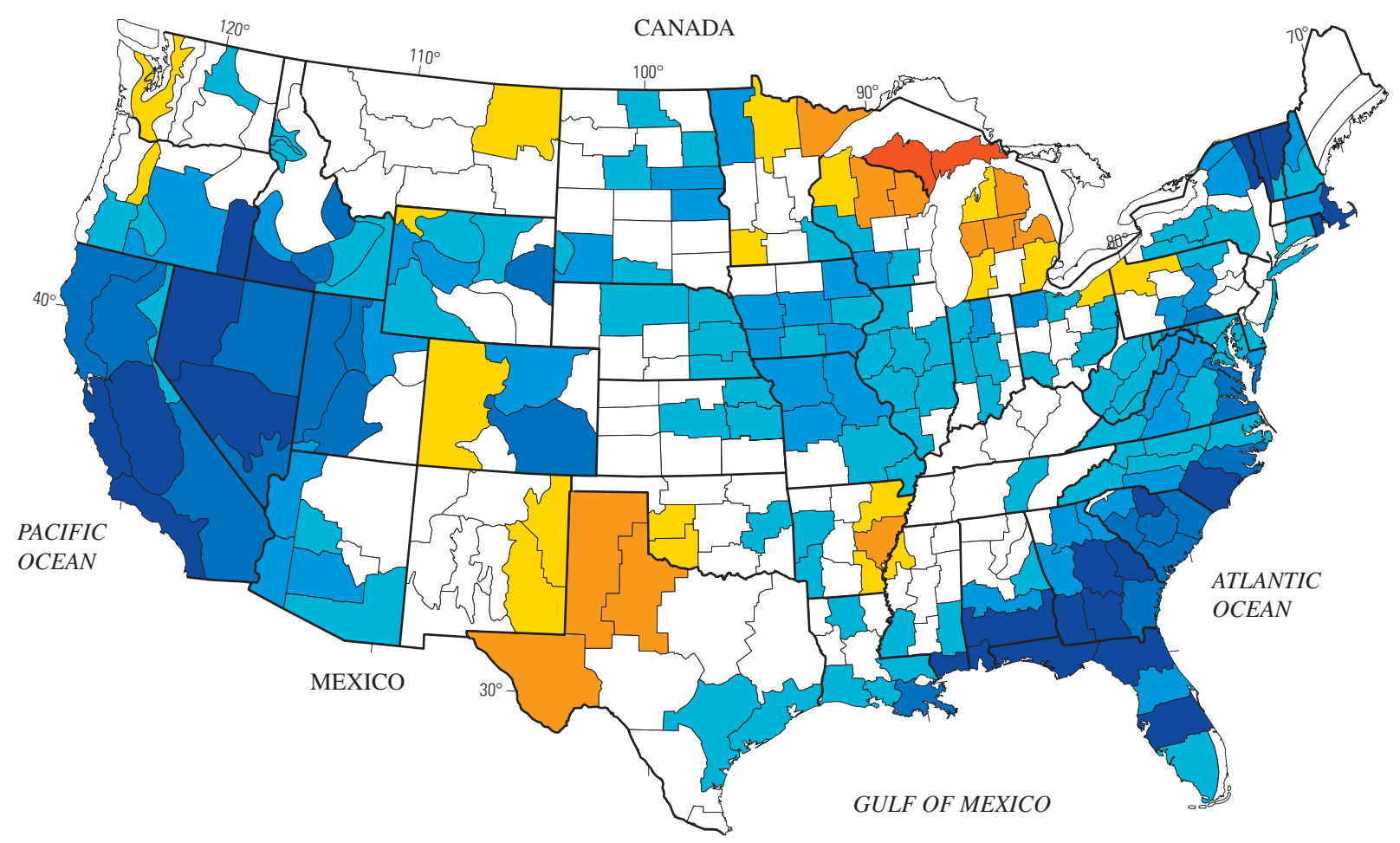

EXPLANATION

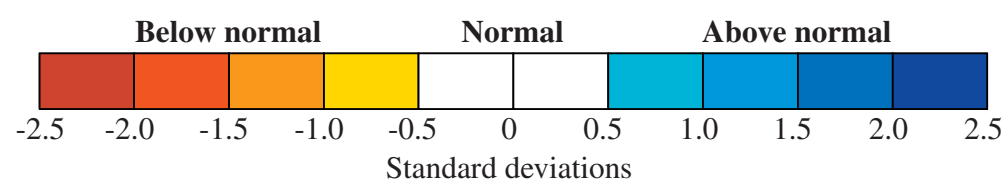

Boundary of National Weather Service meteorological divisions

Figure 12. Standardized 1998 water year precipitation deviations from long-term mean precipitation (1950-95) in conterminous United States (source of data: National Oceanic and Atmospheric Administration, Climate Diagnostics Center, at URL http://www.cdc.noaa.gov/USclimate/USclimdivs.html). 


\section{Summary of Significant Floods in the United States and Puerto Rico, 1994 Through 1998 Water Years}

\section{Significant Floods by State or Territory}

\section{Alabama}

The remnants of Tropical Storm Alberto stalled over the southeastern United States and produced a period of off-and-on torrential rains July 3-7, 1994. The off-and-on rains lasted for 5 days and produced significant flooding on the Pea River, the Choctawhatchee River, Catoma Creek, Conecuh River, Tallapoosa River, and Murder Creek (fig. 13). The torrential rains also produced extensive flash flooding in parts of southeastern Alabama. Two deaths occurred as a result of the excessive rain and flooding. Damage was \$14 to \$20 million (National Oceanic and Atmospheric Administration, 1994b).

Excessive rains of 8 to 14 inches just west of Mobile caused flash flooding on December 18, 1995. A 22-year-old man was killed when he was swept into a culvert while trying to clear debris from a drainage ditch (National Oceanic and Atmospheric Administration, 1995b).

Hurricane Danny moved inland from the Gulf of Mexico across Fort Morgan and into Mobile Bay during the early morning hours of July 18, 1997. The storm remained nearly stationary over Mobile Bay for 8 hours. Danny then crept east, making landfall near Weeks Bay. Danny then drifted north along the Alabama and Florida border, weakening to a depression by July 21. Danny continued to drift north through the State and passed into northern Georgia by July 23. Torrential rainfall fell in extreme southern Alabama. Observing sites reported from 30 to 40 inches across the area with Dauphin Island reporting 36.71 inches. Unofficial estimates of 35 to 40 inches of rainfall (National Oceanic and Atmospheric Administration, 1997a) were reported near the center of Danny as the storm moved across Weeks Bay. Record flooding caused major damage to homes along the Fowl and Fish Rivers. A streamgage on the Fowl River (National Oceanic and Atmospheric
Administration, 1998b) recorded a stage of 12.6 feet, which is 1.5 times higher than the 100-year flood. At a streamgage on the Fish River near Silver Hill (streamgage 02378500, table 2) a stage of 22.8 feet was recorded, making this a 50-year flood. A storm-surge height of 5 to 6.5 feet was recorded.

A vigorous Gulf storm dumped 8 to 14 inches of rain (National Oceanic and Atmospheric Administration, 1998a) across parts of southern Alabama during March 8, 1998. The earthen levee near the city of Elba was breached. A 6-foot wall of water rushed into the city, and 2,000 residents were forced to evacuate. Four people were killed when their vehicles were swept away by floodwaters. The Choctawhatchee River at Newton (streamgage 02361000, table 2) crested near 34.6 feet on March 9. A man drowned when he jumped in to rescue a stranded motorist. Another man drowned when his vehicle was carried into the lake by a collapsing roadway (National Oceanic and Atmospheric Administration, 1998b).

Torrential rains from Tropical Storm/Depression Georges of 8 to 24 inches (National Oceanic and Atmospheric Administration, 1998a) during September 28-29, 1998, produced the worst flooding in southeastern Alabama since Hurricane Opal in 1995 (National Oceanic and Atmospheric Administration, 1998b). The Styx River near Elsanor (streamgage 02377570, table 2) crested at 28.6 feet, which was estimated to be greater than a 100 -year flood.

\section{References}

National Oceanic and Atmospheric Administration (NOAA), 1994a-98a, Climatological data (by State): Asheville, North Carolina, National Climatic Data Center, various months. National Oceanic and Atmospheric Administration (NOAA), 1994b-98b, Storm data (by State): Asheville, North Carolina, National Climatic Data Center, various months. 


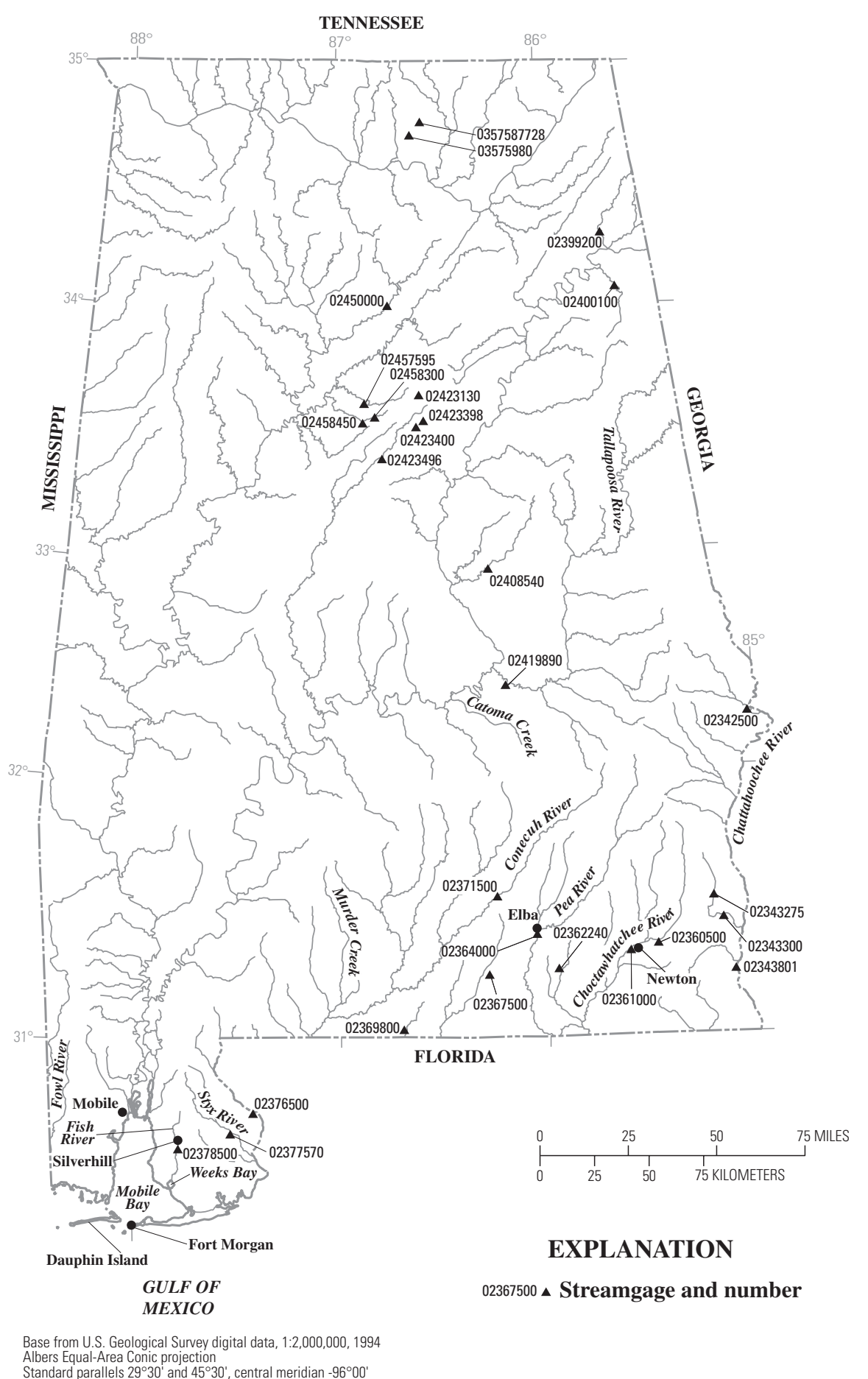

Figure 13. Location of streamgages with significant floods during 1994-98 water years for Alabama. 
Table 2. Maximum stage and discharge for period of record for streamgages having significant floods during 1994-98 water years in Alabama.

$\left[\mathrm{mi}^{2}\right.$, square miles; $\mathrm{ft}$, feet above an arbitrary datum; $\mathrm{ft}^{3} / \mathrm{s}$, cubic feet per second; --, not determined or not applicable; $>$, greater than. Source: Recurrence intervals calculated from U.S. Geological Survey data. Other data from U.S. Geological Survey reports or databases]

\begin{tabular}{|c|c|c|c|c|c|c|c|c|c|c|c|}
\hline \multirow[b]{2}{*}{$\begin{array}{c}\text { Streamgage } \\
\text { number } \\
\text { (fig. 13) }\end{array}$} & \multirow[b]{2}{*}{ Streamgage name } & \multirow[b]{2}{*}{$\begin{array}{c}\text { Total } \\
\text { drainage } \\
\left(\mathrm{mi}^{2}\right)\end{array}$} & \multicolumn{4}{|c|}{$\begin{array}{c}\text { Maximum stage and discharge for period of record } \\
\text { through } 1998 \text { water year }\end{array}$} & \multicolumn{5}{|c|}{ Significant floods $1994-98$ water years } \\
\hline & & & $\begin{array}{l}\text { Period of } \\
\text { record } \\
\text { (water } \\
\text { years) }\end{array}$ & Water year & $\begin{array}{l}\text { Stage } \\
\text { (ft) }\end{array}$ & $\begin{array}{l}\text { Discharge } \\
\qquad\left(\mathrm{ft}^{3} / \mathrm{s}\right)\end{array}$ & $\begin{array}{c}\text { Date } \\
\text { (month/ } \\
\text { day/ } \\
\text { year) }\end{array}$ & $\begin{array}{l}\text { Stage } \\
\text { (ft) }\end{array}$ & $\begin{array}{l}\text { Discharge } \\
\left(\mathrm{ft}^{3} / \mathrm{s}\right)\end{array}$ & $\begin{array}{l}\text { Regulated } \\
\text { during } \\
\text { flood }^{1}\end{array}$ & $\begin{array}{c}\text { Recurrence } \\
\text { interval } \\
\text { (years) }\end{array}$ \\
\hline 02342500 & $\begin{array}{l}\text { Uchee Creek near Fort Mitchell, } \\
\text { AL }\end{array}$ & 322 & $1947-98$ & 1964 & 26.45 & 55,100 & $7 / 8 / 94$ & 23.35 & 25,600 & $\mathrm{~N}$ & 25 \\
\hline 02343275 & Abbie Creek near Abbeville, AL & 48.7 & $\begin{array}{l}1951-80 \\
1990,1994\end{array}$ & 1994 & 13.00 & 30,000 & $7 / 6 / 94$ & 13.00 & 30,000 & $\mathrm{~N}$ & $>100$ \\
\hline 02343300 & Abbie Creek near Haleburg, AL & 146 & 1958-94 & 1994 & 37.00 & 35,000 & $7 / 6 / 94$ & 37.00 & 35,000 & $\mathrm{~N}$ & $>100$ \\
\hline 02343801 & $\begin{array}{l}\text { Chattahoochee River near } \\
\text { Columbia, AL }\end{array}$ & 8,210 & 1975-98 & 1994 & 123.98 & 202,000 & $7 / 7 / 94$ & 123.98 & 202,000 & $\mathrm{Y}$ & -- \\
\hline 02360500 & $\begin{array}{l}\text { East Fork Choctawhatchee River } \\
\text { near Midland City, AL }\end{array}$ & 291 & $\begin{array}{l}\text { 1953-63, } \\
1966-70, \\
1990,1994\end{array}$ & 1994 & 29.30 & 43,000 & $7 / 6 / 94$ & 29.30 & 43,000 & $\mathrm{~N}$ & $>100$ \\
\hline 02361000 & $\begin{array}{l}\text { Choctawhatchee River near } \\
\text { Newton, AL }\end{array}$ & 686 & $\begin{array}{l}1922-27, \\
1929, \\
1935-98\end{array}$ & $\begin{array}{l}1990 \\
1929\end{array}$ & $\begin{array}{l}40.30 \\
42.00\end{array}$ & $\begin{array}{l}87,500 \\
70,000\end{array}$ & $\begin{array}{l}7 / 7 / 94 \\
3 / 9 / 98\end{array}$ & $\begin{array}{l}37.78 \\
34.58\end{array}$ & $\begin{array}{l}60,800 \\
39,200\end{array}$ & $\begin{array}{l}\mathrm{N} \\
\mathrm{N}\end{array}$ & $\begin{array}{r}>100 \\
50\end{array}$ \\
\hline 02362240 & $\begin{array}{l}\text { Little Double Bridges Creek near } \\
\text { Enterprise, AL }\end{array}$ & 21.4 & 1986-98 & 1994 & 16.45 & 14,200 & $7 / 6 / 94$ & 16.45 & 14,200 & $\mathrm{~N}$ & $>100$ \\
\hline 02364000 & Pea River at Elba, AL & 959 & $\begin{array}{l}1929-55 \\
1972-98\end{array}$ & 1929 & 43.50 & 65,000 & $3 / 6 / 98$ & 39.23 & 45,000 & $\mathrm{~N}$ & 35 \\
\hline 02367500 & $\begin{array}{l}\text { Lightwood Knot Creek at Babbie, } \\
\text { AL }\end{array}$ & 114 & $\begin{array}{l}1930-75 \\
1990,1998\end{array}$ & 1998 & 19.54 & 37,900 & $3 / 8 / 98$ & 19.54 & 37,900 & $\mathrm{~N}$ & $>100$ \\
\hline 02369800 & $\begin{array}{l}\text { Blackwater River near } \\
\text { Bradley, AL }\end{array}$ & 87.7 & 1968-98 & 1990 & 25.35 & 24,000 & $3 / 8 / 98$ & 25.30 & 23,500 & $\mathrm{~N}$ & $>100$ \\
\hline 02371500 & Conecuh River at Brantley, AL & 500 & 1929-98 & $\begin{array}{l}1990 \\
1929\end{array}$ & $\begin{array}{l}24.44 \\
26.00\end{array}$ & $\begin{array}{l}25,700 \\
25,000\end{array}$ & $3 / 10 / 98$ & 22.62 & 17,000 & $\mathrm{~N}$ & 15 \\
\hline 02376500 & $\begin{array}{l}\text { Perdido River at Barrineau Park, } \\
\text { FL }\end{array}$ & 394 & $\begin{array}{l}1929 \\
1942-98\end{array}$ & 1998 & 26.30 & 44,000 & 9/29/98 & 26.30 & 44,000 & $\mathrm{~N}$ & $50-100$ \\
\hline 02377570 & Styx River near Elsanor, AL & 192 & 1988-98 & 1998 & 28.60 & 48,000 & $9 / 29 / 98$ & 28.60 & 48,000 & $\mathrm{~N}$ & $>100$ \\
\hline
\end{tabular}


Table 2. Maximum stage and discharge for period of record for streamgages having significant floods during 1994-98 water years in Alabama.—Continued

$\left[\mathrm{mi}^{2}\right.$, square miles; ft, feet above an arbitrary datum; $\mathrm{ft}^{3} / \mathrm{s}$, cubic feet per second; --, not determined or not applicable; >, greater than. Source: Recurrence intervals calculated from U.S. Geological Survey data. Other data from U.S. Geological Survey reports or databases]

\begin{tabular}{|c|c|c|c|c|c|c|c|c|c|c|c|}
\hline \multirow[b]{2}{*}{$\begin{array}{l}\text { Streamgage } \\
\text { number } \\
\text { (fig. 13) }\end{array}$} & \multirow[b]{2}{*}{ Streamgage name } & \multirow{2}{*}{$\begin{array}{l}\text { Total } \\
\text { drainage } \\
\left(\mathrm{mi}^{2}\right)\end{array}$} & \multicolumn{4}{|c|}{$\begin{array}{c}\text { Maximum stage and discharge for period of record } \\
\text { through } 1998 \text { water year }\end{array}$} & \multicolumn{5}{|c|}{ Significant floods $1994-98$ water years } \\
\hline & & & $\begin{array}{l}\text { Period of } \\
\text { record } \\
\text { (water } \\
\text { years) }\end{array}$ & Water year & $\begin{array}{l}\text { Stage } \\
(\mathrm{ft})\end{array}$ & $\begin{array}{l}\text { Discharge } \\
\left(\mathrm{ft}^{3} / \mathrm{s}\right)\end{array}$ & $\begin{array}{l}\text { Date } \\
\text { (month/ } \\
\text { day/ } \\
\text { year) }\end{array}$ & $\begin{array}{l}\text { Stage } \\
(\mathrm{ft})\end{array}$ & $\begin{array}{l}\text { Discharge } \\
\left(\mathrm{ft}^{3} / \mathrm{s}\right)\end{array}$ & $\begin{array}{l}\text { Regulated } \\
\text { during } \\
\text { flood }^{1}\end{array}$ & $\begin{array}{l}\text { Recurrence } \\
\text { interval } \\
\text { (years) }\end{array}$ \\
\hline 02378500 & Fish River near Silver Hill, AL & 55.3 & $\begin{array}{l}1954-69 \\
1971, \\
1987-98\end{array}$ & 1997 & 22.78 & 16,900 & $7 / 20 / 97$ & 22.78 & 16,900 & $\mathrm{~N}$ & 50 \\
\hline 02399200 & Little River near Blue Pond, AL & 199 & $\begin{array}{l}1948, \\
1958-98\end{array}$ & 1985 & 16.98 & 53,800 & $10 / 5 / 95$ & 15.26 & 40,700 & $\mathrm{~N}$ & 50 \\
\hline 02400100 & Terrapin Creek at Ellisville, AL & 252 & $1963-98$ & 1979 & 19.82 & 20,100 & $10 / 5 / 95$ & 19.10 & 16,900 & $\mathrm{~N}$ & 25 \\
\hline 02408540 & $\begin{array}{l}\text { Hatchet Creek below Rockford, } \\
\text { AL }\end{array}$ & 263 & 1981-98 & 1996 & 27.90 & 27,800 & $10 / 5 / 95$ & 27.90 & 27,800 & $\mathrm{~N}$ & $10-25$ \\
\hline 02419890 & $\begin{array}{l}\text { Tallapoosa River near Montgom- } \\
\text { ery-Montgomery Waterworks, } \\
\text { AL }\end{array}$ & 4,646 & 1973-98 & $\begin{array}{l}1996 \\
1990\end{array}$ & $\begin{array}{l}34.59 \\
42.13\end{array}$ & $\begin{array}{r}90,800 \\
--\end{array}$ & $3 / 10 / 98$ & 34.59 & 90,800 & $\mathrm{~N}$ & -- \\
\hline 02423130 & Cahaba River at Trussville, AL & 19.7 & 1989-98 & 1998 & 10.45 & 5,360 & $1 / 7 / 98$ & 10.45 & 5,360 & $\mathrm{~N}$ & -- \\
\hline 02423398 & $\begin{array}{l}\text { Little Cahaba River near Leeds, } \\
\text { AL }\end{array}$ & 19.4 & $\begin{array}{l}1981,1998 \\
1989-98\end{array}$ & 1998 & 11.83 & 2,900 & $1 / 7 / 98$ & 11.83 & 2,900 & $\mathrm{~N}$ & -- \\
\hline 02423400 & $\begin{array}{l}\text { Little Cahaba River near } \\
\text { Jefferson Park, AL }\end{array}$ & 24.4 & 1987-98 & 1996 & 5.08 & 2,900 & $1 / 26 / 96$ & 5.80 & 2,900 & $\mathrm{~N}$ & -- \\
\hline 02423496 & Cahaba River near Hoover, AL & 226 & 1989-98 & 1996 & 33.37 & 12,200 & $3 / 7 / 96$ & 33.37 & 12,200 & $\mathrm{~N}$ & $2-5$ \\
\hline 02450000 & $\begin{array}{l}\text { Mulberry Fork near Garden City, } \\
\text { AL }\end{array}$ & 365 & 1929-98 & 1990 & 25.04 & 66,500 & $2 / 11 / 94$ & 21.97 & 49,600 & $\mathrm{~N}$ & 25 \\
\hline 02457595 & Fivemile Creek near Republic, AL & 51.9 & 1989-98 & 1996 & 15.60 & 8,990 & $1 / 26 / 96$ & 15.60 & 8,990 & $\mathrm{~N}$ & -- \\
\hline 02458300 & $\begin{array}{l}\text { Village Creek at 24th Street at } \\
\text { Birmingham, AL }\end{array}$ & 26.0 & 1989-98 & 1998 & 12.68 & 5,860 & $1 / 7 / 98$ & 12.68 & 5,860 & $\mathrm{~N}$ & -- \\
\hline 02458450 & $\begin{array}{l}\text { Village Creek at Avenue West at } \\
\text { Ensley, AL }\end{array}$ & 33.5 & $\begin{array}{l}1976-79 \\
1989-98\end{array}$ & $\begin{array}{l}1996 \\
1979\end{array}$ & $\begin{array}{l}13.70 \\
14.00\end{array}$ & $\begin{array}{l}6,320 \\
5,450\end{array}$ & $2 / 26 / 96$ & 13.70 & 6,320 & $\mathrm{~N}$ & -- \\
\hline 0357587728 & $\begin{array}{l}\text { Dallas Branch at Coleman Street in } \\
\text { Huntsville, AL }\end{array}$ & 2.99 & 1985-98 & 1994 & 5.20 & 1,150 & $2 / 11 / 94$ & 5.20 & 1,150 & $\mathrm{~N}$ & -- \\
\hline 03575980 & $\begin{array}{l}\text { McDonald Creek at Patton Road } \\
\text { near Huntsville, AL }\end{array}$ & 9.64 & 1985-98 & 1998 & 11.59 & 3,530 & $5 / 7 / 98$ & 11.59 & 3,530 & $\mathrm{~N}$ & $10-25$ \\
\hline
\end{tabular}

${ }^{1}$ Regulated during flood: $\mathrm{N}$, no; $\mathrm{Y}$, yes. 


\section{Alaska}

Southeastern Alaska experiences some of the largest rainfall totals in North America. However, because the Coast Mountains rise so rapidly from the Pacific Ocean, streams are generally short and steep, so regional floods resulting from these intense rains are rare although peak discharge often exceeds 300 cubic feet per second per square mile.

Two storms occurred during August 1994, and each resulted in more than 5 inches of rain (National Oceanic and Atmospheric Administration, 1994) throughout the upper Koyukuk River Basin (fig. 14). Major flooding occurred from Wiseman, in the headwaters, to Hughes, near the mouth of the Koyukuk. Three villages were entirely evacuated, one of which, Alatna, has since been relocated. The area was declared a national and State disaster. The flood had a recurrence interval of greater than 100 years. Peak flows of record were measured at four streamgages across central Alaska.

Remnants of Tropical Storm Oscar struck south-central Alaska on September 19-21, 1995. Rivers from Kodiak Island in the southwest to Valdez in the east to Palmer in the north experienced flows having recurrence intervals of 2 to greater than 100 years (table 3 ). Flood damage was remarkably scattered given that the storm affected such a large area. For example, rivers flowing into Knik Arm of Cook Inlet carried flows greater than the 100-year flood, yet streams in Anchorage did not overtop their banks. Around Cook Inlet, streamflow probably was augmented by snow and glacier melt, which resulted from a very high freezing level. Homes along the Kenai and Resurrection Rivers were inundated, but most structural damage was confined to roads and bridges.

A series of storms in June, July, and August 1997 caused localized flooding in the interior of Alaska, with flood damage to roads along the upper Yukon, upper Tanana, and Chisana Rivers. The Alaska Highway was closed for several days due to bridge abutments being washed out.

\section{Reference}

National Oceanic and Atmospheric Administration (NOAA), 1994, Climatological data (by State): Asheville, North Carolina, National Climatic Data Center, various months. 


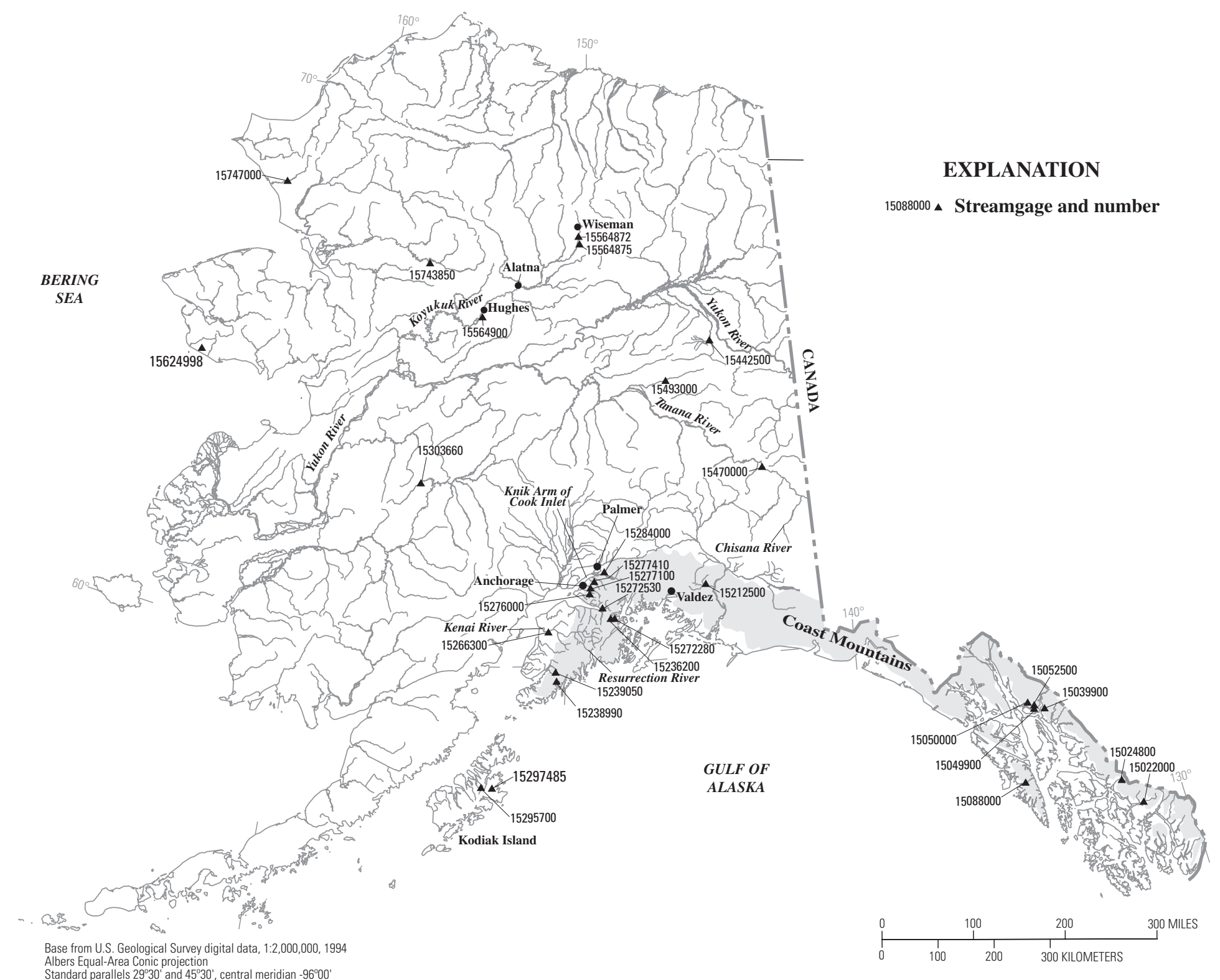

Figure 14. Location of streamgages with significant floods during 1994-98 water years for Alaska. 
Table 3. Maximum stage and discharge for period of record for streamgages having significant floods during 1994-98 water years in Alaska.

$\left[\mathrm{mi}^{2}\right.$, square miles; $\mathrm{ft}$, feet above an arbitrary datum; $\mathrm{ft}^{3} / \mathrm{s}$, cubic feet per second; --, not determined or not applicable; $>$, greater than. Source: Recurrence intervals calculated from U.S. Geological Survey data. Other data from U.S. Geological Survey reports or databases]

\begin{tabular}{|c|c|c|c|c|c|c|c|c|c|c|c|}
\hline \multirow[b]{2}{*}{$\begin{array}{c}\text { Streamgage } \\
\text { number } \\
\text { (fig. 14) }\end{array}$} & \multirow[b]{2}{*}{ Streamgage name } & \multirow[b]{2}{*}{$\begin{array}{l}\text { Total } \\
\text { drainage } \\
\left(\mathrm{mi}^{2}\right)\end{array}$} & \multicolumn{4}{|c|}{$\begin{array}{c}\text { Maximum stage and discharge for period of record } \\
\text { through } 1998 \text { water year }\end{array}$} & \multicolumn{5}{|c|}{ Significant floods $1994-98$ water years } \\
\hline & & & $\begin{array}{l}\text { Period of } \\
\text { record } \\
\text { (water } \\
\text { years) }\end{array}$ & Water year & $\begin{array}{l}\text { Stage } \\
(\mathrm{ft})\end{array}$ & $\begin{array}{l}\text { Discharge } \\
\left(\mathrm{ft}^{3} / \mathrm{s}\right)\end{array}$ & $\begin{array}{c}\text { Date } \\
\text { (month/ } \\
\text { day/ } \\
\text { year) }\end{array}$ & $\begin{array}{l}\text { Stage } \\
(\mathrm{ft})\end{array}$ & $\begin{array}{l}\text { Discharge } \\
\left(\mathrm{ft}^{3} / \mathrm{s}\right)\end{array}$ & $\begin{array}{l}\text { Regulated } \\
\text { during } \\
\text { flood }^{1}\end{array}$ & $\begin{array}{c}\text { Recurrence } \\
\text { interval } \\
\text { (years) }\end{array}$ \\
\hline 15022000 & Harding River near Wrangell, AK & 67.4 & $1952-98$ & $\begin{array}{l}1994 \\
1962\end{array}$ & $\begin{array}{l}16.21 \\
16.22\end{array}$ & $\begin{array}{l}15,300 \\
15,000\end{array}$ & $10 / 26 / 93$ & 16.21 & 175,300 & $\mathrm{~N}$ & $75-100$ \\
\hline 15024800 & Stikine River near Wrangell, AK & 19,920 & 1977-98 & 1994 & 30.60 & 351,000 & $9 / 23 / 94$ & 30.60 & 351,000 & $\mathrm{~N}$ & $50-100$ \\
\hline 15039900 & $\begin{array}{l}\text { Dorothy Lake outlet near Juneau, } \\
\text { AK }\end{array}$ & 11.0 & 1987-98 & 1995 & 13.05 & 990 & $9 / 10 / 95$ & 13.05 & 990 & $\mathrm{~N}$ & $10-25$ \\
\hline 15049900 & Gold Creek near Juneau, AK & 8.41 & 1985-97 & 1996 & 21.71 & 2,810 & $9 / 25 / 96$ & 21.71 & 2,810 & $\mathrm{~N}$ & $25-50$ \\
\hline 15050000 & Gold Creek at Juneau, AK & 9.76 & $\begin{array}{l}\text { 1917-20, } \\
1947-48, \\
1950-82, \\
1991,1994, \\
1996,1998\end{array}$ & 1996 & 8.14 & 2,950 & $9 / 25 / 96$ & 8.14 & 2,950 & $\mathrm{~N}$ & $25-50$ \\
\hline 15052500 & $\begin{array}{l}\text { Mendenhall River near Auke Bay, } \\
\text { AK }\end{array}$ & 85.1 & $1966-98$ & 1995 & 11.18 & 16,000 & $9 / 11 / 95$ & 11.18 & 16,000 & $\mathrm{~N}$ & $50-75$ \\
\hline 15088000 & Sawmill Creek near Sitka, AK & 39.0 & $\begin{array}{l}1921-22, \\
1929-42, \\
1946-57, \\
1994\end{array}$ & $\begin{array}{l}1994 \\
1948\end{array}$ & $-\overline{-}$ & $\begin{array}{r}11,100 \\
7,100\end{array}$ & $11 / 19 / 93$ & -- & 11,100 & $\mathrm{~N}$ & $>100$ \\
\hline 15212500 & Boulder Creek near Tiekel, AK & 9.80 & 1964-98 & $\begin{array}{l}1981 \\
1964\end{array}$ & $\begin{array}{l}11.72 \\
12.28\end{array}$ & $\begin{array}{r}1,330 \\
450\end{array}$ & $9 / 22 / 95$ & 10.58 & 484 & $\mathrm{~N}$ & $10-15$ \\
\hline 15236200 & Shakespeare Creek at Whittier, AK & 1.61 & $\begin{array}{l}1970-80 \\
1984-98\end{array}$ & 1995 & 14.90 & 690 & $9 / 20 / 95$ & 14.90 & 690 & $\mathrm{~N}$ & $25-50$ \\
\hline 15238990 & $\begin{array}{l}\text { Upper Bradley River near Nuka } \\
\text { Glacier near Homer, AK }\end{array}$ & -- & 1980-98 & 1995 & 15.10 & 4,100 & $9 / 20 / 95$ & 15.10 & 4,100 & $\mathrm{Y}$ & -- \\
\hline 15239050 & $\begin{array}{l}\text { Middle Fork Bradley River near } \\
\text { Homer, AK }\end{array}$ & 9.25 & 1980-98 & 1995 & 8.86 & 1,470 & $9 / 20 / 95$ & 8.86 & 1,470 & $\mathrm{~N}$ & $25-50$ \\
\hline
\end{tabular}


Table 3. Maximum stage and discharge for period of record for streamgages having significant floods during 1994-98 water years in Alaska.—Continued

$\left[\mathrm{mi}^{2}\right.$, square miles; ft, feet above an arbitrary datum; $\mathrm{ft}^{3} / \mathrm{s}$, cubic feet per second; --, not determined or not applicable; >, greater than. Source: Recurrence intervals calculated from U.S. Geological Survey data. Other data from U.S. Geological Survey reports or databases]

\begin{tabular}{|c|c|c|c|c|c|c|c|c|c|c|c|}
\hline \multirow{2}{*}{$\begin{array}{c}\text { Streamgage } \\
\text { number } \\
\text { (fig. 14) }\end{array}$} & \multirow[b]{2}{*}{ Streamgage name } & \multirow{2}{*}{$\begin{array}{c}\text { Total } \\
\text { drainage } \\
\left(\mathrm{mi}^{2}\right)\end{array}$} & \multicolumn{4}{|c|}{$\begin{array}{c}\text { Maximum stage and discharge for period of record } \\
\text { through } 1998 \text { water year }\end{array}$} & \multicolumn{5}{|c|}{ Significant floods $1994-98$ water years } \\
\hline & & & $\begin{array}{l}\text { Period of } \\
\text { record } \\
\text { (water } \\
\text { years) }\end{array}$ & Water year & $\begin{array}{l}\text { Stage } \\
(\mathrm{ft})\end{array}$ & $\begin{array}{c}\text { Discharge } \\
\left(\mathrm{ft}^{3} / \mathrm{s}\right)\end{array}$ & $\begin{array}{c}\text { Date } \\
\text { (month/ } \\
\text { day/ } \\
\text { year) }\end{array}$ & $\begin{array}{l}\text { Stage } \\
(\mathrm{ft})\end{array}$ & $\begin{array}{c}\text { Discharge } \\
\left(\mathrm{ft}^{3} / \mathrm{s}\right)\end{array}$ & $\begin{array}{c}\text { Regulated } \\
\text { during } \\
\text { flood }^{1}\end{array}$ & $\begin{array}{c}\text { Recurrence } \\
\text { interval } \\
\text { (years) }\end{array}$ \\
\hline 15266300 & Kenai River at Soldotna, AK & 1,860 & $1965-98$ & 1995 & 14.50 & 42,200 & $9 / 24 / 95$ & 14.50 & 42,200 & $\mathrm{~N}$ & $>100$ \\
\hline 15272280 & $\begin{array}{l}\text { Portage Creek at Portage Lake } \\
\text { outlet near Whittier, AK }\end{array}$ & 40.5 & $\begin{array}{l}\text { 1984, } \\
1989-98\end{array}$ & 1995 & 10.66 & 13,000 & $9 / 20 / 95$ & 10.66 & 13,000 & $\mathrm{~N}$ & $25-50$ \\
\hline 15272530 & California Creek at Girdwood, AK & 7.19 & $\begin{array}{l}1967-84, \\
1986-93, \\
1995\end{array}$ & $\begin{array}{l}1995 \\
1967\end{array}$ & $\begin{array}{l}16.04 \\
20.83\end{array}$ & $\begin{array}{l}760 \\
593\end{array}$ & $9 / 21 / 95$ & 16.04 & 760 & $\mathrm{~N}$ & $30-40$ \\
\hline 15276000 & Ship Creek near Anchorage, AK & 90.5 & $1947-98$ & $\begin{array}{l}1989 \\
1980\end{array}$ & $\begin{array}{l}6.38 \\
8.04\end{array}$ & $\begin{array}{l}2,100 \\
1,080\end{array}$ & $9 / 21 / 95$ & 6.52 & 1,890 & $\mathrm{Y}$ & $30-35$ \\
\hline 15277100 & Eagle River at Eagle River, AK & 192 & $\begin{array}{l}1966-80, \\
1995\end{array}$ & 1995 & 11.10 & 14,000 & 9/21/95 & 11.10 & 14,000 & $\mathrm{~N}$ & $>100$ \\
\hline 15277410 & Peters Creek near Birchwood, AK & 87.8 & $\begin{array}{l}1974-83 \\
1995\end{array}$ & 1995 & 10.40 & 5,000 & $9 / 21 / 95$ & 10.40 & 5,000 & $\mathrm{~N}$ & $>100$ \\
\hline 15284000 & Matanuska River at Palmer, AK & 2,070 & $\begin{array}{l}1949-74, \\
1985-86, \\
1992,1995\end{array}$ & 1971 & 13.60 & 82,100 & 9/22/95 & 13.04 & 46,000 & $\mathrm{~N}$ & $>100$ \\
\hline 15295700 & $\begin{array}{l}\text { Terror River at mouth near Kodiak, } \\
\text { AK }\end{array}$ & 30.7 & $\begin{array}{l}1964-68 \\
1982-98\end{array}$ & 1995 & 7.67 & 10,000 & $9 / 19 / 95$ & 7.67 & 10,000 & $\mathrm{Y}$ & -- \\
\hline 15297485 & $\begin{array}{l}\text { Kizhuyak River near Port Lions, } \\
\text { AK }\end{array}$ & 47.5 & 1980-95 & 1995 & 11.20 & 6,560 & 9/19/95 & 11.20 & 6,560 & $\mathrm{Y}$ & -- \\
\hline 15303660 & Gold Creek at Takotna,AK & 6.31 & 1987-98 & 1998 & 7.49 & 69 & $7 / 8 / 98$ & 7.49 & 69 & $\mathrm{~N}$ & $2-5$ \\
\hline 15442500 & Quartz Creek near Central, AK & 17.2 & $\begin{array}{l}1967, \\
1969-79, \\
1989-98\end{array}$ & 1995 & 23.08 & 700 & $7 / 15 / 95$ & 23.08 & 700 & $\mathrm{~N}$ & $25-50$ \\
\hline 15470000 & $\begin{array}{l}\text { Chisana River at Northway } \\
\text { Junction, AK }\end{array}$ & 3,280 & $\begin{array}{l}1949-71 \\
1997\end{array}$ & 1997 & 13.75 & 14,500 & $8 / 7 / 97$ & 13.75 & 14,500 & $\mathrm{~N}$ & $>100$ \\
\hline
\end{tabular}


$\left[\mathrm{mi}^{2}\right.$, square miles; ft, feet above an arbitrary datum; $\mathrm{ft}^{3} / \mathrm{s}$, cubic feet per second; --, not determined or not applicable; >, greater than. Source: Recurrence intervals calculated from U.S. Geological Survey data. Other data from U.S. Geological Survey reports or databases]

\begin{tabular}{|c|c|c|c|c|c|c|c|c|c|c|c|}
\hline \multirow{2}{*}{$\begin{array}{l}\text { Streamgage } \\
\text { number } \\
\text { (fig. 14) }\end{array}$} & \multirow[b]{2}{*}{ Streamgage name } & \multirow{2}{*}{$\begin{array}{c}\text { Total } \\
\text { drainage } \\
\left(\mathrm{mi}^{2}\right)\end{array}$} & \multicolumn{4}{|c|}{$\begin{array}{c}\text { Maximum stage and discharge for period of record } \\
\text { through } 1998 \text { water year }\end{array}$} & \multicolumn{5}{|c|}{ Significant floods $1994-98$ water years } \\
\hline & & & $\begin{array}{l}\text { Period of } \\
\text { record } \\
\text { (water } \\
\text { years) }\end{array}$ & Water year & $\begin{array}{l}\text { Stage } \\
(\mathrm{ft})\end{array}$ & $\begin{array}{l}\text { Discharge } \\
\left(\mathrm{ft}^{3} / \mathrm{s}\right)\end{array}$ & $\begin{array}{c}\text { Date } \\
\text { (month/ } \\
\text { day/ } \\
\text { year) }\end{array}$ & $\begin{array}{l}\text { Stage } \\
(\mathrm{ft})\end{array}$ & $\begin{array}{l}\text { Discharge } \\
\left(\mathrm{ft}^{3} / \mathrm{s}\right)\end{array}$ & $\begin{array}{l}\text { Regulated } \\
\text { during } \\
\text { flood }^{1}\end{array}$ & $\begin{array}{c}\text { Recurrence } \\
\text { interval } \\
\text { (years) }\end{array}$ \\
\hline \multirow[t]{2}{*}{15493000} & Chena River near Two Rivers, AK & 937 & $1967-98$ & 1992 & 22.04 & 20,000 & $6 / 26 / 94$ & 23.65 & 18,300 & $\mathrm{~N}$ & $10-15$ \\
\hline & & & & 1967 & 26.60 & -- & & & & & \\
\hline 15564872 & Nugget Creek near Wiseman, AK & 9.47 & $\begin{array}{l}1975-88 \\
1990-98\end{array}$ & 1998 & 40.17 & 540 & $5 / 26 / 98$ & 40.17 & 540 & $\mathrm{~N}$ & $25-30$ \\
\hline 15564875 & $\begin{array}{l}\text { Middle Fork Koyukuk River near } \\
\text { Wiseman, AK }\end{array}$ & 1,200 & $\begin{array}{l}1968, \\
1971-80, \\
1984-87, \\
1994\end{array}$ & $\begin{array}{l}1994 \\
1973\end{array}$ & $\begin{array}{l}12.92 \\
13.50\end{array}$ & $\begin{array}{l}42,700 \\
17,100\end{array}$ & $8 / 27 / 94$ & 12.92 & 42,700 & $\mathrm{~N}$ & $>100$ \\
\hline 15564900 & Koyukuk River at Hughes, AK & 18,400 & $\begin{array}{l}1961-82 \\
1994\end{array}$ & 1994 & 34.60 & 330,000 & $8 / 31 / 94$ & 34.60 & 330,000 & $\mathrm{~N}$ & $>100$ \\
\hline 15624998 & $\begin{array}{l}\text { Arctic Creek above tributary near } \\
\text { Nome, AK }\end{array}$ & 1.13 & $\begin{array}{l}\text { 1975, } \\
1979-98\end{array}$ & 1998 & 19.06 & 182 & $8 / 20 / 98$ & 19.06 & 182 & $\mathrm{~N}$ & $25-30$ \\
\hline 15743850 & Dahl Creek near Kobuk, AK & 11.0 & 1986-98 & $\begin{array}{l}1994 \\
1990\end{array}$ & $-\overline{-}-6$ & $\begin{array}{r}1,840 \\
538\end{array}$ & $8 / 17 / 94$ & -- & 1,840 & $\mathrm{~N}$ & $25-50$ \\
\hline 15747000 & $\begin{array}{l}\text { Wulik River below Tutak Creek } \\
\text { near Kivalina, AK }\end{array}$ & 705 & $1985-98$ & 1994 & 12.21 & 38,500 & $8 / 17 / 94$ & 12.21 & 38,500 & $\mathrm{~N}$ & $15-20$ \\
\hline
\end{tabular}

${ }^{1}$ Regulated during flood: N, no; Y, yes. 


\section{Arizona}

Two to 3 inches of rain fell over parts of Scottsdale on October 6, 1993 (National Oceanic and Atmospheric Administration, 1993a). The intense rain caused Indian Bend Wash (fig. 15) to overflow onto city streets and wash over the bridges on Camelback and Indian School Roads. A few motorists had to be rescued from their cars as they tried to cross the flooded wash.

A series of thunderstorms moved through the Yuma area during the early morning hours of August 21, 1994, and as much as 5 inches of rain led to the flooding of four homes about 8 miles south of Yuma. The Yuma County Extension Agent estimated nearly \$1 million in damages, mainly to cotton crops (National Oceanic and Atmospheric Administration, 1994b).

Excessive rains fell on the Salt River and Verde River watersheds during February 13-15, 1995. This, combined with rain falling on snowpack in the higher mountains of central and northern Arizona, led to flooding and flash flooding. The Salt River and Verde River watersheds averaged 1.27 and 2.16 inches of rain, respectively, between the morning of February 13 and the morning of February 15 (National Oceanic and Atmospheric Administration, 1995a). During the night of February 14 , remote rain gages in the Bradshaw Mountains reported as much as 5.12 inches of rain (National Oceanic and Atmospheric Administration, 1995a). Record flows were observed on the Verde River below Tangle Creek (streamgage 09508500, fig. 15) when the flow peaked at 108,000 cubic feet per second (table 4).

Excessive rains on March 6, 1995, produced flash flooding on Bright Angel Creek, and six employees of Phantom Ranch were stranded on an island in the middle of the creek. Rock and soil movement along the south rim of the Grand Canyon caused severe trail damage. The trans-canyon water pipeline running under the trail was broken when a 100-yard-long section of the trail gave way.

A strong Pacific storm on March 5-6, 1995, resulted in excessive rain falling over the central and northern mountains where soils were already saturated from previous rains. Between the afternoon of March 5 and the morning of March 6, 1995, a remote rain gage south of Prescott reported 4.92 inches (National Oceanic and Atmospheric Administration, 1995a). Many other locations received about 2 inches of rain. Nearrecord flows were observed on Oak Creek at Cornville and on Dry Beaver Creek (National Oceanic and Atmospheric Administration, 1995b). The flooding caused an estimated \$1.3 million in damage (National Oceanic and Atmospheric Administration, 1995b).

Excessive rain during March 11-12, 1995, resulted in flooding along Beaver Dam Wash and the Virgin River in the vicinity of Littlefield. Erosion of the banks along the wash caused four mobile homes to be washed downstream. Damage to public property was estimated at $\$ 335,000$ and private property at $\$ 1,290,000$ (National Oceanic and Atmospheric Administration, 1995b).

A series of strong thunderstorms moving through Tucson brought widespread damage on August 11, 1995. Many power poles were knocked over, and roofs were torn off buildings. As much as 4 inches of rain accompanied these storms (National Oceanic and Atmospheric Administration, 1995b). Some areas received 0.75 -inch-diameter hail. Washes in the area were running near bankfull. One woman attempting to drive through a flooded wash was swept to her death.

Eleven hikers and tourists were drowned in a flash flood in a narrow slot canyon 5 miles southeast of Page, on August 12, 1997. A severe thunderstorm 3 to 5 miles upstream produced very intense rain causing a 10- to 30-foot wall of water to crash down Antelope Canyon. The 11 who died were warned not to enter the canyon because of the flood potential from an approaching thunderstorm (National Oceanic and Atmospheric Administration, 1997b).

A flash flood on Phantom Creek killed two people and injured a third person on September 11, 1997. Runoff from the excessive rainfall several miles north of the flash flood site caught the three people as they were crossing Phantom Creek (National Oceanic and Atmospheric Administration, 1997b).

Three members of a Boy Scout troop perished after their sport utility vehicle was swept away on March 28, 1998. The scouts tried to cross a flooded wash near Sunflower (National Oceanic and Atmospheric Administration, 1997b).

A man was killed when he was caught in a flooded wash and drowned in the eastern part of Tucson on August 9, 1998 (National Oceanic and Atmospheric Administration, 1997b).

\section{References}

National Oceanic and Atmospheric Administration (NOAA), 1993a-97a, Climatological data (by State): Asheville, North Carolina, National Climatic Data Center, various months.

National Oceanic and Atmospheric Administration (NOAA), 1993b-97b, Storm data (by State): Asheville, North Carolina, National Climatic Data Center, various months. 


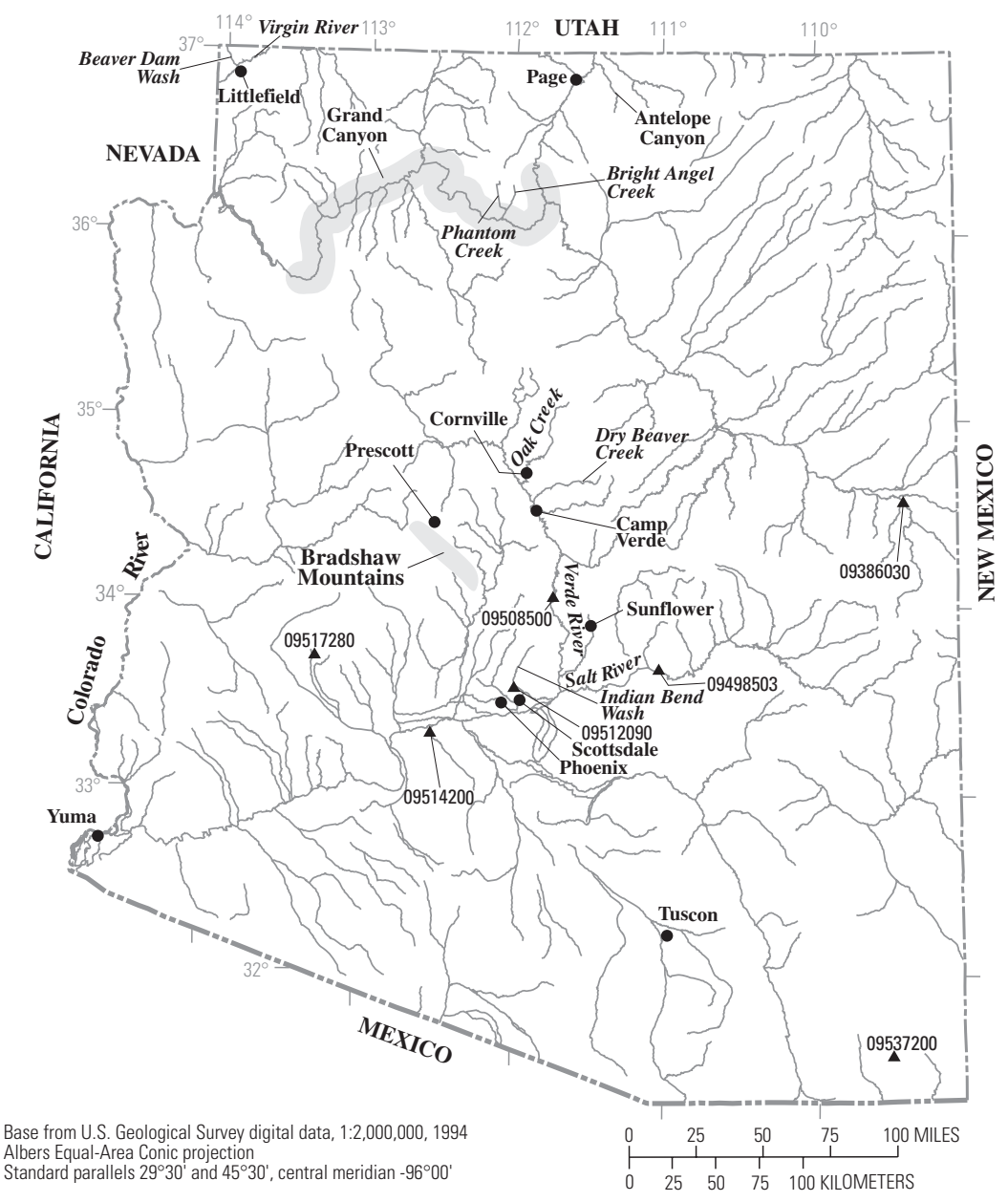

Standard parallels $29^{\circ} 30^{\prime}$ and $45^{\circ} 30^{\prime}$, central meridian $-96^{\circ} 00^{\prime}$

\section{EXPLANATION}

09537200 _Streamgage and number

Figure 15. Location of streamgages with significant floods during 1994-98 water years for Arizona. 
Table 4. Maximum stage and discharge for period of record for streamgages having significant floods during 1994-98 water years in Arizona.

$\left[\mathrm{mi}^{2}\right.$, square miles; $\mathrm{ft}$, feet above an arbitrary datum; $\mathrm{ft}^{3} / \mathrm{s}$, cubic feet per second; --, not determined or not applicable. Source: Recurrence intervals calculated from U.S. Geological Survey data. Other data from U.S. Geological Survey reports or databases]

\begin{tabular}{|c|c|c|c|c|c|c|c|c|c|c|c|}
\hline \multirow[b]{2}{*}{$\begin{array}{c}\text { Streamgage } \\
\text { number } \\
\text { (fig. 15) }\end{array}$} & \multirow[b]{2}{*}{ Streamgage name } & \multirow{2}{*}{$\begin{array}{c}\text { Total } \\
\text { drainage } \\
\left(\mathrm{mi}^{2}\right)\end{array}$} & \multicolumn{4}{|c|}{$\begin{array}{c}\text { Maximum stage and discharge for period of record } \\
\text { through } 1998 \text { water year }\end{array}$} & \multicolumn{5}{|c|}{ Significant floods 1994-98 water years } \\
\hline & & & $\begin{array}{l}\text { Period of } \\
\text { record } \\
\text { (water } \\
\text { years) }\end{array}$ & Water year & $\begin{array}{l}\text { Stage } \\
(\mathrm{ft})\end{array}$ & $\begin{array}{l}\text { Discharge } \\
\left(\mathrm{ft}^{3} / \mathrm{s}\right)\end{array}$ & $\begin{array}{c}\text { Date } \\
\text { (month/ } \\
\text { day/ } \\
\text { year) }\end{array}$ & $\begin{array}{l}\text { Stage } \\
(\mathrm{ft})\end{array}$ & $\begin{array}{l}\text { Discharge } \\
\left(\mathrm{ft}^{3} / \mathrm{s}\right)\end{array}$ & $\begin{array}{c}\text { Regulated } \\
\text { during } \\
\text { flood }^{1}\end{array}$ & $\begin{array}{c}\text { Recurrence } \\
\text { interval } \\
\text { (years) }\end{array}$ \\
\hline 09386030 & $\begin{array}{l}\text { Little Colorado River above Zion } \\
\text { Reservoir near Saint Johns, AZ }\end{array}$ & 1,007 & 1976-98 & 1994 & 4.16 & 590 & $7 / 31 / 94$ & 4.16 & 590 & $\mathrm{Y}$ & -- \\
\hline 09498503 & $\begin{array}{l}\text { South Fork Parker Creek near } \\
\text { Roosevelt, AZ }\end{array}$ & 1.09 & $\begin{array}{l}\text { 1987-92, } \\
1994-98\end{array}$ & 1995 & 4.10 & 87 & $3 / 6 / 95$ & 4.10 & 87 & $\mathrm{~N}$ & $10-25$ \\
\hline 09508500 & $\begin{array}{l}\text { Verde River below Tangle Creek } \\
\text { above Horseshoe Dam, AZ }\end{array}$ & 5,858 & $\begin{array}{l}1891,1906 \\
1916,1920 \\
1925-98\end{array}$ & 1993 & 23.40 & 145,000 & $2 / 15 / 95$ & 21.75 & 108,000 & $\mathrm{~N}$ & 25 \\
\hline 09512090 & $\begin{array}{l}\text { Indian Bend Wash at Shea } \\
\text { Boulevard at Phoenix, AZ }\end{array}$ & 24.5 & $\begin{array}{l}1986-95, \\
1998\end{array}$ & 1994 & 3.89 & 4,700 & $10 / 6 / 93$ & 3.89 & 4,700 & $\mathrm{~N}$ & $25-50$ \\
\hline 09514200 & Waterman Wash near Buckeye, AZ & 420 & $\begin{array}{l}1964-78, \\
1980-93, \\
1996-98\end{array}$ & 1997 & 7.80 & 9,400 & $8 / 8 / 97$ & 7.80 & 9,400 & $\mathrm{~N}$ & 100 \\
\hline 09517280 & Tiger Wash near Aguila, AZ & 85.2 & $\begin{array}{l}1963-79, \\
1983, \\
1991-95, \\
1997-98\end{array}$ & $\begin{array}{l}1997 \\
1970\end{array}$ & $\begin{array}{l}10.17 \\
10.20\end{array}$ & $\begin{array}{l}8,070 \\
4,550\end{array}$ & $9 / 26 / 97$ & 10.17 & 8,070 & $\mathrm{~N}$ & 100 \\
\hline 09537200 & Leslie Creek near McNeal, AZ & 79.1 & $\begin{array}{l}1970-77 \\
1982-98\end{array}$ & 1994 & 9.00 & 5,200 & $9 / 1 / 94$ & 9.00 & 5,200 & $\mathrm{~N}$ & 20 \\
\hline
\end{tabular}

\footnotetext{
${ }^{1}$ Regulated during flood: N, no; Y, yes.
} 


\section{Arkansas}

Flooding was reported across the entire city of Magnolia (fig. 16) on April 4-5, 1997. Rainfall in parts of southwestern Arkansas for a 24-hour period averaged slightly less than 12 inches. Many roads and bridges were washed out, resulting in \$1 million in damage (National Oceanic and Atmospheric Administration, 1997b).

A local man was apparently fishing in the Cross Terre Rouge Creek when he was swept away and drowned by floodwaters on February 11, 1998 (National Oceanic and Atmospheric Administration, 1998b).

Excessive rain caused street flooding throughout Texarkana on May 28, 1998. A total of 10.48 inches of rain fell from the storm, which was a record for that date (National Oceanic and Atmospheric Administration, 1998a). Significant floods for Arkansas are listed in table 5.

\section{References}

National Oceanic and Atmospheric Administration (NOAA), 1997a-98a, Climatological data (by State): Asheville, North Carolina, National Climatic Data Center, various months.

National Oceanic and Atmospheric Administration (NOAA), 1997b-98b, Storm data (by State): Asheville, North Carolina, National Climatic Data Center, various months.

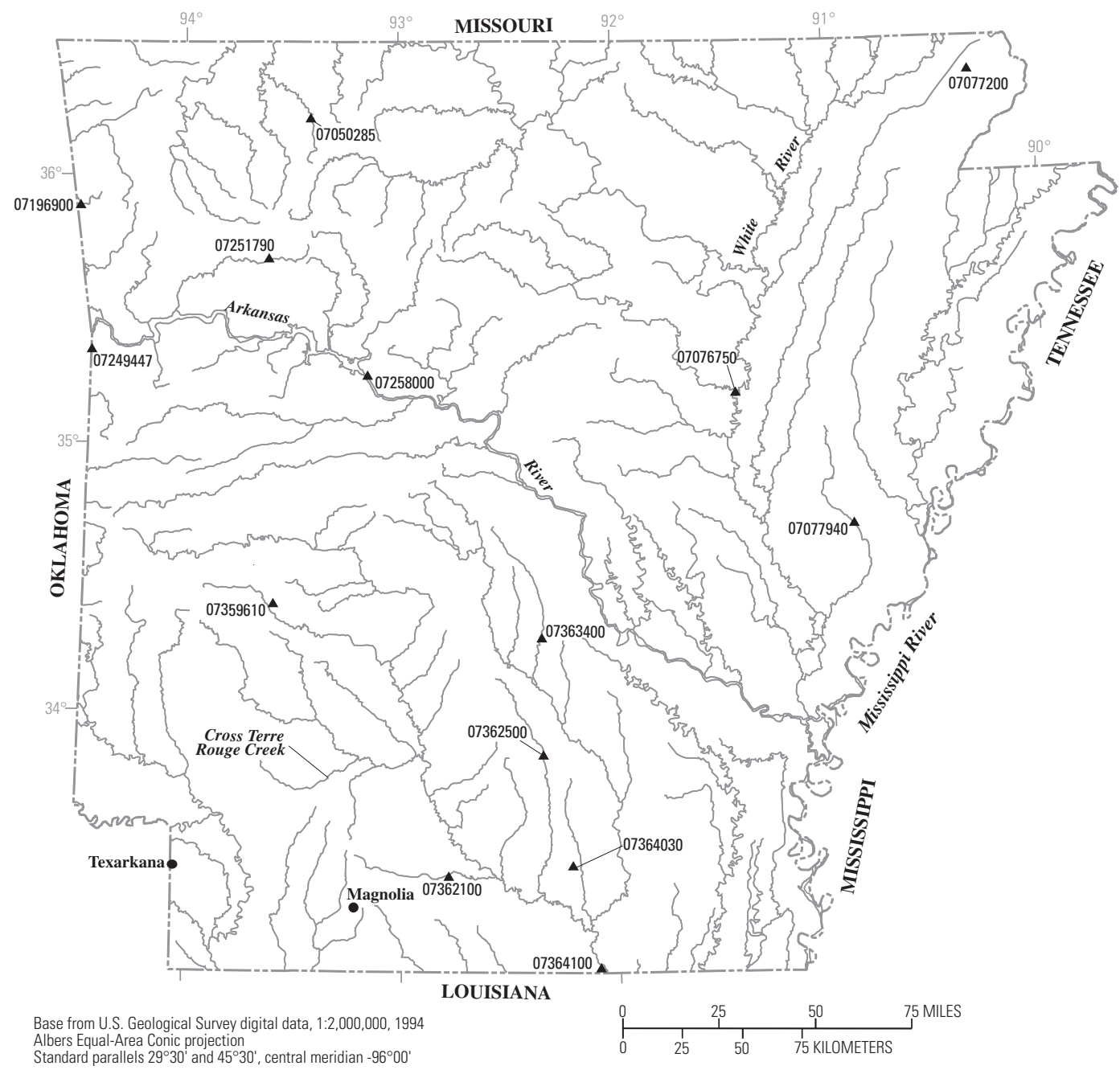

EXPLANATION

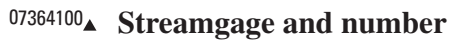

Figure 16. Location of streamgages with significant floods during 1994-98 water years for Arkansas. 
Table 5. Maximum stage and discharge for period of record for streamgages having significant floods during 1994-98 water years in Arkansas.

$\left[\mathrm{mi}^{2}\right.$, square miles; $\mathrm{ft}$, feet above an arbitrary datum; $\mathrm{ft}^{3} / \mathrm{s}$, cubic feet per second; --, not determined or not applicable; <, less than. Source: Recurrence intervals calculated from U.S. Geological Survey data. Other data from U.S. Geological Survey reports or databases]

\begin{tabular}{|c|c|c|c|c|c|c|c|c|c|c|c|}
\hline \multirow{2}{*}{$\begin{array}{l}\text { Streamgage } \\
\text { number } \\
\text { (fig. 16) }\end{array}$} & \multirow[b]{2}{*}{ Streamgage name } & \multirow{2}{*}{$\begin{array}{c}\text { Total } \\
\text { drainage } \\
\left(\mathrm{mi}^{2}\right)\end{array}$} & \multicolumn{4}{|c|}{$\begin{array}{l}\text { Maximum stage and discharge for period of record } \\
\text { through } 1998 \text { water year }\end{array}$} & \multicolumn{5}{|c|}{ Significant floods $1994-98$ water years } \\
\hline & & & $\begin{array}{l}\text { Period of } \\
\text { record } \\
\text { (water } \\
\text { years) }\end{array}$ & Water year & $\begin{array}{l}\text { Stage } \\
(\mathrm{ft})\end{array}$ & $\begin{array}{l}\text { Discharge } \\
\qquad\left(\mathrm{ft}^{3} / \mathrm{s}\right)\end{array}$ & $\begin{array}{c}\text { Date } \\
\text { (month/ } \\
\text { day/year) }\end{array}$ & $\begin{array}{l}\text { Stage } \\
\text { (ft) }\end{array}$ & $\begin{array}{l}\text { Discharge } \\
\left(\mathrm{ft}^{3} / \mathrm{s}\right)\end{array}$ & $\begin{array}{l}\text { Regulated } \\
\text { during } \\
\text { flood }^{1}\end{array}$ & $\begin{array}{c}\text { Recurrence } \\
\text { interval } \\
\text { (years) }\end{array}$ \\
\hline 07050285 & Osage Creek at Osage, AR & 82.3 & $1988-98$ & 1990 & 14.91 & 27,000 & $4 / 22 / 96$ & 11.99 & 13,700 & $\mathrm{~N}$ & $2-5$ \\
\hline 07076750 & White River at Georgetown, AR & 22,387 & 1913-98 & $\begin{array}{l}1983 \\
1949\end{array}$ & $\begin{array}{l}28.87 \\
32.80\end{array}$ & $\begin{array}{r}179,000 \\
--\end{array}$ & $12 / 18 / 94$ & 20.96 & 59,900 & $\mathrm{Y}$ & $<2$ \\
\hline 07077200 & $\begin{array}{l}\text { Big Creek tributary near } \\
\text { Boydsville, AR }\end{array}$ & 1.58 & $1962-98$ & 1998 & 9.94 & 790 & $7 / 25 / 98$ & 9.94 & 790 & $\mathrm{~N}$ & 20 \\
\hline 07077940 & Spring Creek near Aubrey, AR & 38.0 & $\begin{array}{l}\text { 1962-81, } \\
1993-98\end{array}$ & 1997 & 16.11 & 2,050 & $4 / 5 / 97$ & 16.11 & 2,050 & $\mathrm{~N}$ & 10 \\
\hline 07196900 & Baron Fork at Dutch Mills, AR & 40.6 & $1958-98$ & 1986 & 14.81 & 20,900 & $1 / 4 / 98$ & 13.85 & 17,500 & $\mathrm{~N}$ & $5-10$ \\
\hline 07249447 & Mill Creek at Fort Smith, AR & 10.0 & 1981-98 & 1990 & 36.40 & 2,400 & $6 / 10 / 95$ & 32.07 & -- & $\mathrm{N}$ & -- \\
\hline 07251790 & Mulberry River near Oark, AR & 70.2 & 1988-98 & 1993 & 14.72 & 21,500 & 9/26/96 & 14.42 & 20,000 & $\mathrm{~N}$ & $10-25$ \\
\hline 07258000 & $\begin{array}{l}\text { Arkansas River at Dardanelle, } \\
\text { AR }\end{array}$ & 153,670 & $1887-98$ & 1943 & 43.60 & 683,000 & $6 / 16 / 95$ & 33.38 & 205,000 & $\mathrm{Y}$ & 2 \\
\hline 07359610 & $\begin{array}{l}\text { Caddo River near Caddo Gap, } \\
\text { AR }\end{array}$ & 132 & 1989-98 & 1994 & 26.27 & 97,200 & $12 / 3 / 93$ & 26.27 & 97,200 & $\mathrm{~N}$ & $50-100$ \\
\hline 07362100 & $\begin{array}{l}\text { Smackover Creek near } \\
\text { Smackover, AR }\end{array}$ & 385 & 1939-98 & 1974 & 24.97 & 52,700 & $4 / 6 / 97$ & 24.08 & 41,700 & $\mathrm{~N}$ & 50 \\
\hline 07362500 & Moro Creek near Fordyce, AR & 240 & $\begin{array}{l}\text { 1938, } \\
1952-98\end{array}$ & 1958 & 16.47 & 26,800 & $4 / 6 / 97$ & 16.39 & 26,000 & $\mathrm{~N}$ & 50 \\
\hline 07363400 & $\begin{array}{l}\text { Hurricane Creek below Sheridan, } \\
\text { AR }\end{array}$ & 261 & $\begin{array}{l}1938-40, \\
1947-64, \\
1996-98\end{array}$ & $\begin{array}{l}1997 \\
1960\end{array}$ & $\begin{array}{l}16.34 \\
17.60\end{array}$ & 26,400 & $4 / 6 / 97$ & 16.34 & 26,400 & $\mathrm{~N}$ & -- \\
\hline
\end{tabular}


Table 5. Maximum stage and discharge for period of record for streamgages having significant floods during 1994-98 water years in Arkansas. - Continued

$\left[\mathrm{mi}^{2}\right.$, square miles; $\mathrm{ft}$, feet above an arbitrary datum; $\mathrm{ft}^{3} / \mathrm{s}$, cubic feet per second; --, not determined or not applicable; <, less than. Source: Recurrence intervals calculated from U.S. Geological Survey data. Other data from U.S. Geological Survey reports or databases]

\begin{tabular}{|c|c|c|c|c|c|c|c|c|c|c|c|}
\hline \multirow[b]{2}{*}{$\begin{array}{c}\text { Streamgage } \\
\text { number } \\
\text { (fig. 16) }\end{array}$} & \multirow[b]{2}{*}{ Streamgage name } & \multirow[b]{2}{*}{$\begin{array}{l}\text { Total } \\
\text { drainage } \\
\left(\mathrm{mi}^{2}\right)\end{array}$} & \multicolumn{4}{|c|}{$\begin{array}{c}\text { Maximum stage and discharge for period of record } \\
\text { through } 1998 \text { water year }\end{array}$} & \multicolumn{5}{|c|}{ Significant floods $1994-98$ water years } \\
\hline & & & $\begin{array}{l}\text { Period of } \\
\text { record } \\
\text { (water } \\
\text { years) }\end{array}$ & Water year & $\begin{array}{l}\text { Stage } \\
(\mathrm{ft})\end{array}$ & $\begin{array}{l}\text { Discharge } \\
\left(\mathrm{ft}^{3} / \mathrm{s}\right)\end{array}$ & $\begin{array}{c}\text { Date } \\
\text { (month/ } \\
\text { day/year) }\end{array}$ & $\begin{array}{l}\text { Stage } \\
(\mathrm{ft})\end{array}$ & $\begin{array}{l}\text { Discharge } \\
\left(\mathrm{ft}^{3} / \mathrm{s}\right)\end{array}$ & $\begin{array}{c}\text { Regulated } \\
\text { during } \\
\text { flood }^{1}\end{array}$ & $\begin{array}{c}\text { Recurrence } \\
\text { interval } \\
\text { (years) }\end{array}$ \\
\hline 07364030 & $\begin{array}{l}\text { L'aigle Creek tributary near } \\
\text { Hermitage, AR }\end{array}$ & 0.36 & $\begin{array}{l}1963-88, \\
1990, \\
1992-93, \\
1995-98\end{array}$ & 1991 & 7.06 & 260 & $4 / 5 / 97$ & 6.59 & 206 & $\mathrm{~N}$ & $30-40$ \\
\hline 07364100 & $\begin{array}{l}\text { Ouachita River near Louisiana } \\
\text { State line, AR }\end{array}$ & 10,787 & $\begin{array}{l}1912-85 \\
1987-95\end{array}$ & $\begin{array}{l}1971 \\
1945\end{array}$ & $\begin{array}{l}18.66 \\
44.20\end{array}$ & $\begin{array}{r}17,400 \\
--\end{array}$ & $10 / 25 / 94$ & -- & 12,800 & $\mathrm{Y}$ & -- \\
\hline
\end{tabular}

${ }^{1}$ Regulated during flood: N, no; Y, yes. 


\section{California}

Frequent and powerful storms swept over the entire State of California from January to March 1995. El Niño conditions in the Pacific Ocean helped to spawn an unusual series of storms during this time that caused intense, prolonged, and in some cases, unprecedented precipitation across California. This series of storms caused widespread minor to record-breaking floods. Peaks of record occurred at several streamgages. Total damage for the period was estimated to be near $\$ 3$ billion. There were 27 deaths attributed to the storms and flooding (Perry, 2000).

A powerful storm system dropped 3 to 6 inches of rain over portions of Los Angeles (fig. 17C) during the first weeks of January 1995. The Santa Ynez River washed out bridges in the Santa Ynez Valley. Flooding near Santa Barbara caused \$92 million in damage (National Oceanic and Atmospheric Administration, 1995b). Many other cities in southern California had substantial damage from floodwaters. Three days of record-setting rainfall culminated in widespread flooding of small rivers in central California, January 11, 1995. More than 1,000 homes and small businesses were damaged near Sacramento (fig. $17 B$ ) with estimated damage of $\$ 50$ million. One death also occurred when a homeless man was drowned in the flood. Total damage in California from the January 1995 flood exceeded \$350 million (National Oceanic and Atmospheric Administration, 1995b).

Warm rain on top of snow brought large amounts of water from the mountains in Yosemite National Park. The resulting water surge produced the peak of record on the Merced River (streamgage 11264500, table 6). Sierra Nevada 24-hour rainfall reports on the morning of May 16, 1996, ranged from 2.0 to 2.5 inches (National Oceanic and Atmospheric Administration, 1996a). Snowmelt above 10,000 feet in the southern Sierra Nevada Mountains added to the rainfall runoff and caused the Merced River to crest at 8.84 feet, which is 3.1 feet above the stage when minor flooding begins. Damage estimates were in the \$2-3 million range (National Oceanic and Atmospheric Administration, 1996b).

Several periods of rain beginning December 20, 1996, set up saturated conditions in northern California that led to flooding on January 1, 1997, as subtropical air moved in and melted snow up to the 8,000-foot level. As the runoff moved downstream and flood-control dams operated at near-maximum releases, stress on the Sacramento River (fig. 17A) and San Joaquin River (fig. 17B) levees resulted in numerous levee breaks, especially from January 4-10, 1997. Intense rains returned at the end of the month, beginning on the 22nd and lasting through the 27 th. These storms produced flooding problems in the foothills of the Sierra Nevada Mountains and on the valley floor. Levees continued to break in the Sacramento-San Joaquin River delta as the floodwaters headed out to San Francisco Bay. Preliminary data indicated record peak stages were set at nine streamgages in the Sacramento-San Joaquin River system. The particular rivers included the Sacramento, San Joaquin, Feather, Cosumnes, and Toulumne Rivers. The variety and magnitude of the damage were huge. At least 120,000 people were evacuated, and five deaths occurred in interior northern California. Flooding in the estimated 250 square miles that were inundated, damaged or destroyed a minimum of 20,000 homes and 1,500 businesses. Damage was estimated at $\$ 1.6$ billion, much of it in interior northern California. The flooding also virtually destroyed two large fish hatcheries, the Feather River fish hatchery and the San Joaquin fish hatchery (National Oceanic and Atmospheric Administration, 1997b).

Excessive rainfall on a much above-normal snowpack subsequently during the first week of January 1997 also led to floods in the southern Sierra Nevada Mountains as well as the adjacent San Joaquin Valley. Especially hard hit was Yosemite National Park as the Merced River produced the largest flood in 80 years. The water level reached 23.43 feet at the streamgage near Yosemite (streamgage 11266500, table 6), indicating a discharge of 24,600 cubic feet per second on January 3, 1997. One drowning fatality occurred southwest of Yosemite National Park as a vehicle was swept from a roadway. Also adversely affected were Kings Canyon and Sequoia National Parks with substantial road and trail damage (National Oceanic and Atmospheric Administration, 1997b).

A severe thunderstorm packing torrential rains and golfball-size hail pummeled part of the San Bernardino Mountains during September 4, 1997, sending floodwaters rushing down creeks near Forest Falls (fig. 17C). That small community bore the brunt of the damage as tons of mud, boulders, and debris buried roads, mangled automobiles, and battered scores of homes. Total estimated damage was \$3.2 million in Forest Falls (National Oceanic and Atmospheric Administration, 1997b).

A slow-moving, low-pressure center off the coast of southern California spawned late-night showers and thunderstorms on December 6, 1997. Rainfall generally ranged from 4 to 8 inches across the area (National Oceanic and Atmospheric Administration, 1997a), resulting in widespread flooding. The hardest hit area was the coastal plain southwest of the Santa Ana Mountains. It was the most intense rain recorded in at least 70 years. Collapsing hillsides and raging water triggered flooding that forced scores of people to flee their homes, while others climbed atop stalled automobiles to escape the rising waters. Total damage estimates for the Los Angeles area were over \$17 million (National Oceanic and Atmospheric Administration, 1997b).

The Clear Lake region in northern California was hit hard by flooding on February 28, 1998. Clear Lake (fig. 17A) is a very slow-draining natural lake that rose 2.5 feet above flood stage. The town of Clear Lake suffered \$5 million in flood damage (National Oceanic and Atmospheric Administration, 1998b). The flooding continued into March and April 1998.

Flooding in the San Joaquin Valley during the last week in February 1998 caused more than $\$ 40$ million in damage (National Oceanic and Atmospheric Administration, 1998b). A fatality occurred when a man attempted to cross floodwaters on a bicycle. Another death occurred south of Tracy (fig. 17B) when floodwaters swept away a man on a hunting trip after his truck got stuck. Serious flooding also occurred in southern 


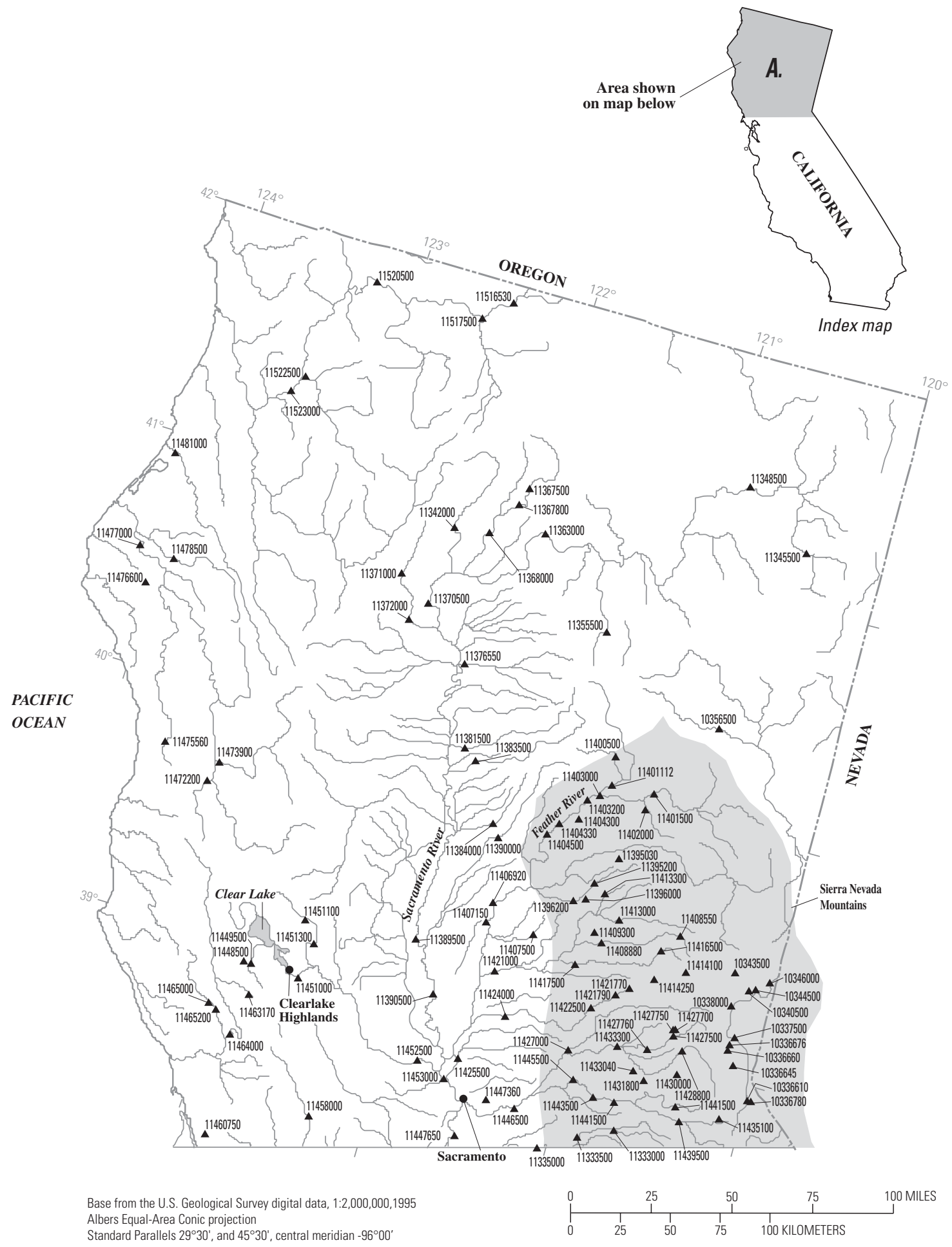

\section{EXPLANATION}

11333500^Streamgage and number

Figure 17. $(A)$ Location of streamgages with significant floods during 1994-98 water years for California. 


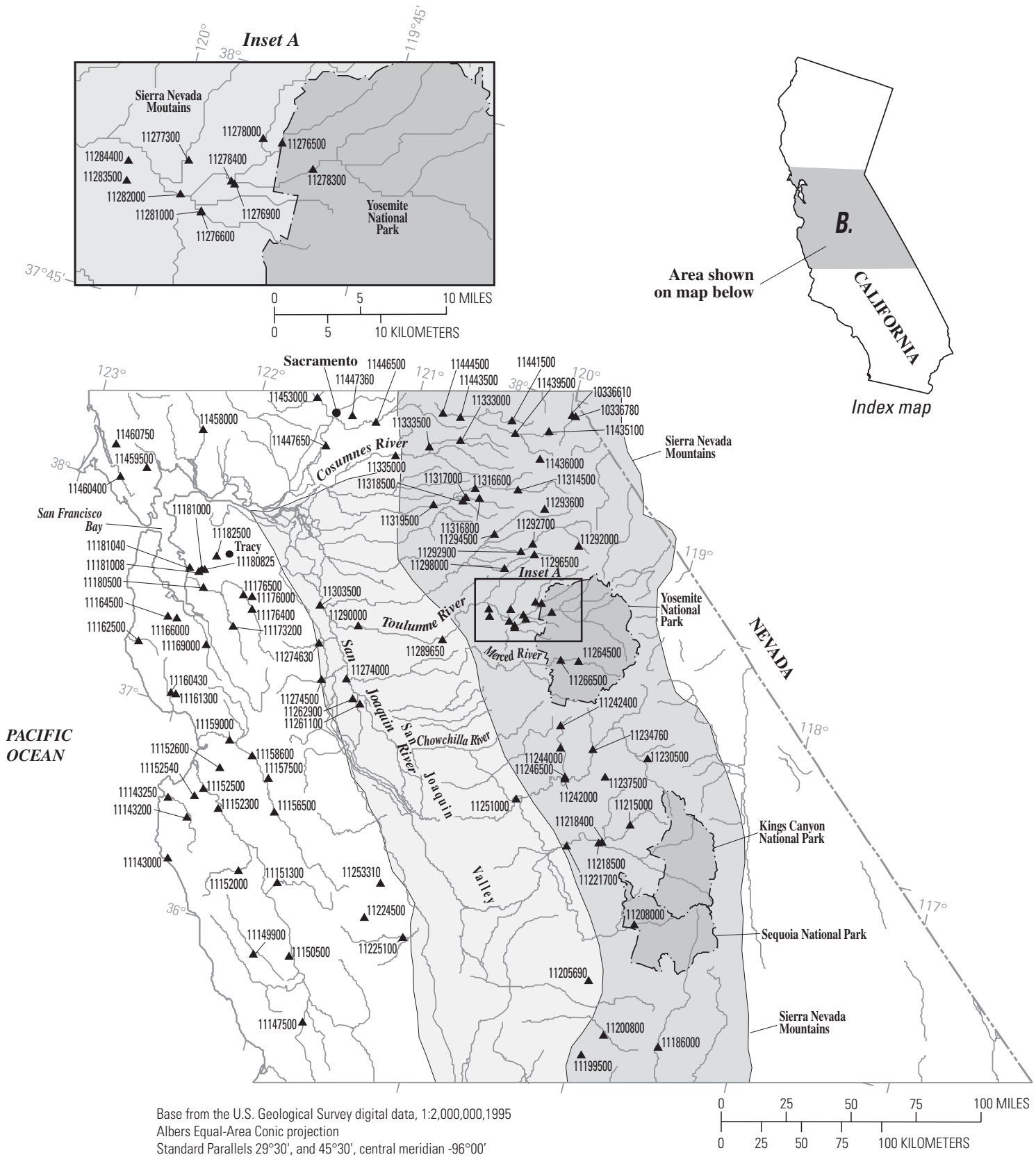

EXPLANATION

$1119950 \_$Streamgage and number

Figure 17. $(B)$ Location of streamgages with significant floods during $1994-98$ water years for California.-Continued 


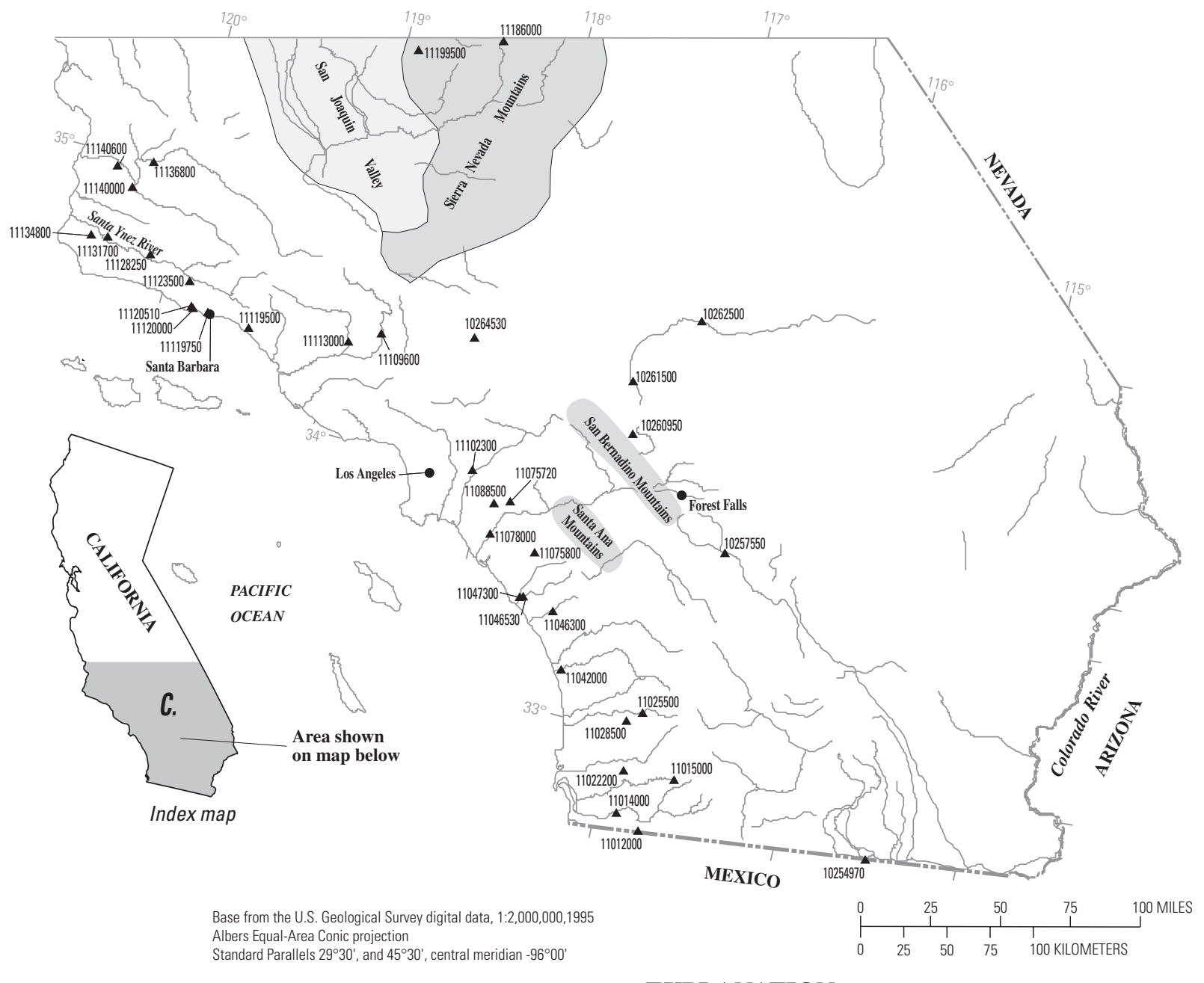

EXPLANATION

11012000 ^Streamgage and number

Figure 17. $(C)$ Location of streamgages with significant floods during 1994-98 water years for California.-Continued

California during February 1998. Damage estimates to residences, businesses, property, infrastructure, agriculture, and the cost of emergency services, and for clean up exceeded $\$ 100$ million (National Oceanic and Atmospheric Administration, 1998b). Flooding, caused by record winter snowpack melt and spring rainstorms, continued in various parts of California from March through June 1998.

\section{References}

National Oceanic and Atmospheric Administration (NOAA), 1995a-98a, Climatological data (by State): Asheville, North Carolina, National Climatic Data Center, various months.

National Oceanic and Atmospheric Administration (NOAA), 1995b-98b, Storm data (by State): Asheville, North Carolina, National Climatic Data Center, various months.

Perry, C.A., 2000, Significant floods in the United States during the 20th century-USGS measures a century of floods: U.S. Geological Survey Fact Sheet 024-00, 4 p. 
Table 6. Maximum stage and discharge for period of record for streamgages having significant floods during 1994-98 water years in California.

$\left[\mathrm{mi}^{2}\right.$, square miles; ft, feet above an arbitrary datum; $\mathrm{ft}^{3} / \mathrm{s}$, cubic feet per second; --, not determined or not applicable; >, greater than. Source: Recurrence intervals calculated from U.S. Geological Survey data. Other data from U.S. Geological Survey reports or databases]

\begin{tabular}{|c|c|c|c|c|c|c|c|c|c|c|c|}
\hline \multirow{2}{*}{$\begin{array}{l}\text { Streamgage } \\
\text { number } \\
\text { (fig. 17) }\end{array}$} & \multirow[b]{2}{*}{ Streamgage name } & \multirow{2}{*}{$\begin{array}{l}\text { Total } \\
\text { drainage } \\
\left(\mathrm{mi}^{2}\right)\end{array}$} & \multicolumn{4}{|c|}{$\begin{array}{l}\text { Maximum stage and discharge for period of record } \\
\text { through } 1998 \text { water year }\end{array}$} & \multicolumn{5}{|c|}{ Significant floods 1994-98 water years } \\
\hline & & & $\begin{array}{l}\text { Period of } \\
\text { record } \\
\text { (water } \\
\text { years) }\end{array}$ & Water year & $\begin{array}{l}\text { Stage } \\
(\mathrm{ft})\end{array}$ & $\begin{array}{l}\text { Discharge } \\
\left(\mathrm{ft}^{3} / \mathrm{s}\right)\end{array}$ & $\begin{array}{l}\text { Date } \\
\text { (month/ } \\
\text { day/ } \\
\text { year) }\end{array}$ & $\begin{array}{l}\text { Stage } \\
(\mathrm{ft})\end{array}$ & $\begin{array}{l}\text { Discharge } \\
\left(\mathrm{ft}^{3} / \mathrm{s}\right)\end{array}$ & $\begin{array}{l}\text { Regulated } \\
\text { during } \\
\text { flood }^{1}\end{array}$ & $\begin{array}{l}\text { Recurrence } \\
\text { interval } \\
\text { (years) }\end{array}$ \\
\hline 10254970 & $\begin{array}{l}\text { New River at international } \\
\text { boundary at Calexico, CA }\end{array}$ & -- & $1982-98$ & 1997 & 14.73 & 833 & $9 / 25 / 97$ & 14.73 & 833 & $\mathrm{~N}$ & $5-10$ \\
\hline 10257550 & $\begin{array}{l}\text { Whitewater River at Windy Point } \\
\text { near Whitewater, CA }\end{array}$ & 264 & $\begin{array}{l}\text { 1985-87, } \\
1990-98\end{array}$ & 1995 & 8.32 & 2,530 & $1 / 10 / 95$ & 8.32 & 2,530 & $\mathrm{Y}$ & $25-50$ \\
\hline 10260950 & $\begin{array}{l}\text { West Fork Mojave River above } \\
\text { Mohave River Forks Reservoir } \\
\text { near Hesperia, CA }\end{array}$ & .30 & $1975-98$ & 1980 & 17.45 & 8,380 & $2 / 20 / 96$ & 6.63 & 2,440 & $\mathrm{Y}$ & -- \\
\hline 10261500 & $\begin{array}{l}\text { Mojave River at lower narrows } \\
\text { near Victorville, CA }\end{array}$ & 513 & 1931-98 & 1938 & 16.70 & 70,600 & $2 / 24 / 98$ & 9.25 & 24,000 & $\mathrm{Y}$ & $10-20$ \\
\hline 10262500 & Mojave River at Barstow, CA & 1,290 & $\begin{array}{l}1931-85 \\
1987-98\end{array}$ & 1938 & 8.60 & 64,300 & $2 / 24 / 98$ & 3.16 & 15,200 & $\mathrm{~N}$ & $10-20$ \\
\hline 10264530 & Pine Creek near Palmdale, CA & 1.78 & $\begin{array}{l}1959-73 \\
1978-91 \\
1996-98\end{array}$ & 1969 & 15.33 & 69 & $--/--/ 98$ & 15.33 & 65 & $\mathrm{~N}$ & $10-25$ \\
\hline 10336610 & $\begin{array}{l}\text { Upper Truckee River at South } \\
\text { Lake Tahoe, CA }\end{array}$ & 54.9 & $\begin{array}{l}1972-74 \\
1978 \\
1980-98\end{array}$ & 1997 & 9.95 & 5,480 & $1 / 2 / 97$ & 9.95 & 5,480 & $\mathrm{~N}$ & $>100$ \\
\hline 10336645 & General Creek near Meeks, CA & 7.44 & $1981-98$ & 1997 & 7.86 & 797 & $1 / 2 / 97$ & 7.86 & 797 & $\mathrm{~N}$ & 20 \\
\hline 10336660 & $\begin{array}{l}\text { Blackwood Creek near Tahoe City, } \\
\text { CA }\end{array}$ & 11.2 & $1961-98$ & 1997 & 9.82 & 2,940 & $1 / 1 / 97$ & 9.82 & 2,940 & $\mathrm{~N}$ & $>100$ \\
\hline 10336676 & $\begin{array}{l}\text { Ward Creek at Highway } 89 \text { near } \\
\text { Tahoe Pines, CA }\end{array}$ & 9.70 & 1973-98 & 1997 & 9.36 & 2,530 & $1 / 1 / 97$ & 9.36 & 2,530 & $\mathrm{~N}$ & $50-75$ \\
\hline 10336780 & $\begin{array}{l}\text { Trout Creek near Tahoe Valley, } \\
\text { CA }\end{array}$ & 36.7 & $1961-98$ & 1963 & 11.14 & 535 & $1 / 2 / 97$ & 9.33 & 535 & $\mathrm{~N}$ & $20-30$ \\
\hline
\end{tabular}


Table 6. Maximum stage and discharge for period of record for streamgages having significant floods during 1994-98 water years in California.-Continued

$\left[\mathrm{mi}^{2}\right.$, square miles; $\mathrm{ft}$, feet above an arbitrary datum; $\mathrm{ft}^{3} / \mathrm{s}$, cubic feet per second; --, not determined or not applicable; >, greater than. Source: Recurrence intervals calculated from U.S. Geological Survey data. Other data from U.S. Geological Survey reports or databases]

\begin{tabular}{|c|c|c|c|c|c|c|c|c|c|c|c|}
\hline \multirow[b]{2}{*}{$\begin{array}{c}\text { Streamgage } \\
\text { number } \\
\text { (fig. 17) }\end{array}$} & \multirow[b]{2}{*}{ Streamgage name } & \multirow[b]{2}{*}{$\begin{array}{c}\text { Total } \\
\text { drainage } \\
\left(\mathrm{mi}^{2}\right)\end{array}$} & \multicolumn{4}{|c|}{$\begin{array}{c}\text { Maximum stage and discharge for period of record } \\
\text { through } 1998 \text { water year }\end{array}$} & \multicolumn{5}{|c|}{ Significant floods $1994-98$ water years } \\
\hline & & & $\begin{array}{l}\text { Period of } \\
\text { record } \\
\text { (water } \\
\text { years) }\end{array}$ & Water year & $\begin{array}{l}\text { Stage } \\
(\mathrm{ft})\end{array}$ & $\begin{array}{l}\text { Discharge } \\
\left(\mathrm{ft}^{3} / \mathrm{s}\right)\end{array}$ & $\begin{array}{c}\text { Date } \\
\text { (month/ } \\
\text { day/ } \\
\text { year) }\end{array}$ & $\begin{array}{l}\text { Stage } \\
(\mathrm{ft})\end{array}$ & $\begin{array}{l}\text { Discharge } \\
\left(\mathrm{ft}^{3} / \mathrm{s}\right)\end{array}$ & $\begin{array}{l}\text { Regulated } \\
\text { during } \\
\text { flood }^{1}\end{array}$ & $\begin{array}{c}\text { Recurrence } \\
\text { interval } \\
\text { (years) }\end{array}$ \\
\hline 10337500 & Truckee River at Tahoe City, CA & 507 & $1901-98$ & 1997 & 9.59 & 2,690 & $\begin{array}{l}5 / 21 / 96 \\
1 / 2 / 97\end{array}$ & $\begin{array}{l}9.07 \\
9.59\end{array}$ & $\begin{array}{l}2,350 \\
2,690\end{array}$ & $\begin{array}{l}\mathrm{Y} \\
\mathrm{Y}\end{array}$ & $\begin{array}{l}20-30 \\
25-50\end{array}$ \\
\hline 10338000 & Truckee River near Truckee, CA & 552 & $\begin{array}{l}1945-61, \\
1963, \\
1977-82, \\
1993-95, \\
1997-98\end{array}$ & 1997 & 9.97 & 11,900 & $1 / 2 / 97$ & 9.97 & 11,900 & $\mathrm{Y}$ & $40-60$ \\
\hline 10340500 & $\begin{array}{l}\text { Prosser Creek below Prosser Creek } \\
\text { Dam near Truckee, CA }\end{array}$ & 52.9 & 1943-98 & $\begin{array}{l}1956 \\
1951\end{array}$ & $\begin{array}{l}10.13 \\
11.01\end{array}$ & $\begin{array}{l}4,560 \\
4,320\end{array}$ & $1 / 3 / 97$ & 6.72 & 2,030 & $\mathrm{Y}$ & $25-50$ \\
\hline 10343500 & Sagehen Creek near Truckee, CA & 10.5 & 1954-98 & 1997 & 5.20 & 1,230 & $1 / 1 / 97$ & 5.20 & 1,230 & $\mathrm{~N}$ & $75-100$ \\
\hline 10344500 & $\begin{array}{l}\text { Little Truckee River below Boca } \\
\text { Dam near Truckee, CA }\end{array}$ & 173 & $\begin{array}{l}1890, \\
1911-15, \\
1940-94, \\
1996-98\end{array}$ & $\begin{array}{l}1951 \\
1963\end{array}$ & $-\overline{-}$ & $\begin{array}{l}5,000 \\
2,590\end{array}$ & $1 / 8 / 97$ & 6.14 & 2,720 & $\mathrm{Y}$ & $20-40$ \\
\hline 10346000 & Truckee River at Farad, CA & 932 & 1900-98 & $\begin{array}{l}1951 \\
1997\end{array}$ & -- & $\begin{array}{l}17,500 \\
14,900\end{array}$ & $1 / 2 / 97$ & 13.13 & 14,900 & $\mathrm{Y}$ & $50-75$ \\
\hline 10356500 & Susan River at Susanville, CA & 184 & $\begin{array}{l}1901, \\
1903-05, \\
1917-21, \\
1951-94, \\
1997\end{array}$ & $\begin{array}{l}1970 \\
1997\end{array}$ & $\begin{array}{r}8.89 \\
11.75\end{array}$ & $\begin{array}{l}5,850 \\
5,150\end{array}$ & $1 / 2 / 97$ & 11.75 & 5,150 & $\mathrm{Y}$ & $20-30$ \\
\hline 11012000 & $\begin{array}{l}\text { Cottonwood Creek above Tecate } \\
\text { Creek near Dulzura, CA }\end{array}$ & 310 & 1937-98 & 1980 & 11.15 & 11,700 & $3 / 6 / 95$ & 11.14 & 11,300 & $\mathrm{Y}$ & $50-75$ \\
\hline 11014000 & Jamul Creek near Jamul, CA & 70.2 & $\begin{array}{l}1940, \\
1942-78, \\
1986-98\end{array}$ & 1995 & 7.59 & 5,870 & $3 / 5 / 95$ & 7.59 & 5,870 & $\mathrm{~N}$ & $40-60$ \\
\hline
\end{tabular}


Table 6. Maximum stage and discharge for period of record for streamgages having significant floods during 1994-98 water years in California.-Continued

$\left[\mathrm{mi}^{2}\right.$, square miles; $\mathrm{ft}$, feet above an arbitrary datum; $\mathrm{ft}^{3} / \mathrm{s}$, cubic feet per second; --, not determined or not applicable; >, greater than. Source: Recurrence intervals calculated from U.S. Geological Survey data. Other data from U.S. Geological Survey reports or databases]

\begin{tabular}{|c|c|c|c|c|c|c|c|c|c|c|c|}
\hline \multirow[b]{2}{*}{$\begin{array}{l}\text { Streamgage } \\
\text { number } \\
\text { (fig. 17) }\end{array}$} & \multirow[b]{2}{*}{ Streamgage name } & \multirow[b]{2}{*}{$\begin{array}{l}\text { Total } \\
\text { drainage } \\
\left(\mathrm{mi}^{2}\right)\end{array}$} & \multicolumn{4}{|c|}{$\begin{array}{c}\text { Maximum stage and discharge for period of record } \\
\text { through } 1998 \text { water year }\end{array}$} & \multicolumn{5}{|c|}{ Significant floods $1994-98$ water years } \\
\hline & & & $\begin{array}{l}\text { Period of } \\
\text { record } \\
\text { (water } \\
\text { years) }\end{array}$ & Water year & $\begin{array}{l}\text { Stage } \\
\text { (ft) }\end{array}$ & $\begin{array}{l}\text { Discharge } \\
\left(\mathrm{ft}^{3} / \mathrm{s}\right)\end{array}$ & $\begin{array}{c}\text { Date } \\
\text { (month/ } \\
\text { day/ } \\
\text { year) }\end{array}$ & $\begin{array}{l}\text { Stage } \\
(\mathrm{ft})\end{array}$ & $\begin{array}{l}\text { Discharge } \\
\left(\mathrm{ft}^{3} / \mathrm{s}\right)\end{array}$ & $\begin{array}{l}\text { Regulated } \\
\text { during } \\
\text { flood }^{1}\end{array}$ & $\begin{array}{l}\text { Recurrence } \\
\text { interval } \\
\text { (years) }\end{array}$ \\
\hline 11015000 & $\begin{array}{l}\text { Sweetwater River near Descanso, } \\
\text { CA }\end{array}$ & 45.4 & $\begin{array}{l}1906-27, \\
1957-98\end{array}$ & $\begin{array}{l}1927 \\
1995\end{array}$ & $\begin{array}{l}13.20 \\
13.22\end{array}$ & $\begin{array}{r}11,200 \\
8,600\end{array}$ & $3 / 5 / 95$ & 13.22 & 8,600 & $\mathrm{~N}$ & $30-40$ \\
\hline 11022200 & $\begin{array}{l}\text { Los Coches Creek near Lakeside, } \\
\text { CA }\end{array}$ & 12.2 & 1984-98 & 1995 & 9.74 & 1,090 & $3 / 5 / 95$ & 9.74 & 1,090 & $\mathrm{~N}$ & $15-25$ \\
\hline 11025500 & $\begin{array}{l}\text { Santa Ysabel Creek near Ramona, } \\
\text { CA }\end{array}$ & 112 & $\begin{array}{l}1912-22, \\
1944-71 \\
1973-98\end{array}$ & $\begin{array}{l}1916 \\
1980\end{array}$ & $\begin{array}{r}6.00 \\
14.25\end{array}$ & $\begin{array}{l}28,400 \\
10,700\end{array}$ & $3 / 5 / 95$ & 12.45 & 8,000 & $\mathrm{Y}$ & $20-30$ \\
\hline 11028500 & $\begin{array}{l}\text { Santa Maria Creek near Ramona, } \\
\text { CA }\end{array}$ & 57.6 & $\begin{array}{l}1914-20 \\
1947-98\end{array}$ & $\begin{array}{l}1980 \\
1916\end{array}$ & $\begin{array}{l}14.39 \\
15.90\end{array}$ & $\begin{array}{r}15,200 \\
7,140\end{array}$ & $3 / 5 / 95$ & 8.80 & 5,270 & $\mathrm{~N}$ & $10-20$ \\
\hline 11042000 & $\begin{array}{l}\text { San Luis Rey River at Oceanside, } \\
\text { CA }\end{array}$ & 557 & $\begin{array}{l}1913-16 \\
1930-42 \\
1947-98\end{array}$ & $\begin{array}{l}1916 \\
1993\end{array}$ & -- & $\begin{array}{l}95,600 \\
25,700\end{array}$ & $3 / 6 / 95$ & 19.97 & 19,500 & $\mathrm{Y}$ & $20-30$ \\
\hline 11046300 & $\begin{array}{l}\text { San Mateo Creek near San } \\
\text { Clemente, CA }\end{array}$ & 80.8 & $\begin{array}{l}1953-67 \\
1969 \\
1994-98\end{array}$ & 1998 & 12.83 & 12,500 & $2 / 23 / 98$ & 12.83 & 12,500 & $\mathrm{~N}$ & $15-25$ \\
\hline 11046530 & $\begin{array}{l}\text { San Juan Creek at La Novia Street } \\
\text { Bridge at San Juan Capistrano, } \\
\text { CA }\end{array}$ & 109 & 1986-98 & 1995 & 20.66 & 25,600 & $3 / 5 / 95$ & 20.66 & 25,600 & $\mathrm{~N}$ & $30-40$ \\
\hline 11047300 & $\begin{array}{l}\text { Arroyo Trabuco at San Juan } \\
\text { Capistrano, CA }\end{array}$ & 54.1 & $\begin{array}{l}1973-77 \\
1984-88 \\
1996-98\end{array}$ & 1998 & 19.81 & 10,000 & $2 / 23 / 98$ & 19.81 & 10,000 & $\mathrm{~N}$ & $50-100$ \\
\hline 11075720 & $\begin{array}{l}\text { Carbon Creek below Carbon } \\
\text { Canyon Dam, CA }\end{array}$ & 19.5 & $1962-98$ & 1983 & 5.11 & 796 & $2 / 24 / 98$ & 4.48 & 573 & $\mathrm{Y}$ & $15-25$ \\
\hline 11075800 & Santiago Creek at Modjeska, CA & 13.0 & $1962-98$ & $\begin{array}{l}1969 \\
1998\end{array}$ & $\begin{array}{l}10.50 \\
12.03\end{array}$ & $\begin{array}{l}6,520 \\
6,200\end{array}$ & $2 / 23 / 98$ & 12.03 & 6,200 & $\mathrm{~N}$ & $15-25$ \\
\hline
\end{tabular}


Table 6. Maximum stage and discharge for period of record for streamgages having significant floods during 1994-98 water years in California.-Continued

$\left[\mathrm{mi}^{2}\right.$, square miles; $\mathrm{ft}$, feet above an arbitrary datum; $\mathrm{ft}^{3} / \mathrm{s}$, cubic feet per second; --, not determined or not applicable; >, greater than. Source: Recurrence intervals calculated from U.S. Geological Survey data. Other data from U.S. Geological Survey reports or databases]

\begin{tabular}{|c|c|c|c|c|c|c|c|c|c|c|c|}
\hline \multirow{2}{*}{$\begin{array}{l}\text { Streamgage } \\
\text { number } \\
\text { (fig. 17) }\end{array}$} & \multirow[b]{2}{*}{ Streamgage name } & \multirow{2}{*}{$\begin{array}{c}\text { Total } \\
\text { drainage } \\
\left(\mathrm{mi}^{2}\right)\end{array}$} & \multicolumn{4}{|c|}{$\begin{array}{c}\text { Maximum stage and discharge for period of record } \\
\text { through } 1998 \text { water year }\end{array}$} & \multicolumn{5}{|c|}{ Significant floods 1994-98 water years } \\
\hline & & & $\begin{array}{l}\text { Period of } \\
\text { record } \\
\text { (water } \\
\text { years) }\end{array}$ & Water year & $\begin{array}{l}\text { Stage } \\
(\mathrm{ft})\end{array}$ & $\begin{array}{l}\text { Discharge } \\
\left(\mathrm{ft}^{3} / \mathrm{s}\right)\end{array}$ & $\begin{array}{c}\text { Date } \\
\text { (month/ } \\
\text { day/ } \\
\text { year) }\end{array}$ & $\begin{array}{l}\text { Stage } \\
(\mathrm{ft})\end{array}$ & $\begin{array}{l}\text { Discharge } \\
\left(\mathrm{ft}^{3} / \mathrm{s}\right)\end{array}$ & $\begin{array}{l}\text { Regulated } \\
\text { during } \\
\text { flood }^{1}\end{array}$ & $\begin{array}{c}\text { Recurrence } \\
\text { interval } \\
\text { (years) }\end{array}$ \\
\hline 11078000 & Santa Ana River at Santa Ana, CA & 1,700 & $\begin{array}{l}1923-30 \\
1932-89, \\
1991-98\end{array}$ & $\begin{array}{l}1938 \\
1981\end{array}$ & $\begin{array}{l}-- \\
16.00\end{array}$ & $\begin{array}{r}46,300 \\
6,200\end{array}$ & $1 / 4 / 95$ & 9.09 & 31,700 & $\mathrm{Y}$ & $30-40$ \\
\hline 11088500 & $\begin{array}{l}\text { Brea Creek below Brea Dam near } \\
\text { Fullerton, CA }\end{array}$ & 21.6 & $\begin{array}{l}1943-80 \\
1982-98\end{array}$ & $\begin{array}{l}1980 \\
1978\end{array}$ & -- & $\begin{array}{l}1,700 \\
1,060\end{array}$ & $2 / 7 / 98$ & 6.03 & 1,390 & $\mathrm{Y}$ & $20-30$ \\
\hline 11102300 & $\begin{array}{l}\text { Rio Hondo below Whittier } \\
\text { Narrows Dam, CA }\end{array}$ & 124 & 1967-98 & 1969 & 13.82 & 38,800 & $1 / 10 / 95$ & 13.80 & 38,700 & $\mathrm{Y}$ & $15-25$ \\
\hline 11109600 & Piru Creek above Lake Piru, CA & 372 & $\begin{array}{l}1938, \\
1956-94, \\
1996-98\end{array}$ & $\begin{array}{l}1998 \\
1969\end{array}$ & $\begin{array}{l}13.38 \\
18.60\end{array}$ & $\begin{array}{l}38,000 \\
31,200\end{array}$ & $2 / 23 / 98$ & 13.38 & 38,000 & $\mathrm{Y}$ & $30-40$ \\
\hline 11113000 & Sespe Creek near Filmore, CA & 251 & $\begin{array}{l}1933-85 \\
1991-92 \\
1994-98\end{array}$ & 1978 & 22.40 & 73,000 & $\begin{array}{l}1 / 10 / 95 \\
2 / 3 / 98\end{array}$ & $\begin{array}{l}16.46 \\
16.31\end{array}$ & $\begin{array}{l}65,000 \\
62,500\end{array}$ & $\begin{array}{l}\mathrm{N} \\
\mathrm{N}\end{array}$ & $\begin{array}{l}20-30 \\
15-25\end{array}$ \\
\hline 11119500 & $\begin{array}{l}\text { Carpinteria Creek near Carpinteria, } \\
\text { CA }\end{array}$ & 13.1 & $\begin{array}{l}1941-77, \\
1979-94, \\
1996-98\end{array}$ & $\begin{array}{l}1972 \\
1969\end{array}$ & $\begin{array}{l}14.10 \\
18.90\end{array}$ & $\begin{array}{l}8,880 \\
4,560\end{array}$ & $2 / 23 / 98$ & 10.77 & 5,930 & $\mathrm{~N}$ & $25-35$ \\
\hline 11119750 & $\begin{array}{l}\text { Mission Creek near Mission Street } \\
\text { near Santa Barbara, CA }\end{array}$ & 8.38 & $\begin{array}{l}1971-94 \\
1996-98\end{array}$ & 1998 & 5.67 & 3,090 & $2 / 23 / 98$ & 5.67 & 3,090 & $\mathrm{~N}$ & $20-30$ \\
\hline 11120000 & Atascadero Creek near Goleta, CA & 18.9 & 1942-98 & $\begin{array}{l}1995 \\
1975\end{array}$ & $\begin{array}{l}12.45 \\
13.30\end{array}$ & $\begin{array}{r}10,200 \\
2,380\end{array}$ & $3 / 10 / 95$ & 12.45 & 10,200 & $\mathrm{~N}$ & $50-75$ \\
\hline 11120510 & San Jose Creek at Goleta, CA & 9.42 & 1971-92, & $\begin{array}{l}1998 \\
1998\end{array}$ & 5.68 & 2,470 & $2 / 7 / 98$ & 5.68 & 2,470 & $\mathrm{~N}$ & $10-20$ \\
\hline 11123500 & $\begin{array}{l}\text { Santa Ynez River below Los } \\
\text { Laureles Canyon near Santa }\end{array}$ & 277 & $\begin{array}{l}1948-94 \\
1996-98\end{array}$ & 1969 & 18.88 & 67,500 & $2 / 23 / 98$ & 17.54 & 55,900 & $\mathrm{Y}$ & $20-30$ \\
\hline
\end{tabular}


Table 6. Maximum stage and discharge for period of record for streamgages having significant floods during 1994-98 water years in California.-Continued

$\left[\mathrm{mi}^{2}\right.$, square miles; $\mathrm{ft}$, feet above an arbitrary datum; $\mathrm{ft}^{3} / \mathrm{s}$, cubic feet per second; --, not determined or not applicable; >, greater than. Source: Recurrence intervals calculated from U.S. Geological Survey data. Other data from U.S. Geological Survey reports or databases]

\begin{tabular}{|c|c|c|c|c|c|c|c|c|c|c|c|}
\hline \multirow{2}{*}{$\begin{array}{c}\text { Streamgage } \\
\text { number } \\
\text { (fig. 17) }\end{array}$} & \multirow[b]{2}{*}{ Streamgage name } & \multirow{2}{*}{$\begin{array}{c}\text { Total } \\
\text { drainage } \\
\left(\mathrm{mi}^{2}\right)\end{array}$} & \multicolumn{4}{|c|}{$\begin{array}{c}\text { Maximum stage and discharge for period of record } \\
\text { through } 1998 \text { water year }\end{array}$} & \multicolumn{5}{|c|}{ Significant floods 1994-98 water years } \\
\hline & & & $\begin{array}{c}\text { Period of } \\
\text { record } \\
\text { (water } \\
\text { years) }\end{array}$ & Water year & $\begin{array}{l}\text { Stage } \\
\text { (ft) }\end{array}$ & $\begin{array}{l}\text { Discharge } \\
\left(\mathrm{ft}^{3} / \mathrm{s}\right)\end{array}$ & $\begin{array}{c}\text { Date } \\
\text { (month/ } \\
\text { day/ } \\
\text { year) }\end{array}$ & $\begin{array}{l}\text { Stage } \\
\text { (ft) }\end{array}$ & $\begin{array}{l}\text { Discharge } \\
\left(\mathrm{ft}^{3} / \mathrm{s}\right)\end{array}$ & $\begin{array}{c}\text { Regulated } \\
\text { during } \\
\text { flood }^{1}\end{array}$ & $\begin{array}{c}\text { Recurrence } \\
\text { interval } \\
\text { (years) }\end{array}$ \\
\hline 11128250 & $\begin{array}{l}\text { Alamo Pintado Creek near } \\
\text { Solvang, CA }\end{array}$ & 29.4 & $\begin{array}{l}1969, \\
\text { 1971-85, } \\
\text { 1990-92, } \\
1995-98\end{array}$ & 1998 & 11.69 & 3,680 & $2 / 3 / 98$ & 11.69 & 3,680 & $\mathrm{~N}$ & $20-30$ \\
\hline 11131700 & Santa Rita Creek near Lompoc, CA & 14.1 & $\begin{array}{l}1976-79 \\
1981-98\end{array}$ & 1998 & 10.57 & 723 & $2 / 23 / 98$ & 10.57 & 723 & $\mathrm{~N}$ & $10-25$ \\
\hline 11134800 & Miguelito Creek at Lompoc, CA & 11.6 & $\begin{array}{l}1969, \\
1971-86, \\
1988-94, \\
1996-98\end{array}$ & $\begin{array}{l}1998 \\
1983\end{array}$ & $\begin{array}{l}4.61 \\
7.63\end{array}$ & $\begin{array}{l}2,660 \\
1,210\end{array}$ & $2 / 3 / 98$ & 4.61 & 2,660 & $\mathrm{~N}$ & $75-100$ \\
\hline 11136800 & $\begin{array}{r}\text { Cuyama River below Buckhorn } \\
\text { Canyon near Santa Maria, CA }\end{array}$ & 886 & $\begin{array}{l}1904-05 \\
1960-98\end{array}$ & 1998 & 14.76 & 26,200 & $2 / 23 / 98$ & 14.76 & 26,200 & $\mathrm{~N}$ & $15-30$ \\
\hline 11140000 & Sisquoc River near Garey, CA & 471 & $\begin{array}{l}1941-53, \\
1955-84, \\
1986-94, \\
1996-98\end{array}$ & $\begin{array}{l}1983 \\
1967\end{array}$ & $\begin{array}{l}11.16 \\
13.50\end{array}$ & $\begin{array}{l}33,600 \\
22,600\end{array}$ & $2 / 3 / 98$ & 10.61 & 29,500 & $\mathrm{~N}$ & $20-30$ \\
\hline 11140600 & $\begin{array}{l}\text { Bradley Ditch near Donovan Road } \\
\text { at Santa Maria, CA }\end{array}$ & 5.47 & $\begin{array}{l}1971-78, \\
1980-92, \\
1998\end{array}$ & $\begin{array}{l}1998 \\
1978\end{array}$ & $\begin{array}{l}5.69 \\
5.85\end{array}$ & $\begin{array}{l}848 \\
379\end{array}$ & $2 / 3 / 98$ & 5.69 & 848 & $\mathrm{~N}$ & $40-60$ \\
\hline 11143000 & Big Sur River near Big Sur, CA & 46.5 & 1951-98 & 1978 & 14.30 & 10,700 & $3 / 10 / 95$ & 11.71 & 6,690 & $\mathrm{~N}$ & $20-30$ \\
\hline 11143200 & $\begin{array}{l}\text { Carmel River at Robles del Rio, } \\
\text { CA }\end{array}$ & 193 & $\begin{array}{l}1956, \\
1958-98\end{array}$ & 1995 & 12.90 & 16,000 & $\begin{array}{l}3 / 10 / 95 \\
2 / 3 / 98\end{array}$ & $\begin{array}{l}12.90 \\
11.08\end{array}$ & $\begin{array}{l}16,000 \\
14,700\end{array}$ & $\begin{array}{l}\mathrm{N} \\
\mathrm{N}\end{array}$ & $\begin{array}{c}>100 \\
75-100\end{array}$ \\
\hline 11143250 & Carmel River near Carmel, CA & 246 & 1963-98 & 1995 & 20.85 & 16,000 & $\begin{array}{l}3 / 10 / 95 \\
2 / 3 / 98\end{array}$ & $\begin{array}{l}20.85 \\
19.35\end{array}$ & $\begin{array}{l}16,000 \\
14,600\end{array}$ & $\begin{array}{l}\mathrm{N} \\
\mathrm{N}\end{array}$ & $\begin{array}{c}75-100 \\
25-50\end{array}$ \\
\hline 11147500 & Salinas River at Paso Robles, CA & 390 & $\begin{array}{l}1938, \\
1940-65, \\
1969-98\end{array}$ & $\begin{array}{l}1995 \\
1969\end{array}$ & $\begin{array}{l}22.99 \\
23.80\end{array}$ & $\begin{array}{l}28,400 \\
28,000\end{array}$ & $3 / 10 / 95$ & 22.99 & 28,400 & $\mathrm{~N}$ & $50-75$ \\
\hline
\end{tabular}


Table 6. Maximum stage and discharge for period of record for streamgages having significant floods during 1994-98 water years in California.-Continued

$\left[\mathrm{mi}^{2}\right.$, square miles; $\mathrm{ft}$, feet above an arbitrary datum; $\mathrm{ft}^{3} / \mathrm{s}$, cubic feet per second; --, not determined or not applicable; >, greater than. Source: Recurrence intervals calculated from U.S. Geological Survey data. Other data from U.S. Geological Survey reports or databases]

\begin{tabular}{|c|c|c|c|c|c|c|c|c|c|c|c|}
\hline \multirow{2}{*}{$\begin{array}{c}\text { Streamgage } \\
\text { number } \\
\text { (fig. 17) }\end{array}$} & \multirow[b]{2}{*}{ Streamgage name } & \multirow{2}{*}{$\begin{array}{c}\text { Total } \\
\text { drainage } \\
\left(\mathrm{mi}^{2}\right)\end{array}$} & \multicolumn{4}{|c|}{$\begin{array}{c}\text { Maximum stage and discharge for period of record } \\
\text { through } 1998 \text { water year }\end{array}$} & \multicolumn{5}{|c|}{ Significant floods $1994-98$ water years } \\
\hline & & & $\begin{array}{l}\text { Period of } \\
\text { record } \\
\text { (water } \\
\text { years) }\end{array}$ & Water year & $\begin{array}{l}\text { Stage } \\
(\mathrm{ft})\end{array}$ & $\begin{array}{l}\text { Discharge } \\
\left(\mathrm{ft}^{3} / \mathrm{s}\right)\end{array}$ & $\begin{array}{c}\text { Date } \\
\text { (month/ } \\
\text { day/ } \\
\text { year) }\end{array}$ & $\begin{array}{l}\text { Stage } \\
(\mathrm{ft})\end{array}$ & $\begin{array}{l}\text { Discharge } \\
\left(\mathrm{ft}^{3} / \mathrm{s}\right)\end{array}$ & $\begin{array}{c}\text { Regulated } \\
\text { during } \\
\text { flood }^{1}\end{array}$ & $\begin{array}{c}\text { Recurrence } \\
\text { interval } \\
\text { (years) }\end{array}$ \\
\hline 11149900 & $\begin{array}{l}\text { San Antonio River near Lockwood, } \\
\text { CA }\end{array}$ & 217 & $1966-98$ & 1995 & 14.25 & 23,600 & $\begin{array}{l}3 / 10 / 95 \\
2 / 3 / 98\end{array}$ & $\begin{array}{l}14.25 \\
14.19\end{array}$ & $\begin{array}{l}23,600 \\
23,100\end{array}$ & $\begin{array}{l}\mathrm{N} \\
\mathrm{N}\end{array}$ & $\begin{array}{l}25-50 \\
25-50\end{array}$ \\
\hline 11150500 & Salinas River near Bradley, CA & 2,535 & 1949-98 & 1995 & 23.44 & 120,000 & $3 / 11 / 95$ & 23.44 & 120,000 & $\mathrm{Y}$ & $50-75$ \\
\hline 11151300 & $\begin{array}{l}\text { San Lorenzo Creek below Bitter- } \\
\text { water Creek near King City, CA }\end{array}$ & 233 & 1959-98 & 1969 & 15.33 & 11,500 & $3 / 10 / 95$ & 14.78 & 11,100 & $\mathrm{~N}$ & $20-30$ \\
\hline 11152000 & Arroyo Seco near Soledad, CA & 244 & 1906-98 & $\begin{array}{l}1958 \\
1914\end{array}$ & $\begin{array}{l}14.40 \\
20.50\end{array}$ & $\begin{array}{l}28,300 \\
17,500\end{array}$ & $3 / 10 / 95$ & 16.44 & 27,300 & $\mathrm{~N}$ & $20-30$ \\
\hline 11152300 & Salinas River near Chualar, CA & 4,042 & 1977-98 & 1995 & 19.70 & 92,000 & $3 / 11 / 95$ & 19.70 & 92,000 & $\mathrm{Y}$ & $75-100$ \\
\hline 11152500 & Salinas River near Spreckels, CA & 4,156 & $\begin{array}{l}1862,1911, \\
1914, \\
1930-56, \\
1958-60, \\
1962-98\end{array}$ & $\begin{array}{l}1995 \\
1862\end{array}$ & $\begin{array}{l}30.29 \\
31.00\end{array}$ & $\begin{array}{r}95,000 \\
--\end{array}$ & $3 / 12 / 95$ & 30.29 & 95,000 & $\mathrm{Y}$ & $25-50$ \\
\hline 11152540 & El Toro Creek near Spreckels, CA & 31.9 & 1962-98 & 1998 & 7.11 & 669 & $\begin{array}{l}3 / 10 / 95 \\
2 / 3 / 98\end{array}$ & $\begin{array}{l}7.08 \\
7.11\end{array}$ & $\begin{array}{l}664 \\
669\end{array}$ & $\begin{array}{l}\mathrm{N} \\
\mathrm{N}\end{array}$ & $\begin{array}{l}10-20 \\
10-20\end{array}$ \\
\hline 11152600 & Gabilan Creek near Salinas, CA & 36.7 & $1971-98$ & $\begin{array}{l}1998 \\
1974\end{array}$ & $\begin{array}{r}5.17 \\
11.13\end{array}$ & $\begin{array}{r}1,030 \\
898\end{array}$ & $2 / 3 / 98$ & 5.17 & 1,030 & $\mathrm{~N}$ & $20-30$ \\
\hline 11156500 & $\begin{array}{l}\text { San Benito River near Willow } \\
\text { Creek School, CA }\end{array}$ & 249 & $\begin{array}{l}1938 \\
1940-98\end{array}$ & 1995 & 14.55 & 9,660 & $3 / 10 / 95$ & 14.55 & 9,660 & $\mathrm{~N}$ & $30-40$ \\
\hline 11157500 & $\begin{array}{l}\text { Tres Pinos Creek near Tres Pinos, } \\
\text { CA }\end{array}$ & 207 & $\begin{array}{l}1938, \\
1940-83, \\
1997-98\end{array}$ & 1998 & 16.00 & 27,200 & $2 / 3 / 98$ & 16.00 & 27,200 & $\mathrm{~N}$ & $75-100$ \\
\hline 11158600 & $\begin{array}{l}\text { San Benito River at Highway } 156 \\
\text { near Hollister, CA }\end{array}$ & 607 & 1971-98 & 1998 & 13.48 & 34,500 & $2 / 3 / 98$ & 13.48 & 34,500 & $\mathrm{~N}$ & $50-75$ \\
\hline 11159000 & Pajaro River at Chittenden, CA & 1,186 & $\begin{array}{l}1938 \\
1940-98\end{array}$ & 1998 & 33.73 & 25,100 & $2 / 3 / 98$ & 33.73 & 25,100 & $\mathrm{~N}$ & $50-75$ \\
\hline
\end{tabular}


Table 6. Maximum stage and discharge for period of record for streamgages having significant floods during 1994-98 water years in California.-Continued

$\left[\mathrm{mi}^{2}\right.$, square miles; $\mathrm{ft}$, feet above an arbitrary datum; $\mathrm{ft}^{3} / \mathrm{s}$, cubic feet per second; --, not determined or not applicable; >, greater than. Source: Recurrence intervals calculated from U.S. Geological Survey data. Other data from U.S. Geological Survey reports or databases]

\begin{tabular}{|c|c|c|c|c|c|c|c|c|c|c|c|}
\hline \multirow{2}{*}{$\begin{array}{l}\text { Streamgage } \\
\text { number } \\
\text { (fig. 17) }\end{array}$} & \multirow[b]{2}{*}{ Streamgage name } & \multirow{2}{*}{$\begin{array}{c}\text { Total } \\
\text { drainage } \\
\left(\mathrm{mi}^{2}\right)\end{array}$} & \multicolumn{4}{|c|}{$\begin{array}{c}\text { Maximum stage and discharge for period of record } \\
\text { through } 1998 \text { water year }\end{array}$} & \multicolumn{5}{|c|}{ Significant floods $1994-98$ water years } \\
\hline & & & $\begin{array}{l}\text { Period of } \\
\text { record } \\
\text { (water } \\
\text { years) }\end{array}$ & Water year & $\begin{array}{l}\text { Stage } \\
(\mathrm{ft})\end{array}$ & $\begin{array}{l}\text { Discharge } \\
\left(\mathrm{ft}^{3} / \mathrm{s}\right)\end{array}$ & $\begin{array}{c}\text { Date } \\
\text { (month/ } \\
\text { day/ } \\
\text { year) }\end{array}$ & $\begin{array}{l}\text { Stage } \\
(\mathrm{ft})\end{array}$ & $\begin{array}{l}\text { Discharge } \\
\left(\mathrm{ft}^{3} / \mathrm{s}\right)\end{array}$ & $\begin{array}{l}\text { Regulated } \\
\text { during } \\
\text { flood }^{1}\end{array}$ & $\begin{array}{l}\text { Recurrence } \\
\text { interval } \\
\text { (years) }\end{array}$ \\
\hline 11160430 & Bean Creek near Scotts Valley, CA & 8.81 & 1989-98 & 1998 & 10.85 & 1,710 & $2 / 3 / 98$ & 10.85 & 1,710 & $\mathrm{~N}$ & $10-15$ \\
\hline 11161300 & $\begin{array}{l}\text { Carbonera Creek at Scotts Valley, } \\
\text { CA }\end{array}$ & 3.60 & 1985-98 & 1997 & 11.89 & 1,620 & $12 / 10 / 96$ & 11.89 & 1,620 & $\mathrm{~N}$ & $50-75$ \\
\hline 11162500 & $\begin{array}{l}\text { Pescadero Creek near Pescadero, } \\
\text { CA }\end{array}$ & 45.9 & $1952-98$ & 1998 & 22.47 & 10,600 & $2 / 3 / 98$ & 22.47 & 10,600 & $\mathrm{~N}$ & $25-50$ \\
\hline 11164500 & $\begin{array}{l}\text { San Francisquito Creek at Stanford } \\
\text { University, CA }\end{array}$ & 37.4 & $\begin{array}{l}1931-41 \\
1951-98\end{array}$ & $\begin{array}{l}1998 \\
1956\end{array}$ & $\begin{array}{l}13.40 \\
13.60\end{array}$ & $\begin{array}{l}7,200 \\
5,560\end{array}$ & 2/3/98 & 13.40 & 7,200 & $\mathrm{~N}$ & $75-100$ \\
\hline 11166000 & Matadero Creek at Palo Alto, CA & 7.26 & $\begin{array}{l}\text { 1953-91, } \\
1993-98\end{array}$ & 1998 & 10.00 & 2,560 & $2 / 2 / 98$ & 10.00 & 2,560 & $\mathrm{~N}$ & $>100$ \\
\hline 11169000 & Guadalupe River at San Jose, CA & 146 & 1930-98 & 1995 & 17.40 & 11,000 & $3 / 10 / 95$ & 17.40 & 11,000 & $\mathrm{Y}$ & $25-50$ \\
\hline 11173200 & Arroyo Hondo near San Jose, CA & 77.1 & $\begin{array}{l}\text { 1969-81, } \\
1995-98\end{array}$ & 1998 & 15.85 & 7,340 & 2/3/98 & 15.85 & 7,340 & $\mathrm{~N}$ & $10-20$ \\
\hline 11176000 & $\begin{array}{l}\text { Arroyo Mocho near Livermore, } \\
\text { CA }\end{array}$ & 38.2 & $\begin{array}{l}1913-30 \\
1956, \\
1964-98\end{array}$ & $\begin{array}{l}1983 \\
1986\end{array}$ & $\begin{array}{r}8.80 \\
10.44\end{array}$ & $\begin{array}{l}2,250 \\
1,660\end{array}$ & $3 / 10 / 95$ & 9.29 & 2,000 & $\mathrm{~N}$ & $10-20$ \\
\hline 11176400 & $\begin{array}{l}\text { Arroyo Valle below Lang Canyon } \\
\text { near Livermore, CA }\end{array}$ & 130 & 1964-98 & $\begin{array}{l}1986 \\
1969\end{array}$ & $\begin{array}{l}7.36 \\
8.90\end{array}$ & $\begin{array}{l}8,790 \\
5,340\end{array}$ & $\begin{array}{l}3 / 10 / 95 \\
2 / 3 / 98\end{array}$ & $\begin{array}{l}7.18 \\
6.73\end{array}$ & $\begin{array}{l}8,490 \\
7,750\end{array}$ & $\begin{array}{l}\mathrm{N} \\
\mathrm{N}\end{array}$ & $\begin{array}{l}10-20 \\
10-20\end{array}$ \\
\hline 11176500 & Arroyo Valley near Livermore, CA & 147 & $\begin{array}{l}1913-24, \\
1926-28 \\
1930,1956 \\
1958-98\end{array}$ & 1956 & 13.93 & 18,200 & $2 / 4 / 98$ & 9.17 & 2,980 & $\mathrm{Y}$ & $15-25$ \\
\hline 11180500 & Dry Creek at Union City, CA & 9.39 & $\begin{array}{l}1917-19 \\
1959-98\end{array}$ & 1995 & 5.32 & 1,680 & $\begin{array}{l}1 / 9 / 95 \\
2 / 3 / 98\end{array}$ & $\begin{array}{l}5.32 \\
5.15\end{array}$ & $\begin{array}{l}1,680 \\
1,500\end{array}$ & $\begin{array}{l}\mathrm{N} \\
\mathrm{N}\end{array}$ & $\begin{array}{l}20-30 \\
15-25\end{array}$ \\
\hline 11180825 & $\begin{array}{l}\text { San Lorenzo Creek above Don } \\
\text { Castro Reservoir near Castro } \\
\text { Valley, CA }\end{array}$ & 18.0 & $\begin{array}{l}1981-91 \\
1993-94 \\
1998\end{array}$ & 1998 & 15.48 & 3,890 & $2 / 3 / 98$ & 15.48 & 3,890 & $\mathrm{~N}$ & $25-35$ \\
\hline
\end{tabular}


Table 6. Maximum stage and discharge for period of record for streamgages having significant floods during 1994-98 water years in California.-Continued

$\left[\mathrm{mi}^{2}\right.$, square miles; ft, feet above an arbitrary datum; $\mathrm{ft}^{3} / \mathrm{s}$, cubic feet per second; --, not determined or not applicable; >, greater than. Source: Recurrence intervals calculated from U.S. Geological Survey data. Other data from U.S. Geological Survey reports or databases]

\begin{tabular}{|c|c|c|c|c|c|c|c|c|c|c|c|}
\hline \multirow{2}{*}{$\begin{array}{l}\text { Streamgage } \\
\text { number } \\
\text { (fig. 17) }\end{array}$} & \multirow[b]{2}{*}{ Streamgage name } & \multirow{2}{*}{$\begin{array}{l}\text { Total } \\
\text { drainage } \\
\left(\mathrm{mi}^{2}\right)\end{array}$} & \multicolumn{4}{|c|}{$\begin{array}{c}\text { Maximum stage and discharge for period of record } \\
\text { through } 1998 \text { water year }\end{array}$} & \multicolumn{5}{|c|}{ Significant floods $1994-98$ water years } \\
\hline & & & $\begin{array}{l}\text { Period of } \\
\text { record } \\
\text { (water } \\
\text { years) }\end{array}$ & Water year & $\begin{array}{l}\text { Stage } \\
\text { (ft) }\end{array}$ & $\begin{array}{l}\text { Discharge } \\
\left(\mathrm{ft}^{3} / \mathrm{s}\right)\end{array}$ & $\begin{array}{l}\text { Date } \\
\text { (month/ } \\
\text { day/ } \\
\text { year) }\end{array}$ & $\begin{array}{l}\text { Stage } \\
(\mathrm{ft})\end{array}$ & $\begin{array}{l}\text { Discharge } \\
\left(\mathrm{ft}^{3} / \mathrm{s}\right)\end{array}$ & $\begin{array}{l}\text { Regulated } \\
\text { during } \\
\text { flood }^{1}\end{array}$ & $\begin{array}{l}\text { Recurrence } \\
\text { interval } \\
\text { (years) }\end{array}$ \\
\hline 11181000 & $\begin{array}{l}\text { San Lorenzo Creek at Hayward, } \\
\text { CA }\end{array}$ & 37.5 & $\begin{array}{l}\text { 1940, 1942, } \\
1947-83, \\
1998\end{array}$ & 1998 & 21.85 & 8,140 & $2 / 3 / 98$ & 21.85 & 8,140 & $\mathrm{~N}$ & $20-30$ \\
\hline 11181008 & $\begin{array}{l}\text { Castro Valley Creek at Hayward, } \\
\text { CA }\end{array}$ & 5.51 & 1972-98 & 1998 & 9.12 & 1,550 & $\begin{array}{l}2 / 2 / 98 \\
1 / 2 / 97\end{array}$ & $\begin{array}{l}9.12 \\
8.15\end{array}$ & $\begin{array}{l}1,550 \\
1,240\end{array}$ & $\begin{array}{l}\mathrm{N} \\
\mathrm{N}\end{array}$ & $\begin{array}{l}50-75 \\
10-20\end{array}$ \\
\hline 11181040 & $\begin{array}{l}\text { San Lorenzo Creek at San Lorenzo, } \\
\text { CA }\end{array}$ & 44.6 & $\begin{array}{l}1968-78 \\
1988-98\end{array}$ & 1998 & 14.27 & 10,300 & $2 / 3 / 98$ & 14.27 & 10,300 & $\mathrm{~N}$ & $75-100$ \\
\hline 11182500 & $\begin{array}{l}\text { San Ramon Creek at San Ramon, } \\
\text { CA }\end{array}$ & 5.89 & 1953-98 & 1963 & 16.98 & 1,600 & $2 / 3 / 98$ & 13.81 & 1,530 & $\mathrm{~N}$ & $20-30$ \\
\hline 11186000 & Kern River near Kernville, CA & 846 & 1912-98 & 1967 & 22.77 & 60,000 & $1 / 3 / 97$ & 16.69 & 46,300 & $\mathrm{Y}$ & $50-75$ \\
\hline 11199500 & White River near Ducor, CA & 90.6 & $\begin{array}{l}1943, \\
1945-53 \\
1972-98\end{array}$ & $\begin{array}{l}1998 \\
1973\end{array}$ & $\begin{array}{l}4.53 \\
6.12\end{array}$ & $\begin{array}{l}2,720 \\
1,330\end{array}$ & $2 / 23 / 98$ & 4.53 & 2,720 & $\mathrm{~N}$ & $30-40$ \\
\hline 11200800 & $\begin{array}{l}\text { Deer Creek near Fountain Springs, } \\
\text { CA }\end{array}$ & 83.3 & $\begin{array}{l}\text { 1967, } \\
1969-98\end{array}$ & 1967 & 12.54 & 5,330 & $\begin{array}{l}1 / 3 / 97 \\
2 / 23 / 98\end{array}$ & $\begin{array}{l}10.32 \\
10.24\end{array}$ & $\begin{array}{l}3,790 \\
3,710\end{array}$ & $\begin{array}{l}\mathrm{N} \\
\mathrm{N}\end{array}$ & $\begin{array}{l}10-20 \\
10-20\end{array}$ \\
\hline 11205690 & Lewis Creek near Lindsay, CA & 21.5 & $\begin{array}{l}1974-88 \\
1992-94 \\
1996-98\end{array}$ & 1997 & 26.75 & 1,550 & $1 / 3 / 97$ & 26.75 & 1,550 & $\mathrm{~N}$ & $30-50$ \\
\hline 11208000 & $\begin{array}{l}\text { Marble Fork Kaweah River at } \\
\text { Potwisha Camp, CA }\end{array}$ & 51.4 & $\begin{array}{l}1950-88 \\
1993-98\end{array}$ & 1956 & 13.40 & 12,500 & $1 / 2 / 97$ & 11.57 & 6,760 & $\mathrm{~N}$ & $25-35$ \\
\hline 11215000 & $\begin{array}{l}\text { North Fork Kings River near Cliff } \\
\text { Camp, CA }\end{array}$ & 181 & $\begin{array}{l}1922-88, \\
1998\end{array}$ & 1938 & 18.00 & 14,000 & $7 / 8 / 98$ & 11.01 & 4,060 & $\mathrm{Y}$ & $15-25$ \\
\hline 11218400 & $\begin{array}{l}\text { North Fork Kings River below } \\
\text { Dinky Creek near Balch Camp, } \\
\text { CA }\end{array}$ & 387 & 1960-98 & 1963 & 19.20 & 27,400 & $1 / 2 / 97$ & 18.43 & 24,900 & $\mathrm{Y}$ & 20 \\
\hline
\end{tabular}


Table 6. Maximum stage and discharge for period of record for streamgages having significant floods during 1994-98 water years in California.-Continued

$\left[\mathrm{mi}^{2}\right.$, square miles; $\mathrm{ft}$, feet above an arbitrary datum; $\mathrm{ft}^{3} / \mathrm{s}$, cubic feet per second; --, not determined or not applicable; >, greater than. Source: Recurrence intervals calculated from U.S. Geological Survey data. Other data from U.S. Geological Survey reports or databases]

\begin{tabular}{|c|c|c|c|c|c|c|c|c|c|c|c|}
\hline \multirow{2}{*}{$\begin{array}{c}\text { Streamgage } \\
\text { number } \\
\text { (fig. 17) }\end{array}$} & \multirow[b]{2}{*}{ Streamgage name } & \multirow{2}{*}{$\begin{array}{c}\text { Total } \\
\text { drainage } \\
\left(\mathrm{mi}^{2}\right)\end{array}$} & \multicolumn{4}{|c|}{$\begin{array}{c}\text { Maximum stage and discharge for period of record } \\
\text { through } 1998 \text { water year }\end{array}$} & \multicolumn{5}{|c|}{ Significant floods $1994-98$ water years } \\
\hline & & & $\begin{array}{l}\text { Period of } \\
\text { record } \\
\text { (water } \\
\text { years) }\end{array}$ & Water year & $\begin{array}{l}\text { Stage } \\
(\mathrm{ft})\end{array}$ & $\begin{array}{l}\text { Discharge } \\
\left(\mathrm{ft}^{3} / \mathrm{s}\right)\end{array}$ & $\begin{array}{c}\text { Date } \\
\text { (month/ } \\
\text { day/ } \\
\text { year) }\end{array}$ & $\begin{array}{l}\text { Stage } \\
(\mathrm{ft})\end{array}$ & $\begin{array}{l}\text { Discharge } \\
\left(\mathrm{ft}^{3} / \mathrm{s}\right)\end{array}$ & $\begin{array}{c}\text { Regulated } \\
\text { during } \\
\text { flood }^{1}\end{array}$ & $\begin{array}{c}\text { Recurrence } \\
\text { interval } \\
\text { (years) }\end{array}$ \\
\hline 11218500 & $\begin{array}{l}\text { Kings River below Northfork near } \\
\text { Trimmer, CA }\end{array}$ & 1,342 & $\begin{array}{l}1951-92, \\
1997\end{array}$ & 1956 & 23.08 & 85,200 & $1 / 2 / 97$ & 21.58 & 73,900 & $\mathrm{Y}$ & $40-50$ \\
\hline 11221700 & Mill Creek near Piedra, CA & 127 & $\begin{array}{l}1958-94 \\
1997\end{array}$ & $\begin{array}{l}1997 \\
1967\end{array}$ & $\begin{array}{l}8.70 \\
9.53\end{array}$ & $\begin{array}{l}12,000 \\
11,000\end{array}$ & $1 / 2 / 97$ & 8.70 & 12,000 & $\mathrm{~N}$ & $10-20$ \\
\hline 11224500 & $\begin{array}{l}\text { Los Gatos Creek above Nunez } \\
\text { Canyon near Coalinga, CA }\end{array}$ & 95.8 & 1946-98 & $\begin{array}{l}1995 \\
1993\end{array}$ & $-\overline{-}+12.08$ & $\begin{array}{l}5,700 \\
3,820\end{array}$ & $3 / 10 / 95$ & -- & 5,700 & $\mathrm{~N}$ & $25-35$ \\
\hline 11225100 & $\begin{array}{l}\text { Los Gatos Creek below Jacalitos } \\
\text { Creek near Coalinga, CA }\end{array}$ & 407 & $\begin{array}{l}1959-67 \\
1969,1995\end{array}$ & $\begin{array}{l}1995 \\
1969\end{array}$ & -- & $\begin{array}{l}25,000 \\
20,200\end{array}$ & $3 / 10 / 95$ & -- & 25,000 & $\mathrm{~N}$ & $>100$ \\
\hline 11230500 & $\begin{array}{l}\text { Bear Creek near Lake Thomas at } \\
\text { Edison, CA }\end{array}$ & 52.5 & $\begin{array}{l}1922-88 \\
1992-98\end{array}$ & 1982 & 8.35 & 3,660 & $1 / 2 / 97$ & 6.84 & 1,760 & $\mathrm{~N}$ & $10-20$ \\
\hline 11234760 & $\begin{array}{l}\text { San Joaquin River above Shakeflat } \\
\text { Creek near Big Creek, CA }\end{array}$ & 1,003 & $\begin{array}{l}1960-88 \\
1992-98\end{array}$ & 1997 & 32.00 & 80,000 & $\begin{array}{l}5 / 16 / 96 \\
1 / 2 / 97\end{array}$ & $\begin{array}{l}20.44 \\
32.00\end{array}$ & $\begin{array}{l}24,700 \\
80,000\end{array}$ & $\begin{array}{l}\mathrm{Y} \\
\mathrm{Y}\end{array}$ & $\begin{array}{l}10-25 \\
>75\end{array}$ \\
\hline 11237500 & $\begin{array}{l}\text { Pitman Creek below Tamarack } \\
\text { Creek, CA }\end{array}$ & 22.9 & $1928-98$ & 1997 & 12.65 & 5,500 & $\begin{array}{l}5 / 16 / 96 \\
1 / 2 / 97\end{array}$ & $\begin{array}{l}11.04 \\
12.65\end{array}$ & $\begin{array}{l}3,540 \\
5,500\end{array}$ & $\begin{array}{l}\mathrm{N} \\
\mathrm{N}\end{array}$ & $\begin{array}{c}25-50 \\
75-100\end{array}$ \\
\hline 11242000 & $\begin{array}{l}\text { San Joaquin River above Willow } \\
\text { Creek near Auberry, CA }\end{array}$ & 1,295 & $1951-98$ & 1997 & 65.17 & 99,200 & $1 / 2 / 97$ & 65.17 & 99,200 & $\mathrm{Y}$ & $>75$ \\
\hline 11242400 & $\begin{array}{l}\text { North Fork Willow Creek near } \\
\text { Sugar Pine, CA }\end{array}$ & 16.9 & 1966-98 & 1980 & 7.41 & 2,750 & $1 / 2 / 97$ & 7.01 & 2,540 & $\mathrm{~N}$ & $20-30$ \\
\hline 11244000 & $\begin{array}{l}\text { North Fork Willow Creek near } \\
\text { Bass Lake, CA }\end{array}$ & 50.8 & $\begin{array}{l}1940-97, \\
1999\end{array}$ & 1997 & 9.10 & 3,770 & $\begin{array}{l}3 / 11 / 95 \\
1 / 2 / 97\end{array}$ & $\overline{--}$ & $\begin{array}{l}2,300 \\
3,770\end{array}$ & $\begin{array}{l}\mathrm{Y} \\
\mathrm{Y}\end{array}$ & $\begin{array}{l}15-25 \\
30-50\end{array}$ \\
\hline 11246500 & $\begin{array}{l}\text { Willow Creek at mouth near } \\
\text { Auberry, CA }\end{array}$ & 130 & $\begin{array}{l}1951-88 \\
1992-98\end{array}$ & $\begin{array}{l}1956 \\
1997\end{array}$ & $\begin{array}{l}28.50 \\
31.65\end{array}$ & $\begin{array}{r}15,700 \\
--\end{array}$ & $3 / 10 / 95$ & 19.38 & 8,460 & $\mathrm{Y}$ & $10-20$ \\
\hline 11251000 & $\begin{array}{l}\text { San Joaquin River below Friant, } \\
\text { CA }\end{array}$ & 1,676 & $\begin{array}{l}1908-17, \\
1920-98\end{array}$ & $\begin{array}{l}1938 \\
1997\end{array}$ & 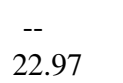 & $\begin{array}{l}77,200 \\
60,300\end{array}$ & $1 / 3 / 97$ & 22.97 & 60,300 & $\mathrm{Y}$ & $>50$ \\
\hline 11253310 & $\begin{array}{l}\text { Cantua Creek near Cantua Creek, } \\
\text { CA }\end{array}$ & 46.4 & 1958-98 & $\begin{array}{l}1983 \\
1995\end{array}$ & $\begin{array}{l}5.72 \\
7.13\end{array}$ & $\begin{array}{l}3,420 \\
2,970\end{array}$ & $3 / 10 / 95$ & 7.13 & 2,970 & $\mathrm{~N}$ & $25-50$ \\
\hline
\end{tabular}


Table 6. Maximum stage and discharge for period of record for streamgages having significant floods during 1994-98 water years in California.-Continued

$\left[\mathrm{mi}^{2}\right.$, square miles; $\mathrm{ft}$, feet above an arbitrary datum; $\mathrm{ft}^{3} / \mathrm{s}$, cubic feet per second; --, not determined or not applicable; >, greater than. Source: Recurrence intervals calculated from U.S. Geological Survey data. Other data from U.S. Geological Survey reports or databases]

\begin{tabular}{|c|c|c|c|c|c|c|c|c|c|c|c|}
\hline \multirow{2}{*}{$\begin{array}{c}\text { Streamgage } \\
\text { number } \\
\text { (fig. 17) }\end{array}$} & \multirow[b]{2}{*}{ Streamgage name } & \multirow{2}{*}{$\begin{array}{c}\text { Total } \\
\text { drainage } \\
\left(\mathrm{mi}^{2}\right)\end{array}$} & \multicolumn{4}{|c|}{$\begin{array}{c}\text { Maximum stage and discharge for period of record } \\
\text { through } 1998 \text { water year }\end{array}$} & \multicolumn{5}{|c|}{ Significant floods $1994-98$ water years } \\
\hline & & & $\begin{array}{l}\text { Period of } \\
\text { record } \\
\text { (water } \\
\text { years) }\end{array}$ & Water year & $\begin{array}{l}\text { Stage } \\
(\mathrm{ft})\end{array}$ & $\begin{array}{l}\text { Discharge } \\
\left(\mathrm{ft}^{3} / \mathrm{s}\right)\end{array}$ & $\begin{array}{c}\text { Date } \\
\text { (month/ } \\
\text { day/ } \\
\text { year) }\end{array}$ & $\begin{array}{l}\text { Stage } \\
(\mathrm{ft})\end{array}$ & $\begin{array}{l}\text { Discharge } \\
\left(\mathrm{ft}^{3} / \mathrm{s}\right)\end{array}$ & $\begin{array}{l}\text { Regulated } \\
\text { during } \\
\text { flood }^{1}\end{array}$ & $\begin{array}{c}\text { Recurrence } \\
\text { interval } \\
\text { (years) }\end{array}$ \\
\hline 11261100 & $\begin{array}{l}\text { Salt Slough at Highway165 near } \\
\text { Stevinson, CA }\end{array}$ & -- & $\begin{array}{l}1986, \\
1988-94, \\
1996-98\end{array}$ & $\begin{array}{l}1998 \\
1997\end{array}$ & $\begin{array}{l}71.25 \\
72.30\end{array}$ & $\begin{array}{l}771 \\
677\end{array}$ & $2 / 17 / 98$ & 71.25 & 771 & $\mathrm{~N}$ & $10-20$ \\
\hline 11262900 & Mud Slough near Gustine, CA & -- & 1986-98 & $\begin{array}{l}1998 \\
1997\end{array}$ & $\begin{array}{l}11.11 \\
12.03\end{array}$ & $\begin{array}{r}1,060 \\
718\end{array}$ & $2 / 8 / 98$ & 11.11 & 1,060 & $\mathrm{~N}$ & $20-40$ \\
\hline 11264500 & $\begin{array}{l}\text { Merced River at Happy Isles } \\
\text { Bridge near Yosemite, CA }\end{array}$ & 181 & $1916-98$ & 1997 & 13.27 & 10,100 & $\begin{array}{l}5 / 16 / 96 \\
1 / 2 / 97\end{array}$ & $\begin{array}{r}8.84 \\
13.27\end{array}$ & $\begin{array}{r}5,900 \\
10,100\end{array}$ & $\begin{array}{l}\mathrm{N} \\
\mathrm{N}\end{array}$ & $\begin{array}{l}<10 \\
50-75\end{array}$ \\
\hline 11266500 & $\begin{array}{l}\text { Merced River at Pohono Bridge } \\
\text { near Yosemite, CA }\end{array}$ & 321 & 1917-98 & 1997 & 23.43 & 24,600 & $1 / 3 / 97$ & 23.43 & 24,600 & $\mathrm{~N}$ & $75-100$ \\
\hline 11274000 & $\begin{array}{l}\text { San Joaquin River near Newman, } \\
\text { CA }\end{array}$ & 9,520 & $\begin{array}{l}1868 \\
1914-98\end{array}$ & 1997 & 66.14 & 36,200 & $1 / 28 / 97$ & 66.14 & 36,200 & $\mathrm{Y}$ & $50-75$ \\
\hline 11274500 & $\begin{array}{l}\text { Orestimba Creek near Newman, } \\
\text { CA }\end{array}$ & 134 & $1932-98$ & $\begin{array}{l}1995 \\
1963\end{array}$ & $\begin{array}{l}9.51 \\
9.72\end{array}$ & $\begin{array}{r}12,000 \\
8,300\end{array}$ & $\begin{array}{l}3 / 10 / 95 \\
2 / 3 / 98\end{array}$ & $\begin{array}{l}9.51 \\
8.80\end{array}$ & $\begin{array}{r}12,000 \\
9,470\end{array}$ & $\begin{array}{l}\mathrm{N} \\
\mathrm{N}\end{array}$ & $\begin{array}{l}40-60 \\
20-30\end{array}$ \\
\hline 11274630 & $\begin{array}{l}\text { Del Puerto Creek near Patterson, } \\
\text { CA }\end{array}$ & 72.6 & 1959-98 & 1998 & 14.92 & 5,270 & $\begin{array}{l}3 / 10 / 95 \\
2 / 3 / 98\end{array}$ & $\begin{array}{l}11.62 \\
14.92\end{array}$ & $\begin{array}{l}3,400 \\
5,270\end{array}$ & $\begin{array}{l}\mathrm{N} \\
\mathrm{N}\end{array}$ & $\begin{array}{l}20-30 \\
50-75\end{array}$ \\
\hline 11276500 & $\begin{array}{l}\text { Tuolumne River near Hetch } \\
\text { Hetchy, CA }\end{array}$ & 457 & $1915-98$ & 1997 & 15.08 & 16,400 & $\begin{array}{l}7 / 9 / 95 \\
1 / 3 / 97\end{array}$ & $\begin{array}{l}14.37 \\
15.08\end{array}$ & $\begin{array}{l}13,600 \\
16,400\end{array}$ & $\begin{array}{l}\mathrm{Y} \\
\mathrm{Y}\end{array}$ & $\begin{array}{c}25-50 \\
75-100\end{array}$ \\
\hline 11276600 & $\begin{array}{l}\text { Tuolumne River above Early } \\
\text { intake near Mather, CA }\end{array}$ & 484 & $\begin{array}{l}\text { 1943, } \\
1971-98\end{array}$ & 1997 & 22.98 & 17,700 & $1 / 3 / 97$ & 22.98 & 17,700 & $\mathrm{Y}$ & $75-100$ \\
\hline 11276900 & $\begin{array}{l}\text { Tuolumne River below Early } \\
\text { intake near Mather, CA }\end{array}$ & 487 & 1967-98 & 1997 & 12.33 & 18,200 & $\begin{array}{l}7 / 9 / 95 \\
1 / 3 / 97\end{array}$ & $\begin{array}{l}11.33 \\
12.33\end{array}$ & $\begin{array}{l}13,800 \\
18,200\end{array}$ & $\begin{array}{l}\mathrm{Y} \\
\mathrm{Y}\end{array}$ & $\begin{array}{c}20-40 \\
>75\end{array}$ \\
\hline 11277300 & $\begin{array}{l}\text { Cherry Creek below Valley Dam } \\
\text { near Hetch Hetchy, CA }\end{array}$ & 118 & 1957-98 & 1996 & 11.15 & 5,120 & $5 / 16 / 96$ & 11.15 & 5,120 & $\mathrm{Y}$ & $25-50$ \\
\hline 11278000 & $\begin{array}{l}\text { Eleanor Creek near Hetch Hetchy, } \\
\text { CA }\end{array}$ & 78.4 & $1915-98$ & 1997 & 26.74 & 19,500 & $1 / 2 / 97$ & 26.74 & 19,500 & $\mathrm{Y}$ & $>75$ \\
\hline
\end{tabular}


Table 6. Maximum stage and discharge for period of record for streamgages having significant floods during 1994-98 water years in California.-Continued

$\left[\mathrm{mi}^{2}\right.$, square miles; $\mathrm{ft}$, feet above an arbitrary datum; $\mathrm{ft}^{3} / \mathrm{s}$, cubic feet per second; --, not determined or not applicable; >, greater than. Source: Recurrence intervals calculated from U.S. Geological Survey data. Other data from U.S. Geological Survey reports or databases]

\begin{tabular}{|c|c|c|c|c|c|c|c|c|c|c|c|}
\hline \multirow[b]{2}{*}{$\begin{array}{l}\text { Streamgage } \\
\text { number } \\
\text { (fig. 17) }\end{array}$} & \multirow[b]{2}{*}{ Streamgage name } & \multirow[b]{2}{*}{$\begin{array}{l}\text { Total } \\
\text { drainage } \\
\left(\mathrm{mi}^{2}\right)\end{array}$} & \multicolumn{4}{|c|}{$\begin{array}{c}\text { Maximum stage and discharge for period of record } \\
\text { through } 1998 \text { water year }\end{array}$} & \multicolumn{5}{|c|}{ Significant floods $1994-98$ water years } \\
\hline & & & $\begin{array}{l}\text { Period of } \\
\text { record } \\
\text { (water } \\
\text { years) }\end{array}$ & Water year & $\begin{array}{l}\text { Stage } \\
(\mathrm{ft})\end{array}$ & $\begin{array}{l}\text { Discharge } \\
\left(\mathrm{ft}^{3} / \mathrm{s}\right)\end{array}$ & $\begin{array}{c}\text { Date } \\
\text { (month/ } \\
\text { day/ } \\
\text { year) }\end{array}$ & $\begin{array}{l}\text { Stage } \\
(\mathrm{ft})\end{array}$ & $\begin{array}{l}\text { Discharge } \\
\left(\mathrm{ft}^{3} / \mathrm{s}\right)\end{array}$ & $\begin{array}{l}\text { Regulated } \\
\text { during } \\
\text { flood }^{1}\end{array}$ & $\begin{array}{l}\text { Recurrence } \\
\text { interval } \\
\text { (years) }\end{array}$ \\
\hline 11278300 & $\begin{array}{l}\text { Cherry Creek near Early intake, } \\
\text { CA }\end{array}$ & 226 & $1957-98$ & 1997 & 18.46 & 33,200 & $1 / 2 / 97$ & 18.46 & 33,200 & $\mathrm{Y}$ & $>75$ \\
\hline 11278400 & $\begin{array}{l}\text { Cherry Creek below Dion River } \\
\text { Holm Power Plant near Mather, } \\
\text { CA }\end{array}$ & 234 & $1963-98$ & 1997 & 25.40 & 33,500 & $1 / 2 / 97$ & 25.40 & 33,500 & $\mathrm{Y}$ & $>75$ \\
\hline 11281000 & $\begin{array}{c}\text { South Fork Tuolumne River near } \\
\text { Oakland Recreation Camp, CA }\end{array}$ & 87.0 & $1923-98$ & $\begin{array}{l}1956 \\
1997\end{array}$ & $\begin{array}{l}10.90 \\
12.51\end{array}$ & $\begin{array}{l}12,000 \\
11,500\end{array}$ & $1 / 3 / 97$ & 12.51 & 12,000 & $\mathrm{~N}$ & $25-50$ \\
\hline 11282000 & $\begin{array}{l}\text { Middle Tuolumne River at } \\
\text { Oakland Recreation Camp, CA }\end{array}$ & 73.5 & 1917-98 & 1997 & 13.02 & 6,300 & $\begin{array}{l}3 / 10 / 95 \\
1 / 3 / 97\end{array}$ & $\begin{array}{l}10.10 \\
13.02\end{array}$ & $\begin{array}{l}3,430 \\
6,300\end{array}$ & $\begin{array}{l}\mathrm{N} \\
\mathrm{N}\end{array}$ & $\begin{array}{l}20-30 \\
>75\end{array}$ \\
\hline 11283500 & $\begin{array}{l}\text { Clavey River near Buck Mead- } \\
\text { ows, CA }\end{array}$ & 144 & $\begin{array}{l}1960-83 \\
1987-94 \\
1997\end{array}$ & 1997 & 28.66 & 47,000 & $1 / 3 / 97$ & 28.66 & 47,000 & $\mathrm{~N}$ & $>75$ \\
\hline 11284400 & $\begin{array}{l}\text { Big Creek above Whites Gulch } \\
\text { near Groveland, CA }\end{array}$ & 16.4 & $\begin{array}{l}1965, \\
1970-98\end{array}$ & 1986 & 7.03 & 2,620 & $1 / 2 / 97$ & 6.94 & 2,500 & $\mathrm{~N}$ & $10-20$ \\
\hline 11289650 & $\begin{array}{l}\text { Tuolumne River below Lagrange } \\
\text { Dam near Lagrange, CA }\end{array}$ & 1,538 & 1971-98 & 1997 & 28.43 & 58,900 & $1 / 3 / 97$ & 28.43 & 58,900 & $\mathrm{Y}$ & $>50$ \\
\hline 11290000 & Tuolumne River at Modesto, CA & 1,884 & $\begin{array}{l}1895,1940, \\
1943-98\end{array}$ & $\begin{array}{l}1951 \\
1997\end{array}$ & $\begin{array}{l}69.19 \\
71.21\end{array}$ & $\begin{array}{l}57,000 \\
55,800\end{array}$ & $1 / 4 / 97$ & 71.21 & 55,800 & $\mathrm{Y}$ & $>50$ \\
\hline 11292000 & $\begin{array}{l}\text { Middle Fork Stanislaus River at } \\
\text { Kennedy Meadows near } \\
\text { Dardanelle, CA }\end{array}$ & 47.5 & $\begin{array}{l}1939-45 \\
1947-98\end{array}$ & 1996 & 8.37 & 3,310 & $1 / 2 / 97$ & $\begin{array}{l}8.37 \\
7.99\end{array}$ & $\begin{array}{l}3,310 \\
2,890\end{array}$ & $\begin{array}{l}\mathrm{Y} \\
\mathrm{Y}\end{array}$ & $\begin{array}{l}>50 \\
>50\end{array}$ \\
\hline 11292700 & $\begin{array}{l}\text { Middle Fork Stanislaus River at } \\
\text { Hells Half Acre Bridge, CA }\end{array}$ & 287 & $1956-98$ & 1997 & 18.02 & 24,600 & & 18.02 & 24,600 & $\mathrm{Y}$ & $50-75$ \\
\hline 11292900 & $\begin{array}{l}\text { Middle Fork Stanislaus River } \\
\text { below Beardsley Dam, CA }\end{array}$ & 316 & 1957-98 & 1997 & 19.31 & 28,200 & $\begin{array}{l}5 / 16 / 96 \\
1 / 2 / 97\end{array}$ & $\begin{array}{l}14.64 \\
19.31\end{array}$ & $\begin{array}{l}15,000 \\
28,200\end{array}$ & $\begin{array}{l}\mathrm{Y} \\
\mathrm{Y}\end{array}$ & $\begin{array}{l}25-50 \\
>75\end{array}$ \\
\hline
\end{tabular}


$\left[\mathrm{mi}^{2}\right.$, square miles; ft, feet above an arbitrary datum; $\mathrm{ft}^{3} / \mathrm{s}$, cubic feet per second; --, not determined or not applicable; >, greater than. Source: Recurrence intervals calculated from U.S. Geological Survey data. Other data from U.S. Geological Survey reports or databases]

\begin{tabular}{|c|c|c|c|c|c|c|c|c|c|c|c|}
\hline \multirow{2}{*}{$\begin{array}{l}\text { Streamgage } \\
\text { number } \\
\text { (fig. 17) }\end{array}$} & \multirow[b]{2}{*}{ Streamgage name } & \multirow{2}{*}{$\begin{array}{l}\text { Total } \\
\text { drainage } \\
\left(\mathrm{mi}^{2}\right)\end{array}$} & \multicolumn{4}{|c|}{$\begin{array}{c}\text { Maximum stage and discharge for period of record } \\
\text { through } 1998 \text { water year }\end{array}$} & \multicolumn{5}{|c|}{ Significant floods $1994-98$ water years } \\
\hline & & & $\begin{array}{l}\text { Period of } \\
\text { record } \\
\text { (water } \\
\text { years) }\end{array}$ & Water year & $\begin{array}{l}\text { Stage } \\
(\mathrm{ft})\end{array}$ & $\begin{array}{l}\text { Discharge } \\
\left(\mathrm{ft}^{3} / \mathrm{s}\right)\end{array}$ & $\begin{array}{l}\text { Date } \\
\text { (month/ } \\
\text { day/ } \\
\text { year) }\end{array}$ & $\begin{array}{l}\text { Stage } \\
(\mathrm{ft})\end{array}$ & $\begin{array}{l}\text { Discharge } \\
\left(\mathrm{ft}^{3} / \mathrm{s}\right)\end{array}$ & $\begin{array}{l}\text { Regulated } \\
\text { during } \\
\text { flood }^{1}\end{array}$ & $\begin{array}{l}\text { Recurrence } \\
\text { interval } \\
\text { (years) }\end{array}$ \\
\hline 11293600 & $\begin{array}{l}\text { North Fork Stanislaus River below } \\
\text { Diversion Dam near Big } \\
\text { Meadows, CA }\end{array}$ & 28.8 & $1988-98$ & 1996 & 7.92 & 3,220 & $5 / 16 / 96$ & 7.92 & 3,220 & $\mathrm{Y}$ & $5-10$ \\
\hline 11294500 & $\begin{array}{l}\text { North Fork Stanislaus River near } \\
\text { Avery, CA }\end{array}$ & 163 & $\begin{array}{l}1915-22 \\
1929-98\end{array}$ & 1963 & 15.00 & 36,000 & $1 / 2 / 97$ & 14.94 & 35,600 & $\mathrm{Y}$ & $25-50$ \\
\hline 11296500 & $\begin{array}{l}\text { South Fork Stanislaus River at } \\
\text { Strawberry, CA }\end{array}$ & 44.8 & $\begin{array}{l}\text { 1912-16, } \\
1939-98\end{array}$ & 1997 & 12.34 & 7,820 & $\begin{array}{l}5 / 16 / 96 \\
1 / 2 / 97\end{array}$ & $\begin{array}{r}8.82 \\
12.34\end{array}$ & $\begin{array}{l}3,510 \\
7,820\end{array}$ & $\begin{array}{l}\mathrm{Y} \\
\mathrm{Y}\end{array}$ & $\begin{array}{l}25-50 \\
>75\end{array}$ \\
\hline 11298000 & $\begin{array}{l}\text { South Fork Stanislaus River near } \\
\text { Long Barn, CA }\end{array}$ & 66.9 & $\begin{array}{l}1938-88 \\
1992-98\end{array}$ & 1997 & 13.03 & 12,900 & $\begin{array}{l}5 / 16 / 96 \\
1 / 2 / 97\end{array}$ & $\begin{array}{r}8.93 \\
13.03\end{array}$ & $\begin{array}{r}4,410 \\
12,900\end{array}$ & $\begin{array}{l}\mathrm{Y} \\
\mathrm{Y}\end{array}$ & $\begin{array}{l}15-25 \\
>75\end{array}$ \\
\hline 11303500 & $\begin{array}{l}\text { San Joaquin River near Vernalis, } \\
\text { CA }\end{array}$ & 13,536 & $\begin{array}{l}1924, \\
1930-98\end{array}$ & $\begin{array}{l}1951 \\
1997\end{array}$ & $-\overline{34.88}$ & $\begin{array}{l}79,000 \\
75,600\end{array}$ & $1 / 5 / 97$ & 34.88 & 75,600 & $\mathrm{Y}$ & $>50$ \\
\hline 11314500 & $\begin{array}{l}\text { North Fork Mokelumne River } \\
\text { below Salt Springs Dam, CA }\end{array}$ & 170 & $\begin{array}{l}1927-33 \\
1935-98\end{array}$ & 1996 & 17.66 & 17,000 & $\begin{array}{l}5 / 16 / 96 \\
1 / 3 / 97\end{array}$ & $\begin{array}{l}17.66 \\
13.04\end{array}$ & $\begin{array}{r}17,000 \\
7,910\end{array}$ & $\begin{array}{l}\mathrm{Y} \\
\mathrm{Y}\end{array}$ & $\begin{array}{c}>75 \\
20-25\end{array}$ \\
\hline 11316600 & $\begin{array}{l}\text { North Fork Mokelumne River } \\
\text { above Tiger Creek, CA }\end{array}$ & 333 & 1987-98 & 1997 & 12.49 & 38,500 & $1 / 2 / 97$ & 12.49 & 38,500 & $\mathrm{Y}$ & $25-50$ \\
\hline 11316800 & Forest Creek near Wilseyville, CA & 20.8 & $1961-98$ & 1986 & 8.12 & 2,020 & $1 / 2 / 97$ & 7.59 & 1,930 & $\mathrm{~N}$ & $30-40$ \\
\hline 11317000 & $\begin{array}{l}\text { Middle Fork Mokelumne River at } \\
\text { West Point, CA }\end{array}$ & 68.4 & 1912-98 & $\begin{array}{l}1997 \\
1914\end{array}$ & $\begin{array}{r}9.28 \\
10.00\end{array}$ & $\begin{array}{l}5,040 \\
2,550\end{array}$ & $1 / 2 / 97$ & 9.28 & 5,040 & $\mathrm{~N}$ & $30-40$ \\
\hline 11318500 & $\begin{array}{l}\text { South Fork Mokelumne } \\
\text { River near West Point, CA }\end{array}$ & 75.1 & 1934-98 & $\begin{array}{l}1997 \\
1956\end{array}$ & $\begin{array}{l}12.72 \\
14.80\end{array}$ & $\begin{array}{l}7,610 \\
6,920\end{array}$ & $1 / 2 / 97$ & 12.72 & 7,610 & $\mathrm{~N}$ & $30-40$ \\
\hline 11319500 & $\begin{array}{l}\text { Mokelumne River near } \\
\text { Mokelumne Hill, CA }\end{array}$ & 544 & $\begin{array}{l}1901 \\
1903-04 \\
1928-98\end{array}$ & 1997 & 25.60 & 41,300 & $1 / 2 / 97$ & 25.60 & 41,300 & $\mathrm{Y}$ & $>100$ \\
\hline 11333000 & Camp Creek near Somerset, CA & 62.6 & $1955-98$ & 1997 & 20.30 & 22,400 & $1 / 2 / 97$ & 20.30 & 22,400 & $\mathrm{Y}$ & $>75$ \\
\hline
\end{tabular}


Table 6. Maximum stage and discharge for period of record for streamgages having significant floods during 1994-98 water years in California.-Continued

$\left[\mathrm{mi}^{2}\right.$, square miles; $\mathrm{ft}$, feet above an arbitrary datum; $\mathrm{ft}^{3} / \mathrm{s}$, cubic feet per second; --, not determined or not applicable; >, greater than. Source: Recurrence intervals calculated from U.S. Geological Survey data. Other data from U.S. Geological Survey reports or databases]

\begin{tabular}{|c|c|c|c|c|c|c|c|c|c|c|c|}
\hline \multirow[b]{2}{*}{$\begin{array}{c}\text { Streamgage } \\
\text { number } \\
\text { (fig. 17) }\end{array}$} & \multirow[b]{2}{*}{ Streamgage name } & \multirow{2}{*}{$\begin{array}{c}\text { Total } \\
\text { drainage } \\
\left(\mathrm{mi}^{2}\right)\end{array}$} & \multicolumn{4}{|c|}{$\begin{array}{c}\text { Maximum stage and discharge for period of record } \\
\text { through } 1998 \text { water year }\end{array}$} & \multicolumn{5}{|c|}{ Significant floods 1994-98 water years } \\
\hline & & & $\begin{array}{c}\text { Period of } \\
\text { record } \\
\text { (water } \\
\text { years) }\end{array}$ & Water year & $\begin{array}{l}\text { Stage } \\
(\mathrm{ft})\end{array}$ & $\begin{array}{l}\text { Discharge } \\
\left(\mathrm{ft}^{3} / \mathrm{s}\right)\end{array}$ & $\begin{array}{c}\text { Date } \\
\text { (month/ } \\
\text { day/ } \\
\text { year) }\end{array}$ & $\begin{array}{l}\text { Stage } \\
(\mathrm{ft})\end{array}$ & $\begin{array}{l}\text { Discharge } \\
\left(\mathrm{ft}^{3} / \mathrm{s}\right)\end{array}$ & $\begin{array}{c}\text { Regulated } \\
\text { during } \\
\text { flood }^{1}\end{array}$ & $\begin{array}{c}\text { Recurrence } \\
\text { interval } \\
\text { (years) }\end{array}$ \\
\hline 11333500 & $\begin{array}{l}\text { North Fork Cosumnes River near } \\
\text { El Dorado, CA }\end{array}$ & 205 & $\begin{array}{l}1912-41, \\
1949-87, \\
1997\end{array}$ & 1997 & 21.40 & 42,000 & $1 / 2 / 97$ & 21.40 & 42,000 & $\mathrm{Y}$ & $>100$ \\
\hline 11335000 & $\begin{array}{l}\text { Cosumnes River at Michigan Bar, } \\
\text { CA }\end{array}$ & 536 & 1907-98 & 1997 & 18.54 & 93,000 & $1 / 2 / 97$ & 18.54 & 93,000 & $\mathrm{~N}$ & $>100$ \\
\hline 11342000 & Sacramento River at Delta, CA & 425 & $1945-98$ & 1974 & 27.20 & 69,800 & $\begin{array}{l}1 / 9 / 95 \\
1 / 1 / 97\end{array}$ & $\begin{array}{l}20.16 \\
25.21\end{array}$ & $\begin{array}{l}43,100 \\
62,300\end{array}$ & $\begin{array}{l}\mathrm{N} \\
\mathrm{N}\end{array}$ & $\begin{array}{l}15-25 \\
50-75\end{array}$ \\
\hline 11345500 & $\begin{array}{l}\text { South Fork Pit River near Likely, } \\
\text { CA }\end{array}$ & 247 & 1932-98 & 1971 & 6.05 & 1,620 & $6 / 12 / 98$ & 5.22 & 1,050 & $\mathrm{~N}$ & $15-25$ \\
\hline 11348500 & Pit River near Canby, CA & 1,431 & $\begin{array}{l}\text { 1904, } \\
1929-98\end{array}$ & 1904 & 15.00 & 13,000 & $1 / 2 / 97$ & 11.82 & 7,280 & $\mathrm{~N}$ & $10-20$ \\
\hline 11355500 & Hat Creek near Hat Creek, CA & 162 & $\begin{array}{l}1926-94 \\
1997\end{array}$ & 1938 & 7.75 & 3,320 & $1 / 3 / 97$ & 7.41 & 1,460 & $\mathrm{~N}$ & $20-25$ \\
\hline 11363000 & Pit River at Big Bend, CA & 4,710 & $1911-98$ & $\begin{array}{l}1970 \\
1986\end{array}$ & $\begin{array}{l}18.17 \\
18.70\end{array}$ & $\begin{array}{l}49,000 \\
43,800\end{array}$ & $1 / 4 / 97$ & 18.36 & 41,200 & $\mathrm{~N}$ & $25-30$ \\
\hline 11367500 & McCloud River near McCloud, CA & 358 & $1932-98$ & 1997 & 11.22 & 15,400 & $1 / 1 / 97$ & 11.22 & 15,400 & $\mathrm{~N}$ & $50-75$ \\
\hline 11367800 & $\begin{array}{l}\text { McCloud River at Ah-Di-Na near } \\
\text { McCloud, CA }\end{array}$ & 427 & $\begin{array}{l}1956, \\
1965-98\end{array}$ & 1997 & 14.77 & 31,700 & $1 / 1 / 97$ & 14.77 & 31,700 & $\mathrm{~N}$ & $50-75$ \\
\hline 11368000 & $\begin{array}{l}\text { McCloud River above Shasta Lake, } \\
\text { CA }\end{array}$ & 604 & 1946-98 & 1997 & 29.00 & 51,300 & $1 / 1 / 97$ & 29.00 & 51,300 & $\mathrm{~N}$ & $50-75$ \\
\hline 11370500 & Sacramento River at Keswick, CA & 6,468 & 1939-98 & $\begin{array}{l}1940 \\
1997\end{array}$ & -- & $\begin{array}{r}186,000 \\
79,200\end{array}$ & $1 / 4 / 97$ & 32.71 & 79,200 & $\mathrm{Y}$ & $20-25$ \\
\hline 11371000 & Clear Creek at French Gulch, CA & 115 & $\begin{array}{l}\text { 1951-93, } \\
1997\end{array}$ & 1974 & 14.99 & 14,600 & $1 / 2 / 97$ & 14.67 & 11,500 & $\mathrm{~N}$ & $40-50$ \\
\hline 11372000 & Clear Creek near Igo, CA & 228 & $1941-98$ & 1956 & 13.75 & 24,500 & $1 / 1 / 97$ & 12.11 & 15,900 & $\mathrm{Y}$ & $40-50$ \\
\hline
\end{tabular}


$\left[\mathrm{mi}^{2}\right.$, square miles; $\mathrm{ft}$, feet above an arbitrary datum; $\mathrm{ft}^{3} / \mathrm{s}$, cubic feet per second; --, not determined or not applicable; $>$, greater than. Source: Recurrence intervals calculated from U.S. Geological Survey data. Other data from U.S. Geological Survey reports or databases]

\begin{tabular}{|c|c|c|c|c|c|c|c|c|c|c|c|}
\hline \multirow{2}{*}{$\begin{array}{l}\text { Streamgage } \\
\text { number } \\
\text { (fig. 17) }\end{array}$} & \multirow[b]{2}{*}{ Streamgage name } & \multirow{2}{*}{$\begin{array}{l}\text { Total } \\
\text { drainage } \\
\left(\mathrm{mi}^{2}\right)\end{array}$} & \multicolumn{4}{|c|}{$\begin{array}{c}\text { Maximum stage and discharge for period of record } \\
\text { through } 1998 \text { water year }\end{array}$} & \multicolumn{5}{|c|}{ Significant floods $1994-98$ water years } \\
\hline & & & $\begin{array}{l}\text { Period of } \\
\text { record } \\
\text { (water } \\
\text { years) }\end{array}$ & Water year & $\begin{array}{l}\text { Stage } \\
(\mathrm{ft})\end{array}$ & $\begin{array}{l}\text { Discharge } \\
\left(\mathrm{ft}^{3} / \mathrm{s}\right)\end{array}$ & $\begin{array}{l}\text { Date } \\
\text { (month/ } \\
\text { day/ } \\
\text { year) }\end{array}$ & $\begin{array}{l}\text { Stage } \\
(\mathrm{ft})\end{array}$ & $\begin{array}{l}\text { Discharge } \\
\left(\mathrm{ft}^{3} / \mathrm{s}\right)\end{array}$ & $\begin{array}{l}\text { Regulated } \\
\text { during } \\
\text { flood }^{1}\end{array}$ & $\begin{array}{l}\text { Recurrence } \\
\text { interval } \\
\text { (years) }\end{array}$ \\
\hline 11376550 & $\begin{array}{l}\text { Battle Creek below Coleman Fish } \\
\text { Hatchery near Cottonwood, CA }\end{array}$ & 357 & $\begin{array}{l}1962-96 \\
1998\end{array}$ & 1970 & 14.75 & 24,300 & $4 / 29 / 95$ & 13.49 & 20,600 & $\mathrm{~N}$ & $20-30$ \\
\hline 11381500 & Mill Creek near Los Molinos, CA & 131 & 1929-98 & 1938 & 23.40 & 36,400 & $1 / 1 / 97$ & 17.10 & 20,600 & $\mathrm{~N}$ & $40-50$ \\
\hline 11383500 & Deer Creek near Vina, CA & 208 & $\begin{array}{l}1912-15 \\
1921-98\end{array}$ & $\begin{array}{l}1997 \\
1938\end{array}$ & $\begin{array}{l}15.56 \\
19.20\end{array}$ & $\begin{array}{l}24,000 \\
23,800\end{array}$ & $1 / 1 / 97$ & 15.56 & 24,000 & $\mathrm{~N}$ & $35-40$ \\
\hline 11384000 & Big Chico Creek near Chico, CA & 72.4 & $\begin{array}{l}1931-86 \\
1997\end{array}$ & 1997 & 15.67 & 13,100 & $1 / 1 / 97$ & 15.67 & 13,100 & $\mathrm{~N}$ & $>100$ \\
\hline 11389500 & Sacramento River at Colusa, CA & 12,090 & 1941-98 & $\begin{array}{l}1983 \\
1942\end{array}$ & $\begin{array}{l}68.50 \\
69.20\end{array}$ & $\begin{array}{l}51,800 \\
49,000\end{array}$ & $2 / 5 / 98$ & 68.02 & 50,300 & $\mathrm{Y}$ & $20-25$ \\
\hline 11390000 & Butte Creek near Chico, CA & 147 & $1931-98$ & 1997 & 15.06 & 35,600 & $1 / 1 / 97$ & 15.06 & 35,600 & $\mathrm{~N}$ & $50-75$ \\
\hline 11390500 & $\begin{array}{l}\text { Sacramento River below Wilkins } \\
\text { Slough near Grimes, CA }\end{array}$ & 12,920 & 1939-98 & $\begin{array}{l}1986 \\
1940\end{array}$ & $\begin{array}{l}52.50 \\
52.75\end{array}$ & $\begin{array}{l}32,700 \\
27,000\end{array}$ & $1 / 4 / 97$ & 52.68 & 31,600 & $\mathrm{Y}$ & $20-25$ \\
\hline 11395030 & $\begin{array}{l}\text { South Fork Feather River below } \\
\text { Little Grass Valley Dam, CA }\end{array}$ & 25.9 & $\begin{array}{l}1928-33 \\
1961-98\end{array}$ & 1997 & 14.80 & 7,370 & $1 / 1 / 97$ & 14.80 & 7,370 & $\mathrm{Y}$ & $75-100$ \\
\hline 11395200 & $\begin{array}{l}\text { South Fork Feather River below } \\
\text { diversion dam near Strawberry } \\
\text { Valley, CA }\end{array}$ & 37.7 & 1961-98 & $\begin{array}{l}1997 \\
1986\end{array}$ & -- & $\begin{array}{r}11,300 \\
8,870\end{array}$ & $1 / 1 / 97$ & -- & 11,300 & $\mathrm{Y}$ & $75-100$ \\
\hline 11396000 & Lost Creek near Clipper Mills, CA & 30.0 & $\begin{array}{l}1928-41 \\
1949-98\end{array}$ & 1997 & 13.50 & 5,760 & $1 / 1 / 97$ & 13.50 & 5,760 & $\mathrm{Y}$ & $>100$ \\
\hline 11396200 & $\begin{array}{l}\text { South Fork Feather River below } \\
\text { Forbestown Dam, CA }\end{array}$ & 87.5 & $1963-98$ & 1997 & 17.64 & 21,800 & $1 / 1 / 97$ & 17.64 & 21,800 & $\mathrm{Y}$ & $>75$ \\
\hline 11400500 & $\begin{array}{l}\text { Butt Creek below Almanor-Butt } \\
\text { Creek Tunnel near Prattville, CA }\end{array}$ & 69.3 & $\begin{array}{l}1937-59 \\
1965-98\end{array}$ & 1997 & 6.22 & 4,080 & $1 / 1 / 97$ & 6.22 & 4,080 & $\mathrm{~N}$ & $50-75$ \\
\hline 11401112 & $\begin{array}{l}\text { North Fork Feather River below } \\
\text { Beldon Dam, CA }\end{array}$ & 612 & 1970-98 & 1997 & 9.17 & 3,460 & $1 / 1 / 97$ & 9.17 & 3,460 & $\mathrm{Y}$ & $20-30$ \\
\hline
\end{tabular}


Table 6. Maximum stage and discharge for period of record for streamgages having significant floods during 1994-98 water years in California.-Continued

$\left[\mathrm{mi}^{2}\right.$, square miles; $\mathrm{ft}$, feet above an arbitrary datum; $\mathrm{ft}^{3} / \mathrm{s}$, cubic feet per second; --, not determined or not applicable; >, greater than. Source: Recurrence intervals calculated from U.S. Geological Survey data. Other data from U.S. Geological Survey reports or databases]

\begin{tabular}{|c|c|c|c|c|c|c|c|c|c|c|c|}
\hline \multirow[b]{2}{*}{$\begin{array}{c}\text { Streamgage } \\
\text { number } \\
\text { (fig. 17) }\end{array}$} & \multirow[b]{2}{*}{ Streamgage name } & \multirow{2}{*}{$\begin{array}{c}\text { Total } \\
\text { drainage } \\
\left(\mathrm{mi}^{2}\right)\end{array}$} & \multicolumn{4}{|c|}{$\begin{array}{c}\text { Maximum stage and discharge for period of record } \\
\text { through } 1998 \text { water year }\end{array}$} & \multicolumn{5}{|c|}{ Significant floods $1994-98$ water years } \\
\hline & & & $\begin{array}{c}\text { Period of } \\
\text { record } \\
\text { (water } \\
\text { years) }\end{array}$ & Water year & $\begin{array}{l}\text { Stage } \\
(\mathrm{ft})\end{array}$ & $\begin{array}{l}\text { Discharge } \\
\left(\mathrm{ft}^{3} / \mathrm{s}\right)\end{array}$ & $\begin{array}{c}\text { Date } \\
\text { (month/ } \\
\text { day/ } \\
\text { year) }\end{array}$ & $\begin{array}{l}\text { Stage } \\
(\mathrm{ft})\end{array}$ & $\begin{array}{l}\text { Discharge } \\
\left(\mathrm{ft}^{3} / \mathrm{s}\right)\end{array}$ & $\begin{array}{c}\text { Regulated } \\
\text { during } \\
\text { flood }^{1}\end{array}$ & $\begin{array}{c}\text { Recurrence } \\
\text { interval } \\
\text { (years) }\end{array}$ \\
\hline 11401500 & $\begin{array}{l}\text { Indian Creek near Crescent Mills, } \\
\text { CA }\end{array}$ & 739 & $\begin{array}{l}\text { 1906-08, } \\
\text { 1912-17, } \\
1931-93, \\
1997\end{array}$ & 1997 & 21.60 & 40,000 & $1 / 1 / 97$ & 21.60 & 40,000 & $\mathrm{~N}$ & $50-75$ \\
\hline 11402000 & $\begin{array}{l}\text { Spanish Creek above Blackhawk } \\
\text { Creek at Keddie, CA }\end{array}$ & 184 & 1934-98 & 1997 & 15.68 & 22,100 & $1 / 2 / 97$ & 15.68 & 22,100 & $\mathrm{~N}$ & $>100$ \\
\hline 11403000 & $\begin{array}{l}\text { East Branch of North Fork Feather } \\
\text { River near Rich Bar, CA }\end{array}$ & 1,025 & $\begin{array}{l}1951-61, \\
1965-82, \\
1997\end{array}$ & $\begin{array}{l}1997 \\
1965\end{array}$ & -- & $\begin{array}{l}88,800 \\
48,300\end{array}$ & $1 / 2 / 97$ & -- & 88,800 & $\mathrm{~N}$ & $>100$ \\
\hline 11403200 & $\begin{array}{l}\text { North Fork Feather River below } \\
\text { Rock Creek Diversion Dam, CA }\end{array}$ & 1,773 & 1986-98 & 1997 & 31.85 & 91,600 & $1 / 2 / 97$ & 31.85 & 91,600 & $\mathrm{Y}$ & $50-75$ \\
\hline 11404300 & $\begin{array}{l}\text { Grizzly Creek below diversion } \\
\text { dam, CA }\end{array}$ & 14.4 & 1986-98 & $\begin{array}{l}1997 \\
1986\end{array}$ & $\begin{array}{l}7.33 \\
9.54\end{array}$ & $\begin{array}{l}6,300 \\
5,870\end{array}$ & $1 / 1 / 97$ & 7.33 & 6,300 & $\mathrm{Y}$ & $>100$ \\
\hline 11404330 & $\begin{array}{l}\text { North Fork Feather River below } \\
\text { Grizzly Creek, CA }\end{array}$ & 1,914 & 1986-98 & 1997 & 29.97 & 115,000 & $1 / 1 / 97$ & 29.97 & 115,000 & $\mathrm{Y}$ & $>100$ \\
\hline 11404500 & $\begin{array}{l}\text { North Fork Feather River at Pulga, } \\
\text { CA }\end{array}$ & 1,953 & $1912-98$ & 1997 & 41.65 & 105,400 & $1 / 1 / 97$ & 41.65 & 105,400 & $\mathrm{Y}$ & $75-100$ \\
\hline 11406920 & $\begin{array}{l}\text { Thermalito Afterbay Release to } \\
\text { Feather River, CA }\end{array}$ & -- & $\begin{array}{l}1986-89 \\
1991-98\end{array}$ & 1997 & 11.45 & 21,600 & $1 / 2 / 97$ & 11.45 & 21,600 & $\mathrm{Y}$ & -- \\
\hline 11407150 & Feather River near Gridley, CA & 3,676 & $\begin{array}{l}1956, \\
1965-98\end{array}$ & $\begin{array}{l}1997 \\
1965\end{array}$ & $\begin{array}{l}100.42 \\
100.43\end{array}$ & $\begin{array}{l}163,000 \\
151,000\end{array}$ & $1 / 2 / 97$ & 100.42 & 163,000 & $\mathrm{Y}$ & $25-50$ \\
\hline 11407500 & $\begin{array}{l}\text { South Honcut Creek near Bangor, } \\
\text { CA }\end{array}$ & 30.6 & $\begin{array}{l}1951-86, \\
1997\end{array}$ & 1965 & 19.25 & 17,600 & $1 / 1 / 97$ & 15.40 & 12,500 & $\mathrm{~N}$ & $75-100$ \\
\hline 11408550 & $\begin{array}{l}\text { Middle Yuba River below Milton } \\
\text { Dam, CA }\end{array}$ & 39.9 & $\begin{array}{l}1926-34, \\
1936-53, \\
1955-64, \\
1988-98\end{array}$ & $\begin{array}{l}1963 \\
1997\end{array}$ & $\begin{array}{r}5.25 \\
17.10\end{array}$ & $\begin{array}{r}10,200 \\
8,610\end{array}$ & $1 / 2 / 97$ & 17.10 & 8,610 & $\mathrm{Y}$ & $25-50$ \\
\hline
\end{tabular}


$\left[\mathrm{mi}^{2}\right.$, square miles; ft, feet above an arbitrary datum; $\mathrm{ft}^{3} / \mathrm{s}$, cubic feet per second; --, not determined or not applicable; >, greater than. Source: Recurrence intervals calculated from U.S. Geological Survey data. Other data from U.S. Geological Survey reports or databases]

\begin{tabular}{|c|c|c|c|c|c|c|c|c|c|c|c|}
\hline \multirow{2}{*}{$\begin{array}{l}\text { Streamgage } \\
\text { number } \\
\text { (fig. 17) }\end{array}$} & \multirow[b]{2}{*}{ Streamgage name } & \multirow{2}{*}{$\begin{array}{c}\text { Total } \\
\text { drainage } \\
\left(\mathrm{mi}^{2}\right)\end{array}$} & \multicolumn{4}{|c|}{$\begin{array}{c}\text { Maximum stage and discharge for period of record } \\
\text { through } 1998 \text { water year }\end{array}$} & \multicolumn{5}{|c|}{ Significant floods $1994-98$ water years } \\
\hline & & & $\begin{array}{l}\text { Period of } \\
\text { record } \\
\text { (water } \\
\text { years) }\end{array}$ & Water year & $\begin{array}{l}\text { Stage } \\
(\mathrm{ft})\end{array}$ & $\begin{array}{l}\text { Discharge } \\
\left(\mathrm{ft}^{3} / \mathrm{s}\right)\end{array}$ & $\begin{array}{c}\text { Date } \\
\text { (month/ } \\
\text { day/ } \\
\text { year) }\end{array}$ & $\begin{array}{l}\text { Stage } \\
(\mathrm{ft})\end{array}$ & $\begin{array}{l}\text { Discharge } \\
\left(\mathrm{ft}^{3} / \mathrm{s}\right)\end{array}$ & $\begin{array}{l}\text { Regulated } \\
\text { during } \\
\text { flood }^{1}\end{array}$ & $\begin{array}{c}\text { Recurrence } \\
\text { interval } \\
\text { (years) }\end{array}$ \\
\hline 11408880 & $\begin{array}{l}\text { Middle Yuba River below Our } \\
\text { House Dam, CA }\end{array}$ & 145 & 1969-98 & 1997 & 30.70 & 27,500 & $1 / 2 / 97$ & 30.70 & 27,500 & $\mathrm{Y}$ & $25-50$ \\
\hline 11409300 & Oregon Creek at Camptonville, CA & 23.0 & 1968-98 & $\begin{array}{l}1997 \\
1986\end{array}$ & $\begin{array}{l}11.31 \\
11.56\end{array}$ & $\begin{array}{l}5,170 \\
4,550\end{array}$ & $1 / 1 / 97$ & 11.31 & 5,170 & $\mathrm{~N}$ & $25-50$ \\
\hline 11413000 & $\begin{array}{l}\text { North Yuba River below Goodyear } \\
\text { Bar, CA }\end{array}$ & 250 & $1931-98$ & 1997 & 25.65 & 45,500 & $1 / 2 / 97$ & 25.65 & 45,500 & $\mathrm{~N}$ & $50-75$ \\
\hline 11413300 & $\begin{array}{l}\text { Slate Creek below diversion dam } \\
\text { near Strawberry, CA }\end{array}$ & 49.4 & $1961-98$ & 1997 & 17.20 & 17,300 & $1 / 1 / 97$ & 17.20 & 17,300 & $\mathrm{Y}$ & $50-75$ \\
\hline 11414100 & $\begin{array}{l}\text { Fordyce Creek below Fordyce } \\
\text { Dam near Cisco, CA }\end{array}$ & 31.7 & 1967-98 & 1974 & 7.90 & 4,660 & $5 / 16 / 96$ & 7.86 & 4,590 & $\mathrm{Y}$ & $25-50$ \\
\hline 11414250 & $\begin{array}{l}\text { South Yuba River at Langs } \\
\text { Crossing near Emigrant Gap, CA }\end{array}$ & 120 & 1966-98 & 1997 & 23.60 & 34,200 & $\begin{array}{l}5 / 16 / 96 \\
1 / 1 / 97\end{array}$ & $\begin{array}{l}20.99 \\
23.60\end{array}$ & $\begin{array}{l}24,000 \\
34,200\end{array}$ & $\begin{array}{l}\mathrm{Y} \\
\mathrm{Y}\end{array}$ & $\begin{array}{l}10-15 \\
20-30\end{array}$ \\
\hline 11416500 & $\begin{array}{l}\text { Canyon Creek below Bowman } \\
\text { Lake, CA }\end{array}$ & 28.3 & 1927-98 & 1997 & 13.01 & 5,500 & $\begin{array}{l}5 / 18 / 96 \\
1 / 2 / 97\end{array}$ & $\begin{array}{r}8.51 \\
13.01\end{array}$ & $\begin{array}{l}3,150 \\
5,500\end{array}$ & $\begin{array}{l}\mathrm{Y} \\
\mathrm{Y}\end{array}$ & $\begin{array}{l}15-25 \\
50-75\end{array}$ \\
\hline 11417500 & $\begin{array}{l}\text { South Yuba River at Jones Bar } \\
\text { near Grass Valley, CA }\end{array}$ & 308 & $\begin{array}{l}1941-48, \\
1956, \\
1960-98\end{array}$ & $\begin{array}{l}1965 \\
1956\end{array}$ & $\begin{array}{l}25.00 \\
28.70\end{array}$ & $\begin{array}{r}53,600 \\
--\end{array}$ & $1 / 1 / 97$ & 24.25 & 49,700 & $\mathrm{Y}$ & $50-100$ \\
\hline 11421000 & Yuba River near Marysville, CA & 1,339 & $\begin{array}{l}1943-44 \\
1946-98\end{array}$ & $\begin{array}{l}1965 \\
1997\end{array}$ & $\begin{array}{l}90.15 \\
91.64\end{array}$ & $\begin{array}{l}180,000 \\
151,000\end{array}$ & $1 / 2 / 97$ & 91.64 & 151,000 & $\mathrm{Y}$ & $25-50$ \\
\hline 11421770 & $\begin{array}{l}\text { Bear River below Drum Afterbay } \\
\text { near Blue Canyon, CA }\end{array}$ & 12.3 & $\begin{array}{l}\text { 1967-96, } \\
1998\end{array}$ & 1982 & 4.64 & 7,530 & $2 / 5 / 96$ & 3.90 & 3,660 & $\mathrm{Y}$ & -- \\
\hline 11421790 & $\begin{array}{l}\text { Bear River below Dutch Flat } \\
\text { Afterbay near Dutch Flat, CA }\end{array}$ & 21.5 & $1966-98$ & $\begin{array}{l}1986 \\
1995\end{array}$ & $-\overline{-}$ & $\begin{array}{l}4,240 \\
2,090\end{array}$ & $1 / 1 / 97$ & -- & 4,070 & $\mathrm{Y}$ & $25-50$ \\
\hline
\end{tabular}


Table 6. Maximum stage and discharge for period of record for streamgages having significant floods during 1994-98 water years in California.-Continued

$\left[\mathrm{mi}^{2}\right.$, square miles; $\mathrm{ft}$, feet above an arbitrary datum; $\mathrm{ft}^{3} / \mathrm{s}$, cubic feet per second; --, not determined or not applicable; >, greater than. Source: Recurrence intervals calculated from U.S. Geological Survey data. Other data from U.S. Geological Survey reports or databases]

\begin{tabular}{|c|c|c|c|c|c|c|c|c|c|c|c|}
\hline \multirow{2}{*}{$\begin{array}{l}\text { Streamgage } \\
\text { number } \\
\text { (fig. 17) }\end{array}$} & \multirow[b]{2}{*}{ Streamgage name } & \multirow{2}{*}{$\begin{array}{l}\text { Total } \\
\text { drainage } \\
\left(\mathrm{mi}^{2}\right)\end{array}$} & \multicolumn{4}{|c|}{$\begin{array}{c}\text { Maximum stage and discharge for period of record } \\
\text { through } 1998 \text { water year }\end{array}$} & \multicolumn{5}{|c|}{ Significant floods 1994-98 water years } \\
\hline & & & $\begin{array}{l}\text { Period of } \\
\text { record } \\
\text { (water } \\
\text { years) }\end{array}$ & Water year & $\begin{array}{l}\text { Stage } \\
\text { (ft) }\end{array}$ & $\begin{array}{l}\text { Discharge } \\
\left(\mathrm{ft}^{3} / \mathrm{s}\right)\end{array}$ & $\begin{array}{l}\text { Date } \\
\text { (month/ } \\
\text { day/ } \\
\text { year) }\end{array}$ & $\begin{array}{l}\text { Stage } \\
\text { (ft) }\end{array}$ & $\begin{array}{l}\text { Discharge } \\
\left(\mathrm{ft}^{3} / \mathrm{s}\right)\end{array}$ & $\begin{array}{l}\text { Regulated } \\
\text { during } \\
\text { flood }^{1}\end{array}$ & $\begin{array}{l}\text { Recurrence } \\
\text { interval } \\
\text { (years) }\end{array}$ \\
\hline 11422500 & $\begin{array}{l}\text { Bear River below Rollins Dam } \\
\text { near Colfax, CA }\end{array}$ & 105 & $\begin{array}{l}1912-13, \\
1916-17, \\
1950-53, \\
1964-94, \\
1996-98\end{array}$ & $\begin{array}{l}1997 \\
1986\end{array}$ & $\begin{array}{l}18.01 \\
20.62\end{array}$ & $\begin{array}{l}34,300 \\
22,500\end{array}$ & $1 / 2 / 97$ & 18.01 & 34,300 & $\mathrm{Y}$ & $>100$ \\
\hline 11424000 & Bear River near Wheatland, CA & 292 & 1929-98 & $\begin{array}{l}1986 \\
1997\end{array}$ & $\begin{array}{l}21.60 \\
23.72\end{array}$ & $\begin{array}{l}48,000 \\
34,900\end{array}$ & $1 / 2 / 97$ & 23.72 & 34,900 & $\mathrm{Y}$ & $25-50$ \\
\hline 11425500 & Sacramento River at Verona, CA & 21,251 & 1930-98 & $\begin{array}{l}1997 \\
1986\end{array}$ & $\begin{array}{l}42.09 \\
42.11\end{array}$ & $\begin{array}{l}94,000 \\
92,900\end{array}$ & $\begin{array}{l}3 / 13 / 95 \\
1 / 2 / 97\end{array}$ & $\begin{array}{l}37.95 \\
42.09\end{array}$ & $\begin{array}{l}81,000 \\
94,000\end{array}$ & $\begin{array}{l}\mathrm{Y} \\
\mathrm{Y}\end{array}$ & $\begin{array}{l}15-25 \\
25-50\end{array}$ \\
\hline 11427000 & $\begin{array}{l}\text { North Fork American River at } \\
\text { North Fork Dam, CA }\end{array}$ & 342 & $1942-98$ & 1965 & 11.87 & 65,400 & $1 / 1 / 97$ & 11.82 & 64,800 & $\mathrm{~N}$ & $25-50$ \\
\hline 11427500 & $\begin{array}{l}\text { Middle Fork American River at } \\
\text { French Meadows, CA }\end{array}$ & 47.9 & $1952-98$ & 1963 & 14.20 & 21,500 & $\begin{array}{l}5 / 16 / 96 \\
1 / 2 / 97\end{array}$ & $\begin{array}{l}11.61 \\
11.17\end{array}$ & $\begin{array}{l}6,050 \\
4,140\end{array}$ & $\begin{array}{l}\mathrm{Y} \\
\mathrm{Y}\end{array}$ & $\begin{array}{l}25-50 \\
15-25\end{array}$ \\
\hline 11427700 & $\begin{array}{l}\text { Duncan Creek near French } \\
\text { Meadows, CA }\end{array}$ & 9.94 & 1961-98 & $\begin{array}{l}1965 \\
1997\end{array}$ & $\begin{array}{l}10.60 \\
10.59\end{array}$ & $\begin{array}{l}3,650 \\
3,630\end{array}$ & $1 / 1 / 97$ & 10.59 & 3,630 & $\mathrm{~N}$ & $20-30$ \\
\hline 11427750 & $\begin{array}{l}\text { Duncan Creek below diversion } \\
\text { dam near French Meadows, CA }\end{array}$ & 10.5 & 1965-98 & $\begin{array}{l}1965 \\
1997\end{array}$ & 8.74 & 3,640 & $1 / 1 / 97$ & $\begin{array}{l}8.59 \\
8.59\end{array}$ & $\begin{array}{l}3,360 \\
3,360\end{array}$ & $\mathrm{Y}$ & $15-25$ \\
\hline 11427760 & $\begin{array}{l}\text { Middle Fork American River } \\
\text { above Middle Fork Power Plant } \\
\text { near Foresthill, CA }\end{array}$ & 87.8 & 1966-98 & 1997 & 14.60 & 13,900 & $1 / 2 / 97$ & 14.60 & 13,900 & $\mathrm{Y}$ & $50-75$ \\
\hline 11428800 & $\begin{array}{l}\text { Rubicon River below Hell Hole } \\
\text { Dam, CA }\end{array}$ & 114 & 1967-98 & 1997 & -- & 28,800 & $\begin{array}{l}5 / 16 / 96 \\
1 / 2 / 97\end{array}$ & -- & $\begin{array}{l}12,300 \\
28,000\end{array}$ & $\begin{array}{l}\mathrm{Y} \\
\mathrm{Y}\end{array}$ & $\begin{array}{l}10-20 \\
20-30\end{array}$ \\
\hline 11430000 & $\begin{array}{l}\text { South Fork Rubicon River below } \\
\text { Gerle Creek near Georgetown, } \\
\text { CA }\end{array}$ & 47.6 & $\begin{array}{l}\text { 1911-14, } \\
1962-98\end{array}$ & 1997 & 12.65 & 12,600 & $1 / 1 / 97$ & 12.65 & 12,600 & $\mathrm{Y}$ & $75-100$ \\
\hline 11431800 & $\begin{array}{r}\text { Pilot Creek above Stumpy } \\
\text { Meadows Reservoir, CA }\end{array}$ & 11.7 & 1961-98 & $\begin{array}{l}1986 \\
1963\end{array}$ & $\begin{array}{l}7.15 \\
8.05\end{array}$ & $\begin{array}{l}3,510 \\
2,070\end{array}$ & $1 / 2 / 97$ & 6.53 & 2,720 & $\mathrm{~N}$ & $20-30$ \\
\hline
\end{tabular}


$\left[\mathrm{mi}^{2}\right.$, square miles; $\mathrm{ft}$, feet above an arbitrary datum; $\mathrm{ft}^{3} / \mathrm{s}$, cubic feet per second; --, not determined or not applicable; $>$, greater than. Source: Recurrence intervals calculated from U.S. Geological Survey data. Other data from U.S. Geological Survey reports or databases]

\begin{tabular}{|c|c|c|c|c|c|c|c|c|c|c|c|}
\hline \multirow{2}{*}{$\begin{array}{c}\text { Streamgage } \\
\text { number } \\
\text { (fig. 17) }\end{array}$} & \multirow[b]{2}{*}{ Streamgage name } & \multirow{2}{*}{$\begin{array}{c}\text { Total } \\
\text { drainage } \\
\left(\mathrm{mi}^{2}\right)\end{array}$} & \multicolumn{4}{|c|}{$\begin{array}{c}\text { Maximum stage and discharge for period of record } \\
\text { through } 1998 \text { water year }\end{array}$} & \multicolumn{5}{|c|}{ Significant floods 1994-98 water years } \\
\hline & & & $\begin{array}{l}\text { Period of } \\
\text { record } \\
\text { (water } \\
\text { years) }\end{array}$ & Water year & $\begin{array}{l}\text { Stage } \\
(\mathrm{ft})\end{array}$ & $\begin{array}{l}\text { Discharge } \\
\left(\mathrm{ft}^{3} / \mathrm{s}\right)\end{array}$ & $\begin{array}{c}\text { Date } \\
\text { (month/ } \\
\text { day/ } \\
\text { year) }\end{array}$ & $\begin{array}{l}\text { Stage } \\
(\mathrm{ft})\end{array}$ & $\begin{array}{l}\text { Discharge } \\
\left(\mathrm{ft}^{3} / \mathrm{s}\right)\end{array}$ & $\begin{array}{l}\text { Regulated } \\
\text { during } \\
\text { flood }^{1}\end{array}$ & $\begin{array}{c}\text { Recurrence } \\
\text { interval } \\
\text { (years) }\end{array}$ \\
\hline 11433040 & $\begin{array}{l}\text { Pilot Creek below Mutton Canyon } \\
\text { near Georgetown, CA }\end{array}$ & 21.1 & 1962-98 & 1997 & 10.95 & 7,830 & $1 / 2 / 97$ & 10.95 & 7,830 & $\mathrm{Y}$ & $75-100$ \\
\hline 11433300 & $\begin{array}{l}\text { Middle Fork American River near } \\
\text { Foresthill, CA }\end{array}$ & 52.4 & 1959-98 & 1965 & 69.00 & 310,000 & $1 / 2 / 97$ & 29.56 & 123,000 & $\mathrm{Y}$ & $>100$ \\
\hline 11435100 & $\begin{array}{l}\text { Pyramid Creek at Twin Bridges, } \\
\text { CA }\end{array}$ & 8.76 & 1971-98 & 1997 & 7.22 & 2,920 & $1 / 2 / 97$ & 7.22 & 2,920 & $\mathrm{Y}$ & $>100$ \\
\hline 11436000 & $\begin{array}{l}\text { Silver Lake outlet near Kirkwood, } \\
\text { CA }\end{array}$ & 15.2 & 1923-98 & 1997 & 7.79 & 2,170 & $\begin{array}{l}5 / 17 / 96 \\
1 / 2 / 97\end{array}$ & $\begin{array}{l}5.52 \\
7.79\end{array}$ & $\begin{array}{r}892 \\
2,170\end{array}$ & $\begin{array}{l}\mathrm{Y} \\
\mathrm{Y}\end{array}$ & $\begin{array}{l}10-20 \\
>100\end{array}$ \\
\hline 11439500 & $\begin{array}{l}\text { South Fork American River near } \\
\text { Kyburz, CA }\end{array}$ & 193 & 1923-98 & 1997 & 14.26 & 25,000 & $1 / 2 / 97$ & 14.26 & 25,000 & $\mathrm{Y}$ & $>100$ \\
\hline 11441500 & $\begin{array}{l}\text { South Fork Silver Creek near Ice } \\
\text { House, CA }\end{array}$ & 27.5 & $1925-98$ & 1996 & 7.64 & 7,530 & $\begin{array}{l}5 / 16 / 96 \\
1 / 2 / 97\end{array}$ & $\begin{array}{l}7.64 \\
7.34\end{array}$ & $\begin{array}{l}7,530 \\
4,440\end{array}$ & $\begin{array}{l}\mathrm{Y} \\
\mathrm{Y}\end{array}$ & $\begin{array}{l}>100 \\
50-75\end{array}$ \\
\hline 11443500 & $\begin{array}{l}\text { South Fork American River near } \\
\text { Camino, CA }\end{array}$ & 493 & 1923-98 & 1997 & -- & 62,300 & $1 / 2 / 97$ & -- & 62,300 & $\mathrm{Y}$ & $>100$ \\
\hline 11444500 & $\begin{array}{l}\text { South Fork American River near } \\
\text { Placerville, CA }\end{array}$ & 598 & $\begin{array}{l}1912-20 \\
1965-98\end{array}$ & $\begin{array}{l}1997 \\
1914\end{array}$ & $-\overline{-}$ & $\begin{array}{l}71,000 \\
15,000\end{array}$ & $1 / 2 / 97$ & -- & 71,000 & $\mathrm{Y}$ & $>100$ \\
\hline 11445500 & $\begin{array}{l}\text { South Fork American River near } \\
\text { Lotus, CA }\end{array}$ & 673 & $\begin{array}{l}1951-95, \\
1997\end{array}$ & 1997 & 26.90 & 90,000 & $1 / 1 / 97$ & 26.90 & 90,000 & $\mathrm{Y}$ & $>100$ \\
\hline 11446500 & American River at Fair Oaks, CA & 1,888 & $\begin{array}{l}1862, \\
1905-17, \\
1919-98\end{array}$ & $\begin{array}{l}1951 \\
1862\end{array}$ & $\begin{array}{l}31.85 \\
38.00\end{array}$ & $\begin{array}{r}180,000 \\
--\end{array}$ & $1 / 2 / 97$ & 26.40 & 117,000 & $\mathrm{Y}$ & $15-25$ \\
\hline 11447360 & $\begin{array}{l}\text { Arcade Creek near Del Paso } \\
\text { Heights, CA }\end{array}$ & 31.4 & $\begin{array}{l}1964-78 \\
1996-98\end{array}$ & 1998 & 15.63 & 3,320 & $2 / 3 / 98$ & 15.63 & 3,320 & $\mathrm{~N}$ & $50-100$ \\
\hline 11447650 & Sacramento River at Freeport, CA & -- & $\begin{array}{l}\text { 1909, } \\
1949-98\end{array}$ & $\begin{array}{l}1986 \\
1951\end{array}$ & $\begin{array}{l}25.00 \\
30.14\end{array}$ & $\begin{array}{l}117,000 \\
104,000\end{array}$ & $1 / 3 / 97$ & 23.83 & 115,000 & $\mathrm{Y}$ & $50-100$ \\
\hline
\end{tabular}


Table 6. Maximum stage and discharge for period of record for streamgages having significant floods during 1994-98 water years in California.-Continued

$\left[\mathrm{mi}^{2}\right.$, square miles; $\mathrm{ft}$, feet above an arbitrary datum; $\mathrm{ft}^{3} / \mathrm{s}$, cubic feet per second; --, not determined or not applicable; >, greater than. Source: Recurrence intervals calculated from U.S. Geological Survey data. Other data from U.S. Geological Survey reports or databases]

\begin{tabular}{|c|c|c|c|c|c|c|c|c|c|c|c|}
\hline \multirow[b]{2}{*}{$\begin{array}{c}\text { Streamgage } \\
\text { number } \\
\text { (fig. 17) }\end{array}$} & \multirow[b]{2}{*}{ Streamgage name } & \multirow{2}{*}{$\begin{array}{c}\text { Total } \\
\text { drainage } \\
\left(\mathrm{mi}^{2}\right)\end{array}$} & \multicolumn{4}{|c|}{$\begin{array}{c}\text { Maximum stage and discharge for period of record } \\
\text { through } 1998 \text { water year }\end{array}$} & \multicolumn{5}{|c|}{ Significant floods 1994-98 water years } \\
\hline & & & $\begin{array}{c}\text { Period of } \\
\text { record } \\
\text { (water } \\
\text { years) }\end{array}$ & Water year & $\begin{array}{l}\text { Stage } \\
(\mathrm{ft})\end{array}$ & $\begin{array}{c}\text { Discharge } \\
\left(\mathrm{ft}^{3} / \mathrm{s}\right)\end{array}$ & $\begin{array}{c}\text { Date } \\
\text { (month/ } \\
\text { day/ } \\
\text { year) }\end{array}$ & $\begin{array}{c}\text { Stage } \\
(\mathrm{ft})\end{array}$ & $\begin{array}{c}\text { Discharge } \\
\left(\mathrm{ft}^{3} / \mathrm{s}\right)\end{array}$ & $\begin{array}{c}\text { Regulated } \\
\text { during } \\
\text { flood }^{1}\end{array}$ & $\begin{array}{c}\text { Recurrence } \\
\text { interval } \\
\text { (years) }\end{array}$ \\
\hline 11448500 & Adobe Creek near Kelseyville, CA & 6.36 & $\begin{array}{l}1955-78, \\
1997\end{array}$ & 1997 & 10.23 & 2,320 & $1 / 1 / 97$ & 10.23 & 2,320 & $\mathrm{~N}$ & $>100$ \\
\hline 11449500 & Kelsey Creek near Kelseyville, CA & 36.6 & $\begin{array}{l}1947-94 \\
1996-98\end{array}$ & 1995 & 13.80 & 8,600 & $\begin{array}{l}3 / 9 / 95 \\
1 / 1 / 97\end{array}$ & $\begin{array}{l}13.80 \\
13.72\end{array}$ & $\begin{array}{l}8,600 \\
8,450\end{array}$ & $\begin{array}{l}\mathrm{N} \\
\mathrm{N}\end{array}$ & $\begin{array}{l}20-30 \\
20-30\end{array}$ \\
\hline 11451000 & Cache Creek near Lower Lake, CA & 528 & $1945-98$ & 1998 & 11.01 & 10,200 & $\begin{array}{l}1 / 1 / 97 \\
2 / 17 / 98\end{array}$ & $\begin{array}{l}10.88 \\
11.01\end{array}$ & $\begin{array}{r}9,730 \\
10,200\end{array}$ & $\begin{array}{l}\mathrm{Y} \\
\mathrm{Y}\end{array}$ & $\begin{array}{l}25-50 \\
50-75\end{array}$ \\
\hline 11451100 & $\begin{array}{l}\text { North Fork Cache Creek at Hough } \\
\text { Spring near Clearlake Oaks, CA }\end{array}$ & 60.2 & $1 \mathrm{fc} 972-98$ & 1997 & 14.14 & 13,200 & $1 / 1 / 97$ & 14.14 & 13,200 & $\mathrm{~N}$ & $25-50$ \\
\hline 11451300 & $\begin{array}{l}\text { North Fork Cache Creek near } \\
\text { Clearlake Oaks, CA }\end{array}$ & 121 & $1986-98$ & $\begin{array}{l}1998 \\
1997\end{array}$ & $\begin{array}{l}10.61 \\
10.62\end{array}$ & $\begin{array}{l}7,950 \\
6,280\end{array}$ & $2 / 11 / 98$ & 10.61 & 7,950 & $\mathrm{Y}$ & $20-30$ \\
\hline 11452500 & Cache Creek at Yolo, CA & 1,139 & 1903-98 & $\begin{array}{l}1958 \\
1995\end{array}$ & $\begin{array}{l}85.35 \\
85.37\end{array}$ & $\begin{array}{l}41,400 \\
36,400\end{array}$ & $3 / 9 / 95$ & 85.37 & 36,400 & $\mathrm{Y}$ & $20-30$ \\
\hline 11453000 & Yolo Bypass near Woodland, CA & -- & $\begin{array}{l}\text { 1942, } \\
1951-97\end{array}$ & 1986 & 34.87 & 374,000 & $1 / 3 / 97$ & 34.84 & 357,000 & Y & $25-50$ \\
\hline 11458000 & Napa River near Napa, CA & 218 & $\begin{array}{l}1930-32 \\
1960-98\end{array}$ & $\begin{array}{l}1986 \\
1995\end{array}$ & $\begin{array}{l}30.20 \\
30.50\end{array}$ & $\begin{array}{l}37,100 \\
32,600\end{array}$ & $3 / 9 / 95$ & 30.50 & 32,600 & $\mathrm{Y}$ & $50-75$ \\
\hline 11459500 & Novato Creek at Novato, CA & 17.6 & 1947-98 & 1982 & 14.52 & 5,000 & $2 / 3 / 98$ & 13.70 & 3,380 & $\mathrm{Y}$ & $20-30$ \\
\hline 11460400 & $\begin{array}{l}\text { Lagunitas Creek at Samuel P. } \\
\text { Taylor State Park, CA }\end{array}$ & 34.3 & 1983-98 & 1998 & 10.00 & 5,830 & $2 / 3 / 98$ & 10.00 & 5,830 & $\mathrm{Y}$ & $50-75$ \\
\hline 11460750 & Walker Creek near Marshall, CA & 31.1 & $\begin{array}{l}\text { 1982, } \\
\text { 1984-98 }\end{array}$ & 1982 & 15.90 & 14,600 & $2 / 2 / 98$ & 14.21 & 10,500 & $\mathrm{Y}$ & $15-25$ \\
\hline 11463170 & $\begin{array}{l}\text { Big Sulphur Creek at Geysers } \\
\text { Resort near Cloverdale, CA }\end{array}$ & 13.1 & 1981-98 & 1997 & 9.78 & 8,010 & $1 / 1 / 97$ & 9.78 & 8,010 & $\mathrm{~N}$ & $>100$ \\
\hline 11464000 & $\begin{array}{l}\text { Russian River near Healdsburg, } \\
\text { CA }\end{array}$ & 793 & $\begin{array}{l}\text { 1938, } \\
1940-98\end{array}$ & $\begin{array}{l}1995 \\
1938\end{array}$ & $\begin{array}{l}26.23 \\
30.80\end{array}$ & $\begin{array}{r}73,000 \\
--\end{array}$ & $1 / 9 / 95$ & 26.23 & 73,000 & $\mathrm{Y}$ & $25-50$ \\
\hline
\end{tabular}


$\left[\mathrm{mi}^{2}\right.$, square miles; $\mathrm{ft}$, feet above an arbitrary datum; $\mathrm{ft}^{3} / \mathrm{s}$, cubic feet per second; --, not determined or not applicable; $>$, greater than. Source: Recurrence intervals calculated from U.S. Geological Survey data. Other data from U.S. Geological Survey reports or databases]

\begin{tabular}{|c|c|c|c|c|c|c|c|c|c|c|c|}
\hline \multirow{2}{*}{$\begin{array}{c}\text { Streamgage } \\
\text { number } \\
\text { (fig. 17) }\end{array}$} & \multirow[b]{2}{*}{ Streamgage name } & \multirow{2}{*}{$\begin{array}{c}\text { Total } \\
\text { drainage } \\
\left(\mathrm{mi}^{2}\right)\end{array}$} & \multicolumn{4}{|c|}{$\begin{array}{c}\text { Maximum stage and discharge for period of record } \\
\text { through } 1998 \text { water year }\end{array}$} & \multicolumn{5}{|c|}{ Significant floods $1994-98$ water years } \\
\hline & & & $\begin{array}{l}\text { Period of } \\
\text { record } \\
\text { (water } \\
\text { years) }\end{array}$ & Water year & $\begin{array}{l}\text { Stage } \\
(\mathrm{ft})\end{array}$ & $\begin{array}{l}\text { Discharge } \\
\left(\mathrm{ft}^{3} / \mathrm{s}\right)\end{array}$ & $\begin{array}{l}\text { Date } \\
\text { (month/ } \\
\text { day/ } \\
\text { year) }\end{array}$ & $\begin{array}{l}\text { Stage } \\
(\mathrm{ft})\end{array}$ & $\begin{array}{l}\text { Discharge } \\
\left(\mathrm{ft}^{3} / \mathrm{s}\right)\end{array}$ & $\begin{array}{l}\text { Regulated } \\
\text { during } \\
\text { flood }^{1}\end{array}$ & $\begin{array}{c}\text { Recurrence } \\
\text { interval } \\
\text { (years) }\end{array}$ \\
\hline 11465000 & $\begin{array}{l}\text { Dry Creek below Warm Springs } \\
\text { Dam near Geyserville, CA }\end{array}$ & 131 & $\begin{array}{l}1940-42 \\
1982-98\end{array}$ & $\begin{array}{l}1940 \\
1998\end{array}$ & $\begin{array}{l}-- \\
10.38\end{array}$ & $\begin{array}{r}22,500 \\
5,590\end{array}$ & $2 / 11 / 98$ & 10.38 & 5,590 & $\mathrm{Y}$ & $10-20$ \\
\hline 11465200 & Dry Creek near Geyserville, CA & 162 & $1960-98$ & 1963 & 17.50 & 32,400 & $1 / 8 / 95$ & 15.48 & 7,600 & $\mathrm{Y}$ & $5-10$ \\
\hline 11472200 & Outlet Creek near Longvale, CA & 161 & $\begin{array}{l}\text { 1957-94, } \\
1997\end{array}$ & 1965 & 30.60 & 77,900 & $1 / 1 / 97$ & 23.20 & 36,700 & $\mathrm{~N}$ & $25-50$ \\
\hline 11473900 & $\begin{array}{l}\text { Middle Fork Eel River near Dos } \\
\text { Rios, CA }\end{array}$ & 745 & 1966-98 & $\begin{array}{l}1997 \\
1966\end{array}$ & $\begin{array}{l}31.46 \\
32.86\end{array}$ & $\begin{array}{r}135,000 \\
70,200\end{array}$ & $1 / 1 / 97$ & 31.46 & 135,000 & $\mathrm{~N}$ & $20-30$ \\
\hline 11475560 & Elder Creek near Branscomb, CA & 6.50 & $\begin{array}{l}\text { 1965, } \\
\text { 1968-98 }\end{array}$ & 1965 & 11.41 & 3,660 & $12 / 30 / 96$ & 9.88 & 2,480 & $\mathrm{~N}$ & $20-30$ \\
\hline 11476600 & Bull Creek near Weott, CA & 28.1 & $1961-98$ & $\begin{array}{l}1997 \\
1965\end{array}$ & $\begin{array}{l}12.84 \\
20.61\end{array}$ & $\begin{array}{l}7,830 \\
6,520\end{array}$ & $12 / 31 / 96$ & 12.84 & 7,830 & $\mathrm{~N}$ & $25-35$ \\
\hline 11477000 & Eel River at Scotia, CA & 3,113 & $\begin{array}{l}1911-15 \\
1917-98\end{array}$ & 1965 & 72.00 & 752,000 & $1 / 9 / 95$ & 51.30 & 368,000 & $\mathrm{~N}$ & $10-20$ \\
\hline 11478500 & $\begin{array}{l}\text { Van Duzen River near Bridgeville, } \\
\text { CA }\end{array}$ & 222 & $1940-98$ & 1965 & 22.60 & 48,700 & $1 / 9 / 95$ & 19.72 & 43,700 & $\mathrm{~N}$ & $20-40$ \\
\hline 11481000 & Mad River near Arcata, CA & 485 & $\begin{array}{l}1911-13 \\
1951-98\end{array}$ & 1965 & 30.71 & 81,000 & $12 / 30 / 95$ & 24.17 & 54,700 & $\mathrm{Y}$ & $5-15$ \\
\hline 11516530 & $\begin{array}{l}\text { Klamath River below Iron Gate } \\
\text { Dam, CA }\end{array}$ & 4,630 & $1961-98$ & 1965 & 13.63 & 29,400 & $1 / 1 / 97$ & 13.08 & 20,500 & $\mathrm{Y}$ & $15-25$ \\
\hline 11517500 & Shasta River near Yreka, CA & 793 & $\begin{array}{l}1934-42 \\
1945-98\end{array}$ & $\begin{array}{l}1965 \\
1938\end{array}$ & $\begin{array}{l}12.92 \\
19.40\end{array}$ & $\begin{array}{r}21,500 \\
1,930\end{array}$ & $1 / 1 / 97$ & 12.27 & 10,900 & $\mathrm{~N}$ & $75-100$ \\
\hline 11520500 & $\begin{array}{l}\text { Klamath River near Seiad Valley, } \\
\text { CA }\end{array}$ & 6,940 & $\begin{array}{l}1913-25, \\
1927, \\
1952-98\end{array}$ & 1965 & 33.75 & 165,000 & $1 / 1 / 97$ & 28.72 & 117,000 & $\mathrm{~N}$ & $20-30$ \\
\hline 11522500 & Salmon River at Somes Bar, CA & 751 & $\begin{array}{l}1912, \\
1914-15, \\
1927-29, \\
1931-98\end{array}$ & 1965 & 43.41 & 133,000 & $1 / 1 / 97$ & 28.46 & 70,800 & $\mathrm{~N}$ & $40-60$ \\
\hline 11523000 & Klamath River at Orleans, CA & 8,475 & $\begin{array}{l}1927-29 \\
1931-98\end{array}$ & 1965 & 76.50 & 307,000 & $1 / 1 / 97$ & 37.79 & 258,000 & $\mathrm{~N}$ & $25-50$ \\
\hline
\end{tabular}




\section{Colorado}

Widespread frontal weather systems crossing Colorado repeatedly produced snow and rain in April and May 1995. Although some streams experienced flooding as a result of rain, the majority of flooding resulted from additions to the mountain snowpack and cool temperatures that delayed the snowmelt runoff until June. When normal summer temperatures occurred, the rapid snowmelt caused widespread flooding with many streamgages recording the peak of record (table 7).

Severe storms brought flooding rain, damaging winds, and large hail the evening of July 9, 1996. From 2 to 4.5 inches of rain fell in a short period of time on the east side of Pueblo (fig. 18), causing widespread flooding of low spots and roof collapses of three businesses (National Oceanic and Atmospheric Administration, 1996a).

Forest fires in Colorado can compound the hazards of flooding. On May 18, 1996, an intense forest fire burned several hundred acres of the Buffalo Creek watershed near the town of Buffalo Creek. On July 12, as much as 4.3 inches of rain fell over the burned area, resulting in a flood that killed two people and caused millions of dollars in damage due to flooding and the removal of massive amounts of sediment eroded from the burned area (National Oceanic and Atmospheric Administration, 1996b).

During the second week of June 1997 intense thunderstorms over the Rocky Mountains and eastward cause widespread flooding in the South Platte, Arkansas, and Colorado River Basins. Several streamgages recorded peaks of record as rainfall and snowmelt combined for record discharges (table 7).

More than 8 inches of rainfall were measured in southwestern Fort Collins during the evening hours of July 28, 1997 (National Oceanic and Atmospheric Administration, 1997a), as a series of storms developed, dumping excessive rain over the same area. A flash flood in Fort Collins killed 5 people and injured 40 others when a 10 - to 15 -foot wall of water surged through two mobile home parks. The wall of water destroyed 108 homes and damaged 481 others, 86 severely. Four women were killed while attempting to evacuate their homes and flee the floodwaters. Another died when she tried to go back into the park to rescue her pets. Floodwaters caused extensive damage at the Colorado State University campus in Fort Collins. Total estimated damage throughout the Fort Collins area was approximately \$190 million (National Oceanic and Atmospheric Administration, 1997b).

Estimated rainfall of 5 to 6 inches was reported north of Weldona during the evening hours of July 29, 1997. The resulting flooding reached a maximum between 10:00 p.m. and 12:00 a.m. when the Weldona Valley Ditch flowed out of its banks. The ensuing floodwaters, as much as 4 feet deep, inundated the entire town of Weldona. Approximately 50 families had to be evacuated, many from their rooftops. Although no dollar estimate was available, 48 homes and at least 30 vehicles were damaged. Several homes had to be condemned. In addition, \$2 million in damage was reported to roads, businesses, and public buildings (National Oceanic and Atmospheric Administration, 1997b).

Thunderstorms drenched the Pawnee National Grasslands, north of Stoneham, with 6 to 8 inches of rainfall July 30, 1997 (National Oceanic and Atmospheric Administration, 1997a). Runoff from the storms eventually turned Pawnee Creek into a huge river. The wall of water, 12 feet high and 200 feet wide, made its way downstream flooding the towns of Atwood and Sterling. At least 13,700 acres of cropland were damaged. Damage estimates of at least $\$ 12.9$ million were reported to property (National Oceanic and Atmospheric Administration, 1997b).

\section{References}

National Oceanic and Atmospheric Administration (NOAA), 1996a-97a, Climatological data (by State): Asheville, North Carolina, National Climatic Data Center, various months. National Oceanic and Atmospheric Administration (NOAA), 1996b-97b, Storm data (by State): Asheville, North Carolina, National Climatic Data Center, various months. 


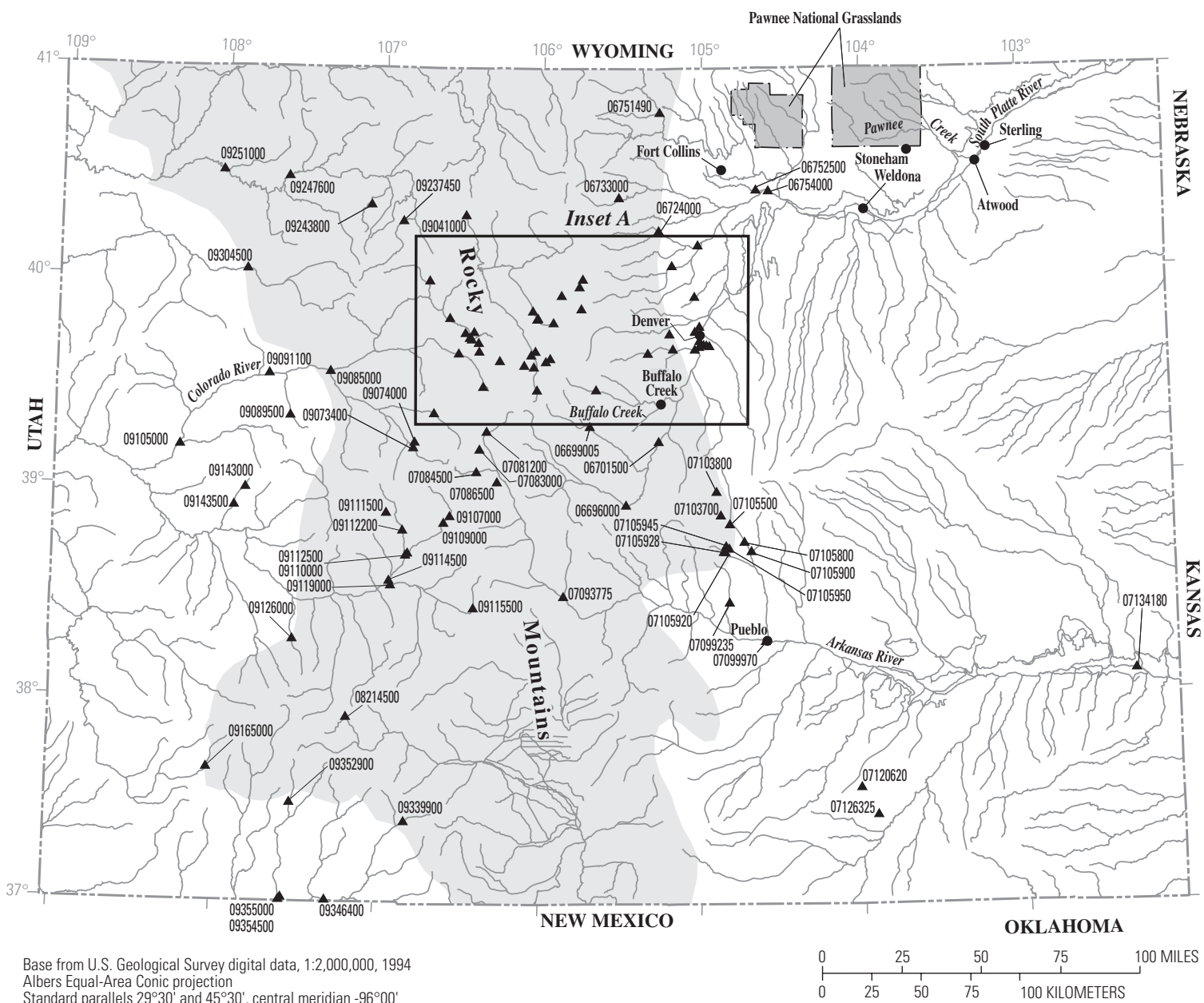

Standard parallels $29^{\circ} 30^{\prime}$ and $45^{\circ} 30^{\prime}$, central meridian $-96^{\circ} 00^{\prime}$

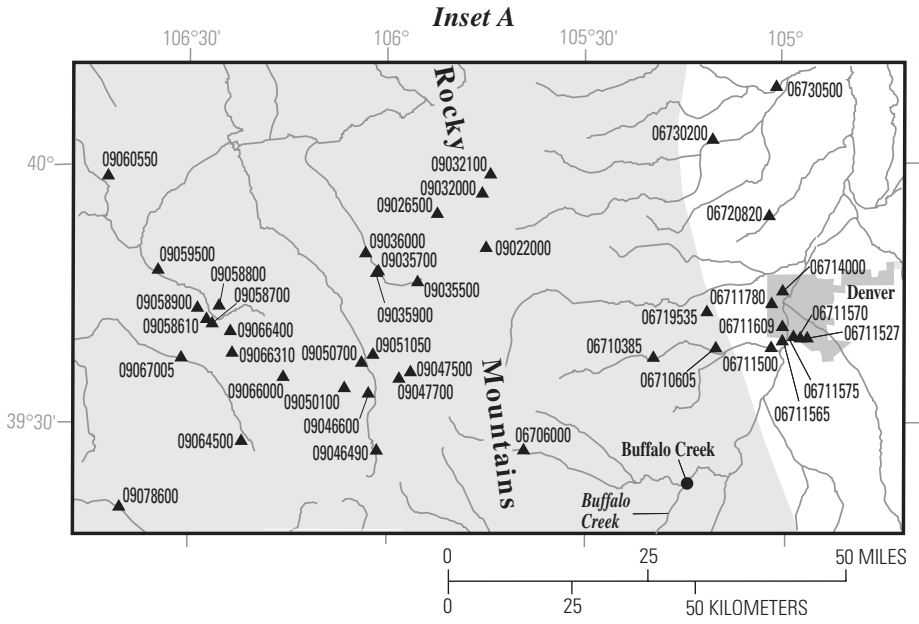

\section{EXPLANATION}

07126325 Streamgage and number

Figure 18. Location of streamgages with significant floods during 1994-98 water years for Colorado. 
Table 7. Maximum stage and discharge for period of record for streamgages having significant floods during 1994-98 water years in Colorado.

$\left[\mathrm{mi}^{2}\right.$, square miles; $\mathrm{ft}$, feet above an arbitrary datum; $\mathrm{ft}^{3} / \mathrm{s}$, cubic feet per second; --, not determined or not applicable; >, greater than. Source: Recurrence intervals calculated from U.S. Geological Survey data. Other data from U.S. Geological Survey reports or databases]

\begin{tabular}{|c|c|c|c|c|c|c|c|c|c|c|c|}
\hline \multirow{2}{*}{$\begin{array}{l}\text { Streamgage } \\
\text { number } \\
\text { (fig. 18) }\end{array}$} & \multirow[b]{2}{*}{ Streamgage name } & \multirow{2}{*}{$\begin{array}{l}\text { Total } \\
\text { drainage } \\
\left(\mathrm{mi}^{2}\right)\end{array}$} & \multicolumn{4}{|c|}{$\begin{array}{l}\text { Maximum stage and discharge for period of record } \\
\text { through } 1998 \text { water year }\end{array}$} & \multicolumn{5}{|c|}{ Significant floods $1994-98$ water years } \\
\hline & & & $\begin{array}{l}\text { Period of } \\
\text { record } \\
\text { (water } \\
\text { years) }\end{array}$ & Water year & $\begin{array}{l}\text { Stage } \\
\text { (ft) }\end{array}$ & $\begin{array}{l}\text { Discharge } \\
\left(\mathrm{ft}^{3} / \mathrm{s}\right)\end{array}$ & $\begin{array}{l}\text { Date } \\
\text { (month/ } \\
\text { day/ } \\
\text { year) }\end{array}$ & $\begin{array}{l}\text { Stage } \\
(\mathrm{ft})\end{array}$ & $\begin{array}{l}\text { Discharge } \\
\left(\mathrm{ft}^{3} / \mathrm{s}\right)\end{array}$ & $\begin{array}{l}\text { Regulated } \\
\text { during } \\
\text { flood }^{1}\end{array}$ & $\begin{array}{l}\text { Recurrence } \\
\text { interval } \\
\text { (years) }\end{array}$ \\
\hline 06696000 & $\begin{array}{l}\text { South Platte River near Lake } \\
\text { George, CO }\end{array}$ & 963 & $\begin{array}{l}1930-89 \\
1992-98\end{array}$ & 1970 & 8.34 & 3,000 & $7 / 4 / 95$ & 5.37 & 997 & $\mathrm{Y}$ & -- \\
\hline 06699005 & $\begin{array}{l}\text { Tarryall Creek below Rock Creek } \\
\text { near Jefferson, CO }\end{array}$ & 230 & $\begin{array}{l}1983-89 \\
1991-92 \\
1994-97\end{array}$ & $\begin{array}{l}1995 \\
1987\end{array}$ & $\begin{array}{l}6.18 \\
7.00\end{array}$ & $\begin{array}{l}850 \\
654\end{array}$ & $6 / 30 / 95$ & 6.18 & 850 & $\mathrm{~N}$ & 25 \\
\hline 06701500 & $\begin{array}{l}\text { South Platte River below } \\
\text { Cheesman Lake, CO }\end{array}$ & 1,752 & $1926-98$ & 1970 & 13.40 & 4,640 & $7 / 2 / 95$ & 8.60 & 2,310 & $\mathrm{Y}$ & -- \\
\hline 06706000 & $\begin{array}{l}\text { North Fork South Platte River } \\
\text { below Geneva Creek at Grant, } \\
\text { CO }\end{array}$ & 127 & $\begin{array}{l}1909-13 \\
1943-89 \\
1991-98\end{array}$ & $\begin{array}{l}1995 \\
1950\end{array}$ & $\begin{array}{l}2.48 \\
4.52\end{array}$ & $\begin{array}{r}1,160 \\
464\end{array}$ & $6 / 18 / 95$ & 2.48 & 1,160 & $\mathrm{Y}$ & 5 \\
\hline 06710385 & Bear Creek above Evergreen, CO & 104 & 1984-98 & $\begin{array}{l}1995 \\
1987\end{array}$ & $\begin{array}{l}5.39 \\
4.02\end{array}$ & $\begin{array}{l}573 \\
312\end{array}$ & $6 / 18 / 95$ & 5.39 & 573 & $\mathrm{~N}$ & 25 \\
\hline 06710605 & $\begin{array}{l}\text { Bear Creek above Bear Creek Lake } \\
\text { near Morrison, CO }\end{array}$ & 176 & $1986-98$ & 1995 & 6.45 & 841 & $6 / 9 / 95$ & 6.45 & 841 & $\mathrm{~N}$ & $5-10$ \\
\hline 06711500 & $\begin{array}{l}\text { Bear Creek at mouth at Sheridan, } \\
\text { CO }\end{array}$ & 260 & $\begin{array}{l}1927-89 \\
1991-98\end{array}$ & 1969 & 9.50 & 8,150 & $6 / 4 / 95$ & 7.50 & 3,550 & $\mathrm{Y}$ & -- \\
\hline 06711565 & $\begin{array}{l}\text { South Platte River at Englewood, } \\
\text { CO }\end{array}$ & 3,387 & $\begin{array}{l}1983-88 \\
1990-98\end{array}$ & 1995 & 7.21 & 9,710 & $6 / 4 / 95$ & 7.21 & 9,710 & $\mathrm{Y}$ & -- \\
\hline 06711570 & $\begin{array}{l}\text { Harvard Gulch at Colorado } \\
\text { Boulevard at Denver, CO }\end{array}$ & -- & $\begin{array}{l}1981-89 \\
1991 \\
1993-98\end{array}$ & $\begin{array}{l}1996 \\
1988\end{array}$ & $\begin{array}{l}13.34 \\
14.02\end{array}$ & $\begin{array}{l}673 \\
597\end{array}$ & 7/12/96 & 13.34 & 673 & $\mathrm{~N}$ & 25 \\
\hline 06711572 & $\begin{array}{l}\text { Harvard Gulch below University } \\
\text { Boulevard at Denver, CO }\end{array}$ & -- & $\begin{array}{l}1981-88 \\
1993-98\end{array}$ & 1996 & 14.55 & 981 & 7/12/96 & 14.55 & 981 & $\mathrm{~N}$ & $10-15$ \\
\hline 06711575 & $\begin{array}{l}\text { Harvard Gulch at Harvard Park at } \\
\text { Denver, CO }\end{array}$ & -- & $\begin{array}{l}\text { 1981-91, } \\
1993-98\end{array}$ & 1996 & 16.25 & 1,100 & 7/12/96 & 16.25 & 1,100 & $\mathrm{~N}$ & 25 \\
\hline
\end{tabular}


Table 7. Maximum stage and discharge for period of record for streamgages having significant floods during 1994-98 water years in Colorado.-Continued

$\left[\mathrm{mi}^{2}\right.$, square miles; $\mathrm{ft}$, feet above an arbitrary datum; $\mathrm{ft}^{3} / \mathrm{s}$, cubic feet per second; --, not determined or not applicable; >, greater than. Source: Recurrence intervals calculated from U.S. Geological Survey data. Other data from U.S. Geological Survey reports or databases]

\begin{tabular}{|c|c|c|c|c|c|c|c|c|c|c|c|}
\hline \multirow[b]{2}{*}{$\begin{array}{c}\text { Streamgage } \\
\text { number } \\
\text { (fig. 18) }\end{array}$} & \multirow[b]{2}{*}{ Streamgage name } & \multirow[b]{2}{*}{$\begin{array}{c}\text { Total } \\
\text { drainage } \\
\left(\mathrm{mi}^{2}\right)\end{array}$} & \multicolumn{4}{|c|}{$\begin{array}{c}\text { Maximum stage and discharge for period of record } \\
\text { through } 1998 \text { water year }\end{array}$} & \multicolumn{5}{|c|}{ Significant floods $1994-98$ water years } \\
\hline & & & $\begin{array}{l}\text { Period of } \\
\text { record } \\
\text { (water } \\
\text { years) }\end{array}$ & Water year & $\begin{array}{l}\text { Stage } \\
(\mathrm{ft})\end{array}$ & $\begin{array}{l}\text { Discharge } \\
\left(\mathrm{ft}^{3} / \mathrm{s}\right)\end{array}$ & $\begin{array}{c}\text { Date } \\
\text { (month/ } \\
\text { day/ } \\
\text { year) }\end{array}$ & $\begin{array}{l}\text { Stage } \\
(\mathrm{ft})\end{array}$ & $\begin{array}{l}\text { Discharge } \\
\left(\mathrm{ft}^{3} / \mathrm{s}\right)\end{array}$ & $\begin{array}{l}\text { Regulated } \\
\text { during } \\
\text { flood }^{1}\end{array}$ & $\begin{array}{c}\text { Recurrence } \\
\text { interval } \\
\text { (years) }\end{array}$ \\
\hline 06711609 & $\begin{array}{l}\text { Sanderson Gulch at Navajo Street } \\
\text { at Denver, CO }\end{array}$ & -- & $\begin{array}{l}\text { 1987-91, } \\
\text { 1993-98 }\end{array}$ & 1998 & 13.05 & 1,230 & $7 / 25 / 98$ & 13.05 & 1,230 & $\mathrm{~N}$ & $25-50$ \\
\hline 06711780 & Lakewood Gulch at Denver, CO & -- & $\begin{array}{l}\text { 1981-91, } \\
1997-96, \\
1998\end{array}$ & $\begin{array}{l}1998 \\
1984\end{array}$ & $\begin{array}{l}14.80 \\
17.24\end{array}$ & $\begin{array}{r}1,180 \\
930\end{array}$ & $8 / 19 / 98$ & 14.80 & 1,180 & $\mathrm{~N}$ & 25 \\
\hline 06714000 & South Platte River at Denver, CO & 3,861 & $\begin{array}{l}\text { 1895-1901, } \\
\text { 1903-06, } \\
1909-98\end{array}$ & 1965 & 15.00 & 40,300 & $7 / 25 / 98$ & 10.90 & 12,600 & $\mathrm{Y}$ & -- \\
\hline 06719535 & $\begin{array}{l}\text { Lena Gulch at upper site at Golden, } \\
\text { CO }\end{array}$ & -- & $\begin{array}{l}\text { 1986-91, } \\
1993-98\end{array}$ & $\begin{array}{l}1997 \\
1991\end{array}$ & $\begin{array}{l}10.93 \\
11.86\end{array}$ & $\begin{array}{l}396 \\
373\end{array}$ & $7 / 27 / 97$ & 10.93 & 396 & $\mathrm{~N}$ & 10 \\
\hline 06720820 & Big Dry Creek at Westminster, CO & 43.8 & $\begin{array}{l}\text { 1987-92, } \\
1994-95, \\
1997-98\end{array}$ & 1997 & 6.08 & 378 & $8 / 4 / 97$ & 6.08 & 378 & Y & -- \\
\hline 06724000 & St. Vrain Creek at Lyons, CO & 212 & $\begin{array}{l}\text { 1888-91, } \\
1895-1998\end{array}$ & $\begin{array}{l}1941 \\
1995\end{array}$ & $-\overline{7}+85$ & $\begin{array}{r}10,500 \\
4,200\end{array}$ & $5 / 29 / 95$ & 7.85 & 4,200 & $\mathrm{~N}$ & 50 \\
\hline 06730200 & $\begin{array}{l}\text { Boulder Creek at North 75th Street } \\
\text { near Boulder, CO }\end{array}$ & 304 & 1987-98 & 1995 & 7.85 & 1,950 & $5 / 17 / 95$ & 7.85 & 1,950 & $\mathrm{Y}$ & -- \\
\hline 06730500 & $\begin{array}{l}\text { Boulder Creek at mouth near } \\
\text { Longmont, } \mathrm{CO}\end{array}$ & 439 & $\begin{array}{l}1927-49, \\
1951-55, \\
1979-90, \\
1992-93, \\
1995-98\end{array}$ & 1938 & 6.94 & 4,410 & $5 / 17 / 95$ & 5.29 & 2,300 & $\mathrm{~N}$ & $10-25$ \\
\hline 06733000 & $\begin{array}{l}\text { Big Thompson River at Estes Park, } \\
\text { CO }\end{array}$ & 137 & 1947-98 & $\begin{array}{l}1982 \\
1965\end{array}$ & $-\overline{-}$ & $\begin{array}{l}5,500 \\
1,640\end{array}$ & $6 / 18 / 95$ & 6.80 & 1,870 & $\mathrm{~N}$ & $25-50$ \\
\hline 06751490 & $\begin{array}{l}\text { North Fork Cache La Poudre River } \\
\text { at Livermore, CO }\end{array}$ & 539 & $\begin{array}{l}\text { 1987-90, } \\
1992-98\end{array}$ & 1995 & 13.17 & 2,170 & $5 / 30 / 95$ & 13.17 & 2,170 & $\mathrm{~N}$ & $10-25$ \\
\hline
\end{tabular}


Table 7. Maximum stage and discharge for period of record for streamgages having significant floods during 1994-98 water years in Colorado.-Continued

$\left[\mathrm{mi}^{2}\right.$, square miles; $\mathrm{ft}$, feet above an arbitrary datum; $\mathrm{ft}^{3} / \mathrm{s}$, cubic feet per second; --, not determined or not applicable; >, greater than. Source: Recurrence intervals calculated from U.S. Geological Survey data. Other data from U.S. Geological Survey reports or databases]

\begin{tabular}{|c|c|c|c|c|c|c|c|c|c|c|c|}
\hline \multirow[b]{2}{*}{$\begin{array}{c}\text { Streamgage } \\
\text { number } \\
\text { (fig. 18) }\end{array}$} & \multirow[b]{2}{*}{ Streamgage name } & \multirow[b]{2}{*}{$\begin{array}{c}\text { Total } \\
\text { drainage } \\
\left(\mathrm{mi}^{2}\right)\end{array}$} & \multicolumn{4}{|c|}{$\begin{array}{c}\text { Maximum stage and discharge for period of record } \\
\text { through } 1998 \text { water year }\end{array}$} & \multicolumn{5}{|c|}{ Significant floods 1994-98 water years } \\
\hline & & & $\begin{array}{l}\text { Period of } \\
\text { record } \\
\text { (water } \\
\text { years) }\end{array}$ & Water year & $\begin{array}{l}\text { Stage } \\
(\mathrm{ft})\end{array}$ & $\begin{array}{l}\text { Discharge } \\
\left(\mathrm{ft}^{3} / \mathrm{s}\right)\end{array}$ & $\begin{array}{c}\text { Date } \\
\text { (month/ } \\
\text { day/ } \\
\text { year) }\end{array}$ & $\begin{array}{l}\text { Stage } \\
(\mathrm{ft})\end{array}$ & $\begin{array}{l}\text { Discharge } \\
\left(\mathrm{ft}^{3} / \mathrm{s}\right)\end{array}$ & $\begin{array}{l}\text { Regulated } \\
\text { during } \\
\text { flood }^{1}\end{array}$ & $\begin{array}{l}\text { Recurrence } \\
\text { interval } \\
\text { (years) }\end{array}$ \\
\hline 06752500 & $\begin{array}{l}\text { Cache La Poudre River near } \\
\text { Greeley, CO }\end{array}$ & 1,877 & $\begin{array}{l}1903, \\
1916-17, \\
1919, \\
1924-98\end{array}$ & 1983 & 8.92 & 6,360 & $6 / 19 / 95$ & 8.30 & 4,050 & $\mathrm{Y}$ & -- \\
\hline 06754000 & $\begin{array}{l}\text { South Platte River near Kersey, } \\
\text { CO }\end{array}$ & 9,598 & $\begin{array}{l}1902-03, \\
1905-12, \\
1914-98\end{array}$ & 1973 & 11.73 & 31,500 & $5 / 31 / 95$ & 11.00 & 22,900 & $\mathrm{Y}$ & -- \\
\hline 07081200 & Arkansas River near Leadville, CO & 98.8 & $\begin{array}{l}1968-83 \\
1990-98\end{array}$ & $\begin{array}{l}1997 \\
1978\end{array}$ & $\begin{array}{l}4.38 \\
4.47\end{array}$ & $\begin{array}{r}1,360 \\
894\end{array}$ & 6/9/97 & 4.38 & 1,360 & $\mathrm{Y}$ & -- \\
\hline 07083000 & Halfmoon Creek near Malta, CO & 23.6 & 1947-98 & $\begin{array}{l}1984 \\
1979\end{array}$ & $\begin{array}{l}3.77 \\
7.76\end{array}$ & $\begin{array}{l}615 \\
274\end{array}$ & $7 / 11 / 95$ & 3.82 & 546 & $\mathrm{~N}$ & 50 \\
\hline 07084500 & $\begin{array}{l}\text { Lake Creek above Twin Lakes } \\
\text { Reservoir, CO }\end{array}$ & 75.0 & $\begin{array}{l}1946-52, \\
1954-58, \\
1960-62, \\
1964-98\end{array}$ & $\begin{array}{l}1978 \\
1990\end{array}$ & $\begin{array}{l}5.08 \\
5.77\end{array}$ & $\begin{array}{l}3,270 \\
1,940\end{array}$ & $6 / 18 / 95$ & 5.06 & 2,900 & $\mathrm{Y}$ & -- \\
\hline 07086500 & $\begin{array}{l}\text { Clear Creek above Clear Creek } \\
\text { Reservoir, CO }\end{array}$ & 67.1 & $\begin{array}{l}1946-52 \\
1954-95\end{array}$ & 1995 & 5.29 & 1,300 & $7 / 12 / 95$ & 5.29 & 1,300 & $\mathrm{~N}$ & 50 \\
\hline 07093775 & $\begin{array}{l}\text { Badger Creek at lower station near } \\
\text { Howard, CO }\end{array}$ & 211 & 1981-98 & $\begin{array}{l}1996 \\
1984\end{array}$ & $\begin{array}{r}.73 \\
8.05\end{array}$ & $\begin{array}{l}2,990 \\
2,470\end{array}$ & $7 / 8 / 96$ & .73 & 2,990 & $\mathrm{~N}$ & $10-20$ \\
\hline 07099235 & Turkey Creek near Stone City, CO & 71.5 & $\begin{array}{l}1979-83, \\
1987-88, \\
1990, \\
1992-95, \\
1997-96, \\
1998\end{array}$ & 1995 & 6.29 & 83 & $5 / 30 / 95$ & 6.29 & 83 & $\mathrm{Y}$ & -- \\
\hline 07099970 & $\begin{array}{l}\text { Arkansas River at Moffat Street at } \\
\text { Pueblo, CO }\end{array}$ & 4,778 & 1989-98 & 1994 & 14.18 & 10,400 & $6 / 3 / 94$ & 14.18 & 10,400 & $\mathrm{~N}$ & 50 \\
\hline
\end{tabular}


$\left[\mathrm{mi}^{2}\right.$, square miles; $\mathrm{ft}$, feet above an arbitrary datum; $\mathrm{ft}^{3} / \mathrm{s}$, cubic feet per second; --, not determined or not applicable; >, greater than. Source: Recurrence intervals calculated from U.S. Geological Survey data. Other data from U.S. Geological Survey reports or databases]

\begin{tabular}{|c|c|c|c|c|c|c|c|c|c|c|c|}
\hline \multirow{2}{*}{$\begin{array}{l}\text { Streamgage } \\
\text { number } \\
\text { (fig. 18) }\end{array}$} & \multirow[b]{2}{*}{ Streamgage name } & \multirow{2}{*}{$\begin{array}{l}\text { Total } \\
\text { drainage } \\
\left(\mathrm{mi}^{2}\right)\end{array}$} & \multicolumn{4}{|c|}{$\begin{array}{c}\text { Maximum stage and discharge for period of record } \\
\text { through } 1998 \text { water year }\end{array}$} & \multicolumn{5}{|c|}{ Significant floods 1994-98 water years } \\
\hline & & & $\begin{array}{l}\text { Period of } \\
\text { record } \\
\text { (water } \\
\text { years) }\end{array}$ & Water year & $\begin{array}{l}\text { Stage } \\
(\mathrm{ft})\end{array}$ & $\begin{array}{l}\text { Discharge } \\
\left(\mathrm{ft}^{3} / \mathrm{s}\right)\end{array}$ & $\begin{array}{l}\text { Date } \\
\text { (month/ } \\
\text { day/ } \\
\text { year) }\end{array}$ & $\begin{array}{l}\text { Stage } \\
(\mathrm{ft})\end{array}$ & $\begin{array}{l}\text { Discharge } \\
\left(\mathrm{ft}^{3} / \mathrm{s}\right)\end{array}$ & $\begin{array}{l}\text { Regulated } \\
\text { during } \\
\text { flood }^{1}\end{array}$ & $\begin{array}{l}\text { Recurrence } \\
\text { interval } \\
\text { (years) }\end{array}$ \\
\hline 07103700 & $\begin{array}{l}\text { Fountain Creek near Colorado } \\
\text { Springs, CO }\end{array}$ & 103 & $1958-98$ & 1964 & 5.27 & 2,630 & $10 / 3 / 94$ & 5.14 & 1,760 & $\mathrm{~N}$ & $10-25$ \\
\hline 07103800 & $\begin{array}{l}\text { West Monument Creek at Air } \\
\text { Force Academy, CO }\end{array}$ & 14.9 & 1970-98 & 1997 & 3.24 & 169 & $6 / 10 / 97$ & 3.24 & 169 & $\mathrm{Y}$ & -- \\
\hline 07105500 & $\begin{array}{l}\text { Fountain Creek at Colorado } \\
\text { Springs, CO }\end{array}$ & 392 & $1976-98$ & 1994 & 12.12 & 10,100 & $9 / 2 / 94$ & 12.12 & 10,100 & $\mathrm{~N}$ & 50 \\
\hline 07105800 & Fountain Creek at Security, CO & 495 & $\begin{array}{l}1965, \\
1967-98\end{array}$ & 1965 & 11.30 & 25,000 & $6 / 14 / 97$ & 10.08 & 10,600 & $\mathrm{~N}$ & 10 \\
\hline 07105900 & $\begin{array}{l}\text { Jimmy Camp Creek at Fountain, } \\
\text { CO }\end{array}$ & 65.6 & 1976-98 & 1994 & 9.51 & 4,810 & $6 / 3 / 94$ & 9.51 & 4,810 & $\mathrm{~N}$ & $10-25$ \\
\hline 07105920 & $\begin{array}{l}\text { Little Fountain Creek above } \\
\text { Keaton Reservoir near Fort } \\
\text { Carson, CO }\end{array}$ & 11.0 & $\begin{array}{l}1979-85 \\
1987-88, \\
1996-98\end{array}$ & 1997 & 4.19 & 914 & $6 / 9 / 97$ & 4.19 & 914 & $\mathrm{~N}$ & $25-50$ \\
\hline 07105928 & $\begin{array}{l}\text { Little Fountain Creek near Fort } \\
\text { Carson, CO }\end{array}$ & 11.8 & $\begin{array}{l}1979-89 \\
1996-98\end{array}$ & 1997 & 7.29 & 914 & 6/10/97 & 7.29 & 914 & $\mathrm{Y}$ & -- \\
\hline 07105945 & $\begin{array}{l}\text { Rock Creek above Fort Carson } \\
\text { Reservation, CO }\end{array}$ & 6.79 & 1979-98 & 1997 & 9.71 & 770 & $6 / 10 / 97$ & 9.71 & 770 & $\mathrm{~N}$ & 25 \\
\hline 07105950 & Rock Creek near Fort Carson, CO & 7.79 & $\begin{array}{l}1979-88 \\
1990-98\end{array}$ & 1997 & 7.28 & 770 & $6 / 10 / 97$ & 7.28 & 770 & $\mathrm{~N}$ & $25-50$ \\
\hline 07120620 & Big Arroyo near Thatcher, CO & -- & $\begin{array}{l}1983-92 \\
1995-98\end{array}$ & 1997 & 5.78 & 1,780 & $8 / 11 / 97$ & 5.78 & 1,780 & $\mathrm{~N}$ & 10 \\
\hline 07126325 & $\begin{array}{l}\text { Taylor Arroyo below Rock } \\
\text { Crossing near Thatcher, CO }\end{array}$ & 48.4 & $\begin{array}{l}1983-89 \\
1991-92 \\
1994-98\end{array}$ & 1998 & 13.71 & 9,090 & 9/30/98 & 13.71 & 9,090 & $\mathrm{~N}$ & $50-100$ \\
\hline 07134180 & Arkansas River near Granada, CO & 23,707 & 1982-98 & $\begin{array}{l}1998 \\
1996\end{array}$ & $\begin{array}{l}11.69 \\
12.38\end{array}$ & $\begin{array}{l}3,590 \\
3,190\end{array}$ & $8 / 1 / 98$ & 11.69 & 3,590 & $\mathrm{Y}$ & -- \\
\hline
\end{tabular}


Table 7. Maximum stage and discharge for period of record for streamgages having significant floods during 1994-98 water years in Colorado.-Continued

$\left[\mathrm{mi}^{2}\right.$, square miles; $\mathrm{ft}$, feet above an arbitrary datum; $\mathrm{ft}^{3} / \mathrm{s}$, cubic feet per second; --, not determined or not applicable; >, greater than. Source: Recurrence intervals calculated from U.S. Geological Survey data. Other data from U.S. Geological Survey reports or databases]

\begin{tabular}{|c|c|c|c|c|c|c|c|c|c|c|c|}
\hline \multirow[b]{2}{*}{$\begin{array}{c}\text { Streamgage } \\
\text { number } \\
\text { (fig. 18) }\end{array}$} & \multirow[b]{2}{*}{ Streamgage name } & \multirow[b]{2}{*}{$\begin{array}{c}\text { Total } \\
\text { drainage } \\
\left(\mathrm{mi}^{2}\right)\end{array}$} & \multicolumn{4}{|c|}{$\begin{array}{c}\text { Maximum stage and discharge for period of record } \\
\text { through } 1998 \text { water year }\end{array}$} & \multicolumn{5}{|c|}{ Significant floods 1994-98 water years } \\
\hline & & & $\begin{array}{c}\text { Period of } \\
\text { record } \\
\text { (water } \\
\text { years) }\end{array}$ & Water year & $\begin{array}{l}\text { Stage } \\
(\mathrm{ft})\end{array}$ & $\begin{array}{l}\text { Discharge } \\
\left(\mathrm{ft}^{3} / \mathrm{s}\right)\end{array}$ & $\begin{array}{c}\text { Date } \\
\text { (month/ } \\
\text { day/ } \\
\text { year) }\end{array}$ & $\begin{array}{l}\text { Stage } \\
(\mathrm{ft})\end{array}$ & $\begin{array}{l}\text { Discharge } \\
\left(\mathrm{ft}^{3} / \mathrm{s}\right)\end{array}$ & $\begin{array}{c}\text { Regulated } \\
\text { during } \\
\text { flood }^{1}\end{array}$ & $\begin{array}{c}\text { Recurrence } \\
\text { interval } \\
\text { (years) }\end{array}$ \\
\hline 08214500 & $\begin{array}{l}\text { North Clear Creek below } \\
\text { Continental Reservoir, CO }\end{array}$ & 51.7 & $\begin{array}{l}\text { 1929-81, } \\
\text { 1983-86, } \\
\text { 1988-91, } \\
1993-98\end{array}$ & $\begin{array}{l}1997 \\
1952\end{array}$ & $\begin{array}{l}3.43 \\
3.66\end{array}$ & $\begin{array}{l}514 \\
362\end{array}$ & $\begin{array}{l}6 / 18 / 95 \\
5 / 15 / 97\end{array}$ & $\begin{array}{l}2.81 \\
3.43\end{array}$ & $\begin{array}{l}380 \\
514\end{array}$ & $\begin{array}{l}\mathrm{Y} \\
\mathrm{Y}\end{array}$ & $\begin{array}{l}- \\
--\end{array}$ \\
\hline 09022000 & $\begin{array}{l}\text { Fraser River at upper station near } \\
\text { Winter Park, CO }\end{array}$ & 10.5 & $\begin{array}{l}\text { 1969-73, } \\
1985-86, \\
1988-90, \\
1992-98\end{array}$ & $\begin{array}{l}1997 \\
1972\end{array}$ & $\begin{array}{l}2.08 \\
2.15\end{array}$ & $\begin{array}{l}291 \\
181\end{array}$ & $6 / 8 / 97$ & 2.08 & 291 & $\mathrm{~N}$ & $>100$ \\
\hline 09026500 & St. Louis Creek near Fraser, CO & 32.9 & $1935-98$ & $\begin{array}{l}1995 \\
1952\end{array}$ & $\begin{array}{l}2.80 \\
2.89\end{array}$ & $\begin{array}{l}558 \\
470\end{array}$ & $\begin{array}{l}6 / 17 / 95 \\
6 / 21 / 96\end{array}$ & $\begin{array}{l}2.80 \\
2.72\end{array}$ & $\begin{array}{l}558 \\
499\end{array}$ & $\begin{array}{l}\mathrm{Y} \\
\mathrm{Y}\end{array}$ & -- \\
\hline 09032000 & Ranch Creek near Fraser, CO & 19.9 & $\begin{array}{l}1935-89 \\
1991-98\end{array}$ & 1997 & 6.71 & 548 & $6 / 4 / 97$ & 6.71 & 548 & $\mathrm{Y}$ & -- \\
\hline 09032100 & Cabin Creek near Fraser, CO & 4.87 & 1984-98 & $\begin{array}{l}1997 \\
1995\end{array}$ & $\begin{array}{l}2.38 \\
2.39\end{array}$ & $\begin{array}{l}162 \\
113\end{array}$ & $6 / 8 / 97$ & 2.38 & 162 & $\mathrm{Y}$ & -- \\
\hline 09035500 & $\begin{array}{l}\text { Williams Fork below Steelman } \\
\text { Creek, CO }\end{array}$ & 16.3 & $\begin{array}{l}1934-41 \\
1966-98\end{array}$ & $\begin{array}{l}1995 \\
1983\end{array}$ & $\begin{array}{l}5.64 \\
5.80\end{array}$ & $\begin{array}{l}516 \\
403\end{array}$ & $7 / 11 / 95$ & 5.64 & 516 & $\mathrm{Y}$ & -- \\
\hline 09035700 & $\begin{array}{l}\text { Williams Fork above Darling } \\
\text { Creek near Leal, CO }\end{array}$ & 35.0 & 1966-98 & $\begin{array}{l}1995 \\
1998\end{array}$ & $\begin{array}{l}6.94 \\
7.18\end{array}$ & $\begin{array}{l}751 \\
297\end{array}$ & $6 / 17 / 95$ & 6.94 & 751 & $\mathrm{Y}$ & -- \\
\hline 09035900 & $\begin{array}{l}\text { South Fork of Williams Fork near } \\
\text { Leal, CO }\end{array}$ & 27.3 & 1966-98 & 1995 & 4.17 & 574 & $6 / 17 / 95$ & 4.17 & 574 & $\mathrm{~N}$ & $50-100$ \\
\hline 09036000 & Williams Fork near Leal, CO & 89.5 & 1934-98 & $\begin{array}{l}1952 \\
1971\end{array}$ & $\begin{array}{l}4.23 \\
4.96\end{array}$ & $\begin{array}{l}1,720 \\
1,300\end{array}$ & $6 / 17 / 95$ & 4.29 & 1,510 & $\mathrm{~N}$ & 25 \\
\hline 09041000 & Muddy Creek near Kremmling, CO & 87.4 & $\begin{array}{l}1938-43 \\
1956-71 \\
1994-98\end{array}$ & 1996 & 8.54 & 1,120 & $5 / 18 / 96$ & 8.54 & 1,120 & $\mathrm{~N}$ & $50-100$ \\
\hline 09046490 & Blue River at Blue River, $\mathrm{CO}$ & 42.4 & 1984-98 & 1995 & 3.23 & 681 & $6 / 18 / 95$ & 3.23 & 681 & $\mathrm{Y}$ & -- \\
\hline
\end{tabular}


$\left[\mathrm{mi}^{2}\right.$, square miles; $\mathrm{ft}$, feet above an arbitrary datum; $\mathrm{ft}^{3} / \mathrm{s}$, cubic feet per second; --, not determined or not applicable; >, greater than. Source: Recurrence intervals calculated from U.S. Geological Survey data. Other data from U.S. Geological Survey reports or databases]

\begin{tabular}{|c|c|c|c|c|c|c|c|c|c|c|c|}
\hline \multirow{2}{*}{$\begin{array}{c}\text { Streamgage } \\
\text { number } \\
\text { (fig. 18) }\end{array}$} & \multirow[b]{2}{*}{ Streamgage name } & \multirow{2}{*}{$\begin{array}{c}\text { Total } \\
\text { drainage } \\
\left(\mathrm{mi}^{2}\right)\end{array}$} & \multicolumn{4}{|c|}{$\begin{array}{c}\text { Maximum stage and discharge for period of record } \\
\text { through } 1998 \text { water year }\end{array}$} & \multicolumn{5}{|c|}{ Significant floods $1994-98$ water years } \\
\hline & & & $\begin{array}{l}\text { Period of } \\
\text { record } \\
\text { (water } \\
\text { years) }\end{array}$ & Water year & $\begin{array}{l}\text { Stage } \\
(\mathrm{ft})\end{array}$ & $\begin{array}{l}\text { Discharge } \\
\left(\mathrm{ft}^{3} / \mathrm{s}\right)\end{array}$ & $\begin{array}{l}\text { Date } \\
\text { (month/ } \\
\text { day/ } \\
\text { year) }\end{array}$ & $\begin{array}{l}\text { Stage } \\
(\mathrm{ft})\end{array}$ & $\begin{array}{l}\text { Discharge } \\
\left(\mathrm{ft}^{3} / \mathrm{s}\right)\end{array}$ & $\begin{array}{l}\text { Regulated } \\
\text { during } \\
\text { flood }^{1}\end{array}$ & $\begin{array}{c}\text { Recurrence } \\
\text { interval } \\
\text { (years) }\end{array}$ \\
\hline 09046600 & Blue River near Dillon, CO & 121 & $1958-98$ & 1995 & 6.91 & 1,390 & $6 / 18 / 95$ & 6.91 & 1,390 & $\mathrm{Y}$ & -- \\
\hline 09047500 & Snake River near Montezuma, CO & 57.7 & $\begin{array}{l}1943-46 \\
1952-98\end{array}$ & $\begin{array}{l}1952 \\
1972\end{array}$ & $\begin{array}{l}3.51 \\
3.88\end{array}$ & $\begin{array}{r}1,250 \\
730\end{array}$ & $6 / 21 / 95$ & 3.71 & 1,110 & $\mathrm{~N}$ & 50 \\
\hline 09047700 & Keystone Gulch near Dillon, CO & 9.10 & $1958-98$ & 1995 & 3.47 & 311 & $\begin{array}{l}6 / 17 / 95 \\
5 / 25 / 96\end{array}$ & $\begin{array}{l}3.47 \\
3.13\end{array}$ & $\begin{array}{l}311 \\
130\end{array}$ & $\begin{array}{l}\mathrm{N} \\
\mathrm{N}\end{array}$ & $\begin{array}{c}>100 \\
25\end{array}$ \\
\hline 09050100 & $\begin{array}{l}\text { Tenmile Creek below North } \\
\text { Tenmile Creek at Frisco, CO }\end{array}$ & 93.3 & $1958-98$ & 1965 & 6.15 & 1,910 & $6 / 17 / 95$ & 5.14 & 1,760 & $\mathrm{~N}$ & 50 \\
\hline 09050700 & Blue River below Dillon, CO & 335 & 1960-98 & $\begin{array}{l}1984 \\
1983\end{array}$ & $\begin{array}{l}3.88 \\
3.95\end{array}$ & $\begin{array}{l}2,010 \\
1,990\end{array}$ & $7 / 14 / 95$ & 3.76 & 2,000 & $\mathrm{Y}$ & -- \\
\hline 09051050 & $\begin{array}{l}\text { Straight Creek below Laskey } \\
\text { Gulch near Dillon, CO }\end{array}$ & 18.3 & 1987-98 & 1995 & 5.78 & 416 & $6 / 17 / 95$ & 5.78 & 416 & $\mathrm{~N}$ & $50-100$ \\
\hline 09058610 & Dickson Creek near Vail, CO & 3.41 & 1972-98 & 1997 & 3.29 & 52 & $6 / 1 / 97$ & 3.29 & 52 & $\mathrm{~N}$ & 25 \\
\hline 09058700 & Freeman Creek near Minturn, CO & 2.94 & 1965-98 & $\begin{array}{l}1984 \\
1976\end{array}$ & $\begin{array}{l}2.21 \\
2.60\end{array}$ & $\begin{array}{l}82 \\
35\end{array}$ & $6 / 3 / 95$ & 2.30 & 58 & $\mathrm{~N}$ & 25 \\
\hline 09058800 & $\begin{array}{l}\text { East Meadow Creek near Minturn, } \\
\text { CO }\end{array}$ & 3.61 & $\begin{array}{l}1965-82, \\
1984-93, \\
1995-98\end{array}$ & $\begin{array}{l}1995 \\
1975\end{array}$ & $\begin{array}{l}1.86 \\
2.13\end{array}$ & $\begin{array}{r}107 \\
74\end{array}$ & $6 / 17 / 95$ & 1.86 & 107 & $\mathrm{~N}$ & $>100$ \\
\hline 09058900 & Moniger Creek near Minturn, CO & .76 & $\begin{array}{l}\text { 1966-74, } \\
1977-85, \\
1987-90, \\
1995-76, \\
1986, \\
1991-94, \\
1997-98\end{array}$ & $\begin{array}{l}1989 \\
1993\end{array}$ & $\begin{array}{l}2.05 \\
2.56\end{array}$ & $\begin{array}{r}29 \\
--\end{array}$ & $--/--/ 94$ & 1.90 & 19 & $\mathrm{~N}$ & 10 \\
\hline 09059500 & Piney River near State Bridge, CO & 86.2 & $\begin{array}{l}1945-83 \\
1986-98\end{array}$ & 1983 & 5.82 & 1,220 & $6 / 1 / 97$ & 5.63 & 1,190 & $\mathrm{~N}$ & 50 \\
\hline
\end{tabular}


Table 7. Maximum stage and discharge for period of record for streamgages having significant floods during 1994-98 water years in Colorado.-Continued

$\left[\mathrm{mi}^{2}\right.$, square miles; $\mathrm{ft}$, feet above an arbitrary datum; $\mathrm{ft}^{3} / \mathrm{s}$, cubic feet per second; --, not determined or not applicable; >, greater than. Source: Recurrence intervals calculated from U.S. Geological Survey data. Other data from U.S. Geological Survey reports or databases]

\begin{tabular}{|c|c|c|c|c|c|c|c|c|c|c|c|}
\hline \multirow[b]{2}{*}{$\begin{array}{c}\text { Streamgage } \\
\text { number } \\
\text { (fig. 18) }\end{array}$} & \multirow[b]{2}{*}{ Streamgage name } & \multirow[b]{2}{*}{$\begin{array}{c}\text { Total } \\
\text { drainage } \\
\left(\mathrm{mi}^{2}\right)\end{array}$} & \multicolumn{4}{|c|}{$\begin{array}{c}\text { Maximum stage and discharge for period of record } \\
\text { through } 1998 \text { water year }\end{array}$} & \multicolumn{5}{|c|}{ Significant floods $1994-98$ water years } \\
\hline & & & $\begin{array}{l}\text { Period of } \\
\text { record } \\
\text { (water } \\
\text { years) }\end{array}$ & Water year & $\begin{array}{l}\text { Stage } \\
(\mathrm{ft})\end{array}$ & $\begin{array}{l}\text { Discharge } \\
\left(\mathrm{ft}^{3} / \mathrm{s}\right)\end{array}$ & $\begin{array}{c}\text { Date } \\
\text { (month/ } \\
\text { day/ } \\
\text { year) }\end{array}$ & $\begin{array}{l}\text { Stage } \\
(\mathrm{ft})\end{array}$ & $\begin{array}{l}\text { Discharge } \\
\left(\mathrm{ft}^{3} / \mathrm{s}\right)\end{array}$ & $\begin{array}{l}\text { Regulated } \\
\text { during } \\
\text { flood }^{1}\end{array}$ & $\begin{array}{l}\text { Recurrence } \\
\text { interval } \\
\text { (years) }\end{array}$ \\
\hline 09060550 & Rock Creek at Crater, CO & 72.6 & $1985-98$ & 1996 & 4.67 & 746 & $5 / 18 / 96$ & 4.67 & 746 & $\mathrm{~N}$ & $25-50$ \\
\hline 09064500 & $\begin{array}{l}\text { Homestake Creek near Red Cliff, } \\
\text { CO }\end{array}$ & 58.2 & $\begin{array}{l}1911-14, \\
1916-18, \\
1944-96, \\
1998-97\end{array}$ & 1918 & 6.20 & 1,300 & $6 / 17 / 95$ & 3.82 & 843 & Y & -- \\
\hline 09066000 & $\begin{array}{l}\text { Black Gore Creek near Minturn, } \\
\text { CO }\end{array}$ & 12.6 & $\begin{array}{l}1948-56, \\
1964-86, \\
1988-98\end{array}$ & $\begin{array}{l}1995 \\
1952\end{array}$ & $\begin{array}{l}5.06 \\
5.42\end{array}$ & $\begin{array}{l}370 \\
365\end{array}$ & $6 / 17 / 95$ & 5.06 & 370 & $\mathrm{~N}$ & $25-50$ \\
\hline 09066310 & $\begin{array}{l}\text { Gore Creek lower station at Vail, } \\
\text { CO }\end{array}$ & 77.1 & 1989-98 & 1997 & 11.50 & 1,840 & $6 / 4 / 97$ & 11.50 & 1,840 & $\mathrm{~N}$ & $10-25$ \\
\hline 09066400 & $\begin{array}{l}\text { Red Sandstone Creek near } \\
\text { Minturn, CO }\end{array}$ & 7.32 & 1964-98 & $\begin{array}{l}1995 \\
1983\end{array}$ & $\begin{array}{l}4.58 \\
4.66\end{array}$ & $\begin{array}{l}223 \\
215\end{array}$ & $6 / 17 / 95$ & 4.58 & 223 & $\mathrm{~N}$ & 25 \\
\hline 09067005 & Eagle River at Avon, CO & 395 & 1989-98 & $\begin{array}{l}1997 \\
1993\end{array}$ & $\begin{array}{l}4.03 \\
5.14\end{array}$ & $\begin{array}{l}3,930 \\
3,860\end{array}$ & 6/6/97 & 4.03 & 3,930 & $\mathrm{~N}$ & 10 \\
\hline 09073400 & $\begin{array}{l}\text { Roaring Fork River near Aspen, } \\
\text { CO }\end{array}$ & 108 & $1965-98$ & 1995 & 5.97 & 2,230 & $7 / 11 / 95$ & 5.97 & 2,230 & $\mathrm{Y}$ & -- \\
\hline 09074000 & Hunter Creek near Aspen, CO & 41.1 & $\begin{array}{l}1950-56, \\
1970-87, \\
1989-98\end{array}$ & $\begin{array}{l}1985 \\
1953\end{array}$ & $\begin{array}{l}2.33 \\
7.02\end{array}$ & $\begin{array}{l}1,170 \\
1,010\end{array}$ & $6 / 17 / 95$ & 3.29 & 1,080 & $\mathrm{~N}$ & 25 \\
\hline 09078600 & $\begin{array}{l}\text { Fryingpan River near } \\
\text { Thomasville, CO }\end{array}$ & 134 & $1976-95$ & 1995 & 4.58 & 1,570 & $6 / 18 / 95$ & 4.58 & 1,570 & $\mathrm{Y}$ & -- \\
\hline 09085000 & $\begin{array}{l}\text { Roaring Fork River at Glenwood } \\
\text { Springs, CO }\end{array}$ & 1,451 & $\begin{array}{l}\text { 1906-09, } \\
\text { 1912, } \\
\text { 1914-81, } \\
1983-98\end{array}$ & $\begin{array}{l}1957 \\
1921\end{array}$ & $\begin{array}{l}8.64 \\
8.70\end{array}$ & $\begin{array}{l}19,000 \\
17,600\end{array}$ & $7 / 13 / 95$ & 8.31 & 13,000 & $\mathrm{Y}$ & -- \\
\hline 09089500 & $\begin{array}{l}\text { West Divide Creek near Raven, } \\
\text { CO }\end{array}$ & 64.6 & $1956-98$ & 1984 & 5.83 & 1,410 & $5 / 15 / 95$ & 4.62 & 912 & $\mathrm{~N}$ & $10-25$ \\
\hline
\end{tabular}


$\left[\mathrm{mi}^{2}\right.$, square miles; $\mathrm{ft}$, feet above an arbitrary datum; $\mathrm{ft}^{3} / \mathrm{s}$, cubic feet per second; --, not determined or not applicable; >, greater than. Source: Recurrence intervals calculated from U.S. Geological Survey data. Other data from U.S. Geological Survey reports or databases]

\begin{tabular}{|c|c|c|c|c|c|c|c|c|c|c|c|}
\hline \multirow{2}{*}{$\begin{array}{l}\text { Streamgage } \\
\text { number } \\
\text { (fig. 18) }\end{array}$} & \multirow[b]{2}{*}{ Streamgage name } & \multirow{2}{*}{$\begin{array}{c}\text { Total } \\
\text { drainage } \\
\left(\mathrm{mi}^{2}\right)\end{array}$} & \multicolumn{4}{|c|}{$\begin{array}{c}\text { Maximum stage and discharge for period of record } \\
\text { through } 1998 \text { water year }\end{array}$} & \multicolumn{5}{|c|}{ Significant floods $1994-98$ water years } \\
\hline & & & $\begin{array}{l}\text { Period of } \\
\text { record } \\
\text { (water } \\
\text { years) }\end{array}$ & Water year & $\begin{array}{l}\text { Stage } \\
(\mathrm{ft})\end{array}$ & $\begin{array}{l}\text { Discharge } \\
\left(\mathrm{ft}^{3} / \mathrm{s}\right)\end{array}$ & $\begin{array}{c}\text { Date } \\
\text { (month/ } \\
\text { day/ } \\
\text { year) }\end{array}$ & $\begin{array}{l}\text { Stage } \\
(\mathrm{ft})\end{array}$ & $\begin{array}{l}\text { Discharge } \\
\left(\mathrm{ft}^{3} / \mathrm{s}\right)\end{array}$ & $\begin{array}{l}\text { Regulated } \\
\text { during } \\
\text { flood }^{1}\end{array}$ & $\begin{array}{c}\text { Recurrence } \\
\text { interval } \\
\text { (years) }\end{array}$ \\
\hline 09091100 & Mamm Creek near Silt, CO & -- & $\begin{array}{l}\text { 1980-91, } \\
1993,1995\end{array}$ & 1995 & 13.47 & 595 & $5 / 29 / 95$ & 13.47 & 595 & $\mathrm{~N}$ & $25-50$ \\
\hline 09105000 & Plateau Creek near Cameo, CO & 592 & $\begin{array}{l}1936-83 \\
1986-98\end{array}$ & $\begin{array}{l}1983 \\
1995\end{array}$ & $\begin{array}{l}8.51 \\
8.73\end{array}$ & $\begin{array}{l}5,010 \\
4,900\end{array}$ & $6 / 16 / 95$ & 8.73 & 4,900 & $\mathrm{~N}$ & $25-50$ \\
\hline 09107000 & Taylor River at Taylor Park, CO & 128 & $\begin{array}{l}1930-34 \\
1988-98\end{array}$ & 1995 & 4.08 & 1,400 & $6 / 18 / 95$ & 4.08 & 1,400 & $\mathrm{~N}$ & $25-50$ \\
\hline 09109000 & $\begin{array}{l}\text { Taylor River below Taylor Park } \\
\text { Reservoir, CO }\end{array}$ & 254 & 1939-98 & 1957 & 7.56 & 2,270 & $7 / 14 / 95$ & 7.22 & 1,910 & $\mathrm{Y}$ & -- \\
\hline 09110000 & Taylor River at Almont, $\mathrm{CO}$ & 477 & $\begin{array}{l}\text { 1913, 1916, } \\
\text { 1918-87, } \\
1989-98\end{array}$ & $\begin{array}{l}1920 \\
1957\end{array}$ & $\begin{array}{l}5.00 \\
5.32\end{array}$ & $\begin{array}{l}3,760 \\
3,590\end{array}$ & $7 / 15 / 95$ & 4.92 & 2,530 & $\mathrm{Y}$ & -- \\
\hline 09111500 & Slate River near Crested Butte, CO & 68.9 & $\begin{array}{l}1940-51 \\
1994-98\end{array}$ & 1995 & 5.84 & 1,550 & $6 / 17 / 95$ & 5.84 & 1,550 & $\mathrm{~N}$ & 50 \\
\hline 09112200 & $\begin{array}{l}\text { East River below Cement Creek } \\
\text { near Crested Butte, } \mathrm{CO}\end{array}$ & 238 & $\begin{array}{l}1964-72, \\
1980-81, \\
1994-98\end{array}$ & $\begin{array}{l}1995 \\
1980\end{array}$ & $\begin{array}{l}5.06 \\
8.30\end{array}$ & $\begin{array}{l}4,350 \\
3,360\end{array}$ & $6 / 18 / 95$ & 5.06 & 4,350 & $\mathrm{~N}$ & $50-100$ \\
\hline 09112500 & East River at Almont, $\mathrm{CO}$ & 289 & $\begin{array}{l}\text { 1913, 1916, } \\
\text { 1918-21, } \\
1935-98\end{array}$ & $\begin{array}{l}1921 \\
1995\end{array}$ & $\begin{array}{l}6.60 \\
8.41\end{array}$ & $\begin{array}{l}6,500 \\
4,430\end{array}$ & $6 / 18 / 95$ & 8.41 & 4,430 & $\mathrm{~N}$ & $10-25$ \\
\hline 09114500 & $\begin{array}{l}\text { Gunnison River near Gunnison, } \\
\text { CO }\end{array}$ & 1,012 & $\begin{array}{l}1912-14, \\
1916-28, \\
1945-98\end{array}$ & $\begin{array}{l}1918 \\
1957\end{array}$ & $-\overline{-}+74$ & $\begin{array}{r}11,400 \\
9,320\end{array}$ & $6 / 18 / 95$ & 5.56 & 7,620 & $\mathrm{~N}$ & 25 \\
\hline 09115500 & Tomichi Creek at Sargents, CO & 149 & $\begin{array}{l}1917-22, \\
1938-72, \\
1993-98\end{array}$ & $\begin{array}{l}1995 \\
1917\end{array}$ & $\begin{array}{l}4.03 \\
4.05\end{array}$ & $\begin{array}{l}964 \\
662\end{array}$ & $6 / 18 / 95$ & 4.03 & 964 & $\mathrm{~N}$ & 50 \\
\hline 09119000 & Tomichi Creek at Gunnison, $\mathrm{CO}$ & 1,061 & $\begin{array}{l}1938-85 \\
1987-98\end{array}$ & 1984 & 5.49 & 4,620 & $6 / 18 / 95$ & 5.14 & 2,420 & $\mathrm{~N}$ & 25 \\
\hline
\end{tabular}


Table 7. Maximum stage and discharge for period of record for streamgages having significant floods during 1994-98 water years in Colorado.-Continued

$\left[\mathrm{mi}^{2}\right.$, square miles; $\mathrm{ft}$, feet above an arbitrary datum; $\mathrm{ft}^{3} / \mathrm{s}$, cubic feet per second; --, not determined or not applicable; >, greater than. Source: Recurrence intervals calculated from U.S. Geological Survey data. Other data from U.S. Geological Survey reports or databases]

\begin{tabular}{|c|c|c|c|c|c|c|c|c|c|c|c|}
\hline \multirow{2}{*}{$\begin{array}{l}\text { Streamgage } \\
\text { number } \\
\text { (fig. 18) }\end{array}$} & \multirow[b]{2}{*}{ Streamgage name } & \multirow{2}{*}{$\begin{array}{c}\text { Total } \\
\text { drainage } \\
\left(\mathrm{mi}^{2}\right)\end{array}$} & \multicolumn{4}{|c|}{$\begin{array}{c}\text { Maximum stage and discharge for period of record } \\
\text { through } 1998 \text { water year }\end{array}$} & \multicolumn{5}{|c|}{ Significant floods 1994-98 water years } \\
\hline & & & $\begin{array}{l}\text { Period of } \\
\text { record } \\
\text { (water } \\
\text { years) }\end{array}$ & Water year & $\begin{array}{l}\text { Stage } \\
(\mathrm{ft})\end{array}$ & $\begin{array}{l}\text { Discharge } \\
\left(\mathrm{ft}^{3} / \mathrm{s}\right)\end{array}$ & $\begin{array}{c}\text { Date } \\
\text { (month/ } \\
\text { day/ } \\
\text { year) }\end{array}$ & $\begin{array}{l}\text { Stage } \\
\text { (ft) }\end{array}$ & $\begin{array}{l}\text { Discharge } \\
\left(\mathrm{ft}^{3} / \mathrm{s}\right)\end{array}$ & $\begin{array}{l}\text { Regulated } \\
\text { during } \\
\text { flood }^{1}\end{array}$ & $\begin{array}{c}\text { Recurrence } \\
\text { interval } \\
\text { (years) }\end{array}$ \\
\hline 09126000 & Cimarron River near Cimarron, $\mathrm{CO}$ & 66.6 & $1955-98$ & 1957 & 8.32 & 1,790 & $6 / 5 / 97$ & 3.91 & 1,620 & $\mathrm{Y}$ & -- \\
\hline 09143000 & Surface Creek near Cedaredge, CO & 27.4 & $1940-98$ & $\begin{array}{l}1995 \\
1957\end{array}$ & $\begin{array}{l}3.79 \\
3.96\end{array}$ & $\begin{array}{l}892 \\
517\end{array}$ & $6 / 15 / 95$ & 3.79 & 892 & $\mathrm{~N}$ & $50-100$ \\
\hline 09143500 & Surface Creek at Cedaredge, CO & 39.0 & $\begin{array}{l}\text { 1917-76, } \\
1978-98\end{array}$ & $\begin{array}{l}1941 \\
1984\end{array}$ & $\begin{array}{l}2.50 \\
3.44\end{array}$ & $\begin{array}{r}1,190 \\
862\end{array}$ & $6 / 15 / 95$ & 3.06 & 764 & $\mathrm{~N}$ & $10-25$ \\
\hline 09165000 & Dolores River below Rico, $\mathrm{CO}$ & 105 & $1952-96$ & $\begin{array}{l}1984 \\
1952\end{array}$ & $\begin{array}{l}5.95 \\
6.15\end{array}$ & $\begin{array}{l}2,170 \\
2,120\end{array}$ & $6 / 17 / 95$ & 5.87 & 2,140 & $\mathrm{~N}$ & $10-25$ \\
\hline 09237450 & $\begin{array}{l}\text { Yampa River above Stagecoach } \\
\text { Reservoir, CO }\end{array}$ & 257 & 1989-98 & 1998 & 5.96 & 765 & $3 / 26 / 98$ & 5.96 & 765 & $\mathrm{~N}$ & $10-25$ \\
\hline 09243800 & Foidel Creek near Oak Creek, CO & 8.61 & $\begin{array}{l}1976-80, \\
1983, \\
1985-98\end{array}$ & 1996 & 4.47 & 65 & $5 / 5 / 96$ & 4.47 & 65 & $\mathrm{~N}$ & 10 \\
\hline 09247600 & Yampa River below Craig, CO & 1,750 & $1985-98$ & 1997 & 10.78 & 12,900 & $6 / 4 / 97$ & 10.78 & 12,900 & $\mathrm{~N}$ & 10 \\
\hline 09251000 & Yampa River near Maybell, CO & 3,410 & $\begin{array}{l}1904-05 \\
1916-98\end{array}$ & 1984 & 12.42 & 25,100 & $6 / 4 / 97$ & 11.18 & 18,800 & $\mathrm{~N}$ & 50 \\
\hline 09304500 & White River near Meeker, CO & 755 & $\begin{array}{l}1901 \\
1910-98\end{array}$ & $\begin{array}{l}1984 \\
1901\end{array}$ & $\begin{array}{l}6.12 \\
6.50\end{array}$ & $\begin{array}{l}6,950 \\
5,000\end{array}$ & $6 / 17 / 95$ & 5.61 & 5,280 & $\mathrm{~N}$ & $10-25$ \\
\hline 09339900 & $\begin{array}{l}\text { East Fork San Juan above Sand } \\
\text { Creek near Pagosa Springs, CO }\end{array}$ & 64.1 & $1957-96$ & 1970 & 6.75 & 2,260 & $6 / 17 / 95$ & 5.93 & 1,290 & $\mathrm{~N}$ & 10 \\
\hline 09346400 & San Juan River near Carracas, CO & 1,230 & $1962-98$ & 1970 & 8.34 & 9,730 & $3 / 6 / 95$ & 8.10 & 8,590 & $\mathrm{~N}$ & $10-25$ \\
\hline 09352900 & Vallecito Creek near Bayfield, CO & 72.1 & $\begin{array}{l}\text { 1963-92, } \\
1994-98\end{array}$ & 1970 & 6.51 & 7,050 & $6 / 15 / 95$ & 3.16 & 1,630 & $\mathrm{~N}$ & 5 \\
\hline 09354500 & Los Pinos River at La Boca, CO & 510 & $\begin{array}{l}1951-85 \\
1987-98\end{array}$ & 1957 & 8.95 & 6,400 & $3 / 6 / 95$ & 7.61 & 3,590 & $\mathrm{~N}$ & 25 \\
\hline 09355000 & Spring Creek at La Boca, CO & 58.0 & $1951-98$ & $\begin{array}{l}1970 \\
1995\end{array}$ & $\begin{array}{l}4.62 \\
5.28\end{array}$ & $\begin{array}{l}1,980 \\
1,740\end{array}$ & $3 / 6 / 95$ & 5.28 & 1,740 & $\mathrm{~N}$ & 50 \\
\hline
\end{tabular}

${ }^{1}$ Regulated during flood: $\mathrm{N}$, no; $\mathrm{Y}$, yes. 


\section{Connecticut}

The excessive rainfall, snowmelt, and ice jams on January 29, 1994, combined to produce floods and flash floods along small rivers and streams throughout Connecticut. The Yantic River (fig. 19) reached a flood stage of 8 feet at Norwich (National Oceanic and Atmospheric Administration, 1994) during the early evening of January 28 and continued to rise to 13 feet in the early morning hours of January 29. The Pomperaug River rose to 4 to 6 feet over bankfull in Southbury (National Oceanic and Atmospheric Administration, 1994) causing streets to be closed and a number of people to be evacuated from their homes. Property damage was reported. An ice jam against a bridge on the Shetucket River in Baltic (National Oceanic and Atmospheric Administration, 1994) resulted in a flash flood when water levels rose 6 feet in a matter of minutes, flooding at least 75 homes and threatening others. A 2.5-mile ice jam was reported on the Shetucket River.
Excessive rain of more than 6 inches on April 16, 1996, caused flooding on the Wepawaug River in Milford, the Quinnipiac River in Southington and Wallingford, the Farm River in East Haven and North Branford, the Muddy River, and Harbor Brook in Meriden (National Oceanic and Atmospheric Administration, 1996). MacKenzie Reservoir, at the headwaters of the Muddy River, overtopped its emergency spillway. Five hundred forty-seven homes and 28 businesses suffered damage. The total uninsured flood damage was about $\$ 1.5$ million (National Oceanic and Atmospheric Administration, 1996). Floods on streamgaged rivers are listed in table 8 .

\section{Reference}

National Oceanic and Atmospheric Administration (NOAA), 1994-96, Storm data (by State): Asheville, North Carolina, National Climatic Data Center, various months.

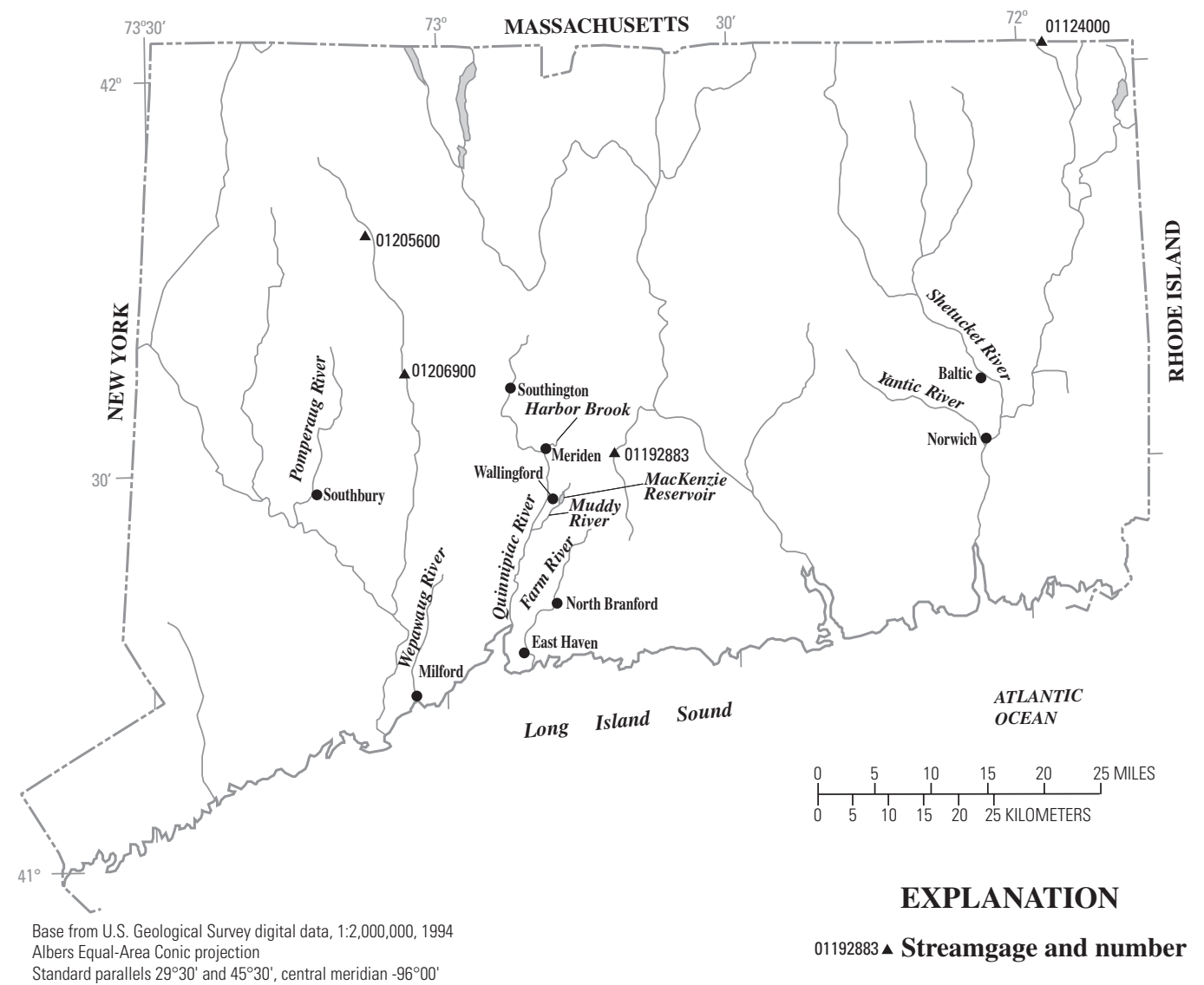

Figure 19. Location of streamgages with significant floods during 1994-98 water years for Connecticut. 
Table 8. Maximum stage and discharge for period of record for streamgages having significant floods during 1994-98 water years in Connecticut.

$\left[\mathrm{mi}^{2}\right.$, square miles; $\mathrm{ft}$, feet above an arbitrary datum; $\mathrm{ft}^{3} / \mathrm{s}$, cubic feet per second; --, not determined or not applicable. Source: Recurrence intervals calculated from U.S. Geological Survey data. Other data from U.S. Geological Survey reports or databases]

\begin{tabular}{|c|c|c|c|c|c|c|c|c|c|c|c|}
\hline \multirow[b]{2}{*}{$\begin{array}{l}\text { Streamgage } \\
\text { number } \\
\text { (fig. 19) }\end{array}$} & \multirow[b]{2}{*}{ Streamgage name } & \multirow[b]{2}{*}{$\begin{array}{c}\text { Total } \\
\text { drainage } \\
\left(\mathrm{mi}^{2}\right)\end{array}$} & \multicolumn{4}{|c|}{$\begin{array}{c}\text { Maximum stage and discharge for period of record } \\
\text { through } 1998 \text { water year }\end{array}$} & \multicolumn{5}{|c|}{ Significant floods $1994-98$ water years } \\
\hline & & & $\begin{array}{l}\text { Period of } \\
\text { record } \\
\text { (water } \\
\text { years) }\end{array}$ & Water year & $\begin{array}{l}\text { Stage } \\
\text { (ft) }\end{array}$ & $\begin{array}{c}\text { Discharge } \\
\left(\mathrm{ft}^{3} / \mathrm{s}\right)\end{array}$ & $\begin{array}{c}\text { Date } \\
\text { (month/ } \\
\text { day/ } \\
\text { year) }\end{array}$ & $\begin{array}{l}\text { Stage } \\
(\mathrm{ft})\end{array}$ & $\begin{array}{c}\text { Discharge } \\
\left(\mathrm{ft}^{3} / \mathrm{s}\right)\end{array}$ & $\begin{array}{c}\text { Regulated } \\
\text { during } \\
\text { flood }^{1}\end{array}$ & $\begin{array}{c}\text { Recurrence } \\
\text { interval } \\
\text { (years) }\end{array}$ \\
\hline 01124000 & Quinebaug River at Quinebaug, CT & 155 & $1932-98$ & 1955 & 18.96 & 49,300 & $3 / 9 / 98$ & 7.50 & 3,810 & $\mathrm{Y}$ & -- \\
\hline 01192883 & $\begin{array}{l}\text { Coginchaug River at Middlefield, } \\
\text { CT }\end{array}$ & 29.8 & $1981-98$ & 1996 & 12.46 & 2,260 & $4 / 16 / 96$ & 12.46 & 2,260 & $\mathrm{Y}$ & -- \\
\hline 01205600 & $\begin{array}{l}\text { West Branch Naugatuck River at } \\
\text { Torrington, CT }\end{array}$ & 33.8 & $\begin{array}{l}\text { 1955, } \\
1957-97\end{array}$ & 1955 & 16.70 & 16,500 & $1 / 27 / 96$ & 8.60 & 3,000 & $\mathrm{Y}$ & -- \\
\hline 01206900 & $\begin{array}{l}\text { Naugatuck River at Thomaston, } \\
\text { CT }\end{array}$ & 99.8 & $1955-98$ & 1955 & 27.00 & 53,400 & $1 / 29 / 94$ & 6.62 & 4,380 & $\mathrm{Y}$ & -- \\
\hline
\end{tabular}

${ }^{1}$ Regulated during flood: N, no; Y, yes. 


\section{Summary of Significant Floods in the United States and Puerto Rico, 1994 Through 1998 Water Years}

\section{Delaware}

An intensifying storm moving offshore late on March 2, 1994, became a true "nor'easter," hammering the Delaware coastline prior to high tide late in the day. For a 2-hour period late on March 2, the storm surge caused extensive beach erosion from South Bethany to Dewey Beach (fig. 20). At Dewey Beach, substantial flood damage was incurred to beachfront property after many dunes were breached by the heavy surf. Damage along the Atlantic Coast of Delaware was estimated at $\$ 500$ thousand to $\$ 1$ million (National Oceanic and Atmospheric Administration, 1994).

On January 19, 1996, excessive rainfall in northern Delaware caused flooding in and near the city of Wilmington. A streamgage $(01480015$, table 9$)$ had a peak of record.

\section{Reference}

National Oceanic and Atmospheric Administration (NOAA), 1994, Storm data (by State): Asheville, North Carolina, National Climatic Data Center, various months.

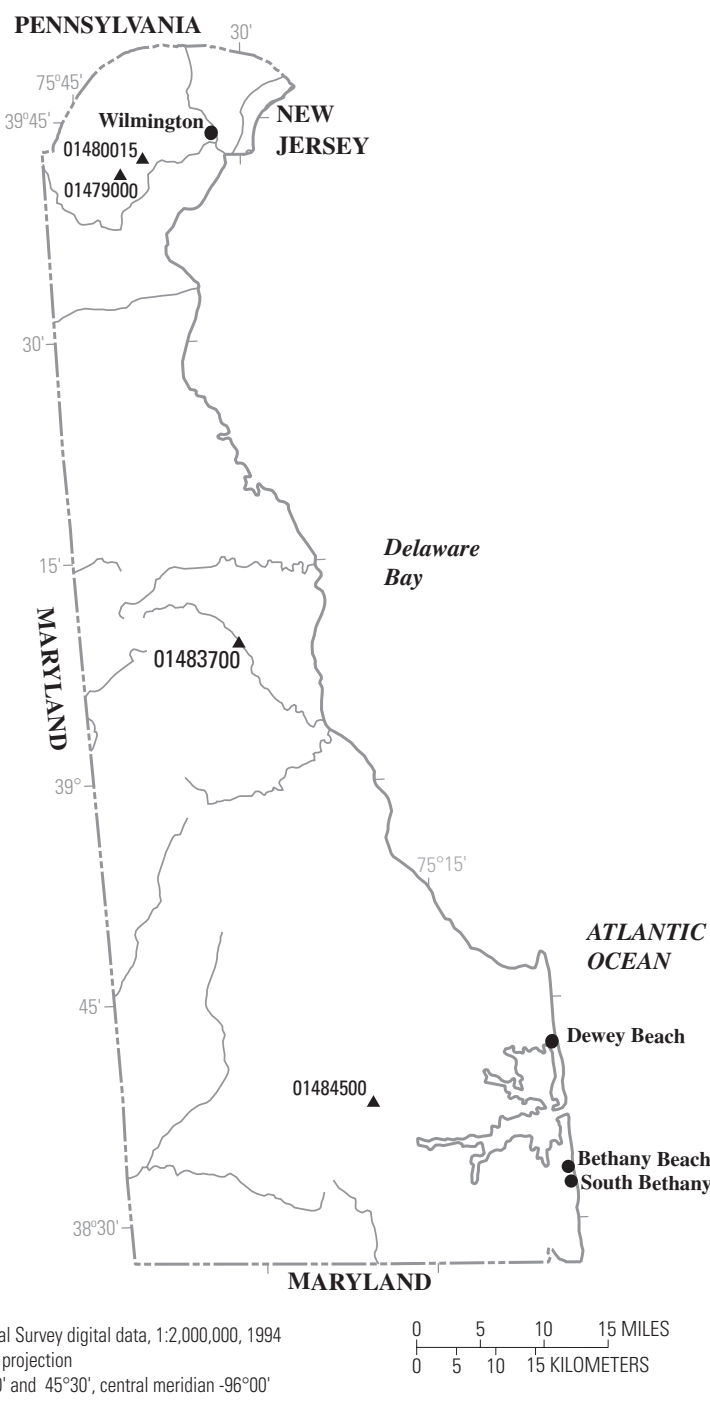

\section{EXPLANATION}

01484500^Streamgage and number

Figure 20. Location of streamgages with significant floods during 1994-98 water years for Delaware. 
Table 9. Maximum stage and discharge for period of record for streamgages having significant floods during 1994-98 water years in Delaware.

$\left[\mathrm{mi}^{2}\right.$, square miles; $\mathrm{ft}$, feet above an arbitrary datum; $\mathrm{ft}^{3} / \mathrm{s}$, cubic feet per second; $>$, greater than; <, less than. Source: Recurrence intervals calculated from U.S. Geological Survey data. Other data from U.S. Geological Survey reports or databases]

\begin{tabular}{|c|c|c|c|c|c|c|c|c|c|c|c|}
\hline \multirow{2}{*}{$\begin{array}{c}\text { Streamgage } \\
\text { number } \\
\text { (fig. 20) }\end{array}$} & \multirow[b]{2}{*}{ Streamgage name } & \multirow{2}{*}{$\begin{array}{c}\text { Total } \\
\text { drainage } \\
\left(\mathrm{mi}^{2}\right)\end{array}$} & \multicolumn{4}{|c|}{$\begin{array}{c}\text { Maximum stage and discharge for period of record } \\
\text { through } 1998 \text { water year }\end{array}$} & \multicolumn{5}{|c|}{ Significant floods 1994-98 water years } \\
\hline & & & $\begin{array}{l}\text { Period of } \\
\text { record } \\
\text { (water } \\
\text { years) }\end{array}$ & Water year & $\begin{array}{l}\text { Stage } \\
\text { (ft) }\end{array}$ & $\begin{array}{l}\text { Discharge } \\
\left(\mathrm{ft}^{3} / \mathrm{s}\right)\end{array}$ & $\begin{array}{c}\text { Date } \\
\text { (month/ } \\
\text { day/ } \\
\text { year) }\end{array}$ & $\begin{array}{l}\text { Stage } \\
\text { (ft) }\end{array}$ & $\begin{array}{l}\text { Discharge } \\
\left(\mathrm{ft}^{3} / \mathrm{s}\right)\end{array}$ & $\begin{array}{c}\text { Regulated } \\
\text { during } \\
\text { flood }^{1}\end{array}$ & $\begin{array}{c}\text { Recurrence } \\
\text { interval } \\
\text { (years) }\end{array}$ \\
\hline 01479000 & $\begin{array}{l}\text { White Clay Creek near Newark, } \\
\text { DE }\end{array}$ & 89.1 & $\begin{array}{l}1932-36, \\
1943-57, \\
1960-98\end{array}$ & $\begin{array}{l}1989 \\
1972\end{array}$ & $\begin{array}{l}16.55 \\
17.74\end{array}$ & $\begin{array}{r}11,600 \\
9,080\end{array}$ & $1 / 19 / 96$ & 15.90 & 9,150 & $\mathrm{~N}$ & $<50$ \\
\hline 01480015 & Red Clay Creek near Stanton, DE & 52.4 & 1989-98 & 1996 & 19.38 & 5,330 & $1 / 19 / 96$ & 19.38 & 5,330 & $\mathrm{~N}$ & $<5$ \\
\hline 01483700 & Saint Jones River at Dover, DE & 31.9 & $1958-98$ & 1960 & 9.45 & 1,900 & $2 / 24 / 98$ & 5.54 & 1,400 & $\mathrm{~N}$ & $>2$ \\
\hline 01484500 & Stockley Branch at Stockley, DE & 5.24 & 1943-98 & 1994 & 5.52 & 303 & $\begin{array}{l}3 / 3 / 94 \\
1 / 28 / 98\end{array}$ & $\begin{array}{l}5.52 \\
5.06\end{array}$ & $\begin{array}{l}303 \\
238\end{array}$ & $\begin{array}{l}\mathrm{N} \\
\mathrm{N}\end{array}$ & $\begin{array}{r}50 \\
>25\end{array}$ \\
\hline
\end{tabular}

TRegulated during flood: $\mathrm{N}, \mathrm{no} ; \mathrm{Y}$, yes. 


\section{Summary of Significant Floods in the United States and Puerto Rico, 1994 Through 1998 Water Years}

\section{Florida}

Tropical Storm Alberto formed in the southeastern Gulf of Mexico on July 1,1994, and moved north crossing the Florida Panhandle near Destin (fig. 21) on July 3. The maximum storm tide was 5 feet, and the immediate rainfall totaled 2 to 7 inches. Tropical depression Alberto meandered around east-central Alabama and west-central Georgia for 72 hours with rainfall that locally exceeded 20 inches in southwest Georgia (National Oceanic and Atmospheric Administration, 1994a). River flooding in Georgia and Alabama spread into the Florida Panhandle on July 5 and, along with 6 to 14 inches of additional rain from the remnants of Alberto (National Oceanic and Atmospheric Administration, 1994a), caused extensive flooding. Flood crests exceeded 100-year recurrence intervals on the Apalachicola (streamgage 02359170) and Chipola (streamgage 02359000, table 10) Rivers. The first flood crest on the Apalachicola River occurred on July 10 and on the Chipola River on July 10-12. Damage to buildings, roads, water systems, and other public property was estimated at $\$ 40$ million (National Oceanic and Atmospheric Administration, 1994b).

Tropical Storm Beryl formed in the northeastern Gulf of Mexico and moved slowly east-northeast, crossing the Florida Panhandle coastline near Panama City on July 15, 1994. The maximum reported storm tide of 2.9 feet and the greatest rainfall total of 10.69 inches were reported at Apalachicola (National Oceanic and Atmospheric Administration, 1994a), but somewhat higher values likely occurred to the east of this location. Beryl weakened to a tropical depression as it moved northeast into extreme southwest Georgia on July 16 . There were no deaths and only one injury directly attributable to Beryl. Damage was estimated at \$5.9 million (National Oceanic and Atmospheric Administration, 1994b).

In November 1994, Tropical Storm Gordon moved slowly west-northwest reaching the lower Florida Keys late on November 15. On November 16, Gordon turned northeast and accelerated, moving inland near Fort Myers in the morning and exiting into the Atlantic Ocean just north of Vero Beach in the late afternoon. After becoming a hurricane off the coast of
North Carolina, then weakening to a tropical depression and moving south and west, Gordon moved ashore a second time just north of Melbourne on November 21. Two persons died when their cars plunged into canals near Miami. Gordon's total damage was estimated at about $\$ 400$ million (National Oceanic and Atmospheric Administration, 1994b).

Moderate to extensive flooding occurred near Fort Myers from Tropical Storm Jerry's outer rain band on August 24 and 25,1995 . Citrus crop losses of $\$ 15$ million resulted from Jerry's excessive rainfall. Approximately $\$ 4$ million worth of property damage occurred (National Oceanic and Atmospheric Administration, 1995b).

Thirty-hour rainfall totals of 8 to 14 inches on September 26-27, 1997 (National Oceanic and Atmospheric Administration, 1997a), near Tampa caused flooding of roads, highways, homes, commercial buildings, and low-lying areas. Damage from the flooding totaled $\$ 5.3$ million (National Oceanic and Atmospheric Administration, 1997b). In St. Petersburg, a 13-year-old died after being swept into a storm drain while playing in floodwaters. A 36-year old man drowned when his truck hydroplaned, flipped over, and slid into a flooded drainage canal east of Arcadia.

Excessive rainfall from a frontal systems caused serious flooding in central and northern Florida including the Panhandle region in February and March 1998. Almost \$400 million in damage resulted from the floods and severe storms throughout the State (National Oceanic and Atmospheric Administration, 1998b).

\section{References}

National Oceanic and Atmospheric Administration (NOAA), 1994a-98a, Climatological data (by State): Asheville, North Carolina, National Climatic Data Center, various months. National Oceanic and Atmospheric Administration (NOAA), 1994b-98b, Storm data (by State): Asheville, North Carolina, National Climatic Data Center, various months. 


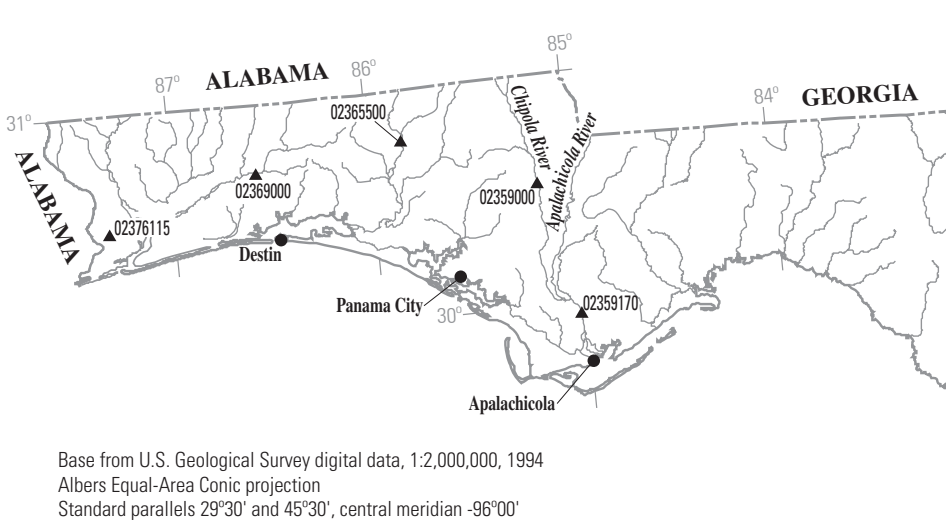

Albers Equal-Area Conic projection
Standard parallels $29^{\circ} 30^{\prime}$ and $45^{\circ} 30^{\prime}$, central meridian $-96^{\circ} 00^{\prime}$

GULF OF MEXICO
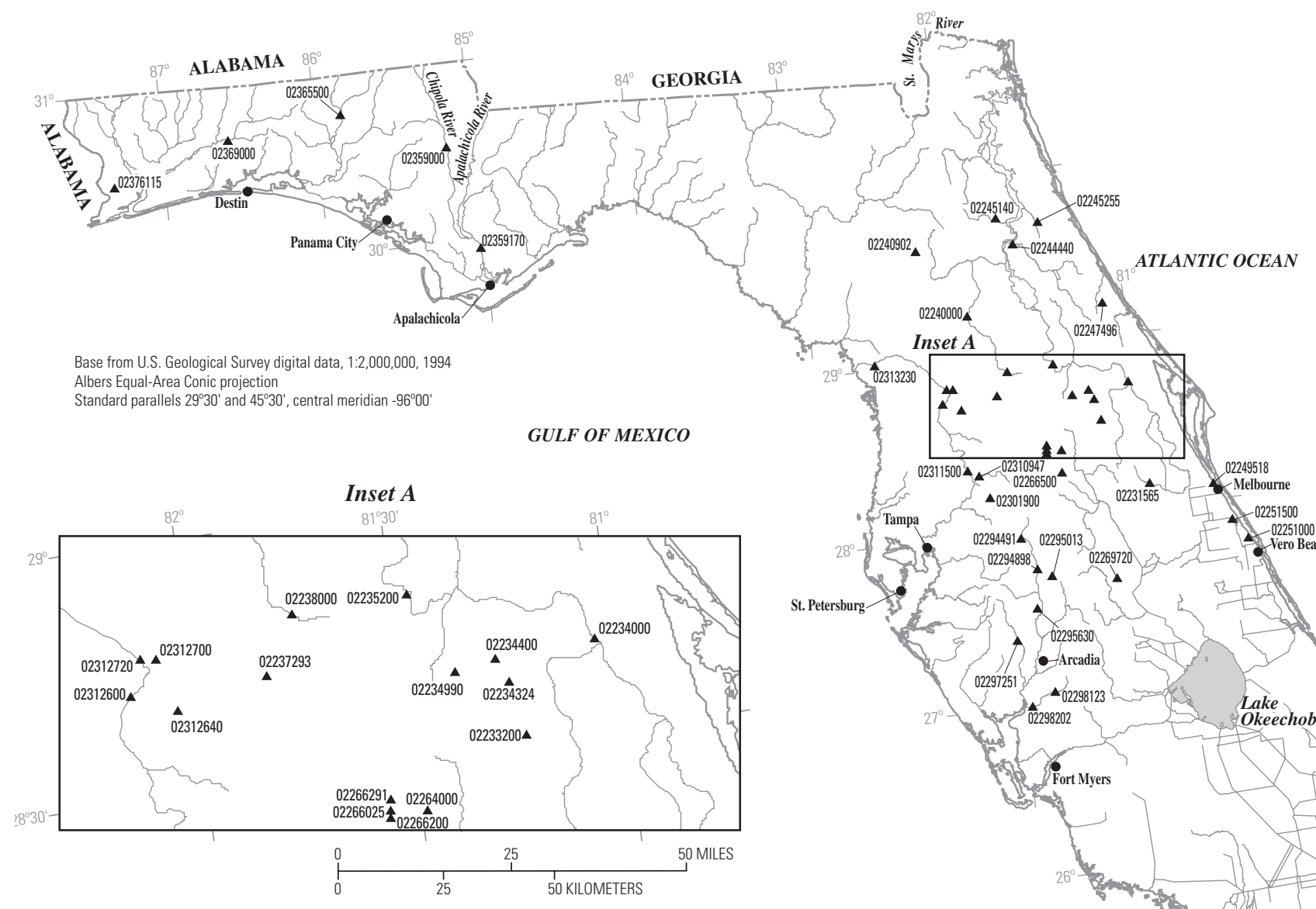

\section{EXPLANATION}

${ }^{02298202} \triangle$ Streamgage and number

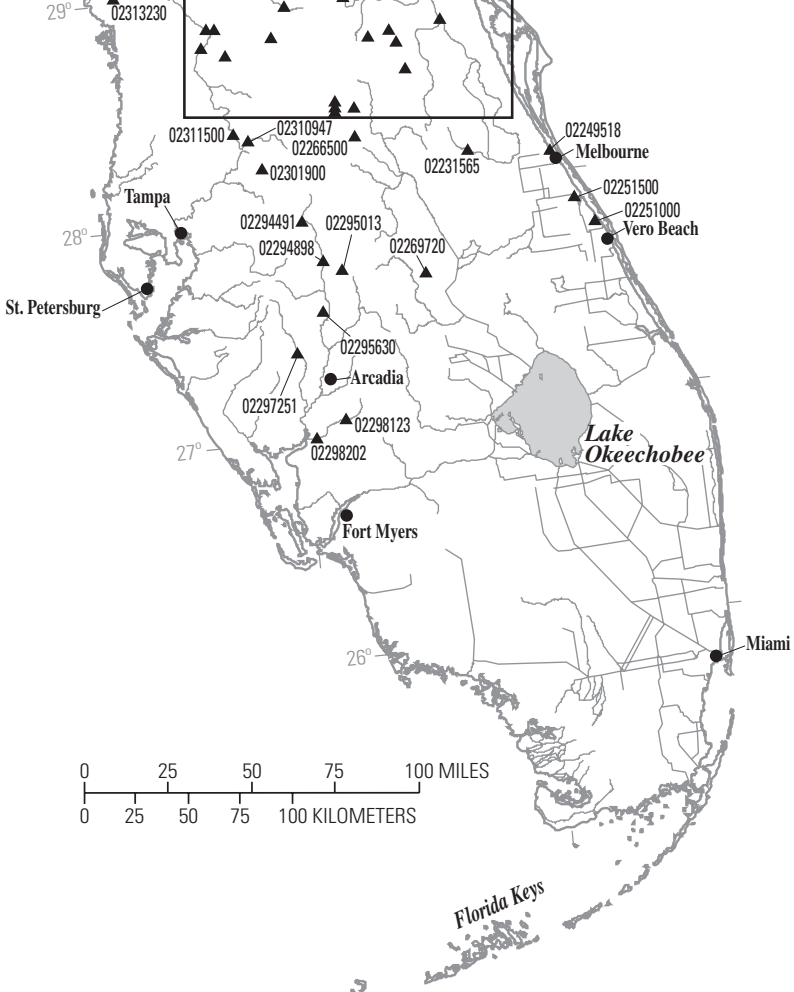

Figure 21. Location of streamgages with significant floods during 1994-98 water years for Florida. 
Table 10. Maximum stage and discharge for period of record for streamgages having significant floods during 1994-98 water years in Florida.

$\left[\mathrm{mi}^{2}\right.$, square miles; $\mathrm{ft}$, feet above an arbitrary datum; $\mathrm{ft}^{3} / \mathrm{s}$, cubic feet per second; --, not determined or not applicable; $>$, greater than. Source: Recurrence intervals calculated from U.S. Geological Survey data. Other data from U.S. Geological Survey reports or databases]

\begin{tabular}{|c|c|c|c|c|c|c|c|c|c|c|c|}
\hline \multirow[b]{2}{*}{$\begin{array}{l}\text { Streamgage } \\
\text { number } \\
\text { (fig. 21) }\end{array}$} & \multirow[b]{2}{*}{ Streamgage name } & \multirow[b]{2}{*}{$\begin{array}{c}\text { Total } \\
\text { drainage } \\
\left(\mathrm{mi}^{2}\right)\end{array}$} & \multicolumn{4}{|c|}{$\begin{array}{c}\text { Maximum stage and discharge for period of record } \\
\text { through } 1998 \text { water year }\end{array}$} & \multicolumn{5}{|c|}{ Significant floods $1994-98$ water years } \\
\hline & & & $\begin{array}{l}\text { Period of } \\
\text { record } \\
\text { (water } \\
\text { years) }\end{array}$ & Water year & $\begin{array}{l}\text { Stage } \\
(\mathrm{ft})\end{array}$ & $\begin{array}{c}\text { Discharge } \\
\left(\mathrm{ft}^{3} / \mathrm{s}\right)\end{array}$ & $\begin{array}{c}\text { Date } \\
\text { (month/ } \\
\text { day/ } \\
\text { year) }\end{array}$ & $\begin{array}{l}\text { Stage } \\
(\mathrm{ft})\end{array}$ & $\begin{array}{c}\text { Discharge } \\
\left(\mathrm{ft}^{3} / \mathrm{s}\right)\end{array}$ & $\begin{array}{l}\text { Regulated } \\
\text { during } \\
\text { flood }\end{array}$ & $\begin{array}{c}\text { Recurrence } \\
\text { interval } \\
\text { (years) }\end{array}$ \\
\hline 02231565 & Crabgrass Creek near Holopaw, FL & 30.2 & $\begin{array}{l}1985, \\
\text { 1987-88, } \\
\text { 1991-95, } \\
\text { 1997-98 }\end{array}$ & 1995 & 8.94 & 1,840 & $11 / 15 / 94$ & 8.94 & 1,840 & $\mathrm{~N}$ & $10-25$ \\
\hline 02233200 & $\begin{array}{l}\text { Little Econlockhatchee River near } \\
\text { Union Park, FL }\end{array}$ & 27.1 & 1960-98 & 1960 & 11.64 & 1,640 & $11 / 17 / 94$ & 11.07 & 1,300 & $\mathrm{~N}$ & $10-25$ \\
\hline 02234000 & $\begin{array}{l}\text { Saint Johns River above Lake } \\
\text { Harney near Geneva, FL }\end{array}$ & 2,043 & 1982-98 & $\begin{array}{l}1995 \\
1993\end{array}$ & $-\overline{-}+7.75$ & $\begin{array}{l}9,880 \\
7,760\end{array}$ & $11 / 22 / 94$ & -- & 9,880 & $\mathrm{~N}$ & $10-25$ \\
\hline 02234324 & Howell Creek near Slavia, FL & 29.2 & $\begin{array}{l}1973-79 \\
1982-98\end{array}$ & $\begin{array}{l}1995 \\
1993\end{array}$ & $\begin{array}{l}36.05 \\
37.98\end{array}$ & $\begin{array}{l}699 \\
454\end{array}$ & $7 / 25 / 95$ & 36.05 & 699 & $\mathrm{~N}$ & $25-50$ \\
\hline 02234400 & Gee Creek near Longwood, FL & 12.8 & $\begin{array}{l}1972-79 \\
1985-98\end{array}$ & 1995 & 16.16 & 459 & $7 / 25 / 95$ & 16.16 & 459 & $\mathrm{~N}$ & $25-50$ \\
\hline 02234990 & $\begin{array}{l}\text { Little Wekiva River near } \\
\text { Altamonte Springs, FL }\end{array}$ & 90.7 & $\begin{array}{l}\text { 1972-79, } \\
1983-98\end{array}$ & 1995 & 30.58 & 1,070 & $11 / 16 / 94$ & 30.58 & 1,070 & $\mathrm{~N}$ & $200-500$ \\
\hline 02235200 & Blackwater Creek near Cassia, FL & 126 & $1962-98$ & 1968 & 9.93 & 749 & $11 / 18 / 94$ & 8.91 & 613 & $\mathrm{~N}$ & $10-25$ \\
\hline 02237293 & $\begin{array}{l}\text { Palatlakaha River at structure M-1 } \\
\text { near Okahumpka, FL }\end{array}$ & 221 & $\begin{array}{l}1971-75 \\
1977-98\end{array}$ & $\begin{array}{l}1998 \\
1977\end{array}$ & $\overline{--}$ & $\begin{array}{r}727 \\
--\end{array}$ & $2 / 20 / 98$ & -- & 727 & $\mathrm{Y}$ & $10-25$ \\
\hline 02238000 & Haines Creek at Lisbon, FL & 648 & $\begin{array}{l}1926, \\
1942-80, \\
1986-98\end{array}$ & $\begin{array}{l}1998 \\
1926\end{array}$ & $\begin{array}{l}-- \\
65.30\end{array}$ & $\begin{array}{r}1,560 \\
--\end{array}$ & $2 / 26 / 98$ & -- & 1,560 & $\mathrm{Y}$ & 100-200 \\
\hline 02240000 & Ocklawaha River near Conner, FL & 1,196 & $\begin{array}{l}1931-46 \\
1964-98\end{array}$ & $\begin{array}{l}1982 \\
1933\end{array}$ & $\begin{array}{l}8.46 \\
9.14\end{array}$ & $\begin{array}{l}4,430 \\
3,700\end{array}$ & 2/17/98 & 7.67 & 3,480 & $\mathrm{~N}$ & 10 \\
\hline 02240902 & Prairie Creek near Gainesville, FL & 114 & $\begin{array}{l}\text { 1979-80, } \\
1994-98\end{array}$ & 1998 & 8.77 & 1,290 & $2 / 25 / 98$ & 8.77 & 1,290 & $\mathrm{~N}$ & $50-100$ \\
\hline 02244440 & Dunns Creek near Satsuma, FL & 585 & $\begin{array}{l}1980, \\
1982-84, \\
1986, \\
1991-98\end{array}$ & 1995 & -- & 8,570 & $9 / 10 / 95$ & -- & 8,570 & $\mathrm{~N}$ & $25-50$ \\
\hline 02245140 & Simms Creek near Bardin, FL & 47.3 & $\begin{array}{l}1974-89 \\
1991-98\end{array}$ & $\begin{array}{l}1997 \\
1974\end{array}$ & $\begin{array}{l}14.96 \\
15.47\end{array}$ & $\begin{array}{l}2,840 \\
1,410\end{array}$ & $10 / 8 / 96$ & 14.96 & 2,840 & $\mathrm{~N}$ & $25-50$ \\
\hline
\end{tabular}


Table 10. Maximum stage and discharge for period of record for streamgages having significant floods during 1994-98 water years in Florida.-Continued

$\left[\mathrm{mi}^{2}\right.$, square miles; ft, feet above an arbitrary datum; $\mathrm{ft}^{3} / \mathrm{s}$, cubic feet per second; --, not determined or not applicable; >, greater than. Source: Recurrence intervals calculated from U.S. Geological Survey data. Other data from U.S. Geological Survey reports or databases]

\begin{tabular}{|c|c|c|c|c|c|c|c|c|c|c|c|}
\hline \multirow[b]{2}{*}{$\begin{array}{l}\text { Streamgage } \\
\text { number } \\
\text { (fig. 21) }\end{array}$} & \multirow[b]{2}{*}{ Streamgage name } & \multirow{2}{*}{$\begin{array}{c}\text { Total } \\
\text { drainage } \\
\left(\mathrm{mi}^{2}\right)\end{array}$} & \multicolumn{4}{|c|}{$\begin{array}{c}\text { Maximum stage and discharge for period of record } \\
\text { through } 1998 \text { water year }\end{array}$} & \multicolumn{5}{|c|}{ Significant floods 1994-98 water years } \\
\hline & & & $\begin{array}{l}\text { Period of } \\
\text { record } \\
\text { (water } \\
\text { years) }\end{array}$ & Water year & $\begin{array}{l}\text { Stage } \\
(\mathrm{ft})\end{array}$ & $\begin{array}{l}\text { Discharge } \\
\left(\mathrm{ft}^{3} / \mathrm{s}\right)\end{array}$ & $\begin{array}{c}\text { Date } \\
\text { (month/ } \\
\text { day/ } \\
\text { year) }\end{array}$ & $\begin{array}{l}\text { Stage } \\
(\mathrm{ft})\end{array}$ & $\begin{array}{l}\text { Discharge } \\
\left(\mathrm{ft}^{3} / \mathrm{s}\right)\end{array}$ & $\begin{array}{l}\text { Regulated } \\
\text { during } \\
\text { flood }^{1}\end{array}$ & $\begin{array}{l}\text { Recurrence } \\
\text { interval } \\
\text { (years) }\end{array}$ \\
\hline 02245255 & Deep Creek near Hastings, FL & 20.7 & $\begin{array}{l}1975-89 \\
1991-98\end{array}$ & 1998 & 8.58 & 880 & $2 / 16 / 98$ & 8.58 & 880 & $\mathrm{~N}$ & $100-200$ \\
\hline 02247496 & $\begin{array}{l}\text { Thayer Canal near Daytona Beach, } \\
\text { FL }\end{array}$ & 33.0 & 1989-98 & 1995 & 26.03 & 283 & $11 / 16 / 94$ & 26.03 & 283 & $\mathrm{~N}$ & $10-25$ \\
\hline 02249518 & $\begin{array}{l}\text { Crane Creek at U.S. Highway } 1 \text { at } \\
\text { Melbourne, FL }\end{array}$ & 18.1 & 1987-98 & 1995 & -- & 2,150 & $8 / 2 / 95$ & -- & 2,150 & $\mathrm{~N}$ & $100-200$ \\
\hline 02251000 & $\begin{array}{l}\text { South Prong Saint Sebastian Creek } \\
\text { near Sebastian, FL }\end{array}$ & 35.0 & $\begin{array}{l}1966-72 \\
1994-98\end{array}$ & 1995 & 19.96 & 2,560 & $11 / 16 / 94$ & 19.96 & 2,560 & $\mathrm{~N}$ & $25-50$ \\
\hline 02251500 & $\begin{array}{l}\text { North Prong Saint Sebastian Creek } \\
\text { near Micco, FL }\end{array}$ & 28.5 & 1987-98 & 1998 & 10.24 & 2,060 & $11 / 14 / 97$ & 10.24 & 2,060 & $\mathrm{~N}$ & $10-25$ \\
\hline 02264000 & Cypress Creek at Vineland, FL & 29.3 & $1946-98$ & 1960 & 4.66 & 309 & $2 / 23 / 98$ & 3.69 & 179 & $\mathrm{~N}$ & $10-25$ \\
\hline 02266025 & $\begin{array}{l}\text { Reedy Creek above S-46 near } \\
\text { Vineland, FL }\end{array}$ & 25.4 & 1987-98 & 1998 & -- & 115 & $2 / 17 / 98$ & -- & 115 & $\mathrm{Y}$ & $10-25$ \\
\hline 02266200 & $\begin{array}{l}\text { Whittenhorse Creek near Vineland, } \\
\text { FL }\end{array}$ & 12.4 & 1967-98 & 1998 & 95.17 & 97 & $12 / 29 / 97$ & 95.17 & 97 & $\mathrm{~N}$ & $25-50$ \\
\hline 02266291 & $\begin{array}{l}\text { Lateral-405 above S-405A near } \\
\text { Doctor Phillips, FL }\end{array}$ & 19.6 & 1987-98 & 1998 & -- & 200 & $12 / 27 / 97$ & -- & 200 & $\mathrm{Y}$ & $25-50$ \\
\hline 02266500 & Reedy Creek near Loughman, FL & 177 & $\begin{array}{l}1940-59 \\
1969-98\end{array}$ & $\begin{array}{l}1998 \\
1969\end{array}$ & $\begin{array}{l}4.22 \\
4.25\end{array}$ & $\begin{array}{r}1,090 \\
790\end{array}$ & $12 / 30 / 97$ & 4.22 & 1,090 & $\mathrm{Y}$ & $>500$ \\
\hline 02269720 & $\begin{array}{l}\text { Morgan Hole Creek near Avon } \\
\text { Park, FL }\end{array}$ & 13.9 & $\begin{array}{l}\text { 1971-75, } \\
\text { 1978-93, } \\
1995, \\
1997-98\end{array}$ & 1998 & 8.23 & 1,420 & $11 / 17 / 97$ & 8.23 & 1,420 & $\mathrm{~N}$ & $10-25$ \\
\hline 02294491 & $\begin{array}{l}\text { Saddle Creek at Structure P-11 } \\
\text { near Bartow, FL }\end{array}$ & 53 & 1964-96 & 1995 & 15.90 & 894 & $\begin{array}{l}9 / 14 / 94 \\
8 / 15 / 95\end{array}$ & $\begin{array}{l}15.10 \\
15.90\end{array}$ & $\begin{array}{l}534 \\
894\end{array}$ & $\mathrm{Y}$ & $\begin{array}{l}2-5 \\
2-5\end{array}$ \\
\hline 02294898 & Peace River at Fort Meade, FL & 480 & 1974-96 & 1995 & 78.54 & 2,150 & $8 / 10 / 95$ & 78.54 & 2,150 & $\mathrm{Y}$ & $2-5$ \\
\hline 02295013 & $\begin{array}{l}\text { Bowlegs Creek near Fort Meade, } \\
\text { FL }\end{array}$ & 47.2 & $\begin{array}{l}1964-68 \\
1991-96\end{array}$ & 1994 & 8.34 & 742 & $9 / 21 / 94$ & 8.34 & 742 & $\mathrm{~N}$ & $5-10$ \\
\hline
\end{tabular}


$\left[\mathrm{mi}^{2}\right.$, square miles; $\mathrm{ft}$, feet above an arbitrary datum; $\mathrm{ft}^{3} / \mathrm{s}$, cubic feet per second; --, not determined or not applicable; >, greater than. Source: Recurrence intervals calculated from U.S. Geological Survey data. Other data from U.S. Geological Survey reports or databases]

\begin{tabular}{|c|c|c|c|c|c|c|c|c|c|c|c|}
\hline \multirow{2}{*}{$\begin{array}{l}\text { Streamgage } \\
\text { number } \\
\text { (fig. 21) }\end{array}$} & \multirow[b]{2}{*}{ Streamgage name } & \multirow{2}{*}{$\begin{array}{c}\text { Total } \\
\text { drainage } \\
\left(\mathrm{mi}^{2}\right)\end{array}$} & \multicolumn{4}{|c|}{$\begin{array}{c}\text { Maximum stage and discharge for period of record } \\
\text { through } 1998 \text { water year }\end{array}$} & \multicolumn{5}{|c|}{ Significant floods $1994-98$ water years } \\
\hline & & & $\begin{array}{c}\text { Period of } \\
\text { record } \\
\text { (water } \\
\text { years) }\end{array}$ & Water year & $\begin{array}{l}\text { Stage } \\
(\mathrm{ft})\end{array}$ & $\begin{array}{l}\text { Discharge } \\
\left(\mathrm{ft}^{3} / \mathrm{s}\right)\end{array}$ & $\begin{array}{l}\text { Date } \\
\text { (month/ } \\
\text { day/ } \\
\text { year) }\end{array}$ & $\begin{array}{l}\text { Stage } \\
(\mathrm{ft})\end{array}$ & $\begin{array}{l}\text { Discharge } \\
\left(\mathrm{ft}^{3} / \mathrm{s}\right)\end{array}$ & $\begin{array}{l}\text { Regulated } \\
\text { during } \\
\text { flood }^{1}\end{array}$ & $\begin{array}{c}\text { Recurrence } \\
\text { interval } \\
\text { (years) }\end{array}$ \\
\hline 02295630 & $\begin{array}{l}\text { Thompson Branch near Wauchula, } \\
\text { FL }\end{array}$ & 5.22 & $\begin{array}{l}1984-91 \\
1993-96\end{array}$ & $\begin{array}{l}1995 \\
1988\end{array}$ & $\begin{array}{r}9.91 \\
10.04\end{array}$ & $\begin{array}{r}396 \\
--\end{array}$ & $8 / 2 / 95$ & 9.91 & 396 & $\mathrm{~N}$ & $5-10$ \\
\hline 02297251 & Horse Creek near Limestone, FL & 130 & $\begin{array}{l}\text { 1981-88, } \\
1990-96\end{array}$ & 1994 & 59.24 & 3,450 & $9 / 16 / 94$ & 59.24 & 3,450 & $\mathrm{~N}$ & $5-10$ \\
\hline 02298123 & Prairie Creek near Fort Ogden, FL & 233 & $\begin{array}{l}1964-68 \\
1978-96\end{array}$ & $\begin{array}{l}1995 \\
1980\end{array}$ & $\begin{array}{l}13.75 \\
14.19\end{array}$ & $\begin{array}{l}5,320 \\
4,280\end{array}$ & $8 / 26 / 95$ & 13.75 & 5,320 & $\mathrm{~N}$ & $25-50$ \\
\hline 02298202 & Shell Creek near Punta Gorda, FL & 373 & $\begin{array}{l}1965-87 \\
1994-96\end{array}$ & 1995 & 7.86 & 8,650 & $6 / 23 / 95$ & 7.86 & 8,650 & $\mathrm{Y}$ & $25-50$ \\
\hline 02301900 & Fox Branch near Socrum, FL & 9.50 & $1965-98$ & $\begin{array}{l}1998 \\
1978\end{array}$ & $\begin{array}{l}8.21 \\
8.22\end{array}$ & $\begin{array}{r}1,790 \\
569\end{array}$ & $12 / 27 / 97$ & 8.21 & 1,790 & $\mathrm{~N}$ & $50-100$ \\
\hline 02310947 & $\begin{array}{l}\text { Withlacoochee River near } \\
\text { Cumpressco, FL }\end{array}$ & 280 & 1968-98 & $\begin{array}{l}1998 \\
1987\end{array}$ & -- & $\begin{array}{l}3,250 \\
2,260\end{array}$ & $12 / 29 / 97$ & -- & 3,250 & $\mathrm{~N}$ & $50-100$ \\
\hline 02311500 & $\begin{array}{l}\text { Withlacoochee River near Dade } \\
\text { City, FL }\end{array}$ & 390 & 1984-98 & 1998 & 77.55 & 3,900 & $12 / 31 / 97$ & 77.55 & 3,900 & $\mathrm{~N}$ & $10-25$ \\
\hline 02312600 & $\begin{array}{l}\text { Withlacoochee River near Floral } \\
\text { City, FL }\end{array}$ & 995 & 1984-98 & 1998 & 44.07 & 5,010 & $1 / 8 / 98$ & 44.07 & 5,010 & $\mathrm{~N}$ & $10-25$ \\
\hline 02312640 & $\begin{array}{l}\text { Jumper Creek Canal near Bushnell, } \\
\text { FL }\end{array}$ & 40.0 & 1964-98 & 1998 & 7.21 & 238 & $2 / 18 / 98$ & 7.21 & 238 & $\mathrm{~N}$ & $25-50$ \\
\hline 02312700 & $\begin{array}{l}\text { Outlet River at Panacoochee } \\
\text { Retreats, FL }\end{array}$ & 420 & 1963-98 & $\begin{array}{l}1998 \\
1970\end{array}$ & $-\overline{4}-49$ & $\begin{array}{l}821 \\
520\end{array}$ & $3 / 21 / 98$ & -- & 821 & $\mathrm{~N}$ & $25-50$ \\
\hline 02312720 & $\begin{array}{l}\text { Withlacoochee River at Wysong } \\
\text { Dam at Carlson, FL }\end{array}$ & 1,520 & $\begin{array}{l}1966-80 \\
1982-98\end{array}$ & 1998 & 41.54 & 4,910 & $1 / 16 / 98$ & 41.54 & 4,910 & $\mathrm{~N}$ & $25-50$ \\
\hline 02313230 & $\begin{array}{l}\text { Withlacoochee River at Inglis Dam } \\
\text { near Dunnellon, FL }\end{array}$ & 2,020 & 1970-98 & 1998 & -- & 6,000 & $3 / 20 / 98$ & -- & 6,000 & $\mathrm{Y}$ & 25 \\
\hline 02359000 & Chipola River near Altha, FL & 781 & $\begin{array}{l}1913, \\
1922-27, \\
1930-31, \\
1944-94, \\
1996\end{array}$ & 1926 & 33.55 & 25,000 & $7 / 11 / 94$ & 29.60 & 14,200 & $\mathrm{~N}$ & $>100$ \\
\hline
\end{tabular}


Table 10. Maximum stage and discharge for period of record for streamgages having significant floods during 1994-98 water years in Florida.—Continued

$\left[\mathrm{mi}^{2}\right.$, square miles; $\mathrm{ft}$, feet above an arbitrary datum; $\mathrm{ft}^{3} / \mathrm{s}$, cubic feet per second; --, not determined or not applicable; >, greater than. Source: Recurrence intervals calculated from U.S. Geological Survey data. Other data from U.S. Geological Survey reports or databases]

\begin{tabular}{|c|c|c|c|c|c|c|c|c|c|c|c|}
\hline \multirow[b]{2}{*}{$\begin{array}{l}\text { Streamgage } \\
\text { number } \\
\text { (fig. 21) }\end{array}$} & \multirow[b]{2}{*}{ Streamgage name } & \multirow[b]{2}{*}{$\begin{array}{c}\text { Total } \\
\text { drainage } \\
\left(\mathrm{mi}^{2}\right)\end{array}$} & \multicolumn{4}{|c|}{$\begin{array}{c}\text { Maximum stage and discharge for period of record } \\
\text { through } 1998 \text { water year }\end{array}$} & \multicolumn{5}{|c|}{ Significant floods $1994-98$ water years } \\
\hline & & & $\begin{array}{l}\text { Period of } \\
\text { record } \\
\text { (water } \\
\text { years) }\end{array}$ & Water year & $\begin{array}{l}\text { Stage } \\
\text { (ft) }\end{array}$ & $\begin{array}{c}\text { Discharge } \\
\left(\mathrm{ft}^{3} / \mathrm{s}\right)\end{array}$ & $\begin{array}{c}\text { Date } \\
\text { (month/ } \\
\text { day/ } \\
\text { year) }\end{array}$ & $\begin{array}{l}\text { Stage } \\
(\mathrm{ft})\end{array}$ & $\begin{array}{c}\text { Discharge } \\
\left(\mathrm{ft}^{3} / \mathrm{s}\right)\end{array}$ & $\begin{array}{c}\text { Regulated } \\
\text { during } \\
\text { flood }^{1}\end{array}$ & $\begin{array}{c}\text { Recurrence } \\
\text { interval } \\
\text { (years) }\end{array}$ \\
\hline 02359170 & $\begin{array}{l}\text { Apalachicola River near Sumatra, } \\
\text { FL }\end{array}$ & 19,200 & $\begin{array}{l}\text { 1978-94, } \\
1996\end{array}$ & 1994 & 15.05 & 221,000 & $7 / 13 / 94$ & 15.05 & 221,000 & $\mathrm{~N}$ & $>100$ \\
\hline 02365500 & $\begin{array}{l}\text { Choctawhatchee River at } \\
\text { Caryville, FL }\end{array}$ & 3,499 & $1928-95$ & 1929 & 27.10 & 206,000 & $7 / 9 / 94$ & 23.85 & 164,000 & $\mathrm{~N}$ & -- \\
\hline 02369000 & Shoal River near Crestview, FL & 3,474 & $\begin{array}{l}\text { 1939-94, } \\
1996\end{array}$ & 1975 & 15.58 & 25,200 & $7 / 8 / 94$ & 14.82 & 24,400 & $\mathrm{~N}$ & -- \\
\hline 02376115 & $\begin{array}{l}\text { Elevenmile Creek near Pensacola, } \\
\text { FL }\end{array}$ & 27.8 & 1988-98 & 1998 & 16.94 & 12,800 & $9 / 28 / 98$ & 16.94 & 12,800 & $\mathrm{~N}$ & -- \\
\hline
\end{tabular}

${ }^{1}$ Regulated during flood: $\mathrm{N}$, no; $\mathrm{Y}$, yes. 


\section{Georgia}

The remnants of Tropical Storm Alberto entered Georgia July 4, 1994, and for the next 2 days the system stalled out in the west-central part of the State. Maximum rainfall of 27.61 inches occurred at Americus (fig. 22) (National Oceanic and Atmospheric Administration, 1994a). The worst flooding occurred along the Flint, Chattahoochee, and Ocmulgee Rivers and their tributaries. More than 50 streamgages in Georgia recorded their peak of record during this flood (table 11). A total of 55 counties were declared Federal disaster areas. The death toll in Georgia was 31, of which most occurred in vehicles that were swept away by floodwaters (Stamey, 1997).

The remnants of Tropical Storm Jerry produced 10 to 14 inches of rain across the southeastern counties of Georgia from August 24 to 27, 1995 (National Oceanic and Atmospheric Administration, 1995a). The excessive rain produced flash flooding, coastal flooding at times of high tide, and river flooding afterwards. Total damage throughout the area was estimated at \$3.5 million (National Oceanic and Atmospheric Administration, 1995b).

An intense Gulf storm produced 5 to 12 inches of rain across much of southwestern Georgia on March 7-9, 1998 (National Oceanic and Atmospheric Administration, 1998a), which caused widespread flooding. Eighteen counties were declared Federal disaster areas. Near Fitzgerald, floodwaters claimed the life of a man whose vehicle was overturned by the floodwaters (National Oceanic and Atmospheric Administration, 1998b).

\section{References}

National Oceanic and Atmospheric Administration (NOAA), 1994a-98a, Climatological data (by State): Asheville, North Carolina, National Climatic Data Center, various months. National Oceanic and Atmospheric Administration (NOAA), 1994b-98b, Storm data (by State): Asheville, North Carolina, National Climatic Data Center, various months.

Stamey, T.C., 1997, Flooding in southeastern United States from Tropical Storm Alberto, July 1994, in Leavesley, G.H., Lins, H.F., Nobilis, Franz, Parker, R.S., Schneider, V.R., and van de Ven, F.H.M., eds., Destructive water-water-caused natural disasters, their abatement and control: International Association of Hydrological Sciences-Association Internationale des Sciences Hydrologiques Publication, v. 239, p. 33-38. 


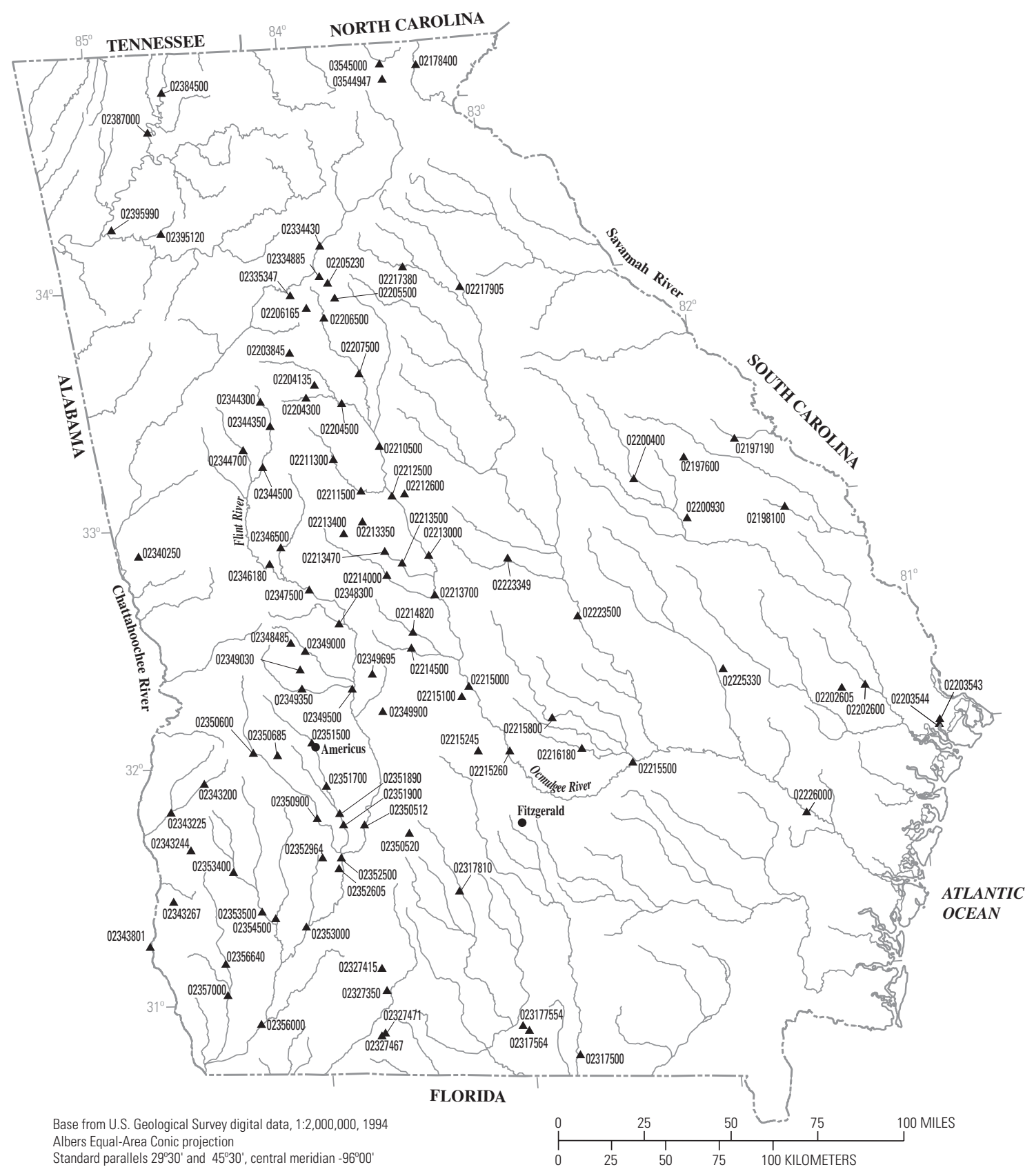

EXPLANATION

02327467\Streamgage and number

Figure 22. Location of streamgages with significant floods during 1994-98 water years for Georgia. 
Table 11. Maximum stage and discharge for period of record for streamgages having significant floods during 1994-98 water years in Georgia.

$\left[\mathrm{mi}^{2}\right.$, square miles; $\mathrm{ft}$, feet above an arbitrary datum; $\mathrm{ft}^{3} / \mathrm{s}$, cubic feet per second; --, not determined or not applicable; $>$, greater than; <, less than. Source: Recurrence intervals calculated from U.S. Geological Survey data. Other data from U.S. Geological Survey reports or databases]

\begin{tabular}{|c|c|c|c|c|c|c|c|c|c|c|c|}
\hline \multirow{2}{*}{$\begin{array}{l}\text { Streamgage } \\
\text { number } \\
\text { (fig. 22) }\end{array}$} & \multirow[b]{2}{*}{ Streamgage name } & \multirow{2}{*}{$\begin{array}{c}\text { Total } \\
\text { drainage } \\
\left(\mathrm{mi}^{2}\right)\end{array}$} & \multicolumn{4}{|c|}{$\begin{array}{c}\text { Maximum stage and discharge for period of record } \\
\text { through } 1998 \text { water year }\end{array}$} & \multicolumn{5}{|c|}{ Significant floods $1994-98$ water years } \\
\hline & & & $\begin{array}{l}\text { Period of } \\
\text { record } \\
\text { (water } \\
\text { years) }\end{array}$ & Water year & $\begin{array}{l}\text { Stage } \\
(\mathrm{ft})\end{array}$ & $\begin{array}{l}\text { Discharge } \\
\left(\mathrm{ft}^{3} / \mathrm{s}\right)\end{array}$ & $\begin{array}{c}\text { Date } \\
\text { (month/ } \\
\text { day/ } \\
\text { year) }\end{array}$ & $\begin{array}{l}\text { Stage } \\
(\mathrm{ft})\end{array}$ & $\begin{array}{l}\text { Discharge } \\
\left(\mathrm{ft}^{3} / \mathrm{s}\right)\end{array}$ & $\begin{array}{l}\text { Regulated } \\
\text { during } \\
\text { flood }^{1}\end{array}$ & $\begin{array}{c}\text { Recurrence } \\
\text { interval } \\
\text { (years) }\end{array}$ \\
\hline 02178400 & Tallulah River near Clayton, GA & 56.6 & $1965-98$ & 1995 & 7.19 & 13,060 & $2 / 16 / 95$ & 7.19 & 13,060 & $\mathrm{~N}$ & $>100$ \\
\hline 02197190 & $\begin{array}{l}\text { McBean Creek at U.S. Route } 25 \\
\text { near McBean, GA }\end{array}$ & 41.4 & 1963-98 & 1991 & 7.52 & 3,160 & 3/9/98 & 5.04 & 586 & $\mathrm{~N}$ & 2 \\
\hline 02197600 & Brushy Creek near Wrens, GA & 28.0 & 1959-98 & 1991 & 14.02 & 11,400 & $2 / 18 / 95$ & 8.32 & 1,970 & $\mathrm{~N}$ & 100 \\
\hline 02198100 & Beaverdam Creek near Sardis, GA & 30.8 & $1987-98$ & 1998 & 8.49 & 4,070 & 3/9/98 & 8.49 & 4,070 & $\mathrm{~N}$ & 25 \\
\hline 02200400 & $\begin{array}{l}\text { Rocky Comfort Creek at State } \\
\text { Route } 88 \text { near Grange, GA }\end{array}$ & 188 & 1979-98 & 1998 & 16.00 & 5,250 & $3 / 10 / 98$ & 16.00 & 5,250 & $\mathrm{~N}$ & 25 \\
\hline 02200930 & Spring Creek near Louisville, GA & 14.2 & $1965-98$ & 1991 & 10.38 & 2,200 & 3/9/98 & 8.04 & 1,430 & $\mathrm{~N}$ & $>50$ \\
\hline 02202600 & Black Creek near Blitchton, GA & 232 & $1980-98$ & 1995 & 15.28 & 11,100 & $10 / 14 / 94$ & 15.28 & 11,100 & $\mathrm{~N}$ & $>100$ \\
\hline 02202605 & $\begin{array}{l}\text { Mill Creek at State Route } 119 \text { near } \\
\text { Pembroke, GA }\end{array}$ & 5.39 & 1979-96 & 1995 & 5.98 & 657 & $10 / 12 / 94$ & 5.98 & 657 & $\mathrm{~N}$ & -- \\
\hline 02203543 & $\begin{array}{l}\text { Wilshire Canal at Tibet Avenue at } \\
\text { Savannah, GA }\end{array}$ & .95 & $1979-96$ & 1996 & 11.87 & 882 & $7 / 5 / 96$ & 11.87 & 882 & $\mathrm{~N}$ & -- \\
\hline 02203544 & $\begin{array}{l}\text { Wilshire Canal tributary at } \\
\text { Windsor Road at Savannah, GA }\end{array}$ & .18 & 1979-96 & 1996 & 6.41 & 127 & $7 / 5 / 96$ & 6.41 & 127 & $\mathrm{~N}$ & -- \\
\hline 02203845 & $\begin{array}{l}\text { Shoal Creek tributary at Glendale } \\
\text { Drive near Atlanta, GA }\end{array}$ & .84 & $\begin{array}{l}1963-65, \\
1973-78, \\
1980-96\end{array}$ & 1994 & 7.19 & 797 & $9 / 18 / 94$ & 7.19 & 797 & $\mathrm{~N}$ & -- \\
\hline 02204135 & $\begin{array}{l}\text { Camp Creek tributary at State } \\
\text { Route } 155 \text { near Stockbridge, GA }\end{array}$ & .28 & 1977-98 & 1994 & 9.02 & 190 & $7 / 5 / 94$ & 9.02 & 190 & $\mathrm{~N}$ & 10 \\
\hline 02204300 & $\begin{array}{l}\text { Little Cotton Indian Creek near } \\
\text { Stockbridge, GA }\end{array}$ & 50.0 & $\begin{array}{l}1951-71 \\
1994\end{array}$ & 1994 & 18.84 & 8,600 & $7 / 5 / 94$ & 18.84 & 8,600 & $\mathrm{~N}$ & 100 \\
\hline 02204500 & South River near McDonough, GA & 456 & $\begin{array}{l}1940-65, \\
1976-82, \\
1994\end{array}$ & 1994 & 28.70 & 41,000 & $7 / 6 / 94$ & 28.70 & 41,000 & $\mathrm{~N}$ & $>100$ \\
\hline 02205230 & $\begin{array}{l}\text { Wolf Creek (Dean Road) near } \\
\text { Suwanee, GA }\end{array}$ & .37 & 1987-98 & 1996 & 8.47 & 220 & $10 / 5 / 95$ & 8.47 & 220 & $\mathrm{~N}$ & -- \\
\hline
\end{tabular}


Table 11. Maximum stage and discharge for period of record for streamgages having significant floods during 1994-98 water years in Georgia.-Continued

$\left[\mathrm{mi}^{2}\right.$, square miles; $\mathrm{ft}$, feet above an arbitrary datum; $\mathrm{ft}^{3} / \mathrm{s}$, cubic feet per second; --, not determined or not applicable; $>$, greater than; <, less than. Source: Recurrence intervals calculated from U.S. Geological Survey data. Other data from U.S. Geological Survey reports or databases]

\begin{tabular}{|c|c|c|c|c|c|c|c|c|c|c|c|}
\hline \multirow[b]{2}{*}{$\begin{array}{l}\text { Streamgage } \\
\text { number } \\
\text { (fig. 22) }\end{array}$} & \multirow[b]{2}{*}{ Streamgage name } & \multirow{2}{*}{$\begin{array}{l}\text { Total } \\
\text { drainage } \\
\left(\mathrm{mi}^{2}\right)\end{array}$} & \multicolumn{4}{|c|}{$\begin{array}{c}\text { Maximum stage and discharge for period of record } \\
\text { through } 1998 \text { water year }\end{array}$} & \multicolumn{5}{|c|}{ Significant floods 1994-98 water years } \\
\hline & & & $\begin{array}{l}\text { Period of } \\
\text { record } \\
\text { (water } \\
\text { years) }\end{array}$ & Water year & $\begin{array}{l}\text { Stage } \\
\text { (ft) }\end{array}$ & $\begin{array}{c}\text { Discharge } \\
\left(\mathrm{ft}^{3} / \mathrm{s}\right)\end{array}$ & $\begin{array}{c}\text { Date } \\
\text { (month/ } \\
\text { day/ } \\
\text { year) }\end{array}$ & $\begin{array}{l}\text { Stage } \\
(\mathrm{ft})\end{array}$ & $\begin{array}{c}\text { Discharge } \\
\left(\mathrm{ft}^{3} / \mathrm{s}\right)\end{array}$ & $\begin{array}{l}\text { Regulated } \\
\text { during } \\
\text { flood }^{1}\end{array}$ & $\begin{array}{c}\text { Recurrence } \\
\text { interval } \\
\text { (years) }\end{array}$ \\
\hline 02205500 & Pew Creek near Lawrenceville, GA & 2.23 & $\begin{array}{l}1954-63 \\
1995-98\end{array}$ & $\begin{array}{l}1998 \\
1996\end{array}$ & $\begin{array}{r}6.57 \\
13.39\end{array}$ & $\begin{array}{r}694 \\
--\end{array}$ & $4 / 9 / 98$ & 6.57 & 694 & $\mathrm{~N}$ & -- \\
\hline 02206165 & $\begin{array}{l}\text { Jackson Creek tributary \#2 near } \\
\text { Lilburn, GA }\end{array}$ & .10 & $\begin{array}{l}1987-92 \\
1994-98\end{array}$ & 1995 & 11.51 & 101 & $9 / 14 / 95$ & 11.51 & 101 & $\mathrm{~N}$ & -- \\
\hline 02206500 & Yellow River near Snellville, GA & 134 & $1943-98$ & 1996 & 19.75 & 12,800 & $10 / 5 / 95$ & 19.75 & 12,800 & $\mathrm{~N}$ & $>100$ \\
\hline 02207500 & Yellow River near Covington, GA & 378 & $\begin{array}{l}1936, \\
1945-65, \\
1976-98\end{array}$ & $\begin{array}{l}1994 \\
1936\end{array}$ & $\begin{array}{l}28.70 \\
29.90\end{array}$ & $\begin{array}{l}41,000 \\
30,000\end{array}$ & $7 / 6 / 94$ & 28.70 & 41,000 & $\mathrm{~N}$ & $>100$ \\
\hline 02210500 & Ocmulgee River near Jackson, GA & 1,420 & $\begin{array}{l}1912,1920 \\
1940-65, \\
1976-82, \\
1988-98\end{array}$ & $\begin{array}{l}1920 \\
1994\end{array}$ & $\begin{array}{l}26.80 \\
26.87\end{array}$ & $\begin{array}{l}69,000 \\
68,500\end{array}$ & $7 / 6 / 94$ & 26.87 & 68,500 & $\mathrm{Y}$ & -- \\
\hline 02211300 & Towaliga River near Jackson, GA & 105 & $\begin{array}{l}1961-83 \\
1990,1994\end{array}$ & 1994 & 26.50 & 20,000 & $7 / 5 / 94$ & 26.50 & 20,000 & $\mathrm{~N}$ & $>100$ \\
\hline 02211500 & Towaliga River near Forsyth, GA & 315 & $\begin{array}{l}\text { 1929-31, } \\
1945-66, \\
1990,1994\end{array}$ & $\begin{array}{l}1994 \\
1966\end{array}$ & $\begin{array}{l}-- \\
22.15\end{array}$ & $\begin{array}{l}60,000 \\
15,000\end{array}$ & $7 / 6 / 94$ & -- & 60,000 & $\mathrm{~N}$ & $<100$ \\
\hline 02212500 & Ocmulgee River at Juliette, GA & 1,960 & $\begin{array}{l}1886, \\
1916-21, \\
1949, \\
1975-88, \\
1990,1994\end{array}$ & 1994 & 41.45 & 100,000 & $7 / 6 / 94$ & 41.45 & 100,000 & $\mathrm{~N}$ & $>100$ \\
\hline 02212600 & Falling Creek near Juliette, GA & 72.2 & 1965-98 & 1994 & 23.25 & 7,920 & $7 / 5 / 94$ & 23.25 & 7,920 & $\mathrm{~N}$ & 25 \\
\hline 02213000 & Ocmulgee River at Macon, GA & 2,240 & $\begin{array}{l}1887 \\
1893-1998\end{array}$ & 1994 & 35.40 & 107,000 & $7 / 6 / 94$ & 35.40 & 107,000 & $\mathrm{~N}$ & $>100$ \\
\hline
\end{tabular}


Table 11. Maximum stage and discharge for period of record for streamgages having significant floods during 1994-98 water years in Georgia.-Continued

$\left[\mathrm{mi}^{2}\right.$, square miles; $\mathrm{ft}$, feet above an arbitrary datum; $\mathrm{ft}^{3} / \mathrm{s}$, cubic feet per second; --, not determined or not applicable; >, greater than; <, less than. Source: Recurrence intervals calculated from U.S Geological Survey data. Other data from U.S. Geological Survey reports or databases]

\begin{tabular}{|c|c|c|c|c|c|c|c|c|c|c|c|}
\hline \multirow[b]{2}{*}{$\begin{array}{c}\text { Streamgage } \\
\text { number } \\
\text { (fig. 22) }\end{array}$} & \multirow[b]{2}{*}{ Streamgage name } & \multirow[b]{2}{*}{$\begin{array}{c}\text { Total } \\
\text { drainage } \\
\left(\mathrm{mi}^{2}\right)\end{array}$} & \multicolumn{4}{|c|}{$\begin{array}{c}\text { Maximum stage and discharge for period of record } \\
\text { through } 1998 \text { water year }\end{array}$} & \multicolumn{5}{|c|}{ Significant floods $1994-98$ water years } \\
\hline & & & $\begin{array}{l}\text { Period of } \\
\text { record } \\
\text { (water } \\
\text { years) }\end{array}$ & Water year & $\begin{array}{l}\text { Stage } \\
(\mathrm{ft})\end{array}$ & $\begin{array}{l}\text { Discharge } \\
\left(\mathrm{ft}^{3} / \mathrm{s}\right)\end{array}$ & $\begin{array}{c}\text { Date } \\
\text { (month/ } \\
\text { day/ } \\
\text { year) }\end{array}$ & $\begin{array}{l}\text { Stage } \\
(\mathrm{ft})\end{array}$ & $\begin{array}{l}\text { Discharge } \\
\left(\mathrm{ft}^{3} / \mathrm{s}\right)\end{array}$ & $\begin{array}{c}\text { Regulated } \\
\text { during } \\
\text { flood }^{1}\end{array}$ & $\begin{array}{c}\text { Recurrence } \\
\text { interval } \\
\text { (years) }\end{array}$ \\
\hline 02213350 & $\begin{array}{l}\text { Tobesofkee Creek below Forsyth, } \\
\text { GA }\end{array}$ & 53.4 & $\begin{array}{l}1963-72, \\
1974-87, \\
1990,1994\end{array}$ & 1994 & 11.99 & 13,000 & $7 / 6 / 94$ & 11.99 & 13,000 & $\mathrm{~N}$ & $>100$ \\
\hline 02213400 & $\begin{array}{l}\text { Little Tobesofkee Creek near } \\
\text { Forsyth, GA }\end{array}$ & 16.8 & $\begin{array}{l}1951-61 \\
1990,1994\end{array}$ & 1994 & 11.00 & 4,900 & $7 / 6 / 94$ & 11.00 & 4,900 & $\mathrm{~N}$ & $<25$ \\
\hline 02213470 & $\begin{array}{l}\text { Tobesofkee Creek above Macon, } \\
\text { GA }\end{array}$ & 156 & $\begin{array}{l}\text { 1967-78, } \\
1990,1994\end{array}$ & 1994 & 26.00 & 31,000 & $7 / 6 / 94$ & 26.00 & 31,000 & $\mathrm{~N}$ & $>100$ \\
\hline 02213500 & Tobesofkee Creek near Macon, GA & 182 & $\begin{array}{l}\text { 1929, } \\
1938-98\end{array}$ & 1994 & 39.50 & 54,000 & $7 / 6 / 94$ & 39.50 & 54,000 & $\mathrm{~N}$ & $>100$ \\
\hline 02213700 & $\begin{array}{l}\text { Ocmulgee River near Warner } \\
\text { Robins, GA }\end{array}$ & 2,690 & $\begin{array}{l}1973-74 \\
1976-97\end{array}$ & 1994 & 21.75 & 105,000 & $7 / 8 / 94$ & 21.75 & 105,000 & $\mathrm{~N}$ & $>100$ \\
\hline 02214000 & $\begin{array}{l}\text { Echeconnee Creek near Macon, } \\
\text { GA }\end{array}$ & 147 & $\begin{array}{l}\text { 1938-43, } \\
1951-78, \\
1990,1994\end{array}$ & 1994 & 20.00 & 64,700 & $7 / 6 / 94$ & 20.00 & 64,700 & $\mathrm{~N}$ & $>100$ \\
\hline 02214500 & Big Indian Creek at Perry, GA & 108 & $\begin{array}{l}\text { 1944-77, } \\
1981,1994\end{array}$ & 1994 & 21.00 & 28,000 & $7 / 6 / 94$ & 21.00 & 28,000 & $\mathrm{~N}$ & $>100$ \\
\hline 02214820 & $\begin{array}{l}\text { Mossy Creek at U.S. } 41 \text { near Perry, } \\
\text { GA }\end{array}$ & 92.9 & $\begin{array}{l}\text { 1979-94, } \\
1996-98\end{array}$ & 1994 & 19.86 & 24,000 & $7 / 6 / 94$ & 19.86 & 24,000 & $\mathrm{~N}$ & $>100$ \\
\hline 02215000 & $\begin{array}{l}\text { Ocmulgee River at Hawkinsville, } \\
\text { GA }\end{array}$ & 3,800 & $\begin{array}{l}1877, \\
1909-80, \\
1983-96\end{array}$ & 1994 & 40.91 & 100,000 & $7 / 9 / 94$ & 40.91 & 100,000 & $\mathrm{~N}$ & $>100$ \\
\hline 02215100 & $\begin{array}{l}\text { Tucsawhatchee Creek near } \\
\text { Hawkinsville, GA }\end{array}$ & 163 & 1984-98 & 1998 & 17.56 & 9,240 & $3 / 9 / 98$ & 17.56 & 9,240 & $\mathrm{~N}$ & 100 \\
\hline 02215245 & $\begin{array}{l}\text { Folsom Creek tributary near } \\
\text { Rochelle, GA }\end{array}$ & 1.44 & 1964-98 & 1970 & 7.16 & 434 & $6 / 7 / 95$ & 6.12 & 380 & $\mathrm{~N}$ & 25 \\
\hline 02215260 & Ocmulgee River at Abbeville, GA & 4,460 & $\begin{array}{l}1902, \\
1905-65, \\
1988-96\end{array}$ & 1994 & 23.10 & 100,000 & $7 / 11 / 94$ & 23.10 & 100,000 & $\mathrm{~N}$ & $>100$ \\
\hline
\end{tabular}


Table 11. Maximum stage and discharge for period of record for streamgages having significant floods during 1994-98 water years in Georgia.-Continued

$\left[\mathrm{mi}^{2}\right.$, square miles; $\mathrm{ft}$, feet above an arbitrary datum; $\mathrm{ft}^{3} / \mathrm{s}$, cubic feet per second; --, not determined or not applicable; $>$, greater than; <, less than. Source: Recurrence intervals calculated from U.S. Geological Survey data. Other data from U.S. Geological Survey reports or databases]

\begin{tabular}{|c|c|c|c|c|c|c|c|c|c|c|c|}
\hline \multirow[b]{2}{*}{$\begin{array}{c}\text { Streamgage } \\
\text { number } \\
\text { (fig. 22) }\end{array}$} & \multirow[b]{2}{*}{ Streamgage name } & \multirow[b]{2}{*}{$\begin{array}{c}\text { Total } \\
\text { drainage } \\
\left(\mathrm{mi}^{2}\right)\end{array}$} & \multicolumn{4}{|c|}{$\begin{array}{c}\text { Maximum stage and discharge for period of record } \\
\text { through } 1998 \text { water year }\end{array}$} & \multicolumn{5}{|c|}{ Significant floods 1994-98 water years } \\
\hline & & & $\begin{array}{l}\text { Period of } \\
\text { record } \\
\text { (water } \\
\text { years) }\end{array}$ & Water year & $\begin{array}{l}\text { Stage } \\
(\mathrm{ft})\end{array}$ & $\begin{array}{l}\text { Discharge } \\
\left(\mathrm{ft}^{3} / \mathrm{s}\right)\end{array}$ & $\begin{array}{c}\text { Date } \\
\text { (month/ } \\
\text { day/ } \\
\text { year) }\end{array}$ & $\begin{array}{l}\text { Stage } \\
(\mathrm{ft})\end{array}$ & $\begin{array}{l}\text { Discharge } \\
\left(\mathrm{ft}^{3} / \mathrm{s}\right)\end{array}$ & $\begin{array}{c}\text { Regulated } \\
\text { during } \\
\text { flood }^{1}\end{array}$ & $\begin{array}{c}\text { Recurrence } \\
\text { interval } \\
\text { (years) }\end{array}$ \\
\hline 02215500 & $\begin{array}{l}\text { Ocmulgee River at Lumber City, } \\
\text { GA }\end{array}$ & 5,180 & $\begin{array}{l}1891 \\
1909-98\end{array}$ & 1925 & 25.70 & 98,400 & $7 / 15 / 94$ & 24.59 & 92,900 & $\mathrm{~N}$ & 100 \\
\hline 02215800 & $\begin{array}{l}\text { Gum Qamp Creek near Chauncey, } \\
\text { GA }\end{array}$ & 221 & 1984-98 & $\begin{array}{l}1998 \\
1991\end{array}$ & $\begin{array}{l}9.73 \\
9.91\end{array}$ & $\begin{array}{l}5,810 \\
4,940\end{array}$ & $3 / 9 / 98$ & 9.73 & 5,810 & $\mathrm{~N}$ & $<25$ \\
\hline 02216180 & $\begin{array}{l}\text { Turnpike Creek (U.S. Highway } \\
319 \& 441 \text { ) near McRae, GA }\end{array}$ & 49.2 & 1983-98 & 1998 & 11.82 & 4,980 & $3 / 9 / 98$ & 11.82 & 4,980 & $\mathrm{~N}$ & $>100$ \\
\hline 02217380 & $\begin{array}{l}\text { Mulberry River (Georgia High- } \\
\text { way 11) near Winder, GA }\end{array}$ & 142 & $\begin{array}{l}\text { 1976, } \\
1984-98\end{array}$ & $\begin{array}{l}1998 \\
1984\end{array}$ & $\begin{array}{l}12.73 \\
13.79\end{array}$ & $\begin{array}{l}6,800 \\
5,710\end{array}$ & $2 / 4 / 98$ & 12.73 & 6,800 & $\mathrm{~N}$ & -- \\
\hline 02217905 & $\begin{array}{l}\text { Tanyard Creek at Baxter Street at } \\
\text { Athens, GA }\end{array}$ & .42 & 1979-96 & 1996 & 9.56 & 821 & $7 / 14 / 96$ & 9.56 & 821 & $\mathrm{~N}$ & -- \\
\hline 02223349 & $\begin{array}{l}\text { Big Sandy Creek tributary near } \\
\text { Irwinton, GA }\end{array}$ & .50 & 1977-98 & 1995 & 3.57 & 87 & $2 / 18 / 95$ & 3.57 & 87 & $\mathrm{~N}$ & 25 \\
\hline 02223500 & Oconee River at Dublin, GA & 4,400 & 1894-1998 & 1936 & 33.00 & 96,700 & $3 / 11 / 98$ & 32.00 & 85,000 & $\mathrm{Y}$ & 25 \\
\hline 02225330 & Beaver Creek near Cobbtown, GA & 9.58 & 1965-98 & $\begin{array}{l}1991 \\
1993\end{array}$ & $\begin{array}{l}8.04 \\
8.25\end{array}$ & $\begin{array}{r}2,030 \\
--\end{array}$ & $3 / 9 / 98$ & 6.48 & 1,050 & $\mathrm{~N}$ & 50 \\
\hline 02226000 & $\begin{array}{l}\text { Altamaha River at Doctortown, } \\
\text { GA }\end{array}$ & 13,600 & 1925-98 & 1925 & 18.60 & 300,000 & $3 / 19 / 98$ & 15.55 & 135,000 & $\mathrm{~N}$ & $<25$ \\
\hline 02317500 & Alapaha River at Statenville, GA & 1,400 & 1928-98 & 1948 & 29.80 & 27,300 & $3 / 15 / 98$ & 29.50 & 18,700 & $\mathrm{~N}$ & 25 \\
\hline 02317564 & $\begin{array}{l}\text { Dukes Bay Canal (SR 94) at } \\
\text { Valdosta, GA }\end{array}$ & 1.27 & 1987-96 & 1995 & 7.27 & 383 & $10 / 2 / 94$ & 7.27 & 383 & $\mathrm{~N}$ & -- \\
\hline 023177554 & $\begin{array}{l}\text { Onemile Branch (Wainwright } \\
\text { Drive) at Valdosta, GA }\end{array}$ & 2.66 & 1987-96 & $\begin{array}{l}1995 \\
1987\end{array}$ & $\begin{array}{l}8.54 \\
8.56\end{array}$ & $\begin{array}{l}886 \\
789\end{array}$ & $10 / 2 / 94$ & 8.54 & 886 & $\mathrm{~N}$ & -- \\
\hline 02317810 & $\begin{array}{l}\text { Arnold Creek tributary near Tifton, } \\
\text { GA }\end{array}$ & .47 & $\begin{array}{l}1965-95 \\
1997-98\end{array}$ & 1995 & 5.46 & 219 & $10 / 3 / 94$ & 5.46 & 219 & $\mathrm{~N}$ & -- \\
\hline 02327350 & $\begin{array}{l}\text { Ochlockonee River tributary near } \\
\text { Coolidge, GA }\end{array}$ & 1.81 & 1965-98 & 1965 & 6.14 & 789 & $10 / 2 / 94$ & 5.49 & 706 & $\mathrm{~N}$ & -- \\
\hline 02327415 & $\begin{array}{l}\text { Little Ochlockonee River at State } \\
\text { Route } 111 \text { near Moultrie, GA }\end{array}$ & 44.8 & $\begin{array}{l}1981-94 \\
1996-98\end{array}$ & 1998 & 10.29 & 6,060 & $3 / 9 / 98$ & 10.29 & 6,060 & $\mathrm{~N}$ & $>100$ \\
\hline
\end{tabular}


Table 11. Maximum stage and discharge for period of record for streamgages having significant floods during 1994-98 water years in Georgia.-Continued

$\left[\mathrm{mi}^{2}\right.$, square miles; $\mathrm{ft}$, feet above an arbitrary datum; $\mathrm{ft}^{3} / \mathrm{s}$, cubic feet per second; --, not determined or not applicable; >, greater than; <, less than. Source: Recurrence intervals calculated from U.S Geological Survey data. Other data from U.S. Geological Survey reports or databases]

\begin{tabular}{|c|c|c|c|c|c|c|c|c|c|c|c|}
\hline \multirow[b]{2}{*}{$\begin{array}{l}\text { Streamgage } \\
\text { number } \\
\text { (fig. 22) }\end{array}$} & \multirow[b]{2}{*}{ Streamgage name } & \multirow[b]{2}{*}{$\begin{array}{l}\text { Total } \\
\text { drainage } \\
\left(\mathrm{mi}^{2}\right)\end{array}$} & \multicolumn{4}{|c|}{$\begin{array}{c}\text { Maximum stage and discharge for period of record } \\
\text { through } 1998 \text { water year }\end{array}$} & \multicolumn{5}{|c|}{ Significant floods $1994-98$ water years } \\
\hline & & & $\begin{array}{l}\text { Period of } \\
\text { record } \\
\text { (water } \\
\text { years) }\end{array}$ & Water year & $\begin{array}{l}\text { Stage } \\
(\mathrm{ft})\end{array}$ & $\begin{array}{l}\text { Discharge } \\
\left(\mathrm{ft}^{3} / \mathrm{s}\right)\end{array}$ & $\begin{array}{l}\text { Date } \\
\text { (month/ } \\
\text { day/ } \\
\text { year) }\end{array}$ & $\begin{array}{l}\text { Stage } \\
(\mathrm{ft})\end{array}$ & $\begin{array}{l}\text { Discharge } \\
\left(\mathrm{ft}^{3} / \mathrm{s}\right)\end{array}$ & $\begin{array}{l}\text { Regulated } \\
\text { during } \\
\text { flood }^{1}\end{array}$ & $\begin{array}{l}\text { Recurrence } \\
\text { interval } \\
\text { (years) }\end{array}$ \\
\hline 02327467 & $\begin{array}{l}\text { Oquina Creek (Wolf Street) at } \\
\text { Thomasville, GA }\end{array}$ & 1.07 & $1986-96$ & 1995 & 6.28 & 366 & $10 / 2 / 94$ & 6.28 & 366 & $\mathrm{~N}$ & -- \\
\hline 02327471 & $\begin{array}{l}\text { Bruces Branch (North Hansell } \\
\text { Street) at Thomasville, GA }\end{array}$ & .12 & $1986-95$ & 1994 & 8.26 & 201 & $8 / 16 / 94$ & 8.26 & 201 & $\mathrm{~N}$ & -- \\
\hline 02334430 & $\begin{array}{l}\text { Chattahoochee River at Buford } \\
\text { Dam near Buford, GA }\end{array}$ & 1,040 & $1972-98$ & $\begin{array}{l}1994 \\
1972\end{array}$ & $\begin{array}{l}5.90 \\
7.88\end{array}$ & $\begin{array}{r}12,100 \\
9,670\end{array}$ & $10 / 25 / 93$ & 5.90 & 12,100 & $\mathrm{Y}$ & -- \\
\hline 02334885 & $\begin{array}{l}\text { Suwanee Creek at U.S. Route } 23 \text { at } \\
\text { Suwanee, GA }\end{array}$ & 46.8 & $1985-98$ & 1996 & 12.04 & 4,350 & $10 / 5 / 95$ & 12.04 & 4,350 & $\mathrm{~N}$ & -- \\
\hline 02335347 & $\begin{array}{l}\text { Crooked Creek tributary \#2 } \\
\text { (Holcomb Bridge Road) near } \\
\text { Norcross, GA }\end{array}$ & .19 & 1987-98 & 1996 & 5.98 & 260 & $10 / 5 / 95$ & 5.98 & 260 & $\mathrm{~N}$ & -- \\
\hline 02340250 & $\begin{array}{l}\text { Flat Shoal Creek (Georgia } \\
\text { Highway 18) near West Point, } \\
\text { GA }\end{array}$ & 204 & $\begin{array}{l}\text { 1948-49, } \\
1961,1969, \\
1971,1981 \\
1984-98\end{array}$ & 1996 & 22.95 & 8,170 & $3 / 8 / 96$ & 22.95 & 8,170 & $\mathrm{~N}$ & $<10$ \\
\hline 02343200 & Pataula Creek near Lumpkin, GA & 70.0 & $\begin{array}{l}1949-78 \\
1990,1994\end{array}$ & 1994 & 12.30 & 17,500 & $7 / 6 / 94$ & 12.30 & 17,500 & $\mathrm{~N}$ & $>100$ \\
\hline 02343225 & $\begin{array}{l}\text { Pataula Creek near Georgetown, } \\
\text { GA }\end{array}$ & 295 & $\begin{array}{l}1949, \\
1951-78 \\
1990,1994\end{array}$ & 1994 & 14.30 & 65,000 & $7 / 6 / 94$ & 14.30 & 65,000 & $\mathrm{~N}$ & $>100$ \\
\hline 02343244 & $\begin{array}{l}\text { Cemochechobee Creek (S 1576) } \\
\text { near Coleman, GA }\end{array}$ & 15.3 & 1984-98 & 1994 & 11.84 & 5,160 & $7 / 4 / 94$ & 11.84 & 5,160 & $\mathrm{~N}$ & $>100$ \\
\hline 02343267 & $\begin{array}{l}\text { Temple Creek at State Route } 39 \\
\text { near Blakely, GA }\end{array}$ & 2.78 & $1978-98$ & 1994 & 6.13 & 746 & $7 / 6 / 94$ & 6.13 & 746 & $\mathrm{~N}$ & $>100$ \\
\hline 02343801 & $\begin{array}{l}\text { Chattahoochee River near } \\
\text { Columbia, AL }\end{array}$ & 8,210 & $1975-98$ & 1994 & 123.98 & 202,000 & 7/7/94 & 123.98 & 202,000 & $\mathrm{Y}$ & -- \\
\hline 02344300 & Camp Creek near Fayetteville, GA & 17.2 & $\begin{array}{l}1961-73, \\
1994\end{array}$ & 1994 & 13.89 & 6,300 & $7 / 5 / 94$ & 13.89 & 6,300 & $\mathrm{~N}$ & $>100$ \\
\hline
\end{tabular}


Table 11. Maximum stage and discharge for period of record for streamgages having significant floods during 1994-98 water years in Georgia.-Continued

$\left[\mathrm{mi}^{2}\right.$, square miles; $\mathrm{ft}$, feet above an arbitrary datum; $\mathrm{ft}^{3} / \mathrm{s}$, cubic feet per second; --, not determined or not applicable; $>$, greater than; <, less than. Source: Recurrence intervals calculated from U.S. Geological Survey data. Other data from U.S. Geological Survey reports or databases]

\begin{tabular}{|c|c|c|c|c|c|c|c|c|c|c|c|}
\hline \multirow{2}{*}{$\begin{array}{l}\text { Streamgage } \\
\text { number } \\
\text { (fig. 22) }\end{array}$} & \multirow[b]{2}{*}{ Streamgage name } & \multirow{2}{*}{$\begin{array}{l}\text { Total } \\
\text { drainage } \\
\left(\mathrm{mi}^{2}\right)\end{array}$} & \multicolumn{4}{|c|}{$\begin{array}{c}\text { Maximum stage and discharge for period of record } \\
\text { through } 1998 \text { water year }\end{array}$} & \multicolumn{5}{|c|}{ Significant floods $1994-98$ water years } \\
\hline & & & $\begin{array}{l}\text { Period of } \\
\text { record } \\
\text { (water } \\
\text { years) }\end{array}$ & Water year & $\begin{array}{l}\text { Stage } \\
(\mathrm{ft})\end{array}$ & $\begin{array}{l}\text { Discharge } \\
\left(\mathrm{ft}^{3} / \mathrm{s}\right)\end{array}$ & $\begin{array}{l}\text { Date } \\
\text { (month/ } \\
\text { day/ } \\
\text { year) }\end{array}$ & $\begin{array}{l}\text { Stage } \\
\text { (ft) }\end{array}$ & $\begin{array}{l}\text { Discharge } \\
\left(\mathrm{ft}^{3} / \mathrm{s}\right)\end{array}$ & $\begin{array}{l}\text { Regulated } \\
\text { during } \\
\text { flood }^{1}\end{array}$ & $\begin{array}{l}\text { Recurrence } \\
\text { interval } \\
\text { (years) }\end{array}$ \\
\hline 02344350 & Flint River near Lovejoy, GA & 130 & 1986-98 & 1994 & 23.60 & 19,000 & $7 / 5 / 94$ & 23.60 & 19,000 & $\mathrm{~N}$ & $>100$ \\
\hline 02344500 & Flint River near Griffin, GA & 272 & $\begin{array}{l}\text { 1929, } \\
1937-98\end{array}$ & 1994 & 24.22 & 31,500 & $7 / 6 / 94$ & 24.22 & 31,500 & $\mathrm{~N}$ & $>100$ \\
\hline 02344700 & Line Creek near Senoia, GA & 101 & $1965-98$ & 1994 & 20.10 & 28,400 & $7 / 5 / 94$ & 20.10 & 28,400 & $\mathrm{~N}$ & $>100$ \\
\hline 02346180 & Flint River near Thomaston, GA & 1,220 & $\begin{array}{l}1900-27, \\
1929, \\
1939-50, \\
1952-56, \\
1961, \\
1967-94\end{array}$ & $\begin{array}{l}1929 \\
1994\end{array}$ & 21.83 & $\begin{array}{l}62,000 \\
55,000\end{array}$ & $7 / 7 / 94$ & 21.83 & 55,000 & $\mathrm{~N}$ & 100 \\
\hline 02346500 & Potato Creek near Thomaston, GA & 186 & $\begin{array}{l}1938-73 \\
1990,1994\end{array}$ & 1994 & 12.00 & 28,000 & $7 / 6 / 94$ & 12.00 & 28,000 & $\mathrm{~N}$ & $>100$ \\
\hline 02347500 & Flint River near Culloden, GA & 1,850 & $\begin{array}{l}1913-27 \\
1929-31 \\
1937-98\end{array}$ & 1994 & 45.73 & 100,000 & $7 / 6 / 94$ & 45.73 & 100,000 & $\mathrm{~N}$ & $>100$ \\
\hline 02348300 & Patsiliga Creek near Reynolds, GA & 139 & $\begin{array}{l}1963-84, \\
1994\end{array}$ & $\begin{array}{l}1994 \\
1964\end{array}$ & $-\overline{9} 9.09$ & $\begin{array}{r}25,000 \\
3,320\end{array}$ & $7 / 6 / 94$ & -- & 25,000 & $\mathrm{~N}$ & $>100$ \\
\hline 02348485 & $\begin{array}{l}\text { Whitewater Creek at Georgia } 137 \\
\text { near Butler, GA }\end{array}$ & 17.3 & 1979-98 & 1994 & 10.78 & 518 & $7 / 5 / 94$ & 10.78 & 518 & $\mathrm{~N}$ & $<50$ \\
\hline 02349000 & $\begin{array}{l}\text { Whitewater Creek below } \\
\text { Rambulette Creek near Butler, } \\
\text { GA }\end{array}$ & 93.4 & $\begin{array}{l}\text { 1944-77, } \\
1981,1994\end{array}$ & 1957 & 7.01 & 2,160 & $7 / 6 / 94$ & 7.00 & 2,150 & $\mathrm{~N}$ & $<50$ \\
\hline 02349030 & $\begin{array}{l}\text { Cedar Creek at U.S. } 19 \text { near } \\
\text { Rupert, GA }\end{array}$ & 41.1 & 1979-98 & 1994 & 7.50 & 2,400 & $7 / 6 / 94$ & 7.50 & 2,400 & $\mathrm{~N}$ & $>100$ \\
\hline 02349350 & $\begin{array}{l}\text { Buck Creek at U.S. } 19 \text { near } \\
\text { Ellaville, GA }\end{array}$ & 146 & 1979-98 & 1994 & 11.31 & 7,800 & $7 / 6 / 94$ & 11.31 & 7,800 & $\mathrm{~N}$ & 100 \\
\hline 02349500 & Flint River at Montezuma, GA & 2,900 & $\begin{array}{l}\text { 1897, } \\
1905-98\end{array}$ & 1994 & 34.11 & 136,000 & $7 / 8 / 94$ & 34.11 & 136,000 & $\mathrm{~N}$ & $>100$ \\
\hline
\end{tabular}


Table 11. Maximum stage and discharge for period of record for streamgages having significant floods during 1994-98 water years in Georgia.-Continued

$\left[\mathrm{mi}^{2}\right.$, square miles; $\mathrm{ft}$, feet above an arbitrary datum; $\mathrm{ft}^{3} / \mathrm{s}$, cubic feet per second; --, not determined or not applicable; >, greater than; <, less than. Source: Recurrence intervals calculated from U.S Geological Survey data. Other data from U.S. Geological Survey reports or databases]

\begin{tabular}{|c|c|c|c|c|c|c|c|c|c|c|c|}
\hline \multirow[b]{2}{*}{$\begin{array}{c}\text { Streamgage } \\
\text { number } \\
\text { (fig. 22) }\end{array}$} & \multirow[b]{2}{*}{ Streamgage name } & \multirow[b]{2}{*}{$\begin{array}{c}\text { Total } \\
\text { drainage } \\
\left(\mathrm{mi}^{2}\right)\end{array}$} & \multicolumn{4}{|c|}{$\begin{array}{c}\text { Maximum stage and discharge for period of record } \\
\text { through } 1998 \text { water year }\end{array}$} & \multicolumn{5}{|c|}{ Significant floods $1994-98$ water years } \\
\hline & & & $\begin{array}{l}\text { Period of } \\
\text { record } \\
\text { (water } \\
\text { years) }\end{array}$ & Water year & $\begin{array}{l}\text { Stage } \\
(\mathrm{ft})\end{array}$ & $\begin{array}{l}\text { Discharge } \\
\left(\mathrm{ft}^{3} / \mathrm{s}\right)\end{array}$ & $\begin{array}{c}\text { Date } \\
\text { (month/ } \\
\text { day/ } \\
\text { year) }\end{array}$ & $\begin{array}{l}\text { Stage } \\
(\mathrm{ft})\end{array}$ & $\begin{array}{l}\text { Discharge } \\
\left(\mathrm{ft}^{3} / \mathrm{s}\right)\end{array}$ & $\begin{array}{c}\text { Regulated } \\
\text { during } \\
\text { flood }^{1}\end{array}$ & $\begin{array}{c}\text { Recurrence } \\
\text { interval } \\
\text { (years) }\end{array}$ \\
\hline 02349695 & $\begin{array}{l}\text { Horsehead Creek at State Route } \\
224 \text { near Montezuma, GA }\end{array}$ & .72 & $1977-98$ & 1994 & 6.96 & 200 & $7 / 6 / 94$ & 6.96 & 200 & $\mathrm{~N}$ & 25 \\
\hline 02349900 & Turkey Creek at Byromville, GA & 45.0 & $1951-98$ & 1994 & 14.29 & 5,820 & $7 / 6 / 94$ & 14.29 & 5,820 & $\mathrm{~N}$ & 100 \\
\hline 02350512 & $\begin{array}{l}\text { Flint River at State Highway } 32 \\
\text { near Oakfield, GA }\end{array}$ & 3,880 & 1988-98 & 1994 & 40.10 & 112,000 & $7 / 10 / 94$ & 40.10 & 112,000 & $\mathrm{~N}$ & $>100$ \\
\hline 02350520 & $\begin{array}{l}\text { Abrams Creek tributary near } \\
\text { Doles, GA }\end{array}$ & 3.77 & $\begin{array}{l}1965-75 \\
1991,1994\end{array}$ & 1994 & 7.06 & 840 & $7 / 6 / 94$ & 7.06 & 840 & $\mathrm{~N}$ & $>100$ \\
\hline 02350600 & $\begin{array}{l}\text { Kinchafoonee Creek at Preston, } \\
\text { GA }\end{array}$ & 197 & $\begin{array}{l}\text { 1943, } \\
\text { 1948-78, } \\
1987-98\end{array}$ & 1990 & 12.16 & 14,500 & $7 / 6 / 94$ & 11.66 & 12,400 & $\mathrm{~N}$ & 100 \\
\hline 02350685 & $\begin{array}{l}\text { Choctahatchee Creek tributary at } \\
\text { U.S. } 280 \text { near Plains, GA }\end{array}$ & .32 & $\begin{array}{l}1977-79 \\
1981-98\end{array}$ & 1994 & 9.25 & 625 & $7 / 6 / 94$ & 9.25 & 625 & $\mathrm{~N}$ & $>100$ \\
\hline 02350900 & $\begin{array}{l}\text { Kinchafoonee Creek near } \\
\text { Dawson, GA }\end{array}$ & 527 & $\begin{array}{l}1943, \\
1948-66, \\
1973, \\
1985-98\end{array}$ & 1994 & 26.56 & 29,500 & 7/7/94 & 26.56 & 29,500 & $\mathrm{~N}$ & $>100$ \\
\hline 02351500 & $\begin{array}{l}\text { Muckalee Creek near Americus, } \\
\text { GA }\end{array}$ & 140 & $\begin{array}{l}1948, \\
1963-83, \\
1994\end{array}$ & 1994 & 19.50 & 33,500 & $7 / 6 / 94$ & 19.50 & 33,500 & $\mathrm{~N}$ & $>100$ \\
\hline 02351700 & $\begin{array}{l}\text { Muckalee Creek near Smithville, } \\
\text { GA }\end{array}$ & 265 & $\begin{array}{l}\text { 1929, 1948, } \\
1951-66, \\
1994\end{array}$ & 1994 & 22.72 & 35,000 & $7 / 6 / 94$ & 22.72 & 35,000 & $\mathrm{~N}$ & $>100$ \\
\hline 02351890 & $\begin{array}{l}\text { Muckalee Creek at State Route } 195 \\
\text { near Leesburg, GA }\end{array}$ & 362 & $\begin{array}{l}1943,1948, \\
1980-98\end{array}$ & $\begin{array}{l}1994 \\
1948\end{array}$ & $\begin{array}{r}29.10 \\
234.66\end{array}$ & $\begin{array}{l}64,400 \\
15,000\end{array}$ & 7/7/94 & 29.10 & 64,400 & $\mathrm{~N}$ & $>100$ \\
\hline 02351900 & $\begin{array}{l}\text { Muckalee Creek near Leesburg, } \\
\text { GA }\end{array}$ & 405 & $\begin{array}{l}1943,1948, \\
1951-65, \\
1980-86, \\
1994\end{array}$ & 1994 & 29.83 & 72,000 & 7/7/94 & 29.83 & 72,000 & $\mathrm{~N}$ & $>100$ \\
\hline
\end{tabular}


Table 11. Maximum stage and discharge for period of record for streamgages having significant floods during 1994-98 water years in Georgia.-Continued

$\left[\mathrm{mi}^{2}\right.$, square miles; $\mathrm{ft}$, feet above an arbitrary datum; $\mathrm{ft}^{3} / \mathrm{s}$, cubic feet per second; --, not determined or not applicable; $>$, greater than; <, less than. Source: Recurrence intervals calculated from U.S. Geological Survey data. Other data from U.S. Geological Survey reports or databases]

\begin{tabular}{|c|c|c|c|c|c|c|c|c|c|c|c|}
\hline \multirow{2}{*}{$\begin{array}{l}\text { Streamgage } \\
\text { number } \\
\text { (fig. 22) }\end{array}$} & \multirow[b]{2}{*}{ Streamgage name } & \multirow{2}{*}{$\begin{array}{c}\text { Total } \\
\text { drainage } \\
\left(\mathrm{mi}^{2}\right)\end{array}$} & \multicolumn{4}{|c|}{$\begin{array}{c}\text { Maximum stage and discharge for period of record } \\
\text { through } 1998 \text { water year }\end{array}$} & \multicolumn{5}{|c|}{ Significant floods $1994-98$ water years } \\
\hline & & & $\begin{array}{l}\text { Period of } \\
\text { record } \\
\text { (water } \\
\text { years) }\end{array}$ & Water year & $\begin{array}{l}\text { Stage } \\
(\mathrm{ft})\end{array}$ & $\begin{array}{l}\text { Discharge } \\
\left(\mathrm{ft}^{3} / \mathrm{s}\right)\end{array}$ & $\begin{array}{c}\text { Date } \\
\text { (month/ } \\
\text { day/ } \\
\text { year) }\end{array}$ & $\begin{array}{l}\text { Stage } \\
(\mathrm{ft})\end{array}$ & $\begin{array}{l}\text { Discharge } \\
\left(\mathrm{ft}^{3} / \mathrm{s}\right)\end{array}$ & $\begin{array}{l}\text { Regulated } \\
\text { during } \\
\text { flood }^{1}\end{array}$ & $\begin{array}{c}\text { Recurrence } \\
\text { interval } \\
\text { (years) }\end{array}$ \\
\hline 02352500 & Flint River at Albany, GA & 5,310 & $1893-1998$ & 1994 & 43.00 & 120,000 & $\begin{array}{l}7 / 11 / 94 \\
3 / 10 / 98\end{array}$ & $\begin{array}{l}43.00 \\
36.92\end{array}$ & $\begin{array}{r}120,000 \\
86,100\end{array}$ & $\begin{array}{l}\mathrm{N} \\
\mathrm{N}\end{array}$ & $\begin{array}{r}>100 \\
90\end{array}$ \\
\hline 02352605 & $\begin{array}{l}\text { Emily Avenue Canal at Albany, } \\
\text { GA }\end{array}$ & .16 & 1987-96 & $\begin{array}{l}1994 \\
1990\end{array}$ & 2.85 & 112 & $8 / 16 / 94$ & $\begin{array}{l}2.85 \\
2.94\end{array}$ & $\begin{array}{r}112 \\
71\end{array}$ & $\mathrm{~N}$ & -- \\
\hline 02352964 & $\begin{array}{l}\text { Percosin Creek tributary at Deans } \\
\text { Drive at Albany, GA }\end{array}$ & .05 & 1987-96 & 1995 & 3.66 & 20 & $7 / 26 / 95$ & 3.66 & 20 & $\mathrm{~N}$ & -- \\
\hline 02353000 & Flint River at Newton, GA & 5,740 & $\begin{array}{l}\text { 1925, 1929, } \\
1938-98\end{array}$ & 1994 & 45.25 & 100,000 & $7 / 13 / 94$ & 45.25 & 100,000 & $\mathrm{~N}$ & $>100$ \\
\hline 02353400 & Pachitla Creek near Edison, GA & 188 & $\begin{array}{l}\text { 1916, 1928, } \\
1948, \\
\text { 1950-78, } \\
1981, \\
1989-98\end{array}$ & 1994 & 14.22 & 43,000 & $\begin{array}{l}7 / 6 / 94 \\
3 / 9 / 98\end{array}$ & $\begin{array}{l}14.22 \\
11.85\end{array}$ & $\begin{array}{l}43,000 \\
17,500\end{array}$ & $\begin{array}{l}\mathrm{N} \\
\mathrm{N}\end{array}$ & $\begin{array}{l}>100 \\
>100\end{array}$ \\
\hline 02353500 & $\begin{array}{l}\text { Ichawaynochaway Creek at } \\
\text { Milford, GA }\end{array}$ & 620 & $\begin{array}{l}\text { 1906-07, } \\
1916,1925, \\
1940-98\end{array}$ & 1994 & 23.20 & 53,000 & $\begin{array}{l}7 / 7 / 94 \\
3 / 9 / 98\end{array}$ & $\begin{array}{l}23.20 \\
19.59\end{array}$ & $\begin{array}{l}53,000 \\
22,800\end{array}$ & $\begin{array}{l}\mathrm{N} \\
\mathrm{N}\end{array}$ & $\begin{array}{l}>100 \\
>100\end{array}$ \\
\hline 02354500 & $\begin{array}{l}\text { Chickasawhatchee Creek at } \\
\text { Elmodel, GA }\end{array}$ & 320 & $\begin{array}{l}1916, \\
1940-49, \\
1952-65, \\
1970-83, \\
1994, \\
1996-98\end{array}$ & 1994 & 20.00 & 16,000 & $7 / 8 / 94$ & 20.00 & 16,000 & $\mathrm{~N}$ & $>100$ \\
\hline 02356000 & Flint River at Bainbridge, GA & 7,570 & $\begin{array}{l}1897 \\
1905-96\end{array}$ & $\begin{array}{l}1994 \\
1925\end{array}$ & $\begin{array}{l}37.20 \\
40.90\end{array}$ & $\begin{array}{l}108,000 \\
101,000\end{array}$ & $7 / 14 / 94$ & 37.20 & 108,000 & $\mathrm{~N}$ & $>100$ \\
\hline 02356640 & $\begin{array}{l}\text { Spring Creek at U.S. } 27 \text { at } \\
\text { Colquitt, GA }\end{array}$ & 281 & $\begin{array}{l}\text { 1981-95, } \\
1997-98\end{array}$ & 1998 & 15.91 & 20,500 & $3 / 10 / 98$ & 15.91 & 20,500 & $\mathrm{~N}$ & $>100$ \\
\hline 02357000 & Spring Creek near Iron City, GA & 485 & $\begin{array}{l}1938-78 \\
1983-98\end{array}$ & 1998 & 24.40 & 34,500 & $3 / 10 / 98$ & 24.40 & 34,500 & $\mathrm{~N}$ & $>100$ \\
\hline
\end{tabular}


Table 11. Maximum stage and discharge for period of record for streamgages having significant floods during 1994-98 water years in Georgia.-Continued

$\left[\mathrm{mi}^{2}\right.$, square miles; $\mathrm{ft}$, feet above an arbitrary datum; $\mathrm{ft}^{3} / \mathrm{s}$, cubic feet per second; --, not determined or not applicable; >, greater than; <, less than. Source: Recurrence intervals calculated from U.S Geological Survey data. Other data from U.S. Geological Survey reports or databases]

\begin{tabular}{|c|c|c|c|c|c|c|c|c|c|c|c|}
\hline \multirow{2}{*}{$\begin{array}{l}\text { Streamgage } \\
\text { number } \\
\text { (fig. 22) }\end{array}$} & \multirow[b]{2}{*}{ Streamgage name } & \multirow{2}{*}{$\begin{array}{c}\text { Total } \\
\text { drainage } \\
\left(\mathrm{mi}^{2}\right)\end{array}$} & \multicolumn{4}{|c|}{$\begin{array}{c}\text { Maximum stage and discharge for period of record } \\
\text { through } 1998 \text { water year }\end{array}$} & \multicolumn{5}{|c|}{ Significant floods 1994-98 water years } \\
\hline & & & $\begin{array}{l}\text { Period of } \\
\text { record } \\
\text { (water } \\
\text { years) }\end{array}$ & Water year & $\begin{array}{l}\text { Stage } \\
\text { (ft) }\end{array}$ & $\begin{array}{c}\text { Discharge } \\
\left(\mathrm{ft}^{3} / \mathrm{s}\right)\end{array}$ & $\begin{array}{c}\text { Date } \\
\text { (month/ } \\
\text { day/ } \\
\text { year) }\end{array}$ & $\begin{array}{l}\text { Stage } \\
(\mathrm{ft})\end{array}$ & $\begin{array}{c}\text { Discharge } \\
\left(\mathrm{ft}^{3} / \mathrm{s}\right)\end{array}$ & $\begin{array}{l}\text { Regulated } \\
\text { during } \\
\text { flood }^{1}\end{array}$ & $\begin{array}{c}\text { Recurrence } \\
\text { interval } \\
\text { (years) }\end{array}$ \\
\hline 02384500 & Conasauga River near Eton, GA & 252 & $1954-58$ & 1990 & 20.50 & 33,200 & $3 / 28 / 94$ & 20.52 & 30,000 & $\mathrm{~N}$ & 100 \\
\hline 02387000 & Conasauga River at Tilton, GA & 687 & $\begin{array}{l}1886 \\
1938-98\end{array}$ & 1886 & 34.00 & 40,000 & $3 / 29 / 94$ & 28.42 & 29,100 & $\mathrm{~N}$ & 25 \\
\hline 02395120 & $\begin{array}{l}\text { Two Run Creek at State Route } 20 \\
\text { near Kingston, GA }\end{array}$ & 33.1 & 1981-98 & 1996 & 8.42 & 3,960 & $1 / 27 / 96$ & 8.42 & 3,960 & $\mathrm{~N}$ & $>10$ \\
\hline 02395990 & $\begin{array}{l}\text { Etowah River tributary at Atteiram } \\
\text { Drive at Rome, GA }\end{array}$ & .37 & 1979-97 & 1997 & 7.53 & 206 & $6 / 28 / 97$ & 7.53 & 206 & $\mathrm{~N}$ & -- \\
\hline 03544947 & Brier Creek near Hiawassee, GA & 1.67 & 1984-98 & 1998 & 4.18 & 990 & $1 / 7 / 98$ & 4.18 & 990 & $\mathrm{~N}$ & $>10$ \\
\hline 03545000 & Hiawassee River at Presley, GA & 45.5 & $1942-98$ & 1952 & 15.24 & 5,700 & $1 / 7 / 98$ & 11.76 & 4,880 & $\mathrm{~N}$ & $>10$ \\
\hline
\end{tabular}

${ }^{1}$ Regulated during flood: N, no; Y, yes. 


\section{Hawaii}

Excessive rains with 8- to 15 -inch totals in 24 hours (National Oceanic and Atmospheric Administration, 1994a) caused localized flash flooding on March 24, 1994, on Maui (fig. 23). Two people were presumed drowned when they were swept away from their vehicle attempting to cross Kauaula Stream.

Night and early morning thundershowers over Oahu on April 12, 1994, caused localized street flooding in central and northern Oahu. As much as 6 inches of rainfall were reported at Wahaiwa (National Oceanic and Atmospheric Administration, 1994a). A group of boy scouts was surprised by a rapid water rise of 2 to 4 feet while walking near a nearly dry creek near Laie, and one was swept away and drowned.

Moisture transported onto the Big Island of Hawaii from the outer shower bands of a tropical depression and enhanced by upslope flow produced intense showers and thunderstorms on August 12, 1994. While extreme runoff occurred from 7 to 15 inches of rainfall in Hilo and the Puna District, greater rainfall likely occurred at ungaged, higher elevations (National Oceanic and Atmospheric Administration, 1994a). Flooding was characterized by some Hilo residents as the worst in more than 40 years. Of an estimated $\$ 3.4$ million in total damage, roads, culverts, bridges, and businesses accounted for $\$ 1.8$ million. Two homes were destroyed, 14 suffered major damage, and 200 had minor damage (National Oceanic and Atmospheric Administration, 1994b). Streamgages with significant floods are listed in table 12 .

\section{References}

National Oceanic and Atmospheric Administration (NOAA), 1994a, Climatological data (by State): Asheville, North Carolina, National Climatic Data Center, various months. National Oceanic and Admospheric Administration (NOAA), 1994b, Storm data (by State): Asheville, North Carolina, National Climatic Data Center, various months.

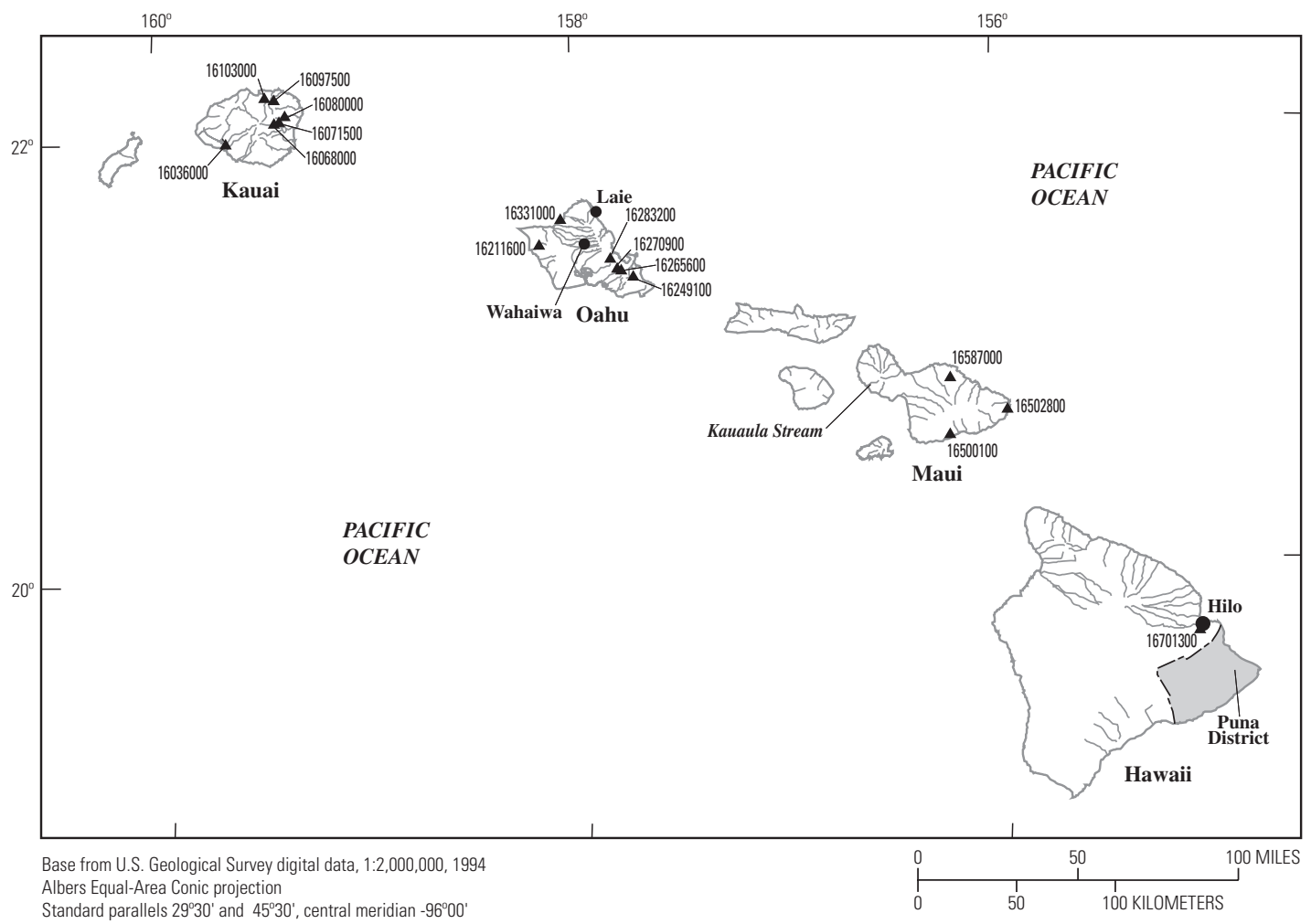

EXPLANATION

$1629100 \triangle$ Streamgage and number

Figure 23. Location of streamgages with significant floods during 1994-98 water years for Hawaii. 
Table 12. Maximum stage and discharge for period of record for streamgages having significant floods during 1994-98 water years in Hawaii.

$\left[\mathrm{mi}^{2}\right.$, square miles; ft, feet above an arbitrary datum; $\mathrm{ft}^{3} / \mathrm{s}$, cubic feet per second; >, greater than. Source: Recurrence intervals calculated from U.S. Geological Survey data. Other data from U.S. Geological Survey reports or databases]

\begin{tabular}{|c|c|c|c|c|c|c|c|c|c|c|c|}
\hline \multirow{2}{*}{$\begin{array}{c}\text { Streamgage } \\
\text { number } \\
\text { (fig. 23) }\end{array}$} & \multirow[b]{2}{*}{ Streamgage name } & \multirow{2}{*}{$\begin{array}{c}\text { Total } \\
\text { drainage } \\
\left(\mathrm{mi}^{2}\right)\end{array}$} & \multicolumn{4}{|c|}{$\begin{array}{l}\text { Maximum stage and discharge for period of record } \\
\text { through } 1998 \text { water year }\end{array}$} & \multicolumn{5}{|c|}{ Significant floods $1994-98$ water years } \\
\hline & & & $\begin{array}{l}\text { Period of } \\
\text { record } \\
\text { (water } \\
\text { years) }\end{array}$ & Water year & $\begin{array}{l}\text { Stage } \\
(\mathrm{ft})\end{array}$ & $\begin{array}{l}\text { Discharge } \\
\left(\mathrm{ft}^{3} / \mathrm{s}\right)\end{array}$ & $\begin{array}{c}\text { Date } \\
\text { (month/ } \\
\text { day/ } \\
\text { year) }\end{array}$ & $\begin{array}{l}\text { Stage } \\
(\mathrm{ft})\end{array}$ & $\begin{array}{l}\text { Discharge } \\
\left(\mathrm{ft}^{3} / \mathrm{s}\right)\end{array}$ & $\begin{array}{l}\text { Regulated } \\
\text { during } \\
\text { flood }^{1}\end{array}$ & $\begin{array}{l}\text { Recurrence } \\
\text { interval } \\
\text { (years) }\end{array}$ \\
\hline 16036000 & $\begin{array}{l}\text { Makaweli River near Waimea, } \\
\text { Kauai, HI }\end{array}$ & 26.0 & $\begin{array}{l}1943, \\
1945-98\end{array}$ & 1975 & 15.51 & 26,000 & $11 / 3 / 95$ & 13.80 & 21,500 & $\mathrm{~N}$ & 20 \\
\hline 16068000 & $\begin{array}{l}\text { East Branch of North Fork Wailua } \\
\text { River near Lihue, Kauai, HI }\end{array}$ & 6.27 & $1916-98$ & 1956 & 14.70 & 18,400 & $\begin{array}{l}2 / 13 / 94 \\
11 / 3 / 95\end{array}$ & $\begin{array}{r}11.70 \\
9.53\end{array}$ & $\begin{array}{r}12,300 \\
7,650\end{array}$ & $\begin{array}{l}\mathrm{N} \\
\mathrm{N}\end{array}$ & $\begin{array}{r}>100 \\
20\end{array}$ \\
\hline 16071500 & $\begin{array}{l}\text { Left Branch Opaekaa Stream near } \\
\text { Kapaa, Kauai, HI }\end{array}$ & .65 & 1961-98 & $\begin{array}{l}1992 \\
1969\end{array}$ & $\begin{array}{l}6.60 \\
7.72\end{array}$ & $\begin{array}{r}1,060 \\
221\end{array}$ & $2 / 13 / 94$ & 6.50 & 1,020 & $\mathrm{~N}$ & 35 \\
\hline 16080000 & $\begin{array}{l}\text { Kapaa Stream at Kapahi Ditch } \\
\text { intake near Kapaa, Kauai, HI }\end{array}$ & 3.86 & $\begin{array}{l}1937, \\
1939-40, \\
1942, \\
1944-75, \\
1977-96\end{array}$ & 1992 & 5.66 & 9,660 & 2/13/94 & 5.14 & 7,580 & $\mathrm{~N}$ & 40 \\
\hline 16097500 & $\begin{array}{l}\text { Halaulani Stream at altitude } \\
400 \text { feet near Kilauea, Kauai, HI }\end{array}$ & 1.90 & 1958-98 & 1994 & 9.76 & 4,140 & 2/13/94 & 9.76 & 4,140 & $\mathrm{~N}$ & 50 \\
\hline 16103000 & $\begin{array}{l}\text { Hanalei River near Hanalei, Kauai, } \\
\text { HI }\end{array}$ & 19.1 & 1962-98 & 1996 & 15.81 & 44,600 & $\begin{array}{l}9 / 30 / 95 \\
11 / 3 / 95\end{array}$ & $\begin{array}{l}14.66 \\
15.81\end{array}$ & $\begin{array}{l}26,600 \\
44,600\end{array}$ & $\begin{array}{l}\mathrm{N} \\
\mathrm{N}\end{array}$ & $\begin{array}{r}5 \\
50\end{array}$ \\
\hline 16211600 & $\begin{array}{l}\text { Makaha Stream near Makaha, } \\
\text { Oahu, HI }\end{array}$ & 2.31 & 1960-98 & 1997 & 9.54 & 2,680 & $11 / 14 / 96$ & 9.54 & 2,680 & $\mathrm{~N}$ & $>100$ \\
\hline 16249100 & $\begin{array}{l}\text { Kaelepulu Stream tributary at } \\
\text { Kailua, Oahu, HI }\end{array}$ & .16 & 1963-98 & 1988 & 7.53 & 467 & $1 / 25 / 96$ & 7.04 & 423 & $\mathrm{~N}$ & 10 \\
\hline 16265600 & $\begin{array}{l}\text { Right Branch of Kamooalii Stream } \\
\text { near Kaneohe, Oahu, HI }\end{array}$ & 1.11 & 1985-97 & $\begin{array}{l}1996 \\
1988\end{array}$ & $\begin{array}{l}11.64 \\
12.40\end{array}$ & $\begin{array}{l}2,800 \\
1,310\end{array}$ & $1 / 25 / 96$ & 11.64 & 2,800 & $\mathrm{~N}$ & $>50$ \\
\hline 16270900 & $\begin{array}{l}\text { Luluku Stream at altitude } 220 \text { feet } \\
\text { near Kaneohe, Oahu, HI }\end{array}$ & .44 & $\begin{array}{l}1967-83, \\
1985-87, \\
1989-98\end{array}$ & $\begin{array}{l}1996 \\
1971\end{array}$ & $\begin{array}{l}4.10 \\
6.18\end{array}$ & $\begin{array}{l}957 \\
651\end{array}$ & $1 / 25 / 96$ & 4.10 & 957 & $\mathrm{~N}$ & 60 \\
\hline
\end{tabular}


Table 12. Maximum stage and discharge for period of record for streamgages having significant floods during 1994-98 water years in Hawaii-Continued.

$\left[\mathrm{mi}^{2}\right.$, square miles; ft, feet above an arbitrary datum; $\mathrm{ft}^{3} / \mathrm{s}$, cubic feet per second; >, greater than. Source: Recurrence intervals calculated from U.S. Geological Survey data. Other data from U.S. Geological Survey reports or databases]

\begin{tabular}{|c|c|c|c|c|c|c|c|c|c|c|c|}
\hline \multirow{2}{*}{$\begin{array}{l}\text { Streamgage } \\
\text { number } \\
\text { (fig. 23) }\end{array}$} & \multirow[b]{2}{*}{ Streamgage name } & \multirow{2}{*}{$\begin{array}{c}\text { Total } \\
\text { drainage } \\
\left(\mathrm{mi}^{2}\right)\end{array}$} & \multicolumn{4}{|c|}{$\begin{array}{c}\text { Maximum stage and discharge for period of record } \\
\text { through } 1998 \text { water year }\end{array}$} & \multicolumn{5}{|c|}{ Significant floods $1994-98$ water years } \\
\hline & & & $\begin{array}{l}\text { Period of } \\
\text { record } \\
\text { (water } \\
\text { years) }\end{array}$ & Water year & $\begin{array}{l}\text { Stage } \\
(\mathrm{ft})\end{array}$ & $\begin{array}{l}\text { Discharge } \\
\left(\mathrm{ft}^{3} / \mathrm{s}\right)\end{array}$ & $\begin{array}{c}\text { Date } \\
\text { (month/ } \\
\text { day/ } \\
\text { year) }\end{array}$ & $\begin{array}{l}\text { Stage } \\
\text { (ft) }\end{array}$ & $\begin{array}{l}\text { Discharge } \\
\qquad\left(\mathrm{ft}^{3} / \mathrm{s}\right)\end{array}$ & $\begin{array}{l}\text { Regulated } \\
\text { during } \\
\text { flood }^{1}\end{array}$ & $\begin{array}{l}\text { Recurrence } \\
\text { interval } \\
\text { (years) }\end{array}$ \\
\hline 16283200 & $\begin{array}{l}\text { Kahaluu Stream near Ahuimanu, } \\
\text { Oahu }\end{array}$ & 0.99 & $1984-98$ & 1994 & 6.05 & 728 & $9 / 18 / 94$ & 6.05 & 728 & $\mathrm{~N}$ & $10-25$ \\
\hline 16331000 & $\begin{array}{l}\text { Waimea Gulch near Kawailoa } \\
\text { Camp, Oahu, HI }\end{array}$ & 2.23 & $1968-98$ & 1980 & 11.20 & 2,030 & $1 / 19 / 97$ & 9.32 & 857 & $\mathrm{~N}$ & 20 \\
\hline 16500100 & $\begin{array}{l}\text { Kepuni Gulch near Kahikinui } \\
\text { House, Maui, HI }\end{array}$ & 1.91 & $\begin{array}{l}\text { 1963-94, } \\
1996-98\end{array}$ & 1994 & 13.68 & 2,320 & 9/18/94 & 13.68 & 2,320 & $\mathrm{~N}$ & 100 \\
\hline 16502800 & $\begin{array}{l}\text { Moomoonui Gulch at Hana, Maui, } \\
\text { HI }\end{array}$ & .90 & 1963-98 & 1993 & 14.71 & 2,480 & $3 / 24 / 94$ & 14.54 & 2,200 & $\mathrm{~N}$ & 15 \\
\hline 16587000 & $\begin{array}{l}\text { Honopou Stream near Huelo, } \\
\text { Maui, HI }\end{array}$ & .64 & $\begin{array}{l}\text { 1911, } \\
\text { 1913-14, } \\
\text { 1916-96, } \\
1998\end{array}$ & 1931 & 7.28 & 5,710 & $7 / 30 / 97$ & 5.79 & 3,030 & $\mathrm{~N}$ & 15 \\
\hline 16701300 & $\begin{array}{l}\text { Waiakea Stream at Hilo, Hawaii, } \\
\text { HI }\end{array}$ & 35.8 & $\begin{array}{l}1969-75, \\
1979, \\
1994-98\end{array}$ & 1994 & 10.90 & 3,670 & $8 / 12 / 94$ & 10.90 & 3,670 & $\mathrm{~N}$ & 25 \\
\hline
\end{tabular}

\footnotetext{
${ }^{1}$ Regulated during flood: N, no; Y, yes.
} 


\section{Summary of Significant Floods in the United States and Puerto Rico, 1994 Through 1998 Water Years}

\section{Idaho}

Significant flooding occurred during the last week of November through the first week of December 1995 in northern Idaho. Warm temperatures, 4 to 7 inches of rainfall (National Oceanic and Atmospheric Administration, 1995a), and snowmelt at elevations above 5,000 feet caused increased runoff levels. Lake Coeur d'Alene (fig. 24) was 9 feet above normal.

Major flooding took place the second week of February 1996 in northern Idaho. Conditions that developed in late January contributed significantly to the flood. Large amounts of snow fell over the lower elevations during the third week of January, especially in northern Idaho where valley locations reported 10 additional inches of snow with as much as 2.5 feet of snow on the ground. During the last week in January temperatures dropped into the single digits for highs and -20 to $-30{ }^{\circ} \mathrm{F}$ for lows (National Oceanic and Atmospheric Administration, 1996a); this resulted in ice buildup along many of the rivers. During the first week of February temperatures drastically warmed, but lows remained below freezing so snowmelt began slowly. Excessive rains also fell on February 6-10 (National Oceanic and Atmospheric Administration, 1996a). Five-day totals were 8.00 inches at Prichard, 6.14 inches at Mullan, and 4.00 inches at other locations. The warm temperatures and excessive rain accelerated low-elevation snowmelt resulting in serious flooding. The ice jams caused serious falls and rises along the rivers as well. The worst flooding occurred in the Clearwater River Basin around the town of Orofino. The flooding of the Palouse, St. Joe, and Coeur d'Alene Rivers along with their tributaries caused major damage. Major ice jams caused serious rises along the North Fork Coeur d'Alene River at Enaville (streamgage 12413000, table 13) and the Coeur d'Alene River near Cataldo (streamgage 12413500, table 13), resulting in the evacuation of the towns of Enaville and Cataldo (National Oceanic and Atmospheric Administration, 1996a). This event is close to the flood of record on the St. Joe River. Total damage was more than \$44 million (National Oceanic and Atmospheric Administration, 1996b).

Above-normal snowfall occurred in northern Idaho during November and December 1996. Most locations had more than 2 to 3 feet of snow with larger amounts at higher elevations. Considerable snow also fell in southern and central Idaho. Wet Pacific storm systems brought precipitation totals two to five times the normal for December. Snowmelt caused by rapidly warming temperatures and persistent rains during the last week of December caused flooding throughout the State during the first week in January 1997. Major flooding occurred on the Weiser, Payette, Snake, and Boise Rivers. The State of Idaho was declared a major disaster area. The total flood damage was $\$ 25$ million. In May 1997 the snow pack at the higher elevations melted resulting in significant flooding in northern Idaho and an additional \$4 million in damage (National Oceanic and Atmospheric Administration, 1997b).

\section{References}

National Oceanic and Atmospheric Administration (NOAA), 1995a-97a, Climatological data (by State): Asheville, North Carolina, National Climatic Data Center, various months.

National Oceanic and Atmospheric Administration (NOAA), 1995b-97b, Storm data (by State): Asheville, North Carolina, National Climatic Data Center, various months. 


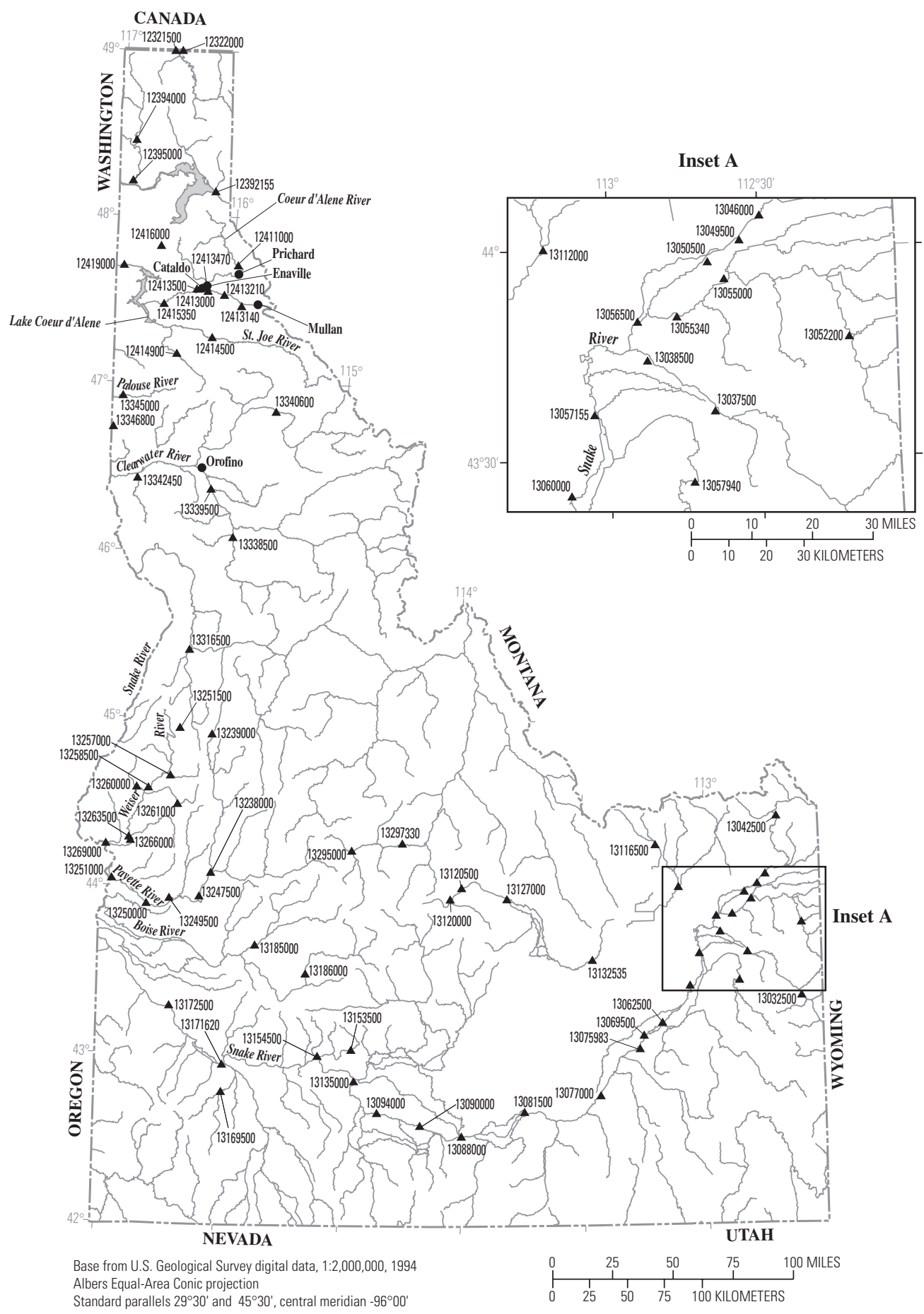

\section{EXPLANATION}

${ }^{13088000} \triangle$ Streamgage and number

Figure 24. Location of streamgages with significant floods during 1994-98 water years for Idaho. 
Table 13. Maximum stage and discharge for period of record for streamgages having significant floods during 1994-98 water years in Idaho.

$\left[\mathrm{mi}^{2}\right.$, square miles; $\mathrm{ft}$, feet above an arbitrary datum; $\mathrm{ft}^{3} / \mathrm{s}$, cubic feet per second; --, not determined or not applicable; $>$, greater than. Source: Recurrence intervals calculated from U.S. Geological Survey data. Other data from U.S. Geological Survey reports or databases]

\begin{tabular}{|c|c|c|c|c|c|c|c|c|c|c|c|}
\hline \multirow{2}{*}{$\begin{array}{l}\text { Streamgage } \\
\text { number } \\
\text { (fig. 24) }\end{array}$} & \multirow[b]{2}{*}{ Streamgage name } & \multirow{2}{*}{$\begin{array}{l}\text { Total } \\
\text { drainage } \\
\left(\mathrm{mi}^{2}\right)\end{array}$} & \multicolumn{4}{|c|}{$\begin{array}{c}\text { Maximum stage and discharge for period of record } \\
\text { through } 1998 \text { water year }\end{array}$} & \multicolumn{5}{|c|}{ Significant floods 1994-98 water years } \\
\hline & & & $\begin{array}{l}\text { Period of } \\
\text { record } \\
\text { (water } \\
\text { years) }\end{array}$ & Water year & $\begin{array}{l}\text { Stage } \\
(\mathrm{ft})\end{array}$ & $\begin{array}{l}\text { Discharge } \\
\qquad\left(\mathrm{ft}^{3} / \mathrm{s}\right)\end{array}$ & $\begin{array}{c}\text { Date } \\
\text { (month/d } \\
\text { ay/year) }\end{array}$ & $\begin{array}{l}\text { Stage } \\
(\mathrm{ft})\end{array}$ & $\begin{array}{l}\text { Discharge } \\
\left(\mathrm{ft}^{3} / \mathrm{s}\right)\end{array}$ & $\begin{array}{l}\text { Regulated } \\
\text { during } \\
\text { flood }^{1}\end{array}$ & $\begin{array}{l}\text { Recurrence } \\
\text { interval } \\
\text { (years) }\end{array}$ \\
\hline 12321500 & Boundary Creek near Porthill, ID & 97 & $1929-98$ & $\begin{array}{l}1997 \\
1998\end{array}$ & $\begin{array}{r}5.88 \\
15.25\end{array}$ & $\begin{array}{l}3,780 \\
2,760\end{array}$ & $\begin{array}{l}4 / 24 / 96 \\
6 / 1 / 97\end{array}$ & $\begin{array}{l}5.86 \\
5.88\end{array}$ & $\begin{array}{l}3,740 \\
3,780\end{array}$ & $\begin{array}{l}\mathrm{N} \\
\mathrm{N}\end{array}$ & $\begin{array}{l}50-75 \\
50-75\end{array}$ \\
\hline 12322000 & Kootenai River at Porthill, ID & 13,700 & $\begin{array}{l}1894 \\
1925-98\end{array}$ & $\begin{array}{l}1948 \\
1894\end{array}$ & $\begin{array}{l}66.16 \\
72.70\end{array}$ & $\begin{array}{r}125,000 \\
--\end{array}$ & $5 / 14 / 97$ & -- & 61,400 & $\mathrm{Y}$ & $2-5$ \\
\hline 12392155 & Lightning Creek at Clark Fork, ID & 115 & 1989-98 & $\begin{array}{l}1996 \\
1998\end{array}$ & $\overline{--}$ & $\begin{array}{l}4,970 \\
3,260\end{array}$ & 2/9/96 & -- & 4,970 & $\mathrm{~N}$ & $25-50$ \\
\hline 12394000 & Priest River near Coolin, ID & 611 & 1949-98 & $\begin{array}{l}1997 \\
1974\end{array}$ & $\begin{array}{l}8.02 \\
8.44\end{array}$ & $\begin{array}{l}9,280 \\
8,900\end{array}$ & $6 / 3 / 97$ & 8.02 & 9,280 & $\mathrm{~N}$ & $>100$ \\
\hline 12395000 & Priest River near Priest River, ID & 902 & $\begin{array}{l}1904, \\
1931-81 \\
1983-98\end{array}$ & 1997 & 9.13 & 10,800 & $5 / 18 / 97$ & 9.13 & 10,800 & $\mathrm{~N}$ & $75-100$ \\
\hline 12411000 & $\begin{array}{l}\text { North Fork Coeur d'Alene River } \\
\text { above Shoshone Creek near } \\
\text { Prichard, ID }\end{array}$ & 335 & $1951-98$ & 1974 & 11.60 & 22,000 & $2 / 9 / 96$ & 10.24 & 17,000 & $\mathrm{~N}$ & $40-50$ \\
\hline 12413000 & $\begin{array}{l}\text { North Fork Coeur d'Alene River at } \\
\text { Enaville, ID }\end{array}$ & 895 & $\begin{array}{l}1912,1934, \\
1938 \\
1940-98\end{array}$ & 1974 & 81.32 & 61,000 & 2/9/96 & 76.95 & 56,600 & $\mathrm{~N}$ & $40-50$ \\
\hline 12413140 & Placer Creek at Wallace, ID & 14.9 & 1968-97 & 1996 & 14.81 & 2,200 & $2 / 9 / 96$ & 14.81 & 2,200 & $\mathrm{Y}$ & $>100$ \\
\hline 12413210 & $\begin{array}{l}\text { South Fork Coeur d'Alene River at } \\
\text { Elizabeth Park near Kellogg, ID }\end{array}$ & -- & 1987-98 & 1996 & 35.50 & 9,600 & $2 / 9 / 96$ & 35.50 & 9,600 & $\mathrm{~N}$ & $>50$ \\
\hline 12413470 & $\begin{array}{l}\text { South Fork Coeur d'Alene River } \\
\text { near Pinehurst, ID }\end{array}$ & 299 & 1988-98 & 1996 & 17.43 & 11,700 & 2/9/96 & 17.43 & 11,700 & $\mathrm{~N}$ & -- \\
\hline 12413500 & $\begin{array}{l}\text { Coeur d'Alene River near Cataldo, } \\
\text { ID }\end{array}$ & 1,223 & $\begin{array}{l}1911-12 \\
1921-72 \\
1974 \\
1987-98\end{array}$ & 1974 & 58.23 & 79,000 & 2/9/96 & 51.62 & 70,000 & $\mathrm{~N}$ & $>100$ \\
\hline 12414500 & St. Joe River at Calder, ID & 1,030 & $\begin{array}{l}1911-12 \\
1921-98\end{array}$ & $\begin{array}{l}1934 \\
1938\end{array}$ & $\begin{array}{l}92.50 \\
93.20\end{array}$ & $\begin{array}{l}53,000 \\
46,000\end{array}$ & 2/9/96 & 15.22 & 39,200 & $\mathrm{~N}$ & $50-75$ \\
\hline 12414900 & St. Maries River near Santa, ID & 275 & 1966-98 & 1996 & 13.75 & 12,300 & $2 / 9 / 96$ & 13.75 & 12,300 & $\mathrm{~N}$ & $50-75$ \\
\hline
\end{tabular}


Table 13. Maximum stage and discharge for period of record for streamgages having significant floods during 1994-98 water years in Idaho.-Continued

$\left[\mathrm{mi}^{2}\right.$, square miles; $\mathrm{ft}$, feet above an arbitrary datum; $\mathrm{ft}^{3} / \mathrm{s}$, cubic feet per second; --, not determined or not applicable; >, greater than. Source: Recurrence intervals calculated from U.S. Geological Survey data. Other data from U.S. Geological Survey reports or databases]

\begin{tabular}{|c|c|c|c|c|c|c|c|c|c|c|c|}
\hline \multirow{2}{*}{$\begin{array}{l}\text { Streamgage } \\
\text { number } \\
\text { (fig. 24) }\end{array}$} & \multirow[b]{2}{*}{ Streamgage name } & \multirow{2}{*}{$\begin{array}{c}\text { Total } \\
\text { drainage } \\
\left(\mathrm{mi}^{2}\right)\end{array}$} & \multicolumn{4}{|c|}{$\begin{array}{c}\text { Maximum stage and discharge for period of record } \\
\text { through } 1998 \text { water year }\end{array}$} & \multicolumn{5}{|c|}{ Significant floods 1994-98 water years } \\
\hline & & & $\begin{array}{c}\text { Period of } \\
\text { record } \\
\text { (water } \\
\text { years) }\end{array}$ & Water year & $\begin{array}{l}\text { Stage } \\
(\mathrm{ft})\end{array}$ & $\begin{array}{l}\text { Discharge } \\
\left(\mathrm{ft}^{3} / \mathrm{s}\right)\end{array}$ & $\begin{array}{c}\text { Date } \\
\text { (month/d } \\
\text { ay/year) }\end{array}$ & $\begin{array}{l}\text { Stage } \\
(\mathrm{ft})\end{array}$ & $\begin{array}{l}\text { Discharge } \\
\left(\mathrm{ft}^{3} / \mathrm{s}\right)\end{array}$ & $\begin{array}{l}\text { Regulated } \\
\text { during } \\
\text { flood }^{1}\end{array}$ & $\begin{array}{c}\text { Recurrence } \\
\text { interval } \\
\text { (years) }\end{array}$ \\
\hline 12415350 & $\begin{array}{l}\text { Wolf Lodge Creek near Coeur } \\
\text { d'Alene, ID }\end{array}$ & 39.4 & $1986-95$ & 1995 & 11.91 & 865 & $2 / 20 / 95$ & 11.91 & 865 & $\mathrm{~N}$ & -- \\
\hline 12416000 & $\begin{array}{l}\text { Hayden Creek below North Fork } \\
\text { near Hayden Lake, ID }\end{array}$ & 22.0 & $\begin{array}{l}1948-53, \\
1959, \\
1962-97\end{array}$ & 1982 & 5.69 & 1,280 & $2 / 9 / 96$ & 5.60 & 1,250 & $\mathrm{~N}$ & $30-40$ \\
\hline 12419000 & Spokane River near Post Falls, ID & 3,840 & 1913-98 & $\begin{array}{l}1934 \\
1917\end{array}$ & $\begin{array}{l}-- \\
79.23\end{array}$ & $\begin{array}{l}50,100 \\
39,800\end{array}$ & $5 / 19 / 97$ & 24.92 & 43,900 & $\mathrm{Y}$ & $20-25$ \\
\hline 13032500 & Snake River near Irwin, ID & 5,225 & $\begin{array}{l}1934-36, \\
1939-41, \\
1949-97\end{array}$ & 1997 & 15.25 & 40,400 & $6 / 19 / 97$ & 15.25 & 40,400 & $\mathrm{Y}$ & $>100$ \\
\hline 13037500 & Snake River near Heise, ID & 5,752 & $1911-98$ & $\begin{array}{l}1927 \\
1997\end{array}$ & -- & $\begin{array}{l}60,000 \\
43,500\end{array}$ & $6 / 13 / 97$ & 11.26 & 43,500 & $\mathrm{Y}$ & $>100$ \\
\hline 13038500 & Snake River at Lorenzo, ID & 5,810 & $\begin{array}{l}\text { 1924-27, } \\
1978-98\end{array}$ & $\begin{array}{l}1927 \\
1997\end{array}$ & $\begin{array}{r}9.85 \\
13.79\end{array}$ & $\begin{array}{l}43,000 \\
38,300\end{array}$ & $6 / 22 / 97$ & 13.79 & 38,300 & $\mathrm{Y}$ & $25-50$ \\
\hline 13042500 & Henrys Fork near Island Park, ID & 481 & 1933-98 & $\begin{array}{l}1984 \\
1946\end{array}$ & $\begin{array}{l}6.06 \\
6.15\end{array}$ & $\begin{array}{l}3,030 \\
2,770\end{array}$ & $5 / 17 / 95$ & 5.77 & 2,870 & $\mathrm{Y}$ & $50-75$ \\
\hline 13046000 & Henrys Fork near Ashton, ID & 1,040 & $\begin{array}{l}\text { 1890-91, } \\
1903-09, \\
1920-98\end{array}$ & $\begin{array}{l}1984 \\
1959\end{array}$ & $\begin{array}{l}6.50 \\
8.34\end{array}$ & $\begin{array}{l}8,140 \\
3,300\end{array}$ & $\begin{array}{l}5 / 12 / 95 \\
5 / 12 / 97\end{array}$ & $\begin{array}{l}5.82 \\
6.02\end{array}$ & $\begin{array}{l}6,090 \\
6,620\end{array}$ & $\begin{array}{l}\mathrm{Y} \\
\mathrm{Y}\end{array}$ & $\begin{array}{l}15-20 \\
35-40\end{array}$ \\
\hline 13049500 & Falls River near Chester, ID & 520 & 1920-98 & 1981 & 7.83 & 7,730 & $5 / 17 / 96$ & 6.56 & 5,760 & $\mathrm{~N}$ & $20-25$ \\
\hline 13050500 & Henrys Fork at Saint Anthony, ID & 1,770 & 1919-98 & 1984 & 8.62 & 13,200 & $5 / 30 / 97$ & 7.57 & 10,600 & $\mathrm{Y}$ & $20-25$ \\
\hline 13052200 & $\begin{array}{l}\text { Teton River above South Leigh } \\
\text { Creek near Driggs, ID }\end{array}$ & 335 & $1962-98$ & $\begin{array}{l}1997 \\
1983\end{array}$ & $\begin{array}{l}5.14 \\
5.17\end{array}$ & $\begin{array}{l}2,980 \\
2,460\end{array}$ & $6 / 11 / 97$ & 5.14 & 2,980 & $\mathrm{~N}$ & $>100$ \\
\hline 13055000 & $\begin{array}{l}\text { Teton River near Saint Anthony, } \\
\text { ID }\end{array}$ & 890 & $\begin{array}{l}1890-93 \\
1903-09, \\
1920-96, \\
1978-98\end{array}$ & 1976 & 42.20 & $1,700,000$ & $6 / 11 / 97$ & 6.78 & 6,360 & $\mathrm{~N}$ & $20-25$ \\
\hline 13055340 & $\begin{array}{l}\text { South Fork Teton River near } \\
\text { Rexburg, ID }\end{array}$ & -- & 1982-98 & 1997 & 10.68 & 3,410 & $6 / 11 / 97$ & 10.68 & 3,410 & $\mathrm{~N}$ & -- \\
\hline
\end{tabular}


$\left[\mathrm{mi}^{2}\right.$, square miles; $\mathrm{ft}$, feet above an arbitrary datum; $\mathrm{ft}^{3} / \mathrm{s}$, cubic feet per second; --, not determined or not applicable; $>$, greater than. Source: Recurrence intervals calculated from U.S. Geological Survey data. Other data from U.S. Geological Survey reports or databases]

\begin{tabular}{|c|c|c|c|c|c|c|c|c|c|c|c|}
\hline \multirow{2}{*}{$\begin{array}{c}\text { Streamgage } \\
\text { number } \\
\text { (fig. 24) }\end{array}$} & \multirow[b]{2}{*}{ Streamgage name } & \multirow{2}{*}{$\begin{array}{c}\text { Total } \\
\text { drainage } \\
\left(\mathrm{mi}^{2}\right)\end{array}$} & \multicolumn{4}{|c|}{$\begin{array}{c}\text { Maximum stage and discharge for period of record } \\
\text { through } 1998 \text { water year }\end{array}$} & \multicolumn{5}{|c|}{ Significant floods 1994-98 water years } \\
\hline & & & $\begin{array}{c}\text { Period of } \\
\text { record } \\
\text { (water } \\
\text { years) }\end{array}$ & Water year & $\begin{array}{l}\text { Stage } \\
(\mathrm{ft})\end{array}$ & $\begin{array}{l}\text { Discharge } \\
\left(\mathrm{ft}^{3} / \mathrm{s}\right)\end{array}$ & $\begin{array}{c}\text { Date } \\
\text { (month/d } \\
\text { ay/year) }\end{array}$ & $\begin{array}{l}\text { Stage } \\
(\mathrm{ft})\end{array}$ & $\begin{array}{l}\text { Discharge } \\
\left(\mathrm{ft}^{3} / \mathrm{s}\right)\end{array}$ & $\begin{array}{c}\text { Regulated } \\
\text { during } \\
\text { flood }^{1}\end{array}$ & $\begin{array}{c}\text { Recurrence } \\
\text { interval } \\
\text { (years) }\end{array}$ \\
\hline 13056500 & Henrys Fork near Rexburg, ID & 2,920 & $\begin{array}{l}1909-23 \\
1925-98\end{array}$ & $\begin{array}{l}1976 \\
1984\end{array}$ & $\begin{array}{l}-- \\
12.05\end{array}$ & $\begin{array}{l}79,000 \\
16,400\end{array}$ & $5 / 18 / 96$ & 11.26 & 13,800 & $\mathrm{Y}$ & $35-40$ \\
\hline 13057155 & $\begin{array}{l}\text { Snake River above Eagle Rock } \\
\text { near Idaho Falls, ID }\end{array}$ & -- & 1988-98 & 1997 & 18.91 & 48,600 & $6 / 16 / 97$ & 18.91 & 48,600 & $\mathrm{~N}$ & -- \\
\hline 13057940 & $\begin{array}{l}\text { Willow Creek below Tex Creek } \\
\text { near Ririe, ID }\end{array}$ & 568 & $\begin{array}{l}1978-79 \\
1986-98\end{array}$ & 1997 & 6.73 & 2,420 & $5 / 7 / 97$ & 6.73 & 2,420 & $\mathrm{~N}$ & -- \\
\hline 13060000 & Snake River near Shelley, ID & 9,790 & $\begin{array}{l}1890, \\
1892-94, \\
1915-98\end{array}$ & $\begin{array}{l}1894 \\
1976\end{array}$ & $\overline{--}$ & $\begin{array}{l}75,000 \\
67,300\end{array}$ & $6 / 17 / 97$ & 16.05 & 47,800 & $\mathrm{Y}$ & $>100$ \\
\hline 13062500 & Snake River at Blackfoot, ID & 9,950 & 1978-98 & 1997 & 13.55 & 43,200 & $6 / 17 / 97$ & 13.55 & 43,200 & $\mathrm{Y}$ & $50-100$ \\
\hline 13069500 & Snake River near Blackfoot, ID & 11,310 & 1911-98 & 1976 & 15.44 & 53,500 & $6 / 18 / 97$ & 14.80 & 42,300 & $\mathrm{Y}$ & $75-100$ \\
\hline 13075983 & $\begin{array}{l}\text { Spring Creek at Sheepskin Road } \\
\text { near Fort Hall, ID }\end{array}$ & -- & 1980-98 & $\begin{array}{l}1998 \\
1995\end{array}$ & $-\overline{-}$ & $\begin{array}{l}605 \\
169\end{array}$ & $6 / 8 / 98$ & -- & 605 & $\mathrm{~N}$ & -- \\
\hline 13077000 & Snake River at Neeley, ID & 13,600 & 1906-98 & $\begin{array}{l}1918 \\
1997\end{array}$ & $\overline{--}$ & $\begin{array}{l}48,400 \\
46,100\end{array}$ & $6 / 19 / 97$ & 11.46 & 46,100 & $\mathrm{Y}$ & $50-75$ \\
\hline 13081500 & Snake River near Minidoka, ID & 15,700 & $\begin{array}{l}1896-99 \\
1901-98\end{array}$ & $\begin{array}{l}1897 \\
1918\end{array}$ & $\begin{array}{l}12.60 \\
16.02\end{array}$ & $\begin{array}{l}47,500 \\
45,900\end{array}$ & $6 / 21 / 97$ & 15.49 & 42,900 & $\mathrm{Y}$ & $25-30$ \\
\hline 13088000 & Snake River at Milner, ID & 17,180 & 1909-98 & $\begin{array}{l}1918 \\
1921\end{array}$ & -- & $\begin{array}{l}40,000 \\
27,000\end{array}$ & $6 / 21 / 97$ & -- & 31,200 & $\mathrm{Y}$ & $40-50$ \\
\hline 13090000 & Snake River near Kimberly, ID & -- & 1924-98 & 1997 & 23.27 & 34,200 & $6 / 21 / 97$ & 23.27 & 34,200 & $\mathrm{Y}$ & $75-100$ \\
\hline 13094000 & Snake River near Buhl, ID & -- & 1947-98 & 1997 & 14.65 & 37,100 & $6 / 22 / 97$ & 14.65 & 37,100 & $\mathrm{Y}$ & $>100$ \\
\hline 13112000 & Camas Creek at Camas, ID & 400 & $1925-98$ & $\begin{array}{l}1998 \\
1984\end{array}$ & $\begin{array}{l}7.49 \\
7.61\end{array}$ & $\begin{array}{l}1,490 \\
1,320\end{array}$ & $\begin{array}{l}5 / 12 / 95 \\
5 / 16 / 98\end{array}$ & $\begin{array}{l}6.91 \\
7.49\end{array}$ & $\begin{array}{l}1,220 \\
1,490\end{array}$ & $\begin{array}{l}\mathrm{N} \\
\mathrm{N}\end{array}$ & $\begin{array}{l}20-25 \\
45-50\end{array}$ \\
\hline 13116500 & $\begin{array}{l}\text { Medicine Lodge Creek near Small, } \\
\text { ID }\end{array}$ & 270 & $\begin{array}{l}1921-23, \\
1941-48, \\
1985-98\end{array}$ & 1995 & 9.09 & 481 & $6 / 19 / 95$ & 9.09 & 481 & $\mathrm{~N}$ & $50-100$ \\
\hline 13120000 & $\begin{array}{l}\text { North Fork Big Lost River at Wild } \\
\text { Horse near Chilly, ID }\end{array}$ & 114 & 1944-98 & $\begin{array}{l}1997 \\
1965\end{array}$ & $\begin{array}{l}5.65 \\
6.39\end{array}$ & $\begin{array}{l}1,560 \\
1,420\end{array}$ & $6 / 5 / 97$ & 5.65 & 1,560 & $\mathrm{~N}$ & $30-35$ \\
\hline
\end{tabular}


Table 13. Maximum stage and discharge for period of record for streamgages having significant floods during 1994-98 water years in Idaho.-Continued

$\left[\mathrm{mi}^{2}\right.$, square miles; $\mathrm{ft}$, feet above an arbitrary datum; $\mathrm{ft}^{3} / \mathrm{s}$, cubic feet per second; --, not determined or not applicable; >, greater than. Source: Recurrence intervals calculated from U.S. Geological Survey data. Other data from U.S. Geological Survey reports or databases]

\begin{tabular}{|c|c|c|c|c|c|c|c|c|c|c|c|}
\hline \multirow{2}{*}{$\begin{array}{l}\text { Streamgage } \\
\text { number } \\
\text { (fig. 24) }\end{array}$} & \multirow[b]{2}{*}{ Streamgage name } & \multirow{2}{*}{$\begin{array}{l}\text { Total } \\
\text { drainage } \\
\left(\mathrm{mi}^{2}\right)\end{array}$} & \multicolumn{4}{|c|}{$\begin{array}{c}\text { Maximum stage and discharge for period of record } \\
\text { through } 1998 \text { water year }\end{array}$} & \multicolumn{5}{|c|}{ Significant floods $1994-98$ water years } \\
\hline & & & $\begin{array}{l}\text { Period of } \\
\text { record } \\
\text { (water } \\
\text { years) }\end{array}$ & Water year & $\begin{array}{l}\text { Stage } \\
(\mathrm{ft})\end{array}$ & $\begin{array}{l}\text { Discharge } \\
\left(\mathrm{ft}^{3} / \mathrm{s}\right)\end{array}$ & $\begin{array}{c}\text { Date } \\
\text { (month/d } \\
\text { ay/year) }\end{array}$ & $\begin{array}{l}\text { Stage } \\
(\mathrm{ft})\end{array}$ & $\begin{array}{l}\text { Discharge } \\
\left(\mathrm{ft}^{3} / \mathrm{s}\right)\end{array}$ & $\begin{array}{l}\text { Regulated } \\
\text { during } \\
\text { flood }^{1}\end{array}$ & $\begin{array}{l}\text { Recurrence } \\
\text { interval } \\
\text { (years) }\end{array}$ \\
\hline 13120500 & $\begin{array}{l}\text { Big Lost River at Howell Ranch } \\
\text { near Chilly, ID }\end{array}$ & 450 & $\begin{array}{l}\text { 1904-14, } \\
1920-98\end{array}$ & 1967 & 6.02 & 4,420 & $7 / 10 / 95$ & 5.17 & 3,690 & $\mathrm{~N}$ & $10-15$ \\
\hline 13127000 & $\begin{array}{l}\text { Big Lost River below Mackay } \\
\text { Reservoir near Mackay, ID }\end{array}$ & 813 & $\begin{array}{l}1904-06 \\
1912-14 \\
1919-98\end{array}$ & 1986 & 6.08 & 2,990 & $7 / 12 / 95$ & 5.65 & 2,880 & $\mathrm{Y}$ & $20-25$ \\
\hline 13132535 & $\begin{array}{l}\text { Big Lost River at Lincoln } \\
\text { Boulevard Bridge near Atomic } \\
\text { City, ID }\end{array}$ & -- & $\begin{array}{l}\text { 1984-91, } \\
1993,1995\end{array}$ & $\begin{array}{l}1995 \\
1993\end{array}$ & 16.56 & $\begin{array}{l}428 \\
288\end{array}$ & $7 / 16 / 95$ & -- & 428 & $\mathrm{~N}$ & -- \\
\hline 13135000 & $\begin{array}{l}\text { Snake River below Lower Salmon } \\
\text { Falls near Hagerman, ID }\end{array}$ & -- & $1938-98$ & 1997 & 18.81 & 38,500 & $6 / 21 / 97$ & 18.81 & 38,500 & $\mathrm{Y}$ & $>100$ \\
\hline 13153500 & Malad River near Bliss, ID & -- & $\begin{array}{l}1899 \\
1985-98\end{array}$ & $\begin{array}{l}1997 \\
1899\end{array}$ & $-\overline{-}$ & $\begin{array}{l}5,390 \\
3,625\end{array}$ & $1 / 2 / 97$ & -- & 5,390 & $\mathrm{~N}$ & -- \\
\hline 13154500 & Snake River at King Hill, ID & 35,800 & 1909-98 & 1918 & 16.30 & 47,200 & $6 / 22 / 97$ & 15.51 & 42,200 & $\mathrm{Y}$ & $>100$ \\
\hline 13169500 & Big Jacks Creek near Bruneau, ID & 253 & $\begin{array}{l}1939-49 \\
1963 \\
1966-98\end{array}$ & $\begin{array}{l}1997 \\
1943\end{array}$ & $\begin{array}{r}5.82 \\
12.40\end{array}$ & $\begin{array}{l}2,950 \\
2,100\end{array}$ & $1 / 2 / 97$ & 5.82 & 2,950 & $\mathrm{~N}$ & $50-75$ \\
\hline 13171620 & $\begin{array}{l}\text { Snake River below CJ Strike Dam } \\
\text { near Grand View, ID }\end{array}$ & 40,800 & $1985-98$ & 1997 & 14.88 & 44,000 & $6 / 20 / 97$ & 14.88 & 44,000 & $\mathrm{Y}$ & -- \\
\hline 13172500 & Snake River near Murphy, ID & 41,900 & 1914-98 & 1918 & 13.95 & 47,300 & $6 / 17 / 97$ & 13.66 & 40,300 & $\mathrm{Y}$ & $20-25$ \\
\hline 13185000 & Boise River near Twin Springs, ID & 830 & $\begin{array}{l}1871-72 \\
1911-98\end{array}$ & $\begin{array}{l}1872 \\
1965\end{array}$ & $\overline{--}$ & $\begin{array}{l}22,700 \\
18,800\end{array}$ & $1 / 1 / 97$ & 10.81 & 12,800 & $\mathrm{~N}$ & $15-20$ \\
\hline 13186000 & $\begin{array}{l}\text { South Fork Boise River near } \\
\text { Featherville, ID }\end{array}$ & 635 & $\begin{array}{l}1943, \\
1945-98\end{array}$ & $\begin{array}{l}1997 \\
1956\end{array}$ & $\begin{array}{l}7.74 \\
8.62\end{array}$ & $\begin{array}{l}8,030 \\
7,580\end{array}$ & $5 / 17 / 97$ & 7.74 & 8,030 & $\mathrm{~N}$ & $20-25$ \\
\hline 13238000 & Payette River near Banks, ID & 1,200 & $\begin{array}{l}1922-73 \\
1997\end{array}$ & 1965 & 15.46 & 20,800 & $1 / 1 / 97$ & -- & 19,100 & $\mathrm{Y}$ & $50-75$ \\
\hline 13239000 & $\begin{array}{l}\text { North Fork Payette River at } \\
\text { McCall, ID }\end{array}$ & 144 & $\begin{array}{l}\text { 1909-17, } \\
1920-98\end{array}$ & 1974 & 8.16 & 4,950 & $\begin{array}{l}5 / 18 / 96 \\
5 / 18 / 97\end{array}$ & $\begin{array}{l}7.82 \\
7.62\end{array}$ & $\begin{array}{l}4,570 \\
4,320\end{array}$ & $\begin{array}{l}\mathrm{Y} \\
\mathrm{Y}\end{array}$ & $\begin{array}{l}30-35 \\
15-20\end{array}$ \\
\hline 13247500 & $\begin{array}{l}\text { Payette River near Horseshoe } \\
\text { Bend, ID }\end{array}$ & 2,230 & $\begin{array}{l}1906-16 \\
1920-98\end{array}$ & 1965 & 16.35 & 27,000 & $1 / 2 / 97$ & 15.63 & 24,400 & $\mathrm{Y}$ & $20-25$ \\
\hline 13249500 & Payette River near Emmett, ID & 2,680 & 1926-98 & 1965 & 15.88 & 32,700 & $1 / 2 / 97$ & 15.30 & 30,500 & $\mathrm{Y}$ & $50-60$ \\
\hline
\end{tabular}


$\left[\mathrm{mi}^{2}\right.$, square miles; ft, feet above an arbitrary datum; $\mathrm{ft}^{3} / \mathrm{s}$, cubic feet per second; --, not determined or not applicable; >, greater than. Source: Recurrence intervals calculated from U.S. Geological Survey data. Other data from U.S. Geological Survey reports or databases]

\begin{tabular}{|c|c|c|c|c|c|c|c|c|c|c|c|}
\hline \multirow{2}{*}{$\begin{array}{l}\text { Streamgage } \\
\text { number } \\
\text { (fig. 24) }\end{array}$} & \multirow[b]{2}{*}{ Streamgage name } & \multirow{2}{*}{$\begin{array}{c}\text { Total } \\
\text { drainage } \\
\left(\mathrm{mi}^{2}\right)\end{array}$} & \multicolumn{4}{|c|}{$\begin{array}{c}\text { Maximum stage and discharge for period of record } \\
\text { through } 1998 \text { water year }\end{array}$} & \multicolumn{5}{|c|}{ Significant floods $1994-98$ water years } \\
\hline & & & $\begin{array}{l}\text { Period of } \\
\text { record } \\
\text { (water } \\
\text { years) }\end{array}$ & Water year & $\begin{array}{l}\text { Stage } \\
(\mathrm{ft})\end{array}$ & $\begin{array}{l}\text { Discharge } \\
\left(\mathrm{ft}^{3} / \mathrm{s}\right)\end{array}$ & $\begin{array}{c}\text { Date } \\
\text { (month/d } \\
\text { ay/year) }\end{array}$ & $\begin{array}{l}\text { Stage } \\
(\mathrm{ft})\end{array}$ & $\begin{array}{l}\text { Discharge } \\
\left(\mathrm{ft}^{3} / \mathrm{s}\right)\end{array}$ & $\begin{array}{c}\text { Regulated } \\
\text { during } \\
\text { flood }^{1}\end{array}$ & $\begin{array}{c}\text { Recurrence } \\
\text { interval } \\
\text { (years) }\end{array}$ \\
\hline 13250000 & Payette River near Letha, ID & 2,760 & $\begin{array}{l}\text { 1979-86, } \\
1994-98\end{array}$ & $\begin{array}{l}1997 \\
1996\end{array}$ & $\begin{array}{l}-- \\
15.97\end{array}$ & $\begin{array}{l}27,000 \\
17,900\end{array}$ & $1 / 2 / 97$ & -- & 27,000 & $\mathrm{Y}$ & $>50$ \\
\hline 13251000 & Payette River near Payette, ID & 3,240 & 1936-98 & $\begin{array}{l}1997 \\
1965\end{array}$ & $\begin{array}{l}-- \\
13.80\end{array}$ & $\begin{array}{l}32,000 \\
30,900\end{array}$ & $1 / 2 / 97$ & -- & 32,000 & $\mathrm{Y}$ & $>100$ \\
\hline 13251500 & Weiser River at Tamarack, ID & 36.5 & $\begin{array}{l}1937-71, \\
1974-75, \\
1997\end{array}$ & $\begin{array}{l}1997 \\
1956\end{array}$ & --17 & $\begin{array}{l}1,520 \\
1,320\end{array}$ & $1 / 1 / 97$ & -- & 1,520 & $\mathrm{~N}$ & $>100$ \\
\hline 13257000 & $\begin{array}{l}\text { Middle Fork Weiser River near } \\
\text { Mesa, ID }\end{array}$ & 86.5 & $\begin{array}{l}\text { 1911-13, } \\
1920-21, \\
1937-49, \\
1956, \\
1981-82, \\
1985-88, \\
1997\end{array}$ & $\begin{array}{l}1997 \\
1948\end{array}$ & $-\overline{4}$ & $\begin{array}{r}2,340 \\
994\end{array}$ & $1 / 1 / 97$ & -- & 2,340 & $\mathrm{~N}$ & $>50$ \\
\hline 13258500 & Weiser River near Cambridge, ID & 605 & 1939-98 & 1997 & 14.54 & 22,800 & $\begin{array}{l}5 / 18 / 96 \\
1 / 1 / 97\end{array}$ & $\begin{array}{l}13.16 \\
14.54\end{array}$ & $\begin{array}{r}8,850 \\
22,800\end{array}$ & $\begin{array}{l}\mathrm{N} \\
\mathrm{N}\end{array}$ & $\begin{array}{r}10-15 \\
>100\end{array}$ \\
\hline 13260000 & Pine Creek near Cambridge, ID & 54.0 & $\begin{array}{l}1939-62 \\
1997\end{array}$ & $\begin{array}{l}1997 \\
1958\end{array}$ & --50 & $\begin{array}{l}970 \\
850\end{array}$ & $1 / 1 / 97$ & -- & 970 & $\mathrm{~N}$ & $50-75$ \\
\hline 13261000 & $\begin{array}{l}\text { Little Weiser River near Indian } \\
\text { Valley, ID }\end{array}$ & 81.9 & $\begin{array}{l}1923-27, \\
1938-71, \\
1997\end{array}$ & $\begin{array}{l}1997 \\
1965\end{array}$ & $-\overline{-}-6$ & $\begin{array}{l}2,230 \\
1,480\end{array}$ & $1 / 1 / 97$ & -- & 2,230 & $\mathrm{~N}$ & $>100$ \\
\hline 13263500 & $\begin{array}{l}\text { Weiser River above Crane Creek } \\
\text { near Weiser, ID }\end{array}$ & 1,160 & $\begin{array}{l}1921-52 \\
1997\end{array}$ & $\begin{array}{l}1997 \\
1932\end{array}$ & $\begin{array}{l}-- \\
10.80\end{array}$ & $\begin{array}{l}31,100 \\
16,900\end{array}$ & $1 / 2 / 97$ & -- & 31,100 & $\mathrm{~N}$ & $>100$ \\
\hline 13266000 & Weiser River near Weiser, ID & 1,460 & $\begin{array}{l}\text { 1890-91, } \\
\text { 1895-1904, } \\
\text { 1911-14, } \\
1953-98\end{array}$ & 1997 & 17.20 & 34,500 & $1 / 2 / 97$ & 17.20 & 34,500 & $\mathrm{~N}$ & $>100$ \\
\hline 13269000 & Snake River at Weiser, ID & 69,200 & 1910-98 & 1952 & 14.67 & 84,500 & $1 / 3 / 97$ & 14.49 & 84,100 & $\mathrm{Y}$ & $15-20$ \\
\hline
\end{tabular}


Table 13. Maximum stage and discharge for period of record for streamgages having significant floods during 1994-98 water years in Idaho.-Continued

$\left[\mathrm{mi}^{2}\right.$, square miles; $\mathrm{ft}$, feet above an arbitrary datum; $\mathrm{ft}^{3} / \mathrm{s}$, cubic feet per second; --, not determined or not applicable; >, greater than. Source: Recurrence intervals calculated from U.S. Geological Survey data. Other data from U.S. Geological Survey reports or databases]

\begin{tabular}{|c|c|c|c|c|c|c|c|c|c|c|c|}
\hline \multirow{2}{*}{$\begin{array}{c}\text { Streamgage } \\
\text { number } \\
\text { (fig. 24) }\end{array}$} & \multirow[b]{2}{*}{ Streamgage name } & \multirow{2}{*}{$\begin{array}{c}\text { Total } \\
\text { drainage } \\
\left(\mathrm{mi}^{2}\right)\end{array}$} & \multicolumn{4}{|c|}{$\begin{array}{c}\text { Maximum stage and discharge for period of record } \\
\text { through } 1998 \text { water year }\end{array}$} & \multicolumn{5}{|c|}{ Significant floods 1994-98 water years } \\
\hline & & & $\begin{array}{l}\text { Period of } \\
\text { record } \\
\text { (water } \\
\text { years) }\end{array}$ & Water year & $\begin{array}{l}\text { Stage } \\
(\mathrm{ft})\end{array}$ & $\begin{array}{c}\text { Discharge } \\
\left(\mathrm{ft}^{3} / \mathrm{s}\right)\end{array}$ & $\begin{array}{c}\text { Date } \\
\text { (month/d } \\
\text { ay/year) }\end{array}$ & $\begin{array}{l}\text { Stage } \\
(\mathrm{ft})\end{array}$ & $\begin{array}{c}\text { Discharge } \\
\left(\mathrm{ft}^{3} / \mathrm{s}\right)\end{array}$ & $\begin{array}{c}\text { Regulated } \\
\text { during } \\
\text { flood }^{1}\end{array}$ & $\begin{array}{c}\text { Recurrence } \\
\text { interval } \\
\text { (years) }\end{array}$ \\
\hline 13295000 & Valley Creek at Stanley, ID & 147 & $\begin{array}{l}\text { 1911-13, } \\
1921-72, \\
1974, \\
1993-98\end{array}$ & $\begin{array}{l}1956 \\
1921\end{array}$ & $\begin{array}{l}3.92 \\
4.40\end{array}$ & $\begin{array}{l}2,000 \\
1,850\end{array}$ & $6 / 10 / 97$ & 3.64 & 1,760 & $\mathrm{~N}$ & $20-25$ \\
\hline 13297330 & Thompson Creek near Clayton, ID & 29.1 & 1973-98 & $\begin{array}{l}1997 \\
1982\end{array}$ & $\begin{array}{l}4.07 \\
5.74\end{array}$ & $\begin{array}{l}442 \\
329\end{array}$ & $5 / 15 / 97$ & 4.07 & 442 & $\mathrm{~N}$ & $20-30$ \\
\hline 13316500 & Little Salmon River at Riggins, ID & 576 & $\begin{array}{l}1948, \\
1951-54, \\
1957-98\end{array}$ & $\begin{array}{l}1974 \\
1997\end{array}$ & $\begin{array}{l}11.05 \\
12.00\end{array}$ & $\begin{array}{l}12,600 \\
10,500\end{array}$ & $1 / 1 / 97$ & 12.00 & 10,500 & $\mathrm{~N}$ & $50-55$ \\
\hline 13338500 & $\begin{array}{l}\text { South Fork Clearwater River at } \\
\text { Stites, ID }\end{array}$ & 1,150 & $\begin{array}{l}1911-12, \\
1964-98\end{array}$ & 1964 & 10.30 & 17,500 & $2 / 7 / 96$ & 8.82 & 12,100 & $\mathrm{~N}$ & $10-15$ \\
\hline 13339500 & Lolo Creek near Greer, ID & 243 & 1980-98 & 1996 & 17.01 & 5,990 & 2/9/96 & 17.01 & 5,990 & $\mathrm{~N}$ & $50-100$ \\
\hline 13340600 & $\begin{array}{l}\text { North Fork Clearwater River near } \\
\text { Canyon Ranger Station, ID }\end{array}$ & 1,360 & 1967-98 & 1996 & 17.58 & 37,500 & $\begin{array}{l}11 / 30 / 95 \\
5 / 17 / 97\end{array}$ & $\begin{array}{l}17.58 \\
17.35\end{array}$ & $\begin{array}{l}37,500 \\
36,200\end{array}$ & $\begin{array}{l}\mathrm{N} \\
\mathrm{N}\end{array}$ & $\begin{array}{l}40-50 \\
30-40\end{array}$ \\
\hline 13342450 & Lapwai Creek near Lapwai, ID & 235 & $1975-98$ & $\begin{array}{l}1996 \\
1986\end{array}$ & $\begin{array}{r}9.70 \\
10.22\end{array}$ & $\begin{array}{l}5,010 \\
3,380\end{array}$ & 2/9/96 & 9.70 & 5,010 & $\mathrm{~N}$ & $20-30$ \\
\hline 13345000 & Palouse River near Potlatch, ID & 317 & $\begin{array}{l}1915-19, \\
1967-98\end{array}$ & 1996 & 22.15 & 14,600 & 2/9/96 & 22.15 & 14,600 & $\mathrm{~N}$ & $>100$ \\
\hline 13346800 & $\begin{array}{l}\text { Paradise Creek at University of } \\
\text { Idaho at Moscow, ID }\end{array}$ & 17.7 & 1979-98 & 1996 & 11.26 & 970 & $2 / 9 / 96$ & 11.26 & 970 & $\mathrm{~N}$ & $30-40$ \\
\hline
\end{tabular}

${ }^{1}$ Regulated during flood: N, no; Y, yes. 


\section{Illinois}

Very intense rainfall fell over most of central Illinois during April 11-12, 1994. Rainfall amounts ranged from 1.40 to 5.28 inches in less than 6 hours at most measurement locations (National Oceanic and Atmospheric Administration, 1994a). Flooding resulted along the Vermilion, Embarras, Sangamon, and Illinois Rivers (fig. 25) and their tributaries due to the very intense rain. Two people died after trying to drive their cars across flooded roadways. Danville's water-treatment plant also flooded causing \$10 million in damage (National Oceanic and Atmospheric Administration, 1994b). Fifteen streamgages had significant flood discharges in April 1994 (table 14). Although flood levels did not reach 1993 crest values, levels still were high enough to cause widespread lowland flooding and flooding in towns near the Illinois and Mississippi Rivers.

The Illinois, Big Muddy, and Sangamon Rivers and many of their tributaries began to rise above flood stage on May 14, 1995, due to excessive amounts of rainfall in the preceding 2 weeks over central and southern Illinois. The flooding continued into June as even more rain fell over the area during the last half of May and first part of June. Damage in and near Lincoln was \$2 million (National Oceanic and Atmospheric Administration, 1995b). The Mississippi River flooded tens of thousands of acres of cropland. Flooding in the Cairo area was considerably worse in 1995 than in 1993 because the Ohio River also was above flood stage. At the confluence of the Ohio and Mississippi Rivers at Cairo, a State park was totally underwater. The flooding on the Mississippi upstream from Cairo did not reach the levels of the 1993 flood. The U.S. Coast Guard halted all barge traffic from Cairo northward for over a week. This action was taken in an effort to prevent wake damage, especially to levees, which were in danger of overtopping. The closure of the river was very costly to the barge industry. The Mississippi River crested at 47 feet, about 14 feet above flood stage, at Cape Girardeau, Missouri, on May 24. The Ohio River crested at 55.6 feet at Cairo on May 25. Extensive flooding of bottom lands resulted as the Ohio River crested well above flood stage. Thousands of acres of crops were submerged. One of the major differences between the floods of 1993 and 1995 was the length of occurrence. The flood of 1993 covered two periods - a spring flood and then the major summer flooding, which continued into September. Although many rivers were still above flood stage at the end of May 1995, river stages fell rapidly and were not above flood stage after June 1995. Another difference was the lack of levees that remained along the rivers. Many agricultural levees destroyed by the flood of 1993 were not rebuilt. Many of the remaining levees were quickly overtopped or washed away in 1995 (National Oceanic and Atmospheric Administration, 1995b).

Slow-moving thunderstorms dumped between 6 and 10 inches of rain near Beardstown on May 8, 1996 (National Oceanic and Atmospheric Administration, 1996a). The resulting flash flooding washed out several roads. Sixty-six homes sustained damage, ranging from water in the basement to structural collapse. No injuries were reported, and damage was estimated at \$2 million (National Oceanic and Atmospheric Administration, 1996b).
The Wabash River was above flood stage for most of May 1996 near Crossville (National Oceanic and Atmospheric Administration, 1996b). On May 7, the river crested at 20.9 feet, 5.9 feet above flood stage. A 9-mile long earthen levee protecting bottomland farm fields was breached at the same time the river crested. This breach resulted in an additional 5,000 acres of flooded cropland near Grayville. The levee near Mt. Carmel was breached. Two counties were declared Federal disaster areas as a result of the flooding.

Record-breaking rainfall over parts of north-central and northeastern Illinois resulted in unprecedented flash flooding July 17-18, 1996. The area of most-intense rain, in excess of 5 inches, fell in a corridor from near Rockford through Aurora and Joliet to the southern suburbs of Chicago (National Oceanic and Atmospheric Administration, 1996a). The 16.91 inches that fell at Aurora set a 24-hour record for the State of Illinois. Much of this area had from 7 to 11 inches of rainfall. Eleven northeastern Illinois counties were declared a Federal disaster. Twenty USGS streamgages recorded all-time record streamflows (table 14). As a result of the flood, a total of $\$ 103$ million in disaster housing grants were issued, $\$ 49$ million in personal property and small business loans were approved, and $\$ 6$ million in small business loans were approved. There also were more than $\$ 2$ million in State grants approved. Crop damage was likely in the tens of millions of dollars. Two deaths were directly attributed to the flood (National Oceanic and Atmospheric Administration, 1996b).

Excessive rainfall occurred on February 20-22, 1997. Between 3 to 4 inches of rain fell over parts of northern Illinois (National Oceanic and Atmospheric Administration, 1997a). Widespread flooding resulted from excessive rains falling on frozen soil. The upper Illinois River crested within a foot of the record from Morris downstream to LaSalle (National Oceanic and Atmospheric Administration, 1997b) on February 22. A 63year-old woman died when she drove her car into a barricaded, flooded viaduct under railroad tracks in a south suburb of Chicago. The single largest effect of the excessive rains was felt along the Rock River where record flooding occurred. Near Joslin (streamgage 05446500 , table 14), the Rock River reached a record crest of 18.88 feet.

A massive flood crest moved down the Ohio River during the first few weeks of March 1997. Approximately 10 inches of rain fell within a 1- to 3-day period in the middle Ohio River Valley from the Louisville, Kentucky, area to Cincinnati, Ohio. This resulted in the worst river flood in about 30 years along the Illinois shore, and one of the five worst on record (National Oceanic and Atmospheric Administration, 1997a).

\section{References}

National Oceanic and Atmospheric Administration (NOAA), 1994a-97a, Climatological data (by State): Asheville, North Carolina, National Climatic Data Center, various months. National Oceanic and Atmospheric Administration (NOAA), 1994b-97b, Storm data (by State): Asheville, North Carolina, National Climatic Data Center, various months. 


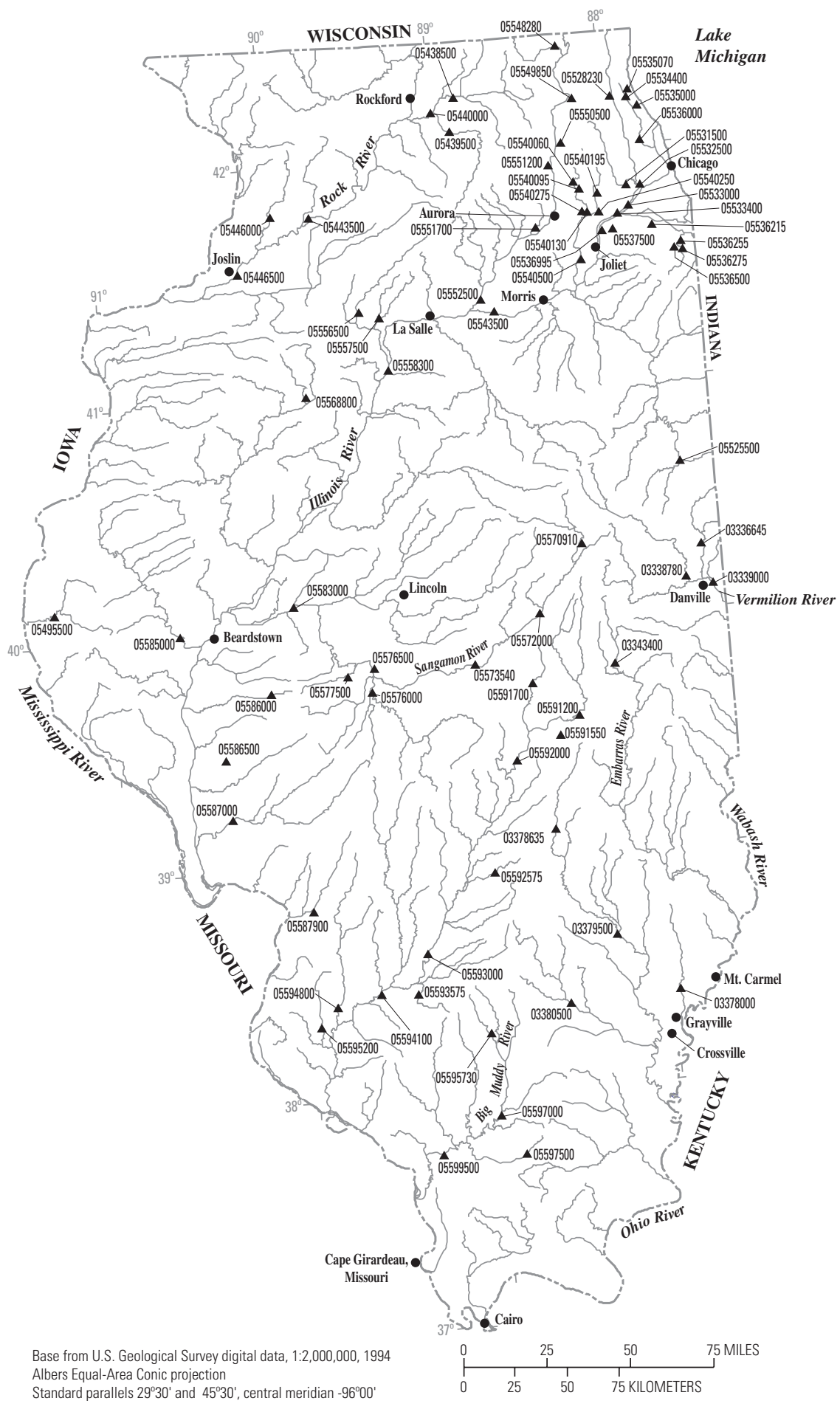

Albers Equal-Area Conic projection
Standard parallels $29^{\circ} 30^{\prime}$ and $45^{\circ} 30^{\prime}$, central meridian $-96^{\circ} 00^{\prime}$

\section{EXPLANATION}

$0559500 \_$Streamgage and number

Figure 25. Location of streamgages with significant floods during 1994-98 water years for Illinois. 
Table 14. Maximum stage and discharge for period of record for streamgages having significant floods during 1994-98 water years in Illinois.

$\left[\mathrm{mi}^{2}\right.$, square miles; $\mathrm{ft}$, feet above an arbitrary datum; $\mathrm{ft}^{3} / \mathrm{s}$, cubic feet per second; --, not determined or not applicable; $>$, greater than; <, less than. Source: Recurrence intervals calculated from U.S. Geological Survey data. Other data from U.S. Geological Survey reports or databases]

\begin{tabular}{|c|c|c|c|c|c|c|c|c|c|c|c|}
\hline \multirow{2}{*}{$\begin{array}{c}\text { Streamgage } \\
\text { number } \\
\text { (fig. 25) }\end{array}$} & \multirow[b]{2}{*}{ Streamgage name } & \multirow{2}{*}{$\begin{array}{c}\text { Total } \\
\text { drainage } \\
\left(\mathrm{mi}^{2}\right)\end{array}$} & \multicolumn{4}{|c|}{$\begin{array}{c}\text { Maximum stage and discharge for period of record } \\
\text { through } 1998 \text { water year }\end{array}$} & \multicolumn{5}{|c|}{ Significant floods $1994-98$ water years } \\
\hline & & & $\begin{array}{l}\text { Period of } \\
\text { record } \\
\text { (water } \\
\text { years) }\end{array}$ & Water year & $\begin{array}{l}\text { Stage } \\
(\mathrm{ft})\end{array}$ & $\begin{array}{l}\text { Discharge } \\
\left(\mathrm{ft}^{3} / \mathrm{s}\right)\end{array}$ & $\begin{array}{c}\text { Date } \\
\text { (month/ } \\
\text { day/ } \\
\text { year) }\end{array}$ & $\begin{array}{l}\text { Stage } \\
(\mathrm{ft})\end{array}$ & $\begin{array}{l}\text { Discharge } \\
\left(\mathrm{ft}^{3} / \mathrm{s}\right)\end{array}$ & $\begin{array}{l}\text { Regulated } \\
\text { during } \\
\text { flood }^{1}\end{array}$ & $\begin{array}{c}\text { Recurrence } \\
\text { interval } \\
\text { (years) }\end{array}$ \\
\hline 03336645 & $\begin{array}{l}\text { Middle Fork Vermilion River } \\
\text { above Oakwood, IL }\end{array}$ & 432 & $1979-98$ & 1994 & 20.46 & 15,500 & $4 / 13 / 94$ & 20.46 & 15,500 & $\mathrm{~N}$ & $25-50$ \\
\hline 03338780 & $\begin{array}{l}\text { North Fork Vermilion River near } \\
\text { Bismarck, IL }\end{array}$ & 262 & 1989-98 & 1994 & 22.45 & 20,100 & $4 / 12 / 94$ & 22.45 & 20,100 & $\mathrm{~N}$ & $5-10$ \\
\hline 03339000 & Vermilion River near Danville, IL & 1,290 & $\begin{array}{l}1915-21, \\
1929, \\
1931-98\end{array}$ & $\begin{array}{l}1939 \\
1994\end{array}$ & $\begin{array}{l}28.59 \\
31.56\end{array}$ & $\begin{array}{l}48,700 \\
47,900\end{array}$ & $4 / 13 / 94$ & 31.56 & 47,900 & $\mathrm{~N}$ & $>100$ \\
\hline 03343400 & Embarras River near Camargo, IL & 186 & $1961-98$ & 1994 & 17.33 & 8,040 & $\begin{array}{l}4 / 12 / 94 \\
5 / 11 / 96\end{array}$ & $\begin{array}{l}17.33 \\
16.43\end{array}$ & $\begin{array}{l}8,040 \\
6,740\end{array}$ & $\begin{array}{l}\mathrm{N} \\
\mathrm{N}\end{array}$ & $\begin{array}{r}50-100 \\
25\end{array}$ \\
\hline 03378000 & Bonpas Creek at Browns, IL & 228 & $1941-98$ & 1961 & 26.04 & 7,500 & $11 / 15 / 93$ & 23.60 & 6,490 & $\mathrm{~N}$ & $25-50$ \\
\hline 03378635 & $\begin{array}{l}\text { Little Wabash River near } \\
\text { Effingham, IL }\end{array}$ & 240 & $1967-98$ & 1996 & 21.19 & 17,800 & $5 / 8 / 96$ & 21.19 & 17,800 & $\mathrm{~N}$ & $>100$ \\
\hline 03379500 & $\begin{array}{l}\text { Little Wabash River below Clay } \\
\text { City, IL }\end{array}$ & 1,131 & $1915-98$ & 1950 & 26.67 & 47,000 & $5 / 19 / 95$ & 26.08 & 43,700 & $\mathrm{~N}$ & 30 \\
\hline 03380500 & Skillet Fork at Wayne City, IL & 464 & $\begin{array}{l}1909-12, \\
1915-21, \\
1929-98\end{array}$ & $\begin{array}{l}1990 \\
1961\end{array}$ & $\begin{array}{l}25.75 \\
25.80\end{array}$ & $\begin{array}{l}59,400 \\
51,000\end{array}$ & $5 / 18 / 95$ & 23.71 & 38,100 & $\mathrm{~N}$ & 40 \\
\hline 05438500 & Kishwaukee River at Belvidere, IL & 538 & 1940-98 & 1994 & 14.19 & 11,900 & $2 / 20 / 94$ & 14.19 & 11,900 & $\mathrm{~N}$ & 25 \\
\hline 05439500 & $\begin{array}{l}\text { South Branch Kishwaukee River } \\
\text { near Fairdale, IL }\end{array}$ & 387 & $\begin{array}{l}1937, \\
1940-98\end{array}$ & 1996 & 13.37 & 25,400 & $\begin{array}{l}2 / 20 / 94 \\
7 / 18 / 96 \\
2 / 21 / 97\end{array}$ & $\begin{array}{l}10.31 \\
13.37 \\
10.71\end{array}$ & $\begin{array}{r}8,790 \\
25,400 \\
9,940\end{array}$ & $\begin{array}{l}\mathrm{N} \\
\mathrm{N} \\
\mathrm{N}\end{array}$ & $\begin{array}{r}10 \\
>100 \\
20\end{array}$ \\
\hline 05440000 & $\begin{array}{l}\text { Kishwaukee River near Perryville, } \\
\text { IL }\end{array}$ & 1,099 & $\begin{array}{l}1938, \\
1940-98\end{array}$ & $\begin{array}{l}1996 \\
1938\end{array}$ & $\begin{array}{l}23.54 \\
23.85\end{array}$ & $\begin{array}{r}24,200 \\
--\end{array}$ & $\begin{array}{l}2 / 21 / 94 \\
7 / 18 / 96 \\
2 / 22 / 97\end{array}$ & $\begin{array}{l}20.71 \\
23.54 \\
19.76\end{array}$ & $\begin{array}{l}17,100 \\
24,200 \\
17,400\end{array}$ & $\begin{array}{l}\mathrm{N} \\
\mathrm{N} \\
\mathrm{N}\end{array}$ & $\begin{array}{r}10-25 \\
50-100 \\
10-25\end{array}$ \\
\hline 05443500 & Rock River at Como, IL & 8,753 & $\begin{array}{l}1915-76, \\
1978-86, \\
1991-98\end{array}$ & 1973 & 15.66 & 59,700 & $5 / 29 / 96$ & 13.58 & 48,300 & $\mathrm{~N}$ & 25 \\
\hline 05446000 & Rock Creek at Morrison, IL & 164 & $\begin{array}{l}\text { 1940-71, } \\
1978-98\end{array}$ & $\begin{array}{l}1946 \\
1997\end{array}$ & $\begin{array}{l}-- \\
17.76\end{array}$ & $\begin{array}{l}5,770 \\
5,050\end{array}$ & $2 / 21 / 97$ & 17.76 & 5,050 & $\mathrm{~N}$ & 100 \\
\hline
\end{tabular}


Table 14. Maximum stage and discharge for period of record for streamgages having significant floods during 1994-98 water years in Illinois.—Continued

$\left[\mathrm{mi}^{2}\right.$, square miles; $\mathrm{ft}$, feet above an arbitrary datum; $\mathrm{ft}^{3} / \mathrm{s}$, cubic feet per second; --, not determined or not applicable; $>$, greater than; <, less than. Source: Recurrence intervals calculated from U.S. Geological Survey data. Other data from U.S. Geological Survey reports or databases]

\begin{tabular}{|c|c|c|c|c|c|c|c|c|c|c|c|}
\hline \multirow{2}{*}{$\begin{array}{l}\text { Streamgage } \\
\text { number } \\
\text { (fig. 25) }\end{array}$} & \multirow[b]{2}{*}{ Streamgage name } & \multirow{2}{*}{$\begin{array}{c}\text { Total } \\
\text { drainage } \\
\left(\mathrm{mi}^{2}\right)\end{array}$} & \multicolumn{4}{|c|}{$\begin{array}{c}\text { Maximum stage and discharge for period of record } \\
\text { through } 1998 \text { water year }\end{array}$} & \multicolumn{5}{|c|}{ Significant floods $1994-98$ water years } \\
\hline & & & $\begin{array}{c}\text { Period of } \\
\text { record } \\
\text { (water } \\
\text { years) }\end{array}$ & Water year & $\begin{array}{l}\text { Stage } \\
\text { (ft) }\end{array}$ & $\begin{array}{l}\text { Discharge } \\
\left(\mathrm{ft}^{3} / \mathrm{s}\right)\end{array}$ & $\begin{array}{c}\text { Date } \\
\text { (month/ } \\
\text { day/ } \\
\text { year) }\end{array}$ & $\begin{array}{l}\text { Stage } \\
(\mathrm{ft})\end{array}$ & $\begin{array}{l}\text { Discharge } \\
\left(\mathrm{ft}^{3} / \mathrm{s}\right)\end{array}$ & $\begin{array}{c}\text { Regulated } \\
\text { during } \\
\text { flood }^{1}\end{array}$ & $\begin{array}{c}\text { Recurrence } \\
\text { interval } \\
\text { (years) }\end{array}$ \\
\hline 05446500 & Rock River near Joslin, IL & 9,549 & $1940-98$ & $\begin{array}{l}1993 \\
1997\end{array}$ & $\begin{array}{l}18.35 \\
18.88\end{array}$ & $\begin{array}{l}46,500 \\
41,000\end{array}$ & $5 / 31 / 96$ & 18.73 & 44,800 & $\mathrm{~N}$ & $10-25$ \\
\hline 05495500 & Bear Creek near Marcelline, IL & 349 & 1944-98 & $\begin{array}{l}1996 \\
1985\end{array}$ & $\begin{array}{l}28.36 \\
28.38\end{array}$ & $\begin{array}{l}35,500 \\
29,500\end{array}$ & $5 / 8 / 96$ & 28.36 & 35,500 & $\mathrm{~N}$ & $>100$ \\
\hline 05525500 & Sugar Creek at Milford, IL & 446 & 1949-98 & $\begin{array}{l}1951 \\
1994\end{array}$ & $\begin{array}{l}20.90 \\
28.16\end{array}$ & $\begin{array}{l}22,900 \\
19,400\end{array}$ & $4 / 12 / 94$ & 28.16 & 19,400 & $\mathrm{~N}$ & $10-25$ \\
\hline 05528230 & Indian Creek at Prairie View, IL & 36.0 & $\begin{array}{l}1960-61, \\
1963-65, \\
1967-71, \\
1973-76, \\
1990-96\end{array}$ & 1996 & 7.10 & 1,800 & $5 / 17 / 96$ & 7.10 & 1,800 & $\mathrm{~N}$ & $200-500$ \\
\hline 05531500 & Salt Creek at Western Springs, IL & 115 & $1946-98$ & 1987 & 10.54 & 3,540 & $2 / 21 / 97$ & 9.35 & 2,510 & $\mathrm{~N}$ & $25-50$ \\
\hline 05532500 & Des Plaines River at Riverside, IL & 630 & $1914-98$ & 1987 & 9.90 & 9,770 & $2 / 22 / 97$ & 8.45 & 6,990 & $\mathrm{~N}$ & 20 \\
\hline 05533000 & $\begin{array}{l}\text { Flag Creek near Willow Springs, } \\
\text { IL }\end{array}$ & 16.5 & 1960-98 & 1961 & 13.71 & 2,680 & $7 / 18 / 96$ & 10.37 & 2,300 & $\mathrm{~N}$ & $25-50$ \\
\hline 05533400 & Sawmill Creek near Lemont, IL & 13.0 & $\begin{array}{l}1961-79 \\
1986-98\end{array}$ & 1996 & 17.53 & 3,070 & $7 / 18 / 96$ & 17.53 & 3,070 & $\mathrm{~N}$ & $200-300$ \\
\hline 05534400 & $\begin{array}{l}\text { North Branch Chicago River at } \\
\text { Bannockburn, IL }\end{array}$ & 15.8 & $\begin{array}{l}1960-76 \\
1996\end{array}$ & 1996 & 17.54 & 3,070 & $7 / 18 / 96$ & 17.54 & 3,070 & $\mathrm{~N}$ & -- \\
\hline 05535000 & Skokie River at Lake Forest, IL & 13.0 & $1952-98$ & $\begin{array}{l}1997 \\
1982\end{array}$ & $\begin{array}{l}7.24 \\
8.35\end{array}$ & $\begin{array}{l}496 \\
435\end{array}$ & $2 / 21 / 97$ & 7.24 & 496 & $\mathrm{~N}$ & $50-100$ \\
\hline 05535070 & $\begin{array}{l}\text { Skokie River near Highland Park, } \\
\text { IL }\end{array}$ & 21.1 & 1967-98 & $\begin{array}{l}1987 \\
1997\end{array}$ & $\begin{array}{l}9.09 \\
9.13\end{array}$ & $\begin{array}{l}895 \\
894\end{array}$ & $2 / 21 / 97$ & 9.13 & 894 & $\mathrm{~N}$ & $50-100$ \\
\hline 05536000 & $\begin{array}{l}\text { North Branch Chicago River at } \\
\text { Niles, IL }\end{array}$ & 100 & 1951-98 & 1987 & 11.35 & 2,590 & $2 / 21 / 97$ & 10.63 & 2,260 & $\mathrm{~N}$ & $25-50$ \\
\hline
\end{tabular}


$\left[\mathrm{mi}^{2}\right.$, square miles; $\mathrm{ft}$, feet above an arbitrary datum; $\mathrm{ft}^{3} / \mathrm{s}$, cubic feet per second; --, not determined or not applicable; $>$, greater than; <, less than. Source: Recurrence intervals calculated from U.S. Geological Survey data. Other data from U.S. Geological Survey reports or databases]

\begin{tabular}{|c|c|c|c|c|c|c|c|c|c|c|c|}
\hline \multirow[b]{2}{*}{$\begin{array}{l}\text { Streamgage } \\
\text { number } \\
\text { (fig. 25) }\end{array}$} & \multirow[b]{2}{*}{ Streamgage name } & \multirow{2}{*}{$\begin{array}{c}\text { Total } \\
\text { drainage } \\
\left(\mathrm{mi}^{2}\right)\end{array}$} & \multicolumn{4}{|c|}{$\begin{array}{c}\text { Maximum stage and discharge for period of record } \\
\text { through } 1998 \text { water year }\end{array}$} & \multicolumn{5}{|c|}{ Significant floods 1994-98 water years } \\
\hline & & & $\begin{array}{l}\text { Period of } \\
\text { record } \\
\text { (water } \\
\text { years) }\end{array}$ & Water year & $\begin{array}{l}\text { Stage } \\
(\mathrm{ft})\end{array}$ & $\begin{array}{l}\text { Discharge } \\
\left(\mathrm{ft}^{3} / \mathrm{s}\right)\end{array}$ & $\begin{array}{c}\text { Date } \\
\text { (month/ } \\
\text { day/ } \\
\text { year) }\end{array}$ & $\begin{array}{l}\text { Stage } \\
(\mathrm{ft})\end{array}$ & $\begin{array}{l}\text { Discharge } \\
\left(\mathrm{ft}^{3} / \mathrm{s}\right)\end{array}$ & $\begin{array}{l}\text { Regulated } \\
\text { during } \\
\text { flood }^{1}\end{array}$ & $\begin{array}{c}\text { Recurrence } \\
\text { interval } \\
\text { (years) }\end{array}$ \\
\hline 05536215 & Thorn Creek at Glenwood, IL & 24.7 & $1950-98$ & $\begin{array}{l}1996 \\
1968\end{array}$ & 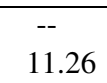 & $\begin{array}{l}2,700 \\
2,600\end{array}$ & $7 / 18 / 96$ & -- & 2,700 & $\mathrm{~N}$ & $25-50$ \\
\hline 05536255 & Butterfield Creek at Flossmoor, IL & 23.5 & $\begin{array}{l}1948-55 \\
1957-98\end{array}$ & 1996 & 12.59 & 2,220 & $7 / 18 / 96$ & 12.59 & 2,220 & $\mathrm{~N}$ & $25-50$ \\
\hline 05536275 & Thorn Creek at Thornton, IL & 104 & 1947-98 & $\begin{array}{l}1957 \\
1981\end{array}$ & $\begin{array}{l}16.00 \\
17.06\end{array}$ & $\begin{array}{l}4,700 \\
4,140\end{array}$ & $7 / 18 / 96$ & 16.24 & 4,470 & $\mathrm{~N}$ & 30 \\
\hline 05536500 & Tinley Creek near Palos Park, IL & 11.2 & $\begin{array}{l}1951-55 \\
1957-98\end{array}$ & $\begin{array}{l}1996 \\
1955\end{array}$ & $\begin{array}{l}10.25 \\
10.30\end{array}$ & $\begin{array}{l}2,010 \\
1,930\end{array}$ & $7 / 18 / 96$ & 10.25 & 2,010 & $\mathrm{~N}$ & $50-100$ \\
\hline 05536995 & $\begin{array}{l}\text { Chicago Sanitary and Ship Canal at } \\
\text { Romeoville, IL }\end{array}$ & 739 & $1985-98$ & $\begin{array}{l}1997 \\
1996\end{array}$ & $\begin{array}{l}23.95 \\
25.08\end{array}$ & $\begin{array}{l}19,466 \\
19,448\end{array}$ & $\begin{array}{l}2 / 21 / 97 \\
7 / 18 / 96\end{array}$ & $\begin{array}{l}23.95 \\
25.08\end{array}$ & $\begin{array}{l}19,466 \\
19,448\end{array}$ & $\begin{array}{l}\mathrm{Y} \\
\mathrm{Y}\end{array}$ & -- \\
\hline 05537500 & Long Run near Lemont, IL & 20.9 & $1951-98$ & 1996 & 11.10 & 5,310 & $7 / 18 / 96$ & 11.10 & 5,310 & $\mathrm{~N}$ & $>500$ \\
\hline 05540060 & Kress Creek at West Chicago, IL & 18.1 & $\begin{array}{l}1961-80 \\
1986-98\end{array}$ & 1996 & 9.24 & 1,980 & $7 / 18 / 96$ & 9.24 & 1,980 & $\mathrm{~N}$ & $>500$ \\
\hline 05540095 & $\begin{array}{l}\text { West Branch Du Page River near } \\
\text { Warrenville, IL }\end{array}$ & 90.4 & 1969-98 & 1996 & 6.41 & 3,470 & $7 / 18 / 96$ & 6.41 & 3,470 & $\mathrm{~N}$ & $200-500$ \\
\hline 05540130 & $\begin{array}{l}\text { West Branch Du Page River near } \\
\text { Naperville, IL }\end{array}$ & 123 & 1989-98 & 1996 & 14.31 & 6,620 & $7 / 18 / 96$ & 14.31 & 6,620 & $\mathrm{~N}$ & $>500$ \\
\hline 05540195 & $\begin{array}{l}\text { Saint Joseph Creek at U.S. } \\
\text { Route } 34 \text { at Lisle, IL }\end{array}$ & 11.1 & 1989-98 & 1996 & 12.89 & 1,280 & $7 / 18 / 96$ & 12.89 & 1,280 & $\mathrm{~N}$ & $>500$ \\
\hline 05540250 & $\begin{array}{l}\text { East Branch Du Page River at } \\
\text { Bolingbrook, IL }\end{array}$ & 75.8 & 1989-98 & 1996 & 23.75 & 3,980 & $7 / 18 / 96$ & 23.75 & 3,980 & $\mathrm{~N}$ & $>500$ \\
\hline 05540275 & $\begin{array}{l}\text { Spring Brook at } 87 \text { th Street near } \\
\text { Naperville, IL }\end{array}$ & 9.90 & 1988-98 & 1996 & 10.77 & 1,750 & 7/18/96 & 10.77 & 1,750 & $\mathrm{~N}$ & $25-50$ \\
\hline 05540500 & Du Page River at Shorewood, IL & 324 & $1941-98$ & 1996 & 14.03 & 17,300 & $7 / 18 / 96$ & 14.03 & 17,300 & $\mathrm{~N}$ & $>500$ \\
\hline
\end{tabular}


Table 14. Maximum stage and discharge for period of record for streamgages having significant floods during 1994-98 water years in Illinois. - Continued

$\left[\mathrm{mi}^{2}\right.$, square miles; $\mathrm{ft}$, feet above an arbitrary datum; $\mathrm{ft}^{3} / \mathrm{s}$, cubic feet per second; --, not determined or not applicable; $>$, greater than; <, less than. Source: Recurrence intervals calculated from U.S. Geological Survey data. Other data from U.S. Geological Survey reports or databases]

\begin{tabular}{|c|c|c|c|c|c|c|c|c|c|c|c|}
\hline \multirow[b]{2}{*}{$\begin{array}{c}\text { Streamgage } \\
\text { number } \\
\text { (fig. 25) }\end{array}$} & \multirow[b]{2}{*}{ Streamgage name } & \multirow[b]{2}{*}{$\begin{array}{c}\text { Total } \\
\text { drainage } \\
\left(\mathrm{mi}^{2}\right)\end{array}$} & \multicolumn{4}{|c|}{$\begin{array}{c}\text { Maximum stage and discharge for period of record } \\
\text { through } 1998 \text { water year }\end{array}$} & \multicolumn{5}{|c|}{ Significant floods 1994-98 water years } \\
\hline & & & $\begin{array}{c}\text { Period of } \\
\text { record } \\
\text { (water } \\
\text { years) }\end{array}$ & Water year & $\begin{array}{l}\text { Stage } \\
(\mathrm{ft})\end{array}$ & $\begin{array}{l}\text { Discharge } \\
\left(\mathrm{ft}^{3} / \mathrm{s}\right)\end{array}$ & $\begin{array}{c}\text { Date } \\
\text { (month/ } \\
\text { day/ } \\
\text { year) }\end{array}$ & $\begin{array}{l}\text { Stage } \\
(\mathrm{ft})\end{array}$ & $\begin{array}{l}\text { Discharge } \\
\left(\mathrm{ft}^{3} / \mathrm{s}\right)\end{array}$ & $\begin{array}{c}\text { Regulated } \\
\text { during } \\
\text { flood }^{1}\end{array}$ & $\begin{array}{c}\text { Recurrence } \\
\text { interval } \\
\text { (years) }\end{array}$ \\
\hline 05543500 & Illinois River at Marseilles, IL & 8,259 & $\begin{array}{l}1892, \\
1894-98, \\
1900, \\
1904-98\end{array}$ & $\begin{array}{l}1997 \\
1922\end{array}$ & $\begin{array}{l}16.85 \\
20.10\end{array}$ & $\begin{array}{l}95,000 \\
60,600\end{array}$ & $2 / 22 / 97$ & 16.85 & 95,000 & $\mathrm{Y}$ & $100-200$ \\
\hline 05548280 & $\begin{array}{l}\text { Nippersink Creek near Spring } \\
\text { Grove, IL }\end{array}$ & 192 & $\begin{array}{l}1960, \\
1966-98\end{array}$ & 1986 & 14.26 & 2,910 & $2 / 21 / 94$ & 13.66 & 2,830 & $\mathrm{~N}$ & $10-25$ \\
\hline 05549850 & $\begin{array}{l}\text { Flint Creek near Fox River Grove, } \\
\text { IL }\end{array}$ & 37.0 & $\begin{array}{l}1960, \\
1962-76, \\
1990-96\end{array}$ & 1996 & 5.88 & 690 & $5 / 21 / 96$ & 5.88 & 690 & $\mathrm{~N}$ & $>500$ \\
\hline 05550500 & Poplar Creek at Elgin, IL & 35.2 & $1952-98$ & 1997 & 6.78 & 1,180 & $2 / 21 / 97$ & 6.78 & 1,180 & $\mathrm{~N}$ & 200 \\
\hline 05551200 & $\begin{array}{l}\text { Ferson Creek near Saint Charles, } \\
\text { IL }\end{array}$ & 51.7 & $\begin{array}{l}1961-83 \\
1985-98\end{array}$ & 1997 & 8.77 & 2,580 & $\begin{array}{l}7 / 18 / 96 \\
2 / 21 / 97\end{array}$ & $\begin{array}{l}7.79 \\
8.77\end{array}$ & $\begin{array}{l}1,990 \\
2,580\end{array}$ & $\begin{array}{l}\mathrm{N} \\
\mathrm{N}\end{array}$ & $\begin{array}{r}10-25 \\
50-100\end{array}$ \\
\hline 05551700 & $\begin{array}{l}\text { Blackberry Creek near Yorkville, } \\
\text { IL }\end{array}$ & 70.2 & $1961-98$ & 1996 & 13.16 & 5,510 & $7 / 18 / 96$ & 13.16 & 5,510 & $\mathrm{~N}$ & $>500$ \\
\hline 05552500 & Fox River at Dayton, IL & 2,642 & $\begin{array}{l}1915-24 \\
1926-98\end{array}$ & $\begin{array}{l}1996 \\
1955\end{array}$ & $\begin{array}{l}24.47 \\
24.63\end{array}$ & $\begin{array}{l}55,400 \\
47,100\end{array}$ & $\begin{array}{l}7 / 19 / 96 \\
2 / 22 / 97\end{array}$ & $\begin{array}{l}24.47 \\
21.46\end{array}$ & $\begin{array}{l}55,400 \\
41,200\end{array}$ & $\begin{array}{l}\mathrm{N} \\
\mathrm{N}\end{array}$ & $\begin{array}{r}>500 \\
200-500\end{array}$ \\
\hline 05556500 & Big Bureau Creek at Princeton, IL & 196 & $1937-98$ & 1974 & 16.01 & 12,500 & $2 / 21 / 97$ & 15.73 & 12,000 & $\mathrm{~N}$ & 50 \\
\hline 05557500 & East Bureau Creek near Bureau, IL & 99.0 & $\begin{array}{l}1937-91 \\
1993-98\end{array}$ & $\begin{array}{l}1997 \\
1938\end{array}$ & $\begin{array}{l}17.09 \\
17.39\end{array}$ & $\begin{array}{l}9,260 \\
6,200\end{array}$ & $2 / 21 / 97$ & 17.09 & 9,260 & $\mathrm{~N}$ & $100-200$ \\
\hline 05558300 & Illinois River at Henry, IL & 13,543 & 1982-98 & $\begin{array}{l}1997 \\
1982\end{array}$ & $\begin{array}{l}27.19 \\
30.75\end{array}$ & $\begin{array}{l}117,000 \\
104,000\end{array}$ & $2 / 23 / 97$ & 27.19 & 117,000 & $\mathrm{Y}$ & -- \\
\hline 05568800 & Indian Creek near Wyoming, IL & 62.7 & 1960-98 & $\begin{array}{l}1974 \\
1997\end{array}$ & $\begin{array}{l}13.81 \\
23.00\end{array}$ & $\begin{array}{l}6,540 \\
4,710\end{array}$ & $2 / 21 / 97$ & 23.00 & 4,710 & $\mathrm{~N}$ & $25-50$ \\
\hline 05570910 & Sangamon River at Fisher, IL & 240 & 1979-98 & 1994 & 21.58 & 13,000 & $4 / 12 / 94$ & 21.58 & 13,000 & $\mathrm{~N}$ & $25-50$ \\
\hline 05572000 & Sangamon River at Monticello, IL & 550 & $\begin{array}{l}1908-13 \\
1915-98\end{array}$ & $\begin{array}{l}1927 \\
1994\end{array}$ & $\begin{array}{l}18.50 \\
19.06\end{array}$ & $\begin{array}{l}19,000 \\
15,900\end{array}$ & 4/13/94 & 19.06 & 15,900 & $\mathrm{~N}$ & 30 \\
\hline
\end{tabular}


$\left[\mathrm{mi}^{2}\right.$, square miles; $\mathrm{ft}$, feet above an arbitrary datum; $\mathrm{ft}^{3} / \mathrm{s}$, cubic feet per second; --, not determined or not applicable; $>$, greater than; <, less than. Source: Recurrence intervals calculated from U.S. Geological Survey data. Other data from U.S. Geological Survey reports or databases]

\begin{tabular}{|c|c|c|c|c|c|c|c|c|c|c|c|}
\hline \multirow{2}{*}{$\begin{array}{l}\text { Streamgage } \\
\text { number } \\
\text { (fig. 25) }\end{array}$} & \multirow[b]{2}{*}{ Streamgage name } & \multirow{2}{*}{$\begin{array}{c}\text { Total } \\
\text { drainage } \\
\left(\mathrm{mi}^{2}\right)\end{array}$} & \multicolumn{4}{|c|}{$\begin{array}{c}\text { Maximum stage and discharge for period of record } \\
\text { through } 1998 \text { water year }\end{array}$} & \multicolumn{5}{|c|}{ Significant floods 1994-98 water years } \\
\hline & & & $\begin{array}{c}\text { Period of } \\
\text { record } \\
\text { (water } \\
\text { years) }\end{array}$ & Water year & $\begin{array}{l}\text { Stage } \\
(\mathrm{ft})\end{array}$ & $\begin{array}{c}\text { Discharge } \\
\left(\mathrm{ft}^{3} / \mathrm{s}\right)\end{array}$ & $\begin{array}{c}\text { Date } \\
\text { (month/ } \\
\text { day/ } \\
\text { year) }\end{array}$ & $\begin{array}{l}\text { Stage } \\
(\mathrm{ft})\end{array}$ & $\begin{array}{c}\text { Discharge } \\
\left(\mathrm{ft}^{3} / \mathrm{s}\right)\end{array}$ & $\begin{array}{l}\text { Regulated } \\
\text { during } \\
\text { flood }^{1}\end{array}$ & $\begin{array}{c}\text { Recurrence } \\
\text { interval } \\
\text { (years) }\end{array}$ \\
\hline 05573540 & $\begin{array}{l}\text { Sangamon River at Route } 48 \text { at } \\
\text { Decatur, IL }\end{array}$ & 938 & $1983-98$ & $\begin{array}{l}1994 \\
1983\end{array}$ & $\begin{array}{l}21.12 \\
23.04\end{array}$ & $\begin{array}{l}17,100 \\
12,400\end{array}$ & $4 / 15 / 94$ & 21.12 & 17,100 & $\mathrm{Y}$ & -- \\
\hline 05576000 & $\begin{array}{l}\text { South Fork Sangamon River near } \\
\text { Rochester, IL }\end{array}$ & 867 & $1950-98$ & 1994 & 32.40 & 20,300 & $4 / 14 / 94$ & 32.40 & 20,300 & $\mathrm{~N}$ & $50-100$ \\
\hline 05576500 & Sangamon River at Riverton, IL & 2,618 & $\begin{array}{l}1908-12, \\
1915-27, \\
1929-31, \\
1933-73, \\
1975-79, \\
1981-98\end{array}$ & $\begin{array}{l}1943 \\
1927\end{array}$ & $\begin{array}{l}31.52 \\
32.04\end{array}$ & $\begin{array}{l}68,700 \\
41,000\end{array}$ & $4 / 13 / 94$ & 28.28 & 46,300 & $\mathrm{~N}$ & $50-100$ \\
\hline 05577500 & Spring Creek at Springfield, IL & 107 & $1948-98$ & 1996 & 16.23 & 10,700 & $5 / 8 / 96$ & 16.23 & 10,700 & $\mathrm{~N}$ & $50-100$ \\
\hline 05583000 & Sangamon River near Oakford, IL & 5,093 & $\begin{array}{l}1910-12, \\
1915-18, \\
1921-22, \\
1926-29, \\
1931-98\end{array}$ & 1943 & 25.63 & 123,000 & $4 / 15 / 94$ & 23.21 & 78,100 & $\mathrm{~N}$ & $50-100$ \\
\hline 05585000 & La Moine River at Ripley, IL & 1,293 & $1921-98$ & $\begin{array}{l}1985 \\
1995\end{array}$ & $\begin{array}{l}29.07 \\
29.15\end{array}$ & $\begin{array}{l}28,000 \\
26,100\end{array}$ & $\begin{array}{l}5 / 18 / 95 \\
5 / 8 / 96\end{array}$ & $\begin{array}{l}29.15 \\
29.07\end{array}$ & $\begin{array}{l}26,100 \\
26,100\end{array}$ & $\begin{array}{l}\mathrm{N} \\
\mathrm{N}\end{array}$ & $\begin{array}{l}25-50 \\
25-50\end{array}$ \\
\hline 05586000 & $\begin{array}{l}\text { North Fork Mauvaise Terre Creek } \\
\text { near Jacksonville, IL }\end{array}$ & 29.1 & $\begin{array}{l}\text { 1950-90, } \\
1992-98\end{array}$ & $\begin{array}{l}1994 \\
1990\end{array}$ & $\begin{array}{l}12.35 \\
12.77\end{array}$ & $\begin{array}{l}7,160 \\
4,600\end{array}$ & $\begin{array}{l}4 / 12 / 94 \\
5 / 8 / 96\end{array}$ & $\begin{array}{l}12.35 \\
12.04\end{array}$ & $\begin{array}{l}7,160 \\
5,670\end{array}$ & $\begin{array}{l}\mathrm{N} \\
\mathrm{N}\end{array}$ & $\begin{array}{r}50-100 \\
25-50\end{array}$ \\
\hline 05586500 & $\begin{array}{l}\text { Hurricane Creek near Roodhouse, } \\
\text { IL }\end{array}$ & 2.30 & $\begin{array}{l}\text { 1951-90, } \\
1992-95\end{array}$ & 1957 & 11.77 & 1,700 & $4 / 12 / 94$ & 10.95 & 995 & $\mathrm{~N}$ & $25-50$ \\
\hline 05587000 & Macoupin Creek near Kane, IL & 868 & $\begin{array}{l}1921-33 \\
1941-98\end{array}$ & $\begin{array}{l}1994 \\
1991\end{array}$ & $\begin{array}{l}28.32 \\
--\end{array}$ & $\begin{array}{l}40,100 \\
13,700\end{array}$ & $4 / 12 / 94$ & 28.32 & 40,100 & $\mathrm{~N}$ & $50-100$ \\
\hline 05587900 & Cahokia Creek at Edwardsville, IL & 212 & 1969-98 & 1979 & 24.74 & 8,200 & $5 / 18 / 95$ & 21.75 & 7,310 & $\mathrm{~N}$ & -- \\
\hline 05591200 & Kaskaskia River at Cooks Mills, IL & 473 & $1971-98$ & 1994 & 17.30 & 9,950 & $4 / 13 / 94$ & 17.30 & 9,950 & $\mathrm{~N}$ & $10-25$ \\
\hline
\end{tabular}


Table 14. Maximum stage and discharge for period of record for streamgages having significant floods during 1994-98 water years in Illinois. - Continued

$\left[\mathrm{mi}^{2}\right.$, square miles; $\mathrm{ft}$, feet above an arbitrary datum; $\mathrm{ft}^{3} / \mathrm{s}$, cubic feet per second; --, not determined or not applicable; $>$, greater than; <, less than. Source: Recurrence intervals calculated from U.S. Geological Survey data. Other data from U.S. Geological Survey reports or databases]

\begin{tabular}{|c|c|c|c|c|c|c|c|c|c|c|c|}
\hline \multirow{2}{*}{$\begin{array}{c}\text { Streamgage } \\
\text { number } \\
\text { (fig. 25) }\end{array}$} & \multirow[b]{2}{*}{ Streamgage name } & \multirow{2}{*}{$\begin{array}{c}\text { Total } \\
\text { drainage } \\
\left(\mathrm{mi}^{2}\right)\end{array}$} & \multicolumn{4}{|c|}{$\begin{array}{c}\text { Maximum stage and discharge for period of record } \\
\text { through } 1998 \text { water year }\end{array}$} & \multicolumn{5}{|c|}{ Significant floods $1994-98$ water years } \\
\hline & & & $\begin{array}{c}\text { Period of } \\
\text { record } \\
\text { (water } \\
\text { years) }\end{array}$ & Water year & $\begin{array}{l}\text { Stage } \\
(\mathrm{ft})\end{array}$ & $\begin{array}{l}\text { Discharge } \\
\left(\mathrm{ft}^{3} / \mathrm{s}\right)\end{array}$ & $\begin{array}{c}\text { Date } \\
\text { (month/ } \\
\text { day/ } \\
\text { year) }\end{array}$ & $\begin{array}{l}\text { Stage } \\
(\mathrm{ft})\end{array}$ & $\begin{array}{l}\text { Discharge } \\
\left(\mathrm{ft}^{3} / \mathrm{s}\right)\end{array}$ & $\begin{array}{l}\text { Regulated } \\
\text { during } \\
\text { flood }^{1}\end{array}$ & $\begin{array}{c}\text { Recurrence } \\
\text { interval } \\
\text { (years) }\end{array}$ \\
\hline 05591550 & Whitley Creek near Allenville, IL & 34.6 & $1980-98$ & 1996 & 12.62 & 3,200 & $4 / 29 / 96$ & 12.62 & 3,200 & $\mathrm{~N}$ & 100 \\
\hline 05591700 & $\begin{array}{l}\text { West Okaw River near Lovington, } \\
\text { IL }\end{array}$ & 112 & 1980-98 & 1996 & 16.40 & 10,300 & $5 / 8 / 96$ & 16.40 & 10,300 & $\mathrm{~N}$ & $100-200$ \\
\hline 05592000 & Kaskaskia River at Shelbyville, IL & 1,054 & $\begin{array}{l}\text { 1908-13, } \\
1939, \\
1941-98\end{array}$ & 1957 & 22.37 & 25,900 & $3 / 22 / 98$ & 12.99 & 5,580 & $\mathrm{Y}$ & $2-5$ \\
\hline 05592575 & $\begin{array}{l}\text { Hickory Creek near Brownstown, } \\
\text { IL }\end{array}$ & 44.2 & 1989-98 & 1994 & 16.43 & 6,250 & $11 / 14 / 93$ & 16.43 & 6,250 & $\mathrm{~N}$ & $5-10$ \\
\hline 05593000 & Kaskaskia River at Carlyle, IL & 2,719 & $\begin{array}{l}1908-12, \\
1915, \\
1930-98\end{array}$ & 1943 & 33.70 & 54,400 & $\begin{array}{l}5 / 10 / 96 \\
4 / 10 / 98\end{array}$ & $\begin{array}{l}23.34 \\
23.39\end{array}$ & $\begin{array}{l}12,300 \\
12,200\end{array}$ & $\begin{array}{l}\mathrm{Y} \\
\mathrm{Y}\end{array}$ & $\begin{array}{l}<2 \\
<2\end{array}$ \\
\hline 05593575 & $\begin{array}{l}\text { Little Crooked Creek near New } \\
\text { Minden, IL }\end{array}$ & 84.3 & 1968-98 & 1995 & 21.76 & 11,900 & $\begin{array}{l}5 / 17 / 95 \\
4 / 29 / 96\end{array}$ & $\begin{array}{l}21.76 \\
21.42\end{array}$ & $\begin{array}{l}11,900 \\
11,300\end{array}$ & $\begin{array}{l}\mathrm{N} \\
\mathrm{N}\end{array}$ & $\begin{array}{l}10-25 \\
10-25\end{array}$ \\
\hline 05594100 & $\begin{array}{l}\text { Kaskaskia River near Venedy } \\
\text { Station, IL }\end{array}$ & 4,393 & 1970-98 & 1995 & 25.79 & 50,300 & $5 / 19 / 95$ & 25.79 & 50,300 & $\mathrm{Y}$ & -- \\
\hline 05594800 & Silver Creek near Freeburg, IL & 464 & $1971-98$ & 1995 & 25.38 & 15,300 & $5 / 19 / 95$ & 25.38 & 15,300 & $\mathrm{~N}$ & $25-50$ \\
\hline 05595200 & Richland Creek near Hecker, IL & 129 & 1970-98 & 1996 & 44.40 & 23,400 & $4 / 29 / 96$ & 44.40 & 23,400 & $\mathrm{~N}$ & $>500$ \\
\hline 05595730 & Rayse Creek near Waltonville, IL & 88.0 & 1980-98 & 1994 & 17.73 & 21,200 & $11 / 14 / 93$ & 17.73 & 21,200 & $\mathrm{~N}$ & $10-25$ \\
\hline 05597000 & Big Muddy River at Plumfield, IL & 794 & $\begin{array}{l}1909-12 \\
1915-98\end{array}$ & $\begin{array}{l}1961 \\
1983\end{array}$ & $\begin{array}{l}29.56 \\
31.84\end{array}$ & $\begin{array}{l}42,900 \\
11,800\end{array}$ & $5 / 1 / 96$ & 31.83 & 14,200 & $\mathrm{Y}$ & $5-10$ \\
\hline 05597500 & $\begin{array}{l}\text { Crab Orchard Creek near Marion, } \\
\text { IL }\end{array}$ & 31.7 & 1952-98 & 1996 & 13.60 & 9,270 & $\begin{array}{l}5 / 11 / 96 \\
5 / 31 / 97\end{array}$ & $\begin{array}{l}13.60 \\
13.21\end{array}$ & $\begin{array}{l}9,270 \\
6,970\end{array}$ & $\begin{array}{l}\mathrm{N} \\
\mathrm{N}\end{array}$ & $\begin{array}{l}>500 \\
>500\end{array}$ \\
\hline 05599500 & $\begin{array}{l}\text { Big Muddy River at Murphysboro, } \\
\text { IL }\end{array}$ & 2,169 & $\begin{array}{l}1916-17, \\
1919, \\
1931-98\end{array}$ & $\begin{array}{l}1996 \\
1961\end{array}$ & $\begin{array}{l}36.33 \\
37.77\end{array}$ & $\begin{array}{l}33,800 \\
33,300\end{array}$ & $5 / 2 / 96$ & 36.33 & 33,800 & $\mathrm{Y}$ & $25-50$ \\
\hline
\end{tabular}

${ }^{1}$ Regulated during flood: $\mathrm{N}$, no; Y, yes. 


\section{Indiana}

Widespread excessive rain and embedded thunderstorms began late April 10, 1994, and continued periodically through April 12, producing anywhere from 3 to 7 inches of rain across much of the State (National Oceanic and Atmospheric Administration, 1994a). As a result, flooding occurred across many parts of Indiana. The main exceptions were in the extreme north and extreme south. Record flooding occurred April 12-15 on Mud Pine and Sugar Creeks, along Big Pine Creek, and along the Vermilion River (fig. 26). The Vermilion River exceeded its record flood of 1939 by 3 to 4 feet (National Oceanic and Atmospheric Administration, 1994b). Other rivers, including the Wabash, White, East Fork White, Tippecanoe, Eel, Maumee, and St. Mary Rivers, exceeded their banks along nearly their entire lengths for a 1 - to 2 -week period. These were the highest floodwaters in many of these rivers since 1991. A man died in floodwaters, apparently when his truck was washed into a ditch by high, fast-moving water east of Medora, Indiana (National Oceanic and Atmospheric Administration, 1994b).

Torrential rains caused the worst flooding since 1959 across many southern Indiana locations during April 1996. Between 5 and 9 inches of rain fell April 28-29 (National Oceanic and Atmospheric Administration, 1996a). The most notable flooding occurred along the Blue River. The Blue River crested at 27.15 feet at Fredericksburg (streamgage 03302800, table 15) and 28.9 feet at Milltown (National Oceanic and Atmospheric Administration, 1996b). Major flooding occurred along the Whitewater River in east-central and southeastern Indiana. The river level below Brookville Lake reached its highest stage since 1968. The excessive rainfall also resulted in the lake level rising to nearly 4 feet above the previous record pool elevation (National Oceanic and Atmospheric Administration, 1996a).

Widespread rainfall amounts of about 10 inches in the middle Ohio River Valley, from around Louisville, Kentucky, to Cincinnati, Ohio, occurred during a 1- to 3-day period during the last week in April 1996 (National Oceanic and Atmospheric Administration, 1996a). The Ohio River rose rapidly and reached a crest of 60.7 feet on May 6 at Markland Dam, which is just across the river in Kentucky. This resulted in a massive flood crest that took a few weeks to travel down the Ohio River. The resultant flood was the worst in about 30 years, and one of the five worst on record.

More than 7 inches of rain fell in a 12-hour period on July 18, 1996, near the city of Huntington, Indiana (National Oceanic and Atmospheric Administration, 1996a), causing flash flooding. Damage to the city alone was estimated at \$1.5 million (National Oceanic and Atmospheric Administration, 1996b). The flooding in this area was the worst since 1959.

\section{References}

National Oceanic and Atmospheric Administration (NOAA), 1994a-96a, Climatological data (by State): Asheville, North Carolina, National Climatic Data Center, various months.

National Oceanic and Atmospheric Administration (NOAA), 1994b-96b, Storm data (by State): Asheville, North Carolina, National Climatic Data Center, various months. 


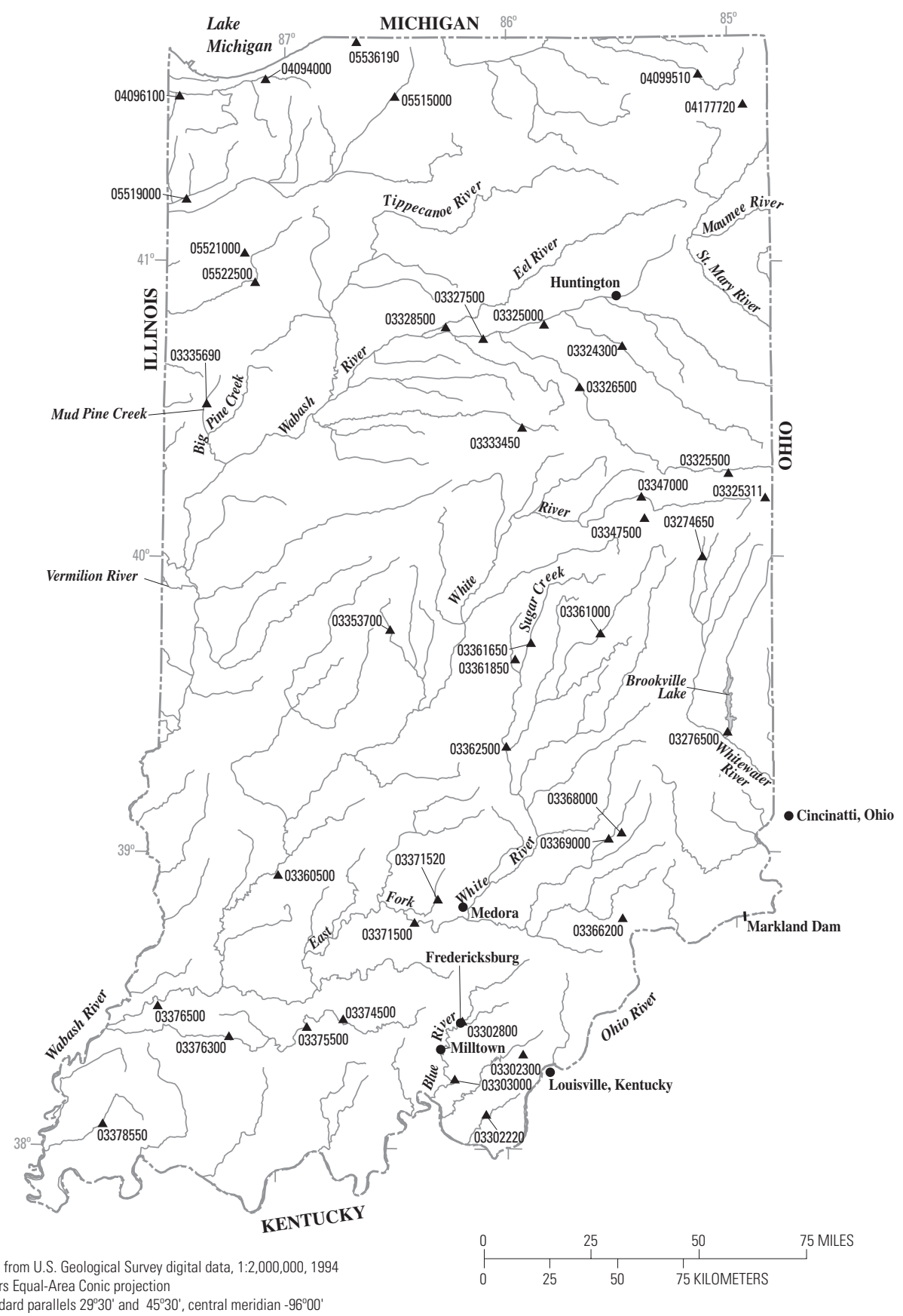

Standard parallels $29^{\circ} 30^{\prime}$ and $45^{\circ} 30^{\prime}$, central meridian $-96^{\circ} 00^{\prime}$

\section{EXPLANATION}

03303000 S Streamgage and number

Figure 26. Location of streamgages with significant floods during 1994-98 water years for Indiana. 
Table 15. Maximum stage and discharge for period of record for streamgages having significant floods during 1994-98 water years in Indiana.

$\left[\mathrm{mi}^{2}\right.$, square miles; $\mathrm{ft}$, feet above an arbitrary datum; $\mathrm{ft}^{3} / \mathrm{s}$, cubic feet per second; --, not determined or not applicable; $>$, greater than. Source: Recurrence intervals calculated from U.S. Geological Survey data. Other data from U.S. Geological Survey reports or databases]

\begin{tabular}{|c|c|c|c|c|c|c|c|c|c|c|c|}
\hline \multirow[b]{2}{*}{$\begin{array}{c}\text { Streamgage } \\
\text { number } \\
\text { (fig. 26) }\end{array}$} & \multirow[b]{2}{*}{ Streamgage name } & \multirow[b]{2}{*}{$\begin{array}{c}\text { Total } \\
\text { drainage } \\
\left(\mathrm{mi}^{2}\right)\end{array}$} & \multicolumn{4}{|c|}{$\begin{array}{c}\text { Maximum stage and discharge for period of record } \\
\text { through } 1998 \text { water year }\end{array}$} & \multicolumn{5}{|c|}{ Significant floods $1994-98$ water years } \\
\hline & & & $\begin{array}{l}\text { Period of } \\
\text { record } \\
\text { (water } \\
\text { years) }\end{array}$ & Water year & $\begin{array}{l}\text { Stage } \\
(\mathrm{ft})\end{array}$ & $\begin{array}{c}\text { Discharge } \\
\left(\mathrm{ft}^{3} / \mathrm{s}\right)\end{array}$ & $\begin{array}{c}\text { Date } \\
\text { (month/ } \\
\text { day/ } \\
\text { year) }\end{array}$ & $\begin{array}{c}\text { Stage } \\
(\mathrm{ft})\end{array}$ & $\begin{array}{c}\text { Discharge } \\
\left(\mathrm{ft}^{3} / \mathrm{s}\right)\end{array}$ & $\begin{array}{l}\text { Regulated } \\
\text { during } \\
\text { flood }^{1}\end{array}$ & $\begin{array}{c}\text { Recurrence } \\
\text { interval } \\
\text { (years) }\end{array}$ \\
\hline 03274650 & $\begin{array}{l}\text { Whitewater River near Economy, } \\
\text { IN }\end{array}$ & 10.4 & 1971-99 & 1994 & 8.91 & 1,120 & $11 / 14 / 93$ & 8.91 & 1,120 & $\mathrm{~N}$ & $10-25$ \\
\hline 03276500 & Whitewater River at Brookville, IN & 1,224 & $\begin{array}{l}1913, \\
1916-20, \\
1924-99\end{array}$ & $\begin{array}{l}1959 \\
1913\end{array}$ & $\begin{array}{l}27.78 \\
39.00\end{array}$ & $\begin{array}{r}81,800 \\
--\end{array}$ & $4 / 29 / 96$ & 21.86 & 49,200 & $\mathrm{Y}$ & $25-50$ \\
\hline 03302220 & $\begin{array}{l}\text { Buck Creek near New } \\
\text { Middletown, IN }\end{array}$ & 65.2 & 1970-99 & 1997 & 17.26 & 20,500 & $3 / 2 / 97$ & 17.26 & 20,500 & $\mathrm{~N}$ & $50-100$ \\
\hline 03302300 & Little Indian Creek near Galena, IN & 16.1 & 1969-99 & 1997 & 9.79 & 6,110 & $3 / 1 / 97$ & 9.79 & 6,110 & $\mathrm{~N}$ & $25-50$ \\
\hline 03302800 & Blue River at Fredericksburg, IN & 283 & 1969-99 & 1996 & 27.15 & 39,000 & $4 / 29 / 96$ & 27.15 & 39,000 & $\mathrm{~N}$ & $>100$ \\
\hline 03303000 & Blue River near White Cloud, IN & 476 & $\begin{array}{l}1910-13, \\
1915-16, \\
1932-99\end{array}$ & 1996 & 23.30 & 29,400 & 4/30/96 & 23.30 & 29,400 & $\mathrm{~N}$ & 50 \\
\hline 03324300 & Salamonie River near Warren, IN & 425 & 1958-99 & $\begin{array}{l}1998 \\
1959\end{array}$ & $\begin{array}{l}16.82 \\
17.05\end{array}$ & $\begin{array}{l}13,500 \\
13,200\end{array}$ & $8 / 5 / 98$ & 16.82 & 13,500 & $\mathrm{~N}$ & $25-50$ \\
\hline 03325000 & Wabash River at Wabash, IN & 1,768 & $\begin{array}{l}1913, \\
1924-99\end{array}$ & $\begin{array}{l}1943 \\
1913\end{array}$ & $\begin{array}{l}24.22 \\
28.70\end{array}$ & $\begin{array}{l}49,600 \\
90,000\end{array}$ & $\begin{array}{l}7 / 19 / 96 \\
7 / 22 / 98\end{array}$ & $\begin{array}{l}18.47 \\
18.98\end{array}$ & $\begin{array}{l}18,800 \\
20,000\end{array}$ & $\begin{array}{l}\mathrm{Y} \\
\mathrm{Y}\end{array}$ & $\begin{array}{l}10-25 \\
10-25\end{array}$ \\
\hline 03325311 & $\begin{array}{l}\text { Little Mississinewa River at Union } \\
\text { City, IN }\end{array}$ & 9.67 & 1983-97 & $\begin{array}{l}1994 \\
1987\end{array}$ & $\begin{array}{l}8.23 \\
8.67\end{array}$ & $\begin{array}{l}625 \\
315\end{array}$ & $11 / 14 / 93$ & 8.23 & 625 & $\mathrm{~N}$ & $10-25$ \\
\hline 03325500 & $\begin{array}{l}\text { Mississinewa River near } \\
\text { Ridgeville, IN }\end{array}$ & 133 & 1947-99 & 1958 & 16.25 & 13,900 & $11 / 14 / 93$ & 15.35 & 10,000 & $\mathrm{~N}$ & $25-50$ \\
\hline 03326500 & Mississinewa River at Marion, IN & 682 & $\begin{array}{l}\text { 1913, } \\
1924-99\end{array}$ & $\begin{array}{l}1927 \\
1913\end{array}$ & $\begin{array}{l}17.40 \\
19.20\end{array}$ & $\begin{array}{r}25,000 \\
--\end{array}$ & $8 / 5 / 98$ & 16.00 & 21,500 & $\mathrm{Y}$ & $10-25$ \\
\hline 03327500 & Wabash River at Peru, IN & 2,686 & $\begin{array}{l}1913, \\
1943-99\end{array}$ & $\begin{array}{l}1943 \\
1913\end{array}$ & $\begin{array}{l}24.46 \\
28.10\end{array}$ & $\begin{array}{r}68,000 \\
115,000\end{array}$ & $7 / 22 / 98$ & -- & 21,100 & $\mathrm{Y}$ & $10-25$ \\
\hline 03328500 & Eel River near Logansport, IN & 789 & 1943-99 & $\begin{array}{l}1985 \\
1943\end{array}$ & $\begin{array}{l}12.68 \\
13.20\end{array}$ & $\begin{array}{l}17,700 \\
17,000\end{array}$ & $7 / 23 / 98$ & 11.77 & 14,800 & $\mathrm{~N}$ & $10-25$ \\
\hline 03333450 & Wildcat Creek near Jerome, IN & 146 & $\begin{array}{l}\text { 1913, } \\
1962-99\end{array}$ & $\begin{array}{l}1992 \\
1913\end{array}$ & $\begin{array}{l}13.31 \\
18.00\end{array}$ & $\begin{array}{r}7,120 \\
--\end{array}$ & $6 / 12 / 98$ & 13.43 & 6,940 & $\mathrm{~N}$ & $10-25$ \\
\hline
\end{tabular}


Table 15. Maximum stage and discharge for period of record for streamgages having significant floods during 1994-98 water years in Indiana.-Continued

$\left[\mathrm{mi}^{2}\right.$, square miles; $\mathrm{ft}$, feet above an arbitrary datum; $\mathrm{ft}^{3} / \mathrm{s}$, cubic feet per second; --, not determined or not applicable; >, greater than. Source: Recurrence intervals calculated from U.S. Geological Survey data. Other data from U.S. Geological Survey reports or databases]

\begin{tabular}{|c|c|c|c|c|c|c|c|c|c|c|c|}
\hline \multirow{2}{*}{$\begin{array}{l}\text { Streamgage } \\
\text { number } \\
\text { (fig. 26) }\end{array}$} & \multirow[b]{2}{*}{ Streamgage name } & \multirow{2}{*}{$\begin{array}{c}\text { Total } \\
\text { drainage } \\
\left(\mathrm{mi}^{2}\right)\end{array}$} & \multicolumn{4}{|c|}{$\begin{array}{c}\text { Maximum stage and discharge for period of record } \\
\text { through } 1998 \text { water year }\end{array}$} & \multicolumn{5}{|c|}{ Significant floods $1994-98$ water years } \\
\hline & & & $\begin{array}{l}\text { Period of } \\
\text { record } \\
\text { (water } \\
\text { years) }\end{array}$ & Water year & $\begin{array}{l}\text { Stage } \\
(\mathrm{ft})\end{array}$ & $\begin{array}{l}\text { Discharge } \\
\left(\mathrm{ft}^{3} / \mathrm{s}\right)\end{array}$ & $\begin{array}{c}\text { Date } \\
\text { (month/ } \\
\text { day/ } \\
\text { year) }\end{array}$ & $\begin{array}{l}\text { Stage } \\
(\mathrm{ft})\end{array}$ & $\begin{array}{l}\text { Discharge } \\
\left(\mathrm{ft}^{3} / \mathrm{s}\right)\end{array}$ & $\begin{array}{c}\text { Regulated } \\
\text { during } \\
\text { flood }^{1}\end{array}$ & $\begin{array}{c}\text { Recurrence } \\
\text { interval } \\
\text { (years) }\end{array}$ \\
\hline 03335690 & Mud Pine Creek near Oxford, IN & 39.4 & $1971-99$ & 1994 & 16.98 & 12,100 & $4 / 12 / 94$ & 16.98 & 12,100 & $\mathrm{~N}$ & $>100$ \\
\hline 03347000 & White River at Muncie, IN & 241 & $\begin{array}{l}\text { 1904, 1913, } \\
1924-29, \\
1931-89, \\
1991-99\end{array}$ & $\begin{array}{l}1964 \\
1913\end{array}$ & $\begin{array}{l}11.98 \\
19.60\end{array}$ & $\begin{array}{r}14,300 \\
--\end{array}$ & $11 / 15 / 93$ & 13.00 & 10,500 & $\mathrm{Y}$ & $10-25$ \\
\hline 03347500 & Buck Creek near Muncie, IN & 35.5 & 1955-99 & 1964 & 13.96 & 1,780 & $11 / 15 / 93$ & 13.69 & 1,730 & $\mathrm{~N}$ & 50 \\
\hline 03353700 & $\begin{array}{l}\text { West Fork White Lick Creek at } \\
\text { Danville, IN }\end{array}$ & 28.8 & 1957-99 & 1957 & 16.00 & 6,660 & $2 / 27 / 97$ & 12.08 & 5,120 & $\mathrm{~N}$ & $25-50$ \\
\hline 03360500 & White River at Newberry, IN & 4,688 & $\begin{array}{l}1897 \\
1908-99\end{array}$ & 1913 & 27.50 & 130,000 & $11 / 18 / 93$ & 25.87 & 105,000 & $\mathrm{Y}$ & 100 \\
\hline 03361000 & Big Blue River at Carthage, IN & 184 & $\begin{array}{l}1949 \\
1951-99\end{array}$ & 1963 & 14.62 & 12,900 & $11 / 14 / 93$ & 12.91 & 8,410 & $\mathrm{~N}$ & $25-50$ \\
\hline 03361650 & Sugar Creek at New Palestine, IN & 93.9 & 1968-99 & $\begin{array}{l}1994 \\
1991\end{array}$ & $\begin{array}{l}10.08 \\
10.31\end{array}$ & $\begin{array}{l}2,340 \\
2,220\end{array}$ & $11 / 14 / 93$ & 10.08 & 2,340 & $\mathrm{~N}$ & $25-50$ \\
\hline 03361850 & Buck Creek at Acton, IN & 78.8 & 1968-99 & 1969 & 14.99 & 7,140 & $11 / 14 / 93$ & 14.44 & 6,310 & $\mathrm{~N}$ & $50-100$ \\
\hline 03362500 & Sugar Creek near Edinburgh, IN & 474 & 1943-99 & 1956 & 18.38 & 27,600 & $11 / 15 / 93$ & 17.05 & 20,500 & $\mathrm{~N}$ & 25 \\
\hline 03366200 & Harberts Creek near Madison, IN & 9.31 & 1969-99 & 1990 & 8.96 & 2,150 & $4 / 29 / 96$ & 7.67 & 1,970 & $\mathrm{~N}$ & $25-50$ \\
\hline 03368000 & Brush Creek near Nebraska, IN & 11.4 & 1956-99 & 1981 & 12.99 & 9,360 & $4 / 16 / 98$ & 12.20 & 5,200 & $\mathrm{~N}$ & 50 \\
\hline 03369000 & $\begin{array}{l}\text { Vernon Fork Muscatatuck River } \\
\text { near Butlerville, IN }\end{array}$ & 85.9 & 1942-99 & 1959 & 25.41 & 26,200 & $4 / 16 / 98$ & 21.54 & 18,500 & $\mathrm{Y}$ & $50-100$ \\
\hline 03371500 & $\begin{array}{l}\text { East Fork White River near } \\
\text { Bedford, IN }\end{array}$ & 3,861 & $\begin{array}{l}1913, \\
1940-99\end{array}$ & 1996 & 36.32 & 80,500 & $5 / 1 / 96$ & 36.32 & 80,500 & $\mathrm{~N}$ & 25 \\
\hline 03371520 & Back Creek at Leesville, IN & 24.1 & $\begin{array}{l}1913, \\
1971-99\end{array}$ & $\begin{array}{l}1973 \\
1913\end{array}$ & $\begin{array}{l}14.00 \\
18.10\end{array}$ & $\begin{array}{r}15,300 \\
--\end{array}$ & $4 / 16 / 98$ & 11.18 & 8,590 & $\mathrm{~N}$ & $10-25$ \\
\hline
\end{tabular}


$\left[\mathrm{mi}^{2}\right.$, square miles; $\mathrm{ft}$, feet above an arbitrary datum; $\mathrm{ft}^{3} / \mathrm{s}$, cubic feet per second; --, not determined or not applicable; >, greater than. Source: Recurrence intervals calculated from U.S. Geological Survey data. Other data from U.S. Geological Survey reports or databases]

\begin{tabular}{|c|c|c|c|c|c|c|c|c|c|c|c|}
\hline \multirow[b]{2}{*}{$\begin{array}{c}\text { Streamgage } \\
\text { number } \\
\text { (fig. 26) }\end{array}$} & \multirow[b]{2}{*}{ Streamgage name } & \multirow[b]{2}{*}{$\begin{array}{c}\text { Total } \\
\text { drainage } \\
\left(\mathrm{mi}^{2}\right)\end{array}$} & \multicolumn{4}{|c|}{$\begin{array}{c}\text { Maximum stage and discharge for period of record } \\
\text { through } 1998 \text { water year }\end{array}$} & \multicolumn{5}{|c|}{ Significant floods $1994-98$ water years } \\
\hline & & & $\begin{array}{l}\text { Period of } \\
\text { record } \\
\text { (water } \\
\text { years) }\end{array}$ & Water year & $\begin{array}{l}\text { Stage } \\
(\mathrm{ft})\end{array}$ & $\begin{array}{l}\text { Discharge } \\
\left(\mathrm{ft}^{3} / \mathrm{s}\right)\end{array}$ & $\begin{array}{c}\text { Date } \\
\text { (month/ } \\
\text { day/ } \\
\text { year) }\end{array}$ & $\begin{array}{l}\text { Stage } \\
(\mathrm{ft})\end{array}$ & $\begin{array}{l}\text { Discharge } \\
\left(\mathrm{ft}^{3} / \mathrm{s}\right)\end{array}$ & $\begin{array}{l}\text { Regulated } \\
\text { during } \\
\text { flood }^{1}\end{array}$ & $\begin{array}{c}\text { Recurrence } \\
\text { interval } \\
\text { (years) }\end{array}$ \\
\hline 03374500 & Patoka River near Cuzco, IN & 170 & $\begin{array}{l}1913, \\
1962-99\end{array}$ & 1964 & 20.02 & 14,700 & $6 / 1 / 96$ & -- & 2,830 & $\mathrm{Y}$ & -- \\
\hline 03375500 & Patoka River at Jasper, IN & 262 & $\begin{array}{l}1913,1937, \\
1948-99\end{array}$ & $\begin{array}{l}1964 \\
1961\end{array}$ & $\begin{array}{l}15.17 \\
20.62\end{array}$ & $\begin{array}{l}14,100 \\
13,700\end{array}$ & $4 / 30 / 96$ & 17.21 & 6,370 & $\mathrm{Y}$ & -- \\
\hline 03376300 & Patoka River at Winslow, IN & 603 & $\begin{array}{l}\text { 1937, 1961, } \\
1964-74, \\
1987-99\end{array}$ & $\begin{array}{l}1964 \\
1937\end{array}$ & $\begin{array}{l}28.84 \\
28.90\end{array}$ & $\begin{array}{r}15,500 \\
--\end{array}$ & $5 / 2 / 96$ & 27.37 & 11,600 & $\mathrm{Y}$ & -- \\
\hline 03376500 & Patoka River near Princeton, IN & 822 & 1935-99 & $\begin{array}{l}1937 \\
1996\end{array}$ & $\overline{24.35}$ & $\begin{array}{l}18,700 \\
13,900\end{array}$ & $5 / 4 / 96$ & 24.35 & 13,900 & $\mathrm{Y}$ & -- \\
\hline 03378550 & Big Creek near Wadesville, IN & 104 & 1966-99 & 1996 & 20.35 & 10,400 & $4 / 29 / 96$ & 20.35 & 10,400 & $\mathrm{~N}$ & $50-100$ \\
\hline 04094000 & Little Calumet River at Porter, IN & 66.2 & 1945-99 & $\begin{array}{l}1991 \\
1955\end{array}$ & $\begin{array}{l}10.93 \\
11.66\end{array}$ & $\begin{array}{l}3,880 \\
3,110\end{array}$ & $5 / 10 / 96$ & 10.63 & 3,400 & $\mathrm{~N}$ & $25-50$ \\
\hline 04096100 & Galena River near Laporte, IN & 17.2 & 1970-99 & $\begin{array}{l}1991 \\
1993\end{array}$ & $-\overline{7}+.04$ & $\begin{array}{l}900 \\
617\end{array}$ & $5 / 10 / 96$ & 7.00 & 874 & $\mathrm{~N}$ & $25-50$ \\
\hline 04099510 & Pigeon Creek near Angola, IN & 106 & 1946-99 & $\begin{array}{l}1996 \\
1950\end{array}$ & $\begin{array}{l}10.92 \\
14.95\end{array}$ & $\begin{array}{r}1,000 \\
744\end{array}$ & $5 / 21 / 96$ & 10.92 & 1,000 & $\mathrm{~N}$ & 100 \\
\hline 04177720 & Fish Creek at Hamilton, IN & 37.5 & 1970-99 & 1996 & 14.49 & 1,510 & $5 / 17 / 96$ & 14.49 & 1,510 & $\mathrm{~N}$ & $>100$ \\
\hline 05515000 & $\begin{array}{l}\text { Kankakee River near North } \\
\text { Liberty, IN }\end{array}$ & 174 & $1951-99$ & $\begin{array}{l}1982 \\
1968\end{array}$ & $\begin{array}{l}9.01 \\
9.04\end{array}$ & $\begin{array}{l}908 \\
629\end{array}$ & $7 / 19 / 96$ & 8.84 & 891 & $\mathrm{~N}$ & 50 \\
\hline 05519000 & Singleton Ditch at Schneider, IN & 123 & $\begin{array}{l}1949-88 \\
1990-99\end{array}$ & $\begin{array}{l}1976 \\
1991\end{array}$ & $\begin{array}{l}11.24 \\
12.54\end{array}$ & $\begin{array}{l}3,550 \\
2,460\end{array}$ & $6 / 16 / 97$ & 12.05 & 2,270 & $\mathrm{~N}$ & $10-25$ \\
\hline 05521000 & Iroquois River at Rosebud, IN & 35.6 & 1949-99 & $\begin{array}{l}1991 \\
1959\end{array}$ & $\begin{array}{l}7.93 \\
8.86\end{array}$ & $\begin{array}{l}656 \\
383\end{array}$ & $10 / 17 / 93$ & 7.28 & 569 & $\mathrm{~N}$ & $25-50$ \\
\hline 05522500 & Iroquois River at Rensselaer, IN & 203 & $\begin{array}{l}\text { 1910, } \\
1949-99\end{array}$ & 1958 & 16.54 & 2,550 & $10 / 18 / 93$ & 15.52 & 2,520 & $\mathrm{~N}$ & $50-100$ \\
\hline 05536190 & Hart Ditch at Munster, IN & 70.7 & 1943-99 & 1991 & 8.72 & 3,010 & $\begin{array}{l}7 / 18 / 96 \\
2 / 21 / 97\end{array}$ & $\begin{array}{l}8.03 \\
8.00\end{array}$ & $\begin{array}{l}2,710 \\
2,700\end{array}$ & $\begin{array}{l}\mathrm{N} \\
\mathrm{N}\end{array}$ & $\begin{array}{l}10-25 \\
10-25\end{array}$ \\
\hline
\end{tabular}




\section{Summary of Significant Floods in the United States and Puerto Rico, 1994 Through 1998 Water Years}

\section{lowa}

Rainfall of 4 to 7 inches was common in central Iowa near Boone (fig. 27) June 16 and 17, 1996 (National Oceanic and Atmospheric Administration, 1996a). Doppler radar precipitation estimates were as high as 8.5 inches in the area. The South Skunk River crested at an all time high discharge. Crop damage was extensive as well with many fields becoming flooded. Total damage in Ames and surrounding areas was believed to be several million dollars (National Oceanic and Atmospheric Administration, 1996b). Intense thunderstorms returned within a week, and additional flooding occurred throughout Iowa. Precipitation totals for some areas were more than 15 inches in 2 days. The flash flooding was considered the worst since the summer of 1993.

Significant ice jams formed along the downstream reaches of the Raccoon River on February 19, 1997. A woman was driving across a flood-plain road south of Des Moines when one of the ice jams suddenly broke. The water rose quickly and swept her vehicle off the road with her in it, and she drowned (National Oceanic and Atmospheric Administration, 1997b).

In June 1998, a strong upper level system, combined with a warm front located to the south of Iowa, set the stage for a major flood. Iowa soils were already saturated before the flood began. Extremely intense rain fell over southwestern and central Iowa from this storm. Southwestern Iowa was the hardest hit as more than 13.18 inches of rain fell at Atlantic on June 14 (National Oceanic and Atmospheric Administration, 1998a). This amount of rain set a State record for the greatest 24-hour rainfall total from an official site. Damage was more than a \$1 million (National Oceanic and Atmospheric Administration, 1998b). The East Nishnabotna River near Atlantic crested at 22.36 feet on June 15 (streamgage 06809210, table 16), missing the all-time record by about 0.5 foot set in September 1972 (Fischer, 1999). Very intense rains of 2 to 4 inches also fell over the Raccoon and South Skunk River Basins, resulting in widespread flooding. Both the North and South Raccoon Rivers flooded. The flood waves from both rivers merged on the main stem to create the second highest crest on record for the Racoon River. Only the great flood of 1993 created higher crests. Following a brief break from the rain on June 16, rainfall resumed June 17-18. Excessive rain fell over just about the entire State on both days, with amounts of 1 to 3 inches of rain on the first night in the Nishnabotna, upper Des Moines, and the downstream parts of the South Skunk River Basins (National Oceanic and Atmospheric Administration, 1998a). These rains fell on already saturated soils and resulted in considerable runoff. On the 18th, the Des Moines metropolitan area was blitzed with massive flooding. Local rains of 1 to 4 inches fell in the city, much of it falling in 1 to 2 hours (National Oceanic and Atmospheric Administration, 1998a). Much of this water was quickly added to the flows on the already high Raccoon River. Cleanup and repair of the damage from this flood alone were estimated at \$12 million (National Oceanic and Atmospheric Administration, 1998b). Numerous rivers south of Des Moines flooded as well. On June 21-23, flooding occurred in the southwestern part of the State. The main rivers affected by the June flood episodes were the East Nishnabotna, Nishnabotna, and Raccoon Rivers, the Des Moines River in the vicinity of Des Moines, and the South Skunk River and Squaw Creek in the vicinity of Ames. The crop-damage estimate from these June 1998 floods totaled more than \$100 million (National Oceanic and Atmospheric Administration, 1998b).

\section{References}

Fischer, E.E., 1999, Flood of June 15-17, 1998, Nishnabotna and East Nishnabotna Rivers, southwest Iowa: U.S. Geological Survey Open-File Report 99-70, 15 p.

National Oceanic and Atmospheric Administration (NOAA), 1996a-98a, Climatological data (by State): Asheville, North Carolina, National Climatic Data Center, various months.

National Oceanic and Atmospheric Administration (NOAA), 1996b-98b, Storm data (by State): Asheville, North Carolina, National Climatic Data Center, various months. 


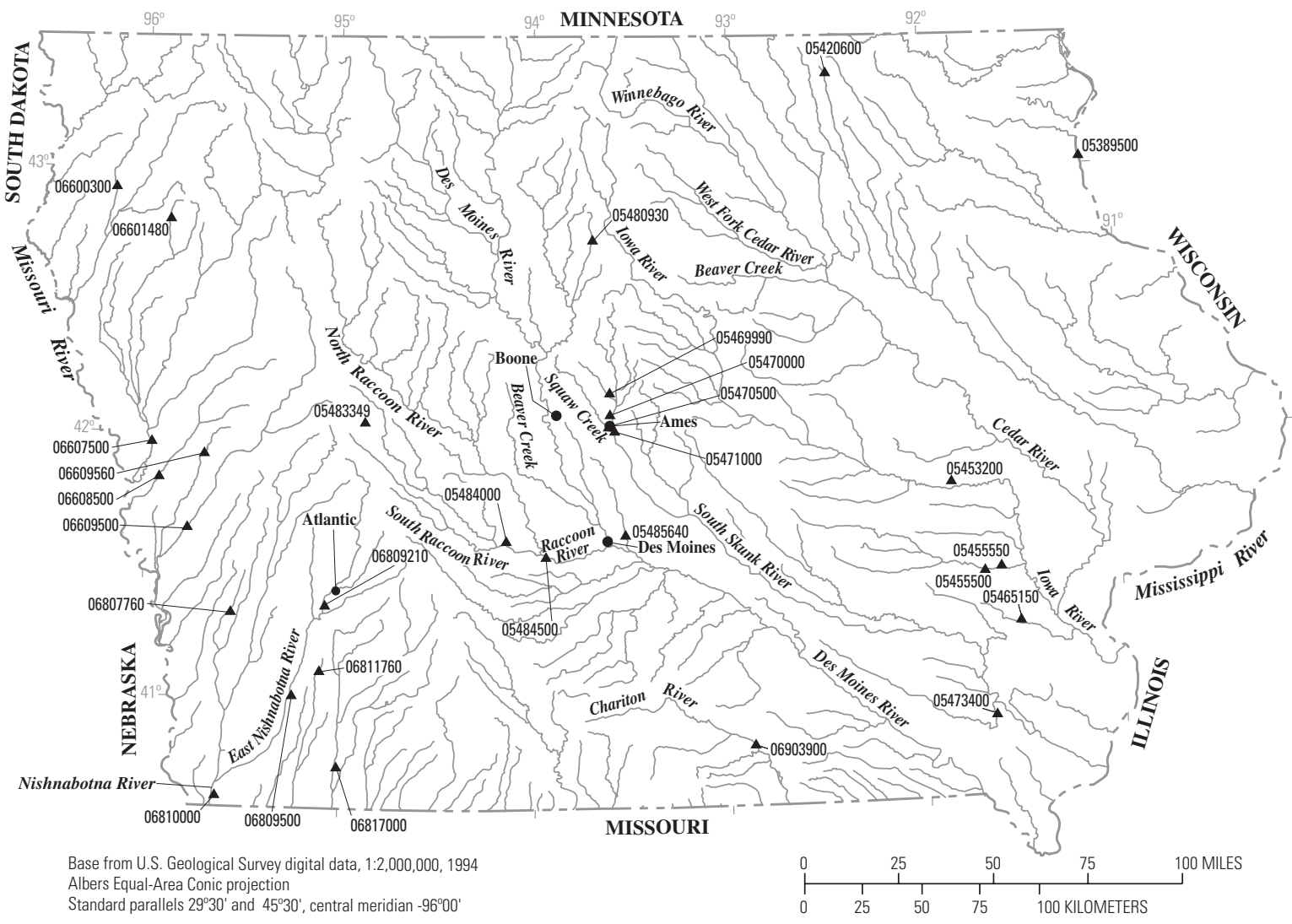

\section{EXPLANATION}

06903900 S Streamgage and number

Figure 27. Location of streamgages with significant floods during 1994-98 water years for lowa. 
Table 16. Maximum stage and discharge for period of record for streamgages having significant floods during 1994-98 water years in lowa.

$\left[\mathrm{mi}^{2}\right.$, square miles; $\mathrm{ft}$, feet above an arbitrary datum; $\mathrm{ft}^{3} / \mathrm{s}$, cubic feet per second; --, not determined or not applicable; $>$, greater than. Source: Recurrence intervals calculated from U.S. Geological Survey data. Other data from U.S. Geological Survey reports or databases]

\begin{tabular}{|c|c|c|c|c|c|c|c|c|c|c|c|}
\hline \multirow{2}{*}{$\begin{array}{c}\text { Streamgage } \\
\text { number } \\
\text { (fig. 27) }\end{array}$} & \multirow[b]{2}{*}{ Streamgage name } & \multirow{2}{*}{$\begin{array}{c}\text { Total } \\
\text { drainage } \\
\left(\mathrm{mi}^{2}\right)\end{array}$} & \multicolumn{4}{|c|}{$\begin{array}{c}\text { Maximum stage and discharge for period of record } \\
\text { through } 1998 \text { water year }\end{array}$} & \multicolumn{5}{|c|}{ Significant floods 1994-98 water years } \\
\hline & & & $\begin{array}{c}\text { Period of } \\
\text { record } \\
\text { (water } \\
\text { years) }\end{array}$ & Water year & $\begin{array}{l}\text { Stage } \\
(\mathrm{ft})\end{array}$ & $\begin{array}{l}\text { Discharge } \\
\left(\mathrm{ft}^{3} / \mathrm{s}\right)\end{array}$ & $\begin{array}{c}\text { Date } \\
\text { (month/ } \\
\text { day/ } \\
\text { year) }\end{array}$ & $\begin{array}{l}\text { Stage } \\
(\mathrm{ft})\end{array}$ & $\begin{array}{l}\text { Discharge } \\
\left(\mathrm{ft}^{3} / \mathrm{s}\right)\end{array}$ & $\begin{array}{l}\text { Regulated } \\
\text { during } \\
\text { flood }^{1}\end{array}$ & $\begin{array}{c}\text { Recurrence } \\
\text { interval } \\
\text { (years) }\end{array}$ \\
\hline 05389500 & Mississippi River at McGregor, IA & 67,500 & $\begin{array}{l}1880 \\
1937-98\end{array}$ & 1965 & 25.38 & 276,000 & $4 / 15 / 97$ & 21.38 & 201,000 & $\mathrm{Y}$ & 25 \\
\hline 05420600 & $\begin{array}{l}\text { Little Wapsipinicon River tributary } \\
\text { near Riceville, IA }\end{array}$ & 1.10 & 1953-98 & 1997 & 5.91 & 3,300 & $3 / 11 / 97$ & 5.91 & 3,300 & $\mathrm{~N}$ & -- \\
\hline 05453200 & Price Creek at Amana, IA & 29.1 & $\begin{array}{l}\text { 1966-86, } \\
1989-98\end{array}$ & 1997 & 87.16 & 3,770 & $2 / 19 / 97$ & 87.16 & 3,770 & $\mathrm{~N}$ & -- \\
\hline 05455500 & English River at Kalona, IA & 573 & $\begin{array}{l}1930 \\
1940-98\end{array}$ & 1993 & 22.55 & 36,100 & $5 / 11 / 96$ & 21.06 & 25,100 & $\mathrm{~N}$ & $35-40$ \\
\hline 05455550 & Bulgers Run near Riverside, IA & 6.31 & $\begin{array}{l}\text { 1965-87, } \\
1989, \\
1992-98\end{array}$ & 1965 & 89.04 & 3,080 & $5 / 10 / 96$ & 88.10 & 2,780 & $\mathrm{~N}$ & -- \\
\hline 05465150 & $\begin{array}{l}\text { North Fork Long Creek at } \\
\text { Ainsworth, IA }\end{array}$ & 30.2 & $1965-98$ & 1997 & 90.44 & 5,000 & $2 / 19 / 97$ & 90.44 & 5,000 & $\mathrm{~N}$ & -- \\
\hline 05469990 & Keigley Branch near Story City, IA & 31.0 & 1966-98 & 1996 & 92.26 & 3,440 & $6 / 17 / 96$ & 92.26 & 3,440 & $\mathrm{~N}$ & $40-60$ \\
\hline 05470000 & South Skunk River near Ames, IA & 315 & $\begin{array}{l}1921-27, \\
1930, \\
1933-98\end{array}$ & 1996 & 15.89 & 14,000 & $7 / 17 / 96$ & 15.89 & 14,000 & $\mathrm{~N}$ & $>500$ \\
\hline 05470500 & Squaw Creek at Ames, IA & 204 & $\begin{array}{l}1918, \\
1920-27, \\
1965-98\end{array}$ & 1993 & 18.54 & 24,300 & $6 / 17 / 96$ & 15.29 & 12,700 & $\mathrm{~N}$ & $60-80$ \\
\hline 05471000 & $\begin{array}{l}\text { South Skunk River below Squaw } \\
\text { Creek near Ames, IA }\end{array}$ & 556 & $\begin{array}{l}1944, \\
1953-79, \\
1990, \\
1992-98\end{array}$ & $\begin{array}{l}1993 \\
1975\end{array}$ & $\begin{array}{l}25.53 \\
25.57\end{array}$ & $\begin{array}{l}26,500 \\
14,700\end{array}$ & $6 / 17 / 96$ & 25.13 & 24,400 & $\mathrm{~N}$ & $>500$ \\
\hline
\end{tabular}


Table 16. Maximum stage and discharge for period of record for streamgages having significant floods during 1994-98 water years in lowa.-Continued

$\left[\mathrm{mi}^{2}\right.$, square miles; $\mathrm{ft}$, feet above an arbitrary datum; $\mathrm{ft}^{3} / \mathrm{s}$, cubic feet per second; --, not determined or not applicable; >, greater than. Source: Recurrence intervals calculated from U.S. Geological Survey data. Other data from U.S. Geological Survey reports or databases]

\begin{tabular}{|c|c|c|c|c|c|c|c|c|c|c|c|}
\hline \multirow[b]{2}{*}{$\begin{array}{l}\text { Streamgage } \\
\text { number } \\
\text { (fig. 27) }\end{array}$} & \multirow[b]{2}{*}{ Streamgage name } & \multirow[b]{2}{*}{$\begin{array}{c}\text { Total } \\
\text { drainage } \\
\left(\mathrm{mi}^{2}\right)\end{array}$} & \multicolumn{4}{|c|}{$\begin{array}{c}\text { Maximum stage and discharge for period of record } \\
\text { through } 1998 \text { water year }\end{array}$} & \multicolumn{5}{|c|}{ Significant floods $1994-98$ water years } \\
\hline & & & $\begin{array}{l}\text { Period of } \\
\text { record } \\
\text { (water } \\
\text { years) }\end{array}$ & Water year & $\begin{array}{l}\text { Stage } \\
(\mathrm{ft})\end{array}$ & $\begin{array}{l}\text { Discharge } \\
\left(\mathrm{ft}^{3} / \mathrm{s}\right)\end{array}$ & $\begin{array}{c}\text { Date } \\
\text { (month/ } \\
\text { day/ } \\
\text { year) }\end{array}$ & $\begin{array}{l}\text { Stage } \\
(\mathrm{ft})\end{array}$ & $\begin{array}{l}\text { Discharge } \\
\left(\mathrm{ft}^{3} / \mathrm{s}\right)\end{array}$ & $\begin{array}{l}\text { Regulated } \\
\text { during } \\
\text { flood }^{1}\end{array}$ & $\begin{array}{c}\text { Recurrence } \\
\text { interval } \\
\text { (years) }\end{array}$ \\
\hline 05473400 & $\begin{array}{l}\text { Cedar Creek near Oakland Mills, } \\
\text { IA }\end{array}$ & 530 & $1979-98$ & $\begin{array}{l}1996 \\
1993\end{array}$ & $\begin{array}{l}21.03 \\
21.27\end{array}$ & $\begin{array}{r}12,300 \\
8,920\end{array}$ & $5 / 28 / 96$ & 21.03 & 12,300 & $\mathrm{~N}$ & $50-75$ \\
\hline 05480930 & White Fox Creek at Clarion, IA & 13.3 & $1966-98$ & $\begin{array}{l}1995 \\
1993\end{array}$ & $\begin{array}{l}92.91 \\
93.59\end{array}$ & $\begin{array}{l}1,700 \\
1,400\end{array}$ & $6 / 29 / 95$ & 92.91 & 1,700 & $\mathrm{~N}$ & -- \\
\hline 05483349 & $\begin{array}{l}\text { Middle Raccoon River tributary at } \\
\text { Carroll, IA }\end{array}$ & 6.58 & 1966-98 & 1996 & 25.88 & 4,600 & $7 / 17 / 96$ & 25.88 & 4,600 & $\mathrm{~N}$ & $75-100$ \\
\hline 05484000 & $\begin{array}{l}\text { South Raccoon River at Redfield, } \\
\text { IA }\end{array}$ & 994 & 1940-98 & $\begin{array}{l}1993 \\
1958\end{array}$ & $\begin{array}{l}26.98 \\
29.04\end{array}$ & $\begin{array}{l}44,000 \\
35,000\end{array}$ & $6 / 15 / 98$ & 24.67 & 35,100 & $\mathrm{~N}$ & $50-70$ \\
\hline 05484500 & Raccoon River at Van Meter, IA & 3,441 & $1915-98$ & 1993 & 26.34 & 70,100 & $6 / 15 / 98$ & 23.29 & 47,400 & $\mathrm{~N}$ & $40-50$ \\
\hline 05485640 & Fourmile Creek at Des Moines, IA & 92.7 & $\begin{array}{l}1972-79 \\
1981-98\end{array}$ & 1998 & 15.00 & 5,600 & $6 / 18 / 98$ & 15.00 & 5,600 & $\mathrm{~N}$ & $10-20$ \\
\hline 06600300 & $\begin{array}{l}\text { West Branch Floyd River near } \\
\text { Struble, IA }\end{array}$ & 180 & $\begin{array}{l}1956-94 \\
1996-98\end{array}$ & $\begin{array}{l}1994 \\
1983\end{array}$ & $\begin{array}{l}15.86 \\
15.86\end{array}$ & $\begin{array}{l}8,920 \\
7,590\end{array}$ & $3 / 4 / 94$ & 15.86 & 8,920 & $\mathrm{~N}$ & $15-20$ \\
\hline 06601480 & $\begin{array}{l}\text { Big Whiskey Slough near } \\
\text { Remsen, IA }\end{array}$ & 12.9 & $\begin{array}{l}1967, \\
1969-71, \\
1973,1975, \\
1978-86, \\
1988-90, \\
1993-98\end{array}$ & $\begin{array}{l}1996 \\
1979\end{array}$ & $\begin{array}{l}93.56 \\
94.87\end{array}$ & $\begin{array}{r}1,210 \\
--\end{array}$ & $6 / 21 / 96$ & 93.56 & 1,210 & $\mathrm{~N}$ & -- \\
\hline 06607500 & Little Sioux River near Turin, IA & 3,526 & 1940-98 & $\begin{array}{l}1996 \\
1971\end{array}$ & $\begin{array}{l}26.99 \\
27.44\end{array}$ & $\begin{array}{l}32,000 \\
30,000\end{array}$ & $6 / 22 / 96$ & 26.99 & 32,000 & $\mathrm{~N}$ & $10-15$ \\
\hline 06608500 & Soldier River at Pisgah, IA & 407 & 1940-98 & 1996 & 28.87 & 34,700 & $7 / 17 / 96$ & 28.87 & 34,700 & $\mathrm{~N}$ & $70-90$ \\
\hline 06609500 & Boyer River at Logan, IA & 871 & $\begin{array}{l}1881, \\
1918-25, \\
1938-98\end{array}$ & $\begin{array}{l}1990 \\
1965\end{array}$ & $\begin{array}{l}22.54 \\
25.22\end{array}$ & $\begin{array}{r}30,800 \\
--\end{array}$ & $7 / 17 / 96$ & 23.87 & 28,700 & $\mathrm{~N}$ & $30-35$ \\
\hline
\end{tabular}


Table 16. Maximum stage and discharge for period of record for streamgages having significant floods during 1994-98 water years in lowa.-Continued

$\left[\mathrm{mi}^{2}\right.$, square miles; $\mathrm{ft}$, feet above an arbitrary datum; $\mathrm{ft}^{3} / \mathrm{s}$, cubic feet per second; --, not determined or not applicable; >, greater than. Source: Recurrence intervals calculated from U.S. Geological Survey data. Other data from U.S. Geological Survey reports or databases]

\begin{tabular}{|c|c|c|c|c|c|c|c|c|c|c|c|}
\hline \multirow{2}{*}{$\begin{array}{c}\text { Streamgage } \\
\text { number } \\
\text { (fig. 27) }\end{array}$} & \multirow[b]{2}{*}{ Streamgage name } & \multirow{2}{*}{$\begin{array}{c}\text { Total } \\
\text { drainage } \\
\left(\mathrm{mi}^{2}\right)\end{array}$} & \multicolumn{4}{|c|}{$\begin{array}{c}\text { Maximum stage and discharge for period of record } \\
\text { through } 1998 \text { water year }\end{array}$} & \multicolumn{5}{|c|}{ Significant floods 1994-98 water years } \\
\hline & & & $\begin{array}{l}\text { Period of } \\
\text { record } \\
\text { (water } \\
\text { years) }\end{array}$ & Water year & $\begin{array}{l}\text { Stage } \\
(\mathrm{ft})\end{array}$ & $\begin{array}{l}\text { Discharge } \\
\left(\mathrm{ft}^{3} / \mathrm{s}\right)\end{array}$ & $\begin{array}{c}\text { Date } \\
\text { (month/ } \\
\text { day/ } \\
\text { year) }\end{array}$ & $\begin{array}{l}\text { Stage } \\
(\mathrm{ft})\end{array}$ & $\begin{array}{l}\text { Discharge } \\
\left(\mathrm{ft}^{3} / \mathrm{s}\right)\end{array}$ & $\begin{array}{l}\text { Regulated } \\
\text { during } \\
\text { flood }^{1}\end{array}$ & $\begin{array}{c}\text { Recurrence } \\
\text { interval } \\
\text { (years) }\end{array}$ \\
\hline 06609560 & Willow Creek near Soldier, IA & 29.1 & $\begin{array}{l}1966-77, \\
1979-98\end{array}$ & 1993 & 84.66 & 6,840 & $7 / 17 / 96$ & 83.02 & 5,660 & $\mathrm{~N}$ & $20-30$ \\
\hline 06807760 & $\begin{array}{l}\text { Middle Silver Creek near } \\
\text { Oakland, IA }\end{array}$ & 25.7 & $1953-98$ & 1998 & 15.63 & 2,540 & $7 / 14 / 98$ & 15.63 & 2,540 & $\mathrm{~N}$ & $60-80$ \\
\hline 06809210 & $\begin{array}{l}\text { East Nishnabotna River near } \\
\text { Atlantic, IA }\end{array}$ & 436 & $\begin{array}{l}1958, \\
1961-98\end{array}$ & $\begin{array}{l}1998 \\
1972\end{array}$ & $\begin{array}{l}22.36 \\
22.81\end{array}$ & $\begin{array}{l}41,400 \\
26,700\end{array}$ & $6 / 15 / 98$ & 22.36 & 41,400 & $\mathrm{~N}$ & $200-300$ \\
\hline 06809500 & $\begin{array}{l}\text { East Nishnabotna River at Red } \\
\text { Oak, IA }\end{array}$ & 894 & $\begin{array}{l}1917-25, \\
1936-98\end{array}$ & 1998 & 29.39 & 60,500 & $6 / 15 / 98$ & 29.39 & 60,500 & $\mathrm{~N}$ & $>500$ \\
\hline 06810000 & $\begin{array}{l}\text { Nishnabotna River above } \\
\text { Hamburg, IA }\end{array}$ & 2,806 & $\begin{array}{l}1917, \\
1922-23, \\
1929-98\end{array}$ & 1998 & 33.18 & 65,100 & $6 / 17 / 98$ & 33.18 & 65,100 & $\mathrm{~N}$ & $>500$ \\
\hline 06811760 & Tarkio River near Elliott, IA & 10.7 & $\begin{array}{l}\text { 1952-87, } \\
\text { 1989-91, } \\
\text { 1993, } \\
\text { 1996-98 }\end{array}$ & 1998 & 14.68 & 5,000 & $6 / 14 / 98$ & 14.68 & 5,000 & $\mathrm{~N}$ & -- \\
\hline 06817000 & Nodaway River at Clarinda, IA & 762 & $\begin{array}{l}1918-25 \\
1936-98\end{array}$ & 1947 & 25.30 & 31,100 & $6 / 15 / 98$ & 23.89 & 30,200 & $\mathrm{~N}$ & 20 \\
\hline 06903900 & Chariton River near Rathbun, IA & 549 & $1957-98$ & 1960 & 25.30 & 21,800 & $12 / 14 / 93$ & 14.94 & 2,780 & $\mathrm{Y}$ & -- \\
\hline
\end{tabular}

Regulated during flood: $\mathrm{N}, \mathrm{no} ; \mathrm{Y}$, yes. 


\section{Kansas}

Approximately 10 inches of rain fell April 9-11, 1994, over much of southeastern Kansas (National Oceanic and Atmospheric Administration, 1994a). At Farlington (fig. 28), rainfall measured 11.25 inches. Many streams and rivers exceeded flood stage throughout the area. The Fall, Marmaton, Neosho, and Verdigris Rivers all exceeded flood stage (National Oceanic and Atmospheric Administration, 1994b). In Fort Scott, the Marmaton River crested almost 10 feet above flood stage. The Neosho River was out of its banks from Iola to the Oklahoma border. Excessive rains returned at the end of April with many of the same streams going out of their banks for the second time (table 17).

Minor to moderate flooding occurred across extreme northeastern and east-central Kansas during May 1995. The floods were caused by above-normal rainfall through the month. The Kansas City area experienced its wettest May in 106 years of record, with a 12.75-inch deluge (National Oceanic and Atmospheric Administration, 1995a). This was 7.71 inches above normal and easily eclipsed the previous record rainfall of 11.00 inches set in 1915. Most rivers only went a few feet above their respective flood stage, but at Osawatomie on the Marais des Cygnes River (National Oceanic and Atmospheric Administration, 1995b), the level topped out 10.6 feet above flood stage. The Missouri River remained above flood stage at Atchison and Leavenworth into June.

Intense rain of 5 to 12 inches fell over parts of extreme southeastern Kansas during September 13-15, 1998 (National Oceanic and Atmospheric Administration, 1998a). Unofficial reports of rainfall as high as 14 inches were reported west of Fort Scott in the Marmaton River Basin. The stage of the Marmaton River at Fort Scott reached 50.05 feet on September 14, which is the second highest stage ever recorded.

\section{References}

National Oceanic and Atmospheric Administration (NOAA), 1994a-98a, Climatological data (by State): Asheville, North Carolina, National Climatic Data Center, various months. National Oceanic and Atmospheric Administration (NOAA), 1994b-98b, Storm data (by State): Asheville, North Carolina, National Climatic Data Center, various months.

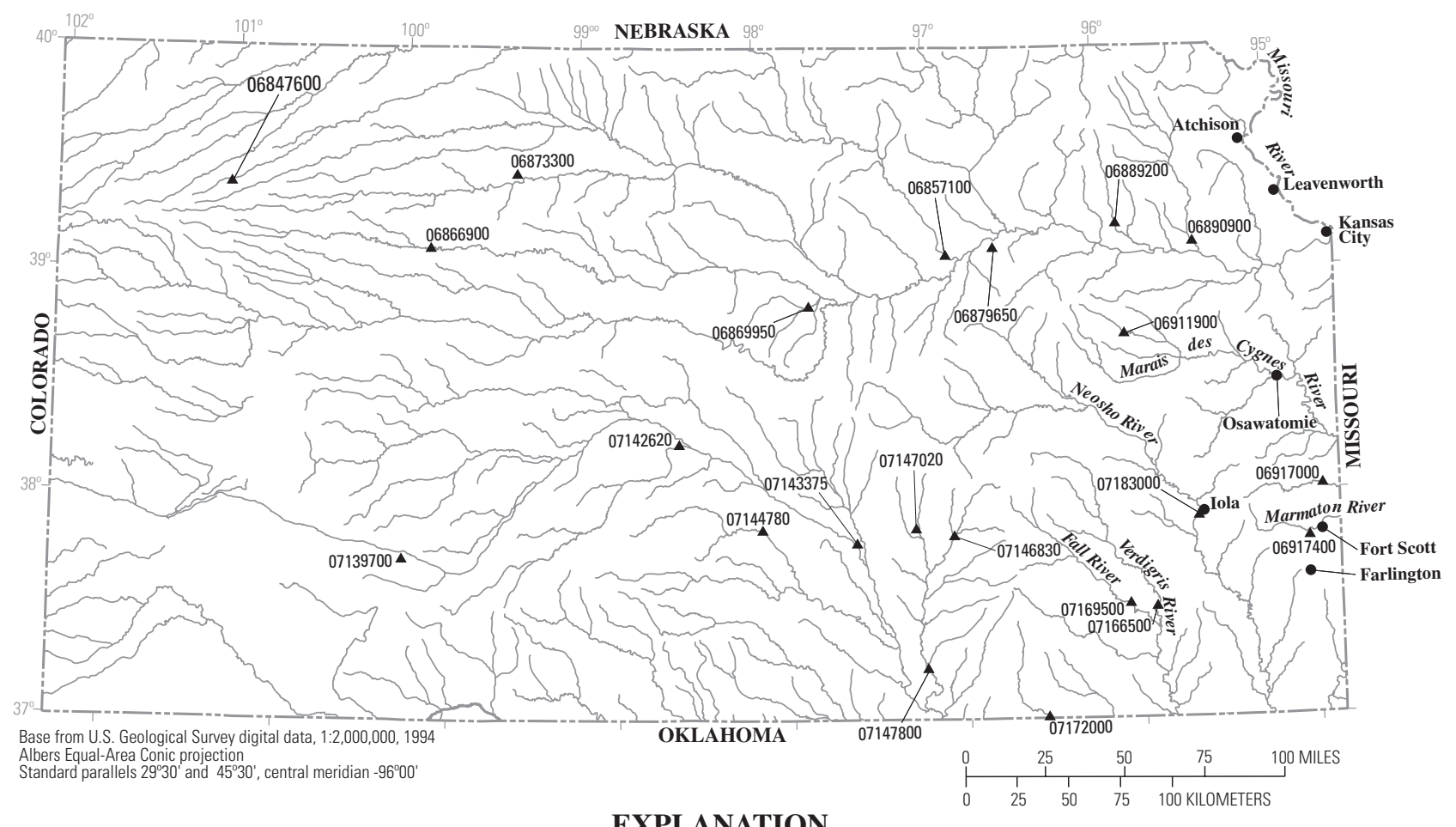

EXPLANATION

07144780 Streamgage and number

Figure 28. Location of streamgages with significant floods during 1994-98 water years for Kansas. 
Table 17. Maximum stage and discharge for period of record for streamgages having significant floods during 1994-98 water years in Kansas.

$\left[\mathrm{mi}^{2}\right.$, square miles; $\mathrm{ft}$, feet above an arbitrary datum; $\mathrm{ft}^{3} / \mathrm{s}$, cubic feet per second; --, not determined or not applicable; >, greater than. Source: Recurrence intervals calculated from U.S. Geological Survey data. Other data from U.S. Geological Survey reports or databases]

\begin{tabular}{|c|c|c|c|c|c|c|c|c|c|c|c|}
\hline \multirow{2}{*}{$\begin{array}{l}\text { Streamgage } \\
\text { number } \\
\text { (fig. 28) }\end{array}$} & \multirow[b]{2}{*}{ Streamgage name } & \multirow{2}{*}{$\begin{array}{c}\text { Total } \\
\text { drainage } \\
\left(\mathrm{mi}^{2}\right)\end{array}$} & \multicolumn{4}{|c|}{$\begin{array}{c}\text { Maximum stage and discharge for period of record } \\
\text { through } 1998 \text { water year }\end{array}$} & \multicolumn{5}{|c|}{ Significant floods 1994-98 water years } \\
\hline & & & $\begin{array}{c}\text { Period of } \\
\text { record } \\
\text { (water } \\
\text { years) }\end{array}$ & Water year & $\begin{array}{l}\text { Stage } \\
(\mathrm{ft})\end{array}$ & $\begin{array}{l}\text { Discharge } \\
\left(\mathrm{ft}^{3} / \mathrm{s}\right)\end{array}$ & $\begin{array}{c}\text { Date } \\
\text { (month/ } \\
\text { day/ } \\
\text { year) }\end{array}$ & $\begin{array}{l}\text { Stage } \\
(\mathrm{ft})\end{array}$ & $\begin{array}{l}\text { Discharge } \\
\left(\mathrm{ft}^{3} / \mathrm{s}\right)\end{array}$ & $\begin{array}{c}\text { Regulated } \\
\text { during } \\
\text { flood }^{1}\end{array}$ & $\begin{array}{c}\text { Recurrence } \\
\text { interval } \\
\text { (years) }\end{array}$ \\
\hline 06847600 & $\begin{array}{l}\text { Prairie Dog Creek tributary at } \\
\text { Colby, KS }\end{array}$ & 7.53 & $1957-97$ & 1975 & 27.44 & 4,300 & $6 / 15 / 96$ & 16.60 & 1,400 & $\mathrm{~N}$ & 25 \\
\hline 06857100 & $\begin{array}{l}\text { Republican River below Milford } \\
\text { Dam, KS }\end{array}$ & 24,890 & 1964-98 & $\begin{array}{l}1993 \\
1964\end{array}$ & $\begin{array}{l}21.52 \\
22.10\end{array}$ & $\begin{array}{l}33,700 \\
17,200\end{array}$ & $12 / 11 / 97$ & 16.03 & 18,700 & Y & -- \\
\hline 06866900 & Saline River near Wakeeney, KS & 696 & $\begin{array}{l}1950, \\
1956-66, \\
1982-98\end{array}$ & $\begin{array}{l}1957 \\
1950\end{array}$ & $\begin{array}{l}19.40 \\
27.00\end{array}$ & $\begin{array}{r}13,000 \\
--\end{array}$ & $9 / 18 / 95$ & 16.20 & 7,040 & $\mathrm{Y}$ & 5 \\
\hline 06869950 & Mulberry Creek near Salina, KS & 261 & $\begin{array}{l}1961-89 \\
1991-98\end{array}$ & 1995 & 27.14 & 8,440 & $5 / 28 / 95$ & 27.14 & 8,440 & $\mathrm{~N}$ & $10-25$ \\
\hline 06873300 & $\begin{array}{l}\text { Ash Creek tributary near Stockton, } \\
\text { KS }\end{array}$ & .89 & $\begin{array}{l}1957-93 \\
1995-98\end{array}$ & $\begin{array}{l}1996 \\
1993\end{array}$ & $\begin{array}{l}14.79 \\
15.54\end{array}$ & $\begin{array}{r}1,380 \\
530\end{array}$ & $8 / 3 / 96$ & 14.79 & 1,380 & $\mathrm{~N}$ & $50-100$ \\
\hline 06879650 & Kings Creek near Manhattan, KS & 4.09 & 1980-98 & 1995 & 13.98 & 10,200 & $5 / 13 / 95$ & 13.98 & 10,200 & $\mathrm{~N}$ & 25 \\
\hline 06889200 & Soldier Creek near Delia, KS & 157 & $\begin{array}{l}\text { 1951, } \\
1959-98\end{array}$ & $\begin{array}{l}1982 \\
1951\end{array}$ & $\begin{array}{l}23.95 \\
24.00\end{array}$ & $\begin{array}{r}29,400 \\
--\end{array}$ & $5 / 13 / 95$ & 23.09 & 19,900 & $\mathrm{~N}$ & 100 \\
\hline 06890900 & $\begin{array}{l}\text { Delaware River below Perry Dam, } \\
\text { KS }\end{array}$ & 1,117 & $1970-98$ & $\begin{array}{l}1995 \\
1970\end{array}$ & $\begin{array}{l}-- \\
--\end{array}$ & $\begin{array}{r}14,000 \\
9,920\end{array}$ & $5 / 31 / 95$ & -- & 14,000 & $\mathrm{Y}$ & -- \\
\hline 06911900 & $\begin{array}{l}\text { Dragoon Creek near Burlingame, } \\
\text { KS }\end{array}$ & 114 & $\begin{array}{l}\text { 1946, } \\
1961-98\end{array}$ & $\begin{array}{l}1977 \\
1946\end{array}$ & $\begin{array}{l}22.05 \\
23.40\end{array}$ & $\begin{array}{r}24,800 \\
--\end{array}$ & $5 / 17 / 95$ & 22.80 & 20,200 & $\mathrm{~N}$ & 50 \\
\hline 06917000 & Little Osage River at Fulton, KS & 295 & 1949-98 & 1987 & 35.21 & 62,800 & $4 / 28 / 94$ & 32.61 & 38,000 & $\mathrm{~N}$ & $50-100$ \\
\hline 06917400 & $\begin{array}{l}\text { Marmaton River tributary near Fort } \\
\text { Scott, KS }\end{array}$ & 2.80 & 1957-98 & 1998 & 17.23 & 2,160 & $9 / 14 / 98$ & 17.23 & 2,160 & $\mathrm{~N}$ & 25 \\
\hline 07139700 & $\begin{array}{l}\text { Arkansas River tributary near } \\
\text { Dodge City, KS }\end{array}$ & 8.66 & $\begin{array}{l}1957-89 \\
1992-98\end{array}$ & 1997 & 16.32 & 1,730 & $9 / 12 / 97$ & 16.32 & 1,730 & $\mathrm{~N}$ & $>100$ \\
\hline 07142620 & $\begin{array}{l}\text { Rattlesnake Creek near Raymond, } \\
\text { KS }\end{array}$ & 1,167 & 1961-98 & 1973 & 8.74 & 2,140 & $5 / 28 / 95$ & 8.37 & 1,530 & $\mathrm{Y}$ & -- \\
\hline
\end{tabular}


Table 17. Maximum stage and discharge for period of record for streamgages having significant floods during 1994-98 water years in Kansas.—Continued

$\left[\mathrm{mi}^{2}\right.$, square miles; $\mathrm{ft}$, feet above an arbitrary datum; $\mathrm{ft}^{3} / \mathrm{s}$, cubic feet per second; --, not determined or not applicable; >, greater than. Source: Recurrence intervals calculated from U.S. Geological Survey data. Other data from U.S. Geological Survey reports or databases]

\begin{tabular}{|c|c|c|c|c|c|c|c|c|c|c|c|}
\hline \multirow{2}{*}{$\begin{array}{l}\text { Streamgage } \\
\text { number } \\
\text { (fig. 28) }\end{array}$} & \multirow[b]{2}{*}{ Streamgage name } & \multirow{2}{*}{$\begin{array}{c}\text { Total } \\
\text { drainage } \\
\left(\mathrm{mi}^{2}\right)\end{array}$} & \multicolumn{4}{|c|}{$\begin{array}{c}\text { Maximum stage and discharge for period of record } \\
\text { through } 1998 \text { water year }\end{array}$} & \multicolumn{5}{|c|}{ Significant floods $1994-98$ water years } \\
\hline & & & $\begin{array}{l}\text { Period of } \\
\text { record } \\
\text { (water } \\
\text { years) }\end{array}$ & Water year & $\begin{array}{l}\text { Stage } \\
(\mathrm{ft})\end{array}$ & $\begin{array}{l}\text { Discharge } \\
\left(\mathrm{ft}^{3} / \mathrm{s}\right)\end{array}$ & $\begin{array}{c}\text { Date } \\
\text { (month/ } \\
\text { day/ } \\
\text { year) }\end{array}$ & $\begin{array}{l}\text { Stage } \\
(\mathrm{ft})\end{array}$ & $\begin{array}{l}\text { Discharge } \\
\left(\mathrm{ft}^{3} / \mathrm{s}\right)\end{array}$ & $\begin{array}{c}\text { Regulated } \\
\text { during } \\
\text { flood }^{1}\end{array}$ & $\begin{array}{c}\text { Recurrence } \\
\text { interval } \\
\text { (years) }\end{array}$ \\
\hline 07143375 & Arkansas River near Maize, KS & 39,110 & 1987-98 & 1993 & 16.84 & 44,900 & $5 / 28 / 95$ & 15.03 & 26,700 & $\mathrm{Y}$ & -- \\
\hline 07144780 & $\begin{array}{l}\text { North Fork Ninnescah River above } \\
\text { Cheney Reservoir, KS }\end{array}$ & 787 & $1966-98$ & 1980 & 11.65 & 87,000 & $5 / 27 / 95$ & 10.93 & 47,900 & $\mathrm{~N}$ & $25-50$ \\
\hline 07146830 & $\begin{array}{l}\text { Walnut River at Highway } 54 \text { east } \\
\text { of El Dorado, KS }\end{array}$ & 350 & 1982-98 & 1995 & 16.90 & 13,000 & $6 / 9 / 95$ & 16.90 & 13,000 & $\mathrm{Y}$ & -- \\
\hline 07147020 & $\begin{array}{l}\text { Whitewater River tributary near } \\
\text { Towanda, KS }\end{array}$ & .17 & 1963-98 & 1995 & 16.59 & 540 & $6 / 9 / 95$ & 16.59 & 540 & $\mathrm{~N}$ & 100 \\
\hline 07147800 & Walnut River at Winfield, KS & 1,880 & $\begin{array}{l}\text { 1898, 1904, } \\
\text { 1915, } \\
1922-98\end{array}$ & $\begin{array}{l}1944 \\
1929\end{array}$ & $\begin{array}{l}38.30 \\
41.00\end{array}$ & $\begin{array}{r}105,000 \\
94,400\end{array}$ & $6 / 10 / 95$ & 37.30 & 85,800 & $\mathrm{Y}$ & $25-50$ \\
\hline 07166500 & Verdigris River near Altoona, KS & 1,138 & 1939-98 & 1951 & 31.09 & 71,000 & $4 / 28 / 94$ & 27.19 & 31,000 & $\mathrm{Y}$ & 5 \\
\hline 07169500 & Fall River at Fredonia, KS & 827 & $\begin{array}{l}\text { 1904, 1923, } \\
\text { 1927-28, } \\
1939-98\end{array}$ & 1945 & 36.17 & 49,000 & $4 / 28 / 94$ & 31.94 & 31,000 & $\mathrm{Y}$ & 5 \\
\hline 07172000 & Caney River near Elgin, KS & 445 & 1939-98 & 1987 & 42.35 & 104,000 & $7 / 3 / 95$ & 29.64 & 43,200 & $\mathrm{~N}$ & 10 \\
\hline 07183000 & Neosho River near Iola, KS & 3,818 & $\begin{array}{l}1885, \\
1895-1904, \\
1918-98\end{array}$ & 1951 & 43.00 & 436,000 & $4 / 28 / 94$ & 27.95 & 37,100 & $\mathrm{Y}$ & $2-5$ \\
\hline
\end{tabular}

\footnotetext{
${ }^{1}$ Regulated during flood: N, no; Y, yes.
} 


\section{Kentucky}

During May 1995, the Mississippi River was well above flood stage, and the Ohio River was also flooding. Near the confluence of these rivers at Wickliffe, Kentucky (fig. 29), the worst flooding occurred since 1973. The bridge over the Ohio River between Cairo, Illinois, and Wickliffe, Kentucky, was closed for the first time since 1973. A state of emergency was declared in four Kentucky counties along the Mississippi River.

Excessive rainfall of 7 to 9 inches of rain fell during a 6hour period in central Kentucky on July 19, 1996 (National Oceanic and Atmospheric Administration, 1996a), which created flash floods that caused more than $\$ 2$ million in property damage (National Oceanic and Atmospheric Administration, 1996b). On July 31, excessive rains just south of Cincinnati, Ohio, created flood conditions on creeks and small rivers in north-central Kentucky. Damage totaled nearly $\$ 7$ million (National Oceanic and Atmospheric Administration, 1996b).

On March 1, 1997, a severe weather situation with tornadoes and very intense rainfall occurred along a nearly stationary front from Texas to West Virginia. Excessive rains totaling more than 12 inches occurred at many locations in Kentucky (National Oceanic and Atmospheric Administration, 1997a). Flooding killed 21 persons, 101 counties were declared Federal disaster areas, and estimated damage in Kentucky was \$250 to 500 million (National Oceanic and Atmospheric Administration, 1997b). The Ohio River crested on March 7 (70.5 feet) at Louisville (streamgage 03294500 , table 18) at about 16 feet above flood stage. The town of Falmouth (population 2,700) was almost totally destroyed (National Oceanic and Atmospheric Administration, 1997b). Record flooding occurred all along the Licking River Basin as 24-hour rainfall amounts beginning early March 1, 1997, totaled from 6 to 10 inches (National Oceanic and Atmospheric Administration, 1997a). The South Fork Licking River at Cynthiana (streamgage 03252500, table 18) crested 8 feet above flood stage at a record 28.03 feet on March 2. The Licking River at Blue Licks Spring crested 22.6 feet above flood stage at a record 47.6 feet on
March 2 (National Oceanic and Atmospheric Administration, 1997b).

The record rainfall lead to major flooding along the Salt River Basin including the Rolling Fork. The Salt River at Shepherdsville (streamgage 03298500 , table 18) crested at 40.9 feet on March 2. This was the worst flooding here since March 1964. The Rolling Fork near Boston (streamgage 03301500, table 18) crested at a record level of 53.2 feet on March 2. The Green River at Rochester crested at 30.7 feet on March 7 (National Oceanic and Atmospheric Administration, 1997b). This is the second highest stage on record. The record rainfall caused the highest stage on the Kentucky River at Frankfort (streamgage 03287500, table 18) since 1978 when the river crested at 45.22 feet on March 3 .

The excessive rains in March 1997 caused the worst flooding on the Ohio River since 1964 and in some spots since 1937. On March 8 the river crested at a record 52.4 feet at Cannelton Dam at Cannelton, Indiana (streamgage 03303280, table 18) and at 50.4 feet at Tell City, Indiana (National Oceanic and Atmospheric Administration, 1997b). The crest at Paducah, nearly 52 feet (National Oceanic and Atmospheric Administration, 1997b), was the highest since 1950, and one of the worst floods on record.

Excessive rainfall from thunderstorms caused flash floods on April 17 and 18 and general flooding on April 19 and 20 in southeastern Kentucky. Flooding was especially severe along the Right and Left Forks of Beaver Creek (National Oceanic and Atmospheric Administration, 1997b).

\section{References}

National Oceanic and Atmospheric Administration (NOAA), 1996a-97a, Climatological data (by State): Asheville, North Carolina, National Climatic Data Center, various months. National Oceanic and Atmospheric Administration (NOAA), 1996b-97b, Storm data (by State): Asheville, North Carolina, National Climatic Data Center, various months. 


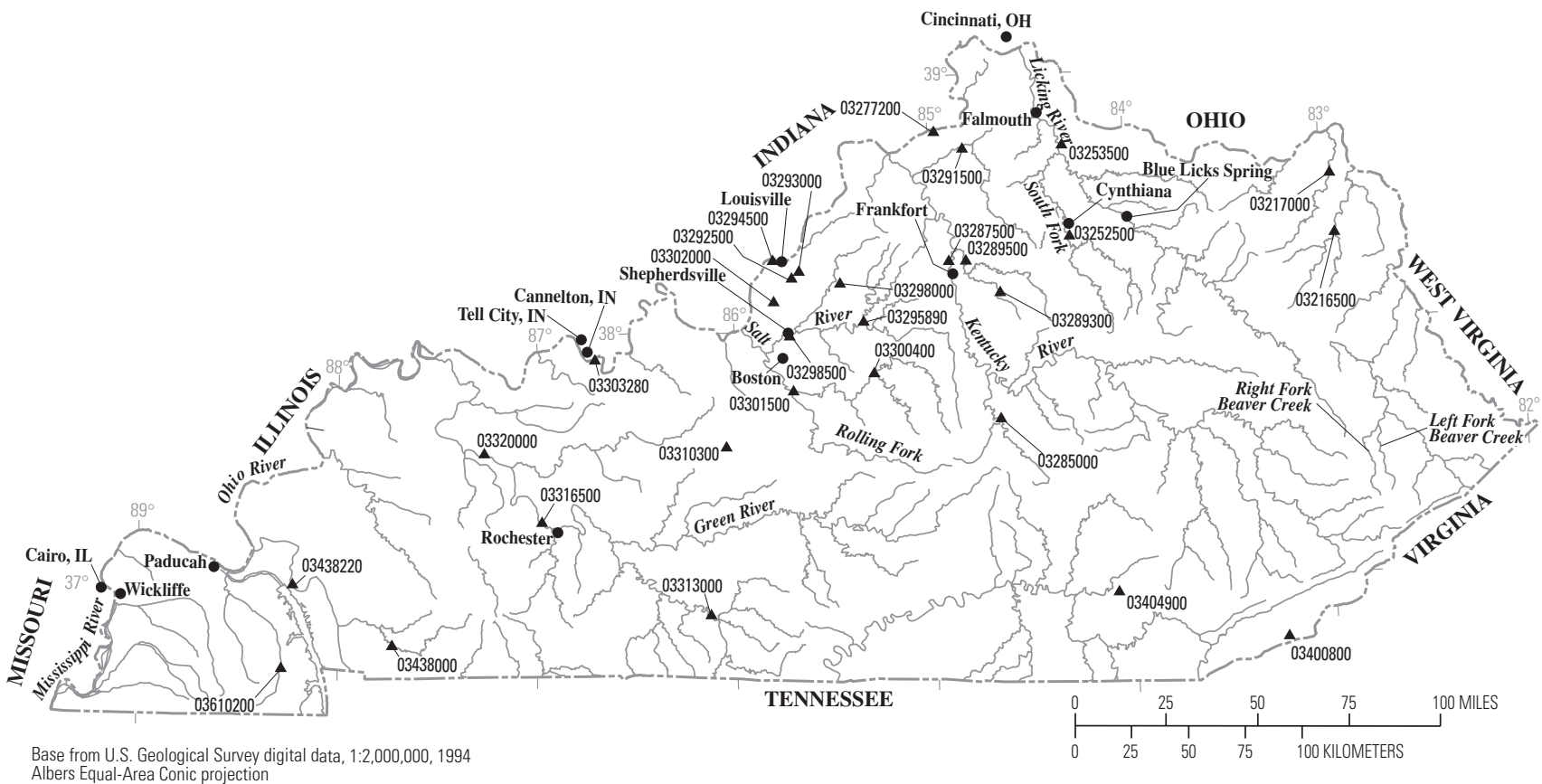

Albers Equal-Area Conic projection
Standard parallels $29^{\circ} 30^{\prime}$ and $45^{\circ} 30^{\prime}$, central meridian $-96^{\circ} 00^{\prime}$

\section{EXPLANATION}

$03313000 \triangle$ Streamgage and number

Figure 29. Location of streamgages with significant floods during 1994-98 water years for Kentucky. 
Table 18. Maximum stage and discharge for period of record for streamgages having significant floods during 1994-98 water years in Kentucky.

$\left[\mathrm{mi}^{2}\right.$, square miles; $\mathrm{ft}$, feet above an arbitrary datum; $\mathrm{ft}^{3} / \mathrm{s}$, cubic feet per second; --, not determined or not applicable; $>$, greater than; <, less than. Source: Recurrence intervals calculated from U.S. Geological Survey data. Other data from U.S. Geological Survey reports or databases]

\begin{tabular}{|c|c|c|c|c|c|c|c|c|c|c|c|}
\hline \multirow{2}{*}{$\begin{array}{l}\text { Streamgage } \\
\text { number } \\
\text { (fig. 29) }\end{array}$} & \multirow[b]{2}{*}{ Streamgage name } & \multirow{2}{*}{$\begin{array}{c}\text { Total } \\
\text { drainage } \\
\left(\mathrm{mi}^{2}\right)\end{array}$} & \multicolumn{4}{|c|}{$\begin{array}{c}\text { Maximum stage and discharge for period of record } \\
\text { through } 1998 \text { water year }\end{array}$} & \multicolumn{5}{|c|}{ Significant floods $1994-98$ water years } \\
\hline & & & $\begin{array}{l}\text { Period of } \\
\text { record } \\
\text { (water } \\
\text { years) }\end{array}$ & Water year & $\begin{array}{l}\text { Stage } \\
(\mathrm{ft})\end{array}$ & $\begin{array}{c}\text { Discharge } \\
\left(\mathrm{ft}^{3} / \mathrm{s}\right)\end{array}$ & $\begin{array}{c}\text { Date } \\
\text { (month/ } \\
\text { day/ } \\
\text { year) }\end{array}$ & $\begin{array}{l}\text { Stage } \\
(\mathrm{ft})\end{array}$ & $\begin{array}{c}\text { Discharge } \\
\left(\mathrm{ft}^{3} / \mathrm{s}\right)\end{array}$ & $\begin{array}{l}\text { Regulated } \\
\text { during } \\
\text { flood }^{1}\end{array}$ & $\begin{array}{c}\text { Recurrence } \\
\text { interval } \\
\text { (years) }\end{array}$ \\
\hline 03216500 & Little Sandy River at Grayson, KY & 400 & $\begin{array}{l}1937, \\
1939-98\end{array}$ & $\begin{array}{l}1950 \\
1997\end{array}$ & $\begin{array}{l}27.53 \\
30.57\end{array}$ & $\begin{array}{l}24,500 \\
16,300\end{array}$ & $3 / 2 / 97$ & 30.57 & 16,300 & $\bar{Y}$ & $5-10$ \\
\hline 03217000 & Tygarts Creek near Greenup, KY & 242 & $\begin{array}{l}\text { 1934, 1937, } \\
1941-98\end{array}$ & 1997 & 23.65 & 34,400 & $3 / 2 / 97$ & 23.65 & 34,400 & $\mathrm{~N}$ & $>100$ \\
\hline 03252500 & $\begin{array}{l}\text { South Fork Licking River at } \\
\text { Cynthiana, KY }\end{array}$ & 621 & $\begin{array}{l}1918-94 \\
1997\end{array}$ & 1997 & 28.03 & 39,000 & $3 / 2 / 97$ & 28.03 & 39,000 & $\mathrm{~N}$ & -- \\
\hline 03253500 & Licking River at Catawba, KY & 3,300 & $\begin{array}{l}1854, \\
1888-1998\end{array}$ & 1997 & 57.57 & 110,000 & $3 / 3 / 97$ & 57.57 & 110,000 & $\mathrm{Y}$ & -- \\
\hline 03277200 & $\begin{array}{l}\text { Ohio River at Markland Dam near } \\
\text { Warsaw, KY }\end{array}$ & 83,170 & 1971-98 & 1997 & 60.72 & 579,000 & $3 / 6 / 97$ & 60.72 & 579,000 & $\mathrm{Y}$ & -- \\
\hline 03285000 & Dix River near Danville, KY & 318 & 1943-98 & $\begin{array}{l}1996 \\
1979\end{array}$ & $\begin{array}{l}18.93 \\
21.81\end{array}$ & $\begin{array}{l}52,400 \\
44,400\end{array}$ & $7 / 20 / 96$ & 18.93 & 52,400 & $\mathrm{~N}$ & $>100$ \\
\hline 03289300 & $\begin{array}{l}\text { South Elkhorn Creek near Midway, } \\
\text { KY }\end{array}$ & 105 & $1983-98$ & 1997 & 26.37 & 12,300 & $3 / 2 / 97$ & 26.37 & 12,300 & $\mathrm{~N}$ & -- \\
\hline 03287500 & Kentucky River at Frankfort, KY & 5,411 & $1895-1998$ & 1978 & 48.47 & 118,000 & $3 / 2 / 97$ & 45.22 & 93,500 & $\mathrm{Y}$ & -- \\
\hline 03289500 & Elkhorn Creek near Frankfort, KY & 473 & $\begin{array}{l}\text { 1916-20, } \\
1932,1937, \\
1940-13, \\
1989-98\end{array}$ & 1997 & 17.96 & 35,900 & $3 / 4 / 97$ & 17.96 & 35,900 & $\mathrm{~N}$ & $>100$ \\
\hline 03291500 & Eagle Creek at Glencoe, KY & 437 & $\begin{array}{l}1913, \\
1915-20, \\
1928-31, \\
1937, \\
1939-87, \\
1989-98\end{array}$ & 1997 & 29.08 & 58,300 & $3 / 2 / 97$ & 29.08 & 58,300 & $\mathrm{~N}$ & $>100$ \\
\hline
\end{tabular}


Table 18. Maximum stage and discharge for period of record for streamgages having significant floods during 1994-98 water years in Kentucky.-Continued

$\left[\mathrm{mi}^{2}\right.$, square miles; $\mathrm{ft}$, feet above an arbitrary datum; $\mathrm{ft}^{3} / \mathrm{s}$, cubic feet per second; --, not determined or not applicable; $>$, greater than; <, less than. Source: Recurrence intervals calculated from U.S. Geological Survey data. Other data from U.S. Geological Survey reports or databases]

\begin{tabular}{|c|c|c|c|c|c|c|c|c|c|c|c|}
\hline \multirow{2}{*}{$\begin{array}{c}\text { Streamgage } \\
\text { number } \\
\text { (fig. 29) }\end{array}$} & \multirow[b]{2}{*}{ Streamgage name } & \multirow{2}{*}{$\begin{array}{c}\text { Total } \\
\text { drainage } \\
\left(\mathrm{mi}^{2}\right)\end{array}$} & \multicolumn{4}{|c|}{$\begin{array}{c}\text { Maximum stage and discharge for period of record } \\
\text { through } 1998 \text { water year }\end{array}$} & \multicolumn{5}{|c|}{ Signific ant floods $1994-98$ water years } \\
\hline & & & $\begin{array}{c}\text { Period of } \\
\text { record } \\
\text { (water } \\
\text { years) }\end{array}$ & Water year & $\begin{array}{l}\text { Stage } \\
(\mathrm{ft})\end{array}$ & $\begin{array}{l}\text { Discharge } \\
\left(\mathrm{ft}^{3} / \mathrm{s}\right)\end{array}$ & $\begin{array}{c}\text { Date } \\
\text { (month/ } \\
\text { day/ } \\
\text { year) }\end{array}$ & $\begin{array}{l}\text { Stage } \\
(\mathrm{ft})\end{array}$ & $\begin{array}{l}\text { Discharge } \\
\left(\mathrm{ft}^{3} / \mathrm{s}\right)\end{array}$ & $\begin{array}{c}\text { Regulated } \\
\text { during } \\
\text { flood }^{1}\end{array}$ & $\begin{array}{c}\text { Recurrence } \\
\text { interval } \\
\text { (years) }\end{array}$ \\
\hline 03292500 & $\begin{array}{l}\text { South Fork Beargrass Creek at } \\
\text { Louisville, KY }\end{array}$ & 17.2 & $\begin{array}{l}1940-83 \\
1988-98\end{array}$ & 1997 & 17.81 & 5,290 & $3 / 2 / 97$ & 17.81 & 5,290 & $\mathrm{~N}$ & $50-100$ \\
\hline 03293000 & $\begin{array}{l}\text { Middle Fork Beargrass Creek at } \\
\text { Louisville, KY }\end{array}$ & 18.9 & $\begin{array}{l}1943, \\
1945-83, \\
1985-98\end{array}$ & $\begin{array}{l}1997 \\
1970\end{array}$ & $\begin{array}{l}8.70 \\
9.60\end{array}$ & $\begin{array}{l}5,900 \\
5,200\end{array}$ & $3 / 2 / 97$ & 8.70 & 5,900 & $\mathrm{~N}$ & $>100$ \\
\hline 03294500 & Ohio River at Louisville, KY & 91,170 & $\begin{array}{l}1832,1848, \\
1858-59, \\
1866-69, \\
1872-1998\end{array}$ & $\begin{array}{l}1937 \\
1997\end{array}$ & $\begin{array}{l}85.44 \\
70.47\end{array}$ & $\begin{array}{r}1,110,000 \\
716,000\end{array}$ & $3 / 6 / 97$ & 70.47 & 716,000 & $\mathrm{Y}$ & -- \\
\hline 03295890 & $\begin{array}{l}\text { Brashears Creek at Taylorsville, } \\
\text { KY }\end{array}$ & 259 & 1982-98 & 1997 & 31.54 & 44,800 & $3 / 2 / 97$ & 31.54 & 44,800 & Y & -- \\
\hline 03298000 & Floyds Fork at Fisherville, KY & 138 & $\begin{array}{l}1937,1943 \\
1945-98\end{array}$ & 1997 & 17.39 & 42,100 & $3 / 2 / 97$ & 17.39 & 42,100 & $\mathrm{~N}$ & $>100$ \\
\hline 03298500 & Salt River at Shepherdsville, KY & 1,197 & 1937-98 & $\begin{array}{l}1964 \\
1937\end{array}$ & $\begin{array}{l}41.50 \\
47.30\end{array}$ & $\begin{array}{l}78,200 \\
71,300\end{array}$ & $3 / 2 / 97$ & 40.92 & 71,300 & $\mathrm{Y}$ & -- \\
\hline 03300400 & Beech Fork at Maud, KY & 436 & $\begin{array}{l}\text { 1964, } \\
1973-98\end{array}$ & 1997 & 27.60 & 41,500 & $3 / 2 / 97$ & 27.60 & 41,500 & $\mathrm{~N}$ & $50-100$ \\
\hline 03301500 & Rolling Fork near Boston, KY & 1,299 & $\begin{array}{l}1937, \\
1939-98\end{array}$ & $\begin{array}{l}1997 \\
1937\end{array}$ & $\begin{array}{l}53.22 \\
55.20\end{array}$ & $\begin{array}{r}69,800 \\
--\end{array}$ & $3 / 3 / 97$ & 53.22 & 69,800 & $\mathrm{~N}$ & $>100$ \\
\hline 03302000 & Pond Creek near Louisville, KY & 64.0 & $\begin{array}{l}\text { 1937, } \\
1945-98\end{array}$ & $\begin{array}{l}1964 \\
1997\end{array}$ & $\begin{array}{l}22.69 \\
25.66\end{array}$ & $\begin{array}{l}8,020 \\
7,800\end{array}$ & $3 / 2 / 97$ & 25.66 & 7,800 & $\mathrm{~N}$ & $>100$ \\
\hline 03303280 & $\begin{array}{l}\text { Ohio River at Cannelton Dam at } \\
\text { Cannelton, IN }\end{array}$ & 97,000 & 1979-98 & 1997 & 52.42 & 735,000 & $3 / 8 / 97$ & 52.42 & 735,000 & $\mathrm{Y}$ & -- \\
\hline 03310300 & Nolin River at White Mills, KY & 357 & 1960-98 & 1997 & 36.46 & 24,500 & $3 / 2 / 97$ & 36.46 & 24,500 & $\mathrm{~N}$ & $50-100$ \\
\hline
\end{tabular}


Table 18. Maximum stage and discharge for period of record for streamgages having significant floods during 1994-98 water years in Kentucky.-Continued

$\left[\mathrm{mi}^{2}\right.$, square miles; $\mathrm{ft}$, feet above an arbitrary datum; $\mathrm{ft}^{3} / \mathrm{s}$, cubic feet per second; --, not determined or not applicable; $>$, greater than; <, less than. Source: Recurrence intervals calculated from U.S. Geological Survey data. Other data from U.S. Geological Survey reports or databases]

\begin{tabular}{|c|c|c|c|c|c|c|c|c|c|c|c|}
\hline \multirow{2}{*}{$\begin{array}{l}\text { Streamgage } \\
\text { number } \\
\text { (fig. 29) }\end{array}$} & \multirow[b]{2}{*}{ Streamgage name } & \multirow{2}{*}{$\begin{array}{c}\text { Total } \\
\text { drainage } \\
\left(\mathrm{mi}^{2}\right)\end{array}$} & \multicolumn{4}{|c|}{$\begin{array}{c}\text { Maximum stage and discharge for period of record } \\
\text { through } 1998 \text { water year }\end{array}$} & \multicolumn{5}{|c|}{ Significant floods 1994-98 water years } \\
\hline & & & $\begin{array}{l}\text { Period of } \\
\text { record } \\
\text { (water } \\
\text { years) }\end{array}$ & Water year & $\begin{array}{l}\text { Stage } \\
(\mathrm{ft})\end{array}$ & $\begin{array}{c}\text { Discharge } \\
\left(\mathrm{ft}^{3} / \mathrm{s}\right)\end{array}$ & $\begin{array}{c}\text { Date } \\
\text { (month/ } \\
\text { day/ } \\
\text { year) }\end{array}$ & $\begin{array}{l}\text { Stage } \\
(\mathrm{ft})\end{array}$ & $\begin{array}{c}\text { Discharge } \\
\left(\mathrm{ft}^{3} / \mathrm{s}\right)\end{array}$ & $\begin{array}{c}\text { Regulated } \\
\text { during } \\
\text { flood }^{1}\end{array}$ & $\begin{array}{c}\text { Recurrence } \\
\text { interval } \\
\text { (years) }\end{array}$ \\
\hline 03313000 & Barren River near Finney, KY & 942 & $\begin{array}{l}1942-50, \\
1961-94\end{array}$ & $\begin{array}{l}1962 \\
1943 \\
1994\end{array}$ & $\begin{array}{l}-- \\
99.50 \\
90.66\end{array}$ & $\begin{array}{r}78,000 \\
23,500 \\
7,470\end{array}$ & $4 / 11 / 94$ & 90.66 & 7,470 & $\mathrm{Y}$ & -- \\
\hline 03316500 & Green River at Paradise, KY & 6,183 & $\begin{array}{l}1940-50, \\
1961-81, \\
1992-98\end{array}$ & $\begin{array}{l}1962 \\
1997\end{array}$ & $\begin{array}{l}40.46 \\
37.63\end{array}$ & $\begin{array}{r}107,000 \\
86,300\end{array}$ & $3 / 7 / 97$ & 37.63 & 83,300 & $\mathrm{Y}$ & -- \\
\hline 03320000 & $\begin{array}{l}\text { Green River at Lock } 2 \text { at Calhoun, } \\
\text { KY }\end{array}$ & 7,566 & $\begin{array}{l}\text { 1913, } \\
1931-98\end{array}$ & $\begin{array}{l}1937 \\
1997\end{array}$ & $\begin{array}{l}43.70 \\
33.23\end{array}$ & $\begin{array}{r}208,000 \\
86,000\end{array}$ & $3 / 7 / 97$ & 33.23 & 86,000 & $\mathrm{Y}$ & -- \\
\hline 03400800 & Martins Fork near Smith, KY & 55.8 & 1968-98 & $\begin{array}{l}1977 \\
1998\end{array}$ & $\begin{array}{l}24.24 \\
14.05\end{array}$ & $\begin{array}{l}9,000 \\
1,520\end{array}$ & $4 / 19 / 98$ & 14.05 & 1,520 & $\mathrm{Y}$ & $<2$ \\
\hline 03404900 & Lynn Camp Creek at Corbin, KY & 53.8 & 1957-98 & $\begin{array}{l}1998 \\
1957\end{array}$ & $\begin{array}{l}14.33 \\
22.50\end{array}$ & $\begin{array}{l}6,820 \\
9,000\end{array}$ & $4 / 17 / 98$ & 14.33 & 6,820 & $\mathrm{~N}$ & $25-50$ \\
\hline 03438000 & Little River near Cadiz, KY & 244 & 1940-98 & 1997 & 26.44 & 37,600 & $3 / 1 / 97$ & 26.44 & 37,600 & $\mathrm{~N}$ & $>100$ \\
\hline 03438220 & $\begin{array}{l}\text { Cumberland River near Grand } \\
\text { Rivers, KY }\end{array}$ & 17,598 & $\begin{array}{l}\text { 1900, } \\
1965-97\end{array}$ & 1997 & 45.72 & 209,000 & $3 / 5 / 97$ & 45.72 & 209,000 & $\mathrm{Y}$ & -- \\
\hline 03610200 & Clarks River at Almo, KY & 134 & 1983-98 & $\begin{array}{l}1997 \\
1991\end{array}$ & $\begin{array}{l}18.35 \\
18.84\end{array}$ & $\begin{array}{l}23,300 \\
16,600\end{array}$ & $3 / 2 / 97$ & 18.35 & 23,300 & $\mathrm{~N}$ & -- \\
\hline
\end{tabular}

${ }^{1}$ Regulated during flood: N, no; Y, yes. 


\section{Louisiana}

A nearly stationary area of rain and embedded thunderstorms produced excessive rain across much of central and southern Louisiana on January 26-27, 1994. Storm totals of 6 to 8 inches were common in a large swath from southwestern and west-central Louisiana into east-central Louisiana (National Oceanic and Atmospheric Administration, 1994a). Within this area, rainfall totals of 8 to 12 inches occurred west of Alexandria (fig. 30), overwhelming drainage capacity and resulting in flash flooding.

Torrential rain of 8 to 12 inches fell across parts of southwestern, west-central, and east-central Louisiana during the night and morning hours of April 11, 1995 (National Oceanic and Atmospheric Administration, 1995a). The Calcasieu River crested at Oakdale on April 12 at 15.7 feet (National Oceanic and Atmospheric Administration, 1995b). In east-central Louisiana, floods occurred on the Amite, Comite, Tickfaw, Tangipahoa, and Tchefuncte Rivers. During May 8-10, 1995, extreme weather conditions, which produced as much as 27.5 inches of rain during a 55-hour period (National Oceanic and
Atmospheric Administration, 1995a), caused the most severe flooding in recent history along coastal areas of the Gulf of Mexico in Mississippi and southeastern Louisiana. At least six people died, and thousands more were left homeless as a result of the intense flooding. At least $\$ 3$ billion in property damage was reported in New Orleans, Louisiana (National Oceanic and Atmospheric Administration, 1995b).

Recurrent thunderstorms during April 1997 caused many streams in Louisiana to flood. The most significant flood was at the Wax Lake outlet at Calumet (streamgage 07381590, table 19). This streamgage recorded the largest discharge since records began in 1942 .

\section{References}

National Oceanic and Atmospheric Administration (NOAA), 1994a-95a, Climatological data (by State): Asheville, North Carolina, National Climatic Data Center, various months. National Oceanic and Atmospheric Administration (NOAA), 1994b-95b, Storm data (by State): Asheville, North Carolina, National Climatic Data Center, various months.

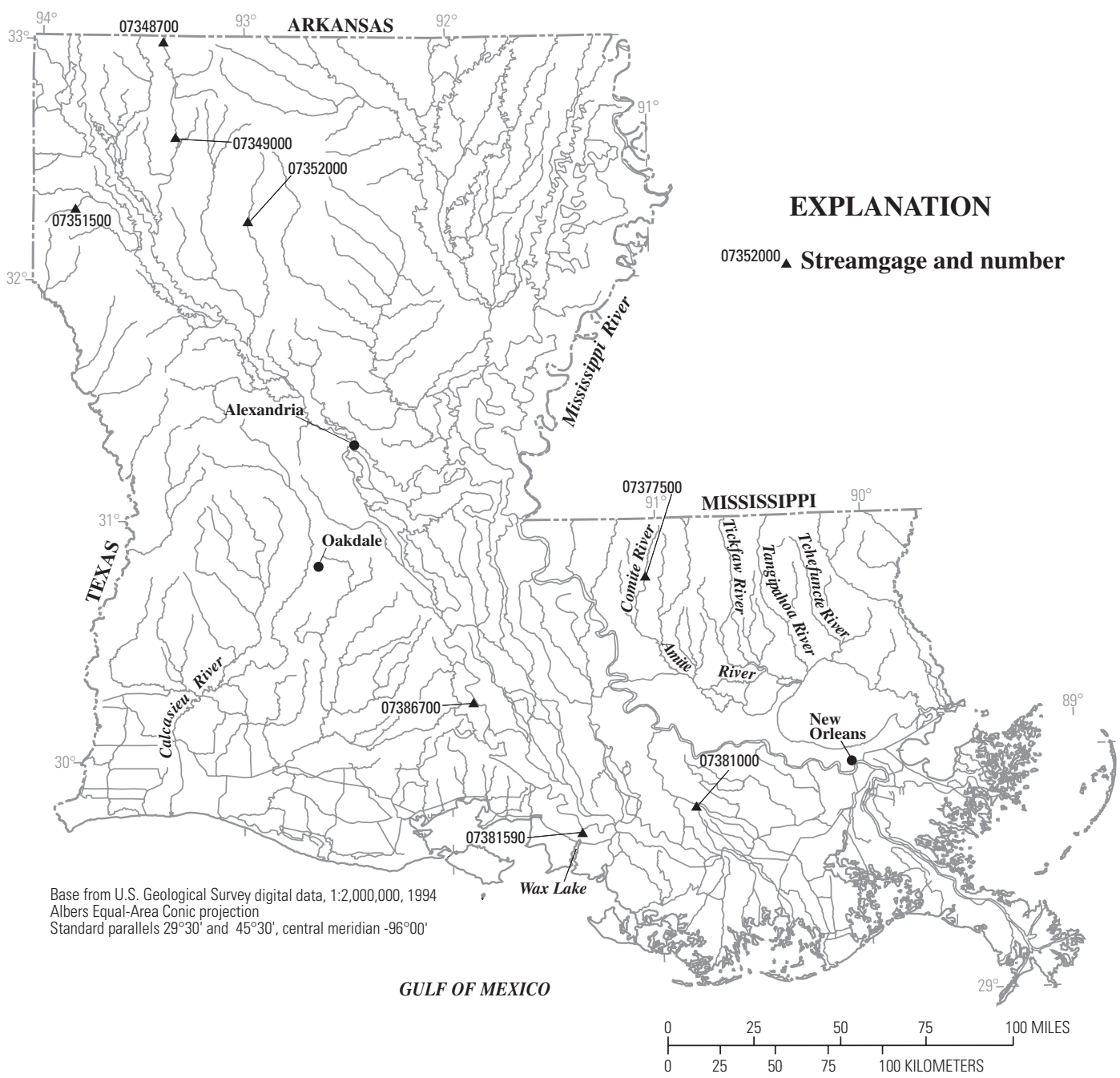

Figure 30. Location of streamgages with significant floods during 1994-98 water years for Louisiana. 
Table 19. Maximum stage and discharge for period of record for streamgages having significant floods during 1994-98 water years in Louisiana.

$\left[\mathrm{mi}^{2}\right.$, square miles; $\mathrm{ft}$, feet above an arbitrary datum; $\mathrm{ft}^{3} / \mathrm{s}$, cubic feet per second; --, not determined or not applicable. Source: Recurrence intervals calculated from U.S. Geological Survey data. Other data from U.S. Geological Survey reports or databases]

\begin{tabular}{|c|c|c|c|c|c|c|c|c|c|c|c|}
\hline \multirow{2}{*}{$\begin{array}{c}\text { Streamgage } \\
\text { number } \\
\text { (fig. 30) }\end{array}$} & \multirow[b]{2}{*}{ Streamgage name } & \multirow{2}{*}{$\begin{array}{c}\text { Total } \\
\text { drainage } \\
\left(\mathrm{mi}^{2}\right)\end{array}$} & \multicolumn{4}{|c|}{$\begin{array}{c}\text { Maximum stage and discharge for period of record } \\
\text { through } 1998 \text { water year }\end{array}$} & \multicolumn{5}{|c|}{ Significant floods 1994-98 water years } \\
\hline & & & $\begin{array}{c}\text { Period of } \\
\text { record } \\
\text { (water } \\
\text { years) }\end{array}$ & Water year & $\begin{array}{l}\text { Stage } \\
(\mathrm{ft})\end{array}$ & $\begin{array}{l}\text { Discharge } \\
\left(\mathrm{ft}^{3} / \mathrm{s}\right)\end{array}$ & $\begin{array}{c}\text { Date } \\
\text { (month/ } \\
\text { day/ } \\
\text { year) }\end{array}$ & $\begin{array}{l}\text { Stage } \\
(\mathrm{ft})\end{array}$ & $\begin{array}{l}\text { Discharge } \\
\left(\mathrm{ft}^{3} / \mathrm{s}\right)\end{array}$ & $\begin{array}{c}\text { Regulated } \\
\text { during } \\
\text { flood }^{1}\end{array}$ & $\begin{array}{c}\text { Recurrence } \\
\text { interval } \\
\text { (years) }\end{array}$ \\
\hline 07348700 & $\begin{array}{l}\text { Bayou Dorcheat near Springhill, } \\
\text { LA }\end{array}$ & 605 & $1958-98$ & $\begin{array}{l}1997 \\
1958\end{array}$ & $\begin{array}{l}21.97 \\
22.79\end{array}$ & $\begin{array}{l}36,700 \\
36,400\end{array}$ & $4 / 6 / 97$ & 21.97 & 36,700 & $\mathrm{~N}$ & -- \\
\hline 07349000 & Bayou Dorcheat near Minden, LA & 1,097 & $\begin{array}{l}\text { 1929-31, } \\
1933, \\
1936-92, \\
1994-98\end{array}$ & $\begin{array}{l}1958 \\
1997\end{array}$ & $\begin{array}{l}24.90 \\
25.12\end{array}$ & $\begin{array}{l}44,800 \\
38,200\end{array}$ & $4 / 8 / 97$ & 25.12 & 38,200 & $\mathrm{~N}$ & -- \\
\hline 07351500 & Cypress Bayou near Keithville, LA & 66.0 & 1939-98 & $\begin{array}{l}1997 \\
1955\end{array}$ & $\begin{array}{l}13.31 \\
13.62\end{array}$ & $\begin{array}{l}26,100 \\
23,700\end{array}$ & $\begin{array}{l}4 / 23 / 95 \\
4 / 5 / 97\end{array}$ & $\begin{array}{l}12.29 \\
13.31\end{array}$ & $\begin{array}{l}15,700 \\
26,100\end{array}$ & $\begin{array}{l}\mathrm{N} \\
\mathrm{N}\end{array}$ & $\begin{array}{l}-- \\
--\end{array}$ \\
\hline 07352000 & Saline Bayou near Lucky, LA & 154 & 1941-98 & 1945 & 12.90 & 13,500 & $4 / 23 / 95$ & 11.45 & 10,800 & $\mathrm{~N}$ & -- \\
\hline 07377500 & $\begin{array}{l}\text { Comite River near Olive Branch, } \\
\text { LA }\end{array}$ & 145 & 1943-98 & $\begin{array}{l}1977 \\
1961\end{array}$ & $\begin{array}{l}22.88 \\
23.37\end{array}$ & $\begin{array}{l}22,400 \\
19,900\end{array}$ & $\begin{array}{l}4 / 12 / 95 \\
4 / 28 / 97\end{array}$ & $\begin{array}{l}18.23 \\
17.81\end{array}$ & $\begin{array}{l}21,400 \\
22,200\end{array}$ & $\begin{array}{l}\mathrm{N} \\
\mathrm{N}\end{array}$ & -- \\
\hline 07381000 & $\begin{array}{l}\text { Bayou Lafourche at Thibodaux, } \\
\text { LA }\end{array}$ & -- & $\begin{array}{l}1966-88 \\
1993-97\end{array}$ & $\begin{array}{l}1995 \\
1973\end{array}$ & $-\overline{-}$ & $\begin{array}{r}1,450 \\
--\end{array}$ & $5 / 9 / 95$ & -- & 1,450 & $\mathrm{Y}$ & -- \\
\hline 07381590 & Wax Lake outlet at Calumet, LA & -- & $\begin{array}{l}1942-85, \\
1988, \\
1990-98\end{array}$ & $\begin{array}{l}1997 \\
1973\end{array}$ & 11.16 & $\begin{array}{r}258,000 \\
--\end{array}$ & $\begin{array}{l}4 / 5 / 97 \\
5 / 18 / 98\end{array}$ & -- & $\begin{array}{l}258,000 \\
197,000\end{array}$ & $\begin{array}{l}\mathrm{Y} \\
\mathrm{Y}\end{array}$ & $\begin{array}{l}-- \\
--\end{array}$ \\
\hline 07386700 & Ruth Canal near Ruth, LA & -- & $\begin{array}{l}1940, \\
1947-85, \\
1997-98\end{array}$ & $\begin{array}{l}1997 \\
1940\end{array}$ & 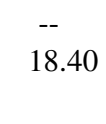 & $\begin{array}{r}802 \\
--\end{array}$ & $4 / 21 / 97$ & -- & 802 & $\mathrm{Y}$ & -- \\
\hline
\end{tabular}

${ }^{1}$ Regulated during flood: N, no; Y, yes. 


\section{Maine}

The town of Fort Fairfield (fig. 31) received its worst flooding in history when ice jams and excessive rains caused the Aroostook River to overflow its banks on April 16, 1994. Floodwaters several feet deep, carrying ice chunks 3 feet thick, caused approximately $\$ 9$ million in damage to businesses and residences (National Oceanic and Atmospheric Administration, 1994b).

Flooding throughout Maine occurred during January 27 30,1996 , when the last and most intense storm in a series of three low-pressure centers moved northeast from the Great Lakes. South to southeast winds brought warm temperatures and excessive rainfall to the area. Rainfall from the storm exceeded 4 inches in the western Maine mountains, with 2 to 3 inches common over much of central Maine (National Oceanic and Atmospheric Administration, 1996a). High runoff and ice jams caused flooding on many rivers and streams from the western mountains to the northeastern corner of the State.

Low pressure moving through western New England caused flooding in the southwestern part of Maine, April 16-18, 1996. Storm damage to non-Federal public property was estimated at \$2.5 million from the storm, including river and coastal flood damage and land-slump damage to public property (National Oceanic and Atmospheric Administration, 1996b). A Federal disaster was declared for five counties in southwestern Maine.

A coastal storm, fed with tropical moisture by the circulation around Hurricane Lily, produced between 8 and 19 inches of rain throughout southwestern Maine during a 30-hour period from October 20-23, 1996 (National Oceanic and Atmospheric Administration, 1996a). Damage was more than \$26 million (National Oceanic and Atmospheric Administration, 1996b). Streamgages on Branch Brook, the Little Ossipee River, and the Presumpscot River experienced their peak of record during this flood (table 20).

Rapidly melting snow, caused by record-breaking warmth, combined with rainfall caused flooding on the Kennebec and Androscoggin Rivers and their tributaries on March 30-31, 1998.

\section{References}

National Oceanic and Atmospheric Administration (NOAA), 1994a-98a, Climatological data (by State): Asheville, North Carolina, National Climatic Data Center, various months. National Oceanic and Atmospheric Administration (NOAA), 1994b-98b, Storm data (by State): Asheville, North Carolina, National Climatic Data Center, various months. 


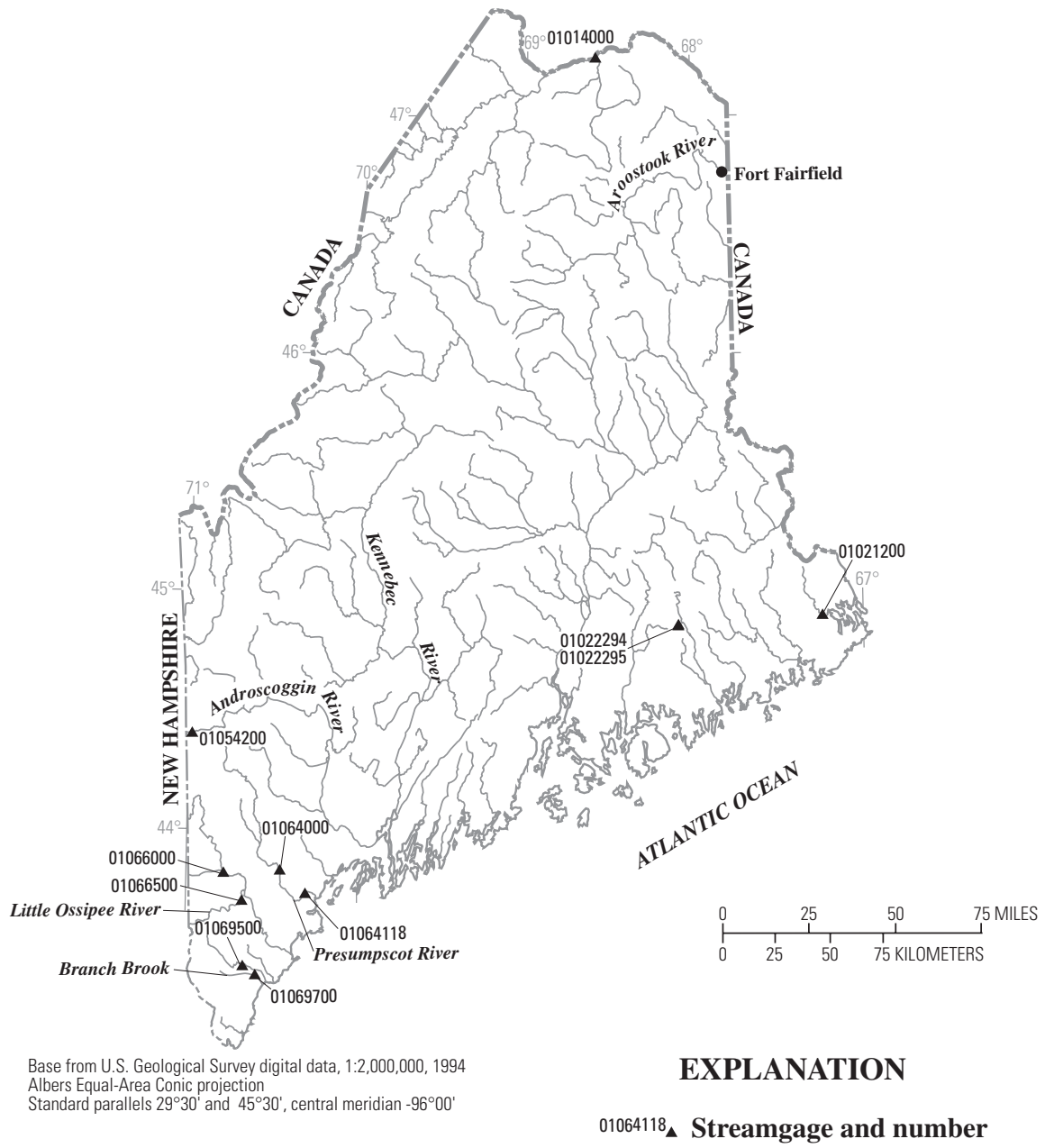

Figure 31. Location of streamgages with significant floods during 1994-98 water years for Maine. 
Table 20. Maximum stage and discharge for period of record for streamgages having significant floods during 1994-98 water years in Maine.

$\left[\mathrm{mi}^{2}\right.$, square miles; $\mathrm{ft}$, feet above an arbitrary datum; $\mathrm{ft}^{3} / \mathrm{s}$, cubic feet per second; --, not determined or not applicable. Source: Recurrence intervals calculated from U.S. Geological Survey data. Other data from U.S. Geological Survey reports or databases]

\begin{tabular}{|c|c|c|c|c|c|c|c|c|c|c|c|}
\hline \multirow[b]{2}{*}{$\begin{array}{l}\text { Streamgage } \\
\text { number } \\
\text { (fig. 31) }\end{array}$} & \multirow[b]{2}{*}{ Streamgage name } & \multirow[b]{2}{*}{$\begin{array}{c}\text { Total } \\
\text { drainage } \\
\left(\mathrm{mi}^{2}\right)\end{array}$} & \multicolumn{4}{|c|}{$\begin{array}{c}\text { Maximum stage and discharge for period of record } \\
\text { through } 1998 \text { water year }\end{array}$} & \multicolumn{5}{|c|}{ Significant floods 1994-98 water years } \\
\hline & & & $\begin{array}{l}\text { Period of } \\
\text { record } \\
\text { (water } \\
\text { years) }\end{array}$ & Water year & $\begin{array}{l}\text { Stage } \\
(\mathrm{ft})\end{array}$ & $\begin{array}{l}\text { Discharge } \\
\left(\mathrm{ft}^{3} / \mathrm{s}\right)\end{array}$ & $\begin{array}{c}\text { Date } \\
\text { (month/ } \\
\text { day/ } \\
\text { year) }\end{array}$ & $\begin{array}{l}\text { Stage } \\
(\mathrm{ft})\end{array}$ & $\begin{array}{l}\text { Discharge } \\
\left(\mathrm{ft}^{3} / \mathrm{s}\right)\end{array}$ & $\begin{array}{l}\text { Regulated } \\
\text { during } \\
\text { flood }^{1}\end{array}$ & $\begin{array}{c}\text { Recurrence } \\
\text { interval } \\
\text { (years) }\end{array}$ \\
\hline 01014000 & $\begin{array}{l}\text { Saint John River below Fish River, } \\
\text { at Fort Kent, ME }\end{array}$ & 5,665 & 1927-98 & 1979 & 27.31 & 151,000 & $4 / 17 / 94$ & 26.60 & 144,000 & $\mathrm{~N}$ & 40 \\
\hline 01021200 & Dennys River at Dennysville, ME & 92.9 & 1956-98 & 1973 & 9.35 & 3,930 & $3 / 10 / 98$ & 8.70 & 3,440 & $\mathrm{Y}$ & 40 \\
\hline 01022294 & $\begin{array}{l}\text { East Branch Bear Brook near } \\
\text { Beddington, ME }\end{array}$ & .04 & $1989-98$ & 1998 & 6.91 & 18.6 & 3/9/98 & 6.91 & 18.6 & $\mathrm{~N}$ & 40 \\
\hline 01022295 & $\begin{array}{l}\text { West Branch Bear Brook near } \\
\text { Beddington, ME }\end{array}$ & .04 & 1989-98 & 1998 & 6.75 & 16.4 & 3/9/98 & 6.75 & 16.4 & $\mathrm{~N}$ & 40 \\
\hline 01054200 & Wild River at Gilead, ME & 69.6 & $\begin{array}{l}1960 \\
1965-98\end{array}$ & 1960 & 15.60 & 28,300 & $10 / 22 / 95$ & 14.84 & 24,500 & $\mathrm{~N}$ & 80 \\
\hline 01064000 & $\begin{array}{l}\text { Presumpscot River at outlet of } \\
\text { Sebago Lake, ME }\end{array}$ & 441 & $1887-1998$ & 1902 & -- & 7,000 & $6 / 17 / 98$ & -- & 3,760 & $\mathrm{Y}$ & 25 \\
\hline 01064118 & $\begin{array}{l}\text { Presumpscot River at Westbrook, } \\
\text { ME }\end{array}$ & 577 & $\begin{array}{l}1976-95 \\
1997\end{array}$ & 1997 & 34.10 & 23,300 & $10 / 22 / 96$ & 34.10 & 23,300 & $\mathrm{Y}$ & 250 \\
\hline 01066000 & Saco River at Cornish, ME & 1,293 & 1917-98 & 1936 & 21.90 & 46,600 & $6 / 18 / 98$ & 15.11 & 27,500 & $\mathrm{~N}$ & 30 \\
\hline 01066500 & $\begin{array}{l}\text { Little Ossipee River near South } \\
\text { Limington, ME }\end{array}$ & 168 & $\begin{array}{l}1936, \\
1941-82, \\
1997\end{array}$ & $\begin{array}{l}1997 \\
1997\end{array}$ & $\begin{array}{l}7.02 \\
7.04\end{array}$ & $\begin{array}{l}5,800 \\
5,760\end{array}$ & $10 / 22 / 96$ & 7.02 & 5,800 & $\mathrm{~N}$ & 30 \\
\hline 01069500 & $\begin{array}{l}\text { Mousam River near West } \\
\text { Kennebunk, ME }\end{array}$ & 99.0 & $\begin{array}{l}1940-84, \\
1997\end{array}$ & $\begin{array}{l}1983 \\
1977\end{array}$ & $\begin{array}{l}5.64 \\
5.82\end{array}$ & $\begin{array}{l}4,020 \\
3,540\end{array}$ & $10 / 22 / 96$ & 5.36 & 3,600 & $\mathrm{Y}$ & 50 \\
\hline 01069700 & $\begin{array}{l}\text { Branch Brook near } \\
\text { Kennebunk, ME }\end{array}$ & 101.7 & $\begin{array}{l}1965-74, \\
1997\end{array}$ & 1997 & 8.08 & 1,020 & $10 / 22 / 96$ & 8.08 & 1,020 & $\mathrm{~N}$ & 40 \\
\hline
\end{tabular}

\footnotetext{
${ }^{1}$ Regulated during flood: N, no; Y, yes.
} 


\section{Maryland}

Unseasonably warm air was drawn northward around the circulation of a deepening storm west of the Appalachian Mountains (fig. 32) on January 18-19, 1996, causing melting of nearly all of a 1- to 2-foot deep snowpack. The melting snow and excessive rains associated with the storm caused widespread, and in some cases, catastrophic flooding and flash flooding on January 19. Flash flooding began at around the same time as the onset of excessive pre-frontal rains. Some areas received between 2 and 4 inches of rain during this period (National Oceanic and Atmospheric Administration, 1996a). Damage throughout Maryland was over \$30 million (National Oceanic and Atmospheric Administration, 1996b). Record floods occurred on Cattail Creek, Monacacy River, Morgan Run, Savage River, Wills Creek, and Youghiogheny River (table 21). The Potomac River was the highest since the Hurricane Agnes flood of 1972.

Torrential convective rainfall moved across north-central Maryland during the early morning hours of June 19, 1996, producing widespread and in some cases catastrophic flooding across the area. Damage estimates were \$7 million (National Oceanic and Atmospheric Administration, 1996b). Rainfall of more than 5.5 inches fell in Emmitsburg, with as much as 13 inches in nearby Gettysburg, Pennsylvania, during the latenight hours. The Monocacy River at Bridgeport (streamgage 01639000, table 21) had the largest flow since 1933 and the largest flood of record. The worst damage was in Emmitsburg, where numerous residences, businesses, and farms were flooded. There was one fatality approximately 3 miles southwest of Emmitsburg, where a woman left her van along Little Owens Creek and was swept away by the floodwaters.

The track of Tropical Storm Fran from south-central through western Virginia on September 6, 1996, allowed gusty southeast winds at and just above the surface to channel water up the Chesapeake Bay and its main tributaries. This became a small-scale storm surge, containing 6-foot waves and tides that ran in some places nearly 6 feet above normal. Torrential rains associated with Tropical Storm Fran caused the rapid onset of river flooding along the headwaters of the Potomac River late on September 6, spreading gradually southeast throughout the entire basin by early September 10. Damage was estimated to be more than \$14 million in the State of Maryland (National Oceanic and Atmospheric Administration, 1996b).

\section{References}

National Oceanic and Atmospheric Administration (NOAA), 1996a, Climatological data (by State): Asheville, North Carolina, National Climatic Data Center, various months.

National Oceanic and Atmospheric Administration (NOAA), 1996b, Storm data (by State): Asheville, North Carolina, National Climatic Data Center, various months.

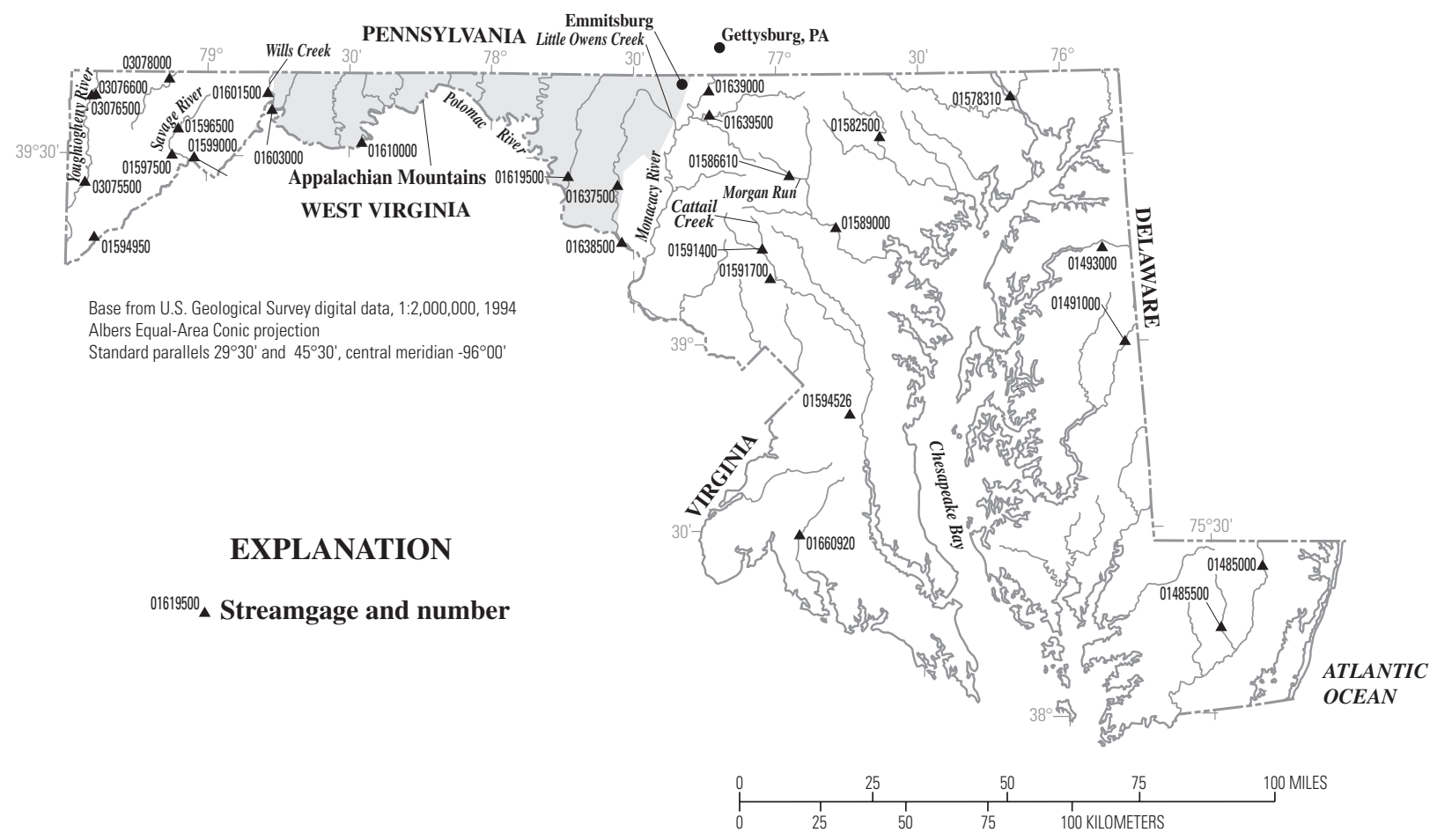

Figure 32. Location of streamgages with significant floods during 1994-98 water years for Maryland. 
$\left[\mathrm{mi}^{2}\right.$, square miles; $\mathrm{ft}$, feet above an arbitrary datum; $\mathrm{ft}$ /s, cubic feet per second; --, not determined or not applicable. Source: Recurrence intervals calculated from U.S. Geological Survey data. Other data from U.S. Geological Survey reports or databases]

\begin{tabular}{|c|c|c|c|c|c|c|c|c|c|c|c|}
\hline \multirow{2}{*}{$\begin{array}{l}\text { Streamgage } \\
\text { number } \\
\text { (fig. 32) }\end{array}$} & \multirow[b]{2}{*}{ Streamgage name } & \multirow{2}{*}{$\begin{array}{c}\text { Total } \\
\text { drainage } \\
\left(\mathrm{mi}^{2}\right)\end{array}$} & \multicolumn{4}{|c|}{$\begin{array}{c}\text { Maximum stage and discharge for period of record } \\
\text { through } 1998 \text { water year }\end{array}$} & \multicolumn{5}{|c|}{ Significant floods $1994-98$ water years } \\
\hline & & & $\begin{array}{l}\text { Period of } \\
\text { record } \\
\text { (water } \\
\text { years) }\end{array}$ & Water year & $\begin{array}{l}\text { Stage } \\
(\mathrm{ft})\end{array}$ & $\begin{array}{l}\text { Discharge } \\
\left(\mathrm{ft}^{3} / \mathrm{s}\right)\end{array}$ & $\begin{array}{c}\text { Date } \\
\text { (month/ } \\
\text { day/ } \\
\text { year) }\end{array}$ & $\begin{array}{l}\text { Stage } \\
(\mathrm{ft})\end{array}$ & $\begin{array}{l}\text { Discharge } \\
\left(\mathrm{ft}^{3} / \mathrm{s}\right)\end{array}$ & $\begin{array}{l}\text { Regulated } \\
\text { during } \\
\text { flood }^{1}\end{array}$ & $\begin{array}{c}\text { Recurrence } \\
\text { interval } \\
\text { (years) }\end{array}$ \\
\hline 01485000 & $\begin{array}{l}\text { Pocomoke River near Willards, } \\
\text { MD }\end{array}$ & 60.5 & $1950-98$ & 1989 & 15.41 & 2,820 & $1 / 29 / 98$ & 13.90 & 1,970 & $\mathrm{~N}$ & $30-35$ \\
\hline 01485500 & $\begin{array}{l}\text { Nassawango Creek near Snow Hill, } \\
\text { MD }\end{array}$ & 44.9 & 1950-98 & $\begin{array}{l}1998 \\
1989\end{array}$ & $\begin{array}{l}8.13 \\
9.07\end{array}$ & $\begin{array}{l}2,300 \\
3,930\end{array}$ & $1 / 29 / 98$ & 8.13 & 2,300 & $\mathrm{~N}$ & 75 \\
\hline 01491000 & $\begin{array}{l}\text { Choptank River near Greensboro, } \\
\text { MD }\end{array}$ & 113 & $1948-98$ & 1967 & 14.47 & 6,970 & $12 / 14 / 96$ & 12.52 & 5,120 & $\mathrm{~N}$ & $15-20$ \\
\hline 01493000 & $\begin{array}{l}\text { Unicorn Branch near Millington, } \\
\text { MD }\end{array}$ & 19.7 & $1948-98$ & $\begin{array}{l}1997 \\
1960\end{array}$ & $\begin{array}{l}6.09 \\
7.17\end{array}$ & $\begin{array}{l}1,160 \\
1,060\end{array}$ & $\begin{array}{l}6 / 19 / 96 \\
12 / 14 / 96\end{array}$ & $\begin{array}{l}5.98 \\
6.09\end{array}$ & $\begin{array}{l}1,090 \\
1,160\end{array}$ & $\begin{array}{l}\mathrm{N} \\
\mathrm{N}\end{array}$ & $\begin{array}{l}15-20 \\
15-20\end{array}$ \\
\hline 01578310 & $\begin{array}{l}\text { Susquehanna River at Conowingo, } \\
\text { MD }\end{array}$ & 27,100 & $1968-98$ & 1972 & 36.83 & $1,130,000$ & $1 / 20 / 96$ & 34.18 & 909,000 & $\mathrm{Y}$ & -- \\
\hline 01582500 & Gunpowder Falls at Glencoe, MD & 160 & 1984-98 & 1994 & 13.72 & 5,180 & $3 / 4 / 94$ & 13.72 & 5,180 & $\mathrm{Y}$ & -- \\
\hline 01586610 & Morgan Run near Louisville, MD & 28.0 & $1983-98$ & 1996 & 8.45 & 3,550 & $1 / 19 / 96$ & 8.45 & 3,550 & $\mathrm{~N}$ & $10-20$ \\
\hline 01589000 & Patapsco River at Hollofield, MD & 285 & $\begin{array}{l}1933, \\
1945-91, \\
1994\end{array}$ & 1972 & 31.30 & 80,600 & $4 / 14 / 94$ & 3.99 & 15,700 & $\mathrm{Y}$ & -- \\
\hline 01591400 & Cattail Creek near Glenwood, MD & 22.9 & 1979-98 & $\begin{array}{l}1996 \\
1979\end{array}$ & $\begin{array}{r}8.96 \\
11.69\end{array}$ & $\begin{array}{l}5,210 \\
4,000\end{array}$ & $1 / 19 / 96$ & 8.96 & 5,210 & $\mathrm{~N}$ & $10-25$ \\
\hline 01591700 & $\begin{array}{l}\text { Hawlings River near Sandy Spring, } \\
\text { MD }\end{array}$ & 27.0 & 1979-98 & 1996 & 9.24 & 5,180 & $1 / 19 / 96$ & 9.24 & 5,180 & $\mathrm{~N}$ & $15-25$ \\
\hline 01594526 & $\begin{array}{l}\text { Western Branch at Upper } \\
\text { Marlboro, MD }\end{array}$ & 89.7 & $\begin{array}{l}1986-89 \\
1993-98\end{array}$ & 1996 & 13.20 & 3,630 & $1 / 19 / 96$ & 13.20 & 3,630 & $\mathrm{~N}$ & $5-10$ \\
\hline 01594950 & $\begin{array}{l}\text { McMillan Fork near Fort } \\
\text { Pendleton, MD }\end{array}$ & 2.30 & 1987-98 & 1994 & 7.23 & 340 & 2/9/94 & 7.23 & 340 & $\mathrm{~N}$ & $10-20$ \\
\hline 01596500 & Savage River near Barton, MD & 49.1 & 1949-98 & 1955 & 8.45 & 7,510 & $9 / 6 / 96$ & 7.97 & 6,620 & $\mathrm{~N}$ & 100 \\
\hline 01597500 & $\begin{array}{l}\text { Savage River below Savage River } \\
\text { dam near Bloomington, MD }\end{array}$ & 106 & 1949-98 & $\begin{array}{l}1996 \\
1986\end{array}$ & $\begin{array}{l}7.81 \\
7.81\end{array}$ & $\begin{array}{l}9,190 \\
8,550\end{array}$ & 9/7/96 & 7.81 & 9,190 & $\mathrm{Y}$ & -- \\
\hline 01599000 & Georges Creek at Franklin, MD & 72.4 & $\begin{array}{l}1924 \\
1931-98\end{array}$ & $\begin{array}{l}1936 \\
1996\end{array}$ & $\begin{array}{r}9.60 \\
12.77\end{array}$ & $\begin{array}{l}8,500 \\
6,500\end{array}$ & $9 / 6 / 96$ & 12.77 & 6,500 & $\mathrm{~N}$ & $45-50$ \\
\hline
\end{tabular}


Table 21. Maximum stage and discharge for period of record for streamgages having significant floods during 1994-98 water years in Maryland.-Continued

$\left[\mathrm{mi}^{2}\right.$, square miles; $\mathrm{ft}$, feet above an arbitrary datum; $\mathrm{ft}^{3} / \mathrm{s}$, cubic feet per second; --, not determined or not applicable. Source: Recurrence intervals calculated from U.S. Geological Survey data. Other data from U.S. Geological Survey reports or databases]

\begin{tabular}{|c|c|c|c|c|c|c|c|c|c|c|c|}
\hline \multirow{2}{*}{$\begin{array}{c}\text { Streamgage } \\
\text { number } \\
\text { (fig. 32) }\end{array}$} & \multirow[b]{2}{*}{ Streamgage name } & \multirow{2}{*}{$\begin{array}{c}\text { Total } \\
\text { drainage } \\
\left(\mathrm{mi}^{2}\right)\end{array}$} & \multicolumn{4}{|c|}{$\begin{array}{c}\text { Maximum stage and discharge for period of record } \\
\text { through } 1998 \text { water year }\end{array}$} & \multicolumn{5}{|c|}{ Signific ant floods $1994-98$ water years } \\
\hline & & & $\begin{array}{c}\text { Period of } \\
\text { record } \\
\text { (water } \\
\text { years) }\end{array}$ & Water year & $\begin{array}{l}\text { Stage } \\
(\mathrm{ft})\end{array}$ & $\begin{array}{l}\text { Discharge } \\
\left(\mathrm{ft}^{3} / \mathrm{s}\right)\end{array}$ & $\begin{array}{c}\text { Date } \\
\text { (month/ } \\
\text { day/ } \\
\text { year) }\end{array}$ & $\begin{array}{l}\text { Stage } \\
(\mathrm{ft})\end{array}$ & $\begin{array}{l}\text { Discharge } \\
\left(\mathrm{ft}^{3} / \mathrm{s}\right)\end{array}$ & $\begin{array}{c}\text { Regulated } \\
\text { during } \\
\text { flood }^{1}\end{array}$ & $\begin{array}{c}\text { Recurrence } \\
\text { interval } \\
\text { (years) }\end{array}$ \\
\hline 01601500 & Wills Creek near Cumberland, MD & 247 & $\begin{array}{l}1924, \\
1930-98\end{array}$ & 1996 & 23.11 & 45,900 & $1 / 19 / 96$ & 23.11 & 45,900 & $\mathrm{~N}$ & 200 \\
\hline 01603000 & $\begin{array}{l}\text { North Branch Potomac River near } \\
\text { Cumberland, MD }\end{array}$ & 877 & $\begin{array}{l}1889,1924, \\
1930-98\end{array}$ & $\begin{array}{l}1936 \\
1889\end{array}$ & $\begin{array}{l}29.10 \\
29.20\end{array}$ & $\begin{array}{l}88,200 \\
89,000\end{array}$ & $1 / 19 / 96$ & 25.56 & 59,200 & $\mathrm{Y}$ & -- \\
\hline 01610000 & Potomac River at Paw Paw, WV & 3,129 & $\begin{array}{l}\text { 1877, 1889, } \\
1924,1928, \\
1936, \\
1939-98\end{array}$ & 1936 & 54.00 & 240,000 & 9/7/96 & 43.45 & 140,000 & $\mathrm{~N}$ & $30-35$ \\
\hline 01619500 & $\begin{array}{l}\text { Antietam Creek near Sharpsburg, } \\
\text { MD }\end{array}$ & 281 & $1928-98$ & 1956 & 16.73 & 12,600 & $1 / 19 / 96$ & 13.71 & 8,960 & $\mathrm{~N}$ & $25-30$ \\
\hline 01637500 & $\begin{array}{l}\text { Catoctin Creek near Middletown, } \\
\text { MD }\end{array}$ & 66.9 & $1948-98$ & 1977 & 14.13 & 12,000 & $1 / 19 / 96$ & 11.72 & 8,560 & $\mathrm{~N}$ & $20-25$ \\
\hline 01638500 & $\begin{array}{l}\text { Potomac River at Point of Rocks, } \\
\text { MD }\end{array}$ & 9,651 & $\begin{array}{l}1889, \\
1895-1998\end{array}$ & 1936 & 41.03 & 480,000 & $1 / 21 / 96$ & 36.34 & 310,000 & $\mathrm{~N}$ & 40 \\
\hline 01639000 & $\begin{array}{l}\text { Monocacy River at Bridgeport, } \\
\text { MD }\end{array}$ & 173 & $\begin{array}{l}1933 \\
1942-98\end{array}$ & 1996 & 25.42 & 24,400 & $6 / 19 / 96$ & 25.42 & 24,400 & $\mathrm{~N}$ & $95-100$ \\
\hline 01639500 & Big Pipe Creek at Bruceville, MD & 102 & 1948-98 & 1975 & 18.98 & 28,000 & $1 / 19 / 96$ & 13.65 & 10,100 & $\mathrm{~N}$ & $20-25$ \\
\hline 01660920 & $\begin{array}{l}\text { Zekiah Swamp Run near } \\
\text { Newtown, MD }\end{array}$ & 79.9 & 1984-98 & $\begin{array}{l}1994 \\
1986\end{array}$ & $\begin{array}{l}5.26 \\
5.74\end{array}$ & $\begin{array}{r}3,380 \\
982\end{array}$ & $3 / 29 / 94$ & 5.26 & 3,380 & $\mathrm{~N}$ & $10-25$ \\
\hline 03075500 & $\begin{array}{l}\text { Youghiogheny River near } \\
\text { Oakland, MD }\end{array}$ & 134 & $\begin{array}{l}1936, \\
1942-98\end{array}$ & $\begin{array}{l}1996 \\
1936\end{array}$ & $\begin{array}{l}13.06 \\
15.30\end{array}$ & 14,100 & $1 / 19 / 96$ & 13.06 & 14,100 & $\mathrm{~N}$ & $50-100$ \\
\hline 03076500 & $\begin{array}{l}\text { Youghiogheny River at } \\
\text { Friendsville, MD }\end{array}$ & 295 & $\begin{array}{l}\text { 1899-1905, } \\
\text { 1923-31, } \\
1940-98\end{array}$ & $\begin{array}{l}1996 \\
1924\end{array}$ & $\begin{array}{r}9.54 \\
14.20\end{array}$ & $\begin{array}{l}16,100 \\
15,600\end{array}$ & $\begin{array}{l}2 / 9 / 94 \\
1 / 19 / 96\end{array}$ & $\begin{array}{l}8.25 \\
9.54\end{array}$ & $\begin{array}{l}12,000 \\
16,100\end{array}$ & $\begin{array}{l}\mathrm{Y} \\
\mathrm{Y}\end{array}$ & $\begin{array}{r}15-20 \\
--\end{array}$ \\
\hline 03076600 & Bear Creek at Friendsville, MD & 48.9 & $1965-98$ & 1971 & 9.60 & 4,650 & $1 / 19 / 96$ & 8.82 & 4,310 & $\mathrm{~N}$ & 50 \\
\hline 03078000 & $\begin{array}{l}\text { Casselman River at Grantsville, } \\
\text { MD }\end{array}$ & 62.5 & 1948-98 & 1955 & 10.70 & 8,400 & $1 / 19 / 96$ & 9.15 & 6,410 & $\mathrm{~N}$ & $50-100$ \\
\hline
\end{tabular}

\footnotetext{
${ }^{1}$ Regulated during flood: N, no; Y, yes.
} 


\section{Massachusetts}

On June 13, 1996, thunderstorms with torrential downpours produced a flash flood that resulted in the worst flooding on the Sawmill River in 100 years of records (fig. 33). The Massachusetts Emergency Management Agency estimated damage from this flood at close to $\$ 2$ million (National Oceanic and Atmospheric Administration, 1996b). Local residents who had lived in the area for many years said this was the worst flooding they had witnessed since the Great New England Hurricane of 1938.

An extensive, slow-moving surface low-pressure system became positioned along the mid-Atlantic Coast during the evening of October 19, 1996. Record-breaking rainfall occurred in northeastern Massachusetts where intense rain bands remained stationary for many hours. Rainfall totals of 8 inches were widespread with this storm (National Oceanic and Atmospheric Administration, 1996a) and resulted in widespread small stream and tributary flooding. A maximum storm total of 13.03 inches was recorded at Newburyport, exceeding the 100-year, 2-day precipitation (National Oceanic and Atmospheric Administration, 1996a). There was widespread severe urban flooding, especially in downtown Boston and from the Greater Boston Metropolitan Area northward as rainfall of historic proportions occurred there. Damage from the excessive rain was in the tens of millions of dollars (National Oceanic and Atmospheric Administration, 1996b). The Shawsheen and Parker Rivers set new flood-of-record discharges (table 22).

On June 12-14, 1998, a very slow-moving storm system moved through southeastern New England. The combination of its slow movement and the presence of tropical moisture across the region caused the storm to produce rainfall of 6 to 12 inches (National Oceanic and Atmospheric Administration, 1998a) over much of eastern Massachusetts, resulting in widespread urban, small stream, and river flooding. Damage was estimated at \$13 million (National Oceanic and Atmospheric Administration, 1998b). Record floods occurred on the Threemile River at North Dighton (streamgage 01109060, table 22).

\section{References}

National Oceanic and Atmospheric Administration (NOAA), 1996a-98a, Climatological data (by State): Asheville, North Carolina, National Climatic Data Center, various months.

National Oceanic and Atmospheric Administration (NOAA), 1996b-98b, Storm data (by State): Asheville, North Carolina, National Climatic Data Center, various months.

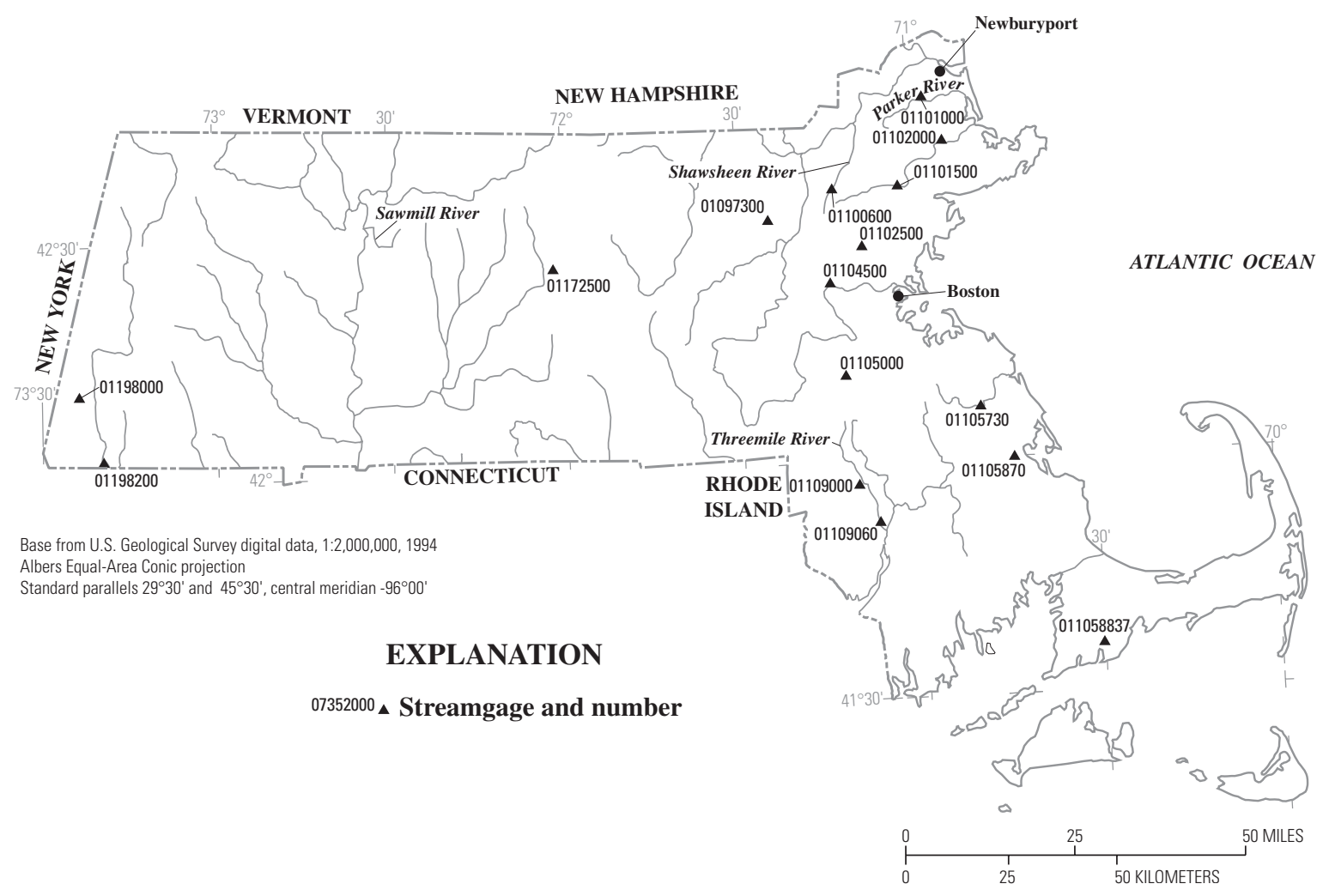

Figure 33. Location of streamgages with significant floods during 1994-98 water years for Massachusetts. 
Table 22. Maximum stage and discharge for period of record for streamgages having significant floods during 1994-98 water years in Massachusetts.

$\left[\mathrm{mi}^{2}\right.$, square miles; $\mathrm{ft}$, feet above an arbitrary datum; $\mathrm{ft}^{3} / \mathrm{s}$, cubic feet per second; --, not determined or not applicable. Source: Recurrence intervals calculated from U.S. Geological Survey data. Other data from U.S. Geological Survey reports or databases]

\begin{tabular}{|c|c|c|c|c|c|c|c|c|c|c|c|}
\hline \multirow[b]{2}{*}{$\begin{array}{c}\text { Streamgage } \\
\text { number } \\
\text { (fig. 33) }\end{array}$} & \multirow[b]{2}{*}{ Streamgage name } & \multirow[b]{2}{*}{$\begin{array}{c}\text { Total } \\
\text { drainage } \\
\left(\mathrm{mi}^{2}\right)\end{array}$} & \multicolumn{4}{|c|}{$\begin{array}{c}\text { Maximum stage and discharge for period of record } \\
\text { through } 1998 \text { water year }\end{array}$} & \multicolumn{5}{|c|}{ Significant floods $1994-98$ water years } \\
\hline & & & $\begin{array}{c}\text { Period of } \\
\text { record } \\
\text { (water } \\
\text { years) }\end{array}$ & Water year & $\begin{array}{l}\text { Stage } \\
(\mathrm{ft})\end{array}$ & $\begin{array}{l}\text { Discharge } \\
\left(\mathrm{ft}^{3} / \mathrm{s}\right)\end{array}$ & $\begin{array}{c}\text { Date } \\
\text { (month/ } \\
\text { day/ } \\
\text { year) }\end{array}$ & $\begin{array}{l}\text { Stage } \\
(\mathrm{ft})\end{array}$ & $\begin{array}{l}\text { Discharge } \\
\left(\mathrm{ft}^{3} / \mathrm{s}\right)\end{array}$ & $\begin{array}{c}\text { Regulated } \\
\text { during } \\
\text { flood }^{1}\end{array}$ & $\begin{array}{c}\text { Recurrence } \\
\text { interval } \\
\text { (years) }\end{array}$ \\
\hline 01097300 & Nashoba Brook near Acton, MA & 12.8 & 1964-98 & $\begin{array}{l}1979 \\
1998\end{array}$ & $\begin{array}{l}5.57 \\
6.89\end{array}$ & $\begin{array}{l}679 \\
293\end{array}$ & $10 / 21 / 96$ & 5.55 & 665 & $\mathrm{~N}$ & 30 \\
\hline 01100600 & $\begin{array}{l}\text { Shawsheen River near } \\
\text { Wilmington, MA }\end{array}$ & 36.5 & 1964-98 & 1997 & 10.49 & 1,850 & $10 / 22 / 96$ & 10.49 & 1,850 & $\mathrm{~N}$ & 70 \\
\hline 01101000 & Parker River at Byfield, MA & 21.3 & $1946-98$ & 1997 & 7.82 & 883 & $10 / 22 / 96$ & 7.82 & 883 & $\mathrm{~N}$ & 150 \\
\hline 01101500 & $\begin{array}{l}\text { Ipswich River at South Middleton, } \\
\text { MA }\end{array}$ & 44.5 & $1938-98$ & $\begin{array}{l}1987 \\
1997\end{array}$ & $\begin{array}{l}7.51 \\
7.88\end{array}$ & $\begin{array}{r}1,010 \\
896\end{array}$ & $10 / 21 / 96$ & 7.88 & 896 & $\mathrm{~N}$ & 30 \\
\hline 01102000 & Ipswich River near Ipswich, MA & 125 & $1931-98$ & 1987 & 9.43 & 3,550 & $10 / 23 / 96$ & 8.98 & 3,120 & $\mathrm{~N}$ & 60 \\
\hline 01102500 & Aberjona River at Winchester, MA & 24.7 & 1940-98 & $\begin{array}{l}1979 \\
1997\end{array}$ & $\begin{array}{l}15.46 \\
16.78\end{array}$ & $\begin{array}{l}1,330 \\
1,150\end{array}$ & $\begin{array}{l}10 / 21 / 96 \\
6 / 14 / 98\end{array}$ & $\begin{array}{l}16.78 \\
15.22\end{array}$ & $\begin{array}{l}1,150 \\
1,070\end{array}$ & $\begin{array}{l}\mathrm{N} \\
\mathrm{N}\end{array}$ & $\begin{array}{l}50 \\
50\end{array}$ \\
\hline 01104500 & Charles River at Waltham, MA & 251 & 1932-98 & 1976 & 6.54 & 4,150 & $10 / 21 / 96$ & 6.05 & 2,990 & $\mathrm{Y}$ & 20 \\
\hline 01105000 & Neponset River at Norwood, MA & 34.7 & $\begin{array}{l}1938 \\
1940-98\end{array}$ & 1955 & 14.65 & 1,490 & $6 / 14 / 98$ & 10.89 & 1,100 & $\mathrm{Y}$ & 50 \\
\hline 01105730 & Indian Head River at Hanover, MA & 30.3 & 1967-98 & 1968 & 7.13 & 1,390 & $10 / 21 / 96$ & 6.76 & 1,290 & $\mathrm{~N}$ & 50 \\
\hline 01105870 & Jones River at Kingston, MA & 19.8 & 1967-98 & $\begin{array}{l}1968 \\
1997\end{array}$ & $\begin{array}{l}4.60 \\
5.18\end{array}$ & $\begin{array}{l}575 \\
343\end{array}$ & $10 / 21 / 96$ & 5.18 & 343 & $\mathrm{Y}$ & 10 \\
\hline 011058837 & $\begin{array}{l}\text { Quashnet River at Waquoit } \\
\text { Village, MA }\end{array}$ & 2.58 & 1989-98 & 1998 & 3.09 & 42 & $7 / 1 / 98$ & 3.09 & 42 & $\mathrm{Y}$ & 10 \\
\hline 01109000 & Wading River near Norton, MA & 43.3 & 1926-98 & 1968 & 11.47 & 1,460 & $6 / 14 / 98$ & 11.47 & 1,220 & $\mathrm{~N}$ & 50 \\
\hline 01109060 & $\begin{array}{l}\text { Threemile River at North Dighton, } \\
\text { MA }\end{array}$ & 84.3 & $1967-98$ & 1998 & 8.89 & 2,870 & $6 / 16 / 98$ & 8.89 & 2,870 & $\mathrm{~N}$ & 50 \\
\hline 01172500 & Ware River near Barre, MA & 55.1 & $\begin{array}{l}1947-94, \\
1996-98\end{array}$ & 1956 & 6.31 & 1,890 & $1 / 30 / 96$ & 5.34 & 1,350 & $\mathrm{Y}$ & $15-20$ \\
\hline 01198000 & $\begin{array}{l}\text { Green River near Great Barrington, } \\
\text { MA }\end{array}$ & 51.0 & $\begin{array}{l}1952-71 \\
1994-96\end{array}$ & 1996 & 9.84 & 6,740 & 7/13/96 & 9.84 & 6,740 & $\mathrm{~N}$ & $>200$ \\
\hline 01198200 & $\begin{array}{l}\text { Konkapot River at Ashley Falls, } \\
\text { MA }\end{array}$ & 61.1 & $\begin{array}{l}1963-71 \\
1994-96\end{array}$ & 1996 & 6.29 & 1,280 & $1 / 28 / 96$ & 6.29 & 1,280 & $\mathrm{~N}$ & 20 \\
\hline
\end{tabular}

${ }^{1}$ Regulated during flood: N, no; Y, yes. 


\section{Michigan}

Repeated, highly localized, excessive rainfall near Juniata, Michigan (fig. 34), on July 8, 1994, resulted in flooding along the Cass River. Damage to roads, bridges, buildings, and so forth was estimated to be approximately $\$ 1.5$ million (National Oceanic and Atmospheric Administration, 1994b).

Melting of a deep snowpack during April 19-26, 1996, triggered flooding on the Escanaba and Ford Rivers and their tributaries in the central part of the Upper Peninsula. Damage was estimated at nearly \$1.6 million (National Oceanic and Atmospheric Administration, 1996b).

On May 10, 1996, 24-hour rainfall totals of 8 to 9 inches southeast of Benton Harbor, Michigan (National Oceanic and Atmospheric Administration, 1996a), caused over \$5 million in damage from flooding (National Oceanic and Atmospheric Administration, 1996b). One week later on May 18, excessive rains on Isle Royale caused the greatest discharge in 33 years of record on Washington Creek at Windigo (streamgage

04001000, table 23).
Severe thunderstorms developed along a warm front across central lower Michigan during the evenings of June 20 and 21, 1997. The storms moved southeast across the Saginaw River Basin and the Thumb Region and produced excessive rainfall. As a result of this weather, flash flooding caused $\$ 19$ million in property damage (National Oceanic and Atmospheric Administration, 1997b). Flash flooding was widespread throughout the Saginaw River Basin and most of eastern Michigan.

\section{References}

National Oceanic and Atmospheric Administration (NOAA), 1994a-97a, Climatological data (by State): Asheville, North Carolina, National Climatic Data Center, various months.

National Oceanic and Atmospheric Administration (NOAA), 1994b-97b, Storm data (by State): Asheville, North Carolina, National Climatic Data Center, various months. 

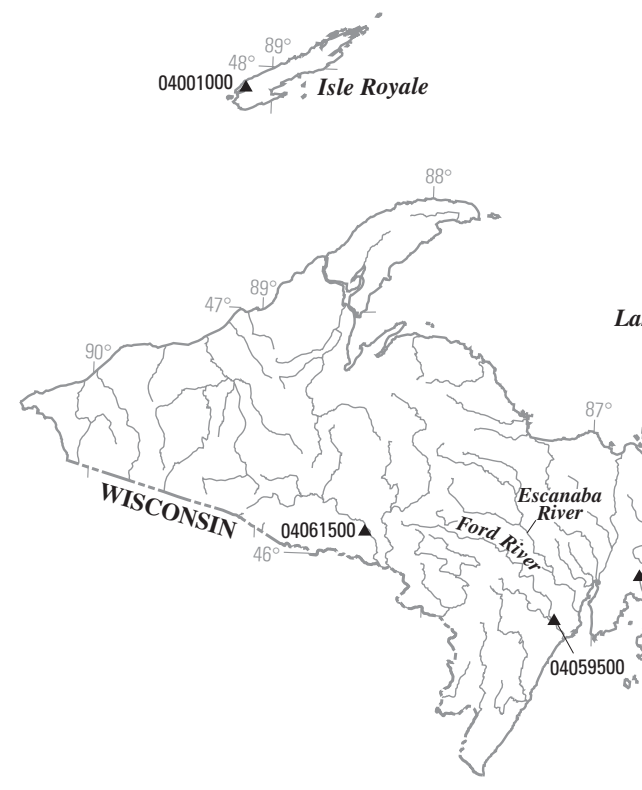

Base from U.S. Geological Survey digital data, 1:2,000,000, 1994 Albers Equal-Area Conic projection

Standard parallels $29^{\circ} 30^{\prime}$ and $45^{\circ} 30^{\prime}$, central meridian $-96^{\circ} 00^{\prime}$

\section{EXPLANATION}

${ }_{04102500}^{\Delta}$ Streamgage and number
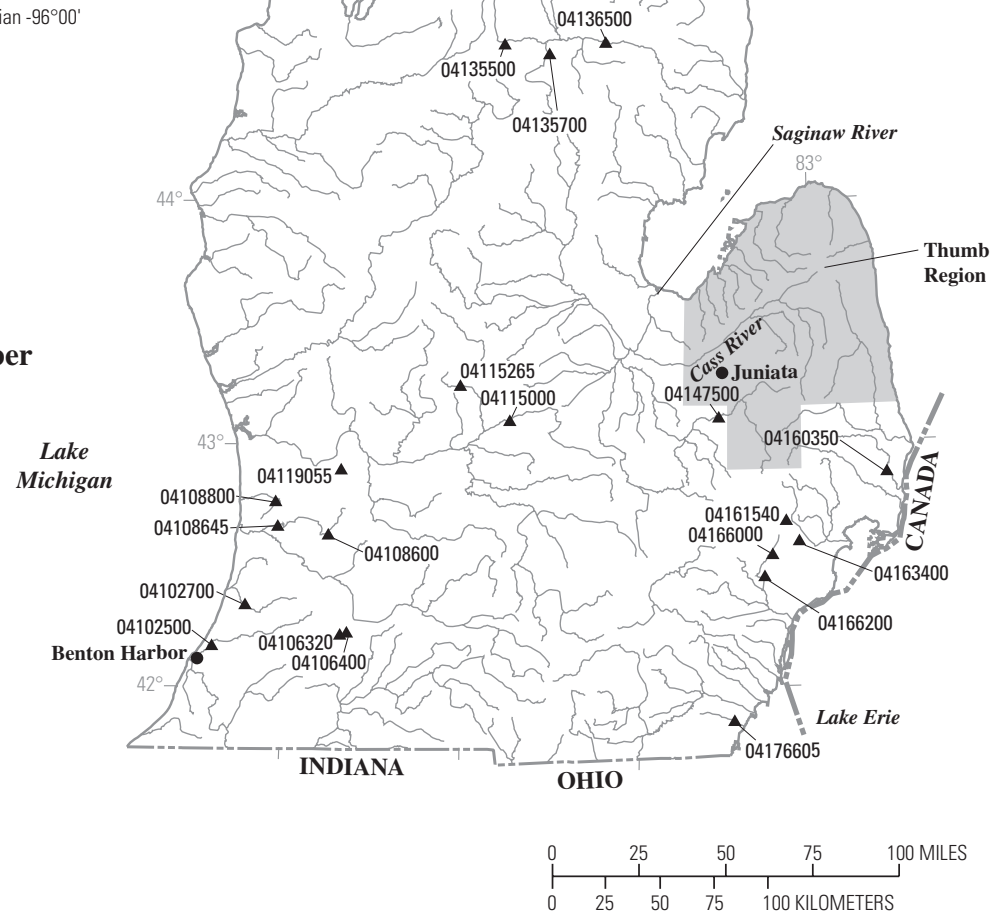

Figure 34. Location of streamgages with significant floods during 1994-98 water years for Michigan. 
$\left[\mathrm{mi}^{2}\right.$, square miles; ft, feet above an arbitrary datum; $\mathrm{ft}^{3} / \mathrm{s}$, cubic feet per second; --, not determined or not applicable; >, greater than. Source: Recurrence intervals calculated from U.S. Geological Survey data. Other data from U.S. Geological Survey reports or databases]

\begin{tabular}{|c|c|c|c|c|c|c|c|c|c|c|c|}
\hline \multirow{2}{*}{$\begin{array}{c}\text { Streamgage } \\
\text { number } \\
\text { (fig. 34) }\end{array}$} & \multirow[b]{2}{*}{ Streamgage name } & \multirow{2}{*}{$\begin{array}{c}\text { Total } \\
\text { drainage } \\
\left(\mathrm{mi}^{2}\right)\end{array}$} & \multicolumn{4}{|c|}{$\begin{array}{c}\text { Maximum stage and discharge for period of record } \\
\text { through } 1998 \text { water year }\end{array}$} & \multicolumn{5}{|c|}{ Significant floods 1994-98 water years } \\
\hline & & & $\begin{array}{c}\text { Period of } \\
\text { record } \\
\text { (water } \\
\text { years) }\end{array}$ & Water year & $\begin{array}{l}\text { Stage } \\
(\mathrm{ft})\end{array}$ & $\begin{array}{l}\text { Discharge } \\
\left(\mathrm{ft}^{3} / \mathrm{s}\right)\end{array}$ & $\begin{array}{c}\text { Date } \\
\text { (month/ } \\
\text { day/ } \\
\text { year) }\end{array}$ & $\begin{array}{l}\text { Stage } \\
(\mathrm{ft})\end{array}$ & $\begin{array}{l}\text { Discharge } \\
\left(\mathrm{ft}^{3} / \mathrm{s}\right)\end{array}$ & $\begin{array}{c}\text { Regulated } \\
\text { during } \\
\text { flood }^{1}\end{array}$ & $\begin{array}{c}\text { Recurrence } \\
\text { interval } \\
\text { (years) }\end{array}$ \\
\hline 04001000 & Washington Creek at Windigo, MI & 13.2 & $1965-98$ & 1996 & 8.17 & 657 & $5 / 18 / 96$ & 8.17 & 657 & $\mathrm{~N}$ & $25-50$ \\
\hline 04057510 & $\begin{array}{l}\text { Sturgeon River near Nahma } \\
\text { Junction, MI }\end{array}$ & 183 & 1967-98 & 1985 & 11.50 & 2,120 & $4 / 26 / 96$ & 11.11 & 1,900 & $\mathrm{~N}$ & $25-50$ \\
\hline 04059500 & Ford River near Hyde, MI & 450 & 1955-98 & 1960 & 8.27 & 7,590 & $4 / 26 / 96$ & 7.45 & 5,630 & $\mathrm{~N}$ & 25 \\
\hline 04061500 & Paint River at Crystal Falls, MI & 597 & $1945-98$ & 1960 & 9.82 & 10,900 & $4 / 26 / 96$ & 7.89 & 7,300 & $\mathrm{~N}$ & 25 \\
\hline 04102500 & Paw Paw River at Riverside, MI & 390 & $1952-98$ & 1987 & 10.90 & 3,580 & $2 / 24 / 97$ & 10.66 & 3,110 & $\mathrm{~N}$ & $25-50$ \\
\hline 04102700 & $\begin{array}{l}\text { South Branch Black River near } \\
\text { Bangor, MI }\end{array}$ & 83.6 & 1967-98 & 1997 & 14.90 & 2,390 & $2 / 21 / 97$ & 14.90 & 2,390 & $\mathrm{~N}$ & $25-50$ \\
\hline 04106320 & $\begin{array}{l}\text { West Fork Portage Creek near } \\
\text { Oshtemo, MI }\end{array}$ & 13.0 & 1973-98 & 1993 & 2.47 & 36 & $10 / 28 / 94$ & 2.42 & 36 & $\mathrm{~N}$ & $25-50$ \\
\hline 04106400 & $\begin{array}{l}\text { West Fork Portage Creek at } \\
\text { Kalamazoo, MI }\end{array}$ & 18.7 & 1960-98 & 1997 & 3.33 & 46 & $6 / 21 / 97$ & 3.33 & 46 & $\mathrm{~N}$ & $10-25$ \\
\hline 04108600 & Rabbit River near Hopkins, MI & 71.4 & $1966-98$ & 1997 & 11.11 & 3,740 & $6 / 21 / 97$ & 11.11 & 3,740 & $\mathrm{~N}$ & $50-100$ \\
\hline 04108645 & Rabbit River at Hamilton, MI & 274 & 1979-98 & 1997 & 21.60 & 12,000 & $6 / 21 / 97$ & 21.60 & 12,000 & $\mathrm{~N}$ & $50-100$ \\
\hline 04108800 & Macatawa River near Zeeland, MI & 65.8 & 1961-98 & 1997 & 16.72 & 8,810 & $6 / 21 / 97$ & 16.72 & 8,810 & $\mathrm{~N}$ & $50-100$ \\
\hline 04115000 & Maple River at Maple Rapids, MI & 434 & $1945-98$ & 1986 & 12.33 & 8,770 & $7 / 8 / 94$ & 10.27 & 5,610 & $\mathrm{~N}$ & 25 \\
\hline 04115265 & Fish Creek near Crystal, MI & 39.7 & $1988-98$ & 1990 & 5.53 & 558 & $2 / 21 / 97$ & 4.99 & 320 & $\mathrm{~N}$ & $10-20$ \\
\hline 04119055 & Plaster Creek at Grand Rapids, MI & 46.6 & 1974-98 & 1997 & 13.43 & 2,300 & $2 / 22 / 97$ & 13.43 & 2,300 & $\mathrm{~N}$ & $25-50$ \\
\hline 04130500 & Black River near Tower, MI & 311 & $1943-98$ & 1960 & 7.13 & 2,340 & $4 / 1 / 98$ & 6.41 & 1,860 & $\mathrm{~N}$ & 25 \\
\hline 04135500 & Au Sable River at Grayling, MI & 110 & $1943-94$ & 1994 & 3.25 & 322 & $7 / 6 / 94$ & 3.25 & 322 & $\mathrm{~N}$ & $>100$ \\
\hline 04135700 & $\begin{array}{l}\text { South Branch Au Sable River near } \\
\text { Luzerne, MI }\end{array}$ & 401 & $\begin{array}{l}1967-89 \\
1991-98\end{array}$ & 1976 & 7.30 & 1,120 & $4 / 7 / 97$ & 6.87 & 940 & $\mathrm{~N}$ & $10-20$ \\
\hline 04136500 & Au Sable River at Mio, MI & 1,361 & 1953-98 & 1998 & 6.37 & 4,380 & $4 / 1 / 98$ & 6.37 & 4,380 & $\mathrm{Y}$ & $25-50$ \\
\hline
\end{tabular}


Table 23. Maximum stage and discharge for period of record for streamgages having significant floods during 1994-98 water years in Michigan.-Continued

$\left[\mathrm{mi}^{2}\right.$, square miles; ft, feet above an arbitrary datum; $\mathrm{ft}^{3} / \mathrm{s}$, cubic feet per second; --, not determined or not applicable; >, greater than. Source: Recurrence intervals calculated from U.S. Geological Survey data. Other data from U.S. Geological Survey reports or databases]

\begin{tabular}{|c|c|c|c|c|c|c|c|c|c|c|c|}
\hline \multirow[b]{2}{*}{$\begin{array}{c}\text { Streamgage } \\
\text { number } \\
\text { (fig. 34) }\end{array}$} & \multirow[b]{2}{*}{ Streamgage name } & \multirow{2}{*}{$\begin{array}{c}\text { Total } \\
\text { drainage } \\
\left(\mathrm{mi}^{2}\right)\end{array}$} & \multicolumn{4}{|c|}{$\begin{array}{c}\text { Maximum stage and discharge for period of record } \\
\text { through } 1998 \text { water year }\end{array}$} & \multicolumn{5}{|c|}{ Significant floods $1994-98$ water years } \\
\hline & & & $\begin{array}{l}\text { Period of } \\
\text { record } \\
\text { (water } \\
\text { years) }\end{array}$ & Water year & $\begin{array}{l}\text { Stage } \\
(\mathrm{ft})\end{array}$ & $\begin{array}{c}\text { Discharge } \\
\left(\mathrm{ft}^{3} / \mathrm{s}\right)\end{array}$ & $\begin{array}{c}\text { Date } \\
\text { (month/ } \\
\text { day/ } \\
\text { year) }\end{array}$ & $\begin{array}{l}\text { Stage } \\
(\mathrm{ft})\end{array}$ & $\begin{array}{c}\text { Discharge } \\
\left(\mathrm{ft}^{3} / \mathrm{s}\right)\end{array}$ & $\begin{array}{c}\text { Regulated } \\
\text { during } \\
\text { flood }^{1}\end{array}$ & $\begin{array}{c}\text { Recurrence } \\
\text { interval } \\
\text { (years) }\end{array}$ \\
\hline 04147500 & Flint River near Otisville, MI & 530 & $\begin{array}{l}1953-89, \\
1991-98\end{array}$ & 1996 & 15.73 & 7,470 & $6 / 24 / 96$ & 15.73 & 7,470 & $\mathrm{~N}$ & $25-50$ \\
\hline 04160350 & Pine River near Rattle Run, MI & 135 & 1974-98 & 1996 & 24.24 & 5,730 & $6 / 22 / 96$ & 24.24 & 5,730 & $\mathrm{~N}$ & $25-50$ \\
\hline 04161540 & Paint Creek at Rochester, MI & 70.9 & 1960-98 & $\begin{array}{l}1968 \\
1965\end{array}$ & $\begin{array}{l}5.22 \\
5.95\end{array}$ & $\begin{array}{l}918 \\
880\end{array}$ & 6/19/96 & -- & 890 & $\mathrm{~N}$ & $10-25$ \\
\hline 04163400 & Plum Brook at Utica, MI & 16.5 & 1966-98 & 1996 & 10.62 & 1,290 & $6 / 18 / 96$ & 10.62 & 1,290 & $\mathrm{~N}$ & $25-50$ \\
\hline 04166000 & River Rouge at Birmingham, MI & 33.3 & $1951-98$ & 1968 & 8.70 & 1,390 & $6 / 18 / 96$ & 7.36 & 1,070 & $\mathrm{~N}$ & 25 \\
\hline 04166200 & Evans Ditch at Southfield, MI & 9.49 & 1959-98 & 1982 & 15.03 & 1,200 & $8 / 6 / 98$ & 12.83 & 962 & $\mathrm{~N}$ & $15-25$ \\
\hline 04176605 & Otter Creek at La Salle, MI & 51.0 & 1988-98 & 1997 & 11.60 & 3,010 & $6 / 2 / 97$ & 11.60 & 3,010 & $\mathrm{~N}$ & $25-50$ \\
\hline
\end{tabular}

${ }^{1}$ Regulated during flood: N, no; Y, yes. 


\section{Minnesota}

Excessive rainfall of between 5 and 15 inches in the region north of Montevideo, Minnesota (fig. 35), resulted in major property and crop damage on July 3, 1995 (National Oceanic and Atmospheric Administration, 1995a). The Chippewa River near Milan (streamgage 05304500, table 24) rose 9 feet on July 3 and 4, and crested at 13.48 feet on July 6 . This was the second highest crest ever for the Chippewa River near Milan.

Moderate to severe flooding occurred on the Red River of the North and many of its tributaries in Minnesota during April 1996. Above-normal precipitation was observed over the basin for the 6 months prior to the flood. In addition, deep snow cover, with drifts as much as 12 feet high, lingered into early April (National Oceanic and Atmospheric Administration, 1996b). This combined with a rapid snowmelt to produce a significant spring flood despite below-average precipitation from mid-March through April. Ice jams contributed to the flooding. The flooding was, for many areas, the worst since 1979 and the most devastating on record. Three streamgages had peaks of record (table 24). Eleven counties were declared Federal disaster areas.

The 1997 spring flooding along the upstream reaches of the Minnesota River and Red River of the North broke many existing flood records in Minnesota. The winter of 1996-97 had extraordinary snowfall with most areas having more than 6 feet of snow, which is two to three times greater than normal. Fargo, North Dakota, had 117 inches of snow compared to an average of 39 inches. Several other cities had in excess of 100 inches (National Oceanic and Atmospheric Administration, 1997a). The western Minnesota towns of Crookston and Madison had 3.08 and 3.14 inches of rain, respectively, on this snowpack on April 5-6 and coupled with warm air temperatures resulted in rapid snowmelt in western Minnesota and eastern North Dakota. Temperatures were approximately 10 degrees above normal in the first week of April. However, during the second and third weeks, temperatures were 20 degrees below normal.
Flood workers had to struggle in the middle of a blizzard at times to save property. Twenty-three streamgages in Minnesota experienced their peak of record during the flood of 1997 (table 24).

The Federal Emergency Management Agency's (FEMA's) estimate of public infrastructure damage in Minnesota from the 1997 flood was approximately \$300 million (Federal Emergency Management Agency, 1997). Before the water receded, 58 of Minnesota's 87 counties were declared Federal disaster areas. The American Red Cross reported that the massive floods affected 23,263 families. Total flood damage and associated economic impacts were estimated to be as high as $\$ 2$ billion (Minnesota Department of Natural Resources, 1997).

Excessive rainfall of as much as 9.5 inches on June 17, 1997, resulted in scattered mud slides and widespread street and basement flooding in the Mankato area. Total damage to the area around Mankato exceeded \$20 million (National Oceanic and Atmospheric Administration, 1997b).

\section{References}

Federal Emergency Management Agency, 1997, 1997 record spring floods-upper Minnesota River and Red River of the North in Minnesota: Minnesota Department of Natural Resources, information available on the Web, accessed January 3, 2005, at http://www.dnr.state.mn.us/ climate/floods/1997/index.html

Minnesota Department of Natural Resources, 1997, 1997 record spring floods: Information available on the Web, accessed January 3, 2005, at http://www.dnr.state.mn.us/ fclimate/floods/1997/index.html

National Oceanic and Atmospheric Administration (NOAA), 1995a-97a, Climatological data (by State): Asheville, North Carolina, National Climatic Data Center, various months. National Oceanic and Atmospheric Administration (NOAA), 1995b-97b, Storm data (by State): Asheville, North Carolina, National Climatic Data Center, various months. 


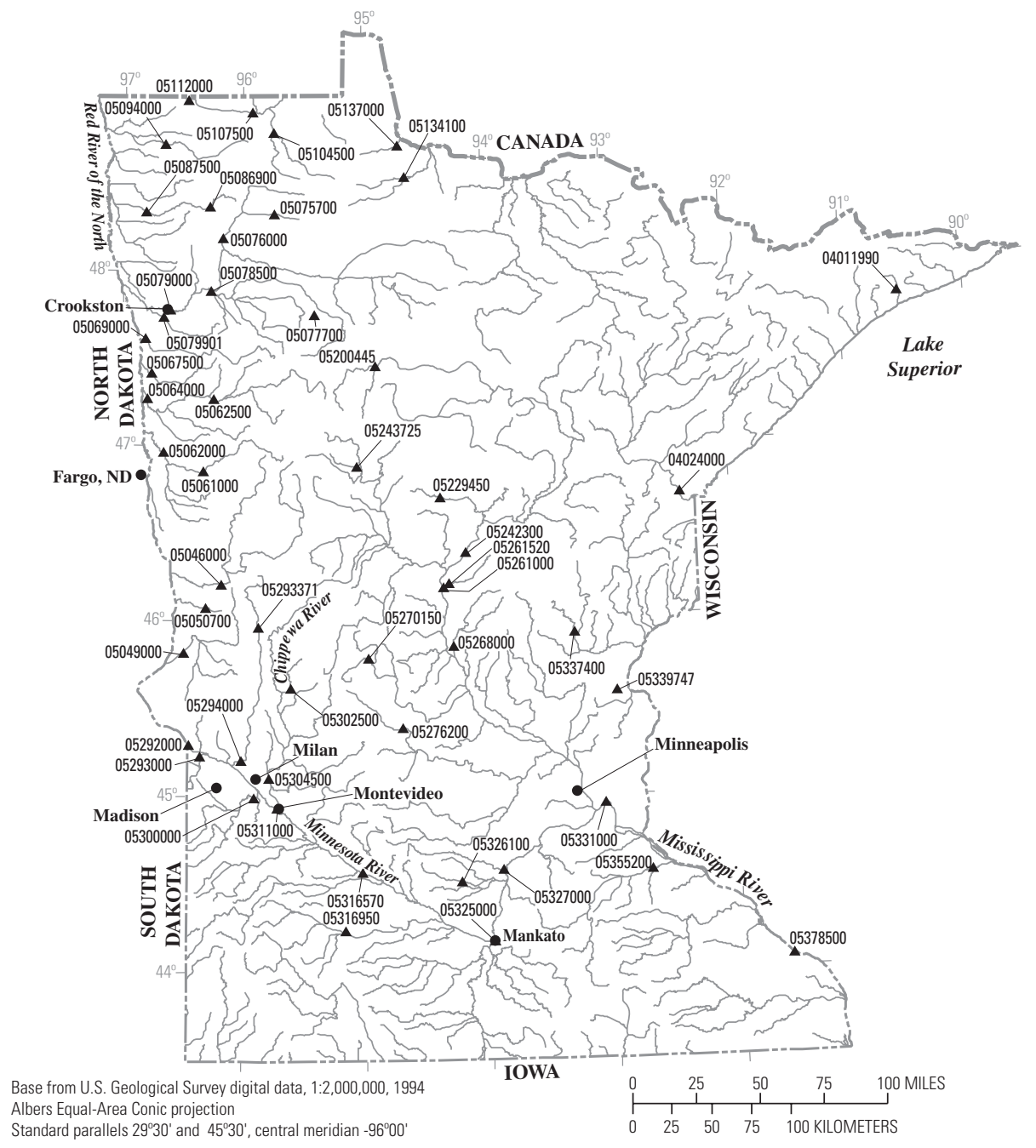

EXPLANATION

$05325000 \_$Streamgage and number

Figure 35. Location of streamgages with significant floods during 1994-98 water years for Minnesota. 
Table 24. Maximum stage and discharge for period of record for streamgages having significant floods during 1994-98 water years in Minnesota.

$\left[\mathrm{mi}^{2}\right.$, square miles; ft, feet above an arbitrary datum; $\mathrm{ft}^{3} / \mathrm{s}$, cubic feet per second; --, not determined or not applicable; $>$, greater than. Source: Recurrence intervals calculated from U.S. Geological Survey data. Other data from U.S. Geological Survey reports or databases]

\begin{tabular}{|c|c|c|c|c|c|c|c|c|c|c|c|}
\hline \multirow{2}{*}{$\begin{array}{c}\text { Streamgage } \\
\text { number } \\
\text { (fig. 35) }\end{array}$} & \multirow[b]{2}{*}{ Streamgage name } & \multirow{2}{*}{$\begin{array}{c}\text { Total } \\
\text { drainage } \\
\left(\mathrm{mi}^{2}\right)\end{array}$} & \multicolumn{4}{|c|}{$\begin{array}{c}\text { Maximum stage and discharge for period of record } \\
\text { through } 1998 \text { water year }\end{array}$} & \multicolumn{5}{|c|}{ Significant floods $1994-98$ water years } \\
\hline & & & $\begin{array}{l}\text { Period of } \\
\text { record } \\
\text { (water } \\
\text { years) }\end{array}$ & Water year & $\begin{array}{l}\text { Stage } \\
\text { (ft) }\end{array}$ & $\begin{array}{l}\text { Discharge } \\
\left(\mathrm{ft}^{3} / \mathrm{s}\right)\end{array}$ & $\begin{array}{c}\text { Date } \\
\text { (month/ } \\
\text { day/ } \\
\text { year) }\end{array}$ & $\begin{array}{l}\text { Stage } \\
\text { (ft) }\end{array}$ & $\begin{array}{l}\text { Discharge } \\
\left(\mathrm{ft}^{3} / \mathrm{s}\right)\end{array}$ & $\begin{array}{l}\text { Regulated } \\
\text { during } \\
\text { flood }^{1}\end{array}$ & $\begin{array}{c}\text { Recurrence } \\
\text { interval } \\
\text { (years) }\end{array}$ \\
\hline 04011990 & $\begin{array}{l}\text { Cascade River at Forest Road } 45 \\
\text { near Grand Marais, MN }\end{array}$ & $\overline{--}$ & $1985-98$ & 1996 & 12.95 & 1,350 & $5 / 19 / 96$ & 12.95 & 1,350 & $\mathrm{~N}$ & $>10$ \\
\hline 04024000 & St. Louis River at Scanlon, MN & 3,430 & 1908-98 & $\begin{array}{l}1950 \\
1979\end{array}$ & $-\overline{-}+93$ & $\begin{array}{l}37,900 \\
34,200\end{array}$ & $4 / 21 / 96$ & -- & 27,600 & $\mathrm{Y}$ & $>10$ \\
\hline 05046000 & $\begin{array}{l}\text { Otter Tail River below Orwell } \\
\text { Dam near Fergus Falls, MN }\end{array}$ & 1,740 & 1931-98 & 1953 & 5.60 & 1,710 & $5 / 22 / 97$ & 4.63 & 1,500 & $\mathrm{Y}$ & $>10$ \\
\hline 05049000 & $\begin{array}{l}\text { Mustinka River above Wheaton, } \\
\text { MN }\end{array}$ & 834 & $\begin{array}{l}1916-17, \\
1919-24, \\
1931-58, \\
1985-98\end{array}$ & 1997 & 23.63 & 8,800 & $4 / 7 / 97$ & 23.63 & 8,800 & $\mathrm{~N}$ & $>25$ \\
\hline 05050700 & Rabbit River near Nashua, MN & 56.1 & 1979-98 & 1997 & 15.76 & 1,640 & $4 / 5 / 97$ & 15.76 & 1,640 & $\mathrm{~N}$ & $>10$ \\
\hline 05061000 & Buffalo River near Hawley, MN & 325 & $\begin{array}{l}1921 \\
1945-98\end{array}$ & $\begin{array}{l}1997 \\
1921\end{array}$ & $\begin{array}{l}10.77 \\
11.30\end{array}$ & $\begin{array}{l}2,360 \\
3,000\end{array}$ & $4 / 6 / 97$ & 10.77 & 2,360 & $\mathrm{~N}$ & $>25$ \\
\hline 05062000 & Buffalo River near Dilworth, MN & 975 & 1931-98 & 1975 & 27.10 & 13,600 & $4 / 6 / 97$ & 27.02 & 8,370 & $\mathrm{~N}$ & $>25$ \\
\hline 05062500 & $\begin{array}{l}\text { Wild Rice River at Twin Valley, } \\
\text { MN }\end{array}$ & 934 & $\begin{array}{l}1909-17 \\
1931-98\end{array}$ & 1997 & 15.91 & 10,000 & $4 / 6 / 97$ & 15.91 & 10,000 & $\mathrm{~N}$ & $>100$ \\
\hline 05064000 & Wild Rice River at Hendrum, MN & 1,560 & 1944-98 & 1997 & 33.85 & 10,600 & $4 / 18 / 97$ & 33.85 & 10,600 & $\mathrm{Y}$ & $>50$ \\
\hline 05067500 & Marsh River near Shelly, MN & 220 & 1944-98 & $\begin{array}{l}1979 \\
1997\end{array}$ & $\begin{array}{l}23.36 \\
25.45\end{array}$ & $\begin{array}{l}4,880 \\
4,300\end{array}$ & 4/18/97 & 25.45 & 4,300 & $\mathrm{Y}$ & $>10$ \\
\hline 05069000 & Sand Hill River at Climax, MN & 420 & 1943-98 & $\begin{array}{l}1965 \\
1997\end{array}$ & $\begin{array}{l}17.81 \\
39.40\end{array}$ & $\begin{array}{l}4,560 \\
4,360\end{array}$ & $\begin{array}{l}4 / 19 / 96 \\
4 / 20 / 97\end{array}$ & $\begin{array}{l}25.17 \\
39.40\end{array}$ & $\begin{array}{l}4,290 \\
4,360\end{array}$ & $\begin{array}{l}\mathrm{N} \\
\mathrm{N}\end{array}$ & $\begin{array}{l}>10 \\
>10\end{array}$ \\
\hline 05075700 & Mud River near Grygla, MN & 170 & 1979-98 & $\begin{array}{l}1996 \\
1997\end{array}$ & $\begin{array}{l}18.57 \\
19.00\end{array}$ & $\begin{array}{l}1,950 \\
1,400\end{array}$ & 4/19/96 & 18.57 & 1,950 & $\mathrm{~N}$ & $>25$ \\
\hline 05076000 & $\begin{array}{l}\text { Thief River near Thief River Falls, } \\
\text { MN }\end{array}$ & 985 & $\begin{array}{l}1909-17, \\
1919-26, \\
1929-98\end{array}$ & 1950 & 17.38 & 5,610 & $4 / 22 / 97$ & 15.20 & 4,120 & $\mathrm{Y}$ & $>10$ \\
\hline 05077700 & Ruffy Brook near Gonvick, MN & 45.2 & $\begin{array}{l}1961-80 \\
1982-98\end{array}$ & $\begin{array}{l}1996 \\
1962\end{array}$ & $\begin{array}{l}5.78 \\
6.70\end{array}$ & $\begin{array}{l}455 \\
364\end{array}$ & 4/19/96 & 5.78 & 455 & $\mathrm{~N}$ & $>10$ \\
\hline 05078500 & $\begin{array}{l}\text { Clearwater River at Red Lake } \\
\text { Falls, MN }\end{array}$ & 1,380 & $\begin{array}{l}\text { 1910-17, } \\
1919, \\
1935-98\end{array}$ & $\begin{array}{l}1979 \\
1995\end{array}$ & $\begin{array}{l}12.38 \\
13.25\end{array}$ & $\begin{array}{r}10,300 \\
3,110\end{array}$ & 4/19/96 & 12.24 & 9,400 & $\mathrm{~N}$ & $>10$ \\
\hline
\end{tabular}


Table 24. Maximum stage and discharge for period of record for streamgages having significant floods during 1994-98 water years in Minnesota.-Continued

$\left[\mathrm{mi}^{2}\right.$, square miles; $\mathrm{ft}$, feet above an arbitrary datum; $\mathrm{ft}^{3} / \mathrm{s}$, cubic feet per second; --, not determined or not applicable; $>$, greater than. Source: Recurrence intervals calculated from U.S. Geological Survey data. Other data from U.S. Geological Survey reports or databases]

\begin{tabular}{|c|c|c|c|c|c|c|c|c|c|c|c|}
\hline \multirow[b]{2}{*}{$\begin{array}{c}\text { Streamgage } \\
\text { number } \\
\text { (fig. 35) }\end{array}$} & \multirow[b]{2}{*}{ Streamgage name } & \multirow[b]{2}{*}{$\begin{array}{c}\text { Total } \\
\text { drainage } \\
\left(\mathrm{mi}^{2}\right)\end{array}$} & \multicolumn{4}{|c|}{$\begin{array}{l}\text { Maximum stage and discharge for period of record } \\
\text { through } 1998 \text { water year }\end{array}$} & \multicolumn{5}{|c|}{ Significant floods 1994-98 water years } \\
\hline & & & $\begin{array}{l}\text { Period of } \\
\text { record } \\
\text { (water } \\
\text { years) }\end{array}$ & Water year & $\begin{array}{l}\text { Stage } \\
(\mathrm{ft})\end{array}$ & $\begin{array}{l}\text { Discharge } \\
\left(\mathrm{ft}^{3} / \mathrm{s}\right)\end{array}$ & $\begin{array}{c}\text { Date } \\
\text { (month/ } \\
\text { day/ } \\
\text { year) }\end{array}$ & $\begin{array}{l}\text { Stage } \\
(\mathrm{ft})\end{array}$ & $\begin{array}{l}\text { Discharge } \\
\left(\mathrm{ft}^{3} / \mathrm{s}\right)\end{array}$ & $\begin{array}{l}\text { Regulated } \\
\text { during } \\
\text { flood }^{1}\end{array}$ & $\begin{array}{c}\text { Recurrence } \\
\text { interval } \\
\text { (years) }\end{array}$ \\
\hline 05079000 & Red Lake River at Crookston, MN & 5,270 & $\begin{array}{l}\text { 1897, 1902, } \\
\text { 1904-20, } \\
1922-98\end{array}$ & 1969 & 27.33 & 28,400 & $\begin{array}{l}4 / 20 / 96 \\
4 / 18 / 97\end{array}$ & $\begin{array}{l}24.84 \\
28.40\end{array}$ & $\begin{array}{l}21,700 \\
28,000\end{array}$ & $\begin{array}{l}\mathrm{N} \\
\mathrm{N}\end{array}$ & $\begin{array}{l}>10 \\
>50\end{array}$ \\
\hline 05079901 & $\begin{array}{l}\text { Burnham Creek near Crookston, } \\
\text { MN }\end{array}$ & -- & 1986-98 & 1997 & 22.63 & 3,000 & $4 / 15 / 97$ & 22.63 & 3,000 & $\mathrm{~N}$ & $>10$ \\
\hline 05086900 & Middle River near Newfolden, MN & 91.1 & 1979-98 & $\begin{array}{l}1996 \\
1997\end{array}$ & $\begin{array}{l}18.31 \\
18.71\end{array}$ & $\begin{array}{l}2,300 \\
2,000\end{array}$ & $5 / 18 / 96$ & 18.31 & 2,300 & $\mathrm{~N}$ & $>25$ \\
\hline 05087500 & Middle River at Argyle, MN & 255 & $\begin{array}{l}1945, \\
1950-98\end{array}$ & 1996 & 18.27 & 5,020 & $\begin{array}{l}5 / 19 / 96 \\
4 / 19 / 97\end{array}$ & $\begin{array}{l}18.27 \\
17.96\end{array}$ & $\begin{array}{l}5,020 \\
4,330\end{array}$ & $\begin{array}{l}\mathrm{N} \\
\mathrm{N}\end{array}$ & $\begin{array}{l}>50 \\
>25\end{array}$ \\
\hline 05094000 & $\begin{array}{l}\text { South Branch Two Rivers at Lake } \\
\text { Bronson, MN }\end{array}$ & 422 & $\begin{array}{l}1929-37, \\
1941-47, \\
1954-98\end{array}$ & 1966 & 18.23 & 5,410 & $\begin{array}{l}4 / 22 / 96 \\
4 / 20 / 97\end{array}$ & $\begin{array}{l}14.45 \\
14.58\end{array}$ & $\begin{array}{l}4,290 \\
4,260\end{array}$ & $\begin{array}{l}\mathrm{N} \\
\mathrm{N}\end{array}$ & $\begin{array}{l}>10 \\
>10\end{array}$ \\
\hline 05104500 & $\begin{array}{l}\text { Roseau River below South Fork } \\
\text { near Malung, MN }\end{array}$ & 430 & $\begin{array}{l}1929-38 \\
1940-98\end{array}$ & $\begin{array}{l}1996 \\
1966\end{array}$ & $\begin{array}{l}-- \\
23.37\end{array}$ & $\begin{array}{l}7,310 \\
5,050\end{array}$ & $5 / 19 / 96$ & -- & 7,310 & $\mathrm{~N}$ & $>50$ \\
\hline 05107500 & Roseau River at Ross, MN & 1,090 & $\begin{array}{l}\text { 1896, 1919, } \\
\text { 1927, } \\
\text { 1929-91, } \\
\text { 1995-98 }\end{array}$ & $\begin{array}{l}1950 \\
1896\end{array}$ & $\begin{array}{l}18.25 \\
19.00\end{array}$ & $\begin{array}{l}6,560 \\
8,200\end{array}$ & $4 / 26 / 97$ & 17.30 & 4,670 & $\mathrm{~N}$ & $>10$ \\
\hline 05112000 & $\begin{array}{l}\text { Roseau River below State Ditch } 51 \\
\text { near Caribou, MN }\end{array}$ & 1,420 & $\begin{array}{l}1917 \\
1920-98\end{array}$ & 1950 & 11.81 & 4,080 & $\begin{array}{l}5 / 18 / 96 \\
5 / 8 / 97\end{array}$ & $\begin{array}{r}10.78 \\
2.00\end{array}$ & $\begin{array}{l}3,350 \\
3,320\end{array}$ & $\begin{array}{l}\mathrm{N} \\
\mathrm{N}\end{array}$ & $\begin{array}{l}>25 \\
>25\end{array}$ \\
\hline 05134100 & $\begin{array}{l}\text { North Branch Rapid River near } \\
\text { Baudette, MN }\end{array}$ & 180 & 1986-98 & 1996 & 13.27 & 1,550 & $5 / 18 / 96$ & 13.27 & 1,550 & $\mathrm{~N}$ & $>10$ \\
\hline 05137000 & $\begin{array}{l}\text { Winter Road River near Baudette, } \\
\text { MN }\end{array}$ & 145 & 1986-98 & 1996 & 15.35 & 2,420 & $5 / 18 / 96$ & 15.35 & 2,420 & $\mathrm{~N}$ & $>25$ \\
\hline 05200445 & Mississippi River at Bemidji, MN & 400 & 1973-98 & 1997 & 13.17 & 1,820 & $4 / 18 / 97$ & 13.17 & 1,820 & $\mathrm{Y}$ & $>25$ \\
\hline 05229450 & Pine River near Pine River, MN & -- & 1986-98 & $\begin{array}{l}1997 \\
1990\end{array}$ & $\begin{array}{l}5.19 \\
5.25\end{array}$ & $\begin{array}{l}1,410 \\
1,100\end{array}$ & $4 / 6 / 97$ & 5.19 & 1,410 & $\mathrm{~N}$ & $>10$ \\
\hline
\end{tabular}


$\left[\mathrm{mi}^{2}\right.$, square miles; ft, feet above an arbitrary datum; $\mathrm{ft}^{3} / \mathrm{s}$, cubic feet per second; --, not determined or not applicable; $>$, greater than. Source: Recurrence intervals calculated from U.S. Geological Survey data. Other data from U.S. Geological Survey reports or databases]

\begin{tabular}{|c|c|c|c|c|c|c|c|c|c|c|c|}
\hline \multirow[b]{2}{*}{$\begin{array}{c}\text { Streamgage } \\
\text { number } \\
\text { (fig. 35) }\end{array}$} & \multirow[b]{2}{*}{ Streamgage name } & \multirow[b]{2}{*}{$\begin{array}{c}\text { Total } \\
\text { drainage } \\
\left(\mathrm{mi}^{2}\right)\end{array}$} & \multicolumn{4}{|c|}{$\begin{array}{c}\text { Maximum stage and discharge for period of record } \\
\text { through } 1998 \text { water year }\end{array}$} & \multicolumn{5}{|c|}{ Significant floods 1994-98 water years } \\
\hline & & & $\begin{array}{l}\text { Period of } \\
\text { record } \\
\text { (water } \\
\text { years) }\end{array}$ & Water year & $\begin{array}{l}\text { Stage } \\
(\mathrm{ft})\end{array}$ & $\begin{array}{l}\text { Discharge } \\
\left(\mathrm{ft}^{3} / \mathrm{s}\right)\end{array}$ & $\begin{array}{c}\text { Date } \\
\text { (month/ } \\
\text { day/ } \\
\text { year) }\end{array}$ & $\begin{array}{l}\text { Stage } \\
(\mathrm{ft})\end{array}$ & $\begin{array}{l}\text { Discharge } \\
\left(\mathrm{ft}^{3} / \mathrm{s}\right)\end{array}$ & $\begin{array}{l}\text { Regulated } \\
\text { during } \\
\text { flood }^{1}\end{array}$ & $\begin{array}{c}\text { Recurrence } \\
\text { interval } \\
\text { (years) }\end{array}$ \\
\hline 05242300 & Mississippi River at Brainerd, MN & 7,320 & $1988-98$ & 1997 & 16.03 & 15,400 & $4 / 10 / 97$ & 16.03 & 15,400 & $\mathrm{Y}$ & $>10$ \\
\hline 05243725 & $\begin{array}{l}\text { Straight River near Park Rapids, } \\
\text { MN }\end{array}$ & 53.2 & 1987-98 & 1997 & 2.33 & 149 & 4/6/97 & 2.33 & 149 & $\mathrm{~N}$ & $>50$ \\
\hline 05261000 & $\begin{array}{l}\text { Mississippi River near Fort Ripley, } \\
\text { MN }\end{array}$ & 11,010 & 1972-98 & 1997 & 14.15 & 32,200 & $4 / 8 / 97$ & 14.15 & 32,200 & $\mathrm{Y}$ & $>25$ \\
\hline 05261520 & $\begin{array}{l}\text { Nokasippi River below Fort } \\
\text { Ripley, MN }\end{array}$ & 178 & 1986-98 & 1997 & 14.93 & 1,030 & $4 / 9 / 97$ & 14.93 & 1,030 & $\mathrm{~N}$ & $>10$ \\
\hline 05268000 & Platte River at Royalton, MN & 432 & $\begin{array}{l}1930-36 \\
1972-98\end{array}$ & $\begin{array}{l}1972 \\
1997\end{array}$ & $\begin{array}{r}7.84 \\
14.72\end{array}$ & $\begin{array}{l}6,850 \\
3,800\end{array}$ & $4 / 5 / 97$ & 14.72 & 3,800 & $\mathrm{~N}$ & $>10$ \\
\hline 05270150 & $\begin{array}{l}\text { Ashley Creek near Sauk Centre, } \\
\text { MN }\end{array}$ & 113 & 1986-98 & 1997 & 17.12 & 740 & $4 / 6 / 97$ & 17.12 & 740 & $\mathrm{~N}$ & $>10$ \\
\hline 05276200 & $\begin{array}{l}\text { North Fork Crow River at } \\
\text { Paynesville, MN }\end{array}$ & 243 & 1973-98 & $\begin{array}{l}1997 \\
1984\end{array}$ & $\begin{array}{r}8.85 \\
10.59\end{array}$ & $\begin{array}{l}2,460 \\
2,300\end{array}$ & $4 / 3 / 97$ & 8.85 & 2,460 & $\mathrm{~N}$ & $>10$ \\
\hline 05292000 & Minnesota River at Ortonville, MN & 1,160 & 1938-98 & $\begin{array}{l}1997 \\
1952\end{array}$ & $\begin{array}{l}12.85 \\
12.92\end{array}$ & $\begin{array}{l}5,070 \\
3,060\end{array}$ & $4 / 10 / 97$ & 12.85 & 5,070 & $\mathrm{Y}$ & $>50$ \\
\hline 05293000 & $\begin{array}{l}\text { Yellow Bank River near Odessa, } \\
\text { MN }\end{array}$ & 459 & 1940-98 & 1969 & 19.07 & 6,970 & $4 / 2 / 97$ & 17.94 & 6,770 & $\mathrm{~N}$ & 45 \\
\hline 05293371 & $\begin{array}{l}\text { Pomme de Terre (site 210) near } \\
\text { Elbow Lake, MN }\end{array}$ & -- & 1986-98 & $\begin{array}{l}1997 \\
1993\end{array}$ & $\begin{array}{l}6.72 \\
7.08\end{array}$ & $\begin{array}{l}550 \\
420\end{array}$ & $4 / 7 / 97$ & 6.72 & 550 & $\mathrm{~N}$ & $>25$ \\
\hline 05294000 & $\begin{array}{l}\text { Pomme de Terre River at } \\
\text { Appleton, MN }\end{array}$ & 905 & 1931-98 & 1997 & 18.13 & 8,890 & $4 / 7 / 97$ & 18.13 & 8,890 & $\mathrm{Y}$ & $>500$ \\
\hline 05300000 & $\begin{array}{l}\text { Lac Qui Parle River near Lac Qui } \\
\text { Parle, MN }\end{array}$ & 960 & $\begin{array}{l}1911-14 \\
1931-98\end{array}$ & $\begin{array}{l}1969 \\
1965\end{array}$ & $\begin{array}{l}18.94 \\
19.37\end{array}$ & $\begin{array}{r}17,100 \\
8,370\end{array}$ & $4 / 7 / 97$ & 17.68 & 13,100 & $\mathrm{~N}$ & $>50$ \\
\hline 05302500 & $\begin{array}{l}\text { Little Chippewa River near } \\
\text { Starbuck, MN }\end{array}$ & 94.1 & 1979-98 & 1997 & 15.45 & 850 & $4 / 6 / 97$ & 15.45 & 850 & $\mathrm{~N}$ & $>100$ \\
\hline 05304500 & Chippewa River near Milan, MN & 1,880 & 1937-98 & 1997 & 18.03 & 14,400 & $\begin{array}{l}7 / 6 / 95 \\
4 / 6 / 97\end{array}$ & $\begin{array}{l}13.48 \\
18.03\end{array}$ & $\begin{array}{r}8,440 \\
14,400\end{array}$ & $\begin{array}{l}\mathrm{N} \\
\mathrm{N}\end{array}$ & $\begin{array}{r}>10 \\
>100\end{array}$ \\
\hline
\end{tabular}


Table 24. Maximum stage and discharge for period of record for streamgages having significant floods during 1994-98 water years in Minnesota.-Continued

$\left[\mathrm{mi}^{2}\right.$, square miles; ft, feet above an arbitrary datum; $\mathrm{ft}^{3} / \mathrm{s}$, cubic feet per second; --, not determined or not applicable; $>$, greater than. Source: Recurrence intervals calculated from U.S. Geological Survey data. Other data from U.S. Geological Survey reports or databases]

\begin{tabular}{|c|c|c|c|c|c|c|c|c|c|c|c|}
\hline \multirow{2}{*}{$\begin{array}{l}\text { Streamgage } \\
\text { number } \\
\text { (fig. 35) }\end{array}$} & \multirow[b]{2}{*}{ Streamgage name } & \multirow{2}{*}{$\begin{array}{c}\text { Total } \\
\text { drainage } \\
\left(\mathrm{mi}^{2}\right)\end{array}$} & \multicolumn{4}{|c|}{$\begin{array}{c}\text { Maximum stage and discharge for period of record } \\
\text { through } 1998 \text { water year }\end{array}$} & \multicolumn{5}{|c|}{ Significant floods $1994-98$ water years } \\
\hline & & & $\begin{array}{l}\text { Period of } \\
\text { record } \\
\text { (water } \\
\text { years) }\end{array}$ & Water year & $\begin{array}{l}\text { Stage } \\
(\mathrm{ft})\end{array}$ & $\begin{array}{l}\text { Discharge } \\
\left(\mathrm{ft}^{3} / \mathrm{s}\right)\end{array}$ & $\begin{array}{c}\text { Date } \\
\text { (month/ } \\
\text { day/ } \\
\text { year) }\end{array}$ & $\begin{array}{l}\text { Stage } \\
(\mathrm{ft})\end{array}$ & $\begin{array}{l}\text { Discharge } \\
\left(\mathrm{ft}^{3} / \mathrm{s}\right)\end{array}$ & $\begin{array}{l}\text { Regulated } \\
\text { during } \\
\text { flood }^{1}\end{array}$ & $\begin{array}{c}\text { Recurrence } \\
\text { interval } \\
\text { (years) }\end{array}$ \\
\hline 05311000 & $\begin{array}{l}\text { Minnesota River at Montevideo, } \\
\text { MN }\end{array}$ & 6,180 & 1910-98 & 1997 & 23.90 & 47,500 & $4 / 6 / 97$ & 23.90 & 47,500 & $\mathrm{Y}$ & $>50$ \\
\hline 05316570 & $\begin{array}{l}\text { Beaver Creek near Beaver Falls, } \\
\text { MN }\end{array}$ & 194 & $\begin{array}{l}1972-80 \\
1982-98\end{array}$ & 1997 & 14.73 & 3,300 & $4 / 2 / 97$ & 14.73 & 3,300 & $\mathrm{~N}$ & $>100$ \\
\hline 05316950 & $\begin{array}{l}\text { Cottonwood River near } \\
\text { Springfield, MN }\end{array}$ & 777 & 1973-98 & 1997 & 28.77 & 7,860 & $3 / 29 / 97$ & 28.77 & 7,860 & $\mathrm{~N}$ & $>5$ \\
\hline 05325000 & Minnesota River at Mankato, MN & 14,900 & $\begin{array}{l}\text { 1881, } \\
1903-98\end{array}$ & $\begin{array}{l}1965 \\
1993\end{array}$ & $\begin{array}{l}29.09 \\
30.11\end{array}$ & $\begin{array}{l}94,100 \\
75,600\end{array}$ & $4 / 10 / 97$ & 27.61 & 79,800 & $\mathrm{Y}$ & $>50$ \\
\hline 05326100 & $\begin{array}{l}\text { Middle Branch Rush River near } \\
\text { Gaylord, MN }\end{array}$ & 67.3 & 1979-98 & $\begin{array}{l}1997 \\
1979\end{array}$ & $\begin{array}{l}19.22 \\
21.00\end{array}$ & $\begin{array}{r}3,200 \\
715\end{array}$ & $3 / 28 / 97$ & 19.22 & 3,200 & $\mathrm{~N}$ & $>100$ \\
\hline 05327000 & $\begin{array}{l}\text { High Island Creek near Henderson, } \\
\text { MN }\end{array}$ & 237 & 1974-98 & $\begin{array}{l}1997 \\
1993\end{array}$ & $\begin{array}{l}9.31 \\
9.72\end{array}$ & $\begin{array}{l}2,830 \\
2,750\end{array}$ & $7 / 25 / 97$ & 9.31 & 2,830 & $\mathrm{~N}$ & $>10$ \\
\hline 05331000 & Mississippi River at St. Paul, MN & 36,800 & $\begin{array}{l}1867-70 \\
1872-1998\end{array}$ & 1965 & 26.01 & 171,000 & $4 / 13 / 97$ & 22.37 & 134,000 & $\mathrm{Y}$ & $>50$ \\
\hline 05337400 & Knife River near Mora, MN & 102 & 1975-98 & 1997 & 6.48 & 1,870 & $4 / 6 / 97$ & 6.48 & 1,870 & $\mathrm{~N}$ & $>5$ \\
\hline 05339747 & Goose Creek at Harris, MN & -- & 1986-98 & 1996 & 7.50 & 295 & $4 / 19 / 96$ & 7.50 & 295 & $\mathrm{~N}$ & $>5$ \\
\hline 05355200 & Cannon River at Welch, MN & 1,340 & $\begin{array}{l}\text { 1888, } \\
\text { 1911-13, } \\
\text { 1931-87, } \\
\text { 1992-98 }\end{array}$ & $\begin{array}{l}1965 \\
1998\end{array}$ & $\begin{array}{l}14.01 \\
15.05\end{array}$ & $\begin{array}{l}36,100 \\
23,500\end{array}$ & $6 / 27 / 98$ & 15.05 & 23,500 & $\mathrm{Y}$ & 50 \\
\hline 05378500 & Mississippi River at Winona, MN & 59,200 & $\begin{array}{l}1879-1922, \\
1924-98\end{array}$ & 1965 & 20.77 & 268,000 & $4 / 11 / 97$ & 18.27 & 194,000 & $\mathrm{Y}$ & $>25$ \\
\hline
\end{tabular}

${ }^{1}$ Regulated during flood: $\mathrm{N}$, no; $\mathrm{Y}$, yes. 


\section{Mississippi}

During May 8-10, 1995, extreme weather conditions, which produced as much as 27.5 inches of rain during a 55-hour period (National Oceanic and Atmospheric Administration, 1995a), caused the most severe flooding in recent history along coastal areas of the Gulf of Mexico in Mississippi and southeastern Louisiana. At least six people died, and thousands more were left homeless as a result of the intense flooding. At least $\$ 3$ billion in property damage was reported in New Orleans, Louisiana, alone, and millions more in damage was reported in the Gulf Coast counties of Mississippi and parishes in southeastern Louisiana as a result of the storm (National Oceanic and Atmospheric Administration, 1995b). In Mississippi, flooding was most severe in the Biloxi and Wolf River Basins (fig. 36, table 25). The response of these rivers to the storm was both rapid and devastating as rivers in the affected basins reached record or near-record stages and returned to below flood stage within 48 hours after the end of the rainfall.

The Mississippi River went above flood stage around March 9, 1997, and crested between March 21 and 26. Considerable damage occurred on the Mississippi side of the river downstream from the confluence of the Mississippi and the Arkansas Rivers (National Oceanic and Atmospheric Administration, 1997b).

Flash flooding occurred due to excessive rainfall totaling 6.40 inches near Lexington, Mississippi, during June 9-10,
1997 (National Oceanic and Atmospheric Administration, 1997a). Mississippi Emergency Management Agency estimated damage at \$3.5 million dollars (National Oceanic and Atmospheric Administration, 1997b). Approximately 1,000 people were evacuated. The town's eastern commercial area was inundated by floodwaters.

On September 28, 1998, Hurricane Georges made landfall just east of Biloxi, Mississippi, with maximum sustained winds of about 105 miles per hour. At landfall, Georges was a strong category 2 hurricane. From September 28-30, 1998, Georges brought torrents of rain to the central Gulf Coast from Gulfport, Mississippi, to the eastern parts of the Florida Panhandle. Rainfall amounts ranging from 15 to more than 25 inches were reported in many areas of southern Alabama, the Florida Panhandle, and the Mississippi Gulf Coast (National Oceanic and Atmospheric Administration, 1998a). Severe flooding occurred in southern Mississippi.

\section{References}

National Oceanic and Atmospheric Administration (NOAA), 1995a-98a, Climatological data (by State): Asheville, North Carolina, National Climatic Data Center, various months.

National Oceanic and Atmospheric Administration (NOAA), 1995b-98b, Storm data (by State): Asheville, North Carolina, National Climatic Data Center, various months. 


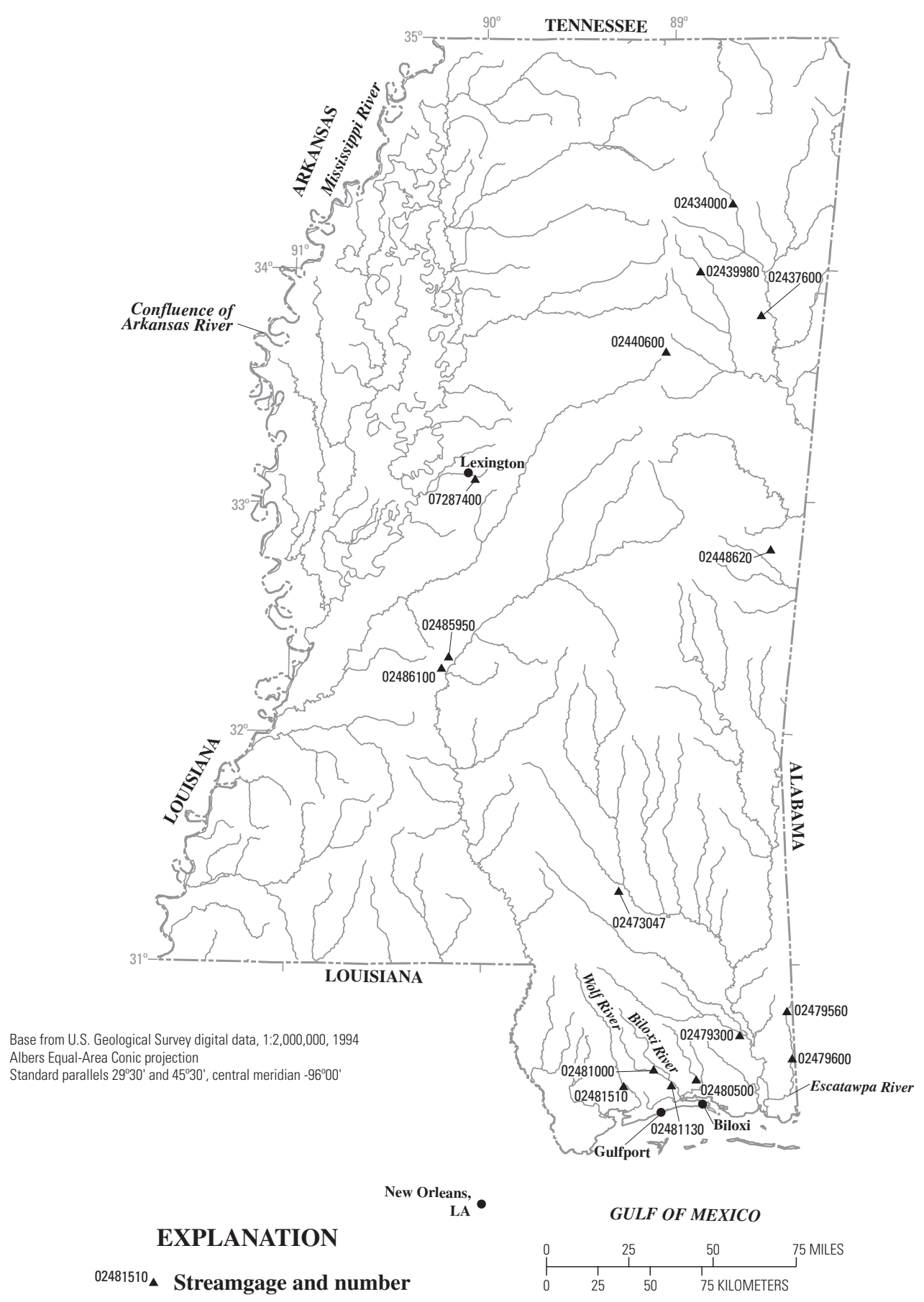

Figure 36. Location of streamgages with significant floods during 1994-98 water years for Mississippi. 
Table 25. Maximum stage and discharge for period of record for streamgages having significant floods during 1994-98 water years in Mississippi.

$\left[\mathrm{mi}^{2}\right.$, square miles; $\mathrm{ft}$, feet above an arbitrary datum; $\mathrm{ft}^{3} / \mathrm{s}$, cubic feet per second; --, not determined or not applicable. Source: Recurrence intervals calculated from U.S. Geological Survey data. Other data from U.S. Geological Survey reports or databases]

\begin{tabular}{|c|c|c|c|c|c|c|c|c|c|c|c|}
\hline \multirow[b]{2}{*}{$\begin{array}{l}\text { Streamgage } \\
\text { number } \\
\text { (fig. 36) }\end{array}$} & \multirow[b]{2}{*}{ Streamgage name } & \multirow[b]{2}{*}{$\begin{array}{c}\text { Total } \\
\text { drainage } \\
\left(\mathrm{mi}^{2}\right)\end{array}$} & \multicolumn{4}{|c|}{$\begin{array}{c}\text { Maximum stage and discharge for period of record } \\
\text { through } 1998 \text { water year }\end{array}$} & \multicolumn{5}{|c|}{ Significant floods $1994-98$ water years } \\
\hline & & & $\begin{array}{l}\text { Period of } \\
\text { record } \\
\text { (water } \\
\text { years) }\end{array}$ & Water year & $\begin{array}{l}\text { Stage } \\
\text { (ft) }\end{array}$ & $\begin{array}{l}\text { Discharge } \\
\left(\mathrm{ft}^{3} / \mathrm{s}\right)\end{array}$ & $\begin{array}{c}\text { Date } \\
\text { (month/ } \\
\text { day/ } \\
\text { year) }\end{array}$ & $\begin{array}{l}\text { Stage } \\
\text { (ft) }\end{array}$ & $\begin{array}{l}\text { Discharge } \\
\left(\mathrm{ft}^{3} / \mathrm{s}\right)\end{array}$ & $\begin{array}{l}\text { Regulated } \\
\text { during } \\
\text { flood }^{1}\end{array}$ & $\begin{array}{c}\text { Recurrence } \\
\text { interval } \\
\text { (years) }\end{array}$ \\
\hline 02434000 & Town Creek at Tupelo, MS & 111 & $\begin{array}{l}1939-46, \\
1949-98\end{array}$ & 1955 & 27.72 & 23,000 & $3 / 2 / 97$ & 26.61 & 20,000 & $\mathrm{~N}$ & $50-100$ \\
\hline 02437600 & James Creek at Aberdeen, MS & 28.4 & $1963-98$ & $\begin{array}{l}1985 \\
1997\end{array}$ & $\begin{array}{l}15.21 \\
25.10\end{array}$ & $\begin{array}{l}6,970 \\
6,770\end{array}$ & $5 / 27 / 97$ & 25.10 & 6,770 & $\mathrm{~N}$ & 20 \\
\hline 02439980 & $\begin{array}{l}\text { Chuquatonchee Creek near } \\
\text { Okalona, MS }\end{array}$ & 68.5 & $\begin{array}{l}\text { 1951, 1953, } \\
\text { 1964-73, } \\
\text { 1975-81, } \\
\text { 1985-87, } \\
1989-95, \\
1998\end{array}$ & 1973 & 16.93 & 15,000 & $3 / 6 / 98$ & 14.56 & 7,060 & $\mathrm{Y}$ & -- \\
\hline 02440600 & Line Creek near Maben, MS & 4.76 & $\begin{array}{l}1952-80 \\
1982-98\end{array}$ & 1984 & 28.33 & 7,540 & $6 / 26 / 94$ & 28.30 & 7,480 & $\mathrm{~N}$ & -- \\
\hline 02448620 & $\begin{array}{l}\text { Flat Scooba Creek tributary near } \\
\text { Scooba, MS }\end{array}$ & .44 & 1967-98 & 1979 & 8.87 & 427 & $1 / 7 / 98$ & 5.74 & 263 & $\mathrm{~N}$ & -- \\
\hline 02473047 & Gordon Creek at Hattiesburg, MS & 8.83 & 1969-98 & 1983 & 61.89 & 6,920 & $1 / 7 / 98$ & 13.84 & 4,920 & $\mathrm{~N}$ & 15 \\
\hline 02479300 & Red Creek near Vestry, MS & 441 & $1959-98$ & 1987 & 21.48 & 28,000 & $9 / 29 / 98$ & 20.89 & 24,300 & $\mathrm{~N}$ & 20 \\
\hline 02479560 & $\begin{array}{l}\text { Escatawpa River near Agricola, } \\
\text { MS }\end{array}$ & 562 & 1974-98 & 1998 & 22.81 & 27,800 & 9/30/98 & 22.81 & 27,800 & $\mathrm{~N}$ & 20 \\
\hline 02479600 & Escatawpa River near Hurley, MS & 646 & $\begin{array}{l}1958-70 \\
1998\end{array}$ & 1998 & 19.30 & 27,500 & 9/30/98 & 19.30 & 27,500 & $\mathrm{~N}$ & 20 \\
\hline 02480500 & Tuxachanie Creek near Biloxi, MS & 92.4 & $\begin{array}{l}1906,1948, \\
1953-98\end{array}$ & 1998 & 26.06 & 20,300 & $\begin{array}{l}9 / 29 / 98 \\
5 / 10 / 95\end{array}$ & $\begin{array}{l}26.06 \\
24.92\end{array}$ & $\begin{array}{l}20,300 \\
16,900\end{array}$ & $\mathrm{~N}$ & $\begin{array}{r}100 \\
50\end{array}$ \\
\hline 02481000 & Biloxi River at Wortham, MS & 96.2 & $\begin{array}{l}1948, \\
1953-98\end{array}$ & 1995 & 28.94 & 13,500 & $5 / 9 / 95$ & 28.94 & 13,500 & $\mathrm{~N}$ & 100 \\
\hline 02481130 & Biloxi River near Lyman, MS & 251 & $\begin{array}{l}1957, \\
1964-85, \\
1987-98\end{array}$ & 1995 & 23.95 & 36,800 & $5 / 10 / 95$ & 23.95 & 36,800 & $\mathrm{~N}$ & 50 \\
\hline 02481510 & Wolf River near Landon, MS & 308 & $1971-98$ & 1995 & 28.85 & 24,500 & $5 / 10 / 95$ & 28.85 & 24,500 & $\mathrm{~N}$ & 50 \\
\hline
\end{tabular}


Table 25. Maximum stage and discharge for period of record for streamgages having significant floods during 1994-98 water years in Mississippi.-Continued

$\left[\mathrm{mi}^{2}\right.$, square miles; $\mathrm{ft}$, feet above an arbitrary datum; $\mathrm{ft}^{3} / \mathrm{s}$, cubic feet per second; --, not determined or not applicable. Source: Recurrence intervals calculated from U.S. Geological Survey data. Other data from U.S. Geological Survey reports or databases]

\begin{tabular}{|c|c|c|c|c|c|c|c|c|c|c|c|}
\hline \multirow{2}{*}{$\begin{array}{c}\text { Streamgage } \\
\text { number } \\
\text { (fig. 36) }\end{array}$} & \multirow[b]{2}{*}{ Streamgage name } & \multirow{2}{*}{$\begin{array}{c}\text { Total } \\
\text { drainage } \\
\left(\mathrm{mi}^{2}\right)\end{array}$} & \multicolumn{4}{|c|}{$\begin{array}{c}\text { Maximum stage and discharge for period of record } \\
\text { through } 1998 \text { water year }\end{array}$} & \multicolumn{5}{|c|}{ Significant floods 1994-98 water years } \\
\hline & & & $\begin{array}{l}\text { Period of } \\
\text { record } \\
\text { (water } \\
\text { years) }\end{array}$ & Water year & $\begin{array}{l}\text { Stage } \\
(\mathrm{ft})\end{array}$ & $\begin{array}{c}\text { Discharge } \\
\left(\mathrm{ft}^{3} / \mathrm{s}\right)\end{array}$ & $\begin{array}{c}\text { Date } \\
\text { (month/ } \\
\text { day/year) }\end{array}$ & $\begin{array}{c}\text { Stage } \\
(\mathrm{ft})\end{array}$ & $\begin{array}{c}\text { Discharge } \\
\left(\mathrm{ft}^{3} / \mathrm{s}\right)\end{array}$ & $\begin{array}{c}\text { Regulated } \\
\text { during } \\
\text { flood }^{1}\end{array}$ & $\begin{array}{c}\text { Recurrence } \\
\text { interval } \\
\text { (years) }\end{array}$ \\
\hline 02485950 & Town Creek at Jackson, MS & 11.4 & $\begin{array}{l}\text { 1885, 1914, } \\
1921, \\
1953-98\end{array}$ & 1921 & 19.00 & 6,000 & $6 / 10 / 97$ & 12.12 & 4,110 & $\mathrm{~N}$ & 20 \\
\hline 02486100 & Lynch Creek at Jackson, MS & 12.0 & 1953-95 & $\begin{array}{l}1995 \\
1953\end{array}$ & $\begin{array}{l}16.30 \\
18.90\end{array}$ & $\begin{array}{l}7,180 \\
7,500\end{array}$ & $4 / 20 / 95$ & 16.30 & 7,180 & $\mathrm{~N}$ & 50 \\
\hline 07287400 & Black Creek at Lexington, MS & 88.1 & 1987-98 & 1997 & 27.89 & 18,600 & $6 / 10 / 97$ & 27.89 & 18,600 & $\mathrm{~N}$ & 25 \\
\hline
\end{tabular}

${ }^{1}$ Regulated during flood: $\mathrm{N}$, no; $\mathrm{Y}$, yes. 


\section{Missouri}

Parts of eastern, southern, and southeastern Missouri received 6 to 10 inches of rain the evening of November 13 and 14, 1993 (National Oceanic and Atmospheric Administration, 1993a). Numerous tributaries of the Missouri and Mississippi Rivers quickly rose above flood stage including the Gasconade, Osage, Meramec, Bourbeuse, Big, Current, and James Rivers (fig. 37). Eight streamgages had the peak of record during this flood (table 26). Estimates of flood damage neared \$50 million, but there were no reported deaths (National Oceanic and Atmospheric Administration, 1993b).

The rains returned to the same areas in Missouri during the second week of April 1994 as 7 to 9 inches of rain fell during a 3-day period (National Oceanic and Atmospheric Administration, 1994a), bringing a quick return to river flooding. Most of the more intense rains were south of the Missouri River. The most serious flooding occurred along the Mississippi, Missouri, Meramec, Bourbeuse, and Big Rivers in eastern Missouri. Damage was over $\$ 10$ million, and three deaths occurred (National Oceanic and Atmospheric Administration 1994b). Rainfall in April averaged from 4 inches above normal at Kansas City to near 8 inches above normal at Columbia. This April was the wettest on record at Columbia, the second wettest at Springfield and St. Louis, and the fourth wettest at Kansas City (National Oceanic and Atmospheric Administration, 1994a).

Serious flooding returned to the Missouri and Mississippi River Valleys in Missouri, during May 1995, 2 short years after the record flood of 1993. While the flood did cause a great deal of hardship and disruption to the economy and daily lives, it was estimated that people, homes, and business affected by the flood in 1995 were 50 percent less than in 1993 (National Oceanic and Atmospheric Administration, 1995b). This was due primarily to the Federally sponsored buyout programs that moved many people out of the flood plains. The flood of May 1995 was triggered by abnormally excessive rain. May rainfall averages across Missouri ranged from 3 inches above normal at Springfield to 9 inches above normal at St. Louis. May 1995 was the wettest May on record for St. Louis where 12.92 inches of rain fell. The spring (March, April, May) of 1995 was the fifth wettest in St. Louis history with a total of 19.44 inches. The majority of serious flooding was on the main stems of the Missouri and Mississippi Rivers, as well as backwater points on tributaries. Nearly \$30 million in damage resulted (National Oceanic and Atmospheric Administration, 1995b). The Mississippi and Missouri Rivers were closed to barge traffic, bringing that industry to a standstill.

A series of thunderstorm complexes produced widespread flooding over central and south-central Missouri on July 26, 1998. Cooperative weather stations reported more than 8 inches of rain at Versailles, Rolla, and Salem (National Oceanic and Atmospheric Administration, 1998a). Flooding caused widespread damage to roads and low-water crossings and bridges.

\section{References}

National Oceanic and Atmospheric Administration (NOAA), 1993a-98a, Climatological data (by State): Asheville, North Carolina, National Climatic Data Center, various months.

National Oceanic and Atmospheric Administration (NOAA), 1993b-98b, Storm data (by State): Asheville, North Carolina, National Climatic Data Center, various months. 


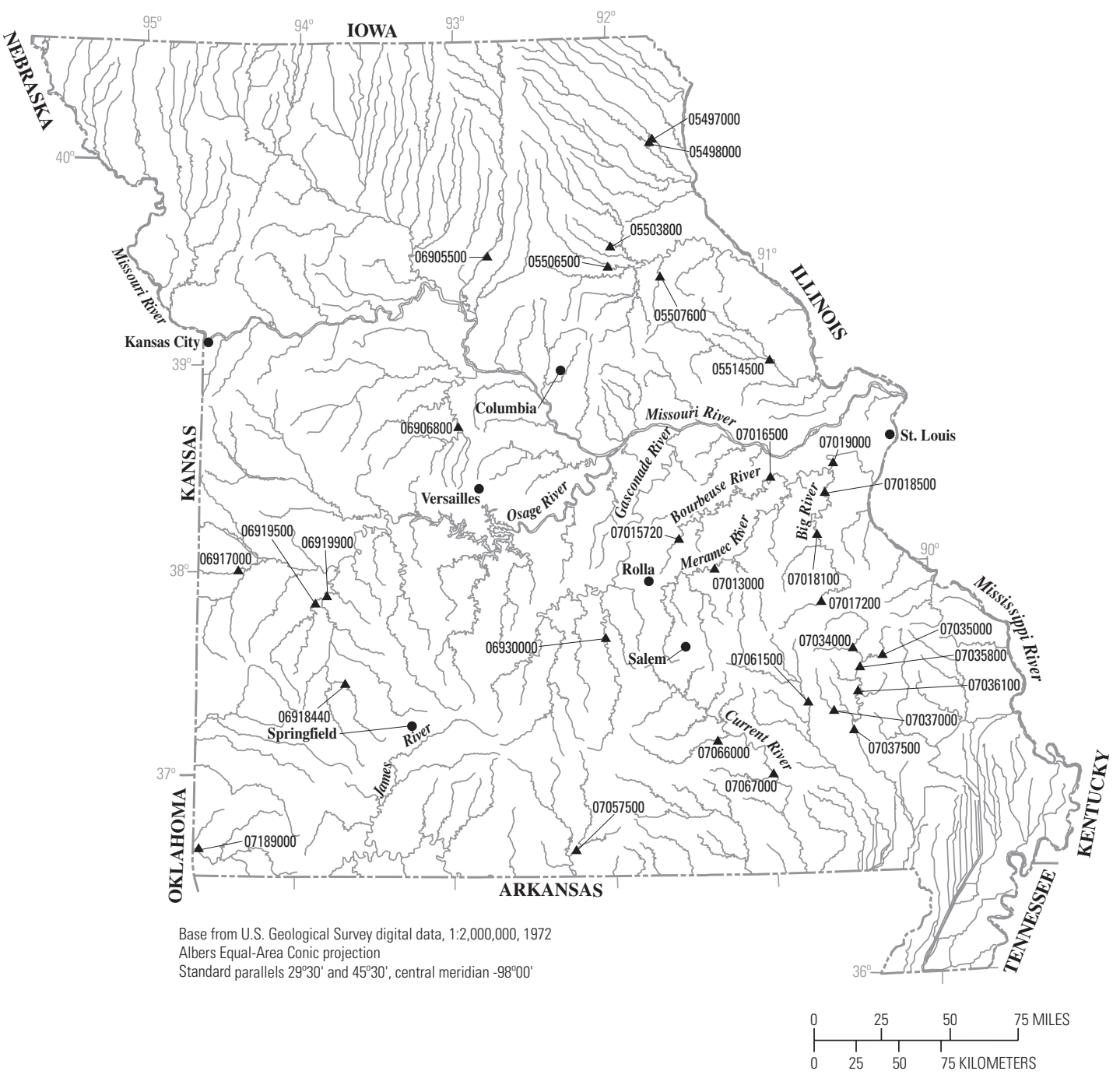

EXPLANATION

${ }_{09537200 \triangle}$ Streamgage and number

Figure 37. Location of streamgages with significant floods during 1994-98 water years for Missouri. 
Table 26. Maximum stage and discharge for period of record for streamgages having significant floods during 1994-98 water years in Missouri.

$\left[\mathrm{mi}^{2}\right.$, square miles; $\mathrm{ft}$, feet above an arbitrary datum; $\mathrm{ft}^{3} / \mathrm{s}$, cubic feet per second; --, not determined or not applicable; >, greater than. Source: Recurrence intervals calculated from U.S. Geological Survey data. Other data from U.S. Geological Survey reports or databases]

\begin{tabular}{|c|c|c|c|c|c|c|c|c|c|c|c|}
\hline \multirow{2}{*}{$\begin{array}{c}\text { Streamgage } \\
\text { number } \\
\text { (fig. 37) }\end{array}$} & \multirow[b]{2}{*}{ Streamgage name } & \multirow{2}{*}{$\begin{array}{c}\text { Total } \\
\text { drainage } \\
\left(\mathrm{mi}^{2}\right)\end{array}$} & \multicolumn{4}{|c|}{$\begin{array}{c}\text { Maximum stage and discharge for period of record } \\
\text { through } 1998 \text { water year }\end{array}$} & \multicolumn{5}{|c|}{ Significant floods 1994-98 water years } \\
\hline & & & $\begin{array}{l}\text { Period of } \\
\text { record } \\
\text { (water } \\
\text { years) }\end{array}$ & Water year & $\begin{array}{l}\text { Stage } \\
\text { (ft) }\end{array}$ & $\begin{array}{l}\text { Discharge } \\
\left(\mathrm{ft}^{3} / \mathrm{s}\right)\end{array}$ & $\begin{array}{c}\text { Date } \\
\text { (month/ } \\
\text { day/ } \\
\text { year) }\end{array}$ & $\begin{array}{l}\text { Stage } \\
\text { (ft) }\end{array}$ & $\begin{array}{l}\text { Discharge } \\
\left(\mathrm{ft}^{3} / \mathrm{s}\right)\end{array}$ & $\begin{array}{c}\text { Regulated } \\
\text { during } \\
\text { flood }^{1}\end{array}$ & $\begin{array}{c}\text { Recurrence } \\
\text { interval } \\
\text { (years) }\end{array}$ \\
\hline 05497000 & $\begin{array}{l}\text { North Fabius River at Monticello, } \\
\text { MO }\end{array}$ & 452 & $1922-98$ & 1973 & 33.03 & 20,700 & $5 / 28 / 96$ & 29.63 & 17,200 & $\mathrm{~N}$ & 10 \\
\hline 05498000 & $\begin{array}{l}\text { Middle Fabius River near } \\
\text { Monticello, MO }\end{array}$ & 393 & 1946-98 & 1973 & 27.14 & 17,700 & $5 / 29 / 96$ & 24.87 & 13,800 & $\mathrm{~N}$ & 5 \\
\hline 05503800 & Crooked Creek near Paris, MO & 80.0 & 1980-98 & 1996 & 13.62 & 9,460 & $5 / 7 / 96$ & 13.62 & 9,460 & $\mathrm{~N}$ & $50-100$ \\
\hline 05506500 & $\begin{array}{l}\text { Middle Fork Salt River at Paris, } \\
\text { MO }\end{array}$ & 356 & 1940-98 & 1973 & 25.21 & 45,000 & $5 / 8 / 96$ & 21.84 & 15,800 & $\mathrm{~N}$ & $10-25$ \\
\hline 05507600 & Lick Creek at Perry, MO & 104 & 1980-98 & 1996 & 22.25 & 11,800 & $5 / 7 / 96$ & 22.25 & 11,800 & $\mathrm{~N}$ & $25-50$ \\
\hline 05514500 & Cuivre River near Troy, MO & 903 & $\begin{array}{l}1922-72, \\
1974-81, \\
1983-98\end{array}$ & 1942 & 33.40 & 120,000 & $4 / 12 / 94$ & 32.47 & 107,000 & $\mathrm{~N}$ & $>500$ \\
\hline 06905500 & $\begin{array}{l}\text { Chariton River near Prairie Hill, } \\
\text { MO }\end{array}$ & 1,870 & 1929-98 & 1996 & 22.33 & 33,600 & $\begin{array}{l}5 / 24 / 95 \\
5 / 27 / 96\end{array}$ & $\begin{array}{l}21.96 \\
22.33\end{array}$ & $\begin{array}{l}31,600 \\
33,600\end{array}$ & $\begin{array}{l}\mathrm{N} \\
\mathrm{N}\end{array}$ & $\begin{array}{l}5 \\
--\end{array}$ \\
\hline 06906800 & Lamine River near Otterville, MO & 543 & 1988-98 & 1995 & 29.43 & 84,900 & $5 / 18 / 95$ & 29.43 & 84,900 & $\mathrm{~N}$ & $>500$ \\
\hline 06917000 & Little Osage River at Fulton, KS & 295 & 1949-98 & 1987 & 35.21 & 62,800 & $4 / 28 / 94$ & 32.61 & 38,000 & $\mathrm{~N}$ & -- \\
\hline 06918440 & Sac River near Dadeville, MO & 257 & $1966-98$ & 1993 & 27.56 & 36,100 & $4 / 11 / 94$ & 20.88 & 13,700 & $\mathrm{~N}$ & 5 \\
\hline 06919500 & $\begin{array}{l}\text { Cedar Creek near Pleasant View, } \\
\text { MO }\end{array}$ & 420 & $\begin{array}{l}1909, \\
1923-26, \\
1943, \\
1949-98\end{array}$ & $\begin{array}{l}1958 \\
1909\end{array}$ & $\begin{array}{l}27.35 \\
27.70\end{array}$ & $\begin{array}{r}37,000 \\
--\end{array}$ & $4 / 12 / 94$ & 27.36 & 36,300 & $\mathrm{~N}$ & 50 \\
\hline 06919900 & $\begin{array}{l}\text { Sac River near Caplinger Mills, } \\
\text { MO }\end{array}$ & 1,810 & $1975-98$ & 1994 & 30.95 & 61,500 & $4 / 12 / 94$ & 30.95 & 61,500 & $\mathrm{Y}$ & -- \\
\hline 06930000 & $\begin{array}{l}\text { Big Piney River near Big Piney, } \\
\text { MO }\end{array}$ & 560 & $\begin{array}{l}1922-70, \\
1972-82, \\
1989-96\end{array}$ & 1943 & 20.70 & 32,700 & $11 / 15 / 93$ & 19.81 & 30,800 & $\mathrm{~N}$ & 10 \\
\hline
\end{tabular}


Table 26. Maximum stage and discharge for period of record for streamgages having significant floods during 1994-98 water years in Missouri.—Continued

$\left[\mathrm{mi}^{2}\right.$, square miles; $\mathrm{ft}$, feet above an arbitrary datum; $\mathrm{ft}^{3} / \mathrm{s}$, cubic feet per second; --, not determined or not applicable; >, greater than. Source: Recurrence intervals calculated from U.S. Geological Survey data. Other data from U.S. Geological Survey reports or databases]

\begin{tabular}{|c|c|c|c|c|c|c|c|c|c|c|c|}
\hline \multirow[b]{2}{*}{$\begin{array}{c}\text { Streamgage } \\
\text { number } \\
\text { (fig. 37) }\end{array}$} & \multirow[b]{2}{*}{ Streamgage name } & \multirow{2}{*}{$\begin{array}{c}\text { Total } \\
\text { drainage } \\
\left(\mathrm{mi}^{2}\right)\end{array}$} & \multicolumn{4}{|c|}{$\begin{array}{c}\text { Maximum stage and discharge for period of record } \\
\text { through } 1998 \text { water year }\end{array}$} & \multicolumn{5}{|c|}{ Significant floods 1994-98 water years } \\
\hline & & & $\begin{array}{l}\text { Period of } \\
\text { record } \\
\text { (water } \\
\text { years) }\end{array}$ & Water year & $\begin{array}{l}\text { Stage } \\
(\mathrm{ft})\end{array}$ & $\begin{array}{c}\text { Discharge } \\
\left(\mathrm{ft}^{3} / \mathrm{s}\right)\end{array}$ & $\begin{array}{c}\text { Date } \\
\text { (month/ } \\
\text { day/ } \\
\text { year) }\end{array}$ & $\begin{array}{l}\text { Stage } \\
(\mathrm{ft})\end{array}$ & $\begin{array}{c}\text { Discharge } \\
\left(\mathrm{ft}^{3} / \mathrm{s}\right)\end{array}$ & $\begin{array}{l}\text { Regulated } \\
\text { during } \\
\text { flood }^{1}\end{array}$ & $\begin{array}{c}\text { Recurrence } \\
\text { interval } \\
\text { (years) }\end{array}$ \\
\hline 07013000 & $\begin{array}{l}\text { Meramec River near Steelville, } \\
\text { MO }\end{array}$ & 781 & $\begin{array}{l}1915, \\
1917-98\end{array}$ & 1998 & 27.22 & 55,800 & $7 / 27 / 98$ & 27.22 & 55,800 & $\mathrm{~N}$ & $10-25$ \\
\hline 07015720 & $\begin{array}{l}\text { Bourbeuse River near High Gate, } \\
\text { MO }\end{array}$ & 135 & $1965-98$ & 1983 & 23.65 & 49,300 & $7 / 26 / 98$ & 23.57 & 34,500 & $\mathrm{~N}$ & $100-500$ \\
\hline 07016500 & Bourbeuse River at Union, MO & 808 & $\begin{array}{l}1897 \\
1915-98\end{array}$ & 1983 & 33.80 & 73,300 & $4 / 13 / 94$ & 27.77 & 42,000 & $\mathrm{~N}$ & $25-50$ \\
\hline 07017200 & Big River at Irondale, MO & 175 & $\begin{array}{l}1965-96 \\
1998\end{array}$ & 1994 & 28.95 & 49,100 & $11 / 14 / 93$ & 28.95 & 49,100 & $\mathrm{~N}$ & $100-500$ \\
\hline 07018100 & Big River near Richwoods, MO & 735 & 1984-98 & $\begin{array}{l}1997 \\
1993\end{array}$ & $\begin{array}{l}28.25 \\
30.33\end{array}$ & $\begin{array}{l}61,500 \\
59,800\end{array}$ & $12 / 10 / 96$ & 28.25 & 61,500 & $\mathrm{~N}$ & $50-100$ \\
\hline 07018500 & Big River at Byrnesville, $\mathrm{MO}$ & 917 & $\begin{array}{l}1915, \\
1923-88, \\
1990, \\
1992-98\end{array}$ & $\begin{array}{l}1993 \\
1915\end{array}$ & $\begin{array}{l}29.37 \\
30.20\end{array}$ & $\begin{array}{l}63,600 \\
80,000\end{array}$ & $11 / 16 / 93$ & 27.61 & 50,200 & $\mathrm{~N}$ & 25 \\
\hline 07019000 & Meramec River near Eureka, MO & 3,788 & $\begin{array}{l}1904-05, \\
1915-16, \\
1922-97\end{array}$ & $\begin{array}{l}1915 \\
1983\end{array}$ & $\begin{array}{l}40.20 \\
42.89\end{array}$ & $\begin{array}{l}175,000 \\
145,000\end{array}$ & $4 / 14 / 94$ & 40.90 & 123,000 & $\mathrm{~N}$ & $10-25$ \\
\hline 07034000 & $\begin{array}{l}\text { Saint Francis River near Roselle, } \\
\text { MO }\end{array}$ & 234 & 1987-97 & 1994 & 26.50 & 45,700 & $11 / 14 / 93$ & 26.50 & 45,700 & $\mathrm{~N}$ & $100-500$ \\
\hline 07035000 & $\begin{array}{l}\text { Little Saint Francis River at } \\
\text { Fredericktown, MO }\end{array}$ & 90.5 & 1984-97 & 1994 & 26.50 & 25,100 & $11 / 14 / 93$ & 26.50 & 25,100 & $\mathrm{~N}$ & $100-500$ \\
\hline 07035800 & $\begin{array}{l}\text { Saint Francis River near Mill } \\
\text { Creek, MO }\end{array}$ & 505 & $\begin{array}{l}\text { 1987-92, } \\
1994-97\end{array}$ & 1994 & 33.10 & 130,000 & $11 / 14 / 93$ & 33.10 & 130,000 & $\mathrm{~N}$ & $>500$ \\
\hline 07036100 & Saint Francis River near Saco, MO & 664 & 1984-97 & 1994 & 36.10 & 161,000 & $11 / 14 / 93$ & 36.10 & 161,000 & $\mathrm{~N}$ & $>500$ \\
\hline 07037000 & Big Creek at Des Arc, MO & 99.6 & 1987-97 & 1994 & 16.85 & 25,700 & $11 / 14 / 93$ & 16.85 & 25,700 & $\mathrm{~N}$ & $100-500$ \\
\hline
\end{tabular}


$\left[\mathrm{mi}^{2}\right.$, square miles; $\mathrm{ft}$, feet above an arbitrary datum; $\mathrm{ft}^{3} / \mathrm{s}$, cubic feet per second; --, not determined or not applicable; >, greater than. Source: Recurrence intervals calculated from U.S. Geological Survey data. Other data from U.S. Geological Survey reports or databases]

\begin{tabular}{|c|c|c|c|c|c|c|c|c|c|c|c|}
\hline \multirow{2}{*}{$\begin{array}{c}\text { Streamgage } \\
\text { number } \\
\text { (fig. 37) }\end{array}$} & \multirow[b]{2}{*}{ Streamgage name } & \multirow{2}{*}{$\begin{array}{c}\text { Total } \\
\text { drainage } \\
\left(\mathrm{mi}^{2}\right)\end{array}$} & \multicolumn{4}{|c|}{$\begin{array}{c}\text { Maximum stage and discharge for period of record } \\
\text { through } 1998 \text { water year }\end{array}$} & \multicolumn{5}{|c|}{ Significant floods $1994-98$ water years } \\
\hline & & & $\begin{array}{l}\text { Period of } \\
\text { record } \\
\text { (water } \\
\text { years) }\end{array}$ & Water year & $\begin{array}{l}\text { Stage } \\
(\mathrm{ft})\end{array}$ & $\begin{array}{l}\text { Discharge } \\
\left(\mathrm{ft}^{3} / \mathrm{s}\right)\end{array}$ & $\begin{array}{c}\text { Date } \\
\text { (month/ } \\
\text { day/ } \\
\text { year) }\end{array}$ & $\begin{array}{l}\text { Stage } \\
(\mathrm{ft})\end{array}$ & $\begin{array}{l}\text { Discharge } \\
\left(\mathrm{ft}^{3} / \mathrm{s}\right)\end{array}$ & $\begin{array}{l}\text { Regulated } \\
\text { during } \\
\text { flood }^{1}\end{array}$ & $\begin{array}{c}\text { Recurrence } \\
\text { interval } \\
\text { (years) }\end{array}$ \\
\hline 07037500 & $\begin{array}{l}\text { Saint Francis River near Patterson, } \\
\text { MO }\end{array}$ & 956 & $\begin{array}{l}1915, \\
1921-75, \\
1977-97\end{array}$ & 1983 & 35.77 & 155,000 & $11 / 15 / 93$ & 34.30 & 133,000 & $\mathrm{~N}$ & $100-500$ \\
\hline 07057500 & $\begin{array}{l}\text { North Fork River near Tecumseh, } \\
\text { MO }\end{array}$ & 561 & $1945-98$ & 1986 & 28.10 & 133,000 & $11 / 14 / 93$ & 24.59 & 71,200 & $\mathrm{~N}$ & 100 \\
\hline 07061500 & Black River near Annapolis, MO & 484 & 1939-98 & 1994 & 27.38 & 109,000 & $11 / 14 / 93$ & 27.38 & 109,000 & $\mathrm{~N}$ & $>500$ \\
\hline 07066000 & Jacks Fork at Eminence, MO & 398 & $\begin{array}{l}\text { 1895, 1904, } \\
1922-98\end{array}$ & $\begin{array}{l}1994 \\
1904 \\
1895\end{array}$ & $\begin{array}{l}17.82 \\
27.00 \\
27.00\end{array}$ & $\begin{array}{r}58,500 \\
-- \\
--\end{array}$ & $11 / 15 / 93$ & 17.82 & 58,500 & $\mathrm{~N}$ & $100-500$ \\
\hline 07067000 & Current River at Van Buren, MO & 1,667 & $\begin{array}{l}1904, \\
1913-91, \\
1993-98\end{array}$ & $\begin{array}{l}1915 \\
1904\end{array}$ & $\begin{array}{l}25.90 \\
29.00\end{array}$ & $\begin{array}{r}125,000 \\
--\end{array}$ & $11 / 15 / 93$ & 27.39 & 92,900 & $\mathrm{~N}$ & $10-25$ \\
\hline 07189000 & Elk River near Tiff City, MO & 872 & 1940-98 & 1941 & 28.40 & 137,000 & $6 / 11 / 95$ & 24.45 & 69,900 & $\mathrm{~N}$ & 25 \\
\hline
\end{tabular}

${ }^{1}$ Regulated during flood: $\mathrm{N}$, no; Y, yes. 


\section{Montana}

As much as 11 inches of rain fell during a 24-hour period in southern Canada and northwestern Montana along the Continental Divide, June 6, 1995 (National Oceanic and Atmospheric Administration, 1995a). As a result, flooding occurred on both sides of the divide. Significant floods occurred on the North Fork Flathead River (fig. 38) as well as many of the smaller rivers and streams of northwestern Montana (table 27).

Warmer temperatures during February 7-11, 1996, after an extended cold and snowy period, caused numerous flooding problems across central Montana during the second week of February. Snowmelt over frozen ground caused streams and rivers to go out of their banks, washing out roads and bridges. Flooding problems ranged from minor inundation of fields to parts of towns being underwater. Ice jams also caused flooding problems as snowmelt ran into frozen streams and rivers. Some of the worst flooding occurred in East Helena where three creeks went out of their banks. Ice jams on the Missouri River caused flooding in Fort Benton. Much of the town was under 1 to 2 feet of water at one point. Seven counties were declared Federal disaster areas. A rapid warming of temperatures, after an extended cold, snowy period, caused ice jams and flooding problems across north-central Montana on March 3-5, 1996. Many small streams went out of their banks, washing out numerous roads. The estimated damage across the region totaled about $\$ 1.5$ million, mostly from washed out roads and bridges (National Oceanic and Atmospheric Administration, 1996b).
Snowmelt flooding caused numerous road closings and road washouts throughout western Montana in May and June 1996. Flooding on the Bitterroot River caused the bridge near Victor to move 6 inches downstream and drop 23 inches. Estimated repair to fix the bridge was $\$ 1$ million, and total damage from the flooding was more than $\$ 2$ million (National Oceanic and Atmospheric Administration, 1996b).

The spring snowmelt for 1997 caused flooding once again in the mountains of Montana. Damage exceeded \$2.5 million (National Oceanic and Atmospheric Administration, 1997b).

Rain falling at the rate of more than 5 inches per hour inundated the town of Culbertson in eastern Montana on July 4, 1998 (National Oceanic and Atmospheric Administration, 1997a). Forty-three homes sustained damage with 20 basements completely filled with water and sewage that backed up during the storm. Two homes were moved completely off of their foundations. Highway 2 was flooded and closed from Poplar to the North Dakota line until the next morning. Damage was more than \$2 million (National Oceanic and Atmospheric Administration, 1997b).

\section{References}

National Oceanic and Atmospheric Administration (NOAA), 1995a-97a, Climatological data (by State): Asheville, North Carolina, National Climatic Data Center, various months.

National Oceanic and Atmospheric Administration (NOAA), 1995b-97b, Storm data (by State): Asheville, North Carolina, National Climatic Data Center, various months. 


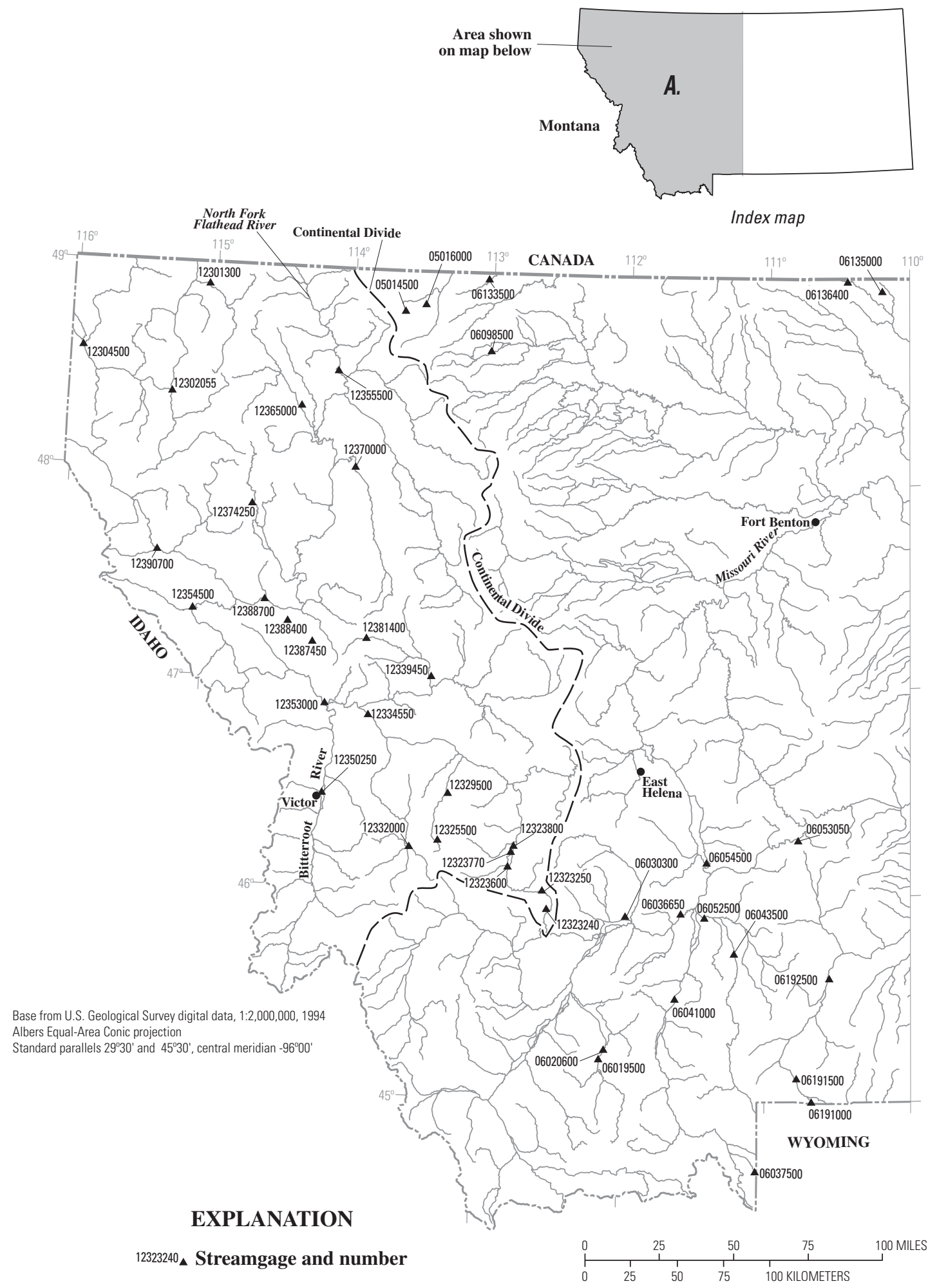

Figure 38. (A) Location of streamgages with significant floods during 1994-98 water years for Montana. 

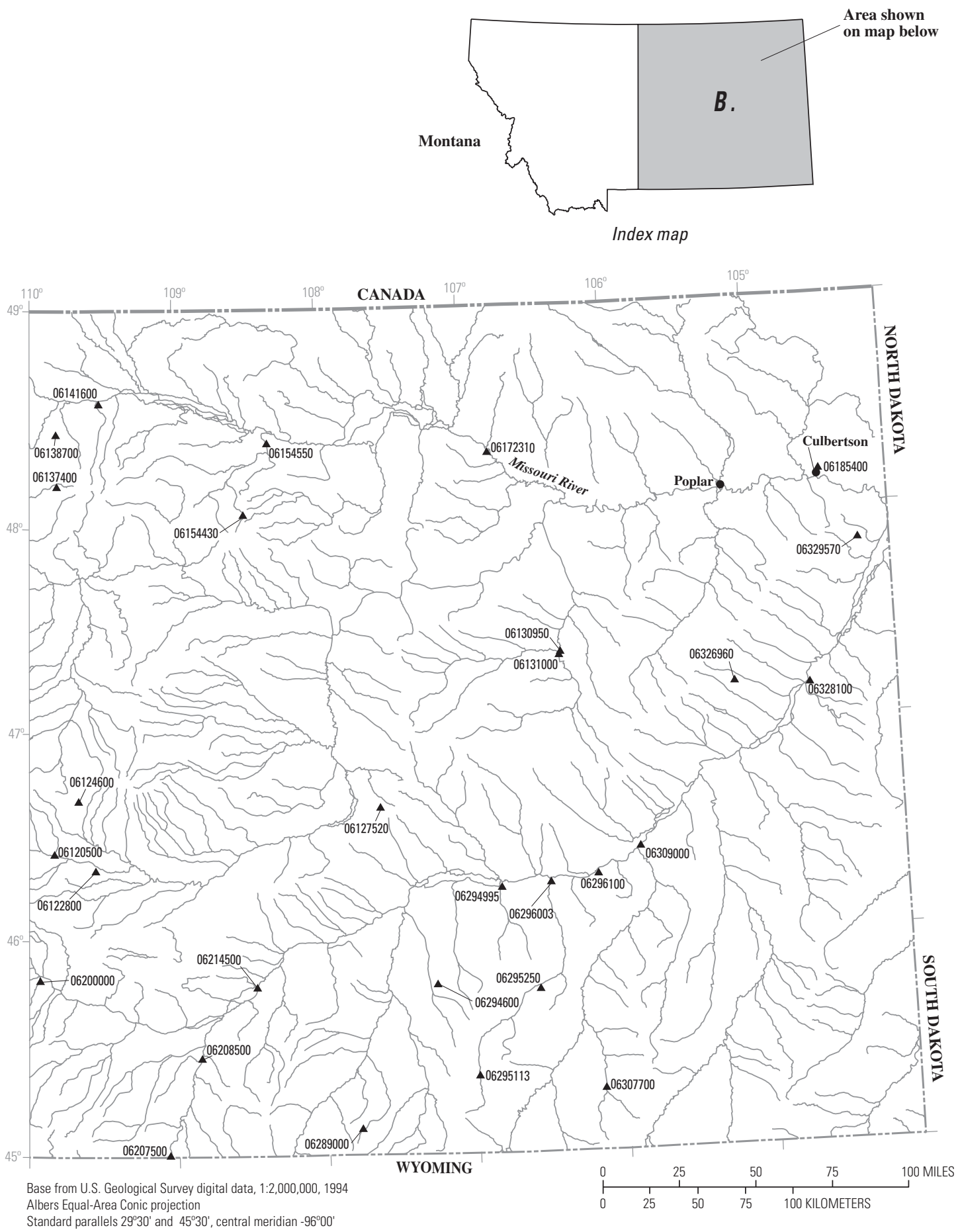

EXPLANATION

06207500 Streamgage and number

Figure 38. (B) Location of streamgages with significant floods during 1994-98 water years for Montana.-Continued 
Table 27. Maximum stage and discharge for period of record for streamgages having significant floods during 1994-98 water years in Montana.

$\left[\mathrm{mi}^{2}\right.$, square miles; $\mathrm{ft}$, feet above an arbitrary datum; $\mathrm{ft}^{3} / \mathrm{s}$, cubic feet per second; --, not determined or not applicable; >, greater than. Source: Recurrence intervals calculated from U.S. Geological Survey data. Other data from U.S. Geological Survey reports or databases]

\begin{tabular}{|c|c|c|c|c|c|c|c|c|c|c|c|}
\hline \multirow[b]{2}{*}{$\begin{array}{c}\text { Streamgage } \\
\text { number } \\
\text { (fig. 38) }\end{array}$} & \multirow[b]{2}{*}{ Streamgage name } & \multirow[b]{2}{*}{$\begin{array}{c}\text { Total } \\
\text { drainage } \\
\left(\mathrm{mi}^{2}\right)\end{array}$} & \multicolumn{4}{|c|}{$\begin{array}{c}\text { Maximum stage and discharge for period of record } \\
\text { through } 1998 \text { water year }\end{array}$} & \multicolumn{5}{|c|}{ Significant floods $1994-98$ water years } \\
\hline & & & $\begin{array}{l}\text { Period of } \\
\text { record } \\
\text { (water } \\
\text { years) }\end{array}$ & Water year & $\begin{array}{l}\text { Stage } \\
(\mathrm{ft})\end{array}$ & $\begin{array}{l}\text { Discharge } \\
\left(\mathrm{ft}^{3} / \mathrm{s}\right)\end{array}$ & $\begin{array}{c}\text { Date } \\
\text { (month/ } \\
\text { day/ } \\
\text { year) }\end{array}$ & $\begin{array}{l}\text { Stage } \\
(\mathrm{ft})\end{array}$ & $\begin{array}{l}\text { Discharge } \\
\left(\mathrm{ft}^{3} / \mathrm{s}\right)\end{array}$ & $\begin{array}{c}\text { Regulated } \\
\text { during } \\
\text { flood }^{1}\end{array}$ & $\begin{array}{c}\text { Recurrence } \\
\text { interval } \\
\text { (years) }\end{array}$ \\
\hline 05014500 & $\begin{array}{l}\text { Swiftcurrent Creek at Many } \\
\text { Glacier, MT }\end{array}$ & 30.9 & $1913-98$ & 1964 & 10.00 & 6,700 & $6 / 7 / 95$ & 7.72 & 3,150 & $\mathrm{~N}$ & $45-50$ \\
\hline 05016000 & $\begin{array}{l}\text { Swiftcurrent Creek at Lake } \\
\text { Sherberne, MT }\end{array}$ & 64.6 & $1913-98$ & 1995 & 8.63 & 2,510 & $6 / 7 / 95$ & 8.63 & 2,510 & $\mathrm{~N}$ & -- \\
\hline 06019500 & $\begin{array}{l}\text { Ruby River above reservoir near } \\
\text { Alder, MT }\end{array}$ & 538 & 1939-98 & 1984 & 6.24 & 3,810 & $6 / 6 / 95$ & 5.14 & 2,060 & $\mathrm{~N}$ & $30-35$ \\
\hline 06020600 & $\begin{array}{l}\text { Ruby River below reservoir near } \\
\text { Alder, MT }\end{array}$ & 596 & $1963-98$ & 1984 & 8.52 & 3,010 & $6 / 7 / 95$ & 6.85 & 1,820 & $\mathrm{Y}$ & -- \\
\hline 06030300 & $\begin{array}{l}\text { Jefferson River tributary number } 2 \\
\text { near Whitehall, MT }\end{array}$ & 4.50 & 1958-98 & 1994 & 8.30 & 425 & $7 / 6 / 94$ & 8.30 & 425 & $\mathrm{~N}$ & $>100$ \\
\hline 06036650 & $\begin{array}{l}\text { Jefferson River near Three Forks, } \\
\text { MT }\end{array}$ & 9,532 & 1979-98 & $\begin{array}{l}1995 \\
1997\end{array}$ & $\begin{array}{l}9.00 \\
9.20\end{array}$ & $\begin{array}{l}17,000 \\
16,700\end{array}$ & $6 / 9 / 95$ & 9.00 & 17,000 & $\mathrm{~N}$ & $10-25$ \\
\hline 06037500 & $\begin{array}{l}\text { Madison River near West } \\
\text { Yellowstone, MT }\end{array}$ & 420 & $\begin{array}{l}1914-17, \\
1919-73, \\
1984-86, \\
1989-98\end{array}$ & 1996 & 3.78 & 2,820 & $\begin{array}{l}5 / 18 / 96 \\
5 / 18 / 97\end{array}$ & $\begin{array}{l}3.78 \\
3.58\end{array}$ & $\begin{array}{l}2,820 \\
2,630\end{array}$ & $\begin{array}{l}\mathrm{N} \\
\mathrm{N}\end{array}$ & $\begin{array}{r}>100 \\
75\end{array}$ \\
\hline 06041000 & $\begin{array}{l}\text { Madison River below Ennis Lake } \\
\text { near McAllister, MT }\end{array}$ & 2,186 & $1943-98$ & 1970 & 8.01 & 9,550 & $6 / 10 / 96$ & 7.60 & 7,980 & $\mathrm{Y}$ & -- \\
\hline 06043500 & $\begin{array}{l}\text { Gallatin River near Gallatin } \\
\text { Gateway, MT }\end{array}$ & 825 & $\begin{array}{l}\text { 1890-94, } \\
1931-81 \\
1985-98\end{array}$ & $\begin{array}{l}1971 \\
1974\end{array}$ & $\begin{array}{l}6.49 \\
7.38\end{array}$ & $\begin{array}{l}9,270 \\
9,100\end{array}$ & $6 / 2 / 97$ & 6.71 & 9,160 & $\mathrm{~N}$ & $25-30$ \\
\hline 06052500 & Gallatin River at Logan, MT & 1,795 & $\begin{array}{l}1895-1900, \\
1902-05, \\
1929-33, \\
1935-98\end{array}$ & $\begin{array}{l}1899 \\
1997\end{array}$ & $-\overline{-}+80$ & $\begin{array}{l}9,840 \\
9,400\end{array}$ & $6 / 8 / 97$ & 9.80 & 9,400 & $\mathrm{~N}$ & $30-35$ \\
\hline
\end{tabular}


Table 27. Maximum stage and discharge for period of record for streamgages having significant floods during 1994-98 water years in Montana.-Continued

$\left[\mathrm{mi}^{2}\right.$, square miles; ft, feet above an arbitrary datum; $\mathrm{ft}^{3} / \mathrm{s}$, cubic feet per second; --, not determined or not applicable; >, greater than. Source: Recurrence intervals calculated from U.S. Geological Survey data. Other data from U.S. Geological Survey reports or databases]

\begin{tabular}{|c|c|c|c|c|c|c|c|c|c|c|c|}
\hline \multirow{2}{*}{$\begin{array}{c}\text { Streamgage } \\
\text { number } \\
\text { (fig. 38) }\end{array}$} & \multirow[b]{2}{*}{ Streamgage name } & \multirow{2}{*}{$\begin{array}{c}\text { Total } \\
\text { drainage } \\
\left(\mathrm{mi}^{2}\right)\end{array}$} & \multicolumn{4}{|c|}{$\begin{array}{c}\text { Maximum stage and discharge for period of record } \\
\text { through } 1998 \text { water year }\end{array}$} & \multicolumn{5}{|c|}{ Significant floods 1994-98 water years } \\
\hline & & & $\begin{array}{l}\text { Period of } \\
\text { record } \\
\text { (water } \\
\text { years) }\end{array}$ & Water year & $\begin{array}{l}\text { Stage } \\
(\mathrm{ft})\end{array}$ & $\begin{array}{l}\text { Discharge } \\
\left(\mathrm{ft}^{3} / \mathrm{s}\right)\end{array}$ & $\begin{array}{c}\text { Date } \\
\text { (month/ } \\
\text { day/ } \\
\text { year) }\end{array}$ & $\begin{array}{l}\text { Stage } \\
(\mathrm{ft})\end{array}$ & $\begin{array}{l}\text { Discharge } \\
\left(\mathrm{ft}^{3} / \mathrm{s}\right)\end{array}$ & $\begin{array}{l}\text { Regulated } \\
\text { during } \\
\text { flood }^{1}\end{array}$ & $\begin{array}{c}\text { Recurrence } \\
\text { interval } \\
\text { (years) }\end{array}$ \\
\hline 06053050 & Lost Creek near Ringling, MT & 9.59 & $1974-98$ & $\begin{array}{l}1996 \\
1986\end{array}$ & $\begin{array}{l}3.62 \\
3.98\end{array}$ & $\begin{array}{r}300 \\
80\end{array}$ & $2 / 8 / 96$ & 3.62 & 300 & $\mathrm{~N}$ & 25 \\
\hline 06054500 & Missouri River at Toston, MT & 14,669 & $\begin{array}{l}1890, \\
1910-16, \\
1941-98\end{array}$ & 1997 & 12.22 & 34,000 & $6 / 12 / 97$ & 12.22 & 34,000 & $\mathrm{~N}$ & $45-50$ \\
\hline 06098500 & $\begin{array}{l}\text { Cut Bank Creek near Browning, } \\
\text { MT }\end{array}$ & 123 & $\begin{array}{l}1918, \\
1920-24, \\
1991-98\end{array}$ & 1995 & 5.59 & 5,480 & $6 / 7 / 95$ & 5.59 & 5,480 & $\mathrm{~N}$ & $25-50$ \\
\hline 06120500 & $\begin{array}{l}\text { Musselshell River at Harlowton, } \\
\text { MT }\end{array}$ & 1,125 & 1909-98 & 1975 & 10.01 & 7,270 & $6 / 12 / 97$ & 8.25 & 3,720 & $\mathrm{~N}$ & 20 \\
\hline 06122800 & $\begin{array}{l}\text { Musselshell River near Shawmut, } \\
\text { MT }\end{array}$ & 1,479 & $1986-97$ & $\begin{array}{l}1997 \\
1991\end{array}$ & $-\overline{-}-65$ & $\begin{array}{l}5,000 \\
3,020\end{array}$ & $6 / 12 / 97$ & -- & 5,000 & $\mathrm{~N}$ & -- \\
\hline 06124600 & $\begin{array}{l}\text { East Fork Roberts Creek tributary } \\
\text { near Judith Gap, MT }\end{array}$ & .74 & 1974-98 & $\begin{array}{l}1997 \\
1974\end{array}$ & $\begin{array}{l}3.87 \\
3.89\end{array}$ & $\begin{array}{l}83 \\
82\end{array}$ & $7 / 20 / 97$ & 3.87 & 83 & $\mathrm{~N}$ & $10-20$ \\
\hline 06127520 & Home Creek near Sumatra, MT & 1.98 & 1973-98 & 1994 & 5.11 & 278 & $3 / 2 / 94$ & 5.11 & 278 & $\mathrm{~N}$ & $25-30$ \\
\hline 06130950 & $\begin{array}{l}\text { Little Dry Creek near Van Norman, } \\
\text { MT }\end{array}$ & 1,224 & $\begin{array}{l}1958-75 \\
1986,1995\end{array}$ & 1995 & 9.20 & 10,000 & --/--/95 & 9.20 & 10,000 & $\mathrm{~N}$ & $>100$ \\
\hline 06131000 & $\begin{array}{l}\text { Big Dry Creek near Van Norman, } \\
\text { MT }\end{array}$ & 2,554 & $\begin{array}{l}1940-48 \\
1950-98\end{array}$ & 1947 & 13.39 & 24,600 & $3 / 3 / 94$ & 10.90 & 15,600 & $\mathrm{~N}$ & $15-20$ \\
\hline
\end{tabular}


$\left[\mathrm{mi}^{2}\right.$, square miles; ft, feet above an arbitrary datum; $\mathrm{ft}^{3} / \mathrm{s}$, cubic feet per second; --, not determined or not applicable; >, greater than. Source: Recurrence intervals calculated from U.S. Geological Survey data. Other data from U.S. Geological Survey reports or databases]

\begin{tabular}{|c|c|c|c|c|c|c|c|c|c|c|c|}
\hline \multirow{2}{*}{$\begin{array}{c}\text { Streamgage } \\
\text { number } \\
\text { (fig. 38) }\end{array}$} & \multirow[b]{2}{*}{ Streamgage name } & \multirow{2}{*}{$\begin{array}{c}\text { Total } \\
\text { drainage } \\
\left(\mathrm{mi}^{2}\right)\end{array}$} & \multicolumn{4}{|c|}{$\begin{array}{c}\text { Maximum stage and discharge for period of record } \\
\text { through } 1998 \text { water year }\end{array}$} & \multicolumn{5}{|c|}{ Significant floods $1994-98$ water years } \\
\hline & & & $\begin{array}{c}\text { Period of } \\
\text { record } \\
\text { (water } \\
\text { years) }\end{array}$ & Water year & $\begin{array}{l}\text { Stage } \\
(\mathrm{ft})\end{array}$ & $\begin{array}{l}\text { Discharge } \\
\left(\mathrm{ft}^{3} / \mathrm{s}\right)\end{array}$ & $\begin{array}{c}\text { Date } \\
\text { (month/ } \\
\text { day/ } \\
\text { year) }\end{array}$ & $\begin{array}{l}\text { Stage } \\
\text { (ft) }\end{array}$ & $\begin{array}{l}\text { Discharge } \\
\left(\mathrm{ft}^{3} / \mathrm{s}\right)\end{array}$ & $\begin{array}{l}\text { Regulated } \\
\text { during } \\
\text { flood }^{1}\end{array}$ & $\begin{array}{c}\text { Recurrence } \\
\text { interval } \\
\text { (years) }\end{array}$ \\
\hline 06133500 & $\begin{array}{l}\text { North Fork Milk River above Saint } \\
\text { Mary Canal near Browning, MT }\end{array}$ & 59.0 & $\begin{array}{l}1911-12, \\
1924, \\
1926-27, \\
1937, \\
1941-42, \\
1944-45, \\
1948, \\
1950-51, \\
1953-98\end{array}$ & 1967 & 7.95 & 3,090 & $3 / 19 / 97$ & 10.50 & 2,860 & $\mathrm{~N}$ & $35-40$ \\
\hline 06135000 & $\begin{array}{l}\text { Milk River at eastern crossing of } \\
\text { international boundary, MT }\end{array}$ & 2,506 & $\begin{array}{l}\text { 1910-11, } \\
\text { 1913-15, } \\
\text { 1917, } \\
\text { 1919-98 }\end{array}$ & 1996 & 15.03 & 12,000 & $\begin{array}{l}3 / 13 / 96 \\
3 / 22 / 97\end{array}$ & $\begin{array}{l}15.03 \\
11.36\end{array}$ & $\begin{array}{l}12,000 \\
10,000\end{array}$ & $\begin{array}{l}\mathrm{N} \\
\mathrm{N}\end{array}$ & $\begin{array}{l}25-50 \\
10-25\end{array}$ \\
\hline 06136400 & $\begin{array}{l}\text { Spring Coulee tributary near } \\
\text { Simpson, MT }\end{array}$ & 2.49 & $\begin{array}{l}\text { 1972, } \\
1974-98\end{array}$ & 1997 & 4.29 & 56 & $2 / 17 / 97$ & 4.29 & 56 & $\mathrm{~N}$ & -- \\
\hline 06137400 & $\begin{array}{l}\text { Big Sandy Creek at reservation } \\
\text { boundary near Rocky Boy, MT }\end{array}$ & 24.7 & $1982-98$ & 1998 & 6.07 & 510 & $6 / 27 / 98$ & 6.07 & 510 & $\mathrm{~N}$ & 25 \\
\hline 06138700 & $\begin{array}{l}\text { South Fork Spring Coulee near } \\
\text { Havre, MT }\end{array}$ & 6.47 & $1960-98$ & $\begin{array}{l}1966 \\
1982\end{array}$ & $\begin{array}{l}4.42 \\
4.70\end{array}$ & $\begin{array}{l}190 \\
145\end{array}$ & $3 / 10 / 96$ & 4.24 & 170 & $\mathrm{~N}$ & $10-25$ \\
\hline 06141600 & $\begin{array}{l}\text { Little Box Elder Creek at mouth } \\
\text { near Havre, MT }\end{array}$ & 95.9 & $\begin{array}{l}\text { 1986-92, } \\
1994-96\end{array}$ & 1996 & 10.30 & 960 & $3 / 11 / 96$ & 10.30 & 960 & $\mathrm{~N}$ & $10-25$ \\
\hline 06154430 & $\begin{array}{l}\text { Lodge Pole Creek at Lodge Pole, } \\
\text { MT }\end{array}$ & 19.5 & 1987-98 & 1997 & 6.02 & 306 & $5 / 26 / 97$ & 6.02 & 306 & $\mathrm{~N}$ & $25-50$ \\
\hline 06154550 & $\begin{array}{l}\text { Peoples Creek below Kuhr Coulee } \\
\text { near Dodson, MT }\end{array}$ & 675 & 1989-98 & 1996 & 13.03 & 1,800 & 3/13/96 & 13.03 & 1,800 & $\mathrm{~N}$ & $5-10$ \\
\hline 06172310 & Milk River at Tampico, MT & 21,078 & $\begin{array}{l}\text { 1974-77, } \\
1988-98\end{array}$ & $\begin{array}{l}1997 \\
1991\end{array}$ & $\overline{--}$ & $\begin{array}{r}11,000 \\
7,240\end{array}$ & $3 / 27 / 97$ & -- & 11,000 & $\mathrm{~N}$ & $5-10$ \\
\hline
\end{tabular}


Table 27. Maximum stage and discharge for period of record for streamgages having significant floods during 1994-98 water years in Montana.-Continued

$\left[\mathrm{mi}^{2}\right.$, square miles; $\mathrm{ft}$, feet above an arbitrary datum; $\mathrm{ft}^{3} / \mathrm{s}$, cubic feet per second; --, not determined or not applicable; >, greater than. Source: Recurrence intervals calculated from U.S. Geological Survey data. Other data from U.S. Geological Survey reports or databases]

\begin{tabular}{|c|c|c|c|c|c|c|c|c|c|c|c|}
\hline \multirow[b]{2}{*}{$\begin{array}{c}\text { Streamgage } \\
\text { number } \\
\text { (fig. 38) }\end{array}$} & \multirow[b]{2}{*}{ Streamgage name } & \multirow{2}{*}{$\begin{array}{c}\text { Total } \\
\text { drainage } \\
\left(\mathrm{mi}^{2}\right)\end{array}$} & \multicolumn{4}{|c|}{$\begin{array}{c}\text { Maximum stage and discharge for period of record } \\
\text { through } 1998 \text { water year }\end{array}$} & \multicolumn{5}{|c|}{ Significant floods 1994-98 water years } \\
\hline & & & $\begin{array}{l}\text { Period of } \\
\text { record } \\
\text { (water } \\
\text { years) }\end{array}$ & Water year & $\begin{array}{l}\text { Stage } \\
(\mathrm{ft})\end{array}$ & $\begin{array}{l}\text { Discharge } \\
\left(\mathrm{ft}^{3} / \mathrm{s}\right)\end{array}$ & $\begin{array}{c}\text { Date } \\
\text { (month/ } \\
\text { day/ } \\
\text { year) }\end{array}$ & $\begin{array}{l}\text { Stage } \\
(\mathrm{ft})\end{array}$ & $\begin{array}{l}\text { Discharge } \\
\left(\mathrm{ft}^{3} / \mathrm{s}\right)\end{array}$ & $\begin{array}{c}\text { Regulated } \\
\text { during } \\
\text { flood }^{1}\end{array}$ & $\begin{array}{c}\text { Recurrence } \\
\text { interval } \\
\text { (years) }\end{array}$ \\
\hline 06185400 & $\begin{array}{l}\text { Missouri River tributary number } 5 \\
\text { at Culbertson, MT }\end{array}$ & 3.67 & $1963-98$ & 1998 & 12.87 & 3,790 & $7 / 4 / 98$ & 12.87 & 3,790 & $\mathrm{~N}$ & $>100$ \\
\hline 06191000 & $\begin{array}{l}\text { Gardner River near Mammoth } \\
\text { Yellowstone National Park, MT }\end{array}$ & 202 & $\begin{array}{l}1939-72 \\
1984-98\end{array}$ & $\begin{array}{l}1956 \\
1997\end{array}$ & $\begin{array}{l}4.46 \\
5.03\end{array}$ & $\begin{array}{l}2,080 \\
2,050\end{array}$ & $6 / 2 / 97$ & 5.03 & 2,050 & $\mathrm{~N}$ & 25 \\
\hline 06191500 & $\begin{array}{l}\text { Yellowstone River at Corwin } \\
\text { Springs, MT }\end{array}$ & 2,623 & $\begin{array}{l}1890-93 \\
1911-98\end{array}$ & $\begin{array}{l}1996 \\
1918\end{array}$ & $\begin{array}{l}10.92 \\
11.50\end{array}$ & $\begin{array}{l}32,200 \\
32,000\end{array}$ & $\begin{array}{l}6 / 10 / 96 \\
6 / 6 / 97\end{array}$ & $\begin{array}{l}10.92 \\
10.91\end{array}$ & $\begin{array}{l}32,200 \\
32,200\end{array}$ & $\begin{array}{l}\mathrm{N} \\
\mathrm{N}\end{array}$ & $\begin{array}{l}100 \\
100\end{array}$ \\
\hline 06192500 & $\begin{array}{l}\text { Yellowstone River near } \\
\text { Livingston, MT }\end{array}$ & 3,551 & $\begin{array}{l}\text { 1897-1905, } \\
\text { 1929-32, } \\
1938-98\end{array}$ & 1997 & 10.72 & 38,000 & $\begin{array}{l}6 / 10 / 96 \\
6 / 6 / 97\end{array}$ & $\begin{array}{r}9.97 \\
10.72\end{array}$ & $\begin{array}{l}37,100 \\
38,000\end{array}$ & $\begin{array}{l}\mathrm{N} \\
\mathrm{N}\end{array}$ & $\begin{array}{l}75-100 \\
75-100\end{array}$ \\
\hline 06200000 & Boulder River at Big Timber, MT & 523 & $\begin{array}{l}1947-53 \\
1955-98\end{array}$ & 1997 & 9.00 & 9,940 & $6 / 5 / 97$ & 9.00 & 9,940 & $\mathrm{~N}$ & 50 \\
\hline 06207500 & $\begin{array}{l}\text { Clarks ForkYellowstone River } \\
\text { near Belfry, MT }\end{array}$ & 1,154 & $1922-98$ & 1981 & 9.97 & 14,800 & $\begin{array}{l}6 / 11 / 96 \\
6 / 11 / 97\end{array}$ & $\begin{array}{l}8.98 \\
8.68\end{array}$ & $\begin{array}{l}11,400 \\
10,900\end{array}$ & $\begin{array}{l}\mathrm{N} \\
\mathrm{N}\end{array}$ & $\begin{array}{r}25 \\
10-25\end{array}$ \\
\hline 06208500 & $\begin{array}{l}\text { Clarks Fork Yellowstone River at } \\
\text { Edgar, MT }\end{array}$ & 2,032 & $\begin{array}{l}1922-32, \\
1934-69, \\
1987-98\end{array}$ & 1997 & 9.30 & 11,100 & $\begin{array}{l}6 / 15 / 96 \\
6 / 12 / 97\end{array}$ & $\begin{array}{l}9.19 \\
9.30\end{array}$ & $\begin{array}{l}11,000 \\
11,100\end{array}$ & $\begin{array}{l}\mathrm{N} \\
\mathrm{N}\end{array}$ & $\begin{array}{r}10-25 \\
25\end{array}$ \\
\hline 06214500 & Yellowstone River at Billings, MT & 11,795 & $\begin{array}{l}\text { 1904-05, } \\
1918, \\
1929-98\end{array}$ & 1997 & 15.00 & 82,000 & $6 / 12 / 97$ & 15.00 & 82,000 & $\mathrm{~N}$ & $>100$ \\
\hline 06289000 & $\begin{array}{l}\text { Little Bighorn River at State line } \\
\text { near Wyola, MT }\end{array}$ & 193 & 1939-98 & 1944 & 4.87 & 2,730 & $6 / 6 / 97$ & 4.35 & 2,100 & $\mathrm{~N}$ & $10-25$ \\
\hline 06294600 & $\begin{array}{l}\text { East Cabin Creek tributary near } \\
\text { Hardin, MT }\end{array}$ & 8.63 & 1973-98 & 1998 & 5.70 & 277 & $6 / 8 / 98$ & 5.70 & 277 & $\mathrm{~N}$ & $25-50$ \\
\hline 06294995 & Armells Creek near Forsyth, MT & 370 & $\begin{array}{l}1975-84 \\
1988-95\end{array}$ & 1994 & 10.25 & 3,990 & $3 / 3 / 94$ & 10.25 & 3,990 & $\mathrm{~N}$ & 25 \\
\hline
\end{tabular}


$\left[\mathrm{mi}^{2}\right.$, square miles; ft, feet above an arbitrary datum; $\mathrm{ft}^{3} / \mathrm{s}$, cubic feet per second; --, not determined or not applicable; >, greater than. Source: Recurrence intervals calculated from U.S. Geological Survey data. Other data from U.S. Geological Survey reports or databases]

\begin{tabular}{|c|c|c|c|c|c|c|c|c|c|c|c|}
\hline \multirow{2}{*}{$\begin{array}{l}\text { Streamgage } \\
\text { number } \\
\text { (fig. 38) }\end{array}$} & \multirow[b]{2}{*}{ Streamgage name } & \multirow{2}{*}{$\begin{array}{c}\text { Total } \\
\text { drainage } \\
\left(\mathrm{mi}^{2}\right)\end{array}$} & \multicolumn{4}{|c|}{$\begin{array}{l}\text { Maximum stage and discharge for period of record } \\
\text { through } 1998 \text { water year }\end{array}$} & \multicolumn{5}{|c|}{ Significant floods 1994-98 water years } \\
\hline & & & $\begin{array}{l}\text { Period of } \\
\text { record } \\
\text { (water } \\
\text { years) }\end{array}$ & Water year & $\begin{array}{l}\text { Stage } \\
(\mathrm{ft})\end{array}$ & $\begin{array}{l}\text { Discharge } \\
\left(\mathrm{ft}^{3} / \mathrm{s}\right)\end{array}$ & $\begin{array}{c}\text { Date } \\
\text { (month/ } \\
\text { day/ } \\
\text { year) }\end{array}$ & $\begin{array}{l}\text { Stage } \\
\text { (ft) }\end{array}$ & $\begin{array}{l}\text { Discharge } \\
\left(\mathrm{ft}^{3} / \mathrm{s}\right)\end{array}$ & $\begin{array}{l}\text { Regulated } \\
\text { during } \\
\text { flood }^{1}\end{array}$ & $\begin{array}{c}\text { Recurrence } \\
\text { interval } \\
\text { (years) }\end{array}$ \\
\hline 06295113 & $\begin{array}{l}\text { Rosebud Creek at reservation } \\
\text { boundary near Kirby, MT }\end{array}$ & 123 & 1980-98 & $\begin{array}{l}1996 \\
1986\end{array}$ & $\begin{array}{l}6.30 \\
6.50\end{array}$ & $\begin{array}{r}219 \\
--\end{array}$ & $3 / 17 / 96$ & 6.30 & 219 & $\mathrm{~N}$ & $25-50$ \\
\hline 06295250 & Rosebud Creek near Colstrip, MT & 799 & $1975-98$ & $\begin{array}{l}1994 \\
1978\end{array}$ & $\begin{array}{l}8.42 \\
9.03\end{array}$ & $\begin{array}{l}754 \\
605\end{array}$ & $3 / 2 / 94$ & 8.42 & 754 & $\mathrm{~N}$ & $25-50$ \\
\hline 06296003 & $\begin{array}{l}\text { Rosebud Creek at mouth near } \\
\text { Rosebud, MT }\end{array}$ & 1,302 & 1975-98 & 1994 & 7.70 & 3,700 & $3 / 4 / 94$ & 7.70 & 3,700 & $\mathrm{~N}$ & $50-100$ \\
\hline 06296100 & Snell Creek near Hathaway, MT & 10.5 & $\begin{array}{l}1963-77, \\
1979, \\
1982-98\end{array}$ & 1994 & 3.70 & 767 & $3 / 2 / 94$ & 3.70 & 767 & $\mathrm{~N}$ & $10-25$ \\
\hline 06307700 & $\begin{array}{l}\text { Cow Creek near Fort Howes } \\
\text { Ranger Station near Otter, MT }\end{array}$ & 8.37 & $1972-98$ & 1995 & 9.21 & 296 & $7 / 15 / 95$ & 9.21 & 296 & $\mathrm{~N}$ & $25-50$ \\
\hline 06309000 & $\begin{array}{l}\text { Yellowstone River at Miles City, } \\
\text { MT }\end{array}$ & 48,253 & $\begin{array}{l}\text { 1923, } \\
1929-98\end{array}$ & $\begin{array}{l}1978 \\
1979\end{array}$ & $\begin{array}{l}16.50 \\
16.62\end{array}$ & $\begin{array}{r}102,000 \\
53,900\end{array}$ & $6 / 15 / 97$ & 14.53 & 83,300 & $\mathrm{~N}$ & $10-25$ \\
\hline 06326960 & $\begin{array}{l}\text { Timber Fork Upper Sevenmile } \\
\text { Creek tributary near Lindsay, MT }\end{array}$ & 1.13 & 1974-98 & 1997 & 10.42 & 250 & $7 / 1 / 97$ & 10.42 & 250 & $\mathrm{~N}$ & $25-50$ \\
\hline 06328100 & $\begin{array}{l}\text { Yellowstone River tributary } \\
\text { number } 6 \text { near Glendive, MT }\end{array}$ & 2.93 & 1974-98 & 1994 & 5.88 & 576 & $6 / 7 / 94$ & 5.88 & 576 & $\mathrm{~N}$ & $25-50$ \\
\hline 06329570 & First Hay Creek near Sidney, MT & 29.1 & 1963-98 & 1997 & 6.42 & 3,530 & $7 / 1 / 97$ & 6.42 & 3,530 & $\mathrm{~N}$ & $50-100$ \\
\hline 12301300 & Tobacco River near Eureka, MT & 440 & $\begin{array}{l}1948, \\
1959-98\end{array}$ & 1991 & 7.16 & 3,180 & $4 / 28 / 97$ & 6.82 & 2,830 & $\mathrm{~N}$ & 25 \\
\hline 12302055 & Fisher River near Libby, MT & 838 & $\begin{array}{l}\text { 1948, } \\
1969-98\end{array}$ & 1996 & 10.35 & 12,000 & 2/9/96 & 10.35 & 12,000 & $\mathrm{~N}$ & 100 \\
\hline 12304500 & Yaak River near Troy, MT & 766 & $\begin{array}{l}\text { 1948, 1954, } \\
1956-98\end{array}$ & 1954 & 11.40 & 13,400 & $5 / 17 / 97$ & 9.58 & 12,600 & $\mathrm{~N}$ & $50-100$ \\
\hline 12323240 & Blacktail Creek at Butte, MT & 95.4 & 1989-98 & 1995 & 5.28 & 303 & $2 / 19 / 95$ & 5.28 & 303 & $\mathrm{~N}$ & $10-25$ \\
\hline
\end{tabular}


Table 27. Maximum stage and discharge for period of record for streamgages having significant floods during 1994-98 water years in Montana.-Continued

$\left[\mathrm{mi}^{2}\right.$, square miles; $\mathrm{ft}$, feet above an arbitrary datum; $\mathrm{ft}^{3} / \mathrm{s}$, cubic feet per second; --, not determined or not applicable; >, greater than. Source: Recurrence intervals calculated from U.S. Geological Survey data. Other data from U.S. Geological Survey reports or databases]

\begin{tabular}{|c|c|c|c|c|c|c|c|c|c|c|c|}
\hline \multirow[b]{2}{*}{$\begin{array}{c}\text { Streamgage } \\
\text { number } \\
\text { (fig. 38) }\end{array}$} & \multirow[b]{2}{*}{ Streamgage name } & \multirow[b]{2}{*}{$\begin{array}{c}\text { Total } \\
\text { drainage } \\
\left(\mathrm{mi}^{2}\right)\end{array}$} & \multicolumn{4}{|c|}{$\begin{array}{c}\text { Maximum stage and discharge for period of record } \\
\text { through } 1998 \text { water year }\end{array}$} & \multicolumn{5}{|c|}{ Significant floods 1994-98 water years } \\
\hline & & & $\begin{array}{c}\text { Period of } \\
\text { record } \\
\text { (water } \\
\text { years) }\end{array}$ & Water year & $\begin{array}{l}\text { Stage } \\
\text { (ft) }\end{array}$ & $\begin{array}{l}\text { Discharge } \\
\left(\mathrm{ft}^{3} / \mathrm{s}\right)\end{array}$ & $\begin{array}{c}\text { Date } \\
\text { (month/ } \\
\text { day/ } \\
\text { year) }\end{array}$ & $\begin{array}{l}\text { Stage } \\
\text { (ft) }\end{array}$ & $\begin{array}{l}\text { Discharge } \\
\left(\mathrm{ft}^{3} / \mathrm{s}\right)\end{array}$ & $\begin{array}{c}\text { Regulated } \\
\text { during } \\
\text { flood }^{1}\end{array}$ & $\begin{array}{c}\text { Recurrence } \\
\text { interval } \\
\text { (years) }\end{array}$ \\
\hline 12323250 & $\begin{array}{l}\text { Silver Bow Creek below Blacktail } \\
\text { Creek at Butte, MT }\end{array}$ & 103 & 1984-98 & 1998 & 8.11 & 447 & $7 / 30 / 98$ & 8.11 & 447 & $\mathrm{~N}$ & 50 \\
\hline 12323600 & $\begin{array}{l}\text { Silver Bow Creek at Opportunity, } \\
\text { MT }\end{array}$ & 284 & 1989-98 & 1996 & 6.91 & 1,300 & $2 / 11 / 96$ & 6.91 & 1,300 & $\mathrm{~N}$ & $25-50$ \\
\hline 12323770 & $\begin{array}{l}\text { Warm Springs Creek at Warm } \\
\text { Springs, MT }\end{array}$ & 163 & 1984-98 & $\begin{array}{l}1997 \\
1984\end{array}$ & $\begin{array}{l}4.55 \\
4.61\end{array}$ & $\begin{array}{l}494 \\
462\end{array}$ & $6 / 5 / 97$ & 4.55 & 494 & $\mathrm{~N}$ & $10-25$ \\
\hline 12323800 & Clark Fork near Galen, MT & 572 & 1989-98 & 1997 & 5.07 & 1,240 & $6 / 7 / 97$ & 5.07 & 1,240 & $\mathrm{~N}$ & -- \\
\hline 12325500 & $\begin{array}{l}\text { Flint Creek near Southern Cross, } \\
\text { MT }\end{array}$ & 52.6 & 1941-98 & $\begin{array}{l}1942 \\
1962\end{array}$ & $\begin{array}{l}1.86 \\
4.56\end{array}$ & $\begin{array}{r}174 \\
35\end{array}$ & $6 / 6 / 95$ & 2.53 & 172 & $\mathrm{~N}$ & -- \\
\hline 12329500 & Flint Creek at Maxville, MT & 208 & 1942-98 & $\begin{array}{l}1943 \\
1974\end{array}$ & $\begin{array}{l}6.79 \\
7.49\end{array}$ & $\begin{array}{r}1,680 \\
680\end{array}$ & 2/9/96 & 6.68 & 1,040 & $\mathrm{~N}$ & $10-25$ \\
\hline 12332000 & $\begin{array}{l}\text { Middle Fork Rock Creek near } \\
\text { Philipsburg, MT }\end{array}$ & 123 & $1938-98$ & 1974 & 5.58 & 1,680 & $6 / 9 / 96$ & 5.41 & 1,540 & $\mathrm{~N}$ & $10-25$ \\
\hline 12334550 & $\begin{array}{l}\text { Clark Fork at Turah Bridge near } \\
\text { Bonner, MT }\end{array}$ & 3,641 & 1986-98 & 1996 & 9.05 & 12,400 & 2/9/96 & 9.05 & 12,400 & $\mathrm{~N}$ & $10-25$ \\
\hline 12339450 & $\begin{array}{l}\text { Clearwater River near Clearwater, } \\
\text { MT }\end{array}$ & 345 & $\begin{array}{l}\text { 1975-92, } \\
1997\end{array}$ & 1997 & 8.52 & 3,800 & $5 / 28 / 97$ & 8.52 & 3,800 & $\mathrm{~N}$ & $50-100$ \\
\hline 12350250 & $\begin{array}{l}\text { Bitterroot River at Bell Crossing } \\
\text { near Victor, MT }\end{array}$ & 1,963 & 1987-98 & 1996 & 10.07 & 18,700 & $6 / 9 / 96$ & 10.07 & 18,700 & $\mathrm{~N}$ & 25 \\
\hline 12353000 & Clark Fork below Missoula, MT & 9,003 & 1930-98 & 1997 & 12.18 & 55,100 & $5 / 18 / 97$ & 12.18 & 55,100 & $\mathrm{~N}$ & $25-50$ \\
\hline 12354500 & Clark Fork at Saint Regis, MT & 10,709 & $\begin{array}{l}1911-23 \\
1929-98\end{array}$ & 1997 & 20.27 & 68,900 & $5 / 18 / 97$ & 20.27 & 68,900 & $\mathrm{~N}$ & $25-50$ \\
\hline 12355500 & $\begin{array}{l}\text { North Fork Flathead River near } \\
\text { Columbia Falls, MT }\end{array}$ & 1,548 & $\begin{array}{l}\text { 1911-17, } \\
1929-98\end{array}$ & 1964 & 18.60 & 69,100 & $6 / 7 / 95$ & 17.07 & 59,200 & $\mathrm{~N}$ & $>100$ \\
\hline
\end{tabular}


$\left[\mathrm{mi}^{2}\right.$, square miles; ft, feet above an arbitrary datum; $\mathrm{ft}^{3} / \mathrm{s}$, cubic feet per second; --, not determined or not applicable; >, greater than. Source: Recurrence intervals calculated from U.S. Geological Survey data. Other data from U.S. Geological Survey reports or databases]

\begin{tabular}{|c|c|c|c|c|c|c|c|c|c|c|c|}
\hline \multirow{2}{*}{$\begin{array}{l}\text { Streamgage } \\
\text { number } \\
\text { (fig. 38) }\end{array}$} & \multirow[b]{2}{*}{ Streamgage name } & \multirow{2}{*}{$\begin{array}{c}\text { Total } \\
\text { drainage } \\
\left(\mathrm{mi}^{2}\right)\end{array}$} & \multicolumn{4}{|c|}{$\begin{array}{l}\text { Maximum stage and discharge for period of record } \\
\text { through } 1998 \text { water year }\end{array}$} & \multicolumn{5}{|c|}{ Significant floods 1994-98 water years } \\
\hline & & & $\begin{array}{l}\text { Period of } \\
\text { record } \\
\text { (water } \\
\text { years) }\end{array}$ & Water year & $\begin{array}{l}\text { Stage } \\
(\mathrm{ft})\end{array}$ & $\begin{array}{l}\text { Discharge } \\
\left(\mathrm{ft}^{3} / \mathrm{s}\right)\end{array}$ & $\begin{array}{c}\text { Date } \\
\text { (month/ } \\
\text { day/ } \\
\text { year) }\end{array}$ & $\begin{array}{l}\text { Stage } \\
(\mathrm{ft})\end{array}$ & $\begin{array}{l}\text { Discharge } \\
\left(\mathrm{ft}^{3} / \mathrm{s}\right)\end{array}$ & $\begin{array}{l}\text { Regulated } \\
\text { during } \\
\text { flood }^{1}\end{array}$ & $\begin{array}{c}\text { Recurrence } \\
\text { interval } \\
\text { (years) }\end{array}$ \\
\hline 12365000 & $\begin{array}{l}\text { Stillwater River near Whitefish, } \\
\text { MT }\end{array}$ & 524 & $\begin{array}{l}1931-50, \\
1964, \\
1973-98\end{array}$ & $\begin{array}{l}1997 \\
1948\end{array}$ & $\begin{array}{l}20.20 \\
20.90\end{array}$ & $\begin{array}{l}4,570 \\
4,330\end{array}$ & $5 / 18 / 97$ & 20.20 & 4,570 & $\mathrm{~N}$ & $50-100$ \\
\hline 12370000 & Qan River near Bigfork, MT & 671 & $1922-98$ & 1974 & 7.34 & 8,890 & $5 / 18 / 97$ & 7.27 & 8,520 & $\mathrm{~N}$ & 50 \\
\hline 12374250 & $\begin{array}{l}\text { Mill Creek above Bassoo Creek } \\
\text { near Niarada, MT }\end{array}$ & 19.6 & $1983-98$ & $\begin{array}{l}1997 \\
1991\end{array}$ & $\begin{array}{l}2.60 \\
6.83\end{array}$ & $\begin{array}{l}173 \\
113\end{array}$ & $4 / 28 / 97$ & 2.60 & 173 & $\mathrm{~N}$ & 25 \\
\hline 12381400 & $\begin{array}{l}\text { South Fork Jocko River near Arlee, } \\
\text { MT }\end{array}$ & 56.0 & $1983-98$ & 1997 & 4.31 & 1,220 & $5 / 17 / 97$ & 4.31 & 1,220 & $\mathrm{~N}$ & $50-100$ \\
\hline 12387450 & Valley Creek near Arlee, MT & 15.3 & 1983-98 & 1997 & 3.04 & 116 & $5 / 16 / 97$ & 3.04 & 116 & $\mathrm{~N}$ & $25-50$ \\
\hline 12388400 & $\begin{array}{l}\text { Revais Creek below West Fork } \\
\text { near Dixon, MT }\end{array}$ & 23.4 & 1983-98 & 1997 & 4.36 & 382 & $6 / 1 / 97$ & 4.36 & 382 & $\mathrm{~N}$ & 50 \\
\hline 12388700 & Flathead River at Perma, MT & 8,795 & 1984-98 & 1997 & 21.65 & 54,700 & $6 / 7 / 97$ & 21.65 & 54,700 & $\mathrm{Y}$ & -- \\
\hline 12390700 & $\begin{array}{l}\text { Prospect Creek at Thompson Falls, } \\
\text { MT }\end{array}$ & 182 & $1956-98$ & 1974 & 9.86 & 5,490 & $2 / 9 / 96$ & 9.54 & 5,160 & $\mathrm{~N}$ & $>100$ \\
\hline
\end{tabular}




\section{Nebraska}

More than 3 inches of rain fell in an hour on July 15, 1994 (National Oceanic and Atmospheric Administration, 1994a), near Scottsbluff (fig. 39), overfilling an irrigation canal. Backups occurred in a sewer system as a result of the runoff, damaging 19 homes. Nearly 200 other homes were flooded due to the storm's runoff, with more than $\$ 1.1$ million in damage (National Oceanic and Atmospheric Administration, 1994b).

Excessive rains of 2 to 4 inches fell across north-central and northeastern Nebraska on May 27-28, 1995, with rapid rises of 2 to 3 feet on area streams and creeks. Flooding occurred on the Elkhorn and Niobrara Rivers and their many tributaries. Damage was more than \$2 million (National Oceanic and Atmospheric Administration, 1995b).

Thunderstorms developed August 22, 1995, over northcentral Nebraska and moved eastward. Continuous redevelopment of thunderstorms resulted in 5- to 8-inch rainfalls in the Niobrara River Basin. Several roads were washed out and numerous bridges suffered structural damage resulting in more than $\$ 1$ million in damage (National Oceanic and Atmospheric Administration, 1995b).

An ice-jam release in March 1996 created a large flood on the Middle Loup River at Dunning (streamgage 06775500, table 28). Isolated intense thunderstorms through the spring and summer of 1996 caused some flash flooding across the State. Buffalo Creek near Overton (streamgage 06769000), Middle Loup River at Dunning (streamgage 06755000), and Maple Creek near Nickerson (streamgage 06800000) had their peaks of record during this period. More than $\$ 9$ million in damage to property and almost $\$ 22$ million damage to crops were reported (National Oceanic and Atmospheric Administration, 1996b).

A series of thunderstorms produced in excess of 7.5 inches of rain west of Kimball during a 5-hour period on May 24, 1997 (National Oceanic and Atmospheric Administration, 1997a). Widespread flooding occurred along Lodgepole Creek and in Sand Draw. More than \$2 million in damage occurred (National Oceanic and Atmospheric Administration 1997b).

\section{References}

National Oceanic and Atmospheric Administration (NOAA), 1994a-97a, Climatological data (by State): Asheville, North Carolina, National Climatic Data Center, various months.

National Oceanic and Atmospheric Administration (NOAA), 1994b-97b, Storm data (by State): Asheville, North Carolina, National Climatic Data Center, various months.

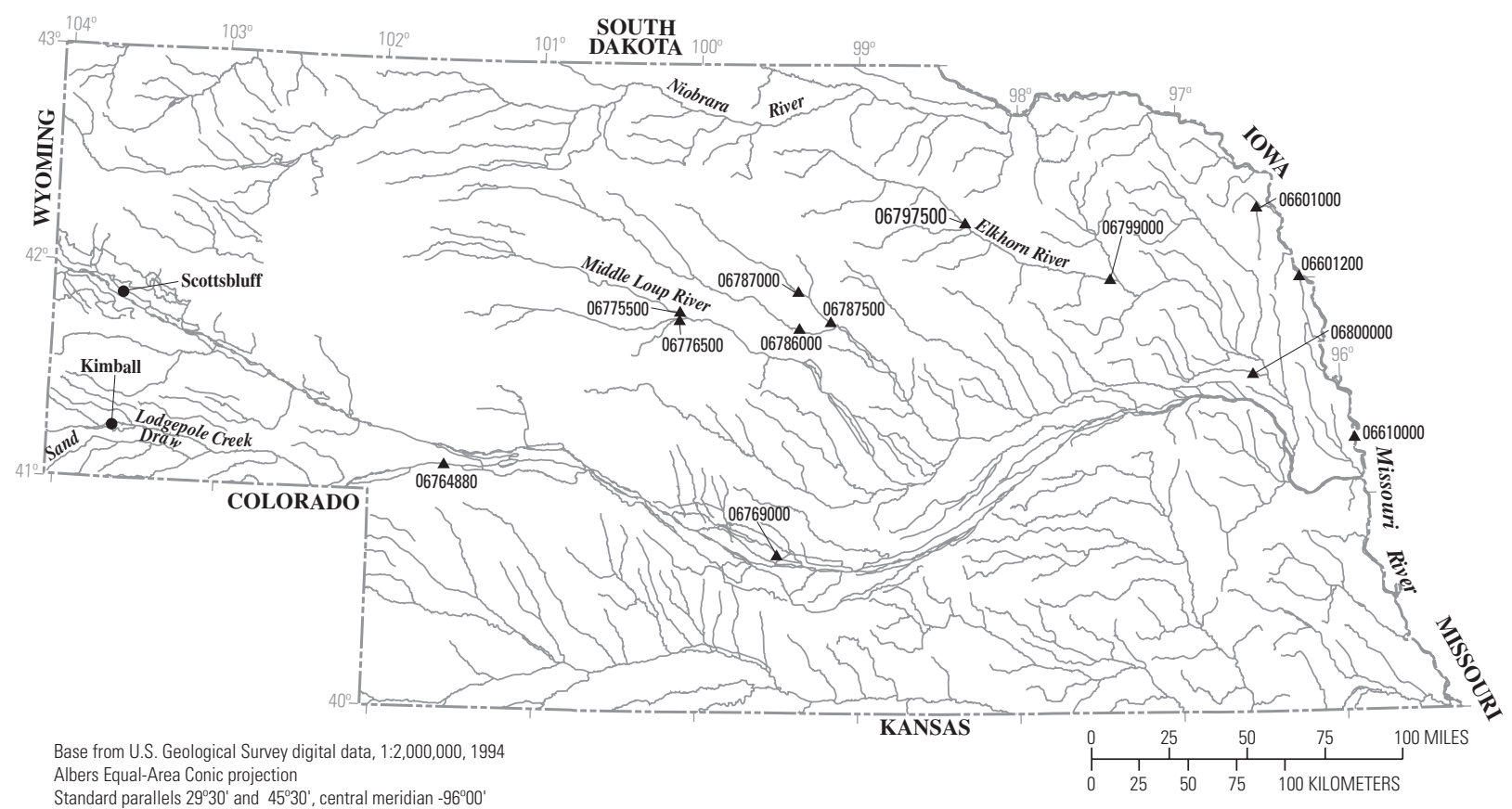

EXPLANATION

06769000 Streamgage and number

Figure 39. Location of streamgages with significant floods during 1994-98 water years for Nebraska. 
Table 28. Maximum stage and discharge for period of record for streamgages having significant floods during 1994-98 water years in Nebraska.

$\left[\mathrm{mi}^{2}\right.$, square miles; $\mathrm{ft}$, feet above an arbitrary datum; $\mathrm{ft}^{3} / \mathrm{s}$, cubic feet per second; --, not determined or not applicable. Source: Recurrence intervals calculated from U.S. Geological Survey data. Other data from U.S. Geological Survey reports or databases]

\begin{tabular}{|c|c|c|c|c|c|c|c|c|c|c|c|}
\hline \multirow[b]{2}{*}{$\begin{array}{c}\text { Streamgage } \\
\text { number } \\
\text { (fig. 39) }\end{array}$} & \multirow[b]{2}{*}{ Streamgage name } & \multirow{2}{*}{$\begin{array}{c}\text { Total } \\
\text { drainage } \\
\left(\mathrm{mi}^{2}\right)\end{array}$} & \multicolumn{4}{|c|}{$\begin{array}{c}\text { Maximum stage and discharge for period of record } \\
\text { through } 1998 \text { water year }\end{array}$} & \multicolumn{5}{|c|}{ Significant floods $1994-98$ water years } \\
\hline & & & $\begin{array}{l}\text { Period of } \\
\text { record } \\
\text { (water } \\
\text { years) }\end{array}$ & Water year & $\begin{array}{l}\text { Stage } \\
(\mathrm{ft})\end{array}$ & $\begin{array}{l}\text { Discharge } \\
\left(\mathrm{ft}^{3} / \mathrm{s}\right)\end{array}$ & $\begin{array}{c}\text { Date } \\
\text { (month/ } \\
\text { day/ } \\
\text { year) }\end{array}$ & $\begin{array}{l}\text { Stage } \\
(\mathrm{ft})\end{array}$ & $\begin{array}{l}\text { Discharge } \\
\left(\mathrm{ft}^{3} / \mathrm{s}\right)\end{array}$ & $\begin{array}{l}\text { Regulated } \\
\text { during } \\
\text { flood }^{1}\end{array}$ & $\begin{array}{c}\text { Recurrence } \\
\text { interval } \\
\text { (years) }\end{array}$ \\
\hline 06601000 & Omaha Creek at Homer, NE & 174 & $\begin{array}{l}1940, \\
1945-98\end{array}$ & 1940 & 32.50 & 51,000 & $7 / 17 / 96$ & 22.30 & 21,500 & $\mathrm{~N}$ & $75-100$ \\
\hline 06601200 & Missouri River at Decatur, NE & 316,200 & 1987-98 & $\begin{array}{l}1997 \\
1996\end{array}$ & $\begin{array}{l}31.99 \\
32.31\end{array}$ & $\begin{array}{r}100,000 \\
83,400\end{array}$ & $4 / 15 / 97$ & 31.99 & 100,000 & $\mathrm{Y}$ & $10-25$ \\
\hline 06610000 & Missouri River at Omaha, NE & 322,800 & 1928-98 & $\begin{array}{l}1952 \\
1993\end{array}$ & $\begin{array}{l}30.20 \\
30.26\end{array}$ & $\begin{array}{l}396,000 \\
115,000\end{array}$ & $6 / 23 / 96$ & 29.50 & 118,000 & $\mathrm{Y}$ & 20 \\
\hline 06764880 & South Platte River at Roscoe, NE & 23,900 & 1982-98 & 1995 & 11.29 & 20,100 & $6 / 6 / 95$ & 11.29 & 20,100 & $\mathrm{Y}$ & $15-35$ \\
\hline 06769000 & Buffalo Creek near Overton, NE & 175 & $\begin{array}{l}1949-58 \\
1996-98\end{array}$ & $\begin{array}{l}1996 \\
1958\end{array}$ & $\begin{array}{r}9.70 \\
10.47\end{array}$ & $\begin{array}{l}387 \\
383\end{array}$ & $8 / 4 / 96$ & 9.70 & 387 & $\mathrm{~N}$ & $5-15$ \\
\hline 06775500 & $\begin{array}{l}\text { Middle Loup River at Dunning, } \\
\text { NE }\end{array}$ & 1,830 & 1946-98 & $\begin{array}{l}1996 \\
1949\end{array}$ & $\begin{array}{l}6.15 \\
7.02\end{array}$ & $\begin{array}{r}2,480 \\
--\end{array}$ & $3 / 25 / 96$ & 6.15 & 2,480 & $\mathrm{~N}$ & $60-85$ \\
\hline 06776500 & Dismal River at Dunning, NE & 2,040 & $\begin{array}{l}1932, \\
1945-95\end{array}$ & $\begin{array}{l}1983 \\
1947\end{array}$ & $\begin{array}{l}2.40 \\
5.21\end{array}$ & $\begin{array}{r}1,290 \\
--\end{array}$ & $5 / 27 / 95$ & 2.37 & 1,060 & $\mathrm{~N}$ & $50-60$ \\
\hline 06786000 & North Loup River at Taylor, NE & 2,350 & 1936-98 & $\begin{array}{l}1995 \\
1957\end{array}$ & $\begin{array}{l}5.59 \\
9.50\end{array}$ & $\begin{array}{r}3,480 \\
--\end{array}$ & $5 / 28 / 95$ & 5.59 & 3,480 & $\mathrm{~N}$ & $50-60$ \\
\hline 06787000 & Calamus River near Harrop, NE & 693 & $\begin{array}{l}1964, \\
1978-97\end{array}$ & $\begin{array}{l}1995 \\
1987\end{array}$ & $\begin{array}{l}4.68 \\
5.34\end{array}$ & $\begin{array}{r}1,380 \\
--\end{array}$ & $5 / 28 / 95$ & 4.68 & 1,380 & $\mathrm{~N}$ & $25-45$ \\
\hline 06787500 & Calamus River near Burwell, NE & 994 & $1940-95$ & $\begin{array}{l}1964 \\
1967\end{array}$ & $\begin{array}{l}4.35 \\
8.90\end{array}$ & $\begin{array}{r}1,790 \\
--\end{array}$ & $5 / 29 / 95$ & 5.43 & 1,380 & $\mathrm{Y}$ & $15-35$ \\
\hline 06797500 & Elkhorn River at Ewing, NE & 1,400 & 1947-98 & $\begin{array}{l}1995 \\
1947\end{array}$ & $\begin{array}{l}11.09 \\
11.32\end{array}$ & $\begin{array}{l}9,050 \\
6,600\end{array}$ & $5 / 29 / 95$ & 11.09 & 9,050 & $\mathrm{~N}$ & $25-30$ \\
\hline 06799000 & Elkhorn River at Norfolk, NE & 2,790 & $\begin{array}{l}1896-1903, \\
1940-98\end{array}$ & $\begin{array}{l}1995 \\
1949\end{array}$ & $\begin{array}{l}13.05 \\
15.63\end{array}$ & $\begin{array}{r}19,200 \\
--\end{array}$ & $5 / 31 / 95$ & 13.05 & 19,200 & $\mathrm{~N}$ & $30-35$ \\
\hline 06800000 & Maple Creek near Nickerson, NE & 369 & $\begin{array}{l}1944, \\
1952-98\end{array}$ & $\begin{array}{l}1996 \\
1984\end{array}$ & $\begin{array}{l}17.33 \\
17.65\end{array}$ & $\begin{array}{r}13,700 \\
6,430\end{array}$ & $8 / 6 / 96$ & 17.33 & 13,700 & $\mathrm{~N}$ & $30-35$ \\
\hline
\end{tabular}

\footnotetext{
${ }^{1}$ Regulated during flood: N, no; Y, yes.
} 


\section{Nevada}

The floods of January 1997 were caused by several factors. First, the Sierra Nevada Mountains and western Nevada had experienced two above-normal precipitation years (1995, 1996). Second, a major winter storm December 21 and 22, 1996, deposited heavy snow in the Sierra Nevada Mountains and western Nevada. Four to 6 feet of snow were common below 7,000 feet in the Sierra Nevada Mountains with as much as 8 feet at the higher elevations. One to 3 feet of snow were found across the valleys of western Nevada (National Oceanic and Atmospheric Administration, 1997a). The following week into New Years' Day showed a dramatic change in the weather pattern. Copious amounts of moisture and warm air were transported from the subtropics into the Sierra Nevada Mountains and western Nevada. The most excessive rainfall occurred January 1 and 2, and snow occurred at elevations as high as 11,700 feet on January 1. An incredible amount of rainfall and snowmelt runoff poured out of the Sierra Nevada from December 30, 1996, to January 6, 1997. For example, an estimated 25 inches of rain and snowmelt runoff occurred during the period in the upper Truckee River Basin in California. These conditions were typical throughout the region and resulted in record flooding on the Truckee, Carson, and Walker Rivers (fig. 40). This flooding caused tremendous amounts of public and private damage throughout the eastern Sierra Nevada Mountains and extreme western Nevada. Total damage was more than $\$ 640$ million, but there were only two reported deaths (National Oceanic and Atmospheric Administration, 1997b).

Nineteen streamgages experienced their peak discharge of record during the first week in January 1997 (table 29).

In addition to strong winds and large hail, redeveloping severe thunderstorms produced intense downpours that resulted in severe flash flooding in the southeastern Las Vegas Valley on August 10, 1997. In Henderson, raging floodwaters turned roads into 3- to 4-foot deep rivers. Water and mudslides closed many roads, including U.S. Highway 95, for several hours. One man was drowned when a strong current swept him under his vehicle as he tried to move it off his neighborhood street. Boulder City and Lake Mead National Recreation Area also were hit hard by thunderstorms producing excessive rain. Damage was estimated at more than $\$ 1$ million for the Lake Mead National Recreation Area and about \$3.4 million for Boulder City (National Oceanic and Atmospheric Administration, 1997b).

\section{References}

National Oceanic and Atmospheric Administration (NOAA), 1997a, Climatological data (by State): Asheville, North Carolina, National Climatic Data Center, various months. National Oceanic and Atmospheric Administration (NOAA), 1997b, Storm data (by State): Asheville, North Carolina, National Climatic Data Center, various months. 


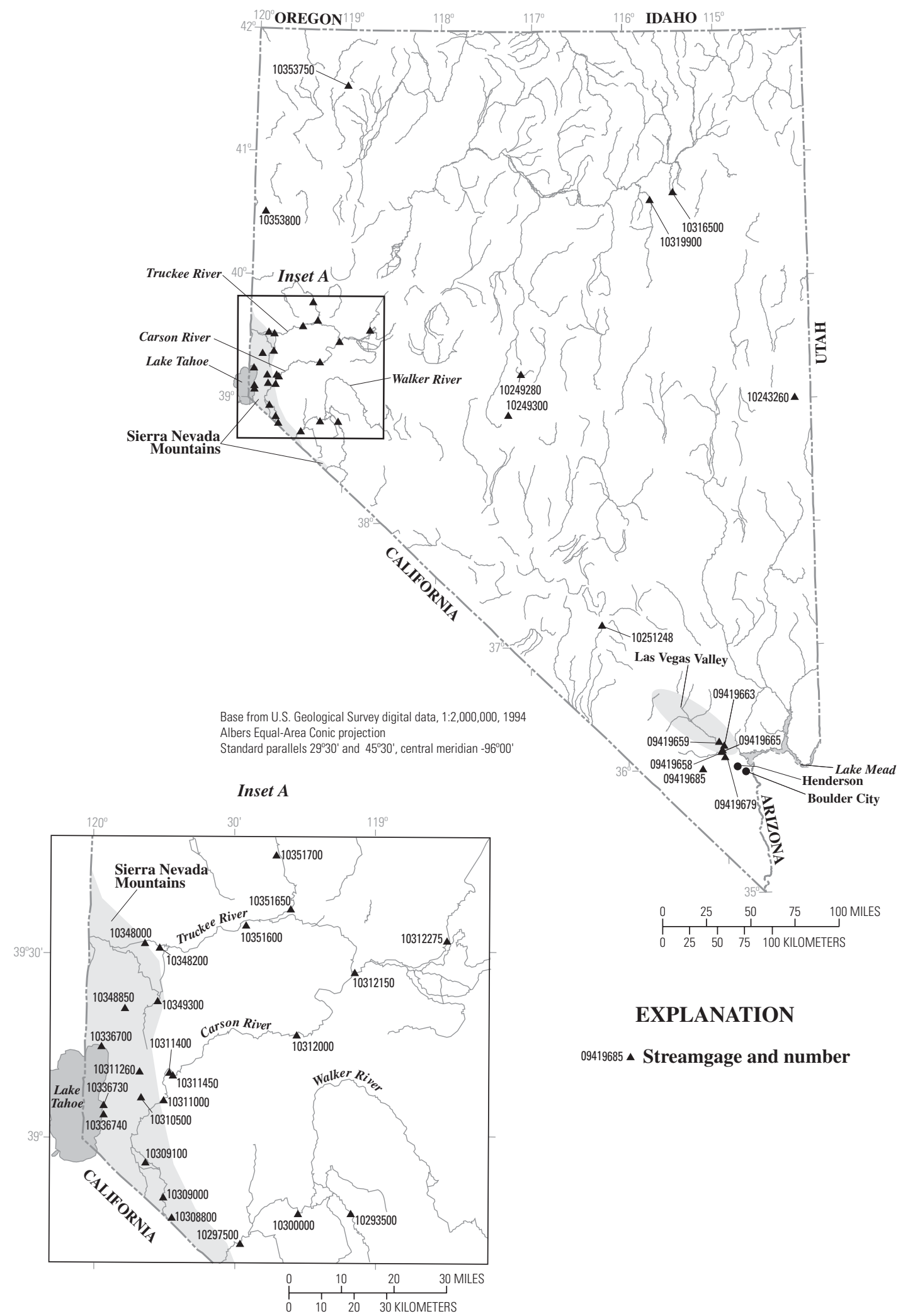

Figure 40. Location of streamgages with significant floods during 1994-98 water years for Nevada. 
Table 29. Maximum stage and discharge for period of record for streamgages having significant floods during 1994-98 water years in Nevada.

$\left[\mathrm{mi}^{2}\right.$, square miles; $\mathrm{ft}$, feet above an arbitrary datum; $\mathrm{ft}^{3} / \mathrm{s}$, cubic feet per second; --, not determined or not applicable; $>$, greater than; <, less than. Source: Recurrence intervals calculated from U.S. Geological Survey data. Other data from U.S. Geological Survey reports or databases]

\begin{tabular}{|c|c|c|c|c|c|c|c|c|c|c|c|}
\hline \multirow{2}{*}{$\begin{array}{l}\text { Streamgage } \\
\text { number } \\
\text { (fig. 40) }\end{array}$} & \multirow[b]{2}{*}{ Streamgage name } & \multirow{2}{*}{$\begin{array}{l}\text { Total } \\
\text { drainage } \\
\left(\mathrm{mi}^{2}\right)\end{array}$} & \multicolumn{4}{|c|}{$\begin{array}{c}\text { Maximum stage and discharge for period of record } \\
\text { through } 1998 \text { water year }\end{array}$} & \multicolumn{5}{|c|}{ Significant floods $1994-98$ water years } \\
\hline & & & $\begin{array}{l}\text { Period of } \\
\text { record } \\
\text { (water } \\
\text { years) }\end{array}$ & Water year & $\begin{array}{l}\text { Stage } \\
(\mathrm{ft})\end{array}$ & $\begin{array}{l}\text { Discharge } \\
\left(\mathrm{ft}^{3} / \mathrm{s}\right)\end{array}$ & $\begin{array}{l}\text { Date } \\
\text { (month/ } \\
\text { day/ } \\
\text { year) }\end{array}$ & $\begin{array}{l}\text { Stage } \\
(\mathrm{ft})\end{array}$ & $\begin{array}{l}\text { Discharge } \\
\left(\mathrm{ft}^{3} / \mathrm{s}\right)\end{array}$ & $\begin{array}{l}\text { Regulated } \\
\text { during } \\
\text { flood }^{1}\end{array}$ & $\begin{array}{l}\text { Recurrence } \\
\text { interval } \\
\text { (years) }\end{array}$ \\
\hline 09419658 & $\begin{array}{l}\text { Las Vegas Wash near Sahara } \\
\text { Avenue near Las Vegas, NV }\end{array}$ & 1,146 & $\begin{array}{l}1975 \\
1988-98\end{array}$ & $\begin{array}{l}1998 \\
1990\end{array}$ & $\begin{array}{l}12.50 \\
16.27\end{array}$ & $\begin{array}{l}4,400 \\
1,960\end{array}$ & $9 / 11 / 98$ & 12.50 & 4,400 & $\mathrm{Y}$ & -- \\
\hline 09419659 & $\begin{array}{l}\text { Sloan Channel tributary at Las } \\
\text { Vegas Boulevard near North Las } \\
\text { Vegas, NV }\end{array}$ & 17.5 & 1989-98 & 1998 & 15.34 & 920 & $9 / 11 / 98$ & 15.34 & 920 & $\mathrm{Y}$ & -- \\
\hline 09419663 & $\begin{array}{l}\text { Las Vegas Wash tributary south of } \\
\text { Nellis Air Force Base, NV }\end{array}$ & 1.20 & $\begin{array}{l}1963-81 \\
1983-98\end{array}$ & 1998 & 21.00 & 600 & $9 / 11 / 98$ & 21.00 & 600 & $\mathrm{Y}$ & -- \\
\hline 09419665 & $\begin{array}{l}\text { Sloan Channel at Charleston } \\
\text { Boulevard near Las Vegas, NV }\end{array}$ & 144 & 1989-98 & $\begin{array}{l}1998 \\
1997\end{array}$ & $\begin{array}{l}11.41 \\
11.72\end{array}$ & $\begin{array}{r}1,230 \\
700\end{array}$ & $9 / 11 / 98$ & 11.41 & 1,230 & $\mathrm{Y}$ & -- \\
\hline 09419679 & $\begin{array}{l}\text { Las Vegas Wasteway near East Las } \\
\text { Vegas, NV }\end{array}$ & -- & $\begin{array}{l}\text { 1980-83, } \\
1985-98\end{array}$ & $\begin{array}{l}1983 \\
1990\end{array}$ & $\begin{array}{l}6.56 \\
6.99\end{array}$ & 630 & $11 / 21 / 96$ & 6.81 & $\begin{array}{l}523 \\
453\end{array}$ & $\mathrm{~N}$ & -- \\
\hline 09419685 & $\begin{array}{l}\text { Bird Springs Wash near Arden, } \\
\text { NV }\end{array}$ & -- & 1987-98 & 1998 & 44.05 & 35 & $7 / 20 / 98$ & 44.05 & 35 & $\mathrm{~N}$ & -- \\
\hline 10243260 & Lehman Creek near Baker, NV & 11.0 & $\begin{array}{l}1948-55 \\
1993-97\end{array}$ & 1995 & 5.01 & 80 & $6 / 29 / 95$ & 5.01 & 80 & $\mathrm{~N}$ & -- \\
\hline 10249280 & $\begin{array}{l}\text { Kingston Creek below Cougar } \\
\text { Canyon near Austin, NV }\end{array}$ & 23.4 & 1967-98 & $\begin{array}{l}1983 \\
1995\end{array}$ & $\begin{array}{l}3.19 \\
3.86\end{array}$ & $\begin{array}{l}385 \\
234\end{array}$ & $6 / 3 / 95$ & 3.86 & 234 & $\mathrm{Y}$ & -- \\
\hline 10249300 & $\begin{array}{l}\text { South Twin River near Round } \\
\text { Mountain, NV }\end{array}$ & 20.0 & 1965-98 & 1983 & 4.39 & 510 & $6 / 3 / 98$ & 3.20 & 148 & $\mathrm{~N}$ & -- \\
\hline 10251248 & $\begin{array}{l}\text { Unnamed tributary-Stockade Wash } \\
\text { near Rattlesnake Ridge, Nevada } \\
\text { Test Site, NV }\end{array}$ & 3.90 & $\begin{array}{l}1984-88 \\
1991-95\end{array}$ & 1995 & 5.49 & 90 & $3 / 11 / 95$ & 5.49 & 90 & $\mathrm{~N}$ & -- \\
\hline 10293500 & $\begin{array}{l}\text { East Walker River above } \\
\text { Strosnider Ditch near Mason, NV }\end{array}$ & 1,100 & $\begin{array}{l}1947-92 \\
1995-98\end{array}$ & $\begin{array}{l}1986 \\
1997\end{array}$ & $\begin{array}{l}7.49 \\
9.61\end{array}$ & $\begin{array}{l}2,820 \\
2,610\end{array}$ & $1 / 4 / 97$ & 9.61 & 2,610 & $\mathrm{Y}$ & $>25$ \\
\hline
\end{tabular}


Table 29. Maximum stage and discharge for period of record for streamgages having significant floods during 1994-98 water years in Nevada.-Continued

$\left[\mathrm{mi}^{2}\right.$, square miles; $\mathrm{ft}$, feet above an arbitrary datum; $\mathrm{ft}^{3} / \mathrm{s}$, cubic feet per second; --, not determined or not applicable; $>$, greater than; <, less than. Source: Recurrence intervals calculated from U.S. Geological Survey data. Other data from U.S. Geological Survey reports or databases]

\begin{tabular}{|c|c|c|c|c|c|c|c|c|c|c|c|}
\hline \multirow{2}{*}{$\begin{array}{l}\text { Streamgage } \\
\text { number } \\
\text { (fig. 40) }\end{array}$} & \multirow[b]{2}{*}{ Streamgage name } & \multirow{2}{*}{$\begin{array}{l}\text { Total } \\
\text { drainage } \\
\left(\mathrm{mi}^{2}\right)\end{array}$} & \multicolumn{4}{|c|}{$\begin{array}{c}\text { Maximum stage and discharge for period of record } \\
\text { through } 1998 \text { water year }\end{array}$} & \multicolumn{5}{|c|}{ Significant floods $1994-98$ water years } \\
\hline & & & $\begin{array}{l}\text { Period of } \\
\text { record } \\
\text { (water } \\
\text { years) }\end{array}$ & Water year & $\begin{array}{l}\text { Stage } \\
\text { (ft) }\end{array}$ & $\begin{array}{l}\text { Discharge } \\
\left(\mathrm{ft}^{3} / \mathrm{s}\right)\end{array}$ & $\begin{array}{l}\text { Date } \\
\text { (month/ } \\
\text { day/ } \\
\text { year) }\end{array}$ & $\begin{array}{l}\text { Stage } \\
\text { (ft) }\end{array}$ & $\begin{array}{l}\text { Discharge } \\
\left(\mathrm{ft}^{3} / \mathrm{s}\right)\end{array}$ & $\begin{array}{l}\text { Regulated } \\
\text { during } \\
\text { flood }^{1}\end{array}$ & $\begin{array}{l}\text { Recurrence } \\
\text { interval } \\
\text { (years) }\end{array}$ \\
\hline 10297500 & $\begin{array}{l}\text { West Walker River at Hoye Bridge } \\
\text { near Wellington, NV }\end{array}$ & 497 & $\begin{array}{l}1921-22 \\
1924-32 \\
1958-98\end{array}$ & 1997 & 13.68 & 11,500 & $1 / 3 / 97$ & 13.68 & 11,500 & $\mathrm{Y}$ & $>100$ \\
\hline 10300000 & $\begin{array}{l}\text { West Walker River near Hudson, } \\
\text { NV }\end{array}$ & 964 & $\begin{array}{l}1915-18 \\
1920-24, \\
1947-92 \\
1995-98\end{array}$ & 1997 & 12.18 & 11,400 & $1 / 3 / 97$ & 12.18 & 11,400 & $\mathrm{Y}$ & $>100$ \\
\hline 10308800 & $\begin{array}{l}\text { Bryant Creek near Gardnerville, } \\
\text { NV }\end{array}$ & 31.5 & $\begin{array}{l}1961-73 \\
1978-80 \\
1995-98\end{array}$ & 1997 & 8.70 & 1,360 & $1 / 2 / 97$ & 8.70 & 1,300 & $\mathrm{~N}$ & $<25$ \\
\hline 10309000 & $\begin{array}{l}\text { East Fork Carson River near } \\
\text { Gardnerville, NV }\end{array}$ & 356 & $\begin{array}{l}\text { 1890-93, } \\
\text { 1901-05, } \\
\text { 1908-10, } \\
\text { 1917, } \\
\text { 1925-28, } \\
\text { 1936-38, } \\
1940-98\end{array}$ & 1997 & 13.00 & 20,300 & $1 / 3 / 97$ & 13.00 & 20,300 & $\mathrm{~N}$ & 100 \\
\hline 10309100 & $\begin{array}{l}\text { East Fork Carson River at Minden, } \\
\text { NV }\end{array}$ & 392 & $\begin{array}{l}1975-84 \\
1994-98\end{array}$ & $\begin{array}{l}1997 \\
1980\end{array}$ & $\begin{array}{l}10.41 \\
11.40\end{array}$ & $\begin{array}{r}10,900 \\
8,000\end{array}$ & $1 / 2 / 97$ & 10.41 & 10,900 & $\mathrm{Y}$ & -- \\
\hline 10310500 & Clear Creek near Carson City, NV & 15.5 & $\begin{array}{l}1948-78 \\
1989-98\end{array}$ & 1997 & 3.94 & 266 & $1 / 2 / 97$ & 3.94 & 266 & $\mathrm{~N}$ & 50 \\
\hline 10311000 & $\begin{array}{l}\text { Carson River near Carson City, } \\
\text { NV }\end{array}$ & 886 & 1939-98 & 1997 & 18.43 & 30,500 & $1 / 3 / 97$ & 18.43 & 30,500 & $\mathrm{~N}$ & $>75$ \\
\hline 10311260 & $\begin{array}{l}\text { Vicee Canyon Creek near } \\
\text { Sagebrush Ranch near Carson } \\
\text { City, NV }\end{array}$ & 1.83 & $\begin{array}{l}1979-80 \\
1982,1984, \\
1986, \\
1991-97\end{array}$ & 1997 & 9.66 & 4,000 & $1 / 2 / 97$ & 9.66 & 4,000 & $\mathrm{~N}$ & $>100$ \\
\hline
\end{tabular}


Table 29. Maximum stage and discharge for period of record for streamgages having significant floods during 1994-98 water years in Nevada.-Continued

$\left[\mathrm{mi}^{2}\right.$, square miles; $\mathrm{ft}$, feet above an arbitrary datum; $\mathrm{ft}^{3} / \mathrm{s}$, cubic feet per second; --, not determined or not applicable; $>$, greater than; <, less than. Source: Recurrence intervals calculated from U.S. Geological Survey data. Other data from U.S. Geological Survey reports or databases]

\begin{tabular}{|c|c|c|c|c|c|c|c|c|c|c|c|}
\hline \multirow[b]{2}{*}{$\begin{array}{c}\text { Streamgage } \\
\text { number } \\
\text { (fig. 40) }\end{array}$} & \multirow[b]{2}{*}{ Streamgage name } & \multirow[b]{2}{*}{$\begin{array}{c}\text { Total } \\
\text { drainage } \\
\left(\mathrm{mi}^{2}\right)\end{array}$} & \multicolumn{4}{|c|}{$\begin{array}{c}\text { Maximum stage and discharge for period of record } \\
\text { through } 1998 \text { water year }\end{array}$} & \multicolumn{5}{|c|}{ Significant floods $1994-98$ water years } \\
\hline & & & $\begin{array}{c}\text { Period of } \\
\text { record } \\
\text { (water } \\
\text { years) }\end{array}$ & Water year & $\begin{array}{l}\text { Stage } \\
\text { (ft) }\end{array}$ & $\begin{array}{l}\text { Discharge } \\
\left(\mathrm{ft}^{3} / \mathrm{s}\right)\end{array}$ & $\begin{array}{c}\text { Date } \\
\text { (month/ } \\
\text { day/ } \\
\text { year) }\end{array}$ & $\begin{array}{l}\text { Stage } \\
\text { (ft) }\end{array}$ & $\begin{array}{l}\text { Discharge } \\
\left(\mathrm{ft}^{3} / \mathrm{s}\right)\end{array}$ & $\begin{array}{c}\text { Regulated } \\
\text { during } \\
\text { flood }^{1}\end{array}$ & $\begin{array}{c}\text { Recurrence } \\
\text { interval } \\
\text { (years) }\end{array}$ \\
\hline 10311400 & $\begin{array}{l}\text { Carson River at Deer Run Road } \\
\text { near Carson City, NV }\end{array}$ & 958 & $\begin{array}{l}1980-85, \\
1991-98\end{array}$ & 1997 & 24.23 & 24,000 & $1 / 3 / 97$ & 24.23 & 24,000 & $\mathrm{~N}$ & $<100$ \\
\hline 10311450 & $\begin{array}{l}\text { Brunswick Canyon near New } \\
\text { Empire, NV }\end{array}$ & 12.7 & $\begin{array}{l}1966-78 \\
1980-98\end{array}$ & 1995 & 5.02 & 245 & $3 / 11 / 95$ & 5.02 & 245 & $\mathrm{~N}$ & $<10$ \\
\hline 10312000 & $\begin{array}{l}\text { Carson River near Fort Churchill, } \\
\text { NV }\end{array}$ & 1,450 & $1911-98$ & 1997 & 15.27 & 22,300 & $1 / 3 / 97$ & 15.27 & 22,300 & $\mathrm{~N}$ & $>100$ \\
\hline 10312150 & $\begin{array}{l}\text { Carson River below Lahontan } \\
\text { Reservoir near Fallon, NV }\end{array}$ & 1,801 & $1980-98$ & 1983 & 8.34 & 3,160 & $5 / 17 / 96$ & 7.78 & 2,430 & $\mathrm{Y}$ & -- \\
\hline 10312275 & $\begin{array}{l}\text { Carson River at Tarzyn Road near } \\
\text { Fallon, NV }\end{array}$ & -- & 1986-98 & $\begin{array}{l}1996 \\
1997\end{array}$ & $\begin{array}{l}6.11 \\
8.73\end{array}$ & $\begin{array}{l}942 \\
821\end{array}$ & $5 / 27 / 96$ & $\begin{array}{l}6.11 \\
8.73\end{array}$ & $\begin{array}{l}942 \\
821\end{array}$ & $\mathrm{Y}$ & -- \\
\hline 10316500 & Lamoille Creek near Lamoille, NV & 24.9 & $\begin{array}{l}\text { 1915-16, } \\
1918-20, \\
1922, \\
1944-98\end{array}$ & $\begin{array}{l}1997 \\
1982\end{array}$ & $\begin{array}{l}5.29 \\
6.23\end{array}$ & $\begin{array}{l}838 \\
829\end{array}$ & $6 / 4 / 97$ & 5.29 & 838 & $\mathrm{~N}$ & -- \\
\hline 10319900 & $\begin{array}{l}\text { South Fork Humboldt River above } \\
\text { Tenmile Creek near Elko, NV }\end{array}$ & 898 & 1989-98 & 1995 & 5.82 & 2,710 & $6 / 3 / 95$ & 5.82 & 2,710 & $\mathrm{~N}$ & -- \\
\hline 10336700 & $\begin{array}{l}\text { Incline Creek near Crystal Bay, } \\
\text { NV }\end{array}$ & 6.69 & $\begin{array}{l}\text { 1970-73, } \\
1975, \\
1988-98\end{array}$ & 1997 & 3.87 & 179 & $1 / 2 / 97$ & 3.87 & 179 & $\mathrm{~N}$ & $<50$ \\
\hline 10336730 & $\begin{array}{l}\text { Glenbrook Creek at Glenbrook, } \\
\text { NV }\end{array}$ & 3.75 & $\begin{array}{l}1972-75 \\
1988-98\end{array}$ & 1997 & 6.46 & 144 & $1 / 2 / 97$ & 6.46 & 144 & $\mathrm{~N}$ & 50 \\
\hline 10336740 & $\begin{array}{l}\text { Logan House Creek near } \\
\text { Glenbrook, NV }\end{array}$ & 2.08 & 1984-98 & 1997 & 4.75 & 11 & $1 / 2 / 97$ & 4.75 & 11 & $\mathrm{~N}$ & $<25$ \\
\hline 10348000 & Truckee River at Reno, NV & 1,067 & $\begin{array}{l}1907-21, \\
1925-26, \\
1931-34, \\
1947-98\end{array}$ & $\begin{array}{l}1956 \\
1997\end{array}$ & $\begin{array}{l}13.63 \\
14.94\end{array}$ & $\begin{array}{l}20,800 \\
18,200\end{array}$ & $1 / 2 / 97$ & 14.94 & 18,200 & $\mathrm{Y}$ & $<50$ \\
\hline
\end{tabular}


$\left[\mathrm{mi}^{2}\right.$, square miles; $\mathrm{ft}$, feet above an arbitrary datum; $\mathrm{ft}^{3} / \mathrm{s}$, cubic feet per second; --, not determined or not applicable; >, greater than; <, less than. Source: Recurrence intervals calculated from U.S. Geological Survey data. Other data from U.S. Geological Survey reports or databases]

\begin{tabular}{|c|c|c|c|c|c|c|c|c|c|c|c|}
\hline \multirow[b]{2}{*}{$\begin{array}{l}\text { Streamgage } \\
\text { number } \\
\text { (fig. 40) }\end{array}$} & \multirow[b]{2}{*}{ Streamgage name } & \multirow[b]{2}{*}{$\begin{array}{c}\text { Total } \\
\text { drainage } \\
\left(\mathrm{mi}^{2}\right)\end{array}$} & \multicolumn{4}{|c|}{$\begin{array}{c}\text { Maximum stage and discharge for period of record } \\
\text { through } 1998 \text { water year }\end{array}$} & \multicolumn{5}{|c|}{ Significant floods $1994-98$ water years } \\
\hline & & & $\begin{array}{c}\text { Period of } \\
\text { record } \\
\text { (water } \\
\text { years) }\end{array}$ & Water year & $\begin{array}{l}\text { Stage } \\
(\mathrm{ft})\end{array}$ & $\begin{array}{l}\text { Discharge } \\
\left(\mathrm{ft}^{3} / \mathrm{s}\right)\end{array}$ & $\begin{array}{c}\text { Date } \\
\text { (month/ } \\
\text { day/ } \\
\text { year) }\end{array}$ & $\begin{array}{l}\text { Stage } \\
(\mathrm{ft})\end{array}$ & $\begin{array}{l}\text { Discharge } \\
\left(\mathrm{ft}^{3} / \mathrm{s}\right)\end{array}$ & $\begin{array}{c}\text { Regulated } \\
\text { during } \\
\text { flood }^{1}\end{array}$ & $\begin{array}{c}\text { Recurrence } \\
\text { interval } \\
\text { (years) }\end{array}$ \\
\hline 10348200 & Truckee River near Sparks, NV & 1,070 & $1978-98$ & $\begin{array}{l}1997 \\
1986\end{array}$ & $\begin{array}{l}-- \\
15.22\end{array}$ & $\begin{array}{l}18,000 \\
14,900\end{array}$ & $1 / 2 / 97$ & -- & 18,000 & $\mathrm{Y}$ & -- \\
\hline 10348850 & $\begin{array}{l}\text { Galena Creek at Galena Creek } \\
\text { State Park, NV }\end{array}$ & 7.69 & 1985-98 & 1997 & 5.54 & 2,610 & $1 / 2 / 97$ & 5.54 & 2,610 & $\mathrm{~N}$ & $<50$ \\
\hline 10349300 & Steamboat Creek at Steamboat, NV & 123 & $1962-98$ & 1986 & 6.79 & 3,600 & $1 / 1 / 97$ & 6.03 & 2,090 & $\mathrm{Y}$ & $>25$ \\
\hline 10351600 & $\begin{array}{l}\text { Truckee River below Derby Dam } \\
\text { near Wadsworth, NV }\end{array}$ & 1,676 & $\begin{array}{l}\text { 1909-10, } \\
1916, \\
1918-98\end{array}$ & 1997 & 14.56 & 19,900 & $1 / 3 / 97$ & 14.56 & 19,900 & $\mathrm{Y}$ & $<50$ \\
\hline 10351650 & Truckee River at Wadsworth, NV & 1,728 & $\begin{array}{l}1966-86, \\
1994-98\end{array}$ & 1997 & 19.62 & 19,100 & $1 / 3 / 97$ & 19.62 & 19,100 & $\mathrm{Y}$ & $<50$ \\
\hline 10351700 & Truckee River near Nixon, NV & 1,827 & $\begin{array}{l}1956, \\
1958-98\end{array}$ & 1997 & 15.28 & 21,200 & $1 / 3 / 97$ & 15.28 & 21,200 & $\mathrm{Y}$ & 50 \\
\hline 10353750 & $\begin{array}{l}\text { Mahogany Creek near Summit } \\
\text { Lake, NV }\end{array}$ & 13.3 & 1988-98 & $\begin{array}{l}1995 \\
1998\end{array}$ & $\begin{array}{l}5.34 \\
5.56\end{array}$ & $\begin{array}{l}50 \\
40\end{array}$ & $6 / 5 / 95$ & 5.34 & 50 & $\mathrm{~N}$ & -- \\
\hline 10353800 & $\begin{array}{l}\text { Smoke Creek below reservoir near } \\
\text { Smoke Creek, NV }\end{array}$ & 50.1 & $\begin{array}{l}\text { 1986, } \\
\text { 1989-98 }\end{array}$ & $\begin{array}{l}1995 \\
1986\end{array}$ & $\begin{array}{l}8.43 \\
9.00\end{array}$ & $\begin{array}{l}4,320 \\
2,270\end{array}$ & $3 / 9 / 95$ & 8.43 & 4,320 & $\mathrm{Y}$ & -- \\
\hline
\end{tabular}

${ }^{1}$ Regulated during flood: N, no; Y, yes. 


\section{New Hampshire}

A coastal storm, which was fed tropical moisture by the circulation around Hurricane Lily, produced 8 to 13 inches of rain throughout parts of central and southern New Hampshire during October 20-23, 1996 (National Oceanic and Atmospheric Administration, 1996a). Many homes and businesses were flooded, roads and bridges were washed out, and several dams were damaged. Total damage was more than $\$ 10.8$ million (National Oceanic and Atmospheric Administration, 1996b). Oyster River near Durham (streamgage 01073000) had a peak of record during this flood (table 30 ).

Three to 8 inches of rain during June 13-15, 1998 (National Oceanic and Atmospheric Administration, 1998a), caused small rivers and streams to rise in central and southern New Hampshire. Many roads were flooded and (or) washed out. Campgrounds and some lakeside homes had to be evacuated. More than \$1 million in damage resulted (National Oceanic and
Atmospheric Administration, 1998b). Excessive rains returned on June 27, 1998. From 3 to possibly as much as 6 inches of rain fell in 12 hours (National Oceanic and Atmospheric Administration, 1998a) causing the Baker River (fig. 41) and its tributaries to rise very rapidly. Bridges were destroyed, vehicles were washed away, and some towns were completely isolated. Damage was near \$1.5 million (National Oceanic and Atmospheric Administration, 1998b).

\section{References}

National Oceanic and Atmospheric Administration (NOAA), 1996a-98a, Climatological data (by State): Asheville, North Carolina, National Climatic Data Center, various months.

National Oceanic and Atmospheric Administration (NOAA), 1996b-98b, Storm data (by State): Asheville, North Carolina, National Climatic Data Center, various months.

\section{EXPLANATION}

$01137500 \_$Streamgage and number
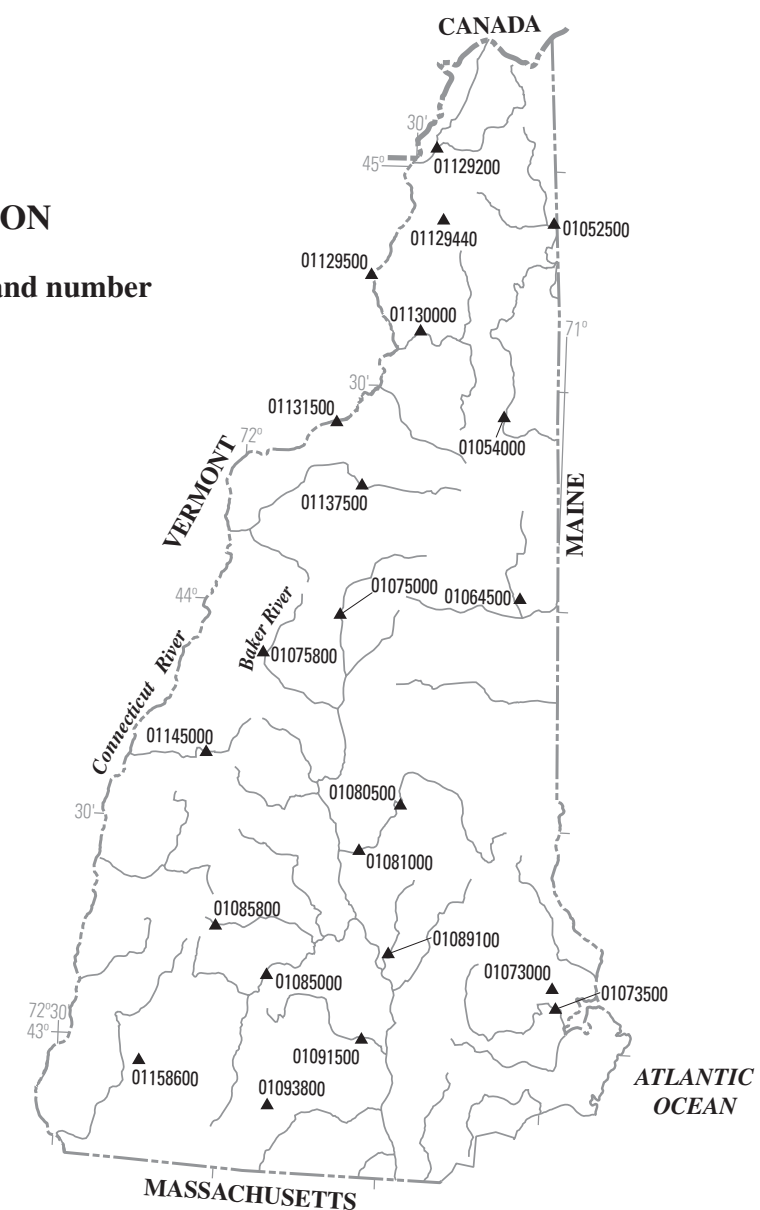

Base from U.S. Geological Survey digital data, 1:2,000,000, 1994 Albers Equal-Area Conic projection

Standard parallels $29^{\circ} 30^{\prime}$ and $45^{\circ} 30^{\prime}$, central meridian $-96^{\circ} 00^{\prime}$

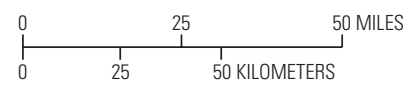

Figure 41. Location of streamgages with significant floods during 1994-98 water years for New Hampshire. 
Table 30. Maximum stage and discharge for period of record for streamgages having significant floods during 1994-98 water years in New Hampshire.

$\left[\mathrm{mi}^{2}\right.$, square miles; ft, feet above an arbitrary datum; $\mathrm{ft}^{3} / \mathrm{s}$, cubic feet per second; --, not determined or not applicable; >, greater than. Source: Recurrence intervals calculated from U.S. Geological Survey data. Other data from U.S. Geological Survey reports or databases]

\begin{tabular}{|c|c|c|c|c|c|c|c|c|c|c|c|}
\hline \multirow[b]{2}{*}{$\begin{array}{l}\text { Streamgage } \\
\text { number } \\
\text { (fig. 41) }\end{array}$} & \multirow[b]{2}{*}{ Streamgage name } & \multirow[b]{2}{*}{$\begin{array}{c}\text { Total } \\
\text { drainage } \\
\left(\mathrm{mi}^{2}\right)\end{array}$} & \multicolumn{4}{|c|}{$\begin{array}{c}\text { Maximum stage and discharge for period of record } \\
\text { through } 1998 \text { water year }\end{array}$} & \multicolumn{5}{|c|}{ Significant floods 1994-98 water years } \\
\hline & & & $\begin{array}{l}\text { Period of } \\
\text { record } \\
\text { (water } \\
\text { years) }\end{array}$ & Water year & $\begin{array}{l}\text { Stage } \\
\text { (ft) }\end{array}$ & $\begin{array}{l}\text { Discharge } \\
\left(\mathrm{ft}^{3} / \mathrm{s}\right)\end{array}$ & $\begin{array}{c}\text { Date } \\
\text { (month/ } \\
\text { day/ } \\
\text { year) }\end{array}$ & $\begin{array}{l}\text { Stage } \\
(\mathrm{ft})\end{array}$ & $\begin{array}{l}\text { Discharge } \\
\left(\mathrm{ft}^{3} / \mathrm{s}\right)\end{array}$ & $\begin{array}{l}\text { Regulated } \\
\text { during } \\
\text { flood }^{1}\end{array}$ & $\begin{array}{c}\text { Recurrence } \\
\text { interval } \\
\text { (years) }\end{array}$ \\
\hline 01052500 & $\begin{array}{l}\text { Diamond River near Wentworth } \\
\text { Location, NH }\end{array}$ & 152 & $1942-98$ & $\begin{array}{l}1998 \\
1981\end{array}$ & $\begin{array}{l}12.11 \\
12.23\end{array}$ & $\begin{array}{r}12,800 \\
3,620\end{array}$ & $3 / 31 / 98$ & 12.11 & 12,800 & $\mathrm{~N}$ & $>100$ \\
\hline 01054000 & $\begin{array}{l}\text { Androscoggin River near Gorham, } \\
\mathrm{NH}\end{array}$ & 1,361 & 1914-98 & $\begin{array}{l}1923 \\
1998\end{array}$ & $\begin{array}{l}-- \\
10.15\end{array}$ & $\begin{array}{l}21,900 \\
20,400\end{array}$ & $4 / 1 / 98$ & 10.15 & 20,400 & $\mathrm{Y}$ & 50 \\
\hline 01064500 & Saco River near Conway, NH & 385 & $\begin{array}{l}1903-09 \\
1910-12, \\
1929-98\end{array}$ & 1998 & 14.67 & 36,500 & $6 / 14 / 98$ & 14.67 & 36,500 & -- & $10-25$ \\
\hline 01073000 & Oyster River near Durham, NH & 12.1 & $1935-98$ & $\begin{array}{l}1997 \\
1936\end{array}$ & $\begin{array}{l}6.67 \\
7.45\end{array}$ & $\begin{array}{r}1,160 \\
548\end{array}$ & $10 / 21 / 96$ & 6.67 & 1,160 & $\mathrm{~N}$ & $>100$ \\
\hline 01073500 & $\begin{array}{l}\text { Lamprey River near Newmarket, } \\
\text { NH }\end{array}$ & 183 & $1935-98$ & 1987 & 15.14 & 7,570 & $10 / 22 / 96$ & 14.59 & 7,080 & $\mathrm{Y}$ & $50-75$ \\
\hline 01075000 & $\begin{array}{l}\text { Pemigewasset River at } \\
\text { Woodstock, NH }\end{array}$ & 193 & $\begin{array}{l}1940-80 \\
1985-98\end{array}$ & 1960 & 16.13 & 47,000 & $10 / 22 / 95$ & 14.09 & 33,500 & $\mathrm{~N}$ & $25-50$ \\
\hline 01075800 & $\begin{array}{l}\text { Stevens Brook near Wentworth, } \\
\text { NH }\end{array}$ & 12.9 & 1964-98 & 1996 & 4.51 & 650 & $11 / 12 / 95$ & 4.51 & 650 & -- & $10-25$ \\
\hline 01080500 & $\begin{array}{l}\text { Lake Winnipesaukee outlet at } \\
\text { Lakeport, NH }\end{array}$ & 363 & $\begin{array}{l}1934-83 \\
1988-98\end{array}$ & 1936 & -- & 2,890 & $6 / 22 / 98$ & -- & 2,620 & $\mathrm{Y}$ & $10-25$ \\
\hline 01081000 & Winnipesaukee River at Tilton, NH & 471 & 1937-98 & 1984 & 8.68 & 4,580 & $6 / 28 / 98$ & 8.04 & 3,940 & $\mathrm{Y}$ & 25 \\
\hline 01085000 & $\begin{array}{l}\text { Contoocook River near Henniker, } \\
\text { NH }\end{array}$ & 368 & $\begin{array}{l}1938, \\
1940-77, \\
1989-98\end{array}$ & 1938 & 21.30 & 22,200 & $10 / 22 / 96$ & 13.18 & 9,840 & $\mathrm{Y}$ & $25-50$ \\
\hline 01085800 & $\begin{array}{l}\text { West Branch Warner River near } \\
\text { Bradford, NH }\end{array}$ & 5.75 & 1963-98 & $\begin{array}{l}1984 \\
1996\end{array}$ & $-\overline{-}$ & $\begin{array}{l}800 \\
690\end{array}$ & $11 / 12 / 95$ & 9.14 & 690 & $\mathrm{~N}$ & $10-25$ \\
\hline 01089100 & $\begin{array}{l}\text { Soucook River at Pembroke Road } \\
\text { near Concord, NH }\end{array}$ & 81.9 & 1989-98 & 1996 & 11.59 & 2,320 & $4 / 17 / 96$ & 11.59 & 2,320 & $\mathrm{~N}$ & $10-25$ \\
\hline
\end{tabular}


Table 30. Maximum stage and discharge for period of record for streamgages having significant floods during 1994-98 water years in New Hampshire.-Continued

$\left[\mathrm{mi}^{2}\right.$, square miles; ft, feet above an arbitrary datum; $\mathrm{ft}^{3} / \mathrm{s}$, cubic feet per second; --, not determined or not applicable; >, greater than. Source: Recurrence intervals calculated from U.S. Geological Survey data. Other data from U.S. Geological Survey reports or databases]

\begin{tabular}{|c|c|c|c|c|c|c|c|c|c|c|c|}
\hline \multirow{2}{*}{$\begin{array}{l}\text { Streamgage } \\
\text { number } \\
\text { (fig. 41) }\end{array}$} & \multirow[b]{2}{*}{ Streamgage name } & \multirow{2}{*}{$\begin{array}{c}\text { Total } \\
\text { drainage } \\
\left(\mathrm{mi}^{2}\right)\end{array}$} & \multicolumn{4}{|c|}{$\begin{array}{l}\text { Maximum stage and discharge for period of record } \\
\text { through } 1998 \text { water year }\end{array}$} & \multicolumn{5}{|c|}{ Significant floods 1994-98 water years } \\
\hline & & & $\begin{array}{c}\text { Period of } \\
\text { record } \\
\text { (water } \\
\text { years) }\end{array}$ & Water year & $\begin{array}{l}\text { Stage } \\
(\mathrm{ft})\end{array}$ & $\begin{array}{l}\text { Discharge } \\
\left(\mathrm{ft}^{3} / \mathrm{s}\right)\end{array}$ & $\begin{array}{c}\text { Date } \\
\text { (month/ } \\
\text { day/ } \\
\text { year) }\end{array}$ & $\begin{array}{l}\text { Stage } \\
(\mathrm{ft})\end{array}$ & $\begin{array}{l}\text { Discharge } \\
\left(\mathrm{ft}^{3} / \mathrm{s}\right)\end{array}$ & $\begin{array}{l}\text { Regulated } \\
\text { during } \\
\text { flood }^{1}\end{array}$ & $\begin{array}{c}\text { Recurrence } \\
\text { interval } \\
\text { (years) }\end{array}$ \\
\hline 01091500 & $\begin{array}{l}\text { Piscataquog River near } \\
\text { Goffstown, NH }\end{array}$ & 202 & $\begin{array}{l}1936,1938 \\
1940-78, \\
1983-98\end{array}$ & 1938 & 17.52 & 21,900 & $10 / 21 / 96$ & 10.61 & 6,470 & $\mathrm{Y}$ & $5-10$ \\
\hline 01093800 & $\begin{array}{l}\text { Stony Brook tributary near } \\
\text { Temple, NH }\end{array}$ & 3.60 & 1964-98 & $\begin{array}{l}1997 \\
1977\end{array}$ & $\begin{array}{l}5.63 \\
7.13\end{array}$ & $\begin{array}{l}648 \\
336\end{array}$ & $10 / 21 / 96$ & 5.63 & 648 & $\mathrm{~N}$ & $25-50$ \\
\hline 01129200 & $\begin{array}{l}\text { Connecticut River below Indian } \\
\text { Stream near Pittsburg, NH }\end{array}$ & 254 & $1957-98$ & 1996 & 7.97 & 5,240 & $\begin{array}{l}4 / 27 / 96 \\
3 / 31 / 98\end{array}$ & $\begin{array}{l}7.97 \\
7.87\end{array}$ & $\begin{array}{l}5,240 \\
5,120\end{array}$ & $\begin{array}{l}\mathrm{Y} \\
\mathrm{Y}\end{array}$ & $\begin{array}{l}50-100 \\
50-100\end{array}$ \\
\hline 01129440 & Mohawk River near Colebrook NH & 36.7 & 1987-98 & 1998 & 10.99 & 4,880 & $3 / 31 / 98$ & 10.99 & 4,880 & $\mathrm{~N}$ & $10-25$ \\
\hline 01129500 & $\begin{array}{l}\text { Connecticut River at North } \\
\text { Stratford, NH }\end{array}$ & 799 & $1931-98$ & $\begin{array}{l}1998 \\
1981\end{array}$ & $\begin{array}{l}15.63 \\
16.40\end{array}$ & $\begin{array}{l}32,300 \\
17,000\end{array}$ & $3 / 31 / 98$ & 15.63 & 32,300 & $\mathrm{Y}$ & $>100$ \\
\hline 01130000 & $\begin{array}{l}\text { Upper Ammonoosuc River near } \\
\text { Groveton, NH }\end{array}$ & 232 & $\begin{array}{l}1936, \\
1941-80, \\
1983-98\end{array}$ & 1969 & 12.01 & 24,100 & $3 / 31 / 98$ & 8.97 & 9,130 & $\mathrm{~N}$ & $25-50$ \\
\hline 01131500 & Connecticut River near Dalton, $\mathrm{NH}$ & 1,514 & 1928-98 & 1936 & 25.60 & 48,300 & $4 / 1 / 98$ & 23.70 & 42,000 & $\mathrm{Y}$ & $25-50$ \\
\hline 01137500 & $\begin{array}{l}\text { Ammonoosuc River at Bethlehem } \\
\text { Junction, NH }\end{array}$ & 87.6 & 1940-98 & 1996 & 12.34 & 11,300 & $11 / 12 / 95$ & 12.34 & 11,300 & $\mathrm{~N}$ & 50 \\
\hline 01145000 & $\begin{array}{l}\text { Mascoma River at West Canaan, } \\
\text { NH }\end{array}$ & 80.5 & $\begin{array}{l}1938, \\
1940-78, \\
1985-98\end{array}$ & $\begin{array}{l}1953 \\
1938\end{array}$ & $\begin{array}{l}8.94 \\
9.60\end{array}$ & $\begin{array}{l}3,780 \\
4,310\end{array}$ & $6 / 27 / 98$ & 8.59 & 3,420 & $\mathrm{~N}$ & $25-50$ \\
\hline 01158600 & $\begin{array}{l}\text { Otter Brook below Otter Brook } \\
\text { Dam near Keene, NH }\end{array}$ & 47.2 & 1959-98 & $\begin{array}{l}1987 \\
1994\end{array}$ & $\begin{array}{l}8.62 \\
8.64\end{array}$ & $\begin{array}{l}752 \\
720\end{array}$ & 4/18/94 & 8.64 & 720 & $\mathrm{Y}$ & $10-25$ \\
\hline
\end{tabular}

${ }^{1}$ Regulated during flood: N, no; Y, yes. 


\section{New Jersey}

The combination of warm temperatures, snowmelt, frozen ground, and basin average precipitation of 1.5 to 2 inches (National Oceanic and Atmospheric Administration, 1994a) caused urban flooding throughout interior southern New Jersey on January 28, 1994. The sudden increase in temperatures led to the formation of a few ice jams on area streams and rivers adding to the flooding problems. The area around Trenton (fig. 42) was the hardest hit with an estimated $\$ 4.5$ million in damage (National Oceanic and Atmospheric Administration, 1994b).

Thunderstorms with torrential downpours remained nearly stationary over the eastern Philadelphia suburbs for about 2 hours on July 14, 1994. Rainfall amounts of 3 to more than 7 inches were reported. In Westmont, 3.25 inches of rain fell within 30 minutes, 4.72 inches within 1 hour, and 6.54 inches in 3 hours. The rainfall rate of 4.72 inches per hour represents a greater than 100-year, 1-hour precipitation event for a single location in southern New Jersey (National Oceanic and Atmospheric Administration, 1994a).

Strong southerly winds ushered very mild and moistureladen air into the State on January 19, 1996. Street flooding and poor drainage became a major problem early on due to the rains, significant snowmelt, and ice jams. The flash flooding of the afternoon and early evening on January 19 led to larger river flooding through January 21 . The worst damage occurred along the Delaware River, which crested at its highest stages since the summer of 1955 (table 31). Widespread flooding along the Raritan River was described as the worst in Manville since Tropical Storm Doria in August 1971. Damage was near \$14 million, and one life was lost (National Oceanic and Atmospheric Administration, 1996b).

Nearly stationary thunderstorms dropped between 3.5 and 7.6 inches of rain within 4 hours northwest of Trenton during the evening of June 12, 1996 (National Oceanic and Atmospheric Administration, 1996a). This caused widespread flash flooding throughout the area. Nearly every roadway was flooded. The 7.6 inches of rain represent about a daily 100-year storm (National Oceanic and Atmospheric Administration, 1996a). Approximately $\$ 8$ million in property damage occurred, most of it in Ewing (National Oceanic and Atmospheric Administration, 1996b).

Rain, intense at times, fell across the northern half of New Jersey during the daylight hours of October 19, 1996. Storm precipitation totals ranged from 5 to nearly 7 inches (National Oceanic and Atmospheric Administration, 1996a). Four streamgages experienced their peak of record during this flood (table 31). Damage was near $\$ 45$ million, but there were no deaths (National Oceanic and Atmospheric Administration, 1996b).

On July 25, 1997, a long period of excessive rain occurred in northern New Jersey as a strong warm front moved slowly north toward the area. In addition, the area was affected by fringe precipitation from Tropical Storm Danny. Excessive rainfall [from 4 to 7 inches with isolated higher amounts (National Oceanic and Atmospheric Administration, 1997a)] caused widespread serious flooding of homes, streets, rivers, streams, and poor drainage areas in and around Newark. Property damage estimates were at least \$3 million (National Oceanic and Atmospheric Administration, 1997b).

Torrential rain from thunderstorms fell across southeastern New Jersey on August 20-21, 1997, as a low-pressure system developed south of Delaware Bay and slowly moved northeast across southern New Jersey. Storm totals included 10.12 inches in Millville and 7.2 inches in Vineland. The storm total of 13.52 inches of rainfall at the Atlantic City International Airport represented a greater than 100-year storm for the area. A 100-year storm for this area is 7.25 inches (National Oceanic and Atmospheric Administration, 1997a). Total damage was estimated at \$54 million, but no deaths occurred (National Oceanic and Atmospheric Administration, 1997b).

\section{References}

National Oceanic and Atmospheric Administration (NOAA), 1994a-97a, Climatological data (by State): Asheville, North Carolina, National Climatic Data Center, various months.

National Oceanic and Atmospheric Administration (NOAA), 1994b-97b, Storm data (by State): Asheville, North Carolina, National Climatic Data Center, various months. 


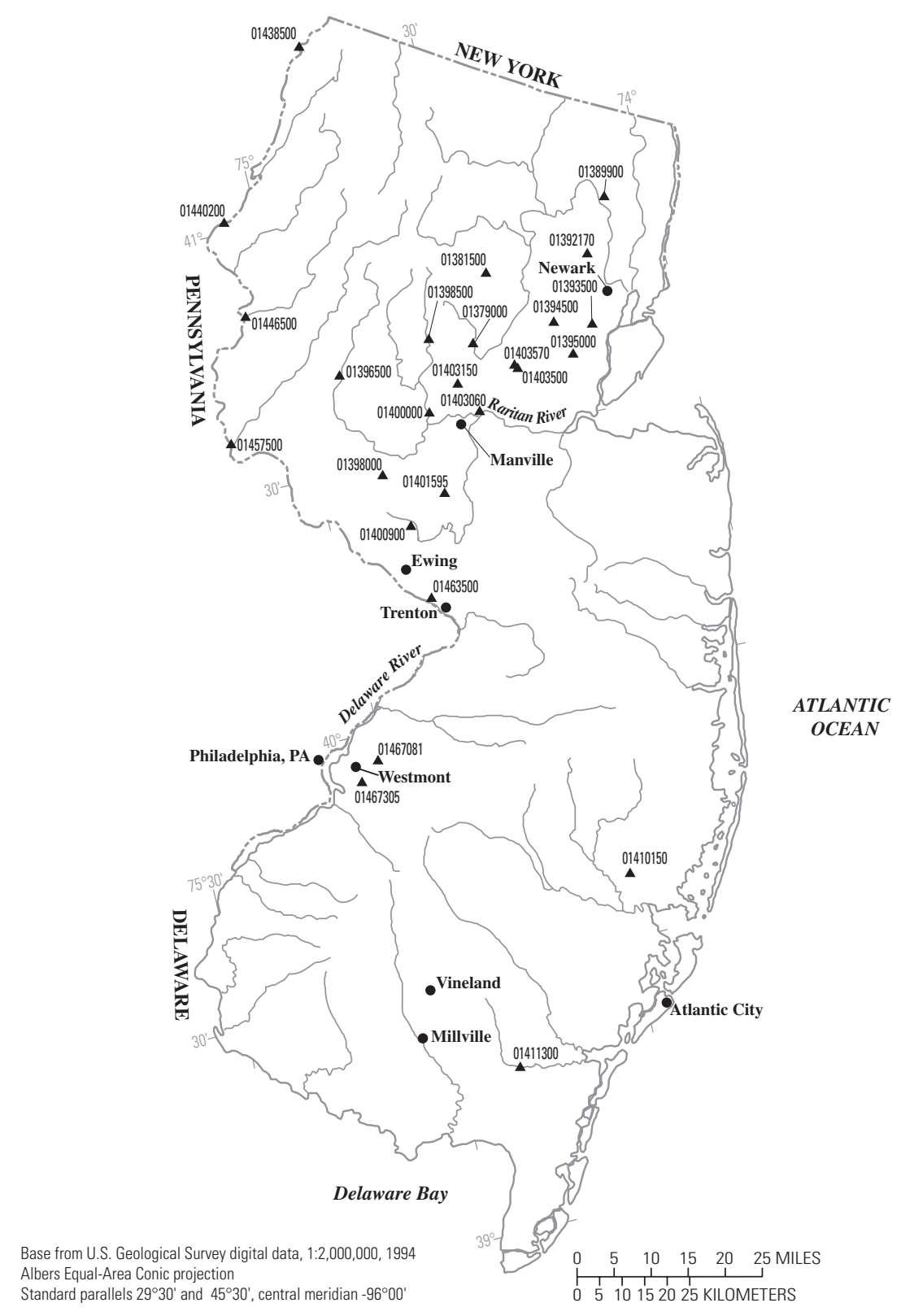

\section{EXPLANATION}

01411300 Streamgage and number

Figure 42. Location of streamgages with significant floods during 1994-98 water years for New Jersey. 
Table 31. Maximum stage and discharge for period of record for streamgages having significant floods during 1994-98 water years in New Jersey.

$\left[\mathrm{mi}^{2}\right.$, square miles; ft, feet above an arbitrary datum; $\mathrm{ft}^{3} / \mathrm{s}$, cubic feet per second; --, not determined or not applicable; >, greater than. Source: Recurrence intervals calculated from U.S. Geological Survey data. Other data from U.S. Geological Survey reports or databases]

\begin{tabular}{|c|c|c|c|c|c|c|c|c|c|c|c|}
\hline \multirow{2}{*}{$\begin{array}{c}\text { Streamgage } \\
\text { number } \\
\text { (fig. 42) }\end{array}$} & \multirow[b]{2}{*}{ Streamgage name } & \multirow{2}{*}{$\begin{array}{c}\text { Total } \\
\text { drainage } \\
\left(\mathrm{mi}^{2}\right)\end{array}$} & \multicolumn{4}{|c|}{$\begin{array}{c}\text { Maximum stage and discharge for period of record } \\
\text { through } 1998 \text { water year }\end{array}$} & \multicolumn{5}{|c|}{ Significant floods 1994-98 water years } \\
\hline & & & $\begin{array}{l}\text { Period of } \\
\text { record } \\
\text { (water } \\
\text { years) }\end{array}$ & Water year & $\begin{array}{l}\text { Stage } \\
\text { (ft) }\end{array}$ & $\begin{array}{l}\text { Discharge } \\
\left(\mathrm{ft}^{3} / \mathrm{s}\right)\end{array}$ & $\begin{array}{c}\text { Date } \\
\text { (month/ } \\
\text { day/ } \\
\text { year) }\end{array}$ & $\begin{array}{l}\text { Stage } \\
(\mathrm{ft})\end{array}$ & $\begin{array}{l}\text { Discharge } \\
\left(\mathrm{ft}^{3} / \mathrm{s}\right)\end{array}$ & $\begin{array}{l}\text { Regulated } \\
\text { during } \\
\text { flood }^{1}\end{array}$ & $\begin{array}{c}\text { Recurrence } \\
\text { interval } \\
\text { (years) }\end{array}$ \\
\hline 01379000 & Passaic River near Millington, NJ & 55.4 & $\begin{array}{l}1904-06 \\
1922-98\end{array}$ & 1997 & 9.89 & 2,290 & $10 / 20 / 96$ & 9.89 & 2,290 & $\mathrm{~N}$ & 80 \\
\hline 01381500 & Whippany River at Morristown, NJ & 29.4 & $1922-98$ & 1971 & 8.60 & 2,800 & $10 / 20 / 96$ & 7.79 & 2,300 & $\mathrm{~N}$ & 40 \\
\hline 01389900 & $\begin{array}{l}\text { Fleischer Brook at Market Street at } \\
\text { Elmwood Park, NJ }\end{array}$ & 1.37 & $\begin{array}{l}\text { 1967-93, } \\
1995-98\end{array}$ & 1978 & 6.47 & 470 & $7 / 8 / 96$ & 2.82 & 1,600 & $\mathrm{~N}$ & -- \\
\hline 01392170 & Third River at Bloomfield, NJ & 7.71 & 1989-98 & 1997 & 7.34 & 1,410 & $10 / 19 / 96$ & 7.34 & 1,410 & $\mathrm{~N}$ & 6 \\
\hline 01393500 & Elizabeth River at Elizabeth, NJ & 20.2 & 1922-98 & 1971 & 18.70 & 4,110 & $7 / 25 / 97$ & -- & 3,080 & $\mathrm{~N}$ & 9 \\
\hline 01394500 & Rahway River near Springfield, NJ & 25.5 & 1938-99 & 1973 & 9.76 & 5,430 & $7 / 25 / 97$ & 9.56 & 5,140 & $\mathrm{~N}$ & 50 \\
\hline 01395000 & Rahway River at Rahway, NJ & 40.9 & $\begin{array}{l}\text { 1922-87, } \\
1989-98\end{array}$ & 1973 & 7.88 & 5,420 & $10 / 19 / 96$ & 7.50 & 4,210 & $\mathrm{~N}$ & 30 \\
\hline 01396500 & $\begin{array}{l}\text { South Branch Raritan River near } \\
\text { High Bridge, NJ }\end{array}$ & 65.3 & $\begin{array}{l}1896,1902, \\
1904, \\
1919-24, \\
1926-98\end{array}$ & $\begin{array}{l}1979 \\
1994\end{array}$ & $\begin{array}{l}12.07 \\
14.26\end{array}$ & $\begin{array}{l}6,910 \\
2,000\end{array}$ & $10 / 20 / 96$ & 11.13 & 4,730 & $\mathrm{~N}$ & 30 \\
\hline 01398000 & Neshanic River at Reaville, NJ & 25.7 & 1931-98 & 1971 & 13.84 & 15,900 & $10 / 19 / 96$ & 12.63 & 11,100 & $\mathrm{~N}$ & 25 \\
\hline 01398500 & $\begin{array}{l}\text { North Branch Raritan River near } \\
\text { Far Hills, NJ }\end{array}$ & 26.2 & $\begin{array}{l}1919 \\
1922-98\end{array}$ & $\begin{array}{l}1971 \\
1919\end{array}$ & $\begin{array}{l}7.28 \\
7.60\end{array}$ & $\begin{array}{l}6,390 \\
7,000\end{array}$ & $10 / 19 / 96$ & 6.65 & 5,090 & $\mathrm{~N}$ & 35 \\
\hline 01400000 & $\begin{array}{l}\text { North Branch Raritan River near } \\
\text { Raritan, NJ }\end{array}$ & 190 & $\begin{array}{l}\text { 1896, } \\
1924-98\end{array}$ & 1971 & 15.47 & 28,600 & $10 / 19 / 96$ & 15.44 & 28,500 & $\mathrm{~N}$ & 100 \\
\hline 01400900 & Stony Brook at Glenmoore, NJ & 17.0 & $1957-95$ & 1971 & 11.02 & 6,100 & $1 / 28 / 94$ & 9.94 & 4,950 & $\mathrm{~N}$ & 35 \\
\hline 01401595 & Rock Brook near Blawenburg, NJ & 9.03 & $\begin{array}{l}1967-76 \\
1978-98\end{array}$ & 1971 & 10.00 & 4,530 & $10 / 19 / 96$ & 8.19 & 3,130 & $\mathrm{~N}$ & 20 \\
\hline 01403060 & $\begin{array}{l}\text { Raritan River below Callo Dam at } \\
\text { Bound Brook, NJ }\end{array}$ & 785 & $\begin{array}{l}1896, \\
1904-09, \\
1936-39, \\
1942, \\
1945-98\end{array}$ & 1971 & 37.47 & 46,100 & $10 / 20 / 96$ & 35.58 & 40,100 & $\mathrm{~N}$ & 25 \\
\hline
\end{tabular}


Table 31. Maximum stage and discharge for period of record for streamgages having significant floods during 1994-98 water years in New Jersey.-Continued

$\left[\mathrm{mi}^{2}\right.$, square miles; ft, feet above an arbitrary datum; $\mathrm{ft}^{3} / \mathrm{s}$, cubic feet per second; --, not determined or not applicable; >, greater than. Source: Recurrence intervals calculated from U.S. Geological Survey data. Other data from U.S. Geological Survey reports or databases]

\begin{tabular}{|c|c|c|c|c|c|c|c|c|c|c|c|}
\hline \multirow{2}{*}{$\begin{array}{c}\text { Streamgage } \\
\text { number } \\
\text { (fig. 42) }\end{array}$} & \multirow[b]{2}{*}{ Streamgage name } & \multirow{2}{*}{$\begin{array}{c}\text { Total } \\
\text { drainage } \\
\left(\mathrm{mi}^{2}\right)\end{array}$} & \multicolumn{4}{|c|}{$\begin{array}{c}\text { Maximum stage and discharge for period of record } \\
\text { through } 1998 \text { water year }\end{array}$} & \multicolumn{5}{|c|}{ Significant floods 1994-98 water years } \\
\hline & & & $\begin{array}{l}\text { Period of } \\
\text { record } \\
\text { (water } \\
\text { years) }\end{array}$ & Water year & $\begin{array}{l}\text { Stage } \\
(\mathrm{ft})\end{array}$ & $\begin{array}{l}\text { Discharge } \\
\left(\mathrm{ft}^{3} / \mathrm{s}\right)\end{array}$ & $\begin{array}{c}\text { Date } \\
\text { (month/ } \\
\text { day/ } \\
\text { year) }\end{array}$ & $\begin{array}{l}\text { Stage } \\
(\mathrm{ft})\end{array}$ & $\begin{array}{l}\text { Discharge } \\
\left(\mathrm{ft}^{3} / \mathrm{s}\right)\end{array}$ & $\begin{array}{l}\text { Regulated } \\
\text { during } \\
\text { flood }^{1}\end{array}$ & $\begin{array}{c}\text { Recurrence } \\
\text { interval } \\
\text { (years) }\end{array}$ \\
\hline 01403150 & $\begin{array}{l}\text { West Branch Middle Brook near } \\
\text { Martinsville, NJ }\end{array}$ & 1.99 & $1980-98$ & 1997 & 6.89 & 700 & $10 / 19 / 96$ & 6.89 & 700 & $\mathrm{~N}$ & 15 \\
\hline 01403500 & Green Brook at Plainfield, NJ & 9.75 & $\begin{array}{l}\text { 1916, 1927, } \\
1936, \\
1938-98\end{array}$ & $\begin{array}{l}1938 \\
1970\end{array}$ & $\begin{array}{l}5.82 \\
6.10\end{array}$ & $\begin{array}{l}2,890 \\
2,050\end{array}$ & $10 / 19 / 96$ & 5.82 & 2,120 & $\mathrm{~N}$ & 30 \\
\hline 01403570 & $\begin{array}{l}\text { Stony Brook at North Plainfield, } \\
\text { NJ }\end{array}$ & 6.88 & $\begin{array}{l}\text { 1938, } \\
\text { 1975-81, } \\
1991-98\end{array}$ & $\begin{array}{l}1997 \\
1938\end{array}$ & $\begin{array}{r}7.35 \\
10.00\end{array}$ & $\begin{array}{r}3,130 \\
--\end{array}$ & $10 / 19 / 96$ & 7.35 & 3,130 & $\mathrm{~N}$ & 30 \\
\hline 01410150 & $\begin{array}{l}\text { East Branch Bass River near } \\
\text { New Gretna, NJ }\end{array}$ & 8.11 & 1978-98 & 1997 & 7.28 & 750 & $8 / 22 / 97$ & 7.28 & 750 & $\mathrm{~N}$ & 100 \\
\hline 01411300 & $\begin{array}{l}\text { Tuckahoe River at Head of River, } \\
\text { NJ }\end{array}$ & 30.8 & $\begin{array}{l}1971-96, \\
1998\end{array}$ & 1997 & 9.09 & 1,340 & $\begin{array}{l}8 / 21 / 97 \\
2 / 5 / 98\end{array}$ & $\begin{array}{l}9.09 \\
6.72\end{array}$ & $\begin{array}{r}1,340 \\
638\end{array}$ & $\begin{array}{l}\mathrm{N} \\
\mathrm{N}\end{array}$ & $\begin{array}{r}>100 \\
40\end{array}$ \\
\hline 01438500 & Delaware River at Montague, $\mathrm{NJ}$ & 3,480 & $\begin{array}{l}1904, \\
1936-98\end{array}$ & $\begin{array}{l}1955 \\
1904\end{array}$ & $\begin{array}{l}35.15 \\
35.50\end{array}$ & $\begin{array}{r}250,000 \\
--\end{array}$ & $1 / 20 / 96$ & 26.66 & 149,000 & $\mathrm{~N}$ & 30 \\
\hline 01440200 & $\begin{array}{l}\text { Delaware River near Delaware } \\
\text { Water Gap, NJ }\end{array}$ & 3,850 & $\begin{array}{l}1955 \\
1965-96\end{array}$ & 1955 & 37.40 & 260,000 & $1 / 20 / 96$ & 24.89 & 155,000 & $\mathrm{~N}$ & 40 \\
\hline 01446500 & Delaware River at Belvidere, $\mathrm{NJ}$ & 4,535 & $\begin{array}{l}\text { 1904, } \\
1923-98\end{array}$ & 1955 & 30.21 & 273,000 & $1 / 20 / 96$ & 22.96 & 158,000 & $\mathrm{~N}$ & 40 \\
\hline 01457500 & Delaware River at Riegelsville, NJ & 6,328 & $\begin{array}{l}1841,1904, \\
1907-98\end{array}$ & 1955 & 38.85 & 340,000 & $1 / 20 / 96$ & 28.72 & 187,000 & $\mathrm{~N}$ & 40 \\
\hline 01463500 & Delaware River at Trenton, NJ & 6,780 & $\begin{array}{l}1902 \\
1904-98\end{array}$ & 1955 & 28.60 & 329,000 & $1 / 20 / 96$ & 22.20 & 179,000 & $\mathrm{~N}$ & 25 \\
\hline 01467081 & $\begin{array}{l}\text { South Branch Pennsauken Creek at } \\
\text { Cherry Hill, NJ }\end{array}$ & 8.98 & $\begin{array}{l}1968-76 \\
1978-98\end{array}$ & 1994 & 11.63 & 1,500 & $7 / 14 / 94$ & 11.63 & 1,500 & $\mathrm{~N}$ & 100 \\
\hline 01467305 & Newton Creek at Collingswood, NJ & 1.33 & $\begin{array}{l}1964-75 \\
1977-98\end{array}$ & 1994 & 6.82 & 328 & $7 / 14 / 94$ & 6.82 & 328 & $\mathrm{~N}$ & 70 \\
\hline
\end{tabular}

\footnotetext{
${ }^{1}$ Regulated during flood: $\mathrm{N}$, no; $\mathrm{Y}$, yes.
} 


\section{New Mexico}

Intense thunderstorms across New Mexico occurred intermittently during May, June, July, and August 1994, and caused six streamgages to experience their peak of record (table 32). On May 31, 1994, the Rio Grande River below Elephant Butte Dam (streamgage 08361000) had its greatest discharge since the flood of 1942 on May 31, 1994.

Intense thunderstorms produced excessive rainfall during June 1995, causing flooding in various parts of the State. Peaks of record were set at two streamgages in June (table 32).

An estimated rainfall of as much as 4.50 inches fell in a 3-hour period shortly after midnight on June 29, 1997 (National Oceanic and Atmospheric Administration, 1997a) near Truth or Consequences (fig. 43). Several roads were washed out or damaged, and several cars were washed into Elephant Butte Reservoir. Considerable damage also took place to utilities and a number of homes and businesses, mainly due to the rapid runoff rather than rising water levels in established drainages. Damage was near \$1 million (National Oceanic and Atmospheric Administration, 1997b).

\section{References}

National Oceanic and Atmospheric Administration (NOAA), 1997a, Climatological data (by State): Asheville, North Carolina, National Climatic Data Center, various months. National Oceanic and Atmospheric Administration (NOAA), 1997b, Storm data (by State): Asheville, North Carolina, National Climatic Data Center, various months.

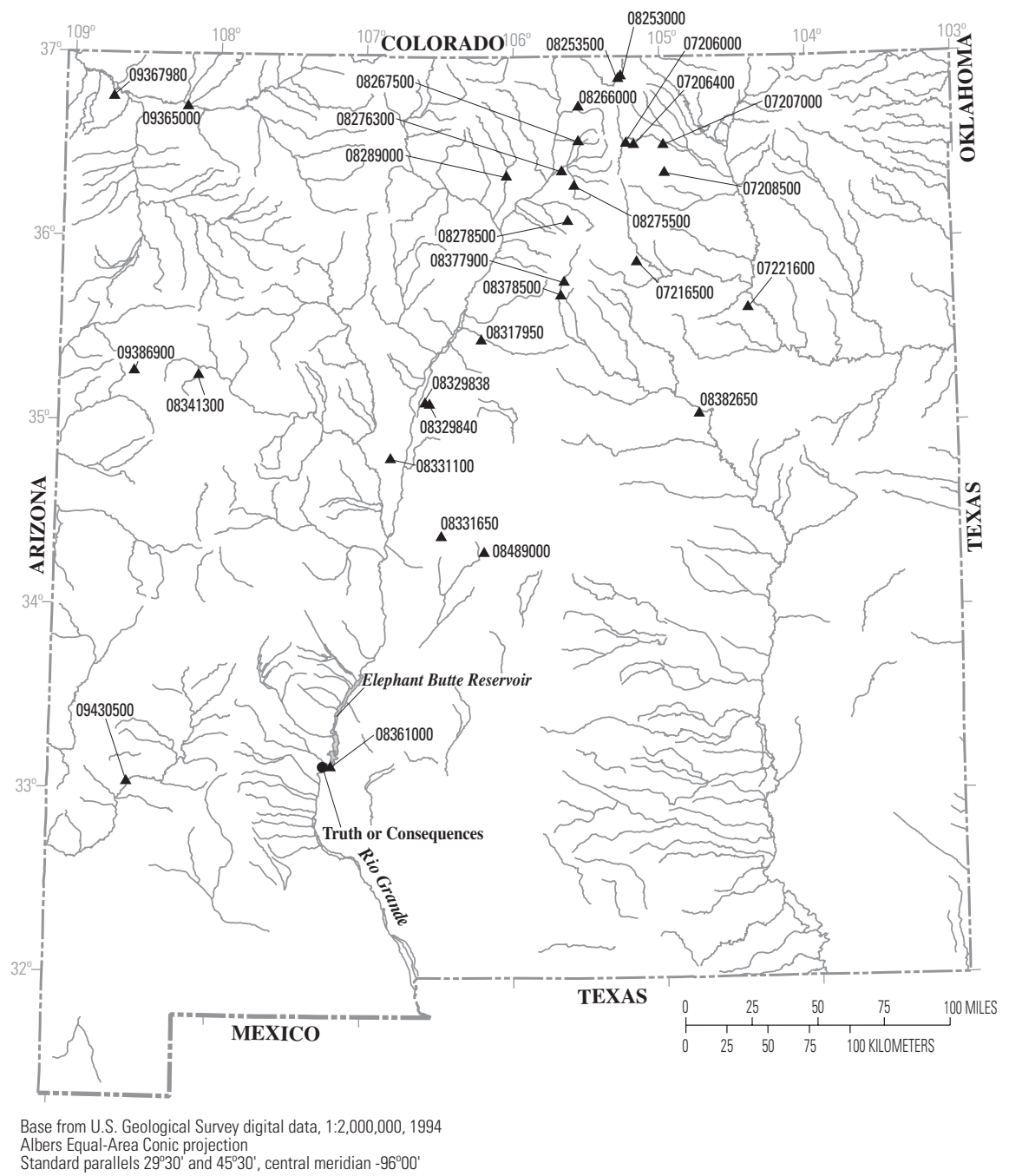

EXPLANATION

$08361000 \triangle$ Streamgage and number

Figure 43. Location of streamgages with significant floods during 1994-98 water years for New Mexico. 
Table 32. Maximum stage and discharge for period of record for streamgages having significant floods during $1994-98$ water years in New Mexico.

$\left[\mathrm{mi}^{2}\right.$, square miles; $\mathrm{ft}$, feet above an arbitrary datum; $\mathrm{ft}^{3} / \mathrm{s}$, cubic feet per second; --, not determined or not applicable. Source: Recurrence intervals calculated from U.S. Geological Survey data. Other data from U.S. Geological Survey reports or databases]

\begin{tabular}{|c|c|c|c|c|c|c|c|c|c|c|c|}
\hline \multirow{2}{*}{$\begin{array}{l}\text { Streamgage } \\
\text { number } \\
\text { (fig. 43) }\end{array}$} & \multirow[b]{2}{*}{ Streamgage name } & \multirow{2}{*}{$\begin{array}{c}\text { Total } \\
\text { drainage } \\
\left(\mathrm{mi}^{2}\right)\end{array}$} & \multicolumn{4}{|c|}{$\begin{array}{c}\text { Maximum stage and discharge for period of record } \\
\text { through } 1998 \text { water year }\end{array}$} & \multicolumn{5}{|c|}{ Significant floods $1994-98$ water years } \\
\hline & & & $\begin{array}{l}\text { Period of } \\
\text { record } \\
\text { (water } \\
\text { years) }\end{array}$ & Water year & $\begin{array}{l}\text { Stage } \\
(\mathrm{ft})\end{array}$ & $\begin{array}{l}\text { Discharge } \\
\left(\mathrm{ft}^{3} / \mathrm{s}\right)\end{array}$ & $\begin{array}{c}\text { Date } \\
\text { (month/ } \\
\text { day/ } \\
\text { year) }\end{array}$ & $\begin{array}{l}\text { Stage } \\
(\mathrm{ft})\end{array}$ & $\begin{array}{l}\text { Discharge } \\
\left(\mathrm{ft}^{3} / \mathrm{s}\right)\end{array}$ & $\begin{array}{c}\text { Regulated } \\
\text { during } \\
\text { flood }^{1}\end{array}$ & $\begin{array}{c}\text { Recurrence } \\
\text { interval } \\
\text { (years) }\end{array}$ \\
\hline 07206000 & $\begin{array}{l}\text { Cimarron River below Eagle Nest } \\
\text { Dam, NM }\end{array}$ & 167 & $1950-98$ & $\begin{array}{l}1994 \\
1955\end{array}$ & $\begin{array}{l}- \\
2.79\end{array}$ & $\begin{array}{l}303 \\
205\end{array}$ & $5 / 23 / 94$ & 3.63 & 303 & $\mathrm{Y}$ & -- \\
\hline 07206400 & Clear Creek near Ute Park, NM & 7.44 & $\begin{array}{l}1962-77, \\
1979-84, \\
1986-96\end{array}$ & 1965 & 3.05 & 151 & $7 / 24 / 94$ & 3.00 & 150 & $\mathrm{~N}$ & 35 \\
\hline 07207000 & $\begin{array}{l}\text { Cimarron River near Cimarron, } \\
\text { NM }\end{array}$ & 294 & 1950-97 & 1965 & 12.42 & 15,500 & $6 / 3 / 95$ & 4.32 & 892 & $\mathrm{Y}$ & -- \\
\hline 07208500 & $\begin{array}{l}\text { Rayado Creek at Sauble Ranch } \\
\text { near Cimarron, NM }\end{array}$ & 65.0 & $\begin{array}{l}1914, \\
1917-20, \\
1924, \\
1928-32, \\
1934-96\end{array}$ & 1965 & 11.50 & 9,000 & $6 / 18 / 95$ & 4.86 & 666 & $\mathrm{~N}$ & 10 \\
\hline 07216500 & Mora River near Golondrinas, NM & 267 & $\begin{array}{l}1916-20 \\
1929-86, \\
1988-98\end{array}$ & $\begin{array}{l}1952 \\
1969\end{array}$ & $\overline{9}-\overline{3}$ & $\begin{array}{r}14,000 \\
3,180\end{array}$ & $5 / 19 / 94$ & 5.82 & 6,180 & $\mathrm{~N}$ & 60 \\
\hline 07221600 & $\begin{array}{l}\text { Lagarita Creek tributary near } \\
\text { Sanchez, NM }\end{array}$ & 1.19 & $\begin{array}{l}\text { 1972, 1982, } \\
1989-96\end{array}$ & 1994 & 5.83 & 1,500 & $5 / 11 / 94$ & 5.83 & 1,500 & $\mathrm{~N}$ & 10 \\
\hline 08253000 & Casias Creek near Costilla, NM & 16.6 & 1937-97 & $\begin{array}{l}1994 \\
1971\end{array}$ & $\begin{array}{l}2.06 \\
2.07\end{array}$ & $\begin{array}{l}196 \\
181\end{array}$ & $5 / 25 / 94$ & 2.06 & 196 & $\mathrm{~N}$ & 50 \\
\hline 08253500 & $\begin{array}{l}\text { Santistevan Creek near Costilla, } \\
\text { NM }\end{array}$ & 2.15 & 1938-98 & $\begin{array}{l}1995 \\
1941\end{array}$ & $\begin{array}{l}1.57 \\
1.73\end{array}$ & $\begin{array}{l}20 \\
18\end{array}$ & $6 / 29 / 95$ & 1.57 & 20 & $\mathrm{~N}$ & 25 \\
\hline 08266000 & Cabresto Creek near Questa, NM & 36.7 & $1944-96$ & 1994 & 5.41 & 289 & $5 / 21 / 94$ & 5.41 & 289 & $\mathrm{Y}$ & -- \\
\hline 08267500 & Rio Hondo near Valdez, NM & 36.2 & 1935-98 & $\begin{array}{l}1941 \\
1979\end{array}$ & $\begin{array}{l}2.73 \\
4.53\end{array}$ & $\begin{array}{l}541 \\
402\end{array}$ & $6 / 20 / 95$ & 3.98 & 460 & $\mathrm{~N}$ & 20 \\
\hline
\end{tabular}


Table 32. Maximum stage and discharge for period of record for streamgages having significant floods during 1994-98 water years in New Mexico.-Continued

$\left[\mathrm{mi}^{2}\right.$, square miles; $\mathrm{ft}$, feet above an arbitrary datum; $\mathrm{ft}^{3} / \mathrm{s}$, cubic feet per second; --, not determined or not applicable. Source: Recurrence intervals calculated from U.S. Geological Survey data. Other data from U.S. Geological Survey reports or databases]

\begin{tabular}{|c|c|c|c|c|c|c|c|c|c|c|c|}
\hline \multirow{2}{*}{$\begin{array}{c}\text { Streamgage } \\
\text { number } \\
\text { (fig. 43) }\end{array}$} & \multirow[b]{2}{*}{ Streamgage name } & \multirow{2}{*}{$\begin{array}{c}\text { Total } \\
\text { drainage } \\
\left(\mathrm{mi}^{2}\right)\end{array}$} & \multicolumn{4}{|c|}{$\begin{array}{c}\text { Maximum stage and discharge for period of record } \\
\text { through } 1998 \text { water year }\end{array}$} & \multicolumn{5}{|c|}{ Significant floods 1994-98 water years } \\
\hline & & & $\begin{array}{l}\text { Period of } \\
\text { record } \\
\text { (water } \\
\text { years) }\end{array}$ & Water year & $\begin{array}{l}\text { Stage } \\
\text { (ft) }\end{array}$ & $\begin{array}{l}\text { Discharge } \\
\left(\mathrm{ft}^{3} / \mathrm{s}\right)\end{array}$ & $\begin{array}{c}\text { Date } \\
\text { (month/ } \\
\text { day/ } \\
\text { year) }\end{array}$ & $\begin{array}{l}\text { Stage } \\
(\mathrm{ft})\end{array}$ & $\begin{array}{l}\text { Discharge } \\
\left(\mathrm{ft}^{3} / \mathrm{s}\right)\end{array}$ & $\begin{array}{l}\text { Regulated } \\
\text { during } \\
\text { flood }^{1}\end{array}$ & $\begin{array}{c}\text { Recurrence } \\
\text { interval } \\
\text { (years) }\end{array}$ \\
\hline 08275500 & $\begin{array}{l}\text { Rio Grande del Rancho near Talpa, } \\
\text { NM }\end{array}$ & 83.0 & 1953-98 & 1991 & 4.16 & 644 & $5 / 20 / 94$ & 3.50 & 529 & $\mathrm{~N}$ & 25 \\
\hline 08276300 & $\begin{array}{l}\text { Rio Pueblo de Taos below Los } \\
\text { Cordovas, NM }\end{array}$ & 380 & 1957-98 & $\begin{array}{l}1994 \\
1991\end{array}$ & $\begin{array}{l}8.88 \\
8.93\end{array}$ & $\begin{array}{l}2,260 \\
1,660\end{array}$ & $5 / 20 / 94$ & 8.88 & 2,260 & $\mathrm{Y}$ & 25 \\
\hline 08278500 & $\begin{array}{l}\text { Rio Santa Barbara near Penasco, } \\
\text { NM }\end{array}$ & 38.0 & $\begin{array}{l}\text { 1954-57, } \\
1992-98\end{array}$ & 1995 & 6.21 & 838 & $6 / 18 / 95$ & 6.21 & 838 & $\mathrm{~N}$ & -- \\
\hline 08289000 & $\begin{array}{l}\text { Rio Ojo Caliente at La Madera, } \\
\text { NM }\end{array}$ & 419 & $1932-98$ & $\begin{array}{l}1998 \\
1994\end{array}$ & $\begin{array}{l}6.00 \\
8.27\end{array}$ & $\begin{array}{l}3,990 \\
3,640\end{array}$ & $\begin{array}{l}8 / 14 / 94 \\
7 / 8 / 98\end{array}$ & $\begin{array}{l}8.27 \\
6.00\end{array}$ & $\begin{array}{l}3,640 \\
3,990\end{array}$ & $\begin{array}{l}\mathrm{N} \\
\mathrm{N}\end{array}$ & $\begin{array}{l}35 \\
70\end{array}$ \\
\hline 08317950 & $\begin{array}{l}\text { Galisteo Creek below Galisteo } \\
\text { Dam, NM }\end{array}$ & 597 & 1971-97 & $\begin{array}{l}1997 \\
1981\end{array}$ & $-\overline{-}$ & $\begin{array}{l}3,460 \\
1,590\end{array}$ & $8 / 24 / 97$ & -- & 3,460 & $\mathrm{Y}$ & -- \\
\hline 08329838 & $\begin{array}{l}\text { South Fork Hahn Arroyo in } \\
\text { Albuquerque, NM }\end{array}$ & 2.03 & $\begin{array}{l}1979-83 \\
1992-98\end{array}$ & 1994 & 4.42 & 574 & $5 / 11 / 94$ & 4.42 & 574 & $\mathrm{~N}$ & -- \\
\hline 08329840 & Hahn Arroyo in Albuquerque, NM & 4.23 & 1979-98 & 1998 & 2.93 & 1,150 & $12 / 27 / 97$ & 2.93 & 1,150 & $\mathrm{~N}$ & -- \\
\hline 08331100 & $\begin{array}{l}\text { Belen Highline Canal tributary } \\
\text { near Los Lunas, NM }\end{array}$ & .16 & $1955-95$ & $\begin{array}{l}1965 \\
1994\end{array}$ & $\overline{-}-\overline{0}-3.30$ & $\begin{array}{l}754 \\
480\end{array}$ & $8 / 15 / 94$ & 7.30 & 480 & $\mathrm{~N}$ & 25 \\
\hline 08331650 & Canada Montoso near Scholle, NM & 35.0 & $\begin{array}{l}1961-80, \\
1982-95, \\
1997\end{array}$ & 1997 & 7.47 & 5,600 & $7 / 31 / 97$ & 7.47 & 5,600 & $\mathrm{~N}$ & 40 \\
\hline 08341300 & $\begin{array}{l}\text { Bluewater Creek above Bluewater } \\
\text { Dam, Bluewater, NM }\end{array}$ & 75.0 & $\begin{array}{l}\text { 1953-71, } \\
\text { 1973-77, } \\
\text { 1989-96, } \\
1998\end{array}$ & 1953 & 8.99 & 3,570 & $3 / 6 / 95$ & 4.45 & 1,940 & $\mathrm{~N}$ & 20 \\
\hline 08361000 & $\begin{array}{l}\text { Rio Grande below Elephant Butte } \\
\text { Dam, NM }\end{array}$ & 29,450 & $1915-98$ & $\begin{array}{l}1942 \\
1998\end{array}$ & $\overline{--}$ & $\begin{array}{l}8,220 \\
2,810\end{array}$ & $5 / 31 / 94$ & -- & 5,270 & $\mathrm{Y}$ & -- \\
\hline
\end{tabular}


Table 32. Maximum stage and discharge for period of record for streamgages having significant floods during 1994-98 water years in New Mexico.-Continued

$\left[\mathrm{mi}^{2}\right.$, square miles; $\mathrm{ft}$, feet above an arbitrary datum; $\mathrm{ft}^{3} / \mathrm{s}$, cubic feet per second; --, not determined or not applicable. Source: Recurrence intervals calculated from U.S. Geological Survey data. Other data from U.S. Geological Survey reports or databases]

\begin{tabular}{|c|c|c|c|c|c|c|c|c|c|c|c|}
\hline \multirow[b]{2}{*}{$\begin{array}{l}\text { Streamgage } \\
\text { number } \\
\text { (fig. 43) }\end{array}$} & \multirow[b]{2}{*}{ Streamgage name } & \multirow[b]{2}{*}{$\begin{array}{c}\text { Total } \\
\text { drainage } \\
\left(\mathrm{mi}^{2}\right)\end{array}$} & \multicolumn{4}{|c|}{$\begin{array}{c}\text { Maximum stage and discharge for period of record } \\
\text { through } 1998 \text { water year }\end{array}$} & \multicolumn{5}{|c|}{ Significant floods 1994-98 water years } \\
\hline & & & $\begin{array}{l}\text { Period of } \\
\text { record } \\
\text { (water } \\
\text { years) }\end{array}$ & Water year & $\begin{array}{l}\text { Stage } \\
\text { (ft) }\end{array}$ & $\begin{array}{c}\text { Discharge } \\
\left(\mathrm{ft}^{3} / \mathrm{s}\right)\end{array}$ & $\begin{array}{c}\text { Date } \\
\text { (month/ } \\
\text { day/ } \\
\text { year) }\end{array}$ & $\begin{array}{l}\text { Stage } \\
(\mathrm{ft})\end{array}$ & $\begin{array}{l}\text { Discharge } \\
\left(\mathrm{ft}^{3} / \mathrm{s}\right)\end{array}$ & $\begin{array}{c}\text { Regulated } \\
\text { during } \\
\text { flood }^{1}\end{array}$ & $\begin{array}{c}\text { Recurrence } \\
\text { interval } \\
\text { (years) }\end{array}$ \\
\hline 08377900 & Rio Mora near Tererro, NM & 53.2 & 1964-98 & $\begin{array}{l}1991 \\
1979\end{array}$ & $\begin{array}{l}4.08 \\
4.15\end{array}$ & $\begin{array}{l}937 \\
820\end{array}$ & $6 / 8 / 97$ & 3.99 & 864 & $\mathrm{~N}$ & 25 \\
\hline 08378500 & Pecos River near Pecos, NM & 189 & $\begin{array}{l}1920-22, \\
1924,1926, \\
1928-98\end{array}$ & 1929 & 6.20 & 4,500 & $6 / 7 / 97$ & 4.79 & 2,060 & $\mathrm{~N}$ & 20 \\
\hline 08382650 & $\begin{array}{l}\text { Pecos River above Santa Rosa } \\
\text { Lake, NM }\end{array}$ & 2,340 & 1976-98 & 1996 & 19.06 & 16,000 & $7 / 11 / 96$ & 19.06 & 16,000 & $\mathrm{~N}$ & 20 \\
\hline 08489000 & Big Draw near Mountainair, NM & 4.06 & $\begin{array}{l}1953-71, \\
1973-74, \\
1978-83, \\
1985-96\end{array}$ & 1954 & 8.68 & 1,710 & $6 / 26 / 96$ & 6.64 & 1,040 & $\mathrm{~N}$ & 20 \\
\hline 09365000 & San Juan River at Farmington, NM & 7,240 & 1924-97 & $\begin{array}{l}1927 \\
1935\end{array}$ & $-\overline{-}-00$ & $\begin{array}{l}68,000 \\
32,800\end{array}$ & $6 / 2 / 97$ & 7.77 & 13,000 & $\mathrm{Y}$ & 4 \\
\hline 09367980 & $\begin{array}{l}\text { Rattlesnake Arroyo near Shiprock, } \\
\text { NM }\end{array}$ & -- & $1980-96$ & 1995 & 6.25 & 3,800 & 9/7/95 & 6.25 & 3,800 & $\mathrm{~N}$ & 20 \\
\hline 09386900 & Rio Nutria near Ramah, NM & 71.4 & 1970-98 & 1995 & 9.34 & 1,850 & $3 / 5 / 95$ & 9.34 & 1,850 & $\mathrm{~N}$ & 30 \\
\hline 09430500 & Gila River near Gila, NM & 1,864 & $1928-98$ & $\begin{array}{l}1985 \\
1941\end{array}$ & $\begin{array}{l}13.00 \\
17.19\end{array}$ & $\begin{array}{l}35,200 \\
25,400\end{array}$ & $9 / 22 / 97$ & 11.18 & 18,200 & $\mathrm{~N}$ & 20 \\
\hline
\end{tabular}

${ }^{1}$ Regulated during flood: N, no; Y, yes. 


\section{New York}

The most disastrous flood in more than 20 years struck central New York from January 19-20, 1996. Significant widespread flooding of streams, rivers, homes and businesses, streets and highways, woodlands, and farmland occurred as a result of rapid snowmelt and nearly simultaneous storm runoff. An area of intense low pressure, which was located over the Mid-Atlantic region on Friday morning January 19, produced unseasonably warm temperatures, high dew points, and strong winds. This resulted in rapid melting of 1 to 3 feet of snow. In addition to the rapid snowmelt, 1 to 3 inches of rain fell as the system moved northeast along the coast (National Oceanic and Atmospheric Administration, 1996a). There were 10 known fatalities. Total damage statewide was more than $\$ 200$ million (National Oceanic and Atmospheric Administration 1996b). Flash floods that began early on January 19 gave rise to mainstem river floods that persisted until the evening of January 21. Near-record river flooding occurred January 19-20 in the Chemung, upper Susquehanna, Hudson, and upper Delaware River Basins (fig. 44). At Waverly, the Susquehanna River crested at 20.35 feet, its third highest level of all time, which was about 1 foot lower than the all time crest of 21.40 feet recorded on March 19, 1946 (National Oceanic and Atmospheric Administration, 1996b). Flooding was so widespread and severe that this event became known as the "Deluge of '96." Thirty-one streamgages experienced their peak of record in New York during this flood (table 33).

On Friday October 18, 1996, a strong low-pressure system developed along a cold front in New Jersey. With a highpressure system in place across northern New England, the low slowly intensified and moved slowly off the southern New
Jersey Coast during October 19. The increasing difference in pressures caused strong and gusty east winds, which also transported abundant moisture from the Atlantic Ocean across the region. Excessive flood-producing rains along with minor-tomajor coastal flooding occurred. More than $\$ 17$ million in damage resulted from coastal and river flooding (National Oceanic and Atmospheric Administration, 1996b).

During November 8-9, 1996, a slow-moving, lowpressure system tracked from northern Pennsylvania to northern New York. This system produced 4 to 5 inches of rain across most of central New York and the northern Catskill Mountains (National Oceanic and Atmospheric Administration, 1996a). The resulting runoff caused flooding along the Mohawk River to the Hudson River. Nearly \$25 million in damage resulted (National Oceanic and Atmospheric Administration, 1996b).

An area of weak low pressure and a moist, unstable air mass over New England and New York resulted in evening and late-night thunderstorms with locally torrential downpours in extreme northern New York during June 25-27, 1998. Approximately \$20 million in damage resulted (National Oceanic and Atmospheric Administration, 1998b).

\section{References}

National Oceanic and Atmospheric Administration (NOAA), 1996a-98a, Climatological data (by State): Asheville, North Carolina, National Climatic Data Center, various months.

National Oceanic and Atmospheric Administration (NOAA), 1996b-98b, Storm data (by State): Asheville, North Carolina, National Climatic Data Center, various months. 

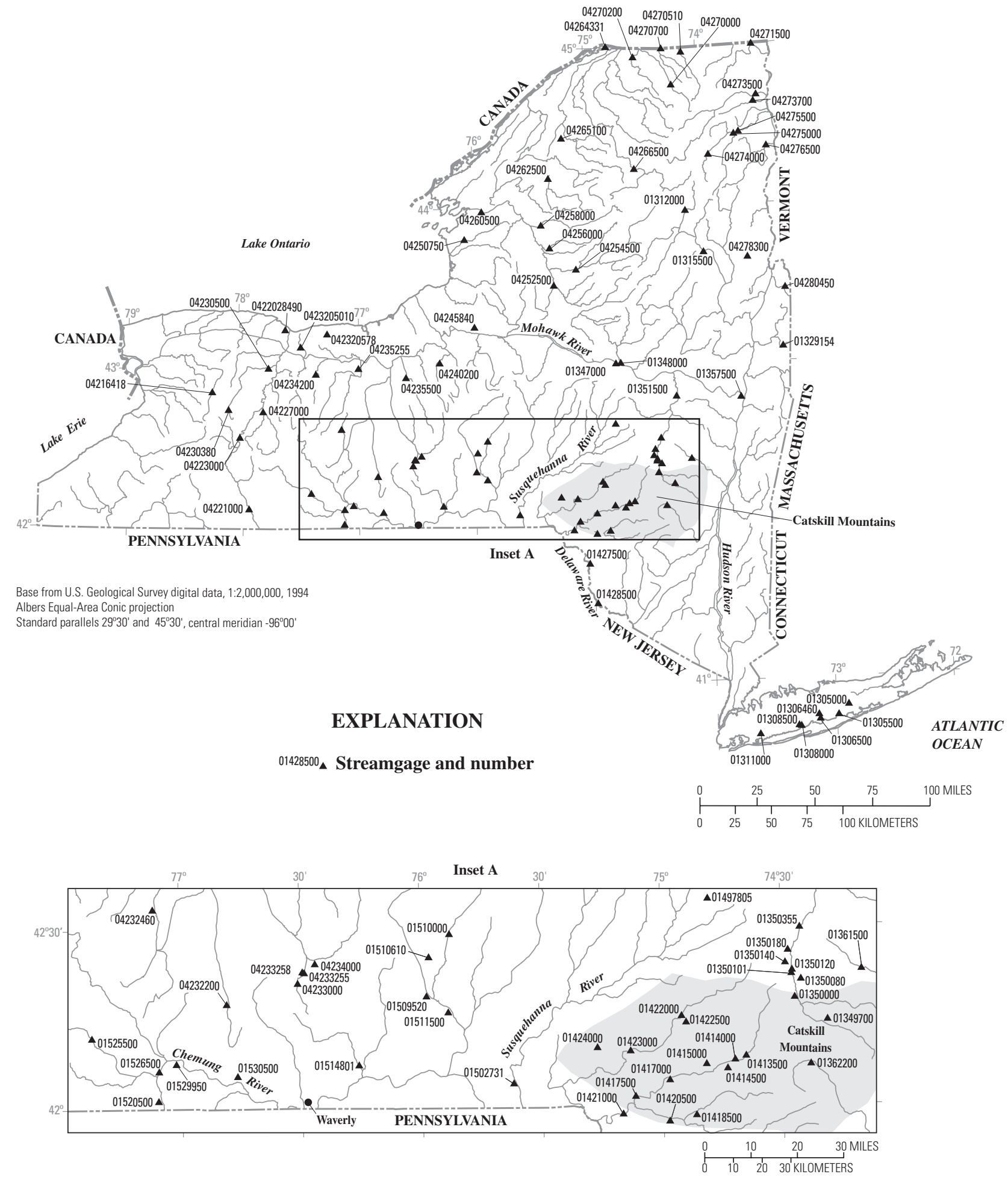

Figure 44. Location of streamgages with significant floods during 1994-98 water years for New York. 
Table 33. Maximum stage and discharge for period of record for streamgages having significant floods during 1994-98 water years in New York.

$\left[\mathrm{mi}^{2}\right.$, square miles; $\mathrm{ft}$, feet above an arbitrary datum; $\mathrm{ft}^{3} / \mathrm{s}$, cubic feet per second; --, not determined or not applicable; >, greater than. Source: Recurrence intervals calculated from U.S. Geological Survey data. Other data from U.S. Geological Survey reports or databases]

\begin{tabular}{|c|c|c|c|c|c|c|c|c|c|c|c|}
\hline \multirow{2}{*}{$\begin{array}{c}\text { Streamgage } \\
\text { number } \\
\text { (fig. 44) }\end{array}$} & \multirow[b]{2}{*}{ Streamgage name } & \multirow{2}{*}{$\begin{array}{c}\text { Total } \\
\text { drainage } \\
\left(\mathrm{mi}^{2}\right)\end{array}$} & \multicolumn{4}{|c|}{$\begin{array}{c}\text { Maximum stage and discharge for period of record } \\
\text { through } 1998 \text { water year }\end{array}$} & \multicolumn{5}{|c|}{ Significant floods 1994-98 water years } \\
\hline & & & $\begin{array}{l}\text { Period of } \\
\text { record } \\
\text { (water } \\
\text { years) }\end{array}$ & Water year & $\begin{array}{l}\text { Stage } \\
(\mathrm{ft})\end{array}$ & $\begin{array}{l}\text { Discharge } \\
\left(\mathrm{ft}^{3} / \mathrm{s}\right)\end{array}$ & $\begin{array}{c}\text { Date } \\
\text { (month/ } \\
\text { day/ } \\
\text { year) }\end{array}$ & $\begin{array}{l}\text { Stage } \\
(\mathrm{ft})\end{array}$ & $\begin{array}{l}\text { Discharge } \\
\left(\mathrm{ft}^{3} / \mathrm{s}\right)\end{array}$ & $\begin{array}{c}\text { Regulated } \\
\text { during } \\
\text { flood }^{1}\end{array}$ & $\begin{array}{c}\text { Recurrence } \\
\text { interval } \\
\text { (years) }\end{array}$ \\
\hline 01305000 & Carmans River at Yaphank, NY & 71.0 & $1943-98$ & 1989 & 2.09 & 143 & $6 / 13 / 98$ & 1.99 & 122 & $\mathrm{~N}$ & 25 \\
\hline 01305500 & Swan River at East Patchogue, NY & 8.60 & 1947-98 & 1990 & 2.71 & 77 & $6 / 13 / 98$ & 2.39 & 69 & $\mathrm{~N}$ & $75-100$ \\
\hline 01306460 & $\begin{array}{l}\text { Connetquot Brook near Central } \\
\text { Islip, NY }\end{array}$ & 18.0 & 1978-98 & 1998 & 3.89 & 155 & $6 / 13 / 98$ & 3.89 & 155 & $\mathrm{~N}$ & $10-25$ \\
\hline 01306500 & $\begin{array}{l}\text { Connetquot River near Oakdale, } \\
\text { NY }\end{array}$ & 24.0 & $\begin{array}{l}\text { 1944-93, } \\
1997-98\end{array}$ & 1956 & -- & 263 & $6 / 13 / 98$ & -- & 174 & $\mathrm{~N}$ & -- \\
\hline 01308000 & Sampawams Creek at Babylon, NY & 22.7 & $1945-98$ & 1998 & 3.73 & 254 & $6 / 13 / 98$ & 3.73 & 254 & $\mathrm{~N}$ & $>100$ \\
\hline 01308500 & Carlls River at Babylon, NY & 35.4 & 1945-98 & 1998 & 2.46 & 336 & $6 / 13 / 98$ & 2.46 & 336 & $\mathrm{~N}$ & 100 \\
\hline 01311000 & Pines Brook at Malverne, NY & 10.0 & 1939-98 & 1994 & 5.28 & 866 & $\begin{array}{l}1 / 28 / 94 \\
10 / 19 / 96\end{array}$ & $\begin{array}{l}5.28 \\
4.81\end{array}$ & $\begin{array}{l}866 \\
578\end{array}$ & $\begin{array}{l}\mathrm{N} \\
\mathrm{N}\end{array}$ & $\begin{array}{r}75-100 \\
25\end{array}$ \\
\hline 01312000 & Hudson River near Newcomb, NY & 192 & 1926-98 & 1998 & 12.84 & 11,500 & $\begin{array}{l}11 / 10 / 96 \\
1 / 9 / 98\end{array}$ & $\begin{array}{r}9.01 \\
12.84\end{array}$ & $\begin{array}{r}5,900 \\
11,500\end{array}$ & $\begin{array}{l}\mathrm{N} \\
\mathrm{N}\end{array}$ & $\begin{array}{r}10 \\
>100\end{array}$ \\
\hline 01315500 & Hudson River at North Creek, NY & 792 & 1908-98 & 1949 & 12.14 & 28,900 & $1 / 9 / 98$ & 11.54 & 26,300 & $\mathrm{Y}$ & 50 \\
\hline 01329154 & Steele Brook at Shushan, NY & 2.85 & 1979-98 & 1996 & 6.56 & 149 & $1 / 19 / 96$ & 6.56 & 149 & $\mathrm{~N}$ & $10-25$ \\
\hline 01347000 & $\begin{array}{l}\text { Mohawk River near Little Falls, } \\
\text { NY }\end{array}$ & 1,342 & $\begin{array}{l}\text { 1901, 1902, } \\
\text { 1904, 1913, } \\
1928-98\end{array}$ & 1913 & -- & 34,800 & $1 / 19 / 96$ & 18.47 & 30,700 & $\mathrm{Y}$ & $75-100$ \\
\hline 01348000 & $\begin{array}{l}\text { East Canada Creek at East Creek, } \\
\text { NY }\end{array}$ & 289 & $\begin{array}{l}1946-96, \\
1998\end{array}$ & 1946 & 9.00 & 24,000 & $\begin{array}{l}1 / 19 / 96 \\
1 / 09 / 98\end{array}$ & $\begin{array}{l}8.32 \\
8.46\end{array}$ & $\begin{array}{l}17,000 \\
17,800\end{array}$ & $\begin{array}{l}\mathrm{N} \\
\mathrm{N}\end{array}$ & $\begin{array}{r}50 \\
50-75\end{array}$ \\
\hline 01349700 & East Kill near Jewett Center, NY & 35.6 & $\begin{array}{l}\text { 1951, 1956, } \\
1960, \\
\text { 1967-68, } \\
\text { 1972-74, } \\
1987, \\
1996-98\end{array}$ & 1996 & 17.00 & 13,500 & $1 / 19 / 96$ & 17.00 & 13,500 & $\mathrm{~N}$ & 25 \\
\hline 01350000 & Schoharie Creek at Prattsville, NY & 237 & $\begin{array}{l}\text { 1904, } \\
\text { 1908-24, } \\
\text { 1926-28, } \\
1930-98\end{array}$ & 1996 & 19.39 & 52,800 & $1 / 19 / 96$ & 19.39 & 52,800 & $\mathrm{~N}$ & $25-50$ \\
\hline
\end{tabular}


Table 33. Maximum stage and discharge for period of record for streamgages having significant floods during 1994-98 water years in New York.—Continued

$\left[\mathrm{mi}^{2}\right.$, square miles; $\mathrm{ft}$, feet above an arbitrary datum; $\mathrm{ft}^{3} / \mathrm{s}$, cubic feet per second; --, not determined or not applicable; >, greater than. Source: Recurrence intervals calculated from U.S. Geological Survey data. Other data from U.S. Geological Survey reports or databases]

\begin{tabular}{|c|c|c|c|c|c|c|c|c|c|c|c|}
\hline \multirow{2}{*}{$\begin{array}{l}\text { Streamgage } \\
\text { number } \\
\text { (fig. 44) }\end{array}$} & \multirow[b]{2}{*}{ Streamgage name } & \multirow{2}{*}{$\begin{array}{c}\text { Total } \\
\text { drainage } \\
\left(\mathrm{mi}^{2}\right)\end{array}$} & \multicolumn{4}{|c|}{$\begin{array}{l}\text { Maximum stage and discharge for period of record } \\
\text { through } 1998 \text { water year }\end{array}$} & \multicolumn{5}{|c|}{ Significant floods $1994-98$ water years } \\
\hline & & & $\begin{array}{l}\text { Period of } \\
\text { record } \\
\text { (water } \\
\text { years) }\end{array}$ & Water year & $\begin{array}{l}\text { Stage } \\
(\mathrm{ft})\end{array}$ & $\begin{array}{l}\text { Discharge } \\
\left(\mathrm{ft}^{3} / \mathrm{s}\right)\end{array}$ & $\begin{array}{c}\text { Date } \\
\text { (month/ } \\
\text { day/ } \\
\text { year) }\end{array}$ & $\begin{array}{l}\text { Stage } \\
(\mathrm{ft})\end{array}$ & $\begin{array}{l}\text { Discharge } \\
\left(\mathrm{ft}^{3} / \mathrm{s}\right)\end{array}$ & $\begin{array}{l}\text { Regulated } \\
\text { during } \\
\text { flood }^{1}\end{array}$ & $\begin{array}{c}\text { Recurrence } \\
\text { interval } \\
\text { (years) }\end{array}$ \\
\hline 01350080 & $\begin{array}{l}\text { Manor Kill at West Conesville near } \\
\text { Gilboa, NY }\end{array}$ & 32.4 & $1987-98$ & 1996 & 10.20 & 5,050 & $1 / 19 / 96$ & 10.20 & 5,050 & $\mathrm{~N}$ & $10-25$ \\
\hline 01350101 & Schoharie Creek at Gilboa, NY & 316 & $\begin{array}{l}\text { 1936, 1938, } \\
1956, \\
1976-98\end{array}$ & 1996 & 30.60 & 70,800 & $1 / 19 / 96$ & 30.60 & 70,800 & $\mathrm{Y}$ & $75-100$ \\
\hline 01350120 & Platter Kill at Gilboa, NY & 10.9 & 1976-98 & 1996 & 6.70 & 1,370 & $1 / 19 / 96$ & 6.70 & 1,370 & $\mathrm{~N}$ & 25 \\
\hline 01350140 & $\begin{array}{l}\text { Mine Kill near North Blenheim, } \\
\text { NY }\end{array}$ & 16.2 & $1975-98$ & 1996 & 5.20 & 2,550 & $1 / 19 / 96$ & 5.20 & 2,550 & $\mathrm{~N}$ & $25-50$ \\
\hline 01350180 & $\begin{array}{l}\text { Schoharie Creek at North } \\
\text { Blenheim, NY }\end{array}$ & 358 & 1971-98 & 1996 & 17.61 & 75,600 & $1 / 19 / 96$ & 17.61 & 75,600 & $\mathrm{Y}$ & $75-100$ \\
\hline 01350355 & $\begin{array}{l}\text { Schoharie Creek at Breakabeen, } \\
\text { NY }\end{array}$ & 444 & 1976-98 & 1996 & 20.51 & 80,200 & $1 / 19 / 96$ & 20.51 & 80,200 & $\mathrm{Y}$ & $75-100$ \\
\hline 01351500 & $\begin{array}{l}\text { Schoharie Creek at Burtonsville, } \\
\text { NY }\end{array}$ & 886 & $1940-98$ & 1996 & 12.88 & 81,600 & $1 / 20 / 96$ & 12.88 & 81,600 & $\mathrm{Y}$ & $>100$ \\
\hline 01357500 & Mohawk River at Cohoes, NY & 3,450 & $1915-98$ & 1964 & 23.15 & 143,000 & $1 / 20 / 96$ & 22.68 & 132,000 & $\mathrm{Y}$ & 100 \\
\hline 01361500 & Catskill Creek at Oak Hill, NY & 98.0 & $\begin{array}{l}\text { 1911-77, } \\
1980, \\
1987-98\end{array}$ & 1987 & 16.60 & 15,400 & $1 / 19 / 96$ & 15.35 & 13,400 & $\mathrm{~N}$ & $25-50$ \\
\hline 01362200 & Esopus Creek at Allaben, NY & 63.7 & $\begin{array}{l}\text { 1951, } \\
1964-98\end{array}$ & 1951 & -- & 20,000 & $1 / 19 / 96$ & 13.58 & 15,000 & $\mathrm{~N}$ & $25-50$ \\
\hline 01413500 & $\begin{array}{l}\text { East Branch Delaware River at } \\
\text { Margaretville, NY }\end{array}$ & 163 & 1937-98 & 1996 & 14.88 & 25,800 & $1 / 19 / 96$ & 14.88 & 25,800 & $\mathrm{~N}$ & $75-100$ \\
\hline 01414000 & Platte Kill at Dunraven, NY & 34.9 & $\begin{array}{l}1942-62 \\
1996-98\end{array}$ & 1996 & 11.20 & 5,690 & $1 / 19 / 96$ & 11.20 & 5,690 & $\mathrm{~N}$ & $>100$ \\
\hline \multirow[t]{2}{*}{01414500} & Mill Brook near Dunraven, NY & 25.2 & 1937-98 & 1996 & 12.56 & 5,380 & $1 / 19 / 96$ & 12.56 & 5,380 & $\mathrm{~N}$ & $75-100$ \\
\hline & & & & & & & $11 / 9 / 96$ & 11.09 & 3,850 & $\mathrm{~N}$ & 25 \\
\hline
\end{tabular}


$\left[\mathrm{mi}^{2}\right.$, square miles; ft, feet above an arbitrary datum; $\mathrm{ft}^{3} / \mathrm{s}$, cubic feet per second; --, not determined or not applicable; >, greater than. Source: Recurrence intervals calculated from U.S. Geological Survey data. Other data from U.S. Geological Survey reports or databases]

\begin{tabular}{|c|c|c|c|c|c|c|c|c|c|c|c|}
\hline \multirow[b]{2}{*}{$\begin{array}{c}\text { Streamgage } \\
\text { number } \\
\text { (fig. 44) }\end{array}$} & \multirow[b]{2}{*}{ Streamgage name } & \multirow[b]{2}{*}{$\begin{array}{c}\text { Total } \\
\text { drainage } \\
\left(\mathrm{mi}^{2}\right)\end{array}$} & \multicolumn{4}{|c|}{$\begin{array}{c}\text { Maximum stage and discharge for period of record } \\
\text { through } 1998 \text { water year }\end{array}$} & \multicolumn{5}{|c|}{ Significant floods $1994-98$ water years } \\
\hline & & & $\begin{array}{l}\text { Period of } \\
\text { record } \\
\text { (water } \\
\text { years) }\end{array}$ & Water year & $\begin{array}{l}\text { Stage } \\
(\mathrm{ft})\end{array}$ & $\begin{array}{l}\text { Discharge } \\
\left(\mathrm{ft}^{3} / \mathrm{s}\right)\end{array}$ & $\begin{array}{c}\text { Date } \\
\text { (month/ } \\
\text { day/ } \\
\text { year) }\end{array}$ & $\begin{array}{l}\text { Stage } \\
(\mathrm{ft})\end{array}$ & $\begin{array}{l}\text { Discharge } \\
\left(\mathrm{ft}^{3} / \mathrm{s}\right)\end{array}$ & $\begin{array}{c}\text { Regulated } \\
\text { during } \\
\text { flood }^{1}\end{array}$ & $\begin{array}{c}\text { Recurrence } \\
\text { interval } \\
\text { (years) }\end{array}$ \\
\hline 01415000 & Tremper Kill near Andes, NY & 33.2 & $1937-98$ & 1996 & 7.69 & 5,000 & $1 / 19 / 96$ & 7.69 & 5,000 & $\mathrm{~N}$ & $75-100$ \\
\hline 01417000 & $\begin{array}{l}\text { East Branch Delaware River at } \\
\text { Downsville, NY }\end{array}$ & 372 & $\begin{array}{l}\text { 1904, } \\
1942-98\end{array}$ & 1904 & 16.00 & 34,000 & $12 / 2 / 96$ & 9.41 & 11,000 & $\mathrm{Y}$ & 25 \\
\hline 01417500 & $\begin{array}{l}\text { East Branch Delaware River at } \\
\text { Harvard, NY }\end{array}$ & 458 & $\begin{array}{l}1904, \\
1935-67, \\
1978-98\end{array}$ & $\begin{array}{l}1938 \\
1904\end{array}$ & $\begin{array}{l}16.93 \\
--\end{array}$ & $\begin{array}{l}31,400 \\
42,000\end{array}$ & $\begin{array}{l}1 / 19 / 96 \\
12 / 2 / 96\end{array}$ & $\begin{array}{l}12.63 \\
12.79\end{array}$ & $\begin{array}{l}12,200 \\
12,400\end{array}$ & $\begin{array}{l}\mathrm{Y} \\
\mathrm{Y}\end{array}$ & $\begin{array}{l}25-50 \\
25-50\end{array}$ \\
\hline 01418500 & Beaver Kill at Craigie Clair, NY & 81.9 & $\begin{array}{l}1937-74, \\
1996\end{array}$ & 1996 & 11.83 & 13,000 & $1 / 19 / 96$ & 11.83 & 13,000 & $\mathrm{~N}$ & $50-75$ \\
\hline 01420500 & Beaver Kill at Cooks Falls, NY & 241 & 1914-98 & 1996 & 17.79 & 42,900 & $1 / 19 / 96$ & 17.79 & 42,900 & $\mathrm{~N}$ & $>100$ \\
\hline 01421000 & $\begin{array}{l}\text { East Branch Delaware River at } \\
\text { Fishs Eddy, NY }\end{array}$ & 784 & $\begin{array}{l}1904, \\
1913-98\end{array}$ & 1904 & 23.60 & 70,000 & $1 / 19 / 96$ & 16.88 & 53,000 & $\mathrm{Y}$ & $>100$ \\
\hline 01422000 & $\begin{array}{l}\text { West Branch Delaware River at } \\
\text { Delhi, NY }\end{array}$ & 142 & $\begin{array}{l}1937-74 \\
1996\end{array}$ & 1996 & 9.80 & 13,000 & $1 / 19 / 96$ & 9.80 & 13,000 & $\mathrm{~N}$ & $>100$ \\
\hline 01422500 & $\begin{array}{l}\text { Little Delaware River near Delhi, } \\
\text { NY }\end{array}$ & 49.8 & $\begin{array}{l}1938-74, \\
1996-98\end{array}$ & 1996 & 8.51 & 6,100 & $\begin{array}{l}1 / 19 / 96 \\
11 / 9 / 96\end{array}$ & $\begin{array}{l}8.51 \\
7.67\end{array}$ & $\begin{array}{l}6,100 \\
4,540\end{array}$ & $\begin{array}{l}\mathrm{N} \\
\mathrm{N}\end{array}$ & $\begin{array}{r}>100 \\
25-50\end{array}$ \\
\hline 01423000 & $\begin{array}{l}\text { West Branch Delaware River at } \\
\text { Walton, NY }\end{array}$ & 332 & 1951-98 & 1996 & 16.36 & 25,000 & $1 / 19 / 96$ & 16.36 & 25,000 & $\mathrm{~N}$ & 50 \\
\hline 01424000 & Trout Creek near Rockroyal, NY & 20.0 & $\begin{array}{l}1952-67, \\
1996\end{array}$ & 1996 & 10.06 & 2,800 & $1 / 19 / 96$ & 10.06 & 2,800 & $\mathrm{~N}$ & 50 \\
\hline 01427500 & Callicoon Creek at Callicoon, NY & 110 & $\begin{array}{l}1940-82 \\
1987-98\end{array}$ & 1947 & 9.68 & 16,000 & $1 / 19 / 96$ & 8.42 & 11,200 & $\mathrm{~N}$ & $25-50$ \\
\hline 01428500 & $\begin{array}{l}\text { Delaware River above Lacka- } \\
\text { waxen River near Barryville, NY }\end{array}$ & 2,020 & 1941-98 & 1955 & 26.40 & 130,000 & $1 / 20 / 96$ & 22.18 & 98,300 & $\mathrm{Y}$ & $50-75$ \\
\hline 01497805 & $\begin{array}{l}\text { Little Elk Creek near Westford, } \\
\text { NY }\end{array}$ & 3.73 & 1978-98 & 1996 & 19.92 & 278 & 1/19/96 & 19.92 & 278 & $\mathrm{~N}$ & $25-50$ \\
\hline
\end{tabular}


Table 33. Maximum stage and discharge for period of record for streamgages having significant floods during 1994-98 water years in New York.-Continued

$\left[\mathrm{mi}^{2}\right.$, square miles; $\mathrm{ft}$, feet above an arbitrary datum; $\mathrm{ft}^{3} / \mathrm{s}$, cubic feet per second; --, not determined or not applicable; >, greater than. Source: Recurrence intervals calculated from U.S. Geological Survey data. Other data from U.S. Geological Survey reports or databases]

\begin{tabular}{|c|c|c|c|c|c|c|c|c|c|c|c|}
\hline \multirow{2}{*}{$\begin{array}{c}\text { Streamgage } \\
\text { number } \\
\text { (fig. 44) }\end{array}$} & \multirow[b]{2}{*}{ Streamgage name } & \multirow{2}{*}{$\begin{array}{c}\text { Total } \\
\text { drainage } \\
\left(\mathrm{mi}^{2}\right)\end{array}$} & \multicolumn{4}{|c|}{$\begin{array}{c}\text { Maximum stage and discharge for period of record } \\
\text { through } 1998 \text { water year }\end{array}$} & \multicolumn{5}{|c|}{ Significant floods 1994-98 water years } \\
\hline & & & $\begin{array}{c}\text { Period of } \\
\text { record } \\
\text { (water } \\
\text { years) }\end{array}$ & Water year & $\begin{array}{l}\text { Stage } \\
\text { (ft) }\end{array}$ & $\begin{array}{l}\text { Discharge } \\
\left(\mathrm{ft}^{3} / \mathrm{s}\right)\end{array}$ & $\begin{array}{c}\text { Date } \\
\text { (month/ } \\
\text { day/ } \\
\text { year) }\end{array}$ & $\begin{array}{l}\text { Stage } \\
\text { (ft) }\end{array}$ & $\begin{array}{l}\text { Discharge } \\
\left(\mathrm{ft}^{3} / \mathrm{s}\right)\end{array}$ & $\begin{array}{c}\text { Regulated } \\
\text { during } \\
\text { flood }^{1}\end{array}$ & $\begin{array}{c}\text { Recurrence } \\
\text { interval } \\
\text { (years) }\end{array}$ \\
\hline 01502731 & $\begin{array}{l}\text { Susquehanna River at Windsor, } \\
\text { NY }\end{array}$ & 1,820 & $1988-98$ & 1996 & 21.22 & 40,000 & $1 / 20 / 96$ & 21.22 & 40,000 & $\mathrm{~N}$ & 10 \\
\hline 01509520 & Tioughnioga River at Lisle, NY & 453 & 1988-98 & $\begin{array}{l}1996 \\
1993\end{array}$ & $\overline{--}$ & $\begin{array}{l}12,900 \\
12,100\end{array}$ & $1 / 20 / 96$ & -- & 12,900 & $\mathrm{~N}$ & 10 \\
\hline 01510000 & Otselic River at Cincinnatus, NY & 147 & $\begin{array}{l}1935, \\
1939-64, \\
1970-98\end{array}$ & $\begin{array}{l}1935 \\
1996\end{array}$ & 10.89 & $\begin{array}{l}9,200 \\
8,000\end{array}$ & $1 / 19 / 96$ & 10.89 & 8,000 & $\mathrm{~N}$ & 25 \\
\hline 01510610 & $\begin{array}{l}\text { Merrill Creek tributary near Texas } \\
\text { Valley, NY }\end{array}$ & 5.32 & $\begin{array}{l}1976-81 \\
1983-98\end{array}$ & 1996 & 6.64 & 1,150 & $1 / 19 / 96$ & 6.64 & 1,150 & $\mathrm{~N}$ & $25-50$ \\
\hline 01511500 & Tioughnioga River at Itaska, NY & 730 & 1930-98 & 1935 & 16.61 & 61,100 & $1 / 19 / 96$ & 10.77 & 20,800 & $\mathrm{Y}$ & $25-50$ \\
\hline 01514801 & $\begin{array}{l}\text { Catatonk Creek northwest of } \\
\text { Owego, NY }\end{array}$ & 151 & 1988-98 & 1996 & 14.83 & 9,740 & $1 / 20 / 96$ & 14.83 & 9,740 & $\mathrm{~N}$ & $25-50$ \\
\hline 01520500 & Tioga River at Lindley, NY & 771 & $1930-98$ & 1972 & 26.27 & 128,000 & $8 / 18 / 94$ & 13.38 & 13,900 & $\mathrm{Y}$ & 10 \\
\hline 01525500 & $\begin{array}{l}\text { Canisteo River at West Cameron, } \\
\text { NY }\end{array}$ & 340 & $\begin{array}{l}1931, \\
1935-72, \\
1974-98\end{array}$ & 1972 & 23.48 & 43,000 & $1 / 19 / 96$ & 20.91 & 29,100 & $\mathrm{Y}$ & $50-75$ \\
\hline 01526500 & Tioga River near Erwins, NY & 1,377 & 1919-98 & 1972 & 26.74 & 190,000 & $1 / 19 / 96$ & 16.98 & 45,600 & $\mathrm{Y}$ & 75 \\
\hline 01529950 & Chemung River at Corning, NY & 2,005 & $\begin{array}{l}\text { 1972, } \\
1975-98\end{array}$ & 1972 & 40.71 & 228,000 & $1 / 19 / 96$ & 25.93 & 61,000 & $\mathrm{Y}$ & $25-50$ \\
\hline 01530500 & Newtown Creek at Elmira, NY & 77.5 & 1938-98 & 1972 & 19.28 & 4,000 & $1 / 19 / 96$ & 16.98 & 3,810 & $\mathrm{Y}$ & 25 \\
\hline 04216418 & Tonawanda Creek at Attica, NY & 76.9 & $\begin{array}{l}\text { 1972, } \\
1978-98\end{array}$ & 1998 & 12.71 & 9,400 & $7 / 8 / 98$ & 12.71 & 9,400 & $\mathrm{~N}$ & $>100$ \\
\hline 0422028490 & $\begin{array}{l}\text { Slater Creek (Latta Road) near } \\
\text { Greece, NY }\end{array}$ & 1.52 & 1989-98 & 1997 & 4.80 & 219 & $10 / 20 / 96$ & 4.80 & 219 & $\mathrm{~N}$ & $10-25$ \\
\hline 04221000 & Genesee River at Wellsville, NY & 288 & $\begin{array}{l}1956-58 \\
1972-98\end{array}$ & 1972 & 20.70 & 38,500 & $1 / 19 / 96$ & 16.13 & 22,700 & $\mathrm{~N}$ & $25-50$ \\
\hline
\end{tabular}


$\left[\mathrm{mi}^{2}\right.$, square miles; ft, feet above an arbitrary datum; $\mathrm{ft}^{3} / \mathrm{s}$, cubic feet per second; --, not determined or not applicable; >, greater than. Source: Recurrence intervals calculated from U.S. Geological Survey data. Other data from U.S. Geological Survey reports or databases]

\begin{tabular}{|c|c|c|c|c|c|c|c|c|c|c|c|}
\hline \multirow[b]{2}{*}{$\begin{array}{l}\text { Streamgage } \\
\text { number } \\
\text { (fig. 44) }\end{array}$} & \multirow[b]{2}{*}{ Streamgage name } & \multirow{2}{*}{$\begin{array}{c}\text { Total } \\
\text { drainage } \\
\left(\mathrm{mi}^{2}\right)\end{array}$} & \multicolumn{4}{|c|}{$\begin{array}{c}\text { Maximum stage and discharge for period of record } \\
\text { through } 1998 \text { water year }\end{array}$} & \multicolumn{5}{|c|}{ Significant floods 1994-98 water years } \\
\hline & & & $\begin{array}{l}\text { Period of } \\
\text { record } \\
\text { (water } \\
\text { years) }\end{array}$ & Water year & $\begin{array}{l}\text { Stage } \\
(\mathrm{ft})\end{array}$ & $\begin{array}{l}\text { Discharge } \\
\left(\mathrm{ft}^{3} / \mathrm{s}\right)\end{array}$ & $\begin{array}{c}\text { Date } \\
\text { (month/ } \\
\text { day/ } \\
\text { year) }\end{array}$ & $\begin{array}{l}\text { Stage } \\
(\mathrm{ft})\end{array}$ & $\begin{array}{l}\text { Discharge } \\
\left(\mathrm{ft}^{3} / \mathrm{s}\right)\end{array}$ & $\begin{array}{c}\text { Regulated } \\
\text { during } \\
\text { flood }^{1}\end{array}$ & $\begin{array}{c}\text { Recurrence } \\
\text { interval } \\
\text { (years) }\end{array}$ \\
\hline 04223000 & Genesee River at Portageville, NY & 984 & $\begin{array}{l}1902, \\
1909-98\end{array}$ & 1972 & 32.25 & 90,000 & $1 / 20 / 96$ & 29.12 & 56,200 & $\mathrm{~N}$ & $25-50$ \\
\hline 04227000 & $\begin{array}{l}\text { Canaseraga Creek at Shakers } \\
\text { Crossing, NY }\end{array}$ & 335 & $\begin{array}{l}\text { 1916-22, } \\
\text { 1959-70, } \\
\text { 1972, } \\
1975-98\end{array}$ & $\begin{array}{l}1972 \\
1916\end{array}$ & 28.92 & $\begin{array}{r}11,200 \\
--\end{array}$ & $1 / 19 / 96$ & 13.01 & 5,510 & $\mathrm{~N}$ & $10-25$ \\
\hline 04230380 & Oatka Creek at Warsaw, NY & 39.1 & 1964-98 & 1998 & 9.90 & 4,110 & $7 / 8 / 98$ & 9.90 & 4,110 & $\mathrm{~N}$ & $50-75$ \\
\hline 04230500 & Oatka Creek at Garbutt, NY & 200 & $1946-98$ & 1960 & 8.64 & 7,050 & $1 / 9 / 98$ & 8.57 & 6,160 & $\mathrm{~N}$ & $25-50$ \\
\hline 0423205010 & $\begin{array}{l}\text { Irondequoit Creek above Blossom } \\
\text { Road near Rochester, NY }\end{array}$ & 142 & 1982-98 & 1998 & 9.95 & 3,300 & $1 / 8 / 98$ & 9.95 & 3,300 & $\mathrm{~N}$ & $75-100$ \\
\hline 042320578 & Bear Creek at Ontario, NY & 6.74 & $\begin{array}{l}1971-73 \\
1975-98\end{array}$ & 1998 & 13.38 & 238 & $1 / 8 / 98$ & 13.38 & 238 & $\mathrm{~N}$ & $25-50$ \\
\hline 04232200 & $\begin{array}{l}\text { Catharine Creek at Montour Falls, } \\
\text { NY }\end{array}$ & 41.1 & $\begin{array}{l}1972, \\
1975-77, \\
1987-98\end{array}$ & 1997 & 8.48 & 4,700 & $11 / 8 / 96$ & 8.48 & 4,700 & $\mathrm{~N}$ & 50 \\
\hline 04232460 & Sugar Creek at Guyanoga, NY & 28.9 & 1966-98 & 1996 & 5.88 & 1,800 & $\begin{array}{l}1 / 19 / 96 \\
1 / 8 / 98\end{array}$ & $\begin{array}{l}5.88 \\
4.98\end{array}$ & $\begin{array}{l}1,800 \\
1,320\end{array}$ & $\begin{array}{l}\mathrm{N} \\
\mathrm{N}\end{array}$ & $\begin{array}{l}25 \\
10\end{array}$ \\
\hline 04233000 & Cayuga Inlet near Ithaca, NY & 35.2 & $\begin{array}{l}\text { 1935, } \\
1937-98\end{array}$ & 1935 & -- & 6,500 & $1 / 19 / 96$ & 7.57 & 4,210 & $\mathrm{~N}$ & $25-50$ \\
\hline 04233255 & Cayuga Inlet at Ithaca, NY & 86.7 & $\begin{array}{l}1935, \\
1971-72, \\
1975-98\end{array}$ & 1935 & -- & 14,000 & $1 / 19 / 96$ & 14.67 & 12,500 & $\mathrm{~N}$ & $25-50$ \\
\hline 04233258 & Coy Glen Creek at Ithaca, NY & 3.56 & $\begin{array}{l}1972, \\
1983-98\end{array}$ & 1996 & 22.23 & 820 & $1 / 19 / 96$ & 22.23 & 820 & $\mathrm{~N}$ & 25 \\
\hline 04234000 & Fall Creek near Ithaca, NY & 126 & 1926-98 & 1935 & 9.52 & 15,500 & $1 / 19 / 96$ & 7.47 & 9,450 & $\mathrm{~N}$ & $50-75$ \\
\hline
\end{tabular}


Table 33. Maximum stage and discharge for period of record for streamgages having significant floods during 1994-98 water years in New York.-Continued

$\left[\mathrm{mi}^{2}\right.$, square miles; $\mathrm{ft}$, feet above an arbitrary datum; $\mathrm{ft}^{3} / \mathrm{s}$, cubic feet per second; --, not determined or not applicable; >, greater than. Source: Recurrence intervals calculated from U.S. Geological Survey data. Other data from U.S. Geological Survey reports or databases]

\begin{tabular}{|c|c|c|c|c|c|c|c|c|c|c|c|}
\hline \multirow{2}{*}{$\begin{array}{c}\text { Streamgage } \\
\text { number } \\
\text { (fig. 44) }\end{array}$} & \multirow[b]{2}{*}{ Streamgage name } & \multirow{2}{*}{$\begin{array}{l}\text { Total } \\
\text { drainage } \\
\left(\mathrm{mi}^{2}\right)\end{array}$} & \multicolumn{4}{|c|}{$\begin{array}{c}\text { Maximum stage and discharge for period of record } \\
\text { through } 1998 \text { water year }\end{array}$} & \multicolumn{5}{|c|}{ Significant floods $1994-98$ water years } \\
\hline & & & $\begin{array}{l}\text { Period of } \\
\text { record } \\
\text { (water } \\
\text { years) }\end{array}$ & Water year & $\begin{array}{l}\text { Stage } \\
(\mathrm{ft})\end{array}$ & $\begin{array}{c}\text { Discharge } \\
\left(\mathrm{ft}^{3} / \mathrm{s}\right)\end{array}$ & $\begin{array}{c}\text { Date } \\
\text { (month/ } \\
\text { day/ } \\
\text { year) }\end{array}$ & $\begin{array}{l}\text { Stage } \\
(\mathrm{ft})\end{array}$ & $\begin{array}{c}\text { Discharge } \\
\left(\mathrm{ft}^{3} / \mathrm{s}\right)\end{array}$ & $\begin{array}{l}\text { Regulated } \\
\text { during } \\
\text { flood }^{1}\end{array}$ & $\begin{array}{c}\text { Recurrence } \\
\text { interval } \\
\text { (years) }\end{array}$ \\
\hline 04234200 & Mud Creek at East Victor, NY & 64.2 & $\begin{array}{l}1958, \\
1961-68, \\
1976-98\end{array}$ & $\begin{array}{l}1991 \\
1972\end{array}$ & $\begin{array}{l}7.22 \\
7.85\end{array}$ & $\begin{array}{l}1,880 \\
1,800\end{array}$ & $1 / 19 / 96$ & 7.09 & 1,810 & $\mathrm{~N}$ & $10-25$ \\
\hline 04235255 & $\begin{array}{l}\text { Canandaigua Outlet tributary near } \\
\text { Alloway, NY }\end{array}$ & 2.94 & 1978-98 & $\begin{array}{l}1996 \\
1998\end{array}$ & $\begin{array}{l}7.34 \\
7.34\end{array}$ & $\begin{array}{l}102 \\
102\end{array}$ & $\begin{array}{l}1 / 19 / 96 \\
1 / 8 / 98\end{array}$ & $\begin{array}{l}7.34 \\
7.34\end{array}$ & $\begin{array}{l}102 \\
102\end{array}$ & $\begin{array}{l}\mathrm{N} \\
\mathrm{N}\end{array}$ & $\begin{array}{l}10 \\
10\end{array}$ \\
\hline 04235500 & Owasco Outlet near Auburn, NY & 206 & $1914-98$ & 1972 & 6.28 & 3,250 & $1 / 9 / 98$ & 4.65 & 2,150 & $\mathrm{Y}$ & $10-25$ \\
\hline 04240200 & Ninemile Creek at Camillus, NY & 84.3 & $\begin{array}{l}1959-82 \\
1989-98\end{array}$ & $\begin{array}{l}1960 \\
1975\end{array}$ & $\begin{array}{r}8.25 \\
10.83\end{array}$ & $\begin{array}{l}2,760 \\
2,120\end{array}$ & $1 / 19 / 96$ & 8.92 & 2,530 & $\mathrm{Y}$ & $10-25$ \\
\hline 04245840 & Scriba Creek near Constantia, NY & 38.4 & $\begin{array}{l}1966-69 \\
1971-98\end{array}$ & $\begin{array}{l}1975 \\
1972\end{array}$ & $\begin{array}{l}7.33 \\
7.42\end{array}$ & $\begin{array}{l}1,310 \\
1,200\end{array}$ & $1 / 8 / 98$ & 7.32 & 1,300 & $\mathrm{~N}$ & $25-50$ \\
\hline 04250750 & Sandy Creek near Adams, NY & 128 & $1958-98$ & 1996 & 11.06 & 7,700 & $1 / 19 / 96$ & 11.06 & 7,700 & $\mathrm{~N}$ & $10-25$ \\
\hline 04252500 & Black River near Boonville, NY & 304 & $1911-98$ & $\begin{array}{l}1985 \\
1982 \\
1913\end{array}$ & $\begin{array}{l}11.41 \\
11.31 \\
12.50\end{array}$ & $\begin{array}{l}12,800 \\
12,800 \\
12,400\end{array}$ & $1 / 9 / 98$ & 11.25 & 12,100 & $\mathrm{~N}$ & $25-50$ \\
\hline 04254500 & Moose River at McKeever, NY & 363 & $\begin{array}{l}1869, \\
1902-70, \\
1982, \\
1985, \\
1987-98\end{array}$ & 1947 & 17.45 & 18,700 & $1 / 8 / 98$ & 14.91 & 14,600 & $\mathrm{~N}$ & $25-50$ \\
\hline 04256000 & $\begin{array}{l}\text { Independence River at } \\
\text { Donnattsburg, NY }\end{array}$ & 88.7 & 1943-98 & 1985 & 13.34 & 9,420 & $1 / 8 / 98$ & 9.53 & 4,340 & $\mathrm{~N}$ & $10-25$ \\
\hline 04258000 & Beaver River at Croghan, NY & 291 & $1931-98$ & 1969 & 6.98 & 5,100 & $1 / 9 / 98$ & 6.56 & 4,440 & $\mathrm{Y}$ & $25-50$ \\
\hline 04260500 & Black River at Watertown, NY & 1,864 & $\begin{array}{l}1869, \\
1897-1913, \\
1917-98\end{array}$ & 1998 & 16.02 & 55,500 & $1 / 10 / 98$ & 16.02 & 55,500 & $\mathrm{Y}$ & $>100$ \\
\hline
\end{tabular}


$\left[\mathrm{mi}^{2}\right.$, square miles; ft, feet above an arbitrary datum; $\mathrm{ft}^{3} / \mathrm{s}$, cubic feet per second; --, not determined or not applicable; >, greater than. Source: Recurrence intervals calculated from U.S. Geological Survey data. Other data from U.S. Geological Survey reports or databases]

\begin{tabular}{|c|c|c|c|c|c|c|c|c|c|c|c|}
\hline \multirow{2}{*}{$\begin{array}{l}\text { Streamgage } \\
\text { number } \\
\text { (fig. 44) }\end{array}$} & \multirow[b]{2}{*}{ Streamgage name } & \multirow{2}{*}{$\begin{array}{c}\text { Total } \\
\text { drainage } \\
\left(\mathrm{mi}^{2}\right)\end{array}$} & \multicolumn{4}{|c|}{$\begin{array}{l}\text { Maximum stage and discharge for period of record } \\
\text { through } 1998 \text { water year }\end{array}$} & \multicolumn{5}{|c|}{ Significant floods 1994-98 water years } \\
\hline & & & $\begin{array}{l}\text { Period of } \\
\text { record } \\
\text { (water } \\
\text { years) }\end{array}$ & Water year & $\begin{array}{l}\text { Stage } \\
(\mathrm{ft})\end{array}$ & $\begin{array}{l}\text { Discharge } \\
\left(\mathrm{ft}^{3} / \mathrm{s}\right)\end{array}$ & $\begin{array}{c}\text { Date } \\
\text { (month/ } \\
\text { day/ } \\
\text { year) }\end{array}$ & $\begin{array}{l}\text { Stage } \\
(\mathrm{ft})\end{array}$ & $\begin{array}{l}\text { Discharge } \\
\left(\mathrm{ft}^{3} / \mathrm{s}\right)\end{array}$ & $\begin{array}{l}\text { Regulated } \\
\text { during } \\
\text { flood }^{1}\end{array}$ & $\begin{array}{c}\text { Recurrence } \\
\text { interval } \\
\text { (years) }\end{array}$ \\
\hline 04262500 & $\begin{array}{l}\text { West Branch Oswegatchie River } \\
\text { near Harrisville, NY }\end{array}$ & 244 & $1917-98$ & 1998 & 10.64 & 8,700 & $1 / 9 / 98$ & 10.64 & 8,700 & $\mathrm{~N}$ & $>100$ \\
\hline 04264331 & $\begin{array}{l}\text { Saint Lawrence River at Cornwall } \\
\text { Ontario near Massena, NY }\end{array}$ & 298,800 & $1918-98$ & 1993 & -- & 378,000 & $3 / 17 / 98$ & -- & $\begin{array}{r}360,000 \\
\text { (Daily) }\end{array}$ & $\mathrm{Y}$ & -- \\
\hline 04265100 & Elm Creek near Hermon, NY & 32.6 & 1959-98 & 1974 & 9.07 & 1,270 & $1 / 19 / 96$ & 8.82 & 1,180 & $\mathrm{~N}$ & 25 \\
\hline 04266500 & Raquette River at Piercefield, NY & 721 & $\begin{array}{l}\text { 1900, } \\
1909-98\end{array}$ & $\begin{array}{l}1993 \\
1972\end{array}$ & $\begin{array}{l}12.04 \\
12.25\end{array}$ & $\begin{array}{l}8,630 \\
8,360\end{array}$ & $4 / 21 / 94$ & 11.50 & 7,660 & $\mathrm{Y}$ & $10-25$ \\
\hline 04270000 & Salmon River at Chasm Falls, NY & 132 & $\begin{array}{l}1926-82 \\
1985, \\
1987-98\end{array}$ & 1985 & 5.63 & 3,700 & $4 / 1 / 98$ & 5.43 & 3,540 & $\mathrm{~N}$ & $75-100$ \\
\hline 04270200 & $\begin{array}{l}\text { Little Salmon River at Bombay, } \\
\text { NY }\end{array}$ & 92.2 & 1959-98 & 1998 & 13.27 & 3,420 & $3 / 31 / 98$ & 13.27 & 3,420 & $\mathrm{~N}$ & $25-50$ \\
\hline 04270510 & $\begin{array}{l}\text { Chateaugay River below } \\
\text { Chateaugay, NY }\end{array}$ & 151 & $\begin{array}{l}\text { 1966-95, } \\
1997-98\end{array}$ & $\begin{array}{l}1998 \\
1966\end{array}$ & $\begin{array}{r}7.54 \\
10.99\end{array}$ & $\begin{array}{r}5,440 \\
--\end{array}$ & $\begin{array}{l}11 / 9 / 96 \\
3 / 31 / 98\end{array}$ & $\begin{array}{l}7.51 \\
7.54\end{array}$ & $\begin{array}{l}5,370 \\
5,440\end{array}$ & $\begin{array}{l}\mathrm{Y} \\
\mathrm{Y}\end{array}$ & $\begin{array}{l}10-25 \\
10-25\end{array}$ \\
\hline 04270700 & Trout River at Trout River, NY & 107 & 1960-98 & 1996 & 9.42 & 6,980 & $7 / 5 / 96$ & 9.42 & 6,980 & $\mathrm{~N}$ & $50-75$ \\
\hline 04271500 & $\begin{array}{l}\text { Great Chazy River at Perry Mills, } \\
\text { NY }\end{array}$ & 243 & $\begin{array}{l}1929-68, \\
1985, \\
1987-98\end{array}$ & 1997 & 12.24 & 9,700 & $\begin{array}{l}11 / 9 / 96 \\
3 / 31 / 98\end{array}$ & $\begin{array}{l}12.24 \\
10.89\end{array}$ & $\begin{array}{l}9,700 \\
7,640\end{array}$ & $\begin{array}{l}\mathrm{N} \\
\mathrm{N}\end{array}$ & $\begin{array}{r}>100 \\
50-75\end{array}$ \\
\hline 04273500 & Saranac River at Plattsburgh, NY & 608 & $\begin{array}{l}\text { 1928, } \\
1944-98\end{array}$ & 1997 & 12.11 & 14,400 & $\begin{array}{l}11 / 09 / 96 \\
4 / 1 / 98\end{array}$ & $\begin{array}{l}12.11 \\
10.73\end{array}$ & $\begin{array}{l}14,400 \\
11,200\end{array}$ & $\begin{array}{l}\mathrm{Y} \\
\mathrm{Y}\end{array}$ & $\begin{array}{r}>100 \\
25\end{array}$ \\
\hline 04273700 & $\begin{array}{l}\text { Salmon River at South Plattsburgh, } \\
\text { NY }\end{array}$ & 63.3 & $\begin{array}{l}1960-86 \\
1990-98\end{array}$ & 1997 & 7.56 & 4,200 & $\begin{array}{l}11 / 9 / 96 \\
6 / 27 / 98\end{array}$ & $\begin{array}{l}7.56 \\
7.54\end{array}$ & $\begin{array}{l}4,200 \\
4,170\end{array}$ & $\begin{array}{l}\mathrm{N} \\
\mathrm{N}\end{array}$ & $\begin{array}{l}50-75 \\
50-75\end{array}$ \\
\hline 04274000 & $\begin{array}{l}\text { West Branch Ausable River near } \\
\text { Lake Placid, NY }\end{array}$ & 116 & $\begin{array}{l}1920-68 \\
1983-98\end{array}$ & 1938 & 12.20 & 10,800 & $11 / 9 / 96$ & 12.09 & 10,600 & $\mathrm{~N}$ & $>100$ \\
\hline 04275000 & $\begin{array}{l}\text { East Branch Ausable River at Au } \\
\text { Sable Forks, NY }\end{array}$ & 198 & $1925-98$ & 1997 & 15.22 & 23,900 & $11 / 9 / 96$ & 15.22 & 23,900 & $\mathrm{~N}$ & $>100$ \\
\hline 04275500 & $\begin{array}{l}\text { Ausable River near Au Sable } \\
\text { Forks, NY }\end{array}$ & 446 & $\begin{array}{l}1911-68 \\
1990-98\end{array}$ & 1997 & 13.83 & 37,400 & $11 / 9 / 96$ & 13.83 & 37,400 & $\mathrm{~N}$ & $>100$ \\
\hline
\end{tabular}


Table 33. Maximum stage and discharge for period of record for streamgages having significant floods during 1994-98 water years in New York.-Continued

$\left[\mathrm{mi}^{2}\right.$, square miles; $\mathrm{ft}$, feet above an arbitrary datum; $\mathrm{ft}^{3} / \mathrm{s}$, cubic feet per second; --, not determined or not applicable; >, greater than. Source: Recurrence intervals calculated from U.S. Geological Survey data. Other data from U.S. Geological Survey reports or databases]

\begin{tabular}{|c|c|c|c|c|c|c|c|c|c|c|c|}
\hline \multirow[b]{2}{*}{$\begin{array}{c}\text { Streamgage } \\
\text { number } \\
\text { (fig. 44) }\end{array}$} & \multirow[b]{2}{*}{ Streamgage name } & \multirow[b]{2}{*}{$\begin{array}{c}\text { Total } \\
\text { drainage } \\
\left(\mathrm{mi}^{2}\right)\end{array}$} & \multicolumn{4}{|c|}{$\begin{array}{c}\text { Maximum stage and discharge for period of record } \\
\text { through } 1998 \text { water year }\end{array}$} & \multicolumn{5}{|c|}{ Significant floods $1994-98$ water years } \\
\hline & & & $\begin{array}{l}\text { Period of } \\
\text { record } \\
\text { (water } \\
\text { years) }\end{array}$ & Water year & $\begin{array}{l}\text { Stage } \\
(\mathrm{ft})\end{array}$ & $\begin{array}{c}\text { Discharge } \\
\left(\mathrm{ft}^{3} / \mathrm{s}\right)\end{array}$ & $\begin{array}{c}\text { Date } \\
\text { (month/ } \\
\text { day/ } \\
\text { year) }\end{array}$ & $\begin{array}{l}\text { Stage } \\
\text { (ft) }\end{array}$ & $\begin{array}{c}\text { Discharge } \\
\left(\mathrm{ft}^{3} / \mathrm{s}\right)\end{array}$ & $\begin{array}{c}\text { Regulated } \\
\text { during } \\
\text { flood }^{1}\end{array}$ & $\begin{array}{c}\text { Recurrence } \\
\text { interval } \\
\text { (years) }\end{array}$ \\
\hline 04276500 & Bouquet River at Willsboro, NY & 270 & $\begin{array}{l}1924-68, \\
1980,1985, \\
1987-98\end{array}$ & 1997 & 10.93 & 12,300 & $11 / 9 / 96$ & 10.93 & 12,300 & $\mathrm{~N}$ & $50-75$ \\
\hline 04278300 & $\begin{array}{l}\text { Northwest Bay Brook near Bolton } \\
\text { Landing, NY }\end{array}$ & 22.0 & 1966-98 & 1996 & 6.57 & 1,950 & $1 / 19 / 96$ & 6.57 & 1,950 & $\mathrm{~N}$ & $25-50$ \\
\hline 04280450 & $\begin{array}{l}\text { Mettawee River near Middle } \\
\text { Granville, NY }\end{array}$ & 167 & $\begin{array}{l}1984 \\
1990-98\end{array}$ & 1996 & 10.69 & 7,600 & $1 / 20 / 96$ & 10.69 & 7,600 & $\mathrm{~N}$ & 10 \\
\hline
\end{tabular}

${ }^{1}$ Regulated during flood: N, no; Y, yes. 


\section{North Carolina}

Rainfall amounts of 5 to 7 inches (National Oceanic and Atmospheric Administration, 1994a) caused serious flooding in the western mountains of North Carolina on March 27, 1994. Bridges were washed away and roads closed. Mudslides were widespread.

Remnants of Tropical Storm Beryl dropped 3 to 4 inches of rain west of Cape Fear (fig. 45) on August 18, 1994 (National Oceanic and Atmospheric Administration, 1994a). The town of Southport sustained nearly $\$ 3$ million in damage (National Oceanic and Atmospheric Administration, 1994b).

Hurricane Gordon was a major weather factor along the North Carolina coast during October 17-18, 1994. The storm remained offshore but caused significant flooding and strong winds along the central and northern Carolina coast. The flooding began on Hatteras Island, with about 12 inches of overwash (National Oceanic and Atmospheric Administration, 1994b).

More than 11 inches of rain fell during a 2-day period causing widespread flooding in western North Carolina, January 14-15, 1995. Three Boy Scouts drowned in Pisgah National Forest. They were attempting to cross a footbridge and were swept away in the swollen creek. Two other men drowned. Overall damage was estimated at \$2.5 million (National Oceanic and Atmospheric Administration, 1995b).

Flash flooding developed rapidly during the evening of September 4, 1996, as stationary thunderstorms dumped between 10 and 15 inches of rain in a small area of Hickory Nut Gorge (National Oceanic and Atmospheric Administration, 1996a). A wall of water rushed down the valley of the upper Broad River carrying campsites, RVs, mobile homes, trees, and debris downstream.

Hurricane Fran made landfall near Cape Fear, on September 5, 1996, and was the worst natural economic disaster to occur in North Carolina history. In North Carolina, wind and flooding damage exceeded $\$ 5$ billion (National Oceanic and Atmospheric Administration, 1996b). The copious rainfall produced many severe flash and river floods. The flooding on the Haw River at Haw River (streamgage 02096500, table 34), the Tar River at Louisburg (streamgage 02081747), and at Rocky Mount (streamgage 02082585, table 34) exceeded the highest flood on record. A massive evacuation occurred in Goldsboro where residents of 550 homes had to be moved to public shelters. Excessive rain from Hurricane Fran moved downstream along the Neuse River and, combined with a large amount of debris, led to serious flooding. The Neuse River at Kinston (streamgage 02089500, table 34) crested at 23.3 feet, more than 9 feet above flood stage (National Oceanic and Atmospheric Administration, 1996b).

The remnants of Hurricane Fran produced widespread wind damage and flash flooding in north-central and parts of northwestern North Carolina during the late evening hours of September 5 and morning hours of September 6, 1996. Excessive rainfall forced creeks and streams to leave their banks, flooding roads and damaging crops, roads, and vehicles.

Strong thunderstorms occurred in the early morning of July 23, 1997, between Charlotte and Raleigh. These storms developed ahead of the remnants of Hurricane Danny. Between 5 and 10 inches of rain fell in a few hours (National Oceanic and Atmospheric Administration, 1997a) causing widespread flooding. Two women died after their vehicles became trapped in floodwaters. A young girl perished when she went to play in the water and was swept away. Damage in this area was more than \$11 million (National Oceanic and Atmospheric Administration, 1997b).

\section{References}

National Oceanic and Atmospheric Administration (NOAA), 1994a-97a, Climatological data (by State): Asheville, North Carolina, National Climatic Data Center, various months. National Oceanic and Atmospheric Administration (NOAA), 1994b-97b, Storm data (by State): Asheville, North Carolina, National Climatic Data Center, various months. 


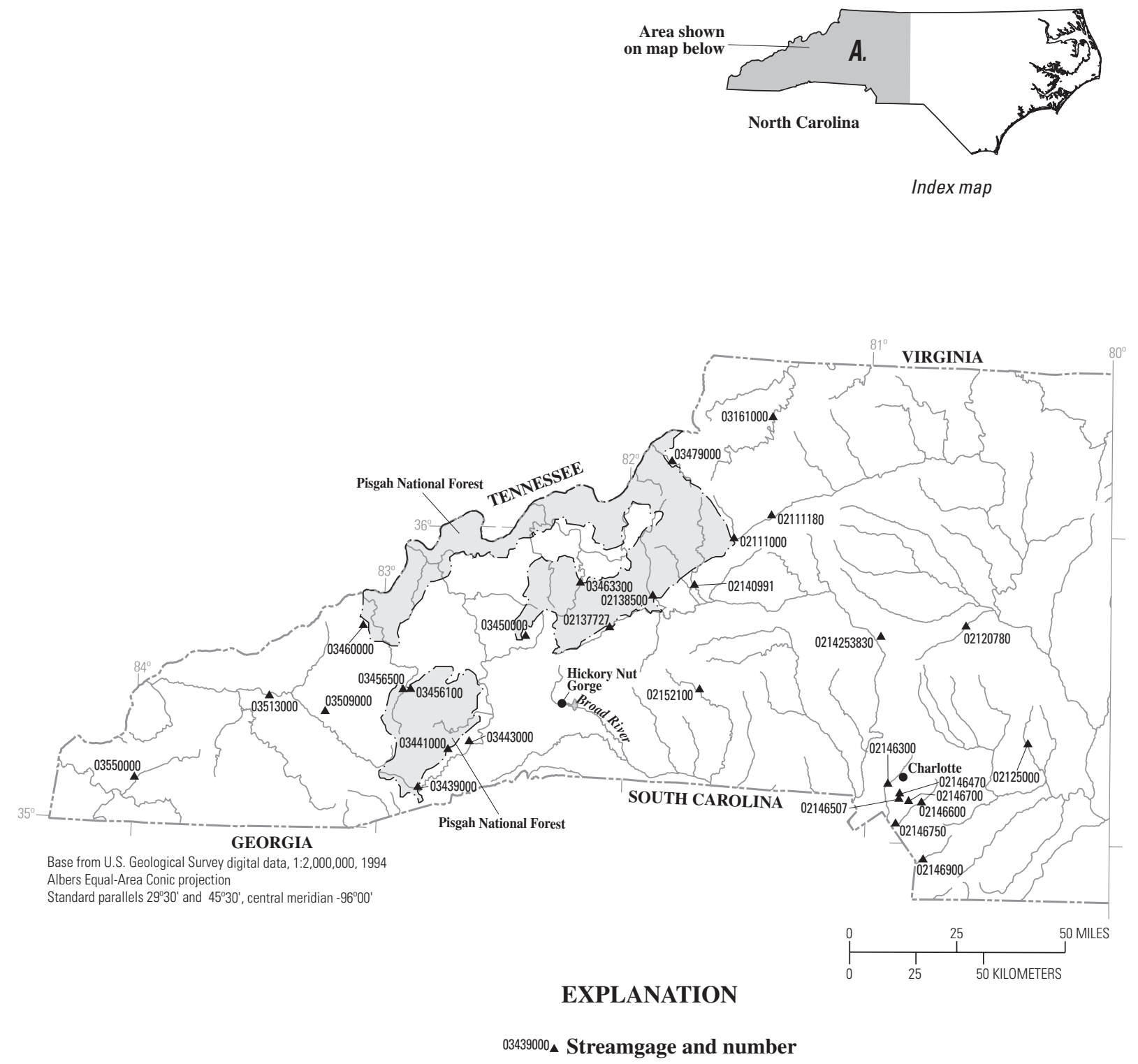

Figure 45. (A) Location of streamgages with significant floods during 1994-98 water years for North Carolina. 

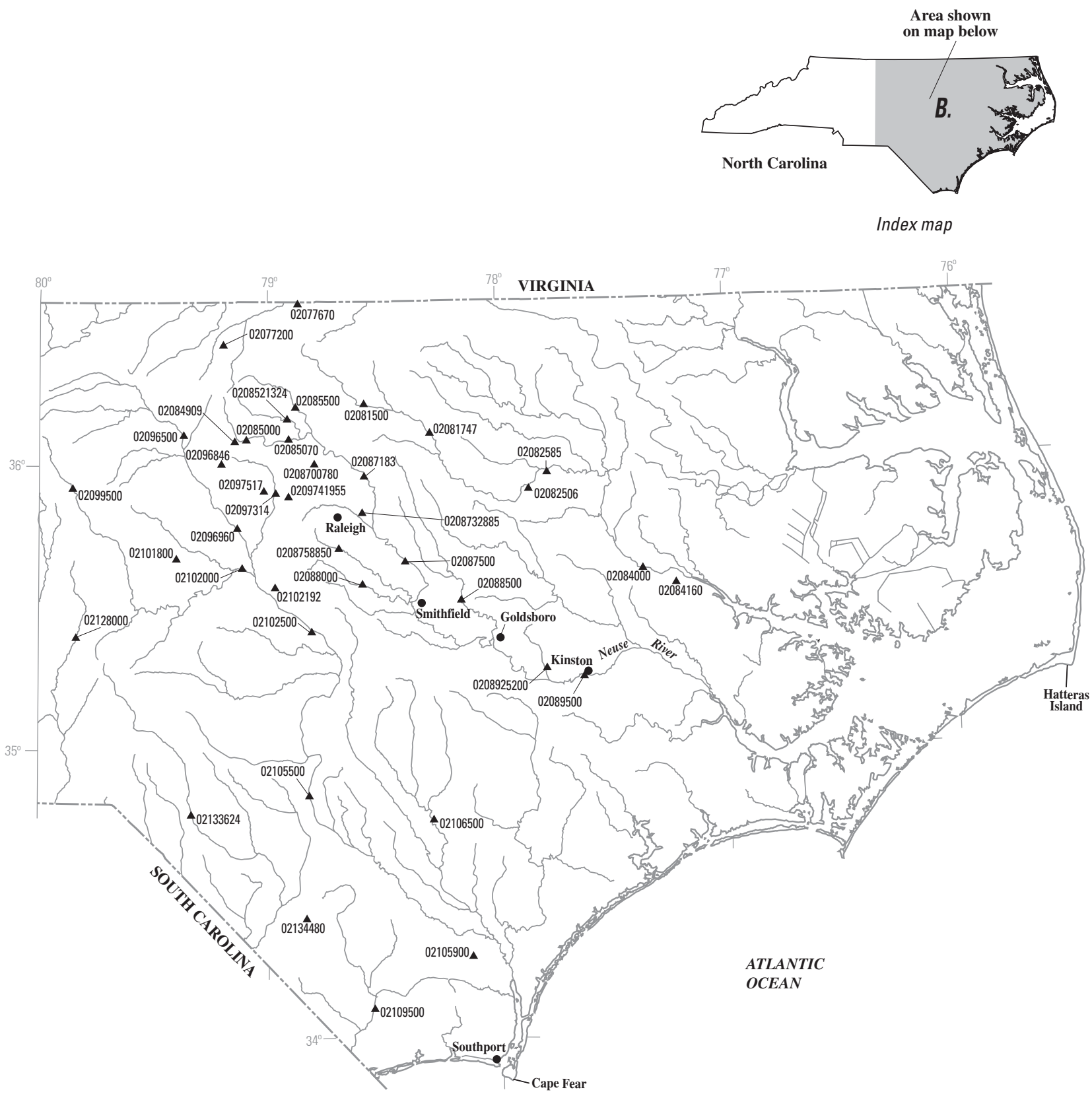

Base from U.S. Geological Survey digital data, 1:2,000,000, 1994 Albers Equal-Area Conic projection

Standard parallels $29^{\circ} 30^{\prime}$ and $45^{\circ} 30^{\prime}$, central meridian $-96^{\circ} 00^{\prime}$

\section{EXPLANATION}

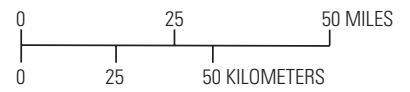

$02109500 \triangle$ Streamgage and number

Figure 45. (B) Location of streamgages with significant floods during 1994-98 water years for North Carolina.-Continued 
Table 34. Maximum stage and discharge for period of record for streamgages having significant floods during 1994-98 water years in North Carolina.

$\left[\mathrm{mi}^{2}\right.$, square miles; $\mathrm{ft}$, feet above an arbitrary datum; $\mathrm{ft}^{3} / \mathrm{s}$, cubic feet per second; --, not determined or not applicable; $>$, greater than; <, less than. Source: Recurrence intervals calculated from U.S. Geological Survey data. Other data from U.S. Geological Survey reports or databases]

\begin{tabular}{|c|c|c|c|c|c|c|c|c|c|c|c|}
\hline \multirow{2}{*}{$\begin{array}{c}\text { Streamgage } \\
\text { number } \\
\text { (fig. 45) }\end{array}$} & \multirow[b]{2}{*}{ Streamgage name } & \multirow{2}{*}{$\begin{array}{c}\text { Total } \\
\text { drainage } \\
\left(\mathrm{mi}^{2}\right)\end{array}$} & \multicolumn{4}{|c|}{$\begin{array}{c}\text { Maximum stage and discharge for period of record } \\
\text { through } 1998 \text { water year }\end{array}$} & \multicolumn{5}{|c|}{ Significant floods $1994-98$ water years } \\
\hline & & & $\begin{array}{l}\text { Period of } \\
\text { record } \\
\text { (water } \\
\text { years) }\end{array}$ & Water year & $\begin{array}{l}\text { Stage } \\
(\mathrm{ft})\end{array}$ & $\begin{array}{l}\text { Discharge } \\
\left(\mathrm{ft}^{3} / \mathrm{s}\right)\end{array}$ & $\begin{array}{c}\text { Date } \\
\text { (month/ } \\
\text { day/year) }\end{array}$ & $\begin{array}{l}\text { Stage } \\
\text { (ft) }\end{array}$ & $\begin{array}{l}\text { Discharge } \\
\left(\mathrm{ft}^{3} / \mathrm{s}\right)\end{array}$ & $\begin{array}{l}\text { Regulated } \\
\text { during } \\
\text { flood }^{1}\end{array}$ & $\begin{array}{c}\text { Recurrence } \\
\text { interval } \\
\text { (years) }\end{array}$ \\
\hline 02077200 & Hyco Creek near Leasburg, NC & 45.9 & $\begin{array}{l}1965-92 \\
1994-97\end{array}$ & $\begin{array}{l}1996 \\
1995\end{array}$ & $\begin{array}{l}40.47 \\
48.53\end{array}$ & $\begin{array}{r}9,140 \\
--\end{array}$ & $9 / 6 / 96$ & 40.47 & 9,140 & $\mathrm{~N}$ & $25-50$ \\
\hline 02077670 & Mayo Creek near Bethel Hill, NC & 53.5 & 1978-98 & 1978 & 10.83 & 3,950 & $9 / 6 / 96$ & 10.83 & 3,040 & $\mathrm{Y}$ & 25 \\
\hline 02081500 & Tar River near Tar River, NC & 167 & 1940-98 & 1996 & 24.06 & 19,900 & $\begin{array}{l}9 / 6 / 96 \\
3 / 19 / 98\end{array}$ & $\begin{array}{l}24.06 \\
19.36\end{array}$ & $\begin{array}{l}19,900 \\
13,200\end{array}$ & $\begin{array}{l}\mathrm{N} \\
\mathrm{N}\end{array}$ & $\begin{array}{r}50-100 \\
10-25\end{array}$ \\
\hline 02081747 & Tar River at Louisburg, NC & 427 & 1964-98 & 1996 & 25.34 & 21,100 & $\begin{array}{l}9 / 6 / 96 \\
3 / 20 / 98\end{array}$ & $\begin{array}{l}25.34 \\
24.62\end{array}$ & $\begin{array}{l}21,100 \\
18,500\end{array}$ & $\begin{array}{l}\mathrm{N} \\
\mathrm{N}\end{array}$ & $\begin{array}{r}>100 \\
50-100\end{array}$ \\
\hline 02082506 & $\begin{array}{l}\text { Tar River below Tar River } \\
\text { Reservoir near Rocky Mount, NC }\end{array}$ & 777 & 1973-98 & 1998 & 23.67 & 14,700 & $3 / 23 / 98$ & 23.67 & 14,700 & $\mathrm{Y}$ & $>500$ \\
\hline 02082585 & Tar River at Rocky Mount, NC & 925 & 1977-98 & 1996 & 25.88 & 15,100 & $9 / 12 / 96$ & 25.88 & 15,100 & $\mathrm{Y}$ & $>500$ \\
\hline 02084000 & Tar River at Greenville, NC & 2,620 & $\begin{array}{l}1888, \\
1906-76, \\
1997-98\end{array}$ & 1919 & 24.50 & 46,500 & $\begin{array}{l}5 / 7 / 97 \\
3 / 28 / 98\end{array}$ & $\begin{array}{l}14.05 \\
18.08\end{array}$ & $\begin{array}{l}13,900 \\
25,500\end{array}$ & $\begin{array}{l}\mathrm{N} \\
\mathrm{N}\end{array}$ & $\begin{array}{l}-- \\
--\end{array}$ \\
\hline 02084160 & Chicod Creek near Simpson, NC & 45.0 & $\begin{array}{l}1976-87 \\
1992-98\end{array}$ & 1998 & 13.45 & 3,150 & $8 / 27 / 98$ & 13.45 & 3,150 & $\mathrm{~N}$ & 25 \\
\hline 02084909 & Sevenmile Creek near Efland, NC & 14.1 & $1988-98$ & 1996 & 15.47 & 3,440 & $9 / 6 / 96$ & 15.47 & 3,440 & $\mathrm{~N}$ & $>100$ \\
\hline 02085000 & Eno River at Hillsborough, NC & 66.0 & $\begin{array}{l}1928, \\
1930-71, \\
1986-98\end{array}$ & $\begin{array}{l}1945 \\
1996\end{array}$ & $\begin{array}{l}20.01 \\
21.13\end{array}$ & $\begin{array}{l}11,000 \\
10,800\end{array}$ & $9 / 6 / 96$ & 21.13 & 10,800 & $\mathrm{~N}$ & $>100$ \\
\hline 02085070 & Eno River near Durham, NC & 141 & 1964-98 & 1996 & 23.58 & 14,700 & $\begin{array}{l}9 / 6 / 96 \\
3 / 19 / 98\end{array}$ & $\begin{array}{l}23.58 \\
20.76\end{array}$ & $\begin{array}{l}14,700 \\
10,800\end{array}$ & $\begin{array}{l}\mathrm{N} \\
\mathrm{N}\end{array}$ & $\begin{array}{r}10-25 \\
<10\end{array}$ \\
\hline 0208521324 & $\begin{array}{l}\text { Little River at State Road } 1461 \\
\text { near Orange Factory, NC }\end{array}$ & 78.2 & 1988-98 & 1996 & 13.26 & 11,600 & 9/6/96 & 13.26 & 11,600 & $\mathrm{~N}$ & 25 \\
\hline 02085500 & Flat River at Bahama, NC & 149 & $1926-98$ & 1996 & 17.26 & 33,800 & $\begin{array}{l}9 / 6 / 96 \\
3 / 19 / 98\end{array}$ & $\begin{array}{l}17.26 \\
12.05\end{array}$ & $\begin{array}{l}33,800 \\
16,700\end{array}$ & $\begin{array}{l}\mathrm{N} \\
\mathrm{N}\end{array}$ & $\begin{array}{c}200-500 \\
10-25\end{array}$ \\
\hline 0208700780 & $\begin{array}{l}\text { Little Lick Creek above State Road } \\
1814 \text { near Oak Grove, NC }\end{array}$ & 10.1 & 1983-95 & 1995 & 9.33 & 1,830 & $6 / 29 / 95$ & 9.33 & 1,830 & $\mathrm{~N}$ & $10-25$ \\
\hline
\end{tabular}


Table 34. Maximum stage and discharge for period of record for streamgages having significant floods during 1994-98 water years in North Carolina.-Continued

$\left[\mathrm{mi}^{2}\right.$, square miles; $\mathrm{ft}$, feet above an arbitrary datum; $\mathrm{ft}^{3} / \mathrm{s}$, cubic feet per second; --, not determined or not applicable; $>$, greater than; <, less than. Source: Recurrence intervals calculated from U.S. Geological Survey data. Other data from U.S. Geological Survey reports or databases]

\begin{tabular}{|c|c|c|c|c|c|c|c|c|c|c|c|}
\hline \multirow{2}{*}{$\begin{array}{c}\text { Streamgage } \\
\text { number } \\
\text { (fig. 45) }\end{array}$} & \multirow[b]{2}{*}{ Streamgage name } & \multirow{2}{*}{$\begin{array}{c}\text { Total } \\
\text { drainage } \\
\left(\mathrm{mi}^{2}\right)\end{array}$} & \multicolumn{4}{|c|}{$\begin{array}{c}\text { Maximum stage and discharge for period of record } \\
\text { through } 1998 \text { water year }\end{array}$} & \multicolumn{5}{|c|}{ Significant floods $1994-98$ water years } \\
\hline & & & $\begin{array}{l}\text { Period of } \\
\text { record } \\
\text { (water } \\
\text { years) }\end{array}$ & Water year & $\begin{array}{l}\text { Stage } \\
(\mathrm{ft})\end{array}$ & $\begin{array}{l}\text { Discharge } \\
\left(\mathrm{ft}^{3} / \mathrm{s}\right)\end{array}$ & $\begin{array}{c}\text { Date } \\
\text { (month/ } \\
\text { day/year) }\end{array}$ & $\begin{array}{l}\text { Stage } \\
(\mathrm{ft})\end{array}$ & $\begin{array}{l}\text { Discharge } \\
\left(\mathrm{ft}^{3} / \mathrm{s}\right)\end{array}$ & $\begin{array}{l}\text { Regulated } \\
\text { during } \\
\text { flood }^{1}\end{array}$ & $\begin{array}{c}\text { Recurrence } \\
\text { interval } \\
\text { (years) }\end{array}$ \\
\hline 02087183 & Neuse River near Falls, NC & 771 & $\begin{array}{l}1945, \\
1971-98\end{array}$ & 1975 & 25.21 & 13,600 & $9 / 16 / 96$ & 8.05 & 7,650 & $\mathrm{Y}$ & $25-50$ \\
\hline 0208732885 & Marsh Creek near New Hope, NC & 6.84 & 1984-98 & $\begin{array}{l}1995 \\
1996\end{array}$ & $\begin{array}{l}12.84 \\
13.33\end{array}$ & $\begin{array}{l}4,140 \\
3,900\end{array}$ & $8 / 27 / 95$ & 12.84 & 4,140 & $\mathrm{~N}$ & 25 \\
\hline 02087500 & Neuse River near Clayton, NC & 1,150 & $\begin{array}{l}\text { 1919, } \\
1928-98\end{array}$ & 1945 & 22.12 & 22,900 & $9 / 7 / 96$ & 20.12 & 19,700 & $\mathrm{Y}$ & $50-100$ \\
\hline 0208758850 & $\begin{array}{l}\text { Swift Creek near McCullars } \\
\text { Crossroads, NC }\end{array}$ & 35.8 & 1989-98 & 1996 & 14.15 & 6,790 & $9 / 6 / 96$ & 14.15 & 6,790 & $\mathrm{~N}$ & $25-50$ \\
\hline 02088000 & Middle Creek near Clayton, NC & 83.5 & 1940-98 & 1996 & 14.88 & 11,900 & $9 / 6 / 96$ & 14.88 & 11,900 & $\mathrm{~N}$ & $200-500$ \\
\hline 02088500 & Little River near Princeton, NC & 232 & $\begin{array}{l}\text { 1919, 1924, } \\
1928, \\
1930-98\end{array}$ & $\begin{array}{l}1965 \\
1924\end{array}$ & $\begin{array}{l}13.94 \\
14.90\end{array}$ & $\begin{array}{r}7,150 \\
--\end{array}$ & $\begin{array}{l}9 / 9 / 96 \\
3 / 11 / 98\end{array}$ & $\begin{array}{l}13.32 \\
13.51\end{array}$ & $\begin{array}{l}5,480 \\
5,150\end{array}$ & $\begin{array}{l}\mathrm{N} \\
\mathrm{N}\end{array}$ & $\begin{array}{r}25 \\
10-25\end{array}$ \\
\hline 0208925200 & Bear Creek at Mays Store, NC & 57.7 & 1988-98 & 1997 & 9.50 & 1,550 & $10 / 9 / 96$ & 9.50 & 1,550 & $\mathrm{~N}$ & $<5$ \\
\hline 02089500 & Neuse River at Kinston, NC & 2,692 & $\begin{array}{l}1919,1924, \\
1928-98\end{array}$ & 1919 & 25.00 & 39,000 & $9 / 17 / 96$ & 23.26 & 27,100 & $\mathrm{Y}$ & $50-100$ \\
\hline 02096500 & Haw River at Haw River, NC & 606 & $1929-98$ & 1996 & 32.83 & 51,400 & $\begin{array}{l}6 / 29 / 95 \\
9 / 6 / 96\end{array}$ & $\begin{array}{l}28.46 \\
32.83\end{array}$ & $\begin{array}{l}29,500 \\
51,400\end{array}$ & $\begin{array}{l}\mathrm{N} \\
\mathrm{N}\end{array}$ & $\begin{array}{r}10-25 \\
200\end{array}$ \\
\hline 02096846 & $\begin{array}{l}\text { Cane Creek near Orange Grove, } \\
\text { NC }\end{array}$ & 7.54 & 1989-98 & 1996 & 7.90 & 2,060 & $9 / 6 / 96$ & 7.90 & 2,060 & $\mathrm{~N}$ & 25 \\
\hline 02096960 & Haw River near Bynum, NC & 1,275 & 1974-98 & 1996 & 21.76 & 76,700 & $9 / 6 / 96$ & 21.76 & 76,700 & $\mathrm{~N}$ & $>100$ \\
\hline 02097314 & New Hope Creek near Blands, NC & 75.9 & 1983-98 & 1996 & 14.05 & 12,700 & $9 / 6 / 96$ & 14.05 & 12,700 & $\mathrm{~N}$ & $100-200$ \\
\hline 0209741955 & $\begin{array}{l}\text { Northeast Creek at State Road } \\
1100 \text { near Genlee, NC }\end{array}$ & 21.1 & $\begin{array}{l}1983-93 \\
1996-98\end{array}$ & 1996 & 13.92 & 5,140 & $9 / 6 / 96$ & 13.92 & 5,140 & $\mathrm{~N}$ & $25-50$ \\
\hline 02097517 & $\begin{array}{l}\text { Morgan Creek near Chapel Hill, } \\
\text { NC }\end{array}$ & 41.0 & 1983-98 & 1996 & 16.18 & 4,210 & $9 / 6 / 96$ & 16.18 & 4,210 & $\mathrm{~N}$ & 50 \\
\hline 02099500 & Deep River near Randleman, NC & 125 & $\begin{array}{l}1929-31 \\
1934-98\end{array}$ & 1947 & 32.20 & 20,000 & $9 / 6 / 96$ & 28.75 & 15,600 & $\mathrm{~N}$ & $50-100$ \\
\hline
\end{tabular}


Table 34. Maximum stage and discharge for period of record for streamgages having significant floods during 1994-98 water years in North Carolina.-Continued

$\left[\mathrm{mi}^{2}\right.$, square miles; $\mathrm{ft}$, feet above an arbitrary datum; $\mathrm{ft}^{3} / \mathrm{s}$, cubic feet per second; --, not determined or not applicable; $>$, greater than; <, less than. Source: Recurrence intervals calculated from U.S. Geological Survey data. Other data from U.S. Geological Survey reports or databases]

\begin{tabular}{|c|c|c|c|c|c|c|c|c|c|c|c|}
\hline \multirow[b]{2}{*}{$\begin{array}{c}\text { Streamgage } \\
\text { number } \\
\text { (fig. 45) }\end{array}$} & \multirow[b]{2}{*}{ Streamgage name } & \multirow[b]{2}{*}{$\begin{array}{c}\text { Total } \\
\text { drainage } \\
\left(\mathrm{mi}^{2}\right)\end{array}$} & \multicolumn{4}{|c|}{$\begin{array}{c}\text { Maximum stage and discharge for period of record } \\
\text { through } 1998 \text { water year }\end{array}$} & \multicolumn{5}{|c|}{ Significant floods 1994-98 water years } \\
\hline & & & $\begin{array}{l}\text { Period of } \\
\text { record } \\
\text { (water } \\
\text { years) }\end{array}$ & Water year & $\begin{array}{l}\text { Stage } \\
\text { (ft) }\end{array}$ & $\begin{array}{l}\text { Discharge } \\
\left(\mathrm{ft}^{3} / \mathrm{s}\right)\end{array}$ & $\begin{array}{c}\text { Date } \\
\text { (month/ } \\
\text { day/year) }\end{array}$ & $\begin{array}{l}\text { Stage } \\
\text { (ft) }\end{array}$ & $\begin{array}{l}\text { Discharge } \\
\left(\mathrm{ft}^{3} / \mathrm{s}\right)\end{array}$ & $\begin{array}{c}\text { Regulated } \\
\text { during } \\
\text { flood }^{1}\end{array}$ & $\begin{array}{c}\text { Recurrence } \\
\text { interval } \\
\text { (years) }\end{array}$ \\
\hline 02101800 & $\begin{array}{l}\text { Tick Creek near Mount Vernon } \\
\text { Springs, NC }\end{array}$ & 15.5 & $\begin{array}{l}\text { 1959-81, } \\
1994-98\end{array}$ & 1996 & 13.41 & 4,010 & $9 / 6 / 96$ & 13.41 & 4,010 & $\mathrm{~N}$ & 25 \\
\hline 02102000 & Deep River at Moncure, NC & 1,434 & $1931-98$ & 1945 & 17.20 & 80,300 & $9 / 6 / 96$ & 12.94 & 47,900 & $\mathrm{~N}$ & 50 \\
\hline 02102192 & Buckhorn Creek near Corinth, NC & 76.3 & 1973-98 & 1973 & 20.02 & 6,920 & 9/6/96 & 16.79 & 4,300 & $\mathrm{Y}$ & $25-50$ \\
\hline 02102500 & Cape Fear River at Lillington, NC & 3,464 & 1924-98 & 1945 & 33.19 & 150,000 & 9/7/96 & 18.97 & 51,800 & $\mathrm{Y}$ & 50 \\
\hline 02105500 & $\begin{array}{l}\text { Cape Fear River at Wilm O Huske } \\
\text { Lock near Tarheel, NC }\end{array}$ & 4,852 & $\begin{array}{l}1938-94 \\
1997-98\end{array}$ & $\begin{array}{l}1952 \\
1945\end{array}$ & $\begin{array}{l}29.92 \\
43.44\end{array}$ & $\begin{array}{r}70,600 \\
--\end{array}$ & $3 / 22 / 98$ & 26.28 & 47,100 & $\mathrm{Y}$ & $>500$ \\
\hline 02105900 & Hood Creek near Leland, NC & 21.6 & $\begin{array}{l}\text { 1953-73, } \\
1994-98\end{array}$ & 1998 & 11.53 & 2,650 & $8 / 27 / 98$ & 11.53 & 2,650 & $\mathrm{~N}$ & $25-50$ \\
\hline 02106500 & Black River near Tomahawk, NC & 676 & $\begin{array}{l}1928,1945, \\
1948, \\
1952-98\end{array}$ & $\begin{array}{l}1984 \\
1928\end{array}$ & $\begin{array}{l}22.08 \\
47.00\end{array}$ & $\begin{array}{l}17,500 \\
11,400\end{array}$ & 9/10/96 & 21.31 & 13,700 & $\mathrm{~N}$ & $25-50$ \\
\hline 02109500 & Waccamaw River at Freeland, NC & 680 & 1940-98 & 1996 & 17.02 & 12,400 & $9 / 12 / 96$ & 17.02 & 12,400 & $\mathrm{~N}$ & 50 \\
\hline 02111000 & Yadkin River at Patterson, NC & 28.8 & $\begin{array}{l}1940-43 \\
1945-98\end{array}$ & 1973 & 12.70 & 16,200 & $8 / 17 / 94$ & 9.38 & 5,750 & $\mathrm{~N}$ & $10-25$ \\
\hline 02111180 & Elk Creek at Elkville, NC & 48.1 & $\begin{array}{l}1940 \\
1966-98\end{array}$ & 1940 & 22.00 & 70,000 & $8 / 17 / 94$ & 12.02 & 18,700 & $\mathrm{~N}$ & 25 \\
\hline 02120780 & Second Creek near Barber, NC & 118 & 1980-98 & 1995 & 17.28 & 8,560 & $8 / 28 / 95$ & 17.28 & 8,560 & $\mathrm{~N}$ & $100-200$ \\
\hline 02125000 & Big Bear Creek near Richfield, NC & 55.6 & 1955-98 & 1997 & 16.54 & 11,400 & $7 / 23 / 97$ & 16.54 & 11,400 & $\mathrm{~N}$ & 25 \\
\hline 02128000 & Little River near Star, NC & 106 & $\begin{array}{l}\text { 1955-93, } \\
1995-98\end{array}$ & 1997 & 18.60 & 15,400 & $\begin{array}{l}7 / 23 / 97 \\
3 / 19 / 98\end{array}$ & $\begin{array}{l}18.60 \\
15.84\end{array}$ & $\begin{array}{l}15,400 \\
11,300\end{array}$ & $\begin{array}{l}\mathrm{N} \\
\mathrm{N}\end{array}$ & $\begin{array}{c}200-500 \\
50-100\end{array}$ \\
\hline 02133624 & Lumber River near Maxton, NC & 365 & $\begin{array}{l}\text { 1988-92, } \\
1994-98\end{array}$ & 1998 & 13.52 & 3,380 & $3 / 22 / 98$ & 13.52 & 3,380 & $\mathrm{~N}$ & $10-25$ \\
\hline 02134480 & Big Swamp near Tarheel, NC & 229 & $\begin{array}{l}1986-95 \\
1997-98\end{array}$ & $\begin{array}{l}1998 \\
1993\end{array}$ & $\begin{array}{l}13.10 \\
13.34\end{array}$ & $\begin{array}{l}3,980 \\
2,840\end{array}$ & $3 / 11 / 98$ & 13.10 & 3,980 & $\mathrm{~N}$ & $10-25$ \\
\hline 02137727 & $\begin{array}{l}\text { Catawba River near Pleasant } \\
\text { Gardens, NC }\end{array}$ & 127 & $1981-98$ & 1994 & 15.22 & 13,700 & $8 / 17 / 94$ & 15.22 & 13,700 & $\mathrm{~N}$ & $10-25$ \\
\hline
\end{tabular}


$\left[\mathrm{mi}^{2}\right.$, square miles; $\mathrm{ft}$, feet above an arbitrary datum; $\mathrm{ft}^{3} / \mathrm{s}$, cubic feet per second; --, not determined or not applicable; >, greater than; <, less than. Source: Recurrence intervals calculated from U.S. Geological Survey data. Other data from U.S. Geological Survey reports or databases]

\begin{tabular}{|c|c|c|c|c|c|c|c|c|c|c|c|}
\hline \multirow{2}{*}{$\begin{array}{l}\text { Streamgage } \\
\text { number } \\
\text { (fig. 45) }\end{array}$} & \multirow[b]{2}{*}{ Streamgage name } & \multirow{2}{*}{$\begin{array}{c}\text { Total } \\
\text { drainage } \\
\left(\mathrm{mi}^{2}\right)\end{array}$} & \multicolumn{4}{|c|}{$\begin{array}{l}\text { Maximum stage and discharge for period of record } \\
\text { through } 1998 \text { water year }\end{array}$} & \multicolumn{5}{|c|}{ Significant floods 1994-98 water years } \\
\hline & & & $\begin{array}{l}\text { Period of } \\
\text { record } \\
\text { (water } \\
\text { years) }\end{array}$ & Water year & $\begin{array}{l}\text { Stage } \\
\text { (ft) }\end{array}$ & $\begin{array}{l}\text { Discharge } \\
\left(\mathrm{ft}^{3} / \mathrm{s}\right)\end{array}$ & $\begin{array}{c}\text { Date } \\
\text { (month/ } \\
\text { day/year) }\end{array}$ & $\begin{array}{l}\text { Stage } \\
(\mathrm{ft})\end{array}$ & $\begin{array}{l}\text { Discharge } \\
\left(\mathrm{ft}^{3} / \mathrm{s}\right)\end{array}$ & $\begin{array}{l}\text { Regulated } \\
\text { during } \\
\text { flood }^{1}\end{array}$ & $\begin{array}{c}\text { Recurrence } \\
\text { interval } \\
\text { (years) }\end{array}$ \\
\hline 02138500 & Linville River near Nebo, NC & 66.7 & $\begin{array}{l}\text { 1916, } \\
1923-98\end{array}$ & 1940 & 11.40 & 39,500 & $8 / 17 / 94$ & 10.52 & 18,600 & $\mathrm{~N}$ & 25 \\
\hline 02140991 & Johns River at Arneys Store, NC & 201 & $1986-98$ & 1994 & 25.23 & 42,300 & $8 / 17 / 94$ & 25.23 & 42,300 & $\mathrm{~N}$ & $25-50$ \\
\hline 0214253830 & $\begin{array}{l}\text { Norwood Creek near Troutman, } \\
\text { NC }\end{array}$ & 7.18 & 1984-98 & 1997 & 9.20 & 1,480 & $4 / 28 / 97$ & 9.20 & 1,480 & $\mathrm{~N}$ & $10-25$ \\
\hline 02146300 & Irwin Creek near Charlotte, NC & 30.7 & 1963-98 & 1997 & 20.38 & 11,600 & $7 / 23 / 97$ & 20.38 & 11,600 & $\mathrm{~N}$ & 500 \\
\hline 02146470 & $\begin{array}{l}\text { Little Hope Creek at Seneca Place, } \\
\text { Charlotte, NC }\end{array}$ & 2.63 & $\begin{array}{l}\text { 1967-72, } \\
\text { 1983-86, } \\
\text { 1988-90, } \\
1995-98\end{array}$ & 1997 & 8.50 & 1,700 & $7 / 23 / 97$ & 8.50 & 1,700 & $\mathrm{~N}$ & 50 \\
\hline 02146507 & $\begin{array}{l}\text { Little Sugar Creek at Archdale } \\
\text { Drive at Charlotte, NC }\end{array}$ & 42.6 & 1978-98 & 1997 & 15.06 & 13,600 & $7 / 23 / 97$ & 15.06 & 13,600 & $\mathrm{~N}$ & 500 \\
\hline 02146600 & $\begin{array}{l}\text { McAlpine Creek at Sardis Road } \\
\text { near Charlotte, NC }\end{array}$ & 39.6 & 1962-98 & 1995 & 17.79 & 9,040 & $8 / 27 / 95$ & 17.79 & 9,040 & $\mathrm{~N}$ & $>200$ \\
\hline 02146700 & $\begin{array}{l}\text { McMullen Creek at Sharon View } \\
\text { Road near Charlotte, NC }\end{array}$ & 6.95 & $1963-98$ & 1995 & 11.03 & 3,470 & $\begin{array}{l}8 / 27 / 95 \\
7 / 27 / 98\end{array}$ & $\begin{array}{l}11.03 \\
10.64\end{array}$ & $\begin{array}{l}3,470 \\
3,230\end{array}$ & $\begin{array}{l}\mathrm{N} \\
\mathrm{N}\end{array}$ & $\begin{array}{r}50 \\
25-50\end{array}$ \\
\hline 02146750 & $\begin{array}{l}\text { McAlpine Creek below McMullen } \\
\text { Creek near Pineville, NC }\end{array}$ & 92.4 & 1975-98 & 1995 & 19.40 & 12,500 & $8 / 27 / 95$ & 19.40 & 12,500 & $\mathrm{~N}$ & 200 \\
\hline 02146900 & $\begin{array}{l}\text { Twelve Mile Creek near Waxhaw, } \\
\text { NC }\end{array}$ & 76.5 & $\begin{array}{l}1949 \\
1961-98\end{array}$ & $\begin{array}{l}1995 \\
1949\end{array}$ & $\begin{array}{l}21.94 \\
23.60\end{array}$ & $\begin{array}{r}9,970 \\
--\end{array}$ & $8 / 27 / 95$ & 21.94 & 9,970 & $\mathrm{~N}$ & $50-100$ \\
\hline 02152100 & First Broad River near Casar, NC & 60.5 & $\begin{array}{l}\text { 1960-95, } \\
\text { 1997-98 }\end{array}$ & $\begin{array}{l}1995 \\
1976\end{array}$ & $\begin{array}{l}15.58 \\
16.70\end{array}$ & $\begin{array}{l}7,790 \\
7,760\end{array}$ & $1 / 14 / 95$ & 15.58 & 7,790 & $\mathrm{~N}$ & $10-25$ \\
\hline 03161000 & $\begin{array}{l}\text { South Fork New River near } \\
\text { Jefferson, NC }\end{array}$ & 205 & $\begin{array}{l}1916, \\
1925-26, \\
1929-41, \\
1943-98\end{array}$ & 1940 & 22.50 & 52,800 & $1 / 15 / 95$ & 13.73 & 21,000 & $\mathrm{~N}$ & 50 \\
\hline 03439000 & $\begin{array}{l}\text { French Broad River at Rosman, } \\
\text { NC }\end{array}$ & 67.9 & $\begin{array}{l}\text { 1908-09, } \\
\text { 1916, 1928, } \\
\text { 1936-89, } \\
1991-98\end{array}$ & 1965 & 14.95 & 13,500 & 8/17/94 & 14.12 & 11,500 & $\mathrm{~N}$ & 50 \\
\hline
\end{tabular}


Table 34. Maximum stage and discharge for period of record for streamgages having significant floods during 1994-98 water years in North Carolina.-Continued

$\left[\mathrm{mi}^{2}\right.$, square miles; $\mathrm{ft}$, feet above an arbitrary datum; $\mathrm{ft}^{3} / \mathrm{s}$, cubic feet per second; --, not determined or not applicable; $>$, greater than; <, less than. Source: Recurrence intervals calculated from U.S. Geological Survey data. Other data from U.S. Geological Survey reports or databases]

\begin{tabular}{|c|c|c|c|c|c|c|c|c|c|c|c|}
\hline \multirow{2}{*}{$\begin{array}{c}\text { Streamgage } \\
\text { number } \\
\text { (fig. 45) }\end{array}$} & \multirow[b]{2}{*}{ Streamgage name } & \multirow{2}{*}{$\begin{array}{c}\text { Total } \\
\text { drainage } \\
\left(\mathrm{mi}^{2}\right)\end{array}$} & \multicolumn{4}{|c|}{$\begin{array}{c}\text { Maximum stage and discharge for period of record } \\
\text { through } 1998 \text { water year }\end{array}$} & \multicolumn{5}{|c|}{ Significant floods $1994-98$ water years } \\
\hline & & & $\begin{array}{l}\text { Period of } \\
\text { record } \\
\text { (water } \\
\text { years) }\end{array}$ & Water year & $\begin{array}{l}\text { Stage } \\
(\mathrm{ft})\end{array}$ & $\begin{array}{l}\text { Discharge } \\
\left(\mathrm{ft}^{3} / \mathrm{s}\right)\end{array}$ & $\begin{array}{c}\text { Date } \\
\text { (month/ } \\
\text { day/year) }\end{array}$ & $\begin{array}{l}\text { Stage } \\
(\mathrm{ft})\end{array}$ & $\begin{array}{l}\text { Discharge } \\
\left(\mathrm{ft}^{3} / \mathrm{s}\right)\end{array}$ & $\begin{array}{l}\text { Regulated } \\
\text { during } \\
\text { flood }^{1}\end{array}$ & $\begin{array}{c}\text { Recurrence } \\
\text { interval } \\
\text { (years) }\end{array}$ \\
\hline 03441000 & Davidson River near Brevard, NC & 40.4 & $\begin{array}{l}\text { 1876, 1916, } \\
1919, \\
1921-90, \\
1924-98\end{array}$ & $\begin{array}{l}1928 \\
1994\end{array}$ & $\begin{array}{l}11.80 \\
12.08\end{array}$ & $\begin{array}{l}8,400 \\
7,620\end{array}$ & $8 / 17 / 94$ & 12.08 & 7,620 & $\mathrm{~N}$ & $25-50$ \\
\hline 03443000 & $\begin{array}{l}\text { French Broad River at Blantyre, } \\
\text { NC }\end{array}$ & 296 & $\begin{array}{l}1875-76, \\
1880,1893, \\
1899, \\
1901-02, \\
1905-06, \\
1910,1916, \\
1919, \\
1921-98\end{array}$ & $\begin{array}{l}1965 \\
1916\end{array}$ & $\begin{array}{l}25.50 \\
27.10\end{array}$ & $\begin{array}{r}30,000 \\
--\end{array}$ & $\begin{array}{l}8 / 18 / 94 \\
1 / 8 / 98\end{array}$ & $\begin{array}{l}23.81 \\
22.61\end{array}$ & $\begin{array}{l}21,200 \\
19,400\end{array}$ & $\begin{array}{l}\mathrm{N} \\
\mathrm{N}\end{array}$ & $\begin{array}{r}50 \\
25-50\end{array}$ \\
\hline 03450000 & $\begin{array}{l}\text { Beetree Creek near Swannanoa, } \\
\text { NC }\end{array}$ & 5.46 & $\begin{array}{l}1927-75, \\
1978, \\
1980-81, \\
1986, \\
1988-98\end{array}$ & 1940 & 6.20 & 1,370 & $8 / 17 / 94$ & 5.75 & 1,040 & $\mathrm{~N}$ & $50-100$ \\
\hline 03456100 & $\begin{array}{l}\text { West Fork Pigeon River at Bethel, } \\
\text { NC }\end{array}$ & 58.4 & 1981-98 & 1994 & 12.63 & 11,700 & $8 / 17 / 94$ & 12.63 & 11,700 & $\mathrm{Y}$ & $25-50$ \\
\hline 03456500 & $\begin{array}{l}\text { East Fork Pigeon River near } \\
\text { Canton, NC }\end{array}$ & 51.5 & $1955-98$ & 1973 & 11.19 & 12,000 & $8 / 17 / 94$ & 10.70 & 11,100 & $\mathrm{~N}$ & $10-25$ \\
\hline 03460000 & $\begin{array}{l}\text { Cataloochee Creek near } \\
\text { Cataloochee, } \mathrm{NC}\end{array}$ & 49.2 & $\begin{array}{l}1935-52 \\
1963-98\end{array}$ & 1963 & 8.08 & 5,080 & $3 / 27 / 94$ & 7.55 & 4,280 & $\mathrm{~N}$ & $10-25$ \\
\hline 03463300 & South Toe River near Celo, NC & 43.3 & $1958-98$ & 1978 & 17.41 & 32,900 & $8 / 17 / 94$ & 10.49 & 15,600 & $\mathrm{~N}$ & $10-25$ \\
\hline 03479000 & $\begin{array}{l}\text { Watauga River near Sugar Grove, } \\
\text { NC }\end{array}$ & 92.1 & $\begin{array}{l}\text { 1916, } \\
1940-98\end{array}$ & 1940 & 29.60 & 50,800 & $1 / 14 / 95$ & 20.46 & 20,500 & $\mathrm{~N}$ & 25 \\
\hline 03509000 & Scott Creek above Sylva, NC & 51.0 & $\begin{array}{l}1940, \\
1942-75 \\
1993-95\end{array}$ & $\begin{array}{l}1994 \\
1973\end{array}$ & $\begin{array}{l}6.77 \\
8.78\end{array}$ & $\begin{array}{l}4,440 \\
2,800\end{array}$ & $3 / 27 / 94$ & 6.77 & 4,440 & $\mathrm{~N}$ & 200 \\
\hline 03513000 & $\begin{array}{l}\text { Tuckasegee River at Bryson City, } \\
\text { NC }\end{array}$ & 655 & $\begin{array}{l}\text { 1898-1982, } \\
\text { 1984-95, } \\
1997-98\end{array}$ & 1940 & 15.96 & 61,600 & $3 / 28 / 94$ & 14.25 & 33,300 & $\mathrm{Y}$ & $10-25$ \\
\hline
\end{tabular}


$\left[\mathrm{mi}^{2}\right.$, square miles; $\mathrm{ft}$, feet above an arbitrary datum; $\mathrm{ft}^{3} / \mathrm{s}$, cubic feet per second; --, not determined or not applicable; $>$, greater than; <, less than. Source: Recurrence intervals calculated from U.S. Geological Survey data. Other data from U.S. Geological Survey reports or databases]

\begin{tabular}{|c|c|c|c|c|c|c|c|c|c|c|c|}
\hline \multirow[b]{2}{*}{$\begin{array}{l}\text { Streamgage } \\
\text { number } \\
\text { (fig. 45) }\end{array}$} & \multirow[b]{2}{*}{ Streamgage name } & \multirow{2}{*}{$\begin{array}{c}\text { Total } \\
\text { drainage } \\
\left(\mathrm{mi}^{2}\right)\end{array}$} & \multicolumn{4}{|c|}{$\begin{array}{c}\text { Maximum stage and discharge for period of record } \\
\text { through } 1998 \text { water year }\end{array}$} & \multicolumn{5}{|c|}{ Significant floods 1994-98 water years } \\
\hline & & & $\begin{array}{l}\text { Period of } \\
\text { record } \\
\text { (water } \\
\text { years) }\end{array}$ & Water year & $\begin{array}{l}\text { Stage } \\
(\mathrm{ft})\end{array}$ & $\begin{array}{c}\text { Discharge } \\
\left(\mathrm{ft}^{3} / \mathrm{s}\right)\end{array}$ & $\begin{array}{c}\text { Date } \\
\text { (month/ } \\
\text { day/year) }\end{array}$ & $\begin{array}{l}\text { Stage } \\
(\mathrm{ft})\end{array}$ & $\begin{array}{c}\text { Discharge } \\
\left(\mathrm{ft}^{3} / \mathrm{s}\right)\end{array}$ & $\begin{array}{l}\text { Regulated } \\
\text { during } \\
\text { flood }^{1}\end{array}$ & $\begin{array}{c}\text { Recurrence } \\
\text { interval } \\
\text { (years) }\end{array}$ \\
\hline 03550000 & Valley River at Tomotla, NC & 104 & $\begin{array}{l}1898, \\
1905-09, \\
1915-17, \\
1919-98\end{array}$ & $\begin{array}{l}1907 \\
1898\end{array}$ & $\begin{array}{l}20.50 \\
21.20\end{array}$ & $\begin{array}{l}18,000 \\
20,000\end{array}$ & $\begin{array}{l}3 / 27 / 94 \\
2 / 16 / 95\end{array}$ & $\begin{array}{l}17.10 \\
17.15\end{array}$ & $\begin{array}{l}11,900 \\
12,100\end{array}$ & $\begin{array}{l}\mathrm{N} \\
\mathrm{N}\end{array}$ & $\begin{array}{r}25-50 \\
50\end{array}$ \\
\hline
\end{tabular}


Table 29. Maximum stage and discharge for period of record for streamgages having significant floods during 1994-98 water years in Nevada.

$\left[\mathrm{mi}^{2}\right.$, square miles; $\mathrm{ft}$, feet above an arbitrary datum; $\mathrm{ft}^{3} / \mathrm{s}$, cubic feet per second; --, not determined or not applicable; $>$, greater than; <, less than. Source: Recurrence intervals calculated from U.S. Geological Survey data. Other data from U.S. Geological Survey reports or databases]

\begin{tabular}{|c|c|c|c|c|c|c|c|c|c|c|c|}
\hline \multirow{2}{*}{$\begin{array}{l}\text { Streamgage } \\
\text { number } \\
\text { (fig. 40) }\end{array}$} & \multirow[b]{2}{*}{ Streamgage name } & \multirow{2}{*}{$\begin{array}{l}\text { Total } \\
\text { drainage } \\
\left(\mathrm{mi}^{2}\right)\end{array}$} & \multicolumn{4}{|c|}{$\begin{array}{c}\text { Maximum stage and discharge for period of record } \\
\text { through } 1998 \text { water year }\end{array}$} & \multicolumn{5}{|c|}{ Significant floods $1994-98$ water years } \\
\hline & & & $\begin{array}{l}\text { Period of } \\
\text { record } \\
\text { (water } \\
\text { years) }\end{array}$ & Water year & $\begin{array}{l}\text { Stage } \\
(\mathrm{ft})\end{array}$ & $\begin{array}{l}\text { Discharge } \\
\left(\mathrm{ft}^{3} / \mathrm{s}\right)\end{array}$ & $\begin{array}{l}\text { Date } \\
\text { (month/ } \\
\text { day/ } \\
\text { year) }\end{array}$ & $\begin{array}{l}\text { Stage } \\
(\mathrm{ft})\end{array}$ & $\begin{array}{l}\text { Discharge } \\
\left(\mathrm{ft}^{3} / \mathrm{s}\right)\end{array}$ & $\begin{array}{l}\text { Regulated } \\
\text { during } \\
\text { flood }^{1}\end{array}$ & $\begin{array}{l}\text { Recurrence } \\
\text { interval } \\
\text { (years) }\end{array}$ \\
\hline 09419658 & $\begin{array}{l}\text { Las Vegas Wash near Sahara } \\
\text { Avenue near Las Vegas, NV }\end{array}$ & 1,146 & $\begin{array}{l}1975 \\
1988-98\end{array}$ & $\begin{array}{l}1998 \\
1990\end{array}$ & $\begin{array}{l}12.50 \\
16.27\end{array}$ & $\begin{array}{l}4,400 \\
1,960\end{array}$ & $9 / 11 / 98$ & 12.50 & 4,400 & $\mathrm{Y}$ & -- \\
\hline 09419659 & $\begin{array}{l}\text { Sloan Channel tributary at Las } \\
\text { Vegas Boulevard near North Las } \\
\text { Vegas, NV }\end{array}$ & 17.5 & 1989-98 & 1998 & 15.34 & 920 & $9 / 11 / 98$ & 15.34 & 920 & $\mathrm{Y}$ & -- \\
\hline 09419663 & $\begin{array}{l}\text { Las Vegas Wash tributary south of } \\
\text { Nellis Air Force Base, NV }\end{array}$ & 1.20 & $\begin{array}{l}1963-81 \\
1983-98\end{array}$ & 1998 & 21.00 & 600 & $9 / 11 / 98$ & 21.00 & 600 & $\mathrm{Y}$ & -- \\
\hline 09419665 & $\begin{array}{l}\text { Sloan Channel at Charleston } \\
\text { Boulevard near Las Vegas, NV }\end{array}$ & 144 & 1989-98 & $\begin{array}{l}1998 \\
1997\end{array}$ & $\begin{array}{l}11.41 \\
11.72\end{array}$ & $\begin{array}{r}1,230 \\
700\end{array}$ & $9 / 11 / 98$ & 11.41 & 1,230 & $\mathrm{Y}$ & -- \\
\hline 09419679 & $\begin{array}{l}\text { Las Vegas Wasteway near East Las } \\
\text { Vegas, NV }\end{array}$ & -- & $\begin{array}{l}\text { 1980-83, } \\
1985-98\end{array}$ & $\begin{array}{l}1983 \\
1990\end{array}$ & $\begin{array}{l}6.56 \\
6.99\end{array}$ & 630 & $11 / 21 / 96$ & 6.81 & $\begin{array}{l}523 \\
453\end{array}$ & $\mathrm{~N}$ & -- \\
\hline 09419685 & $\begin{array}{l}\text { Bird Springs Wash near Arden, } \\
\text { NV }\end{array}$ & -- & 1987-98 & 1998 & 44.05 & 35 & $7 / 20 / 98$ & 44.05 & 35 & $\mathrm{~N}$ & -- \\
\hline 10243260 & Lehman Creek near Baker, NV & 11.0 & $\begin{array}{l}1948-55 \\
1993-97\end{array}$ & 1995 & 5.01 & 80 & $6 / 29 / 95$ & 5.01 & 80 & $\mathrm{~N}$ & -- \\
\hline 10249280 & $\begin{array}{l}\text { Kingston Creek below Cougar } \\
\text { Canyon near Austin, NV }\end{array}$ & 23.4 & 1967-98 & $\begin{array}{l}1983 \\
1995\end{array}$ & $\begin{array}{l}3.19 \\
3.86\end{array}$ & $\begin{array}{l}385 \\
234\end{array}$ & $6 / 3 / 95$ & 3.86 & 234 & $\mathrm{Y}$ & -- \\
\hline 10249300 & $\begin{array}{l}\text { South Twin River near Round } \\
\text { Mountain, NV }\end{array}$ & 20.0 & 1965-98 & 1983 & 4.39 & 510 & $6 / 3 / 98$ & 3.20 & 148 & $\mathrm{~N}$ & -- \\
\hline 10251248 & $\begin{array}{l}\text { Unnamed tributary-Stockade Wash } \\
\text { near Rattlesnake Ridge, Nevada } \\
\text { Test Site, NV }\end{array}$ & 3.90 & $\begin{array}{l}1984-88 \\
1991-95\end{array}$ & 1995 & 5.49 & 90 & $3 / 11 / 95$ & 5.49 & 90 & $\mathrm{~N}$ & -- \\
\hline 10293500 & $\begin{array}{l}\text { East Walker River above } \\
\text { Strosnider Ditch near Mason, NV }\end{array}$ & 1,100 & $\begin{array}{l}1947-92 \\
1995-98\end{array}$ & $\begin{array}{l}1986 \\
1997\end{array}$ & $\begin{array}{l}7.49 \\
9.61\end{array}$ & $\begin{array}{l}2,820 \\
2,610\end{array}$ & $1 / 4 / 97$ & 9.61 & 2,610 & $\mathrm{Y}$ & $>25$ \\
\hline
\end{tabular}


Table 29. Maximum stage and discharge for period of record for streamgages having significant floods during 1994-98 water years in Nevada.-Continued

$\left[\mathrm{mi}^{2}\right.$, square miles; $\mathrm{ft}$, feet above an arbitrary datum; $\mathrm{ft}^{3} / \mathrm{s}$, cubic feet per second; --, not determined or not applicable; $>$, greater than; <, less than. Source: Recurrence intervals calculated from U.S. Geological Survey data. Other data from U.S. Geological Survey reports or databases]

\begin{tabular}{|c|c|c|c|c|c|c|c|c|c|c|c|}
\hline \multirow{2}{*}{$\begin{array}{l}\text { Streamgage } \\
\text { number } \\
\text { (fig. 40) }\end{array}$} & \multirow[b]{2}{*}{ Streamgage name } & \multirow{2}{*}{$\begin{array}{l}\text { Total } \\
\text { drainage } \\
\left(\mathrm{mi}^{2}\right)\end{array}$} & \multicolumn{4}{|c|}{$\begin{array}{c}\text { Maximum stage and discharge for period of record } \\
\text { through } 1998 \text { water year }\end{array}$} & \multicolumn{5}{|c|}{ Significant floods $1994-98$ water years } \\
\hline & & & $\begin{array}{l}\text { Period of } \\
\text { record } \\
\text { (water } \\
\text { years) }\end{array}$ & Water year & $\begin{array}{l}\text { Stage } \\
\text { (ft) }\end{array}$ & $\begin{array}{l}\text { Discharge } \\
\left(\mathrm{ft}^{3} / \mathrm{s}\right)\end{array}$ & $\begin{array}{l}\text { Date } \\
\text { (month/ } \\
\text { day/ } \\
\text { year) }\end{array}$ & $\begin{array}{l}\text { Stage } \\
\text { (ft) }\end{array}$ & $\begin{array}{l}\text { Discharge } \\
\left(\mathrm{ft}^{3} / \mathrm{s}\right)\end{array}$ & $\begin{array}{l}\text { Regulated } \\
\text { during } \\
\text { flood }^{1}\end{array}$ & $\begin{array}{l}\text { Recurrence } \\
\text { interval } \\
\text { (years) }\end{array}$ \\
\hline 10297500 & $\begin{array}{l}\text { West Walker River at Hoye Bridge } \\
\text { near Wellington, NV }\end{array}$ & 497 & $\begin{array}{l}1921-22 \\
1924-32 \\
1958-98\end{array}$ & 1997 & 13.68 & 11,500 & $1 / 3 / 97$ & 13.68 & 11,500 & $\mathrm{Y}$ & $>100$ \\
\hline 10300000 & $\begin{array}{l}\text { West Walker River near Hudson, } \\
\text { NV }\end{array}$ & 964 & $\begin{array}{l}1915-18 \\
1920-24, \\
1947-92 \\
1995-98\end{array}$ & 1997 & 12.18 & 11,400 & $1 / 3 / 97$ & 12.18 & 11,400 & $\mathrm{Y}$ & $>100$ \\
\hline 10308800 & $\begin{array}{l}\text { Bryant Creek near Gardnerville, } \\
\text { NV }\end{array}$ & 31.5 & $\begin{array}{l}1961-73 \\
1978-80 \\
1995-98\end{array}$ & 1997 & 8.70 & 1,360 & $1 / 2 / 97$ & 8.70 & 1,300 & $\mathrm{~N}$ & $<25$ \\
\hline 10309000 & $\begin{array}{l}\text { East Fork Carson River near } \\
\text { Gardnerville, NV }\end{array}$ & 356 & $\begin{array}{l}\text { 1890-93, } \\
\text { 1901-05, } \\
\text { 1908-10, } \\
\text { 1917, } \\
\text { 1925-28, } \\
\text { 1936-38, } \\
1940-98\end{array}$ & 1997 & 13.00 & 20,300 & $1 / 3 / 97$ & 13.00 & 20,300 & $\mathrm{~N}$ & 100 \\
\hline 10309100 & $\begin{array}{l}\text { East Fork Carson River at Minden, } \\
\text { NV }\end{array}$ & 392 & $\begin{array}{l}1975-84 \\
1994-98\end{array}$ & $\begin{array}{l}1997 \\
1980\end{array}$ & $\begin{array}{l}10.41 \\
11.40\end{array}$ & $\begin{array}{r}10,900 \\
8,000\end{array}$ & $1 / 2 / 97$ & 10.41 & 10,900 & $\mathrm{Y}$ & -- \\
\hline 10310500 & Clear Creek near Carson City, NV & 15.5 & $\begin{array}{l}1948-78 \\
1989-98\end{array}$ & 1997 & 3.94 & 266 & $1 / 2 / 97$ & 3.94 & 266 & $\mathrm{~N}$ & 50 \\
\hline 10311000 & $\begin{array}{l}\text { Carson River near Carson City, } \\
\text { NV }\end{array}$ & 886 & 1939-98 & 1997 & 18.43 & 30,500 & $1 / 3 / 97$ & 18.43 & 30,500 & $\mathrm{~N}$ & $>75$ \\
\hline 10311260 & $\begin{array}{l}\text { Vicee Canyon Creek near } \\
\text { Sagebrush Ranch near Carson } \\
\text { City, NV }\end{array}$ & 1.83 & $\begin{array}{l}1979-80 \\
1982,1984, \\
1986, \\
1991-97\end{array}$ & 1997 & 9.66 & 4,000 & $1 / 2 / 97$ & 9.66 & 4,000 & $\mathrm{~N}$ & $>100$ \\
\hline
\end{tabular}


Table 29. Maximum stage and discharge for period of record for streamgages having significant floods during 1994-98 water years in Nevada.-Continued

$\left[\mathrm{mi}^{2}\right.$, square miles; $\mathrm{ft}$, feet above an arbitrary datum; $\mathrm{ft}^{3} / \mathrm{s}$, cubic feet per second; --, not determined or not applicable; $>$, greater than; <, less than. Source: Recurrence intervals calculated from U.S. Geological Survey data. Other data from U.S. Geological Survey reports or databases]

\begin{tabular}{|c|c|c|c|c|c|c|c|c|c|c|c|}
\hline \multirow[b]{2}{*}{$\begin{array}{c}\text { Streamgage } \\
\text { number } \\
\text { (fig. 40) }\end{array}$} & \multirow[b]{2}{*}{ Streamgage name } & \multirow[b]{2}{*}{$\begin{array}{c}\text { Total } \\
\text { drainage } \\
\left(\mathrm{mi}^{2}\right)\end{array}$} & \multicolumn{4}{|c|}{$\begin{array}{c}\text { Maximum stage and discharge for period of record } \\
\text { through } 1998 \text { water year }\end{array}$} & \multicolumn{5}{|c|}{ Significant floods $1994-98$ water years } \\
\hline & & & $\begin{array}{c}\text { Period of } \\
\text { record } \\
\text { (water } \\
\text { years) }\end{array}$ & Water year & $\begin{array}{l}\text { Stage } \\
\text { (ft) }\end{array}$ & $\begin{array}{l}\text { Discharge } \\
\left(\mathrm{ft}^{3} / \mathrm{s}\right)\end{array}$ & $\begin{array}{c}\text { Date } \\
\text { (month/ } \\
\text { day/ } \\
\text { year) }\end{array}$ & $\begin{array}{l}\text { Stage } \\
\text { (ft) }\end{array}$ & $\begin{array}{l}\text { Discharge } \\
\left(\mathrm{ft}^{3} / \mathrm{s}\right)\end{array}$ & $\begin{array}{c}\text { Regulated } \\
\text { during } \\
\text { flood }^{1}\end{array}$ & $\begin{array}{c}\text { Recurrence } \\
\text { interval } \\
\text { (years) }\end{array}$ \\
\hline 10311400 & $\begin{array}{l}\text { Carson River at Deer Run Road } \\
\text { near Carson City, NV }\end{array}$ & 958 & $\begin{array}{l}1980-85, \\
1991-98\end{array}$ & 1997 & 24.23 & 24,000 & $1 / 3 / 97$ & 24.23 & 24,000 & $\mathrm{~N}$ & $<100$ \\
\hline 10311450 & $\begin{array}{l}\text { Brunswick Canyon near New } \\
\text { Empire, NV }\end{array}$ & 12.7 & $\begin{array}{l}1966-78 \\
1980-98\end{array}$ & 1995 & 5.02 & 245 & $3 / 11 / 95$ & 5.02 & 245 & $\mathrm{~N}$ & $<10$ \\
\hline 10312000 & $\begin{array}{l}\text { Carson River near Fort Churchill, } \\
\text { NV }\end{array}$ & 1,450 & $1911-98$ & 1997 & 15.27 & 22,300 & $1 / 3 / 97$ & 15.27 & 22,300 & $\mathrm{~N}$ & $>100$ \\
\hline 10312150 & $\begin{array}{l}\text { Carson River below Lahontan } \\
\text { Reservoir near Fallon, NV }\end{array}$ & 1,801 & $1980-98$ & 1983 & 8.34 & 3,160 & $5 / 17 / 96$ & 7.78 & 2,430 & $\mathrm{Y}$ & -- \\
\hline 10312275 & $\begin{array}{l}\text { Carson River at Tarzyn Road near } \\
\text { Fallon, NV }\end{array}$ & -- & 1986-98 & $\begin{array}{l}1996 \\
1997\end{array}$ & $\begin{array}{l}6.11 \\
8.73\end{array}$ & $\begin{array}{l}942 \\
821\end{array}$ & $5 / 27 / 96$ & $\begin{array}{l}6.11 \\
8.73\end{array}$ & $\begin{array}{l}942 \\
821\end{array}$ & $\mathrm{Y}$ & -- \\
\hline 10316500 & Lamoille Creek near Lamoille, NV & 24.9 & $\begin{array}{l}\text { 1915-16, } \\
1918-20, \\
1922, \\
1944-98\end{array}$ & $\begin{array}{l}1997 \\
1982\end{array}$ & $\begin{array}{l}5.29 \\
6.23\end{array}$ & $\begin{array}{l}838 \\
829\end{array}$ & $6 / 4 / 97$ & 5.29 & 838 & $\mathrm{~N}$ & -- \\
\hline 10319900 & $\begin{array}{l}\text { South Fork Humboldt River above } \\
\text { Tenmile Creek near Elko, NV }\end{array}$ & 898 & 1989-98 & 1995 & 5.82 & 2,710 & $6 / 3 / 95$ & 5.82 & 2,710 & $\mathrm{~N}$ & -- \\
\hline 10336700 & $\begin{array}{l}\text { Incline Creek near Crystal Bay, } \\
\text { NV }\end{array}$ & 6.69 & $\begin{array}{l}\text { 1970-73, } \\
1975, \\
1988-98\end{array}$ & 1997 & 3.87 & 179 & $1 / 2 / 97$ & 3.87 & 179 & $\mathrm{~N}$ & $<50$ \\
\hline 10336730 & $\begin{array}{l}\text { Glenbrook Creek at Glenbrook, } \\
\text { NV }\end{array}$ & 3.75 & $\begin{array}{l}1972-75 \\
1988-98\end{array}$ & 1997 & 6.46 & 144 & $1 / 2 / 97$ & 6.46 & 144 & $\mathrm{~N}$ & 50 \\
\hline 10336740 & $\begin{array}{l}\text { Logan House Creek near } \\
\text { Glenbrook, NV }\end{array}$ & 2.08 & 1984-98 & 1997 & 4.75 & 11 & $1 / 2 / 97$ & 4.75 & 11 & $\mathrm{~N}$ & $<25$ \\
\hline 10348000 & Truckee River at Reno, NV & 1,067 & $\begin{array}{l}1907-21, \\
1925-26, \\
1931-34, \\
1947-98\end{array}$ & $\begin{array}{l}1956 \\
1997\end{array}$ & $\begin{array}{l}13.63 \\
14.94\end{array}$ & $\begin{array}{l}20,800 \\
18,200\end{array}$ & $1 / 2 / 97$ & 14.94 & 18,200 & $\mathrm{Y}$ & $<50$ \\
\hline
\end{tabular}


$\left[\mathrm{mi}^{2}\right.$, square miles; $\mathrm{ft}$, feet above an arbitrary datum; $\mathrm{ft}^{3} / \mathrm{s}$, cubic feet per second; --, not determined or not applicable; >, greater than; <, less than. Source: Recurrence intervals calculated from U.S. Geological Survey data. Other data from U.S. Geological Survey reports or databases]

\begin{tabular}{|c|c|c|c|c|c|c|c|c|c|c|c|}
\hline \multirow[b]{2}{*}{$\begin{array}{l}\text { Streamgage } \\
\text { number } \\
\text { (fig. 40) }\end{array}$} & \multirow[b]{2}{*}{ Streamgage name } & \multirow[b]{2}{*}{$\begin{array}{c}\text { Total } \\
\text { drainage } \\
\left(\mathrm{mi}^{2}\right)\end{array}$} & \multicolumn{4}{|c|}{$\begin{array}{c}\text { Maximum stage and discharge for period of record } \\
\text { through } 1998 \text { water year }\end{array}$} & \multicolumn{5}{|c|}{ Significant floods $1994-98$ water years } \\
\hline & & & $\begin{array}{c}\text { Period of } \\
\text { record } \\
\text { (water } \\
\text { years) }\end{array}$ & Water year & $\begin{array}{l}\text { Stage } \\
(\mathrm{ft})\end{array}$ & $\begin{array}{l}\text { Discharge } \\
\left(\mathrm{ft}^{3} / \mathrm{s}\right)\end{array}$ & $\begin{array}{c}\text { Date } \\
\text { (month/ } \\
\text { day/ } \\
\text { year) }\end{array}$ & $\begin{array}{l}\text { Stage } \\
(\mathrm{ft})\end{array}$ & $\begin{array}{l}\text { Discharge } \\
\left(\mathrm{ft}^{3} / \mathrm{s}\right)\end{array}$ & $\begin{array}{c}\text { Regulated } \\
\text { during } \\
\text { flood }^{1}\end{array}$ & $\begin{array}{c}\text { Recurrence } \\
\text { interval } \\
\text { (years) }\end{array}$ \\
\hline 10348200 & Truckee River near Sparks, NV & 1,070 & $1978-98$ & $\begin{array}{l}1997 \\
1986\end{array}$ & $\begin{array}{l}-- \\
15.22\end{array}$ & $\begin{array}{l}18,000 \\
14,900\end{array}$ & $1 / 2 / 97$ & -- & 18,000 & $\mathrm{Y}$ & -- \\
\hline 10348850 & $\begin{array}{l}\text { Galena Creek at Galena Creek } \\
\text { State Park, NV }\end{array}$ & 7.69 & 1985-98 & 1997 & 5.54 & 2,610 & $1 / 2 / 97$ & 5.54 & 2,610 & $\mathrm{~N}$ & $<50$ \\
\hline 10349300 & Steamboat Creek at Steamboat, NV & 123 & $1962-98$ & 1986 & 6.79 & 3,600 & $1 / 1 / 97$ & 6.03 & 2,090 & $\mathrm{Y}$ & $>25$ \\
\hline 10351600 & $\begin{array}{l}\text { Truckee River below Derby Dam } \\
\text { near Wadsworth, NV }\end{array}$ & 1,676 & $\begin{array}{l}\text { 1909-10, } \\
1916, \\
1918-98\end{array}$ & 1997 & 14.56 & 19,900 & $1 / 3 / 97$ & 14.56 & 19,900 & $\mathrm{Y}$ & $<50$ \\
\hline 10351650 & Truckee River at Wadsworth, NV & 1,728 & $\begin{array}{l}1966-86, \\
1994-98\end{array}$ & 1997 & 19.62 & 19,100 & $1 / 3 / 97$ & 19.62 & 19,100 & $\mathrm{Y}$ & $<50$ \\
\hline 10351700 & Truckee River near Nixon, NV & 1,827 & $\begin{array}{l}1956, \\
1958-98\end{array}$ & 1997 & 15.28 & 21,200 & $1 / 3 / 97$ & 15.28 & 21,200 & $\mathrm{Y}$ & 50 \\
\hline 10353750 & $\begin{array}{l}\text { Mahogany Creek near Summit } \\
\text { Lake, NV }\end{array}$ & 13.3 & 1988-98 & $\begin{array}{l}1995 \\
1998\end{array}$ & $\begin{array}{l}5.34 \\
5.56\end{array}$ & $\begin{array}{l}50 \\
40\end{array}$ & $6 / 5 / 95$ & 5.34 & 50 & $\mathrm{~N}$ & -- \\
\hline 10353800 & $\begin{array}{l}\text { Smoke Creek below reservoir near } \\
\text { Smoke Creek, NV }\end{array}$ & 50.1 & $\begin{array}{l}\text { 1986, } \\
\text { 1989-98 }\end{array}$ & $\begin{array}{l}1995 \\
1986\end{array}$ & $\begin{array}{l}8.43 \\
9.00\end{array}$ & $\begin{array}{l}4,320 \\
2,270\end{array}$ & $3 / 9 / 95$ & 8.43 & 4,320 & $\mathrm{Y}$ & -- \\
\hline
\end{tabular}

${ }^{1}$ Regulated during flood: N, no; Y, yes. 


\section{New Hampshire}

A coastal storm, which was fed tropical moisture by the circulation around Hurricane Lily, produced 8 to 13 inches of rain throughout parts of central and southern New Hampshire during October 20-23, 1996 (National Oceanic and Atmospheric Administration, 1996a). Many homes and businesses were flooded, roads and bridges were washed out, and several dams were damaged. Total damage was more than $\$ 10.8$ million (National Oceanic and Atmospheric Administration, 1996b). Oyster River near Durham (streamgage 01073000) had a peak of record during this flood (table 30 ).

Three to 8 inches of rain during June 13-15, 1998 (National Oceanic and Atmospheric Administration, 1998a), caused small rivers and streams to rise in central and southern New Hampshire. Many roads were flooded and (or) washed out. Campgrounds and some lakeside homes had to be evacuated. More than \$1 million in damage resulted (National Oceanic and
Atmospheric Administration, 1998b). Excessive rains returned on June 27, 1998. From 3 to possibly as much as 6 inches of rain fell in 12 hours (National Oceanic and Atmospheric Administration, 1998a) causing the Baker River (fig. 41) and its tributaries to rise very rapidly. Bridges were destroyed, vehicles were washed away, and some towns were completely isolated. Damage was near \$1.5 million (National Oceanic and Atmospheric Administration, 1998b).

\section{References}

National Oceanic and Atmospheric Administration (NOAA), 1996a-98a, Climatological data (by State): Asheville, North Carolina, National Climatic Data Center, various months.

National Oceanic and Atmospheric Administration (NOAA), 1996b-98b, Storm data (by State): Asheville, North Carolina, National Climatic Data Center, various months.

\section{EXPLANATION}

$01137500 \_$Streamgage and number
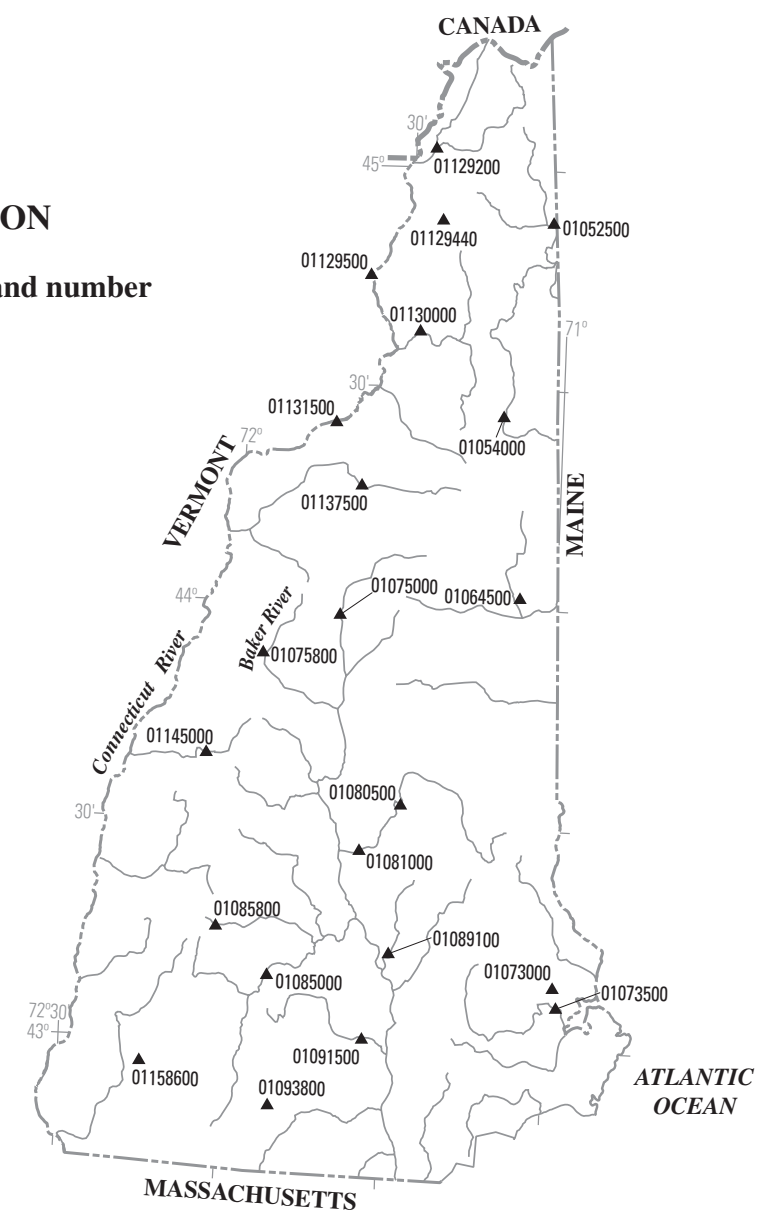

Base from U.S. Geological Survey digital data, 1:2,000,000, 1994 Albers Equal-Area Conic projection

Standard parallels $29^{\circ} 30^{\prime}$ and $45^{\circ} 30^{\prime}$, central meridian $-96^{\circ} 00^{\prime}$

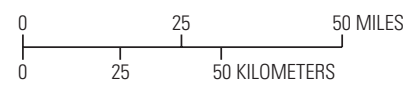

Figure 41. Location of streamgages with significant floods during 1994-98 water years for New Hampshire. 
Table 30. Maximum stage and discharge for period of record for streamgages having significant floods during 1994-98 water years in New Hampshire.

$\left[\mathrm{mi}^{2}\right.$, square miles; ft, feet above an arbitrary datum; $\mathrm{ft}^{3} / \mathrm{s}$, cubic feet per second; --, not determined or not applicable; >, greater than. Source: Recurrence intervals calculated from U.S. Geological Survey data. Other data from U.S. Geological Survey reports or databases]

\begin{tabular}{|c|c|c|c|c|c|c|c|c|c|c|c|}
\hline \multirow[b]{2}{*}{$\begin{array}{l}\text { Streamgage } \\
\text { number } \\
\text { (fig. 41) }\end{array}$} & \multirow[b]{2}{*}{ Streamgage name } & \multirow[b]{2}{*}{$\begin{array}{c}\text { Total } \\
\text { drainage } \\
\left(\mathrm{mi}^{2}\right)\end{array}$} & \multicolumn{4}{|c|}{$\begin{array}{c}\text { Maximum stage and discharge for period of record } \\
\text { through } 1998 \text { water year }\end{array}$} & \multicolumn{5}{|c|}{ Significant floods 1994-98 water years } \\
\hline & & & $\begin{array}{l}\text { Period of } \\
\text { record } \\
\text { (water } \\
\text { years) }\end{array}$ & Water year & $\begin{array}{l}\text { Stage } \\
\text { (ft) }\end{array}$ & $\begin{array}{l}\text { Discharge } \\
\left(\mathrm{ft}^{3} / \mathrm{s}\right)\end{array}$ & $\begin{array}{c}\text { Date } \\
\text { (month/ } \\
\text { day/ } \\
\text { year) }\end{array}$ & $\begin{array}{l}\text { Stage } \\
(\mathrm{ft})\end{array}$ & $\begin{array}{l}\text { Discharge } \\
\left(\mathrm{ft}^{3} / \mathrm{s}\right)\end{array}$ & $\begin{array}{l}\text { Regulated } \\
\text { during } \\
\text { flood }^{1}\end{array}$ & $\begin{array}{c}\text { Recurrence } \\
\text { interval } \\
\text { (years) }\end{array}$ \\
\hline 01052500 & $\begin{array}{l}\text { Diamond River near Wentworth } \\
\text { Location, NH }\end{array}$ & 152 & $1942-98$ & $\begin{array}{l}1998 \\
1981\end{array}$ & $\begin{array}{l}12.11 \\
12.23\end{array}$ & $\begin{array}{r}12,800 \\
3,620\end{array}$ & $3 / 31 / 98$ & 12.11 & 12,800 & $\mathrm{~N}$ & $>100$ \\
\hline 01054000 & $\begin{array}{l}\text { Androscoggin River near Gorham, } \\
\mathrm{NH}\end{array}$ & 1,361 & 1914-98 & $\begin{array}{l}1923 \\
1998\end{array}$ & $\begin{array}{l}-- \\
10.15\end{array}$ & $\begin{array}{l}21,900 \\
20,400\end{array}$ & $4 / 1 / 98$ & 10.15 & 20,400 & $\mathrm{Y}$ & 50 \\
\hline 01064500 & Saco River near Conway, NH & 385 & $\begin{array}{l}1903-09 \\
1910-12, \\
1929-98\end{array}$ & 1998 & 14.67 & 36,500 & $6 / 14 / 98$ & 14.67 & 36,500 & -- & $10-25$ \\
\hline 01073000 & Oyster River near Durham, NH & 12.1 & $1935-98$ & $\begin{array}{l}1997 \\
1936\end{array}$ & $\begin{array}{l}6.67 \\
7.45\end{array}$ & $\begin{array}{r}1,160 \\
548\end{array}$ & $10 / 21 / 96$ & 6.67 & 1,160 & $\mathrm{~N}$ & $>100$ \\
\hline 01073500 & $\begin{array}{l}\text { Lamprey River near Newmarket, } \\
\text { NH }\end{array}$ & 183 & $1935-98$ & 1987 & 15.14 & 7,570 & $10 / 22 / 96$ & 14.59 & 7,080 & $\mathrm{Y}$ & $50-75$ \\
\hline 01075000 & $\begin{array}{l}\text { Pemigewasset River at } \\
\text { Woodstock, NH }\end{array}$ & 193 & $\begin{array}{l}1940-80 \\
1985-98\end{array}$ & 1960 & 16.13 & 47,000 & $10 / 22 / 95$ & 14.09 & 33,500 & $\mathrm{~N}$ & $25-50$ \\
\hline 01075800 & $\begin{array}{l}\text { Stevens Brook near Wentworth, } \\
\text { NH }\end{array}$ & 12.9 & 1964-98 & 1996 & 4.51 & 650 & $11 / 12 / 95$ & 4.51 & 650 & -- & $10-25$ \\
\hline 01080500 & $\begin{array}{l}\text { Lake Winnipesaukee outlet at } \\
\text { Lakeport, NH }\end{array}$ & 363 & $\begin{array}{l}1934-83 \\
1988-98\end{array}$ & 1936 & -- & 2,890 & $6 / 22 / 98$ & -- & 2,620 & $\mathrm{Y}$ & $10-25$ \\
\hline 01081000 & Winnipesaukee River at Tilton, NH & 471 & 1937-98 & 1984 & 8.68 & 4,580 & $6 / 28 / 98$ & 8.04 & 3,940 & $\mathrm{Y}$ & 25 \\
\hline 01085000 & $\begin{array}{l}\text { Contoocook River near Henniker, } \\
\text { NH }\end{array}$ & 368 & $\begin{array}{l}1938, \\
1940-77, \\
1989-98\end{array}$ & 1938 & 21.30 & 22,200 & $10 / 22 / 96$ & 13.18 & 9,840 & $\mathrm{Y}$ & $25-50$ \\
\hline 01085800 & $\begin{array}{l}\text { West Branch Warner River near } \\
\text { Bradford, NH }\end{array}$ & 5.75 & 1963-98 & $\begin{array}{l}1984 \\
1996\end{array}$ & $-\overline{-}$ & $\begin{array}{l}800 \\
690\end{array}$ & $11 / 12 / 95$ & 9.14 & 690 & $\mathrm{~N}$ & $10-25$ \\
\hline 01089100 & $\begin{array}{l}\text { Soucook River at Pembroke Road } \\
\text { near Concord, NH }\end{array}$ & 81.9 & 1989-98 & 1996 & 11.59 & 2,320 & $4 / 17 / 96$ & 11.59 & 2,320 & $\mathrm{~N}$ & $10-25$ \\
\hline
\end{tabular}


Table 30. Maximum stage and discharge for period of record for streamgages having significant floods during 1994-98 water years in New Hampshire.-Continued

$\left[\mathrm{mi}^{2}\right.$, square miles; ft, feet above an arbitrary datum; $\mathrm{ft}^{3} / \mathrm{s}$, cubic feet per second; --, not determined or not applicable; >, greater than. Source: Recurrence intervals calculated from U.S. Geological Survey data. Other data from U.S. Geological Survey reports or databases]

\begin{tabular}{|c|c|c|c|c|c|c|c|c|c|c|c|}
\hline \multirow{2}{*}{$\begin{array}{l}\text { Streamgage } \\
\text { number } \\
\text { (fig. 41) }\end{array}$} & \multirow[b]{2}{*}{ Streamgage name } & \multirow{2}{*}{$\begin{array}{c}\text { Total } \\
\text { drainage } \\
\left(\mathrm{mi}^{2}\right)\end{array}$} & \multicolumn{4}{|c|}{$\begin{array}{l}\text { Maximum stage and discharge for period of record } \\
\text { through } 1998 \text { water year }\end{array}$} & \multicolumn{5}{|c|}{ Significant floods 1994-98 water years } \\
\hline & & & $\begin{array}{c}\text { Period of } \\
\text { record } \\
\text { (water } \\
\text { years) }\end{array}$ & Water year & $\begin{array}{l}\text { Stage } \\
(\mathrm{ft})\end{array}$ & $\begin{array}{l}\text { Discharge } \\
\left(\mathrm{ft}^{3} / \mathrm{s}\right)\end{array}$ & $\begin{array}{c}\text { Date } \\
\text { (month/ } \\
\text { day/ } \\
\text { year) }\end{array}$ & $\begin{array}{l}\text { Stage } \\
(\mathrm{ft})\end{array}$ & $\begin{array}{l}\text { Discharge } \\
\left(\mathrm{ft}^{3} / \mathrm{s}\right)\end{array}$ & $\begin{array}{l}\text { Regulated } \\
\text { during } \\
\text { flood }^{1}\end{array}$ & $\begin{array}{c}\text { Recurrence } \\
\text { interval } \\
\text { (years) }\end{array}$ \\
\hline 01091500 & $\begin{array}{l}\text { Piscataquog River near } \\
\text { Goffstown, NH }\end{array}$ & 202 & $\begin{array}{l}1936,1938 \\
1940-78, \\
1983-98\end{array}$ & 1938 & 17.52 & 21,900 & $10 / 21 / 96$ & 10.61 & 6,470 & $\mathrm{Y}$ & $5-10$ \\
\hline 01093800 & $\begin{array}{l}\text { Stony Brook tributary near } \\
\text { Temple, NH }\end{array}$ & 3.60 & 1964-98 & $\begin{array}{l}1997 \\
1977\end{array}$ & $\begin{array}{l}5.63 \\
7.13\end{array}$ & $\begin{array}{l}648 \\
336\end{array}$ & $10 / 21 / 96$ & 5.63 & 648 & $\mathrm{~N}$ & $25-50$ \\
\hline 01129200 & $\begin{array}{l}\text { Connecticut River below Indian } \\
\text { Stream near Pittsburg, NH }\end{array}$ & 254 & $1957-98$ & 1996 & 7.97 & 5,240 & $\begin{array}{l}4 / 27 / 96 \\
3 / 31 / 98\end{array}$ & $\begin{array}{l}7.97 \\
7.87\end{array}$ & $\begin{array}{l}5,240 \\
5,120\end{array}$ & $\begin{array}{l}\mathrm{Y} \\
\mathrm{Y}\end{array}$ & $\begin{array}{l}50-100 \\
50-100\end{array}$ \\
\hline 01129440 & Mohawk River near Colebrook NH & 36.7 & 1987-98 & 1998 & 10.99 & 4,880 & $3 / 31 / 98$ & 10.99 & 4,880 & $\mathrm{~N}$ & $10-25$ \\
\hline 01129500 & $\begin{array}{l}\text { Connecticut River at North } \\
\text { Stratford, NH }\end{array}$ & 799 & $1931-98$ & $\begin{array}{l}1998 \\
1981\end{array}$ & $\begin{array}{l}15.63 \\
16.40\end{array}$ & $\begin{array}{l}32,300 \\
17,000\end{array}$ & $3 / 31 / 98$ & 15.63 & 32,300 & $\mathrm{Y}$ & $>100$ \\
\hline 01130000 & $\begin{array}{l}\text { Upper Ammonoosuc River near } \\
\text { Groveton, NH }\end{array}$ & 232 & $\begin{array}{l}1936, \\
1941-80, \\
1983-98\end{array}$ & 1969 & 12.01 & 24,100 & $3 / 31 / 98$ & 8.97 & 9,130 & $\mathrm{~N}$ & $25-50$ \\
\hline 01131500 & Connecticut River near Dalton, $\mathrm{NH}$ & 1,514 & 1928-98 & 1936 & 25.60 & 48,300 & $4 / 1 / 98$ & 23.70 & 42,000 & $\mathrm{Y}$ & $25-50$ \\
\hline 01137500 & $\begin{array}{l}\text { Ammonoosuc River at Bethlehem } \\
\text { Junction, NH }\end{array}$ & 87.6 & 1940-98 & 1996 & 12.34 & 11,300 & $11 / 12 / 95$ & 12.34 & 11,300 & $\mathrm{~N}$ & 50 \\
\hline 01145000 & $\begin{array}{l}\text { Mascoma River at West Canaan, } \\
\text { NH }\end{array}$ & 80.5 & $\begin{array}{l}1938, \\
1940-78, \\
1985-98\end{array}$ & $\begin{array}{l}1953 \\
1938\end{array}$ & $\begin{array}{l}8.94 \\
9.60\end{array}$ & $\begin{array}{l}3,780 \\
4,310\end{array}$ & $6 / 27 / 98$ & 8.59 & 3,420 & $\mathrm{~N}$ & $25-50$ \\
\hline 01158600 & $\begin{array}{l}\text { Otter Brook below Otter Brook } \\
\text { Dam near Keene, NH }\end{array}$ & 47.2 & 1959-98 & $\begin{array}{l}1987 \\
1994\end{array}$ & $\begin{array}{l}8.62 \\
8.64\end{array}$ & $\begin{array}{l}752 \\
720\end{array}$ & 4/18/94 & 8.64 & 720 & $\mathrm{Y}$ & $10-25$ \\
\hline
\end{tabular}

${ }^{1}$ Regulated during flood: N, no; Y, yes. 


\section{New Jersey}

The combination of warm temperatures, snowmelt, frozen ground, and basin average precipitation of 1.5 to 2 inches (National Oceanic and Atmospheric Administration, 1994a) caused urban flooding throughout interior southern New Jersey on January 28, 1994. The sudden increase in temperatures led to the formation of a few ice jams on area streams and rivers adding to the flooding problems. The area around Trenton (fig. 42) was the hardest hit with an estimated $\$ 4.5$ million in damage (National Oceanic and Atmospheric Administration, 1994b).

Thunderstorms with torrential downpours remained nearly stationary over the eastern Philadelphia suburbs for about 2 hours on July 14, 1994. Rainfall amounts of 3 to more than 7 inches were reported. In Westmont, 3.25 inches of rain fell within 30 minutes, 4.72 inches within 1 hour, and 6.54 inches in 3 hours. The rainfall rate of 4.72 inches per hour represents a greater than 100-year, 1-hour precipitation event for a single location in southern New Jersey (National Oceanic and Atmospheric Administration, 1994a).

Strong southerly winds ushered very mild and moistureladen air into the State on January 19, 1996. Street flooding and poor drainage became a major problem early on due to the rains, significant snowmelt, and ice jams. The flash flooding of the afternoon and early evening on January 19 led to larger river flooding through January 21 . The worst damage occurred along the Delaware River, which crested at its highest stages since the summer of 1955 (table 31). Widespread flooding along the Raritan River was described as the worst in Manville since Tropical Storm Doria in August 1971. Damage was near \$14 million, and one life was lost (National Oceanic and Atmospheric Administration, 1996b).

Nearly stationary thunderstorms dropped between 3.5 and 7.6 inches of rain within 4 hours northwest of Trenton during the evening of June 12, 1996 (National Oceanic and Atmospheric Administration, 1996a). This caused widespread flash flooding throughout the area. Nearly every roadway was flooded. The 7.6 inches of rain represent about a daily 100-year storm (National Oceanic and Atmospheric Administration, 1996a). Approximately $\$ 8$ million in property damage occurred, most of it in Ewing (National Oceanic and Atmospheric Administration, 1996b).

Rain, intense at times, fell across the northern half of New Jersey during the daylight hours of October 19, 1996. Storm precipitation totals ranged from 5 to nearly 7 inches (National Oceanic and Atmospheric Administration, 1996a). Four streamgages experienced their peak of record during this flood (table 31). Damage was near $\$ 45$ million, but there were no deaths (National Oceanic and Atmospheric Administration, 1996b).

On July 25, 1997, a long period of excessive rain occurred in northern New Jersey as a strong warm front moved slowly north toward the area. In addition, the area was affected by fringe precipitation from Tropical Storm Danny. Excessive rainfall [from 4 to 7 inches with isolated higher amounts (National Oceanic and Atmospheric Administration, 1997a)] caused widespread serious flooding of homes, streets, rivers, streams, and poor drainage areas in and around Newark. Property damage estimates were at least \$3 million (National Oceanic and Atmospheric Administration, 1997b).

Torrential rain from thunderstorms fell across southeastern New Jersey on August 20-21, 1997, as a low-pressure system developed south of Delaware Bay and slowly moved northeast across southern New Jersey. Storm totals included 10.12 inches in Millville and 7.2 inches in Vineland. The storm total of 13.52 inches of rainfall at the Atlantic City International Airport represented a greater than 100-year storm for the area. A 100-year storm for this area is 7.25 inches (National Oceanic and Atmospheric Administration, 1997a). Total damage was estimated at \$54 million, but no deaths occurred (National Oceanic and Atmospheric Administration, 1997b).

\section{References}

National Oceanic and Atmospheric Administration (NOAA), 1994a-97a, Climatological data (by State): Asheville, North Carolina, National Climatic Data Center, various months.

National Oceanic and Atmospheric Administration (NOAA), 1994b-97b, Storm data (by State): Asheville, North Carolina, National Climatic Data Center, various months. 


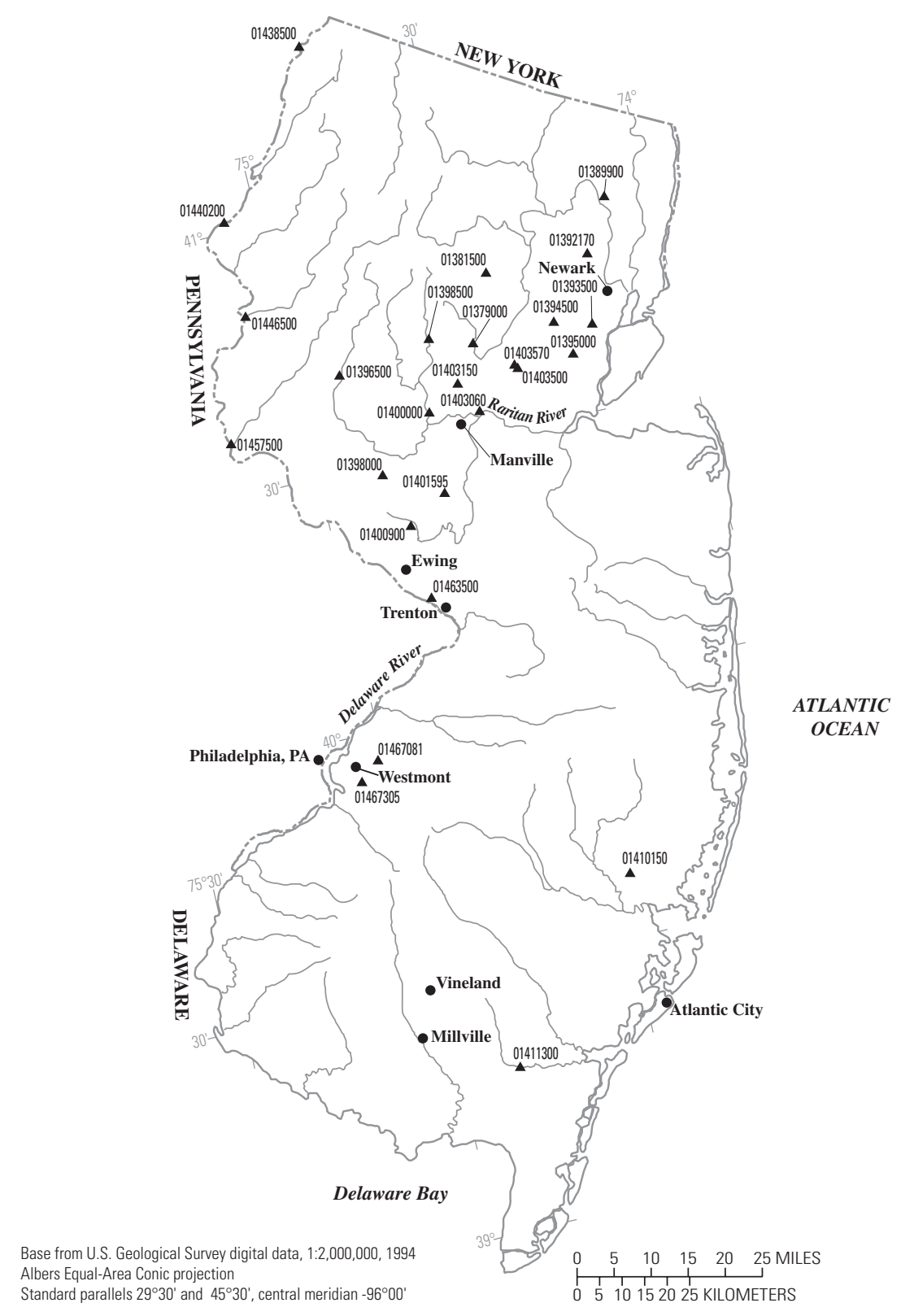

\section{EXPLANATION}

01411300 Streamgage and number

Figure 42. Location of streamgages with significant floods during 1994-98 water years for New Jersey. 
Table 31. Maximum stage and discharge for period of record for streamgages having significant floods during 1994-98 water years in New Jersey.

$\left[\mathrm{mi}^{2}\right.$, square miles; ft, feet above an arbitrary datum; $\mathrm{ft}^{3} / \mathrm{s}$, cubic feet per second; --, not determined or not applicable; >, greater than. Source: Recurrence intervals calculated from U.S. Geological Survey data. Other data from U.S. Geological Survey reports or databases]

\begin{tabular}{|c|c|c|c|c|c|c|c|c|c|c|c|}
\hline \multirow{2}{*}{$\begin{array}{c}\text { Streamgage } \\
\text { number } \\
\text { (fig. 42) }\end{array}$} & \multirow[b]{2}{*}{ Streamgage name } & \multirow{2}{*}{$\begin{array}{c}\text { Total } \\
\text { drainage } \\
\left(\mathrm{mi}^{2}\right)\end{array}$} & \multicolumn{4}{|c|}{$\begin{array}{c}\text { Maximum stage and discharge for period of record } \\
\text { through } 1998 \text { water year }\end{array}$} & \multicolumn{5}{|c|}{ Significant floods 1994-98 water years } \\
\hline & & & $\begin{array}{l}\text { Period of } \\
\text { record } \\
\text { (water } \\
\text { years) }\end{array}$ & Water year & $\begin{array}{l}\text { Stage } \\
\text { (ft) }\end{array}$ & $\begin{array}{l}\text { Discharge } \\
\left(\mathrm{ft}^{3} / \mathrm{s}\right)\end{array}$ & $\begin{array}{c}\text { Date } \\
\text { (month/ } \\
\text { day/ } \\
\text { year) }\end{array}$ & $\begin{array}{l}\text { Stage } \\
(\mathrm{ft})\end{array}$ & $\begin{array}{l}\text { Discharge } \\
\left(\mathrm{ft}^{3} / \mathrm{s}\right)\end{array}$ & $\begin{array}{l}\text { Regulated } \\
\text { during } \\
\text { flood }^{1}\end{array}$ & $\begin{array}{c}\text { Recurrence } \\
\text { interval } \\
\text { (years) }\end{array}$ \\
\hline 01379000 & Passaic River near Millington, NJ & 55.4 & $\begin{array}{l}1904-06 \\
1922-98\end{array}$ & 1997 & 9.89 & 2,290 & $10 / 20 / 96$ & 9.89 & 2,290 & $\mathrm{~N}$ & 80 \\
\hline 01381500 & Whippany River at Morristown, NJ & 29.4 & $1922-98$ & 1971 & 8.60 & 2,800 & $10 / 20 / 96$ & 7.79 & 2,300 & $\mathrm{~N}$ & 40 \\
\hline 01389900 & $\begin{array}{l}\text { Fleischer Brook at Market Street at } \\
\text { Elmwood Park, NJ }\end{array}$ & 1.37 & $\begin{array}{l}\text { 1967-93, } \\
1995-98\end{array}$ & 1978 & 6.47 & 470 & $7 / 8 / 96$ & 2.82 & 1,600 & $\mathrm{~N}$ & -- \\
\hline 01392170 & Third River at Bloomfield, NJ & 7.71 & 1989-98 & 1997 & 7.34 & 1,410 & $10 / 19 / 96$ & 7.34 & 1,410 & $\mathrm{~N}$ & 6 \\
\hline 01393500 & Elizabeth River at Elizabeth, NJ & 20.2 & 1922-98 & 1971 & 18.70 & 4,110 & $7 / 25 / 97$ & -- & 3,080 & $\mathrm{~N}$ & 9 \\
\hline 01394500 & Rahway River near Springfield, NJ & 25.5 & 1938-99 & 1973 & 9.76 & 5,430 & $7 / 25 / 97$ & 9.56 & 5,140 & $\mathrm{~N}$ & 50 \\
\hline 01395000 & Rahway River at Rahway, NJ & 40.9 & $\begin{array}{l}\text { 1922-87, } \\
1989-98\end{array}$ & 1973 & 7.88 & 5,420 & $10 / 19 / 96$ & 7.50 & 4,210 & $\mathrm{~N}$ & 30 \\
\hline 01396500 & $\begin{array}{l}\text { South Branch Raritan River near } \\
\text { High Bridge, NJ }\end{array}$ & 65.3 & $\begin{array}{l}1896,1902, \\
1904, \\
1919-24, \\
1926-98\end{array}$ & $\begin{array}{l}1979 \\
1994\end{array}$ & $\begin{array}{l}12.07 \\
14.26\end{array}$ & $\begin{array}{l}6,910 \\
2,000\end{array}$ & $10 / 20 / 96$ & 11.13 & 4,730 & $\mathrm{~N}$ & 30 \\
\hline 01398000 & Neshanic River at Reaville, NJ & 25.7 & 1931-98 & 1971 & 13.84 & 15,900 & $10 / 19 / 96$ & 12.63 & 11,100 & $\mathrm{~N}$ & 25 \\
\hline 01398500 & $\begin{array}{l}\text { North Branch Raritan River near } \\
\text { Far Hills, NJ }\end{array}$ & 26.2 & $\begin{array}{l}1919 \\
1922-98\end{array}$ & $\begin{array}{l}1971 \\
1919\end{array}$ & $\begin{array}{l}7.28 \\
7.60\end{array}$ & $\begin{array}{l}6,390 \\
7,000\end{array}$ & $10 / 19 / 96$ & 6.65 & 5,090 & $\mathrm{~N}$ & 35 \\
\hline 01400000 & $\begin{array}{l}\text { North Branch Raritan River near } \\
\text { Raritan, NJ }\end{array}$ & 190 & $\begin{array}{l}\text { 1896, } \\
1924-98\end{array}$ & 1971 & 15.47 & 28,600 & $10 / 19 / 96$ & 15.44 & 28,500 & $\mathrm{~N}$ & 100 \\
\hline 01400900 & Stony Brook at Glenmoore, NJ & 17.0 & $1957-95$ & 1971 & 11.02 & 6,100 & $1 / 28 / 94$ & 9.94 & 4,950 & $\mathrm{~N}$ & 35 \\
\hline 01401595 & Rock Brook near Blawenburg, NJ & 9.03 & $\begin{array}{l}1967-76 \\
1978-98\end{array}$ & 1971 & 10.00 & 4,530 & $10 / 19 / 96$ & 8.19 & 3,130 & $\mathrm{~N}$ & 20 \\
\hline 01403060 & $\begin{array}{l}\text { Raritan River below Callo Dam at } \\
\text { Bound Brook, NJ }\end{array}$ & 785 & $\begin{array}{l}1896, \\
1904-09, \\
1936-39, \\
1942, \\
1945-98\end{array}$ & 1971 & 37.47 & 46,100 & $10 / 20 / 96$ & 35.58 & 40,100 & $\mathrm{~N}$ & 25 \\
\hline
\end{tabular}


Table 31. Maximum stage and discharge for period of record for streamgages having significant floods during 1994-98 water years in New Jersey.-Continued

$\left[\mathrm{mi}^{2}\right.$, square miles; ft, feet above an arbitrary datum; $\mathrm{ft}^{3} / \mathrm{s}$, cubic feet per second; --, not determined or not applicable; >, greater than. Source: Recurrence intervals calculated from U.S. Geological Survey data. Other data from U.S. Geological Survey reports or databases]

\begin{tabular}{|c|c|c|c|c|c|c|c|c|c|c|c|}
\hline \multirow{2}{*}{$\begin{array}{c}\text { Streamgage } \\
\text { number } \\
\text { (fig. 42) }\end{array}$} & \multirow[b]{2}{*}{ Streamgage name } & \multirow{2}{*}{$\begin{array}{c}\text { Total } \\
\text { drainage } \\
\left(\mathrm{mi}^{2}\right)\end{array}$} & \multicolumn{4}{|c|}{$\begin{array}{c}\text { Maximum stage and discharge for period of record } \\
\text { through } 1998 \text { water year }\end{array}$} & \multicolumn{5}{|c|}{ Significant floods 1994-98 water years } \\
\hline & & & $\begin{array}{l}\text { Period of } \\
\text { record } \\
\text { (water } \\
\text { years) }\end{array}$ & Water year & $\begin{array}{l}\text { Stage } \\
(\mathrm{ft})\end{array}$ & $\begin{array}{l}\text { Discharge } \\
\left(\mathrm{ft}^{3} / \mathrm{s}\right)\end{array}$ & $\begin{array}{c}\text { Date } \\
\text { (month/ } \\
\text { day/ } \\
\text { year) }\end{array}$ & $\begin{array}{l}\text { Stage } \\
(\mathrm{ft})\end{array}$ & $\begin{array}{l}\text { Discharge } \\
\left(\mathrm{ft}^{3} / \mathrm{s}\right)\end{array}$ & $\begin{array}{l}\text { Regulated } \\
\text { during } \\
\text { flood }^{1}\end{array}$ & $\begin{array}{c}\text { Recurrence } \\
\text { interval } \\
\text { (years) }\end{array}$ \\
\hline 01403150 & $\begin{array}{l}\text { West Branch Middle Brook near } \\
\text { Martinsville, NJ }\end{array}$ & 1.99 & $1980-98$ & 1997 & 6.89 & 700 & $10 / 19 / 96$ & 6.89 & 700 & $\mathrm{~N}$ & 15 \\
\hline 01403500 & Green Brook at Plainfield, NJ & 9.75 & $\begin{array}{l}\text { 1916, 1927, } \\
1936, \\
1938-98\end{array}$ & $\begin{array}{l}1938 \\
1970\end{array}$ & $\begin{array}{l}5.82 \\
6.10\end{array}$ & $\begin{array}{l}2,890 \\
2,050\end{array}$ & $10 / 19 / 96$ & 5.82 & 2,120 & $\mathrm{~N}$ & 30 \\
\hline 01403570 & $\begin{array}{l}\text { Stony Brook at North Plainfield, } \\
\text { NJ }\end{array}$ & 6.88 & $\begin{array}{l}\text { 1938, } \\
\text { 1975-81, } \\
1991-98\end{array}$ & $\begin{array}{l}1997 \\
1938\end{array}$ & $\begin{array}{r}7.35 \\
10.00\end{array}$ & $\begin{array}{r}3,130 \\
--\end{array}$ & $10 / 19 / 96$ & 7.35 & 3,130 & $\mathrm{~N}$ & 30 \\
\hline 01410150 & $\begin{array}{l}\text { East Branch Bass River near } \\
\text { New Gretna, NJ }\end{array}$ & 8.11 & 1978-98 & 1997 & 7.28 & 750 & $8 / 22 / 97$ & 7.28 & 750 & $\mathrm{~N}$ & 100 \\
\hline 01411300 & $\begin{array}{l}\text { Tuckahoe River at Head of River, } \\
\text { NJ }\end{array}$ & 30.8 & $\begin{array}{l}1971-96, \\
1998\end{array}$ & 1997 & 9.09 & 1,340 & $\begin{array}{l}8 / 21 / 97 \\
2 / 5 / 98\end{array}$ & $\begin{array}{l}9.09 \\
6.72\end{array}$ & $\begin{array}{r}1,340 \\
638\end{array}$ & $\begin{array}{l}\mathrm{N} \\
\mathrm{N}\end{array}$ & $\begin{array}{r}>100 \\
40\end{array}$ \\
\hline 01438500 & Delaware River at Montague, $\mathrm{NJ}$ & 3,480 & $\begin{array}{l}1904, \\
1936-98\end{array}$ & $\begin{array}{l}1955 \\
1904\end{array}$ & $\begin{array}{l}35.15 \\
35.50\end{array}$ & $\begin{array}{r}250,000 \\
--\end{array}$ & $1 / 20 / 96$ & 26.66 & 149,000 & $\mathrm{~N}$ & 30 \\
\hline 01440200 & $\begin{array}{l}\text { Delaware River near Delaware } \\
\text { Water Gap, NJ }\end{array}$ & 3,850 & $\begin{array}{l}1955 \\
1965-96\end{array}$ & 1955 & 37.40 & 260,000 & $1 / 20 / 96$ & 24.89 & 155,000 & $\mathrm{~N}$ & 40 \\
\hline 01446500 & Delaware River at Belvidere, $\mathrm{NJ}$ & 4,535 & $\begin{array}{l}\text { 1904, } \\
1923-98\end{array}$ & 1955 & 30.21 & 273,000 & $1 / 20 / 96$ & 22.96 & 158,000 & $\mathrm{~N}$ & 40 \\
\hline 01457500 & Delaware River at Riegelsville, NJ & 6,328 & $\begin{array}{l}1841,1904, \\
1907-98\end{array}$ & 1955 & 38.85 & 340,000 & $1 / 20 / 96$ & 28.72 & 187,000 & $\mathrm{~N}$ & 40 \\
\hline 01463500 & Delaware River at Trenton, NJ & 6,780 & $\begin{array}{l}1902 \\
1904-98\end{array}$ & 1955 & 28.60 & 329,000 & $1 / 20 / 96$ & 22.20 & 179,000 & $\mathrm{~N}$ & 25 \\
\hline 01467081 & $\begin{array}{l}\text { South Branch Pennsauken Creek at } \\
\text { Cherry Hill, NJ }\end{array}$ & 8.98 & $\begin{array}{l}1968-76 \\
1978-98\end{array}$ & 1994 & 11.63 & 1,500 & $7 / 14 / 94$ & 11.63 & 1,500 & $\mathrm{~N}$ & 100 \\
\hline 01467305 & Newton Creek at Collingswood, NJ & 1.33 & $\begin{array}{l}1964-75 \\
1977-98\end{array}$ & 1994 & 6.82 & 328 & $7 / 14 / 94$ & 6.82 & 328 & $\mathrm{~N}$ & 70 \\
\hline
\end{tabular}

\footnotetext{
${ }^{1}$ Regulated during flood: $\mathrm{N}$, no; $\mathrm{Y}$, yes.
} 


\section{New Mexico}

Intense thunderstorms across New Mexico occurred intermittently during May, June, July, and August 1994, and caused six streamgages to experience their peak of record (table 32). On May 31, 1994, the Rio Grande River below Elephant Butte Dam (streamgage 08361000) had its greatest discharge since the flood of 1942 on May 31, 1994.

Intense thunderstorms produced excessive rainfall during June 1995, causing flooding in various parts of the State. Peaks of record were set at two streamgages in June (table 32).

An estimated rainfall of as much as 4.50 inches fell in a 3-hour period shortly after midnight on June 29, 1997 (National Oceanic and Atmospheric Administration, 1997a) near Truth or Consequences (fig. 43). Several roads were washed out or damaged, and several cars were washed into Elephant Butte Reservoir. Considerable damage also took place to utilities and a number of homes and businesses, mainly due to the rapid runoff rather than rising water levels in established drainages. Damage was near \$1 million (National Oceanic and Atmospheric Administration, 1997b).

\section{References}

National Oceanic and Atmospheric Administration (NOAA), 1997a, Climatological data (by State): Asheville, North Carolina, National Climatic Data Center, various months. National Oceanic and Atmospheric Administration (NOAA), 1997b, Storm data (by State): Asheville, North Carolina, National Climatic Data Center, various months.

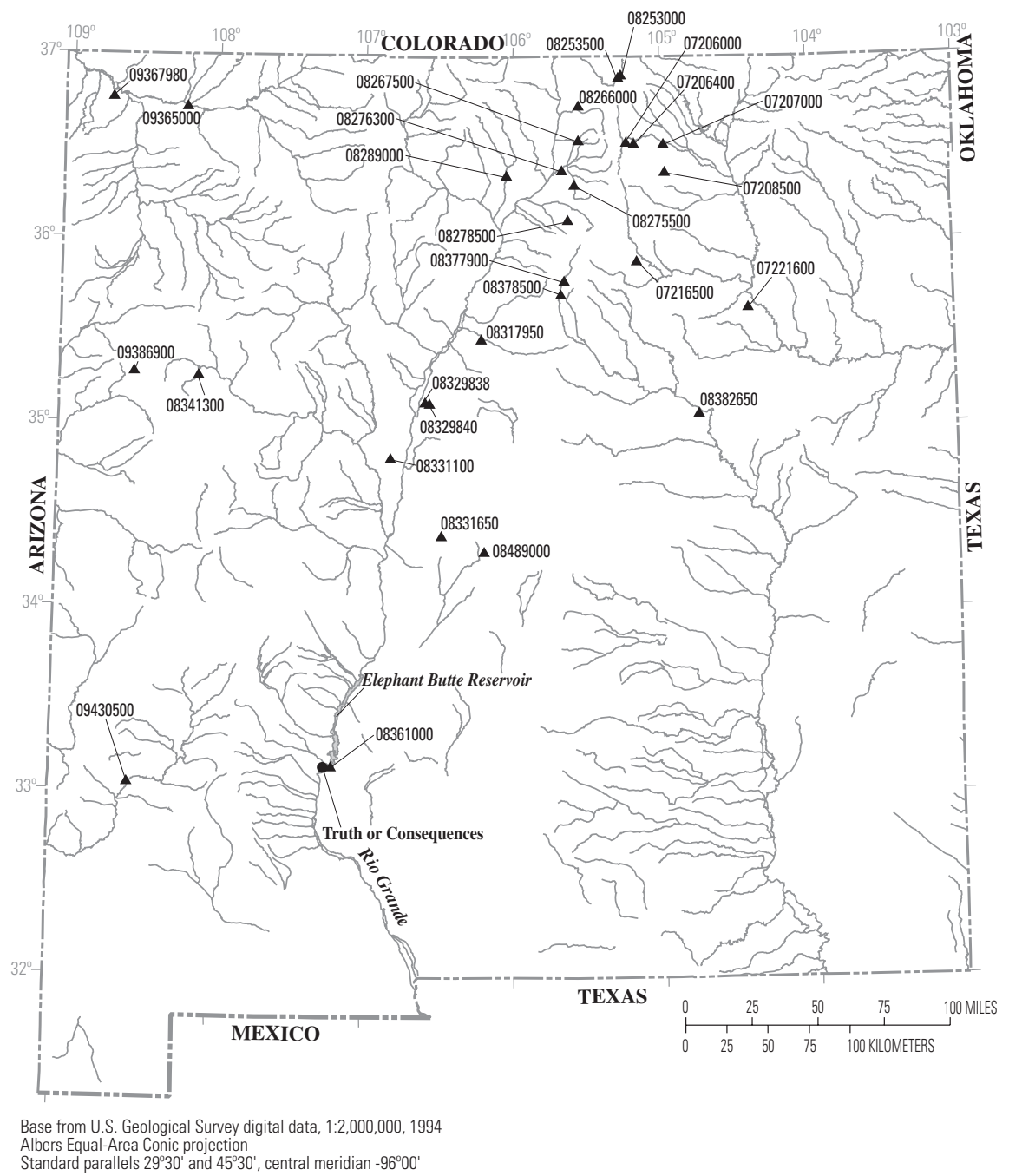

EXPLANATION

$08361000 \triangle$ Streamgage and number

Figure 43. Location of streamgages with significant floods during 1994-98 water years for New Mexico. 
Table 32. Maximum stage and discharge for period of record for streamgages having significant floods during $1994-98$ water years in New Mexico.

$\left[\mathrm{mi}^{2}\right.$, square miles; $\mathrm{ft}$, feet above an arbitrary datum; $\mathrm{ft}^{3} / \mathrm{s}$, cubic feet per second; --, not determined or not applicable. Source: Recurrence intervals calculated from U.S. Geological Survey data. Other data from U.S. Geological Survey reports or databases]

\begin{tabular}{|c|c|c|c|c|c|c|c|c|c|c|c|}
\hline \multirow{2}{*}{$\begin{array}{l}\text { Streamgage } \\
\text { number } \\
\text { (fig. 43) }\end{array}$} & \multirow[b]{2}{*}{ Streamgage name } & \multirow{2}{*}{$\begin{array}{c}\text { Total } \\
\text { drainage } \\
\left(\mathrm{mi}^{2}\right)\end{array}$} & \multicolumn{4}{|c|}{$\begin{array}{c}\text { Maximum stage and discharge for period of record } \\
\text { through } 1998 \text { water year }\end{array}$} & \multicolumn{5}{|c|}{ Significant floods $1994-98$ water years } \\
\hline & & & $\begin{array}{l}\text { Period of } \\
\text { record } \\
\text { (water } \\
\text { years) }\end{array}$ & Water year & $\begin{array}{l}\text { Stage } \\
(\mathrm{ft})\end{array}$ & $\begin{array}{l}\text { Discharge } \\
\left(\mathrm{ft}^{3} / \mathrm{s}\right)\end{array}$ & $\begin{array}{c}\text { Date } \\
\text { (month/ } \\
\text { day/ } \\
\text { year) }\end{array}$ & $\begin{array}{l}\text { Stage } \\
(\mathrm{ft})\end{array}$ & $\begin{array}{l}\text { Discharge } \\
\left(\mathrm{ft}^{3} / \mathrm{s}\right)\end{array}$ & $\begin{array}{c}\text { Regulated } \\
\text { during } \\
\text { flood }^{1}\end{array}$ & $\begin{array}{c}\text { Recurrence } \\
\text { interval } \\
\text { (years) }\end{array}$ \\
\hline 07206000 & $\begin{array}{l}\text { Cimarron River below Eagle Nest } \\
\text { Dam, NM }\end{array}$ & 167 & $1950-98$ & $\begin{array}{l}1994 \\
1955\end{array}$ & $\begin{array}{l}- \\
2.79\end{array}$ & $\begin{array}{l}303 \\
205\end{array}$ & $5 / 23 / 94$ & 3.63 & 303 & $\mathrm{Y}$ & -- \\
\hline 07206400 & Clear Creek near Ute Park, NM & 7.44 & $\begin{array}{l}1962-77, \\
1979-84, \\
1986-96\end{array}$ & 1965 & 3.05 & 151 & $7 / 24 / 94$ & 3.00 & 150 & $\mathrm{~N}$ & 35 \\
\hline 07207000 & $\begin{array}{l}\text { Cimarron River near Cimarron, } \\
\text { NM }\end{array}$ & 294 & 1950-97 & 1965 & 12.42 & 15,500 & $6 / 3 / 95$ & 4.32 & 892 & $\mathrm{Y}$ & -- \\
\hline 07208500 & $\begin{array}{l}\text { Rayado Creek at Sauble Ranch } \\
\text { near Cimarron, NM }\end{array}$ & 65.0 & $\begin{array}{l}1914, \\
1917-20, \\
1924, \\
1928-32, \\
1934-96\end{array}$ & 1965 & 11.50 & 9,000 & $6 / 18 / 95$ & 4.86 & 666 & $\mathrm{~N}$ & 10 \\
\hline 07216500 & Mora River near Golondrinas, NM & 267 & $\begin{array}{l}1916-20 \\
1929-86, \\
1988-98\end{array}$ & $\begin{array}{l}1952 \\
1969\end{array}$ & $\overline{9}-\overline{3}$ & $\begin{array}{r}14,000 \\
3,180\end{array}$ & $5 / 19 / 94$ & 5.82 & 6,180 & $\mathrm{~N}$ & 60 \\
\hline 07221600 & $\begin{array}{l}\text { Lagarita Creek tributary near } \\
\text { Sanchez, NM }\end{array}$ & 1.19 & $\begin{array}{l}\text { 1972, 1982, } \\
1989-96\end{array}$ & 1994 & 5.83 & 1,500 & $5 / 11 / 94$ & 5.83 & 1,500 & $\mathrm{~N}$ & 10 \\
\hline 08253000 & Casias Creek near Costilla, NM & 16.6 & 1937-97 & $\begin{array}{l}1994 \\
1971\end{array}$ & $\begin{array}{l}2.06 \\
2.07\end{array}$ & $\begin{array}{l}196 \\
181\end{array}$ & $5 / 25 / 94$ & 2.06 & 196 & $\mathrm{~N}$ & 50 \\
\hline 08253500 & $\begin{array}{l}\text { Santistevan Creek near Costilla, } \\
\text { NM }\end{array}$ & 2.15 & 1938-98 & $\begin{array}{l}1995 \\
1941\end{array}$ & $\begin{array}{l}1.57 \\
1.73\end{array}$ & $\begin{array}{l}20 \\
18\end{array}$ & $6 / 29 / 95$ & 1.57 & 20 & $\mathrm{~N}$ & 25 \\
\hline 08266000 & Cabresto Creek near Questa, NM & 36.7 & $1944-96$ & 1994 & 5.41 & 289 & $5 / 21 / 94$ & 5.41 & 289 & $\mathrm{Y}$ & -- \\
\hline 08267500 & Rio Hondo near Valdez, NM & 36.2 & 1935-98 & $\begin{array}{l}1941 \\
1979\end{array}$ & $\begin{array}{l}2.73 \\
4.53\end{array}$ & $\begin{array}{l}541 \\
402\end{array}$ & $6 / 20 / 95$ & 3.98 & 460 & $\mathrm{~N}$ & 20 \\
\hline
\end{tabular}


Table 32. Maximum stage and discharge for period of record for streamgages having significant floods during 1994-98 water years in New Mexico.-Continued

$\left[\mathrm{mi}^{2}\right.$, square miles; $\mathrm{ft}$, feet above an arbitrary datum; $\mathrm{ft}^{3} / \mathrm{s}$, cubic feet per second; --, not determined or not applicable. Source: Recurrence intervals calculated from U.S. Geological Survey data. Other data from U.S. Geological Survey reports or databases]

\begin{tabular}{|c|c|c|c|c|c|c|c|c|c|c|c|}
\hline \multirow{2}{*}{$\begin{array}{c}\text { Streamgage } \\
\text { number } \\
\text { (fig. 43) }\end{array}$} & \multirow[b]{2}{*}{ Streamgage name } & \multirow{2}{*}{$\begin{array}{c}\text { Total } \\
\text { drainage } \\
\left(\mathrm{mi}^{2}\right)\end{array}$} & \multicolumn{4}{|c|}{$\begin{array}{c}\text { Maximum stage and discharge for period of record } \\
\text { through } 1998 \text { water year }\end{array}$} & \multicolumn{5}{|c|}{ Significant floods 1994-98 water years } \\
\hline & & & $\begin{array}{l}\text { Period of } \\
\text { record } \\
\text { (water } \\
\text { years) }\end{array}$ & Water year & $\begin{array}{l}\text { Stage } \\
\text { (ft) }\end{array}$ & $\begin{array}{l}\text { Discharge } \\
\left(\mathrm{ft}^{3} / \mathrm{s}\right)\end{array}$ & $\begin{array}{c}\text { Date } \\
\text { (month/ } \\
\text { day/ } \\
\text { year) }\end{array}$ & $\begin{array}{l}\text { Stage } \\
(\mathrm{ft})\end{array}$ & $\begin{array}{l}\text { Discharge } \\
\left(\mathrm{ft}^{3} / \mathrm{s}\right)\end{array}$ & $\begin{array}{l}\text { Regulated } \\
\text { during } \\
\text { flood }^{1}\end{array}$ & $\begin{array}{c}\text { Recurrence } \\
\text { interval } \\
\text { (years) }\end{array}$ \\
\hline 08275500 & $\begin{array}{l}\text { Rio Grande del Rancho near Talpa, } \\
\text { NM }\end{array}$ & 83.0 & 1953-98 & 1991 & 4.16 & 644 & $5 / 20 / 94$ & 3.50 & 529 & $\mathrm{~N}$ & 25 \\
\hline 08276300 & $\begin{array}{l}\text { Rio Pueblo de Taos below Los } \\
\text { Cordovas, NM }\end{array}$ & 380 & 1957-98 & $\begin{array}{l}1994 \\
1991\end{array}$ & $\begin{array}{l}8.88 \\
8.93\end{array}$ & $\begin{array}{l}2,260 \\
1,660\end{array}$ & $5 / 20 / 94$ & 8.88 & 2,260 & $\mathrm{Y}$ & 25 \\
\hline 08278500 & $\begin{array}{l}\text { Rio Santa Barbara near Penasco, } \\
\text { NM }\end{array}$ & 38.0 & $\begin{array}{l}\text { 1954-57, } \\
1992-98\end{array}$ & 1995 & 6.21 & 838 & $6 / 18 / 95$ & 6.21 & 838 & $\mathrm{~N}$ & -- \\
\hline 08289000 & $\begin{array}{l}\text { Rio Ojo Caliente at La Madera, } \\
\text { NM }\end{array}$ & 419 & $1932-98$ & $\begin{array}{l}1998 \\
1994\end{array}$ & $\begin{array}{l}6.00 \\
8.27\end{array}$ & $\begin{array}{l}3,990 \\
3,640\end{array}$ & $\begin{array}{l}8 / 14 / 94 \\
7 / 8 / 98\end{array}$ & $\begin{array}{l}8.27 \\
6.00\end{array}$ & $\begin{array}{l}3,640 \\
3,990\end{array}$ & $\begin{array}{l}\mathrm{N} \\
\mathrm{N}\end{array}$ & $\begin{array}{l}35 \\
70\end{array}$ \\
\hline 08317950 & $\begin{array}{l}\text { Galisteo Creek below Galisteo } \\
\text { Dam, NM }\end{array}$ & 597 & 1971-97 & $\begin{array}{l}1997 \\
1981\end{array}$ & $-\overline{-}$ & $\begin{array}{l}3,460 \\
1,590\end{array}$ & $8 / 24 / 97$ & -- & 3,460 & $\mathrm{Y}$ & -- \\
\hline 08329838 & $\begin{array}{l}\text { South Fork Hahn Arroyo in } \\
\text { Albuquerque, NM }\end{array}$ & 2.03 & $\begin{array}{l}1979-83 \\
1992-98\end{array}$ & 1994 & 4.42 & 574 & $5 / 11 / 94$ & 4.42 & 574 & $\mathrm{~N}$ & -- \\
\hline 08329840 & Hahn Arroyo in Albuquerque, NM & 4.23 & 1979-98 & 1998 & 2.93 & 1,150 & $12 / 27 / 97$ & 2.93 & 1,150 & $\mathrm{~N}$ & -- \\
\hline 08331100 & $\begin{array}{l}\text { Belen Highline Canal tributary } \\
\text { near Los Lunas, NM }\end{array}$ & .16 & $1955-95$ & $\begin{array}{l}1965 \\
1994\end{array}$ & $\overline{-}-\overline{0}-3.30$ & $\begin{array}{l}754 \\
480\end{array}$ & $8 / 15 / 94$ & 7.30 & 480 & $\mathrm{~N}$ & 25 \\
\hline 08331650 & Canada Montoso near Scholle, NM & 35.0 & $\begin{array}{l}1961-80, \\
1982-95, \\
1997\end{array}$ & 1997 & 7.47 & 5,600 & $7 / 31 / 97$ & 7.47 & 5,600 & $\mathrm{~N}$ & 40 \\
\hline 08341300 & $\begin{array}{l}\text { Bluewater Creek above Bluewater } \\
\text { Dam, Bluewater, NM }\end{array}$ & 75.0 & $\begin{array}{l}\text { 1953-71, } \\
\text { 1973-77, } \\
\text { 1989-96, } \\
1998\end{array}$ & 1953 & 8.99 & 3,570 & $3 / 6 / 95$ & 4.45 & 1,940 & $\mathrm{~N}$ & 20 \\
\hline 08361000 & $\begin{array}{l}\text { Rio Grande below Elephant Butte } \\
\text { Dam, NM }\end{array}$ & 29,450 & $1915-98$ & $\begin{array}{l}1942 \\
1998\end{array}$ & $\overline{--}$ & $\begin{array}{l}8,220 \\
2,810\end{array}$ & $5 / 31 / 94$ & -- & 5,270 & $\mathrm{Y}$ & -- \\
\hline
\end{tabular}


Table 32. Maximum stage and discharge for period of record for streamgages having significant floods during 1994-98 water years in New Mexico.-Continued

$\left[\mathrm{mi}^{2}\right.$, square miles; $\mathrm{ft}$, feet above an arbitrary datum; $\mathrm{ft}^{3} / \mathrm{s}$, cubic feet per second; --, not determined or not applicable. Source: Recurrence intervals calculated from U.S. Geological Survey data. Other data from U.S. Geological Survey reports or databases]

\begin{tabular}{|c|c|c|c|c|c|c|c|c|c|c|c|}
\hline \multirow[b]{2}{*}{$\begin{array}{l}\text { Streamgage } \\
\text { number } \\
\text { (fig. 43) }\end{array}$} & \multirow[b]{2}{*}{ Streamgage name } & \multirow[b]{2}{*}{$\begin{array}{c}\text { Total } \\
\text { drainage } \\
\left(\mathrm{mi}^{2}\right)\end{array}$} & \multicolumn{4}{|c|}{$\begin{array}{c}\text { Maximum stage and discharge for period of record } \\
\text { through } 1998 \text { water year }\end{array}$} & \multicolumn{5}{|c|}{ Significant floods 1994-98 water years } \\
\hline & & & $\begin{array}{l}\text { Period of } \\
\text { record } \\
\text { (water } \\
\text { years) }\end{array}$ & Water year & $\begin{array}{l}\text { Stage } \\
\text { (ft) }\end{array}$ & $\begin{array}{c}\text { Discharge } \\
\left(\mathrm{ft}^{3} / \mathrm{s}\right)\end{array}$ & $\begin{array}{c}\text { Date } \\
\text { (month/ } \\
\text { day/ } \\
\text { year) }\end{array}$ & $\begin{array}{l}\text { Stage } \\
(\mathrm{ft})\end{array}$ & $\begin{array}{l}\text { Discharge } \\
\left(\mathrm{ft}^{3} / \mathrm{s}\right)\end{array}$ & $\begin{array}{c}\text { Regulated } \\
\text { during } \\
\text { flood }^{1}\end{array}$ & $\begin{array}{c}\text { Recurrence } \\
\text { interval } \\
\text { (years) }\end{array}$ \\
\hline 08377900 & Rio Mora near Tererro, NM & 53.2 & 1964-98 & $\begin{array}{l}1991 \\
1979\end{array}$ & $\begin{array}{l}4.08 \\
4.15\end{array}$ & $\begin{array}{l}937 \\
820\end{array}$ & $6 / 8 / 97$ & 3.99 & 864 & $\mathrm{~N}$ & 25 \\
\hline 08378500 & Pecos River near Pecos, NM & 189 & $\begin{array}{l}1920-22, \\
1924,1926, \\
1928-98\end{array}$ & 1929 & 6.20 & 4,500 & $6 / 7 / 97$ & 4.79 & 2,060 & $\mathrm{~N}$ & 20 \\
\hline 08382650 & $\begin{array}{l}\text { Pecos River above Santa Rosa } \\
\text { Lake, NM }\end{array}$ & 2,340 & 1976-98 & 1996 & 19.06 & 16,000 & $7 / 11 / 96$ & 19.06 & 16,000 & $\mathrm{~N}$ & 20 \\
\hline 08489000 & Big Draw near Mountainair, NM & 4.06 & $\begin{array}{l}1953-71, \\
1973-74, \\
1978-83, \\
1985-96\end{array}$ & 1954 & 8.68 & 1,710 & $6 / 26 / 96$ & 6.64 & 1,040 & $\mathrm{~N}$ & 20 \\
\hline 09365000 & San Juan River at Farmington, NM & 7,240 & 1924-97 & $\begin{array}{l}1927 \\
1935\end{array}$ & $-\overline{-}-00$ & $\begin{array}{l}68,000 \\
32,800\end{array}$ & $6 / 2 / 97$ & 7.77 & 13,000 & $\mathrm{Y}$ & 4 \\
\hline 09367980 & $\begin{array}{l}\text { Rattlesnake Arroyo near Shiprock, } \\
\text { NM }\end{array}$ & -- & $1980-96$ & 1995 & 6.25 & 3,800 & 9/7/95 & 6.25 & 3,800 & $\mathrm{~N}$ & 20 \\
\hline 09386900 & Rio Nutria near Ramah, NM & 71.4 & 1970-98 & 1995 & 9.34 & 1,850 & $3 / 5 / 95$ & 9.34 & 1,850 & $\mathrm{~N}$ & 30 \\
\hline 09430500 & Gila River near Gila, NM & 1,864 & $1928-98$ & $\begin{array}{l}1985 \\
1941\end{array}$ & $\begin{array}{l}13.00 \\
17.19\end{array}$ & $\begin{array}{l}35,200 \\
25,400\end{array}$ & $9 / 22 / 97$ & 11.18 & 18,200 & $\mathrm{~N}$ & 20 \\
\hline
\end{tabular}

${ }^{1}$ Regulated during flood: N, no; Y, yes. 


\section{New York}

The most disastrous flood in more than 20 years struck central New York from January 19-20, 1996. Significant widespread flooding of streams, rivers, homes and businesses, streets and highways, woodlands, and farmland occurred as a result of rapid snowmelt and nearly simultaneous storm runoff. An area of intense low pressure, which was located over the Mid-Atlantic region on Friday morning January 19, produced unseasonably warm temperatures, high dew points, and strong winds. This resulted in rapid melting of 1 to 3 feet of snow. In addition to the rapid snowmelt, 1 to 3 inches of rain fell as the system moved northeast along the coast (National Oceanic and Atmospheric Administration, 1996a). There were 10 known fatalities. Total damage statewide was more than $\$ 200$ million (National Oceanic and Atmospheric Administration 1996b). Flash floods that began early on January 19 gave rise to mainstem river floods that persisted until the evening of January 21. Near-record river flooding occurred January 19-20 in the Chemung, upper Susquehanna, Hudson, and upper Delaware River Basins (fig. 44). At Waverly, the Susquehanna River crested at 20.35 feet, its third highest level of all time, which was about 1 foot lower than the all time crest of 21.40 feet recorded on March 19, 1946 (National Oceanic and Atmospheric Administration, 1996b). Flooding was so widespread and severe that this event became known as the "Deluge of '96." Thirty-one streamgages experienced their peak of record in New York during this flood (table 33).

On Friday October 18, 1996, a strong low-pressure system developed along a cold front in New Jersey. With a highpressure system in place across northern New England, the low slowly intensified and moved slowly off the southern New
Jersey Coast during October 19. The increasing difference in pressures caused strong and gusty east winds, which also transported abundant moisture from the Atlantic Ocean across the region. Excessive flood-producing rains along with minor-tomajor coastal flooding occurred. More than $\$ 17$ million in damage resulted from coastal and river flooding (National Oceanic and Atmospheric Administration, 1996b).

During November 8-9, 1996, a slow-moving, lowpressure system tracked from northern Pennsylvania to northern New York. This system produced 4 to 5 inches of rain across most of central New York and the northern Catskill Mountains (National Oceanic and Atmospheric Administration, 1996a). The resulting runoff caused flooding along the Mohawk River to the Hudson River. Nearly \$25 million in damage resulted (National Oceanic and Atmospheric Administration, 1996b).

An area of weak low pressure and a moist, unstable air mass over New England and New York resulted in evening and late-night thunderstorms with locally torrential downpours in extreme northern New York during June 25-27, 1998. Approximately \$20 million in damage resulted (National Oceanic and Atmospheric Administration, 1998b).

\section{References}

National Oceanic and Atmospheric Administration (NOAA), 1996a-98a, Climatological data (by State): Asheville, North Carolina, National Climatic Data Center, various months.

National Oceanic and Atmospheric Administration (NOAA), 1996b-98b, Storm data (by State): Asheville, North Carolina, National Climatic Data Center, various months. 

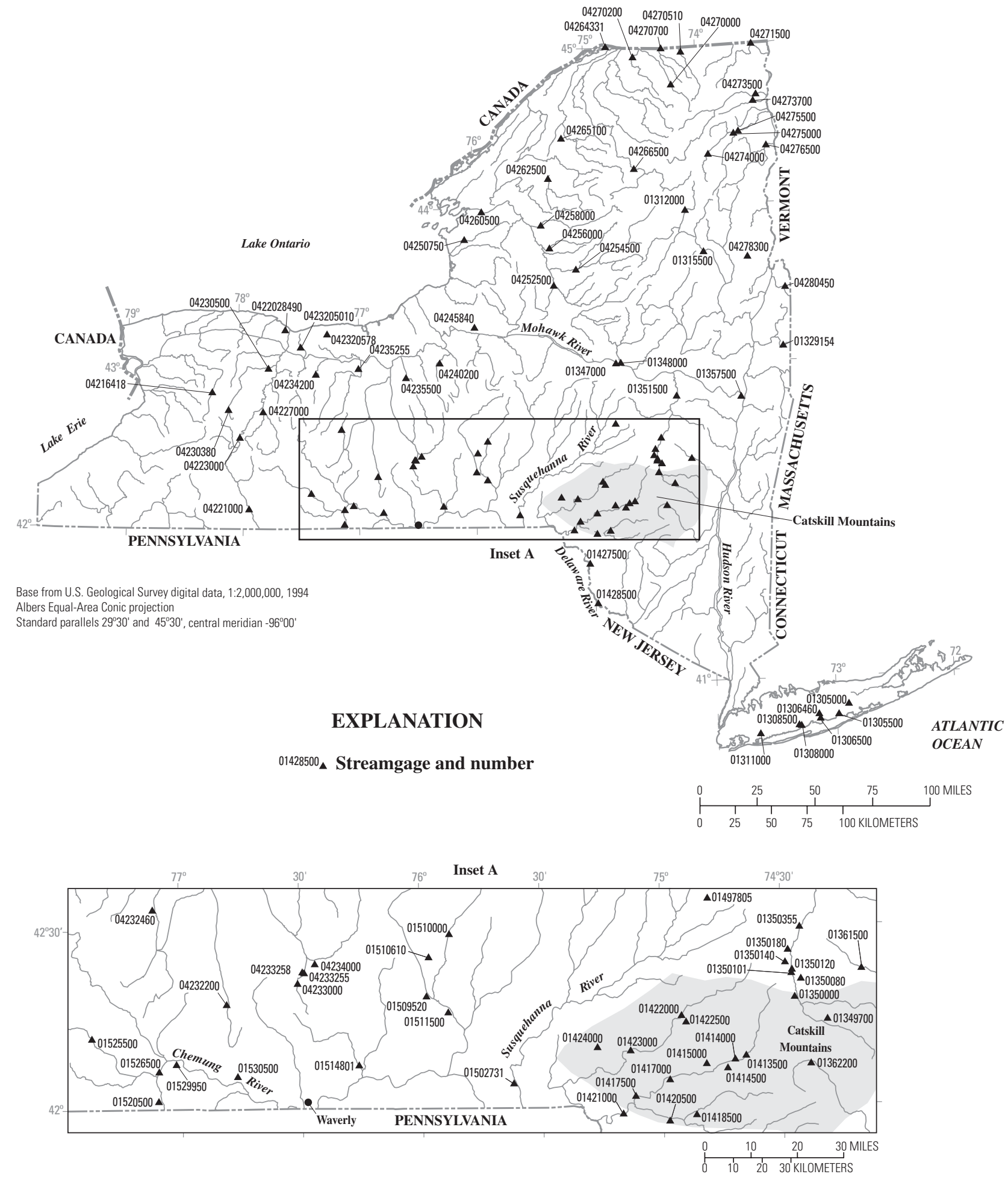

Figure 44. Location of streamgages with significant floods during 1994-98 water years for New York. 
Table 33. Maximum stage and discharge for period of record for streamgages having significant floods during 1994-98 water years in New York.

$\left[\mathrm{mi}^{2}\right.$, square miles; $\mathrm{ft}$, feet above an arbitrary datum; $\mathrm{ft}^{3} / \mathrm{s}$, cubic feet per second; --, not determined or not applicable; >, greater than. Source: Recurrence intervals calculated from U.S. Geological Survey data. Other data from U.S. Geological Survey reports or databases]

\begin{tabular}{|c|c|c|c|c|c|c|c|c|c|c|c|}
\hline \multirow{2}{*}{$\begin{array}{c}\text { Streamgage } \\
\text { number } \\
\text { (fig. 44) }\end{array}$} & \multirow[b]{2}{*}{ Streamgage name } & \multirow{2}{*}{$\begin{array}{c}\text { Total } \\
\text { drainage } \\
\left(\mathrm{mi}^{2}\right)\end{array}$} & \multicolumn{4}{|c|}{$\begin{array}{c}\text { Maximum stage and discharge for period of record } \\
\text { through } 1998 \text { water year }\end{array}$} & \multicolumn{5}{|c|}{ Significant floods 1994-98 water years } \\
\hline & & & $\begin{array}{l}\text { Period of } \\
\text { record } \\
\text { (water } \\
\text { years) }\end{array}$ & Water year & $\begin{array}{l}\text { Stage } \\
(\mathrm{ft})\end{array}$ & $\begin{array}{l}\text { Discharge } \\
\left(\mathrm{ft}^{3} / \mathrm{s}\right)\end{array}$ & $\begin{array}{c}\text { Date } \\
\text { (month/ } \\
\text { day/ } \\
\text { year) }\end{array}$ & $\begin{array}{l}\text { Stage } \\
(\mathrm{ft})\end{array}$ & $\begin{array}{l}\text { Discharge } \\
\left(\mathrm{ft}^{3} / \mathrm{s}\right)\end{array}$ & $\begin{array}{c}\text { Regulated } \\
\text { during } \\
\text { flood }^{1}\end{array}$ & $\begin{array}{c}\text { Recurrence } \\
\text { interval } \\
\text { (years) }\end{array}$ \\
\hline 01305000 & Carmans River at Yaphank, NY & 71.0 & $1943-98$ & 1989 & 2.09 & 143 & $6 / 13 / 98$ & 1.99 & 122 & $\mathrm{~N}$ & 25 \\
\hline 01305500 & Swan River at East Patchogue, NY & 8.60 & 1947-98 & 1990 & 2.71 & 77 & $6 / 13 / 98$ & 2.39 & 69 & $\mathrm{~N}$ & $75-100$ \\
\hline 01306460 & $\begin{array}{l}\text { Connetquot Brook near Central } \\
\text { Islip, NY }\end{array}$ & 18.0 & 1978-98 & 1998 & 3.89 & 155 & $6 / 13 / 98$ & 3.89 & 155 & $\mathrm{~N}$ & $10-25$ \\
\hline 01306500 & $\begin{array}{l}\text { Connetquot River near Oakdale, } \\
\text { NY }\end{array}$ & 24.0 & $\begin{array}{l}\text { 1944-93, } \\
1997-98\end{array}$ & 1956 & -- & 263 & $6 / 13 / 98$ & -- & 174 & $\mathrm{~N}$ & -- \\
\hline 01308000 & Sampawams Creek at Babylon, NY & 22.7 & $1945-98$ & 1998 & 3.73 & 254 & $6 / 13 / 98$ & 3.73 & 254 & $\mathrm{~N}$ & $>100$ \\
\hline 01308500 & Carlls River at Babylon, NY & 35.4 & 1945-98 & 1998 & 2.46 & 336 & $6 / 13 / 98$ & 2.46 & 336 & $\mathrm{~N}$ & 100 \\
\hline 01311000 & Pines Brook at Malverne, NY & 10.0 & 1939-98 & 1994 & 5.28 & 866 & $\begin{array}{l}1 / 28 / 94 \\
10 / 19 / 96\end{array}$ & $\begin{array}{l}5.28 \\
4.81\end{array}$ & $\begin{array}{l}866 \\
578\end{array}$ & $\begin{array}{l}\mathrm{N} \\
\mathrm{N}\end{array}$ & $\begin{array}{r}75-100 \\
25\end{array}$ \\
\hline 01312000 & Hudson River near Newcomb, NY & 192 & 1926-98 & 1998 & 12.84 & 11,500 & $\begin{array}{l}11 / 10 / 96 \\
1 / 9 / 98\end{array}$ & $\begin{array}{r}9.01 \\
12.84\end{array}$ & $\begin{array}{r}5,900 \\
11,500\end{array}$ & $\begin{array}{l}\mathrm{N} \\
\mathrm{N}\end{array}$ & $\begin{array}{r}10 \\
>100\end{array}$ \\
\hline 01315500 & Hudson River at North Creek, NY & 792 & 1908-98 & 1949 & 12.14 & 28,900 & $1 / 9 / 98$ & 11.54 & 26,300 & $\mathrm{Y}$ & 50 \\
\hline 01329154 & Steele Brook at Shushan, NY & 2.85 & 1979-98 & 1996 & 6.56 & 149 & $1 / 19 / 96$ & 6.56 & 149 & $\mathrm{~N}$ & $10-25$ \\
\hline 01347000 & $\begin{array}{l}\text { Mohawk River near Little Falls, } \\
\text { NY }\end{array}$ & 1,342 & $\begin{array}{l}\text { 1901, 1902, } \\
\text { 1904, 1913, } \\
1928-98\end{array}$ & 1913 & -- & 34,800 & $1 / 19 / 96$ & 18.47 & 30,700 & $\mathrm{Y}$ & $75-100$ \\
\hline 01348000 & $\begin{array}{l}\text { East Canada Creek at East Creek, } \\
\text { NY }\end{array}$ & 289 & $\begin{array}{l}1946-96, \\
1998\end{array}$ & 1946 & 9.00 & 24,000 & $\begin{array}{l}1 / 19 / 96 \\
1 / 09 / 98\end{array}$ & $\begin{array}{l}8.32 \\
8.46\end{array}$ & $\begin{array}{l}17,000 \\
17,800\end{array}$ & $\begin{array}{l}\mathrm{N} \\
\mathrm{N}\end{array}$ & $\begin{array}{r}50 \\
50-75\end{array}$ \\
\hline 01349700 & East Kill near Jewett Center, NY & 35.6 & $\begin{array}{l}\text { 1951, 1956, } \\
1960, \\
\text { 1967-68, } \\
\text { 1972-74, } \\
1987, \\
1996-98\end{array}$ & 1996 & 17.00 & 13,500 & $1 / 19 / 96$ & 17.00 & 13,500 & $\mathrm{~N}$ & 25 \\
\hline 01350000 & Schoharie Creek at Prattsville, NY & 237 & $\begin{array}{l}\text { 1904, } \\
\text { 1908-24, } \\
\text { 1926-28, } \\
1930-98\end{array}$ & 1996 & 19.39 & 52,800 & $1 / 19 / 96$ & 19.39 & 52,800 & $\mathrm{~N}$ & $25-50$ \\
\hline
\end{tabular}


Table 33. Maximum stage and discharge for period of record for streamgages having significant floods during 1994-98 water years in New York.—Continued

$\left[\mathrm{mi}^{2}\right.$, square miles; $\mathrm{ft}$, feet above an arbitrary datum; $\mathrm{ft}^{3} / \mathrm{s}$, cubic feet per second; --, not determined or not applicable; >, greater than. Source: Recurrence intervals calculated from U.S. Geological Survey data. Other data from U.S. Geological Survey reports or databases]

\begin{tabular}{|c|c|c|c|c|c|c|c|c|c|c|c|}
\hline \multirow{2}{*}{$\begin{array}{l}\text { Streamgage } \\
\text { number } \\
\text { (fig. 44) }\end{array}$} & \multirow[b]{2}{*}{ Streamgage name } & \multirow{2}{*}{$\begin{array}{c}\text { Total } \\
\text { drainage } \\
\left(\mathrm{mi}^{2}\right)\end{array}$} & \multicolumn{4}{|c|}{$\begin{array}{l}\text { Maximum stage and discharge for period of record } \\
\text { through } 1998 \text { water year }\end{array}$} & \multicolumn{5}{|c|}{ Significant floods $1994-98$ water years } \\
\hline & & & $\begin{array}{l}\text { Period of } \\
\text { record } \\
\text { (water } \\
\text { years) }\end{array}$ & Water year & $\begin{array}{l}\text { Stage } \\
(\mathrm{ft})\end{array}$ & $\begin{array}{l}\text { Discharge } \\
\left(\mathrm{ft}^{3} / \mathrm{s}\right)\end{array}$ & $\begin{array}{c}\text { Date } \\
\text { (month/ } \\
\text { day/ } \\
\text { year) }\end{array}$ & $\begin{array}{l}\text { Stage } \\
(\mathrm{ft})\end{array}$ & $\begin{array}{l}\text { Discharge } \\
\left(\mathrm{ft}^{3} / \mathrm{s}\right)\end{array}$ & $\begin{array}{l}\text { Regulated } \\
\text { during } \\
\text { flood }^{1}\end{array}$ & $\begin{array}{c}\text { Recurrence } \\
\text { interval } \\
\text { (years) }\end{array}$ \\
\hline 01350080 & $\begin{array}{l}\text { Manor Kill at West Conesville near } \\
\text { Gilboa, NY }\end{array}$ & 32.4 & $1987-98$ & 1996 & 10.20 & 5,050 & $1 / 19 / 96$ & 10.20 & 5,050 & $\mathrm{~N}$ & $10-25$ \\
\hline 01350101 & Schoharie Creek at Gilboa, NY & 316 & $\begin{array}{l}\text { 1936, 1938, } \\
1956, \\
1976-98\end{array}$ & 1996 & 30.60 & 70,800 & $1 / 19 / 96$ & 30.60 & 70,800 & $\mathrm{Y}$ & $75-100$ \\
\hline 01350120 & Platter Kill at Gilboa, NY & 10.9 & 1976-98 & 1996 & 6.70 & 1,370 & $1 / 19 / 96$ & 6.70 & 1,370 & $\mathrm{~N}$ & 25 \\
\hline 01350140 & $\begin{array}{l}\text { Mine Kill near North Blenheim, } \\
\text { NY }\end{array}$ & 16.2 & $1975-98$ & 1996 & 5.20 & 2,550 & $1 / 19 / 96$ & 5.20 & 2,550 & $\mathrm{~N}$ & $25-50$ \\
\hline 01350180 & $\begin{array}{l}\text { Schoharie Creek at North } \\
\text { Blenheim, NY }\end{array}$ & 358 & 1971-98 & 1996 & 17.61 & 75,600 & $1 / 19 / 96$ & 17.61 & 75,600 & $\mathrm{Y}$ & $75-100$ \\
\hline 01350355 & $\begin{array}{l}\text { Schoharie Creek at Breakabeen, } \\
\text { NY }\end{array}$ & 444 & 1976-98 & 1996 & 20.51 & 80,200 & $1 / 19 / 96$ & 20.51 & 80,200 & $\mathrm{Y}$ & $75-100$ \\
\hline 01351500 & $\begin{array}{l}\text { Schoharie Creek at Burtonsville, } \\
\text { NY }\end{array}$ & 886 & $1940-98$ & 1996 & 12.88 & 81,600 & $1 / 20 / 96$ & 12.88 & 81,600 & $\mathrm{Y}$ & $>100$ \\
\hline 01357500 & Mohawk River at Cohoes, NY & 3,450 & $1915-98$ & 1964 & 23.15 & 143,000 & $1 / 20 / 96$ & 22.68 & 132,000 & $\mathrm{Y}$ & 100 \\
\hline 01361500 & Catskill Creek at Oak Hill, NY & 98.0 & $\begin{array}{l}\text { 1911-77, } \\
1980, \\
1987-98\end{array}$ & 1987 & 16.60 & 15,400 & $1 / 19 / 96$ & 15.35 & 13,400 & $\mathrm{~N}$ & $25-50$ \\
\hline 01362200 & Esopus Creek at Allaben, NY & 63.7 & $\begin{array}{l}\text { 1951, } \\
1964-98\end{array}$ & 1951 & -- & 20,000 & $1 / 19 / 96$ & 13.58 & 15,000 & $\mathrm{~N}$ & $25-50$ \\
\hline 01413500 & $\begin{array}{l}\text { East Branch Delaware River at } \\
\text { Margaretville, NY }\end{array}$ & 163 & 1937-98 & 1996 & 14.88 & 25,800 & $1 / 19 / 96$ & 14.88 & 25,800 & $\mathrm{~N}$ & $75-100$ \\
\hline 01414000 & Platte Kill at Dunraven, NY & 34.9 & $\begin{array}{l}1942-62 \\
1996-98\end{array}$ & 1996 & 11.20 & 5,690 & $1 / 19 / 96$ & 11.20 & 5,690 & $\mathrm{~N}$ & $>100$ \\
\hline \multirow[t]{2}{*}{01414500} & Mill Brook near Dunraven, NY & 25.2 & 1937-98 & 1996 & 12.56 & 5,380 & $1 / 19 / 96$ & 12.56 & 5,380 & $\mathrm{~N}$ & $75-100$ \\
\hline & & & & & & & $11 / 9 / 96$ & 11.09 & 3,850 & $\mathrm{~N}$ & 25 \\
\hline
\end{tabular}


$\left[\mathrm{mi}^{2}\right.$, square miles; ft, feet above an arbitrary datum; $\mathrm{ft}^{3} / \mathrm{s}$, cubic feet per second; --, not determined or not applicable; >, greater than. Source: Recurrence intervals calculated from U.S. Geological Survey data. Other data from U.S. Geological Survey reports or databases]

\begin{tabular}{|c|c|c|c|c|c|c|c|c|c|c|c|}
\hline \multirow[b]{2}{*}{$\begin{array}{c}\text { Streamgage } \\
\text { number } \\
\text { (fig. 44) }\end{array}$} & \multirow[b]{2}{*}{ Streamgage name } & \multirow[b]{2}{*}{$\begin{array}{c}\text { Total } \\
\text { drainage } \\
\left(\mathrm{mi}^{2}\right)\end{array}$} & \multicolumn{4}{|c|}{$\begin{array}{c}\text { Maximum stage and discharge for period of record } \\
\text { through } 1998 \text { water year }\end{array}$} & \multicolumn{5}{|c|}{ Significant floods $1994-98$ water years } \\
\hline & & & $\begin{array}{l}\text { Period of } \\
\text { record } \\
\text { (water } \\
\text { years) }\end{array}$ & Water year & $\begin{array}{l}\text { Stage } \\
(\mathrm{ft})\end{array}$ & $\begin{array}{l}\text { Discharge } \\
\left(\mathrm{ft}^{3} / \mathrm{s}\right)\end{array}$ & $\begin{array}{c}\text { Date } \\
\text { (month/ } \\
\text { day/ } \\
\text { year) }\end{array}$ & $\begin{array}{l}\text { Stage } \\
(\mathrm{ft})\end{array}$ & $\begin{array}{l}\text { Discharge } \\
\left(\mathrm{ft}^{3} / \mathrm{s}\right)\end{array}$ & $\begin{array}{c}\text { Regulated } \\
\text { during } \\
\text { flood }^{1}\end{array}$ & $\begin{array}{c}\text { Recurrence } \\
\text { interval } \\
\text { (years) }\end{array}$ \\
\hline 01415000 & Tremper Kill near Andes, NY & 33.2 & $1937-98$ & 1996 & 7.69 & 5,000 & $1 / 19 / 96$ & 7.69 & 5,000 & $\mathrm{~N}$ & $75-100$ \\
\hline 01417000 & $\begin{array}{l}\text { East Branch Delaware River at } \\
\text { Downsville, NY }\end{array}$ & 372 & $\begin{array}{l}\text { 1904, } \\
1942-98\end{array}$ & 1904 & 16.00 & 34,000 & $12 / 2 / 96$ & 9.41 & 11,000 & $\mathrm{Y}$ & 25 \\
\hline 01417500 & $\begin{array}{l}\text { East Branch Delaware River at } \\
\text { Harvard, NY }\end{array}$ & 458 & $\begin{array}{l}1904, \\
1935-67, \\
1978-98\end{array}$ & $\begin{array}{l}1938 \\
1904\end{array}$ & $\begin{array}{l}16.93 \\
--\end{array}$ & $\begin{array}{l}31,400 \\
42,000\end{array}$ & $\begin{array}{l}1 / 19 / 96 \\
12 / 2 / 96\end{array}$ & $\begin{array}{l}12.63 \\
12.79\end{array}$ & $\begin{array}{l}12,200 \\
12,400\end{array}$ & $\begin{array}{l}\mathrm{Y} \\
\mathrm{Y}\end{array}$ & $\begin{array}{l}25-50 \\
25-50\end{array}$ \\
\hline 01418500 & Beaver Kill at Craigie Clair, NY & 81.9 & $\begin{array}{l}1937-74, \\
1996\end{array}$ & 1996 & 11.83 & 13,000 & $1 / 19 / 96$ & 11.83 & 13,000 & $\mathrm{~N}$ & $50-75$ \\
\hline 01420500 & Beaver Kill at Cooks Falls, NY & 241 & 1914-98 & 1996 & 17.79 & 42,900 & $1 / 19 / 96$ & 17.79 & 42,900 & $\mathrm{~N}$ & $>100$ \\
\hline 01421000 & $\begin{array}{l}\text { East Branch Delaware River at } \\
\text { Fishs Eddy, NY }\end{array}$ & 784 & $\begin{array}{l}1904, \\
1913-98\end{array}$ & 1904 & 23.60 & 70,000 & $1 / 19 / 96$ & 16.88 & 53,000 & $\mathrm{Y}$ & $>100$ \\
\hline 01422000 & $\begin{array}{l}\text { West Branch Delaware River at } \\
\text { Delhi, NY }\end{array}$ & 142 & $\begin{array}{l}1937-74 \\
1996\end{array}$ & 1996 & 9.80 & 13,000 & $1 / 19 / 96$ & 9.80 & 13,000 & $\mathrm{~N}$ & $>100$ \\
\hline 01422500 & $\begin{array}{l}\text { Little Delaware River near Delhi, } \\
\text { NY }\end{array}$ & 49.8 & $\begin{array}{l}1938-74, \\
1996-98\end{array}$ & 1996 & 8.51 & 6,100 & $\begin{array}{l}1 / 19 / 96 \\
11 / 9 / 96\end{array}$ & $\begin{array}{l}8.51 \\
7.67\end{array}$ & $\begin{array}{l}6,100 \\
4,540\end{array}$ & $\begin{array}{l}\mathrm{N} \\
\mathrm{N}\end{array}$ & $\begin{array}{r}>100 \\
25-50\end{array}$ \\
\hline 01423000 & $\begin{array}{l}\text { West Branch Delaware River at } \\
\text { Walton, NY }\end{array}$ & 332 & 1951-98 & 1996 & 16.36 & 25,000 & $1 / 19 / 96$ & 16.36 & 25,000 & $\mathrm{~N}$ & 50 \\
\hline 01424000 & Trout Creek near Rockroyal, NY & 20.0 & $\begin{array}{l}1952-67, \\
1996\end{array}$ & 1996 & 10.06 & 2,800 & $1 / 19 / 96$ & 10.06 & 2,800 & $\mathrm{~N}$ & 50 \\
\hline 01427500 & Callicoon Creek at Callicoon, NY & 110 & $\begin{array}{l}1940-82 \\
1987-98\end{array}$ & 1947 & 9.68 & 16,000 & $1 / 19 / 96$ & 8.42 & 11,200 & $\mathrm{~N}$ & $25-50$ \\
\hline 01428500 & $\begin{array}{l}\text { Delaware River above Lacka- } \\
\text { waxen River near Barryville, NY }\end{array}$ & 2,020 & 1941-98 & 1955 & 26.40 & 130,000 & $1 / 20 / 96$ & 22.18 & 98,300 & $\mathrm{Y}$ & $50-75$ \\
\hline 01497805 & $\begin{array}{l}\text { Little Elk Creek near Westford, } \\
\text { NY }\end{array}$ & 3.73 & 1978-98 & 1996 & 19.92 & 278 & 1/19/96 & 19.92 & 278 & $\mathrm{~N}$ & $25-50$ \\
\hline
\end{tabular}


Table 33. Maximum stage and discharge for period of record for streamgages having significant floods during 1994-98 water years in New York.-Continued

$\left[\mathrm{mi}^{2}\right.$, square miles; $\mathrm{ft}$, feet above an arbitrary datum; $\mathrm{ft}^{3} / \mathrm{s}$, cubic feet per second; --, not determined or not applicable; >, greater than. Source: Recurrence intervals calculated from U.S. Geological Survey data. Other data from U.S. Geological Survey reports or databases]

\begin{tabular}{|c|c|c|c|c|c|c|c|c|c|c|c|}
\hline \multirow{2}{*}{$\begin{array}{c}\text { Streamgage } \\
\text { number } \\
\text { (fig. 44) }\end{array}$} & \multirow[b]{2}{*}{ Streamgage name } & \multirow{2}{*}{$\begin{array}{c}\text { Total } \\
\text { drainage } \\
\left(\mathrm{mi}^{2}\right)\end{array}$} & \multicolumn{4}{|c|}{$\begin{array}{c}\text { Maximum stage and discharge for period of record } \\
\text { through } 1998 \text { water year }\end{array}$} & \multicolumn{5}{|c|}{ Significant floods 1994-98 water years } \\
\hline & & & $\begin{array}{c}\text { Period of } \\
\text { record } \\
\text { (water } \\
\text { years) }\end{array}$ & Water year & $\begin{array}{l}\text { Stage } \\
\text { (ft) }\end{array}$ & $\begin{array}{l}\text { Discharge } \\
\left(\mathrm{ft}^{3} / \mathrm{s}\right)\end{array}$ & $\begin{array}{c}\text { Date } \\
\text { (month/ } \\
\text { day/ } \\
\text { year) }\end{array}$ & $\begin{array}{l}\text { Stage } \\
\text { (ft) }\end{array}$ & $\begin{array}{l}\text { Discharge } \\
\left(\mathrm{ft}^{3} / \mathrm{s}\right)\end{array}$ & $\begin{array}{c}\text { Regulated } \\
\text { during } \\
\text { flood }^{1}\end{array}$ & $\begin{array}{c}\text { Recurrence } \\
\text { interval } \\
\text { (years) }\end{array}$ \\
\hline 01502731 & $\begin{array}{l}\text { Susquehanna River at Windsor, } \\
\text { NY }\end{array}$ & 1,820 & $1988-98$ & 1996 & 21.22 & 40,000 & $1 / 20 / 96$ & 21.22 & 40,000 & $\mathrm{~N}$ & 10 \\
\hline 01509520 & Tioughnioga River at Lisle, NY & 453 & 1988-98 & $\begin{array}{l}1996 \\
1993\end{array}$ & $\overline{--}$ & $\begin{array}{l}12,900 \\
12,100\end{array}$ & $1 / 20 / 96$ & -- & 12,900 & $\mathrm{~N}$ & 10 \\
\hline 01510000 & Otselic River at Cincinnatus, NY & 147 & $\begin{array}{l}1935, \\
1939-64, \\
1970-98\end{array}$ & $\begin{array}{l}1935 \\
1996\end{array}$ & 10.89 & $\begin{array}{l}9,200 \\
8,000\end{array}$ & $1 / 19 / 96$ & 10.89 & 8,000 & $\mathrm{~N}$ & 25 \\
\hline 01510610 & $\begin{array}{l}\text { Merrill Creek tributary near Texas } \\
\text { Valley, NY }\end{array}$ & 5.32 & $\begin{array}{l}1976-81 \\
1983-98\end{array}$ & 1996 & 6.64 & 1,150 & $1 / 19 / 96$ & 6.64 & 1,150 & $\mathrm{~N}$ & $25-50$ \\
\hline 01511500 & Tioughnioga River at Itaska, NY & 730 & 1930-98 & 1935 & 16.61 & 61,100 & $1 / 19 / 96$ & 10.77 & 20,800 & $\mathrm{Y}$ & $25-50$ \\
\hline 01514801 & $\begin{array}{l}\text { Catatonk Creek northwest of } \\
\text { Owego, NY }\end{array}$ & 151 & 1988-98 & 1996 & 14.83 & 9,740 & $1 / 20 / 96$ & 14.83 & 9,740 & $\mathrm{~N}$ & $25-50$ \\
\hline 01520500 & Tioga River at Lindley, NY & 771 & $1930-98$ & 1972 & 26.27 & 128,000 & $8 / 18 / 94$ & 13.38 & 13,900 & $\mathrm{Y}$ & 10 \\
\hline 01525500 & $\begin{array}{l}\text { Canisteo River at West Cameron, } \\
\text { NY }\end{array}$ & 340 & $\begin{array}{l}1931, \\
1935-72, \\
1974-98\end{array}$ & 1972 & 23.48 & 43,000 & $1 / 19 / 96$ & 20.91 & 29,100 & $\mathrm{Y}$ & $50-75$ \\
\hline 01526500 & Tioga River near Erwins, NY & 1,377 & 1919-98 & 1972 & 26.74 & 190,000 & $1 / 19 / 96$ & 16.98 & 45,600 & $\mathrm{Y}$ & 75 \\
\hline 01529950 & Chemung River at Corning, NY & 2,005 & $\begin{array}{l}\text { 1972, } \\
1975-98\end{array}$ & 1972 & 40.71 & 228,000 & $1 / 19 / 96$ & 25.93 & 61,000 & $\mathrm{Y}$ & $25-50$ \\
\hline 01530500 & Newtown Creek at Elmira, NY & 77.5 & 1938-98 & 1972 & 19.28 & 4,000 & $1 / 19 / 96$ & 16.98 & 3,810 & $\mathrm{Y}$ & 25 \\
\hline 04216418 & Tonawanda Creek at Attica, NY & 76.9 & $\begin{array}{l}\text { 1972, } \\
1978-98\end{array}$ & 1998 & 12.71 & 9,400 & $7 / 8 / 98$ & 12.71 & 9,400 & $\mathrm{~N}$ & $>100$ \\
\hline 0422028490 & $\begin{array}{l}\text { Slater Creek (Latta Road) near } \\
\text { Greece, NY }\end{array}$ & 1.52 & 1989-98 & 1997 & 4.80 & 219 & $10 / 20 / 96$ & 4.80 & 219 & $\mathrm{~N}$ & $10-25$ \\
\hline 04221000 & Genesee River at Wellsville, NY & 288 & $\begin{array}{l}1956-58 \\
1972-98\end{array}$ & 1972 & 20.70 & 38,500 & $1 / 19 / 96$ & 16.13 & 22,700 & $\mathrm{~N}$ & $25-50$ \\
\hline
\end{tabular}


$\left[\mathrm{mi}^{2}\right.$, square miles; ft, feet above an arbitrary datum; $\mathrm{ft}^{3} / \mathrm{s}$, cubic feet per second; --, not determined or not applicable; >, greater than. Source: Recurrence intervals calculated from U.S. Geological Survey data. Other data from U.S. Geological Survey reports or databases]

\begin{tabular}{|c|c|c|c|c|c|c|c|c|c|c|c|}
\hline \multirow[b]{2}{*}{$\begin{array}{l}\text { Streamgage } \\
\text { number } \\
\text { (fig. 44) }\end{array}$} & \multirow[b]{2}{*}{ Streamgage name } & \multirow{2}{*}{$\begin{array}{c}\text { Total } \\
\text { drainage } \\
\left(\mathrm{mi}^{2}\right)\end{array}$} & \multicolumn{4}{|c|}{$\begin{array}{c}\text { Maximum stage and discharge for period of record } \\
\text { through } 1998 \text { water year }\end{array}$} & \multicolumn{5}{|c|}{ Significant floods 1994-98 water years } \\
\hline & & & $\begin{array}{l}\text { Period of } \\
\text { record } \\
\text { (water } \\
\text { years) }\end{array}$ & Water year & $\begin{array}{l}\text { Stage } \\
(\mathrm{ft})\end{array}$ & $\begin{array}{l}\text { Discharge } \\
\left(\mathrm{ft}^{3} / \mathrm{s}\right)\end{array}$ & $\begin{array}{c}\text { Date } \\
\text { (month/ } \\
\text { day/ } \\
\text { year) }\end{array}$ & $\begin{array}{l}\text { Stage } \\
(\mathrm{ft})\end{array}$ & $\begin{array}{l}\text { Discharge } \\
\left(\mathrm{ft}^{3} / \mathrm{s}\right)\end{array}$ & $\begin{array}{c}\text { Regulated } \\
\text { during } \\
\text { flood }^{1}\end{array}$ & $\begin{array}{c}\text { Recurrence } \\
\text { interval } \\
\text { (years) }\end{array}$ \\
\hline 04223000 & Genesee River at Portageville, NY & 984 & $\begin{array}{l}1902, \\
1909-98\end{array}$ & 1972 & 32.25 & 90,000 & $1 / 20 / 96$ & 29.12 & 56,200 & $\mathrm{~N}$ & $25-50$ \\
\hline 04227000 & $\begin{array}{l}\text { Canaseraga Creek at Shakers } \\
\text { Crossing, NY }\end{array}$ & 335 & $\begin{array}{l}\text { 1916-22, } \\
\text { 1959-70, } \\
\text { 1972, } \\
1975-98\end{array}$ & $\begin{array}{l}1972 \\
1916\end{array}$ & 28.92 & $\begin{array}{r}11,200 \\
--\end{array}$ & $1 / 19 / 96$ & 13.01 & 5,510 & $\mathrm{~N}$ & $10-25$ \\
\hline 04230380 & Oatka Creek at Warsaw, NY & 39.1 & 1964-98 & 1998 & 9.90 & 4,110 & $7 / 8 / 98$ & 9.90 & 4,110 & $\mathrm{~N}$ & $50-75$ \\
\hline 04230500 & Oatka Creek at Garbutt, NY & 200 & $1946-98$ & 1960 & 8.64 & 7,050 & $1 / 9 / 98$ & 8.57 & 6,160 & $\mathrm{~N}$ & $25-50$ \\
\hline 0423205010 & $\begin{array}{l}\text { Irondequoit Creek above Blossom } \\
\text { Road near Rochester, NY }\end{array}$ & 142 & 1982-98 & 1998 & 9.95 & 3,300 & $1 / 8 / 98$ & 9.95 & 3,300 & $\mathrm{~N}$ & $75-100$ \\
\hline 042320578 & Bear Creek at Ontario, NY & 6.74 & $\begin{array}{l}1971-73 \\
1975-98\end{array}$ & 1998 & 13.38 & 238 & $1 / 8 / 98$ & 13.38 & 238 & $\mathrm{~N}$ & $25-50$ \\
\hline 04232200 & $\begin{array}{l}\text { Catharine Creek at Montour Falls, } \\
\text { NY }\end{array}$ & 41.1 & $\begin{array}{l}1972, \\
1975-77, \\
1987-98\end{array}$ & 1997 & 8.48 & 4,700 & $11 / 8 / 96$ & 8.48 & 4,700 & $\mathrm{~N}$ & 50 \\
\hline 04232460 & Sugar Creek at Guyanoga, NY & 28.9 & 1966-98 & 1996 & 5.88 & 1,800 & $\begin{array}{l}1 / 19 / 96 \\
1 / 8 / 98\end{array}$ & $\begin{array}{l}5.88 \\
4.98\end{array}$ & $\begin{array}{l}1,800 \\
1,320\end{array}$ & $\begin{array}{l}\mathrm{N} \\
\mathrm{N}\end{array}$ & $\begin{array}{l}25 \\
10\end{array}$ \\
\hline 04233000 & Cayuga Inlet near Ithaca, NY & 35.2 & $\begin{array}{l}\text { 1935, } \\
1937-98\end{array}$ & 1935 & -- & 6,500 & $1 / 19 / 96$ & 7.57 & 4,210 & $\mathrm{~N}$ & $25-50$ \\
\hline 04233255 & Cayuga Inlet at Ithaca, NY & 86.7 & $\begin{array}{l}1935, \\
1971-72, \\
1975-98\end{array}$ & 1935 & -- & 14,000 & $1 / 19 / 96$ & 14.67 & 12,500 & $\mathrm{~N}$ & $25-50$ \\
\hline 04233258 & Coy Glen Creek at Ithaca, NY & 3.56 & $\begin{array}{l}1972, \\
1983-98\end{array}$ & 1996 & 22.23 & 820 & $1 / 19 / 96$ & 22.23 & 820 & $\mathrm{~N}$ & 25 \\
\hline 04234000 & Fall Creek near Ithaca, NY & 126 & 1926-98 & 1935 & 9.52 & 15,500 & $1 / 19 / 96$ & 7.47 & 9,450 & $\mathrm{~N}$ & $50-75$ \\
\hline
\end{tabular}


Table 33. Maximum stage and discharge for period of record for streamgages having significant floods during 1994-98 water years in New York.-Continued

$\left[\mathrm{mi}^{2}\right.$, square miles; $\mathrm{ft}$, feet above an arbitrary datum; $\mathrm{ft}^{3} / \mathrm{s}$, cubic feet per second; --, not determined or not applicable; >, greater than. Source: Recurrence intervals calculated from U.S. Geological Survey data. Other data from U.S. Geological Survey reports or databases]

\begin{tabular}{|c|c|c|c|c|c|c|c|c|c|c|c|}
\hline \multirow{2}{*}{$\begin{array}{c}\text { Streamgage } \\
\text { number } \\
\text { (fig. 44) }\end{array}$} & \multirow[b]{2}{*}{ Streamgage name } & \multirow{2}{*}{$\begin{array}{l}\text { Total } \\
\text { drainage } \\
\left(\mathrm{mi}^{2}\right)\end{array}$} & \multicolumn{4}{|c|}{$\begin{array}{c}\text { Maximum stage and discharge for period of record } \\
\text { through } 1998 \text { water year }\end{array}$} & \multicolumn{5}{|c|}{ Significant floods $1994-98$ water years } \\
\hline & & & $\begin{array}{l}\text { Period of } \\
\text { record } \\
\text { (water } \\
\text { years) }\end{array}$ & Water year & $\begin{array}{l}\text { Stage } \\
(\mathrm{ft})\end{array}$ & $\begin{array}{c}\text { Discharge } \\
\left(\mathrm{ft}^{3} / \mathrm{s}\right)\end{array}$ & $\begin{array}{c}\text { Date } \\
\text { (month/ } \\
\text { day/ } \\
\text { year) }\end{array}$ & $\begin{array}{l}\text { Stage } \\
(\mathrm{ft})\end{array}$ & $\begin{array}{c}\text { Discharge } \\
\left(\mathrm{ft}^{3} / \mathrm{s}\right)\end{array}$ & $\begin{array}{l}\text { Regulated } \\
\text { during } \\
\text { flood }^{1}\end{array}$ & $\begin{array}{c}\text { Recurrence } \\
\text { interval } \\
\text { (years) }\end{array}$ \\
\hline 04234200 & Mud Creek at East Victor, NY & 64.2 & $\begin{array}{l}1958, \\
1961-68, \\
1976-98\end{array}$ & $\begin{array}{l}1991 \\
1972\end{array}$ & $\begin{array}{l}7.22 \\
7.85\end{array}$ & $\begin{array}{l}1,880 \\
1,800\end{array}$ & $1 / 19 / 96$ & 7.09 & 1,810 & $\mathrm{~N}$ & $10-25$ \\
\hline 04235255 & $\begin{array}{l}\text { Canandaigua Outlet tributary near } \\
\text { Alloway, NY }\end{array}$ & 2.94 & 1978-98 & $\begin{array}{l}1996 \\
1998\end{array}$ & $\begin{array}{l}7.34 \\
7.34\end{array}$ & $\begin{array}{l}102 \\
102\end{array}$ & $\begin{array}{l}1 / 19 / 96 \\
1 / 8 / 98\end{array}$ & $\begin{array}{l}7.34 \\
7.34\end{array}$ & $\begin{array}{l}102 \\
102\end{array}$ & $\begin{array}{l}\mathrm{N} \\
\mathrm{N}\end{array}$ & $\begin{array}{l}10 \\
10\end{array}$ \\
\hline 04235500 & Owasco Outlet near Auburn, NY & 206 & $1914-98$ & 1972 & 6.28 & 3,250 & $1 / 9 / 98$ & 4.65 & 2,150 & $\mathrm{Y}$ & $10-25$ \\
\hline 04240200 & Ninemile Creek at Camillus, NY & 84.3 & $\begin{array}{l}1959-82 \\
1989-98\end{array}$ & $\begin{array}{l}1960 \\
1975\end{array}$ & $\begin{array}{r}8.25 \\
10.83\end{array}$ & $\begin{array}{l}2,760 \\
2,120\end{array}$ & $1 / 19 / 96$ & 8.92 & 2,530 & $\mathrm{Y}$ & $10-25$ \\
\hline 04245840 & Scriba Creek near Constantia, NY & 38.4 & $\begin{array}{l}1966-69 \\
1971-98\end{array}$ & $\begin{array}{l}1975 \\
1972\end{array}$ & $\begin{array}{l}7.33 \\
7.42\end{array}$ & $\begin{array}{l}1,310 \\
1,200\end{array}$ & $1 / 8 / 98$ & 7.32 & 1,300 & $\mathrm{~N}$ & $25-50$ \\
\hline 04250750 & Sandy Creek near Adams, NY & 128 & $1958-98$ & 1996 & 11.06 & 7,700 & $1 / 19 / 96$ & 11.06 & 7,700 & $\mathrm{~N}$ & $10-25$ \\
\hline 04252500 & Black River near Boonville, NY & 304 & $1911-98$ & $\begin{array}{l}1985 \\
1982 \\
1913\end{array}$ & $\begin{array}{l}11.41 \\
11.31 \\
12.50\end{array}$ & $\begin{array}{l}12,800 \\
12,800 \\
12,400\end{array}$ & $1 / 9 / 98$ & 11.25 & 12,100 & $\mathrm{~N}$ & $25-50$ \\
\hline 04254500 & Moose River at McKeever, NY & 363 & $\begin{array}{l}1869, \\
1902-70, \\
1982, \\
1985, \\
1987-98\end{array}$ & 1947 & 17.45 & 18,700 & $1 / 8 / 98$ & 14.91 & 14,600 & $\mathrm{~N}$ & $25-50$ \\
\hline 04256000 & $\begin{array}{l}\text { Independence River at } \\
\text { Donnattsburg, NY }\end{array}$ & 88.7 & 1943-98 & 1985 & 13.34 & 9,420 & $1 / 8 / 98$ & 9.53 & 4,340 & $\mathrm{~N}$ & $10-25$ \\
\hline 04258000 & Beaver River at Croghan, NY & 291 & $1931-98$ & 1969 & 6.98 & 5,100 & $1 / 9 / 98$ & 6.56 & 4,440 & $\mathrm{Y}$ & $25-50$ \\
\hline 04260500 & Black River at Watertown, NY & 1,864 & $\begin{array}{l}1869, \\
1897-1913, \\
1917-98\end{array}$ & 1998 & 16.02 & 55,500 & $1 / 10 / 98$ & 16.02 & 55,500 & $\mathrm{Y}$ & $>100$ \\
\hline
\end{tabular}


$\left[\mathrm{mi}^{2}\right.$, square miles; ft, feet above an arbitrary datum; $\mathrm{ft}^{3} / \mathrm{s}$, cubic feet per second; --, not determined or not applicable; >, greater than. Source: Recurrence intervals calculated from U.S. Geological Survey data. Other data from U.S. Geological Survey reports or databases]

\begin{tabular}{|c|c|c|c|c|c|c|c|c|c|c|c|}
\hline \multirow{2}{*}{$\begin{array}{l}\text { Streamgage } \\
\text { number } \\
\text { (fig. 44) }\end{array}$} & \multirow[b]{2}{*}{ Streamgage name } & \multirow{2}{*}{$\begin{array}{c}\text { Total } \\
\text { drainage } \\
\left(\mathrm{mi}^{2}\right)\end{array}$} & \multicolumn{4}{|c|}{$\begin{array}{l}\text { Maximum stage and discharge for period of record } \\
\text { through } 1998 \text { water year }\end{array}$} & \multicolumn{5}{|c|}{ Significant floods 1994-98 water years } \\
\hline & & & $\begin{array}{l}\text { Period of } \\
\text { record } \\
\text { (water } \\
\text { years) }\end{array}$ & Water year & $\begin{array}{l}\text { Stage } \\
(\mathrm{ft})\end{array}$ & $\begin{array}{l}\text { Discharge } \\
\left(\mathrm{ft}^{3} / \mathrm{s}\right)\end{array}$ & $\begin{array}{c}\text { Date } \\
\text { (month/ } \\
\text { day/ } \\
\text { year) }\end{array}$ & $\begin{array}{l}\text { Stage } \\
(\mathrm{ft})\end{array}$ & $\begin{array}{l}\text { Discharge } \\
\left(\mathrm{ft}^{3} / \mathrm{s}\right)\end{array}$ & $\begin{array}{l}\text { Regulated } \\
\text { during } \\
\text { flood }^{1}\end{array}$ & $\begin{array}{c}\text { Recurrence } \\
\text { interval } \\
\text { (years) }\end{array}$ \\
\hline 04262500 & $\begin{array}{l}\text { West Branch Oswegatchie River } \\
\text { near Harrisville, NY }\end{array}$ & 244 & $1917-98$ & 1998 & 10.64 & 8,700 & $1 / 9 / 98$ & 10.64 & 8,700 & $\mathrm{~N}$ & $>100$ \\
\hline 04264331 & $\begin{array}{l}\text { Saint Lawrence River at Cornwall } \\
\text { Ontario near Massena, NY }\end{array}$ & 298,800 & $1918-98$ & 1993 & -- & 378,000 & $3 / 17 / 98$ & -- & $\begin{array}{r}360,000 \\
\text { (Daily) }\end{array}$ & $\mathrm{Y}$ & -- \\
\hline 04265100 & Elm Creek near Hermon, NY & 32.6 & 1959-98 & 1974 & 9.07 & 1,270 & $1 / 19 / 96$ & 8.82 & 1,180 & $\mathrm{~N}$ & 25 \\
\hline 04266500 & Raquette River at Piercefield, NY & 721 & $\begin{array}{l}\text { 1900, } \\
1909-98\end{array}$ & $\begin{array}{l}1993 \\
1972\end{array}$ & $\begin{array}{l}12.04 \\
12.25\end{array}$ & $\begin{array}{l}8,630 \\
8,360\end{array}$ & $4 / 21 / 94$ & 11.50 & 7,660 & $\mathrm{Y}$ & $10-25$ \\
\hline 04270000 & Salmon River at Chasm Falls, NY & 132 & $\begin{array}{l}1926-82 \\
1985, \\
1987-98\end{array}$ & 1985 & 5.63 & 3,700 & $4 / 1 / 98$ & 5.43 & 3,540 & $\mathrm{~N}$ & $75-100$ \\
\hline 04270200 & $\begin{array}{l}\text { Little Salmon River at Bombay, } \\
\text { NY }\end{array}$ & 92.2 & 1959-98 & 1998 & 13.27 & 3,420 & $3 / 31 / 98$ & 13.27 & 3,420 & $\mathrm{~N}$ & $25-50$ \\
\hline 04270510 & $\begin{array}{l}\text { Chateaugay River below } \\
\text { Chateaugay, NY }\end{array}$ & 151 & $\begin{array}{l}\text { 1966-95, } \\
1997-98\end{array}$ & $\begin{array}{l}1998 \\
1966\end{array}$ & $\begin{array}{r}7.54 \\
10.99\end{array}$ & $\begin{array}{r}5,440 \\
--\end{array}$ & $\begin{array}{l}11 / 9 / 96 \\
3 / 31 / 98\end{array}$ & $\begin{array}{l}7.51 \\
7.54\end{array}$ & $\begin{array}{l}5,370 \\
5,440\end{array}$ & $\begin{array}{l}\mathrm{Y} \\
\mathrm{Y}\end{array}$ & $\begin{array}{l}10-25 \\
10-25\end{array}$ \\
\hline 04270700 & Trout River at Trout River, NY & 107 & 1960-98 & 1996 & 9.42 & 6,980 & $7 / 5 / 96$ & 9.42 & 6,980 & $\mathrm{~N}$ & $50-75$ \\
\hline 04271500 & $\begin{array}{l}\text { Great Chazy River at Perry Mills, } \\
\text { NY }\end{array}$ & 243 & $\begin{array}{l}1929-68, \\
1985, \\
1987-98\end{array}$ & 1997 & 12.24 & 9,700 & $\begin{array}{l}11 / 9 / 96 \\
3 / 31 / 98\end{array}$ & $\begin{array}{l}12.24 \\
10.89\end{array}$ & $\begin{array}{l}9,700 \\
7,640\end{array}$ & $\begin{array}{l}\mathrm{N} \\
\mathrm{N}\end{array}$ & $\begin{array}{r}>100 \\
50-75\end{array}$ \\
\hline 04273500 & Saranac River at Plattsburgh, NY & 608 & $\begin{array}{l}\text { 1928, } \\
1944-98\end{array}$ & 1997 & 12.11 & 14,400 & $\begin{array}{l}11 / 09 / 96 \\
4 / 1 / 98\end{array}$ & $\begin{array}{l}12.11 \\
10.73\end{array}$ & $\begin{array}{l}14,400 \\
11,200\end{array}$ & $\begin{array}{l}\mathrm{Y} \\
\mathrm{Y}\end{array}$ & $\begin{array}{r}>100 \\
25\end{array}$ \\
\hline 04273700 & $\begin{array}{l}\text { Salmon River at South Plattsburgh, } \\
\text { NY }\end{array}$ & 63.3 & $\begin{array}{l}1960-86 \\
1990-98\end{array}$ & 1997 & 7.56 & 4,200 & $\begin{array}{l}11 / 9 / 96 \\
6 / 27 / 98\end{array}$ & $\begin{array}{l}7.56 \\
7.54\end{array}$ & $\begin{array}{l}4,200 \\
4,170\end{array}$ & $\begin{array}{l}\mathrm{N} \\
\mathrm{N}\end{array}$ & $\begin{array}{l}50-75 \\
50-75\end{array}$ \\
\hline 04274000 & $\begin{array}{l}\text { West Branch Ausable River near } \\
\text { Lake Placid, NY }\end{array}$ & 116 & $\begin{array}{l}1920-68 \\
1983-98\end{array}$ & 1938 & 12.20 & 10,800 & $11 / 9 / 96$ & 12.09 & 10,600 & $\mathrm{~N}$ & $>100$ \\
\hline 04275000 & $\begin{array}{l}\text { East Branch Ausable River at Au } \\
\text { Sable Forks, NY }\end{array}$ & 198 & $1925-98$ & 1997 & 15.22 & 23,900 & $11 / 9 / 96$ & 15.22 & 23,900 & $\mathrm{~N}$ & $>100$ \\
\hline 04275500 & $\begin{array}{l}\text { Ausable River near Au Sable } \\
\text { Forks, NY }\end{array}$ & 446 & $\begin{array}{l}1911-68 \\
1990-98\end{array}$ & 1997 & 13.83 & 37,400 & $11 / 9 / 96$ & 13.83 & 37,400 & $\mathrm{~N}$ & $>100$ \\
\hline
\end{tabular}


Table 33. Maximum stage and discharge for period of record for streamgages having significant floods during 1994-98 water years in New York.-Continued

$\left[\mathrm{mi}^{2}\right.$, square miles; $\mathrm{ft}$, feet above an arbitrary datum; $\mathrm{ft}^{3} / \mathrm{s}$, cubic feet per second; --, not determined or not applicable; >, greater than. Source: Recurrence intervals calculated from U.S. Geological Survey data. Other data from U.S. Geological Survey reports or databases]

\begin{tabular}{|c|c|c|c|c|c|c|c|c|c|c|c|}
\hline \multirow[b]{2}{*}{$\begin{array}{c}\text { Streamgage } \\
\text { number } \\
\text { (fig. 44) }\end{array}$} & \multirow[b]{2}{*}{ Streamgage name } & \multirow[b]{2}{*}{$\begin{array}{c}\text { Total } \\
\text { drainage } \\
\left(\mathrm{mi}^{2}\right)\end{array}$} & \multicolumn{4}{|c|}{$\begin{array}{c}\text { Maximum stage and discharge for period of record } \\
\text { through } 1998 \text { water year }\end{array}$} & \multicolumn{5}{|c|}{ Significant floods $1994-98$ water years } \\
\hline & & & $\begin{array}{l}\text { Period of } \\
\text { record } \\
\text { (water } \\
\text { years) }\end{array}$ & Water year & $\begin{array}{l}\text { Stage } \\
(\mathrm{ft})\end{array}$ & $\begin{array}{c}\text { Discharge } \\
\left(\mathrm{ft}^{3} / \mathrm{s}\right)\end{array}$ & $\begin{array}{c}\text { Date } \\
\text { (month/ } \\
\text { day/ } \\
\text { year) }\end{array}$ & $\begin{array}{l}\text { Stage } \\
\text { (ft) }\end{array}$ & $\begin{array}{c}\text { Discharge } \\
\left(\mathrm{ft}^{3} / \mathrm{s}\right)\end{array}$ & $\begin{array}{c}\text { Regulated } \\
\text { during } \\
\text { flood }^{1}\end{array}$ & $\begin{array}{c}\text { Recurrence } \\
\text { interval } \\
\text { (years) }\end{array}$ \\
\hline 04276500 & Bouquet River at Willsboro, NY & 270 & $\begin{array}{l}1924-68, \\
1980,1985, \\
1987-98\end{array}$ & 1997 & 10.93 & 12,300 & $11 / 9 / 96$ & 10.93 & 12,300 & $\mathrm{~N}$ & $50-75$ \\
\hline 04278300 & $\begin{array}{l}\text { Northwest Bay Brook near Bolton } \\
\text { Landing, NY }\end{array}$ & 22.0 & 1966-98 & 1996 & 6.57 & 1,950 & $1 / 19 / 96$ & 6.57 & 1,950 & $\mathrm{~N}$ & $25-50$ \\
\hline 04280450 & $\begin{array}{l}\text { Mettawee River near Middle } \\
\text { Granville, NY }\end{array}$ & 167 & $\begin{array}{l}1984 \\
1990-98\end{array}$ & 1996 & 10.69 & 7,600 & $1 / 20 / 96$ & 10.69 & 7,600 & $\mathrm{~N}$ & 10 \\
\hline
\end{tabular}

${ }^{1}$ Regulated during flood: N, no; Y, yes. 


\section{North Carolina}

Rainfall amounts of 5 to 7 inches (National Oceanic and Atmospheric Administration, 1994a) caused serious flooding in the western mountains of North Carolina on March 27, 1994. Bridges were washed away and roads closed. Mudslides were widespread.

Remnants of Tropical Storm Beryl dropped 3 to 4 inches of rain west of Cape Fear (fig. 45) on August 18, 1994 (National Oceanic and Atmospheric Administration, 1994a). The town of Southport sustained nearly $\$ 3$ million in damage (National Oceanic and Atmospheric Administration, 1994b).

Hurricane Gordon was a major weather factor along the North Carolina coast during October 17-18, 1994. The storm remained offshore but caused significant flooding and strong winds along the central and northern Carolina coast. The flooding began on Hatteras Island, with about 12 inches of overwash (National Oceanic and Atmospheric Administration, 1994b).

More than 11 inches of rain fell during a 2-day period causing widespread flooding in western North Carolina, January 14-15, 1995. Three Boy Scouts drowned in Pisgah National Forest. They were attempting to cross a footbridge and were swept away in the swollen creek. Two other men drowned. Overall damage was estimated at \$2.5 million (National Oceanic and Atmospheric Administration, 1995b).

Flash flooding developed rapidly during the evening of September 4, 1996, as stationary thunderstorms dumped between 10 and 15 inches of rain in a small area of Hickory Nut Gorge (National Oceanic and Atmospheric Administration, 1996a). A wall of water rushed down the valley of the upper Broad River carrying campsites, RVs, mobile homes, trees, and debris downstream.

Hurricane Fran made landfall near Cape Fear, on September 5, 1996, and was the worst natural economic disaster to occur in North Carolina history. In North Carolina, wind and flooding damage exceeded $\$ 5$ billion (National Oceanic and Atmospheric Administration, 1996b). The copious rainfall produced many severe flash and river floods. The flooding on the Haw River at Haw River (streamgage 02096500, table 34), the Tar River at Louisburg (streamgage 02081747), and at Rocky Mount (streamgage 02082585, table 34) exceeded the highest flood on record. A massive evacuation occurred in Goldsboro where residents of 550 homes had to be moved to public shelters. Excessive rain from Hurricane Fran moved downstream along the Neuse River and, combined with a large amount of debris, led to serious flooding. The Neuse River at Kinston (streamgage 02089500, table 34) crested at 23.3 feet, more than 9 feet above flood stage (National Oceanic and Atmospheric Administration, 1996b).

The remnants of Hurricane Fran produced widespread wind damage and flash flooding in north-central and parts of northwestern North Carolina during the late evening hours of September 5 and morning hours of September 6, 1996. Excessive rainfall forced creeks and streams to leave their banks, flooding roads and damaging crops, roads, and vehicles.

Strong thunderstorms occurred in the early morning of July 23, 1997, between Charlotte and Raleigh. These storms developed ahead of the remnants of Hurricane Danny. Between 5 and 10 inches of rain fell in a few hours (National Oceanic and Atmospheric Administration, 1997a) causing widespread flooding. Two women died after their vehicles became trapped in floodwaters. A young girl perished when she went to play in the water and was swept away. Damage in this area was more than \$11 million (National Oceanic and Atmospheric Administration, 1997b).

\section{References}

National Oceanic and Atmospheric Administration (NOAA), 1994a-97a, Climatological data (by State): Asheville, North Carolina, National Climatic Data Center, various months. National Oceanic and Atmospheric Administration (NOAA), 1994b-97b, Storm data (by State): Asheville, North Carolina, National Climatic Data Center, various months. 


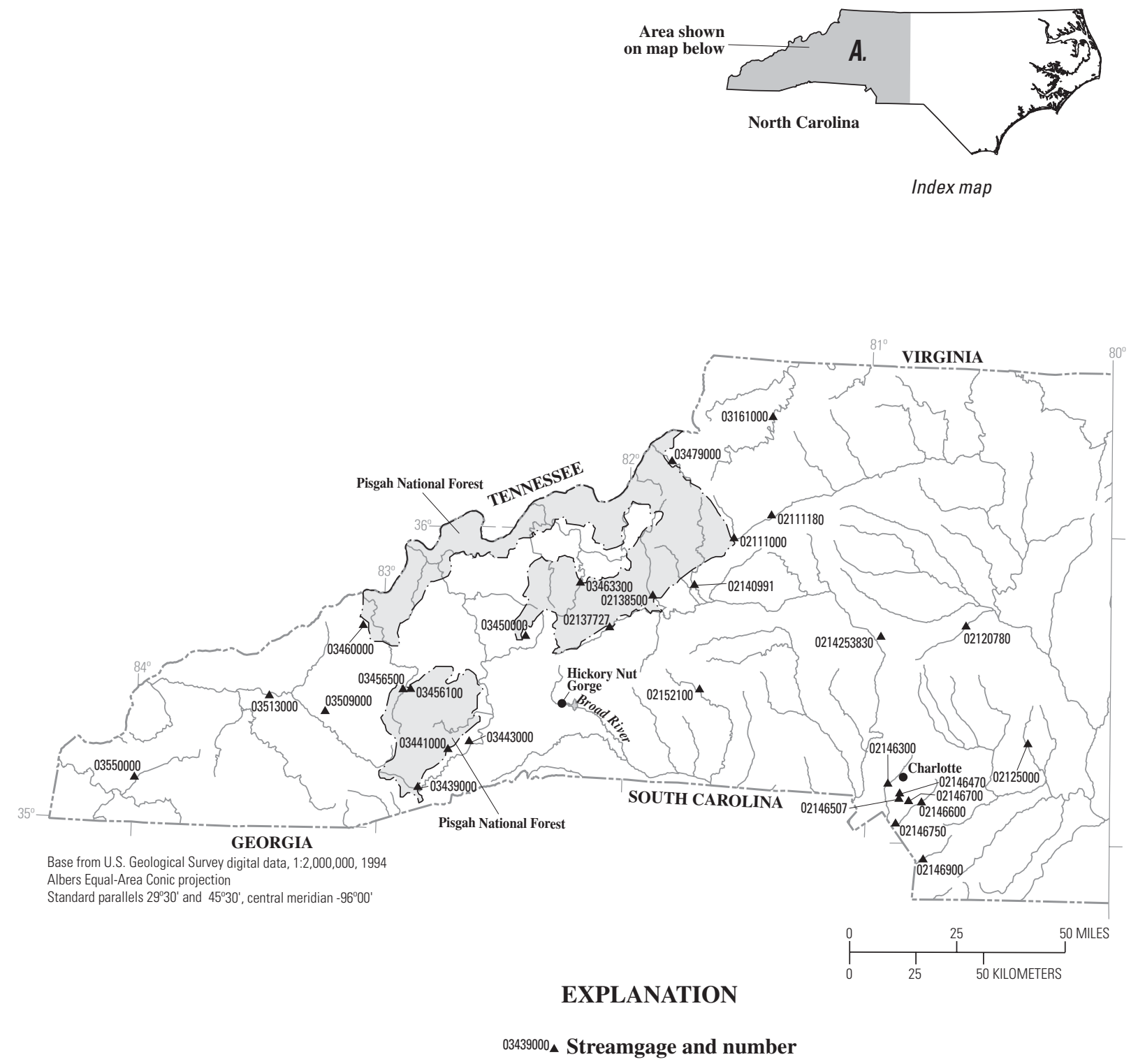

Figure 45. (A) Location of streamgages with significant floods during 1994-98 water years for North Carolina. 

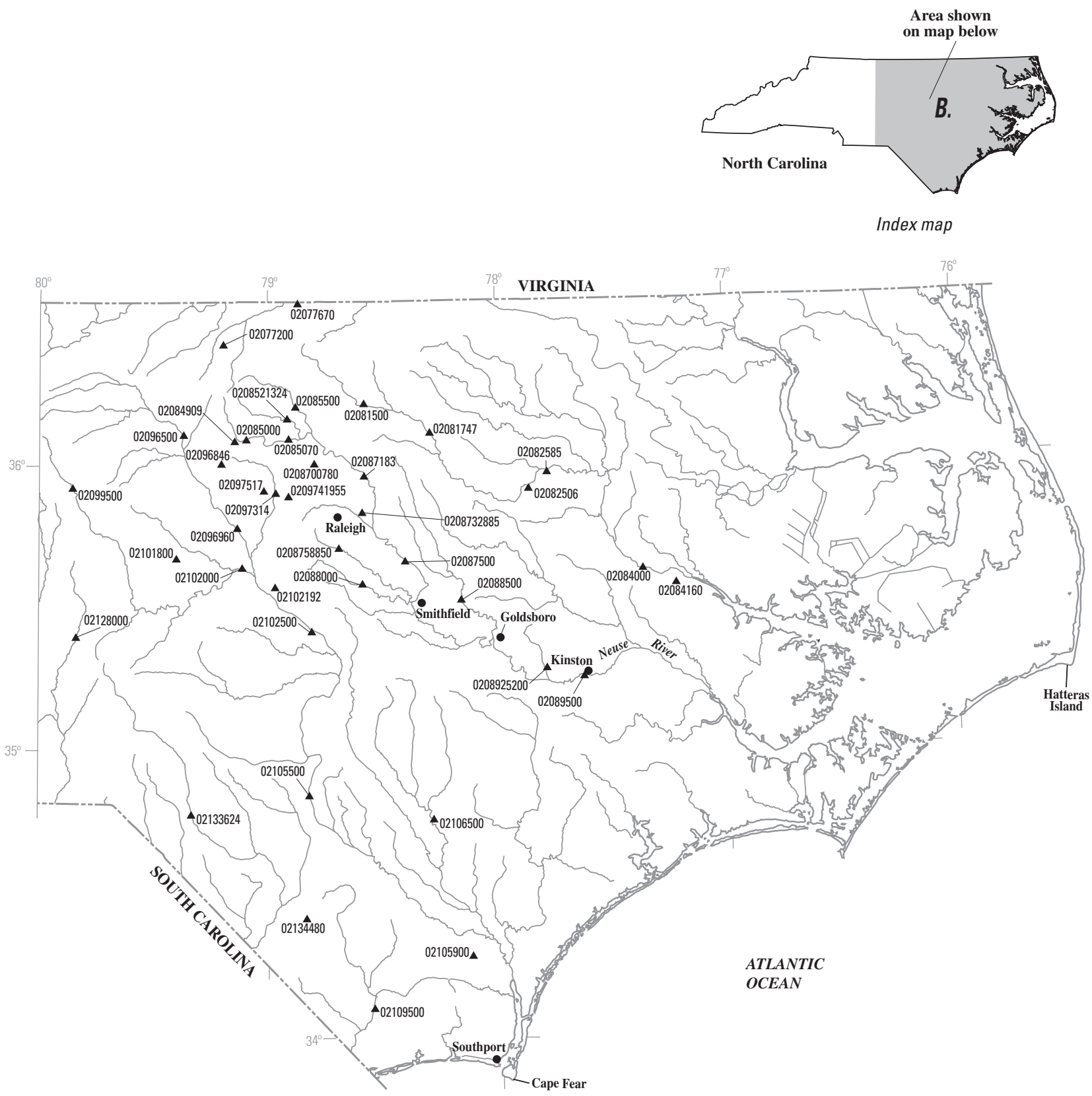

Base from U.S. Geological Survey digital data, 1:2,000,000, 1994 Albers Equal-Area Conic projection

Standard parallels $29^{\circ} 30^{\prime}$ and $45^{\circ} 30^{\prime}$, central meridian $-96^{\circ} 00^{\prime}$

\section{EXPLANATION}

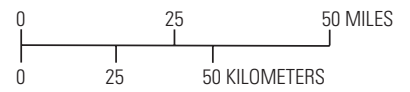

$02109500 \triangle$ Streamgage and number

Figure 45. (B) Location of streamgages with significant floods during 1994-98 water years for North Carolina.-Continued 
Table 34. Maximum stage and discharge for period of record for streamgages having significant floods during 1994-98 water years in North Carolina.

$\left[\mathrm{mi}^{2}\right.$, square miles; $\mathrm{ft}$, feet above an arbitrary datum; $\mathrm{ft}^{3} / \mathrm{s}$, cubic feet per second; --, not determined or not applicable; $>$, greater than; <, less than. Source: Recurrence intervals calculated from U.S. Geological Survey data. Other data from U.S. Geological Survey reports or databases]

\begin{tabular}{|c|c|c|c|c|c|c|c|c|c|c|c|}
\hline \multirow{2}{*}{$\begin{array}{c}\text { Streamgage } \\
\text { number } \\
\text { (fig. 45) }\end{array}$} & \multirow[b]{2}{*}{ Streamgage name } & \multirow{2}{*}{$\begin{array}{c}\text { Total } \\
\text { drainage } \\
\left(\mathrm{mi}^{2}\right)\end{array}$} & \multicolumn{4}{|c|}{$\begin{array}{c}\text { Maximum stage and discharge for period of record } \\
\text { through } 1998 \text { water year }\end{array}$} & \multicolumn{5}{|c|}{ Significant floods $1994-98$ water years } \\
\hline & & & $\begin{array}{l}\text { Period of } \\
\text { record } \\
\text { (water } \\
\text { years) }\end{array}$ & Water year & $\begin{array}{l}\text { Stage } \\
(\mathrm{ft})\end{array}$ & $\begin{array}{l}\text { Discharge } \\
\left(\mathrm{ft}^{3} / \mathrm{s}\right)\end{array}$ & $\begin{array}{c}\text { Date } \\
\text { (month/ } \\
\text { day/year) }\end{array}$ & $\begin{array}{l}\text { Stage } \\
\text { (ft) }\end{array}$ & $\begin{array}{l}\text { Discharge } \\
\left(\mathrm{ft}^{3} / \mathrm{s}\right)\end{array}$ & $\begin{array}{l}\text { Regulated } \\
\text { during } \\
\text { flood }^{1}\end{array}$ & $\begin{array}{c}\text { Recurrence } \\
\text { interval } \\
\text { (years) }\end{array}$ \\
\hline 02077200 & Hyco Creek near Leasburg, NC & 45.9 & $\begin{array}{l}1965-92 \\
1994-97\end{array}$ & $\begin{array}{l}1996 \\
1995\end{array}$ & $\begin{array}{l}40.47 \\
48.53\end{array}$ & $\begin{array}{r}9,140 \\
--\end{array}$ & $9 / 6 / 96$ & 40.47 & 9,140 & $\mathrm{~N}$ & $25-50$ \\
\hline 02077670 & Mayo Creek near Bethel Hill, NC & 53.5 & 1978-98 & 1978 & 10.83 & 3,950 & $9 / 6 / 96$ & 10.83 & 3,040 & $\mathrm{Y}$ & 25 \\
\hline 02081500 & Tar River near Tar River, NC & 167 & 1940-98 & 1996 & 24.06 & 19,900 & $\begin{array}{l}9 / 6 / 96 \\
3 / 19 / 98\end{array}$ & $\begin{array}{l}24.06 \\
19.36\end{array}$ & $\begin{array}{l}19,900 \\
13,200\end{array}$ & $\begin{array}{l}\mathrm{N} \\
\mathrm{N}\end{array}$ & $\begin{array}{r}50-100 \\
10-25\end{array}$ \\
\hline 02081747 & Tar River at Louisburg, NC & 427 & 1964-98 & 1996 & 25.34 & 21,100 & $\begin{array}{l}9 / 6 / 96 \\
3 / 20 / 98\end{array}$ & $\begin{array}{l}25.34 \\
24.62\end{array}$ & $\begin{array}{l}21,100 \\
18,500\end{array}$ & $\begin{array}{l}\mathrm{N} \\
\mathrm{N}\end{array}$ & $\begin{array}{r}>100 \\
50-100\end{array}$ \\
\hline 02082506 & $\begin{array}{l}\text { Tar River below Tar River } \\
\text { Reservoir near Rocky Mount, NC }\end{array}$ & 777 & 1973-98 & 1998 & 23.67 & 14,700 & $3 / 23 / 98$ & 23.67 & 14,700 & $\mathrm{Y}$ & $>500$ \\
\hline 02082585 & Tar River at Rocky Mount, NC & 925 & 1977-98 & 1996 & 25.88 & 15,100 & $9 / 12 / 96$ & 25.88 & 15,100 & $\mathrm{Y}$ & $>500$ \\
\hline 02084000 & Tar River at Greenville, NC & 2,620 & $\begin{array}{l}1888, \\
1906-76, \\
1997-98\end{array}$ & 1919 & 24.50 & 46,500 & $\begin{array}{l}5 / 7 / 97 \\
3 / 28 / 98\end{array}$ & $\begin{array}{l}14.05 \\
18.08\end{array}$ & $\begin{array}{l}13,900 \\
25,500\end{array}$ & $\begin{array}{l}\mathrm{N} \\
\mathrm{N}\end{array}$ & $\begin{array}{l}-- \\
--\end{array}$ \\
\hline 02084160 & Chicod Creek near Simpson, NC & 45.0 & $\begin{array}{l}1976-87 \\
1992-98\end{array}$ & 1998 & 13.45 & 3,150 & $8 / 27 / 98$ & 13.45 & 3,150 & $\mathrm{~N}$ & 25 \\
\hline 02084909 & Sevenmile Creek near Efland, NC & 14.1 & $1988-98$ & 1996 & 15.47 & 3,440 & $9 / 6 / 96$ & 15.47 & 3,440 & $\mathrm{~N}$ & $>100$ \\
\hline 02085000 & Eno River at Hillsborough, NC & 66.0 & $\begin{array}{l}1928, \\
1930-71, \\
1986-98\end{array}$ & $\begin{array}{l}1945 \\
1996\end{array}$ & $\begin{array}{l}20.01 \\
21.13\end{array}$ & $\begin{array}{l}11,000 \\
10,800\end{array}$ & $9 / 6 / 96$ & 21.13 & 10,800 & $\mathrm{~N}$ & $>100$ \\
\hline 02085070 & Eno River near Durham, NC & 141 & 1964-98 & 1996 & 23.58 & 14,700 & $\begin{array}{l}9 / 6 / 96 \\
3 / 19 / 98\end{array}$ & $\begin{array}{l}23.58 \\
20.76\end{array}$ & $\begin{array}{l}14,700 \\
10,800\end{array}$ & $\begin{array}{l}\mathrm{N} \\
\mathrm{N}\end{array}$ & $\begin{array}{r}10-25 \\
<10\end{array}$ \\
\hline 0208521324 & $\begin{array}{l}\text { Little River at State Road } 1461 \\
\text { near Orange Factory, NC }\end{array}$ & 78.2 & 1988-98 & 1996 & 13.26 & 11,600 & 9/6/96 & 13.26 & 11,600 & $\mathrm{~N}$ & 25 \\
\hline 02085500 & Flat River at Bahama, NC & 149 & $1926-98$ & 1996 & 17.26 & 33,800 & $\begin{array}{l}9 / 6 / 96 \\
3 / 19 / 98\end{array}$ & $\begin{array}{l}17.26 \\
12.05\end{array}$ & $\begin{array}{l}33,800 \\
16,700\end{array}$ & $\begin{array}{l}\mathrm{N} \\
\mathrm{N}\end{array}$ & $\begin{array}{c}200-500 \\
10-25\end{array}$ \\
\hline 0208700780 & $\begin{array}{l}\text { Little Lick Creek above State Road } \\
1814 \text { near Oak Grove, NC }\end{array}$ & 10.1 & 1983-95 & 1995 & 9.33 & 1,830 & $6 / 29 / 95$ & 9.33 & 1,830 & $\mathrm{~N}$ & $10-25$ \\
\hline
\end{tabular}


Table 34. Maximum stage and discharge for period of record for streamgages having significant floods during 1994-98 water years in North Carolina.-Continued

$\left[\mathrm{mi}^{2}\right.$, square miles; $\mathrm{ft}$, feet above an arbitrary datum; $\mathrm{ft}^{3} / \mathrm{s}$, cubic feet per second; --, not determined or not applicable; $>$, greater than; <, less than. Source: Recurrence intervals calculated from U.S. Geological Survey data. Other data from U.S. Geological Survey reports or databases]

\begin{tabular}{|c|c|c|c|c|c|c|c|c|c|c|c|}
\hline \multirow{2}{*}{$\begin{array}{c}\text { Streamgage } \\
\text { number } \\
\text { (fig. 45) }\end{array}$} & \multirow[b]{2}{*}{ Streamgage name } & \multirow{2}{*}{$\begin{array}{c}\text { Total } \\
\text { drainage } \\
\left(\mathrm{mi}^{2}\right)\end{array}$} & \multicolumn{4}{|c|}{$\begin{array}{c}\text { Maximum stage and discharge for period of record } \\
\text { through } 1998 \text { water year }\end{array}$} & \multicolumn{5}{|c|}{ Significant floods $1994-98$ water years } \\
\hline & & & $\begin{array}{l}\text { Period of } \\
\text { record } \\
\text { (water } \\
\text { years) }\end{array}$ & Water year & $\begin{array}{l}\text { Stage } \\
(\mathrm{ft})\end{array}$ & $\begin{array}{l}\text { Discharge } \\
\left(\mathrm{ft}^{3} / \mathrm{s}\right)\end{array}$ & $\begin{array}{c}\text { Date } \\
\text { (month/ } \\
\text { day/year) }\end{array}$ & $\begin{array}{l}\text { Stage } \\
(\mathrm{ft})\end{array}$ & $\begin{array}{l}\text { Discharge } \\
\left(\mathrm{ft}^{3} / \mathrm{s}\right)\end{array}$ & $\begin{array}{l}\text { Regulated } \\
\text { during } \\
\text { flood }^{1}\end{array}$ & $\begin{array}{c}\text { Recurrence } \\
\text { interval } \\
\text { (years) }\end{array}$ \\
\hline 02087183 & Neuse River near Falls, NC & 771 & $\begin{array}{l}1945, \\
1971-98\end{array}$ & 1975 & 25.21 & 13,600 & $9 / 16 / 96$ & 8.05 & 7,650 & $\mathrm{Y}$ & $25-50$ \\
\hline 0208732885 & Marsh Creek near New Hope, NC & 6.84 & 1984-98 & $\begin{array}{l}1995 \\
1996\end{array}$ & $\begin{array}{l}12.84 \\
13.33\end{array}$ & $\begin{array}{l}4,140 \\
3,900\end{array}$ & $8 / 27 / 95$ & 12.84 & 4,140 & $\mathrm{~N}$ & 25 \\
\hline 02087500 & Neuse River near Clayton, NC & 1,150 & $\begin{array}{l}\text { 1919, } \\
1928-98\end{array}$ & 1945 & 22.12 & 22,900 & $9 / 7 / 96$ & 20.12 & 19,700 & $\mathrm{Y}$ & $50-100$ \\
\hline 0208758850 & $\begin{array}{l}\text { Swift Creek near McCullars } \\
\text { Crossroads, NC }\end{array}$ & 35.8 & 1989-98 & 1996 & 14.15 & 6,790 & $9 / 6 / 96$ & 14.15 & 6,790 & $\mathrm{~N}$ & $25-50$ \\
\hline 02088000 & Middle Creek near Clayton, NC & 83.5 & 1940-98 & 1996 & 14.88 & 11,900 & $9 / 6 / 96$ & 14.88 & 11,900 & $\mathrm{~N}$ & $200-500$ \\
\hline 02088500 & Little River near Princeton, NC & 232 & $\begin{array}{l}\text { 1919, 1924, } \\
1928, \\
1930-98\end{array}$ & $\begin{array}{l}1965 \\
1924\end{array}$ & $\begin{array}{l}13.94 \\
14.90\end{array}$ & $\begin{array}{r}7,150 \\
--\end{array}$ & $\begin{array}{l}9 / 9 / 96 \\
3 / 11 / 98\end{array}$ & $\begin{array}{l}13.32 \\
13.51\end{array}$ & $\begin{array}{l}5,480 \\
5,150\end{array}$ & $\begin{array}{l}\mathrm{N} \\
\mathrm{N}\end{array}$ & $\begin{array}{r}25 \\
10-25\end{array}$ \\
\hline 0208925200 & Bear Creek at Mays Store, NC & 57.7 & 1988-98 & 1997 & 9.50 & 1,550 & $10 / 9 / 96$ & 9.50 & 1,550 & $\mathrm{~N}$ & $<5$ \\
\hline 02089500 & Neuse River at Kinston, NC & 2,692 & $\begin{array}{l}1919,1924, \\
1928-98\end{array}$ & 1919 & 25.00 & 39,000 & $9 / 17 / 96$ & 23.26 & 27,100 & $\mathrm{Y}$ & $50-100$ \\
\hline 02096500 & Haw River at Haw River, NC & 606 & $1929-98$ & 1996 & 32.83 & 51,400 & $\begin{array}{l}6 / 29 / 95 \\
9 / 6 / 96\end{array}$ & $\begin{array}{l}28.46 \\
32.83\end{array}$ & $\begin{array}{l}29,500 \\
51,400\end{array}$ & $\begin{array}{l}\mathrm{N} \\
\mathrm{N}\end{array}$ & $\begin{array}{r}10-25 \\
200\end{array}$ \\
\hline 02096846 & $\begin{array}{l}\text { Cane Creek near Orange Grove, } \\
\text { NC }\end{array}$ & 7.54 & 1989-98 & 1996 & 7.90 & 2,060 & $9 / 6 / 96$ & 7.90 & 2,060 & $\mathrm{~N}$ & 25 \\
\hline 02096960 & Haw River near Bynum, NC & 1,275 & 1974-98 & 1996 & 21.76 & 76,700 & $9 / 6 / 96$ & 21.76 & 76,700 & $\mathrm{~N}$ & $>100$ \\
\hline 02097314 & New Hope Creek near Blands, NC & 75.9 & 1983-98 & 1996 & 14.05 & 12,700 & $9 / 6 / 96$ & 14.05 & 12,700 & $\mathrm{~N}$ & $100-200$ \\
\hline 0209741955 & $\begin{array}{l}\text { Northeast Creek at State Road } \\
1100 \text { near Genlee, NC }\end{array}$ & 21.1 & $\begin{array}{l}1983-93 \\
1996-98\end{array}$ & 1996 & 13.92 & 5,140 & $9 / 6 / 96$ & 13.92 & 5,140 & $\mathrm{~N}$ & $25-50$ \\
\hline 02097517 & $\begin{array}{l}\text { Morgan Creek near Chapel Hill, } \\
\text { NC }\end{array}$ & 41.0 & 1983-98 & 1996 & 16.18 & 4,210 & $9 / 6 / 96$ & 16.18 & 4,210 & $\mathrm{~N}$ & 50 \\
\hline 02099500 & Deep River near Randleman, NC & 125 & $\begin{array}{l}1929-31 \\
1934-98\end{array}$ & 1947 & 32.20 & 20,000 & $9 / 6 / 96$ & 28.75 & 15,600 & $\mathrm{~N}$ & $50-100$ \\
\hline
\end{tabular}


Table 34. Maximum stage and discharge for period of record for streamgages having significant floods during 1994-98 water years in North Carolina.-Continued

$\left[\mathrm{mi}^{2}\right.$, square miles; $\mathrm{ft}$, feet above an arbitrary datum; $\mathrm{ft}^{3} / \mathrm{s}$, cubic feet per second; --, not determined or not applicable; $>$, greater than; <, less than. Source: Recurrence intervals calculated from U.S. Geological Survey data. Other data from U.S. Geological Survey reports or databases]

\begin{tabular}{|c|c|c|c|c|c|c|c|c|c|c|c|}
\hline \multirow[b]{2}{*}{$\begin{array}{c}\text { Streamgage } \\
\text { number } \\
\text { (fig. 45) }\end{array}$} & \multirow[b]{2}{*}{ Streamgage name } & \multirow[b]{2}{*}{$\begin{array}{c}\text { Total } \\
\text { drainage } \\
\left(\mathrm{mi}^{2}\right)\end{array}$} & \multicolumn{4}{|c|}{$\begin{array}{c}\text { Maximum stage and discharge for period of record } \\
\text { through } 1998 \text { water year }\end{array}$} & \multicolumn{5}{|c|}{ Significant floods 1994-98 water years } \\
\hline & & & $\begin{array}{l}\text { Period of } \\
\text { record } \\
\text { (water } \\
\text { years) }\end{array}$ & Water year & $\begin{array}{l}\text { Stage } \\
\text { (ft) }\end{array}$ & $\begin{array}{l}\text { Discharge } \\
\left(\mathrm{ft}^{3} / \mathrm{s}\right)\end{array}$ & $\begin{array}{c}\text { Date } \\
\text { (month/ } \\
\text { day/year) }\end{array}$ & $\begin{array}{l}\text { Stage } \\
\text { (ft) }\end{array}$ & $\begin{array}{l}\text { Discharge } \\
\left(\mathrm{ft}^{3} / \mathrm{s}\right)\end{array}$ & $\begin{array}{c}\text { Regulated } \\
\text { during } \\
\text { flood }^{1}\end{array}$ & $\begin{array}{c}\text { Recurrence } \\
\text { interval } \\
\text { (years) }\end{array}$ \\
\hline 02101800 & $\begin{array}{l}\text { Tick Creek near Mount Vernon } \\
\text { Springs, NC }\end{array}$ & 15.5 & $\begin{array}{l}\text { 1959-81, } \\
1994-98\end{array}$ & 1996 & 13.41 & 4,010 & $9 / 6 / 96$ & 13.41 & 4,010 & $\mathrm{~N}$ & 25 \\
\hline 02102000 & Deep River at Moncure, NC & 1,434 & $1931-98$ & 1945 & 17.20 & 80,300 & $9 / 6 / 96$ & 12.94 & 47,900 & $\mathrm{~N}$ & 50 \\
\hline 02102192 & Buckhorn Creek near Corinth, NC & 76.3 & 1973-98 & 1973 & 20.02 & 6,920 & 9/6/96 & 16.79 & 4,300 & $\mathrm{Y}$ & $25-50$ \\
\hline 02102500 & Cape Fear River at Lillington, NC & 3,464 & 1924-98 & 1945 & 33.19 & 150,000 & 9/7/96 & 18.97 & 51,800 & $\mathrm{Y}$ & 50 \\
\hline 02105500 & $\begin{array}{l}\text { Cape Fear River at Wilm O Huske } \\
\text { Lock near Tarheel, NC }\end{array}$ & 4,852 & $\begin{array}{l}1938-94 \\
1997-98\end{array}$ & $\begin{array}{l}1952 \\
1945\end{array}$ & $\begin{array}{l}29.92 \\
43.44\end{array}$ & $\begin{array}{r}70,600 \\
--\end{array}$ & $3 / 22 / 98$ & 26.28 & 47,100 & $\mathrm{Y}$ & $>500$ \\
\hline 02105900 & Hood Creek near Leland, NC & 21.6 & $\begin{array}{l}\text { 1953-73, } \\
1994-98\end{array}$ & 1998 & 11.53 & 2,650 & $8 / 27 / 98$ & 11.53 & 2,650 & $\mathrm{~N}$ & $25-50$ \\
\hline 02106500 & Black River near Tomahawk, NC & 676 & $\begin{array}{l}1928,1945, \\
1948, \\
1952-98\end{array}$ & $\begin{array}{l}1984 \\
1928\end{array}$ & $\begin{array}{l}22.08 \\
47.00\end{array}$ & $\begin{array}{l}17,500 \\
11,400\end{array}$ & 9/10/96 & 21.31 & 13,700 & $\mathrm{~N}$ & $25-50$ \\
\hline 02109500 & Waccamaw River at Freeland, NC & 680 & 1940-98 & 1996 & 17.02 & 12,400 & $9 / 12 / 96$ & 17.02 & 12,400 & $\mathrm{~N}$ & 50 \\
\hline 02111000 & Yadkin River at Patterson, NC & 28.8 & $\begin{array}{l}1940-43 \\
1945-98\end{array}$ & 1973 & 12.70 & 16,200 & $8 / 17 / 94$ & 9.38 & 5,750 & $\mathrm{~N}$ & $10-25$ \\
\hline 02111180 & Elk Creek at Elkville, NC & 48.1 & $\begin{array}{l}1940 \\
1966-98\end{array}$ & 1940 & 22.00 & 70,000 & $8 / 17 / 94$ & 12.02 & 18,700 & $\mathrm{~N}$ & 25 \\
\hline 02120780 & Second Creek near Barber, NC & 118 & 1980-98 & 1995 & 17.28 & 8,560 & $8 / 28 / 95$ & 17.28 & 8,560 & $\mathrm{~N}$ & $100-200$ \\
\hline 02125000 & Big Bear Creek near Richfield, NC & 55.6 & 1955-98 & 1997 & 16.54 & 11,400 & $7 / 23 / 97$ & 16.54 & 11,400 & $\mathrm{~N}$ & 25 \\
\hline 02128000 & Little River near Star, NC & 106 & $\begin{array}{l}\text { 1955-93, } \\
1995-98\end{array}$ & 1997 & 18.60 & 15,400 & $\begin{array}{l}7 / 23 / 97 \\
3 / 19 / 98\end{array}$ & $\begin{array}{l}18.60 \\
15.84\end{array}$ & $\begin{array}{l}15,400 \\
11,300\end{array}$ & $\begin{array}{l}\mathrm{N} \\
\mathrm{N}\end{array}$ & $\begin{array}{c}200-500 \\
50-100\end{array}$ \\
\hline 02133624 & Lumber River near Maxton, NC & 365 & $\begin{array}{l}\text { 1988-92, } \\
1994-98\end{array}$ & 1998 & 13.52 & 3,380 & $3 / 22 / 98$ & 13.52 & 3,380 & $\mathrm{~N}$ & $10-25$ \\
\hline 02134480 & Big Swamp near Tarheel, NC & 229 & $\begin{array}{l}1986-95 \\
1997-98\end{array}$ & $\begin{array}{l}1998 \\
1993\end{array}$ & $\begin{array}{l}13.10 \\
13.34\end{array}$ & $\begin{array}{l}3,980 \\
2,840\end{array}$ & $3 / 11 / 98$ & 13.10 & 3,980 & $\mathrm{~N}$ & $10-25$ \\
\hline 02137727 & $\begin{array}{l}\text { Catawba River near Pleasant } \\
\text { Gardens, NC }\end{array}$ & 127 & $1981-98$ & 1994 & 15.22 & 13,700 & $8 / 17 / 94$ & 15.22 & 13,700 & $\mathrm{~N}$ & $10-25$ \\
\hline
\end{tabular}


$\left[\mathrm{mi}^{2}\right.$, square miles; $\mathrm{ft}$, feet above an arbitrary datum; $\mathrm{ft}^{3} / \mathrm{s}$, cubic feet per second; --, not determined or not applicable; >, greater than; <, less than. Source: Recurrence intervals calculated from U.S. Geological Survey data. Other data from U.S. Geological Survey reports or databases]

\begin{tabular}{|c|c|c|c|c|c|c|c|c|c|c|c|}
\hline \multirow{2}{*}{$\begin{array}{l}\text { Streamgage } \\
\text { number } \\
\text { (fig. 45) }\end{array}$} & \multirow[b]{2}{*}{ Streamgage name } & \multirow{2}{*}{$\begin{array}{c}\text { Total } \\
\text { drainage } \\
\left(\mathrm{mi}^{2}\right)\end{array}$} & \multicolumn{4}{|c|}{$\begin{array}{l}\text { Maximum stage and discharge for period of record } \\
\text { through } 1998 \text { water year }\end{array}$} & \multicolumn{5}{|c|}{ Significant floods 1994-98 water years } \\
\hline & & & $\begin{array}{l}\text { Period of } \\
\text { record } \\
\text { (water } \\
\text { years) }\end{array}$ & Water year & $\begin{array}{l}\text { Stage } \\
\text { (ft) }\end{array}$ & $\begin{array}{l}\text { Discharge } \\
\left(\mathrm{ft}^{3} / \mathrm{s}\right)\end{array}$ & $\begin{array}{c}\text { Date } \\
\text { (month/ } \\
\text { day/year) }\end{array}$ & $\begin{array}{l}\text { Stage } \\
(\mathrm{ft})\end{array}$ & $\begin{array}{l}\text { Discharge } \\
\left(\mathrm{ft}^{3} / \mathrm{s}\right)\end{array}$ & $\begin{array}{l}\text { Regulated } \\
\text { during } \\
\text { flood }^{1}\end{array}$ & $\begin{array}{c}\text { Recurrence } \\
\text { interval } \\
\text { (years) }\end{array}$ \\
\hline 02138500 & Linville River near Nebo, NC & 66.7 & $\begin{array}{l}\text { 1916, } \\
1923-98\end{array}$ & 1940 & 11.40 & 39,500 & $8 / 17 / 94$ & 10.52 & 18,600 & $\mathrm{~N}$ & 25 \\
\hline 02140991 & Johns River at Arneys Store, NC & 201 & $1986-98$ & 1994 & 25.23 & 42,300 & $8 / 17 / 94$ & 25.23 & 42,300 & $\mathrm{~N}$ & $25-50$ \\
\hline 0214253830 & $\begin{array}{l}\text { Norwood Creek near Troutman, } \\
\text { NC }\end{array}$ & 7.18 & 1984-98 & 1997 & 9.20 & 1,480 & $4 / 28 / 97$ & 9.20 & 1,480 & $\mathrm{~N}$ & $10-25$ \\
\hline 02146300 & Irwin Creek near Charlotte, NC & 30.7 & 1963-98 & 1997 & 20.38 & 11,600 & $7 / 23 / 97$ & 20.38 & 11,600 & $\mathrm{~N}$ & 500 \\
\hline 02146470 & $\begin{array}{l}\text { Little Hope Creek at Seneca Place, } \\
\text { Charlotte, NC }\end{array}$ & 2.63 & $\begin{array}{l}\text { 1967-72, } \\
\text { 1983-86, } \\
\text { 1988-90, } \\
1995-98\end{array}$ & 1997 & 8.50 & 1,700 & $7 / 23 / 97$ & 8.50 & 1,700 & $\mathrm{~N}$ & 50 \\
\hline 02146507 & $\begin{array}{l}\text { Little Sugar Creek at Archdale } \\
\text { Drive at Charlotte, NC }\end{array}$ & 42.6 & 1978-98 & 1997 & 15.06 & 13,600 & $7 / 23 / 97$ & 15.06 & 13,600 & $\mathrm{~N}$ & 500 \\
\hline 02146600 & $\begin{array}{l}\text { McAlpine Creek at Sardis Road } \\
\text { near Charlotte, NC }\end{array}$ & 39.6 & 1962-98 & 1995 & 17.79 & 9,040 & $8 / 27 / 95$ & 17.79 & 9,040 & $\mathrm{~N}$ & $>200$ \\
\hline 02146700 & $\begin{array}{l}\text { McMullen Creek at Sharon View } \\
\text { Road near Charlotte, NC }\end{array}$ & 6.95 & $1963-98$ & 1995 & 11.03 & 3,470 & $\begin{array}{l}8 / 27 / 95 \\
7 / 27 / 98\end{array}$ & $\begin{array}{l}11.03 \\
10.64\end{array}$ & $\begin{array}{l}3,470 \\
3,230\end{array}$ & $\begin{array}{l}\mathrm{N} \\
\mathrm{N}\end{array}$ & $\begin{array}{r}50 \\
25-50\end{array}$ \\
\hline 02146750 & $\begin{array}{l}\text { McAlpine Creek below McMullen } \\
\text { Creek near Pineville, NC }\end{array}$ & 92.4 & 1975-98 & 1995 & 19.40 & 12,500 & $8 / 27 / 95$ & 19.40 & 12,500 & $\mathrm{~N}$ & 200 \\
\hline 02146900 & $\begin{array}{l}\text { Twelve Mile Creek near Waxhaw, } \\
\text { NC }\end{array}$ & 76.5 & $\begin{array}{l}1949 \\
1961-98\end{array}$ & $\begin{array}{l}1995 \\
1949\end{array}$ & $\begin{array}{l}21.94 \\
23.60\end{array}$ & $\begin{array}{r}9,970 \\
--\end{array}$ & $8 / 27 / 95$ & 21.94 & 9,970 & $\mathrm{~N}$ & $50-100$ \\
\hline 02152100 & First Broad River near Casar, NC & 60.5 & $\begin{array}{l}\text { 1960-95, } \\
\text { 1997-98 }\end{array}$ & $\begin{array}{l}1995 \\
1976\end{array}$ & $\begin{array}{l}15.58 \\
16.70\end{array}$ & $\begin{array}{l}7,790 \\
7,760\end{array}$ & $1 / 14 / 95$ & 15.58 & 7,790 & $\mathrm{~N}$ & $10-25$ \\
\hline 03161000 & $\begin{array}{l}\text { South Fork New River near } \\
\text { Jefferson, NC }\end{array}$ & 205 & $\begin{array}{l}1916, \\
1925-26, \\
1929-41, \\
1943-98\end{array}$ & 1940 & 22.50 & 52,800 & $1 / 15 / 95$ & 13.73 & 21,000 & $\mathrm{~N}$ & 50 \\
\hline 03439000 & $\begin{array}{l}\text { French Broad River at Rosman, } \\
\text { NC }\end{array}$ & 67.9 & $\begin{array}{l}\text { 1908-09, } \\
\text { 1916, 1928, } \\
\text { 1936-89, } \\
1991-98\end{array}$ & 1965 & 14.95 & 13,500 & 8/17/94 & 14.12 & 11,500 & $\mathrm{~N}$ & 50 \\
\hline
\end{tabular}


Table 34. Maximum stage and discharge for period of record for streamgages having significant floods during 1994-98 water years in North Carolina.-Continued

$\left[\mathrm{mi}^{2}\right.$, square miles; $\mathrm{ft}$, feet above an arbitrary datum; $\mathrm{ft}^{3} / \mathrm{s}$, cubic feet per second; --, not determined or not applicable; $>$, greater than; <, less than. Source: Recurrence intervals calculated from U.S. Geological Survey data. Other data from U.S. Geological Survey reports or databases]

\begin{tabular}{|c|c|c|c|c|c|c|c|c|c|c|c|}
\hline \multirow{2}{*}{$\begin{array}{c}\text { Streamgage } \\
\text { number } \\
\text { (fig. 45) }\end{array}$} & \multirow[b]{2}{*}{ Streamgage name } & \multirow{2}{*}{$\begin{array}{c}\text { Total } \\
\text { drainage } \\
\left(\mathrm{mi}^{2}\right)\end{array}$} & \multicolumn{4}{|c|}{$\begin{array}{c}\text { Maximum stage and discharge for period of record } \\
\text { through } 1998 \text { water year }\end{array}$} & \multicolumn{5}{|c|}{ Significant floods $1994-98$ water years } \\
\hline & & & $\begin{array}{l}\text { Period of } \\
\text { record } \\
\text { (water } \\
\text { years) }\end{array}$ & Water year & $\begin{array}{l}\text { Stage } \\
(\mathrm{ft})\end{array}$ & $\begin{array}{l}\text { Discharge } \\
\left(\mathrm{ft}^{3} / \mathrm{s}\right)\end{array}$ & $\begin{array}{c}\text { Date } \\
\text { (month/ } \\
\text { day/year) }\end{array}$ & $\begin{array}{l}\text { Stage } \\
(\mathrm{ft})\end{array}$ & $\begin{array}{l}\text { Discharge } \\
\left(\mathrm{ft}^{3} / \mathrm{s}\right)\end{array}$ & $\begin{array}{l}\text { Regulated } \\
\text { during } \\
\text { flood }^{1}\end{array}$ & $\begin{array}{c}\text { Recurrence } \\
\text { interval } \\
\text { (years) }\end{array}$ \\
\hline 03441000 & Davidson River near Brevard, NC & 40.4 & $\begin{array}{l}\text { 1876, 1916, } \\
1919, \\
1921-90, \\
1924-98\end{array}$ & $\begin{array}{l}1928 \\
1994\end{array}$ & $\begin{array}{l}11.80 \\
12.08\end{array}$ & $\begin{array}{l}8,400 \\
7,620\end{array}$ & $8 / 17 / 94$ & 12.08 & 7,620 & $\mathrm{~N}$ & $25-50$ \\
\hline 03443000 & $\begin{array}{l}\text { French Broad River at Blantyre, } \\
\text { NC }\end{array}$ & 296 & $\begin{array}{l}1875-76, \\
1880,1893, \\
1899, \\
1901-02, \\
1905-06, \\
1910,1916, \\
1919, \\
1921-98\end{array}$ & $\begin{array}{l}1965 \\
1916\end{array}$ & $\begin{array}{l}25.50 \\
27.10\end{array}$ & $\begin{array}{r}30,000 \\
--\end{array}$ & $\begin{array}{l}8 / 18 / 94 \\
1 / 8 / 98\end{array}$ & $\begin{array}{l}23.81 \\
22.61\end{array}$ & $\begin{array}{l}21,200 \\
19,400\end{array}$ & $\begin{array}{l}\mathrm{N} \\
\mathrm{N}\end{array}$ & $\begin{array}{r}50 \\
25-50\end{array}$ \\
\hline 03450000 & $\begin{array}{l}\text { Beetree Creek near Swannanoa, } \\
\text { NC }\end{array}$ & 5.46 & $\begin{array}{l}1927-75, \\
1978, \\
1980-81, \\
1986, \\
1988-98\end{array}$ & 1940 & 6.20 & 1,370 & $8 / 17 / 94$ & 5.75 & 1,040 & $\mathrm{~N}$ & $50-100$ \\
\hline 03456100 & $\begin{array}{l}\text { West Fork Pigeon River at Bethel, } \\
\text { NC }\end{array}$ & 58.4 & 1981-98 & 1994 & 12.63 & 11,700 & $8 / 17 / 94$ & 12.63 & 11,700 & $\mathrm{Y}$ & $25-50$ \\
\hline 03456500 & $\begin{array}{l}\text { East Fork Pigeon River near } \\
\text { Canton, NC }\end{array}$ & 51.5 & $1955-98$ & 1973 & 11.19 & 12,000 & $8 / 17 / 94$ & 10.70 & 11,100 & $\mathrm{~N}$ & $10-25$ \\
\hline 03460000 & $\begin{array}{l}\text { Cataloochee Creek near } \\
\text { Cataloochee, } \mathrm{NC}\end{array}$ & 49.2 & $\begin{array}{l}1935-52 \\
1963-98\end{array}$ & 1963 & 8.08 & 5,080 & $3 / 27 / 94$ & 7.55 & 4,280 & $\mathrm{~N}$ & $10-25$ \\
\hline 03463300 & South Toe River near Celo, NC & 43.3 & $1958-98$ & 1978 & 17.41 & 32,900 & $8 / 17 / 94$ & 10.49 & 15,600 & $\mathrm{~N}$ & $10-25$ \\
\hline 03479000 & $\begin{array}{l}\text { Watauga River near Sugar Grove, } \\
\text { NC }\end{array}$ & 92.1 & $\begin{array}{l}\text { 1916, } \\
1940-98\end{array}$ & 1940 & 29.60 & 50,800 & $1 / 14 / 95$ & 20.46 & 20,500 & $\mathrm{~N}$ & 25 \\
\hline 03509000 & Scott Creek above Sylva, NC & 51.0 & $\begin{array}{l}1940, \\
1942-75 \\
1993-95\end{array}$ & $\begin{array}{l}1994 \\
1973\end{array}$ & $\begin{array}{l}6.77 \\
8.78\end{array}$ & $\begin{array}{l}4,440 \\
2,800\end{array}$ & $3 / 27 / 94$ & 6.77 & 4,440 & $\mathrm{~N}$ & 200 \\
\hline 03513000 & $\begin{array}{l}\text { Tuckasegee River at Bryson City, } \\
\text { NC }\end{array}$ & 655 & $\begin{array}{l}\text { 1898-1982, } \\
\text { 1984-95, } \\
1997-98\end{array}$ & 1940 & 15.96 & 61,600 & $3 / 28 / 94$ & 14.25 & 33,300 & $\mathrm{Y}$ & $10-25$ \\
\hline
\end{tabular}


$\left[\mathrm{mi}^{2}\right.$, square miles; $\mathrm{ft}$, feet above an arbitrary datum; $\mathrm{ft}^{3} / \mathrm{s}$, cubic feet per second; --, not determined or not applicable; $>$, greater than; <, less than. Source: Recurrence intervals calculated from U.S. Geological Survey data. Other data from U.S. Geological Survey reports or databases]

\begin{tabular}{|c|c|c|c|c|c|c|c|c|c|c|c|}
\hline \multirow[b]{2}{*}{$\begin{array}{l}\text { Streamgage } \\
\text { number } \\
\text { (fig. 45) }\end{array}$} & \multirow[b]{2}{*}{ Streamgage name } & \multirow{2}{*}{$\begin{array}{c}\text { Total } \\
\text { drainage } \\
\left(\mathrm{mi}^{2}\right)\end{array}$} & \multicolumn{4}{|c|}{$\begin{array}{c}\text { Maximum stage and discharge for period of record } \\
\text { through } 1998 \text { water year }\end{array}$} & \multicolumn{5}{|c|}{ Significant floods 1994-98 water years } \\
\hline & & & $\begin{array}{l}\text { Period of } \\
\text { record } \\
\text { (water } \\
\text { years) }\end{array}$ & Water year & $\begin{array}{l}\text { Stage } \\
(\mathrm{ft})\end{array}$ & $\begin{array}{c}\text { Discharge } \\
\left(\mathrm{ft}^{3} / \mathrm{s}\right)\end{array}$ & $\begin{array}{c}\text { Date } \\
\text { (month/ } \\
\text { day/year) }\end{array}$ & $\begin{array}{l}\text { Stage } \\
(\mathrm{ft})\end{array}$ & $\begin{array}{c}\text { Discharge } \\
\left(\mathrm{ft}^{3} / \mathrm{s}\right)\end{array}$ & $\begin{array}{l}\text { Regulated } \\
\text { during } \\
\text { flood }^{1}\end{array}$ & $\begin{array}{c}\text { Recurrence } \\
\text { interval } \\
\text { (years) }\end{array}$ \\
\hline 03550000 & Valley River at Tomotla, NC & 104 & $\begin{array}{l}1898, \\
1905-09, \\
1915-17, \\
1919-98\end{array}$ & $\begin{array}{l}1907 \\
1898\end{array}$ & $\begin{array}{l}20.50 \\
21.20\end{array}$ & $\begin{array}{l}18,000 \\
20,000\end{array}$ & $\begin{array}{l}3 / 27 / 94 \\
2 / 16 / 95\end{array}$ & $\begin{array}{l}17.10 \\
17.15\end{array}$ & $\begin{array}{l}11,900 \\
12,100\end{array}$ & $\begin{array}{l}\mathrm{N} \\
\mathrm{N}\end{array}$ & $\begin{array}{r}25-50 \\
50\end{array}$ \\
\hline
\end{tabular}




\section{North Dakota}

During the winter of 1993-94, above-average precipitation in Montana resulted in deeper than normal snowpacks in river basins that drain east into North Dakota. In January 1994, record snowfalls occurred in much of the western part of North Dakota. During February and March, additional snowfalls of several inches added to the ever-increasing snowpack. By early March, a rapid warm-up caused snowmelt and ice jams to occur on several rivers and streams in eastern Montana and western North Dakota. Flooding occurred along the Yellowstone River (fig. 46). High water flowed eastward into the Missouri River and caused record stages along the way. A maximum daily gage height of 26.60 feet for the period of record occurred on March 8, 1994, on the Missouri River near Williston (streamgage 06330000 , table 35). About 10,000 acres of prime farmland were flooded in the northwestern part of the State. Property and crop damage in eastern Montana and western North Dakota were estimated to be about $\$ 5$ million (National Oceanic and Atmospheric Administration, 1994b).

During the spring of 1996, alternating warm and cold periods resulted in multiple snowmelt discharges in February, March, and April over all of the State except the northeastern one-third. By having the snowmelt runoff in three separate months rather than one, few record discharges resulted.

Excessive runoff in the upper Sheyenne River Basin primarily occurred during April 1996. Because of the sudden increase of runoff into Lake Ashtabula, high release rates were required to avoid an uncontrolled flow through the emergency spillway. A peak stage of 36.46 feet occurred on April 20, 1996, at the Sheyenne River below Baldhill Dam (streamgage 05058000 , table 35 ), which is 0.20 foot higher than the previous record set in 1979. A peak stage of 19.20 feet occurred on April 13, 1996, on the Sheyenne River at Lisbon (streamgage 05058700 , table 35 ), which is 0.16 foot higher than the previous record set in 1975 .

The harsh winter of 1996-97, combined with the 1997 spring floods, caused the worst natural disaster in recent history for North Dakota, eastern South Dakota, and western Minnesota. Above-normal snowfall in central and eastern North Dakota during the winter of 1996-97 and a blizzard on April 56,1997 , caused the worst flooding in the Red River of the North and Missouri River Basins in more than 100 years.

The heaviest snowfalls occurred along the main stems of the Red River of the North and the Missouri River and were about 300 percent greater than normal. About 117 inches of

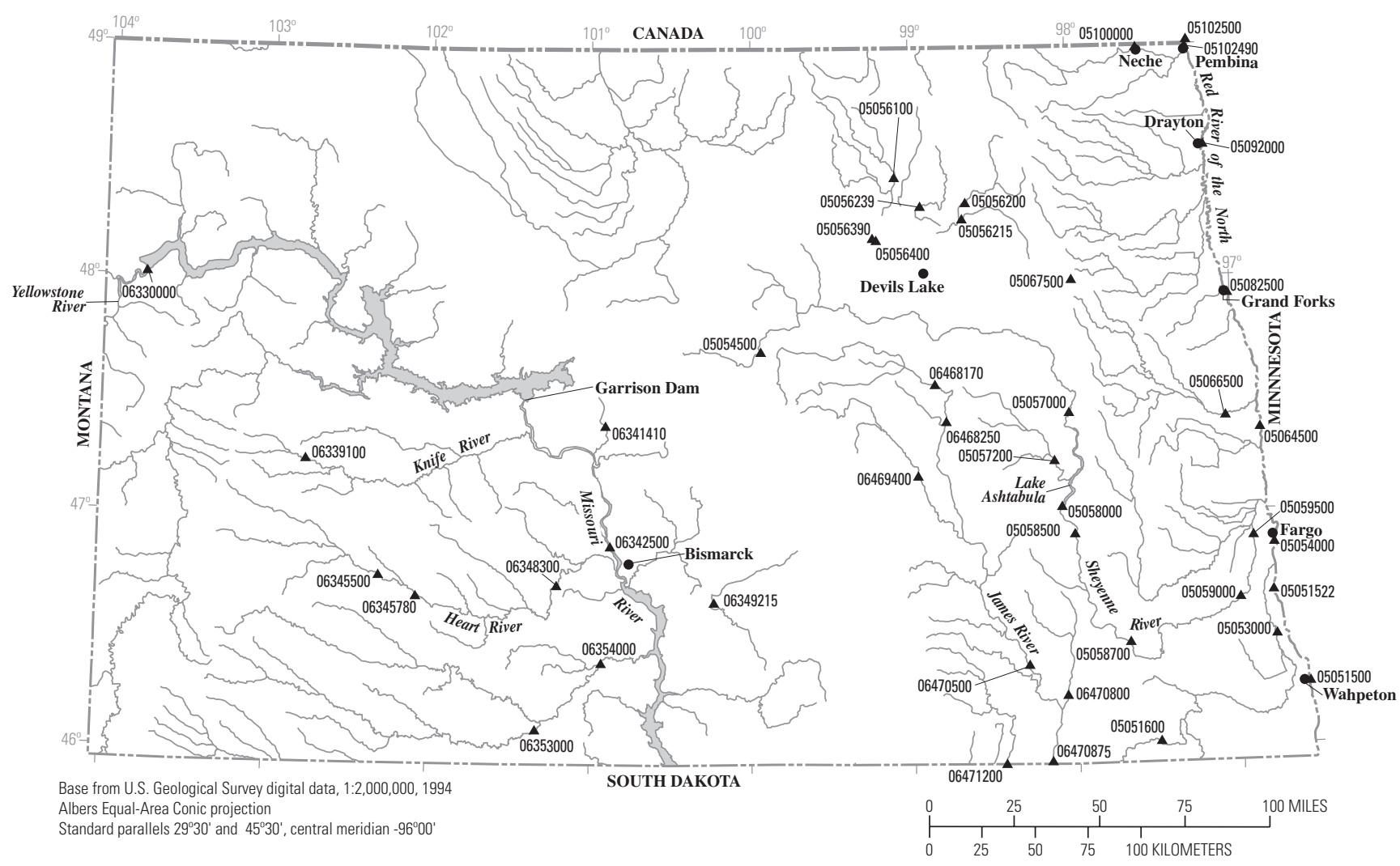

EXPLANATION

$06353000 \_$Streamgage and number

Figure 46. Location of streamgages with significant floods during 1994-98 water years for North Dakota. 
snow were recorded in Fargo, 96 inches in Grand Forks, and 101 inches in Bismarck (National Oceanic and Atmospheric Administration, 1996a). Elsewhere in the region, snowfalls were well above seasonal averages. Melting of the snowpack and thawing of ice began in late March on rivers and streams in the southern and western parts of the State. Flows were inhibited by a blizzard that occurred on April 5-6, 1997. The blizzard brought a severe drop in temperatures, winds up to 70 miles per hour, and up to 2 feet of snow with drifts many feet higher in several areas. In southeastern North Dakota, the blizzard was preceded by wind-driven rain and sleet. The wind and ice toppled trees and power lines, leaving thousands of people without power for days. Thousands of people were forced to flee their homes, some permanently, as floodwaters and severe weather caused over $\$ 5$ billion in damage to the region (National Oceanic and Atmospheric Administration, 1997b).

The Red River of the North is one of the few rivers in the United States to flow directly north into Canada. The basin flood plain lies in a glacial lakebed and is relatively flat (less than 0.5-foot drop in elevation per mile in the reach downstream from Grand Forks, North Dakota). Because of the flat basin, the shallow river channel, and the northerly flow, the timing of spring thaw and snowmelt can greatly aggravate flooding in the basin. Snow and ice in the headwaters of the Red River of the North begin to melt first, when areas downstream remain largely frozen. The melt pattern can cause ice jams to form, and substantial backwater can occur as flow moves northward toward a still-frozen river channel.

Two peak stages occurred in Wahpeton, which is located in the southern part of the Red River of the North Basin. On April 6, 1997, the stage of the Red River of the North at Wahpeton (streamgage 05051500, table 35) was 19.42 feet, which is 1.47 feet higher than the record set in 1989. Because of the additional moisture from the April 5-6, 1997, blizzard, a record flow of 12,800 cubic feet per second and corresponding stage of 19.25 feet was recorded 9 days later. On April 17, 1997, the peak stage of the Red River of the North at Fargo (streamgage 05054000 , table 35), about 96 river miles north of Wahpeton, was 39.57 feet, and the peak flow was 28,000 cubic feet per second. On April 18, 1997, the peak stage at Fargo was 39.72 feet, which exceeded the record set 100 years earlier, and the peak flow was 27,700 cubic feet per second.

High flows continued to move downstream in the Red River of the North. On April 18, 1997, the peak stage of the Red River of the North at Grand Forks (streamgage 05082500, table 35) was 52.04 feet, which is 1.84 feet higher than the record set in 1897, and the peak flow was 137,000 cubic feet per second. The peak flow was unusual because it resulted from the convergence of flows from the Red Lake River in Minnesota, flows from the main channel, and breakout flows from the Red River of the North that were conveyed by old Red River of the North oxbows. Breakout flows occurred upstream from Grand Forks when plugs in the upstream end of the oxbows either were overtopped or washed away, which caused a flow of about 25,000 cubic feet per second to arrive at the confluence of the Red Lake River and the Red River of the North at Grand Forks.
The flow of 25,000 cubic feet per second coincided with the peak flow of the two rivers. To compound problems in Grand Forks, a fire on April 19, 1997, demolished several buildings in the flooded city. The flooding made it extremely difficult for firefighters to reach the fires and put them out. Except for emergency personnel, Grand Forks and its sister city, East Grand Forks, Minnesota, were completely evacuated at this time.

On April 24, 1997, the peak stage at the Red River of the North at Drayton (streamgage 05092000, table 35) was 45.55 feet, which is 1.89 feet higher than the record set in 1979, and the peak flow was 124,000 cubic feet per second. At the Pembina River at Neche (streamgage 05100000 , table 35), the peak stage was 24.51 feet, which is 0.87 foot higher than the record set in 1979, and the flow was 12,800 cubic feet per second. Six days later, the peak flow was 15,100 cubic feet per second, and the stage was 24.20 feet. On April 27, 1997, USGS personnel measured 141,000 cubic feet per second in the Red River of the North at Pembina (streamgage 05102490, table 35), which is located about 2 miles upstream from the international boundary with Canada. The previous maximum discharge of this century at the Canadian streamgage, Red River at Emerson, Manitoba (streamgage 05102500), located about 1 mile downstream from the border was 95,500 cubic feet per second on May 13, 1950.

Devils Lake Basin is a 3,810-square-mile closed subbasin within the Red River of the North Basin. Devils Lake discharges no water until the lake level reaches 1,459 feet above NAVD 88, the lowest natural outlet elevation. Since 1993, the lake level has risen rapidly in response to above-normal precipitation and runoff. The rising water has inundated homes, businesses, and agricultural lands and has caused roads to be closed or raised in elevation. On April 16, 1997, Devils Lake reached 1,438.4 feet above NAVD 88, equaling the previous record set in 1867. Because of the excessive runoff during the spring of 1997 , the lake level continued rising to a maximum daily elevation of 1,442.97 feet above NAVD 88 on July 26, 1997, the highest level in at least 130 years (Wiche, 1998). In 1997, record peak stages or flows occurred at several streamgages within the Devils Lake Basin (table 35).

Flooding also occurred in the Missouri River Basin during 1997. Heavy snowpacks in North Dakota, Montana, and South Dakota caused high flows throughout the basin. Flow in the Missouri River main stem is controlled by six dams. Garrison Dam, located in central North Dakota about 75 river miles north of Bismarck, was completed in 1953. On July 13, 1997, the peak stage of the Missouri River at Bismarck, North Dakota (streamgage 06342500, table 35), was 14.00 feet, which is 0.58 foot less than the post-Garrison Dam record set on December 18,1979 . The peak flow of 59,500 cubic feet per second on July 25, 1997, was maintained for several weeks by U.S. Army Corps of Engineer personnel to accommodate the high water moving through the system. Although high flow on the Missouri River main stem caused little damage in North Dakota, the high flows on tributaries to the Missouri River, such as the Knife, Heart, and James Rivers, caused flooding in several communities in North Dakota. New records for peak stage and peak 
flow occurred at several streamgages in the upper Missouri River Basin during the spring of 1997 (table 35).

\section{References}

National Oceanic and Atmospheric Administration (NOAA), 1994a-97a, Climatological data (by State): Asheville, North Carolina, National Climatic Data Center, various months.
National Oceanic and Atmospheric Administration (NOAA), 1994b-97b, Storm data (by State): Asheville, North Carolina, National Climatic Data Center, various months.

Wiche, G.J., 1998, Lake levels, streamflow, and surface-water quality in the Devils Lake area, North Dakota, through 1997: U.S. Geological Survey Fact Sheet 033-98, 4 p. 
Table 35. Maximum stage and discharge for period of record for streamgages having significant floods during 1994-98 water years in North Dakota.

$\left[\mathrm{mi}^{2}\right.$, square miles; $\mathrm{ft}$, feet above an arbitrary datum; $\mathrm{ft}^{3} / \mathrm{s}$, cubic feet per second; --, not determined or not applicable. Source: Recurrence intervals calculated from U.S. Geological Survey data. Other data from U.S. Geological Survey reports or databases]

\begin{tabular}{|c|c|c|c|c|c|c|c|c|c|c|c|}
\hline \multirow{2}{*}{$\begin{array}{c}\text { Streamgage } \\
\text { number } \\
\text { (fig. 46) }\end{array}$} & \multirow[b]{2}{*}{ Streamgage name } & \multirow{2}{*}{$\begin{array}{c}\text { Total } \\
\text { drainage } \\
\left(\mathrm{mi}^{2}\right)\end{array}$} & \multicolumn{4}{|c|}{$\begin{array}{c}\text { Maximum stage and discharge for period of record } \\
\text { through } 1998 \text { water year }\end{array}$} & \multicolumn{5}{|c|}{ Significant floods 1994-98 water years } \\
\hline & & & $\begin{array}{l}\text { Period of } \\
\text { record } \\
\text { (water } \\
\text { years) }\end{array}$ & Water year & $\begin{array}{l}\text { Stage } \\
(\mathrm{ft})\end{array}$ & $\begin{array}{l}\text { Discharge } \\
\left(\mathrm{ft}^{3} / \mathrm{s}\right)\end{array}$ & $\begin{array}{c}\text { Date } \\
\text { (month/d } \\
\text { ay/year) }\end{array}$ & $\begin{array}{l}\text { Stage } \\
(\mathrm{ft})\end{array}$ & $\begin{array}{l}\text { Discharge } \\
\left(\mathrm{ft}^{3} / \mathrm{s}\right)\end{array}$ & $\begin{array}{c}\text { Regulated } \\
\text { during } \\
\text { flood }^{1}\end{array}$ & $\begin{array}{c}\text { Recurrence } \\
\text { interval } \\
\text { (years) }\end{array}$ \\
\hline 05051500 & $\begin{array}{l}\text { Red River of the North at } \\
\text { Wahpeton, ND }\end{array}$ & 4,010 & $\begin{array}{l}1897, \\
1942-98\end{array}$ & $\begin{array}{l}1997 \\
1997\end{array}$ & $\begin{array}{l}19.25 \\
19.42\end{array}$ & $\begin{array}{r}12,800 \\
--\end{array}$ & $4 / 15 / 97$ & 19.25 & 12,800 & $\mathrm{Y}$ & 100 \\
\hline 05051522 & $\begin{array}{l}\text { Red River of the North at Hickson, } \\
\text { ND }\end{array}$ & 4,300 & 1976-98 & $\begin{array}{l}1997 \\
1997\end{array}$ & $\begin{array}{l}36.85 \\
37.60\end{array}$ & $\begin{array}{r}13,300 \\
--\end{array}$ & 4/14/97 & 36.85 & 13,300 & $\mathrm{Y}$ & $50-150$ \\
\hline 05051600 & Wild Rice River near Rutland, ND & 546 & 1960-98 & 1997 & 10.11 & 2,700 & $\begin{array}{l}4 / 3 / 97 \\
6 / 29 / 98\end{array}$ & $\begin{array}{r}10.11 \\
8.40\end{array}$ & $\begin{array}{l}2,700 \\
1,590\end{array}$ & $\begin{array}{l}\mathrm{N} \\
\mathrm{N}\end{array}$ & $\begin{array}{r}100 \\
25\end{array}$ \\
\hline 05053000 & $\begin{array}{l}\text { Wild Rice River near } \\
\text { Abercrombie, ND }\end{array}$ & 2,080 & $\begin{array}{l}\text { 1897, } \\
\text { 1933-98 }\end{array}$ & $\begin{array}{l}1969 \\
1897\end{array}$ & $\begin{array}{l}24.58 \\
27.50\end{array}$ & $\begin{array}{r}9,540 \\
--\end{array}$ & $4 / 16 / 97$ & 25.40 & 9,470 & $\mathrm{Y}$ & 25 \\
\hline 05054000 & $\begin{array}{l}\text { Red River of the North at Fargo, } \\
\text { ND }\end{array}$ & 6,800 & $\begin{array}{l}1882,1897 \\
1902-98\end{array}$ & $\begin{array}{l}1997 \\
1997\end{array}$ & $\begin{array}{l}39.57 \\
39.72\end{array}$ & $\begin{array}{l}28,000 \\
27,700\end{array}$ & $\begin{array}{l}4 / 17 / 97 \\
4 / 18 / 97\end{array}$ & $\begin{array}{l}39.57 \\
39.72\end{array}$ & $\begin{array}{l}28,000 \\
27,700\end{array}$ & $\begin{array}{l}\mathrm{Y} \\
\mathrm{Y}\end{array}$ & $\begin{array}{l}50-100 \\
50-100\end{array}$ \\
\hline 05054500 & Sheyenne River above Harvey, ND & 424 & 1956-98 & $\begin{array}{l}1979 \\
1996\end{array}$ & $\begin{array}{r}9.45 \\
10.30\end{array}$ & $\begin{array}{r}1,000 \\
570\end{array}$ & $4 / 12 / 96$ & 10.30 & 570 & $\mathrm{~N}$ & $10-25$ \\
\hline 05056100 & Mauvais Coulee near Cando, ND & 387 & $\begin{array}{l}1954, \\
1956-90, \\
1992-98\end{array}$ & 1997 & 11.68 & 3,000 & $4 / 21 / 97$ & 11.68 & 3,000 & $\mathrm{~N}$ & $10-50$ \\
\hline 05056200 & Edmore Coulee near Edmore, ND & 382 & 1956-98 & 1997 & 87.95 & 1,830 & $4 / 24 / 97$ & 87.95 & 1,830 & $\mathrm{~N}$ & $10-50$ \\
\hline 05056215 & $\begin{array}{l}\text { Edmore Coulee tributary near } \\
\text { Webster, ND }\end{array}$ & 148 & $\begin{array}{l}1988-89 \\
1991-98\end{array}$ & $\begin{array}{l}1997 \\
1993\end{array}$ & $\begin{array}{l}74.41 \\
75.06\end{array}$ & $\begin{array}{l}1,390 \\
1,330\end{array}$ & $\begin{array}{l}4 / 25 / 97 \\
8 / 2 / 93\end{array}$ & $\begin{array}{l}74.41 \\
75.06\end{array}$ & $\begin{array}{l}1,390 \\
1,330\end{array}$ & $\begin{array}{l}\mathrm{N} \\
\mathrm{N}\end{array}$ & $\begin{array}{l}-- \\
--\end{array}$ \\
\hline 05056239 & $\begin{array}{l}\text { Starkweather Coulee near } \\
\text { Webster, ND }\end{array}$ & 310 & 1980-98 & $\begin{array}{l}1997 \\
1987\end{array}$ & $\begin{array}{l}7.75 \\
8.50\end{array}$ & $\begin{array}{l}782 \\
570\end{array}$ & $4 / 27 / 97$ & 7.75 & 782 & $\mathrm{~N}$ & $25-100$ \\
\hline 05056390 & Little Coulee near Brinsmade, ND & 350 & $\begin{array}{l}1976-89 \\
1992-97\end{array}$ & 1997 & 11.47 & 439 & $4 / 26 / 97$ & 11.47 & 439 & $\mathrm{~N}$ & -- \\
\hline 05056400 & $\begin{array}{l}\text { Big Coulee near Churchs Ferry, } \\
\text { ND }\end{array}$ & 2,510 & $\begin{array}{l}1950-89 \\
1992-97\end{array}$ & 1997 & 9.26 & 2,280 & $\begin{array}{l}4 / 23 / 95 \\
5 / 4 / 97\end{array}$ & $\begin{array}{l}7.62 \\
9.26\end{array}$ & $\begin{array}{l}1,450 \\
2,280\end{array}$ & $\begin{array}{l}\mathrm{Y} \\
\mathrm{Y}\end{array}$ & $25-100$ \\
\hline 05057000 & $\begin{array}{l}\text { Sheyenne River near Cooperstown, } \\
\text { ND }\end{array}$ & 6,470 & 1945-98 & $\begin{array}{l}1950 \\
1996\end{array}$ & $\begin{array}{l}18.69 \\
19.13\end{array}$ & $\begin{array}{l}7,830 \\
6,760\end{array}$ & $4 / 18 / 96$ & 19.13 & 6,760 & $\mathrm{~N}$ & 25 \\
\hline 05057200 & Baldhill Creek near Dazey, ND & 691 & $\begin{array}{l}1950, \\
1956-98\end{array}$ & 1979 & 17.78 & 9,000 & $4 / 3 / 97$ & 11.56 & 2,780 & $\mathrm{~N}$ & $10-25$ \\
\hline
\end{tabular}


Table 35. Maximum stage and discharge for period of record for streamgages having significant floods during 1994-98 water years in North Dakota.-Continued

$\left[\mathrm{mi}^{2}\right.$, square miles; $\mathrm{ft}$, feet above an arbitrary datum; $\mathrm{ft}^{3} / \mathrm{s}$, cubic feet per second; --, not determined or not applicable. Source: Recurrence intervals calculated from U.S. Geological Survey data. Other data from U.S. Geological Survey reports or databases]

\begin{tabular}{|c|c|c|c|c|c|c|c|c|c|c|c|}
\hline \multirow{2}{*}{$\begin{array}{c}\text { Streamgage } \\
\text { number } \\
\text { (fig. 46) }\end{array}$} & \multirow[b]{2}{*}{ Streamgage name } & \multirow{2}{*}{$\begin{array}{c}\text { Total } \\
\text { drainage } \\
\left(\mathrm{mi}^{2}\right)\end{array}$} & \multicolumn{4}{|c|}{$\begin{array}{c}\text { Maximum stage and discharge for period of record } \\
\text { through } 1998 \text { water year }\end{array}$} & \multicolumn{5}{|c|}{ Significant floods 1994-98 water years } \\
\hline & & & $\begin{array}{l}\text { Period of } \\
\text { record } \\
\text { (water } \\
\text { years) }\end{array}$ & Water year & $\begin{array}{l}\text { Stage } \\
(\mathrm{ft})\end{array}$ & $\begin{array}{l}\text { Discharge } \\
\left(\mathrm{ft}^{3} / \mathrm{s}\right)\end{array}$ & $\begin{array}{c}\text { Date } \\
\text { (month/d } \\
\text { ay/year) }\end{array}$ & $\begin{array}{l}\text { Stage } \\
(\mathrm{ft})\end{array}$ & $\begin{array}{l}\text { Discharge } \\
\left(\mathrm{ft}^{3} / \mathrm{s}\right)\end{array}$ & $\begin{array}{l}\text { Regulated } \\
\text { during } \\
\text { flood }^{1}\end{array}$ & $\begin{array}{c}\text { Recurrence } \\
\text { interval } \\
\text { (years) }\end{array}$ \\
\hline 05058000 & $\begin{array}{l}\text { Sheyenne River below Baldhill } \\
\text { Dam, ND }\end{array}$ & 7,470 & $\begin{array}{l}1948, \\
1950-98\end{array}$ & 1996 & 36.46 & 5,460 & $4 / 20 / 96$ & 36.46 & 5,460 & $\mathrm{Y}$ & $10-25$ \\
\hline 05058500 & Sheyenne River at Valley City, ND & 7,810 & $\begin{array}{l}1882,1897, \\
1919, \\
1938-75, \\
1980-98\end{array}$ & $\begin{array}{l}1996 \\
1882\end{array}$ & $\begin{array}{l}18.78 \\
20.00\end{array}$ & $\begin{array}{r}5,250 \\
--\end{array}$ & $\begin{array}{l}4 / 21 / 96 \\
4 / 19 / 97\end{array}$ & $\begin{array}{l}18.78 \\
18.01\end{array}$ & $\begin{array}{l}5,250 \\
4,810\end{array}$ & $\begin{array}{l}\mathrm{Y} \\
\mathrm{Y}\end{array}$ & $\begin{array}{r}25 \\
10-25\end{array}$ \\
\hline 05058700 & Sheyenne River at Lisbon, ND & 8,190 & $\begin{array}{l}1950 \\
1957-98\end{array}$ & $\begin{array}{l}1997 \\
1975\end{array}$ & 19.29 & 5,670 & $\begin{array}{l}4 / 23 / 97 \\
4 / 13 / 96\end{array}$ & $\begin{array}{l}19.29 \\
19.20\end{array}$ & $\begin{array}{l}5,670 \\
5,060\end{array}$ & $\begin{array}{l}\mathrm{Y} \\
\mathrm{Y}\end{array}$ & $\begin{array}{l}10-25 \\
10-25\end{array}$ \\
\hline 05059000 & Sheyenne River near Kindred, ND & 8,800 & $\begin{array}{l}1947, \\
1950-98\end{array}$ & $\begin{array}{l}1997 \\
1997\end{array}$ & $\begin{array}{l}21.38 \\
22.33\end{array}$ & $\begin{array}{l}5,970 \\
3,600\end{array}$ & $\begin{array}{l}4 / 30 / 96 \\
4 / 27 / 97\end{array}$ & $\begin{array}{l}20.65 \\
21.38\end{array}$ & $\begin{array}{l}5,100 \\
5,970\end{array}$ & $\begin{array}{l}\mathrm{Y} \\
\mathrm{Y}\end{array}$ & $\begin{array}{l}10-25 \\
25-50\end{array}$ \\
\hline 05059500 & Sheyenne River at West Fargo, ND & 8,870 & $\begin{array}{l}\text { 1903-06, } \\
1919, \\
1930-94, \\
1996-98\end{array}$ & $\begin{array}{l}1997 \\
1997\end{array}$ & $\begin{array}{l}22.68 \\
22.90\end{array}$ & $\begin{array}{r}4,810 \\
--\end{array}$ & $\begin{array}{l}5 / 2 / 96 \\
4 / 19 / 97\end{array}$ & $-\overline{-}$ & $\begin{array}{l}4,240 \\
4,810\end{array}$ & $\begin{array}{l}\mathrm{Y} \\
\mathrm{Y}\end{array}$ & -- \\
\hline 05064500 & $\begin{array}{l}\text { Red River of the North at Halstad, } \\
\text { MN }\end{array}$ & 21,800 & $\begin{array}{l}1936-37 \\
1942-98\end{array}$ & 1997 & 40.74 & 71,500 & 4/19/97 & 40.74 & 71,500 & $\mathrm{Y}$ & $100-200$ \\
\hline 05066500 & Goose River at Hillsboro, ND & 1,203 & $\begin{array}{l}1882,1897, \\
1904,1916, \\
1931-98\end{array}$ & 1979 & 16.76 & 14,800 & $4 / 6 / 97$ & 15.62 & 8,520 & $\mathrm{~N}$ & $10-25$ \\
\hline 05067500 & Marsh River near Shelly, MN & 151 & 1944-98 & $\begin{array}{l}1979 \\
1997\end{array}$ & $\begin{array}{l}23.36 \\
25.45\end{array}$ & $\begin{array}{l}4,880 \\
4,300\end{array}$ & $4 / 18 / 97$ & 25.45 & 4,300 & $\mathrm{~N}$ & $10-25$ \\
\hline 05082500 & $\begin{array}{l}\text { Red River of the North at Grand } \\
\text { Forks, ND }\end{array}$ & 30,100 & $1882-1998$ & $\begin{array}{l}1997 \\
1997\end{array}$ & $\begin{array}{l}52.04 \\
54.35\end{array}$ & $\begin{array}{l}137,000 \\
114,000\end{array}$ & $\begin{array}{l}4 / 21 / 96 \\
4 / 18 / 97 \\
4 / 22 / 97\end{array}$ & $\begin{array}{l}45.93 \\
52.04 \\
54.35\end{array}$ & $\begin{array}{r}58,400 \\
137,000 \\
114,000\end{array}$ & $\begin{array}{l}\mathrm{Y} \\
\mathrm{Y} \\
\mathrm{Y}\end{array}$ & $\begin{array}{c}10-25 \\
100-200\end{array}$ \\
\hline 05092000 & $\begin{array}{l}\text { Red River of the North at Drayton, } \\
\text { ND }\end{array}$ & 34,800 & $\begin{array}{l}1897, \\
1936-37 \\
1941-98\end{array}$ & 1997 & 45.55 & 124,000 & $4 / 24 / 97$ & 45.55 & 124,000 & $\mathrm{Y}$ & $100-200$ \\
\hline 05100000 & Pembina River at Neche, ND & 3,410 & $\begin{array}{l}1904-08 \\
1910-15, \\
1919-98\end{array}$ & $\begin{array}{l}1997 \\
1997\end{array}$ & $\begin{array}{l}24.20 \\
24.51\end{array}$ & $\begin{array}{l}15,100 \\
12,800\end{array}$ & $\begin{array}{l}4 / 23 / 95 \\
4 / 27 / 97\end{array}$ & $\begin{array}{l}23.30 \\
24.20\end{array}$ & $\begin{array}{r}8,500 \\
15,100\end{array}$ & $\begin{array}{l}\mathrm{N} \\
\mathrm{N}\end{array}$ & $\begin{array}{r}10-25 \\
50-100\end{array}$ \\
\hline
\end{tabular}


$\left[\mathrm{mi}^{2}\right.$, square miles; $\mathrm{ft}$, feet above an arbitrary datum; $\mathrm{ft}^{3} / \mathrm{s}$, cubic feet per second; --, not determined or not applicable. Source: Recurrence intervals calculated from U.S. Geological Survey data. Other data from U.S. Geological Survey reports or databases]

\begin{tabular}{|c|c|c|c|c|c|c|c|c|c|c|c|}
\hline \multirow{2}{*}{$\begin{array}{c}\text { Streamgage } \\
\text { number } \\
\text { (fig. 46) }\end{array}$} & \multirow[b]{2}{*}{ Streamgage name } & \multirow{2}{*}{$\begin{array}{c}\text { Total } \\
\text { drainage } \\
\left(\mathrm{mi}^{2}\right)\end{array}$} & \multicolumn{4}{|c|}{$\begin{array}{c}\text { Maximum stage and discharge for period of record } \\
\text { through } 1998 \text { water year }\end{array}$} & \multicolumn{5}{|c|}{ Significant floods $1994-98$ water years } \\
\hline & & & $\begin{array}{l}\text { Period of } \\
\text { record } \\
\text { (water } \\
\text { years) }\end{array}$ & Water year & $\begin{array}{l}\text { Stage } \\
(\mathrm{ft})\end{array}$ & $\begin{array}{l}\text { Discharge } \\
\left(\mathrm{ft}^{3} / \mathrm{s}\right)\end{array}$ & $\begin{array}{c}\text { Date } \\
\text { (month/d } \\
\text { ay/year) }\end{array}$ & $\begin{array}{l}\text { Stage } \\
(\mathrm{ft})\end{array}$ & $\begin{array}{l}\text { Discharge } \\
\left(\mathrm{ft}^{3} / \mathrm{s}\right)\end{array}$ & $\begin{array}{l}\text { Regulated } \\
\text { during } \\
\text { flood }^{1}\end{array}$ & $\begin{array}{c}\text { Recurrence } \\
\text { interval } \\
\text { (years) }\end{array}$ \\
\hline 05102490 & $\begin{array}{l}\text { Red River of the North at Pembina, } \\
\text { ND }\end{array}$ & 36,400 & $1985-98$ & 1997 & 794.39 & 141,000 & $\begin{array}{l}4 / 26 / 97 \\
4 / 26 / 96\end{array}$ & $\begin{array}{l}794.39 \\
790.95\end{array}$ & $\begin{array}{r}141,000 \\
66,400\end{array}$ & $\begin{array}{l}\mathrm{Y} \\
\mathrm{Y}\end{array}$ & $\begin{array}{c}100-200 \\
10-25\end{array}$ \\
\hline 05102500 & $\begin{array}{l}\text { Red River of the North at Emerson, } \\
\text { Manitoba }\end{array}$ & 40,200 & 1913-98 & 1997 & 92.41 & 133,000 & $\begin{array}{l}4 / 26 / 97 \\
4 / 26 / 96\end{array}$ & $\begin{array}{l}92.41 \\
89.10\end{array}$ & $\begin{array}{r}133,000 \\
66,700\end{array}$ & $\begin{array}{l}\mathrm{Y} \\
\mathrm{Y}\end{array}$ & $\begin{array}{c}100-200 \\
10-25\end{array}$ \\
\hline 06339100 & Knife River at Manning, ND & 205 & 1968-98 & 1997 & 17.05 & 3,600 & $3 / 21 / 97$ & 17.05 & 3,600 & $\mathrm{~N}$ & $10-25$ \\
\hline 06341410 & Turtle Creek above Washburn, ND & 350 & $1987-98$ & 1996 & 7.28 & 954 & $3 / 12 / 96$ & 7.28 & 954 & $\mathrm{~N}$ & -- \\
\hline 06342500 & Missouri River at Bismarck, ND & 186,400 & 1929-98 & 1952 & 27.90 & 500,000 & $\begin{array}{l}7 / 13 / 97 \\
7 / 25 / 97\end{array}$ & $\begin{array}{l}14.00 \\
13.98\end{array}$ & $\begin{array}{l}59,100 \\
59,500\end{array}$ & $\begin{array}{l}\mathrm{Y} \\
\mathrm{Y}\end{array}$ & $\begin{array}{l}50-100 \\
50-100\end{array}$ \\
\hline 06345500 & Heart River near Richardton, ND & 1,240 & $\begin{array}{l}1905-21, \\
1938, \\
1943-98\end{array}$ & $\begin{array}{l}1950 \\
1912\end{array}$ & $\begin{array}{l}28.05 \\
38.50\end{array}$ & $\begin{array}{r}23,400 \\
4,500\end{array}$ & $3 / 23 / 97$ & -- & 13,000 & $\mathrm{Y}$ & $25-50$ \\
\hline 06345780 & $\begin{array}{l}\text { Heart River above Lake Tschida } \\
\text { near Glen Ullin, ND }\end{array}$ & 1,530 & 1988-98 & $\begin{array}{l}1997 \\
1997\end{array}$ & -- & $\begin{array}{r}11,500 \\
4,540\end{array}$ & $3 / 22 / 97$ & -- & 11,500 & $\mathrm{Y}$ & -- \\
\hline 06348300 & $\begin{array}{l}\text { Heart River at Stark Bridge near } \\
\text { Judson, ND }\end{array}$ & 2,930 & 1989-98 & 1997 & 21.90 & 18,000 & $3 / 23 / 97$ & 21.90 & 18,000 & $\mathrm{Y}$ & -- \\
\hline 06349215 & $\begin{array}{l}\text { Long Lake Creek above Long Lake } \\
\text { near Moffit, ND }\end{array}$ & 280 & 1989-98 & 1997 & 12.99 & 3,200 & $3 / 29 / 97$ & 12.99 & 3,200 & $\mathrm{~N}$ & -- \\
\hline 06353000 & Cedar Creek near Raleigh, ND & 1,750 & $\begin{array}{l}1939, \\
1962-98\end{array}$ & 1997 & 17.05 & 14,600 & $3 / 24 / 97$ & 17.05 & 14,600 & $\mathrm{~N}$ & $50-100$ \\
\hline 06354000 & Cannonball River at Breien, ND & 4,100 & $\begin{array}{l}1906-08, \\
1912-18, \\
1922,1924, \\
1928-98\end{array}$ & 1950 & 22.30 & 94,800 & $3 / 25 / 97$ & 20.82 & 31,100 & $\mathrm{~N}$ & $10-25$ \\
\hline 06468170 & James River near Grace City, ND & 1,060 & 1969-98 & 1997 & 14.77 & 4,000 & $4 / 3 / 97$ & 14.77 & 4,000 & $\mathrm{~N}$ & $10-20$ \\
\hline 06468250 & $\begin{array}{l}\text { James River above Arrowwood } \\
\text { Lake near Kensal, ND }\end{array}$ & 1,200 & $1986-98$ & 1997 & 13.00 & 4,700 & $4 / 5 / 97$ & 13.00 & 4,700 & $\mathrm{~N}$ & -- \\
\hline 06469400 & Pipestem Creek near Pingree, ND & 700 & 1974-98 & $\begin{array}{l}1997 \\
1995\end{array}$ & $\begin{array}{l}11.37 \\
11.70\end{array}$ & $\begin{array}{l}3,400 \\
3,180\end{array}$ & $4 / 19 / 97$ & 11.37 & 3,400 & $\mathrm{~N}$ & $10-25$ \\
\hline 06470500 & James River at Lamoure, ND & 4,390 & 1950-98 & 1969 & 16.17 & 6,800 & $4 / 1 / 97$ & 16.09 & 6,500 & $\mathrm{Y}$ & $15-45$ \\
\hline
\end{tabular}


Table 35. Maximum stage and discharge for period of record for streamgages having significant floods during 1994-98 water years in North Dakota.-Continued

$\left[\mathrm{mi}^{2}\right.$, square miles; $\mathrm{ft}$, feet above an arbitrary datum; $\mathrm{ft}^{3} / \mathrm{s}$, cubic feet per second; --, not determined or not applicable. Source: Recurrence intervals calculated from U.S. Geological Survey data. Other data from U.S. Geological Survey reports or databases]

\begin{tabular}{|c|c|c|c|c|c|c|c|c|c|c|c|}
\hline \multirow{2}{*}{$\begin{array}{c}\text { Streamgage } \\
\text { number } \\
\text { (fig. 46) }\end{array}$} & \multirow[b]{2}{*}{ Streamgage name } & \multirow{2}{*}{$\begin{array}{c}\text { Total } \\
\text { drainage } \\
\left(\mathrm{mi}^{2}\right)\end{array}$} & \multicolumn{4}{|c|}{$\begin{array}{c}\text { Maximum stage and discharge for period of record } \\
\text { through } 1998 \text { water year }\end{array}$} & \multicolumn{5}{|c|}{ Significant floods 1994-98 water years } \\
\hline & & & $\begin{array}{l}\text { Period of } \\
\text { record } \\
\text { (water } \\
\text { years) }\end{array}$ & Water year & $\begin{array}{l}\text { Stage } \\
(\mathrm{ft})\end{array}$ & $\begin{array}{c}\text { Discharge } \\
\left(\mathrm{ft}^{3} / \mathrm{s}\right)\end{array}$ & $\begin{array}{c}\text { Date } \\
\text { (month/d } \\
\text { ay/year) }\end{array}$ & $\begin{array}{l}\text { Stage } \\
(\mathrm{ft})\end{array}$ & $\begin{array}{l}\text { Discharge } \\
\left(\mathrm{ft}^{3} / \mathrm{s}\right)\end{array}$ & $\begin{array}{l}\text { Regulated } \\
\text { during } \\
\text { flood }^{1}\end{array}$ & $\begin{array}{c}\text { Recurrence } \\
\text { interval } \\
\text { (years) }\end{array}$ \\
\hline 06470800 & Bear Creek near Oakes, ND & 365 & 1977-98 & 1998 & 11.75 & 1,730 & $6 / 28 / 98$ & 11.75 & 1,730 & $\mathrm{~N}$ & $10-25$ \\
\hline 06470875 & $\begin{array}{l}\text { James River at Dakota Lake Dam } \\
\text { near Ludden, ND }\end{array}$ & 5,480 & 1982-98 & 1997 & 17.86 & 7,500 & $4 / 6 / 97$ & 17.86 & 7,500 & $\mathrm{Y}$ & $25-100$ \\
\hline 06471200 & $\begin{array}{l}\text { Maple River at North Dakota- } \\
\text { South Dakota State line, ND }\end{array}$ & 716 & 1957-98 & $\begin{array}{l}1969 \\
1997\end{array}$ & $\begin{array}{l}15.22 \\
16.19\end{array}$ & $\begin{array}{l}5,930 \\
5,300\end{array}$ & $3 / 29 / 97$ & 16.19 & 5,300 & $\mathrm{~N}$ & $20-45$ \\
\hline
\end{tabular}

1 Regulated during flood: N, no; Y, yes. 


\section{Summary of Significant Floods in the United States and Puerto Rico, 1994 Through 1998 Water Years}

\section{Ohio}

Two to 3 inches of rain (National Oceanic and Atmospheric Administration, 1994a) on top of saturated ground produced widespread flooding of streams, streets, basements, and poor drainage areas in northeastern Ohio during the middle of April 1994. Damage was near \$25 million, and one death occurred (National Oceanic and Atmospheric Administration, 1994b).

Nearly stationary thunderstorms in central and northern Ohio resulted in rainfall totals of 4 to 11 inches during August 7-9, 1995 (National Oceanic and Atmospheric Administration, 1995a). Damage from the August flooding was more than $\$ 13$ million (National Oceanic and Atmospheric Administration, 1995b).

As a result of rain and melted snow in Ohio, Pennsylvania, and West Virginia, the Ohio River (fig. 47) crested 3 to 6 feet above flood stage from Marietta to Ironton during January 1930, 1996. Damage from this flood was more than $\$ 10$ million, but no lives were lost (National Oceanic and Atmospheric Administration, 1996a). The Ohio River at Cincinnati (National Oceanic and Atmospheric Administration, 1996b) eventually crested 5.3 feet above flood stage at a stage of 57.3 feet during the evening of January 24. This was the highest crest in Cincinnati since March 1979.

Showers and thunderstorms dumped 4 to 12 inches of rain across southern Ohio during March 1-3, 1997 (National Oceanic and Atmospheric Administration 1997a). Five people were killed by the flooding in Ohio from this storm, and there was more than \$200 million in damage (National Oceanic and Atmospheric Administration, 1997b). Sixteen counties were declared Federal disaster areas. The Ohio River rose rapidly reaching a crest stage of 59.8 feet (9.8 feet above flood stage) at
Portsmouth (National Oceanic and Atmospheric Administration, 1997b) on March 4. Farther downstream at Meldahl Dam (National Oceanic and Atmospheric Administration, 1997b), the river crested at 61.3 feet ( 10.3 feet above flood stage) on March 6. In Cincinnati, the Ohio River crested at 64.7 feet on March 5 (National Oceanic and Atmospheric Administration, 1997b). Many towns were flooded from Portsmouth to Cincinnati, and thousands of people were evacuated from their homes for several days.

Severe thunderstorms bringing damaging winds and torrential rains affected much of east-central Ohio, western Pennsylvania, and northern West Virginia during June 26-30, 1998. These storms caused eight deaths and several injuries, along with more than $\$ 98$ million in damage across Ohio. Damage to crops was an additional $\$ 70$ million (National Oceanic and Atmospheric Administration, 1998b). Travel across this area was nearly impossible, as many roads were underwater. Four streamgages recorded the highest discharge of record during this June flood, and two more peaks of record occurred during local floods from intense thunderstorms in August 1998 (table 36). The August 1998 floods caused more than $\$ 3$ million in damage (National Oceanic and Atmospheric Administration, 1998b).

\section{References}

National Oceanic and Atmospheric Administration (NOAA), 1994a-98a, Climatological data (by State): Asheville, North Carolina, National Climatic Data Center, various months.

National Oceanic and Atmospheric Administration (NOAA), 1994b-98b, Storm data (by State): Asheville, North Carolina, National Climatic Data Center, various months. 


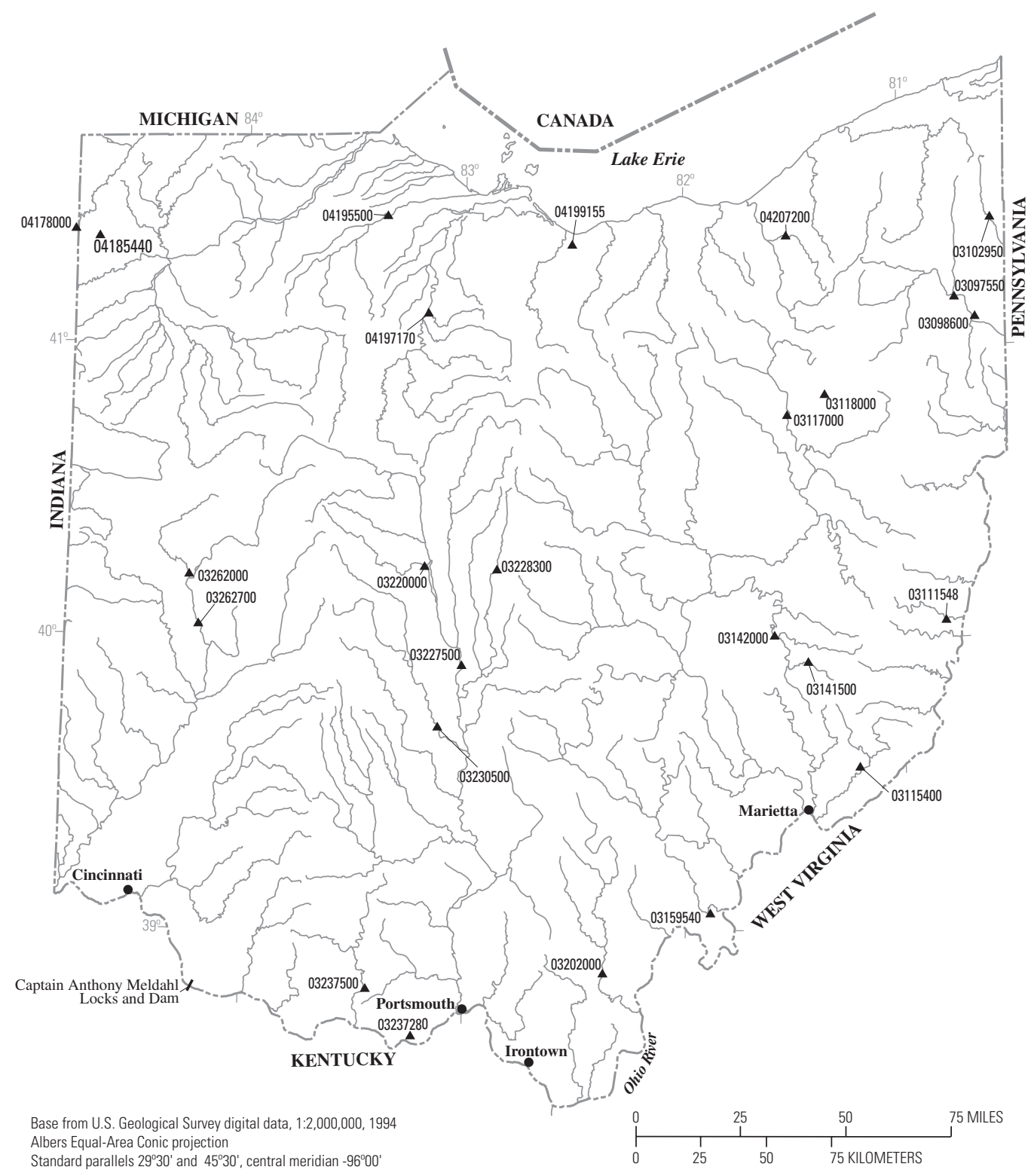

\section{EXPLANATION}

$03237280 \triangle$ Streamgage and number

Figure 47. Location of streamgages with significant floods during 1994-98 water years for Ohio. 
Table 36. Maximum stage and discharge for period of record for streamgages having significant floods during 1994-98 water years in Ohio.

$\left[\mathrm{mi}^{2}\right.$, square miles; ft, feet above an arbitrary datum; $\mathrm{ft}^{3} / \mathrm{s}$, cubic feet per second; --, not determined or not applicable; >, greater than. Source: Recurrence intervals calculated from U.S. Geological Survey data. Other data from U.S. Geological Survey reports or databases]

\begin{tabular}{|c|c|c|c|c|c|c|c|c|c|c|c|}
\hline \multirow{2}{*}{$\begin{array}{c}\text { Streamgage } \\
\text { number } \\
\text { (fig. 47) }\end{array}$} & \multirow[b]{2}{*}{ Streamgage name } & \multirow{2}{*}{$\begin{array}{c}\text { Total } \\
\text { drainage } \\
\left(\mathrm{mi}^{2}\right)\end{array}$} & \multicolumn{4}{|c|}{$\begin{array}{c}\text { Maximum stage and discharge for period of record } \\
\text { through } 1998 \text { water year }\end{array}$} & \multicolumn{5}{|c|}{ Significant floods 1994-98 water years } \\
\hline & & & $\begin{array}{l}\text { Period of } \\
\text { record } \\
\text { (water } \\
\text { years) }\end{array}$ & Water year & $\begin{array}{l}\text { Stage } \\
\text { (ft) }\end{array}$ & $\begin{array}{l}\text { Discharge } \\
\left(\mathrm{ft}^{3} / \mathrm{s}\right)\end{array}$ & $\begin{array}{c}\text { Date } \\
\text { (month/ } \\
\text { day/ } \\
\text { year) }\end{array}$ & $\begin{array}{l}\text { Stage } \\
\text { (ft) }\end{array}$ & $\begin{array}{l}\text { Discharge } \\
\left(\mathrm{ft}^{3} / \mathrm{s}\right)\end{array}$ & $\begin{array}{l}\text { Regulated } \\
\text { during } \\
\text { flood }^{1}\end{array}$ & $\begin{array}{c}\text { Recurrence } \\
\text { interval } \\
\text { (years) }\end{array}$ \\
\hline 03097550 & $\begin{array}{l}\text { Mahoning River at Ohio Edison } \\
\text { Power Plant at Niles, OH }\end{array}$ & 854 & 1988-98 & 1994 & 13.35 & 9,760 & $4 / 13 / 94$ & 13.35 & 9,760 & $\mathrm{Y}$ & -- \\
\hline 03098600 & $\begin{array}{l}\text { Mahoning River below West } \\
\text { Avenue at Youngstown, } \mathrm{OH}\end{array}$ & 978 & 1988-98 & 1994 & 15.44 & 11,900 & $4 / 13 / 94$ & 15.44 & 11,900 & $\mathrm{Y}$ & -- \\
\hline 03102950 & Pymatuning Creek at Kinsman, $\mathrm{OH}$ & 96.7 & 1966-96 & 1986 & 12.40 & 2,740 & $1 / 19 / 96$ & 12.28 & 2,560 & $\mathrm{~N}$ & $10-25$ \\
\hline 03111548 & Wheeling Creek below Blaine, $\mathrm{OH}$ & 97.7 & $\begin{array}{l}\text { 1983-87, } \\
1989-98\end{array}$ & 1998 & 8.21 & 5,470 & $6 / 28 / 98$ & 8.21 & 5,470 & $\mathrm{~N}$ & 10 \\
\hline 03115400 & $\begin{array}{l}\text { Little Muskingum River at } \\
\text { Bloomfield, OH }\end{array}$ & 210 & $\begin{array}{l}1959-81 \\
1996-98\end{array}$ & 1998 & 30.78 & 32,300 & $6 / 28 / 98$ & 30.78 & 32,300 & $\mathrm{~N}$ & $>100$ \\
\hline 03117000 & $\begin{array}{l}\text { Tuscarawas River at Massillon, } \\
\text { OH }\end{array}$ & 518 & 1939-98 & 1969 & 16.43 & 10,700 & $4 / 13 / 94$ & 12.77 & 6,490 & $\mathrm{Y}$ & -- \\
\hline 03118000 & $\begin{array}{l}\text { Main Branch Nimishillen Creek at } \\
\text { Canton, OH }\end{array}$ & 43.1 & 1942-98 & $\begin{array}{l}1959 \\
1994\end{array}$ & $\begin{array}{l}6.50 \\
6.62\end{array}$ & $\begin{array}{l}2,470 \\
1,810\end{array}$ & $4 / 13 / 94$ & 6.62 & 1,810 & $\mathrm{~N}$ & $25-50$ \\
\hline 03141500 & $\begin{array}{l}\text { Seneca Fork below Senecaville } \\
\text { Dam near Senecaville, } \mathrm{OH}\end{array}$ & 118 & $\begin{array}{l}\text { 1939-91, } \\
\text { 1995-96, } \\
1998\end{array}$ & $\begin{array}{l}1998 \\
1949\end{array}$ & $\begin{array}{r}9.51 \\
10.35\end{array}$ & $\begin{array}{l}977 \\
718\end{array}$ & $6 / 28 / 98$ & 9.51 & 977 & $\mathrm{Y}$ & -- \\
\hline 03142000 & Wills Creek at Cambridge, $\mathrm{OH}$ & 406 & $\begin{array}{l}1927-28 \\
1938-98\end{array}$ & 1998 & 26.91 & 11,400 & $6 / 29 / 98$ & 26.91 & 11,400 & $\mathrm{Y}$ & $50-100$ \\
\hline 03159540 & Shade River near Ter, OH & 156 & 1966-98 & 1997 & 31.44 & 15,600 & $3 / 2 / 97$ & 31.44 & 15,600 & $\mathrm{~N}$ & $>100$ \\
\hline 03202000 & Raccoon Creek at Adamsville, $\mathrm{OH}$ & 585 & $\begin{array}{l}1916-35, \\
1937, \\
1939-85, \\
1992-98\end{array}$ & $\begin{array}{l}1968 \\
1997\end{array}$ & $\begin{array}{l}28.69 \\
29.11\end{array}$ & $\begin{array}{l}20,000 \\
16,500\end{array}$ & $3 / 3 / 97$ & 29.11 & 16,500 & $\mathrm{~N}$ & $25-50$ \\
\hline 03220000 & Mill Creek near Bellepoint, $\mathrm{OH}$ & 178 & $\begin{array}{l}1913 \\
1943-98\end{array}$ & $\begin{array}{l}1997 \\
1913\end{array}$ & $\begin{array}{l}14.45 \\
18.00\end{array}$ & $\begin{array}{r}21,800 \\
--\end{array}$ & $6 / 2 / 97$ & 14.45 & 21,800 & $\mathrm{~N}$ & $>100$ \\
\hline
\end{tabular}


Table 36. Maximum stage and discharge for period of record for streamgages having significant floods during 1994-98 water years in 0hio.-Continued

$\left[\mathrm{mi}^{2}\right.$, square miles; $\mathrm{ft}$, feet above an arbitrary datum; $\mathrm{ft}^{3} / \mathrm{s}$, cubic feet per second; --, not determined or not applicable; $>$, greater than. Source: Recurrence intervals calculated from U.S. Geological Survey data. Other data from U.S. Geological Survey reports or databases]

\begin{tabular}{|c|c|c|c|c|c|c|c|c|c|c|c|}
\hline \multirow{2}{*}{$\begin{array}{l}\text { Streamgage } \\
\text { number } \\
\text { (fig. 47) }\end{array}$} & \multirow[b]{2}{*}{ Streamgage name } & \multirow{2}{*}{$\begin{array}{c}\text { Total } \\
\text { drainage } \\
\left(\mathrm{mi}^{2}\right)\end{array}$} & \multicolumn{4}{|c|}{$\begin{array}{l}\text { Maximum stage and discharge for period of record } \\
\text { through } 1998 \text { water year }\end{array}$} & \multicolumn{5}{|c|}{ Significant floods $1994-98$ water years } \\
\hline & & & $\begin{array}{l}\text { Period of } \\
\text { record } \\
\text { (water } \\
\text { years) }\end{array}$ & Water year & $\begin{array}{l}\text { Stage } \\
(\mathrm{ft})\end{array}$ & $\begin{array}{l}\text { Discharge } \\
\left(\mathrm{ft}^{3} / \mathrm{s}\right)\end{array}$ & $\begin{array}{c}\text { Date } \\
\text { (month/ } \\
\text { day/ } \\
\text { year) }\end{array}$ & $\begin{array}{l}\text { Stage } \\
(\mathrm{ft})\end{array}$ & $\begin{array}{l}\text { Discharge } \\
\left(\mathrm{ft}^{3} / \mathrm{s}\right)\end{array}$ & $\begin{array}{l}\text { Regulated } \\
\text { during } \\
\text { flood }^{1}\end{array}$ & $\begin{array}{c}\text { Recurrence } \\
\text { interval } \\
\text { (years) }\end{array}$ \\
\hline 03227500 & Scioto River at Columbus, $\mathrm{OH}$ & 1,629 & $\begin{array}{l}1913, \\
1921-98\end{array}$ & 1959 & 27.22 & 68,200 & $6 / 2 / 97$ & 24.78 & 43,800 & $\mathrm{Y}$ & -- \\
\hline 03228300 & Big Walnut Creek at Sunbury, OH & 101 & 1989-98 & $\begin{array}{l}1997 \\
1991\end{array}$ & $\begin{array}{l}11.20 \\
11.86\end{array}$ & $\begin{array}{l}6,700 \\
5,690\end{array}$ & $6 / 1 / 97$ & 11.20 & 6,700 & $\mathrm{~N}$ & -- \\
\hline 03230500 & $\begin{array}{l}\text { Big Darby Creek at Darbyville, } \\
\text { OH }\end{array}$ & 534 & $\begin{array}{l}1922-36 \\
1938-98\end{array}$ & 1959 & 17.94 & 49,000 & $6 / 3 / 97$ & 15.62 & 23,700 & $\mathrm{~N}$ & $25-50$ \\
\hline 03237280 & Upper Twin Creek at McGaw, OH & 12.2 & $\begin{array}{l}1960 \\
1964-98\end{array}$ & 1960 & 11.62 & 7,320 & $3 / 2 / 97$ & 10.01 & 4,430 & $\mathrm{~N}$ & $50-100$ \\
\hline 03237500 & $\begin{array}{l}\text { Ohio Brush Creek near West } \\
\text { Union, } \mathrm{OH}\end{array}$ & 387 & $\begin{array}{l}1927-35 \\
1941-98\end{array}$ & 1997 & 31.15 & 77,700 & $3 / 2 / 97$ & 31.15 & 77,700 & $\mathrm{~N}$ & $>100$ \\
\hline 03262000 & Loramie Creek at Lockington, $\mathrm{OH}$ & 257 & $\begin{array}{l}1913, \\
1916-98\end{array}$ & 1913 & 91.60 & 25,600 & $8 / 8 / 95$ & 84.59 & 6,130 & Y & -- \\
\hline 03262700 & Great Miami River at Troy, $\mathrm{OH}$ & 926 & $\begin{array}{l}1958, \\
1963-88, \\
1991-98\end{array}$ & $\begin{array}{l}1995 \\
1958\end{array}$ & $\begin{array}{l}16.02 \\
16.40\end{array}$ & $\begin{array}{l}21,700 \\
21,000\end{array}$ & $8 / 8 / 95$ & 16.02 & 21,700 & $\mathrm{Y}$ & -- \\
\hline 04178000 & $\begin{array}{l}\text { Saint Joseph River near Newville, } \\
\text { IN }\end{array}$ & 610 & $\begin{array}{l}1947-96 \\
1998\end{array}$ & 1996 & 17.74 & 10,400 & $5 / 18 / 96$ & 17.74 & 10,400 & $\mathrm{~N}$ & -- \\
\hline 04185440 & $\begin{array}{l}\text { Unnamed tributary to Lost Creek } \\
\text { near Farmers, OH }\end{array}$ & 4.23 & 1986-98 & 1998 & 7.59 & 1,770 & $8 / 25 / 98$ & 7.59 & 1,770 & $\mathrm{~N}$ & -- \\
\hline 04195500 & Portage River at Woodville, $\mathrm{OH}$ & 428 & $\begin{array}{l}1913, \\
1929-35, \\
1940-98\end{array}$ & 1913 & 17.00 & 17,000 & $8 / 27 / 98$ & 13.98 & 11,500 & $\mathrm{~N}$ & 25 \\
\hline 04197170 & Rock Creek at Tiffin, $\mathrm{OH}$ & 34.6 & $\begin{array}{l}1983-86 \\
1988-98\end{array}$ & 1998 & 8.96 & 2,640 & $8 / 26 / 98$ & 8.96 & 2,640 & $\mathrm{~N}$ & -- \\
\hline 04199155 & $\begin{array}{l}\text { Old Womans Creek at Berlin Road } \\
\text { near Huron, OH }\end{array}$ & 22.1 & $\begin{array}{l}\text { 1988-94, } \\
1996-98\end{array}$ & 1997 & 11.81 & 1,940 & 2/27/97 & 11.81 & 1,940 & $\mathrm{~N}$ & -- \\
\hline 04207200 & Tinkers Creek at Bedford, $\mathrm{OH}$ & 83.9 & $1963-98$ & 1969 & 10.10 & 7,220 & $8 / 13 / 94$ & 9.81 & 6,750 & $\mathrm{~N}$ & $>100$ \\
\hline
\end{tabular}

\footnotetext{
${ }^{1}$ Regulated during flood: $\mathrm{N}$, no; $\mathrm{Y}$, yes.
} 


\section{Oklahoma}

High water along the Neosho River in northeastern Oklahoma forced the evacuation of about 50 homes in Miami (fig. 48). Most areas of Oklahoma received locally intense rain and large hail from thunderstorms on April 1995 (National Oceanic and Atmospheric Administration, 1995a). Severe thunderstorms with a 3-day rainfall of about 8 inches in many areas moved across Oklahoma intermittently during both May and June 1995 (National Oceanic and Atmospheric Administration, 1995a). Runoff from the intense rains caused flash floods on small streams and larger regional floods in the larger basins. Flood-prone areas of Guthrie, Kingfisher, and Miami were inundated. On June 7, 1995, the Red River near Terral (streamgage 07315500 , table 37 ) reached its highest discharge since records began in 1935. Sixteen of the 23 significant floods for Oklahoma during the 1994 through 1998 water years occurred during May and June 1995. Damage was more than \$5 million (National Oceanic and Atmospheric Administration, 1995b).

Widespread excessive rainfall caused flooding in September 1996 due to two storm systems - the remnants of the Pacific Tropical Storm Fausto during the middle of the month and a frontal system that produced localized flash flooding and caused five deaths at the end of the month. Flooding associated with Fausto was most notable in west-central Oklahoma in the Clinton area with more than 6 inches of rain (National Oceanic and Atmospheric Administration, 1996a). Severe flash flooding occurred in northeastern Oklahoma on September 25-26, 1996, with more than 8 inches of rain in many areas (National Oceanic and Atmospheric Administration, 1996a). One person drowned in Pryor; a mother and three children died near Tahlequah when their car was swept off a road (National Oceanic and Atmospheric Administration, 1996b).

Slow-moving thunderstorms moved repeatedly over parts of northern and western Oklahoma causing extensive flash flooding during September 22-23, 1997. Rainfall amounts were about 9 inches at some locations (National Oceanic and Atmospheric Administration, 1997a), causing severe localized flooding in Cherokee, Tipton, Hobart, and west of Clinton. Flooding also occurred along the Salt Fork of the Arkansas River near Tonkawa and the Chikaskia River near Blackwell (National Oceanic and Atmospheric Administration, 1997b).

\section{References}

National Oceanic and Atmospheric Administration (NOAA), 1995a-97a, Climatological data (by State): Asheville, North Carolina, National Climatic Data Center, various months.

National Oceanic and Atmospheric Administration (NOAA), 1995b-97b, Storm data (by State): Asheville, North Carolina, National Climatic Data Center, various months.

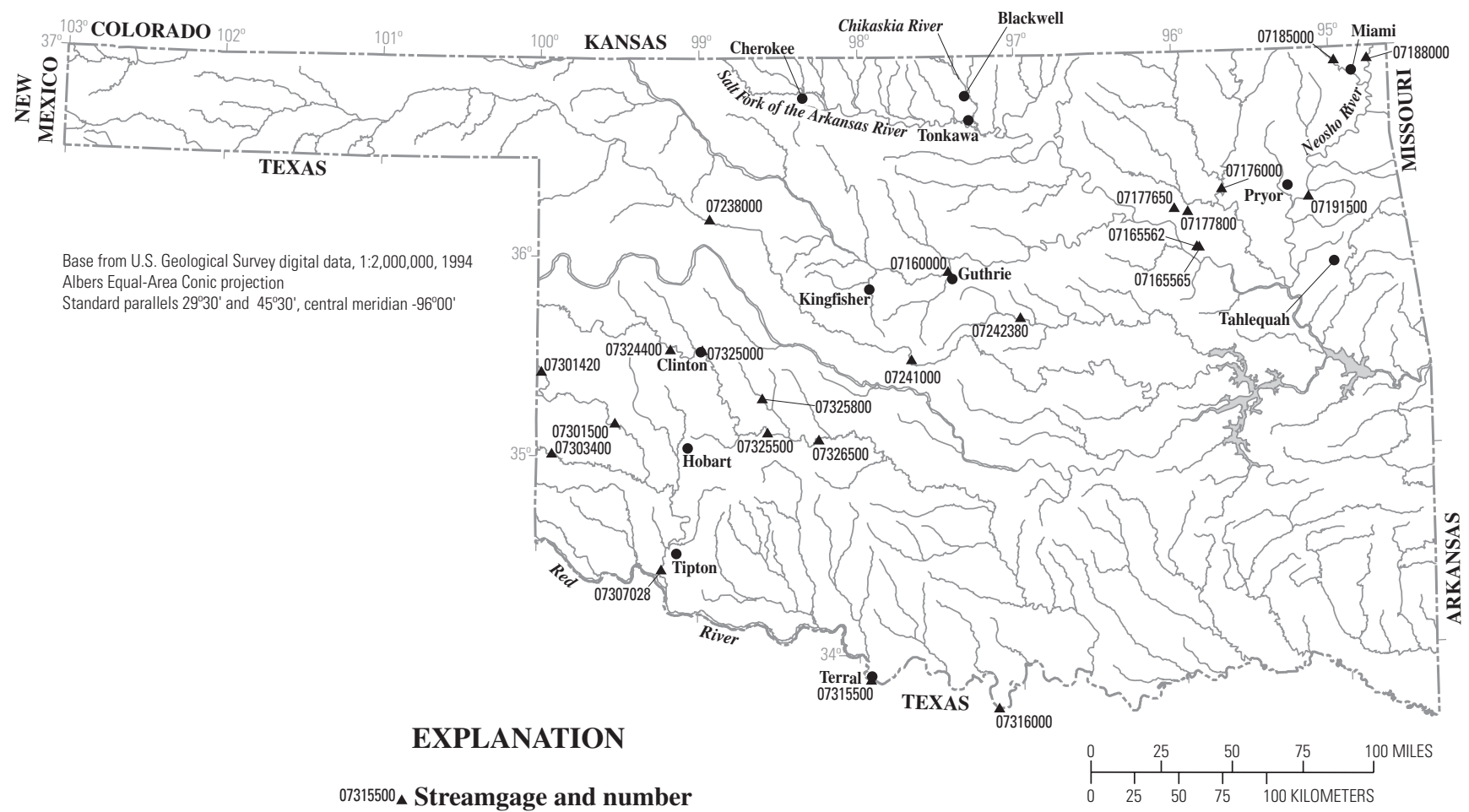

Figure 48. Location of streamgages with significant floods during 1994-98 water years for Oklahoma. 
Table 37. Maximum stage and discharge for period of record for streamgages having significant floods during 1994-98 water years in 0klahoma.

$\left[\mathrm{mi}^{2}\right.$, square miles; $\mathrm{ft}$, feet above an arbitrary datum; $\mathrm{ft}^{3} / \mathrm{s}$, cubic feet per second; --, not determined or not applicable; $>$, greater than. Source: Recurrence intervals calculated from U.S. Geological Survey data. Other data from U.S. Geological Survey reports or databases]

\begin{tabular}{|c|c|c|c|c|c|c|c|c|c|c|c|}
\hline \multirow{2}{*}{$\begin{array}{l}\text { Streamgage } \\
\text { number } \\
\text { (fig. 48) }\end{array}$} & \multirow[b]{2}{*}{ Streamgage name } & \multirow{2}{*}{$\begin{array}{c}\text { Total } \\
\text { drainage } \\
\left(\mathrm{mi}^{2}\right)\end{array}$} & \multicolumn{4}{|c|}{$\begin{array}{c}\text { Maximum stage and discharge for period of record } \\
\text { through } 1998 \text { water year }\end{array}$} & \multicolumn{5}{|c|}{ Significant floods 1994-98 water years } \\
\hline & & & $\begin{array}{c}\text { Period of } \\
\text { record } \\
\text { (water } \\
\text { years) }\end{array}$ & Water year & $\begin{array}{l}\text { Stage } \\
(\mathrm{ft})\end{array}$ & $\begin{array}{l}\text { Discharge } \\
\left(\mathrm{ft}^{3} / \mathrm{s}\right)\end{array}$ & $\begin{array}{c}\text { Date } \\
\text { (month/ } \\
\text { day/ } \\
\text { year) }\end{array}$ & $\begin{array}{l}\text { Stage } \\
(\mathrm{ft})\end{array}$ & $\begin{array}{l}\text { Discharge } \\
\left(\mathrm{ft}^{3} / \mathrm{s}\right)\end{array}$ & $\begin{array}{c}\text { Regulated } \\
\text { during } \\
\text { flood }^{1}\end{array}$ & $\begin{array}{c}\text { Recurrence } \\
\text { interval } \\
\text { (years) }\end{array}$ \\
\hline 07160000 & Cimarron River near Guthrie, OK & 16,892 & $\begin{array}{l}1935, \\
1937-76, \\
1983-98\end{array}$ & $\begin{array}{l}1957 \\
1987\end{array}$ & $\begin{array}{l}18.58 \\
20.71\end{array}$ & $\begin{array}{l}158,000 \\
116,000\end{array}$ & $6 / 10 / 95$ & 17.18 & 93,300 & $\mathrm{~N}$ & $15-20$ \\
\hline 07165562 & $\begin{array}{l}\text { Haikey Creek at 101st Street South } \\
\text { at Tulsa, OK }\end{array}$ & 17.8 & 1988-98 & 1995 & 17.42 & 6,470 & $4 / 10 / 95$ & 17.42 & 6,470 & $\mathrm{~N}$ & 10 \\
\hline 07165565 & $\begin{array}{l}\text { Little Haikey Creek at 101st Street } \\
\text { South at Tulsa, OK }\end{array}$ & 5.45 & 1987-98 & 1995 & 16.82 & 1,930 & $6 / 29 / 95$ & 16.82 & 1,930 & $\mathrm{~N}$ & 10 \\
\hline 07176000 & $\begin{array}{l}\text { Verdigris River near Claremore, } \\
\text { OK }\end{array}$ & 6,534 & $1935-98$ & 1943 & 55.05 & 182,000 & 7/7/95 & 29.76 & 40,800 & $\mathrm{Y}$ & 10 \\
\hline 07177650 & $\begin{array}{l}\text { Flat Rock Creek at Cincinnati } \\
\text { Avenue at Tulsa, OK }\end{array}$ & 8.20 & 1987-98 & 1995 & 12.82 & 4,220 & $6 / 9 / 95$ & 12.82 & 4,220 & $\mathrm{~N}$ & $10-25$ \\
\hline 07177800 & Coal Creek at Tulsa, OK & 7.53 & 1989-98 & 1995 & 14.18 & 5,190 & $6 / 23 / 95$ & 14.18 & 5,190 & $\mathrm{~N}$ & $10-25$ \\
\hline 07185000 & Neosho River near Commerce, OK & 5,876 & $\begin{array}{l}\text { 1904, 1927, } \\
1935,1938, \\
1939-98\end{array}$ & 1951 & 34.03 & 267,000 & 4/13/94 & 25.67 & 106,000 & $\mathrm{Y}$ & 50 \\
\hline 07188000 & Spring River near Quapaw, OK & 2,510 & $\begin{array}{l}1935 \\
1939-98\end{array}$ & 1993 & 46.60 & 230,000 & 4/13/94 & 34.38 & 107,000 & $\mathrm{~N}$ & $15-20$ \\
\hline 07191500 & Neosho River near Chouteau, OK & 11,534 & $\begin{array}{l}1927, \\
1937-58, \\
1961, \\
1963-98\end{array}$ & 1943 & 45.00 & 400,000 & $6 / 11 / 95$ & 36.29 & 164,000 & $\mathrm{Y}$ & $30-35$ \\
\hline 07238000 & $\begin{array}{l}\text { North Canadian River near Seiling, } \\
\text { OK }\end{array}$ & 12,261 & $\begin{array}{l}1924, \\
1946-98\end{array}$ & $\begin{array}{l}1951 \\
1924\end{array}$ & $\begin{array}{l}15.61 \\
16.40\end{array}$ & $\begin{array}{r}33,000 \\
--\end{array}$ & 9/23/97 & 14.86 & 7,200 & $\mathrm{Y}$ & $25-35$ \\
\hline
\end{tabular}


$\left[\mathrm{mi}^{2}\right.$, square miles; ft, feet above an arbitrary datum; $\mathrm{ft}^{3} / \mathrm{s}$, cubic feet per second; --, not determined or not applicable; $>$, greater than. Source: Recurrence intervals calculated from U.S. Geological Survey data. Other data from U.S. Geological Survey reports or databases]

\begin{tabular}{|c|c|c|c|c|c|c|c|c|c|c|c|}
\hline \multirow[b]{2}{*}{$\begin{array}{l}\text { Streamgage } \\
\text { number } \\
\text { (fig. 48) }\end{array}$} & \multirow[b]{2}{*}{ Streamgage name } & \multirow[b]{2}{*}{$\begin{array}{l}\text { Total } \\
\text { drainage } \\
\left(\mathrm{mi}^{2}\right)\end{array}$} & \multicolumn{4}{|c|}{$\begin{array}{l}\text { Maximum stage and discharge for period of record } \\
\text { through } 1998 \text { water year }\end{array}$} & \multicolumn{5}{|c|}{ Signific ant floods $1994-98$ water years } \\
\hline & & & $\begin{array}{l}\text { Period of } \\
\text { record } \\
\text { (water } \\
\text { years) }\end{array}$ & Water year & $\begin{array}{l}\text { Stage } \\
(\mathrm{ft})\end{array}$ & $\begin{array}{l}\text { Discharge } \\
\left(\mathrm{ft}^{3} / \mathrm{s}\right)\end{array}$ & $\begin{array}{c}\text { Date } \\
\text { (month/ } \\
\text { day/ } \\
\text { year) }\end{array}$ & $\begin{array}{l}\text { Stage } \\
(\mathrm{ft})\end{array}$ & $\begin{array}{l}\text { Discharge } \\
\left(\mathrm{ft}^{3} / \mathrm{s}\right)\end{array}$ & $\begin{array}{l}\text { Regulated } \\
\text { during } \\
\text { flood }^{1}\end{array}$ & $\begin{array}{l}\text { Recurrence } \\
\text { interval } \\
\text { (years) }\end{array}$ \\
\hline 07241000 & $\begin{array}{l}\text { North Canadian River below Lake } \\
\text { Overholser near Oklahoma City, } \\
\text { OK }\end{array}$ & 13,222 & $\begin{array}{l}1921, \\
1923-24, \\
1952-68, \\
1969-72, \\
1973-87, \\
1988-98\end{array}$ & 1924 & 40.90 & 135,000 & $6 / 11 / 95$ & 25.05 & 19,500 & $\mathrm{Y}$ & $15-20$ \\
\hline 07242380 & Deep Fork near Warwick, OK & 532 & $1983-98$ & $\begin{array}{l}1983 \\
1995\end{array}$ & $\begin{array}{l}22.05 \\
21.28\end{array}$ & $\begin{array}{l}28,700 \\
34,600\end{array}$ & $6 / 9 / 95$ & 21.28 & 34,600 & Y & 25 \\
\hline 07301420 & $\begin{array}{l}\text { Sweetwater Creek near } \\
\text { Sweetwater, OK }\end{array}$ & 424 & 1986-98 & 1995 & 15.89 & 1,940 & $6 / 3 / 95$ & 15.89 & 1,940 & $\mathrm{~N}$ & $10-25$ \\
\hline 07301500 & $\begin{array}{l}\text { North Fork Red River near Carter, } \\
\text { OK }\end{array}$ & 2,337 & $\begin{array}{l}1904-07, \\
1928, \\
1930-32, \\
1935, \\
1938-98\end{array}$ & $\begin{array}{l}1959 \\
1995\end{array}$ & $\begin{array}{l}13.42 \\
15.08\end{array}$ & $\begin{array}{l}53,400 \\
34,800\end{array}$ & $6 / 4 / 95$ & 15.08 & 34,800 & $\mathrm{~N}$ & $30-35$ \\
\hline 07303400 & $\begin{array}{l}\text { Elm Fork of North Fork Red River } \\
\text { near Carl, OK }\end{array}$ & 416 & $\begin{array}{l}1959-79 \\
1995-98\end{array}$ & 1995 & 18.80 & 62,300 & $6 / 3 / 95$ & 18.80 & 62,300 & $\mathrm{~N}$ & $>100$ \\
\hline 07307028 & $\begin{array}{l}\text { North Fork Red River near Tipton, } \\
\text { OK }\end{array}$ & 4,691 & 1985-98 & 1993 & 19.18 & 51,200 & $6 / 6 / 95$ & 18.41 & 40,700 & $\mathrm{Y}$ & $25-50$ \\
\hline 07315500 & Red River near Terral, OK & 28,723 & $\begin{array}{l}1935, \\
1938-80, \\
1981-83, \\
1986-91, \\
1993-98\end{array}$ & $\begin{array}{l}1995 \\
1987\end{array}$ & $\begin{array}{l}30.56 \\
32.65\end{array}$ & $\begin{array}{l}236,000 \\
225,000\end{array}$ & $6 / 7 / 95$ & 30.56 & 236,000 & $\mathrm{Y}$ & $>100$ \\
\hline 07316000 & Red River near Gainesville, TX & 30,782 & 1935-98 & 1987 & 40.08 & 265,000 & $6 / 13 / 95$ & 36.63 & 169,000 & $\mathrm{Y}$ & $10-15$ \\
\hline 07324400 & Washita River near Foss, OK & 1,551 & $\begin{array}{l}1956-58 \\
1961-87 \\
1989-98\end{array}$ & $\begin{array}{l}1958 \\
1959\end{array}$ & $\begin{array}{l}16.90 \\
23.40\end{array}$ & $\begin{array}{r}3,660 \\
--\end{array}$ & $9 / 15 / 96$ & 21.24 & 2,990 & $\mathrm{Y}$ & $75-85$ \\
\hline 07325000 & Washita River near Clinton, OK & 1,977 & 1934-98 & 1934 & 33.90 & 90,000 & $9 / 15 / 96$ & 26.24 & 10,800 & $\mathrm{Y}$ & $30-35$ \\
\hline
\end{tabular}


Table 37. Maximum stage and discharge for period of record for streamgages having significant floods during 1994-98 water years in 0klahoma.-Continued

$\left[\mathrm{mi}^{2}\right.$, square miles; ft, feet above an arbitrary datum; $\mathrm{ft}^{3} / \mathrm{s}$, cubic feet per second; --, not determined or not applicable; $>$, greater than. Source: Recurrence intervals calculated from U.S. Geological Survey data. Other data from U.S. Geological Survey reports or databases]

\begin{tabular}{|c|c|c|c|c|c|c|c|c|c|c|c|}
\hline \multirow{2}{*}{$\begin{array}{c}\text { Streamgage } \\
\text { number } \\
\text { (fig. 48) }\end{array}$} & \multirow[b]{2}{*}{ Streamgage name } & \multirow{2}{*}{$\begin{array}{c}\text { Total } \\
\text { drainage } \\
\left(\mathrm{mi}^{2}\right)\end{array}$} & \multicolumn{4}{|c|}{$\begin{array}{c}\text { Maximum stage and discharge for period of record } \\
\text { through } 1998 \text { water year }\end{array}$} & \multicolumn{5}{|c|}{ Significant floods 1994-98 water years } \\
\hline & & & $\begin{array}{l}\text { Period of } \\
\text { record } \\
\text { (water } \\
\text { years) }\end{array}$ & Water year & $\begin{array}{l}\text { Stage } \\
\text { (ft) }\end{array}$ & $\begin{array}{c}\text { Discharge } \\
\left(\mathrm{ft}^{3} / \mathrm{s}\right)\end{array}$ & $\begin{array}{c}\text { Date } \\
\text { (month/ } \\
\text { day/ } \\
\text { year) }\end{array}$ & $\begin{array}{l}\text { Stage } \\
\text { (ft) }\end{array}$ & $\begin{array}{c}\text { Discharge } \\
\left(\mathrm{ft}^{3} / \mathrm{s}\right)\end{array}$ & $\begin{array}{c}\text { Regulated } \\
\text { during } \\
\text { flood }^{1}\end{array}$ & $\begin{array}{c}\text { Recurrence } \\
\text { interval } \\
\text { (years) }\end{array}$ \\
\hline 07325500 & Washita River at Carnegie, OK & 3,129 & $\begin{array}{l}\text { 1913, 1921, } \\
\text { 1923-24, } \\
\text { 1934-36, } \\
1937-98\end{array}$ & $\begin{array}{l}1949 \\
1995\end{array}$ & $\begin{array}{l}26.21 \\
31.50\end{array}$ & $\begin{array}{l}50,000 \\
40,200\end{array}$ & $6 / 5 / 95$ & 31.50 & 40,200 & $\mathrm{Y}$ & $40-45$ \\
\hline 07325800 & Cobb Creek near Eakly, OK & 132 & $1968-98$ & $\begin{array}{l}1995 \\
1986\end{array}$ & $\begin{array}{l}22.05 \\
24.38\end{array}$ & $\begin{array}{l}12,000 \\
10,000\end{array}$ & $6 / 4 / 95$ & 22.05 & 12,000 & $\mathrm{~N}$ & 25 \\
\hline 07326500 & Washita River at Anadarko, OK & 3,656 & $\begin{array}{l}1902-08 \\
1924-25, \\
1935-37 \\
1993-98\end{array}$ & 1995 & 25.37 & 52,800 & $6 / 6 / 95$ & 25.37 & 52,800 & $\mathrm{Y}$ & $50-55$ \\
\hline
\end{tabular}

Regulated during flood: $\mathrm{N}, \mathrm{no} ; \mathrm{Y}$, yes. 


\section{Oregon}

A subtropical, moisture-laden Pacific front stalled over northwestern Oregon on November 10, 1995. Storm total rainfall ranged from about 3 inches in the Willamette River Valley to 4 inches in the Cascade Range and 5 inches in the Coast Range. The most serious damages occurred in the Portland area and along the Zig Zag River in the foothills of Mt. Hood (fig. 49) (National Oceanic and Atmospheric Administration, 1995b).

Runoff from excessive rains and melting mountain snow caused major floods on many northern and eastern Oregon rivers beginning on February 6, 1996. The first rivers reached flood stage on the morning of February 6 when the most intense rain was still falling. Streamflow measured at 27 streamgages set all-time high river discharges, and 4 more set stage records during February 1996 (table 38). Many smaller ungaged streams also left their banks during this period. Statewide damage was estimated at over $\$ 400$ million, with an estimated 5,000 homes destroyed, and seven people lost their lives as a direct result of flooding (National Oceanic and Atmospheric Administration, 1996b). Most rivers had receded to below flood stage by February 14; however, the lower Willamette River fluctuated around flood stage, and the downstream reaches of the Columbia River remained above flood stage until the end of February.

Moist southwest flow aloft produced moderate to excessive rain and strong winds over southwestern and northern Oregon during November 17-21, 1996. Storm total rainfall ranged from 8 to 12 inches on the coast with local amounts near 20 inches, while 3 to 7 inches of rain fell inland (National Oceanic and Atmospheric Administration, 1996a). Flooding was extensive in southwestern Oregon. The rainfall amount and rate produced numerous mudslides into homes and across highways. Many highways were closed due to slides, and the northbound lane of I-5 near Roseburg collapsed. Major rivers, such as the downstream reaches of the Willamette River, remained above flood stage until November 23. Damage estimates were nearly $\$ 45$ million, and there were five deaths from drowning (National Oceanic and Atmospheric Administration, 1996b).

A series of storm systems dumped excessive amounts of rain over southern Oregon during December 7-10, 1996. A 24-hour rainfall total of 4 to 8 inches fell along the coast and 2 to 6 inches inland (National Oceanic and Atmospheric Administration, 1996a). Most major rivers and many of the small streams in southwest Oregon flooded. Mudslides and flooding closed many roads and highways. Damage was over $\$ 8$ million (National Oceanic and Atmospheric Administration, 1996b).

A warm southwest flow pushed temperatures above $60{ }^{\circ} \mathrm{F}$ on New Years Eve Day, 1997, with the freezing level lifting to elevations above 10,000 feet. Melting snow and copious rain drove rivers and streams well above flood stage across southern Oregon from New Years Day to January 5, with widespread flooding through January 3. The total basin rainfall for the New Years Day storm averaged 2 to 4 inches, with rainfall amounts in excess of 8 inches (National Oceanic and Atmospheric Administration, 1997a). Six streamgages recorded their peak of record during this flood (table 38). Damage was nearly $\$ 89$ million (National Oceanic and Atmospheric Administration, 1997b).

\section{References}

National Oceanic and Atmospheric Administration (NOAA), 1995a-97a, Climatological data (by State): Asheville, North Carolina, National Climatic Data Center, various months. National Oceanic and Atmospheric Administration (NOAA), 1995b-97b, Storm data (by State): Asheville, North Carolina, National Climatic Data Center, various months. 


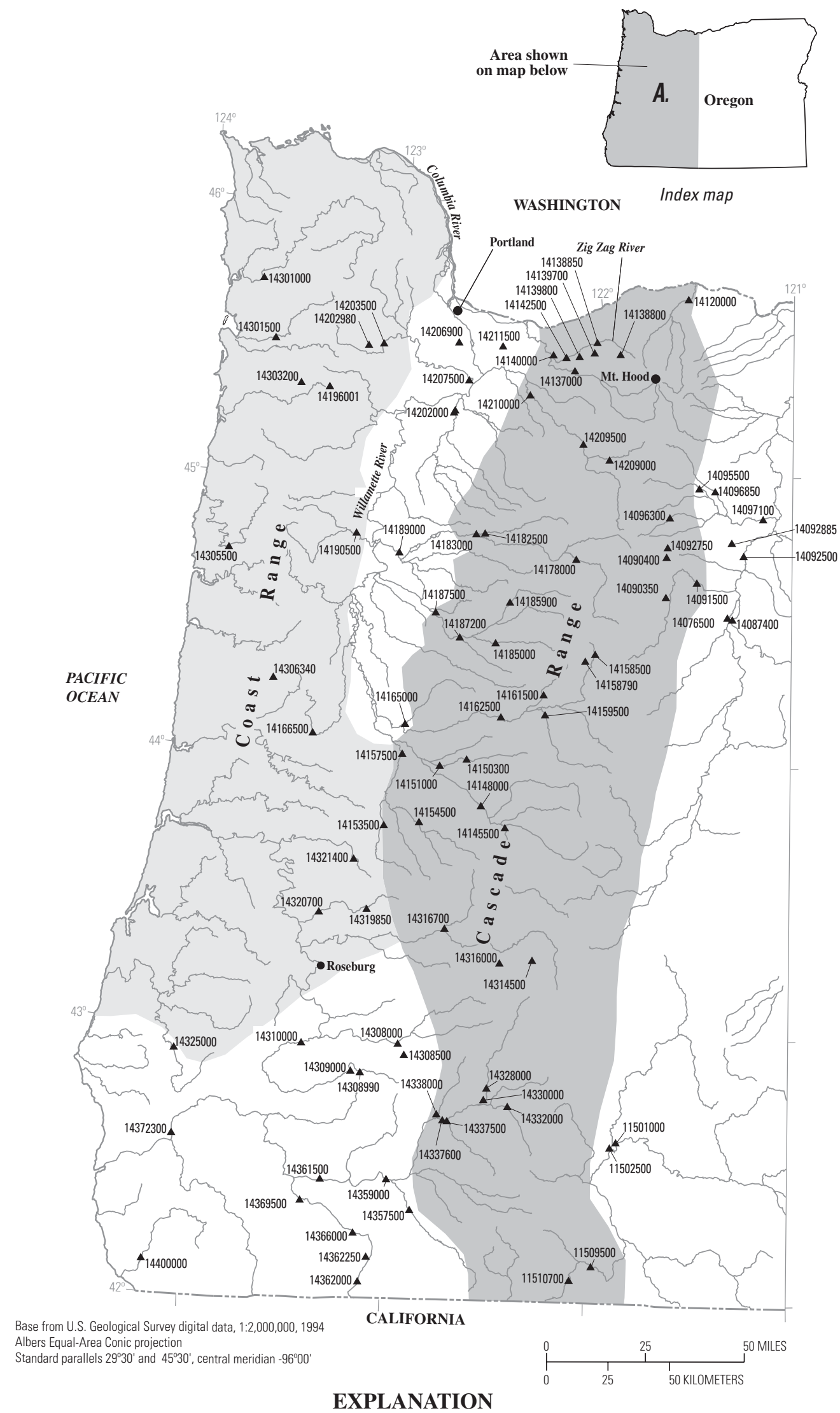

$11510700 \triangle$ Streamgage and number

Figure 49. (A) Location of streamgages with significant floods during 1994-98 water years for Oregon. 


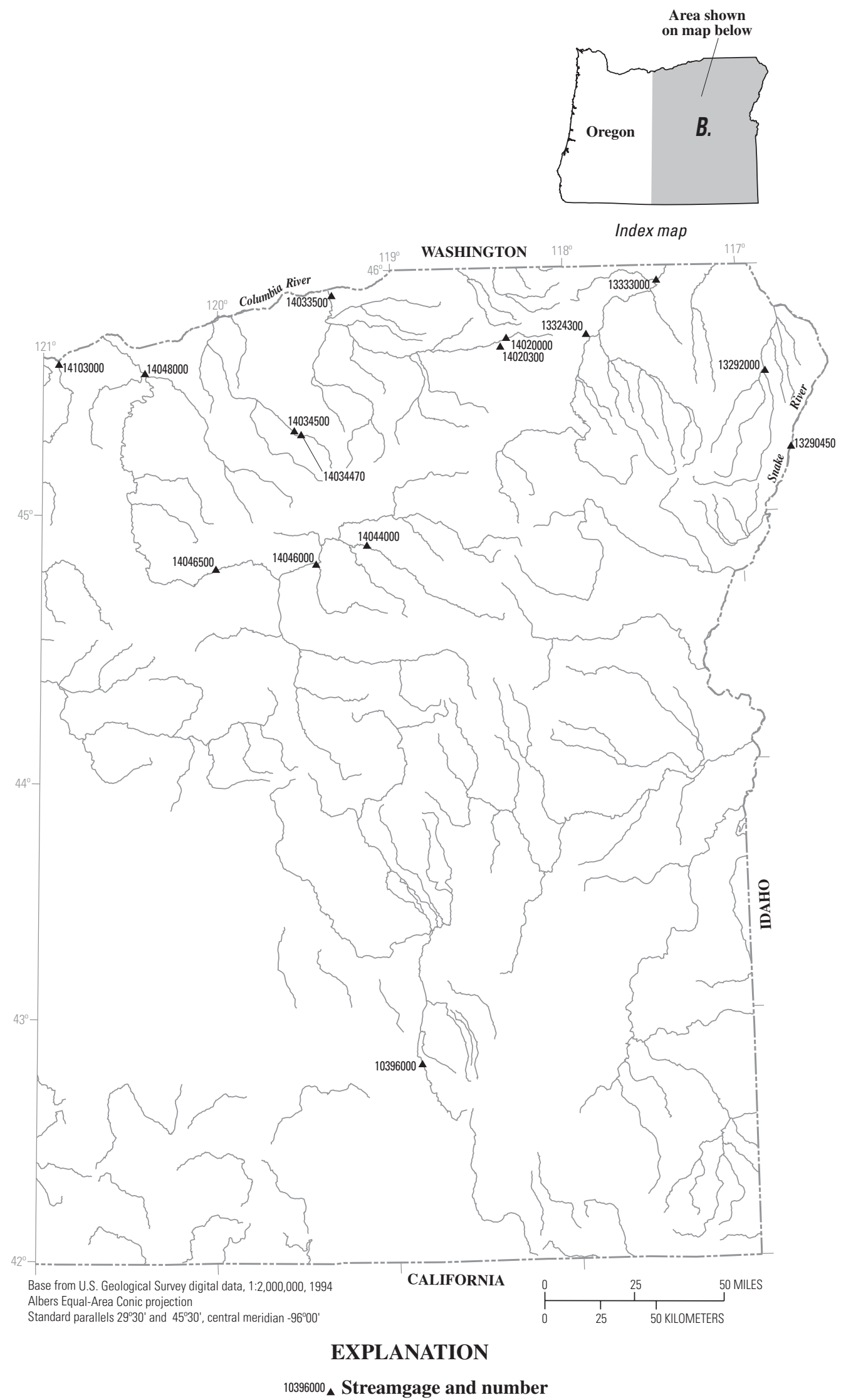

Figure 49. (B) Location of streamgages with significant floods during 1994-98 water years for Oregon.-Continued 
Table 38. Maximum stage and discharge for period of record for streamgages having significant floods during 1994-98 water years in Oregon.

$\left[\mathrm{mi}^{2}\right.$, square miles; $\mathrm{ft}$, feet above an arbitrary datum; $\mathrm{ft}^{3} / \mathrm{s}$, cubic feet per second; --, not determined or not applicable; >, greater than. Source: Recurrence intervals calculated from U.S. Geological Survey data. Other data from U.S. Geological Survey reports or databases]

\begin{tabular}{|c|c|c|c|c|c|c|c|c|c|c|c|}
\hline \multirow{2}{*}{$\begin{array}{l}\text { Streamgage } \\
\text { number } \\
\text { (fig. 49) }\end{array}$} & \multirow[b]{2}{*}{ Streamgage name } & \multirow{2}{*}{$\begin{array}{l}\text { Total } \\
\text { drainage } \\
\left(\mathrm{mi}^{2}\right)\end{array}$} & \multicolumn{4}{|c|}{$\begin{array}{c}\text { Maximum stage and discharge for period of record } \\
\text { through } 1998 \text { water year }\end{array}$} & \multicolumn{5}{|c|}{ Significant floods $1994-98$ water years } \\
\hline & & & $\begin{array}{l}\text { Period of } \\
\text { record } \\
\text { (water } \\
\text { years) }\end{array}$ & Water year & $\begin{array}{l}\text { Stage } \\
(\mathrm{ft})\end{array}$ & $\begin{array}{l}\text { Discharge } \\
\left(\mathrm{ft}^{3} / \mathrm{s}\right)\end{array}$ & $\begin{array}{l}\text { Date } \\
\text { (month/ } \\
\text { day/ } \\
\text { year) }\end{array}$ & $\begin{array}{l}\text { Stage } \\
(\mathrm{ft})\end{array}$ & $\begin{array}{l}\text { Discharge } \\
\left(\mathrm{ft}^{3} / \mathrm{s}\right)\end{array}$ & $\begin{array}{l}\text { Regulated } \\
\text { during } \\
\text { flood }^{1}\end{array}$ & $\begin{array}{l}\text { Recurrence } \\
\text { interval } \\
\text { (years) }\end{array}$ \\
\hline 10396000 & $\begin{array}{l}\text { Donner Und Blitzen River near } \\
\text { Frenchglen, OR }\end{array}$ & 200 & $\begin{array}{l}1911-16, \\
1918-21, \\
1930, \\
1938-98\end{array}$ & 1978 & 7.15 & 4,270 & $5 / 14 / 98$ & 6.32 & 3,110 & $\mathrm{~N}$ & 15 \\
\hline 11501000 & Sprague River near Chiloquin, OR & 1,580 & $1921-98$ & 1965 & 10.37 & 14,900 & $1 / 5 / 97$ & 9.05 & 10,800 & $\mathrm{~N}$ & $40-50$ \\
\hline 11502500 & $\begin{array}{l}\text { Williamson River below Sprague } \\
\text { River near Chiloquin, OR }\end{array}$ & 3,000 & $1917-98$ & $\begin{array}{l}1997 \\
1965\end{array}$ & $\begin{array}{l}10.27 \\
10.56\end{array}$ & $\begin{array}{l}17,100 \\
16,100\end{array}$ & $1 / 5 / 97$ & 10.27 & 17,100 & $\mathrm{~N}$ & $75-100$ \\
\hline 11509500 & Klamath River at Keno, OR & 3,920 & $\begin{array}{l}1905-13 \\
1930-98\end{array}$ & $\begin{array}{l}1986 \\
1907\end{array}$ & $\begin{array}{l}12.82 \\
14.40\end{array}$ & $\begin{array}{r}10,300 \\
5,220\end{array}$ & $\begin{array}{l}2 / 22 / 96 \\
1 / 3 / 97\end{array}$ & $\begin{array}{l}12.41 \\
12.60\end{array}$ & $\begin{array}{l}9,520 \\
9,870\end{array}$ & $\begin{array}{l}\mathrm{Y} \\
\mathrm{Y}\end{array}$ & -- \\
\hline 11510700 & $\begin{array}{l}\text { Klamath River below John C. } \\
\text { Boyle Power Plant near Keno, } \\
\text { OR }\end{array}$ & 4,080 & 1959-98 & 1996 & 9.50 & 11,600 & $\begin{array}{l}2 / 21 / 96 \\
1 / 3 / 97\end{array}$ & $\begin{array}{l}9.50 \\
9.44\end{array}$ & $\begin{array}{l}11,600 \\
11,400\end{array}$ & $\begin{array}{l}\mathrm{Y} \\
\mathrm{Y}\end{array}$ & -- \\
\hline 13290450 & $\begin{array}{l}\text { Snake River at Hells Canyon Dam, } \\
\text { Idaho-Oregon State line, OR }\end{array}$ & 73,300 & 1966-98 & 1997 & 86.17 & 103,000 & $1 / 2 / 97$ & 86.17 & 103,000 & $\mathrm{Y}$ & -- \\
\hline 13292000 & Imnaha River at Imnaha, OR & 622 & 1929-98 & 1997 & 11.44 & 20,200 & $1 / 1 / 97$ & 11.44 & 20,200 & $\mathrm{~N}$ & $>100$ \\
\hline 13324300 & $\begin{array}{l}\text { Lookingglass Creek near Looking } \\
\text { Glass, OR }\end{array}$ & 78.3 & 1983-98 & 1996 & 7.41 & 2,120 & 2/9/96 & 7.41 & 2,120 & $\mathrm{~N}$ & $>25$ \\
\hline 13333000 & Grande Ronde River at Troy, OR & 3,275 & $1945-98$ & 1996 & 13.76 & 51,800 & $\begin{array}{l}2 / 9 / 96 \\
1 / 1 / 97\end{array}$ & $\begin{array}{l}13.76 \\
12.65\end{array}$ & $\begin{array}{l}51,800 \\
36,700\end{array}$ & $\begin{array}{l}\mathrm{N} \\
\mathrm{N}\end{array}$ & $\begin{array}{r}>100 \\
25\end{array}$ \\
\hline 14020000 & $\begin{array}{l}\text { Umatilla River above Meacham } \\
\text { Creek near Gibbon, OR }\end{array}$ & 131 & $1933-98$ & $\begin{array}{l}1996 \\
1965\end{array}$ & $\begin{array}{l}9.40 \\
9.50\end{array}$ & $\begin{array}{l}6,220 \\
4,910\end{array}$ & $\begin{array}{l}11 / 28 / 95 \\
1 / 1 / 97\end{array}$ & $\begin{array}{l}9.40 \\
8.76\end{array}$ & $\begin{array}{l}6,220 \\
5,230\end{array}$ & $\begin{array}{l}\mathrm{N} \\
\mathrm{N}\end{array}$ & $\begin{array}{l}50 \\
25\end{array}$ \\
\hline 14020300 & Meacham Creek at Gibbon, OR & 176 & $1976-98$ & 1996 & 7.67 & 5,930 & $11 / 28 / 95$ & 7.67 & 5,930 & $\mathrm{~N}$ & 20 \\
\hline 14033500 & Umatilla River near Umatilla, OR & 2,290 & 1904-98 & $\begin{array}{l}1965 \\
1906\end{array}$ & $\begin{array}{l}10.75 \\
11.00\end{array}$ & $\begin{array}{l}19,800 \\
19,600\end{array}$ & $2 / 10 / 96$ & 8.49 & 16,300 & $\mathrm{Y}$ & 30 \\
\hline 14034470 & $\begin{array}{l}\text { Willow Creek above Willow Creek } \\
\text { Lake near Heppner, OR }\end{array}$ & 67.6 & 1983-98 & 1997 & 9.60 & 544 & $2 / 1 / 97$ & 9.60 & 544 & $\mathrm{~N}$ & $10-25$ \\
\hline
\end{tabular}


Table 38. Maximum stage and discharge for period of record for streamgages having significant floods during 1994-98 water years in Oregon.-Continued

$\left[\mathrm{mi}^{2}\right.$, square miles; ft, feet above an arbitrary datum; $\mathrm{ft}^{3} / \mathrm{s}$, cubic feet per second; --, not determined or not applicable; >, greater than. Source: Recurrence intervals calculated from U.S. Geological Survey data. Other data from U.S. Geological Survey reports or databases]

\begin{tabular}{|c|c|c|c|c|c|c|c|c|c|c|c|}
\hline \multirow[b]{2}{*}{$\begin{array}{c}\text { Streamgage } \\
\text { number } \\
\text { (fig. 49) }\end{array}$} & \multirow[b]{2}{*}{ Streamgage name } & \multirow[b]{2}{*}{$\begin{array}{c}\text { Total } \\
\text { drainage } \\
\left(\mathrm{mi}^{2}\right)\end{array}$} & \multicolumn{4}{|c|}{$\begin{array}{c}\text { Maximum stage and discharge for period of record } \\
\text { through } 1998 \text { water year }\end{array}$} & \multicolumn{5}{|c|}{ Significant floods $1994-98$ water years } \\
\hline & & & $\begin{array}{l}\text { Period of } \\
\text { record } \\
\text { (water } \\
\text { years) }\end{array}$ & Water year & $\begin{array}{l}\text { Stage } \\
(\mathrm{ft})\end{array}$ & $\begin{array}{l}\text { Discharge } \\
\left(\mathrm{ft}^{3} / \mathrm{s}\right)\end{array}$ & $\begin{array}{c}\text { Date } \\
\text { (month/ } \\
\text { day/ } \\
\text { year) }\end{array}$ & $\begin{array}{l}\text { Stage } \\
(\mathrm{ft})\end{array}$ & $\begin{array}{l}\text { Discharge } \\
\left(\mathrm{ft}^{3} / \mathrm{s}\right)\end{array}$ & $\begin{array}{l}\text { Regulated } \\
\text { during } \\
\text { flood }^{1}\end{array}$ & $\begin{array}{c}\text { Recurrence } \\
\text { interval } \\
\text { (years) }\end{array}$ \\
\hline 14034500 & Willow Creek at Heppner, OR & 96.8 & $\begin{array}{l}1949, \\
1952-90, \\
1992-98\end{array}$ & $\begin{array}{l}1949 \\
1957\end{array}$ & $\begin{array}{l}- \\
6.15\end{array}$ & $\begin{array}{r}1,700 \\
812\end{array}$ & $5 / 11 / 95$ & 5.22 & 333 & $\mathrm{Y}$ & -- \\
\hline 14044000 & $\begin{array}{l}\text { Middle Fork John Day River at } \\
\text { Ritter, OR }\end{array}$ & 515 & 1930-98 & 1965 & 8.39 & 4,730 & $1 / 1 / 97$ & 8.24 & 4,540 & $\mathrm{~N}$ & 50 \\
\hline 14046000 & $\begin{array}{l}\text { North Fork John Day River at } \\
\text { Monument, OR }\end{array}$ & 2,520 & $1925-98$ & 1965 & 18.45 & 33,400 & $\begin{array}{l}2 / 9 / 96 \\
1 / 1 / 97\end{array}$ & $\begin{array}{l}14.63 \\
17.56\end{array}$ & $\begin{array}{l}21,700 \\
30,400\end{array}$ & $\begin{array}{l}\mathrm{N} \\
\mathrm{N}\end{array}$ & $\begin{array}{l}15 \\
50\end{array}$ \\
\hline 14046500 & $\begin{array}{l}\text { John Day River at Service Creek, } \\
\text { OR }\end{array}$ & 5,090 & $\begin{array}{l}\text { 1926, } \\
1930-98\end{array}$ & 1965 & 17.85 & 40,200 & $1 / 1 / 97$ & 16.49 & 35,200 & $\mathrm{~N}$ & 35 \\
\hline 14048000 & $\begin{array}{l}\text { John Day River at McDonald } \\
\text { Ferry, OR }\end{array}$ & 7,580 & $\begin{array}{l}1905-08, \\
1910-96, \\
1998\end{array}$ & 1965 & 13.59 & 42,800 & $2 / 10 / 96$ & 13.16 & 31,300 & $\mathrm{~N}$ & 25 \\
\hline 14076500 & Deschutes River near Culver, OR & 2,705 & 1953-98 & 1965 & 10.00 & 6,680 & $1 / 31 / 97$ & 7.89 & 3,910 & Y & 25 \\
\hline 14087400 & $\begin{array}{l}\text { Crooked River below Opal Springs } \\
\text { near Culver, OR }\end{array}$ & 4,300 & $1962-98$ & 1965 & 9.36 & 6,660 & $1 / 2 / 97$ & 9.08 & 6,350 & $\mathrm{Y}$ & -- \\
\hline 14090350 & $\begin{array}{l}\text { Jefferson Creek near Camp } \\
\text { Sherman, OR }\end{array}$ & 27.8 & 1984-98 & 1996 & 3.94 & 730 & 2/7/96 & 3.94 & 730 & $\mathrm{~N}$ & $25-50$ \\
\hline 14090400 & $\begin{array}{l}\text { Whitewater River near Camp } \\
\text { Sherman, OR }\end{array}$ & 22.8 & 1983-98 & $\begin{array}{l}1996 \\
1998\end{array}$ & $\overline{--}$ & $\begin{array}{r}2,320 \\
683\end{array}$ & $2 / 7 / 96$ & -- & 2,320 & $\mathrm{~N}$ & $50-100$ \\
\hline 14091500 & $\begin{array}{l}\text { Metolius River near Grandview, } \\
\text { OR }\end{array}$ & 316 & $\begin{array}{l}1913, \\
1922-98\end{array}$ & 1996 & 7.38 & 8,430 & 2/7/96 & 7.38 & 8,430 & $\mathrm{~N}$ & $>100$ \\
\hline 14092500 & Deschutes River near Madras, OR & 7,820 & 1924-98 & 1983 & 7.70 & 22,500 & $2 / 8 / 96$ & 7.08 & 19,100 & $\mathrm{Y}$ & -- \\
\hline 14092750 & $\begin{array}{l}\text { Shitike Creek at Peters Pasture } \\
\text { near Warm Springs, OR }\end{array}$ & 22.9 & 1983-98 & 1996 & 6.66 & 2,430 & 2/7/96 & 6.66 & 2,430 & $\mathrm{~N}$ & $25-50$ \\
\hline 14092885 & $\begin{array}{l}\text { Shitike Creek below Wolford } \\
\text { Canyon near Warm Springs, OR }\end{array}$ & 75.8 & $1975-96$ & 1996 & 7.93 & 3,600 & 2/7/96 & 7.93 & 3,600 & $\mathrm{~N}$ & 50 \\
\hline 14095500 & $\begin{array}{l}\text { Warm Springs River near } \\
\text { Simnasho, OR }\end{array}$ & 107 & 1984-98 & 1996 & 6.98 & 4,670 & $2 / 7 / 96$ & 6.98 & 4,670 & $\mathrm{~N}$ & $50-100$ \\
\hline
\end{tabular}


Table 38. Maximum stage and discharge for period of record for streamgages having significant floods during 1994-98 water years in Oregon.-Continued

$\left[\mathrm{mi}^{2}\right.$, square miles; $\mathrm{ft}$, feet above an arbitrary datum; $\mathrm{ft}^{3} / \mathrm{s}$, cubic feet per second; --, not determined or not applicable; >, greater than. Source: Recurrence intervals calculated from U.S. Geological Survey data. Other data from U.S. Geological Survey reports or databases]

\begin{tabular}{|c|c|c|c|c|c|c|c|c|c|c|c|}
\hline \multirow[b]{2}{*}{$\begin{array}{l}\text { Streamgage } \\
\text { number } \\
\text { (fig. 49) }\end{array}$} & \multirow[b]{2}{*}{ Streamgage name } & \multirow[b]{2}{*}{$\begin{array}{l}\text { Total } \\
\text { drainage } \\
\left(\mathrm{mi}^{2}\right)\end{array}$} & \multicolumn{4}{|c|}{$\begin{array}{c}\text { Maximum stage and discharge for period of record } \\
\text { through } 1998 \text { water year }\end{array}$} & \multicolumn{5}{|c|}{ Significant floods $1994-98$ water years } \\
\hline & & & $\begin{array}{l}\text { Period of } \\
\text { record } \\
\text { (water } \\
\text { years) }\end{array}$ & Water year & $\begin{array}{l}\text { Stage } \\
\text { (ft) }\end{array}$ & $\begin{array}{l}\text { Discharge } \\
\left(\mathrm{ft}^{3} / \mathrm{s}\right)\end{array}$ & $\begin{array}{c}\text { Date } \\
\text { (month/ } \\
\text { day/ } \\
\text { year) }\end{array}$ & $\begin{array}{l}\text { Stage } \\
(\mathrm{ft})\end{array}$ & $\begin{array}{l}\text { Discharge } \\
\left(\mathrm{ft}^{3} / \mathrm{s}\right)\end{array}$ & $\begin{array}{l}\text { Regulated } \\
\text { during } \\
\text { flood }^{1}\end{array}$ & $\begin{array}{l}\text { Recurrence } \\
\text { interval } \\
\text { (years) }\end{array}$ \\
\hline 14096300 & $\begin{array}{l}\text { Mill Creek near Badger Butte near } \\
\text { Warm Springs, OR }\end{array}$ & 26.8 & $1984-98$ & 1996 & 8.42 & 1,300 & $2 / 7 / 96$ & 8.42 & 1,300 & $\mathrm{~N}$ & $25-50$ \\
\hline 14096850 & $\begin{array}{l}\text { Beaver Creek below Quartz Creek } \\
\text { near Simnasho, OR }\end{array}$ & 145 & 1984-98 & 1996 & 10.57 & 5,760 & $2 / 7 / 96$ & 10.57 & 5,760 & $\mathrm{~N}$ & $20-30$ \\
\hline 14097100 & $\begin{array}{l}\text { Warm Springs River near Kah- } \\
\text { neeta Hot Springs, OR }\end{array}$ & 526 & 1973-98 & 1996 & 14.32 & 22,600 & $2 / 7 / 96$ & 14.32 & 22,600 & $\mathrm{~N}$ & $50-100$ \\
\hline 14103000 & $\begin{array}{l}\text { Deschutes River at Moody near } \\
\text { Biggs, OR }\end{array}$ & 10,500 & $\begin{array}{l}\text { 1898, } \\
1907-98\end{array}$ & 1996 & 12.08 & 70,300 & $2 / 8 / 96$ & 12.08 & 70,300 & $\mathrm{Y}$ & $>100$ \\
\hline 14120000 & $\begin{array}{l}\text { Hood River at Tucker Bridge near } \\
\text { Hood River, OR }\end{array}$ & 279 & $\begin{array}{l}1898-99, \\
1914, \\
1916-17, \\
1965-75, \\
1977-98\end{array}$ & 1965 & 20.60 & 33,200 & $2 / 7 / 96$ & 17.11 & 23,300 & $\mathrm{~N}$ & $<25$ \\
\hline 14137000 & Sandy River near Marmot, OR & 263 & 1912-98 & 1965 & 17.05 & 61,400 & $2 / 7 / 96$ & 20.40 & 48,100 & $\mathrm{~N}$ & $75-100$ \\
\hline 14138800 & $\begin{array}{l}\text { Blazed Alder Creek near } \\
\text { Rhododendron, OR }\end{array}$ & 8.17 & 1964-98 & 1965 & 8.25 & 2,610 & $2 / 7 / 96$ & 7.05 & 2,020 & $\mathrm{~N}$ & 25 \\
\hline 14138850 & $\begin{array}{l}\text { Bull Run River near Multnomah } \\
\text { Falls, OR }\end{array}$ & 47.9 & 1967-98 & 1996 & 13.60 & 9,140 & $2 / 6 / 96$ & 13.60 & 9,140 & $\mathrm{~N}$ & $30-40$ \\
\hline 14139700 & Cedar Creek near Brightwood, OR & 7.93 & 1965-98 & 1965 & 7.20 & 1,990 & $2 / 7 / 96$ & 5.40 & 1,720 & $\mathrm{~N}$ & 15 \\
\hline 14139800 & $\begin{array}{l}\text { South Fork Bull Run River near } \\
\text { Bull Run, OR }\end{array}$ & 15.4 & $1975-98$ & 1996 & 9.54 & 3,630 & $2 / 7 / 96$ & 9.54 & 3,630 & $\mathrm{~N}$ & 50 \\
\hline 14140000 & Bull Run River near Bull Run, OR & 107 & 1908-98 & $\begin{array}{l}1965 \\
1996\end{array}$ & $-\overline{16.00}$ & $\begin{array}{l}25,100 \\
20,200\end{array}$ & 2/7/96 & 16.00 & 20,200 & $\mathrm{Y}$ & 35 \\
\hline 14142500 & $\begin{array}{l}\text { Sandy River below Bull Run River } \\
\text { near Bull Run, OR }\end{array}$ & 436 & $\begin{array}{l}1911-14 \\
1930-66 \\
1985-98\end{array}$ & $\begin{array}{l}1965 \\
1996\end{array}$ & $\begin{array}{l}22.30 \\
22.59\end{array}$ & $\begin{array}{l}84,400 \\
68,600\end{array}$ & 2/7/96 & 22.59 & 68,600 & $\mathrm{Y}$ & 50 \\
\hline 14145500 & $\begin{array}{l}\text { Middle Fork Willamette River } \\
\text { above Salt Creek near Oakridge, } \\
\text { OR }\end{array}$ & 392 & $\begin{array}{l}1914, \\
1936-98\end{array}$ & $\begin{array}{l}1946 \\
1956\end{array}$ & $\begin{array}{l}12.06 \\
12.71\end{array}$ & $\begin{array}{l}34,000 \\
33,300\end{array}$ & $1 / 2 / 97$ & 8.38 & 8,610 & $\mathrm{Y}$ & -- \\
\hline
\end{tabular}


$\left[\mathrm{mi}^{2}\right.$, square miles; ft, feet above an arbitrary datum; $\mathrm{ft}^{3} / \mathrm{s}$, cubic feet per second; --, not determined or not applicable; >, greater than. Source: Recurrence intervals calculated from U.S. Geological Survey data. Other data from U.S. Geological Survey reports or databases]

\begin{tabular}{|c|c|c|c|c|c|c|c|c|c|c|c|}
\hline \multirow{2}{*}{$\begin{array}{l}\text { Streamgage } \\
\text { number } \\
\text { (fig. 49) }\end{array}$} & \multirow[b]{2}{*}{ Streamgage name } & \multirow{2}{*}{$\begin{array}{l}\text { Total } \\
\text { drainage } \\
\left(\mathrm{mi}^{2}\right)\end{array}$} & \multicolumn{4}{|c|}{$\begin{array}{c}\text { Maximum stage and discharge for period of record } \\
\text { through } 1998 \text { water year }\end{array}$} & \multicolumn{5}{|c|}{ Significant floods $1994-98$ water years } \\
\hline & & & $\begin{array}{l}\text { Period of } \\
\text { record } \\
\text { (water } \\
\text { years) }\end{array}$ & Water year & $\begin{array}{l}\text { Stage } \\
(\mathrm{ft})\end{array}$ & $\begin{array}{l}\text { Discharge } \\
\left(\mathrm{ft}^{3} / \mathrm{s}\right)\end{array}$ & $\begin{array}{l}\text { Date } \\
\text { (month/ } \\
\text { day/ } \\
\text { year) }\end{array}$ & $\begin{array}{l}\text { Stage } \\
(\mathrm{ft})\end{array}$ & $\begin{array}{l}\text { Discharge } \\
\left(\mathrm{ft}^{3} / \mathrm{s}\right)\end{array}$ & $\begin{array}{l}\text { Regulated } \\
\text { during } \\
\text { flood }^{1}\end{array}$ & $\begin{array}{l}\text { Recurrence } \\
\text { interval } \\
\text { (years) }\end{array}$ \\
\hline 14148000 & $\begin{array}{l}\text { Middle Fork Willamette River } \\
\text { below North Fork near Oakridge, } \\
\text { OR }\end{array}$ & 924 & $\begin{array}{l}\text { 1912, } \\
1924-98\end{array}$ & 1946 & 18.80 & 81,800 & $11 / 19 / 96$ & 10.34 & 40,800 & $\mathrm{Y}$ & -- \\
\hline 14150300 & Fall Creek near Lowell, OR & 118 & 1964-98 & 1997 & 12.48 & 12,700 & $11 / 19 / 96$ & 12.48 & 12,700 & $\mathrm{~N}$ & 15 \\
\hline 14151000 & $\begin{array}{l}\text { Fall Creek below Winberry Creek } \\
\text { near Fall Creek, OR }\end{array}$ & 186 & $1936-98$ & 1957 & 18.80 & 24,700 & $12 / 27 / 96$ & 8.01 & 4,640 & $\mathrm{Y}$ & -- \\
\hline 14153500 & $\begin{array}{l}\text { Coast Fork Willamette River } \\
\text { below Cottage Grove Dam, OR }\end{array}$ & 104 & 1939-98 & 1965 & 11.83 & 5,910 & $12 / 12 / 96$ & 9.16 & 3,420 & $\mathrm{Y}$ & -- \\
\hline 14154500 & $\begin{array}{l}\text { Row River above Pitcher Creek } \\
\text { near Dorena, OR }\end{array}$ & 211 & $1936-98$ & 1965 & 18.19 & 33,100 & $11 / 18 / 96$ & 17.25 & 30,100 & $\mathrm{~N}$ & 100 \\
\hline 14157500 & $\begin{array}{l}\text { Coast Fork Willamette River near } \\
\text { Goshen, OR }\end{array}$ & 642 & $\begin{array}{l}1906-12 \\
1951-98\end{array}$ & 1910 & 19.50 & 58,500 & $11 / 19 / 96$ & 17.17 & 33,400 & $\mathrm{Y}$ & -- \\
\hline 14158500 & $\begin{array}{l}\text { McKenzie River at outlet of Clear } \\
\text { Lake, OR }\end{array}$ & 92.4 & $\begin{array}{l}1913-15 \\
1948-98\end{array}$ & $\begin{array}{l}1965 \\
1913\end{array}$ & $\begin{array}{r}8.15 \\
10.69\end{array}$ & $\begin{array}{l}3,300 \\
1,130\end{array}$ & $2 / 7 / 96$ & 7.01 & 2,600 & $\mathrm{Y}$ & $15-20$ \\
\hline 14158790 & $\begin{array}{l}\text { Smith River above Smith River } \\
\text { Reservoir near Belknap Springs, } \\
\text { OR }\end{array}$ & 16.2 & $1961-98$ & 1965 & 11.90 & 5,160 & $2 / 7 / 96$ & 9.48 & 2,960 & $\mathrm{~N}$ & 30 \\
\hline 14159500 & $\begin{array}{l}\text { South Fork McKenzie River near } \\
\text { Rainbow, OR }\end{array}$ & 208 & 1948-98 & $\begin{array}{l}1957 \\
1946\end{array}$ & $\begin{array}{l}8.66 \\
9.30\end{array}$ & $\begin{array}{l}17,600 \\
24,500\end{array}$ & $\begin{array}{l}2 / 13 / 96 \\
1 / 6 / 97\end{array}$ & $\begin{array}{l}5.42 \\
5.39\end{array}$ & $\begin{array}{l}6,650 \\
6,570\end{array}$ & $\begin{array}{l}\mathrm{Y} \\
\mathrm{Y}\end{array}$ & -- \\
\hline 14161500 & $\begin{array}{l}\text { Lookout Creek near Blue River, } \\
\text { OR }\end{array}$ & 24.1 & $\begin{array}{l}1950-55 \\
1964-98\end{array}$ & 1996 & 10.03 & 8,000 & $2 / 7 / 96$ & 10.03 & 8,000 & $\mathrm{~N}$ & $>100$ \\
\hline 14162500 & McKenzie River near Vida, OR & 930 & $1925-98$ & 1946 & 17.70 & 64,400 & $2 / 7 / 96$ & 10.05 & 30,900 & $\mathrm{Y}$ & -- \\
\hline 14165000 & $\begin{array}{l}\text { Mohawk River near Springfield, } \\
\text { OR }\end{array}$ & 177 & $\begin{array}{l}1936-52 \\
1956 \\
1964-97\end{array}$ & 1996 & 23.11 & 13,500 & $2 / 7 / 96$ & 23.11 & 13,500 & $\mathrm{~N}$ & 30 \\
\hline 14166500 & Long Tom River near Noti, OR & 89.3 & 1936-98 & 1956 & 20.17 & 6,990 & $2 / 7 / 96$ & 19.98 & 6,780 & $\mathrm{~N}$ & 25 \\
\hline 14178000 & $\begin{array}{l}\text { North Santiam River below } \\
\text { Boulder Creek near Detroit, OR }\end{array}$ & 216 & $\begin{array}{l}\text { 1907-09, } \\
1929-98\end{array}$ & 1965 & 13.76 & 26,700 & $2 / 7 / 96$ & 11.78 & 24,000 & $\mathrm{~N}$ & $60-80$ \\
\hline
\end{tabular}


Table 38. Maximum stage and discharge for period of record for streamgages having significant floods during 1994-98 water years in Oregon.-Continued

$\left[\mathrm{mi}^{2}\right.$, square miles; $\mathrm{ft}$, feet above an arbitrary datum; $\mathrm{ft}^{3} / \mathrm{s}$, cubic feet per second; --, not determined or not applicable; >, greater than. Source: Recurrence intervals calculated from U.S. Geological Survey data. Other data from U.S. Geological Survey reports or databases]

\begin{tabular}{|c|c|c|c|c|c|c|c|c|c|c|c|}
\hline \multirow[b]{2}{*}{$\begin{array}{c}\text { Streamgage } \\
\text { number } \\
\text { (fig. 49) }\end{array}$} & \multirow[b]{2}{*}{ Streamgage name } & \multirow{2}{*}{$\begin{array}{c}\text { Total } \\
\text { drainage } \\
\left(\mathrm{mi}^{2}\right)\end{array}$} & \multicolumn{4}{|c|}{$\begin{array}{c}\text { Maximum stage and discharge for period of record } \\
\text { through } 1998 \text { water year }\end{array}$} & \multicolumn{5}{|c|}{ Significant floods $1994-98$ water years } \\
\hline & & & $\begin{array}{c}\text { Period of } \\
\text { record } \\
\text { (water } \\
\text { years) }\end{array}$ & Water year & $\begin{array}{l}\text { Stage } \\
(\mathrm{ft})\end{array}$ & $\begin{array}{l}\text { Discharge } \\
\left(\mathrm{ft}^{3} / \mathrm{s}\right)\end{array}$ & $\begin{array}{c}\text { Date } \\
\text { (month/ } \\
\text { day/ } \\
\text { year) }\end{array}$ & $\begin{array}{l}\text { Stage } \\
(\mathrm{ft})\end{array}$ & $\begin{array}{l}\text { Discharge } \\
\left(\mathrm{ft}^{3} / \mathrm{s}\right)\end{array}$ & $\begin{array}{c}\text { Regulated } \\
\text { during } \\
\text { flood }^{1}\end{array}$ & $\begin{array}{c}\text { Recurrence } \\
\text { interval } \\
\text { (years) }\end{array}$ \\
\hline 14182500 & $\begin{array}{l}\text { Little North Santiam River near } \\
\text { Mehama, OR }\end{array}$ & 112 & $1932-98$ & 1965 & 16.73 & 36,000 & $2 / 7 / 96$ & 15.29 & 33,900 & $\mathrm{~N}$ & $60-80$ \\
\hline 14183000 & $\begin{array}{l}\text { North Santiam River at Mehama, } \\
\text { OR }\end{array}$ & 655 & $\begin{array}{l}1906-07, \\
1911-14, \\
1922-98\end{array}$ & $\begin{array}{l}1946 \\
1922 \\
1923\end{array}$ & $\begin{array}{l}15.37 \\
17.50 \\
17.50\end{array}$ & $\begin{array}{l}76,600 \\
62,900 \\
62,900\end{array}$ & 2/7/96 & 13.40 & 53,800 & $\mathrm{Y}$ & -- \\
\hline 14185000 & $\begin{array}{l}\text { South Santiam River below } \\
\text { Cascadia, OR }\end{array}$ & 174 & 1936-98 & $\begin{array}{l}1996 \\
1965\end{array}$ & $\begin{array}{l}18.11 \\
19.68\end{array}$ & $\begin{array}{l}31,700 \\
27,600\end{array}$ & 2/7/96 & 18.11 & 31,700 & $\mathrm{~N}$ & $50-70$ \\
\hline 14185900 & $\begin{array}{l}\text { Quartzville Creek near Cascadia, } \\
\text { OR }\end{array}$ & 99.2 & 1964-98 & $\begin{array}{l}1965 \\
1996\end{array}$ & 20.54 & $\begin{array}{l}36,500 \\
23,700\end{array}$ & $2 / 7 / 96$ & 20.54 & 23,700 & $\mathrm{~N}$ & 25 \\
\hline 14187200 & $\begin{array}{l}\text { South Santiam River near Foster, } \\
\text { OR }\end{array}$ & 557 & 1974-98 & 1996 & 18.74 & 28,700 & 2/7/96 & 18.74 & 28,700 & $\mathrm{Y}$ & -- \\
\hline 14187500 & $\begin{array}{l}\text { South Santiam River at Waterloo, } \\
\text { OR }\end{array}$ & 640 & $\begin{array}{l}1906, \\
1924-98\end{array}$ & 1965 & 24.50 & 95,200 & 2/7/96 & 13.09 & 29,200 & $\mathrm{Y}$ & -- \\
\hline 14189000 & Santiam River at Jefferson, OR & 1,790 & $\begin{array}{l}1908-16, \\
1922, \\
1940-98\end{array}$ & $\begin{array}{l}1922 \\
1965\end{array}$ & $\begin{array}{l}22.50 \\
24.22\end{array}$ & $\begin{array}{l}202,000 \\
197,000\end{array}$ & $2 / 7 / 96$ & 23.25 & 168,000 & $\mathrm{Y}$ & -- \\
\hline 14190500 & Luckiamute River near Suver, OR & 240 & $\begin{array}{l}1906-11 \\
1941-98\end{array}$ & 1965 & 34.52 & 32,900 & $2 / 8 / 96$ & 33.00 & 24,800 & $\mathrm{~N}$ & 25 \\
\hline 14196001 & $\begin{array}{l}\text { Haskins Creek below reservoir } \\
\text { near McMinnville, OR }\end{array}$ & 6.90 & 1952-98 & 1996 & 6.01 & 1,050 & $2 / 8 / 96$ & 6.01 & 1,050 & $\mathrm{Y}$ & $>50$ \\
\hline 14202000 & Pudding River at Aurora, OR & 479 & $\begin{array}{l}1929-65 \\
1994-97\end{array}$ & 1996 & 30.72 & 43,700 & $2 / 8 / 96$ & 30.72 & 43,700 & $\mathrm{~N}$ & $50-100$ \\
\hline 14202980 & $\begin{array}{l}\text { Scoggins Creek below Henry Hagg } \\
\text { Lake near Gaston, OR }\end{array}$ & 38.8 & $1975-98$ & $\begin{array}{l}1996 \\
1991\end{array}$ & $\begin{array}{l}16.88 \\
18.01\end{array}$ & $\begin{array}{l}2,210 \\
2,050\end{array}$ & $4 / 23 / 96$ & 16.88 & 2,210 & $\mathrm{Y}$ & -- \\
\hline 14203500 & Tualatin River near Dilley, OR & 125 & 1940-98 & 1965 & 19.34 & 17,100 & $2 / 8 / 96$ & 19.06 & 10,100 & $\mathrm{Y}$ & -- \\
\hline 14206900 & $\begin{array}{l}\text { Fanno Creek at 56th Avenue at } \\
\text { Portland, OR }\end{array}$ & 2.37 & $\begin{array}{l}1974-78 \\
1991-98\end{array}$ & 1996 & 13.20 & 733 & $2 / 8 / 96$ & 13.20 & 733 & $\mathrm{~N}$ & $25-50$ \\
\hline 14207500 & Tualatin River at West Linn, OR & 706 & 1929-98 & 1996 & 18.32 & 26,400 & $2 / 10 / 96$ & 18.32 & 26,400 & $\mathrm{Y}$ & -- \\
\hline
\end{tabular}


$\left[\mathrm{mi}^{2}\right.$, square miles; ft, feet above an arbitrary datum; $\mathrm{ft}^{3} / \mathrm{s}$, cubic feet per second; --, not determined or not applicable; >, greater than. Source: Recurrence intervals calculated from U.S. Geological Survey data. Other data from U.S. Geological Survey reports or databases]

\begin{tabular}{|c|c|c|c|c|c|c|c|c|c|c|c|}
\hline \multirow{2}{*}{$\begin{array}{c}\text { Streamgage } \\
\text { number } \\
\text { (fig. 49) }\end{array}$} & \multirow[b]{2}{*}{ Streamgage name } & \multirow{2}{*}{$\begin{array}{c}\text { Total } \\
\text { drainage } \\
\left(\mathrm{mi}^{2}\right)\end{array}$} & \multicolumn{4}{|c|}{$\begin{array}{c}\text { Maximum stage and discharge for period of record } \\
\text { through } 1998 \text { water year }\end{array}$} & \multicolumn{5}{|c|}{ Significant floods $1994-98$ water years } \\
\hline & & & $\begin{array}{l}\text { Period of } \\
\text { record } \\
\text { (water } \\
\text { years) }\end{array}$ & Water year & $\begin{array}{l}\text { Stage } \\
(\mathrm{ft})\end{array}$ & $\begin{array}{l}\text { Discharge } \\
\left(\mathrm{ft}^{3} / \mathrm{s}\right)\end{array}$ & $\begin{array}{c}\text { Date } \\
\text { (month/ } \\
\text { day/ } \\
\text { year) }\end{array}$ & $\begin{array}{l}\text { Stage } \\
(\mathrm{ft})\end{array}$ & $\begin{array}{l}\text { Discharge } \\
\qquad\left(\mathrm{ft}^{3} / \mathrm{s}\right)\end{array}$ & $\begin{array}{c}\text { Regulated } \\
\text { during } \\
\text { flood }^{1}\end{array}$ & $\begin{array}{c}\text { Recurrence } \\
\text { interval } \\
\text { (years) }\end{array}$ \\
\hline 14209000 & $\begin{array}{l}\text { Oak Grove Fork above powerplant } \\
\text { intake, OR }\end{array}$ & 124 & 1910-98 & $\begin{array}{l}1923 \\
1997\end{array}$ & $\begin{array}{l}5.45 \\
8.52\end{array}$ & $\begin{array}{l}5,000 \\
2,620\end{array}$ & $2 / 7 / 96$ & 6.18 & 4,530 & $\mathrm{Y}$ & -- \\
\hline 14209500 & $\begin{array}{l}\text { Clackamas River above Three } \\
\text { Lynx Creek, OR }\end{array}$ & 479 & $\begin{array}{l}1910-13 \\
1922-98\end{array}$ & 1965 & 21.70 & 68,200 & 2/7/96 & 18.09 & 46,600 & $\mathrm{Y}$ & $40-50$ \\
\hline 14210000 & Clackamas River at Estacada, OR & 671 & 1909-98 & $\begin{array}{l}1965 \\
1996\end{array}$ & $\begin{array}{l}18.36 \\
27.57\end{array}$ & $\begin{array}{l}86,900 \\
68,900\end{array}$ & $2 / 7 / 96$ & 27.57 & 68,900 & $\mathrm{Y}$ & $40-50$ \\
\hline 14211500 & Johnson Creek at Sycamore, OR & 26.5 & $1941-98$ & $\begin{array}{l}1965 \\
1997\end{array}$ & $\begin{array}{l}14.68 \\
15.30\end{array}$ & $\begin{array}{l}2,620 \\
2,550\end{array}$ & $\begin{array}{l}2 / 7 / 96 \\
11 / 19 / 96\end{array}$ & $\begin{array}{l}14.28 \\
15.30\end{array}$ & $\begin{array}{l}2,350 \\
2,550\end{array}$ & $\begin{array}{l}\mathrm{N} \\
\mathrm{N}\end{array}$ & $\begin{array}{l}15 \\
25\end{array}$ \\
\hline 14301000 & Nehalem River near Foss, OR & 667 & 1940-98 & 1996 & 29.56 & 70,300 & 2/8/96 & 29.56 & 70,300 & $\mathrm{~N}$ & $>100$ \\
\hline 14301500 & Wilson River near Tillamook, OR & 161 & $\begin{array}{l}1915, \\
1932-98\end{array}$ & $\begin{array}{l}1972 \\
1965\end{array}$ & $\begin{array}{l}16.91 \\
20.26\end{array}$ & $\begin{array}{l}36,000 \\
32,100\end{array}$ & $2 / 8 / 96$ & 19.51 & 35,000 & $\mathrm{~N}$ & 50 \\
\hline 14303200 & Tucca Creek near Blaine, OR & 3.09 & 1984-98 & $\begin{array}{l}1996 \\
1997\end{array}$ & $\begin{array}{l}4.30 \\
5.09\end{array}$ & $\begin{array}{l}680 \\
400\end{array}$ & $2 / 6 / 96$ & 4.30 & 680 & $\mathrm{~N}$ & $>50$ \\
\hline 14305500 & Siletz River at Siletz, OR & 202 & $\begin{array}{l}1906-12 \\
1925-98\end{array}$ & $\begin{array}{l}1996 \\
1965\end{array}$ & $\begin{array}{l}24.49 \\
27.32\end{array}$ & $\begin{array}{l}34,700 \\
32,200\end{array}$ & $2 / 7 / 96$ & 24.49 & 34,700 & $\mathrm{~N}$ & 50 \\
\hline 14306340 & $\begin{array}{l}\text { East Fork Lobster Creek near } \\
\text { Alsea, OR }\end{array}$ & 5.70 & 1984-98 & 1996 & 5.37 & 1,360 & $2 / 7 / 96$ & 5.37 & 1,360 & $\mathrm{~N}$ & $25-50$ \\
\hline 14308000 & South Umpqua River at Tiller, OR & 449 & $\begin{array}{l}\text { 1911, } \\
1940-98\end{array}$ & 1965 & 25.72 & 60,200 & $11 / 18 / 96$ & 22.17 & 46,000 & $\mathrm{~N}$ & 25 \\
\hline 14308500 & Elk Creek near Drew, OR & 54.4 & $\begin{array}{l}1955-82 \\
1987-98\end{array}$ & 1995 & 11.09 & 9,120 & $1 / 9 / 95$ & 11.09 & 9,120 & $\mathrm{~N}$ & $20-25$ \\
\hline 14308990 & $\begin{array}{l}\text { Cow Creek above Galesville } \\
\text { Reservoir near Azalea, OR }\end{array}$ & 64.7 & 1986-98 & 1995 & 12.04 & 6,980 & $1 / 9 / 95$ & 12.04 & 6,980 & $\mathrm{~N}$ & $10-25$ \\
\hline 14309000 & Cow Creek near Azalea, OR & 78.0 & $\begin{array}{l}1928-31 \\
1933-98\end{array}$ & 1974 & 16.40 & 10,600 & $1 / 2 / 97$ & 9.69 & 2,490 & $\mathrm{Y}$ & -- \\
\hline 14310000 & Cow Creek near Riddle, OR & 456 & $\begin{array}{l}1951 \\
1955-98\end{array}$ & 1951 & 28.50 & 41,100 & $1 / 9 / 95$ & 26.22 & 34,400 & $\mathrm{Y}$ & 10 \\
\hline
\end{tabular}


Table 38. Maximum stage and discharge for period of record for streamgages having significant floods during 1994-98 water years in Oregon.-Continued

$\left[\mathrm{mi}^{2}\right.$, square miles; $\mathrm{ft}$, feet above an arbitrary datum; $\mathrm{ft}^{3} / \mathrm{s}$, cubic feet per second; --, not determined or not applicable; >, greater than. Source: Recurrence intervals calculated from U.S. Geological Survey data. Other data from U.S. Geological Survey reports or databases]

\begin{tabular}{|c|c|c|c|c|c|c|c|c|c|c|c|}
\hline \multirow{2}{*}{$\begin{array}{c}\text { Streamgage } \\
\text { number } \\
\text { (fig. 49) }\end{array}$} & \multirow[b]{2}{*}{ Streamgage name } & \multirow{2}{*}{$\begin{array}{l}\text { Total } \\
\text { drainage } \\
\left(\mathrm{mi}^{2}\right)\end{array}$} & \multicolumn{4}{|c|}{$\begin{array}{l}\text { Maximum stage and discharge for period of record } \\
\text { through } 1998 \text { water year }\end{array}$} & \multicolumn{5}{|c|}{ Significant floods $1994-98$ water years } \\
\hline & & & $\begin{array}{l}\text { Period of } \\
\text { record } \\
\text { (water } \\
\text { years) }\end{array}$ & Water year & $\begin{array}{l}\text { Stage } \\
\text { (ft) }\end{array}$ & $\begin{array}{l}\text { Discharge } \\
\qquad\left(\mathrm{ft}^{3} / \mathrm{s}\right)\end{array}$ & $\begin{array}{c}\text { Date } \\
\text { (month/ } \\
\text { day/ } \\
\text { year) }\end{array}$ & $\begin{array}{l}\text { Stage } \\
\text { (ft) }\end{array}$ & $\begin{array}{l}\text { Discharge } \\
\left(\mathrm{ft}^{3} / \mathrm{s}\right)\end{array}$ & $\begin{array}{l}\text { Regulated } \\
\text { during } \\
\text { flood }^{1}\end{array}$ & $\begin{array}{l}\text { Recurrence } \\
\text { interval } \\
\text { (years) }\end{array}$ \\
\hline 14314500 & $\begin{array}{l}\text { Clearwater River above Trap } \\
\text { Creek near Toketee Falls, OR }\end{array}$ & 41.6 & $1928-98$ & $\begin{array}{l}1965 \\
1997\end{array}$ & $\begin{array}{ll}- \\
- \\
6.92\end{array}$ & $\begin{array}{r}1,020 \\
768\end{array}$ & $1 / 1 / 97$ & 6.92 & 768 & $\mathrm{Y}$ & -- \\
\hline 14316000 & $\begin{array}{c}\text { Fish Creek at Big Camas Ranger } \\
\text { Station near Toketee Falls, OR }\end{array}$ & 68.8 & 1948-98 & $\begin{array}{l}1965 \\
1956\end{array}$ & 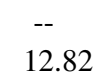 & $\begin{array}{r}12,100 \\
9,880\end{array}$ & $11 / 18 / 96$ & 10.66 & 6,240 & $\mathrm{Y}$ & 15 \\
\hline 14316700 & Steamboat Creek near Glide, OR & 227 & $1956-98$ & 1965 & 25.60 & 51,000 & $11 / 18 / 96$ & 19.54 & 31,400 & $\mathrm{~N}$ & $20-25$ \\
\hline 14319850 & Gassy Creek near Nonpareil, OR & 9.19 & 1989-98 & 1997 & 6.59 & 1,940 & $11 / 18 / 96$ & 6.59 & 1,940 & $\mathrm{~N}$ & $10-25$ \\
\hline 14320700 & $\begin{array}{l}\text { Calapooya Creek near Oakland, } \\
\text { OR }\end{array}$ & 210 & $\begin{array}{l}1956-73 \\
1987-98\end{array}$ & 1997 & 21.62 & 27,100 & $11 / 18 / 96$ & 21.62 & 27,100 & $\mathrm{~N}$ & 25 \\
\hline 14321400 & Elk Creek near Elkhead, OR & 28.7 & 1987-98 & 1997 & 10.82 & 6,670 & $11 / 18 / 96$ & 10.82 & 6,670 & $\mathrm{~N}$ & $25-50$ \\
\hline 14325000 & $\begin{array}{l}\text { South Fork Coquille River at } \\
\text { Powers, OR }\end{array}$ & 169 & $\begin{array}{l}\text { 1917-26, } \\
1929-98\end{array}$ & 1965 & 26.51 & 48,900 & $11 / 18 / 96$ & 21.93 & 38,500 & $\mathrm{~N}$ & 50 \\
\hline 14328000 & Rogue River above Prospect, OR & 312 & $\begin{array}{l}\text { 1909, 1911, } \\
1924-98\end{array}$ & 1965 & 11.55 & 22,400 & $1 / 1 / 97$ & 8.38 & 12,000 & $\mathrm{~N}$ & 20 \\
\hline 14330000 & Rogue River below Prospect, OR & 379 & $\begin{array}{l}\text { 1914-30, } \\
1969-98\end{array}$ & 1997 & 8.15 & 12,200 & $1 / 1 / 97$ & 8.15 & 12,200 & $\mathrm{~N}$ & 25 \\
\hline 14332000 & $\begin{array}{l}\text { South Fork Rogue River near } \\
\text { Prospect, OR }\end{array}$ & 83.8 & $\begin{array}{l}1925-31 \\
1950-98\end{array}$ & $\begin{array}{l}1965 \\
1956\end{array}$ & $-\overline{-}$ & $\begin{array}{l}7,010 \\
3,180\end{array}$ & $12 / 30 / 95$ & 7.13 & 4,030 & $\mathrm{~N}$ & 25 \\
\hline 14337500 & Big Butte Creek near McLeod, OR & 245 & $\begin{array}{l}1946-57 \\
1968-98\end{array}$ & $\begin{array}{l}1956 \\
1972\end{array}$ & $\begin{array}{l}12.75 \\
13.21\end{array}$ & $\begin{array}{l}8,950 \\
8,170\end{array}$ & $1 / 1 / 97$ & 12.61 & 7,390 & $\mathrm{~N}$ & $15-20$ \\
\hline 14337600 & Rogue River near McLeod, OR & 938 & $1965-98$ & 1965 & 20.35 & 74,300 & $1 / 7 / 97$ & 9.01 & 17,400 & Y & -- \\
\hline 14338000 & Elk Creek near Trail, OR & 129 & 1946-98 & 1965 & 18.84 & 19,200 & $1 / 1 / 97$ & 12.00 & 11,300 & $\mathrm{~N}$ & 15 \\
\hline 14357500 & Bear Creek at Medford, OR & 289 & 1916-98 & 1997 & 14.69 & 17,600 & $1 / 1 / 97$ & 14.69 & 17,600 & $\mathrm{Y}$ & $50-100$ \\
\hline 14359000 & $\begin{array}{l}\text { Rogue River at Raygold near } \\
\text { Central Point, OR }\end{array}$ & 2,053 & 1906-98 & $\begin{array}{l}1965 \\
1927\end{array}$ & $\begin{array}{l}23.43 \\
24.80\end{array}$ & $\begin{array}{l}131,000 \\
110,000\end{array}$ & $1 / 1 / 97$ & 17.17 & 70,500 & $\mathrm{Y}$ & -- \\
\hline 14361500 & Rogue River at Grants Pass, OR & 2,459 & 1939-98 & 1965 & 34.15 & 152,000 & $1 / 1 / 97$ & 26.49 & 90,800 & $\mathrm{Y}$ & -- \\
\hline
\end{tabular}


$\left[\mathrm{mi}^{2}\right.$, square miles; ft, feet above an arbitrary datum; $\mathrm{ft}^{3} / \mathrm{s}$, cubic feet per second; --, not determined or not applicable; >, greater than. Source: Recurrence intervals calculated from U.S. Geological Survey data. Other data from U.S. Geological Survey reports or databases]

\begin{tabular}{|c|c|c|c|c|c|c|c|c|c|c|c|}
\hline \multirow{2}{*}{$\begin{array}{l}\text { Streamgage } \\
\text { number } \\
\text { (fig. 49) }\end{array}$} & \multirow[b]{2}{*}{ Streamgage name } & \multirow{2}{*}{$\begin{array}{c}\text { Total } \\
\text { drainage } \\
\left(\mathrm{mi}^{2}\right)\end{array}$} & \multicolumn{4}{|c|}{$\begin{array}{l}\text { Maximum stage and discharge for period of record } \\
\text { through } 1998 \text { water year }\end{array}$} & \multicolumn{5}{|c|}{ Significant floods 1994-98 water years } \\
\hline & & & $\begin{array}{l}\text { Period of } \\
\text { record } \\
\text { (water } \\
\text { years) }\end{array}$ & Water year & $\begin{array}{l}\text { Stage } \\
(\mathrm{ft})\end{array}$ & $\begin{array}{l}\text { Discharge } \\
\left(\mathrm{ft}^{3} / \mathrm{s}\right)\end{array}$ & $\begin{array}{c}\text { Date } \\
\text { (month/ } \\
\text { day/ } \\
\text { year) }\end{array}$ & $\begin{array}{l}\text { Stage } \\
(\mathrm{ft})\end{array}$ & $\begin{array}{l}\text { Discharge } \\
\left(\mathrm{ft}^{3} / \mathrm{s}\right)\end{array}$ & $\begin{array}{l}\text { Regulated } \\
\text { during } \\
\text { flood }^{1}\end{array}$ & $\begin{array}{c}\text { Recurrence } \\
\text { interval } \\
\text { (years) }\end{array}$ \\
\hline 14362000 & Applegate River near Copper, OR & 225 & 1939-98 & $\begin{array}{l}1974 \\
1965\end{array}$ & $\begin{array}{l}25.38 \\
26.00\end{array}$ & $\begin{array}{l}29,800 \\
29,000\end{array}$ & $1 / 1 / 97$ & 16.23 & 18,800 & $\mathrm{Y}$ & -- \\
\hline 14362250 & Star Gulch near Ruch, OR & 16.0 & 1984-98 & 1997 & 5.43 & 1,050 & $1 / 1 / 97$ & 5.43 & 1,050 & $\mathrm{~N}$ & $>50$ \\
\hline 14366000 & $\begin{array}{l}\text { Applegate River near Applegate, } \\
\text { OR }\end{array}$ & 483 & 1939-98 & 1974 & 20.41 & 37,200 & $1 / 1 / 97$ & 17.90 & 29,700 & $\mathrm{Y}$ & -- \\
\hline 14369500 & $\begin{array}{l}\text { Applegate River near Wilderville, } \\
\text { OR }\end{array}$ & 698 & $\begin{array}{l}1939-55 \\
1979-98\end{array}$ & 1956 & 20.30 & 66,500 & $1 / 2 / 97$ & 19.38 & 44,000 & Y & -- \\
\hline 14372300 & Rogue River near Agness, OR & 3,939 & $1961-98$ & 1965 & 68.03 & 290,000 & $1 / 2 / 97$ & 39.60 & 241,000 & $\mathrm{Y}$ & -- \\
\hline 14400000 & Chetco River near Brookings, OR & 271 & 1970-98 & 1997 & 28.56 & 76,100 & $11 / 19 / 96$ & 28.56 & 76,100 & $\mathrm{~N}$ & 50 \\
\hline
\end{tabular}

Regulated during flood: N, no; Y, yes. 


\section{Pennsylvania}

Slow-moving thunderstorms with excessive rainfall occurred several times during the summer months of 1994 including the remnants of Tropical Storm Beryl in August. These excessive rains caused small-stream flash flooding across the State. Although no records were set for gage heights or stream discharges, extensive damage occurred from the floods. Total damage was estimated to be nearly $\$ 19$ million (National Oceanic and Atmospheric Administration, 1994b). There were no deaths caused by the flooding during this period.

Severe flooding occurred near York (fig. 50) on July 4, 1995, as about 4.5 inches of rain fell within 3 hours (National Oceanic and Atmospheric Administration, 1995a). Total damage was estimated at $\$ 8$ million (National Oceanic and Atmospheric Administration, 1995b). This was the worst flood damage in York since the remnants of Hurricane Eloise in September 1975.

The most disastrous flood in more than 20 years struck Pennsylvania during the early morning of January 19 through the evening of January 21, 1996. Nearly all of Pennsylvania experienced flooding of unusual magnitude beginning in the early morning of January 19 with rapid flooding of headwater basins of western Pennsylvania and ending as major flooding along the rivers of the State on January 20-21. Two major snowstorms set the stage for the great flood. The snowstorm of January 7-8 had been termed the Blizzard of ' 96 and was followed by a second major storm on January 12-13. Overnight on January 18 and 19, excessive rain fell atop snow that had begun to melt rapidly due to high winds and warm temperatures. Many locations had in excess of 2 inches of rain, with the hardest hit areas receiving 3 inches in less than 12 hours (National Oceanic and Atmospheric Administration, 1996a). Snowpack from the severe January snowstorms held water equivalents of 2 to 4 inches across much of the region, and much of the water was released during the period of excessive rain. Major rivers and streams responded quite rapidly, and ice jams caused significant problems in many areas. The flooding was as bad as that from Hurricane Eloise in 1975 and in a few areas was even worse than the record floods of Hurricane Agnes in 1972. Record discharges were measured at 29 streamgages throughout the State (table 39). In the hardest hit areas of central Pennsylvania, 18 deaths were attributed to the flooding. Many of the deaths occurred when people drove into flooded areas and became stranded. Because of strong winds, temperatures in the teens, and ice-cold water, there was little time to rescue people caught in the flood. Estimates of damage from the flooding and the prior week's blizzard totaled $\$ 700$ million for the State (National Oceanic and Atmospheric Administration, 1996b). An estimated $\$ 760$ million also was lost to commerce during the 2 weeks. The Pennsylvania Department of Transportation further estimated damage to roads and bridges at $\$ 500$ million (National Oceanic and Atmospheric Administration, 1996b). Intense thunderstorms over southern Pennsylvania produced as much as 12 inches of rainfall during the evening of June 18, 1996. Major flash floods caused more than \$26 million in damage, and three persons lost their lives. Slow-moving thunderstorms caused flash flooding during the early morning of July 19, with 4 to 6 inches of rainfall over northwestern Pennsylvania (National Oceanic and Atmospheric Administration, 1996a). The greatest damage occurred near and west of Punxsutawney. More than $\$ 225$ million in damage to homes, businesses, roads, and bridges occurred from this storm, and there were two deaths (National Oceanic and Atmospheric Administration, 1996b).

Tropical moisture left behind by the remnants of Hurricane Fran was a major contributor to the torrential downpours from thunderstorms in south-central Pennsylvania, southwest of Harrisburg, on September 8, 1996. As much as 10 inches of rainfall within a 3-hour period caused widespread flash flooding and resulted in the deaths of two persons and about \$20 million dollars in property damage (National Oceanic and Atmospheric Administration, 1996b). A little more than a week later 7 inches of rainfall caused flash flooding near Erie (National Oceanic and Atmospheric Administration, 1996a), where $\$ 5$ million in damage occurred (National Oceanic and Atmospheric Administration, 1996b).

\section{References}

National Oceanic and Atmospheric Administration (NOAA), 1994a-96a, Climatological data (by State): Asheville, North Carolina, National Climatic Data Center, various months. National Oceanic and Atmospheric Administration (NOAA), 1994b-96b, Storm data (by State): Asheville, North Carolina, National Climatic Data Center, various months. 


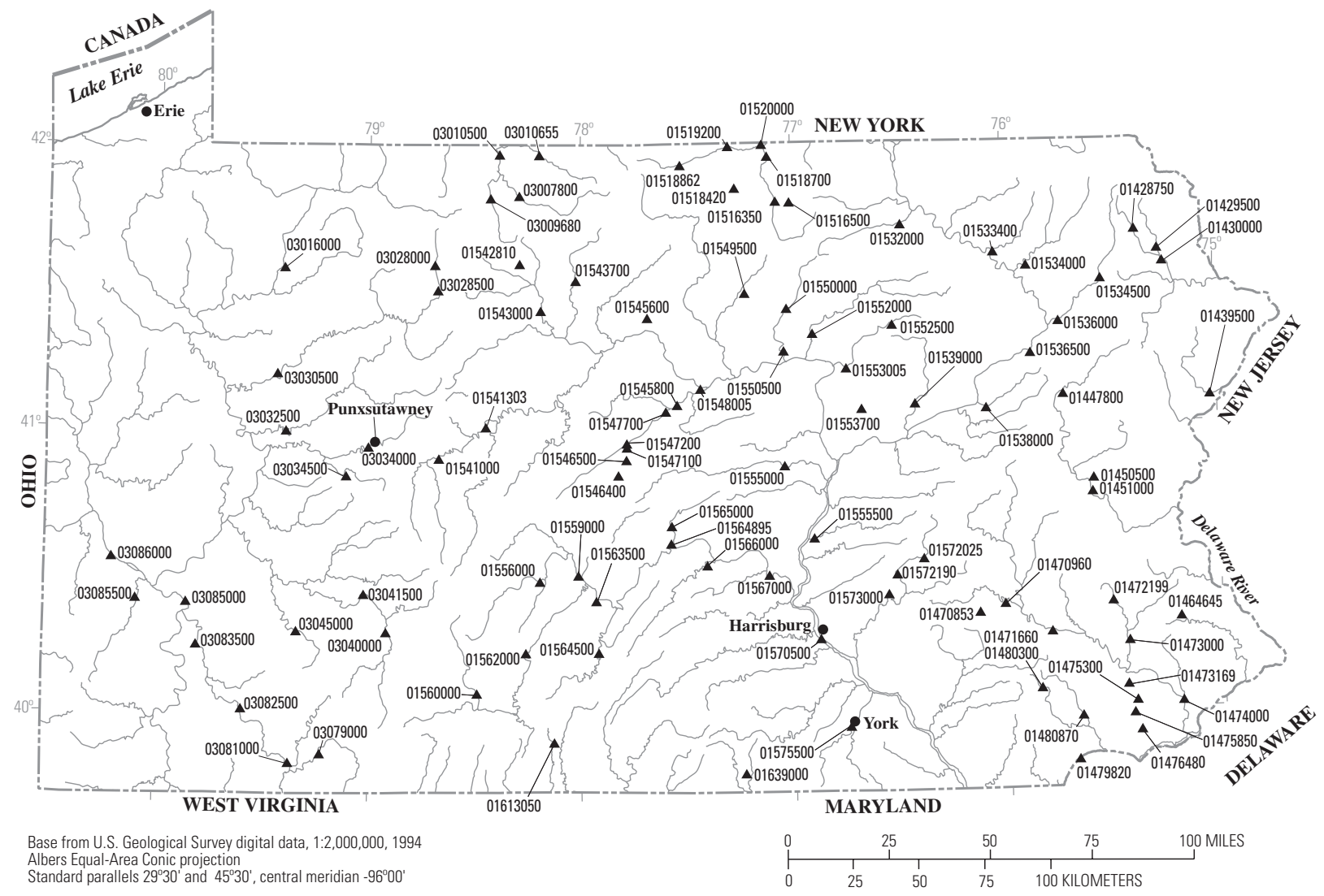

\section{EXPLANATION}

01613050 _ Streamgage and number

Figure 50. Location of streamgages with significant floods during 1994-98 water years for Pennsylvania. 
Table 39. Maximum stage and discharge for period of record for streamgages having significant floods during $1994-98$ water years in Pennsylvania.

$\left[\mathrm{mi}^{2}\right.$, square miles; $\mathrm{ft}$, feet above an arbitrary datum; $\mathrm{ft}^{3} / \mathrm{s}$, cubic feet per second; --, not determined or not applicable; $>$, greater than; <, less than. Source: Recurrence intervals calculated from U.S. Geological Survey data. Other data from U.S. Geological Survey reports or databases]

\begin{tabular}{|c|c|c|c|c|c|c|c|c|c|c|c|}
\hline \multirow{2}{*}{$\begin{array}{l}\text { Streamgage } \\
\text { number } \\
\text { (fig. 50) }\end{array}$} & \multirow[b]{2}{*}{ Streamgage name } & \multirow{2}{*}{$\begin{array}{l}\text { Total } \\
\text { drainage } \\
\left(\mathrm{mi}^{2}\right)\end{array}$} & \multicolumn{4}{|c|}{$\begin{array}{l}\text { Maximum stage and discharge for period of record } \\
\text { through } 1998 \text { water year }\end{array}$} & \multicolumn{5}{|c|}{ Significant floods $1994-98$ water years } \\
\hline & & & $\begin{array}{l}\text { Period of } \\
\text { record } \\
\text { (water } \\
\text { years) }\end{array}$ & Water year & $\begin{array}{l}\text { Stage } \\
(\mathrm{ft})\end{array}$ & $\begin{array}{l}\text { Discharge } \\
\left(\mathrm{ft}^{3} / \mathrm{s}\right)\end{array}$ & $\begin{array}{c}\text { Date } \\
\text { (month/d } \\
\text { ay/year) }\end{array}$ & $\begin{array}{l}\text { Stage } \\
(\mathrm{ft})\end{array}$ & $\begin{array}{l}\text { Discharge } \\
\left(\mathrm{ft}^{3} / \mathrm{s}\right)\end{array}$ & $\begin{array}{l}\text { Regulated } \\
\text { during } \\
\text { flood }^{1}\end{array}$ & $\begin{array}{l}\text { Recurrence } \\
\text { interval } \\
\text { (years) }\end{array}$ \\
\hline 01428750 & $\begin{array}{l}\text { West Branch Lackawaxen River } \\
\text { near Aldenville, PA }\end{array}$ & 40.6 & $1975-98$ & 1996 & 8.00 & 4,340 & $1 / 19 / 96$ & 8.00 & 4,340 & $\mathrm{~N}$ & $25-50$ \\
\hline 01429500 & Dyberry Creek near Honesdale, PA & 64.6 & 1944-98 & 1952 & 14.60 & 15,500 & $1 / 20 / 96$ & 7.32 & 2,600 & $\mathrm{Y}$ & $<10$ \\
\hline 01430000 & $\begin{array}{l}\text { Lackawaxen River near Honesdale, } \\
\text { PA }\end{array}$ & 164 & $\begin{array}{l}1942, \\
1949-69, \\
1974-82, \\
1984-94, \\
1996-98\end{array}$ & 1942 & 24.50 & 34,000 & $1 / 19 / 96$ & 8.49 & 7,180 & $\mathrm{Y}$ & $<10$ \\
\hline 01439500 & Bush Kill at Shoemakers, PA & 117 & 1909-98 & 1955 & 13.95 & 23,400 & $1 / 27 / 96$ & 7.01 & 4,990 & $\mathrm{~N}$ & $>10$ \\
\hline 01447800 & $\begin{array}{l}\text { Lehigh River below Frances E. } \\
\text { Walter Reservoir near White } \\
\text { Haven, PA }\end{array}$ & 290 & $\begin{array}{l}1955, \\
1958-98\end{array}$ & $\begin{array}{l}1955 \\
1958\end{array}$ & $-\overline{9} 85$ & $\begin{array}{l}54,200 \\
13,800\end{array}$ & $1 / 29 / 96$ & 8.79 & 11,500 & $\mathrm{Y}$ & $<10$ \\
\hline 01450500 & $\begin{array}{l}\text { Aquashicola Creek at Palmerton, } \\
\text { PA }\end{array}$ & 76.7 & 1940-98 & 1945 & 13.63 & 11,700 & $1 / 19 / 96$ & 12.64 & 9,760 & $\mathrm{~N}$ & $50-100$ \\
\hline 01451000 & Lehigh River at Walnutport, PA & 889 & $\begin{array}{l}1942 \\
1947-98\end{array}$ & $\begin{array}{l}1955 \\
1942\end{array}$ & $\begin{array}{l}17.68 \\
20.60\end{array}$ & $\begin{array}{r}77,800 \\
--\end{array}$ & $1 / 19 / 96$ & 12.32 & 40,100 & $\mathrm{Y}$ & 25 \\
\hline 01464645 & $\begin{array}{l}\text { North Branch Neshaminy Creek } \\
\text { below Lake Galena near New } \\
\text { Britain, PA }\end{array}$ & 16.2 & 1986-98 & 1997 & 4.77 & 2,060 & $10 / 19 / 96$ & 4.77 & 2,060 & $\mathrm{Y}$ & $10-25$ \\
\hline 01470853 & Furnace Creek at Robesonia, PA & 4.18 & 1983-98 & $\begin{array}{l}1994 \\
1984\end{array}$ & $\begin{array}{l}3.79 \\
4.70\end{array}$ & $\begin{array}{l}537 \\
227\end{array}$ & $11 / 28 / 93$ & 3.79 & 537 & $\mathrm{~N}$ & $10-25$ \\
\hline 01470960 & $\begin{array}{l}\text { Tulpehocken Creek at Blue Marsh } \\
\text { damsite near Readin, PA }\end{array}$ & 175 & 1965-98 & 1972 & 18.70 & 16,100 & $12 / 6 / 93$ & 7.73 & 4,060 & $\mathrm{Y}$ & $<10$ \\
\hline 01471660 & Schuylkill River at Birdsboro, PA & 976 & $\begin{array}{l}1984-94, \\
1996\end{array}$ & 1996 & 157.59 & 27,900 & $1 / 20 / 96$ & 157.59 & 27,900 & $\mathrm{~N}$ & $<10$ \\
\hline 01472199 & $\begin{array}{l}\text { West Branch Perkiomen Creek at } \\
\text { Hillegass, PA }\end{array}$ & 23.0 & 1982-98 & $\begin{array}{l}1996 \\
1989\end{array}$ & $\begin{array}{l}6.34 \\
9.55\end{array}$ & $\begin{array}{l}4,610 \\
1,190\end{array}$ & $1 / 19 / 96$ & 6.34 & 4,610 & $\mathrm{~N}$ & $25-50$ \\
\hline 01473000 & Perkiomen Creek at Graterford, PA & 279 & $1915-98$ & 1935 & 18.26 & 39,900 & $1 / 19 / 96$ & 16.45 & 32,500 & $\mathrm{~N}$ & $25-50$ \\
\hline
\end{tabular}


Table 39. Maximum stage and discharge for period of record for streamgages having significant floods during 1994-98 water years in Pennsylvania.-Continued

$\left[\mathrm{mi}^{2}\right.$, square miles; $\mathrm{ft}$, feet above an arbitrary datum; $\mathrm{ft}^{3} / \mathrm{s}$, cubic feet per second; --, not determined or not applicable; $>$, greater than; <, less than. Source: Recurrence intervals calculated from U.S. Geological Survey data. Other data from U.S. Geological Survey reports or databases]

\begin{tabular}{|c|c|c|c|c|c|c|c|c|c|c|c|}
\hline \multirow[b]{2}{*}{$\begin{array}{l}\text { Streamgage } \\
\text { number } \\
\text { (fig. 50) }\end{array}$} & \multirow[b]{2}{*}{ Streamgage name } & \multirow{2}{*}{$\begin{array}{l}\text { Total } \\
\text { drainage } \\
\left(\mathrm{mi}^{2}\right)\end{array}$} & \multicolumn{4}{|c|}{$\begin{array}{c}\text { Maximum stage and discharge for period of record } \\
\text { through } 1998 \text { water year }\end{array}$} & \multicolumn{5}{|c|}{ Significant floods 1994-98 water years } \\
\hline & & & $\begin{array}{l}\text { Period of } \\
\text { record } \\
\text { (water } \\
\text { years) }\end{array}$ & Water year & $\begin{array}{l}\text { Stage } \\
(\mathrm{ft})\end{array}$ & $\begin{array}{c}\text { Discharge } \\
\left(\mathrm{ft}^{3} / \mathrm{s}\right)\end{array}$ & $\begin{array}{c}\text { Date } \\
\text { (month/d } \\
\text { ay/year) }\end{array}$ & $\begin{array}{l}\text { Stage } \\
(\mathrm{ft})\end{array}$ & $\begin{array}{c}\text { Discharge } \\
\left(\mathrm{ft}^{3} / \mathrm{s}\right)\end{array}$ & $\begin{array}{l}\text { Regulated } \\
\text { during } \\
\text { flood }^{1}\end{array}$ & $\begin{array}{l}\text { Recurrence } \\
\text { interval } \\
\text { (years) }\end{array}$ \\
\hline 01473169 & $\begin{array}{l}\text { Valley Creek at Pennsylvania } \\
\text { Turnpike Bridge near Valley } \\
\text { Forge, PA }\end{array}$ & 20.8 & $1983-98$ & 1997 & 10.54 & 2,200 & $10 / 19 / 96$ & 10.54 & 2,200 & $\mathrm{~N}$ & $<10$ \\
\hline 01474000 & $\begin{array}{l}\text { Wissahickon Creek at mouth, } \\
\text { Philadelphia, PA }\end{array}$ & 64.0 & 1966-98 & 1973 & 7.92 & 6,870 & $1 / 19 / 96$ & 7.60 & 6,240 & $\mathrm{~N}$ & $<10$ \\
\hline 01475300 & $\begin{array}{l}\text { Darby Creek at Waterloo Mills } \\
\text { near Devon, PA }\end{array}$ & 5.15 & 1972-97 & 1997 & 6.87 & 1,920 & $10 / 19 / 96$ & 6.87 & 1,920 & $\mathrm{~N}$ & 25 \\
\hline 01475850 & $\begin{array}{l}\text { Crum Creek near Newtown } \\
\text { Square, PA }\end{array}$ & 15.8 & 1977-98 & 1997 & 9.62 & 2,380 & $10 / 19 / 96$ & 9.62 & 2,380 & $\mathrm{~N}$ & $10-25$ \\
\hline 01476480 & Ridley Creek at Media, PA & 30.5 & $\begin{array}{l}\text { 1987-95, } \\
1997-98\end{array}$ & 1994 & 9.93 & 3,700 & $1 / 28 / 94$ & 9.93 & 3,700 & $\mathrm{~N}$ & $10-25$ \\
\hline 01479820 & $\begin{array}{l}\text { Red Clay Creek near Kennett } \\
\text { Square, PA }\end{array}$ & 28.3 & 1988-98 & 1996 & 9.22 & 3,760 & $1 / 19 / 96$ & 9.22 & 3,760 & $\mathrm{~N}$ & $10-25$ \\
\hline 01480300 & $\begin{array}{l}\text { West Branch Brandywine Creek } \\
\text { near Honey Brook, PA }\end{array}$ & 18.7 & 1960-98 & 1996 & 11.62 & 8,920 & $1 / 19 / 96$ & 11.62 & 8,920 & $\mathrm{~N}$ & $>25$ \\
\hline 01480870 & $\begin{array}{l}\text { East Branch Brandywine Creek } \\
\text { below Downingtown, PA }\end{array}$ & 89.9 & $1972-98$ & 1997 & 12.60 & 6,700 & $10 / 19 / 96$ & 12.60 & 6,700 & $\mathrm{Y}$ & $10-25$ \\
\hline 01516350 & Tioga River near Mansfield, PA & 153 & $\begin{array}{l}1972,1975, \\
1977-98\end{array}$ & $\begin{array}{l}1996 \\
1975\end{array}$ & $\begin{array}{l}18.87 \\
20.13\end{array}$ & $\begin{array}{l}38,900 \\
18,000\end{array}$ & $1 / 19 / 96$ & 18.87 & 38,900 & $\mathrm{~N}$ & $<50$ \\
\hline 01516500 & Corey Creek near Mainesburg, PA & 12.2 & $1955-98$ & 1972 & 10.44 & 5,580 & $8 / 18 / 94$ & 10.15 & 4,970 & $\mathrm{~N}$ & $50-100$ \\
\hline 01518420 & $\begin{array}{l}\text { Crooked Creek below Catlin } \\
\text { Hollow at Middlebury Center, } \\
\text { PA }\end{array}$ & 74.3 & 1985-98 & 1997 & 51.93 & 15,300 & $11 / 08 / 96$ & 51.93 & 15,300 & $\mathrm{~N}$ & $<25$ \\
\hline 01518700 & Tioga River at Tioga Junction, PA & 446 & 1976-98 & 1976 & 22.12 & 48,000 & $8 / 18 / 94$ & 15.42 & 11,200 & $\mathrm{Y}$ & 10 \\
\hline 01518862 & $\begin{array}{l}\text { Cowanesque River at Westfield, } \\
\text { PA }\end{array}$ & 90.6 & 1984-98 & 1996 & 11.10 & 13,000 & $1 / 19 / 96$ & 11.10 & 13,000 & $\mathrm{~N}$ & $10-25$ \\
\hline 01519200 & Cowanesque River at Elkland, PA & 235 & 1980-98 & 1996 & 30.20 & 28,000 & $1 / 19 / 96$ & 30.20 & 28,000 & $\mathrm{~N}$ & $25-50$ \\
\hline
\end{tabular}


Table 39. Maximum stage and discharge for period of record for streamgages having significant floods during 1994-98 water years in Pennsylvania.-Continued

$\left[\mathrm{mi}^{2}\right.$, square miles; $\mathrm{ft}$, feet above an arbitrary datum; $\mathrm{ft}^{3} / \mathrm{s}$, cubic feet per second; --, not determined or not applicable; $>$, greater than; <, less than. Source: Recurrence intervals calculated from U.S. Geological Survey data. Other data from U.S. Geological Survey reports or databases]

\begin{tabular}{|c|c|c|c|c|c|c|c|c|c|c|c|}
\hline \multirow{2}{*}{$\begin{array}{c}\text { Streamgage } \\
\text { number } \\
\text { (fig. 50) }\end{array}$} & \multirow[b]{2}{*}{ Streamgage name } & \multirow{2}{*}{$\begin{array}{c}\text { Total } \\
\text { drainage } \\
\left(\mathrm{mi}^{2}\right)\end{array}$} & \multicolumn{4}{|c|}{$\begin{array}{l}\text { Maximum stage and discharge for period of record } \\
\text { through } 1998 \text { water year }\end{array}$} & \multicolumn{5}{|c|}{ Significant floods $1994-98$ water years } \\
\hline & & & $\begin{array}{l}\text { Period of } \\
\text { record } \\
\text { (water } \\
\text { years) }\end{array}$ & Water year & $\begin{array}{l}\text { Stage } \\
(\mathrm{ft})\end{array}$ & $\begin{array}{l}\text { Discharge } \\
\left(\mathrm{ft}^{3} / \mathrm{s}\right)\end{array}$ & $\begin{array}{c}\text { Date } \\
\text { (month/d } \\
\text { ay/year) }\end{array}$ & $\begin{array}{l}\text { Stage } \\
(\mathrm{ft})\end{array}$ & $\begin{array}{l}\text { Discharge } \\
\left(\mathrm{ft}^{3} / \mathrm{s}\right)\end{array}$ & $\begin{array}{c}\text { Regulated } \\
\text { during } \\
\text { flood }^{1}\end{array}$ & $\begin{array}{c}\text { Recurrence } \\
\text { interval } \\
\text { (years) }\end{array}$ \\
\hline 01520000 & $\begin{array}{l}\text { Cowanesque River near } \\
\text { Lawrenceville, PA }\end{array}$ & 298 & $1952-98$ & 1975 & 18.13 & 43,700 & $1 / 23 / 96$ & 12.41 & 6,580 & $\mathrm{Y}$ & $<10$ \\
\hline 01532000 & $\begin{array}{l}\text { Towanda Creek near Monroeton, } \\
\text { PA }\end{array}$ & 215 & 1914-98 & $\begin{array}{l}1972 \\
1996\end{array}$ & $\begin{array}{l}16.90 \\
20.86\end{array}$ & $\begin{array}{l}74,000 \\
67,900\end{array}$ & $1 / 19 / 96$ & 20.86 & 67,900 & $\mathrm{~N}$ & $>100$ \\
\hline 01533400 & $\begin{array}{l}\text { Susquehanna River at Meshoppen, } \\
\text { PA }\end{array}$ & 8,720 & 1977-98 & 1996 & 36.34 & 226,000 & $1 / 20 / 96$ & 36.34 & 226,000 & $\mathrm{~N}$ & $>25$ \\
\hline 01534000 & $\begin{array}{l}\text { Tunkhannock Creek near } \\
\text { Tunkhannock, PA }\end{array}$ & 383 & 1914-98 & 1996 & 19.97 & 30,300 & $1 / 19 / 96$ & 19.97 & 30,300 & $\mathrm{~N}$ & $>25$ \\
\hline 01534500 & Lackawanna River at Archbald, PA & 108 & 1940-98 & 1942 & 10.58 & 9,510 & $1 / 19 / 96$ & 9.32 & 7,190 & Y & $<50$ \\
\hline 01536000 & $\begin{array}{l}\text { Lackawanna River at Old Forge, } \\
\text { PA }\end{array}$ & 332 & 1939-98 & 1955 & 20.05 & 31,000 & $1 / 19 / 96$ & 15.58 & 22,300 & $\mathrm{Y}$ & 50 \\
\hline 01536500 & $\begin{array}{l}\text { Susquehanna River at Wilkes } \\
\text { Barre, PA }\end{array}$ & 9,960 & $\begin{array}{l}1787,1807, \\
1809,1833, \\
1865, \\
1891-1998\end{array}$ & 1972 & 40.91 & 345,000 & $1 / 20 / 96$ & 34.45 & 221,000 & $\mathrm{~N}$ & $10-25$ \\
\hline 01538000 & $\begin{array}{l}\text { Wapwallopen Creek near } \\
\text { Wapwallopen, PA }\end{array}$ & 43.8 & 1920-98 & 1972 & 11.04 & 5,410 & $1 / 19 / 96$ & 10.35 & 4,650 & $\mathrm{~N}$ & $<100$ \\
\hline 01539000 & $\begin{array}{l}\text { Fishing Creek near Bloomsburg, } \\
\text { PA }\end{array}$ & 274 & $\begin{array}{l}1936, \\
1939-98\end{array}$ & 1972 & 15.18 & 30,900 & $1 / 19 / 96$ & 12.86 & 21,300 & $\mathrm{~N}$ & $<25$ \\
\hline 01541000 & $\begin{array}{l}\text { West Branch Susquehanna River at } \\
\text { Bower, PA }\end{array}$ & 315 & $\begin{array}{l}1889 \\
1914-98\end{array}$ & 1936 & 19.74 & 31,500 & 7/19/96 & 17.24 & 22,000 & $\mathrm{~N}$ & $50-100$ \\
\hline 01541303 & $\begin{array}{l}\text { West Branch Susquehanna River at } \\
\text { Hyde, PA }\end{array}$ & 474 & $\begin{array}{l}1964, \\
1979-98\end{array}$ & 1964 & 18.10 & 19,400 & $1 / 19 / 96$ & 11.16 & 7,630 & $\mathrm{Y}$ & $<10$ \\
\hline 01542810 & Waldy Run near Emporium, PA & 5.24 & 1964-98 & 1967 & 6.32 & 828 & $1 / 19 / 96$ & 6.24 & 698 & $\mathrm{~N}$ & 50 \\
\hline 01543000 & $\begin{array}{l}\text { Driftwood Branch Sinnemahoning } \\
\text { Creek at Sterling Run, PA }\end{array}$ & 272 & 1914-98 & 1942 & 14.70 & 47,800 & $1 / 19 / 96$ & 11.17 & 26,700 & $\mathrm{~N}$ & $>25$ \\
\hline 01543700 & $\begin{array}{l}\text { First Fork Sinnemahoning Creek at } \\
\text { Wharton, PA }\end{array}$ & 182 & 1984-98 & 1996 & 15.37 & 15,400 & $1 / 19 / 96$ & 15.37 & 15,400 & $\mathrm{~N}$ & 50 \\
\hline 01545600 & $\begin{array}{l}\text { Young Womans Creek near } \\
\text { Renovo, PA }\end{array}$ & 46.2 & 1965-98 & 1972 & 7.98 & 5,370 & $8 / 18 / 94$ & 6.92 & 3,890 & $\mathrm{~N}$ & $>25$ \\
\hline
\end{tabular}


$\left[\mathrm{mi}^{2}\right.$, square miles; $\mathrm{ft}$, feet above an arbitrary datum; $\mathrm{ft}^{3} / \mathrm{s}$, cubic feet per second; --, not determined or not applicable; $>$, greater than; <, less than. Source: Recurrence intervals calculated from U.S. Geological Survey data. Other data from U.S. Geological Survey reports or databases]

\begin{tabular}{|c|c|c|c|c|c|c|c|c|c|c|c|}
\hline \multirow{2}{*}{$\begin{array}{c}\text { Streamgage } \\
\text { number } \\
\text { (fig. 50) }\end{array}$} & \multirow[b]{2}{*}{ Streamgage name } & \multirow{2}{*}{$\begin{array}{c}\text { Total } \\
\text { drainage } \\
\left(\mathrm{mi}^{2}\right)\end{array}$} & \multicolumn{4}{|c|}{$\begin{array}{c}\text { Maximum stage and discharge for period of record } \\
\text { through } 1998 \text { water year }\end{array}$} & \multicolumn{5}{|c|}{ Significant floods $1994-98$ water years } \\
\hline & & & $\begin{array}{l}\text { Period of } \\
\text { record } \\
\text { (water } \\
\text { years) }\end{array}$ & Water year & $\begin{array}{l}\text { Stage } \\
(\mathrm{ft})\end{array}$ & $\begin{array}{l}\text { Discharge } \\
\left(\mathrm{ft}^{3} / \mathrm{s}\right)\end{array}$ & $\begin{array}{c}\text { Date } \\
\text { (month/d } \\
\text { ay/year) }\end{array}$ & $\begin{array}{l}\text { Stage } \\
(\mathrm{ft})\end{array}$ & $\begin{array}{l}\text { Discharge } \\
\left(\mathrm{ft}^{3} / \mathrm{s}\right)\end{array}$ & $\begin{array}{c}\text { Regulated } \\
\text { during } \\
\text { flood }^{1}\end{array}$ & $\begin{array}{c}\text { Recurrence } \\
\text { interval } \\
\text { (years) }\end{array}$ \\
\hline 01545800 & $\begin{array}{l}\text { West Branch Susquehanna River at } \\
\text { Lock Haven, PA }\end{array}$ & 3,345 & $1975-98$ & 1996 & 25.76 & 93,900 & $1 / 20 / 96$ & 25.76 & 93,900 & $\mathrm{~N}$ & $25-50$ \\
\hline 01546400 & Spring Creek at Houserville, PA & 58.5 & 1985-98 & 1996 & 10.05 & 2,370 & $1 / 19 / 96$ & 10.05 & 2,370 & $\mathrm{~N}$ & $>50$ \\
\hline 01546500 & Spring Creek near Axemann, PA & 87.2 & $\begin{array}{l}1936 \\
1941-98\end{array}$ & 1936 & 8.60 & 8,400 & $1 / 19 / 96$ & 6.42 & 3,490 & $\mathrm{~N}$ & $>25$ \\
\hline 01547100 & Spring Creek at Milesburg, PA & 142 & 1967-98 & 1972 & 13.20 & 8,170 & $1 / 19 / 96$ & 10.78 & 4,780 & $\mathrm{~N}$ & $<25$ \\
\hline 01547200 & $\begin{array}{l}\text { Bald Eagle Creek below Spring } \\
\text { Creek at Milesburg, PA }\end{array}$ & 265 & $1956-98$ & 1972 & 11.67 & 21,300 & $1 / 19 / 96$ & 10.47 & 16,800 & $\mathrm{~N}$ & $25-50$ \\
\hline 01547700 & Marsh Creek at Blanchard, PA & 44.1 & $1956-98$ & 1984 & 7.85 & 6,900 & $1 / 19 / 96$ & 7.43 & 5,890 & $\mathrm{~N}$ & $<50$ \\
\hline 01548005 & $\begin{array}{l}\text { Bald Eagle Creek near Beech } \\
\text { Creek Station, PA }\end{array}$ & 562 & 1985-98 & 1996 & 15.62 & 12,600 & $1 / 19 / 96$ & 15.62 & 12,600 & $\mathrm{Y}$ & $10-25$ \\
\hline 01549500 & $\begin{array}{l}\text { Blockhouse Creek near English } \\
\text { Center, PA }\end{array}$ & 37.7 & $\begin{array}{l}1936, \\
1941-98\end{array}$ & 1972 & 9.34 & 6,260 & $1 / 19 / 96$ & 8.84 & 5,540 & $\mathrm{~N}$ & $>25$ \\
\hline 01550000 & $\begin{array}{l}\text { Lycoming Creek near Trout Run, } \\
\text { PA }\end{array}$ & 173 & 1914-98 & 1996 & 22.68 & 32,000 & $1 / 19 / 96$ & 22.68 & 32,000 & $\mathrm{~N}$ & $>100$ \\
\hline 01550500 & $\begin{array}{l}\text { Lycoming Creek near } \\
\text { Williamsport, PA }\end{array}$ & 268 & $\begin{array}{l}1909-12 \\
1988-90 \\
1995-98\end{array}$ & 1996 & 18.69 & 45,000 & $1 / 19 / 96$ & 18.69 & 45,000 & $\mathrm{~N}$ & -- \\
\hline 01552000 & $\begin{array}{l}\text { Loyalsock Creek at Loyalsockville, } \\
\text { PA }\end{array}$ & 443 & 1926-98 & 1996 & 17.93 & 55,800 & $1 / 19 / 96$ & 17.93 & 55,800 & $\mathrm{~N}$ & $>100$ \\
\hline 01552500 & Muncy Creek near Sonestown, PA & 23.8 & $\begin{array}{l}1936 \\
1941-98\end{array}$ & $\begin{array}{l}1972 \\
1936\end{array}$ & $\begin{array}{l}8.94 \\
9.30\end{array}$ & $\begin{array}{r}8,260 \\
--\end{array}$ & $1 / 19 / 96$ & 8.70 & 7,560 & $\mathrm{~N}$ & $<50$ \\
\hline 01553005 & Muncy Creek near Muncy, PA & 209 & 1989-98 & 1996 & 20.57 & 43,000 & $1 / 19 / 96$ & 20.57 & 43,000 & $\mathrm{~N}$ & -- \\
\hline 01553700 & $\begin{array}{l}\text { Chillisquaque Creek at } \\
\text { Washingtonville, PA }\end{array}$ & 51.3 & 1980-98 & 1996 & 11.27 & 3,770 & $1 / 19 / 96$ & 11.27 & 3,770 & $\mathrm{~N}$ & $<25$ \\
\hline 01555000 & Penns Creek at Penns Creek, PA & 301 & $1930-98$ & 1972 & 14.85 & 34,600 & $1 / 19 / 96$ & 13.74 & 25,300 & $\mathrm{~N}$ & $>50$ \\
\hline 01555500 & $\begin{array}{l}\text { East Mahantango Creek near } \\
\text { Dalmatia, PA }\end{array}$ & 162 & $\begin{array}{l}1930-93 \\
1995-98\end{array}$ & 1972 & 26.62 & 69,900 & $1 / 19 / 96$ & 17.47 & 21,200 & $\mathrm{~N}$ & $<50$ \\
\hline
\end{tabular}


Table 39. Maximum stage and discharge for period of record for streamgages having significant floods during 1994-98 water years in Pennsylvania.-Continued

$\left[\mathrm{mi}^{2}\right.$, square miles; $\mathrm{ft}$, feet above an arbitrary datum; $\mathrm{ft}^{3} / \mathrm{s}$, cubic feet per second; --, not determined or not applicable; $>$, greater than; <, less than. Source: Recurrence intervals calculated from U.S. Geological Survey data. Other data from U.S. Geological Survey reports or databases]

\begin{tabular}{|c|c|c|c|c|c|c|c|c|c|c|c|}
\hline \multirow{2}{*}{$\begin{array}{c}\text { Streamgage } \\
\text { number } \\
\text { (fig. 50) }\end{array}$} & \multirow[b]{2}{*}{ Streamgage name } & \multirow{2}{*}{$\begin{array}{c}\text { Total } \\
\text { drainage } \\
\left(\mathrm{mi}^{2}\right)\end{array}$} & \multicolumn{4}{|c|}{$\begin{array}{c}\text { Maximum stage and discharge for period of record } \\
\text { through } 1998 \text { water year }\end{array}$} & \multicolumn{5}{|c|}{ Significant floods 1994-98 water years } \\
\hline & & & $\begin{array}{l}\text { Period of } \\
\text { record } \\
\text { (water } \\
\text { years) }\end{array}$ & Water year & $\begin{array}{l}\text { Stage } \\
\text { (ft) }\end{array}$ & $\begin{array}{l}\text { Discharge } \\
\left(\mathrm{ft}^{3} / \mathrm{s}\right)\end{array}$ & $\begin{array}{c}\text { Date } \\
\text { (month/d } \\
\text { ay/year) }\end{array}$ & $\begin{array}{l}\text { Stage } \\
\text { (ft) }\end{array}$ & $\begin{array}{l}\text { Discharge } \\
\left(\mathrm{ft}^{3} / \mathrm{s}\right)\end{array}$ & $\begin{array}{c}\text { Regulated } \\
\text { during } \\
\text { flood }^{1}\end{array}$ & $\begin{array}{c}\text { Recurrence } \\
\text { interval } \\
\text { (years) }\end{array}$ \\
\hline 01556000 & $\begin{array}{l}\text { Frankstown Branch Juniata River } \\
\text { at Williamsburg, PA }\end{array}$ & 291 & $\begin{array}{l}1889 \\
1917-98\end{array}$ & $\begin{array}{l}1936 \\
1996\end{array}$ & $\begin{array}{l}18.58 \\
19.35\end{array}$ & $\begin{array}{l}30,000 \\
17,800\end{array}$ & $1 / 19 / 96$ & 19.35 & 17,800 & $\mathrm{~N}$ & $>25$ \\
\hline 01559000 & Juniata River at Huntingdon, PA & 816 & $\begin{array}{l}1896-98, \\
1900-22, \\
1924-29, \\
1931-38, \\
1942-98\end{array}$ & 1936 & 21.87 & 81,000 & $1 / 19 / 96$ & 15.95 & 36,500 & $\mathrm{~N}$ & $<25$ \\
\hline 01560000 & Dunning Creek at Belden, PA & 172 & $\begin{array}{l}\text { 1936, } \\
1940-98\end{array}$ & $\begin{array}{l}1977 \\
1936\end{array}$ & $\begin{array}{l}14.15 \\
17.81\end{array}$ & $\begin{array}{l}19,400 \\
16,900\end{array}$ & $1 / 19 / 96$ & 13.46 & 15,700 & $\mathrm{~N}$ & 50 \\
\hline 01562000 & $\begin{array}{l}\text { Raystown Branch Juniata River at } \\
\text { Saxton, PA }\end{array}$ & 756 & $\begin{array}{l}1889 \\
1912-98\end{array}$ & 1936 & 24.54 & 80,500 & $1 / 20 / 96$ & 19.63 & 45,800 & $\mathrm{~N}$ & $50-100$ \\
\hline 01563500 & $\begin{array}{l}\text { Juniata River at Mapleton Depot, } \\
\text { PA }\end{array}$ & 2,030 & $\begin{array}{l}1936, \\
1938-98\end{array}$ & 1936 & 38.20 & 165,000 & $1 / 20 / 96$ & 22.47 & 57,600 & $\mathrm{Y}$ & $10-25$ \\
\hline 01564500 & $\begin{array}{l}\text { Aughwick Creek near Three } \\
\text { Springs, PA }\end{array}$ & 205 & $\begin{array}{l}1889 \\
1939-98\end{array}$ & 1996 & 20.85 & 32,600 & $1 / 19 / 96$ & 20.85 & 32,600 & $\mathrm{~N}$ & $>100$ \\
\hline 01564895 & Juniata River at Lewistown, PA & 2,519 & 1989-98 & 1996 & 31.64 & 74,400 & $1 / 20 / 96$ & 31.64 & 74,400 & $\mathrm{Y}$ & -- \\
\hline 01565000 & $\begin{array}{l}\text { Kishacoquillas Creek at } \\
\text { Reedsville, PA }\end{array}$ & 164 & $\begin{array}{l}1936, \\
1940-70, \\
1972, \\
1984-85, \\
1989-98\end{array}$ & 1972 & 16.17 & 16,400 & $1 / 19 / 96$ & 14.20 & 12,400 & $\mathrm{~N}$ & 50 \\
\hline 01566000 & $\begin{array}{l}\text { Tuscarora Creek near Port Royal, } \\
\text { PA }\end{array}$ & 214 & $\begin{array}{l}1889, \\
1912-58, \\
1972, \\
1988-90, \\
1996-98\end{array}$ & $\begin{array}{l}1996 \\
1972\end{array}$ & $\begin{array}{l}21.27 \\
25.10\end{array}$ & $\begin{array}{r}25,000 \\
--\end{array}$ & $9 / 7 / 96$ & 21.27 & 25,000 & $\mathrm{~N}$ & $>100$ \\
\hline 01567000 & Juniata River at Newport, PA & 3,354 & $\begin{array}{l}1889, \\
1899-1998\end{array}$ & 1889 & 35.90 & 209,000 & $1 / 20 / 96$ & 24.69 & 103,000 & $\mathrm{Y}$ & $10-25$ \\
\hline
\end{tabular}


$\left[\mathrm{mi}^{2}\right.$, square miles; $\mathrm{ft}$, feet above an arbitrary datum; $\mathrm{ft}^{3} / \mathrm{s}$, cubic feet per second; --, not determined or not applicable; $>$, greater than; <, less than. Source: Recurrence intervals calculated from U.S. Geological Survey data. Other data from U.S. Geological Survey reports or databases]

\begin{tabular}{|c|c|c|c|c|c|c|c|c|c|c|c|}
\hline \multirow{2}{*}{$\begin{array}{c}\text { Streamgage } \\
\text { number } \\
\text { (fig. 50) }\end{array}$} & \multirow[b]{2}{*}{ Streamgage name } & \multirow{2}{*}{$\begin{array}{c}\text { Total } \\
\text { drainage } \\
\left(\mathrm{mi}^{2}\right)\end{array}$} & \multicolumn{4}{|c|}{$\begin{array}{c}\text { Maximum stage and discharge for period of record } \\
\text { through } 1998 \text { water year }\end{array}$} & \multicolumn{5}{|c|}{ Significant floods $1994-98$ water years } \\
\hline & & & $\begin{array}{l}\text { Period of } \\
\text { record } \\
\text { (water } \\
\text { years) }\end{array}$ & Water year & $\begin{array}{l}\text { Stage } \\
(\mathrm{ft})\end{array}$ & $\begin{array}{l}\text { Discharge } \\
\left(\mathrm{ft}^{3} / \mathrm{s}\right)\end{array}$ & $\begin{array}{c}\text { Date } \\
\text { (month/d } \\
\text { ay/year) }\end{array}$ & $\begin{array}{l}\text { Stage } \\
(\mathrm{ft})\end{array}$ & $\begin{array}{l}\text { Discharge } \\
\left(\mathrm{ft}^{3} / \mathrm{s}\right)\end{array}$ & $\begin{array}{l}\text { Regulated } \\
\text { during } \\
\text { flood }^{1}\end{array}$ & $\begin{array}{c}\text { Recurrence } \\
\text { interval } \\
\text { (years) }\end{array}$ \\
\hline 01570500 & $\begin{array}{l}\text { Susquehanna River at Harrisburg, } \\
\text { PA }\end{array}$ & 24,100 & $\begin{array}{l}\text { 1787, 1846, } \\
1865,1868, \\
1886,1889, \\
1891-1998\end{array}$ & 1972 & 32.57 & $1,020,000$ & $1 / 21 / 96$ & 24.66 & 568,000 & $\mathrm{~N}$ & $>25$ \\
\hline 01572025 & $\begin{array}{l}\text { Swatara Creek near Pine Grove, } \\
\text { PA }\end{array}$ & 116 & 1989-98 & 1994 & 14.17 & 5,880 & $11 / 28 / 93$ & 14.17 & 5,880 & $\mathrm{~N}$ & -- \\
\hline 01572190 & Swatara Creek near Inwood, PA & 167 & 1989-98 & 1994 & 16.20 & 9,510 & $11 / 28 / 93$ & 16.20 & 9,510 & $\mathrm{~N}$ & -- \\
\hline 01573000 & $\begin{array}{l}\text { Swatara Creek at Harper Tavern, } \\
\text { PA }\end{array}$ & 337 & 1919-98 & 1972 & 23.72 & 66,700 & $1 / 20 / 96$ & 15.67 & 19,800 & $\mathrm{~N}$ & $10-25$ \\
\hline 01575500 & Codorus Creek near York, PA & 222 & $\begin{array}{l}1933 \\
1940-98\end{array}$ & 1972 & 26.36 & 30,000 & $1 / 19 / 96$ & 14.91 & 9,890 & $\mathrm{Y}$ & $<10$ \\
\hline 01613050 & $\begin{array}{l}\text { Tonoloway Creek near Needmore, } \\
\text { PA }\end{array}$ & 10.7 & $\begin{array}{l}\text { 1963-93, } \\
\text { 1995-96, } \\
1998\end{array}$ & $\begin{array}{l}1972 \\
1996\end{array}$ & $\begin{array}{l}9.17 \\
9.48\end{array}$ & $\begin{array}{l}1,300 \\
1,250\end{array}$ & $1 / 19 / 96$ & 9.48 & 1,250 & $\mathrm{~N}$ & 25 \\
\hline 01639000 & Plum Run at Round Top, PA & 1.65 & $\begin{array}{l}1933 \\
1942-98\end{array}$ & 1996 & 25.42 & 24,400 & $6 / 19 / 96$ & 25.42 & 24,400 & $\mathrm{~N}$ & -- \\
\hline 03007800 & $\begin{array}{l}\text { Allegheny River at Port Allegany, } \\
\text { PA }\end{array}$ & 248 & $1975-98$ & 1996 & 15.37 & 12,600 & $1 / 19 / 96$ & 15.37 & 12,600 & $\mathrm{~N}$ & $50-100$ \\
\hline 03009680 & Potato Creek at Smethport, PA & 160 & $1975-97$ & 1996 & 13.19 & 8,160 & $1 / 19 / 96$ & 13.19 & 8,160 & $\mathrm{~N}$ & $<25$ \\
\hline 03010500 & Allegheny River at Eldred, PA & 550 & $1916-98$ & 1972 & 29.05 & 65,400 & $1 / 20 / 96$ & 21.88 & 20,600 & $\mathrm{~N}$ & $10-25$ \\
\hline 03010655 & $\begin{array}{l}\text { Oswayo Creek at Shinglehouse, } \\
\text { PA }\end{array}$ & 98.7 & $\begin{array}{l}1972 \\
1975-98\end{array}$ & 1996 & 12.74 & 4,660 & $1 / 19 / 96$ & 12.74 & 4,660 & $\mathrm{~N}$ & 50 \\
\hline 03016000 & $\begin{array}{l}\text { Allegheny River at West Hickory, } \\
\text { PA }\end{array}$ & 3,660 & $\begin{array}{l}1942-84 \\
1986-95 \\
1997-98\end{array}$ & 1956 & 17.20 & 101,000 & $1 / 8 / 98$ & 11.19 & 43,100 & $\mathrm{Y}$ & 10 \\
\hline 03028000 & $\begin{array}{l}\text { West Branch Clarion River at } \\
\text { Wilcox, PA }\end{array}$ & 63.0 & 1954-98 & 1996 & 10.23 & 5,590 & $1 / 19 / 96$ & 10.23 & 5,590 & $\mathrm{~N}$ & $>25$ \\
\hline 03028500 & Clarion River at Johnsonburg, PA & 204 & $\begin{array}{l}1942, \\
1946-94, \\
1996-98\end{array}$ & $\begin{array}{l}1996 \\
1942\end{array}$ & $\begin{array}{l}10.14 \\
16.70\end{array}$ & $\begin{array}{r}12,800 \\
--\end{array}$ & $1 / 19 / 96$ & 10.14 & 12,800 & $\mathrm{Y}$ & $>100$ \\
\hline
\end{tabular}


Table 39. Maximum stage and discharge for period of record for streamgages having significant floods during 1994-98 water years in Pennsylvania.-Continued

$\left[\mathrm{mi}^{2}\right.$, square miles; $\mathrm{ft}$, feet above an arbitrary datum; $\mathrm{ft}^{3} / \mathrm{s}$, cubic feet per second; --, not determined or not applicable; $>$, greater than; <, less than. Source: Recurrence intervals calculated from U.S. Geological Survey data. Other data from U.S. Geological Survey reports or databases]

\begin{tabular}{|c|c|c|c|c|c|c|c|c|c|c|c|}
\hline \multirow[b]{2}{*}{$\begin{array}{l}\text { Streamgage } \\
\text { number } \\
\text { (fig. 50) }\end{array}$} & \multirow[b]{2}{*}{ Streamgage name } & \multirow{2}{*}{$\begin{array}{c}\text { Total } \\
\text { drainage } \\
\left(\mathrm{mi}^{2}\right)\end{array}$} & \multicolumn{4}{|c|}{$\begin{array}{c}\text { Maximum stage and discharge for period of record } \\
\text { through } 1998 \text { water year }\end{array}$} & \multicolumn{5}{|c|}{ Significant floods $1994-98$ water years } \\
\hline & & & $\begin{array}{l}\text { Period of } \\
\text { record } \\
\text { (water } \\
\text { years) }\end{array}$ & Water year & $\begin{array}{l}\text { Stage } \\
(\mathrm{ft})\end{array}$ & $\begin{array}{l}\text { Discharge } \\
\left(\mathrm{ft}^{3} / \mathrm{s}\right)\end{array}$ & $\begin{array}{c}\text { Date } \\
\text { (month/d } \\
\text { ay/year) }\end{array}$ & $\begin{array}{l}\text { Stage } \\
(\mathrm{ft})\end{array}$ & $\begin{array}{l}\text { Discharge } \\
\left(\mathrm{ft}^{3} / \mathrm{s}\right)\end{array}$ & $\begin{array}{c}\text { Regulated } \\
\text { during } \\
\text { flood }^{1}\end{array}$ & $\begin{array}{c}\text { Recurrence } \\
\text { interval } \\
\text { (years) }\end{array}$ \\
\hline 03030500 & Clarion River near Piney, PA & 951 & $\begin{array}{l}1936, \\
1948-98\end{array}$ & 1972 & 28.24 & 74,500 & $7 / 19 / 96$ & 25.22 & 62,400 & $\mathrm{~N}$ & $50-100$ \\
\hline 03032500 & $\begin{array}{l}\text { Redbank Creek at Saint Charles, } \\
\text { PA }\end{array}$ & 528 & $\begin{array}{l}1910-93 \\
1995-98\end{array}$ & 1996 & 23.90 & 66,300 & $7 / 19 / 96$ & 23.90 & 66,300 & $\mathrm{~N}$ & $>100$ \\
\hline 03034000 & $\begin{array}{l}\text { Mahoning Creek at } \\
\text { Punxsutawney, PA }\end{array}$ & 158 & $\begin{array}{l}\text { 1936, } \\
\text { 1939-98 }\end{array}$ & 1996 & 18.38 & 20,400 & $7 / 19 / 96$ & 18.38 & 20,400 & $\mathrm{~N}$ & $>100$ \\
\hline 03034500 & $\begin{array}{l}\text { Little Mahoning Creek at } \\
\text { McCormick, PA }\end{array}$ & 87.4 & 1940-98 & 1996 & 14.46 & 10,600 & $7 / 19 / 96$ & 14.46 & 10,600 & $\mathrm{~N}$ & $>100$ \\
\hline 03040000 & Stonycreek River at Ferndale, PA & 451 & $\begin{array}{l}\text { 1914-36, } \\
1939-98\end{array}$ & $\begin{array}{l}1936 \\
1977\end{array}$ & $-\overline{-}-23.21$ & $\begin{array}{l}59,000 \\
48,000\end{array}$ & $1 / 19 / 96$ & 17.64 & 41,600 & $\mathrm{~N}$ & $50-100$ \\
\hline 03041500 & Conemaugh River at Seward, PA & 715 & $\begin{array}{l}1936, \\
1939-98\end{array}$ & 1977 & 27.06 & 115,000 & $1 / 19 / 96$ & 20.68 & 59,900 & $\mathrm{~N}$ & $>25$ \\
\hline 03045000 & Loyalhanna Creek at Kingston, PA & 172 & 1940-98 & 1955 & 15.80 & 29,700 & $1 / 19 / 96$ & 13.37 & 18,200 & $\mathrm{~N}$ & $25-50$ \\
\hline 03079000 & Casselman River at Markleton, PA & 382 & 1915-98 & $\begin{array}{l}1955 \\
1936\end{array}$ & $\begin{array}{l}14.06 \\
16.40\end{array}$ & $\begin{array}{l}50,000 \\
35,800\end{array}$ & $1 / 19 / 96$ & 13.26 & 45,000 & $\mathrm{~N}$ & $>100$ \\
\hline 03081000 & $\begin{array}{l}\text { Youghiogheny River below } \\
\text { Confluence, PA }\end{array}$ & 1,029 & $\begin{array}{l}\text { 1936, } \\
1941-98\end{array}$ & 1936 & 21.60 & 85,000 & $1 / 19 / 96$ & 17.74 & 48,500 & $\mathrm{Y}$ & $<100$ \\
\hline 03082500 & $\begin{array}{l}\text { Youghiogheny River at } \\
\text { Connellsville, PA }\end{array}$ & 1,326 & $\begin{array}{l}1860,1888, \\
1891-99, \\
1901-98\end{array}$ & 1955 & 21.96 & 103,000 & $1 / 19 / 96$ & 18.65 & 65,300 & $\mathrm{Y}$ & $25-50$ \\
\hline 03083500 & $\begin{array}{l}\text { Youghiogheny River at } \\
\text { Sutersville, PA }\end{array}$ & 1,715 & $1921-98$ & 1955 & 32.50 & 108,000 & $1 / 19 / 96$ & 28.19 & 84,100 & $\mathrm{Y}$ & $<50$ \\
\hline 03085000 & $\begin{array}{l}\text { Monongahela River at Braddock, } \\
\text { PA }\end{array}$ & 7,337 & $\begin{array}{l}1936, \\
1939-98\end{array}$ & $\begin{array}{l}1996 \\
1972\end{array}$ & $\begin{array}{l}29.07 \\
31.39\end{array}$ & $\begin{array}{l}210,000 \\
180,000\end{array}$ & $1 / 20 / 96$ & 29.07 & 210,000 & $\mathrm{Y}$ & $<50$ \\
\hline 03085500 & Chartiers Creek at Carnegie, PA & 257 & $\begin{array}{l}1916-33, \\
1936, \\
1941-98\end{array}$ & 1956 & 17.37 & 13,500 & $1 / 28 / 94$ & 11.94 & 11,800 & $\mathrm{~N}$ & $<25$ \\
\hline 03086000 & Ohio River at Sewickley, PA & 19,500 & 1934-98 & 1936 & 34.75 & 574,000 & $1 / 20 / 96$ & 33.34 & 372,000 & $\mathrm{Y}$ & $<25$ \\
\hline
\end{tabular}




\section{Puerto Rico}

Thunderstorms developed over the north-central interior slopes of Puerto Rico on November 9, 1995, dumping 6.70 inches of rain at Corozal (fig. 51). One nonofficial rain gage recorded 13.50 inches of rain (National Oceanic and Atmospheric Administration, 1995a). Civil defense agencies reported numerous mudslides in and around Corozal. Río Cibuco below Corozal (streamgage 50038320, table 40) reached its highest discharge since records began in 1970 .

Rainfall amounts associated with the eastern circulation of Hurricane Hortense produced widespread flooding and caused 21 deaths across the interior sections of Puerto Rico on September 9-10, 1996. The eastern interior municipalities received as much as 22 inches of rain (National Oceanic and Atmospheric Administration, 1996a). Creeks and streams island-wide reached very high stages and produced very serious flooding. Seven streamgages measured their peak of record during this flood (table 40). Río Grande de Loíza at Caguas (streamgage 50055000, table 40) had its largest discharge since 1945.

During September 20-22, 1998, Hurricane Georges passed directly over Puerto Rico with wind gusts as high as 150 miles per hour and rainfall amounts up to 27 inches (National Oceanic and Atmospheric Administration, 1998a). Total damage was over $\$ 2$ billion from the wind and rain, but no lives were lost (National Oceanic and Atmospheric Administration, 1998b). Ten streamgages recorded their largest discharges (table 40).

\section{References}

National Oceanic and Atmospheric Administration (NOAA), 1995a-98a, Climatological data (by State): Asheville, North Carolina, National Climatic Data Center, various months.

National Oceanic and Atmospheric Administration (NOAA), 1995b-98b, Storm data (by State): Asheville, North Carolina, National Climatic Data Center, various months.

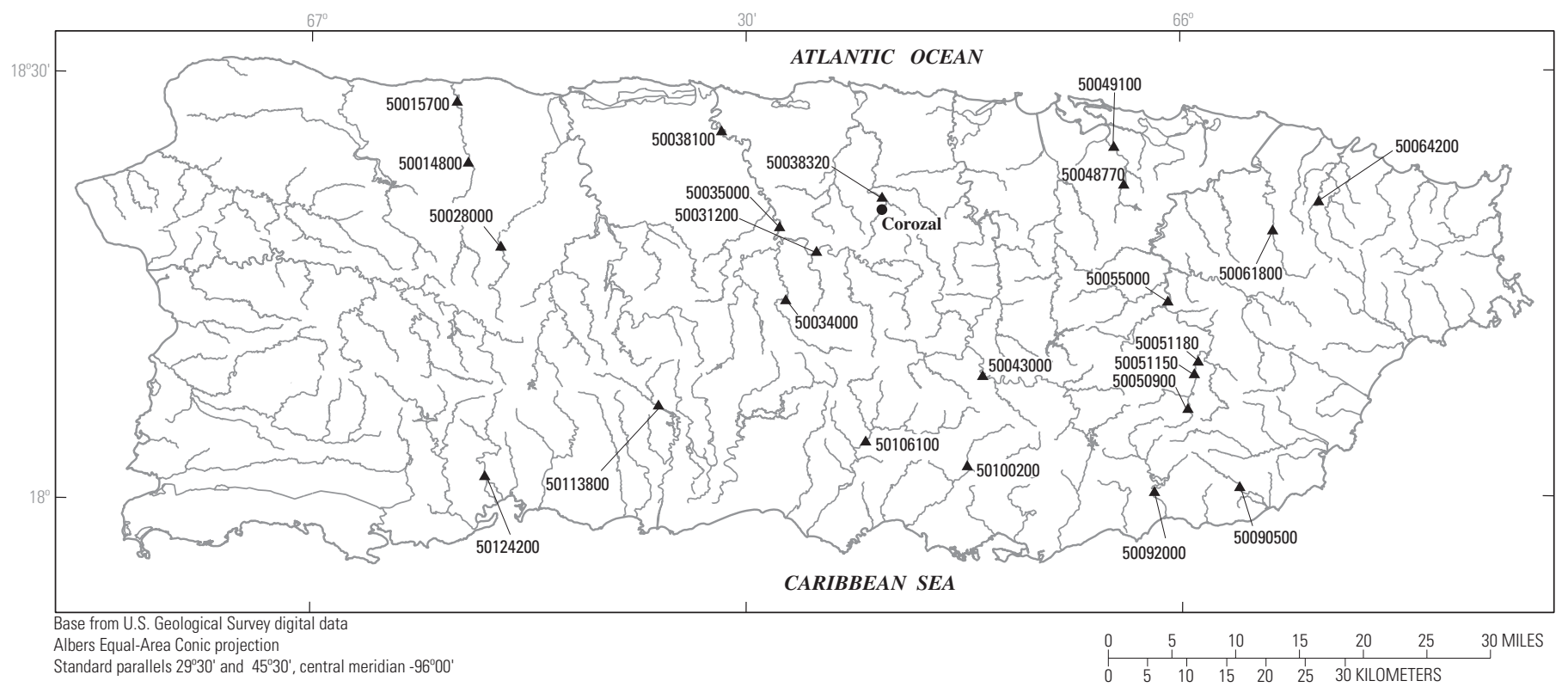

EXPLANATION

50124200_Streamgage and number

Figure 51. Location of streamgages with significant floods during 1994-98 water years for Puerto Rico. 
Table 40. Maximum stage and discharge for period of record for streamgages having significant floods during 1994-98 water years in Puerto Rico.

$\left[\mathrm{mi}^{2}\right.$, square miles; $\mathrm{ft}$, feet above an arbitrary datum; $\mathrm{ft}^{3} / \mathrm{s}$, cubic feet per second; --, not determined or not applicable; >, greater than. Source: Recurrence intervals calculated from U.S. Geological Survey data. Other data from U.S. Geological Survey reports or databases]

\begin{tabular}{|c|c|c|c|c|c|c|c|c|c|c|c|}
\hline \multirow{2}{*}{$\begin{array}{c}\text { Streamgage } \\
\text { number } \\
\text { (fig. 51) }\end{array}$} & \multirow[b]{2}{*}{ Streamgage name } & \multirow{2}{*}{$\begin{array}{c}\text { Total } \\
\text { drainage } \\
\left(\mathrm{mi}^{2}\right)\end{array}$} & \multicolumn{4}{|c|}{$\begin{array}{c}\text { Maximum stage and discharge for period of record } \\
\text { through } 1998 \text { water year }\end{array}$} & \multicolumn{5}{|c|}{ Significant floods 1994-98 water years } \\
\hline & & & $\begin{array}{l}\text { Period of } \\
\text { record } \\
\text { (water } \\
\text { years) }\end{array}$ & Water year & $\begin{array}{l}\text { Stage } \\
\text { (ft) }\end{array}$ & $\begin{array}{l}\text { Discharge } \\
\left(\mathrm{ft}^{3} / \mathrm{s}\right)\end{array}$ & $\begin{array}{c}\text { Date } \\
\text { (month/ } \\
\text { day/year) }\end{array}$ & $\begin{array}{l}\text { Stage } \\
(\mathrm{ft})\end{array}$ & $\begin{array}{l}\text { Discharge } \\
\left(\mathrm{ft}^{3} / \mathrm{s}\right)\end{array}$ & $\begin{array}{c}\text { Regulated } \\
\text { during } \\
\text { flood }^{1}\end{array}$ & $\begin{array}{c}\text { Recurrence } \\
\text { interval } \\
\text { (years) }\end{array}$ \\
\hline 50014800 & Rio Camuy near Bayaney, PR & -- & 1984-98 & 1998 & 21.69 & 11,600 & $9 / 22 / 98$ & 21.69 & 11,600 & $\mathrm{~N}$ & $>50$ \\
\hline 50015700 & Rio Camuy near Hatillo, PR & -- & 1984-96 & 1996 & 25.18 & 11,400 & 9/10/96 & 25.18 & 11,400 & $\mathrm{~N}$ & $10-25$ \\
\hline 50028000 & Rio Tanama near Utuado, PR & 18.4 & 1960-98 & 1998 & 21.24 & 23,500 & 9/22/98 & 21.24 & 23,500 & $\mathrm{~N}$ & $>100$ \\
\hline 50031200 & $\begin{array}{l}\text { Rio Grande de Manati near } \\
\text { Morovis, PR }\end{array}$ & 55.2 & 1965-98 & $\begin{array}{l}1985 \\
1971\end{array}$ & $\begin{array}{l}17.89 \\
20.30\end{array}$ & $\begin{array}{l}48,000 \\
35,000\end{array}$ & 9/10/96 & 18.83 & 47,700 & $\mathrm{~N}$ & $40-50$ \\
\hline 50034000 & Rio Bauta near Orocovis, PR & 16.7 & $\begin{array}{l}\text { 1970-82, } \\
1989-98\end{array}$ & 1998 & 25.93 & 28,200 & 9/22/98 & 25.93 & 28,200 & $\mathrm{~N}$ & $50-100$ \\
\hline 50035000 & $\begin{array}{l}\text { Rio Grande de Manati at Ciales, } \\
\text { PR }\end{array}$ & 128 & $\begin{array}{l}1899,1928, \\
1932, \\
1949-53, \\
1956, \\
1958-98\end{array}$ & $\begin{array}{l}1996 \\
1899\end{array}$ & $\begin{array}{l}25.20 \\
50.00\end{array}$ & $\begin{array}{r}128,000 \\
--\end{array}$ & $\begin{array}{l}9 / 10 / 96 \\
9 / 21 / 98\end{array}$ & $\begin{array}{l}25.20 \\
22.38\end{array}$ & $\begin{array}{r}128,000 \\
78,900\end{array}$ & $\begin{array}{l}\mathrm{N} \\
\mathrm{N}\end{array}$ & $\begin{array}{r}50-100 \\
20\end{array}$ \\
\hline 50038100 & $\begin{array}{l}\text { Rio Grande de Manati at } \\
\text { Highway } 2 \text { near Manati, PR }\end{array}$ & 197 & $\begin{array}{l}\text { 1928, 1932, } \\
1945, \\
1959-63, \\
1965-66, \\
1968-98\end{array}$ & $\begin{array}{l}1998 \\
1996\end{array}$ & $\begin{array}{l}34.90 \\
36.39\end{array}$ & $\begin{array}{r}136,000 \\
--\end{array}$ & $9 / 22 / 98$ & 34.90 & 136,000 & $\mathrm{~N}$ & $40-50$ \\
\hline 50038320 & Rio Cibuco below Corozal, PR & 15.1 & 1970-98 & 1996 & 22.35 & 20,400 & $11 / 9 / 95$ & 22.35 & 20,400 & $\mathrm{~N}$ & $50-100$ \\
\hline 50043000 & $\begin{array}{l}\text { Rio de La Plata at Proyecto La } \\
\text { Plata, PR }\end{array}$ & 54.8 & $\begin{array}{l}\text { 1960-92, } \\
1996\end{array}$ & 1992 & 36.39 & 73,600 & $9 / 10 / 96$ & 34.10 & 66,000 & $\mathrm{~N}$ & $\begin{array}{r}30-40 \\
--\end{array}$ \\
\hline 50048770 & Rio Piedras at El Senorial, PR & 7.49 & 1988-98 & $\begin{array}{l}1996 \\
1988\end{array}$ & $\begin{array}{l}15.46 \\
16.08\end{array}$ & $\begin{array}{l}5,390 \\
4,680\end{array}$ & $9 / 10 / 96$ & 15.46 & 5,390 & $\mathrm{~N}$ & $10-25$ \\
\hline
\end{tabular}


Table 40. Maximum stage and discharge for period of record for streamgages having significant floods during 1994-98 water years in Puerto Rico.-Continued

$\left[\mathrm{mi}^{2}\right.$, square miles; ft, feet above an arbitrary datum; $\mathrm{ft}^{3} / \mathrm{s}$, cubic feet per second; --, not determined or not applicable; >, greater than. Source: Recurrence intervals calculated from U.S. Geological Survey data. Other data from U.S. Geological Survey reports or databases]

\begin{tabular}{|c|c|c|c|c|c|c|c|c|c|c|c|}
\hline \multirow[b]{2}{*}{$\begin{array}{l}\text { Streamgage } \\
\text { number } \\
\text { (fig. 51) }\end{array}$} & \multirow[b]{2}{*}{ Streamgage name } & \multirow{2}{*}{$\begin{array}{c}\text { Total } \\
\text { drainage } \\
\left(\mathrm{mi}^{2}\right)\end{array}$} & \multicolumn{4}{|c|}{$\begin{array}{c}\text { Maximum stage and discharge for period of record } \\
\text { through } 1998 \text { water year }\end{array}$} & \multicolumn{5}{|c|}{ Significant floods 1994-98 water years } \\
\hline & & & $\begin{array}{l}\text { Period of } \\
\text { record } \\
\text { (water } \\
\text { years) }\end{array}$ & Water year & $\begin{array}{l}\text { Stage } \\
(\mathrm{ft})\end{array}$ & $\begin{array}{l}\text { Discharge } \\
\left(\mathrm{ft}^{3} / \mathrm{s}\right)\end{array}$ & $\begin{array}{c}\text { Date } \\
\text { (month/ } \\
\text { day/year) }\end{array}$ & $\begin{array}{l}\text { Stage } \\
\text { (ft) }\end{array}$ & $\begin{array}{l}\text { Discharge } \\
\left(\mathrm{ft}^{3} / \mathrm{s}\right)\end{array}$ & $\begin{array}{l}\text { Regulated } \\
\text { during } \\
\text { flood }^{1}\end{array}$ & $\begin{array}{c}\text { Recurrence } \\
\text { interval } \\
\text { (years) }\end{array}$ \\
\hline 50049100 & Rio Piedras at Hato Rey, PR & 15.2 & $\begin{array}{l}\text { 1970, } \\
\text { 1972-74, } \\
\text { 1976-82, } \\
1988-98\end{array}$ & 1996 & 22.11 & 10,500 & $9 / 10 / 96$ & 22.11 & 10,500 & $\mathrm{~N}$ & $10-25$ \\
\hline 50050900 & $\begin{array}{l}\text { Rio Grande de Loiza at Quebrada } \\
\text { Arenas, PR }\end{array}$ & 6.00 & 1978-98 & 1998 & 26.37 & 45,000 & $9 / 21 / 98$ & 26.37 & 45,000 & $\mathrm{~N}$ & $50-100$ \\
\hline 50051150 & Quebrada Blanca at Jagual, PR & 3.25 & 1985-98 & 1996 & 14.64 & 7,610 & 9/10/96 & 14.64 & 7,610 & $\mathrm{~N}$ & $10-25$ \\
\hline 50051180 & $\begin{array}{l}\text { Quebrada Salvatierra near San } \\
\text { Lorenzo, PR }\end{array}$ & 3.74 & 1984-98 & 1996 & 20.86 & 15,000 & 9/10/96 & 20.86 & 15,000 & $\mathrm{~N}$ & $25-50$ \\
\hline 50055000 & Rio Grande de Loiza at Caguas, PR & 89.8 & $\begin{array}{l}1945, \\
1960-98\end{array}$ & 1945 & 33.20 & 85,000 & $9 / 10 / 96$ & 32.32 & 83,000 & $\mathrm{~N}$ & $50-100$ \\
\hline 50061800 & $\begin{array}{l}\text { Rio Canovanas near Campo Rico, } \\
\text { PR }\end{array}$ & 9.84 & 1968-98 & 1998 & 15.90 & 17,300 & $9 / 21 / 98$ & 15.90 & 17,300 & $\mathrm{~N}$ & $50-100$ \\
\hline 50064200 & Rio Grande near El Verde, PR & 7.31 & $\begin{array}{l}1968-75 \\
1977-82, \\
1991-98\end{array}$ & 1998 & 19.30 & 22,000 & $9 / 21 / 98$ & 19.30 & 22,000 & $\mathrm{~N}$ & $50-100$ \\
\hline 50090500 & Rio Maunabo at Lizas, PR & 5.38 & $\begin{array}{l}1971-85 \\
1991-98\end{array}$ & 1994 & 17.46 & 9,950 & $9 / 20 / 94$ & 17.46 & 9,950 & $\mathrm{~N}$ & $10-25$ \\
\hline 50092000 & $\begin{array}{l}\text { Rio Grande de Patillas near } \\
\text { Patillas, PR }\end{array}$ & 18.3 & 1966-98 & $\begin{array}{l}1992 \\
1998\end{array}$ & -- & $\begin{array}{r}30,900 \\
--\end{array}$ & $\begin{array}{l}9 / 10 / 96 \\
9 / 21 / 98\end{array}$ & $\begin{array}{l}22.55 \\
24.36\end{array}$ & $\begin{array}{r}22,400 \\
--\end{array}$ & $\begin{array}{l}\mathrm{N} \\
\mathrm{N}\end{array}$ & $\begin{array}{r}30-40 \\
--\end{array}$ \\
\hline 50100200 & Rio Lapa near Rabo del Buey, PR & 10.0 & $\begin{array}{l}\text { 1971, } \\
\text { 1989-98 }\end{array}$ & 1996 & 18.65 & 18,100 & $9 / 10 / 96$ & 18.65 & 18,100 & $\mathrm{~N}$ & $10-25$ \\
\hline 50106100 & Rio Coamo at Coamo, PR & 43.5 & $1987-98$ & 1998 & 25.94 & 52,700 & $9 / 21 / 98$ & 25.94 & 52,700 & $\mathrm{~N}$ & $>50$ \\
\hline 50113800 & $\begin{array}{l}\text { Rio Cerrillos above Lago Cerrillos } \\
\text { near Ponce, PR }\end{array}$ & 11.9 & 1989-98 & 1998 & 12.42 & 16,200 & $9 / 21 / 98$ & 12.42 & 16,200 & $\mathrm{~N}$ & $10-25$ \\
\hline 50124200 & $\begin{array}{l}\text { Rio Guayanilla near Guayanilla, } \\
\text { PR }\end{array}$ & 18.9 & 1981-98 & 1998 & 21.88 & 18,700 & $9 / 22 / 98$ & 21.88 & 18,700 & $\mathrm{~N}$ & $25-50$ \\
\hline
\end{tabular}

${ }^{1}$ Regulated during flood: N, no; Y, yes. 


\section{Rhode Island}

A strong low-pressure system that moved to the northeast along the Mid-Atlantic Coast on February 18, 1998, brought excessive rainfall, isolated flash floods, and thunderstorms to central and southern Rhode Island. Rainfall totals for this storm ranged from 2.0 to 3.5 inches during a 12-hour period (National Oceanic and Atmospheric Administration, 1998). In Middletown (fig. 52), the Maidford River rose out of its banks and flooded part of a neighborhood.

During June 12-14, 1998, a very slow-moving, complex storm system moved through southeastern New England. The combination of its slow movement and the presence of tropical moisture across the region produced rainfall of 6 to 8 inches over much of Rhode Island. The most excessive rainfall amounts of 7 to 8 inches occurred in the northeast corner of the State (National Oceanic and Atmospheric Administration, 1998). Numerous small streams flooded over their banks. The streamgage on Ten Mile River at Pawtucket Avenue at East Providence (streamgage 01109403, table 41) recorded its peak of record on June 15.

A slow-moving warm front with an abundance of tropical moisture produced several hours of torrential rain with amounts totaling 3 to 6 inches during the morning and early afternoon of June 30, 1998 (National Oceanic and Atmospheric Administration, 1998). During the evening, more thunderstorm activity produced flooding and the highest discharge since 1936 on the Woonasquatucket River at Centerdale (streamgage 01114500, table 41).

\section{Reference}

National Oceanic and Atmospheric Administration (NOAA), 1998, Climatological data (by State): Asheville, North Carolina, National Climatic Data Center, various months.

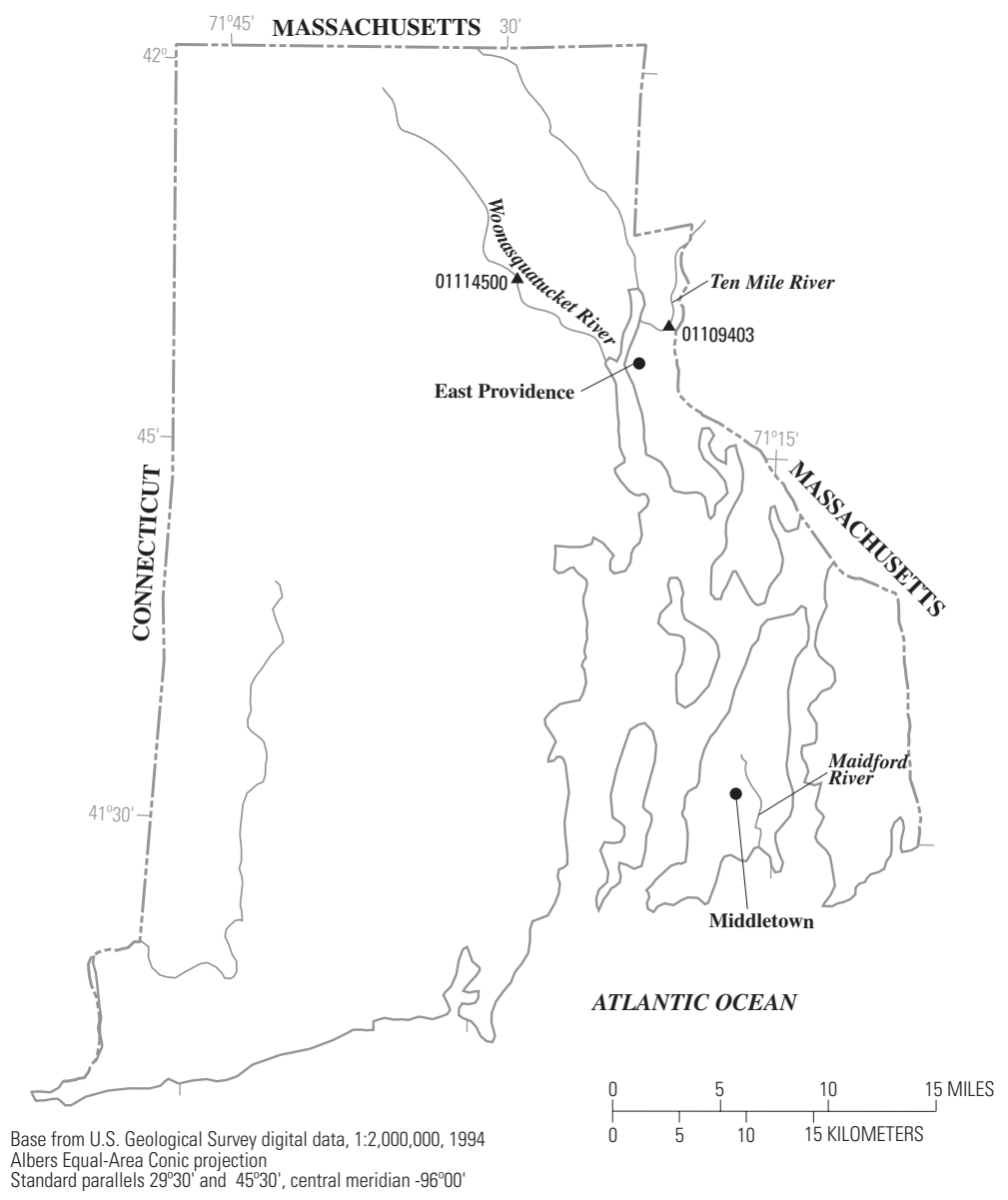

\section{EXPLANATION}

$01114500 \_$Streamgage and number

Figure 52. Location of streamgages with significant floods during 1994-98 water years for Rhode Island. 
Table 41. Maximum stage and discharge for period of record for streamgages having significant floods during 1994-98 water years in Rhode Island.

$\left[\mathrm{mi}^{2}\right.$, square miles; $\mathrm{ft}$, feet above an arbitrary datum; $\mathrm{ft}^{3} / \mathrm{s}$, cubic feet per second. Source: Recurrence intervals calculated from U.S. Geological Survey data. Other data from U.S. Geological Survey reports or databases]

\begin{tabular}{|c|c|c|c|c|c|c|c|c|c|c|c|}
\hline \multirow[b]{2}{*}{$\begin{array}{l}\text { Streamgage } \\
\text { number } \\
\text { (fig. 52) }\end{array}$} & \multirow[b]{2}{*}{ Streamgage name } & \multirow[b]{2}{*}{$\begin{array}{c}\text { Total } \\
\text { drainage } \\
\left(\mathrm{mi}^{2}\right)\end{array}$} & \multicolumn{4}{|c|}{$\begin{array}{c}\text { Maximum stage and discharge for period of record } \\
\text { through } 1998 \text { water year }\end{array}$} & \multicolumn{5}{|c|}{ Significant floods $1994-98$ water years } \\
\hline & & & $\begin{array}{c}\text { Period of } \\
\text { record } \\
\text { (water } \\
\text { years) }\end{array}$ & Water year & $\begin{array}{l}\text { Stage } \\
(\mathrm{ft})\end{array}$ & $\begin{array}{c}\text { Discharge } \\
\left(\mathrm{ft}^{3} / \mathrm{s}\right)\end{array}$ & $\begin{array}{c}\text { Date } \\
\text { (month/ } \\
\text { day/ } \\
\text { year) }\end{array}$ & $\begin{array}{l}\text { Stage } \\
(\mathrm{ft})\end{array}$ & $\begin{array}{c}\text { Discharge } \\
\left(\mathrm{ft}^{3} / \mathrm{s}\right)\end{array}$ & $\begin{array}{c}\text { Regulated } \\
\text { during } \\
\text { flood }^{1}\end{array}$ & $\begin{array}{c}\text { Recurrence } \\
\text { interval } \\
\text { (years) }\end{array}$ \\
\hline 01109403 & $\begin{array}{l}\text { Ten Mile River at Pawtucket } \\
\text { Avenue at East Providence, RI }\end{array}$ & 53.1 & $1987-98$ & 1998 & 8.50 & 1,450 & $6 / 15 / 98$ & 8.50 & 1,450 & $\mathrm{Y}$ & 25 \\
\hline 01114500 & $\begin{array}{l}\text { Woonasquatucket River at } \\
\text { Centerdale, RI }\end{array}$ & 38.3 & $\begin{array}{l}\text { 1936, } \\
1942-98\end{array}$ & $\begin{array}{l}1998 \\
1968\end{array}$ & $\begin{array}{l}7.26 \\
7.75\end{array}$ & $\begin{array}{l}1,520 \\
1,440\end{array}$ & $6 / 30 / 98$ & 7.26 & 1,520 & $\mathrm{Y}$ & 50 \\
\hline
\end{tabular}

${ }^{1}$ Regulated during flood: N, no; Y, yes. 


\section{South Carolina}

Excessive rains of more than 6 inches within 24 hours caused flash flooding early the morning of June 27, 1994 (National Oceanic and Atmospheric Administration, 1994a). The flash flooding affected several creeks in and near Lexington (fig. 53) and caused $\$ 5.0$ million in property damage (National Oceanic and Atmospheric Administration, 1994b).

Rainfall associated with Tropical Storm Beryl totalled 4 or 5 inches in the Piedmont area and 12 inches in the mountains on August 17, 1994 (National Oceanic and Atmospheric Administration, 1994a). This rainfall caused severe flooding near Greenville and Spartanburg. Damage was more than \$1.5 million to property and more than $\$ 10$ million to crops. No deaths were reported (National Oceanic and Atmospheric Administration, 1994b).

Several episodes of intense rainfall during the fall of 1994 caused flash floods and coastal flooding in South Carolina. Record-breaking rains of more than 13 inches in 24 hours fell near Beaufort on October 3, 1994. A 24-hour total of 11.5 inches on northern Hilton Head Island broke the official all-time rainfall records at that location (National Oceanic and Atmospheric Administration, 1994a). Scattered areas of flash flooding were followed by coastal flooding. Excessive rainfall occurred again on October 13 in and around Charleston with 4 to 8 inches falling (National Oceanic and Atmospheric Administration, 1994a). More than \$27 million in damage resulted from the flooding (National Oceanic and Atmospheric Administration, 1994b).
The remnants of Tropical Storm Jerry moved slowly eastward across the State during August 24-28, 1995, and produced unusually excessive rains statewide with amounts mostly varying from 8 to more than 12 inches in some locations (National Oceanic and Atmospheric Administration, 1995a). The excessive rain produced flash flooding in flood-prone areas, general flooding on many rivers, broken dams, flooded streets, homes, and low-lying farmland. Damage was more than \$20 million (National Oceanic and Atmospheric Administration, 1995b). Statewide damage to roads and bridges was estimated by the South Carolina Department of Transportation to be $\$ 4.5$ million. Eleven streamgages recorded their highest discharge ever during this flood (table 42).

A flash flood occurred late in the evening on August 14, 1998, following 4 to 5 inches of rainfall in a short period (National Oceanic and Atmospheric Administration, 1998a). The flash flood affected several creeks near Spartanburg. There were nearly $\$ 2.5$ million in damage (National Oceanic and Atmospheric Administration, 1998b).

\section{References}

National Oceanic and Atmospheric Administration (NOAA), 1994a-98a, Climatological data (by State): Asheville, North Carolina, National Climatic Data Center, various months.

National Oceanic and Atmospheric Administration (NOAA), 1994b-98b, Storm data (by State): Asheville, North Carolina, National Climatic Data Center, various months. 


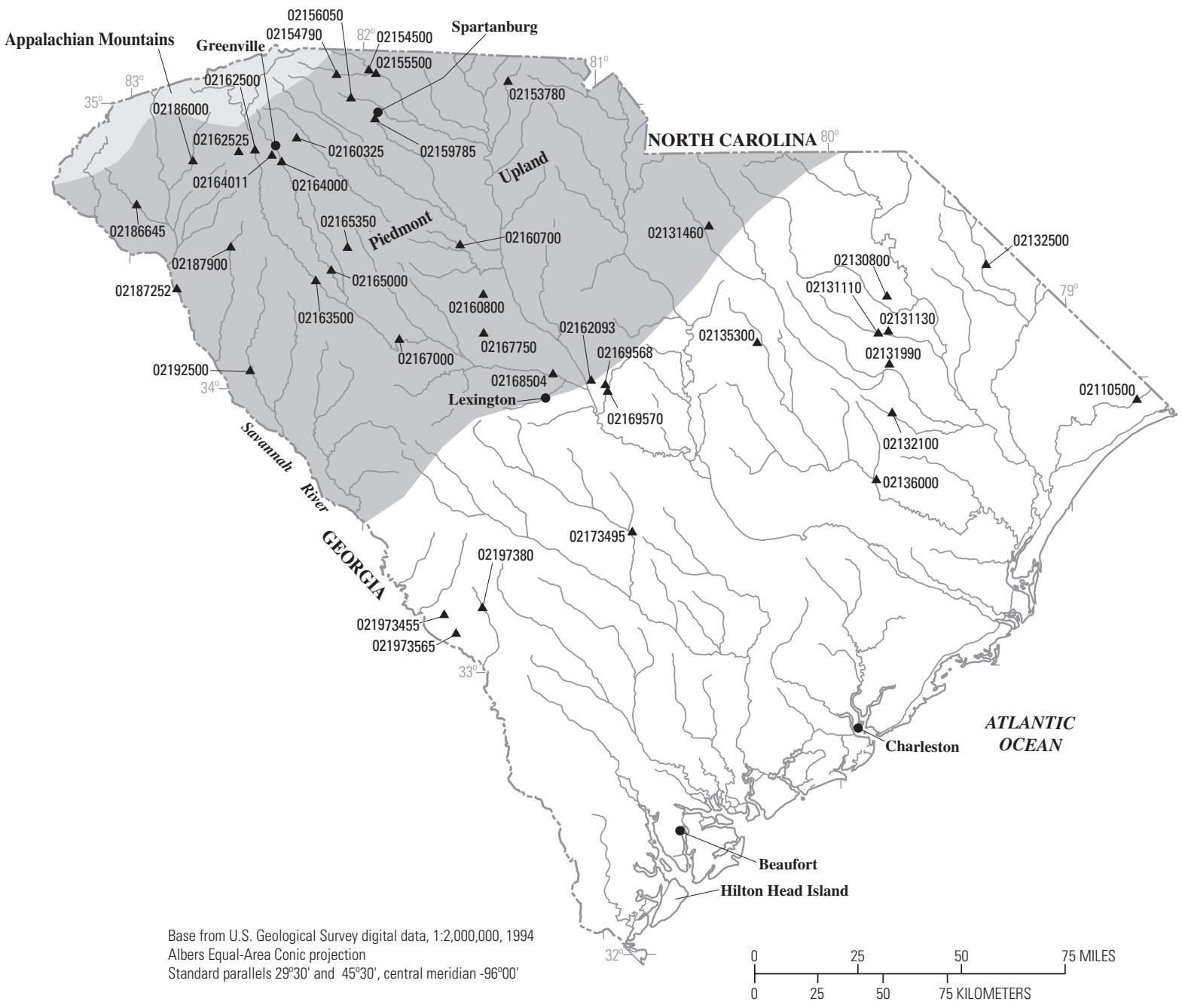

\section{EXPLANATION}

02197345 Streamgage and number

Figure 53. Location of streamgages with significant floods during 1994-98 water years for South Carolina. 
Table 42. Maximum stage and discharge for period of record for streamgages having significant floods during 1994-98 water years in South Carolina.

$\left[\mathrm{mi}^{2}\right.$, square miles; $\mathrm{ft}$, feet above an arbitrary datum; $\mathrm{ft}^{3} / \mathrm{s}$, cubic feet per second; --, not determined or not applicable; >, greater than. Source: Recurrence intervals calculated from U.S. Geological Survey data. Other data from U.S. Geological Survey reports or databases]

\begin{tabular}{|c|c|c|c|c|c|c|c|c|c|c|c|}
\hline \multirow{2}{*}{$\begin{array}{c}\text { Streamgage } \\
\text { number } \\
\text { (fig. 53) }\end{array}$} & \multirow[b]{2}{*}{ Streamgage name } & \multirow{2}{*}{$\begin{array}{l}\text { Total } \\
\text { drainage } \\
\left(\mathrm{mi}^{2}\right)\end{array}$} & \multicolumn{4}{|c|}{$\begin{array}{c}\text { Maximum stage and discharge for period of record } \\
\text { through } 1998 \text { water year }\end{array}$} & \multicolumn{5}{|c|}{ Significant floods $1994-98$ water years } \\
\hline & & & $\begin{array}{l}\text { Period of } \\
\text { record } \\
\text { (water } \\
\text { years) }\end{array}$ & Water year & $\begin{array}{l}\text { Stage } \\
(\mathrm{ft})\end{array}$ & $\begin{array}{l}\text { Discharge } \\
\left(\mathrm{ft}^{3} / \mathrm{s}\right)\end{array}$ & $\begin{array}{c}\text { Date } \\
\text { (month/ } \\
\text { day/ } \\
\text { year) }\end{array}$ & $\begin{array}{l}\text { Stage } \\
(\mathrm{ft})\end{array}$ & $\begin{array}{l}\text { Discharge } \\
\left(\mathrm{ft}^{3} / \mathrm{s}\right)\end{array}$ & $\begin{array}{l}\text { Regulated } \\
\text { during } \\
\text { flood }^{1}\end{array}$ & $\begin{array}{c}\text { Recurrence } \\
\text { interval } \\
\text { (years) }\end{array}$ \\
\hline 02110500 & Waccamaw River near Longs, SC & 1,110 & $1951-98$ & $\begin{array}{l}1981 \\
1996\end{array}$ & $\begin{array}{l}14.87 \\
14.95\end{array}$ & $\begin{array}{l}16,200 \\
15,800\end{array}$ & $9 / 15 / 96$ & 14.95 & 15,800 & $\mathrm{~N}$ & $10-25$ \\
\hline 02130800 & Backswamp near Darlington, SC & 6.22 & 1976-98 & 1995 & 12.21 & 800 & $12 / 24 / 94$ & 12.21 & 800 & $\mathrm{~N}$ & $50-100$ \\
\hline 02131110 & Jeffries Creek above Florence, SC & 46.6 & 1968-98 & 1995 & 10.72 & 3,220 & $12 / 24 / 94$ & 10.72 & 3,220 & $\mathrm{~N}$ & $50-100$ \\
\hline 02131130 & $\begin{array}{l}\text { Gully Branch at Cherokee Road at } \\
\text { Florence, SC }\end{array}$ & 1.92 & 1985-98 & 1996 & 6.37 & 765 & $9 / 11 / 96$ & 6.37 & 765 & $\mathrm{~N}$ & $10-25$ \\
\hline 02131460 & Neds Creek near Kershaw, SC & 3.98 & $\begin{array}{l}1977-78, \\
1980-82, \\
1984-86, \\
1989, \\
1991-96\end{array}$ & 1996 & 6.98 & 238 & $6 / 10 / 95$ & 6.98 & 238 & $\mathrm{~N}$ & -- \\
\hline 02131990 & Carter Creek at Effingham, SC & 8.28 & $\begin{array}{l}1969-84 \\
1987-98\end{array}$ & 1995 & 9.61 & 1,440 & $12 / 24 / 94$ & 9.61 & 1,440 & $\mathrm{~N}$ & -- \\
\hline 02132100 & $\begin{array}{l}\text { Two Mile Branch near Lake City, } \\
\text { SC }\end{array}$ & 19.0 & 1976-98 & 1995 & 10.19 & 2,400 & $12 / 24 / 94$ & 10.19 & 2,400 & $\mathrm{~N}$ & $>100$ \\
\hline 02132500 & $\begin{array}{l}\text { Little Pee Dee River near Dillon, } \\
\text { SC }\end{array}$ & 524 & 1940-98 & 1945 & 14.64 & 9,810 & $2 / 24 / 95$ & 12.73 & 6,760 & $\mathrm{~N}$ & 25 \\
\hline 02135300 & $\begin{array}{l}\text { Scape Ore Swamp near } \\
\text { Bishopville, SC }\end{array}$ & 96.0 & 1969-98 & 1991 & 11.80 & 4,500 & $12 / 24 / 94$ & 9.86 & 2,580 & $\mathrm{~N}$ & $25-50$ \\
\hline 02136000 & Black River at Kingstree, SC & 1,252 & $1893-1998$ & 1973 & 19.77 & 58,000 & $12 / 28 / 94$ & 15.35 & 22,100 & $\mathrm{~N}$ & $10-25$ \\
\hline 02153780 & $\begin{array}{l}\text { Clarks Fork Creek near Smyrna, } \\
\text { SC }\end{array}$ & 24.1 & 1981-98 & 1995 & 13.77 & 2,100 & $8 / 27 / 95$ & 13.77 & 2,100 & $\mathrm{~N}$ & -- \\
\hline 02154500 & $\begin{array}{l}\text { North Pacolet River at Fingerville, } \\
\text { SC }\end{array}$ & 116 & 1931-98 & 1940 & 27.13 & 12,500 & $8 / 28 / 95$ & 21.37 & 8,160 & $\mathrm{~N}$ & $10-25$ \\
\hline 02154790 & $\begin{array}{l}\text { South Pacolet River near } \\
\text { Campobello, SC }\end{array}$ & 55.4 & 1989-98 & 1995 & 11.33 & 5,170 & $8 / 27 / 95$ & 11.33 & 5,170 & $\mathrm{~N}$ & $25-50$ \\
\hline 02155500 & Pacolet River near Fingerville, SC & 212 & $\begin{array}{l}1903 \\
1931-98\end{array}$ & $\begin{array}{l}1940 \\
1903\end{array}$ & $\begin{array}{l}22.43 \\
46.00\end{array}$ & $\begin{array}{r}22,800 \\
--\end{array}$ & $8 / 27 / 95$ & 15.58 & 13,700 & $\mathrm{Y}$ & -- \\
\hline
\end{tabular}


Table 42. Maximum stage and discharge for period of record for streamgages having significant floods during 1994-98 water years in South Carolina.-Continued

$\left[\mathrm{mi}^{2}\right.$, square miles; $\mathrm{ft}$, feet above an arbitrary datum; $\mathrm{ft}^{3} / \mathrm{s}$, cubic feet per second; --, not determined or not applicable; >, greater than. Source: Recurrence intervals calculated from U.S. Geological Survey data. Other data from U.S. Geological Survey reports or databases]

\begin{tabular}{|c|c|c|c|c|c|c|c|c|c|c|c|}
\hline \multirow{2}{*}{$\begin{array}{c}\text { Streamgage } \\
\text { number } \\
\text { (fig. 53) }\end{array}$} & \multirow[b]{2}{*}{ Streamgage name } & \multirow{2}{*}{$\begin{array}{c}\text { Total } \\
\text { drainage } \\
\left(\mathrm{mi}^{2}\right)\end{array}$} & \multicolumn{4}{|c|}{$\begin{array}{c}\text { Maximum stage and discharge for period of record } \\
\text { through } 1998 \text { water year }\end{array}$} & \multicolumn{5}{|c|}{ Significant floods 1994-98 water years } \\
\hline & & & $\begin{array}{l}\text { Period of } \\
\text { record } \\
\text { (water } \\
\text { years) }\end{array}$ & Water year & $\begin{array}{l}\text { Stage } \\
\text { (ft) }\end{array}$ & $\begin{array}{l}\text { Discharge } \\
\left(\mathrm{ft}^{3} / \mathrm{s}\right)\end{array}$ & $\begin{array}{c}\text { Date } \\
\text { (month/ } \\
\text { day/ } \\
\text { year) }\end{array}$ & $\begin{array}{l}\text { Stage } \\
(\mathrm{ft})\end{array}$ & $\begin{array}{l}\text { Discharge } \\
\left(\mathrm{ft}^{3} / \mathrm{s}\right)\end{array}$ & $\begin{array}{l}\text { Regulated } \\
\text { during } \\
\text { flood }^{1}\end{array}$ & $\begin{array}{c}\text { Recurrence } \\
\text { interval } \\
\text { (years) }\end{array}$ \\
\hline 02156050 & $\begin{array}{l}\text { Lawsons Fork Creek at Dewey } \\
\text { Plant near Inman, SC }\end{array}$ & 6.46 & 1980-98 & $\begin{array}{l}1994 \\
1980\end{array}$ & $\begin{array}{l}7.33 \\
7.86\end{array}$ & $\begin{array}{l}563 \\
213\end{array}$ & $8 / 17 / 94$ & 7.33 & 563 & $\mathrm{~N}$ & -- \\
\hline 02159785 & $\begin{array}{l}\text { Tributary to Fairforest Creek at } \\
\text { Spartanburg, SC }\end{array}$ & .52 & 1987-98 & 1994 & 5.19 & 243 & $6 / 28 / 94$ & 5.19 & 243 & $\mathrm{~N}$ & $50-100$ \\
\hline 02160325 & Brushy Creek near Greenville, SC & 9.05 & 1986-98 & 1995 & 14.10 & -- & $8 / 27 / 95$ & 14.10 & -- & $\mathrm{N}$ & -- \\
\hline 02160700 & Enoree River at Whitmire, SC & 444 & 1974-98 & 1995 & 37.32 & 31,200 & $8 / 28 / 95$ & 37.32 & 31,200 & $\mathrm{~N}$ & $>100$ \\
\hline 02160800 & Second Creek near Pomaria, SC & 1.87 & $\begin{array}{l}\text { 1977-89, } \\
1991, \\
1993-98\end{array}$ & 1995 & 8.43 & 1,090 & $8 / 26 / 95$ & 8.43 & 1,090 & $\mathrm{~N}$ & -- \\
\hline 02162093 & $\begin{array}{l}\text { Smith Branch at North Main Street } \\
\text { at Columbia, SC }\end{array}$ & 5.67 & 1977-98 & 1995 & 11.69 & 2,120 & $6 / 11 / 95$ & 11.69 & 2,120 & $\mathrm{~N}$ & $10-25$ \\
\hline 02162500 & Saluda River near Greenville, SC & 295 & $\begin{array}{l}1942-78 \\
1981-82 \\
1984-98\end{array}$ & 1950 & 19.38 & 11,000 & $8 / 27 / 95$ & 15.59 & 8,550 & $\mathrm{~N}$ & $10-25$ \\
\hline 02162525 & $\begin{array}{l}\text { Hamilton Creek (Road 135) near } \\
\text { Easley, SC }\end{array}$ & 1.60 & $\begin{array}{l}1983, \\
1988-90, \\
1993-98\end{array}$ & 1995 & 8.11 & 835 & $8 / 27 / 95$ & 8.11 & 835 & $\mathrm{~N}$ & -- \\
\hline 02163500 & Saluda River near Ware Shoals, SC & 580 & 1939-98 & 1995 & 22.95 & 20,900 & $8 / 27 / 95$ & 22.95 & 20,900 & $\mathrm{~N}$ & $25-50$ \\
\hline 02164000 & Reedy River near Greenville, SC & 48.6 & $\begin{array}{l}1942-75, \\
1977, \\
1987-98\end{array}$ & 1995 & 11.88 & 5,400 & $8 / 27 / 95$ & 11.88 & 5,400 & $\mathrm{~N}$ & $50-100$ \\
\hline 02164011 & $\begin{array}{l}\text { Brushy Creek at Grove Road at } \\
\text { Greenville, SC }\end{array}$ & 2.82 & 1985-98 & 1993 & 9.18 & 1,740 & $1 / 8 / 98$ & 7.57 & 1,250 & $\mathrm{~N}$ & $2-5$ \\
\hline 02165000 & Reedy River near Ware Shoals, SC & 236 & $\begin{array}{l}1940-59 \\
1961-98\end{array}$ & $\begin{array}{l}1973 \\
1995\end{array}$ & $\begin{array}{l}15.40 \\
18.71\end{array}$ & $\begin{array}{r}11,000 \\
9,980\end{array}$ & $8 / 28 / 95$ & 18.71 & 9,980 & $\mathrm{~N}$ & $10-25$ \\
\hline
\end{tabular}


Table 42. Maximum stage and discharge for period of record for streamgages having significant floods during 1994-98 water years in South Carolina.-Continued

$\left[\mathrm{mi}^{2}\right.$, square miles; ft, feet above an arbitrary datum; $\mathrm{ft}^{3} / \mathrm{s}$, cubic feet per second; --, not determined or not applicable; >, greater than. Source: Recurrence intervals calculated from U.S. Geological Survey data. Other data from U.S. Geological Survey reports or databases]

\begin{tabular}{|c|c|c|c|c|c|c|c|c|c|c|c|}
\hline \multirow{2}{*}{$\begin{array}{c}\text { Streamgage } \\
\text { number } \\
\text { (fig. 53) }\end{array}$} & \multirow[b]{2}{*}{ Streamgage name } & \multirow{2}{*}{$\begin{array}{c}\text { Total } \\
\text { drainage } \\
\left(\mathrm{mi}^{2}\right)\end{array}$} & \multicolumn{4}{|c|}{$\begin{array}{c}\text { Maximum stage and discharge for period of record } \\
\text { through } 1998 \text { water year }\end{array}$} & \multicolumn{5}{|c|}{ Significant floods 1994-98 water years } \\
\hline & & & $\begin{array}{l}\text { Period of } \\
\text { record } \\
\text { (water } \\
\text { years) }\end{array}$ & Water year & $\begin{array}{l}\text { Stage } \\
(\mathrm{ft})\end{array}$ & $\begin{array}{l}\text { Discharge } \\
\left(\mathrm{ft}^{3} / \mathrm{s}\right)\end{array}$ & $\begin{array}{c}\text { Date } \\
\text { (month/ } \\
\text { day/ } \\
\text { year) }\end{array}$ & $\begin{array}{l}\text { Stage } \\
(\mathrm{ft})\end{array}$ & $\begin{array}{l}\text { Discharge } \\
\left(\mathrm{ft}^{3} / \mathrm{s}\right)\end{array}$ & $\begin{array}{c}\text { Regulated } \\
\text { during } \\
\text { flood }^{1}\end{array}$ & $\begin{array}{c}\text { Recurrence } \\
\text { interval } \\
\text { (years) }\end{array}$ \\
\hline 02165350 & $\begin{array}{l}\text { Dirty Creek tributary near Laurens, } \\
\text { SC }\end{array}$ & 1.21 & $\begin{array}{l}\text { 1978, 1980, } \\
\text { 1984-91, } \\
\text { 1993-98 }\end{array}$ & $\begin{array}{l}1994 \\
1995\end{array}$ & $\begin{array}{l}7.27 \\
8.76\end{array}$ & $\begin{array}{r}252 \\
--\end{array}$ & $8 / 27 / 95$ & 8.76 & -- & $\mathrm{N}$ & -- \\
\hline 02167000 & Saluda River at Chappells, SC & 1,360 & $\begin{array}{l}1888 \\
1906-98\end{array}$ & $\begin{array}{l}1930 \\
1908\end{array}$ & $\begin{array}{l}31.50 \\
34.70\end{array}$ & $\begin{array}{r}63,700 \\
--\end{array}$ & $8 / 28 / 95$ & 27.22 & 38,000 & $\mathrm{Y}$ & -- \\
\hline 02167750 & $\begin{array}{l}\text { Camping Creek tributary near } \\
\text { Prosperity, SC }\end{array}$ & .52 & $\begin{array}{l}\text { 1974-87, } \\
1989-98\end{array}$ & $\begin{array}{l}1995 \\
1991\end{array}$ & $\begin{array}{l}6.64 \\
7.22\end{array}$ & $\begin{array}{r}135 \\
--\end{array}$ & $8 / 27 / 95$ & 6.64 & 135 & $\mathrm{~N}$ & 50 \\
\hline 02168504 & $\begin{array}{l}\text { Saluda River below Lake Murray } \\
\text { Dam near Columbia, SC }\end{array}$ & 2,420 & 1989-98 & 1996 & 15.85 & 22,000 & $3 / 9 / 96$ & 15.85 & 22,000 & $\mathrm{Y}$ & -- \\
\hline 02169568 & Pen Branch at Columbia, SC & 2.26 & 1986-98 & 1997 & 9.10 & 2,350 & $7 / 24 / 97$ & 9.10 & 2,350 & $\mathrm{~N}$ & $>100$ \\
\hline 02169570 & Gills Creek at Columbia, SC & 59.6 & 1967-98 & $\begin{array}{l}1979 \\
1997\end{array}$ & $\begin{array}{l}8.66 \\
9.43\end{array}$ & $\begin{array}{l}2,880 \\
2,480\end{array}$ & $7 / 24 / 97$ & 9.43 & 2,480 & $\mathrm{Y}$ & -- \\
\hline 02173495 & $\begin{array}{l}\text { Sunnyside Canal at Orangeburg, } \\
\text { SC }\end{array}$ & 1.07 & 1986-98 & 1995 & 7.38 & 2,980 & $1 / 7 / 95$ & 7.38 & 2,980 & $\mathrm{~N}$ & $>100$ \\
\hline 02186000 & $\begin{array}{l}\text { Twelvemile Creek near Liberty, } \\
\text { SC }\end{array}$ & 106 & $\begin{array}{l}1955-64 \\
1990-98\end{array}$ & 1998 & 13.46 & 6,730 & $1 / 8 / 98$ & 13.46 & 6,730 & $\mathrm{~N}$ & 25 \\
\hline 02186645 & Coneross Creek near Seneca, SC & 65.4 & 1989-98 & 1994 & 15.26 & 3,590 & $8 / 17 / 94$ & 15.26 & 3,590 & $\mathrm{~N}$ & $5-10$ \\
\hline 02187252 & $\begin{array}{l}\text { Savannah River below Hartwell } \\
\text { Lake near Hartwell, GA }\end{array}$ & 2,090 & 1985-98 & $\begin{array}{l}1997 \\
1994\end{array}$ & $\begin{array}{l}12.80 \\
17.18\end{array}$ & $\begin{array}{r}39,00 \\
--\end{array}$ & $3 / 27 / 97$ & 12.80 & 39,000 & $\mathrm{Y}$ & -- \\
\hline 02187900 & $\begin{array}{l}\text { Broadway Creek near Anderson, } \\
\text { SC }\end{array}$ & 26.4 & $\begin{array}{l}1977-79, \\
1981-82, \\
1984-98\end{array}$ & 1995 & 15.81 & 2,720 & $8 / 27 / 95$ & 15.81 & 2,720 & $\mathrm{~N}$ & $25-50$ \\
\hline 02192500 & $\begin{array}{l}\text { Little River near Mount Carmel, } \\
\text { SC }\end{array}$ & 217 & $\begin{array}{l}1940-79, \\
1981-82, \\
1984-85, \\
1987-98\end{array}$ & 1940 & 29.60 & 20,800 & $8 / 27 / 95$ & 26.46 & 14,800 & $\mathrm{~N}$ & $50-100$ \\
\hline 021973455 & $\begin{array}{l}\text { Indian Grave Branch at Savannah } \\
\text { River site, SC }\end{array}$ & 2.06 & 1987-96 & $\begin{array}{l}1996 \\
1991\end{array}$ & $\begin{array}{l}3.99 \\
5.90\end{array}$ & $\begin{array}{r}52 \\
--\end{array}$ & $6 / 14 / 96$ & 3.99 & 52 & $\mathrm{Y}$ & -- \\
\hline 021973565 & $\begin{array}{l}\text { Steel Creek at Road A at Savannah } \\
\text { River site, SC }\end{array}$ & -- & $\begin{array}{l}\text { 1985-86, } \\
1988-98\end{array}$ & $\begin{array}{l}1998 \\
1991\end{array}$ & $\begin{array}{l}4.32 \\
4.32\end{array}$ & $\begin{array}{r}602 \\
--\end{array}$ & $3 / 9 / 98$ & 4.32 & 602 & $\mathrm{Y}$ & -- \\
\hline 02197380 & $\begin{array}{l}\text { Lower Three Runs below Par Pond } \\
\text { at Savannah River site, SC }\end{array}$ & 36.7 & $\begin{array}{l}1980-82 \\
1987-98\end{array}$ & 1998 & 6.43 & 603 & $3 / 5 / 98$ & 6.43 & 603 & $\mathrm{Y}$ & -- \\
\hline
\end{tabular}




\section{Summary of Significant Floods in the United States and Puerto Rico, 1994 Through 1998 Water Years}

\section{South Dakota}

Flooding occurred along the James River Basin in South Dakota (fig. 54) through the month of April 1995. Abovenormal precipitation in March and April and rapid snowmelt from two significant winter storms in April kept the James River above flood stage throughout the month. Water levels were 3 to 6 feet above flood stage at the beginning of the month and 2 to 9 feet above flood stage at the end of the month. A significant amount of farmland and several roads were flooded throughout the month. Flooding along the James River continued from the end of April through all of May. An all-time record stage was recorded at Huron (streamgage 06476000, table 43) on May 19, and the river farther south reached levels not far below the all-time record. The most excessive and most widespread rain occurred on May 8-9 when 1 to 5 inches fell (National Oceanic and Atmospheric Administration, 1995a). Record streamflow occurred at 20 streamgages throughout South Dakota with many occurring in the Black Hills Region (table 43). More than $\$ 3.5$ million in damage occurred (National Oceanic and Atmospheric Administration, 1995b).

Late March 1997 flooding from snowmelt of near-record to record snowpack occurred across parts of central and northcentral South Dakota and most of northeastern South Dakota through the month of April. Much of the snowpack across northeastern South Dakota melted in the first week of April. The massive amount of water flooded many stretches of county and township roads as well as many State and Federal highways. The inundated sections of roads either were broken up or washed out. Hundreds of culverts were blown out or damaged, and many bridges either were damaged or washed out by ice flows and the high water. Many long-term residents said this was the worst flooding they had seen in their lifetimes. Little precipitation through mid- to late April allowed for a significant reduction in the flooding, although much of the area remained flooded into May. The total damage estimate for the March and April flooding, which included road, home, and sewer- and water-system damage, was \$35 million (National Oceanic and Atmospheric Administration, 1997b). The record discharges on the James River in 1995 were nearly doubled during the flood of 1997. The record flooding on the James River in March and April continued a slow recession through the month of May. Thousands of acres of farmland and pastureland remained flooded by the James River through May. As a result, large economic losses were incurred from the inability to plant the flooded acres.

Excessive rain of 2 to 4 inches, with some amounts nearing 5 inches, fell across a large part northeastern South Dakota mainly on the evening of May 11, 1998 (National Oceanic and Atmospheric Administration, 1998a). This round of excessive rain only exacerbated the already extensive flooding occurring from many years of above-normal precipitation. Some residents of Blue Dog Lake said they had never seen the lake so high in more than 35 years of living there. Damage was almost \$4 million (National Oceanic and Atmospheric Administration, 1998b).

\section{References}

National Oceanic and Atmospheric Administration (NOAA), 1995a-98a, Climatological data (by State): Asheville, North Carolina, National Climatic Data Center, various months.

National Oceanic and Atmospheric Administration (NOAA), 1995b-98b, Storm data (by State): Asheville, North Carolina, National Climatic Data Center, various months. 


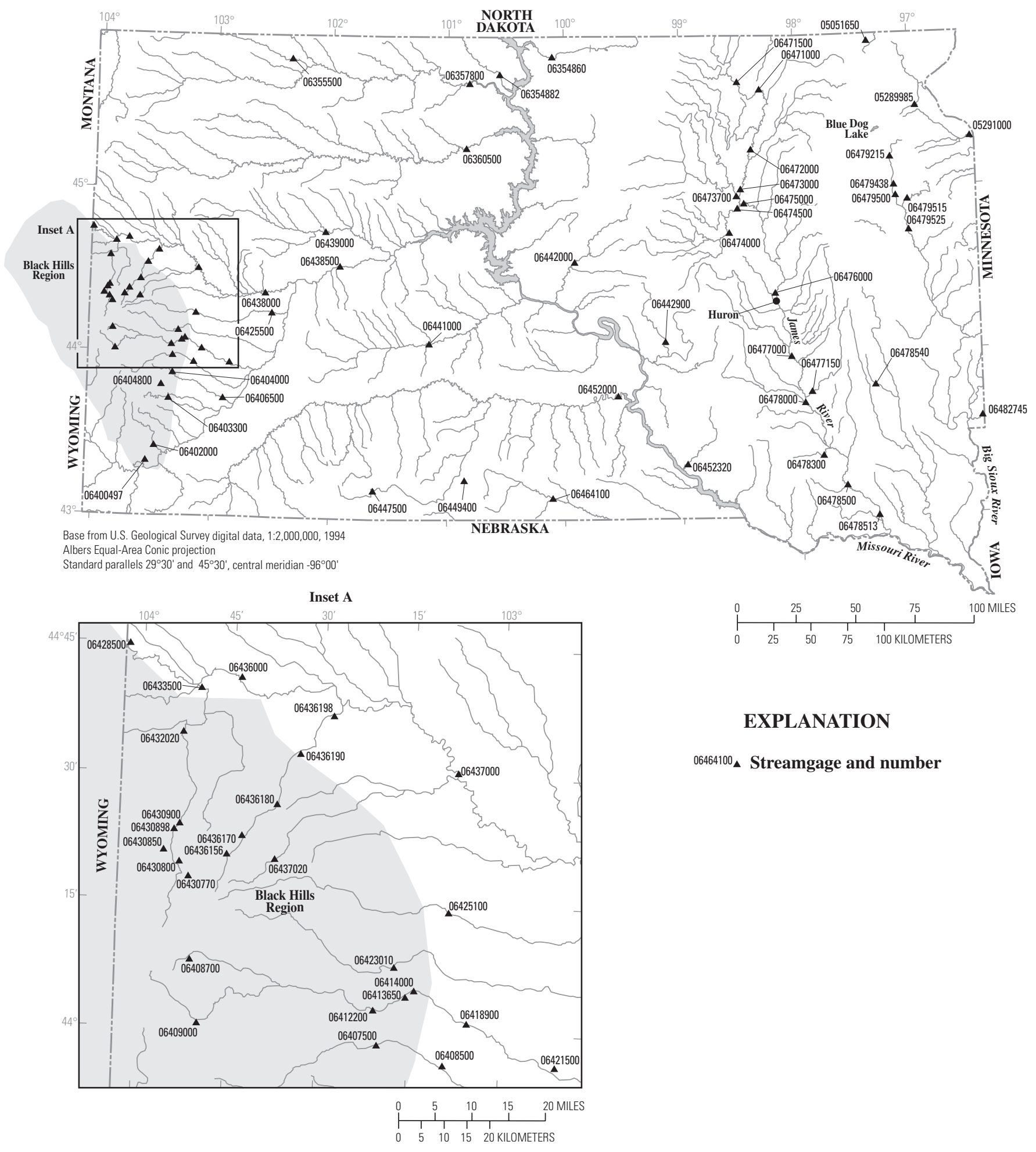

Figure 54. Location of streamgages with significant floods during 1994-98 water years for South Dakota. 
Table 43. Maximum stage and discharge for period of record for streamgages having significant floods during 1994-98 water years in South Dakota.

$\left[\mathrm{mi}^{2}\right.$, square miles; ft, feet above an arbitrary datum; $\mathrm{ft}^{3} / \mathrm{s}$, cubic feet per second; --, not determined or not applicable; >, greater than. Source: Recurrence intervals calculated from U.S. Geological Survey data. Other data from U.S. Geological Survey reports or databases]

\begin{tabular}{|c|c|c|c|c|c|c|c|c|c|c|c|}
\hline \multirow{2}{*}{$\begin{array}{c}\text { Streamgage } \\
\text { number } \\
\text { (fig. 54) }\end{array}$} & \multirow[b]{2}{*}{ Streamgage name } & \multirow{2}{*}{$\begin{array}{c}\text { Total } \\
\text { drainage } \\
\left(\mathrm{mi}^{2}\right)\end{array}$} & \multicolumn{4}{|c|}{$\begin{array}{c}\text { Maximum stage and discharge for period of record } \\
\text { through } 1998 \text { water year }\end{array}$} & \multicolumn{5}{|c|}{ Significant floods $1994-98$ water years } \\
\hline & & & $\begin{array}{c}\text { Period of } \\
\text { record } \\
\text { (water } \\
\text { years) }\end{array}$ & Water year & $\begin{array}{l}\text { Stage } \\
\text { (ft) }\end{array}$ & $\begin{array}{l}\text { Discharge } \\
\left(\mathrm{ft}^{3} / \mathrm{s}\right)\end{array}$ & $\begin{array}{c}\text { Date } \\
\text { (month/ } \\
\text { day/ } \\
\text { year) }\end{array}$ & $\begin{array}{l}\text { Stage } \\
\text { (ft) }\end{array}$ & $\begin{array}{l}\text { Discharge } \\
\left(\mathrm{ft}^{3} / \mathrm{s}\right)\end{array}$ & $\begin{array}{c}\text { Regulated } \\
\text { during } \\
\text { flood }^{1}\end{array}$ & $\begin{array}{c}\text { Recurrence } \\
\text { interval } \\
\text { (years) }\end{array}$ \\
\hline 05051650 & La Belle Creek near Veblen, SD & 8.74 & $1988-98$ & $\begin{array}{l}1996 \\
1997\end{array}$ & $\begin{array}{r}7.34 \\
10.60\end{array}$ & $\begin{array}{l}664 \\
100\end{array}$ & $\begin{array}{l}5 / 18 / 96 \\
4 / 4 / 97\end{array}$ & $\begin{array}{r}7.34 \\
10.60\end{array}$ & $\begin{array}{l}664 \\
100\end{array}$ & $\begin{array}{l}\mathrm{N} \\
\mathrm{N}\end{array}$ & $\begin{array}{r}5-20 \\
--\end{array}$ \\
\hline 05289985 & Big Coulee Creek near Peever, SD & 12.1 & 1988-98 & $\begin{array}{l}1998 \\
1995\end{array}$ & $\begin{array}{l}7.42 \\
9.08\end{array}$ & $\begin{array}{l}614 \\
300\end{array}$ & $\begin{array}{l}5 / 12 / 98 \\
3 / 11 / 95\end{array}$ & $\begin{array}{l}7.42 \\
9.08\end{array}$ & $\begin{array}{l}614 \\
300\end{array}$ & $\begin{array}{l}\mathrm{N} \\
\mathrm{N}\end{array}$ & $\begin{array}{r}5-15 \\
--\end{array}$ \\
\hline 05291000 & $\begin{array}{l}\text { Whetstone River near Big Stone } \\
\text { City, SD }\end{array}$ & 39 & $\begin{array}{l}1910-12, \\
1919, \\
1931-98\end{array}$ & 1919 & 26.00 & 29,000 & $4 / 6 / 97$ & 14.21 & 7,930 & $\mathrm{~N}$ & $15-25$ \\
\hline 06354860 & Spring Creek near Herreid, SD & 440 & $\begin{array}{l}\text { 1963-87, } \\
1989-97\end{array}$ & 1987 & 13.38 & 4,540 & $3 / 31 / 97$ & 12.64 & 2,680 & $\mathrm{~N}$ & $10-20$ \\
\hline 06354882 & Oak Creek near Wakpala, SD & 356 & 1985-98 & 1997 & 19.62 & 7,500 & $3 / 27 / 97$ & 19.62 & 7,500 & $\mathrm{~N}$ & $20-60$ \\
\hline 06355500 & $\begin{array}{l}\text { North Fork Grand River near } \\
\text { White Butte, SD }\end{array}$ & 1,190 & 1946-98 & 1950 & 20.00 & 30,900 & $3 / 21 / 97$ & 11.00 & 4,000 & $\mathrm{Y}$ & $15-20$ \\
\hline 06357800 & Grand River at Little Eagle, SD & 5,370 & 1959-98 & $\begin{array}{l}1987 \\
1972\end{array}$ & $\begin{array}{l}19.16 \\
21.01\end{array}$ & $\begin{array}{l}31,000 \\
15,000\end{array}$ & $3 / 27 / 97$ & 16.96 & 20,900 & $\mathrm{Y}$ & $20-35$ \\
\hline 06360500 & Moreau River near Whitehorse, SD & 4,880 & $\begin{array}{l}1953, \\
1955-98\end{array}$ & 1997 & 26.93 & 29,700 & $3 / 23 / 97$ & 26.93 & 29,700 & $\mathrm{~N}$ & $25-40$ \\
\hline 06400497 & $\begin{array}{l}\text { Cascade Springs near Hot Springs, } \\
\text { SD }\end{array}$ & .47 & 1977-96 & 1996 & 10.82 & 247 & $8 / 9 / 96$ & 10.82 & 247 & $\mathrm{~N}$ & $>100$ \\
\hline 06402000 & Fall River at Hot Springs, SD & 137 & 1938-98 & 1947 & 11.12 & 8,300 & $7 / 19 / 97$ & 4.36 & 1,170 & Y & $20-35$ \\
\hline 06403300 & French Creek above Fairburn, SD & 105 & 1982-98 & 1995 & 4.08 & 1,060 & $5 / 8 / 95$ & 4.08 & 1,060 & $\mathrm{~N}$ & $20-45$ \\
\hline 06404000 & Battle Creek near Keystone, SD & 66.0 & $\begin{array}{l}1946-47, \\
1962-98\end{array}$ & 1972 & 14.50 & 26,200 & $5 / 8 / 95$ & 7.17 & 1,690 & $\mathrm{~N}$ & $5-10$ \\
\hline 06404800 & $\begin{array}{l}\text { Grace Coolidge Creek near } \\
\text { Hayward, SD }\end{array}$ & 7.48 & 1989-98 & 1995 & 7.57 & 337 & $5 / 8 / 95$ & 7.57 & 337 & $\mathrm{~N}$ & $10-20$ \\
\hline 06406500 & Battle Creek below Hermosa, SD & 285 & 1989-98 & 1995 & 9.30 & 1,360 & $5 / 9 / 95$ & 9.30 & 1,360 & $\mathrm{~N}$ & $2-10$ \\
\hline
\end{tabular}


Table 43. Maximum stage and discharge for period of record for streamgages having significant floods during 1994-98 water years in South Dakota.-Continued

$\left[\mathrm{mi}^{2}\right.$, square miles; ft, feet above an arbitrary datum; $\mathrm{ft}^{3} / \mathrm{s}$, cubic feet per second; --, not determined or not applicable; >, greater than. Source: Recurrence intervals calculated from U.S. Geological Survey data. Other data from U.S. Geological Survey reports or databases]

\begin{tabular}{|c|c|c|c|c|c|c|c|c|c|c|c|}
\hline \multirow{2}{*}{$\begin{array}{l}\text { Streamgage } \\
\text { number } \\
\text { (fig. 54) }\end{array}$} & \multirow[b]{2}{*}{ Streamgage name } & \multirow{2}{*}{$\begin{array}{l}\text { Total } \\
\text { drainage } \\
\left(\mathrm{mi}^{2}\right)\end{array}$} & \multicolumn{4}{|c|}{$\begin{array}{c}\text { Maximum stage and discharge for period of record } \\
\text { through } 1998 \text { water year }\end{array}$} & \multicolumn{5}{|c|}{ Significant floods $1994-98$ water years } \\
\hline & & & $\begin{array}{l}\text { Period of } \\
\text { record } \\
\text { (water } \\
\text { years) }\end{array}$ & Water year & $\begin{array}{l}\text { Stage } \\
(\mathrm{ft})\end{array}$ & $\begin{array}{l}\text { Discharge } \\
\left(\mathrm{ft}^{3} / \mathrm{s}\right)\end{array}$ & $\begin{array}{l}\text { Date } \\
\text { (month/ } \\
\text { day/ } \\
\text { year) }\end{array}$ & $\begin{array}{l}\text { Stage } \\
(\mathrm{ft})\end{array}$ & $\begin{array}{l}\text { Discharge } \\
\left(\mathrm{ft}^{3} / \mathrm{s}\right)\end{array}$ & $\begin{array}{l}\text { Regulated } \\
\text { during } \\
\text { flood }^{1}\end{array}$ & $\begin{array}{l}\text { Recurrence } \\
\text { interval } \\
\text { (years) }\end{array}$ \\
\hline 06407500 & Spring Creek near Keystone, SD & 163 & 1987-98 & 1995 & 7.96 & 913 & $5 / 9 / 95$ & 7.96 & 913 & $\mathrm{~N}$ & $10-20$ \\
\hline 06408500 & Spring Creek near Hermosa, SD & 199 & $1950-98$ & $\begin{array}{l}1972 \\
1996\end{array}$ & $\begin{array}{l}-- \\
12.24\end{array}$ & $\begin{array}{r}13,400 \\
6,910\end{array}$ & $5 / 30 / 96$ & 12.24 & 6,910 & $\mathrm{~N}$ & $35-60$ \\
\hline 06408700 & Rhoads Fork near Rochford, SD & 7.95 & 1982-98 & 1997 & 4.00 & 10 & $7 / 24 / 97$ & 4.00 & 10 & $\mathrm{~N}$ & $2-10$ \\
\hline 06409000 & Castle Creek near Hill City, SD & 79.2 & 1949-98 & $\begin{array}{l}1952 \\
1984\end{array}$ & $\begin{array}{l}5.81 \\
6.31\end{array}$ & $\begin{array}{r}1,120 \\
122\end{array}$ & $4 / 9 / 96$ & 3.69 & 271 & $\mathrm{~N}$ & $15-20$ \\
\hline 06412200 & $\begin{array}{l}\text { Rapid Creek above Victoria Creek } \\
\text { near Rapid City, SD }\end{array}$ & 355 & 1989-98 & 1997 & 8.38 & 1,180 & $6 / 2 / 97$ & 8.38 & 1,180 & $\mathrm{Y}$ & $10-30$ \\
\hline 06413650 & $\begin{array}{l}\text { Lime Creek at mouth at Rapid } \\
\text { City, SD }\end{array}$ & 10.0 & $\begin{array}{l}1981-83 \\
1988-98\end{array}$ & 1997 & 4.97 & 365 & $6 / 2 / 97$ & 4.97 & 365 & $\mathrm{~N}$ & $10-20$ \\
\hline 06414000 & Rapid Creek at Rapid City, SD & 410 & $\begin{array}{l}\text { 1905-06, } \\
1943-98\end{array}$ & 1972 & 19.66 & 50,000 & $6 / 2 / 97$ & 11.18 & 3,190 & $\mathrm{Y}$ & $10-20$ \\
\hline 06418900 & $\begin{array}{l}\text { Rapid Creek below sewage } \\
\text { treatment plant near Rapid City, } \\
\text { SD }\end{array}$ & 452 & 1982-98 & 1997 & 10.05 & 2,260 & $6 / 3 / 97$ & 10.05 & 2,260 & $\mathrm{Y}$ & $25-50$ \\
\hline 06421500 & Rapid Creek near Farmingdale, SD & 602 & $\begin{array}{l}1947-58 \\
1960-98\end{array}$ & 1972 & 11.85 & 7,320 & $5 / 27 / 96$ & 10.77 & 3,830 & $\mathrm{Y}$ & $30-45$ \\
\hline 06423010 & $\begin{array}{l}\text { Boxelder Creek near Rapid City, } \\
\text { SD }\end{array}$ & 128 & $1981-98$ & $\begin{array}{l}1995 \\
1996\end{array}$ & $\begin{array}{l}33.09 \\
33.46\end{array}$ & $\begin{array}{l}1,080 \\
1,060\end{array}$ & $\begin{array}{l}5 / 10 / 95 \\
5 / 31 / 96\end{array}$ & $\begin{array}{l}33.09 \\
33.46\end{array}$ & $\begin{array}{l}1,080 \\
1,060\end{array}$ & $\begin{array}{l}\mathrm{N} \\
\mathrm{N}\end{array}$ & $\begin{array}{l}10-20 \\
10-20\end{array}$ \\
\hline 06425100 & Elk Creek near Rapid City, SD & 190 & $\begin{array}{l}\text { 1979-93, } \\
1995-98\end{array}$ & 1996 & 12.77 & 3,120 & $5 / 27 / 96$ & 12.77 & 3,120 & $\mathrm{~N}$ & $5-15$ \\
\hline 06425500 & Elk Creek near Elm Springs, SD & 540 & 1950-98 & $\begin{array}{l}1952 \\
1997\end{array}$ & $\begin{array}{l}-- \\
16.22\end{array}$ & $\begin{array}{l}8,540 \\
3,000\end{array}$ & $5 / 28 / 96$ & 14.85 & 7,660 & $\mathrm{~N}$ & $10-15$ \\
\hline 06428500 & $\begin{array}{l}\text { Belle Fourche River at Wyoming- } \\
\text { South Dakota State line }\end{array}$ & 3,280 & 1947-98 & 1995 & 16.33 & 6,320 & $\begin{array}{l}5 / 10 / 95 \\
3 / 14 / 96\end{array}$ & $\begin{array}{l}16.33 \\
15.58\end{array}$ & $\begin{array}{l}6,320 \\
5,050\end{array}$ & $\begin{array}{l}\mathrm{Y} \\
\mathrm{Y}\end{array}$ & $\begin{array}{l}40-80 \\
20-35\end{array}$ \\
\hline
\end{tabular}


$\left[\mathrm{mi}^{2}\right.$, square miles; ft, feet above an arbitrary datum; $\mathrm{ft}^{3} / \mathrm{s}$, cubic feet per second; --, not determined or not applicable; >, greater than. Source: Recurrence intervals calculated from U.S. Geological Survey data. Other data from U.S. Geological Survey reports or databases]

\begin{tabular}{|c|c|c|c|c|c|c|c|c|c|c|c|}
\hline \multirow[b]{2}{*}{$\begin{array}{l}\text { Streamgage } \\
\text { number } \\
\text { (fig. 54) }\end{array}$} & \multirow[b]{2}{*}{ Streamgage name } & \multirow{2}{*}{$\begin{array}{c}\text { Total } \\
\text { drainage } \\
\left(\mathrm{mi}^{2}\right)\end{array}$} & \multicolumn{4}{|c|}{$\begin{array}{c}\text { Maximum stage and discharge for period of record } \\
\text { through } 1998 \text { water year }\end{array}$} & \multicolumn{5}{|c|}{ Significant floods 1994-98 water years } \\
\hline & & & $\begin{array}{l}\text { Period of } \\
\text { record } \\
\text { (water } \\
\text { years) }\end{array}$ & Water year & $\begin{array}{l}\text { Stage } \\
(\mathrm{ft})\end{array}$ & $\begin{array}{l}\text { Discharge } \\
\left(\mathrm{ft}^{3} / \mathrm{s}\right)\end{array}$ & $\begin{array}{c}\text { Date } \\
\text { (month/ } \\
\text { day/ } \\
\text { year) }\end{array}$ & $\begin{array}{l}\text { Stage } \\
(\mathrm{ft})\end{array}$ & $\begin{array}{l}\text { Discharge } \\
\left(\mathrm{ft}^{3} / \mathrm{s}\right)\end{array}$ & $\begin{array}{l}\text { Regulated } \\
\text { during } \\
\text { flood }^{1}\end{array}$ & $\begin{array}{c}\text { Recurrence } \\
\text { interval } \\
\text { (years) }\end{array}$ \\
\hline 06430770 & Spearfish Creek near Lead, SD & 63.5 & $1989-98$ & 1998 & 8.39 & 181 & $8 / 20 / 98$ & 8.39 & 181 & $\mathrm{Y}$ & $10-20$ \\
\hline 06430800 & Annie Creek near Lead, SD & 3.55 & 1989-98 & 1995 & 6.12 & 270 & $5 / 8 / 95$ & 6.12 & 270 & $\mathrm{~N}$ & $25-55$ \\
\hline 06430850 & $\begin{array}{l}\text { Little Spearfish Creek near Lead, } \\
\text { SD }\end{array}$ & 25.8 & 1989-98 & 1995 & -- & 61 & $5 / 10 / 95$ & -- & 61 & $\mathrm{~N}$ & $10-25$ \\
\hline 06430898 & Squaw Creek near Spearfish, SD & 6.95 & 1989-98 & 1995 & 9.47 & 860 & $5 / 8 / 95$ & 9.47 & 860 & $\mathrm{~N}$ & $25-100$ \\
\hline 06430900 & $\begin{array}{l}\text { Spearfish Creek above Spearfish, } \\
\text { SD }\end{array}$ & 139 & 1989-98 & 1995 & 7.42 & 2,890 & $5 / 8 / 95$ & 7.42 & 2,890 & $\mathrm{Y}$ & $40-120$ \\
\hline 06432020 & $\begin{array}{l}\text { Spearfish Creek below Spearfish, } \\
\text { SD }\end{array}$ & 204 & 1989-98 & 1995 & 7.37 & 1,590 & $5 / 9 / 95$ & 7.37 & 1,590 & $\mathrm{Y}$ & $25-70$ \\
\hline 06433500 & Hay Creek at Belle Fourche, SD & 121 & 1954-96 & 1995 & 10.23 & 1,280 & $5 / 9 / 95$ & 10.23 & 1,280 & $\mathrm{~N}$ & $40-80$ \\
\hline 06436000 & $\begin{array}{l}\text { Belle Fourche River near Fruitdale, } \\
\text { SD }\end{array}$ & 4,540 & $1946-98$ & 1982 & 14.32 & 12,700 & $5 / 10 / 95$ & 14.09 & 12,200 & $\mathrm{Y}$ & $15-35$ \\
\hline 06436156 & Whitetail Creek at Lead, SD & 6.15 & 1989-98 & 1995 & 6.67 & 507 & $5 / 8 / 95$ & 6.67 & 507 & $\mathrm{~N}$ & $20-45$ \\
\hline 06436170 & $\begin{array}{l}\text { Whitewood Creek at Deadwood, } \\
\text { SD }\end{array}$ & 40.6 & $1982-95$ & 1995 & 10.63 & 3,540 & $5 / 8 / 95$ & 10.63 & 3,540 & $\mathrm{~N}$ & $10-20$ \\
\hline 06436180 & $\begin{array}{l}\text { Whitewood Creek above } \\
\text { Whitewood, SD }\end{array}$ & 56.3 & $1983-98$ & 1995 & 9.06 & 3,800 & $5 / 8 / 95$ & 9.06 & 3,800 & $\mathrm{~N}$ & $30-60$ \\
\hline 06436190 & $\begin{array}{l}\text { Whitewood Creek near } \\
\text { Whitewood, SD }\end{array}$ & 77.4 & 1982-98 & 1995 & 6.01 & 3,930 & $5 / 8 / 95$ & 6.01 & 3,930 & $\mathrm{~N}$ & $15-25$ \\
\hline 06436198 & Whitewood Creek above Vale, SD & 102 & 1983-98 & 1995 & 5.72 & 4,250 & $5 / 8 / 95$ & 5.72 & 4,250 & $\mathrm{~N}$ & $10-20$ \\
\hline 06437000 & $\begin{array}{l}\text { Belle Fourche River near Sturgis, } \\
\text { SD }\end{array}$ & 5,870 & 1946-98 & 1982 & 19.10 & 36,400 & $5 / 10 / 95$ & 17.10 & 20,000 & $\mathrm{Y}$ & $15-25$ \\
\hline 06437020 & $\begin{array}{l}\text { Bear Butte Creek near Deadwood, } \\
\text { SD }\end{array}$ & 16.6 & 1989-98 & 1995 & 8.34 & 1,590 & $5 / 8 / 95$ & 8.34 & 1,590 & $\mathrm{~N}$ & $15-30$ \\
\hline
\end{tabular}


Table 43. Maximum stage and discharge for period of record for streamgages having significant floods during 1994-98 water years in South Dakota.-Continued

$\left[\mathrm{mi}^{2}\right.$, square miles; $\mathrm{ft}$, feet above an arbitrary datum; $\mathrm{ft}^{3} / \mathrm{s}$, cubic feet per second; --, not determined or not applicable; >, greater than. Source: Recurrence intervals calculated from U.S. Geological Survey data. Other data from U.S. Geological Survey reports or databases]

\begin{tabular}{|c|c|c|c|c|c|c|c|c|c|c|c|}
\hline \multirow{2}{*}{$\begin{array}{l}\text { Streamgage } \\
\text { number } \\
\text { (fig. 54) }\end{array}$} & \multirow[b]{2}{*}{ Streamgage name } & \multirow{2}{*}{$\begin{array}{c}\text { Total } \\
\text { drainage } \\
\left(\mathrm{mi}^{2}\right)\end{array}$} & \multicolumn{4}{|c|}{$\begin{array}{l}\text { Maximum stage and discharge for period of record } \\
\text { through } 1998 \text { water year }\end{array}$} & \multicolumn{5}{|c|}{ Significant floods 1994-98 water years } \\
\hline & & & $\begin{array}{l}\text { Period of } \\
\text { record } \\
\text { (water } \\
\text { years) }\end{array}$ & Water year & $\begin{array}{l}\text { Stage } \\
(\mathrm{ft})\end{array}$ & $\begin{array}{l}\text { Discharge } \\
\left(\mathrm{ft}^{3} / \mathrm{s}\right)\end{array}$ & $\begin{array}{c}\text { Date } \\
\text { (month/ } \\
\text { day/ } \\
\text { year) }\end{array}$ & $\begin{array}{l}\text { Stage } \\
(\mathrm{ft})\end{array}$ & $\begin{array}{l}\text { Discharge } \\
\left(\mathrm{ft}^{3} / \mathrm{s}\right)\end{array}$ & $\begin{array}{l}\text { Regulated } \\
\text { during } \\
\text { flood }^{1}\end{array}$ & $\begin{array}{l}\text { Recurrence } \\
\text { interval } \\
\text { (years) }\end{array}$ \\
\hline 06438000 & $\begin{array}{l}\text { Belle Fourche River near Elm } \\
\text { Springs, SD }\end{array}$ & 7,210 & $\begin{array}{l}1927, \\
1929-98\end{array}$ & $\begin{array}{l}1964 \\
1927\end{array}$ & $\begin{array}{l}15.90 \\
21.80\end{array}$ & $\begin{array}{r}45,100 \\
--\end{array}$ & $5 / 27 / 96$ & 17.28 & 39,900 & $\mathrm{Y}$ & $15-20$ \\
\hline 06438500 & $\begin{array}{l}\text { Cheyenne River near Plainview, } \\
\text { SD }\end{array}$ & 21,600 & $\begin{array}{l}\text { 1920, 1927, } \\
\text { 1951-81, } \\
1995-98\end{array}$ & 1996 & 22.10 & 69,700 & $5 / 28 / 96$ & 22.10 & 69,700 & $\mathrm{Y}$ & $30-60$ \\
\hline 06439000 & Cherry Creek near Plainview, SD & 1,190 & $1946-98$ & 1952 & 22.63 & 17,500 & $5 / 29 / 96$ & 19.43 & 9,430 & $\mathrm{~N}$ & $15-25$ \\
\hline 06441000 & Bad River near Midland, SD & 1,460 & $1946-98$ & 1967 & 24.44 & 29,400 & $5 / 29 / 96$ & 22.99 & 16,300 & $\mathrm{~N}$ & $25-30$ \\
\hline 06442000 & $\begin{array}{l}\text { Medicine Knoll Creek near Blunt, } \\
\text { SD }\end{array}$ & 317 & $\begin{array}{l}1917, \\
1950-97\end{array}$ & $\begin{array}{l}1991 \\
1917\end{array}$ & $\begin{array}{l}12.98 \\
15.00\end{array}$ & $\begin{array}{r}5,000 \\
--\end{array}$ & $3 / 28 / 97$ & 13.15 & 4,000 & $\mathrm{~N}$ & $20-30$ \\
\hline 06442900 & Elm Creek near Gann Valley, SD & 381 & $\begin{array}{l}1988-92 \\
1994-98\end{array}$ & 1997 & 15.58 & 3,440 & $3 / 28 / 97$ & 15.58 & 3,440 & $\mathrm{~N}$ & $15-40$ \\
\hline 06447500 & Little White River near Martin, SD & 310 & $\begin{array}{l}1932, \\
1938-40, \\
1962-98\end{array}$ & 1997 & 13.48 & 1,300 & $6 / 4 / 97$ & 13.48 & 1,300 & $\mathrm{~N}$ & $25-40$ \\
\hline 06449400 & Rosebud Creek at Rosebud, SD & 50.8 & $1975-97$ & 1995 & 10.53 & 670 & $6 / 23 / 95$ & 10.53 & 670 & $\mathrm{~N}$ & $15-20$ \\
\hline 06452000 & White River near Oacoma, SD & 10,200 & 1929-98 & $\begin{array}{l}1952 \\
1994\end{array}$ & 24.70 & $\begin{array}{l}51,900 \\
25,000\end{array}$ & $6 / 5 / 97$ & 18.58 & 33,700 & $\mathrm{~N}$ & $15-20$ \\
\hline 06452320 & Platte Creek near Platte, SD & 741 & 1989-98 & 1995 & 11.29 & 2,600 & $5 / 11 / 95$ & 11.29 & 2,600 & $\mathrm{~N}$ & $2-10$ \\
\hline 06464100 & $\begin{array}{l}\text { Keya Paha River near Keyapaha, } \\
\text { SD }\end{array}$ & 466 & 1982-98 & 1997 & 9.48 & 1,020 & $2 / 20 / 97$ & 9.48 & 1,020 & $\mathrm{~N}$ & $5-15$ \\
\hline 06471000 & James River at Columbia, SD & 5,857 & 1946-98 & $\begin{array}{l}1950 \\
1997\end{array}$ & $\begin{array}{l}16.89 \\
18.63\end{array}$ & $\begin{array}{l}5,420 \\
4,130\end{array}$ & $4 / 30 / 97$ & 18.63 & 4,130 & $\mathrm{Y}$ & $25-50$ \\
\hline 06471500 & Elm River at Westport, SD & 1,493 & $1947-98$ & 1969 & 22.11 & 12,600 & $3 / 30 / 97$ & 21.56 & 9,380 & $\mathrm{~N}$ & $15-25$ \\
\hline
\end{tabular}


$\left[\mathrm{mi}^{2}\right.$, square miles; ft, feet above an arbitrary datum; $\mathrm{ft}^{3} / \mathrm{s}$, cubic feet per second; --, not determined or not applicable; >, greater than. Source: Recurrence intervals calculated from U.S. Geological Survey data. Other data from U.S. Geological Survey reports or databases]

\begin{tabular}{|c|c|c|c|c|c|c|c|c|c|c|c|}
\hline \multirow[b]{2}{*}{$\begin{array}{l}\text { Streamgage } \\
\text { number } \\
\text { (fig. 54) }\end{array}$} & \multirow[b]{2}{*}{ Streamgage name } & \multirow{2}{*}{$\begin{array}{c}\text { Total } \\
\text { drainage } \\
\left(\mathrm{mi}^{2}\right)\end{array}$} & \multicolumn{4}{|c|}{$\begin{array}{l}\text { Maximum stage and discharge for period of record } \\
\text { through } 1998 \text { water year }\end{array}$} & \multicolumn{5}{|c|}{ Significant floods $1994-98$ water years } \\
\hline & & & $\begin{array}{l}\text { Period of } \\
\text { record } \\
\text { (water } \\
\text { years) }\end{array}$ & Water year & $\begin{array}{l}\text { Stage } \\
(\mathrm{ft})\end{array}$ & $\begin{array}{l}\text { Discharge } \\
\left(\mathrm{ft}^{3} / \mathrm{s}\right)\end{array}$ & $\begin{array}{c}\text { Date } \\
\text { (month/ } \\
\text { day/ } \\
\text { year) }\end{array}$ & $\begin{array}{l}\text { Stage } \\
(\mathrm{ft})\end{array}$ & $\begin{array}{l}\text { Discharge } \\
\left(\mathrm{ft}^{3} / \mathrm{s}\right)\end{array}$ & $\begin{array}{l}\text { Regulated } \\
\text { during } \\
\text { flood }^{1}\end{array}$ & $\begin{array}{c}\text { Recurrence } \\
\text { interval } \\
\text { (years) }\end{array}$ \\
\hline 06472000 & James River near Stratford, SD & 8,865 & $\begin{array}{l}\text { 1950-72, } \\
1977,1995, \\
1997\end{array}$ & $\begin{array}{l}1997 \\
1995\end{array}$ & $\begin{array}{l}19.48 \\
19.86\end{array}$ & $\begin{array}{r}8,400 \\
--\end{array}$ & $4 / 6 / 97$ & 19.48 & 8,400 & $\mathrm{~N}$ & $>100$ \\
\hline 06473000 & James River at Ashton, SD & 9,742 & 1946-98 & 1997 & 25.03 & 9,150 & $4 / 23 / 97$ & 25.03 & 9,150 & $\mathrm{~N}$ & $70-125$ \\
\hline 06473700 & Snake Creek near Ashton, SD & 2,657 & $\begin{array}{l}1956-72, \\
1977-79, \\
1985-89, \\
1997\end{array}$ & 1997 & 20.74 & 15,000 & $4 / 1 / 97$ & 20.74 & 15,000 & $\mathrm{~N}$ & $60-150$ \\
\hline 06474000 & Turtle Creek near Tulare, SD & 1,124 & $\begin{array}{l}1954-56, \\
1966-81, \\
1985-98\end{array}$ & 1997 & 18.80 & 13,500 & $3 / 28 / 97$ & 18.80 & 13,500 & $\mathrm{~N}$ & $30-60$ \\
\hline 06474500 & Turtle Creek at Redfield, SD & 1,481 & $\begin{array}{l}1946-72, \\
1997\end{array}$ & 1997 & 18.32 & 13,500 & $3 / 29 / 97$ & 18.32 & 13,500 & $\mathrm{~N}$ & $35-70$ \\
\hline 06475000 & James River near Redfield, SD & 13,911 & $1950-98$ & 1997 & 29.92 & 17,000 & $\begin{array}{l}5 / 15 / 95 \\
4 / 3 / 97\end{array}$ & $\begin{array}{l}26.26 \\
29.92\end{array}$ & $\begin{array}{r}9,800 \\
17,000\end{array}$ & $\begin{array}{l}\mathrm{N} \\
\mathrm{N}\end{array}$ & $\begin{array}{r}45-70 \\
>150\end{array}$ \\
\hline 06476000 & James River at Huron, SD & 15,869 & $\begin{array}{l}\text { 1881, 1922, } \\
\text { 1929-32, } \\
\text { 1944-98 }\end{array}$ & 1997 & 21.28 & 23,400 & $\begin{array}{l}5 / 19 / 95 \\
4 / 6 / 97\end{array}$ & $\begin{array}{l}16.86 \\
21.28\end{array}$ & $\begin{array}{l}10,000 \\
23,400\end{array}$ & $\begin{array}{l}\mathrm{N} \\
\mathrm{N}\end{array}$ & $\begin{array}{r}25-40 \\
>250\end{array}$ \\
\hline 06477000 & James River near Forestburg, SD & 17,590 & $\begin{array}{l}\text { 1920, 1922, } \\
1950-98\end{array}$ & 1997 & 20.61 & 25,600 & $\begin{array}{l}5 / 18 / 95 \\
4 / 6 / 97\end{array}$ & $\begin{array}{l}17.08 \\
20.61\end{array}$ & $\begin{array}{l}13,000 \\
25,600\end{array}$ & $\begin{array}{l}\mathrm{N} \\
\mathrm{N}\end{array}$ & $\begin{array}{r}25-40 \\
>125\end{array}$ \\
\hline 06477150 & Rock Creek near Fulton, SD & 240 & $\begin{array}{l}\text { 1967-79, } \\
\text { 1989-98 }\end{array}$ & $\begin{array}{l}1997 \\
1993\end{array}$ & $\begin{array}{l}13.74 \\
14.34\end{array}$ & $\begin{array}{l}3,120 \\
1,880\end{array}$ & $3 / 29 / 97$ & 13.74 & 3,120 & $\mathrm{~N}$ & $10-20$ \\
\hline 06478000 & James River near Mitchell, SD & 19,064 & $\begin{array}{l}\text { 1954-58, } \\
1966-72, \\
1995,1997\end{array}$ & 1997 & 23.14 & 28,000 & 4/7/97 & 23.14 & 28,000 & $\mathrm{~N}$ & $>100$ \\
\hline
\end{tabular}


Table 43. Maximum stage and discharge for period of record for streamgages having significant floods during 1994-98 water years in South Dakota.-Continued

$\left[\mathrm{mi}^{2}\right.$, square miles; $\mathrm{ft}$, feet above an arbitrary datum; $\mathrm{ft}^{3} / \mathrm{s}$, cubic feet per second; --, not determined or not applicable; >, greater than. Source: Recurrence intervals calculated from U.S. Geological Survey data. Other data from U.S. Geological Survey reports or databases]

\begin{tabular}{|c|c|c|c|c|c|c|c|c|c|c|c|}
\hline \multirow[b]{2}{*}{$\begin{array}{l}\text { Streamgage } \\
\text { number } \\
\text { (fig. 54) }\end{array}$} & \multirow[b]{2}{*}{ Streamgage name } & \multirow[b]{2}{*}{$\begin{array}{c}\text { Total } \\
\text { drainage } \\
\left(\mathrm{mi}^{2}\right)\end{array}$} & \multicolumn{4}{|c|}{$\begin{array}{c}\text { Maximum stage and discharge for period of record } \\
\text { through } 1998 \text { water year }\end{array}$} & \multicolumn{5}{|c|}{ Significant floods $1994-98$ water years } \\
\hline & & & $\begin{array}{l}\text { Period of } \\
\text { record } \\
\text { (water } \\
\text { years) }\end{array}$ & Water year & $\begin{array}{l}\text { Stage } \\
\text { (ft) }\end{array}$ & $\begin{array}{c}\text { Discharge } \\
\left(\mathrm{ft}^{3} / \mathrm{s}\right)\end{array}$ & $\begin{array}{c}\text { Date } \\
\text { (month/ } \\
\text { day/ } \\
\text { year) }\end{array}$ & $\begin{array}{l}\text { Stage } \\
(\mathrm{ft})\end{array}$ & $\begin{array}{c}\text { Discharge } \\
\left(\mathrm{ft}^{3} / \mathrm{s}\right)\end{array}$ & $\begin{array}{l}\text { Regulated } \\
\text { during } \\
\text { flood }^{1}\end{array}$ & $\begin{array}{c}\text { Recurrence } \\
\text { interval } \\
\text { (years) }\end{array}$ \\
\hline 06478300 & Dry Creek near Parkston, SD & 97.2 & $\begin{array}{l}\text { 1956-80, } \\
1989-97\end{array}$ & 1960 & 12.70 & 4,210 & $5 / 27 / 95$ & 9.89 & 2,900 & $\mathrm{~N}$ & $15-25$ \\
\hline 06478500 & James River near Scotland, SD & 20,653 & 1929-98 & 1984 & 20.45 & 29,400 & $\begin{array}{l}5 / 29 / 95 \\
4 / 9 / 97\end{array}$ & $\begin{array}{l}19.39 \\
19.87\end{array}$ & $\begin{array}{l}18,100 \\
28,000\end{array}$ & $\begin{array}{l}\mathrm{N} \\
\mathrm{N}\end{array}$ & $\begin{array}{r}25-35 \\
60-100\end{array}$ \\
\hline 06478513 & James River near Yankton, SD & 20,942 & 1982-98 & $\begin{array}{l}1997 \\
1984\end{array}$ & $\begin{array}{l}22.94 \\
24.34\end{array}$ & $\begin{array}{l}28,800 \\
26,400\end{array}$ & 4/9/97 & 22.94 & 28,800 & $\mathrm{~N}$ & $60-100$ \\
\hline 06478540 & $\begin{array}{l}\text { Little Vermillion River near Salem, } \\
\text { SD }\end{array}$ & 78.6 & 1967-98 & 1993 & 11.95 & 3,300 & $3 / 28 / 97$ & 10.01 & 1,560 & $\mathrm{~N}$ & $20-40$ \\
\hline 06479215 & Big Sioux River near Florence, SD & 638 & 1984-98 & 1997 & 9.32 & 2,000 & $4 / 4 / 97$ & 9.32 & 2,000 & $\mathrm{~N}$ & $15-30$ \\
\hline 06479438 & $\begin{array}{l}\text { Big Sioux River near Watertown, } \\
\text { SD }\end{array}$ & 1,007 & 1973-98 & 1997 & 12.09 & 7,820 & $4 / 5 / 97$ & 12.09 & 7,820 & $\mathrm{~N}$ & $45-100$ \\
\hline 06479500 & Big Sioux River at Watertown, SD & 1,129 & $\begin{array}{l}1946-72, \\
1997\end{array}$ & 1997 & 12.49 & 5,800 & 4/6/97 & 12.49 & 5,800 & $\mathrm{~N}$ & $>250$ \\
\hline 06479515 & Willow Creek near Watertown, SD & 110 & $\begin{array}{l}1972-86, \\
1997\end{array}$ & 1997 & 10.93 & 3,650 & $4 / 5 / 97$ & 10.93 & 3,650 & $\mathrm{~N}$ & $20-60$ \\
\hline 06479525 & $\begin{array}{l}\text { Big Sioux River near Castlewood, } \\
\text { SD }\end{array}$ & 1,997 & 1977-98 & 1997 & 12.87 & 4,300 & $4 / 11 / 97$ & 12.87 & 4,300 & $\mathrm{~N}$ & $>150$ \\
\hline 06482745 & $\begin{array}{l}\text { Beaver Creek at Valley Springs, } \\
\text { SD }\end{array}$ & 104 & 1986-96 & 1994 & 24.89 & 2,280 & $6 / 13 / 94$ & 24.89 & 2,280 & $\mathrm{~N}$ & $5-15$ \\
\hline
\end{tabular}

${ }^{1}$ Regulated during flood: N, no; Y, yes. 


\section{Tennessee}

Torrential rainfall of more than 7 inches in some areas occurred across the eastern one-third of Tennessee on March 27, 1994 (National Oceanic and Atmospheric Administration, 1994a) resulting in widespread flash flooding across that part of the State. Record discharges were measured at five streamgages (table 44). Three people were killed due to flash flooding. A 19-year-old man was killed near Sevierville (fig. 55) when he tried to cross a swollen creek. A woman was killed in Seymour when the car she was in washed off the road. In all, nearly 200 people were evacuated, more than 1,000 homes were affected by the floods, numerous roads and bridges were damaged or destroyed, and several mudslides and rockslides occurred. Damage totaled \$155 million (National Oceanic and Atmospheric Administration, 1994b).

The Mississippi River went above flood stage on April 12, 1994, at Caruthersville, Missouri, producing flooding in northwest Tennessee. Several roads were closed for a few days due to the flooding. Overnight thunderstorms produced localized flash flooding in western Tennessee on April 28, 1994 (National Oceanic and Atmospheric Administration, 1994a).

Excessive rains across the Midwest forced the Mississippi River and some of its tributaries above flood stage in western Tennessee during the end of May 1995. About 35 miles of roads were underwater, and more than 145,000 acres of crops were flooded (National Oceanic and Atmospheric Administration, 1995b).

Excessive rain fell on ground already saturated from previous rains on August 11, 1996. Seventy-seven homes, 20 businesses, 4 public buildings, and 3 churches were extensively damaged in Chattanooga. Approximately \$2 million in damage resulted (National Oceanic and Atmospheric Administration, 1996b).

Excessive rainfall during the first few days of March 1997 along with rivers that were already high caused prolonged flooding along the Mississippi and Tennessee Rivers and their tributaries. The Mississippi River reached levels that had not been seen since 1937. Numerous roads were closed for days. Near Jackson, two persons were killed when their car was swept off a flooded road. One person was killed near Memphis while attempting to cross a flooded bridge. One man also was killed near Union City when he fell out of a boat helping people out of their homes. Total damage was more than \$22 million (National Oceanic and Atmospheric Administration, 1997b).

An estimated 8 inches of rain fell at Lawrenceburg on July 13-14, 1998, with 4 inches of rain falling in about 1 hour on July 13 (National Oceanic and Atmospheric Administration, 1998a). Two people were killed, and 20 were injured from the resulting flood. Damage was more than \$4 million with 122 homes damaged or destroyed; 13 mobile homes and several small bridges were swept away. The water and sewage plant for Lawrenceburg was left inoperable (National Oceanic and Atmospheric Administration, 1998b).

\section{References}

National Oceanic and Atmospheric Administration (NOAA), 1994a-98a, Climatological data (by State): Asheville, North Carolina, National Climatic Data Center, various months.

National Oceanic and Atmospheric Administration (NOAA), 1994b-98b, Storm data (by State): Asheville, North Carolina, National Climatic Data Center, various months.

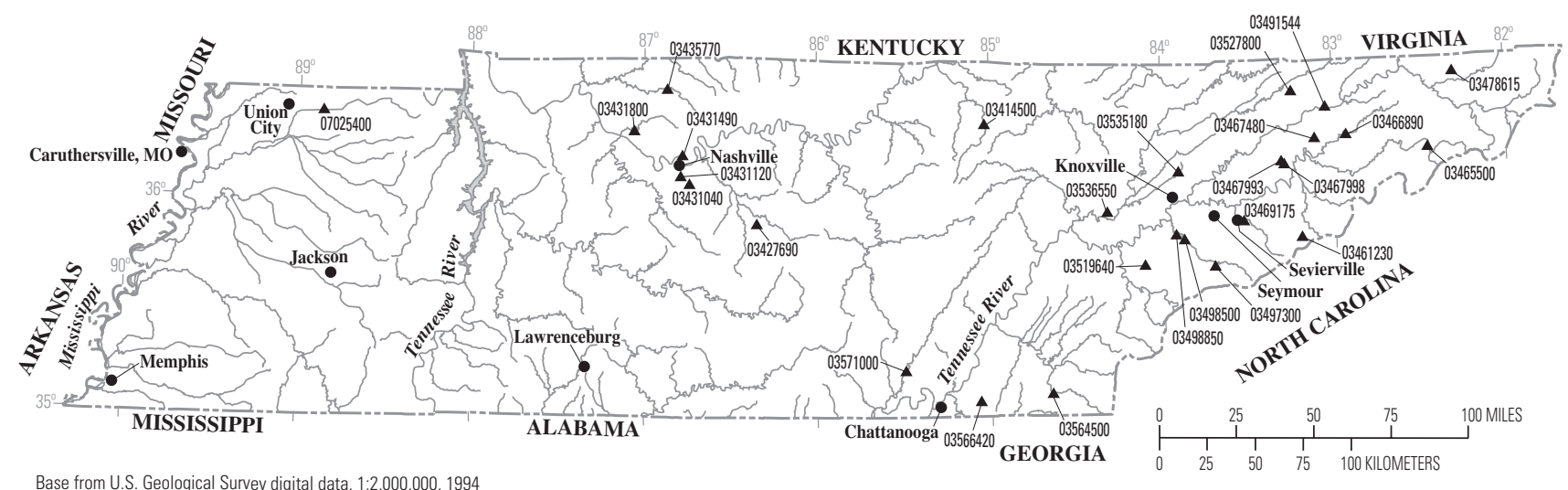

digital data, $1: 2,000,000,1994$ Albers Equal-Area Conic projection

Standard parallels $29^{\circ} 30^{\prime}$ and $45^{\circ} 30^{\prime}$, central meridian $-96^{\circ} 00^{\prime}$

\section{EXPLANATION}

$0^{03571000} \triangle$ Streamgage and number

Figure 55. Location of streamgages with significant floods during 1994-98 water years for Tennessee. 
Table 44. Maximum stage and discharge for period of record for streamgages having significant floods during 1994-98 water years in Tennessee.

$\left[\mathrm{mi}^{2}\right.$, square miles; $\mathrm{ft}$, feet above an arbitrary datum; $\mathrm{ft}^{3} / \mathrm{s}$, cubic feet per second; --, not determined or not applicable; >, greater than. Source: Recurrence intervals calculated from U.S. Geological Survey data. Other data from U.S. Geological Survey reports or databases]

\begin{tabular}{|c|c|c|c|c|c|c|c|c|c|c|c|}
\hline \multirow{2}{*}{$\begin{array}{l}\text { Streamgage } \\
\text { number } \\
\text { (fig. 55) }\end{array}$} & \multirow[b]{2}{*}{ Streamgage name } & \multirow{2}{*}{$\begin{array}{c}\text { Total } \\
\text { drainage } \\
\left(\mathrm{mi}^{2}\right)\end{array}$} & \multicolumn{4}{|c|}{$\begin{array}{c}\text { Maximum stage and discharge for period of record } \\
\text { through } 1998 \text { water year }\end{array}$} & \multicolumn{5}{|c|}{ Significant floods 1994-98 water years } \\
\hline & & & $\begin{array}{l}\text { Period of } \\
\text { record } \\
\text { (water } \\
\text { years) }\end{array}$ & Water year & $\begin{array}{l}\text { Stage } \\
(\mathrm{ft})\end{array}$ & $\begin{array}{l}\text { Discharge } \\
\left(\mathrm{ft}^{3} / \mathrm{s}\right)\end{array}$ & $\begin{array}{c}\text { Date } \\
\text { (month/ } \\
\text { day/ } \\
\text { year) }\end{array}$ & $\begin{array}{l}\text { Stage } \\
(\mathrm{ft})\end{array}$ & $\begin{array}{l}\text { Discharge } \\
\left(\mathrm{ft}^{3} / \mathrm{s}\right)\end{array}$ & $\begin{array}{l}\text { Regulated } \\
\text { during } \\
\text { flood }^{1}\end{array}$ & $\begin{array}{l}\text { Recurrence } \\
\text { interval } \\
\text { (years) }\end{array}$ \\
\hline 03414500 & $\begin{array}{l}\text { East Fork Obey River near } \\
\text { Jamestown, TN }\end{array}$ & 202 & $\begin{array}{l}1929 \\
1943-98\end{array}$ & $\begin{array}{l}1973 \\
1929\end{array}$ & $\begin{array}{l}30.46 \\
30.70\end{array}$ & $\begin{array}{r}44,800 \\
--\end{array}$ & $11 / 30 / 96$ & 29.11 & 39,800 & $\mathrm{~N}$ & 25 \\
\hline 03427690 & $\begin{array}{l}\text { Bushman Creek at Pitts Lane Ford } \\
\text { near Compton, TN }\end{array}$ & 9.67 & 1989-98 & 1996 & 7.24 & 2,020 & $7 / 21 / 96$ & 7.24 & 2,020 & $\mathrm{~N}$ & 25 \\
\hline 03431040 & $\begin{array}{l}\text { Sevenmile Creek at Blackman } \\
\text { Road near Nashville, TN }\end{array}$ & 12.2 & $1965-98$ & 1998 & 10.57 & 10,500 & $6 / 4 / 98$ & 10.57 & 10,500 & $\mathrm{~N}$ & $>100$ \\
\hline 03431120 & $\begin{array}{l}\text { West Fork Browns Creek at Gen } \\
\text { Bates Drive at Nashville, TN }\end{array}$ & 3.30 & $1965-98$ & 1975 & 7.00 & 2,110 & $11 / 27 / 94$ & 6.67 & 1,840 & $\mathrm{~N}$ & 10 \\
\hline 03431490 & Pages Branch at Avondale, TN & 2.01 & 1977-98 & $\begin{array}{l}1995 \\
1998\end{array}$ & $\begin{array}{l}6.03 \\
6.32\end{array}$ & $\begin{array}{l}2,800 \\
1,430\end{array}$ & $11 / 27 / 94$ & 6.03 & 2,800 & $\mathrm{~N}$ & 25 \\
\hline 03431800 & $\begin{array}{l}\text { Sycamore Creek near Ashland } \\
\text { City, TN }\end{array}$ & 97.2 & $\begin{array}{l}\text { 1962-87, } \\
1989-98\end{array}$ & 1989 & 13.50 & 18,500 & $3 / 3 / 97$ & 13.45 & 18,200 & $\mathrm{~N}$ & $15-20$ \\
\hline 03435770 & $\begin{array}{l}\text { Sulphur Fork Red River above } \\
\text { Springfield, TN }\end{array}$ & 65.6 & 1976-97 & 1997 & 14.52 & 12,100 & $3 / 3 / 97$ & 14.52 & 12,100 & $\mathrm{~N}$ & $10-15$ \\
\hline 03461230 & Caney Creek near Cosby, TN & 1.62 & 1967-98 & 1996 & 6.45 & 275 & $1 / 26 / 96$ & 6.45 & 275 & $\mathrm{~N}$ & $30-35$ \\
\hline 03465500 & $\begin{array}{l}\text { Nolichucky River at Embreeville, } \\
\text { TN }\end{array}$ & 805 & $\begin{array}{l}\text { 1901, } \\
1921-98\end{array}$ & 1901 & 24.00 & 120,000 & $1 / 15 / 95$ & 15.53 & 64,000 & $\mathrm{~N}$ & 25 \\
\hline 03466890 & Lick Creek near Albany, TN & 172 & $1985-98$ & 1994 & 17.41 & 10,800 & $3 / 27 / 94$ & 17.41 & 10,800 & $\mathrm{~N}$ & 100 \\
\hline 03467480 & Bent Creek at Taylor Gap, TN & 2.18 & 1986-98 & 1994 & 15.56 & 2,550 & $3 / 27 / 94$ & 15.56 & 2,550 & $\mathrm{~N}$ & 10 \\
\hline 03467993 & $\begin{array}{l}\text { Cedar Creek near Valley Home, } \\
\text { TN }\end{array}$ & 2.01 & $1986-98$ & 1997 & 13.38 & 210 & $4 / 29 / 97$ & 13.38 & 210 & $\mathrm{~N}$ & $20-25$ \\
\hline 03467998 & Sinking Fork at White Pine, TN & 6.38 & 1986-98 & 1998 & 7.25 & 1,600 & $4 / 17 / 98$ & 7.25 & 1,600 & $\mathrm{~N}$ & $15-20$ \\
\hline 03469175 & $\begin{array}{l}\text { Little Pigeon River above } \\
\text { Sevierville, TN }\end{array}$ & 184 & 1989-98 & 1994 & 17.50 & 19,700 & $3 / 28 / 94$ & 17.50 & 19,700 & $\mathrm{~N}$ & $25-50$ \\
\hline 03478615 & Evans Creek near Blountville, TN & 2.50 & 1984-98 & 1996 & 12.68 & 98 & $5 / 25 / 96$ & 12.68 & 98 & $\mathrm{~N}$ & $5-10$ \\
\hline
\end{tabular}


Table 44. Maximum stage and discharge for period of record for streamgages having significant floods during 1994-98 water years in Tennessee.-Continued

$\left[\mathrm{mi}^{2}\right.$, square miles; ft, feet above an arbitrary datum; $\mathrm{ft}^{3} / \mathrm{s}$, cubic feet per second; --, not determined or not applicable; >, greater than. Source: Recurrence intervals calculated from U.S. Geological Survey data. Other data from U.S. Geological Survey reports or databases]

\begin{tabular}{|c|c|c|c|c|c|c|c|c|c|c|c|}
\hline \multirow[b]{2}{*}{$\begin{array}{c}\text { Streamgage } \\
\text { number } \\
\text { (fig. 55) }\end{array}$} & \multirow[b]{2}{*}{ Streamgage name } & \multirow[b]{2}{*}{$\begin{array}{c}\text { Total } \\
\text { drainage } \\
\left(\mathrm{mi}^{2}\right)\end{array}$} & \multicolumn{4}{|c|}{$\begin{array}{c}\text { Maximum stage and discharge for period of record } \\
\text { through } 1998 \text { water year }\end{array}$} & \multicolumn{5}{|c|}{ Significant floods $1994-98$ water years } \\
\hline & & & $\begin{array}{l}\text { Period of } \\
\text { record } \\
\text { (water } \\
\text { years) }\end{array}$ & Water year & $\begin{array}{l}\text { Stage } \\
(\mathrm{ft})\end{array}$ & $\begin{array}{c}\text { Discharge } \\
\left(\mathrm{ft}^{3} / \mathrm{s}\right)\end{array}$ & $\begin{array}{c}\text { Date } \\
\text { (month/ } \\
\text { day/ } \\
\text { year) }\end{array}$ & $\begin{array}{l}\text { Stage } \\
\text { (ft) }\end{array}$ & $\begin{array}{c}\text { Discharge } \\
\left(\mathrm{ft}^{3} / \mathrm{s}\right)\end{array}$ & $\begin{array}{c}\text { Regulated } \\
\text { during } \\
\text { flood }^{1}\end{array}$ & $\begin{array}{c}\text { Recurrence } \\
\text { interval } \\
\text { (years) }\end{array}$ \\
\hline 03491544 & $\begin{array}{l}\text { Crockett Creek below Rogersville, } \\
\text { TN }\end{array}$ & 4.67 & 1989-98 & 1998 & 5.39 & 996 & $4 / 17 / 98$ & 5.39 & 996 & $\mathrm{~N}$ & $10-25$ \\
\hline 03497300 & Little River above Townsend, TN & 106 & 1964-98 & 1994 & 15.75 & 27,100 & $3 / 27 / 94$ & 15.75 & 27,100 & $\mathrm{~N}$ & $>100$ \\
\hline 03498500 & Little River near Maryville, TN & 269 & $\begin{array}{l}\text { 1875, 1896, } \\
1920, \\
1951-98\end{array}$ & $\begin{array}{l}1875 \\
1896\end{array}$ & $\begin{array}{l}31.00 \\
31.00\end{array}$ & $\begin{array}{l}50,000 \\
36,000\end{array}$ & $3 / 28 / 94$ & 27.95 & 42,100 & $\mathrm{~N}$ & 100 \\
\hline 03498850 & Little River near Alcoa, TN & 300 & 1986-98 & 1994 & 25.63 & 28,000 & $\begin{array}{l}3 / 28 / 94 \\
4 / 19 / 98\end{array}$ & $\begin{array}{l}25.63 \\
19.58\end{array}$ & $\begin{array}{l}28,000 \\
13,000\end{array}$ & $\begin{array}{l}\mathrm{N} \\
\mathrm{N}\end{array}$ & $\begin{array}{r}25-50 \\
--\end{array}$ \\
\hline 03519640 & Baker Creek near Greenback, TN & 16.0 & 1966-98 & 1998 & 10.61 & 4,100 & $4 / 17 / 98$ & 10.61 & 4,100 & $\mathrm{~N}$ & 50 \\
\hline 03527800 & Big War Creek at Luther, TN & 22.3 & 1986-98 & 1998 & 10.61 & 4,100 & $4 / 17 / 98$ & 10.61 & 4,100 & $\mathrm{~N}$ & $25-50$ \\
\hline 03535180 & $\begin{array}{l}\text { Willow Fork near Halls } \\
\text { Crossroads, TN }\end{array}$ & 3.23 & 1967-98 & 1998 & 8.40 & 990 & $4 / 17 / 98$ & 8.40 & 990 & $\mathrm{~N}$ & $25-30$ \\
\hline 03536550 & $\begin{array}{l}\text { Whiteoak Creek below Melton } \\
\text { Valley Drive near Oak Ridge, TN }\end{array}$ & 3.28 & 1985-96 & 1996 & 6.55 & 728 & $5 / 26 / 96$ & 6.55 & 728 & $\mathrm{~N}$ & $10-25$ \\
\hline 03564500 & Ocoee River at Parksville, TN & 595 & $\begin{array}{l}1907, \\
1912-16, \\
1922-94\end{array}$ & $\begin{array}{l}1990 \\
1907\end{array}$ & $\begin{array}{l}24.76 \\
--\end{array}$ & $\begin{array}{l}61,800 \\
65,000\end{array}$ & $3 / 27 / 94$ & 22.36 & 42,000 & $\mathrm{Y}$ & $75-100$ \\
\hline 03566420 & $\begin{array}{l}\text { Wolftever Creek near Ooltewah, } \\
\text { TN }\end{array}$ & 18.8 & $1965-98$ & 1973 & 9.75 & 7,300 & $3 / 28 / 94$ & 9.08 & 5,110 & $\mathrm{~N}$ & $40-45$ \\
\hline 03571000 & $\begin{array}{l}\text { Sequatchie River near Whitwell, } \\
\text { TN }\end{array}$ & 402 & $\begin{array}{l}1867 \\
1921-94\end{array}$ & $\begin{array}{l}1991 \\
1867\end{array}$ & $\begin{array}{l}18.02 \\
19.00\end{array}$ & $\begin{array}{r}35,400 \\
--\end{array}$ & $2 / 11 / 94$ & 16.68 & 25,100 & $\mathrm{~N}$ & $20-25$ \\
\hline 07025400 & $\begin{array}{l}\text { North Fork Obion River near } \\
\text { Martin, TN }\end{array}$ & 372 & $\begin{array}{l}\text { 1939-67, } \\
1997-98\end{array}$ & 1958 & 23.05 & 30,300 & $3 / 2 / 97$ & 22.98 & 27,000 & $\mathrm{~N}$ & $25-50$ \\
\hline
\end{tabular}

${ }^{1}$ Regulated during flood: $\mathrm{N}$, no; $\mathrm{Y}$, yes. 


\section{Texas}

Excessive rains began falling late October 17, 1994, in southeastern Texas from Austin to Livingston (fig. 56). Rainfall amounts overnight ranged from 10 to 18 inches in 16 counties (National Oceanic and Atmospheric Administration, 1994a), and most of these areas experienced flash flooding during the night. During the night, five lives were lost in the flash floods in three separate instances. During the day, September 18, an additional seven people drowned in the flood, bringing the 2-day total to 12 people. On October 19, the storms moved farther south and began affecting the counties along the coast of the Gulf of Mexico. Over the next 2 days, an additional five people died as a result of the floods. Total rainfall for the entire storm generally ranged from 10 to 20 inches, with Liberty recording 30.50 inches of rain during the storm (National Oceanic and Atmospheric Administration, 1994a). Before the flash-floodproducing rains had ended, flooding of rivers, creeks, and bayous had begun and would continue, in some areas, to the end of October.

The Trinity River experienced extensive flooding from just upstream from Lake Livingston to the mouth of the Trinity River at Trinity Bay. A record-high elevation on Lake Livingston was recorded on October 17, which resulted in a record release from the dam of more than 110,000 cubic feet per second. This record release, in combination with an additional inflow of 45,000 cubic feet per second downstream from the dam, led to a record flood along the Trinity River downstream from Lake Livingston. The Trinity River at Liberty (streamgage 08067000, table 45) set a new record crest on October 18, and when a levee system failed, much of the city of Liberty was flooded.

The San Jacinto River and 24 other streamgages in southeast Texas were also at record heights during the flood (table 45). Lake Conroe reached a record elevation on the afternoon of October 17, which resulted in record releases from the lake and also resulted in record flood stages along the San Jacinto River all the way to Lake Houston. The excessive inflow resulted in a record elevation at Lake Houston of 52.76 feet, which is 3 feet higher than the previous record. The uncontrolled spillway at Lake Houston released an estimated 354,000 cubic feet per second into the lower San Jacinto River that flows into the Houston Ship Channel. The tremendous flows caused four fuel pipelines to rupture resulting in a fuel spill in the river and subsequent fires. The floods on the Brazos River lasted until October 27, 1994.

In summary, 17 people lost their lives during the flood, more than 13,000 people had to be evacuated, and more than 22,000 homes received flood damage. Total damage to homes and businesses was approximately $\$ 800$ million, while another $\$ 100$ million in damage occurred to roads and bridges throughout southeast Texas (National Oceanic and Atmospheric

Administration, 1994b). During the height of the flood, several major highways leading into and out of Houston were impassable due to high water.

On May 5, 1995, more than 3 inches of rain in 30 minutes and as much as 5 inches in 1 hour caused massive flash flooding across the city of Dallas (National Oceanic and Atmospheric Administration, 1995a). Seventeen people drowned, most were in cars trying to cross flooded intersections, low-lying areas, or flooded creeks. The flooding destroyed four single-family homes and damaged 93, destroyed 166 multi-family housing units, and damaged 54 businesses. Excessive rainfall continued into June 1995, especially along the Red River Valley. The peak of 48 years of record occurred on the Red River near Burkburnett (streamgage 07308500, table 45) on June 6, 1995.

Tropical Storm Josephine caused coastal flooding along the upper Texas coast on October 28, 1996. Tides ranged from 2 to 5 feet above predicted levels and caused substantial beach erosion and damaged beach houses and low-lying coastal roads. Seven homes were destroyed, and 75 to 80 homes were damaged. Rainfall of as much as 13 inches in the headwaters of the Nueces River on October 27-28 (National Oceanic and Atmospheric Administration, 1996a) caused the river to rise rapidly. The Nueces River crested at 24.88 feet (13.88 feet above flood stage) just south of Uvalde (streamgage 08192000, table 45). This was the highest reading since 1962. Flooding also occurred along the Frio River due to the excessive rainfall. Total damage was more than \$18 million (National Oceanic and Atmospheric Administration, 1996b).

An upper level, low-pressure system was stationary over south-central Texas during June 21-23, 1997. Spiral rain bands around the low repeatedly moved over the same areas, bringing continuous flooding rains. Several river flood warnings were issued along the Llano and Colorado Rivers. The Llano River at Llano (streamgage 08151500, table 45) crested at 38.86 feet.

This crest was the highest since 1935. Damage was more than \$47 million, and there were three deaths (National Oceanic and Atmospheric Administration, 1997b).

During August 21-26, 1998, Tropical Storm Charley spread excessive rainfall over much of south-central Texas. Thunderstorms produced rainfall rates approaching 5 inches per hour (National Oceanic and Atmospheric Administration, 1998a). Flooding occurred on the Frio, Nueces, and Rio Grande Rivers. Del Rio received nearly all of its average annual precipitation (18 inches) during a 2-day period (National Oceanic and Atmospheric Administration, 1998a). Flooding occurred downstream from Del Rio on the Rio Grande. More than \$43 million in damage and 13 deaths resulted from Tropical Storm Charley (National Oceanic and Atmospheric Administration, 1998b).

Tropical Storm Frances came onshore near the same location as Tropical Storm Charley on September 10, 1998. However, Frances tracked to the north over Dallas, dropping from 4 to 12 inches of rainfall in central Texas (National Oceanic and Atmospheric Administration, 1998a). Some significant floods occurred near Houston, and damage primarily was due to high winds and coastal flooding with some inland flooding.

\section{References}

National Oceanic and Atmospheric Administration (NOAA), 1994a-98a, Climatological data (by State): Asheville, North Carolina, National Climatic Data Center, various months.

National Oceanic and Atmospheric Administration (NOAA), 1994b-98b, Storm data (by State): Asheville, North Carolina, National Climatic Data Center, various months. 


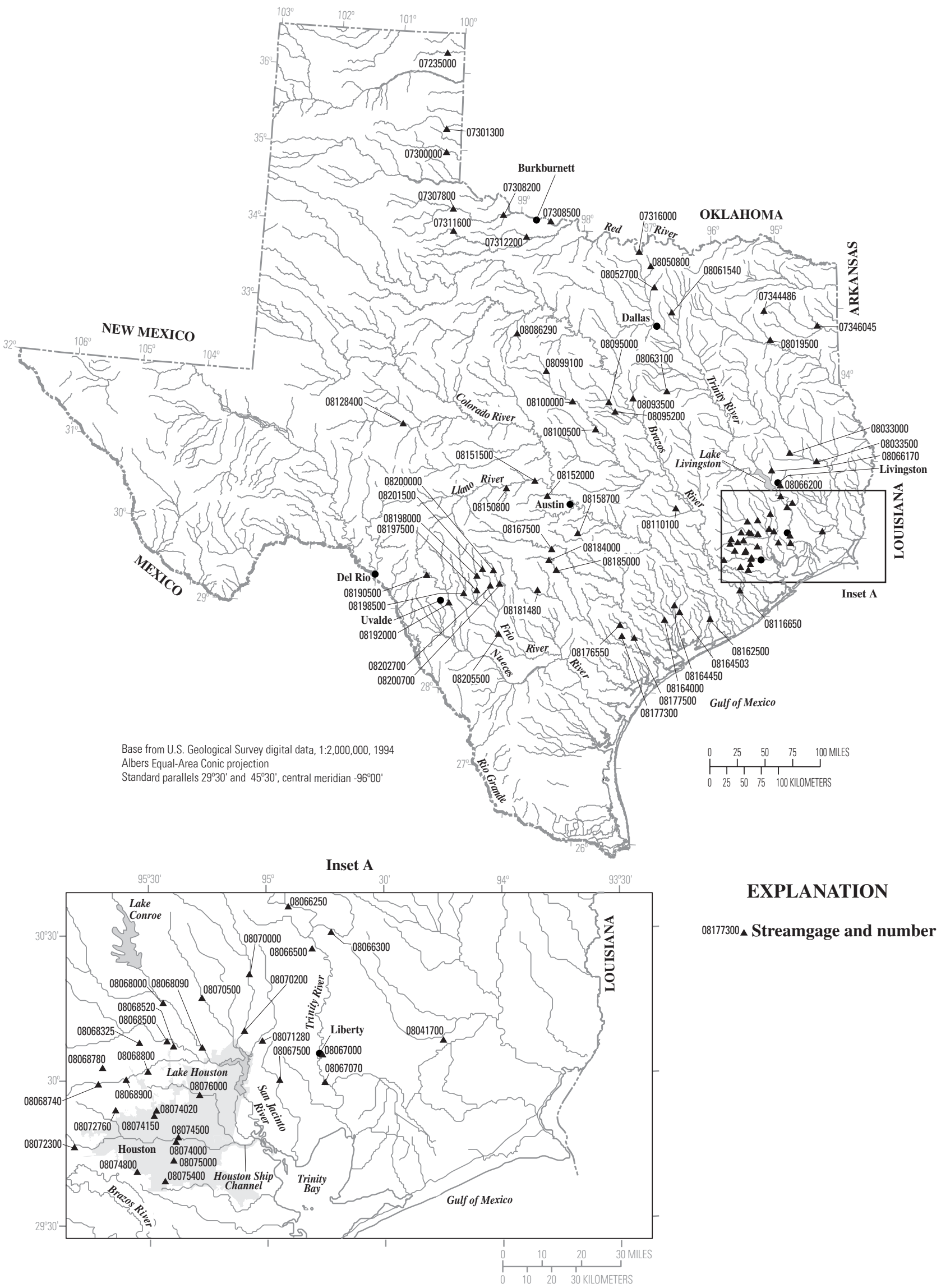

Figure 56. Location of streamgages with significant floods during 1994-98 water years for Texas. 
Table 45. Maximum stage and discharge for period of record for streamgages having significant floods during 1994-98 water years in Texas.

$\left[\mathrm{mi}^{2}\right.$, square miles; $\mathrm{ft}$, feet above an arbitrary datum; $\mathrm{ft}^{3} / \mathrm{s}$, cubic feet per second; --, not determined or not applicable; $>$, greater than. Source: Recurrence intervals calculated from U.S. Geological Survey data. Other data from U.S. Geological Survey reports or databases]

\begin{tabular}{|c|c|c|c|c|c|c|c|c|c|c|c|}
\hline \multirow{2}{*}{$\begin{array}{l}\text { Streamgage } \\
\text { number } \\
\text { (fig. 56) }\end{array}$} & \multirow[b]{2}{*}{ Streamgage name } & \multirow{2}{*}{$\begin{array}{c}\text { Total } \\
\text { drainage } \\
\left(\mathrm{mi}^{2}\right)\end{array}$} & \multicolumn{4}{|c|}{$\begin{array}{c}\text { Maximum stage and discharge for period of record } \\
\text { through } 1998 \text { water year }\end{array}$} & \multicolumn{5}{|c|}{ Significant floods $1994-98$ water years } \\
\hline & & & $\begin{array}{l}\text { Period of } \\
\text { record } \\
\text { (water } \\
\text { years) }\end{array}$ & Water year & $\begin{array}{l}\text { Stage } \\
(\mathrm{ft})\end{array}$ & $\begin{array}{l}\text { Discharge } \\
\left(\mathrm{ft}^{3} / \mathrm{s}\right)\end{array}$ & $\begin{array}{c}\text { Date } \\
\text { (month/d } \\
\text { ay/year) }\end{array}$ & $\begin{array}{l}\text { Stage } \\
\text { (ft) }\end{array}$ & $\begin{array}{l}\text { Discharge } \\
\left(\mathrm{ft}^{3} / \mathrm{s}\right)\end{array}$ & $\begin{array}{l}\text { Regulated } \\
\text { during } \\
\text { flood }\end{array}$ & $\begin{array}{c}\text { Recurrence } \\
\text { interval } \\
\text { (years) }\end{array}$ \\
\hline 07235000 & Wolf Creek at Lipscomb, TX & 697 & $\begin{array}{l}1938-42, \\
1962-98\end{array}$ & $\begin{array}{l}1942 \\
1996\end{array}$ & $\begin{array}{l}11.57 \\
12.44\end{array}$ & $\begin{array}{l}20,000 \\
10,300\end{array}$ & $9 / 19 / 96$ & 12.44 & 10,300 & $\mathrm{Y}$ & 10 \\
\hline 07300000 & $\begin{array}{l}\text { Salt Fork Red River near } \\
\text { Wellington, TX }\end{array}$ & 1,222 & 1953-98 & 1957 & 19.00 & 146,000 & $4 / 3 / 97$ & 17.10 & 81,100 & $\mathrm{Y}$ & 15 \\
\hline 07301300 & $\begin{array}{l}\text { North Fork Red River near } \\
\text { Shamrock, TX }\end{array}$ & 1,082 & 1964-97 & 1995 & 8.49 & 25,600 & $6 / 3 / 95$ & 8.49 & 25,600 & $\mathrm{~N}$ & $>100$ \\
\hline 07307800 & Pease River near Childress, TX & 2,754 & $\begin{array}{l}1960-62 \\
1968-98\end{array}$ & 1995 & 17.12 & 28,500 & $6 / 5 / 95$ & 17.12 & 28,500 & $\mathrm{~N}$ & $>100$ \\
\hline 07308200 & Pease River near Vernon, TX & 3,488 & $\begin{array}{l}\text { 1960-89, } \\
1991, \\
1993-98\end{array}$ & 1984 & 20.15 & 40,500 & $8 / 2 / 95$ & 19.23 & 32,800 & $\mathrm{~N}$ & 20 \\
\hline 07308500 & Red River near Burkburnett, TX & 20,570 & $1960-98$ & $\begin{array}{l}1995 \\
1984\end{array}$ & $\begin{array}{l}16.61 \\
16.90\end{array}$ & $\begin{array}{l}174,000 \\
166,000\end{array}$ & $6 / 6 / 95$ & 16.61 & 174,000 & $\mathrm{~N}$ & $>100$ \\
\hline 07311600 & $\begin{array}{l}\text { North Wichita River near Paducah, } \\
\text { TX }\end{array}$ & 540 & $\begin{array}{l}1962-82 \\
1995-98\end{array}$ & 1995 & 19.76 & 18,100 & $6 / 5 / 95$ & 19.76 & 18,100 & $\mathrm{~N}$ & $>100$ \\
\hline 07312200 & Beaver Creek near Electra, TX & 652 & 1961-98 & $\begin{array}{l}1995 \\
1987\end{array}$ & $\begin{array}{l}34.87 \\
34.94\end{array}$ & $\begin{array}{l}11,700 \\
11,600\end{array}$ & $8 / 3 / 95$ & 34.87 & 11,700 & Y & 75 \\
\hline 07316000 & Red River near Gainesville, TX & 30,782 & 1936-98 & 1987 & 40.08 & 265,000 & $6 / 13 / 95$ & 36.63 & 169,000 & $\mathrm{Y}$ & -- \\
\hline 07344486 & Brushy Creek at Scroggins, TX & 23.4 & 1979-98 & 1995 & 14.96 & 10,700 & $11 / 5 / 94$ & 14.96 & 10,700 & $\mathrm{~N}$ & $>100$ \\
\hline 07346045 & $\begin{array}{l}\text { Black Cypress Bayou at Jefferson, } \\
\text { TX }\end{array}$ & 365 & 1969-98 & 1988 & 19.34 & 11,600 & $4 / 29 / 97$ & 18.88 & 10,100 & $\mathrm{~N}$ & 20 \\
\hline 08019500 & $\begin{array}{l}\text { Big Sandy Creek near Big Sandy, } \\
\text { TX }\end{array}$ & 231 & 1939-98 & 1945 & 24.10 & 24,000 & $11 / 7 / 94$ & 17.89 & 6,040 & $\mathrm{Y}$ & 5 \\
\hline 08033000 & Neches River near Diboll, TX & 2,724 & $\begin{array}{l}\text { 1884, 1900, } \\
\text { 1924-25, } \\
1940-98\end{array}$ & 1884 & 21.00 & 110,000 & $10 / 17 / 94$ & 18.16 & 44,900 & $\mathrm{Y}$ & 15 \\
\hline 08033500 & Neches River near Rockland, TX & 3,636 & $\begin{array}{l}\text { 1884, } \\
\text { 1904-11, } \\
\text { 1914-98 }\end{array}$ & 1884 & 34.90 & 62,000 & $10 / 20 / 94$ & 33.29 & 42,300 & $\mathrm{Y}$ & 15 \\
\hline
\end{tabular}


Table 45. Maximum stage and discharge for period of record for streamgages having significant floods during 1994-98 water years in Texas. - Continued

$\left[\mathrm{mi}^{2}\right.$, square miles; ft, feet above an arbitrary datum; $\mathrm{ft}^{3} / \mathrm{s}$, cubic feet per second; --, not determined or not applicable; >, greater than. Source: Recurrence intervals calculated from U.S. Geological Survey data. Other data from U.S. Geological Survey reports or databases]

\begin{tabular}{|c|c|c|c|c|c|c|c|c|c|c|c|}
\hline \multirow{2}{*}{$\begin{array}{c}\text { Streamgage } \\
\text { number } \\
\text { (fig. 56) }\end{array}$} & \multirow[b]{2}{*}{ Streamgage name } & \multirow{2}{*}{$\begin{array}{l}\text { Total } \\
\text { drainage } \\
\left(\mathrm{mi}^{2}\right)\end{array}$} & \multicolumn{4}{|c|}{$\begin{array}{c}\text { Maximum stage and discharge for period of record } \\
\text { through } 1998 \text { water year }\end{array}$} & \multicolumn{5}{|c|}{ Significant floods $1994-98$ water years } \\
\hline & & & $\begin{array}{l}\text { Period of } \\
\text { record } \\
\text { (water } \\
\text { years) }\end{array}$ & Water year & $\begin{array}{l}\text { Stage } \\
\text { (ft) }\end{array}$ & $\begin{array}{l}\text { Discharge } \\
\left(\mathrm{ft}^{3} / \mathrm{s}\right)\end{array}$ & $\begin{array}{c}\text { Date } \\
\text { (month/d } \\
\text { ay/year) }\end{array}$ & $\begin{array}{l}\text { Stage } \\
(\mathrm{ft})\end{array}$ & $\begin{array}{l}\text { Discharge } \\
\left(\mathrm{ft}^{3} / \mathrm{s}\right)\end{array}$ & $\begin{array}{l}\text { Regulated } \\
\text { during } \\
\text { flood }^{1}\end{array}$ & $\begin{array}{l}\text { Recurrence } \\
\text { interval } \\
\text { (years) }\end{array}$ \\
\hline 08041700 & $\begin{array}{l}\text { Pine Island Bayou near Sour Lake, } \\
\text { TX }\end{array}$ & 336 & $1968-98$ & 1995 & 37.50 & 48,800 & $10 / 20 / 94$ & 37.50 & 48,800 & $\mathrm{~N}$ & $>100$ \\
\hline 08050800 & $\begin{array}{l}\text { Timber Creek near Collinsville, } \\
\text { TX }\end{array}$ & 38.8 & 1986-98 & 1994 & 14.94 & 13,300 & $7 / 10 / 94$ & 14.94 & 13,300 & $\mathrm{~N}$ & 20 \\
\hline 08052700 & Little Elm Creek near Aubrey, TX & 75.5 & $\begin{array}{l}\text { 1957-76, } \\
1980-98\end{array}$ & 1994 & 18.27 & 36,200 & $7 / 11 / 94$ & 18.27 & 36,200 & $\mathrm{Y}$ & $>100$ \\
\hline 08061540 & Rowlett Creek near Sachse, TX & 120 & 1969-98 & 1998 & 28.07 & 32,200 & $1 / 5 / 98$ & 28.07 & 32,200 & $\mathrm{~N}$ & 20 \\
\hline 08063100 & Richland Creek near Dawson, TX & 333 & $\begin{array}{l}1961-93 \\
1995-98\end{array}$ & $\begin{array}{l}1962 \\
1998\end{array}$ & $\begin{array}{l}21.64 \\
22.61\end{array}$ & $\begin{array}{r}15,800 \\
3,790\end{array}$ & $1 / 6 / 98$ & 22.61 & 3,790 & $\mathrm{Y}$ & -- \\
\hline 08066170 & $\begin{array}{l}\text { Kickapoo Creek near Onalaska, } \\
\text { TX }\end{array}$ & 57.0 & $1966-98$ & 1995 & 41.85 & 84,600 & $10 / 17 / 94$ & 41.85 & 84,600 & $\mathrm{~N}$ & $>100$ \\
\hline 08066200 & $\begin{array}{l}\text { Long King Creek at Livingston, } \\
\text { TX }\end{array}$ & 141 & 1963-98 & 1995 & 30.49 & 50,900 & $10 / 17 / 94$ & 30.49 & 50,900 & $\mathrm{~N}$ & $>100$ \\
\hline 08066250 & Trinity River near Goodrich, TX & 16,844 & 1966-98 & 1995 & 48.97 & 125,000 & $10 / 18 / 94$ & 48.97 & 125,000 & $\mathrm{Y}$ & -- \\
\hline 08066300 & Menard Creek near Rye, TX & 152 & $1966-98$ & 1995 & 31.12 & 13,700 & $10 / 17 / 94$ & 31.12 & 13,700 & $\mathrm{~N}$ & 40 \\
\hline 08066500 & Trinity River at Romayor, TX & 17,186 & 1924-98 & 1995 & 42.70 & 122,000 & $10 / 19 / 94$ & 42.70 & 122,000 & $\mathrm{Y}$ & 75 \\
\hline 08067000 & Trinity River at Liberty, TX & 17,468 & $\begin{array}{l}1940-70 \\
1973-98\end{array}$ & 1995 & 31.00 & 135,000 & $10 / 18 / 94$ & 31.00 & 135,000 & $\mathrm{Y}$ & 75 \\
\hline 08067070 & $\begin{array}{l}\text { Coastal Water Authority Canal } \\
\text { near Dayton, TX }\end{array}$ & -- & 1982-98 & 1998 & 3.07 & 1,220 & $6 / 2 / 98$ & 3.07 & 1,220 & $\mathrm{Y}$ & -- \\
\hline 08067500 & Cedar Bayou near Crosby, TX & 64.9 & $1972-98$ & 1995 & 28.33 & 7,800 & $10 / 18 / 94$ & 28.33 & 7,800 & $\mathrm{~N}$ & $>100$ \\
\hline 08068000 & $\begin{array}{l}\text { West Fork San Jacinto River near } \\
\text { Conroe, TX }\end{array}$ & 828 & $\begin{array}{l}1913, \\
1924-27, \\
1940-98\end{array}$ & 1995 & 32.30 & 115,000 & $10 / 18 / 94$ & 32.30 & 115,000 & $\mathrm{Y}$ & 50 \\
\hline 08068090 & $\begin{array}{l}\text { West Fork San Jacinto River above } \\
\text { Lake Houston near Porter, TX }\end{array}$ & 962 & $1985-98$ & 1995 & 40.10 & 130,000 & $10 / 18 / 94$ & 40.10 & 130,000 & $\mathrm{Y}$ & -- \\
\hline
\end{tabular}


Table 45. Maximum stage and discharge for period of record for streamgages having significant floods during 1994-98 water years in Texas. - Continued

$\left[\mathrm{mi}^{2}\right.$, square miles; $\mathrm{ft}$, feet above an arbitrary datum; $\mathrm{ft}^{3} / \mathrm{s}$, cubic feet per second; --, not determined or not applicable; >, greater than. Source: Recurrence intervals calculated from U.S. Geological Survey data. Other data from U.S. Geological Survey reports or databases]

\begin{tabular}{|c|c|c|c|c|c|c|c|c|c|c|c|}
\hline \multirow[b]{2}{*}{$\begin{array}{c}\text { Streamgage } \\
\text { number } \\
\text { (fig. 56) }\end{array}$} & \multirow[b]{2}{*}{ Streamgage name } & \multirow[b]{2}{*}{$\begin{array}{c}\text { Total } \\
\text { drainage } \\
\left(\mathrm{mi}^{2}\right)\end{array}$} & \multicolumn{4}{|c|}{$\begin{array}{c}\text { Maximum stage and discharge for period of record } \\
\text { through } 1998 \text { water year }\end{array}$} & \multicolumn{5}{|c|}{ Significant floods $1994-98$ water years } \\
\hline & & & $\begin{array}{l}\text { Period of } \\
\text { record } \\
\text { (water } \\
\text { years) }\end{array}$ & Water year & $\begin{array}{l}\text { Stage } \\
(\mathrm{ft})\end{array}$ & $\begin{array}{c}\text { Discharge } \\
\left(\mathrm{ft}^{3} / \mathrm{s}\right)\end{array}$ & $\begin{array}{c}\text { Date } \\
\text { (month/d } \\
\text { ay/year) }\end{array}$ & $\begin{array}{l}\text { Stage } \\
(\mathrm{ft})\end{array}$ & $\begin{array}{c}\text { Discharge } \\
\left(\mathrm{ft}^{3} / \mathrm{s}\right)\end{array}$ & $\begin{array}{c}\text { Regulated } \\
\text { during } \\
\text { flood }^{1}\end{array}$ & $\begin{array}{c}\text { Recurrence } \\
\text { interval } \\
\text { (years) }\end{array}$ \\
\hline 08068325 & Willow Creek near Tomball, TX & 41.0 & $\begin{array}{l}1984, \\
1986-87, \\
1989-98\end{array}$ & $\begin{array}{l}1998 \\
1995\end{array}$ & $\begin{array}{l}29.36 \\
31.81\end{array}$ & $\begin{array}{r}1,920 \\
--\end{array}$ & $1 / 7 / 98$ & 29.36 & 1,920 & $\mathrm{~N}$ & -- \\
\hline 08068500 & Spring Creek near Spring, TX & 409 & $\begin{array}{l}\text { 1929, } \\
1939-98\end{array}$ & 1995 & 44.05 & 78,800 & $10 / 18 / 94$ & 44.05 & 78,800 & $\mathrm{~N}$ & $>100$ \\
\hline 08068520 & Spring Creek at Spring, TX & 419 & $\begin{array}{l}\text { 1929, } \\
1939-95\end{array}$ & 1995 & 44.05 & 78,800 & $10 / 18 / 94$ & 44.05 & 78,800 & $\mathrm{~N}$ & $>100$ \\
\hline 08068740 & $\begin{array}{l}\text { Cypress Creek at House-Hahl } \\
\text { Road near Cypress, TX }\end{array}$ & 131 & 1975-98 & 1995 & 47.61 & 5,200 & $10 / 19 / 94$ & 47.61 & 5,200 & $\mathrm{~N}$ & $>100$ \\
\hline 08068780 & $\begin{array}{l}\text { Little Cypress Creek near Cypress, } \\
\text { TX }\end{array}$ & 41.0 & 1983-98 & 1995 & 81.41 & 4,520 & $10 / 18 / 94$ & 81.41 & 4,520 & $\mathrm{~N}$ & 25 \\
\hline 08068800 & $\begin{array}{l}\text { Cypress Creek at Grant Road near } \\
\text { Cypress, TX }\end{array}$ & 214 & 1983-98 & 1995 & 47.38 & 10,500 & $10 / 18 / 94$ & 47.38 & 10,500 & $\mathrm{~N}$ & $>100$ \\
\hline 08068900 & $\begin{array}{l}\text { Cypress Creek at Stuebner-Airline } \\
\text { Road near Westfield, TX }\end{array}$ & 248 & $\begin{array}{l}\text { 1984-85, } \\
1988-98\end{array}$ & 1995 & 39.61 & 11,300 & $10 / 19 / 94$ & 39.61 & 11,300 & $\mathrm{~N}$ & -- \\
\hline 08070000 & $\begin{array}{l}\text { East Fork San Jacinto River near } \\
\text { Cleveland, TX }\end{array}$ & 325 & $\begin{array}{l}1935 \\
1940-98\end{array}$ & 1995 & 24.57 & 63,000 & $10 / 18 / 94$ & 24.57 & 63,000 & $\mathrm{~N}$ & 100 \\
\hline 08070200 & $\begin{array}{l}\text { East Fork San Jacinto River near } \\
\text { New Caney, TX }\end{array}$ & 388 & 1985-98 & 1995 & 33.00 & 74,100 & $10 / 19 / 94$ & 33.00 & 74,100 & $\mathrm{~N}$ & $>100$ \\
\hline 08070500 & Caney Creek near Splendora, TX & 105 & 1944-98 & 1995 & 26.40 & 36,000 & $10 / 17 / 94$ & 26.40 & 36,000 & $\mathrm{~N}$ & $>100$ \\
\hline 08071280 & $\begin{array}{l}\text { Luce Bayou above Lake Houston } \\
\text { near Huffman, TX }\end{array}$ & 218 & 1985-98 & 1995 & 35.08 & 25,900 & $10 / 18 / 94$ & 35.08 & 25,900 & $\mathrm{~N}$ & 25 \\
\hline 08072300 & Buffalo Bayou near Katy, TX & 63.3 & 1978-98 & 1994 & 38.85 & 3,780 & $2 / 21 / 94$ & 38.85 & 3,780 & $\mathrm{~N}$ & 50 \\
\hline 08072760 & $\begin{array}{l}\text { Langham Creek at West Little } \\
\text { York Road near Addicks, TX }\end{array}$ & 24.6 & 1981-98 & $\begin{array}{l}1997 \\
1981\end{array}$ & $\begin{array}{l}22.62 \\
22.80\end{array}$ & $\begin{array}{l}1,820 \\
1,000\end{array}$ & $5 / 24 / 97$ & 22.62 & 1,820 & $\mathrm{~N}$ & 10 \\
\hline 08074000 & Buffalo Bayou at Houston, TX & 358 & $\begin{array}{l}\text { 1929, } \\
1936-98\end{array}$ & 1936 & 49.00 & 40,000 & $9 / 11 / 98$ & 36.33 & 13,400 & $\mathrm{Y}$ & -- \\
\hline 08074020 & $\begin{array}{l}\text { Whiteoak Bayou at Alabonson } \\
\text { Road at Houston, TX }\end{array}$ & -- & $\begin{array}{l}\text { 1984, } \\
1986-98\end{array}$ & $\begin{array}{l}1998 \\
1992\end{array}$ & $\begin{array}{l}48.54 \\
49.58\end{array}$ & $\begin{array}{r}13,300 \\
8,610\end{array}$ & $9 / 11 / 98$ & 48.54 & 13,300 & $\mathrm{~N}$ & 50 \\
\hline
\end{tabular}


$\left[\mathrm{mi}^{2}\right.$, square miles; $\mathrm{ft}$, feet above an arbitrary datum; $\mathrm{ft}^{3} / \mathrm{s}$, cubic feet per second; --, not determined or not applicable; >, greater than. Source: Recurrence intervals calculated from U.S. Geological Survey data. Other data from U.S. Geological Survey reports or databases]

\begin{tabular}{|c|c|c|c|c|c|c|c|c|c|c|c|}
\hline \multirow{2}{*}{$\begin{array}{c}\text { Streamgage } \\
\text { number } \\
\text { (fig. 56) }\end{array}$} & \multirow[b]{2}{*}{ Streamgage name } & \multirow{2}{*}{$\begin{array}{c}\text { Total } \\
\text { drainage } \\
\left(\mathrm{mi}^{2}\right)\end{array}$} & \multicolumn{4}{|c|}{$\begin{array}{c}\text { Maximum stage and discharge for period of record } \\
\text { through } 1998 \text { water year }\end{array}$} & \multicolumn{5}{|c|}{ Significant floods 1994-98 water years } \\
\hline & & & $\begin{array}{l}\text { Period of } \\
\text { record } \\
\text { (water } \\
\text { years) }\end{array}$ & Water year & $\begin{array}{l}\text { Stage } \\
(\mathrm{ft})\end{array}$ & $\begin{array}{l}\text { Discharge } \\
\left(\mathrm{ft}^{3} / \mathrm{s}\right)\end{array}$ & $\begin{array}{c}\text { Date } \\
\text { (month/d } \\
\text { ay/year) }\end{array}$ & $\begin{array}{l}\text { Stage } \\
(\mathrm{ft})\end{array}$ & $\begin{array}{l}\text { Discharge } \\
\left(\mathrm{ft}^{3} / \mathrm{s}\right)\end{array}$ & $\begin{array}{c}\text { Regulated } \\
\text { during } \\
\text { flood }^{1}\end{array}$ & $\begin{array}{c}\text { Recurrence } \\
\text { interval } \\
\text { (years) }\end{array}$ \\
\hline 08074150 & $\begin{array}{l}\text { Cole Creek at Deihl Road, } \\
\text { Houston, TX }\end{array}$ & 7.50 & $\begin{array}{l}\text { 1964-86, } \\
1990-98\end{array}$ & 1998 & 80.86 & 2,860 & $9 / 11 / 98$ & 80.86 & 2,860 & $\mathrm{~N}$ & -- \\
\hline 08074500 & Whiteoak Bayou at Houston, TX & 86.3 & $\begin{array}{l}\text { 1929, } \\
1936-98\end{array}$ & $\begin{array}{l}1992 \\
1936\end{array}$ & $\begin{array}{l}50.43 \\
51.50\end{array}$ & $\begin{array}{l}25,100 \\
14,750\end{array}$ & $9 / 11 / 98$ & 47.06 & 21,200 & $\mathrm{~N}$ & -- \\
\hline 08074800 & $\begin{array}{l}\text { Keegans Bayou at Roark Road } \\
\text { near Houston, TX }\end{array}$ & 12.7 & 1965-98 & 1992 & 75.91 & 4,880 & $10 / 18 / 94$ & 75.87 & 4,850 & $\mathrm{~N}$ & -- \\
\hline 08075000 & Brays Bayou at Houston, TX & 94.9 & $\begin{array}{l}1929, \\
1936-98\end{array}$ & 1976 & 52.13 & 29,000 & $10 / 18 / 94$ & 51.02 & 27,000 & $\mathrm{~N}$ & -- \\
\hline 08075400 & $\begin{array}{l}\text { Sims Bayou at Hiram Clarke } \\
\text { Street, Houston, TX }\end{array}$ & 20.2 & $1965-98$ & $\begin{array}{l}1995 \\
1976\end{array}$ & $\begin{array}{l}54.65 \\
57.12\end{array}$ & $\begin{array}{l}7,510 \\
4,500\end{array}$ & $10 / 18 / 94$ & 54.65 & 7,510 & $\mathrm{~N}$ & -- \\
\hline 08076000 & Greens Bayou near Houston, TX & 68.7 & 1953-98 & 1989 & 66.04 & 16,500 & $5 / 24 / 97$ & 62.77 & 13,100 & $\mathrm{~N}$ & -- \\
\hline 08086290 & $\begin{array}{l}\text { Big Sandy Creek above } \\
\text { Breckenridge, TX }\end{array}$ & 280 & 1962-98 & 1982 & 28.60 & 80,000 & $9 / 15 / 96$ & 26.53 & 15,000 & $\mathrm{~N}$ & 50 \\
\hline 08093500 & Aquilla Creek near Aquilla, TX & 308 & $\begin{array}{l}\text { 1936, } \\
1939-98\end{array}$ & 1936 & 33.00 & 74,200 & $12 / 21 / 97$ & 28.38 & 14,200 & $\mathrm{Y}$ & 5 \\
\hline 08095000 & $\begin{array}{l}\text { North Bosque River near Clifton, } \\
\text { TX }\end{array}$ & 968 & 1924-98 & 1992 & 38.30 & 200,000 & $3 / 16 / 98$ & 34.88 & 137,000 & $\mathrm{Y}$ & $>100$ \\
\hline 08095200 & $\begin{array}{l}\text { North Bosque River at Valley } \\
\text { Mills, TX }\end{array}$ & 1,146 & 1960-98 & 1992 & 44.60 & 220,000 & $3 / 16 / 98$ & 39.40 & 92,000 & $\mathrm{Y}$ & 10 \\
\hline 08099100 & Leon River near De Leon, TX & 479 & 1961-98 & 1990 & 19.00 & 24,500 & $6 / 24 / 97$ & 16.37 & 10,500 & $\mathrm{Y}$ & -- \\
\hline 08100000 & Leon River near Hamilton, TX & 1,891 & $\begin{array}{l}1925-31 \\
1962-98\end{array}$ & 1992 & 35.02 & 32,100 & 2/20/97 & 33.42 & 27,200 & $\mathrm{Y}$ & -- \\
\hline 08100500 & Leon River at Gatesville, TX & 2,342 & $\begin{array}{l}1908 \\
1951-98\end{array}$ & $\begin{array}{l}1992 \\
1908\end{array}$ & $\begin{array}{l}35.00 \\
35.00\end{array}$ & $\begin{array}{l}68,000 \\
70,000\end{array}$ & $2 / 24 / 97$ & 31.04 & 31,000 & $\mathrm{Y}$ & 10 \\
\hline 08110100 & Davidson Creek near Lyons, TX & 195 & 1963-98 & 1995 & 19.33 & 26,400 & $10 / 17 / 94$ & 19.33 & 26,400 & $\mathrm{~N}$ & 100 \\
\hline 08116650 & Brazos River near Rosharon, TX & 45,339 & $\begin{array}{l}1967-80 \\
1984-98\end{array}$ & $\begin{array}{l}1995 \\
1992\end{array}$ & $\begin{array}{l}51.82 \\
51.89\end{array}$ & $\begin{array}{l}84,400 \\
82,700\end{array}$ & $10 / 22 / 94$ & 51.82 & 84,400 & $\mathrm{Y}$ & -- \\
\hline 08128400 & $\begin{array}{l}\text { Middle Concho River above } \\
\text { Tankersley, TX }\end{array}$ & 2,084 & 1961-98 & 1974 & 24.98 & 15,500 & $8 / 29 / 96$ & 24.30 & 14,400 & $\mathrm{~N}$ & 25 \\
\hline
\end{tabular}


Table 45. Maximum stage and discharge for period of record for streamgages having significant floods during 1994-98 water years in Texas. - Continued

$\left[\mathrm{mi}^{2}\right.$, square miles; $\mathrm{ft}$, feet above an arbitrary datum; $\mathrm{ft}^{3} / \mathrm{s}$, cubic feet per second; --, not determined or not applicable; >, greater than. Source: Recurrence intervals calculated from U.S. Geological Survey data. Other data from U.S. Geological Survey reports or databases]

\begin{tabular}{|c|c|c|c|c|c|c|c|c|c|c|c|}
\hline \multirow[b]{2}{*}{$\begin{array}{c}\text { Streamgage } \\
\text { number } \\
\text { (fig. 56) }\end{array}$} & \multirow[b]{2}{*}{ Streamgage name } & \multirow[b]{2}{*}{$\begin{array}{c}\text { Total } \\
\text { drainage } \\
\left(\mathrm{mi}^{2}\right)\end{array}$} & \multicolumn{4}{|c|}{$\begin{array}{c}\text { Maximum stage and discharge for period of record } \\
\text { through } 1998 \text { water year }\end{array}$} & \multicolumn{5}{|c|}{ Significant floods $1994-98$ water years } \\
\hline & & & $\begin{array}{c}\text { Period of } \\
\text { record } \\
\text { (water } \\
\text { years) }\end{array}$ & Water year & $\begin{array}{l}\text { Stage } \\
\text { (ft) }\end{array}$ & $\begin{array}{c}\text { Discharge } \\
\left(\mathrm{ft}^{3} / \mathrm{s}\right)\end{array}$ & $\begin{array}{c}\text { Date } \\
\text { (month/d } \\
\text { ay/year) }\end{array}$ & $\begin{array}{l}\text { Stage } \\
(\mathrm{ft})\end{array}$ & $\begin{array}{l}\text { Discharge } \\
\left(\mathrm{ft}^{3} / \mathrm{s}\right)\end{array}$ & $\begin{array}{c}\text { Regulated } \\
\text { during } \\
\text { flood }^{1}\end{array}$ & $\begin{array}{c}\text { Recurrence } \\
\text { interval } \\
\text { (years) }\end{array}$ \\
\hline 08150800 & Beaver Creek near Mason, TX & 215 & 1964-98 & 1978 & 24.00 & 66,900 & $10 / 28 / 96$ & 19.45 & 45,900 & $\mathrm{~N}$ & 25 \\
\hline 08151500 & Llano River at Llano, TX & 4,197 & $\begin{array}{l}1935 \\
1940-98\end{array}$ & 1935 & 41.50 & 380,000 & $6 / 23 / 97$ & 38.86 & 260,000 & $\mathrm{~N}$ & 50 \\
\hline 08152000 & Sandy Creek near Kingsland, TX & 346 & $\begin{array}{l}1952, \\
1967-95, \\
1998\end{array}$ & 1952 & 34.20 & 163,000 & $5 / 29 / 95$ & 31.22 & 107,000 & $\mathrm{~N}$ & $>100$ \\
\hline 08158700 & Onion Creek near Driftwood, TX & 124 & $\begin{array}{l}\text { 1941, } \\
\text { 1980-94, } \\
\text { 1996-98 }\end{array}$ & 1997 & 17.56 & 10,000 & $6 / 9 / 97$ & 17.56 & 10,000 & $\mathrm{~N}$ & 5 \\
\hline 08162500 & Colorado River near Bay City, TX & 42,240 & $\begin{array}{l}1940 \\
1948-98\end{array}$ & $\begin{array}{l}1960 \\
1940\end{array}$ & $\begin{array}{l}46.40 \\
46.60\end{array}$ & $\begin{array}{l}84,100 \\
83,300\end{array}$ & $10 / 20 / 94$ & 38.67 & 71,100 & $\mathrm{Y}$ & -- \\
\hline 08164000 & Lavaca River near Edna, TX & 817 & $\begin{array}{l}1936, \\
1939-98\end{array}$ & 1995 & 35.49 & 150,000 & $10 / 19 / 94$ & 35.49 & 150,000 & $\mathrm{~N}$ & $>100$ \\
\hline 08164450 & Sandy Creek near Louise, TX & 289 & 1978-98 & 1995 & 28.45 & 24,900 & $10 / 19 / 94$ & 28.45 & 24,900 & $\mathrm{~N}$ & $>100$ \\
\hline 08164503 & $\begin{array}{l}\text { West Mustang Creek near Ganado, } \\
\text { TX }\end{array}$ & 178 & $1978-98$ & 1995 & 28.39 & 20,000 & $10 / 19 / 94$ & 28.39 & 20,000 & $\mathrm{~N}$ & $>100$ \\
\hline 08167500 & $\begin{array}{l}\text { Guadalupe River near Spring } \\
\text { Branch, TX }\end{array}$ & 1,315 & $\begin{array}{l}\text { 1923-92, } \\
1994-98\end{array}$ & 1978 & 45.25 & 160,000 & $6 / 22 / 97$ & 45.12 & 116,000 & $\mathrm{~N}$ & 50 \\
\hline 08176550 & Fifteenmile Creek near Weser, TX & 167 & 1985-98 & 1997 & 26.68 & 19,400 & $6 / 21 / 97$ & 26.68 & 19,400 & $\mathrm{~N}$ & -- \\
\hline 08177300 & $\begin{array}{l}\text { Perdido Creek at Farm Road } 622 \\
\text { near Fannin, TX }\end{array}$ & 28.0 & 1979-98 & 1997 & 17.89 & 26,510 & $4 / 4 / 97$ & 17.89 & 26,510 & $\mathrm{~N}$ & 25 \\
\hline 08177500 & Coleto Creek near Victoria, TX & 514 & $\begin{array}{l}\text { 1939-54, } \\
1967, \\
1979-98\end{array}$ & 1967 & 42.00 & 236,000 & $4 / 4 / 97$ & 32.05 & 50,100 & $\mathrm{Y}$ & 10 \\
\hline 08181480 & $\begin{array}{l}\text { Leon Creek at Interstate } \\
\text { Highway } 35 \text { at San Antonio, TX }\end{array}$ & 219 & $1985-98$ & 1997 & 24.60 & 27,900 & $6 / 22 / 97$ & 24.60 & 27,900 & $\mathrm{~N}$ & -- \\
\hline 08184000 & Cibolo Creek near Bulverde, TX & 198 & $\begin{array}{l}1946-65 \\
1997\end{array}$ & $\begin{array}{l}1997 \\
1958\end{array}$ & $-\overline{-} 22.50$ & $\begin{array}{l}61,600 \\
21,100\end{array}$ & $6 / 22 / 97$ & -- & 61,600 & $\mathrm{~N}$ & $>100$ \\
\hline 08185000 & Cibolo Creek at Selma, TX & 274 & 1946-98 & 1997 & 29.73 & 69,600 & $6 / 22 / 97$ & 29.73 & 69,600 & $\mathrm{Y}$ & 50 \\
\hline
\end{tabular}


$\left[\mathrm{mi}^{2}\right.$, square miles; ft, feet above an arbitrary datum; $\mathrm{ft}^{3} / \mathrm{s}$, cubic feet per second; --, not determined or not applicable; >, greater than. Source: Recurrence intervals calculated from U.S. Geological Survey data. Other data from U.S. Geological Survey reports or databases]

\begin{tabular}{|c|c|c|c|c|c|c|c|c|c|c|c|}
\hline \multirow{2}{*}{$\begin{array}{l}\text { Streamgage } \\
\text { number } \\
\text { (fig. 56) }\end{array}$} & \multirow[b]{2}{*}{ Streamgage name } & \multirow{2}{*}{$\begin{array}{c}\text { Total } \\
\text { drainage } \\
\left(\mathrm{mi}^{2}\right)\end{array}$} & \multicolumn{4}{|c|}{$\begin{array}{c}\text { Maximum stage and discharge for period of record } \\
\text { through } 1998 \text { water year }\end{array}$} & \multicolumn{5}{|c|}{ Significant floods $1994-98$ water years } \\
\hline & & & $\begin{array}{l}\text { Period of } \\
\text { record } \\
\text { (water } \\
\text { years) }\end{array}$ & Water year & $\begin{array}{l}\text { Stage } \\
\text { (ft) }\end{array}$ & $\begin{array}{l}\text { Discharge } \\
\left(\mathrm{ft}^{3} / \mathrm{s}\right)\end{array}$ & $\begin{array}{c}\text { Date } \\
\text { (month/d } \\
\text { ay/year) }\end{array}$ & $\begin{array}{l}\text { Stage } \\
(\mathrm{ft})\end{array}$ & $\begin{array}{l}\text { Discharge } \\
\left(\mathrm{ft}^{3} / \mathrm{s}\right)\end{array}$ & $\begin{array}{l}\text { Regulated } \\
\text { during } \\
\text { flood }^{1}\end{array}$ & $\begin{array}{c}\text { Recurrence } \\
\text { interval } \\
\text { (years) }\end{array}$ \\
\hline 08190500 & $\begin{array}{l}\text { West Nueces River near } \\
\text { Brackettville, TX }\end{array}$ & 694 & $\begin{array}{l}1935, \\
1940-50, \\
1957-98\end{array}$ & 1935 & 40.00 & 550,000 & $10 / 28 / 96$ & 30.74 & 230,000 & $\mathrm{~N}$ & 50 \\
\hline 08192000 & Nueces River below Uvalde, TX & 1,861 & 1928-98 & 1935 & 49.40 & 616,000 & $10 / 28 / 96$ & 24.88 & 201,000 & $\mathrm{~N}$ & 25 \\
\hline 08197500 & $\begin{array}{l}\text { Frio River below Dry Frio River } \\
\text { near Uvalde, TX }\end{array}$ & 631 & $1952-98$ & 1997 & 25.09 & 100,000 & $6 / 22 / 97$ & 25.09 & 100,000 & $\mathrm{~N}$ & 50 \\
\hline 08198000 & Sabinal River near Sabinal, TX & 206 & 1943-98 & $\begin{array}{l}1958 \\
1997\end{array}$ & $\begin{array}{l}24.60 \\
28.50\end{array}$ & $\begin{array}{l}55,200 \\
52,500\end{array}$ & $6 / 22 / 97$ & 28.50 & 52,500 & $\mathrm{~N}$ & 100 \\
\hline 08198500 & Sabinal River at Sabinal, TX & 241 & $\begin{array}{l}1932, \\
1953-98\end{array}$ & 1997 & 35.86 & 93,500 & $6 / 22 / 97$ & 35.86 & 93,500 & $\mathrm{~N}$ & $>100$ \\
\hline 08200000 & Hondo Creek near Tarpley, TX & 95.6 & $\begin{array}{l}1932, \\
1953-98\end{array}$ & 1997 & 29.64 & 76,900 & $6 / 22 / 97$ & 29.64 & 76,900 & $\mathrm{~N}$ & 100 \\
\hline 08200700 & $\begin{array}{l}\text { Hondo Creek at King Waterhole } \\
\text { near Hondo, TX }\end{array}$ & 149 & 1961-98 & 1997 & 18.96 & 63,600 & $6 / 22 / 97$ & 18.96 & 63,600 & $\mathrm{~N}$ & 100 \\
\hline 08201500 & $\begin{array}{l}\text { Seco Creek at Miller Ranch near } \\
\text { Utopia, TX }\end{array}$ & 45.0 & $\begin{array}{l}1958 \\
1962-98\end{array}$ & 1997 & 17.70 & 64,900 & $6 / 22 / 97$ & 17.70 & 64,900 & $\mathrm{~N}$ & $>100$ \\
\hline 08202700 & $\begin{array}{l}\text { Seco Creek at Rowe Ranch near } \\
\text { D'Hanis, TX }\end{array}$ & 168 & $\begin{array}{l}1932, \\
1961-98\end{array}$ & 1997 & 30.62 & 51,400 & $6 / 22 / 97$ & 30.62 & 51,400 & $\mathrm{~N}$ & 50 \\
\hline 08205500 & Frio River near Derby, TX & 3,429 & 1916-98 & 1932 & 29.45 & 230,000 & $6 / 24 / 97$ & 21.77 & 56,400 & $\mathrm{~N}$ & 50 \\
\hline
\end{tabular}

${ }^{1}$ Regulated during flood: N, no; Y, yes. 


\section{Utah}

A powerful Pacific storm system affected Utah during March 1995. Total storm snowfall in the northern mountains included 25 inches at Alta and 21 inches at Park City (fig. 57). In the southern mountains, Brian Head recorded 38 inches of snow (National Oceanic and Atmospheric Administration, 1995a). In southwestern Utah, the combination of excessive rain, deep snow, and already saturated soils produced significant flooding. Flooding began first on the Santa Clara River after Gunlock Reservoir began to overflow. A record flow of 3,500 cubic feet per second was measured at the Gunlock Reservoir spillway (National Oceanic and Atmospheric Administration, 1995b). Damage estimates to public and private property in Washington County were \$1.4 million (National Oceanic and Atmospheric Administration, 1995b). Snowmelt and thunderstorm activity throughout Utah caused 10 streamgages to experience their peak of record (table 46) in 1995.

Intense downpours occurred across southern Utah on September 14, 1996. A flash flood occurred in a portion of White Canyon, about 10 miles northwest of Natural Bridges National Monument. The floodwaters claimed the life of a 16-year-old girl (National Oceanic and Atmospheric Administration, 1996b).

Excessive rains fell in southwest Utah during

November 21-22, 1996. Some of the worst flooding in the State's history was reported near Washington. The mountains of southwest Utah received 2 to 4 inches of rainfall, and valley locations had from 1 to 2 inches (National Oceanic and Atmospheric Administration, 1996a). Rainfall rates of as much as 1 inch per hour were measured. Although the damage was more than $\$ 5$ million, there were only two known injuries. Excessive rains returned in January 1997 to the same part of southwestern Utah, and another \$5 million in damage resulted (National Oceanic and Atmospheric Administration, 1997b).

On July 22, 1998, a flash flood moved down Canal Creek Canyon just south of Spring City. Several roads were damaged, and four bridges were washed out. Crops were ruined in the area as well (National Oceanic and Atmospheric Administration, 1998b).

A 10-year-old girl drowned on September 5, 1998, in a flash flood in Ice Cream Canyon, located in the southern part of Glen Canyon National Recreation Area, when 0.87 inch of rain fell in a short period of time in the area (National Oceanic and Atmospheric Administration, 1998b).

\section{References}

National Oceanic and Atmospheric Administration (NOAA), 1995a-98a, Climatological data (by State): Asheville, North Carolina, National Climatic Data Center, various months. National Oceanic and Atmospheric Administration (NOAA), 1995b-98b, Storm data (by State): Asheville, North Carolina, National Climatic Data Center, various months. 


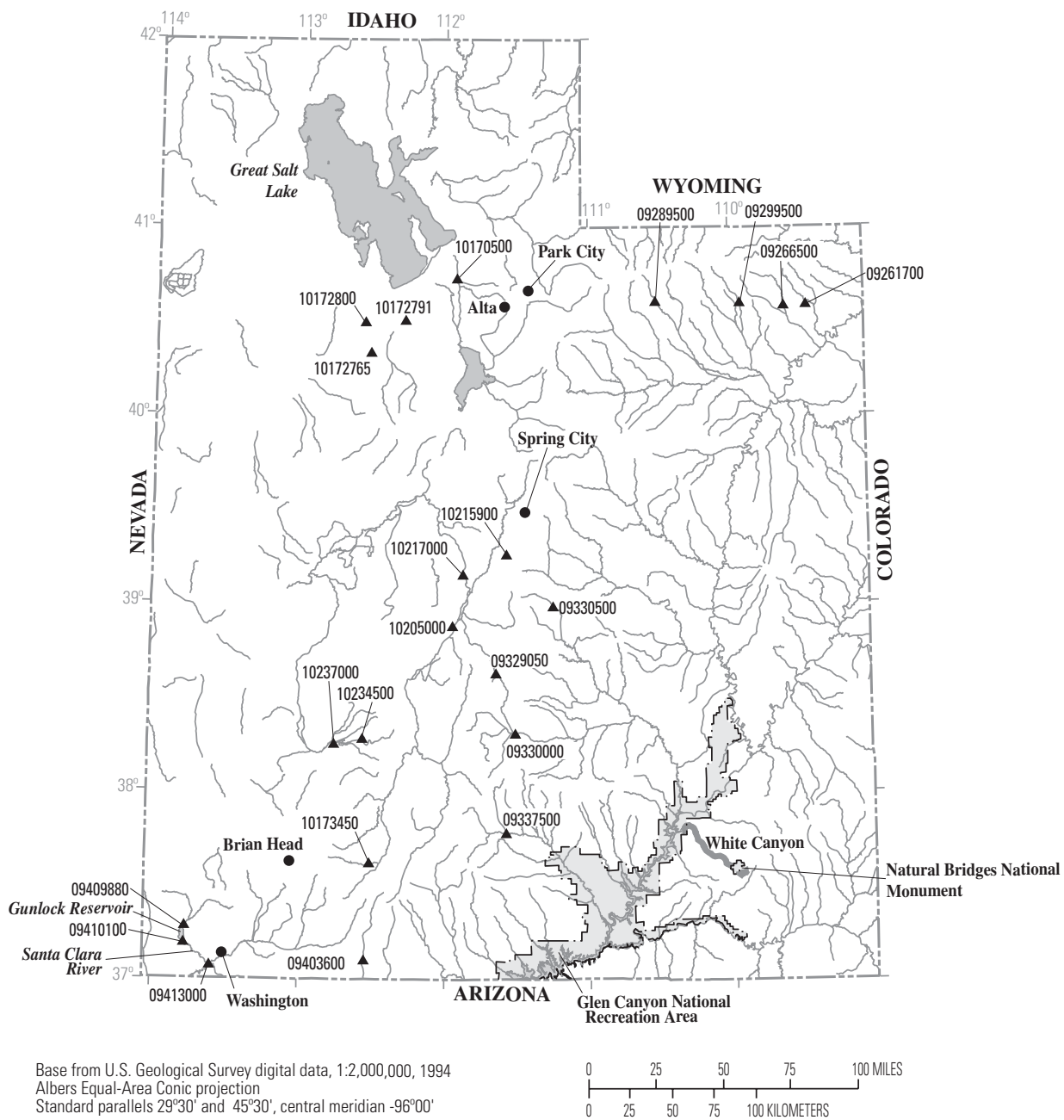

\section{EXPLANATION}

09403600 S Streamgage and number

Figure 57. Location of streamgages with significant floods during 1994-98 water years for Utah. 
Table 46. Maximum stage and discharge for period of record for streamgages having significant floods during 1994-98 water years in Utah.

$\left[\mathrm{mi}^{2}\right.$, square miles; $\mathrm{ft}$, feet above an arbitrary datum; $\mathrm{ft}^{3} / \mathrm{s}$, cubic feet per second; --, not determined or not applicable; $>$, greater than. Source: Recurrence intervals calculated from U.S. Geological Survey data. Other data from U.S. Geological Survey reports or databases]

\begin{tabular}{|c|c|c|c|c|c|c|c|c|c|c|c|}
\hline \multirow{2}{*}{$\begin{array}{l}\text { Streamgage } \\
\text { number } \\
\text { (fig. 57) }\end{array}$} & \multirow[b]{2}{*}{ Streamgage name } & \multirow{2}{*}{$\begin{array}{c}\text { Total } \\
\text { drainage } \\
\left(\mathrm{mi}^{2}\right)\end{array}$} & \multicolumn{4}{|c|}{$\begin{array}{c}\text { Maximum stage and discharge for period of record } \\
\text { through } 1998 \text { water year }\end{array}$} & \multicolumn{5}{|c|}{ Significant floods $1994-98$ water years } \\
\hline & & & $\begin{array}{l}\text { Period of } \\
\text { record } \\
\text { (water } \\
\text { years) }\end{array}$ & Water year & $\begin{array}{l}\text { Stage } \\
(\mathrm{ft})\end{array}$ & $\begin{array}{l}\text { Discharge } \\
\left(\mathrm{ft}^{3} / \mathrm{s}\right)\end{array}$ & $\begin{array}{c}\text { Date } \\
\text { (month/ } \\
\text { day/ } \\
\text { year) }\end{array}$ & $\begin{array}{l}\text { Stage } \\
(\mathrm{ft})\end{array}$ & $\begin{array}{l}\text { Discharge } \\
\left(\mathrm{ft}^{3} / \mathrm{s}\right)\end{array}$ & $\begin{array}{l}\text { Regulated } \\
\text { during } \\
\text { flood }^{1}\end{array}$ & $\begin{array}{c}\text { Recurrence } \\
\text { interval } \\
\text { (years) }\end{array}$ \\
\hline 09261700 & $\begin{array}{l}\text { Big Brush Creek above Red Fleet } \\
\text { Reservoir near Vernal, UT }\end{array}$ & 77.2 & $1980-98$ & $\begin{array}{l}1998 \\
1980\end{array}$ & $\begin{array}{l}2.09 \\
3.06\end{array}$ & $\begin{array}{l}384 \\
314\end{array}$ & $5 / 22 / 98$ & 2.09 & 384 & $\mathrm{Y}$ & $10-25$ \\
\hline 09266500 & Ashley Creek near Vernal, UT & 101 & $\begin{array}{l}1912 \\
1914-98\end{array}$ & 1995 & 5.64 & 4,100 & $6 / 15 / 95$ & 5.64 & 4,100 & $\mathrm{~N}$ & $\sim 500$ \\
\hline 09289500 & $\begin{array}{l}\text { Lake Fork River above Moon Lake } \\
\text { near Mountain Home, UT }\end{array}$ & 77.9 & $\begin{array}{l}1933-34 \\
1943-55 \\
1964-98\end{array}$ & 1995 & 6.44 & 2,740 & $6 / 27 / 95$ & 6.44 & 2,740 & $\mathrm{~N}$ & $75-100$ \\
\hline 09299500 & $\begin{array}{l}\text { Whiterocks River near Whiterocks, } \\
\text { UT }\end{array}$ & 109 & $\begin{array}{l}\text { 1902-03, } \\
1909, \\
1918-25, \\
1927, \\
1930-98\end{array}$ & 1983 & 5.28 & 4,640 & $6 / 26 / 95$ & 6.98 & 2,820 & $\mathrm{~N}$ & $75-90$ \\
\hline 09329050 & $\begin{array}{l}\text { Seven Mile Creek near Fish Lake, } \\
\text { UT }\end{array}$ & 24 & $1965-98$ & $\begin{array}{l}1995 \\
1984\end{array}$ & $\begin{array}{l}3.52 \\
4.03\end{array}$ & $\begin{array}{l}424 \\
369\end{array}$ & $6 / 12 / 95$ & 3.52 & 424 & $\mathrm{~N}$ & $40-50$ \\
\hline 09330000 & Fremont River near Bicknell, UT & 751 & $\begin{array}{l}1938-43 \\
1945-58 \\
1977-97\end{array}$ & 1997 & 7.02 & 1,360 & $3 / 21 / 97$ & 7.02 & 1,360 & $\mathrm{Y}$ & $50-60$ \\
\hline 09330500 & Muddy Creek near Emery, UT & 105 & $\begin{array}{l}1909, \\
1911-14, \\
1949-98\end{array}$ & 1952 & 11.14 & 3,340 & $8 / 11 / 95$ & 10.05 & 3,140 & $\mathrm{~N}$ & $50-60$ \\
\hline 09337500 & Escalante River near Escalante, UT & 320 & $\begin{array}{l}1910-12, \\
1943-55, \\
1972-98\end{array}$ & 1998 & 11.05 & 4,550 & $8 / 24 / 98$ & 11.05 & 4,550 & $\mathrm{~N}$ & $35-40$ \\
\hline 09403600 & Kanab Creek near Kanab, UT & 198 & 1979-98 & 1961 & 15.70 & 3,030 & $1 / 3 / 97$ & 7.97 & 2,300 & $\mathrm{~N}$ & $5-10$ \\
\hline 09409880 & Santa Clara River at Gunlock, UT & 271 & $1970-98$ & 1995 & 8.07 & 2,830 & $3 / 11 / 95$ & 8.07 & 2,830 & $\mathrm{Y}$ & $20-30$ \\
\hline
\end{tabular}


Table 46. Maximum stage and discharge for period of record for streamgages having significant floods during 1994-98 water years in Utah.-Continued

$\left[\mathrm{mi}^{2}\right.$, square miles; ft, feet above an arbitrary datum; $\mathrm{ft}^{3} / \mathrm{s}$, cubic feet per second; --, not determined or not applicable; >, greater than. Source: Recurrence intervals calculated from U.S. Geological Survey data. Other data from U.S. Geological Survey reports or databases]

\begin{tabular}{|c|c|c|c|c|c|c|c|c|c|c|c|}
\hline \multirow{2}{*}{$\begin{array}{c}\text { Streamgage } \\
\text { number } \\
\text { (fig. 57) }\end{array}$} & \multirow[b]{2}{*}{ Streamgage name } & \multirow{2}{*}{$\begin{array}{c}\text { Total } \\
\text { drainage } \\
\left(\mathrm{mi}^{2}\right)\end{array}$} & \multicolumn{4}{|c|}{$\begin{array}{c}\text { Maximum stage and discharge for period of record } \\
\text { through } 1998 \text { water year }\end{array}$} & \multicolumn{5}{|c|}{ Significant floods $1994-98$ water years } \\
\hline & & & $\begin{array}{c}\text { Period of } \\
\text { record } \\
\text { (water } \\
\text { years) }\end{array}$ & Water year & $\begin{array}{l}\text { Stage } \\
(\mathrm{ft})\end{array}$ & $\begin{array}{l}\text { Discharge } \\
\left(\mathrm{ft}^{3} / \mathrm{s}\right)\end{array}$ & $\begin{array}{c}\text { Date } \\
\text { (month/ } \\
\text { day/ } \\
\text { year) }\end{array}$ & $\begin{array}{l}\text { Stage } \\
(\mathrm{ft})\end{array}$ & $\begin{array}{l}\text { Discharge } \\
\left(\mathrm{ft}^{3} / \mathrm{s}\right)\end{array}$ & $\begin{array}{l}\text { Regulated } \\
\text { during } \\
\text { flood }^{1}\end{array}$ & $\begin{array}{c}\text { Recurrence } \\
\text { interval } \\
\text { (years) }\end{array}$ \\
\hline 09410100 & $\begin{array}{l}\text { Santa Clara River below Winsor } \\
\text { Dam near Santa Clara, UT }\end{array}$ & 378 & $1972-98$ & 1995 & 20.17 & 3,460 & $3 / 12 / 95$ & 20.17 & 3,460 & $\mathrm{Y}$ & $30-40$ \\
\hline 09413000 & $\begin{array}{l}\text { Santa Clara River at St. George, } \\
\text { UT }\end{array}$ & 541 & $\begin{array}{l}1951-56 \\
1985-98\end{array}$ & 1995 & 14.60 & 6,000 & $3 / 12 / 95$ & 14.60 & 6,000 & $\mathrm{Y}$ & $10-25$ \\
\hline 10170500 & $\begin{array}{l}\text { Surplus Canal at Salt Lake City, } \\
\text { UT }\end{array}$ & -- & $1943-98$ & 1984 & 8.91 & 4,410 & $6 / 17 / 98$ & 16.79 & 3,500 & $\mathrm{Y}$ & -- \\
\hline 10172765 & $\begin{array}{l}\text { Clover Creek above Big Hollow } \\
\text { near Clover, UT }\end{array}$ & 6.71 & 1985-98 & 1995 & 2.26 & 47 & $6 / 6 / 95$ & 2.26 & 47 & $\mathrm{~N}$ & $20-30$ \\
\hline 10172791 & $\begin{array}{l}\text { Settlement Creek above reservoir } \\
\text { near Tooele, UT }\end{array}$ & 16.8 & 1989-98 & $\begin{array}{l}1995 \\
1990\end{array}$ & $\begin{array}{l}4.41 \\
4.59\end{array}$ & $\begin{array}{r}67 \\
7\end{array}$ & $6 / 15 / 95$ & 4.41 & 67 & $\mathrm{~N}$ & $5-10$ \\
\hline 10172800 & $\begin{array}{l}\text { South Willow Creek near } \\
\text { Grantsville, UT }\end{array}$ & 4.19 & $1963-98$ & 1998 & 2.45 & 118 & $7 / 24 / 98$ & 2.45 & 118 & $\mathrm{~N}$ & $75-100$ \\
\hline 10173450 & $\begin{array}{l}\text { Mammoth Creek above West } \\
\text { Hatch Ditch near Hatch, UT }\end{array}$ & 105 & $1965-98$ & 1983 & 5.13 & 838 & $6 / 14 / 95$ & 4.79 & 654 & $\mathrm{~N}$ & $5-10$ \\
\hline 10205000 & Sevier River near Sigurd, UT & 3,375 & $1915-98$ & $\begin{array}{l}1922 \\
1995\end{array}$ & $\begin{array}{l}6.10 \\
8.33\end{array}$ & $\begin{array}{l}2,400 \\
1,310\end{array}$ & $6 / 15 / 95$ & 8.33 & 1,310 & $\mathrm{Y}$ & $25-30$ \\
\hline 10215900 & $\begin{array}{l}\text { Manti Creek below Dugway Creek } \\
\text { near Manti, UT }\end{array}$ & 26.4 & $\begin{array}{l}\text { 1965-74, } \\
1979-98\end{array}$ & 1995 & 5.49 & 705 & $6 / 28 / 95$ & 5.49 & 705 & $\mathrm{~N}$ & $25-30$ \\
\hline 10217000 & $\begin{array}{l}\text { Sevier River below San Pitch River } \\
\text { near Gunnison, UT }\end{array}$ & 4,921 & 1918-98 & $\begin{array}{l}1984 \\
1995\end{array}$ & $-\overline{-}$ & $\begin{array}{l}5,400 \\
3,290\end{array}$ & $6 / 15 / 95$ & 9.17 & 3,290 & $\mathrm{Y}$ & $40-50$ \\
\hline 10234500 & Beaver River near Beaver, UT & 91.0 & 1914-98 & $\begin{array}{l}1936 \\
1937\end{array}$ & $\begin{array}{l}7.27 \\
7.95\end{array}$ & $\begin{array}{r}1,080 \\
749\end{array}$ & $6 / 15 / 95$ & 3.16 & 1,050 & $\mathrm{~N}$ & $25-30$ \\
\hline 10237000 & Beaver River at Adamsville, UT & 303 & 1914-98 & $\begin{array}{l}1995 \\
1980\end{array}$ & $\begin{array}{l}5.52 \\
7.04\end{array}$ & $\begin{array}{r}1,870 \\
728\end{array}$ & $6 / 6 / 95$ & 5.52 & 1,870 & $\mathrm{Y}$ & $75-100$ \\
\hline
\end{tabular}

${ }^{1}$ Regulated during flood: N, no; $\mathrm{Y}$, yes. 


\section{Vermont}

High water occurred at Lake Champlain in northwestern Vermont (fig. 58) starting in April 1994, which resulted in flood damage to homes and roadways, shoreline erosion, and washing of debris into the lake and onto lakeshores. Compared to the record flood in 1993, water levels peaked about 6 inches lower, and there was much less damage. The worst damage occurred on the morning of April 19 when a thick ice pack on the lake broke up as strong south winds pushed masses of ice northward on the lake. The ice caused an estimated $\$ 500,000$ to $\$ 700,000$ in damage when it sheared off 11 high-voltage utility poles (National Oceanic and Atmospheric Administration, 1994b).

A cold front moved southeast from Canada during the afternoon of August 4, 1995, and focused the most intense rains across northern Vermont. More than 5.5 inches of rain fell from the remnants of Tropical Storm Dean as a result of tropical moisture moving northeast along the front (National Oceanic and Atmospheric Administration, 1995a). A woman was killed when her car went into a culvert, which had been washed out. Road washouts and closures were reported in many areas across Vermont. Extensive crop damage also was reported. Total damage was more than \$5 million (National Oceanic and Atmospheric Administration, 1995b).

A strong storm system moved in from the Great Lakes to Vermont on January 18, 1996. The circulation associated with this storm resulted in above-normal temperatures, strong winds, and flooding due to snowmelt and rainfall. Two deaths were attributed to the flooding. Damage was more than $\$ 30$ million (National Oceanic and Atmospheric Administration, 1996b).

A very moist and unstable atmosphere remained over Vermont on June 13, 1996. This produced a second straight day of torrential rains from training thunderstorms in the Grafton area, which resulted in disastrous flash flooding. Grafton was completely isolated with all roads becoming impassable. The Saxtons River reached its third highest stage of record. Several bridges were severely damaged, and 14 homes were affected by the flooding with 3 homes sustaining severe damage. Total damage was \$1.5 million (National Oceanic and Atmospheric Administration, 1996b).

A cold front stalled across northern Vermont during the morning hours of July 15, 1997, and focused excessive convective rain east of St. Albans during the morning of July 15. This resulted in numerous road and bridge washouts. Extensive flooding of tributary rivers was followed by flooding of larger rivers. Damage in northwestern Vermont was more than \$8 million (National Oceanic and Atmospheric Administration, 1997b).

An area of low pressure tracked across New York and New England on June 26-27, 1998. Excessive convective rains fell with 3 to 8 inches across the midsection of Vermont. Extensive flooding occurred, especially along the Mad River, White River, and Ayers Brook (table 47). Damage was \$13 million (National Oceanic and Atmospheric Administration, 1998b).

A cold front moved across the region on August 11-12, 1998. With a warm, humid air mass in place, thunderstorms resulted in torrential downpours during the night. Small streams and brooks quickly rose out of their banks. Road flooding was especially severe in St. Albans.

\section{References}

National Oceanic and Atmospheric Administration (NOAA), 1994a-98a, Climatological data (by State): Asheville, North Carolina, National Climatic Data Center, various months. National Oceanic and Atmospheric Administration (NOAA), 1994b-98b, Storm data (by State): Asheville, North Carolina, National Climatic Data Center, various months. 


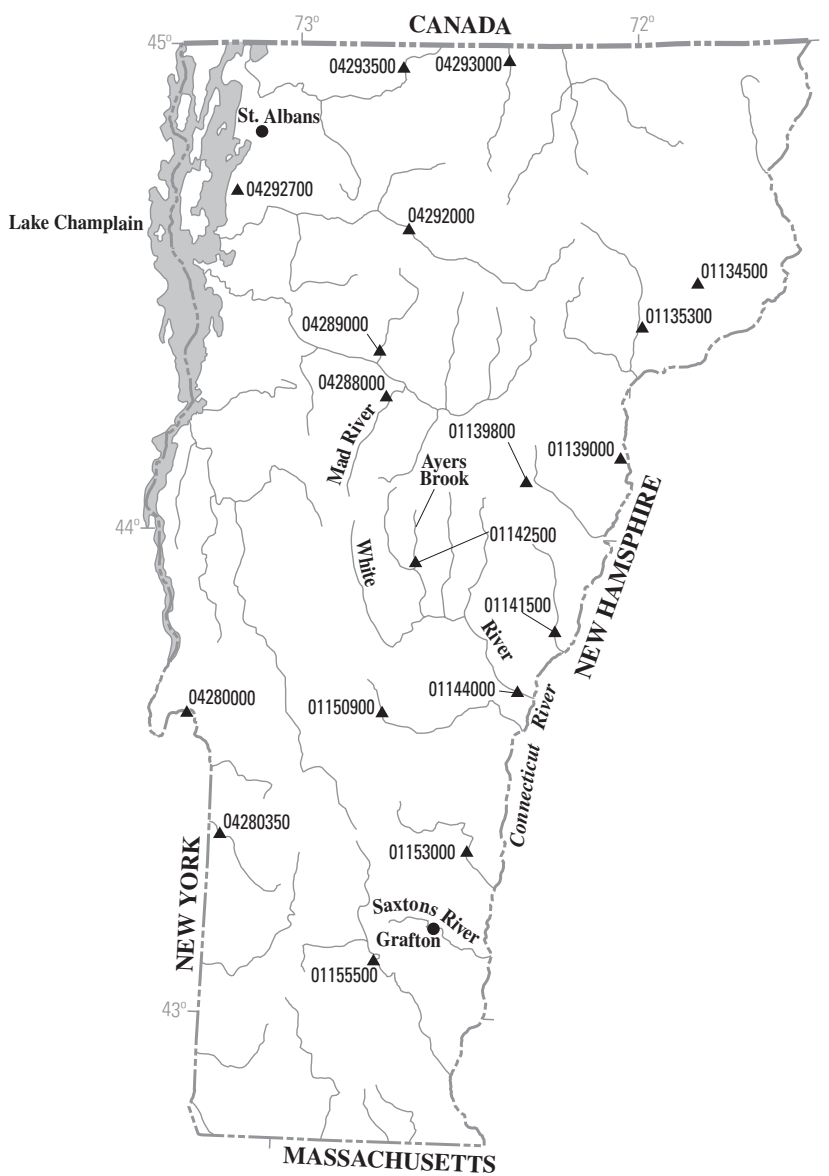

Base from U.S. Geological Survey digital data, 1:2,000,000, 1994

Albers Equal-Area Conic projection

Standard parallels $29^{\circ} 30^{\prime}$ and $45^{\circ} 30^{\prime}$, central meridian $-96^{\circ} 00^{\prime}$

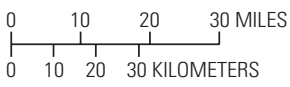

\section{EXPLANATION}

01155500 Streamgage and number

Figure 58. Location of streamgages with significant floods during 1994-98 water years for Vermont. 
Table 47. Maximum stage and discharge for period of record for streamgages having significant floods during 1994-98 water years in Vermont.

$\left[\mathrm{mi}^{2}\right.$, square miles; $\mathrm{ft}$, feet above an arbitrary datum; $\mathrm{ft}^{3} / \mathrm{s}$, cubic feet per second; --, not determined or not applicable; $>$, greater than; <, less than. Source: Recurrence intervals calculated from U.S. Geological Survey data. Other data from U.S. Geological Survey reports or databases]

\begin{tabular}{|c|c|c|c|c|c|c|c|c|c|c|c|}
\hline \multirow{2}{*}{$\begin{array}{l}\text { Streamgage } \\
\text { number } \\
\text { (fig. 58) }\end{array}$} & \multirow[b]{2}{*}{ Streamgage name } & \multirow{2}{*}{$\begin{array}{c}\text { Total } \\
\text { drainage } \\
\left(\mathrm{mi}^{2}\right)\end{array}$} & \multicolumn{4}{|c|}{$\begin{array}{c}\text { Maximum stage and discharge for period of record } \\
\text { through } 1998 \text { water year }\end{array}$} & \multicolumn{5}{|c|}{ Significant floods 1994-98 water years } \\
\hline & & & $\begin{array}{c}\text { Period of } \\
\text { record } \\
\text { (water } \\
\text { years) }\end{array}$ & Water year & $\begin{array}{l}\text { Stage } \\
(\mathrm{ft})\end{array}$ & $\begin{array}{l}\text { Discharge } \\
\left(\mathrm{ft}^{3} / \mathrm{s}\right)\end{array}$ & $\begin{array}{c}\text { Date } \\
\text { (month/ } \\
\text { day/ } \\
\text { year) }\end{array}$ & $\begin{array}{l}\text { Stage } \\
(\mathrm{ft})\end{array}$ & $\begin{array}{l}\text { Discharge } \\
\left(\mathrm{ft}^{3} / \mathrm{s}\right)\end{array}$ & $\begin{array}{c}\text { Regulated } \\
\text { during } \\
\text { flood }^{1}\end{array}$ & $\begin{array}{c}\text { Recurrence } \\
\text { interval } \\
\text { (years) }\end{array}$ \\
\hline 01134500 & Moose River at Victory, VT & 75.2 & $1947-98$ & 1973 & 12.04 & 4,940 & $8 / 6 / 95$ & 11.72 & 4,540 & $\mathrm{~N}$ & $50-75$ \\
\hline 01135300 & $\begin{array}{l}\text { Sleepers River near St. Johnsbury, } \\
\text { VT }\end{array}$ & 42.9 & 1990-98 & 1998 & 7.11 & 7,570 & $8 / 12 / 98$ & 7.11 & 7,570 & -- & $>100$ \\
\hline 01139000 & Wells River at Wells River, VT & 98.4 & $1941-98$ & 1973 & 9.82 & 5,970 & $7 / 15 / 97$ & 8.54 & 4,560 & $\mathrm{~N}$ & $25-50$ \\
\hline 01139800 & $\begin{array}{l}\text { East Orange Branch at East } \\
\text { Orange, VT }\end{array}$ & 8.95 & $1958-98$ & 1996 & 5.42 & 626 & $1 / 19 / 96$ & 5.42 & 626 & -- & $10-25$ \\
\hline 01141500 & $\begin{array}{l}\text { Ompompanoosuc River at Union } \\
\text { Village, VT }\end{array}$ & 130 & $\begin{array}{l}1928, \\
1941-98\end{array}$ & $\begin{array}{l}1947 \\
1928\end{array}$ & $\begin{array}{r}9.65 \\
14.50\end{array}$ & $\begin{array}{r}4,800 \\
--\end{array}$ & $\begin{array}{l}4 / 19 / 97 \\
4 / 6 / 98\end{array}$ & $\begin{array}{r}10.03 \\
9.97\end{array}$ & $\begin{array}{l}2,440 \\
2,360\end{array}$ & $\begin{array}{l}\mathrm{Y} \\
\mathrm{Y}\end{array}$ & $\begin{array}{l}<10 \\
<10\end{array}$ \\
\hline 01142500 & Ayers Brook at Randolph, VT & 30.5 & $\begin{array}{l}1928 \\
1940-98\end{array}$ & $\begin{array}{l}1998 \\
1928\end{array}$ & $\begin{array}{l}11.93 \\
16.00\end{array}$ & $\begin{array}{r}3,480 \\
--\end{array}$ & $6 / 27 / 98$ & 11.93 & 3,480 & $\mathrm{~N}$ & $>200$ \\
\hline 01144000 & White River at West Hartford, VT & 690 & $1915-98$ & 1998 & 17.38 & 34,500 & $6 / 27 / 98$ & 17.38 & 34,500 & -- & $10-25$ \\
\hline 01150900 & $\begin{array}{l}\text { Ottauquechee River near West } \\
\text { Bridgewater, VT }\end{array}$ & 23.4 & $1985-98$ & $\begin{array}{l}1996 \\
1987\end{array}$ & $\begin{array}{l}7.33 \\
7.78\end{array}$ & $\begin{array}{l}1,960 \\
1,270\end{array}$ & $10 / 22 / 95$ & 7.33 & 1,960 & $\mathrm{~N}$ & 25 \\
\hline 01153000 & $\begin{array}{l}\text { Black River at North Springfield, } \\
\text { VT }\end{array}$ & 158 & $1930-98$ & 1938 & 17.68 & 15,500 & $4 / 6 / 98$ & 7.93 & 4,150 & $\mathrm{Y}$ & -- \\
\hline 01155500 & West River at Jamaica, VT & 179 & $1947-98$ & 1949 & 14.87 & 29,500 & $\begin{array}{l}4 / 23 / 96 \\
4 / 6 / 98\end{array}$ & $\begin{array}{l}9.47 \\
9.49\end{array}$ & $\begin{array}{l}5,840 \\
5,900\end{array}$ & $\begin{array}{l}\mathrm{Y} \\
\mathrm{Y}\end{array}$ & -- \\
\hline 04280000 & $\begin{array}{l}\text { Poultney River below Fair Haven, } \\
\text { VT }\end{array}$ & 187 & 1929-98 & 1945 & 24.36 & 14,800 & $1 / 20 / 96$ & 20.91 & 10,200 & $\mathrm{Y}$ & $25-50$ \\
\hline 04280350 & Mettawee River near Pawlet, VT & 70.2 & $1985-98$ & 1996 & 6.31 & 4,480 & $1 / 19 / 96$ & 6.31 & 4,480 & $\mathrm{~N}$ & $10-25$ \\
\hline 04288000 & Mad River near Moretown, VT & 139 & $1928-98$ & 1928 & 19.40 & 23,000 & $6 / 27 / 98$ & 14.13 & 14,500 & $\mathrm{~N}$ & 50 \\
\hline 04289000 & Little River near Waterbury, VT & 111 & $1936-98$ & 1936 & 19.38 & 6,520 & $8 / 6 / 95$ & 14.81 & 4,410 & $\mathrm{Y}$ & $25-50$ \\
\hline
\end{tabular}


Table 47. Maximum stage and discharge for period of record for streamgages having significant floods during 1994-98 water years in Vermont.-Continued

$\left[\mathrm{mi}^{2}\right.$, square miles; $\mathrm{ft}$, feet above an arbitrary datum; $\mathrm{ft}^{3} / \mathrm{s}$, cubic feet per second; --, not determined or not applicable; $>$, greater than; <, less than. Source: Recurrence intervals calculated from U.S. Geological Survey data. Other data from U.S. Geological Survey reports or databases]

\begin{tabular}{|c|c|c|c|c|c|c|c|c|c|c|c|}
\hline \multirow[b]{2}{*}{$\begin{array}{l}\text { Streamgage } \\
\text { number } \\
\text { (fig. 58) }\end{array}$} & \multirow[b]{2}{*}{ Streamgage name } & \multirow{2}{*}{$\begin{array}{c}\text { Total } \\
\text { drainage } \\
\left(\mathrm{mi}^{2}\right)\end{array}$} & \multicolumn{4}{|c|}{$\begin{array}{c}\text { Maximum stage and discharge for period of record } \\
\text { through } 1998 \text { water year }\end{array}$} & \multicolumn{5}{|c|}{ Significant floods $1994-98$ water years } \\
\hline & & & $\begin{array}{l}\text { Period of } \\
\text { record } \\
\text { (water } \\
\text { years) }\end{array}$ & Water year & $\begin{array}{l}\text { Stage } \\
(\mathrm{ft})\end{array}$ & $\begin{array}{l}\text { Discharge } \\
\left(\mathrm{ft}^{3} / \mathrm{s}\right)\end{array}$ & $\begin{array}{c}\text { Date } \\
\text { (month/ } \\
\text { day/ } \\
\text { year) }\end{array}$ & $\begin{array}{l}\text { Stage } \\
(\mathrm{ft})\end{array}$ & $\begin{array}{l}\text { Discharge } \\
\left(\mathrm{ft}^{3} / \mathrm{s}\right)\end{array}$ & $\begin{array}{l}\text { Regulated } \\
\text { during } \\
\text { flood }^{1}\end{array}$ & $\begin{array}{c}\text { Recurrence } \\
\text { interval } \\
\text { (years) }\end{array}$ \\
\hline 04292000 & Lamoille River at Johnson, VT & 310 & $\begin{array}{l}1912-13 \\
1929-98\end{array}$ & 1995 & 19.88 & 19,000 & $8 / 6 / 95$ & 19.88 & 19,000 & $\mathrm{~N}$ & $>200$ \\
\hline 04292700 & $\begin{array}{l}\text { Stone Bridge Brook near Georgia } \\
\text { Plains, VT }\end{array}$ & 8.45 & $\begin{array}{l}1963-74 \\
1990-98\end{array}$ & 1996 & 8.59 & 1,030 & $1 / 19 / 96$ & 8.59 & 1,030 & $\mathrm{~N}$ & $50-100$ \\
\hline 04293000 & $\begin{array}{l}\text { Missisquoi River near North Troy, } \\
\text { VT }\end{array}$ & 131 & $1932-98$ & 1997 & 13.84 & 8,940 & $\begin{array}{l}7 / 15 / 97 \\
3 / 31 / 98\end{array}$ & $\begin{array}{l}13.84 \\
12.68\end{array}$ & $\begin{array}{l}8,940 \\
8,890\end{array}$ & $\begin{array}{l}\mathrm{N} \\
\mathrm{N}\end{array}$ & $\begin{array}{l}50-100 \\
50-100\end{array}$ \\
\hline 04293500 & $\begin{array}{l}\text { Missisquoi River near East } \\
\text { Berkshire, VT }\end{array}$ & 479 & $\begin{array}{l}1912-19, \\
1921-23, \\
1928-98\end{array}$ & 1928 & 23.10 & 45,000 & $3 / 31 / 98$ & 17.09 & 20,400 & $\mathrm{~N}$ & $25-50$ \\
\hline
\end{tabular}

${ }^{1}$ Regulated during flood: N, no; Y, yes. 


\section{Virginia}

In February 1994, a developing storm over the southeastern United States brought excessive precipitation to extreme southwestern Virginia after 2 to 3 inches of rain had exacerbated flooding conditions a day earlier. One to three additional inches of rain fell into already swollen creeks, streams, and rivers, creating flash flooding beginning late on February 10 and continuing through February 11 (National Oceanic and Atmospheric Administration, 1994a).

A slow-moving frontal system, with several low-pressure waves riding along it, produced moderate to excessive rainfall during the last week of March 1994. Rainfall totals ranged from 3 to 6 inches across the State, with the largest amounts occurring across the extreme southwestern areas (National Oceanic and Atmospheric Administration, 1994a). The rain, falling on saturated ground, produced widespread flooding across the western one-half of the State, mainly on March 28 and 29. One death occurred northwest of Richmond (fig. 59) when the transportation director for the public schools drowned while checking water depth on area roadways (National Oceanic and Atmospheric Administration, 1994b).

Torrential rainfall caused by thunderstorms in southcentral and southwestern Virginia occurred June 22-23, 1995. The storms produced major flash flooding that resulted in two fatalities and three injuries along the Maury, James, Roanoke, and Dan Rivers (fig. 59, inset A). Excessive rainfall continued over the central and northern Shenandoah Valley June 27-28, 1995. The excessive rain, falling on saturated soil, caused catastrophic flooding and flash flooding, resulting in three deaths, at least 20 injured, $\$ 50$ million in private and public property damage, and nearly $\$ 100$ million in agricultural damage (National Oceanic and Atmospheric Administration, 1995b). Eight Virginia counties were declared Federal disaster areas. The most severe flooding occurred near Madison, where rainfall at higher elevations exceeded 20 inches in a 12-hour period (National Oceanic and Atmospheric Administration, 1995a). Flooding was most extreme along the Rapidan and Rappahannock River Basins. Flooding along the Rapidan River near Ruckersville (streamgage 01665500, table 48) was deemed to be greater than a 500-year event. Although the streamgage was washed away, a watermark estimate at the Ruckersville site was 31.3 feet, exceeding the previous record set in October 1942 by 10.5 feet.
Near Fredericksburg (streamgage 01668000, table 48), the flood stage on the Rappahannock River was exceeded by nearly 7 feet (National Oceanic and Atmospheric Administration, 1995b).

In January 1996, precipitation combined with unseasonably warm, humid air caused nearly all of the snowpack, estimated at between 6 and 15 inches and as much as 2 feet or more at higher elevations, to melt in a 12-hour period (National Oceanic and Atmospheric Administration, 1996a). The unseasonably warm air was drawn northward around the circulation of a deepening storm west of the Appalachian Mountains during January 18-19. The melting snow and additional rainfall produced widespread flooding and flash flooding on January 19. A pre-frontal line of intense showers and embedded thunderstorms moved into the region shortly after dawn on January 19, causing widespread flash flooding. Snowmelt, combined with 1 to 3 inches of rain (some locations received nearly 5 inches) (National Oceanic and Atmospheric Administration, 1996a), caused the worst regional flooding in more than 10 years. Five deaths occurred as a result of the flooding, and damage estimates were more than $\$ 67$ million (National Oceanic and Atmospheric Administration, 1996b).

On September 5, 1996, Hurricane Fran made landfall on the North Carolina coast near Cape Fear (fig. 45). As a category 3 hurricane, Fran had 115-mile-per-hour sustained winds, a 13foot storm surge along the Virginia coast, and excessive rains of as much as 16 inches in Virginia and West Virginia. The center of the storm passed from south to north through the center of Virginia. Five deaths occurred from drowning, and property damage estimates from flooding were more than $\$ 41$ million in Virginia (National Oceanic and Atmospheric Administration, 1996b). Twenty-one streamgages recorded their highest discharge of record during this widespread flood (table 48).

\section{References}

National Oceanic and Atmospheric Administration (NOAA), 1994a-96a, Climatological data (by State): Asheville, North Carolina, National Climatic Data Center, various months.

National Oceanic and Atmospheric Administration (NOAA), 1994b-96b, Storm data (by State): Asheville, North Carolina, National Climatic Data Center, various months. 


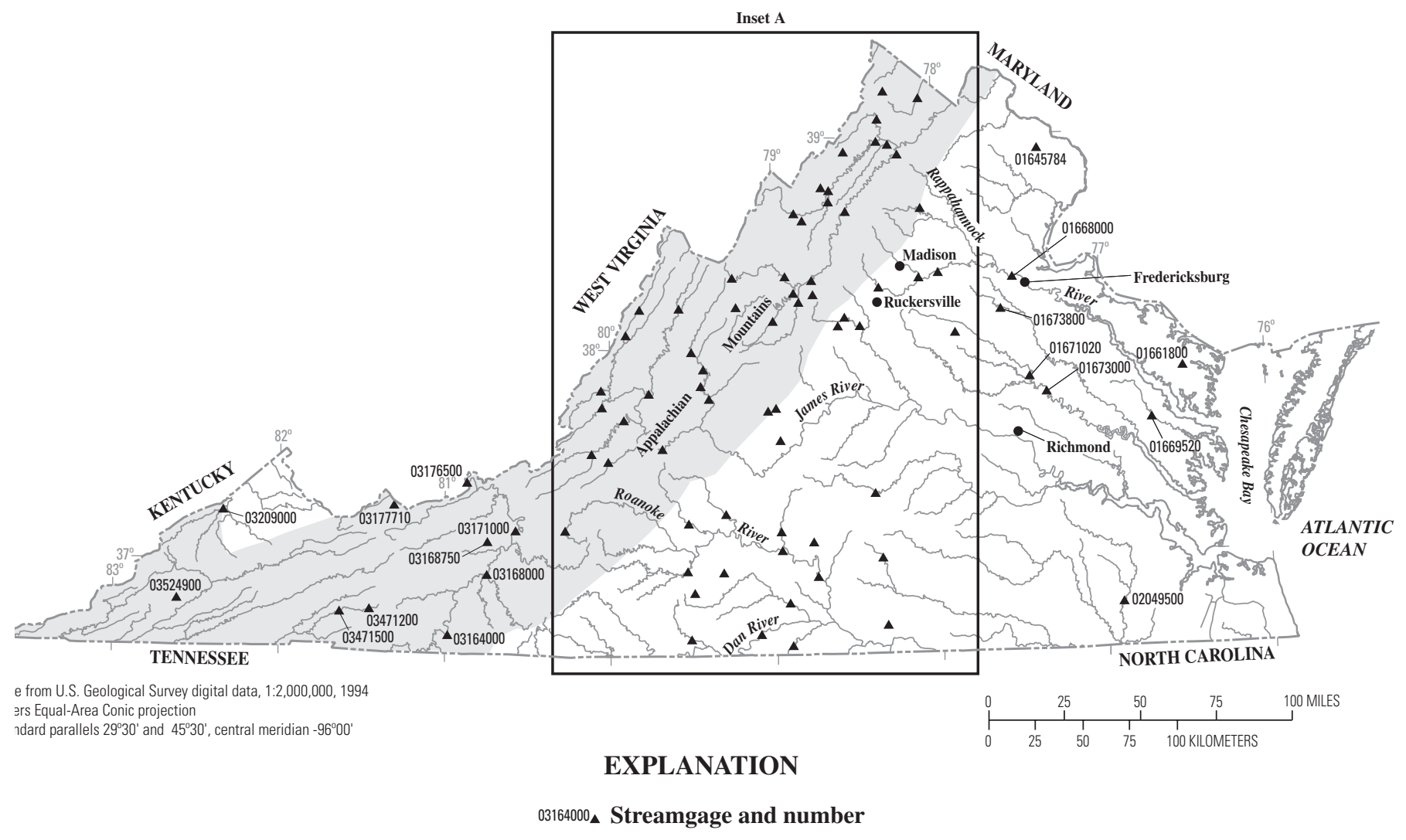

Figure 59. Location of streamgages with significant floods during 1994-98 water years for Virginia. 
Inset A

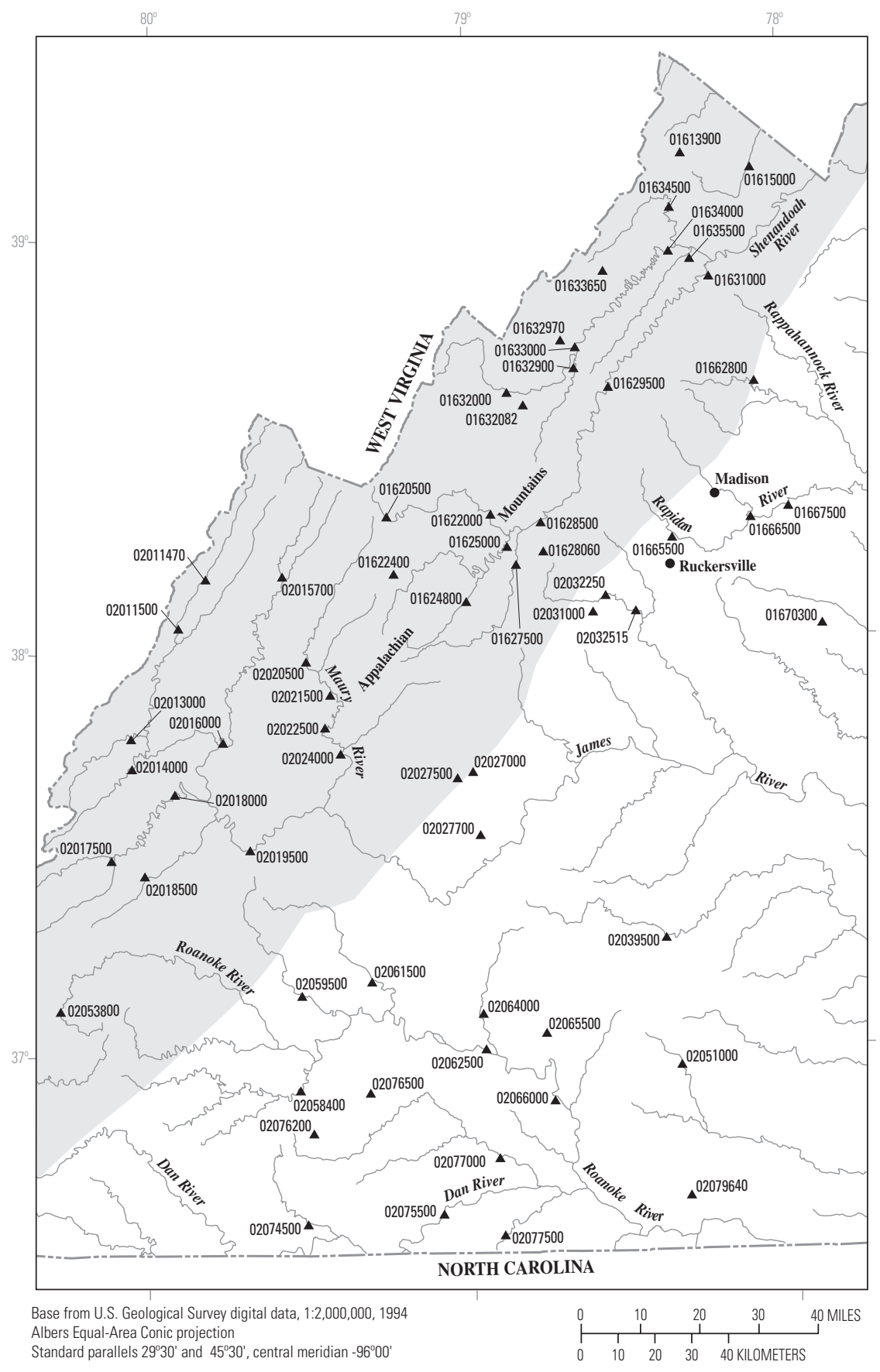

EXPLANATION

02074500_ Streamgage and number

Figure 59. Location of streamgages with significant floods during 1994-98 water years for Virginia.-Continued 
Table 48. Maximum stage and discharge for period of record for streamgages having significant floods during 1994-98 water years in Virginia.

$\left[\mathrm{mi}^{2}\right.$, square miles; $\mathrm{ft}$, feet above an arbitrary datum; $\mathrm{ft}^{3} / \mathrm{s}$, cubic feet per second; --, not determined or not applicable; >, greater than. Source: Recurrence intervals calculated from U.S. Geological Survey data. Other data from U.S. Geological Survey reports or databases]

\begin{tabular}{|c|c|c|c|c|c|c|c|c|c|c|c|}
\hline \multirow[b]{2}{*}{$\begin{array}{c}\text { Streamgage } \\
\text { number } \\
\text { (fig. 59) }\end{array}$} & \multirow[b]{2}{*}{ Streamgage name } & \multirow[b]{2}{*}{$\begin{array}{c}\text { Total } \\
\text { drainage } \\
\left(\mathrm{mi}^{2}\right)\end{array}$} & \multicolumn{4}{|c|}{$\begin{array}{c}\text { Maximum stage and discharge for period of record } \\
\text { through } 1998 \text { water year }\end{array}$} & \multicolumn{5}{|c|}{ Significant floods 1994-98 water years } \\
\hline & & & $\begin{array}{l}\text { Period of } \\
\text { record } \\
\text { (water } \\
\text { years) }\end{array}$ & Water year & $\begin{array}{l}\text { Stage } \\
(\mathrm{ft})\end{array}$ & $\begin{array}{l}\text { Discharge } \\
\left(\mathrm{ft}^{3} / \mathrm{s}\right)\end{array}$ & $\begin{array}{c}\text { Date } \\
\text { (month/d } \\
\text { ay/year) }\end{array}$ & $\begin{array}{l}\text { Stage } \\
(\mathrm{ft})\end{array}$ & $\begin{array}{l}\text { Discharge } \\
\left(\mathrm{ft}^{3} / \mathrm{s}\right)\end{array}$ & $\begin{array}{l}\text { Regulated } \\
\text { during } \\
\text { flood }^{1}\end{array}$ & $\begin{array}{c}\text { Recurrence } \\
\text { interval } \\
\text { (years) }\end{array}$ \\
\hline 01613900 & Hogue Creek near Hayfield, VA & 15.0 & $1961-98$ & 1996 & 9.71 & 4,090 & $9 / 6 / 96$ & 9.71 & 4,090 & $\mathrm{~N}$ & $100-200$ \\
\hline 01615000 & $\begin{array}{l}\text { Opequon Creek near Berryville, } \\
\text { VA }\end{array}$ & 57.4 & $1943-97$ & $\begin{array}{l}1988 \\
1943\end{array}$ & $\begin{array}{l}13.49 \\
18.40\end{array}$ & $\begin{array}{r}12,600 \\
--\end{array}$ & $1 / 19 / 96$ & 13.30 & 11,900 & $\mathrm{~N}$ & 100 \\
\hline 01620500 & North River near Stokesville, VA & 17.2 & 1947-98 & $\begin{array}{l}1949 \\
1986\end{array}$ & $\begin{array}{l}10.90 \\
19.80\end{array}$ & $\begin{array}{l}9,530 \\
7,600\end{array}$ & $9 / 6 / 96$ & 9.79 & 3,750 & $\mathrm{~N}$ & $25-50$ \\
\hline 01622000 & North River near Burketown, VA & 379 & $\begin{array}{l}1924, \\
1927-72, \\
1976-98\end{array}$ & 1996 & 36.70 & 70,400 & $9 / 6 / 96$ & 36.70 & 70,400 & $\mathrm{~N}$ & 200 \\
\hline 01622400 & $\begin{array}{l}\text { Buffalo Branch tributary O } 2 \text { near } \\
\text { Christians, VA }\end{array}$ & .49 & 1967-96 & 1996 & 7.68 & 244 & $9 / 6 / 96$ & 7.68 & 244 & $\mathrm{~N}$ & $50-100$ \\
\hline 01624800 & $\begin{array}{l}\text { Christians Creek near Fisherville, } \\
\text { VA }\end{array}$ & 70.1 & $\begin{array}{l}1968-90 \\
1992-97\end{array}$ & 1996 & 16.14 & 16,200 & $9 / 6 / 96$ & 16.14 & 16,200 & $\mathrm{~N}$ & $>500$ \\
\hline 01625000 & Middle River near Grottoes, VA & 375 & $\begin{array}{l}\text { 1924, } \\
1928-98\end{array}$ & 1996 & 35.62 & 44,300 & 9/7/96 & 35.62 & 44,300 & $\mathrm{~N}$ & 200 \\
\hline 01627500 & South River at Harriston, VA & 212 & $\begin{array}{l}1870,1878, \\
1924, \\
1926-51, \\
1969-98\end{array}$ & $\begin{array}{l}1996 \\
1870\end{array}$ & $\begin{array}{l}15.57 \\
18.80\end{array}$ & $\begin{array}{r}28,900 \\
--\end{array}$ & $9 / 6 / 96$ & 15.57 & 28,900 & $\mathrm{~N}$ & 100 \\
\hline 01628060 & White Oak Run near Grottoes, VA & 1.94 & $1980-96$ & 1996 & 6.25 & 530 & 9/6/96 & 6.25 & 530 & $\mathrm{~N}$ & -- \\
\hline 01628500 & $\begin{array}{l}\text { South Fork Shenandoah River near } \\
\text { Lynnwood, VA }\end{array}$ & 1,084 & $1931-98$ & 1996 & 30.84 & 107,000 & $9 / 7 / 96$ & 30.84 & 107,000 & $\mathrm{~N}$ & 100 \\
\hline 01629500 & $\begin{array}{l}\text { South Fork Shenandoah River near } \\
\text { Luray, VA }\end{array}$ & 1,377 & $\begin{array}{l}\text { 1897, 1924, } \\
1926-30, \\
1936, \\
1939-51, \\
1979-98\end{array}$ & 1996 & 26.95 & 112,000 & $9 / 7 / 96$ & 26.95 & 112,000 & $\mathrm{~N}$ & $50-100$ \\
\hline 01631000 & $\begin{array}{l}\text { South Fork Shenandoah River at } \\
\text { Front Royal, VA }\end{array}$ & 1,642 & $\begin{array}{l}1900-05 \\
1931-98\end{array}$ & 1943 & 34.80 & 130,000 & 9/7/96 & 32.57 & 121,000 & $\mathrm{~N}$ & $50-100$ \\
\hline
\end{tabular}


Table 48. Maximum stage and discharge for period of record for streamgages having significant floods during 1994-98 water years in Virginia.-Continued

$\left[\mathrm{mi}^{2}\right.$, square miles; $\mathrm{ft}$, feet above an arbitrary datum; $\mathrm{ft}^{3} / \mathrm{s}$, cubic feet per second; --, not determined or not applicable; >, greater than. Source: Recurrence intervals calculated from U.S. Geological Survey data. Other data from U.S. Geological Survey reports or databases]

\begin{tabular}{|c|c|c|c|c|c|c|c|c|c|c|c|}
\hline \multirow{2}{*}{$\begin{array}{l}\text { Streamgage } \\
\text { number } \\
\text { (fig. 59) }\end{array}$} & \multirow[b]{2}{*}{ Streamgage name } & \multirow{2}{*}{$\begin{array}{c}\text { Total } \\
\text { drainage } \\
\left(\mathrm{mi}^{2}\right)\end{array}$} & \multicolumn{4}{|c|}{$\begin{array}{c}\text { Maximum stage and discharge for period of record } \\
\text { through } 1998 \text { water year }\end{array}$} & \multicolumn{5}{|c|}{ Significant floods 1994-98 water years } \\
\hline & & & $\begin{array}{l}\text { Period of } \\
\text { record } \\
\text { (water } \\
\text { years) }\end{array}$ & Water year & $\begin{array}{l}\text { Stage } \\
(\mathrm{ft})\end{array}$ & $\begin{array}{l}\text { Discharge } \\
\left(\mathrm{ft}^{3} / \mathrm{s}\right)\end{array}$ & $\begin{array}{c}\text { Date } \\
\text { (month/d } \\
\text { ay/year) }\end{array}$ & $\begin{array}{l}\text { Stage } \\
(\mathrm{ft})\end{array}$ & $\begin{array}{l}\text { Discharge } \\
\left(\mathrm{ft}^{3} / \mathrm{s}\right)\end{array}$ & $\begin{array}{l}\text { Regulated } \\
\text { during } \\
\text { flood }^{1}\end{array}$ & $\begin{array}{c}\text { Recurrence } \\
\text { interval } \\
\text { (years) }\end{array}$ \\
\hline 01632000 & $\begin{array}{l}\text { North Fork Shenandoah River at } \\
\text { Cootes Store, VA }\end{array}$ & 210 & $\begin{array}{l}\text { 1878, 1889, } \\
1924, \\
1926-98\end{array}$ & 1996 & 27.86 & 63,400 & $9 / 6 / 96$ & 27.86 & 63,400 & $\mathrm{~N}$ & $>500$ \\
\hline 01632082 & Linville Creek at Broadway, VA & 45.5 & 1986-98 & 1996 & 13.23 & 17,800 & $9 / 6 / 96$ & 13.23 & 17,800 & $\mathrm{~N}$ & -- \\
\hline 01632900 & Smith Creek near New Market, VA & 93.2 & 1960-98 & 1996 & 17.62 & 12,400 & 9/6/96 & 17.62 & 12,400 & $\mathrm{~N}$ & $50-100$ \\
\hline 01632970 & $\begin{array}{l}\text { Crooked Run near Mount Jackson, } \\
\text { VA }\end{array}$ & 6.49 & 1972-96 & 1996 & 11.34 & 5,700 & $1 / 19 / 96$ & 11.34 & 5,700 & $\mathrm{~N}$ & $>500$ \\
\hline 01633000 & $\begin{array}{l}\text { North Fork Shenandoah River at } \\
\text { Mount Jackson, VA }\end{array}$ & 506 & 1943-98 & 1996 & 22.17 & 103,000 & $9 / 6 / 96$ & 22.17 & 103,000 & $\mathrm{~N}$ & $>500$ \\
\hline 01633650 & Pughs Run near Woodstock, VA & 3.66 & $1971-96$ & 1996 & 13.39 & 1,100 & 9/6/96 & 13.39 & 1,100 & $\mathrm{~N}$ & 100 \\
\hline 01634000 & $\begin{array}{l}\text { North Fork Shenandoah River near } \\
\text { Strasburg, VA }\end{array}$ & 768 & $1926-98$ & 1996 & 32.27 & 114,000 & $9 / 7 / 96$ & 32.27 & 114,000 & $\mathrm{~N}$ & $>500$ \\
\hline 01634500 & Cedar Creek near Winchester, VA & 103 & $\begin{array}{l}\text { 1936, } \\
1938-98\end{array}$ & 1943 & 27.00 & 22,000 & $9 / 6 / 96$ & 23.40 & 20,800 & $\mathrm{~N}$ & $50-100$ \\
\hline 01635500 & Passage Creek near Buckton, VA & 87.8 & 1933-98 & 1996 & 15.89 & 23,000 & $9 / 6 / 96$ & 15.89 & 23,000 & $\mathrm{~N}$ & 200 \\
\hline 01645784 & Snakeden Branch at Reston, VA & .79 & $\begin{array}{l}1972-78 \\
1985-96\end{array}$ & 1996 & 7.62 & 1,050 & $9 / 6 / 96$ & 7.62 & 1,050 & $\mathrm{~N}$ & 50 \\
\hline 01661800 & $\begin{array}{l}\text { Bush Mill Stream near Heathsville, } \\
\text { VA }\end{array}$ & 6.82 & 1964-96 & 1979 & 8.52 & 714 & $3 / 3 / 94$ & 8.10 & 625 & $\mathrm{~N}$ & $25-50$ \\
\hline 01662800 & Battle Run near Laurel Mills, VA & 27.6 & $\begin{array}{l}\text { 1959-95, } \\
1998\end{array}$ & 1995 & 14.40 & 9,120 & $6 / 27 / 95$ & 14.40 & 9,120 & $\mathrm{~N}$ & 100 \\
\hline 01665500 & $\begin{array}{l}\text { Rapidan River near Ruckersville, } \\
\text { VA }\end{array}$ & 114 & $\begin{array}{l}\text { 1943-81, } \\
1983-96\end{array}$ & 1995 & 31.30 & 106,000 & $6 / 27 / 95$ & 31.30 & 106,000 & $\mathrm{~N}$ & $>500$ \\
\hline 01666500 & $\begin{array}{l}\text { Robinson River near Locust Dale, } \\
\text { VA }\end{array}$ & 179 & 1943-98 & $\begin{array}{l}1995 \\
1996\end{array}$ & $\begin{array}{l}22.93 \\
23.92\end{array}$ & $\begin{array}{l}25,400 \\
22,100\end{array}$ & $6 / 27 / 95$ & 22.93 & 25,400 & $\mathrm{~N}$ & $25-50$ \\
\hline 01667500 & Rapidan River near Culpeper, VA & 472 & $1931-98$ & 1995 & 30.40 & 59,300 & $6 / 28 / 95$ & 30.40 & 59,300 & $\mathrm{~N}$ & 100 \\
\hline 01668000 & $\begin{array}{l}\text { Rappahannock River near } \\
\text { Fredericksburg, VA }\end{array}$ & 1,596 & 1908-98 & 1943 & 25.90 & 140,000 & $9 / 7 / 96$ & 17.97 & 74,100 & $\mathrm{~N}$ & 25 \\
\hline
\end{tabular}


$\left[\mathrm{mi}^{2}\right.$, square miles; ft, feet above an arbitrary datum; $\mathrm{ft}^{3} / \mathrm{s}$, cubic feet per second; --, not determined or not applicable; >, greater than. Source: Recurrence intervals calculated from U.S. Geological Survey data. Other data from U.S. Geological Survey reports or databases]

\begin{tabular}{|c|c|c|c|c|c|c|c|c|c|c|c|}
\hline \multirow{2}{*}{$\begin{array}{c}\text { Streamgage } \\
\text { number } \\
\text { (fig. 59) }\end{array}$} & \multirow[b]{2}{*}{ Streamgage name } & \multirow{2}{*}{$\begin{array}{c}\text { Total } \\
\text { drainage } \\
\left(\mathrm{mi}^{2}\right)\end{array}$} & \multicolumn{4}{|c|}{$\begin{array}{c}\text { Maximum stage and discharge for period of record } \\
\text { through } 1998 \text { water year }\end{array}$} & \multicolumn{5}{|c|}{ Significant floods 1994-98 water years } \\
\hline & & & $\begin{array}{l}\text { Period of } \\
\text { record } \\
\text { (water } \\
\text { years) }\end{array}$ & Water year & $\begin{array}{l}\text { Stage } \\
(\mathrm{ft})\end{array}$ & $\begin{array}{l}\text { Discharge } \\
\left(\mathrm{ft}^{3} / \mathrm{s}\right)\end{array}$ & $\begin{array}{c}\text { Date } \\
\text { (month/d } \\
\text { ay/year) }\end{array}$ & $\begin{array}{l}\text { Stage } \\
(\mathrm{ft})\end{array}$ & $\begin{array}{l}\text { Discharge } \\
\left(\mathrm{ft}^{3} / \mathrm{s}\right)\end{array}$ & $\begin{array}{c}\text { Regulated } \\
\text { during } \\
\text { flood }^{1}\end{array}$ & $\begin{array}{c}\text { Recurrence } \\
\text { interval } \\
\text { (years) }\end{array}$ \\
\hline 01669520 & Dragon Swamp at Mascot, VA & 108 & $1982-98$ & 1998 & 9.39 & 2,800 & $2 / 6 / 98$ & 9.39 & 2,800 & $\mathrm{~N}$ & -- \\
\hline 01670300 & Contrary Creek near Mineral, VA & 5.53 & 1976-96 & 1994 & 6.94 & 7,050 & $11 / 28 / 93$ & 6.94 & 7,050 & $\mathrm{~N}$ & $>500$ \\
\hline 01671020 & $\begin{array}{l}\text { North Anna River at Hart Corner } \\
\text { near Doswell, VA }\end{array}$ & 463 & 1980-98 & 1994 & 21.80 & 12,000 & $3 / 29 / 94$ & 21.80 & 12,000 & $\mathrm{~N}$ & -- \\
\hline 01673000 & $\begin{array}{l}\text { Pamunkey River near Hanover, } \\
\text { VA }\end{array}$ & 1,081 & $\begin{array}{l}1928, \\
1942-92, \\
1994-98\end{array}$ & $\begin{array}{l}1969 \\
1928\end{array}$ & $\begin{array}{l}31.12 \\
32.60\end{array}$ & $\begin{array}{r}40,300 \\
--\end{array}$ & $3 / 31 / 94$ & 25.16 & 21,200 & $\mathrm{~N}$ & 25 \\
\hline 01673800 & Po River near Spotsylvania, VA & 77.4 & $1963-98$ & 1972 & 19.03 & 10,900 & $11 / 28 / 93$ & 17.10 & 8,070 & $\mathrm{~N}$ & 50 \\
\hline 02011470 & Back Creek at Sunrise, VA & 76.1 & 1985-98 & 1996 & 11.99 & 5,690 & $1 / 19 / 96$ & 11.99 & 5,690 & $\mathrm{Y}$ & -- \\
\hline 02011500 & $\begin{array}{l}\text { Back Creek near Mountain Grove, } \\
\text { VA }\end{array}$ & 134 & $\begin{array}{l}1913, \\
1951-98\end{array}$ & $\begin{array}{l}1996 \\
1913\end{array}$ & $\begin{array}{l}12.41 \\
17.00\end{array}$ & $\begin{array}{r}18,400 \\
--\end{array}$ & $1 / 19 / 96$ & 12.41 & 18,400 & $\mathrm{Y}$ & -- \\
\hline 02013000 & Dunlap Creek near Covington, VA & 164 & $\begin{array}{l}1913 \\
1929-98\end{array}$ & $\begin{array}{l}1972 \\
1913\end{array}$ & $\begin{array}{l}15.65 \\
18.00\end{array}$ & $\begin{array}{r}27,400 \\
--\end{array}$ & $1 / 19 / 96$ & 13.33 & 17,100 & $\mathrm{~N}$ & 50 \\
\hline 02014000 & Potts Creek near Covington, VA & 153 & $\begin{array}{l}1878,1913, \\
1929-56, \\
1966-81, \\
1983-98\end{array}$ & 1986 & 13.46 & 15,400 & $1 / 19 / 96$ & 11.32 & 9,860 & $\mathrm{~N}$ & 25 \\
\hline 02015700 & $\begin{array}{r}\text { Bullpasture River at } \\
\text { Williamsville, VA }\end{array}$ & 110 & $1961-98$ & 1986 & 14.39 & 22,900 & $9 / 6 / 96$ & 12.50 & 21,600 & $\mathrm{~N}$ & 200 \\
\hline 02016000 & $\begin{array}{l}\text { Cowpasture River near Clifton } \\
\text { Forge, VA }\end{array}$ & 461 & $\begin{array}{l}1913, \\
1926-98\end{array}$ & 1913 & 20.80 & 45,000 & 9/7/96 & 16.91 & 30,100 & $\mathrm{~N}$ & $25-50$ \\
\hline 02017500 & Johns Creek at New Castle, VA & 104 & 1927-98 & $\begin{array}{l}1935 \\
1972\end{array}$ & $\begin{array}{l}10.80 \\
12.48\end{array}$ & $\begin{array}{l}8,000 \\
7,960\end{array}$ & $1 / 19 / 96$ & 11.78 & 6,760 & $\mathrm{~N}$ & $10-25$ \\
\hline 02018000 & Craig Creek at Parr, VA & 329 & 1926-98 & 1986 & 24.76 & 58,500 & $1 / 19 / 96$ & 16.71 & 21,600 & $\mathrm{~N}$ & $25-50$ \\
\hline 02018500 & Catawba Creek near Catawba, VA & 34.3 & $\begin{array}{l}\text { 1940, } \\
1944-98\end{array}$ & 1986 & 19.19 & 21,200 & $6 / 28 / 95$ & 11.34 & 8,640 & $\mathrm{~N}$ & 50 \\
\hline 02019500 & James River at Buchanan, VA & 2,075 & $\begin{array}{l}1878,1886, \\
1889, \\
1893-1998\end{array}$ & 1986 & 38.84 & 179,000 & $1 / 20 / 96$ & 29.24 & 103,000 & $\mathrm{Y}$ & -- \\
\hline
\end{tabular}


Table 48. Maximum stage and discharge for period of record for streamgages having significant floods during 1994-98 water years in Virginia.-Continued

$\left[\mathrm{mi}^{2}\right.$, square miles; $\mathrm{ft}$, feet above an arbitrary datum; $\mathrm{ft}^{3} / \mathrm{s}$, cubic feet per second; --, not determined or not applicable; >, greater than. Source: Recurrence intervals calculated from U.S. Geological Survey data. Other data from U.S. Geological Survey reports or databases]

\begin{tabular}{|c|c|c|c|c|c|c|c|c|c|c|c|}
\hline \multirow[b]{2}{*}{$\begin{array}{l}\text { Streamgage } \\
\text { number } \\
\text { (fig. 59) }\end{array}$} & \multirow[b]{2}{*}{ Streamgage name } & \multirow{2}{*}{$\begin{array}{c}\text { Total } \\
\text { drainage } \\
\left(\mathrm{mi}^{2}\right)\end{array}$} & \multicolumn{4}{|c|}{$\begin{array}{c}\text { Maximum stage and discharge for period of record } \\
\text { through } 1998 \text { water year }\end{array}$} & \multicolumn{5}{|c|}{ Significant floods $1994-98$ water years } \\
\hline & & & $\begin{array}{l}\text { Period of } \\
\text { record } \\
\text { (water } \\
\text { years) }\end{array}$ & Water year & $\begin{array}{l}\text { Stage } \\
(\mathrm{ft})\end{array}$ & $\begin{array}{l}\text { Discharge } \\
\left(\mathrm{ft}^{3} / \mathrm{s}\right)\end{array}$ & $\begin{array}{c}\text { Date } \\
\text { (month/d } \\
\text { ay/year) }\end{array}$ & $\begin{array}{l}\text { Stage } \\
(\mathrm{ft})\end{array}$ & $\begin{array}{l}\text { Discharge } \\
\left(\mathrm{ft}^{3} / \mathrm{s}\right)\end{array}$ & $\begin{array}{c}\text { Regulated } \\
\text { during } \\
\text { flood }^{1}\end{array}$ & $\begin{array}{c}\text { Recurrence } \\
\text { interval } \\
\text { (years) }\end{array}$ \\
\hline 02020500 & $\begin{array}{l}\text { Calfpasture River above Mill } \\
\text { Creek at Goshen, VA }\end{array}$ & 144 & $1939-96$ & 1986 & 20.23 & 56,300 & $9 / 6 / 96$ & 16.38 & 35,800 & $\mathrm{~N}$ & $100-200$ \\
\hline 02021500 & $\begin{array}{l}\text { Maury River at Rockbridge Baths, } \\
\text { VA }\end{array}$ & 329 & 1929-98 & 1986 & 19.19 & 87,700 & $9 / 6 / 96$ & 14.28 & 42,000 & $\mathrm{~N}$ & $50-100$ \\
\hline 02022500 & Kerrs Creek near Lexington, VA & 35.0 & 1927-98 & $\begin{array}{l}1950 \\
1995\end{array}$ & $\begin{array}{l}13.80 \\
15.44\end{array}$ & $\begin{array}{l}23,000 \\
21,700\end{array}$ & $6 / 28 / 95$ & 15.44 & 21,700 & $\mathrm{~N}$ & $200-500$ \\
\hline 02024000 & Maury River near Buena Vista, VA & 646 & $\begin{array}{l}\text { 1936, } \\
1939-98\end{array}$ & 1969 & 31.23 & 105,000 & $6 / 28 / 95$ & 19.48 & 37,500 & $\mathrm{~N}$ & $25-50$ \\
\hline 02027000 & Tye River near Lovingston, VA & 92.8 & 1939-98 & 1969 & 29.00 & 80,000 & $9 / 6 / 96$ & 16.05 & 17,100 & $\mathrm{~N}$ & 50 \\
\hline 02027500 & Piney River at Piney River, VA & 47.6 & 1949-98 & 1969 & 13.80 & 38,000 & $9 / 6 / 96$ & 12.85 & 27,400 & $\mathrm{~N}$ & $200-500$ \\
\hline 02027700 & $\begin{array}{l}\text { Buffalo River tributary near } \\
\text { Amherst, VA }\end{array}$ & .46 & $\begin{array}{l}1966-78 \\
1980-96\end{array}$ & $\begin{array}{l}1985 \\
1996\end{array}$ & $\begin{array}{l}2.96 \\
7.33\end{array}$ & $\begin{array}{l}720 \\
196\end{array}$ & 9/6/96 & 7.33 & 196 & $\mathrm{~N}$ & $10-25$ \\
\hline 02031000 & $\begin{array}{l}\text { Mechums River near White Hall, } \\
\text { VA }\end{array}$ & 95.4 & $\begin{array}{l}\text { 1943-51, } \\
1959, \\
1979-98\end{array}$ & 1943 & 30.30 & 20,000 & $9 / 6 / 96$ & 24.79 & 14,200 & $\mathrm{~N}$ & 25 \\
\hline 02032250 & $\begin{array}{l}\text { Moormans River near Free Union, } \\
\text { VA }\end{array}$ & 74.6 & $\begin{array}{l}\text { 1972, } \\
1979-97\end{array}$ & 1995 & 22.28 & 19,100 & $6 / 28 / 95$ & 22.28 & 19,100 & $\mathrm{~N}$ & 25 \\
\hline 02032515 & $\begin{array}{l}\text { South Fork Rivanna River near } \\
\text { Charlottesville, VA }\end{array}$ & 260 & 1979-97 & 1996 & 24.39 & 16,100 & $9 / 6 / 96$ & 24.39 & 16,100 & $\mathrm{Y}$ & -- \\
\hline 02039500 & $\begin{array}{l}\text { Appomattox River at Farmville, } \\
\text { VA }\end{array}$ & 303 & 1926-98 & 1972 & 29.70 & 33,100 & 9/7/96 & 24.02 & 17,900 & $\mathrm{~N}$ & $25-50$ \\
\hline 02049500 & $\begin{array}{l}\text { Blackwater River near Franklin, } \\
\text { VA }\end{array}$ & 617 & $\begin{array}{l}1940 \\
1942-98\end{array}$ & 1940 & 22.00 & 21,000 & 2/7/98 & 15.27 & 7,250 & $\mathrm{~N}$ & $10-25$ \\
\hline 02051000 & $\begin{array}{l}\text { North Meherrin River near } \\
\text { Lunenburg, VA }\end{array}$ & 55.6 & $\begin{array}{l}1940, \\
1947-75, \\
1977-80, \\
1982-98\end{array}$ & $\begin{array}{l}1972 \\
1940\end{array}$ & $\begin{array}{l}28.30 \\
48.00\end{array}$ & $\begin{array}{r}14,400 \\
--\end{array}$ & $9 / 6 / 96$ & 22.94 & 6,390 & $\mathrm{~N}$ & $10-25$ \\
\hline 02053800 & $\begin{array}{l}\text { South Fork Roanoke River near } \\
\text { Shawsville, VA }\end{array}$ & 110 & 1961-98 & 1972 & 11.12 & 14,200 & $9 / 6 / 96$ & 8.88 & 9,690 & $\mathrm{~N}$ & $10-25$ \\
\hline 02058400 & Pigg River near Sandy Level, VA & 350 & 1964-98 & 1987 & 31.12 & 65,600 & 9/6/96 & 27.32 & 33,700 & $\mathrm{~N}$ & $25-50$ \\
\hline
\end{tabular}


Table 48. Maximum stage and discharge for period of record for streamgages having significant floods during 1994-98 water years in Virginia.-Continued

$\left[\mathrm{mi}^{2}\right.$, square miles; $\mathrm{ft}$, feet above an arbitrary datum; $\mathrm{ft}^{3} / \mathrm{s}$, cubic feet per second; --, not determined or not applicable; >, greater than. Source: Recurrence intervals calculated from U.S. Geological Survey data. Other data from U.S. Geological Survey reports or databases]

\begin{tabular}{|c|c|c|c|c|c|c|c|c|c|c|c|}
\hline \multirow{2}{*}{$\begin{array}{c}\text { Streamgage } \\
\text { number } \\
\text { (fig. 59) }\end{array}$} & \multirow[b]{2}{*}{ Streamgage name } & \multirow{2}{*}{$\begin{array}{c}\text { Total } \\
\text { drainage } \\
\left(\mathrm{mi}^{2}\right)\end{array}$} & \multicolumn{4}{|c|}{$\begin{array}{c}\text { Maximum stage and discharge for period of record } \\
\text { through } 1998 \text { water year }\end{array}$} & \multicolumn{5}{|c|}{ Significant floods 1994-98 water years } \\
\hline & & & $\begin{array}{c}\text { Period of } \\
\text { record } \\
\text { (water } \\
\text { years) }\end{array}$ & Water year & $\begin{array}{l}\text { Stage } \\
(\mathrm{ft})\end{array}$ & $\begin{array}{l}\text { Discharge } \\
\left(\mathrm{ft}^{3} / \mathrm{s}\right)\end{array}$ & $\begin{array}{c}\text { Date } \\
\text { (month/d } \\
\text { ay/year) }\end{array}$ & $\begin{array}{l}\text { Stage } \\
(\mathrm{ft})\end{array}$ & $\begin{array}{l}\text { Discharge } \\
\left(\mathrm{ft}^{3} / \mathrm{s}\right)\end{array}$ & $\begin{array}{c}\text { Regulated } \\
\text { during } \\
\text { flood }^{1}\end{array}$ & $\begin{array}{c}\text { Recurrence } \\
\text { interval } \\
\text { (years) }\end{array}$ \\
\hline 02059500 & Goose Creek near Huddleston, VA & 188 & $\begin{array}{l}1924, \\
1926-27, \\
1930-98\end{array}$ & 1987 & 37.49 & 53,200 & $6 / 29 / 95$ & 25.80 & 20,400 & $\mathrm{~N}$ & $10-25$ \\
\hline 02061500 & Big Otter River near Evington, VA & 320 & 1937-98 & 1995 & 29.93 & 45,900 & $\begin{array}{l}6 / 23 / 95 \\
9 / 6 / 96\end{array}$ & $\begin{array}{l}29.93 \\
22.98\end{array}$ & $\begin{array}{l}45,900 \\
29,200\end{array}$ & $\begin{array}{l}\mathrm{N} \\
\mathrm{N}\end{array}$ & $\begin{array}{r}50-100 \\
25\end{array}$ \\
\hline 02062500 & $\begin{array}{l}\text { Roanoke (Staunton) River at } \\
\text { Brookneal, VA }\end{array}$ & 2,415 & $\begin{array}{l}1878, \\
1924-76, \\
1978-98\end{array}$ & 1940 & 46.00 & 130,000 & 9/7/96 & 38.78 & 80,300 & $\mathrm{Y}$ & -- \\
\hline 02064000 & Falling River near Naruna, VA & 173 & $\begin{array}{l}\text { 1930-34, } \\
1940, \\
1942-98\end{array}$ & 1996 & 36.14 & 62,800 & $9 / 6 / 96$ & 36.14 & 62,800 & $\mathrm{~N}$ & $>500$ \\
\hline 02065500 & Cub Creek at Phenix, VA & 98.0 & $\begin{array}{l}1940 \\
1947-98\end{array}$ & 1996 & 21.89 & 15,200 & $9 / 6 / 96$ & 21.89 & 15,200 & $\mathrm{~N}$ & $200-500$ \\
\hline 02066000 & $\begin{array}{l}\text { Roanoke (Staunton) River at } \\
\text { Randolph, VA }\end{array}$ & 2,977 & $\begin{array}{l}1878, \\
1901-13, \\
1915-98\end{array}$ & 1940 & 41.60 & 150,000 & $9 / 7 / 96$ & 34.94 & 89,300 & $\mathrm{Y}$ & -- \\
\hline 02074500 & Sandy River near Danville, VA & 112 & 1930-98 & 1940 & 17.38 & 23,000 & $9 / 6 / 96$ & 11.06 & 13,000 & $\mathrm{~N}$ & 25 \\
\hline 02075500 & Dan River at Paces, VA & 2,550 & $\begin{array}{l}1940, \\
1951-75, \\
1977-98\end{array}$ & 1972 & 33.15 & 64,800 & $9 / 7 / 96$ & 31.43 & 56,500 & $\mathrm{~N}$ & $25-50$ \\
\hline 02076200 & Bearskin Creek near Chatham, VA & 4.06 & $\begin{array}{l}1967-78 \\
1980-84 \\
1986-96\end{array}$ & 1995 & 19.90 & 2,850 & $6 / 29 / 95$ & 19.90 & 2,850 & $\mathrm{~N}$ & $50-100$ \\
\hline 02076500 & Georges Creek near Gretna, VA & 9.24 & 1950-97 & 1996 & 10.02 & 2,260 & $9 / 6 / 96$ & 10.02 & 2,260 & $\mathrm{~N}$ & 50 \\
\hline 02077000 & Banister River at Halifax, VA & 547 & $\begin{array}{l}\text { 1905, } \\
1929-98\end{array}$ & 1944 & 40.80 & 50,000 & $9 / 7 / 96$ & 33.45 & 23,900 & $\mathrm{~N}$ & $25-50$ \\
\hline 02077500 & Hyco River near Denniston, VA & 289 & $\begin{array}{l}1928, \\
1930-34, \\
1945, \\
1951-98\end{array}$ & $\begin{array}{l}1975 \\
1928\end{array}$ & $\begin{array}{l}24.27 \\
26.40\end{array}$ & $\begin{array}{r}10,800 \\
--\end{array}$ & $9 / 7 / 96$ & 23.16 & 9,430 & $\mathrm{Y}$ & -- \\
\hline 02079640 & Allen Creek near Boydton, VA & 53.4 & 1962-96 & 1996 & 22.93 & 6,870 & $9 / 6 / 96$ & 22.93 & 6,870 & $\mathrm{~N}$ & 50 \\
\hline
\end{tabular}


Table 48. Maximum stage and discharge for period of record for streamgages having significant floods during 1994-98 water years in Virginia.-Continued

$\left[\mathrm{mi}^{2}\right.$, square miles; $\mathrm{ft}$, feet above an arbitrary datum; $\mathrm{ft}^{3} / \mathrm{s}$, cubic feet per second; --, not determined or not applicable; >, greater than. Source: Recurrence intervals calculated from U.S. Geological Survey data. Other data from U.S. Geological Survey reports or databases]

\begin{tabular}{|c|c|c|c|c|c|c|c|c|c|c|c|}
\hline \multirow{2}{*}{$\begin{array}{c}\text { Streamgage } \\
\text { number } \\
\text { (fig. 59) }\end{array}$} & \multirow[b]{2}{*}{ Streamgage name } & \multirow{2}{*}{$\begin{array}{c}\text { Total } \\
\text { drainage } \\
\left(\mathrm{mi}^{2}\right)\end{array}$} & \multicolumn{4}{|c|}{$\begin{array}{c}\text { Maximum stage and discharge for period of record } \\
\text { through } 1998 \text { water year }\end{array}$} & \multicolumn{5}{|c|}{ Signific ant floods $1994-98$ water years } \\
\hline & & & $\begin{array}{c}\text { Period of } \\
\text { record } \\
\text { (water } \\
\text { years) }\end{array}$ & Water year & $\begin{array}{l}\text { Stage } \\
(\mathrm{ft})\end{array}$ & $\begin{array}{l}\text { Discharge } \\
\left(\mathrm{ft}^{3} / \mathrm{s}\right)\end{array}$ & $\begin{array}{c}\text { Date } \\
\text { (month/d } \\
\text { ay/year) }\end{array}$ & $\begin{array}{l}\text { Stage } \\
(\mathrm{ft})\end{array}$ & $\begin{array}{l}\text { Discharge } \\
\left(\mathrm{ft}^{3} / \mathrm{s}\right)\end{array}$ & $\begin{array}{l}\text { Regulated } \\
\text { during } \\
\text { flood }^{1}\end{array}$ & $\begin{array}{c}\text { Recurrence } \\
\text { interval } \\
\text { (years) }\end{array}$ \\
\hline 03164000 & New River near Galax, VA & 1,131 & $1930-98$ & 1940 & 25.70 & 141,000 & $1 / 15 / 95$ & 15.23 & 68,700 & $\mathrm{~N}$ & $50-100$ \\
\hline 03168000 & New River at Allisonia, VA & 2,202 & 1930-98 & 1940 & 23.42 & 185,000 & $1 / 15 / 95$ & 16.56 & 108,000 & $\mathrm{~N}$ & 100 \\
\hline 03168750 & $\begin{array}{l}\text { Thorne Springs Branch near } \\
\text { Dublin, VA }\end{array}$ & 4.77 & $1957-96$ & $\begin{array}{l}1995 \\
1973\end{array}$ & $\begin{array}{l}7.41 \\
8.01\end{array}$ & $\begin{array}{l}3,760 \\
2,200\end{array}$ & 7/7/95 & 7.41 & 3,760 & $\mathrm{~N}$ & $>500$ \\
\hline 03171000 & New River at Radford, VA & 2,748 & $\begin{array}{l}1878, \\
1896-1998\end{array}$ & 1940 & 35.96 & 218,000 & $1 / 15 / 95$ & 24.04 & 108,000 & $\mathrm{Y}$ & -- \\
\hline 03176500 & New River at Glen Lyn, VA & 3,768 & $\begin{array}{l}1878 \\
1915-98\end{array}$ & 1878 & 33.10 & 240,000 & $1 / 16 / 95$ & 19.83 & 125,000 & $\mathrm{Y}$ & -- \\
\hline 03177710 & Bluestone River at Falls Mills, VA & 44.2 & $1981-97$ & 1996 & 8.66 & 1,560 & $1 / 27 / 96$ & 8.66 & 1,560 & $\mathrm{~N}$ & -- \\
\hline 03209000 & $\begin{array}{l}\text { Pound River below Flannagan } \\
\text { Dam near Haysi, VA }\end{array}$ & 221 & $\begin{array}{l}1920 \\
1927-98\end{array}$ & $\begin{array}{l}1929 \\
1957\end{array}$ & $\begin{array}{l}16.50 \\
18.65\end{array}$ & $\begin{array}{l}30,000 \\
27,300\end{array}$ & $4 / 14 / 94$ & 7.96 & 4,240 & $\mathrm{Y}$ & -- \\
\hline 03471200 & $\begin{array}{l}\text { South Fork Holston River at Teas, } \\
\text { VA }\end{array}$ & 31.1 & $\begin{array}{l}1967-80 \\
1982-95\end{array}$ & 1994 & 17.61 & 7,660 & 2/11/94 & 17.61 & 7,660 & $\mathrm{~N}$ & $200-500$ \\
\hline 03471500 & $\begin{array}{l}\text { South Fork Holston River at } \\
\text { Riverside near Chilhowie, VA }\end{array}$ & 76.1 & $\begin{array}{l}1908-09 \\
1921-31 \\
1942-98\end{array}$ & 1978 & 10.20 & 9,600 & $1 / 15 / 95$ & 8.68 & 5,820 & $\mathrm{~N}$ & 50 \\
\hline 03524900 & Stony Creek at Ka, VA & 30.9 & $1981-96$ & 1994 & 7.81 & 10,800 & $3 / 28 / 94$ & 7.81 & 10,800 & $\mathrm{~N}$ & -- \\
\hline
\end{tabular}

Regulated during flood: $\mathrm{N}, \mathrm{no} ; \mathrm{Y}$, yes. 


\section{Washington}

Flooding occurred on every major river in western Washington in late November 1995. The Skagit River near Concrete (streamgage 12194000, table 49) had its greatest discharge since 1921. Other major floods were on the Snoqualmie River, which was 7 feet above flood stage, and the Nooksack River, which was 6 feet above flood stage (National Oceanic and Atmospheric Administration, 1995a). Most rivers crested near or at record levels. In the towns of Duvall and Carnation (fig. 60, inset A), 15,000 people were stranded as the flooded rivers covered the few roads in and out of the towns. A state of emergency was declared in 16 counties in the State, and an estimated \$3 million was needed for road repairs. Property damage was more than $\$ 10$ million (National Oceanic and Atmospheric Administration, 1995b). For the month of November, most areas had twice their normal average rainfall, and many areas fell just short of setting records for the wettest November on record (National Oceanic and Atmospheric Administration, 1995a).

Some of the worst flooding in more than 60 years took place in Washington during February 7-10, 1996. Damage statewide was estimated at \$223 million (National Oceanic and Atmospheric Administration, 1996b). The Chehalis, Yakima, Cowlitz, and Klickitat Rivers all had record discharges (table 49). A state of emergency was declared in 13 counties. Overall it was the wettest winter season ever. The normal, winter precipitation at Seattle-Tacoma International Airport (Sea Tac) is 21.11 inches, the previous record was 30.6 inches, and for 1995-96 the total was 32.46 inches (National Oceanic and Atmospheric Administration, 1995a; 1996a). Thirty-eight streamgages had record discharges during February 1996 (table 49).

The 1996-97 winter had excessive precipitation and snowmelt periods that caused flooding throughout the State. In January 1997, the runoff from melting snow and rain caused mudslides and sinkholes in the Seattle area. The cleanup from the mudslides and repairs to roads and bridges was estimated to be \$20 million (National Oceanic and Atmospheric Administration, 1997b). Lake Sammamish was about 7 feet above normal, its highest level since 1962, and many lawns and docks were flooded. Flooding occurred in western Washington in February and in eastern Washington during March through May as the deep snowpack melted.

\section{References}

National Oceanic and Atmospheric Administration (NOAA), 1995a-97a, Climatological data (by State): Asheville, North Carolina, National Climatic Data Center, various months. National Oceanic and Atmospheric Administration (NOAA), 1995b-97b, Storm data (by State): Asheville, North Carolina, National Climatic Data Center, various months. 


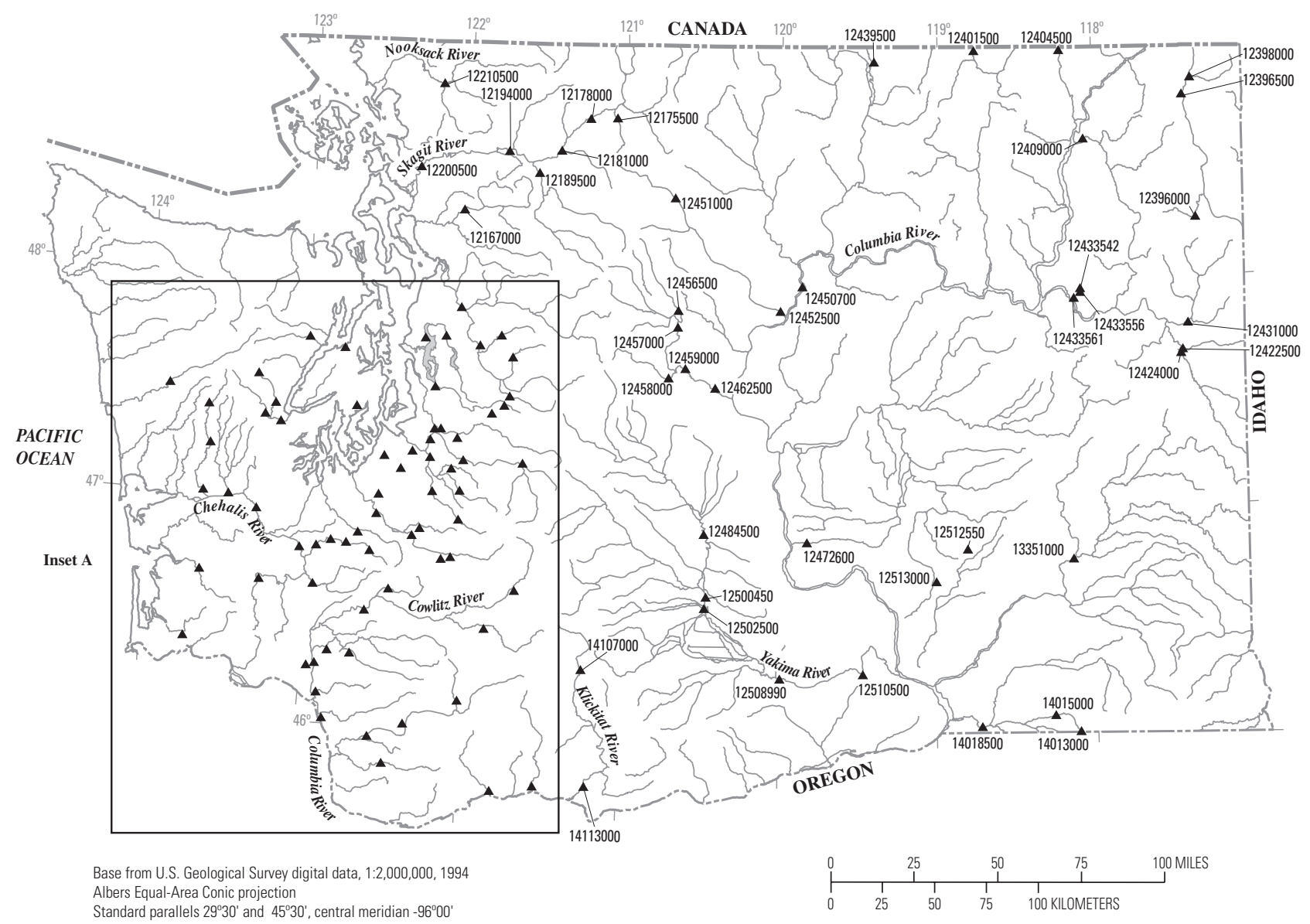

EXPLANATION

12510500 S Streamgage and number

Figure 60. Location of streamgages with significant floods during 1994-98 water years for Washington. 
Inset A

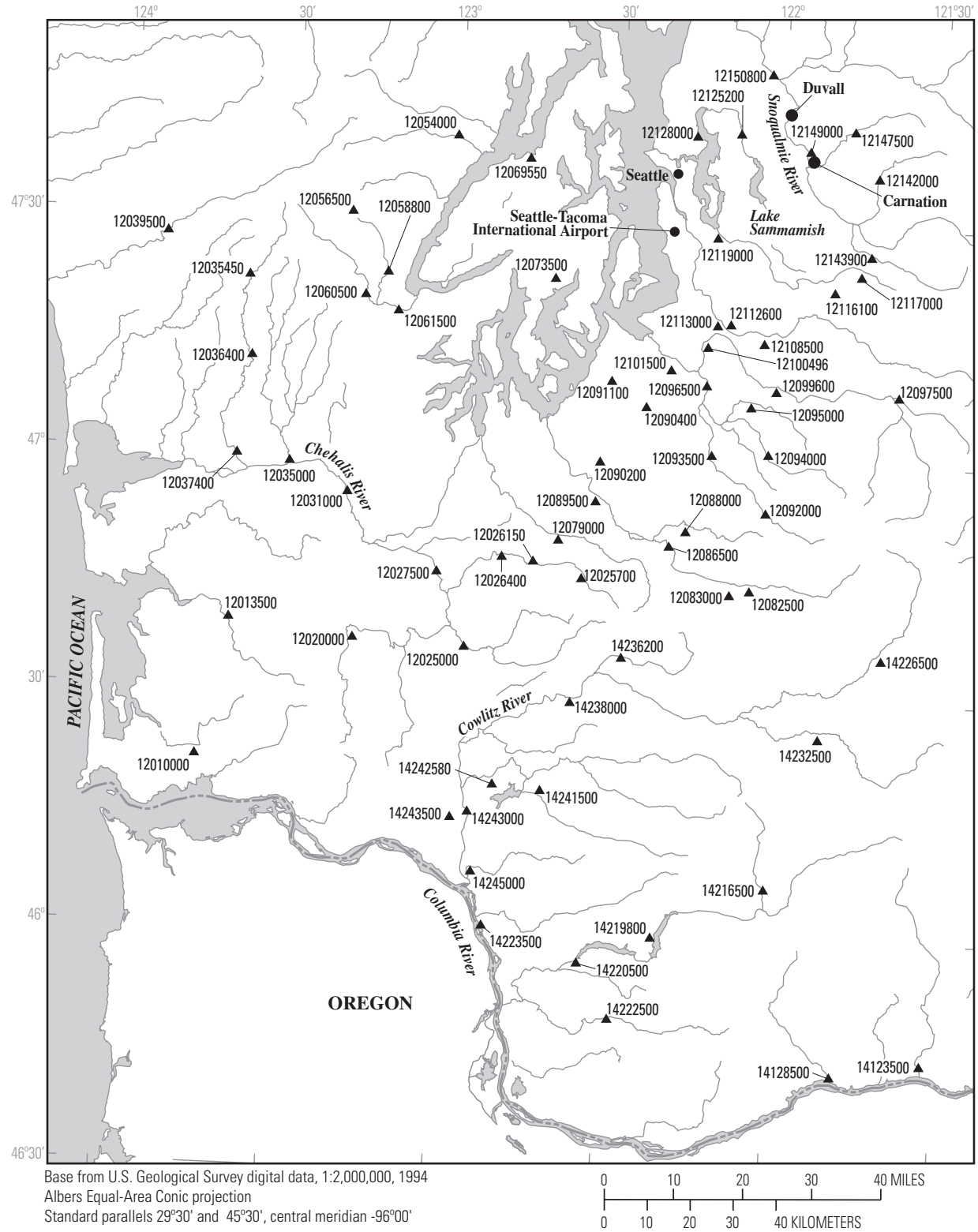

EXPLANATION

14222500 _Streamgage and number

Figure 60. Location of streamgages with significant floods during 1994-98 water years for Washington.-Continued 
Table 49. Maximum stage and discharge for period of record for streamgages having significant floods during 1994-98 water years in Washington.

$\left[\mathrm{mi}^{2}\right.$, square miles; ft, feet above an arbitrary datum; $\mathrm{ft}^{3} / \mathrm{s}$, cubic feet per second; --, not determined or not applicable; >, greater than. Source: Recurrence intervals calculated from U.S. Geological Survey data. Other data from U.S. Geological Survey reports or databases]

\begin{tabular}{|c|c|c|c|c|c|c|c|c|c|c|c|}
\hline \multirow{2}{*}{$\begin{array}{l}\text { Streamgage } \\
\text { number } \\
\text { (fig. 60) }\end{array}$} & \multirow[b]{2}{*}{ Streamgage name } & \multirow{2}{*}{$\begin{array}{l}\text { Total } \\
\text { drainage } \\
\left(\mathrm{mi}^{2}\right)\end{array}$} & \multicolumn{4}{|c|}{$\begin{array}{l}\text { Maximum stage and discharge for period of record } \\
\text { through } 1998 \text { water year }\end{array}$} & \multicolumn{5}{|c|}{ Significant floods $1994-98$ water years } \\
\hline & & & $\begin{array}{l}\text { Period of } \\
\text { record } \\
\text { (water } \\
\text { years) }\end{array}$ & Water year & $\begin{array}{l}\text { Stage } \\
(\mathrm{ft})\end{array}$ & $\begin{array}{l}\text { Discharge } \\
\qquad\left(\mathrm{ft}^{3} / \mathrm{s}\right)\end{array}$ & $\begin{array}{c}\text { Date } \\
\text { (month/d } \\
\text { ay/year) }\end{array}$ & $\begin{array}{l}\text { Stage } \\
(\mathrm{ft})\end{array}$ & $\begin{array}{l}\text { Discharge } \\
\left(\mathrm{ft}^{3} / \mathrm{s}\right)\end{array}$ & $\begin{array}{l}\text { Regulated } \\
\text { during } \\
\text { flood }^{1}\end{array}$ & $\begin{array}{l}\text { Recurrence } \\
\text { interval } \\
\text { (years) }\end{array}$ \\
\hline 12010000 & Naselle River near Naselle, WA & 54.8 & $1930-98$ & 1997 & 19.26 & 12,600 & $3 / 18 / 97$ & 19.26 & 12,600 & $\mathrm{~N}$ & $>100$ \\
\hline 12013500 & Willapa River near Willapa, WA & 130 & $\begin{array}{l}1949-56 \\
1958-59 \\
1962-98\end{array}$ & 1995 & 27.28 & 14,800 & $\begin{array}{l}12 / 20 / 94 \\
3 / 19 / 97\end{array}$ & $\begin{array}{l}27.28 \\
24.53\end{array}$ & $\begin{array}{l}14,800 \\
12,100\end{array}$ & $\begin{array}{l}\mathrm{N} \\
\mathrm{N}\end{array}$ & $\begin{array}{r}50-100 \\
10-25\end{array}$ \\
\hline 12020000 & Chehalis River near Doty, WA & 113 & 1940-98 & 1996 & 20.37 & 28,900 & $2 / 8 / 96$ & 20.37 & 28,900 & $\mathrm{~N}$ & $50-100$ \\
\hline 12025000 & $\begin{array}{l}\text { Newaukum River near Chehalis, } \\
\text { WA }\end{array}$ & 155 & $\begin{array}{l}1929-31 \\
1943-81 \\
1983-98\end{array}$ & $\begin{array}{l}1996 \\
1954\end{array}$ & $\begin{array}{l}13.54 \\
13.62\end{array}$ & $\begin{array}{r}13,300 \\
7,880\end{array}$ & $2 / 8 / 96$ & 13.54 & 13,300 & $\mathrm{~N}$ & $>100$ \\
\hline 12025700 & $\begin{array}{l}\text { Skookumchuck River near Vail, } \\
\text { WA }\end{array}$ & 40.0 & $1968-98$ & 1996 & 11.24 & 8,350 & $2 / 8 / 96$ & 11.24 & 8,350 & $\mathrm{~N}$ & $25-50$ \\
\hline 12026150 & $\begin{array}{l}\text { Skookumchuck River below } \\
\text { Bloody Run Creek near } \\
\text { Centralia, WA }\end{array}$ & 65.9 & $\begin{array}{l}1930-33 \\
1940-98\end{array}$ & $\begin{array}{l}1996 \\
1954\end{array}$ & $\begin{array}{l}13.41 \\
48.59\end{array}$ & $\begin{array}{l}9,020 \\
6,710\end{array}$ & $2 / 8 / 96$ & 13.41 & 9,020 & Y & -- \\
\hline 12026400 & $\begin{array}{l}\text { Skookumchuck River near Bucoda, } \\
\text { WA }\end{array}$ & 112 & 1968-98 & 1996 & 17.87 & 11,300 & $2 / 8 / 96$ & 17.87 & 11,300 & $\mathrm{Y}$ & -- \\
\hline 12027500 & $\begin{array}{l}\text { Chehalis River near Grand Mound, } \\
\text { WA }\end{array}$ & 895 & 1929-98 & 1996 & 19.98 & 74,800 & 2/9/96 & 19.98 & 74,800 & $\mathrm{~N}$ & $>100$ \\
\hline 12031000 & Chehalis River at Porter, WA & 1,294 & $\begin{array}{l}1947-85 \\
1987-98\end{array}$ & 1996 & 25.22 & 80,700 & $2 / 9 / 96$ & 25.22 & 80,700 & $\mathrm{~N}$ & $>100$ \\
\hline 12035000 & Satsop River near Satsop, WA & 299 & $1930-98$ & $\begin{array}{l}1997 \\
1935\end{array}$ & $\begin{array}{l}38.87 \\
38.90\end{array}$ & $\begin{array}{l}63,600 \\
46,600\end{array}$ & $\begin{array}{l}12 / 20 / 94 \\
3 / 19 / 97\end{array}$ & $\begin{array}{l}37.28 \\
38.87\end{array}$ & $\begin{array}{l}50,600 \\
63,600\end{array}$ & $\begin{array}{l}\mathrm{N} \\
\mathrm{N}\end{array}$ & $\begin{array}{r}50-100 \\
>100\end{array}$ \\
\hline 12035450 & Big Creek near Grisdale, WA & 9.57 & 1973-97 & $\begin{array}{l}1997 \\
1987\end{array}$ & $\begin{array}{l}8.17 \\
9.20\end{array}$ & $\begin{array}{l}4,000 \\
3,340\end{array}$ & $3 / 19 / 97$ & 8.17 & 4,000 & $\mathrm{~N}$ & $25-50$ \\
\hline 12036400 & Schafer Creek near Grisdale, WA & 12.1 & 1987-97 & 1997 & 13.57 & 4,100 & $3 / 19 / 97$ & 13.57 & 4,100 & $\mathrm{~N}$ & $50-100$ \\
\hline 12037400 & $\begin{array}{l}\text { Wynoochee River above Black } \\
\text { Creek near Montesano, WA }\end{array}$ & 155 & 1957-98 & $\begin{array}{l}1997 \\
1957\end{array}$ & $\begin{array}{l}20.21 \\
20.54\end{array}$ & $\begin{array}{l}25,600 \\
24,500\end{array}$ & $3 / 19 / 97$ & 20.21 & 25,600 & $\mathrm{Y}$ & $10-25$ \\
\hline 12039500 & $\begin{array}{l}\text { Quinault River at Quinault Lake, } \\
\text { WA }\end{array}$ & 264 & $\begin{array}{l}1910 \\
1912-22 \\
1926-98\end{array}$ & 1910 & 22.00 & 52,600 & $3 / 19 / 97$ & 19.72 & 46,400 & $\mathrm{~N}$ & $25-50$ \\
\hline
\end{tabular}


Table 49. Maximum stage and discharge for period of record for streamgages having significant floods during 1994-98 water years in Washington.-Continued

$\left[\mathrm{mi}^{2}\right.$, square miles; ft, feet above an arbitrary datum; $\mathrm{ft}^{3} / \mathrm{s}$, cubic feet per second; --, not determined or not applicable; >, greater than. Source: Recurrence intervals calculated from U.S. Geological Survey data. Other data from U.S. Geological Survey reports or databases]

\begin{tabular}{|c|c|c|c|c|c|c|c|c|c|c|c|}
\hline \multirow{2}{*}{$\begin{array}{l}\text { Streamgage } \\
\text { number } \\
\text { (fig. 60) }\end{array}$} & \multirow[b]{2}{*}{ Streamgage name } & \multirow{2}{*}{$\begin{array}{l}\text { Total } \\
\text { drainage } \\
\left(\mathrm{mi}^{2}\right)\end{array}$} & \multicolumn{4}{|c|}{$\begin{array}{c}\text { Maximum stage and discharge for period of record } \\
\text { through } 1998 \text { water year }\end{array}$} & \multicolumn{5}{|c|}{ Significant floods 1994-98 water years } \\
\hline & & & $\begin{array}{l}\text { Period of } \\
\text { record } \\
\text { (water } \\
\text { years) }\end{array}$ & Water year & $\begin{array}{l}\text { Stage } \\
(\mathrm{ft})\end{array}$ & $\begin{array}{l}\text { Discharge } \\
\left(\mathrm{ft}^{3} / \mathrm{s}\right)\end{array}$ & $\begin{array}{c}\text { Date } \\
\text { (month/d } \\
\text { ay/year) }\end{array}$ & $\begin{array}{l}\text { Stage } \\
\text { (ft) }\end{array}$ & $\begin{array}{l}\text { Discharge } \\
\left(\mathrm{ft}^{3} / \mathrm{s}\right)\end{array}$ & $\begin{array}{l}\text { Regulated } \\
\text { during } \\
\text { flood }^{1}\end{array}$ & $\begin{array}{l}\text { Recurrence } \\
\text { interval } \\
\text { (years) }\end{array}$ \\
\hline 12054000 & $\begin{array}{l}\text { Duckabush River near Brinnon, } \\
\text { WA }\end{array}$ & 66.5 & $1939-98$ & $\begin{array}{l}1996 \\
1950\end{array}$ & $\begin{array}{r}8.62 \\
10.06\end{array}$ & $\begin{array}{l}9,240 \\
8,960\end{array}$ & $12 / 12 / 95$ & 8.62 & 9,240 & $\mathrm{~N}$ & $25-50$ \\
\hline 12056500 & $\begin{array}{l}\text { North Fork Skokomish River } \\
\text { below Staircase Rapids near } \\
\text { Hoodsport, WA }\end{array}$ & 57.2 & 1925-98 & 1935 & 14.40 & 27,000 & $12 / 20 / 94$ & 10.49 & 14,800 & $\mathrm{~N}$ & $10-25$ \\
\hline 12058800 & $\begin{array}{l}\text { North Fork Skokomish River } \\
\text { below Lower Cushman Dam near } \\
\text { Potlatch, WA }\end{array}$ & -- & 1989-98 & 1996 & 10.97 & 3,680 & $12 / 19 / 95$ & 10.97 & 3,680 & $\mathrm{Y}$ & -- \\
\hline 12060500 & $\begin{array}{l}\text { South Fork Skokomish River near } \\
\text { Union, WA }\end{array}$ & 76.3 & $\begin{array}{l}\text { 1932-84, } \\
1996-98\end{array}$ & $\begin{array}{l}1997 \\
1935\end{array}$ & $\begin{array}{r}8.98 \\
11.00\end{array}$ & $\begin{array}{l}24,400 \\
21,600\end{array}$ & $3 / 19 / 97$ & 8.98 & 24,400 & $\mathrm{~N}$ & $50-100$ \\
\hline 12061500 & $\begin{array}{l}\text { Skokomish River near Potlatch, } \\
\text { WA }\end{array}$ & 227 & $\begin{array}{l}1934, \\
1944-98\end{array}$ & $\begin{array}{l}1991 \\
1997\end{array}$ & $\begin{array}{l}16.80 \\
17.75\end{array}$ & $\begin{array}{r}36,600 \\
--\end{array}$ & $\begin{array}{l}12 / 10 / 94 \\
3 / 19 / 97\end{array}$ & $\begin{array}{l}17.48 \\
17.75\end{array}$ & $\begin{array}{r}30,000 \\
--\end{array}$ & $\begin{array}{l}\mathrm{Y} \\
\mathrm{Y}\end{array}$ & -- \\
\hline 12069550 & Big Beef Creek near Seabeck, WA & 13.8 & $\begin{array}{l}1970-81 \\
1994-98\end{array}$ & 1997 & 6.97 & 1,840 & $1 / 1 / 97$ & 6.97 & 1,840 & $\mathrm{~N}$ & $>100$ \\
\hline 12073500 & Huge Creek near Wauna, WA & 6.47 & $\begin{array}{l}1948-69 \\
1978-98\end{array}$ & $\begin{array}{l}1991 \\
1997\end{array}$ & $\begin{array}{l}5.13 \\
5.76\end{array}$ & $\begin{array}{l}547 \\
526\end{array}$ & $3 / 19 / 97$ & 5.76 & 526 & $\mathrm{~N}$ & $25-50$ \\
\hline 12079000 & Deschutes River near Rainier, WA & 89.8 & $\begin{array}{l}1950-79 \\
1981-82 \\
1988-98\end{array}$ & 1990 & 17.01 & 9,600 & 2/8/96 & 15.74 & 7,850 & $\mathrm{~N}$ & $25-50$ \\
\hline 12082500 & Nisqually River near National, WA & 133 & 1943-98 & 1996 & 12.18 & 21,200 & $2 / 8 / 96$ & 12.18 & 21,200 & $\mathrm{~N}$ & $50-100$ \\
\hline 12083000 & Mineral Creek near Mineral, WA & 75.2 & $1943-98$ & $\begin{array}{l}1996 \\
1990\end{array}$ & $\begin{array}{l}12.89 \\
13.56\end{array}$ & $\begin{array}{l}14,900 \\
13,800\end{array}$ & $2 / 8 / 96$ & 12.89 & 14,900 & $\mathrm{~N}$ & $>100$ \\
\hline 12086500 & Nisqually River at La Grande, WA & 292 & $\begin{array}{l}1907-08 \\
1910-11, \\
1920-31 \\
1945-98\end{array}$ & 1996 & 15.30 & 39,500 & $2 / 8 / 96$ & 15.30 & 39,500 & $\mathrm{Y}$ & -- \\
\hline
\end{tabular}


Table 49. Maximum stage and discharge for period of record for streamgages having significant floods during 1994-98 water years in Washington.-Continued

$\left[\mathrm{mi}^{2}\right.$, square miles; $\mathrm{ft}$, feet above an arbitrary datum; $\mathrm{ft}^{3} / \mathrm{s}$, cubic feet per second; --, not determined or not applicable; >, greater than. Source: Recurrence intervals calculated from U.S. Geological Survey data. Other data from U.S. Geological Survey reports or databases]

\begin{tabular}{|c|c|c|c|c|c|c|c|c|c|c|c|}
\hline \multirow[b]{2}{*}{$\begin{array}{c}\text { Streamgage } \\
\text { number } \\
\text { (fig. 60) }\end{array}$} & \multirow[b]{2}{*}{ Streamgage name } & \multirow[b]{2}{*}{$\begin{array}{c}\text { Total } \\
\text { drainage } \\
\left(\mathrm{mi}^{2}\right)\end{array}$} & \multicolumn{4}{|c|}{$\begin{array}{c}\text { Maximum stage and discharge for period of record } \\
\text { through } 1998 \text { water year }\end{array}$} & \multicolumn{5}{|c|}{ Significant floods $1994-98$ water years } \\
\hline & & & $\begin{array}{c}\text { Period of } \\
\text { record } \\
\text { (water } \\
\text { years) }\end{array}$ & Water year & $\begin{array}{l}\text { Stage } \\
(\mathrm{ft})\end{array}$ & $\begin{array}{l}\text { Discharge } \\
\left(\mathrm{ft}^{3} / \mathrm{s}\right)\end{array}$ & $\begin{array}{c}\text { Date } \\
\text { (month/d } \\
\text { ay/year) }\end{array}$ & $\begin{array}{l}\text { Stage } \\
\text { (ft) }\end{array}$ & $\begin{array}{l}\text { Discharge } \\
\left(\mathrm{ft}^{3} / \mathrm{s}\right)\end{array}$ & $\begin{array}{c}\text { Regulated } \\
\text { during } \\
\text { flood }^{1}\end{array}$ & $\begin{array}{c}\text { Recurrence } \\
\text { interval } \\
\text { (years) }\end{array}$ \\
\hline 12088000 & Ohop Creek near Eatonville, WA & 34.5 & $\begin{array}{l}1928-32, \\
1942-74, \\
1993-98\end{array}$ & 1996 & 8.76 & 2,620 & $2 / 8 / 96$ & 8.76 & 2,620 & $\mathrm{~N}$ & $>100$ \\
\hline 12089500 & Nisqually River at McKenna, WA & 517 & $\begin{array}{l}1948-68 \\
1978-98\end{array}$ & 1996 & 17.13 & 50,000 & $2 / 8 / 96$ & 17.13 & 50,000 & $\mathrm{Y}$ & -- \\
\hline 12090200 & Muck Creek at Roy, WA & 86.8 & $\begin{array}{l}1957-76 \\
1996\end{array}$ & $\begin{array}{l}1996 \\
1972\end{array}$ & $-\overline{-}$ & $\begin{array}{r}1,670 \\
692\end{array}$ & $2 / 8 / 96$ & -- & 1,670 & $\mathrm{~N}$ & $>100$ \\
\hline 12090400 & $\begin{array}{l}\text { North Fork Clover Creek near } \\
\text { Parkland, WA }\end{array}$ & 6.25 & $\begin{array}{l}1960-75 \\
1995-98\end{array}$ & $\begin{array}{l}1995 \\
1960\end{array}$ & $\begin{array}{l}10.05 \\
14.78\end{array}$ & $\begin{array}{l}371 \\
153\end{array}$ & $2 / 19 / 95$ & 10.05 & 371 & $\mathrm{~N}$ & $>100$ \\
\hline 12091100 & Flett Creek at Tacoma, WA & 8.01 & 1960-98 & 1996 & 3.08 & 203 & $2 / 8 / 96$ & 3.08 & 203 & $\mathrm{Y}$ & -- \\
\hline 12092000 & Puyallup River near Electron, WA & 92.8 & $\begin{array}{l}1912-26, \\
1945-49, \\
1958-93, \\
1995-98\end{array}$ & $\begin{array}{l}1996 \\
1960\end{array}$ & $\begin{array}{l}10.94 \\
11.90\end{array}$ & $\begin{array}{l}16,000 \\
10,800\end{array}$ & $2 / 8 / 96$ & 10.94 & 16,000 & $\mathrm{~N}$ & $>100$ \\
\hline 12093500 & Puyallup River near Orting, WA & 172 & 1932-98 & 1996 & 11.37 & 18,300 & $2 / 8 / 96$ & 11.37 & 18,300 & $\mathrm{~N}$ & $>100$ \\
\hline 12094000 & Carbon River near Fairfax, WA & 78.9 & $\begin{array}{l}1930-78 \\
1991-98\end{array}$ & $\begin{array}{l}1991 \\
1996\end{array}$ & $\begin{array}{l}-- \\
15.85\end{array}$ & $\begin{array}{l}13,000 \\
12,000\end{array}$ & $2 / 8 / 96$ & 15.85 & 12,000 & $\mathrm{~N}$ & $25-50$ \\
\hline 12095000 & $\begin{array}{l}\text { South Prairie Creek at South } \\
\text { Prairie, WA }\end{array}$ & 79.5 & $\begin{array}{l}1950-79 \\
1988-98\end{array}$ & 1996 & 35.14 & 8,170 & $2 / 8 / 96$ & 35.14 & 8,170 & $\mathrm{~N}$ & $50-100$ \\
\hline 12096500 & Puyallup River at Alderton, WA & 438 & $\begin{array}{l}1916-27 \\
1944-57 \\
1996-97\end{array}$ & 1996 & 61.15 & 41,500 & $2 / 9 / 96$ & 61.15 & 41,500 & $\mathrm{~N}$ & $>100$ \\
\hline 12097500 & $\begin{array}{l}\text { Greenwater River at Greenwater, } \\
\text { WA }\end{array}$ & 73.5 & $\begin{array}{l}1912, \\
1930-78, \\
1993-98\end{array}$ & 1978 & 9.80 & 10,500 & $2 / 8 / 96$ & 8.94 & 5,900 & $\mathrm{~N}$ & $25-50$ \\
\hline 12099600 & Boise Creek at Buckley, WA & 15.4 & 1978-98 & $\begin{array}{l}1996 \\
1984\end{array}$ & $\begin{array}{l}4.26 \\
5.18\end{array}$ & $\begin{array}{r}1,200 \\
972\end{array}$ & $2 / 8 / 96$ & 4.26 & 1,200 & $\mathrm{~N}$ & $25-50$ \\
\hline 12100496 & White River near Auburn, WA & 464 & 1988-98 & 1996 & 83.15 & 15,000 & $2 / 10 / 96$ & 83.15 & 15,000 & $\mathrm{Y}$ & -- \\
\hline 12101500 & Puyallup River at Puyallup, WA & 948 & $1915-98$ & $\begin{array}{l}1934 \\
1918\end{array}$ & $\begin{array}{l}21.40 \\
34.15\end{array}$ & $\begin{array}{l}57,000 \\
40,500\end{array}$ & 2/9/96 & 29.77 & 46,700 & $\mathrm{Y}$ & 40 \\
\hline
\end{tabular}


$\left[\mathrm{mi}^{2}\right.$, square miles; ft, feet above an arbitrary datum; $\mathrm{ft}^{3} / \mathrm{s}$, cubic feet per second; --, not determined or not applicable; >, greater than. Source: Recurrence intervals calculated from U.S. Geological Survey data. Other data from U.S. Geological Survey reports or databases]

\begin{tabular}{|c|c|c|c|c|c|c|c|c|c|c|c|}
\hline \multirow{2}{*}{$\begin{array}{l}\text { Streamgage } \\
\text { number } \\
\text { (fig. 60) }\end{array}$} & \multirow[b]{2}{*}{ Streamgage name } & \multirow{2}{*}{$\begin{array}{l}\text { Total } \\
\text { drainage } \\
\left(\mathrm{mi}^{2}\right)\end{array}$} & \multicolumn{4}{|c|}{$\begin{array}{l}\text { Maximum stage and discharge for period of record } \\
\text { through } 1998 \text { water year }\end{array}$} & \multicolumn{5}{|c|}{ Significant floods $1994-98$ water years } \\
\hline & & & $\begin{array}{l}\text { Period of } \\
\text { record } \\
\text { (water } \\
\text { years) }\end{array}$ & Water year & $\begin{array}{l}\text { Stage } \\
(\mathrm{ft})\end{array}$ & $\begin{array}{l}\text { Discharge } \\
\qquad\left(\mathrm{ft}^{3} / \mathrm{s}\right)\end{array}$ & $\begin{array}{c}\text { Date } \\
\text { (month/d } \\
\text { ay/year) }\end{array}$ & $\begin{array}{l}\text { Stage } \\
\text { (ft) }\end{array}$ & $\begin{array}{l}\text { Discharge } \\
\left(\mathrm{ft}^{3} / \mathrm{s}\right)\end{array}$ & $\begin{array}{l}\text { Regulated } \\
\text { during } \\
\text { flood }^{1}\end{array}$ & $\begin{array}{c}\text { Recurrence } \\
\text { interval } \\
\text { (years) }\end{array}$ \\
\hline 12108500 & $\begin{array}{l}\text { Newaukum Creek near Black } \\
\text { Diamond, WA }\end{array}$ & 27.4 & $1945-98$ & 1996 & 3.95 & 2,640 & $2 / 8 / 96$ & 3.95 & 2,640 & $\mathrm{~N}$ & $>100$ \\
\hline 12112600 & $\begin{array}{l}\text { Big Soos Creek above hatchery } \\
\text { near Auburn, WA }\end{array}$ & 66.7 & 1961-98 & 1996 & 8.88 & 4,200 & $2 / 9 / 96$ & 8.88 & 4,200 & $\mathrm{~N}$ & $>100$ \\
\hline 12113000 & Green River near Auburn, WA & 399 & 1937-98 & 1960 & 69.75 & 28,100 & 2/8/96 & 63.62 & 12,400 & $\mathrm{Y}$ & -- \\
\hline 12116100 & $\begin{array}{l}\text { Canyon Creek near Cedar Falls, } \\
\text { WA }\end{array}$ & .19 & 1946-98 & 1976 & 2.22 & 131 & $12 / 4 / 95$ & 2.05 & 107 & $\mathrm{~N}$ & $25-50$ \\
\hline 12117000 & Taylor Creek near Selleck, WA & 17.2 & 1957-98 & $\begin{array}{l}1996 \\
1965\end{array}$ & $\begin{array}{l}5.53 \\
5.78\end{array}$ & $\begin{array}{l}3,130 \\
2,730\end{array}$ & $2 / 8 / 96$ & 5.53 & 3,130 & $\mathrm{~N}$ & $50-100$ \\
\hline 12119000 & Cedar River at Renton, WA & 184 & $\begin{array}{l}1907, \\
1946-50, \\
1952-98\end{array}$ & 1991 & 17.13 & 10,600 & $11 / 30 / 95$ & 16.04 & 7,650 & $\mathrm{Y}$ & -- \\
\hline 12125200 & $\begin{array}{l}\text { Sammamish River near } \\
\text { Woodinville, WA }\end{array}$ & 157 & 1966-98 & 1997 & 26.93 & 2,870 & $\begin{array}{l}2 / 9 / 96 \\
1 / 1 / 97\end{array}$ & $\begin{array}{l}25.76 \\
26.93\end{array}$ & $\begin{array}{l}2,470 \\
2,870\end{array}$ & $\begin{array}{l}\mathrm{N} \\
\mathrm{N}\end{array}$ & $\begin{array}{r}10-25 \\
50-100\end{array}$ \\
\hline 12128000 & Thornton Creek near Seattle, WA & 12.1 & $\begin{array}{l}1946, \\
1962-68, \\
1996-98\end{array}$ & 1997 & 5.26 & 423 & $12 / 31 / 96$ & 5.26 & 423 & $\mathrm{~N}$ & -- \\
\hline 12142000 & $\begin{array}{l}\text { North Fork Snoqualmie River near } \\
\text { Snoqualmie Falls, WA }\end{array}$ & 64.0 & $\begin{array}{l}1930-54, \\
1956-57, \\
1959-98\end{array}$ & 1932 & 17.50 & 15,800 & $11 / 29 / 95$ & 12.82 & 14,500 & $\mathrm{~N}$ & $10-25$ \\
\hline 12143900 & Boxley Creek near Edgewick, WA & 3.64 & 1982-98 & 1996 & 5.20 & 256 & $12 / 3 / 95$ & 5.20 & 256 & $\mathrm{~N}$ & $10-25$ \\
\hline 12147500 & $\begin{array}{l}\text { North Fork Tolt River near } \\
\text { Carnation, WA }\end{array}$ & 39.9 & $\begin{array}{l}1953-65 \\
1968-98\end{array}$ & 1960 & 13.15 & 9,560 & $11 / 29 / 95$ & 12.74 & 8,280 & $\mathrm{~N}$ & $10-25$ \\
\hline 12149000 & $\begin{array}{l}\text { Snoqualmie River near Carnation, } \\
\text { WA }\end{array}$ & 603 & 1930-98 & 1991 & 60.70 & 65,200 & 2/9/96 & 60.34 & 61,600 & $\mathrm{~N}$ & $10-25$ \\
\hline 12150800 & $\begin{array}{l}\text { Snohomish River near Monroe, } \\
\text { WA }\end{array}$ & 1,537 & 1964-98 & 1991 & 25.30 & 150,000 & $11 / 30 / 95$ & 24.10 & 132,000 & $\mathrm{~N}$ & $25-50$ \\
\hline 12167000 & $\begin{array}{l}\text { North Fork Stillaguamish River } \\
\text { near Arlington, WA }\end{array}$ & 262 & 1929-98 & 1991 & 15.20 & 36,700 & $\begin{array}{l}2 / 8 / 96 \\
3 / 19 / 97\end{array}$ & $\begin{array}{l}14.84 \\
14.81\end{array}$ & $\begin{array}{l}34,600 \\
34,400\end{array}$ & $\begin{array}{l}\mathrm{N} \\
\mathrm{N}\end{array}$ & $\begin{array}{l}10-25 \\
10-25\end{array}$ \\
\hline
\end{tabular}


Table 49. Maximum stage and discharge for period of record for streamgages having significant floods during 1994-98 water years in Washington.-Continued

$\left[\mathrm{mi}^{2}\right.$, square miles; $\mathrm{ft}$, feet above an arbitrary datum; $\mathrm{ft}^{3} / \mathrm{s}$, cubic feet per second; --, not determined or not applicable; >, greater than. Source: Recurrence intervals calculated from U.S. Geological Survey data. Other data from U.S. Geological Survey reports or databases]

\begin{tabular}{|c|c|c|c|c|c|c|c|c|c|c|c|}
\hline \multirow{2}{*}{$\begin{array}{c}\text { Streamgage } \\
\text { number } \\
\text { (fig. 60) }\end{array}$} & \multirow[b]{2}{*}{ Streamgage name } & \multirow{2}{*}{$\begin{array}{c}\text { Total } \\
\text { drainage } \\
\left(\mathrm{mi}^{2}\right)\end{array}$} & \multicolumn{4}{|c|}{$\begin{array}{c}\text { Maximum stage and discharge for period of record } \\
\text { through } 1998 \text { water year }\end{array}$} & \multicolumn{5}{|c|}{ Significant floods $1994-98$ water years } \\
\hline & & & $\begin{array}{l}\text { Period of } \\
\text { record } \\
\text { (water } \\
\text { years) }\end{array}$ & Water year & $\begin{array}{l}\text { Stage } \\
(\mathrm{ft})\end{array}$ & $\begin{array}{l}\text { Discharge } \\
\qquad\left(\mathrm{ft}^{3} / \mathrm{s}\right)\end{array}$ & $\begin{array}{c}\text { Date } \\
\text { (month/d } \\
\text { ay/year) }\end{array}$ & $\begin{array}{l}\text { Stage } \\
\text { (ft) }\end{array}$ & $\begin{array}{l}\text { Discharge } \\
\left(\mathrm{ft}^{3} / \mathrm{s}\right)\end{array}$ & $\begin{array}{l}\text { Regulated } \\
\text { during } \\
\text { flood }^{1}\end{array}$ & $\begin{array}{l}\text { Recurrence } \\
\text { interval } \\
\text { (years) }\end{array}$ \\
\hline 12175500 & $\begin{array}{l}\text { Thunder Creek near Newhalem, } \\
\text { WA }\end{array}$ & 105 & $1920-98$ & $\begin{array}{l}1922 \\
1981\end{array}$ & $\overline{14.50}$ & $\begin{array}{l}15,400 \\
14,500\end{array}$ & $11 / 29 / 95$ & 12.76 & 10,900 & $\mathrm{~N}$ & $10-25$ \\
\hline 12178000 & Skagit River at Newhalem, WA & 1,175 & $\begin{array}{l}1815,1856, \\
1898, \\
1909-14, \\
1921-98\end{array}$ & $\begin{array}{l}1856 \\
1922\end{array}$ & 94.80 & $\begin{array}{l}95,000 \\
60,000\end{array}$ & $12 / 2 / 95$ & 91.14 & 32,300 & $\mathrm{Y}$ & -- \\
\hline 12181000 & Skagit River at Marblemount, WA & 1,381 & $\begin{array}{l}1944, \\
1947-57, \\
1976-98\end{array}$ & 1996 & 13.73 & 62,300 & $11 / 29 / 95$ & 13.73 & 62,300 & $\mathrm{Y}$ & -- \\
\hline 12189500 & Sauk River near Sauk, WA & 714 & $\begin{array}{l}\text { 1912, } \\
1929-98\end{array}$ & 1981 & 18.24 & 98,600 & $11 / 8 / 95$ & 16.57 & 79,000 & $\mathrm{~N}$ & $25-50$ \\
\hline 12194000 & Skagit River near Concrete, WA & 2,737 & $\begin{array}{l}\text { 1815, 1856, } \\
1898,1910, \\
1918,1922, \\
1925-98\end{array}$ & 1815 & 69.30 & 500,000 & $11 / 29 / 95$ & 41.57 & 160,000 & $\mathrm{Y}$ & -- \\
\hline 12200500 & $\begin{array}{l}\text { Skagit River near Mount Vernon, } \\
\text { WA }\end{array}$ & 3,093 & $\begin{array}{l}1907, \\
1941-98\end{array}$ & 1991 & 37.37 & 152,000 & $11 / 30 / 95$ & 37.34 & 141,000 & $\mathrm{Y}$ & -- \\
\hline 12210500 & Nooksack River at Deming, WA & 584 & $\begin{array}{l}\text { 1908, 1910, } \\
\text { 1932, } \\
\text { 1935-48, } \\
1950-98\end{array}$ & $\begin{array}{l}1932 \\
1908\end{array}$ & $\begin{array}{l}16.80 \\
20.00\end{array}$ & $\begin{array}{r}49,300 \\
--\end{array}$ & $11 / 29 / 95$ & 14.80 & 48,900 & $\mathrm{~N}$ & $50-100$ \\
\hline 12396000 & Calispell Creek near Dalkena, WA & 68.3 & $\begin{array}{l}1951-77, \\
1979-93, \\
1995-98\end{array}$ & 1974 & 14.38 & 3,190 & $4 / 23 / 96$ & 9.21 & 1,190 & $\mathrm{~N}$ & $10-25$ \\
\hline 12396500 & $\begin{array}{l}\text { Pend Oreille River Bel Box } \\
\text { Canyon near Ione, WA }\end{array}$ & 24,900 & $\begin{array}{l}1948 \\
1953-98\end{array}$ & 1972 & 34.54 & 136,000 & $6 / 7 / 97$ & 34.44 & 134,000 & $\mathrm{Y}$ & -- \\
\hline 12398000 & $\begin{array}{l}\text { Sullivan Creek at Metaline Falls, } \\
\text { WA }\end{array}$ & 142 & $\begin{array}{l}1954-68, \\
1970, \\
1994-98\end{array}$ & $\begin{array}{l}1997 \\
1961\end{array}$ & $\begin{array}{l}4.38 \\
6.20\end{array}$ & $\begin{array}{l}4,350 \\
2,020\end{array}$ & $6 / 1 / 97$ & 4.38 & 4,350 & $\mathrm{~N}$ & $>100$ \\
\hline 12401500 & Kettle River near Ferry, WA & 2,200 & 1929-98 & 1948 & 21.15 & 21,200 & $6 / 1 / 97$ & 20.85 & 19,600 & $\mathrm{~N}$ & $50-100$ \\
\hline
\end{tabular}


$\left[\mathrm{mi}^{2}\right.$, square miles; ft, feet above an arbitrary datum; $\mathrm{ft}^{3} / \mathrm{s}$, cubic feet per second; --, not determined or not applicable; >, greater than. Source: Recurrence intervals calculated from U.S. Geological Survey data. Other data from U.S. Geological Survey reports or databases]

\begin{tabular}{|c|c|c|c|c|c|c|c|c|c|c|c|}
\hline \multirow{2}{*}{$\begin{array}{c}\text { Streamgage } \\
\text { number } \\
\text { (fig. 60) }\end{array}$} & \multirow[b]{2}{*}{ Streamgage name } & \multirow{2}{*}{$\begin{array}{l}\text { Total } \\
\text { drainage } \\
\left(\mathrm{mi}^{2}\right)\end{array}$} & \multicolumn{4}{|c|}{$\begin{array}{l}\text { Maximum stage and discharge for period of record } \\
\text { through } 1998 \text { water year }\end{array}$} & \multicolumn{5}{|c|}{ Significant floods $1994-98$ water years } \\
\hline & & & $\begin{array}{l}\text { Period of } \\
\text { record } \\
\text { (water } \\
\text { years) }\end{array}$ & Water year & $\begin{array}{l}\text { Stage } \\
(\mathrm{ft})\end{array}$ & $\begin{array}{l}\text { Discharge } \\
\left(\mathrm{ft}^{3} / \mathrm{s}\right)\end{array}$ & $\begin{array}{c}\text { Date } \\
\text { (month/d } \\
\text { ay/year) }\end{array}$ & $\begin{array}{l}\text { Stage } \\
(\mathrm{ft})\end{array}$ & $\begin{array}{l}\text { Discharge } \\
\left(\mathrm{ft}^{3} / \mathrm{s}\right)\end{array}$ & $\begin{array}{l}\text { Regulated } \\
\text { during } \\
\text { flood }^{1}\end{array}$ & $\begin{array}{c}\text { Recurrence } \\
\text { interval } \\
\text { (years) }\end{array}$ \\
\hline 12404500 & Kettle River near Laurier, WA & 3,800 & $\begin{array}{l}1894 \\
1930-98\end{array}$ & $\begin{array}{l}1948 \\
1894\end{array}$ & $\begin{array}{l}17.25 \\
22.00\end{array}$ & $\begin{array}{r}35,000 \\
--\end{array}$ & $5 / 17 / 97$ & 15.87 & 30,800 & $\mathrm{~N}$ & $25-50$ \\
\hline 12409000 & Colville River at Kettle Falls, WA & 1,007 & 1923-98 & $\begin{array}{l}1974 \\
1956\end{array}$ & $\begin{array}{r}9.84 \\
10.17\end{array}$ & $\begin{array}{l}3,440 \\
3,230\end{array}$ & $4 / 28 / 97$ & 9.44 & 2,860 & $\mathrm{~N}$ & $25-50$ \\
\hline 12422500 & Spokane River at Spokane, WA & 4,290 & 1891-1998 & $\begin{array}{l}1894 \\
1934\end{array}$ & 29.75 & $\begin{array}{l}49,000 \\
47,800\end{array}$ & $5 / 19 / 97$ & 29.06 & 42,600 & $\mathrm{Y}$ & -- \\
\hline 12424000 & Hangman Creek at Spokane, WA & 689 & $1948-98$ & 1997 & 14.31 & 21,200 & $1 / 1 / 97$ & 14.31 & 21,200 & $\mathrm{~N}$ & $50-100$ \\
\hline 12431000 & $\begin{array}{l}\text { Little Spokane River at Dartford, } \\
\text { WA }\end{array}$ & 665 & $\begin{array}{l}1929-32 \\
1947-98\end{array}$ & 1997 & 8.27 & 4,110 & $3 / 21 / 97$ & 8.27 & 4,110 & $\mathrm{~N}$ & $>100$ \\
\hline 12433542 & $\begin{array}{l}\text { Blue Creek above Midnite Mine } \\
\text { drainage near Wellpinit, WA }\end{array}$ & 6.00 & 1985-98 & 1997 & 3.22 & 65 & $3 / 22 / 97$ & 3.22 & 65 & $\mathrm{~N}$ & $50-100$ \\
\hline 12433556 & $\begin{array}{l}\text { Midnite Mine drainage near } \\
\text { Wellpinit, WA }\end{array}$ & 1.30 & 1985-94, & 1997 & 1.78 & 6 & $3 / 19 / 97$ & 1.78 & 6 & $\mathrm{Y}$ & $25-50$ \\
\hline 12433561 & $\begin{array}{l}\text { Blue Ceek River near mouth near } \\
\text { Wellpinit, WA }\end{array}$ & 19.1 & $1985-98$ & 1997 & 4.87 & 180 & $3 / 19 / 97$ & 4.87 & 180 & Y & $25-50$ \\
\hline 12439500 & Okanogan River at Oroville, WA & 3,195 & $1943-98$ & 1997 & 14.88 & 3,770 & $6 / 7 / 97$ & 14.88 & 3,770 & $\mathrm{Y}$ & -- \\
\hline 12450700 & $\begin{array}{l}\text { Columbia River below Wells Dam, } \\
\text { WA }\end{array}$ & 86,100 & $1968-98$ & 1972 & -- & 402,000 & $6 / 12 / 97$ & -- & 362,000 & $\mathrm{Y}$ & -- \\
\hline 12451000 & Stehekin River at Stehekin, WA & 321 & $\begin{array}{l}1911-15, \\
1927-98\end{array}$ & 1996 & 29.58 & 20,900 & $11 / 29 / 95$ & 29.58 & 20,900 & $\mathrm{~N}$ & $>100$ \\
\hline 12452500 & Chelan River at Chelan, WA & 924 & $\begin{array}{l}\text { 1904-93, } \\
1995-98\end{array}$ & $\begin{array}{l}1982 \\
1968\end{array}$ & $\begin{array}{l}-- \\
--\end{array}$ & $\begin{array}{l}18,400 \\
18,400\end{array}$ & $11 / 30 / 95$ & -- & 17,000 & $\mathrm{Y}$ & -- \\
\hline 12456500 & Chiwawa River near Plain, WA & 170 & $\begin{array}{l}1914, \\
1937-49, \\
1955-57, \\
1991-98\end{array}$ & 1996 & 9.36 & 7,030 & $11 / 30 / 95$ & 9.36 & 7,030 & $\mathrm{~N}$ & $25-50$ \\
\hline 12457000 & Wenatchee River at Plain, WA & 591 & $\begin{array}{l}1911-29, \\
1932-79, \\
1990-98\end{array}$ & 1996 & 14.97 & 36,100 & $11 / 30 / 95$ & 14.97 & 36,100 & $\mathrm{~N}$ & $>100$ \\
\hline
\end{tabular}


Table 49. Maximum stage and discharge for period of record for streamgages having significant floods during 1994-98 water years in Washington.-Continued

$\left[\mathrm{mi}^{2}\right.$, square miles; $\mathrm{ft}$, feet above an arbitrary datum; $\mathrm{ft}^{3} / \mathrm{s}$, cubic feet per second; --, not determined or not applicable; >, greater than. Source: Recurrence intervals calculated from U.S. Geological Survey data. Other data from U.S. Geological Survey reports or databases]

\begin{tabular}{|c|c|c|c|c|c|c|c|c|c|c|c|}
\hline \multirow{2}{*}{$\begin{array}{c}\text { Streamgage } \\
\text { number } \\
\text { (fig. 60) }\end{array}$} & \multirow[b]{2}{*}{ Streamgage name } & \multirow{2}{*}{$\begin{array}{c}\text { Total } \\
\text { drainage } \\
\left(\mathrm{mi}^{2}\right)\end{array}$} & \multicolumn{4}{|c|}{$\begin{array}{c}\text { Maximum stage and discharge for period of record } \\
\text { through } 1998 \text { water year }\end{array}$} & \multicolumn{5}{|c|}{ Significant floods $1994-98$ water years } \\
\hline & & & $\begin{array}{l}\text { Period of } \\
\text { record } \\
\text { (water } \\
\text { years) }\end{array}$ & Water year & $\begin{array}{l}\text { Stage } \\
(\mathrm{ft})\end{array}$ & $\begin{array}{l}\text { Discharge } \\
\left(\mathrm{ft}^{3} / \mathrm{s}\right)\end{array}$ & $\begin{array}{c}\text { Date } \\
\text { (month/d } \\
\text { ay/year) }\end{array}$ & $\begin{array}{l}\text { Stage } \\
(\mathrm{ft})\end{array}$ & $\begin{array}{l}\text { Discharge } \\
\left(\mathrm{ft}^{3} / \mathrm{s}\right)\end{array}$ & $\begin{array}{l}\text { Regulated } \\
\text { during } \\
\text { flood }^{1}\end{array}$ & $\begin{array}{c}\text { Recurrence } \\
\text { interval } \\
\text { (years) }\end{array}$ \\
\hline 12458000 & $\begin{array}{l}\text { Icicle Creek above Snow Creek } \\
\text { near Leavenworth, WA }\end{array}$ & 193 & $\begin{array}{l}1912-14, \\
1937-79, \\
1994-98\end{array}$ & 1996 & 16.04 & 19,800 & $11 / 29 / 95$ & 16.04 & 19,800 & $\mathrm{~N}$ & $>100$ \\
\hline 12459000 & Wenatchee River at Peshastin, WA & 1,000 & $1929-98$ & 1996 & 17.89 & 41,300 & $11 / 30 / 95$ & 17.89 & 41,300 & $\mathrm{~N}$ & $>100$ \\
\hline 12462500 & Wenatchee River at Monitor, WA & 1,301 & $1963-98$ & 1996 & 30.02 & 47,500 & $11 / 30 / 95$ & 30.02 & 47,500 & $\mathrm{~N}$ & $>100$ \\
\hline 12472600 & Crab Creek near Beverly, WA & 4,840 & 1960-98 & 1980 & 6.46 & 936 & $1 / 4 / 97$ & 5.10 & 512 & $\mathrm{Y}$ & -- \\
\hline 12484500 & Yakima River at Umtanum, WA & 1,594 & $\begin{array}{l}1907-17, \\
1919-21, \\
1923, \\
1925-27, \\
1929-98\end{array}$ & $\begin{array}{l}1934 \\
1907\end{array}$ & -- & $\begin{array}{l}32,200 \\
41,000\end{array}$ & 2/9/96 & 38.77 & 27,200 & $\mathrm{Y}$ & -- \\
\hline 12500450 & $\begin{array}{l}\text { Yakima River above Ahtanum } \\
\text { Creek at Union Gap, WA }\end{array}$ & 3,479 & 1967-98 & 1996 & 53.88 & 53,300 & $2 / 9 / 96$ & 53.88 & 53,300 & $\mathrm{Y}$ & -- \\
\hline 12502500 & Ahtanum Creek at Union Gap, WA & 173 & $\begin{array}{l}\text { 1908, 1910, } \\
1912-14, \\
1952, \\
1960-98\end{array}$ & $\begin{array}{l}1974 \\
1996\end{array}$ & $\begin{array}{l}10.36 \\
13.50\end{array}$ & $\begin{array}{l}3,100 \\
2,660\end{array}$ & $2 / 9 / 96$ & 13.50 & 2,660 & $\mathrm{~N}$ & $50-100$ \\
\hline 12508990 & Yakima River at Mabton, WA & 5,359 & $1971-98$ & 1996 & 28.18 & 49,500 & $2 / 10 / 96$ & 28.18 & 49,500 & $\mathrm{Y}$ & -- \\
\hline 12510500 & Yakima River at Kiona, WA & 5,615 & $\begin{array}{l}1878, \\
1897-1914, \\
1934-98\end{array}$ & 1934 & 21.57 & 67,000 & $2 / 11 / 96$ & 20.98 & 49,400 & $\mathrm{Y}$ & -- \\
\hline 12512550 & $\begin{array}{l}\text { Providence Coulee near } \\
\text { Cunningham, WA }\end{array}$ & 52.1 & $\begin{array}{l}\text { 1978-91, } \\
\text { 1993-94, } \\
1996-98\end{array}$ & 1997 & 16.18 & 1,000 & $1 / 31 / 97$ & 16.18 & 1,000 & $\mathrm{~N}$ & $>100$ \\
\hline 12513000 & Esquatzel Coulee at Connell, WA & 234 & $\begin{array}{l}\text { 1953-94, } \\
1996-98\end{array}$ & $\begin{array}{l}1956 \\
1997\end{array}$ & $\begin{array}{l}12.68 \\
19.86\end{array}$ & $\begin{array}{l}5,560 \\
2,820\end{array}$ & $1 / 31 / 97$ & 19.86 & 2,820 & $\mathrm{~N}$ & 25 \\
\hline 13351000 & Palouse River at Hooper, WA & 2,500 & $\begin{array}{l}1898-99, \\
1901-07, \\
1909-16, \\
1948, \\
1951-98\end{array}$ & 1963 & 19.13 & 33,500 & $2 / 9 / 96$ & 17.95 & 28,000 & $\mathrm{~N}$ & 40 \\
\hline
\end{tabular}


$\left[\mathrm{mi}^{2}\right.$, square miles; ft, feet above an arbitrary datum; $\mathrm{ft}^{3} / \mathrm{s}$, cubic feet per second; --, not determined or not applicable; >, greater than. Source: Recurrence intervals calculated from U.S. Geological Survey data. Other data from U.S. Geological Survey reports or databases]

\begin{tabular}{|c|c|c|c|c|c|c|c|c|c|c|c|}
\hline \multirow{2}{*}{$\begin{array}{c}\text { Streamgage } \\
\text { number } \\
\text { (fig. 60) }\end{array}$} & \multirow[b]{2}{*}{ Streamgage name } & \multirow{2}{*}{$\begin{array}{c}\text { Total } \\
\text { drainage } \\
\left(\mathrm{mi}^{2}\right)\end{array}$} & \multicolumn{4}{|c|}{$\begin{array}{c}\text { Maximum stage and discharge for period of record } \\
\text { through } 1998 \text { water year }\end{array}$} & \multicolumn{5}{|c|}{ Significant floods $1994-98$ water years } \\
\hline & & & $\begin{array}{c}\text { Period of } \\
\text { record } \\
\text { (water } \\
\text { years) }\end{array}$ & Water year & $\begin{array}{l}\text { Stage } \\
(\mathrm{ft})\end{array}$ & $\begin{array}{l}\text { Discharge } \\
\left(\mathrm{ft}^{3} / \mathrm{s}\right)\end{array}$ & $\begin{array}{c}\text { Date } \\
\text { (month/d } \\
\text { ay/year) }\end{array}$ & $\begin{array}{l}\text { Stage } \\
(\mathrm{ft})\end{array}$ & $\begin{array}{l}\text { Discharge } \\
\left(\mathrm{ft}^{3} / \mathrm{s}\right)\end{array}$ & $\begin{array}{c}\text { Regulated } \\
\text { during } \\
\text { flood }^{1}\end{array}$ & $\begin{array}{c}\text { Recurrence } \\
\text { interval } \\
\text { (years) }\end{array}$ \\
\hline 14013000 & Mill Creek near Walla Walla, WA & 59.6 & $\begin{array}{l}1914-17 \\
1940-98\end{array}$ & 1996 & 20.43 & 6,350 & $2 / 9 / 96$ & 20.43 & 6,350 & $\mathrm{~N}$ & $>100$ \\
\hline 14015000 & Mill Creek at Walla Walla, WA & 95.7 & $\begin{array}{l}1942-48 \\
1950-98\end{array}$ & 1996 & 6.89 & 4,190 & $2 / 9 / 96$ & 6.89 & 4,190 & $\mathrm{Y}$ & -- \\
\hline 14018500 & $\begin{array}{l}\text { Walla Walla River near Touchet, } \\
\text { WA }\end{array}$ & 1,657 & $\begin{array}{l}1949 \\
1952-98\end{array}$ & $\begin{array}{l}1965 \\
1996\end{array}$ & $\begin{array}{l}18.90 \\
20.58\end{array}$ & $\begin{array}{l}33,400 \\
32,500\end{array}$ & $2 / 10 / 96$ & 20.58 & 32,500 & $\mathrm{~N}$ & $>100$ \\
\hline 14107000 & $\begin{array}{l}\text { Klickitat River above West Fork } \\
\text { near Glenwood, WA }\end{array}$ & 151 & $\begin{array}{l}1945-78 \\
1992-98\end{array}$ & 1996 & 5.70 & 5,500 & $2 / 8 / 96$ & 5.70 & 5,500 & $\mathrm{~N}$ & $>100$ \\
\hline 14113000 & Klickitat River near Pitt, WA & 1,297 & $\begin{array}{l}1910-12 \\
1929-98\end{array}$ & 1996 & 17.90 & 51,000 & $2 / 8 / 96$ & 17.90 & 51,000 & $\mathrm{~N}$ & $>100$ \\
\hline 14123500 & $\begin{array}{l}\text { White Salmon River near } \\
\text { Underwood, WA }\end{array}$ & 386 & $\begin{array}{l}1916-30 \\
1936-98\end{array}$ & 1996 & 19.16 & 45,200 & $2 / 8 / 96$ & 19.16 & 45,200 & $\mathrm{~N}$ & $>100$ \\
\hline 14128500 & Wind River near Carson, WA & 225 & $\begin{array}{l}1935-79 \\
1996-97\end{array}$ & 1996 & 23.04 & 53,600 & $2 / 8 / 96$ & 23.04 & 53,600 & $\mathrm{~N}$ & $>100$ \\
\hline 14216500 & $\begin{array}{l}\text { Muddy River below Clear Creek } \\
\text { near Cougar, WA }\end{array}$ & 131 & $\begin{array}{l}1928-34 \\
1955-73 \\
1984-98\end{array}$ & 1996 & 33.26 & 30,600 & $2 / 8 / 96$ & 33.26 & 30,600 & $\mathrm{~N}$ & $>100$ \\
\hline 14219800 & Speelyai Creek near Cougar, WA & 12.6 & 1960-98 & $\begin{array}{l}1963 \\
1996\end{array}$ & $-\overline{8} .12$ & $\begin{array}{l}3,600 \\
3,300\end{array}$ & $2 / 8 / 96$ & 8.12 & 3,300 & $\mathrm{~N}$ & $10-25$ \\
\hline 14220500 & Lewis River at Ariel, WA & 731 & 1924-98 & 1934 & 35.00 & 129,000 & $2 / 8 / 96$ & 27.38 & 86,400 & $\mathrm{Y}$ & -- \\
\hline 14222500 & $\begin{array}{l}\text { East Fork Lewis River near } \\
\text { Heisson, WA }\end{array}$ & 125 & $1930-98$ & 1996 & 25.26 & 28,600 & $2 / 8 / 96$ & 25.26 & 28,600 & $\mathrm{~N}$ & $>100$ \\
\hline 14223500 & $\begin{array}{l}\text { Kalama River below Italian Creek } \\
\text { near Kalama, WA }\end{array}$ & 198 & $\begin{array}{l}1947-79 \\
1996\end{array}$ & $\begin{array}{l}1996 \\
1972\end{array}$ & $\begin{array}{l}-- \\
15.80\end{array}$ & $\begin{array}{l}24,000 \\
17,900\end{array}$ & $2 / 8 / 96$ & -- & 24,000 & $\mathrm{~N}$ & $>100$ \\
\hline 14226500 & Cowlitz River at Packwood, WA & 287 & $\begin{array}{l}1912-20 \\
1930-98\end{array}$ & $\begin{array}{l}1934 \\
1978\end{array}$ & $\begin{array}{l}13.00 \\
13.73\end{array}$ & $\begin{array}{l}36,600 \\
36,200\end{array}$ & $2 / 8 / 96$ & 10.86 & 32,900 & $\mathrm{~N}$ & $25-50$ \\
\hline 14232500 & Cispus River near Randle, WA & 321 & $\begin{array}{l}1911-12, \\
1930-41 \\
1943-96\end{array}$ & $\begin{array}{l}1996 \\
1934\end{array}$ & $\begin{array}{l}11.50 \\
12.70\end{array}$ & $\begin{array}{l}31,600 \\
20,000\end{array}$ & $2 / 8 / 96$ & 11.50 & 31,600 & $\mathrm{~N}$ & $>100$ \\
\hline
\end{tabular}


Table 49. Maximum stage and discharge for period of record for streamgages having significant floods during 1994-98 water years in Washington.—Continued

$\left[\mathrm{mi}^{2}\right.$, square miles; $\mathrm{ft}$, feet above an arbitrary datum; $\mathrm{ft}^{3} / \mathrm{s}$, cubic feet per second; --, not determined or not applicable; >, greater than. Source: Recurrence intervals calculated from U.S. Geological Survey data. Other data from U.S. Geological Survey reports or databases]

\begin{tabular}{|c|c|c|c|c|c|c|c|c|c|c|c|}
\hline \multirow[b]{2}{*}{$\begin{array}{c}\text { Streamgage } \\
\text { number } \\
\text { (fig. 60) }\end{array}$} & \multirow[b]{2}{*}{ Streamgage name } & \multirow[b]{2}{*}{$\begin{array}{c}\text { Total } \\
\text { drainage } \\
\left(\mathrm{mi}^{2}\right)\end{array}$} & \multicolumn{4}{|c|}{$\begin{array}{c}\text { Maximum stage and discharge for period of record } \\
\text { through } 1998 \text { water year }\end{array}$} & \multicolumn{5}{|c|}{ Significant floods $1994-98$ water years } \\
\hline & & & $\begin{array}{c}\text { Period of } \\
\text { record } \\
\text { (water } \\
\text { years) }\end{array}$ & Water year & $\begin{array}{l}\text { Stage } \\
(\mathrm{ft})\end{array}$ & $\begin{array}{l}\text { Discharge } \\
\left(\mathrm{ft}^{3} / \mathrm{s}\right)\end{array}$ & $\begin{array}{c}\text { Date } \\
\text { (month/d } \\
\text { ay/year) }\end{array}$ & $\begin{array}{l}\text { Stage } \\
\text { (ft) }\end{array}$ & $\begin{array}{l}\text { Discharge } \\
\left(\mathrm{ft}^{3} / \mathrm{s}\right)\end{array}$ & $\begin{array}{c}\text { Regulated } \\
\text { during } \\
\text { flood }^{1}\end{array}$ & $\begin{array}{c}\text { Recurrence } \\
\text { interval } \\
\text { (years) }\end{array}$ \\
\hline 14236200 & $\begin{array}{l}\text { Tilton River above Bear Canyon } \\
\text { Creek near Cinebar, WA }\end{array}$ & 141 & $1957-98$ & 1996 & 17.90 & 27,100 & $2 / 8 / 96$ & 17.90 & 27,100 & $\mathrm{~N}$ & $50-100$ \\
\hline 14238000 & $\begin{array}{l}\text { Cowlitz River below Mayfield } \\
\text { Dam, WA }\end{array}$ & 1,400 & $\begin{array}{l}1912, \\
1935-98\end{array}$ & 1996 & 26.19 & 68,400 & $11 / 28 / 95$ & 26.19 & 68,400 & $\mathrm{Y}$ & -- \\
\hline 14241500 & $\begin{array}{l}\text { South Fork Toutle River at Toutle, } \\
\text { WA }\end{array}$ & 120 & $\begin{array}{l}1940-57 \\
1996-98\end{array}$ & $\begin{array}{l}1997 \\
1954\end{array}$ & $\begin{array}{l}27.01 \\
58.91\end{array}$ & $\begin{array}{l}15,500 \\
14,300\end{array}$ & $1 / 1 / 97$ & 27.01 & 15,500 & $\mathrm{~N}$ & -- \\
\hline 14242580 & $\begin{array}{l}\text { Toutle River at Tower Road near } \\
\text { Silver Lake, WA }\end{array}$ & 496 & 1982-98 & $\begin{array}{l}1996 \\
1983\end{array}$ & $\begin{array}{l}24.91 \\
28.03\end{array}$ & $\begin{array}{l}61,800 \\
38,000\end{array}$ & $2 / 8 / 96$ & 24.91 & 61,800 & $\mathrm{~N}$ & -- \\
\hline 14243000 & Cowlitz River at Castle Rock, WA & 2,238 & $1927-98$ & $\begin{array}{l}1934 \\
1998\end{array}$ & $\begin{array}{l}31.60 \\
41.30\end{array}$ & $\begin{array}{r}139,000 \\
30,800\end{array}$ & $2 / 8 / 96$ & 32.11 & 112,000 & $\mathrm{Y}$ & $>100$ \\
\hline 14243500 & $\begin{array}{l}\text { Delameter Creek near Castle Rock, } \\
\text { WA }\end{array}$ & 19.6 & $\begin{array}{l}1950-69 \\
1996\end{array}$ & $\begin{array}{l}1996 \\
1963\end{array}$ & $-\overline{-}-5.53$ & $\begin{array}{l}3,500 \\
2,420\end{array}$ & $2 / 8 / 96$ & -- & 3,500 & $\mathrm{~N}$ & $50-100$ \\
\hline 14245000 & Coweman River near Kelso, WA & 119 & $\begin{array}{l}1950-84, \\
1996\end{array}$ & $\begin{array}{l}1996 \\
1978\end{array}$ & $\begin{array}{l}-- \\
14.67\end{array}$ & $\begin{array}{r}11,700 \\
9,940\end{array}$ & $2 / 8 / 96$ & -- & 11,700 & $\mathrm{~N}$ & $50-100$ \\
\hline
\end{tabular}

Regulated during flood: $\mathrm{N}, \mathrm{no} ; \mathrm{Y}$, yes. 


\section{West Virginia}

Excessive rains of 2 to 5 inches fell mainly over central and northern West Virginia during February 8-9, 1994

(National Oceanic and Atmospheric Administration, 1994a). Small-stream flooding was widespread late on the night of February 8-9. Moderate to major river flooding occurred along the Tygart Valley, Buckhannon, Cheat, and West Fork Little Kanawha Rivers (fig. 61). The crest on the Tygart Valley River at Philippi (streamgage 03054500, table 50) reached 27.13 feet on February 9, the second highest stage in the 20th century. Damage was more than $\$ 6$ million (National Oceanic and Atmospheric Administration, 1994b).

A stationary front, combined with an upper level disturbance, caused excessive rains across southern West Virginia on June 27-28, 1995. Six inches of rain caused severe flooding and flash flooding near Summersville (National Oceanic and Atmospheric Administration, 1995a). Four to 6 inches of rain near Princeton flooded and washed out many roads and bridges, stranded motorists in their vehicles, caused mud slides, damaged and destroyed crops, and damaged buildings. Total damage was more than \$8 million (National Oceanic and Atmospheric Administration, 1995b).

On January 19, 1996, a combination of unseasonably warm, humid air over a dense snowpack, estimated between 12 and 18 inches and as much as 2 feet at higher elevations (National Oceanic and Atmospheric Administration, 1996a), caused nearly all of the snow to melt in a 12-hour period. The unseasonably warm air was drawn northward around the circulation of a deepening storm west of the Appalachian Mountains on January 18-19. The melting snow, combined with downpours, produced serious flooding over West Virginia. The most serious flooding was reported in the upstream reaches of the Potomac River. Total damage from this flood was estimated to be more than \$61 million (National Oceanic and Atmospheric Administration, 1996b). There was one death. The Cheat River, in the northern mountains of West Virginia and one of the flashiest rivers in the country, was the first river to reach flood stage during this widespread flood. Flooding from the Allegheny and Monongahela Rivers in Pennsylvania, met nearly simultaneously in Pittsburgh, Pennsylvania, and then flowed down the Ohio River. Widespread 1- to 2-inch rains, plus runoff from snowmelt in the West Virginia and Maryland mountains, combined to create major flooding along the Ohio River. Six streamgages recorded their highest discharge for their periods of record in West Virginia (table 50) in January 1996. The flood-control projects held water back from the main-stem Ohio River to help reduce flood damage there.

On May 15-16, 1996, training thunderstorms, producing rainfall totals of 2.5 to 3.5 inches in 6 hours (National Oceanic and Atmospheric Administration, 1996a), moved southeast across southern West Virginia. A 57-year-old woman from Whitman died when she tried to leave her home and wade through the floodwaters. Two other women drowned trying to flee their flooded home in Cassity. The Tygart Valley River had significant floods (table 50). Most of the more than \$7 million in damage across southern West Virginia was caused by the floodwaters from small streams and creeks (National Oceanic and Atmospheric Administration, 1996b).

Excessive rainfall associated with the remnants of Hurricane Fran on September 6, 1996, caused considerable and in some cases record flooding along rivers and tributaries in the Potomac River Basin. A thin band of 11 to 14 inches of rain (National Oceanic and Atmospheric Administration, 1996a) falling along higher ridges ran off into the South Branch Potomac River. Damage was excessive, especially along the South Branch Potomac. The $\$ 5$ million in reported property damage included $\$ 3.5$ million to 227 residences and \$1.5 million to public buildings. Two persons died from the floods (National Oceanic and Atmospheric Administration, 1996b).

Showers and thunderstorms hit the western lowlands three separate times during March 1-2, 1997. Rainfall totals of 5 to 7.5 inches were measured (National Oceanic and Atmospheric Administration, 1997a). The full spectrum of flooding occurred. Sixteen West Virginia counties were declared Federal disaster areas. There was more than $\$ 13$ million in property damage, and three persons drowned in the flooding (National Oceanic and Atmospheric Administration, 1997b).

A series of thunderstorms produced flash floods across central and north-central West Virginia during June 27-30, 1998. Nearly $\$ 30$ million in damage and two deaths resulted from the flash floods (National Oceanic and Atmospheric Administration, 1998b).

\section{References}

National Oceanic and Atmospheric Administration (NOAA), 1994a-98a, Climatological data (by State): Asheville, North Carolina, National Climatic Data Center, various months.

National Oceanic and Atmospheric Administration (NOAA), 1994b-98b, Storm data (by State): Asheville, North Carolina, National Climatic Data Center, various months. 

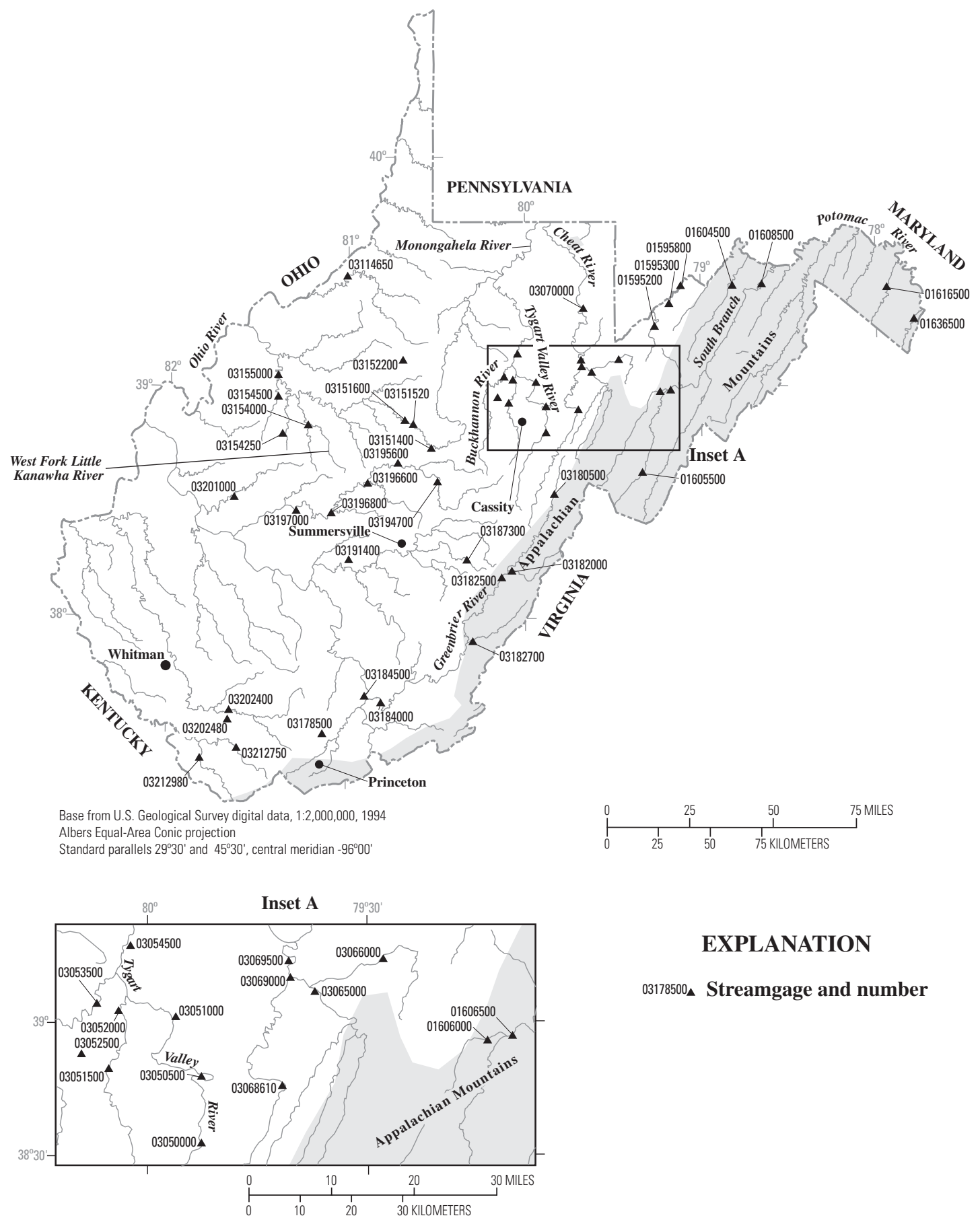

EXPLANATION

03178500 Streamgage and number

Figure 61. Location of streamgages with significant floods during 1994-98 water years for West Virginia. 
Table 50. Maximum stage and discharge for period of record for streamgages having significant floods during 1994-98 water years in West Virginia.

$\left[\mathrm{mi}^{2}\right.$, square miles; $\mathrm{ft}$, feet above an arbitrary datum; $\mathrm{ft}^{3} / \mathrm{s}$, cubic feet per second; --, not determined or not applicable; >, greater than. Source: Recurrence intervals calculated from U.S. Geological Survey data. Other data from U.S. Geological Survey reports or databases]

\begin{tabular}{|c|c|c|c|c|c|c|c|c|c|c|c|}
\hline \multirow[b]{2}{*}{$\begin{array}{c}\text { Streamgage } \\
\text { number } \\
\text { (fig. 61) }\end{array}$} & \multirow[b]{2}{*}{ Streamgage name } & \multirow[b]{2}{*}{$\begin{array}{c}\text { Total } \\
\text { drainage } \\
\left(\mathrm{mi}^{2}\right)\end{array}$} & \multicolumn{4}{|c|}{$\begin{array}{c}\text { Maximum stage and discharge for period of record } \\
\text { through } 1998 \text { water year }\end{array}$} & \multicolumn{5}{|c|}{ Significant floods 1994-98 water years } \\
\hline & & & $\begin{array}{l}\text { Period of } \\
\text { record } \\
\text { (water } \\
\text { years) }\end{array}$ & Water year & $\begin{array}{l}\text { Stage } \\
\text { (ft) }\end{array}$ & $\begin{array}{l}\text { Discharge } \\
\left(\mathrm{ft}^{3} / \mathrm{s}\right)\end{array}$ & $\begin{array}{c}\text { Date } \\
\text { (month/d } \\
\text { ay/year) }\end{array}$ & $\begin{array}{l}\text { Stage } \\
(\mathrm{ft})\end{array}$ & $\begin{array}{l}\text { Discharge } \\
\left(\mathrm{ft}^{3} / \mathrm{s}\right)\end{array}$ & $\begin{array}{c}\text { Regulated } \\
\text { during } \\
\text { flood }^{1}\end{array}$ & $\begin{array}{c}\text { Recurrence } \\
\text { interval } \\
\text { (years) }\end{array}$ \\
\hline 01595200 & $\begin{array}{l}\text { Stony River near Mount Storm, } \\
\text { WV }\end{array}$ & 249 & $1962-98$ & 1986 & 16.41 & 14,000 & $9 / 6 / 96$ & 12.55 & 7,750 & $\mathrm{Y}$ & -- \\
\hline 01595300 & Abram Creek at Oakmont, WV & 42.6 & $\begin{array}{l}1955, \\
1957-82, \\
1996\end{array}$ & $\begin{array}{l}1996 \\
1955\end{array}$ & $-\overline{-} 9.82$ & $\begin{array}{r}17,500 \\
3,830\end{array}$ & $9 / 6 / 96$ & -- & 17,500 & $\mathrm{~N}$ & $>500$ \\
\hline 01595800 & $\begin{array}{l}\text { North Branch Potomac River at } \\
\text { Barnum, WV }\end{array}$ & 266 & $\begin{array}{l}\text { 1967-95, } \\
1997-99\end{array}$ & 1978 & 13.37 & 27,100 & $3 / 30 / 95$ & 8.92 & 8,830 & $\mathrm{Y}$ & -- \\
\hline 01604500 & $\begin{array}{l}\text { Patterson Creek near Headsville, } \\
\text { WV }\end{array}$ & 211 & 1939-98 & $\begin{array}{l}1955 \\
1943\end{array}$ & $\begin{array}{l}12.20 \\
13.00\end{array}$ & $\begin{array}{l}16,000 \\
15,300\end{array}$ & $1 / 19 / 96$ & 11.97 & 14,400 & $\mathrm{~N}$ & $25-50$ \\
\hline 01605500 & $\begin{array}{l}\text { South Branch Potomac River at } \\
\text { Franklin, WV }\end{array}$ & 179 & $\begin{array}{l}1936, \\
1941-69, \\
1977-98\end{array}$ & 1986 & 22.58 & 44,000 & $9 / 6 / 96$ & 19.16 & 34,000 & $\mathrm{~N}$ & 200 \\
\hline 01606000 & $\begin{array}{l}\text { North Fork South Branch Potomac } \\
\text { River at Cabins, WV }\end{array}$ & 335 & $\begin{array}{l}1936, \\
1940-80, \\
1986,1996\end{array}$ & $\begin{array}{l}1986 \\
1949\end{array}$ & $-\overline{-}$ & $\begin{array}{l}90,000 \\
50,000\end{array}$ & $9 / 6 / 96$ & -- & 8,000 & $\mathrm{~N}$ & 2 \\
\hline 01606500 & $\begin{array}{l}\text { South Branch Potomac River near } \\
\text { Petersburg, WV }\end{array}$ & 676 & $\begin{array}{l}\text { 1878, 1924, } \\
1929-98\end{array}$ & $\begin{array}{l}1986 \\
1949\end{array}$ & $\begin{array}{l}21.80 \\
22.83\end{array}$ & $\begin{array}{r}130,000 \\
62,000\end{array}$ & $9 / 6 / 96$ & 22.20 & 113,000 & $\mathrm{~N}$ & $200-500$ \\
\hline 01608500 & $\begin{array}{l}\text { South Branch Potomac River near } \\
\text { Springfield, WV }\end{array}$ & 1,486 & $\begin{array}{l}\text { 1878, } \\
\text { 1900-01, } \\
\text { 1904-06, } \\
\text { 1929-98 }\end{array}$ & 1986 & 44.22 & 240,000 & 9/7/96 & 34.98 & 147,000 & $\mathrm{~N}$ & $50-100$ \\
\hline 01616500 & $\begin{array}{l}\text { Opequon Creek near Martinsburg, } \\
\text { WV }\end{array}$ & 273 & $\begin{array}{l}\text { 1906, 1936, } \\
1948-98\end{array}$ & 1996 & 18.76 & 23,400 & $1 / 20 / 96$ & 18.76 & 23,400 & $\mathrm{~N}$ & 50 \\
\hline 01636500 & Shenandoah River at Millville, WV & 3,022 & $\begin{array}{l}1870, \\
1896-98, \\
1900-01, \\
1903-08, \\
1924, \\
1929-98\end{array}$ & 1943 & 32.40 & 230,000 & 9/8/96 & 26.84 & 156,000 & $\mathrm{~N}$ & $50-100$ \\
\hline
\end{tabular}


Table 50. Maximum stage and discharge for period of record for streamgages having significant floods during 1994-98 water years in West Virginia.-Continued

$\left[\mathrm{mi}^{2}\right.$, square miles; ft, feet above an arbitrary datum; $\mathrm{ft}^{3} / \mathrm{s}$, cubic feet per second; --, not determined or not applicable; >, greater than. Source: Recurrence intervals calculated from U.S. Geological Survey data. Other data from U.S. Geological Survey reports or databases]

\begin{tabular}{|c|c|c|c|c|c|c|c|c|c|c|c|}
\hline \multirow{2}{*}{$\begin{array}{c}\text { Streamgage } \\
\text { number } \\
\text { (fig. 61) }\end{array}$} & \multirow[b]{2}{*}{ Streamgage name } & \multirow{2}{*}{$\begin{array}{c}\text { Total } \\
\text { drainage } \\
\left(\mathrm{mi}^{2}\right)\end{array}$} & \multicolumn{4}{|c|}{$\begin{array}{l}\text { Maximum stage and discharge for period of record } \\
\text { through } 1998 \text { water year }\end{array}$} & \multicolumn{5}{|c|}{ Significant floods 1994-98 water years } \\
\hline & & & $\begin{array}{l}\text { Period of } \\
\text { record } \\
\text { (water } \\
\text { years) }\end{array}$ & Water year & $\begin{array}{l}\text { Stage } \\
(\mathrm{ft})\end{array}$ & $\begin{array}{l}\text { Discharge } \\
\left(\mathrm{ft}^{3} / \mathrm{s}\right)\end{array}$ & $\begin{array}{c}\text { Date } \\
\text { (month/d } \\
\text { ay/year) }\end{array}$ & $\begin{array}{l}\text { Stage } \\
(\mathrm{ft})\end{array}$ & $\begin{array}{l}\text { Discharge } \\
\left(\mathrm{ft}^{3} / \mathrm{s}\right)\end{array}$ & $\begin{array}{c}\text { Regulated } \\
\text { during } \\
\text { flood }^{1}\end{array}$ & $\begin{array}{c}\text { Recurrence } \\
\text { interval } \\
\text { (years) }\end{array}$ \\
\hline 03050000 & $\begin{array}{l}\text { Tygart Valley River near Dailey, } \\
\text { WV }\end{array}$ & 185 & $\begin{array}{l}\text { 1916-76, } \\
1986, \\
1989-98\end{array}$ & $\begin{array}{l}1986 \\
1932\end{array}$ & $\begin{array}{l}16.60 \\
17.20\end{array}$ & $\begin{array}{l}22,000 \\
13,100\end{array}$ & $\begin{array}{l}5 / 17 / 96 \\
6 / 19 / 98\end{array}$ & $\begin{array}{l}15.65 \\
14.65\end{array}$ & $\begin{array}{l}19,900 \\
15,900\end{array}$ & $\begin{array}{l}\mathrm{N} \\
\mathrm{N}\end{array}$ & $\begin{array}{r}100-200 \\
50\end{array}$ \\
\hline 03050500 & $\begin{array}{l}\text { Tygart Valley River near Elkins, } \\
\text { WV }\end{array}$ & 271 & $1945-98$ & 1986 & 22.81 & 23,500 & $5 / 17 / 96$ & 18.66 & 14,500 & $\mathrm{~N}$ & $25-50$ \\
\hline 03051000 & $\begin{array}{l}\text { Tygart Valley River at Belington, } \\
\text { WV }\end{array}$ & 406 & $\begin{array}{l}1888 \\
1908-98\end{array}$ & 1986 & 23.65 & 29,500 & $\begin{array}{l}2 / 9 / 94 \\
5 / 17 / 96\end{array}$ & $\begin{array}{l}19.64 \\
20.19\end{array}$ & $\begin{array}{l}20,000 \\
21,200\end{array}$ & $\begin{array}{l}\mathrm{N} \\
\mathrm{N}\end{array}$ & $\begin{array}{r}25-50 \\
50\end{array}$ \\
\hline 03051500 & Middle Fork River at Midvale, WV & 122 & $\begin{array}{l}1916-42, \\
1986,1996\end{array}$ & $\begin{array}{l}1986 \\
1939\end{array}$ & 18.50 & $\begin{array}{l}14,000 \\
11,400\end{array}$ & $5 / 17 / 96$ & -- & 14,000 & $\mathrm{~N}$ & $50-100$ \\
\hline 03052000 & Middle Fork River at Audra, WV & 148 & $\begin{array}{l}\text { 1943-79, } \\
1986, \\
1989-98\end{array}$ & 1986 & 15.80 & 17,100 & $5 / 17 / 96$ & 15.60 & 16,700 & $\mathrm{~N}$ & $50-100$ \\
\hline 03052500 & Sand Run near Buckhannon, WV & 14.3 & 1947-98 & 1986 & 8.34 & 3,200 & $\begin{array}{l}2 / 9 / 94 \\
5 / 17 / 96\end{array}$ & $\begin{array}{l}7.18 \\
7.98\end{array}$ & $\begin{array}{l}2,120 \\
2,860\end{array}$ & $\begin{array}{l}\mathrm{N} \\
\mathrm{N}\end{array}$ & $\begin{array}{r}10-25 \\
50\end{array}$ \\
\hline 03053500 & Buckhannon River at Hall, WV & 277 & $\begin{array}{l}1908, \\
1916-98\end{array}$ & 1986 & 16.88 & 15,000 & $2 / 9 / 94$ & 15.59 & 13,300 & $\mathrm{~N}$ & 25 \\
\hline 03054500 & $\begin{array}{l}\text { Tygart Valley River at Philippi, } \\
\text { WV }\end{array}$ & 914 & $\begin{array}{l}1912, \\
1941-98\end{array}$ & 1986 & 31.83 & 61,000 & $\begin{array}{l}2 / 9 / 94 \\
5 / 17 / 96\end{array}$ & $\begin{array}{l}27.13 \\
27.50\end{array}$ & $\begin{array}{l}47,400 \\
48,400\end{array}$ & $\begin{array}{l}\mathrm{N} \\
\mathrm{N}\end{array}$ & $\begin{array}{r}50 \\
50-100\end{array}$ \\
\hline 03065000 & Dry Fork at Hendricks, WV & 349 & $1941-98$ & 1986 & 20.74 & 100,000 & $1 / 19 / 96$ & 14.17 & 41,000 & $\mathrm{~N}$ & $25-50$ \\
\hline 03066000 & Blackwater River at Davis, WV & 85.9 & 1922-98 & 1986 & 17.67 & 12,500 & 2/9/94 & 13.60 & 7,660 & $\mathrm{~N}$ & $25-50$ \\
\hline 03068610 & Taylor Run at Bowden, WV & 5.06 & $\begin{array}{l}1974-82 \\
1992-98\end{array}$ & 1996 & 8.00 & 600 & $7 / 31 / 96$ & 8.00 & 600 & $\mathrm{~N}$ & 25 \\
\hline 03069000 & Shavers Fork at Parsons, WV & 213 & $\begin{array}{l}\text { 1888, 1907, } \\
\text { 1911-26, } \\
1941-98\end{array}$ & 1986 & 19.86 & 43,000 & $5 / 17 / 96$ & 14.02 & 22,500 & $\mathrm{~N}$ & 50 \\
\hline 03069500 & Cheat River near Parsons, WV & 722 & $\begin{array}{l}1844,1888 \\
1914-98\end{array}$ & 1986 & 24.30 & 170,000 & $1 / 19 / 96$ & 19.84 & 90,100 & $\mathrm{~N}$ & 100 \\
\hline
\end{tabular}


$\left[\mathrm{mi}^{2}\right.$, square miles; $\mathrm{ft}$, feet above an arbitrary datum; $\mathrm{ft}^{3} / \mathrm{s}$, cubic feet per second; --, not determined or not applicable; >, greater than. Source: Recurrence intervals calculated from U.S. Geological Survey data. Other data from U.S. Geological Survey reports or databases]

\begin{tabular}{|c|c|c|c|c|c|c|c|c|c|c|c|}
\hline \multirow{2}{*}{$\begin{array}{c}\text { Streamgage } \\
\text { number } \\
\text { (fig. 61) }\end{array}$} & \multirow[b]{2}{*}{ Streamgage name } & \multirow{2}{*}{$\begin{array}{c}\text { Total } \\
\text { drainage } \\
\left(\mathrm{mi}^{2}\right)\end{array}$} & \multicolumn{4}{|c|}{$\begin{array}{c}\text { Maximum stage and discharge for period of record } \\
\text { through } 1998 \text { water year }\end{array}$} & \multicolumn{5}{|c|}{ Significant floods 1994-98 water years } \\
\hline & & & $\begin{array}{l}\text { Period of } \\
\text { record } \\
\text { (water } \\
\text { years) }\end{array}$ & Water year & $\begin{array}{l}\text { Stage } \\
(\mathrm{ft})\end{array}$ & $\begin{array}{l}\text { Discharge } \\
\left(\mathrm{ft}^{3} / \mathrm{s}\right)\end{array}$ & $\begin{array}{c}\text { Date } \\
\text { (month/d } \\
\text { ay/year) }\end{array}$ & $\begin{array}{l}\text { Stage } \\
(\mathrm{ft})\end{array}$ & $\begin{array}{l}\text { Discharge } \\
\left(\mathrm{ft}^{3} / \mathrm{s}\right)\end{array}$ & $\begin{array}{c}\text { Regulated } \\
\text { during } \\
\text { flood }^{1}\end{array}$ & $\begin{array}{c}\text { Recurrence } \\
\text { interval } \\
\text { (years) }\end{array}$ \\
\hline 03070000 & Cheat River at Rowlesburg, WV & 939 & $\begin{array}{l}\text { 1844, 1888, } \\
1921, \\
1924-96\end{array}$ & 1986 & 34.91 & 190,000 & $1 / 19 / 96$ & 25.88 & 114,000 & $\mathrm{~N}$ & 100 \\
\hline 03114650 & Buffalo Run near Little, WV & 4.19 & $\begin{array}{l}\text { 1969-77, } \\
1994-98\end{array}$ & $\begin{array}{l}1996 \\
1998\end{array}$ & $\begin{array}{l}12.53 \\
13.30\end{array}$ & $\begin{array}{l}2,420 \\
1,900\end{array}$ & 7/19/96 & 12.53 & 2,420 & $\mathrm{~N}$ & $50-100$ \\
\hline 03151400 & $\begin{array}{l}\text { Little Kanawha River near } \\
\text { Wildcat, WV }\end{array}$ & 112 & $\begin{array}{l}1975-83 \\
1986-98\end{array}$ & 1996 & 18.47 & 19,600 & $7 / 31 / 96$ & 18.47 & 19,600 & $\mathrm{~N}$ & $200-500$ \\
\hline 03151520 & $\begin{array}{l}\text { Little Kanawha River below } \\
\text { Burnsville Dam, WV }\end{array}$ & 163 & 1977-98 & $\begin{array}{l}1996 \\
1977\end{array}$ & $\begin{array}{r}8.19 \\
58.81\end{array}$ & $\begin{array}{l}2,540 \\
2,530\end{array}$ & $8 / 6 / 96$ & 8.19 & 2,540 & $\mathrm{Y}$ & -- \\
\hline 03151600 & $\begin{array}{l}\text { Little Kanawha River at } \\
\text { Burnsville, WV }\end{array}$ & 248 & $\begin{array}{l}1975-83 \\
1994-98\end{array}$ & 1996 & 14.82 & 6,100 & $7 / 31 / 96$ & 14.82 & 6,100 & $\mathrm{Y}$ & -- \\
\hline 03152200 & Buck Run near Leopold, WV & 2.91 & $\begin{array}{l}\text { 1970-77, } \\
1994-98\end{array}$ & 1998 & 11.45 & 1,290 & $6 / 28 / 98$ & 11.45 & 1,290 & $\mathrm{~N}$ & $200-500$ \\
\hline 03154000 & $\begin{array}{l}\text { West Fork Little Kanawha River at } \\
\text { Rocksdale, WV }\end{array}$ & 205 & $\begin{array}{l}1929-31 \\
1938-98\end{array}$ & $\begin{array}{l}1939 \\
1997\end{array}$ & $\begin{array}{l}30.30 \\
31.55\end{array}$ & $\begin{array}{l}20,200 \\
15,000\end{array}$ & $3 / 2 / 97$ & 31.55 & 15,000 & $\mathrm{~N}$ & 25 \\
\hline 03154250 & Tanner Run at Spencer, WV & 2.82 & $\begin{array}{l}1970-77 \\
1994-98\end{array}$ & 1995 & 8.20 & 1,520 & $5 / 14 / 95$ & 8.20 & 1,520 & $\mathrm{~N}$ & $50-100$ \\
\hline 03154500 & Reedy Creek near Reedy, WV & 79.4 & $\begin{array}{l}1952-78 \\
1997\end{array}$ & 1997 & 15.37 & 7,260 & 3/--/97 & 15.37 & 7,260 & $\mathrm{~N}$ & $10-25$ \\
\hline 03155000 & $\begin{array}{l}\text { Little Kanawha River at Palestine, } \\
\text { WV }\end{array}$ & 1,516 & $\begin{array}{l}1897, \\
1916-22, \\
1939-98\end{array}$ & $\begin{array}{l}1939 \\
1967\end{array}$ & $\begin{array}{l}32.25 \\
39.14\end{array}$ & $\begin{array}{l}53,000 \\
50,700\end{array}$ & $3 / 2 / 97$ & -- & 48,100 & $\mathrm{Y}$ & $10-25$ \\
\hline 03178500 & $\begin{array}{l}\text { Camp Creek near Camp Creek, } \\
\text { WV }\end{array}$ & 32.0 & $\begin{array}{l}1947-71 \\
1994-98\end{array}$ & 1996 & 6.77 & 5,610 & $1 / 19 / 96$ & 6.77 & 5,610 & $\mathrm{~N}$ & 100 \\
\hline 03180500 & Greenbrier River at Durbin, WV & 133 & 1944-98 & 1986 & 15.82 & 37,100 & $5 / 18 / 96$ & 15.52 & 35,600 & $\mathrm{~N}$ & $>500$ \\
\hline 03182000 & Knapp Creek at Marlinton, WV & 108 & $\begin{array}{l}1946-58, \\
1986,1989, \\
1994-98\end{array}$ & 1996 & 19.55 & 22,000 & $1 / 19 / 96$ & 19.55 & 22,000 & $\mathrm{~N}$ & $200-500$ \\
\hline 03182500 & Greenbrier River at Buckeye, WV & 540 & 1930-98 & 1986 & 23.20 & 82,000 & $1 / 19 / 96$ & 20.93 & 61,500 & $\mathrm{~N}$ & $100-200$ \\
\hline
\end{tabular}


Table 50. Maximum stage and discharge for period of record for streamgages having significant floods during 1994-98 water years in West Virginia.-Continued

$\left[\mathrm{mi}^{2}\right.$, square miles; $\mathrm{ft}$, feet above an arbitrary datum; $\mathrm{ft}^{3} / \mathrm{s}$, cubic feet per second; --, not determined or not applicable; >, greater than. Source: Recurrence intervals calculated from U.S. Geological Survey data. Other data from U.S. Geological Survey reports or databases]

\begin{tabular}{|c|c|c|c|c|c|c|c|c|c|c|c|}
\hline \multirow{2}{*}{$\begin{array}{c}\text { Streamgage } \\
\text { number } \\
\text { (fig. 61) }\end{array}$} & \multirow[b]{2}{*}{ Streamgage name } & \multirow{2}{*}{$\begin{array}{l}\text { Total } \\
\text { drainage } \\
\left(\mathrm{mi}^{2}\right)\end{array}$} & \multicolumn{4}{|c|}{$\begin{array}{c}\text { Maximum stage and discharge for period of record } \\
\text { through } 1998 \text { water year }\end{array}$} & \multicolumn{5}{|c|}{ Significant floods $1994-98$ water years } \\
\hline & & & $\begin{array}{l}\text { Period of } \\
\text { record } \\
\text { (water } \\
\text { years) }\end{array}$ & Water year & $\begin{array}{l}\text { Stage } \\
(\mathrm{ft})\end{array}$ & $\begin{array}{l}\text { Discharge } \\
\left(\mathrm{ft}^{3} / \mathrm{s}\right)\end{array}$ & $\begin{array}{c}\text { Date } \\
\text { (month/d } \\
\text { ay/year) }\end{array}$ & $\begin{array}{l}\text { Stage } \\
(\mathrm{ft})\end{array}$ & $\begin{array}{l}\text { Discharge } \\
\left(\mathrm{ft}^{3} / \mathrm{s}\right)\end{array}$ & $\begin{array}{l}\text { Regulated } \\
\text { during } \\
\text { flood }^{1}\end{array}$ & $\begin{array}{c}\text { Recurrence } \\
\text { interval } \\
\text { (years) }\end{array}$ \\
\hline 03182700 & Anthony Creek near Anthony, WV & 144 & $\begin{array}{l}1972-82, \\
1996\end{array}$ & 1996 & 21.35 & 27,400 & $1 / 23 / 96$ & 21.35 & 27,400 & $\mathrm{~N}$ & $200-500$ \\
\hline 03184000 & Greenbrier River at Hilldale, WV & 1,619 & $1936-98$ & 1996 & 26.88 & 93,000 & $1 / 20 / 96$ & 26.88 & 93,000 & $\mathrm{~N}$ & $100-200$ \\
\hline 03184500 & New River at Hinton, WV & 6,256 & $1937-98$ & 1940 & 18.97 & 246,000 & $1 / 20 / 96$ & 10.68 & 91,700 & $\mathrm{Y}$ & -- \\
\hline 03187300 & $\begin{array}{l}\text { North Fork Cranberry River near } \\
\text { Hillsboro, WV }\end{array}$ & 9.78 & $\begin{array}{l}1969-82 \\
1994-98\end{array}$ & 1996 & 6.20 & 2,300 & $1 / 19 / 96$ & 6.20 & 2,300 & $\mathrm{~N}$ & 50 \\
\hline 03191400 & $\begin{array}{l}\text { Laurel Creek near Summersville, } \\
\text { WV }\end{array}$ & 4.28 & $\begin{array}{l}\text { 1966-77, } \\
1994-98\end{array}$ & 1995 & 13.20 & 1,900 & $6 / 27 / 95$ & 13.20 & 1,900 & $\mathrm{~N}$ & 500 \\
\hline 03194700 & $\begin{array}{l}\text { Elk River below Webster Springs, } \\
\text { WV }\end{array}$ & 266 & $\begin{array}{l}\text { 1861, 1896, } \\
1930-83, \\
1986-98\end{array}$ & $\begin{array}{l}1986 \\
1861\end{array}$ & $\begin{array}{l}17.20 \\
26.34\end{array}$ & $\begin{array}{r}38,000 \\
--\end{array}$ & $1 / 19 / 96$ & 15.14 & 28,600 & $\mathrm{~N}$ & 50 \\
\hline 03195600 & Granny Creek at Sutton, WV & 6.98 & $\begin{array}{l}1966-77 \\
1994-98\end{array}$ & 1994 & 14.91 & 1,650 & $5 / 7 / 94$ & 14.91 & 1,650 & $\mathrm{~N}$ & 50 \\
\hline 03196600 & Elk River near Frametown, WV & 751 & 1959-98 & 1996 & 20.39 & 30,300 & $\begin{array}{l}7 / 31 / 96 \\
3 / 1 / 97\end{array}$ & $\begin{array}{l}20.39 \\
16.65\end{array}$ & $\begin{array}{l}30,300 \\
22,800\end{array}$ & $\begin{array}{l}\mathrm{Y} \\
\mathrm{Y}\end{array}$ & $\begin{array}{l}-- \\
--\end{array}$ \\
\hline 03196800 & Elk River at Clay, WV & 992 & $1916-98$ & 1967 & 22.80 & 48,000 & $3 / 1 / 97$ & 21.98 & 45,100 & $\mathrm{Y}$ & -- \\
\hline 03197000 & Elk River at Queen Shoals, WV & 1,145 & $\begin{array}{l}1918 \\
1929-98\end{array}$ & $\begin{array}{l}1932 \\
1918\end{array}$ & $\begin{array}{l}29.20 \\
37.20\end{array}$ & $\begin{array}{r}72,000 \\
--\end{array}$ & $3 / 2 / 97$ & 25.36 & 47,000 & $\mathrm{Y}$ & -- \\
\hline 03201000 & $\begin{array}{l}\text { Pocatalico River at Sissonville, } \\
\text { WV }\end{array}$ & 238 & $\begin{array}{l}\text { 1909-16, } \\
1931, \\
1938-78, \\
1980, \\
1997-98\end{array}$ & $\begin{array}{l}1939 \\
1998\end{array}$ & $\begin{array}{l}34.40 \\
35.70\end{array}$ & $\begin{array}{l}15,500 \\
14,600\end{array}$ & $6 / 29 / 98$ & 35.70 & 14,600 & $\mathrm{~N}$ & $25-50$ \\
\hline 03202400 & $\begin{array}{l}\text { Guyandotte River near } \\
\text { Baileysville, WV }\end{array}$ & 306 & 1969-98 & 1977 & 26.89 & 36,700 & $5 / 16 / 96$ & 22.18 & 22,500 & $\mathrm{~N}$ & $10-25$ \\
\hline 03202480 & Brier Creek at Fanrock, WV & 7.34 & $\begin{array}{l}\text { 1970-77, } \\
1994-98\end{array}$ & $\begin{array}{l}1996 \\
1977\end{array}$ & $\overline{--}$ & $\begin{array}{r}1,200 \\
980\end{array}$ & $5 / 16 / 96$ & -- & 1,200 & $\mathrm{~N}$ & 25 \\
\hline 03212750 & Tug Fork at Welch, WV & 174 & 1986-98 & 1996 & 13.23 & 6,550 & $5 / 16 / 96$ & 13.23 & 6,550 & $\mathrm{~N}$ & 10 \\
\hline 03212980 & Dry Fork at Beartown, WV & 209 & 1986-98 & 1998 & 11.88 & 9,840 & $3 / 21 / 98$ & 11.88 & 9,840 & $\mathrm{~N}$ & $10-25$ \\
\hline
\end{tabular}

'Regulated during flood: $\mathrm{N}$, no; $\mathrm{Y}$, yes. 


\section{Wisconsin}

Excessive rainfall from thunderstorms occurred September 15, 1994, across most of north-central Wisconsin and caused flooding on the Lily, Namekagon, Flambeau, and Spirit Rivers (fig. 62). Six streamgages recorded their highest discharges of record (table 51). Damage was more than $\$ 62$ million (National Oceanic and Atmospheric Administration, 1994b).

Excessive rainfall of 4 to 8 inches fell across most of central and east-central Wisconsin, and parts of northeastern Wisconsin on June 16-18, 1996. Amounts during the 3-day period ranged from 2 to 7 inches in most areas (National Oceanic and Atmospheric Administration, 1996a). Runoff from the excessive rainfall caused rivers to rise, with several peaking well above flood stage. Property damage was more than $\$ 23$ million, and crop damage estimates amounted to at least \$56 million (National Oceanic and Atmospheric Administration, 1996b). Some of the larger lakes recorded their highest levels ever. Lake Monona and Lake Kegonsa eventually reached new record levels on June 19 (National Oceanic and Atmospheric Administration, 1996a). About 1,275 homes had varying amounts of flood damage as rains of 1 to 2 inches fell on saturated soil (National Oceanic and Atmospheric Administration, 1996a; 1996b), resulting in flash flooding in the Madison area. Emergency managers noted that this was the worst flash flooding seen in the Madison area for 30 years. On June 17, 4.51 inches of rain fell at the Madison airport, making it the wettest June day ever. A total of 13.52 inches of rain fell at Port Washington during June 16-18, with a 1-day record maximum rainfall of 7.68 inches occurring on June 18 (National Oceanic and Atmospheric Administration, 1996a).

In extreme southern Wisconsin, rainfall amounts during a 5-hour period on July 18, 1996, generally totaled 10 to 12 inches. As much as 13.50 inches fell about 6 miles northwest of Monroe (National Oceanic and Atmospheric Administration, 1996a). A bridge west of Browntown was washed away as water depths on the road reached 4 feet. Damage was more than $\$ 6$ million (National Oceanic and Atmospheric Administration, 1996b).

Severe flash flooding occurred in southeastern Wisconsin including Milwaukee as rainfall amounts of as much as 10 inches fell during a 30-hour period ending on June 21, 1997 (National Oceanic and Atmospheric Administration, 1997a). The flash flooding was greater than the 100-year flood
(National Oceanic and Atmospheric Administration, 1997b). No one was injured or killed by the floodwaters, thanks to superb rescue efforts by local law enforcement officials and firefighters. About 9,600 homes in the county had minor damage, 137 had major damage, and 15 were destroyed. Property losses were more than \$84 million (National Oceanic and Atmospheric Administration, 1997b). Four streamgages recorded their highest discharge ever during this flood (table 51). Areas along and near the Menomonee River experienced moderate to major flooding to nearby roads, homes, and businesses. In Menomonee Falls, several homes had significant structural damage, and about 50 homes had significant damage.

A series of slow-moving thunderstorms produced a total of 6 to 10 inches of rainfall (National Oceanic and Atmospheric Administration, 1998a) and caused flash flooding as they moved through the Janesville and Milton areas on August 5, 1998. Flash floods also hit the area from Sheboygan Falls to Sheboygan during the early morning hours of August 5. Sheboygan had the worst flooding, with estimated damage of about $\$ 6$ million to roads and bridges. The city school district suffered damage of $\$ 1.2$ million (National Oceanic and Atmospheric Administration, 1998b).

Flash floods ravaged parts of Milwaukee and the counties to the west during the afternoon and evening hours of August 6, 1998. For Milwaukee, it was the second year in row for devastating floods. The damage was not as bad as that in June 1997, but for the county to the west it was the worst flooding ever. The flash flood was the result of 5 to 9 inches of rain falling in addition to 1 to 3 inches that fell during the previous 2 days. Maximum storm rainfall was 8.90 inches in Milwaukee (National Oceanic and Atmospheric Administration, 1998a). Damage was more than $\$ 82$ million (National Oceanic and Atmospheric Administration, 1998b). One 13-year-old boy was injured as he was swept into a culvert and submerged for 15 minutes by floodwaters.

\section{References}

National Oceanic and Atmospheric Administration (NOAA), 1994a-98a, Climatological data (by State): Asheville, North Carolina, National Climatic Data Center, various months.

National Oceanic and Atmospheric Administration (NOAA), 1994b-98b, Storm data (by State): Asheville, North Carolina, National Climatic Data Center, various months. 


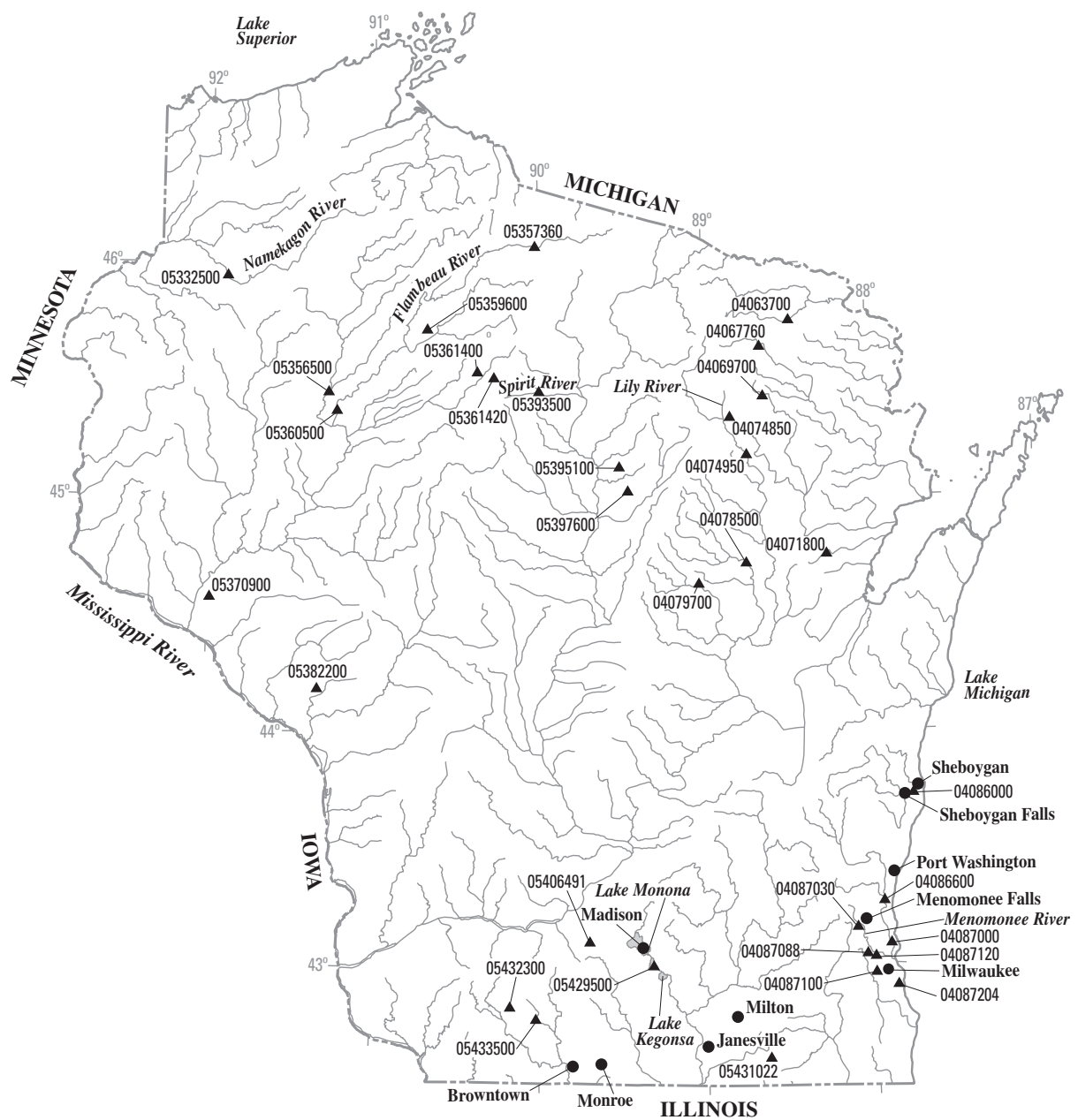

Base from U.S. Geological Survey digital data, 1:2,000,000, 1994 Albers Equal-Area Conic projection

Standard parallels $29^{\circ} 30^{\prime}$ and $45^{\circ} 30^{\prime}$, central meridian $-96^{\circ} 00^{\prime}$

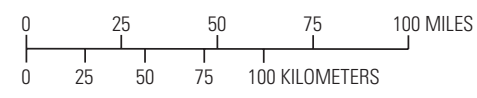

EXPLANATION

05431022 Streamgage and number

Figure 62. Location of streamgages with significant floods during 1994-98 water years for Wisconsin. 
Table 51. Maximum stage and discharge for period of record for streamgages having significant floods during 1994-98 water years in Wisconsin.

$\left[\mathrm{mi}^{2}\right.$, square miles; ft, feet above an arbitrary datum; $\mathrm{ft}^{3} / \mathrm{s}$, cubic feet per second; --, not determined or not applicable; >, greater than. Source: Recurrence intervals calculated from U.S. Geological Survey data. Other data from U.S. Geological Survey reports or databases]

\begin{tabular}{|c|c|c|c|c|c|c|c|c|c|c|c|}
\hline \multirow[b]{2}{*}{$\begin{array}{c}\text { Streamgage } \\
\text { number } \\
\text { (fig. 62) }\end{array}$} & \multirow[b]{2}{*}{ Streamgage name } & \multirow[b]{2}{*}{$\begin{array}{c}\text { Total } \\
\text { drainage } \\
\left(\mathrm{mi}^{2}\right)\end{array}$} & \multicolumn{4}{|c|}{$\begin{array}{c}\text { Maximum stage and discharge for period of record } \\
\text { through } 1998 \text { water year }\end{array}$} & \multicolumn{5}{|c|}{ Significant floods $1994-98$ water years } \\
\hline & & & $\begin{array}{l}\text { Period of } \\
\text { record } \\
\text { (water } \\
\text { years) }\end{array}$ & Water year & $\begin{array}{l}\text { Stage } \\
\text { (ft) }\end{array}$ & $\begin{array}{c}\text { Discharge } \\
\left(\mathrm{ft}^{3} / \mathrm{s}\right)\end{array}$ & $\begin{array}{c}\text { Date } \\
\text { (month/ } \\
\text { day/ } \\
\text { year) }\end{array}$ & $\begin{array}{l}\text { Stage } \\
(\mathrm{ft})\end{array}$ & $\begin{array}{c}\text { Discharge } \\
\left(\mathrm{ft}^{3} / \mathrm{s}\right)\end{array}$ & $\begin{array}{l}\text { Regulated } \\
\text { during } \\
\text { flood }^{1}\end{array}$ & $\begin{array}{c}\text { Recurrence } \\
\text { interval } \\
\text { (years) }\end{array}$ \\
\hline 04063700 & Popple River near Fence, WI & 139 & $1964-98$ & $\begin{array}{l}1979 \\
1996\end{array}$ & $\begin{array}{l}4.52 \\
4.70\end{array}$ & $\begin{array}{l}1,640 \\
1,490\end{array}$ & $4 / 26 / 96$ & 4.70 & 1,490 & $\mathrm{~N}$ & $30-50$ \\
\hline 04067760 & Peshtigo River near Cavour, WI & 150 & 1970-98 & 1996 & 15.78 & 1,600 & $4 / 21 / 96$ & 15.78 & 1,600 & $\mathrm{~N}$ & $20-30$ \\
\hline 04069700 & $\begin{array}{l}\text { North Branch Oconto River near } \\
\text { Wabeno, WI }\end{array}$ & 34.1 & 1970-98 & 1996 & 14.21 & 621 & $4 / 20 / 96$ & 14.21 & 621 & $\mathrm{~N}$ & 100 \\
\hline 04071800 & Pensaukee River near Pulaski, WI & 48.8 & $\begin{array}{l}\text { 1961-93 } \\
1995-98\end{array}$ & $\begin{array}{l}1996 \\
1973\end{array}$ & $\begin{array}{l}16.96 \\
17.10\end{array}$ & $\begin{array}{l}1,810 \\
1,700\end{array}$ & $6 / 18 / 96$ & 16.96 & 1,810 & $\mathrm{~N}$ & 25 \\
\hline 04074850 & Lily River near Lily, WI & 45.6 & $\begin{array}{l}\text { 1970-76, } \\
1978-98\end{array}$ & $\begin{array}{l}1994 \\
1975\end{array}$ & $\begin{array}{l}10.55 \\
11.00\end{array}$ & $\begin{array}{l}173 \\
158\end{array}$ & $9 / 15 / 94$ & 10.55 & 173 & $\mathrm{~N}$ & $10-20$ \\
\hline 04074950 & Wolf River at Langlade, WI & 463 & $\begin{array}{l}1968-79 \\
1981-98\end{array}$ & 1996 & 10.40 & 2,440 & $4 / 26 / 96$ & 10.40 & 2,440 & $\mathrm{~N}$ & $30-50$ \\
\hline 04078500 & $\begin{array}{l}\text { Embarrass River near Embarrass, } \\
\text { WI }\end{array}$ & 384 & $\begin{array}{l}1920-85 \\
1994-98\end{array}$ & 1965 & 12.13 & 7,080 & $6 / 19 / 96$ & 10.81 & 4,830 & $\mathrm{~N}$ & $10-20$ \\
\hline 04079700 & $\begin{array}{l}\text { Spaulding Creek near Big Falls, } \\
\text { WI }\end{array}$ & 5.57 & 1959-98 & 1960 & 11.64 & 101 & $6 / 18 / 96$ & 11.56 & 93 & $\mathrm{~N}$ & 25 \\
\hline 04086000 & $\begin{array}{l}\text { Sheboygan River at Sheboygan, } \\
\text { WI }\end{array}$ & 418 & $\begin{array}{l}1917-23 \\
1951-98\end{array}$ & 1998 & 12.02 & 7,820 & $8 / 6 / 98$ & 12.02 & 7,820 & $\mathrm{~N}$ & $25-30$ \\
\hline 04086600 & $\begin{array}{l}\text { Milwaukee River near Cedarburg, } \\
\text { WI }\end{array}$ & 607 & 1982-98 & 1996 & 12.88 & 5,500 & $6 / 18 / 96$ & 12.88 & 5,500 & $\mathrm{~N}$ & $10-25$ \\
\hline 04087000 & $\begin{array}{l}\text { Milwaukee River at Milwaukee, } \\
\text { WI }\end{array}$ & 696 & 1915-98 & 1997 & 10.00 & 16,500 & $6 / 21 / 97$ & 10.00 & 16,500 & $\mathrm{~N}$ & $>100$ \\
\hline 04087030 & $\begin{array}{l}\text { Menomonee River at Menomonee } \\
\text { Falls, WI }\end{array}$ & 34.7 & $\begin{array}{l}\text { 1975-77, } \\
1980-98\end{array}$ & 1997 & 8.31 & 1,500 & $6 / 21 / 97$ & 8.31 & 1,500 & $\mathrm{~N}$ & $25-50$ \\
\hline 04087088 & $\begin{array}{l}\text { Underwood Creek at Wauwatosa, } \\
\text { WI }\end{array}$ & 18.2 & 1979-98 & 1998 & 13.10 & 7,500 & $8 / 6 / 98$ & 13.10 & 7,500 & $\mathrm{~N}$ & $50-100$ \\
\hline 04087100 & Honey Creek at Milwaukee, WI & 3.26 & 1959-98 & 1997 & 22.70 & 1,100 & $6 / 21 / 97$ & 22.70 & 1,100 & $\mathrm{~N}$ & $75-100$ \\
\hline 04087120 & $\begin{array}{l}\text { Menomonee River at Wauwatosa, } \\
\text { WI }\end{array}$ & 123 & 1962-98 & 1997 & 18.64 & 13,500 & $6 / 21 / 97$ & 18.64 & 13,500 & $\mathrm{~N}$ & $30-50$ \\
\hline
\end{tabular}


Table 51. Maximum stage and discharge for period of record for streamgages having significant floods during 1994-98 water years in Wisconsin.-Continued

$\left[\mathrm{mi}^{2}\right.$, square miles; $\mathrm{ft}$, feet above an arbitrary datum; $\mathrm{ft}^{3} / \mathrm{s}$, cubic feet per second; --, not determined or not applicable; $>$, greater than. Source: Recurrence intervals calculated from U.S. Geological Survey data. Other data from U.S. Geological Survey reports or databases]

\begin{tabular}{|c|c|c|c|c|c|c|c|c|c|c|c|}
\hline \multirow{2}{*}{$\begin{array}{l}\text { Streamgage } \\
\text { number } \\
\text { (fig. 62) }\end{array}$} & \multirow[b]{2}{*}{ Streamgage name } & \multirow{2}{*}{$\begin{array}{c}\text { Total } \\
\text { drainage } \\
\left(\mathrm{mi}^{2}\right)\end{array}$} & \multicolumn{4}{|c|}{$\begin{array}{l}\text { Maximum stage and discharge for period of record } \\
\text { through } 1998 \text { water year }\end{array}$} & \multicolumn{5}{|c|}{ Significant floods 1994-98 water years } \\
\hline & & & $\begin{array}{l}\text { Period of } \\
\text { record } \\
\text { (water } \\
\text { years) }\end{array}$ & Water year & $\begin{array}{l}\text { Stage } \\
(\mathrm{ft})\end{array}$ & $\begin{array}{l}\text { Discharge } \\
\left(\mathrm{ft}^{3} / \mathrm{s}\right)\end{array}$ & $\begin{array}{c}\text { Date } \\
\text { (month/ } \\
\text { day/ } \\
\text { year) }\end{array}$ & $\begin{array}{l}\text { Stage } \\
(\mathrm{ft})\end{array}$ & $\begin{array}{l}\text { Discharge } \\
\left(\mathrm{ft}^{3} / \mathrm{s}\right)\end{array}$ & $\begin{array}{l}\text { Regulated } \\
\text { during } \\
\text { flood }^{1}\end{array}$ & $\begin{array}{c}\text { Recurrence } \\
\text { interval } \\
\text { (years) }\end{array}$ \\
\hline 04087204 & $\begin{array}{l}\text { Oak Creek at South Milwaukee, } \\
\text { WI }\end{array}$ & 25.0 & $1964-98$ & 1986 & 9.88 & 1,140 & $6 / 21 / 97$ & 9.71 & 1,110 & $\mathrm{~N}$ & $30-50$ \\
\hline 05332500 & Namekagon River near Trego, WI & 488 & $\begin{array}{l}1928-70 \\
1988-98\end{array}$ & 1941 & -- & 5,200 & 9/17/94 & -- & 3,060 & $\mathrm{Y}$ & $25-30$ \\
\hline 05356500 & Chippewa River near Bruce, WI & 1,650 & 1914-98 & $\begin{array}{l}1994 \\
1941\end{array}$ & $\begin{array}{l}18.12 \\
20.46\end{array}$ & $\begin{array}{l}29,000 \\
25,800\end{array}$ & $9 / 17 / 94$ & 18.12 & 29,000 & $\mathrm{Y}$ & $>100$ \\
\hline 05357360 & Bear River near Powell, WI & 120 & $1970-98$ & 1996 & 13.06 & 730 & $4 / 26 / 96$ & 13.06 & 730 & $\mathrm{~N}$ & $10-25$ \\
\hline 05359600 & Price Creek near Phillips, WI & 16.9 & $1958-98$ & 1994 & 17.43 & 552 & $9 / 15 / 94$ & 17.43 & 552 & $\mathrm{~N}$ & $>100$ \\
\hline 05360500 & Flambeau River near Bruce, WI & 1,860 & $1952-98$ & 1994 & 12.44 & 24,100 & $\begin{array}{l}9 / 16 / 94 \\
4 / 21 / 96\end{array}$ & $\begin{array}{l}12.44 \\
10.56\end{array}$ & $\begin{array}{l}24,100 \\
17,900\end{array}$ & $\begin{array}{l}\text { Y } \\
\text { Y }\end{array}$ & $\begin{array}{l}60-70 \\
15-20\end{array}$ \\
\hline 05361400 & Hay Creek near Prentice, WI & 22.6 & $1961-98$ & $\begin{array}{l}1994 \\
1967\end{array}$ & $\begin{array}{l}15.39 \\
15.41\end{array}$ & $\begin{array}{r}1,650 \\
975\end{array}$ & $9 / 16 / 94$ & 15.39 & 1,650 & $\mathrm{~N}$ & $>100$ \\
\hline 05361420 & Douglas Creek near Prentice, WI & 25.2 & 1970-98 & 1994 & 17.66 & 1,620 & $9 / 15 / 94$ & 17.66 & 1,620 & $\mathrm{~N}$ & $>100$ \\
\hline 05370900 & Spring Creek near Durand, WI & 6.45 & 1962-98 & 1975 & 15.71 & 860 & 7/7/94 & 14.10 & 450 & $\mathrm{~N}$ & $10-20$ \\
\hline 05382200 & French Creek near Ettrick, WI & 14.7 & $\begin{array}{l}\text { 1960-71, } \\
1989-98\end{array}$ & $\begin{array}{l}1998 \\
1967\end{array}$ & $\begin{array}{l}12.14 \\
13.07\end{array}$ & $\begin{array}{l}2,450 \\
1,300\end{array}$ & $6 / 27 / 98$ & 12.14 & 2,450 & $\mathrm{~N}$ & $25-50$ \\
\hline 05393500 & Spirit River at Spirit Falls, WI & 81.6 & $1942-98$ & 1942 & 10.00 & 4,180 & $9 / 15 / 94$ & 8.66 & 3,990 & $\mathrm{~N}$ & $75-100$ \\
\hline 05395100 & $\begin{array}{l}\text { Trappe River tributary near } \\
\text { Merrill, WI }\end{array}$ & 1.58 & 1959-98 & 1995 & 17.79 & 396 & $8 / 15 / 95$ & 17.79 & 396 & $\mathrm{~N}$ & $20-25$ \\
\hline 05397600 & Big Sandy Creek near Wausau, WI & 11.5 & 1959-98 & 1959 & 15.18 & 2,120 & $8 / 15 / 95$ & 14.46 & 1,600 & $\mathrm{~N}$ & $30-40$ \\
\hline 05406491 & $\begin{array}{l}\text { Garfoot Creek near Cross Plains, } \\
\text { WI }\end{array}$ & 5.39 & $\begin{array}{l}1985-86 \\
1990-98\end{array}$ & $\begin{array}{l}1998 \\
1993\end{array}$ & $\begin{array}{l}6.78 \\
7.57\end{array}$ & $\begin{array}{l}212 \\
111\end{array}$ & $3 / 31 / 98$ & 6.78 & 212 & $\mathrm{~N}$ & $50-100$ \\
\hline 05429500 & Yahara River near McFarland, WI & 327 & $\begin{array}{l}1931-71 \\
1974-98\end{array}$ & $\begin{array}{l}1959 \\
1996\end{array}$ & $\begin{array}{l}5.82 \\
6.66\end{array}$ & $\begin{array}{l}867 \\
778\end{array}$ & $6 / 19 / 96$ & 6.66 & 778 & $\mathrm{Y}$ & $50-60$ \\
\hline
\end{tabular}


$\left[\mathrm{mi}^{2}\right.$, square miles; ft, feet above an arbitrary datum; $\mathrm{ft}^{3} / \mathrm{s}$, cubic feet per second; --, not determined or not applicable; >, greater than. Source: Recurrence intervals calculated from U.S. Geological Survey data. Other data from U.S. Geological Survey reports or databases]

\begin{tabular}{|c|c|c|c|c|c|c|c|c|c|c|c|}
\hline \multirow{2}{*}{$\begin{array}{l}\text { Streamgage } \\
\text { number } \\
\text { (fig. 62) }\end{array}$} & \multirow[b]{2}{*}{ Streamgage name } & \multirow{2}{*}{$\begin{array}{c}\text { Total } \\
\text { drainage } \\
\left(\mathrm{mi}^{2}\right)\end{array}$} & \multicolumn{4}{|c|}{$\begin{array}{c}\text { Maximum stage and discharge for period of record } \\
\text { through } 1998 \text { water year }\end{array}$} & \multicolumn{5}{|c|}{ Significant floods $1994-98$ water years } \\
\hline & & & $\begin{array}{l}\text { Period of } \\
\text { record } \\
\text { (water } \\
\text { years) }\end{array}$ & Water year & $\begin{array}{l}\text { Stage } \\
(\mathrm{ft})\end{array}$ & $\begin{array}{l}\text { Discharge } \\
\left(\mathrm{ft}^{3} / \mathrm{s}\right)\end{array}$ & $\begin{array}{c}\text { Date } \\
\text { (month/ } \\
\text { day/ } \\
\text { year) }\end{array}$ & $\begin{array}{l}\text { Stage } \\
(\mathrm{ft})\end{array}$ & $\begin{array}{l}\text { Discharge } \\
\left(\mathrm{ft}^{3} / \mathrm{s}\right)\end{array}$ & $\begin{array}{l}\text { Regulated } \\
\text { during } \\
\text { flood }^{1}\end{array}$ & $\begin{array}{c}\text { Recurrence } \\
\text { interval } \\
\text { (years) }\end{array}$ \\
\hline 05431022 & $\begin{array}{l}\text { Delavan Lake outlet at Borg Road } \\
\text { near Delavan, WI }\end{array}$ & 42.1 & $1984-98$ & $\begin{array}{l}1994 \\
1998\end{array}$ & $\begin{array}{l}8.27 \\
8.35\end{array}$ & $\begin{array}{l}473 \\
325\end{array}$ & $2 / 22 / 94$ & 8.27 & 473 & $\mathrm{~N}$ & $10-25$ \\
\hline 05432300 & $\begin{array}{l}\text { Rock Branch near Mineral Point, } \\
\text { WI }\end{array}$ & 4.83 & 1959-98 & 1993 & 22.63 & 3,100 & $5 / 24 / 94$ & 17.74 & 1,570 & $\mathrm{~N}$ & $25-35$ \\
\hline 05433500 & $\begin{array}{l}\text { Yellowstone River near } \\
\text { Blanchardville, WI }\end{array}$ & 28.5 & $\begin{array}{l}1955-73 \\
1975-98\end{array}$ & 1990 & 11.40 & 8,500 & $6 / 17 / 96$ & 10.74 & 5,100 & $\mathrm{~N}$ & $10-15$ \\
\hline
\end{tabular}

Regulated during flood: N, no; $\mathrm{Y}$, yes. 


\section{Wyoming}

Early on the morning of June 7, 1994, 4 to 5 inches of rain fell southwest of Kaycee (National Oceanic and Atmospheric Administration, 1994a) causing flash flooding on the Middle Fork Powder River (fig. 63). Several ranches sustained damage to irrigation equipment and fences. Only minor structural damage was reported at Kaycee.

On June 14, 1995, prolonged flooding occurred in central Wyoming as warm temperatures in the mountains brought a rapid snowmelt. Streams in the Wind River Basin overflowed their banks and caused considerable flooding. The flooding at Riverton occurred on the far south side of town. There was one fatality due to the flooding (National Oceanic and Atmospheric Administration, 1995b).

During snowmelt in May 1996 several rivers in Yellowstone National Park had their largest discharge of record (table 52). During July and August of 1996 several episodes of excessive rain caused by thunderstorms resulted in urban flooding in Cheyenne, Wheatland, and Chugwater.

Snowmelt in May and June 1997 caused significant flooding in Wyoming. The Snake River near Alpine, Wyoming (streamgage 13022500, table 52), had its largest discharge since 1937. Floods also occurred on the Wind River at Riverton (streamgage 06228000, table 52) and the Encampment River above Hog Park Creek near Encampment (streamgage 06623800, table 52).

\section{References}

National Oceanic and Atmospheric Administration (NOAA), 1994a-96a, Climatological data (by State): Asheville, North Carolina, National Climatic Data Center, various months.

National Oceanic and Atmospheric Administration (NOAA), 1994b-96b, Storm data (by State): Asheville, North Carolina, National Climatic Data Center, various months.

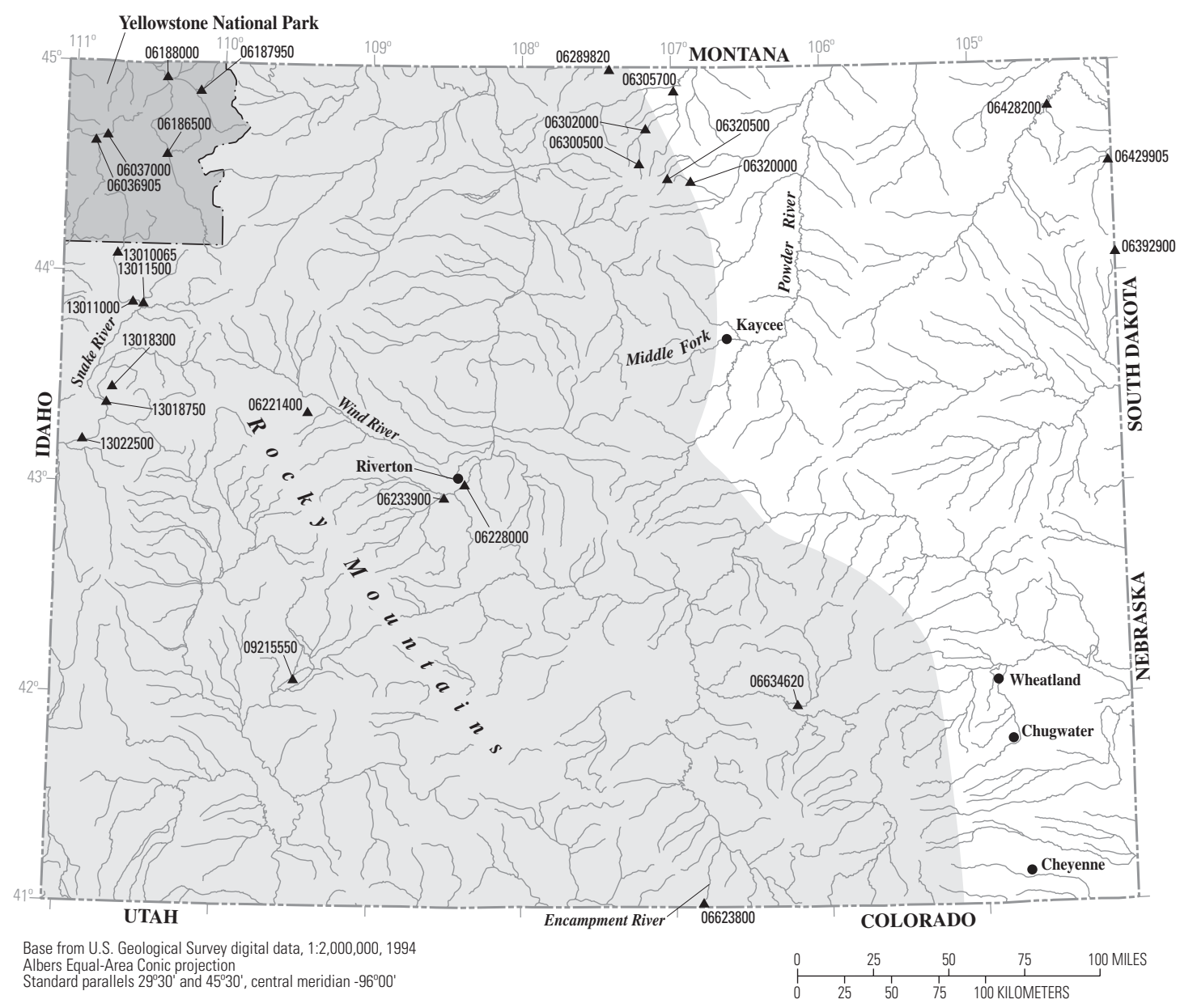

EXPLANATION

06623800 $\triangle$ Streamgage and number

Figure 63. Location of streamgages with significant floods during 1994-98 water years for Wyoming. 
Table 52. Maximum stage and discharge for period of record for streamgages having significant floods during 1994-98 water years in Wyoming.

$\left[\mathrm{mi}^{2}\right.$, square miles; $\mathrm{ft}$, feet above an arbitrary datum; $\mathrm{ft}^{3} / \mathrm{s}$, cubic feet per second; --, not determined or not applicable; >, greater than. Source: Recurrence intervals calculated from U.S. Geological Survey data. Other data from U.S. Geological Survey reports or databases]

\begin{tabular}{|c|c|c|c|c|c|c|c|c|c|c|c|}
\hline \multirow{2}{*}{$\begin{array}{l}\text { Streamgage } \\
\text { number } \\
\text { (fig. 63) }\end{array}$} & \multirow[b]{2}{*}{ Streamgage name } & \multirow{2}{*}{$\begin{array}{l}\text { Total } \\
\text { drainage } \\
\left(\mathrm{mi}^{2}\right)\end{array}$} & \multicolumn{4}{|c|}{$\begin{array}{c}\text { Maximum stage and discharge for period of record } \\
\text { through } 1998 \text { water year }\end{array}$} & \multicolumn{5}{|c|}{ Significant floods 1994-98 water years } \\
\hline & & & $\begin{array}{l}\text { Period of } \\
\text { record } \\
\text { (water } \\
\text { years) }\end{array}$ & Water year & $\begin{array}{l}\text { Stage } \\
(\mathrm{ft})\end{array}$ & $\begin{array}{l}\text { Discharge } \\
\left(\mathrm{ft}^{3} / \mathrm{s}\right)\end{array}$ & $\begin{array}{l}\text { Date } \\
\text { (month/ } \\
\text { day/ } \\
\text { year) }\end{array}$ & $\begin{array}{l}\text { Stage } \\
(\mathrm{ft})\end{array}$ & $\begin{array}{l}\text { Discharge } \\
\left(\mathrm{ft}^{3} / \mathrm{s}\right)\end{array}$ & $\begin{array}{l}\text { Regulated } \\
\text { during } \\
\text { flood }^{1}\end{array}$ & $\begin{array}{l}\text { Recurrence } \\
\text { interval } \\
\text { (years) }\end{array}$ \\
\hline 06036905 & $\begin{array}{l}\text { Firehole River near West } \\
\text { Yellowstone, MT }\end{array}$ & 282 & $1984-96$ & 1996 & 6.10 & 2,050 & $5 / 18 / 96$ & 6.10 & 2,050 & $\mathrm{~N}$ & $25-50$ \\
\hline 06037000 & $\begin{array}{l}\text { Gibbon River near West } \\
\text { Yellowstone, MT }\end{array}$ & 118 & 1984-96 & 1996 & 5.30 & 1,500 & $5 / 18 / 96$ & 5.30 & 1,500 & $\mathrm{~N}$ & $10-25$ \\
\hline 06186500 & $\begin{array}{l}\text { Yellowstone River at Yellowstone } \\
\text { Lake outlet, Yellowstone } \\
\text { National Park, WY }\end{array}$ & 1,006 & $\begin{array}{l}1923-82 \\
1984-86 \\
1989-98\end{array}$ & 1997 & 8.90 & 9,950 & $\begin{array}{l}6 / 28 / 96 \\
6 / 18 / 97\end{array}$ & $\begin{array}{l}8.25 \\
8.90\end{array}$ & $\begin{array}{l}8,770 \\
9,950\end{array}$ & $\begin{array}{l}\mathrm{N} \\
\mathrm{N}\end{array}$ & $\begin{array}{r}25-50 \\
50-100\end{array}$ \\
\hline 06187950 & $\begin{array}{l}\text { Soda Butte Creek near Lamar } \\
\text { Ranger Station, Yellowstone } \\
\text { National Park, WY }\end{array}$ & 99.0 & 1989-98 & $\begin{array}{l}1996 \\
1998\end{array}$ & $\begin{array}{l}5.61 \\
5.87\end{array}$ & $\begin{array}{r}2,450 \\
997\end{array}$ & $6 / 8 / 96$ & 5.61 & 2,450 & $\mathrm{~N}$ & $10-25$ \\
\hline 06188000 & $\begin{array}{l}\text { Lamar River near Tower Falls } \\
\text { Ranger Station, Yellowstone } \\
\text { National Park, WY }\end{array}$ & 660 & $\begin{array}{l}1923-69 \\
1985-86 \\
1989-98\end{array}$ & 1996 & 12.15 & 19,500 & $\begin{array}{l}6 / 6 / 95 \\
6 / 10 / 96 \\
6 / 5 / 97\end{array}$ & $\begin{array}{r}9.95 \\
12.15 \\
11.46\end{array}$ & $\begin{array}{l}14,000 \\
19,500 \\
17,500\end{array}$ & $\begin{array}{l}\mathrm{N} \\
\mathrm{N} \\
\mathrm{N}\end{array}$ & $\begin{array}{r}25-50 \\
>100 \\
100\end{array}$ \\
\hline 06221400 & $\begin{array}{l}\text { Dinwoody Creek above lakes near } \\
\text { Burris, WY }\end{array}$ & 88.2 & $\begin{array}{l}\text { 1918, 1956, } \\
1958-78 \\
1989 \\
1997-98\end{array}$ & $\begin{array}{l}1995 \\
1963\end{array}$ & $\begin{array}{l}4.50 \\
4.57\end{array}$ & $\begin{array}{l}1,510 \\
1,270\end{array}$ & $\begin{array}{l}7 / 13 / 95 \\
6 / 9 / 97\end{array}$ & $\begin{array}{l}4.50 \\
4.35\end{array}$ & $\begin{array}{l}1,510 \\
1,490\end{array}$ & $\begin{array}{l}\mathrm{N} \\
\mathrm{N}\end{array}$ & $\begin{array}{l}30-50 \\
20-30\end{array}$ \\
\hline 06228000 & Wind River at Riverton, WY & 2,309 & $\begin{array}{l}\text { 1906, 1908, } \\
1911-98\end{array}$ & $\begin{array}{l}1935 \\
1997\end{array}$ & $\begin{array}{r}8.15 \\
10.86\end{array}$ & $\begin{array}{l}13,300 \\
10,100\end{array}$ & $6 / 10 / 97$ & 10.86 & 10,100 & $\mathrm{Y}$ & 20 \\
\hline 06233900 & $\begin{array}{l}\text { Popo Agie River near Arapahoe, } \\
\text { WY }\end{array}$ & 796 & 1980-95 & 1995 & 10.22 & 4,760 & $6 / 17 / 95$ & 10.22 & 4,760 & $\mathrm{Y}$ & $10-25$ \\
\hline 06289820 & East Pass Creek near Dayton, WY & 21.7 & 1983-98 & $\begin{array}{l}1995 \\
1997\end{array}$ & $\begin{array}{l}4.47 \\
6.45\end{array}$ & $\begin{array}{l}511 \\
117\end{array}$ & $5 / 9 / 95$ & 4.47 & 511 & $\mathrm{Y}$ & $>50$ \\
\hline 06300500 & $\begin{array}{l}\text { East Fork Big Goose Creek near } \\
\text { Big Horn, WY }\end{array}$ & 20.1 & $\begin{array}{l}1954-83 \\
1985-98\end{array}$ & $\begin{array}{l}1963 \\
1995\end{array}$ & $\begin{array}{l}3.59 \\
6.69\end{array}$ & $\begin{array}{l}1,230 \\
1,140\end{array}$ & $6 / 15 / 95$ & 6.69 & 1,140 & $\mathrm{~N}$ & $40-50$ \\
\hline
\end{tabular}


Table 52. Maximum stage and discharge for period of record for streamgages having significant floods during 1994-98 water years in Wyoming. - Continued

$\left[\mathrm{mi}^{2}\right.$, square miles; $\mathrm{ft}$, feet above an arbitrary datum; $\mathrm{ft}^{3} / \mathrm{s}$, cubic feet per second; --, not determined or not applicable; >, greater than. Source: Recurrence intervals calculated from U.S. Geological Survey data. Other data from U.S. Geological Survey reports or databases]

\begin{tabular}{|c|c|c|c|c|c|c|c|c|c|c|c|}
\hline \multirow{2}{*}{$\begin{array}{l}\text { Streamgage } \\
\text { number } \\
\text { (fig. 63) }\end{array}$} & \multirow[b]{2}{*}{ Streamgage name } & \multirow{2}{*}{$\begin{array}{l}\text { Total } \\
\text { drainage } \\
\left(\mathrm{mi}^{2}\right)\end{array}$} & \multicolumn{4}{|c|}{$\begin{array}{c}\text { Maximum stage and discharge for period of record } \\
\text { through } 1998 \text { water year }\end{array}$} & \multicolumn{5}{|c|}{ Significant floods 1994-98 water years } \\
\hline & & & $\begin{array}{l}\text { Period of } \\
\text { record } \\
\text { (water } \\
\text { years) }\end{array}$ & Water year & $\begin{array}{l}\text { Stage } \\
(\mathrm{ft})\end{array}$ & $\begin{array}{l}\text { Discharge } \\
\left(\mathrm{ft}^{3} / \mathrm{s}\right)\end{array}$ & $\begin{array}{l}\text { Date } \\
\text { (month/ } \\
\text { day/ } \\
\text { year) }\end{array}$ & $\begin{array}{l}\text { Stage } \\
(\mathrm{ft})\end{array}$ & $\begin{array}{l}\text { Discharge } \\
\left(\mathrm{ft}^{3} / \mathrm{s}\right)\end{array}$ & $\begin{array}{l}\text { Regulated } \\
\text { during } \\
\text { flood }^{1}\end{array}$ & $\begin{array}{l}\text { Recurrence } \\
\text { interval } \\
\text { (years) }\end{array}$ \\
\hline 06302000 & $\begin{array}{l}\text { Big Goose Creek near Sheridan, } \\
\text { WY }\end{array}$ & 120 & $1930-98$ & 1963 & 5.83 & 3,160 & $6 / 17 / 95$ & 4.65 & 2,090 & $\mathrm{Y}$ & 30 \\
\hline 06305700 & Goose Creek near Acme, WY & 411 & 1984-98 & $\begin{array}{l}1995 \\
1994\end{array}$ & $\begin{array}{l}7.30 \\
7.51\end{array}$ & $\begin{array}{l}3,330 \\
1,490\end{array}$ & $6 / 17 / 95$ & 7.30 & 3,330 & $\mathrm{Y}$ & $75-100$ \\
\hline 06320000 & Rock Creek near Buffalo, WY & 60.0 & $1941-98$ & 1997 & 8.80 & 2,080 & $6 / 8 / 97$ & 8.80 & 2,080 & $\mathrm{Y}$ & $30-35$ \\
\hline 06320500 & $\begin{array}{l}\text { South Piney Creek at Willow Park, } \\
\text { WY }\end{array}$ & 33.6 & $\begin{array}{l}1946, \\
1948-57, \\
1960-72, \\
1975-83, \\
1985-98\end{array}$ & $\begin{array}{l}1963 \\
1997\end{array}$ & $\begin{array}{l}4.68 \\
5.27\end{array}$ & $\begin{array}{l}1,620 \\
1,220\end{array}$ & $6 / 8 / 97$ & 5.27 & 1,220 & $\mathrm{Y}$ & $35-50$ \\
\hline 06392900 & $\begin{array}{l}\text { Beaver Creek at Mallo Camp near } \\
\text { Four Corners, WY }\end{array}$ & 10.3 & $\begin{array}{l}1975-80 \\
1982-83 \\
1991-96\end{array}$ & $\begin{array}{l}1994 \\
1975\end{array}$ & $\begin{array}{l}2.14 \\
5.40\end{array}$ & $\begin{array}{r}103 \\
21\end{array}$ & $4 / 22 / 94$ & 2.14 & 103 & $\mathrm{~N}$ & $15-30$ \\
\hline 06428200 & $\begin{array}{l}\text { Belle Fourche River near Alva, } \\
\text { WY }\end{array}$ & 2,948 & 1989-98 & 1995 & 6.76 & 2,690 & $5 / 8 / 95$ & 6.76 & 2,690 & $\mathrm{Y}$ & -- \\
\hline 06429905 & $\begin{array}{l}\text { Sand Creek near Ranch A near } \\
\text { Beulah, WY }\end{array}$ & 267 & $\begin{array}{l}\text { 1977-83 } \\
1991-96\end{array}$ & $\begin{array}{l}1995 \\
1982\end{array}$ & $\begin{array}{l}3.80 \\
7.35\end{array}$ & $\begin{array}{r}1,230 \\
514\end{array}$ & $5 / 8 / 95$ & 3.80 & 1,230 & $\mathrm{~N}$ & $>50$ \\
\hline 06623800 & $\begin{array}{l}\text { Encampment River above Hog } \\
\text { Park Creek near Encampment, } \\
\text { WY }\end{array}$ & 72.7 & $1965-98$ & $\begin{array}{l}1997 \\
1970\end{array}$ & $\begin{array}{l}4.94 \\
5.01\end{array}$ & $\begin{array}{l}1,490 \\
1,180\end{array}$ & $6 / 2 / 97$ & 4.94 & 1,490 & $\mathrm{~N}$ & 10 \\
\hline 06634620 & $\begin{array}{l}\text { Little Medicine Bow River at } \\
\text { Boles Spring near Medicine } \\
\text { Bow, WY }\end{array}$ & 969 & 1984-97 & 1997 & 7.66 & 1,900 & $3 / 20 / 97$ & 7.66 & 1,900 & $\mathrm{~N}$ & 5 \\
\hline 09215550 & $\begin{array}{l}\text { Big Sandy River below Farson, } \\
\text { WY }\end{array}$ & 1,097 & 1982-98 & 1995 & 7.44 & 1,400 & $6 / 18 / 95$ & 7.44 & 1,400 & $\mathrm{Y}$ & -- \\
\hline 13010065 & $\begin{array}{l}\text { Snake River above Jackson Lake at } \\
\text { Flagg Ranch, WY }\end{array}$ & 486 & 1984-97 & 1996 & 10.75 & 15,000 & $6 / 5 / 96$ & 10.75 & 15,000 & $\mathrm{~N}$ & $10-25$ \\
\hline 13011000 & Snake River near Moran, WY & 807 & $\begin{array}{l}\text { 1904-67, } \\
1971-97\end{array}$ & $\begin{array}{l}1918 \\
1943\end{array}$ & 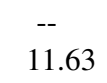 & $\begin{array}{l}15,100 \\
13,300\end{array}$ & $6 / 11 / 97$ & 10.96 & 12,100 & $\mathrm{Y}$ & 20 \\
\hline
\end{tabular}


$\left[\mathrm{mi}^{2}\right.$, square miles; ft, feet above an arbitrary datum; $\mathrm{ft}^{3} / \mathrm{s}$, cubic feet per second; --, not determined or not applicable; >, greater than. Source: Recurrence intervals calculated from U.S. Geological Survey data. Other data from U.S. Geological Survey reports or databases]

\begin{tabular}{|c|c|c|c|c|c|c|c|c|c|c|c|}
\hline \multirow{2}{*}{$\begin{array}{l}\text { Streamgage } \\
\text { number } \\
\text { (fig. 63) }\end{array}$} & \multirow[b]{2}{*}{ Streamgage name } & \multirow{2}{*}{$\begin{array}{c}\text { Total } \\
\text { drainage } \\
\left(\mathrm{mi}^{2}\right)\end{array}$} & \multicolumn{4}{|c|}{$\begin{array}{c}\text { Maximum stage and discharge for period of record } \\
\text { through } 1998 \text { water year }\end{array}$} & \multicolumn{5}{|c|}{ Significant floods $1994-98$ water years } \\
\hline & & & $\begin{array}{l}\text { Period of } \\
\text { record } \\
\text { (water } \\
\text { years) }\end{array}$ & Water year & $\begin{array}{l}\text { Stage } \\
(\mathrm{ft})\end{array}$ & $\begin{array}{l}\text { Discharge } \\
\left(\mathrm{ft}^{3} / \mathrm{s}\right)\end{array}$ & $\begin{array}{c}\text { Date } \\
\text { (month/ } \\
\text { day/ } \\
\text { year) }\end{array}$ & $\begin{array}{l}\text { Stage } \\
(\mathrm{ft})\end{array}$ & $\begin{array}{l}\text { Discharge } \\
\left(\mathrm{ft}^{3} / \mathrm{s}\right)\end{array}$ & $\begin{array}{l}\text { Regulated } \\
\text { during } \\
\text { flood }^{1}\end{array}$ & $\begin{array}{c}\text { Recurrence } \\
\text { interval } \\
\text { (years) }\end{array}$ \\
\hline 13011500 & Pacific Creek at Moran, WY & 169 & $\begin{array}{l}1918, \\
1945-75, \\
1978-97\end{array}$ & $\begin{array}{l}1983 \\
1997\end{array}$ & $\begin{array}{l}6.33 \\
7.56\end{array}$ & $\begin{array}{l}5,350 \\
4,890\end{array}$ & $6 / 5 / 97$ & 7.56 & 4,890 & $\mathrm{~N}$ & $40-60$ \\
\hline 13018300 & Cache Creek near Jackson, WY & 10.6 & 1945-98 & $\begin{array}{l}1971 \\
1996\end{array}$ & $\begin{array}{l}3.90 \\
4.30\end{array}$ & $\begin{array}{l}225 \\
166\end{array}$ & $6 / 10 / 96$ & 4.30 & 166 & $\mathrm{~N}$ & 20 \\
\hline 13018750 & $\begin{array}{l}\text { Snake River below Flat Creek near } \\
\text { Jackson, WY }\end{array}$ & 2,627 & 1978-97 & 1997 & 11.66 & 32,000 & $6 / 11 / 97$ & 11.66 & 32,000 & $\mathrm{Y}$ & $30-70$ \\
\hline 13022500 & $\begin{array}{l}\text { Snake River above reservoir near } \\
\text { Alpine, WY }\end{array}$ & 3,465 & $\begin{array}{l}1937-38 \\
1953-67 \\
1971-97\end{array}$ & $\begin{array}{l}1997 \\
1974\end{array}$ & $-\overline{-}$ & $\begin{array}{l}38,300 \\
28,600\end{array}$ & $\begin{array}{l}6 / 11 / 96 \\
6 / 11 / 97\end{array}$ & -- & $\begin{array}{l}31,600 \\
38,300\end{array}$ & $\begin{array}{l}\mathrm{Y} \\
\mathrm{Y}\end{array}$ & $\begin{array}{r}20-30 \\
100\end{array}$ \\
\hline
\end{tabular}

${ }^{1}$ Regulated during flood: N, no; Y, yes. 


\section{Index}

\section{A}

absorption, definition, ix

Alabama, 7, 11, 14, 20-23, 74, 148

Dauphin Island, 20

Elba, 20

Fort Morgan, 20

Mobile, 9, 20

Mobile Bay, 20

Weeks Bay, 20

Alaska, 7, 24-28

Alaska Highway, 24

Alatna, 24

Anchorage, 24

Coast Mountains, 24

Cook Inlet, 24

Hughes, 24

Kodiak Island, 24

Palmer, 24

Valdez, 24

Wiseman, 24

Alatna,

Alaska, 24

Alexandria,

Louisiana, 128

Allegheny River,

Pennsylvania, 298

Alta,

Utah, 269

Americus,

Georgia, 80

Ames,

Iowa, 115

Amite River,

Louisiana, 128

Anchorage,

Alaska, 24

Androscoggin River,

Maine, 130

Antelope Canyon,

Arizona, 29

Apalachicola,

Florida, 74

Apalachicola River,

Florida, 74

Appalachian Mountains, 133, 277, 298

Arcadia,

Florida, 74

Arizona, 16, 29-31

Antelope Canyon, 29
Arizona-Continued

Bradshaw Mountains, 29

Grand Canyon, 29

Littlefield, 29

Page, 29

Phantom Ranch, 29

Prescott, 29

Scottsdale, 29

Sunflower, 29

Tuscon, 29

Yuma, 29

Arkansas, 14, 32-34

Magnolia, 32

Texarkana, 32

Arkansas River,

Colorado, 59

Mississippi, 148

Oklahoma, 216

Salt Fork of the, 216

Aroostook River,

Maine, 130

Atlantic,

Iowa, 115

Atlantic City, New Jersey, 178

Atwood,

Colorado, 59

Aurora,

Illinois, 102

Austin,

Texas, 261

Ayers Brook,

Vermont, 273

B

Baker River, New Hampshire, 175

bank, definition, ix

Beardstown, Illinois, 102

Beaufort,

South Carolina, 245

Beaver Creek,

Kentucky, 123

Left Fork, 123

Right Fork, 123

Beaver Dam Wash, Arizona, 29

Benton Harbor, Michigan, 138

Big Muddy River, Illinois, 102 
Big Pine Creek, Indiana, 110

Big River,

Missouri, 7, 148

Biloxi, Mississippi, 148

Bismarck, North Dakota, 206

Bitterroot River, Montana, 157

Black Hills Region, South Dakota, 250

Blue Dog Lake, South Dakota, 250

Blue Licks Springs, Kentucky, 123

Blue River, at Fredericksburg, 110 at Milltown, 110 Indiana, 110

Boise River, Idaho, 94

Boone, Iowa, 115

Boston, Massachusetts, 136

Boulder City, Nevada, 169

Bourbeuse River, Missouri, 7, 152

Bradshaw Mountains, Arizona, 29

Branch Brook, Maine, 130

Brazos River, Texas, 261

Brian Head, Utah, 270

Bright Angel Creek, Arizona, 29

Broad River, North Carolina, 196

Brookville Lake, Indiana, 110

Browntown, Wisconsin, 304

Buckhannon River, West Virginia, 298

Buffalo Creek, Colorado, 59 near Overton, 167 Nebraska, 167
C

Cairo, Illinois, 14, 102, 123

Calcasieu River, at Oakdale, 128

Louisiana, 128

California, 13-14, 17, 35-58

Clear Lake, 35

Forest Falls, 35

Kings Canyon National Park, 35

Los Angeles, 17, 35

Sacramento, 35

San Francisco Bay, 17, 35

Santa Barbara, 35

Sequoia National Park, 35

Tracy, 35

Yosmite, 35

Yosmite National Park, 35

Calumet, Louisiana, 128

Canada, 13, 157, 206, 273

Canal Creek Canyon, Utah, 270

Cannelton, Indiana, 123

Cannelton Dam, Indiana, 123

Cape Fear, North Carolina, 196, 277

Cape Girardeau, Missouri, 102

Carnation, Washington, 286

Carson River, Nevada, 169

Caruthersville, Tennessee, 258

Cascade Range, Oregon, 220

Cassity, West Virginia, 298

Cass River, Michigan, 138

Cataldo, Idaho, 94

Catoma Creek, Alabama, 17

Catskill Mountains, New York, 186

Cattail Creek, Maryland, 133

Charleston, South Carolina, 245 
Charlotte,

North Carolina, 196

Chattahoochee River,

Georgia, 80

Chattanooga,

Tennessee, 258

Cheat River,

West Virginia, 298

Chehalis River,

Washington, 286

Chemung River, New York, 186

Cherokee, Oklahoma, 216

Chesapeake Bay, 133

Cheyenne, Wyoming, 309

Chicago, Illinois, 102

Chipola River, Florida, 74

Chippewa River, Minnesota, 142 near Milan, 142

Chisana River, Alaska, 24

Chikaskia River, near Blackwell, 216 Oklahoma, 216

Choctawhatchee River, Alabama, 20 at Newton, 20

Chugwater, Wyoming, 309

Cincinnati, Ohio, 102, 110, 123

Clear Lake, California, 35

Clearwater River, Idaho, 94

Clinton, Oklahoma, 216

Coast Mountains, Alaska, 24

Coast Range, Oregon, 220

Coeur d'Alene River, Idaho, 94 near Cataldo, 94 North Fork at Enaville, 94

Colorado, 9, 16, 59-69

Atwood, 59

Buffalo Creek, 59

Fort Collins, 59

Pawnee National Grasslands, 59

Pueblo, 59

Sterling, 59

Weldona, 59
Colorado River, 13

Colorado, 59

Texas, 262

Columbia,

Missouri, 152

Columbia River, Oregon, 220

Comite River, Louisiana, 128

Conecuh River, Alabama, 17

Connecticut, 70-71

Baltic, 70

East Haven, 70

MacKenzie Reservoir, 70

Meriden, 70

Milford, 70

North Branford, 70

Norwich, 70

Southbury, 70

Southington, 70

Wallingford, 70

Consumnes River, California, 35

Continental Divide, 157

convective precipitation, definition, ix

Cook Inlet,

Alaska, 24

Corozal,

Puerto Rico, 240

Cowlitz River,

Washington, 286

Crookston,

Minnesota, 142

Cross Terre Rouge Creek, Arkansas, 32

Crossville, Illinois, 102

Cuba, 17

cubic feet per second, definition, ix

Culbertson, Montana, 157

current meter, definition, ix

Current River, Missouri, 7, 152

Cynthiana, Kentucky, 123

D

Dallas, Texas, 9, 261

Dan River,

Virginia, 277

Danville,

Illinois, 102 
Dauphin Island, Alabama, 20

Delaware, 7, 13, 72-73 Dewey Beach, 72

South Bethany, 72

Wilmington, 72

Delaware Bay, 178

Delaware River, New Jersey, 178 New York, 186

Del Rio,

Texas, 261

Des Moines, Iowa, 115

Des Moines River, Iowa, 115

Destin,

Florida, 74

Devils Lake, North Dakota, 206

Dewey Beach, Delaware, 72

discharge, definition, ix measurements, 2-3

drainage area, definition, ix measurement, 3

drainage basin, definition, ix

Dry Beaver Creek, Arizona, 29

Duvall, Washington, 286

\section{E}

East Grand Forks, Minnesota, 206

East Haven, Connecticut, 70

East Helena, Montana, 157

East Nishnabotna River, near Atlantic, 115

Iowa, 115

Eel River, Indiana, 7, 110

Elba,

Alabama, 20

Elephant Butte Reservoir, New Mexico, 182

Elkhorn River, Nebraska, 167

El Nino, 35 definition, ix

Embarras River, Illinois, 7, 102
Emmitsburg, Maryland, 133

Enaville, Idaho, 94

Encampment River, above Hog Park Creek, 309

Wyoming, 309

envelope curve, 5

Erie,

Pennsylvania, 231

Escanaba River, Michigan, 138

Ewing, New Jersey, 178

$\mathbf{F}$

Fall River, Kansas, 120

Falmouth, Kentucky, 123

Fargo, North Dakota, 142, 206

Farlington, Kansas, 120

Farm River, Connecticut, 70

Feather River, California, 35

Fish River, Alabama, 20 near Silver Hill, 20

Fitzgerald, Georgia, 80

Flambeau River, Wisconsin, 304

Flathead River, Montana, 157 North Fork, 157

Flint River, Georgia, 80

flood,

flood, damages, 1 definition, ix envelope curve, 5 probability, 3 relative magnitude, 3,5 summary reports, 2 summary tables, 3-4

flood plain, definition, ix

flood stage, definition, ix determination, 2

Florida, 7, 9, 14, 17, 20, 74-79, 148 Apalachicola, 74 Arcadia, 74 
Florida-Continued

Destin, 74

Fort Myers, 74

Melbourne, 74

Miami, 74

Panama City, 74

St. Petersburg, 74

Tampa, 74

Vero Beach, 74

Florida Keys, 74

Ford River,

Michigan, 138

Forest Falls,

California, 35

Fort Benton, Montana, 157

Fort Collins,

Colorado, 59

Fort Fairfield,

Maine, 130

Fort Morgan,

Alabama, 20

Fort Myers,

Florida, 74

Fort Scott, Kansas, 120

Fowl River, Alabama, 20

Fredericksburg, Indiana, 110

Virginia, 277

Frio River,

Texas, 261

G

Garrison Dam, North Dakota, 206

Gasconade River, Missouri, 7, 152

Georgia, 7, 9, 11, 14, 17, 20, 74, 80-90

Americus, 80

Fitzgerald, 80

Gettysburg,

Pennsylvania, 133

Glen Canyon National Recreation Area, Utah, 269

Goldsboro, North Carolina, 196

Grafton, Vermont, 273

Grand Canyon, Arizona, 29

Grand Forks, North Dakota, 206

Grayville, Illinois, 102

Great Lakes, 130, 273

Great New England Hurricane, 136
Green River,

at Rochester, 123

Kentucky, 123

Greenville,

South Carolina, 245

Gulf Coast, 7, 17, 148, 261

Gulf of Mexico, 17, 74, 128, 148

Gulfport, Mississippi, 148

Gunlock Reservoir, Utah, 269

Guthrie,

Oklahoma, 216

H

Harbor Brook,

Connecticut, 70

Harrisburg,

Pennsylvania, 231

Hatteras Island, North Carolina, 196

Hawaii, 91-93

Big Island, 91

Hilo, 91

Laie, 91

Maui, 91

Oahu, 91

Puna District, 91

Wahaiwa, 91

Haw River,

at Haw River, 196

North Carolina, 196

Heart River,

North Dakota, 206

Henderson,

Nevada, 169

Hickory Nut Gorge, North Carolina, 196

Hilo,

Hawaii, 91

Hilton Head Island, South Carolina, 245

Hispaniola, 17

Hobart,

Oklahoma, 216

Houston,

Texas, 9, 261

Houston Ship Channel,

Texas, 261

Hudson River,

New York, 186

Hughes,

Alaska, 24

human factors, 1

Huntington,

Indiana, 110

Hurricane Agnes, 133, 231

Hurricane Allison, 9 
Hurricane Bertha, 13

Hurricane Bonnie, 17

Hurricane Danny, 14, 20, 196

Hurricane Earl, 17

Hurricane Eloise, 231

Hurricane Erin, 9

Hurricane Fran, 13, 196, 231, 277, 298

Hurricane Georges, 17, 148, 240

Hurricane Gordon, 196

Hurricane Hortense, 13, 240

Hurricane Lily, 130, 175

Hurricane Marilyn, 9

Hurricane Opal, 13, 20

\section{I}

Ice Cream Canyon,

Utah, 269

Idaho, 11, 13-14, 94-101

Cataldo, 94

Enaville, 94

Lake Coeur d'Alene, 94

Orofino, 94

Prichard, 94

Illinois, 7, 9, 13, 102-109

Aurora, 102

Beardsville, 102

Cairo, 102, 123

Chicago, 102

Danville, 102

Grayville, 102

Joliet, 102

Joslin, 102

LaSalle, 102

Lincoln, 102

Morris, 102

Mt. Carmel, 102

Rockford, 102

Illinois River,

Illinois, 7, 102

Indiana, 7, 14, 110-114

Brookville Lake, 110

Cannelton, 123

Cannelton Dam, 123

Huntington, 110

Medora, 110

Tell City, 123

Indian Bend Wash,

Arizona, 29

indirect measurements, 2-3

Iola,

Kansas, 120

Iowa, 115-119

Ames, 115

Atlantic, 115

Boone, 115

Des Moines, 115
Isle Royale, Michigan, 138

isohyetal map, definition, ix

J

Jackson,

Tennessee, 258

James River,

at Huron, 250

Missouri, 7, 152

North Dakota, 206

South Dakota, 250

Virginia, 277

Janesville,

Wisconsin, 304

Joliet,

Illinois, 102

Joslin, Illinois, 102

Juniata, Michigan, 138

K

Kansas, 120-122

Atchison, 120

Farlington, 120

Fort Scott, 120

Iola, 120

Kansas City, 120

Leavenworth, 120

Osawatomie, 120

Kansas City,

Kansas, 120

Missouri, 152

Kauaula Stream, Maui, Hawaii, 91

Kaycee, Wyoming, 309

Kenai River, Alaska, 24

Kennebec River, Maine, 130

Kentucky, 9, 13-14, 123-127

Falmouth, 123

Louisville, 102, 110, 123

Markland Dam, 110

Paducah, 123

Wickliffe, 123

Kentucky River, at Frankfort, 123

Kimball, Nebraska, 167

Kingfisher, Oklahoma, 216

Kings Canyon National Park, California, 35 
Klickitat River,

Washington, 286

Knife River,

North Dakota, 206

Kodiak Island, Alaska, 24

Koyukuk River, Alaska, 7, 24

L

Laie,

Hawaii, 91

Lake Ashtabula, North Dakota, 205

Lake Champlain, Vermont, 273

Lake Coeur d'Alene, Idaho, 94

Lake Conroe, Texas, 261

Lake Erie, 13

Lake Houston, Texas, 261

Lake Kegonsa, Wisconsin, 304

Lake Livingston Texas, 261

Lake Powell, 13

Lake Mead, 13

Lake Mead National Recreation Area, Nevada, 169

Lake Monona,

Wisconsin, 304

Lake Sammamish, Washington, 286

LaSalle, Illinois, 102

Las Vegas Valley, Nevada, 169

Lexington, Mississippi, 148

South Carolina, 245

Liberty,

Texas, 262

Licking River, at Blue Licks Springs, 123

Kentucky, 14, 123

South Fork at Cynthiana, 123

Lily River,

Wisconsin, 304

Lincoln,

Illinois, 102

Little Kanawha River, West Fork, 298

West Virginia, 298

Little Ossipee River, Maine, 130
Little Owens Creek, Maryland, 133

Livingston, Texas, 261

Llano River, Texas, 261

Lodgepole Creek, Nebraska, 167

Los Angeles,

California, 17, 35

Louisiana, 128-129, 148

Alexandria, 128

Calumet, 128

Delta, 14

New Orleans, 128, 148

Louisville,

Kentucky, 102, 110, 123

M

MacKenzie Reservoir, Connecticut, 70

Madison,

Minnesota, 142

Virginia, 277

Wisconsin, 304

Mad River,

Vermont, 273

Magnolia, Arkansas, 32

Maidford River, Rhode Island, 243

Maine, 17, 130-132

Fort Fairfield, 130

Mankato,

Minnesota, 142

Manville,

New Jersey, 178

Maple Creek, near Nickerson, 167

Nebraska, 167

Marais des Cygnes River, Kansas, 120

Markland Dam, Kentucky, 110

Marmaton River, Kansas, 120

Maryland, 13, 133-135, 298 Emmitsburg, 133

Massachusetts, 136-137

Boston, 136

Newburyport, 136

Maui,

Hawaii, 91

Maumee River, Indiana, 7, 110

Maury River, Virginia, 277 
Medora,

Indiana, 110

Melbourne,

Florida, 74

Meldahl Dam,

Ohio, 212

Memphis,

Tennessee, 258

Menomonee Falls,

Wisconsin, 304

Menomonee River,

Wisconsin, 304

Meramec River,

Missouri, 7, 152

Merced River,

California, 35

Meriden,

Connecticut, 70

meteorological factors, 1

Miami,

Florida, 74

Oklahoma, 212

Michigan, 138-141

Benton Harbor, 138

Isle Royale, 138

Juniata, 138

Thumb Region, 138

Upper Peninsula, 138

Middle Loup River, at Dunning, 167

Nebraska, 167

Middletown,

Rhode Island, 243

Millville,

New Jersey, 178

Milton,

Wisconsin, 304

Milwaukee,

Wisconsin, 304

Minnesota, 14, 142-147, 205

Crookston, 142

East Grand Forks, 206

Gulfport, 148

Madison, 142

Mankato, 142

Montevideo, 142

Minnesota River,

Minnesota, 142

Mississippi, 7, 14, 17, 128, 148-151

Biloxi, 148

Lexington, 148

Mississippi River, 7, 9, 102, 123, 148, 152, 258

Missouri, 7, 9, 14, 152-156

Cape Girardeau, 102

Caruthersville, 258

Columbia, 152
Missouri-Continued

Rolla, 152

Salem, 152

Springfield, 152

St. Louis, 152

Versailles, 152

Missouri River, 120, 152, 157, 205-207

at Bismarck, 206

near Williston, 205

North Dakota, 205-207

Mobile,

Alabama, 9, 20

Mobile Bay,

Alabama, 20

Mohawk River,

New York, 186

Monacacy River, at Bridgeport, 133

Maryland, 133

Monongahela River, Pennsylvania, 298

Monroe,

Wisconsin, 304

Montana, 11, 14, 157-166, 205-206

Culbertson, 157

East Helena, 157

Fort Benton, 157

Poplar, 157

Victor, 157

Montevideo,

Minnesota, 142

Morgan Run, Maryland, 133

Morris,

Illinois, 102

Mt. Carmel,

Illinois, 102

Mt. Hood,

Oregon, 220

Muddy River, Connecticut, 70

Mud Pine Creek, Indiana, 110

Murder Creek, Alabama, 17

$\mathbf{N}$

Namekagon River, Wisconsin, 304

National Water Information System (NWIS), 1

Natural Bridges National Monument, Utah, 270

Nebraska, 167-168

Scottsbluff, 167

Neosho River,

Kansas, 120 
Neosho River-Continued

Oklahoma, 216

Neuces River, Texas, 261

Neuse River, at Kinston, 196 North Carolina, 196

Nevada, 14, 169-174

Boulder City, 169

Henderson, 169

Lake Mead National Recreation Area, 169

Las Vegas Valley, 169

Newark,

New Jersey, 178

Newburyport,

Massachusetts, 136

New England, 130, 136, 186, 243, 273

New Hampshire, 175-177

New Jersey, 13, 17, 178-181, 186

Atlantic City, 178

Ewing, 178

Manville, 178

Millville, 178

Newark, 178

Trenton, 178

Vineland, 178

Westmont, 178

New Mexico, 182-185

Elephant Butte Reservoir, 182

Truth or Consequences, 182

New Orleans,

Louisiana, 128, 148

New York, 11, 13, 186-195, 273

Niobrara River,

Nebraska, 167

Nishnabotna River,

Iowa, 115

Nooksack River,

Washington, 286

North Branford, Connecticut, 70

North Carolina, 7, 9, 11, 13-14, 17, 74, 196-204

Cape Fear, 196, 277

Charlotte, 196

Goldsboro, 196

Hatteras Island, 196

Hickory Nut Gorge, 196

Pisgah National Forest, 196

Raleigh, 196

Southport, 196

North Dakota, 7, 14, 142. 157, 205-211

Bismarck, 206

Fargo, 142, 206

Garrison Dam, 206

Grand Forks, 206
North Dakota-Continued

Lake Ashtabula, 205

Wahpeton, 206

North Fork Coeur d'Alene River, Idaho, 94

North Raccoon River, Iowa, 115

Norwich,

Connecticut, 70

0

Oahu,

Hawaii, 91

Oak Creek,

Arizona, 29

at Cornville, 29

Ocmulgee River,

Georgia, 80

Ohio, 14, 212-215

Cincinnati, 102, 110, 123, 212

Meldahl Dam, 212

Portsmouth, 212

Ohio River, 9, 14, 102, 110, 123, 212, 298

at Cincinnati, 212

at Portsmouth, 212

Ohio, 212

Ohio River Valley, 13, 102, 110

Oklahoma, 17, 216-219

Cherokee, 216

Clinton, 216

Guthrie, 216

Hobart, 216

Kingfisher, 216

Miami, 216

Pryor, 216

Tahlequah, 216

Tipton, 216

Oregon, 11, 13-14, 220-230

Mt. Hood, 220

Portland, 220

Roseburg, 220

Willamette River Valley, 220

Orofino,

Idaho, 94

Osage River,

Missouri, 7, 152

overland flow,

definition, ix

Oyster River,

near Durham, 175

New Hampshire, 175

$\mathbf{P}$

Paducah,

Kentucky, 123 
Page,

Arizona, 29

Palmer, Alaska, 24

Palouse River, Idaho, 94

Panama City, Florida, 74

Park City, Utah, 269

Parker River, Massachusetts, 136

Pawnee Creek, Colorado, 59

Pawnee National Grasslands, Colorado, 59

Payette River, Idaho, 94

peak flow, ix

Pea River,

Alabama, 20

Pembina River, at Neche, 206

North Dakota, 206

Pennsylvania, 13-14, 186, 212, 231-239, 298

Erie, 231

Gettysburg, 133

Harrisburg, 231

Philadelphia, 178

Pittsburg, 14

Punxsutawney, 231

York, 231

Phantom Creek,

Arizona, 29

Phantom Ranch,

Arizona, 29

Philadelphia, Pennsylvania, 178

Pisgah National Forest, North Carolina, 196

Pittsburg,

Pennsylvania, 14, 298

percent change of occurrence, 3,5

period of record, 3

physiographic features, 1

Pomperaug River, Connecticut, 70

Poplar, Montana, 157

Portland,

Oregon, 220

Portsmouth, Ohio, 212

Port Washington, Wisconsin, 304

Potomac River, Maryland, 133
Potomac River-Continued

South Branch, 298

West Virginia, 298

Powder River,

Middle Fork, 309

Wyoming, 309

Prescott,

Arizona, 29

Presumpscot River, Maine, 130

Prichard,

Idaho, 94

Princeton,

West Virginia, 298

Pryor,

Oklahoma, 216

Pueblo, Colorado, 59

Puerto Rico, 9, 13, 17, 240-242 Corozal, 240

Puna District, Hawaii, 91

Punxsutawney, Pennsylvania, 231

$\mathbf{0}$

Quinnipiac River, Connecticut, 70

$\mathbf{R}$

Racoon River, Iowa, 115

Raleigh, North Carolina, 196

Rapidan River, near Ruckersville, 277

Virginia, 277

Rappahannock River, Virginia, 277

Raritan River, New Jersey, 178

recurrence interval, 3, 5

Red Lake River, Minnesota, 206

Red River, at Emerson, Manitoba, 206 near Burkburnett, 261

near Terral, 216

Oklahoma, 216

Texas, 261

Red River of the North, 14, 142, 205-206

at Drayton, 206

at Fargo, 206

at Grand Forks, 206

at Pembina, 206

at Wahpeton, 206 
regulation, definition, ix

reservoir, definition, ix

Resurrection River, Alaska, 24

Rhode Island, 243-244

Middletown, 243

Richmond, Virginia, 277

Río Cibuco, below Corozal, 240

Puerto Rico, 240

Río Grande de Loíza, at Caguas, 240

Puerto Rico, 240

Río Grande River, below Elephant Butte Dam, 182

New Mexico, 182

Texas, 261

Riverton, Wyoming, 309

Roanoke River, Virginia, 277

Rockford, Illinois, 102

Rock River, Illinois, 102

Rocky Mountains, 7, 59

Rolla, Missouri, 152

Rolling Fork River, at Shepherdsville, 123

Kentucky, 123

near Boston, 123

Roseburg,

Oregon, 220

runoff,

definition, ix

\section{S}

Sacramento, California, 35

Sacramento River, California, 35

Saginaw River, Michigan, 138

Salem, Missouri, 152

Salt Fork of the Arkansas River, near Tonkawa, 216

Oklahoma, 216

Salt River,

Arizona, 29

Kentucky, 123
San Bernardino Mountains, California, 35

Sand Draw, Nebraska, 167

San Francisco Bay, 17, 35

Sangamon River, Illinois, 7, 102

San Jacinto River, Texas, 261

San Joaquin River, California, 35

San Joaquin Valley, California, 35

Santa Ana Mountains, California, 35

Santa Barbara, California, 35

Santa Clara River, Utah, 269

Santa Ynez River, California, 35

Santa Ynez Valley, California, 35

Savage River, Maryland, 133

Sawmill River, Massachusetts, 136

Saxton River, Vermont, 273

Scottsbluff, Nebraska, 167

Scottsdale, Arizona, 29

Seattle, Washington, 13, 286

Sequoia National Park, California, 35

Sevierville, Tennessee, 258

Seymour, Tennessee, 258

Shawsheen River, Massachusetts, 133

Sheboygan, Wisconsin, 304

Sheboygan Falls, Wisconsin, 304

Shenandoah Valley, Virginia, 277

Shetucket River, Connecticut, 70 in Baltic, 70

Sheyenne River, at Lisbon, 205 below Baldhill Dam, 205 North Dakota, 205 
Sierra Nevada Mountains, 35, 169

Skagit River, near Concrete, 286

Washington, 286

Snake River,

Idaho, 94

near Alpine, 309

Wyoming, 309

Snoqualmie River,

Washington, 286

South Bethany,

Delaware, 72

Southbury,

Connecticut, 70

South Carolina, 7, 9, 13-14, 17, 245-249

Beaufort, 245

Charleston, 245

Greenville, 245

Hilton Head Island, 245

Lexington, 245

Piedmont area, 245

Spartanburg, 245

South Dakota, 14, 206, 250-257

Blue Dog Lake, 250

Southington,

Connecticut, 70

South Platte River,

Colorado, 59

Southport,

North Carolina, 196

South Skunk River,

Iowa, 115

Spartanburg,

South Carolina, 245

Spirit River,

Wisconsin, 304

Spring City,

Utah, 269

Springfield,

Missouri, 152

Squaw Creek,

Iowa, 115

stage,

definition, ix

stage-discharge curve, definition, ix

stage-discharge relation, definition, ix

example, 2

St. Albans,

Vermont, 273

Standardized Precipitation Index, 5 definition, ix

Sterling,

Colorado, 59

St. Joe River,

Idaho, 94
St. Louis,

Missouri, 152

St. Mary River, Indiana, 7, 110

St. Petersburg, Florida, 74

streamflow, definition, $\mathrm{x}$

streamgage, definition, $\mathrm{x}$

Styx River,

Alabama, 20

near Elsanor, 20

Sugar Creek, Indiana, 110

Summersville, West Virginia, 298

Sunflower,

Arizona, 29

surface water, definition, $x$

Susquehanna River, at Waverly, 196

New York, 196

$\mathbf{T}$

Tacoma, Washington, 286

Tahlequah, Oklahoma, 216

Tallapoosa River, Alabama, 17

Tampa, Florida, 74

Tanana River, Alaska, 24

Tangipahoa River, Louisiana, 128

Tar River, at Rocky Mount, 196 at Louisburg, 196 North Carolina, 196

Tchefuncte River, Louisiana, 128

Tell City, Indiana, 123

Tenmile River at Pawtucket Avenue at East Providence, 243 Rhode Island, 243

Tennessee, 7, 14, 258-260

Chattanooga, 258

Jackson, 258

Lawrenceburg, 258

Memphis, 258

Sevierville, 258

Seymour, 258

Union City, 258 
Tennessee River, Tennessee, 258

Texarkana, Arkansas, 32

Texas, 17, 123, 261-268

Austin, 261

Dallas, 9, 261

Del Rio, 261

Houston, 9

Houston Ship Channel, 261

Lake Conroe, 261

Lake Houston, 261

Lake Livingston, 261

Liberty, 261

Livingston, 261

Trinity Bay, 261

Uvalde, 261

Threemile River, at North Dighton, 136

Massachusetts, 136

Thumb Region, Michigan, 138

Tickfaw River, Louisiana, 128

Tippecanoe River, Indiana, 7, 110

Tipton, Oklahoma, 216

Toulumne River, California, 35

Tracy, California, 35

Trenton, New Jersey, 178

Trinity Bay, Texas, 261

Trinity River, at Liberty, 261 at Trinity Bay, 261

Texas, 261

Tropical Storm Alberto, 7, 20, 74, 80

Tropical Storm Beryl, 7, 74, 196, 231, 245

Tropical Storm Charlie, 17, 261

Tropical Storm Danny, 178

Tropical Storm Dean, 9, 273

Tropical Storm Doria, 178

Tropical Storm Fausto, 216

Tropical Storm Fran, 133

Tropical Storm Frances, 17, 261

Tropical Storm Georges, 20

Tropical Storm Gordon, 74

Tropical Storm Jerry, 9, 74, 80, 245

Tropical Storm Josephine, 261

Tropical Storm Oscar, 24

Truckee River, Nevada, 169
Truckee River Basin, California, 169

Tuscon, Arizona, 29

Tygart Valley River, at Philippi, 298

West Virginia, 298

U

Union City, Tennessee, 258

unit discharge, 5

Utah, 269-272

Alta, 269

Brian Head, 269

Canal Creek Canyon, 269

Glen Canyon National Recreation Area, 269

Gunlock Reservoir, 269

Ice Cream Canyon, 269

Natural Bridges National Monument, 269

Park City, 269

Spring City, 269

Washington County, 269

White Canyon, 269

Uvalde,

Texas, 261

V

Valdez,

Alaska, 24

Verde River, Arizona, 29

below Tangle Creek, 29

Verdigris River, Kansas, 120

Vermilion River, Illinois, 7, 102

Indiana, 7, 110

Vermont, 13, 273-276

Grafton, 274

Lake Champlain, 273

St. Albans, 273

Vero Beach,

Florida, 74

Versailles,

Missouri, 152

Victor,

Montana, 157

Vineland,

New Jersey, 178

Virginia, 13-14, 133, 277-285

Fredericksburg, 277

Madison, 277

Richmond, 277

Shenandoah Valley, 277

Virgin Islands, 13 
Virgin River,

Arizona, 29

\section{W}

Wabash River, Illinois, 102 Indiana, 7, 110 near Crossville, 102

Wahaiwa, Oahu, Hawaii, 91

Walker River, Nevada, 169

Wallingford, Connecticut, 70

Washington, 11, 13-14, 286-297

Carnation, 286

Duvall, 286

Lake Sammamish, 286

Seattle, 13, 286

Tacoma, 286

Utah, 269

Washington County, Utah, 269

Washington Creek, at Windigo, 138

Michigan, 138

water equivalent of snow, definition, $\mathrm{x}$

water year, definition, $x$

Wax Lake, Louisiana, 128

Weeks Bay,

Alabama, 20

Weiser River, Idaho, 94

Weldona, Colorado, 59

Weldona Valley Ditch, Colorado, 59

Wepawaug River, Connecticut, 70 in Milford, 70

Westmont, New Jersey, 178

West Virginia, 7, 9, 11, 13-14, 123, 212, 277, 298-303

Cassity, 298

Princeton, 298

Summersville, 298

Whitman, 298

Wheatland, Wyoming, 309

White Canyon, Utah, 269

White River, East Fork, 110
White River-Continued

Indiana, 7, 110

Vermont, 273

Whitewater River, Indiana, 110

Whitman,

West Virginia, 298

Wickliffe,

Kentucky, 123

Willamette River, Oregon, 220

Wills Creek, Maryland, 133

Wilmington,

Delaware, 72

Wind River, at Riverton, 309

Wyoming, 309

Wisconsin, 304-308

Browntown, 304

Janesville, 304

Lake Kegonsa, 304

Lake Monona, 304

Madison, 304

Menomonee Falls, 304

Milton, 304

Milwaukee, 304

Monroe, 304

Port Washington, 304

Sheboygan, 304

Sheboygan Falls, 304

Wiseman,

Alaska, 24

Wolf River,

Mississippi, 148

Woonasquatucket River,

at Centerdale, 243

Rhode Island, 243

Wyoming, 9, 11, 13, 309-312

Cheyenne, 309

Chugwater, 309

Encampment, 309

Kaycee, 309

Riverton, 309

Wheatland, 309

Yellowstone National Park, 309

Y

Yakima River,

Washington, 286

Yantic River,

Connecticut, 70

Yellowstone National Park, Wyoming, 309

Yellowstone River, North Dakota, 205 
York,

Pennsylvania, 231

Yosemite,

California, 35

Yosemite National Park,

California, 35

Youghiogheny River,

Maryland, 133
Yukon River, Alaska, 24

Yuma,

Arizona, 29

Z

Zig Zag River, Oregon, 220 